\title{
Detecting Incidents on Uninterrupted Flow Facilities: Bayesian-Monte Carlo Models
}

\author{
A thesis submitted to \\ the Faculty of Graduate and Postdoctoral Affairs \\ in partial fulfillment of the requirements for the degree of Doctor of \\ Philosophy in Civil Engineering
}

by

Adnan Hammoud

B.Sc. (1994) University of Benghazi, Benghazi, Libya

MASc. (2010) Carleton University, Ottawa, Ontario

Department of Civil and Environmental Engineering

Carleton University

Ottawa-Carleton Institute of Civil and Environmental Engineering

(C) 2017, Adnan Hammoud 


\section{Acknowledgments}

I am deeply grateful to almighty God who enabled me to bring this thesis to its current successful stage.

I would like to take the opportunity to convey my thanks and sincere appreciation to my supervisor, Professor Ata Khan for his inspiration, his constant encouragement and support enabled me to continue the study.

I would like to extend my thanks to members of the thesis proposal examination committee, their valuable suggestions helped me to clarify my argument and better prepare the final thesis. I would also like to thank Carleton University family and especially the Civil and Environmental Engineering Department team.

I would like to thank Mr. Rajab M. R. Legnain for his invaluable help during the construction phases of the Matlab code, and to ONE-ITS Toronto system service team, especially Mr. Mohamed Elshenawy for facilitating the accesses to HW400 series database and providing much of the incident and traffic data.

Special thanks to my family, particularly to my father for his kind prayer for me, my wife, who supported me to overcome so many difficulties during all these years with unconditional endurance and love. My children deserve more care and time than I will ever give to them. 


\section{Abstract}

Incidents on freeways and other major highways during high traffic volume conditions cause capacity bottlenecks. If incidents are detected quickly and are managed promptly, traffic delay can be reduced, and secondary accidents can be avoided. There are opportunities to capture real-time data and use these in incident detection algorithms designed specifically for alerting the traffic management centre about an incident to be the likely cause of a rapid drop in speed and formation of stationary and/or moving queues. Although over the years, a number of algorithms have been developed for this purpose, further advances are needed for reducing false alarms and improve the accuracy and speed of detecting incidents.

An original incident detection model based on Bayesian, Montecarlo, and reliability methods was formulated, calibrated and verified for uninterrupted flow facilities. The inputs to the model were obtained from Montecarlo simulation and archived Highway 401 (Greater Toronto Area) incident and detector-based traffic flow data. The detectors served as a proxy for road-side units (RSU) of a future connected infrastructure-vehicle system.

The incident detection model is built into an algorithm with the capability to identify patterns of traffic flow caused by incidents under high traffic volume conditions. The model and algorithm are designed to work with data obtainable from highway sensors/detectors and RSU. With minor changes to the code, data from probe vehicles and crowd-sources can be used as well.

The algorithm includes a number of criteria that narrow the choice of likely causes of the rapid drop in speed both spatially and temporally. The results achieved suggest high 
success rate in minimizing false alarms and improving detection rate under applicable traffic conditions.

A Matlab-based test bed was developed for testing models, and simulated incidents were used for conducting additional tests on the predictive capability of the algorithm/model. The results are in line with those obtained from archived actual incident data.

It is expected that the use of the algorithm and its constituent model will contribute to reduced delay, and improved safety (e.g. reduction of secondary collisions). 


\section{Table of Contents}

I.

II.

Acknowledgments......................................................................................

II.

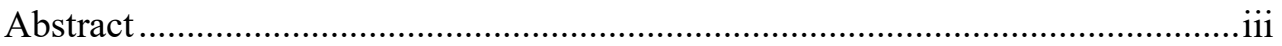

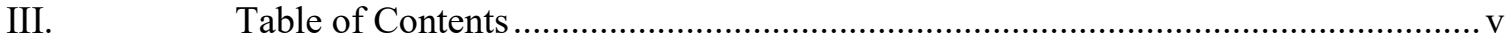

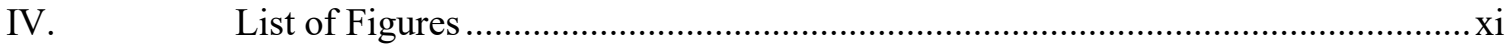

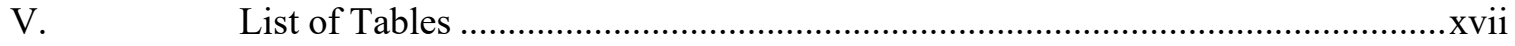

$1 \quad$ Problem Identification, Definition and Research Approach ................................. 1

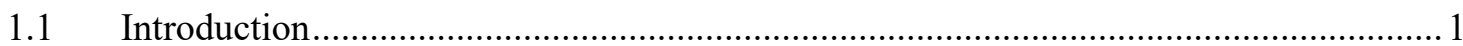

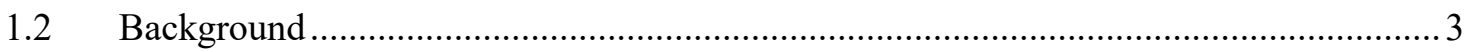

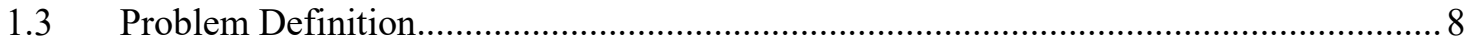

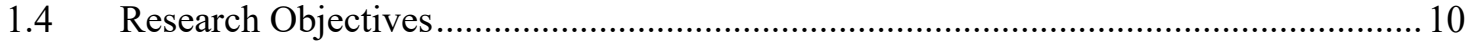

1.5 Scope

1.6 Limitations of the Research Approach and the Product............................................... 12

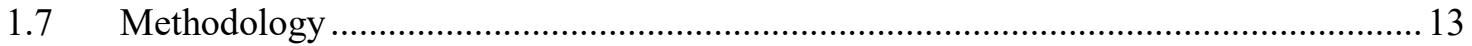

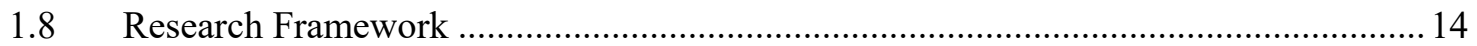

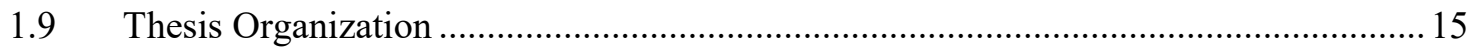

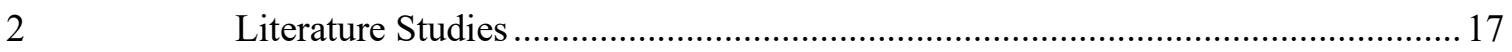

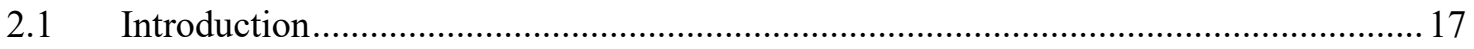

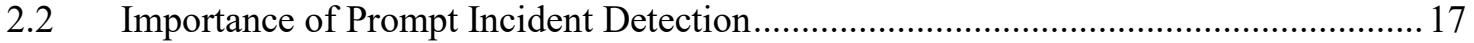

2.3 How Detecting Incidents in this Research differs from Past/ Current Practice? ........... 18

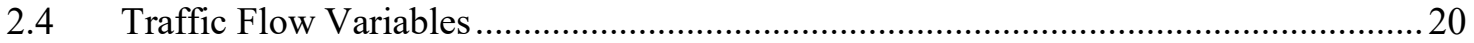

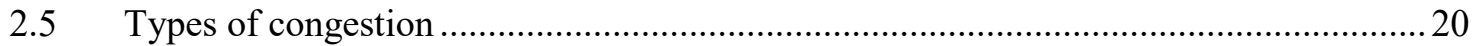

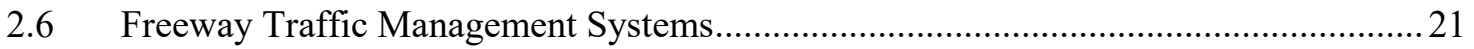

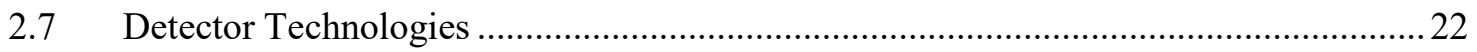

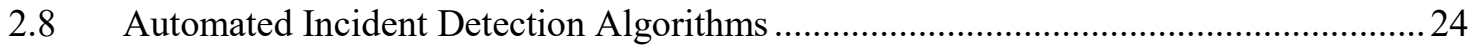

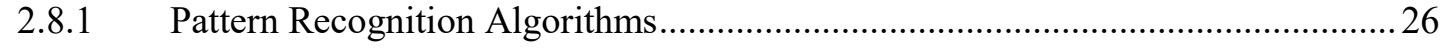

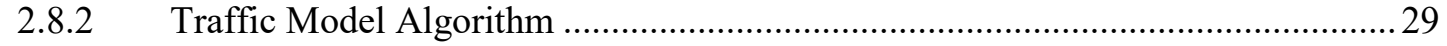

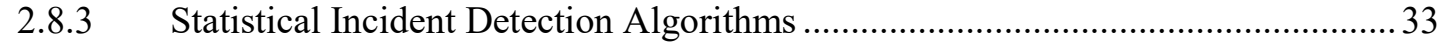

2.9 Performance Measures of Incident Detection Algorithms ...........................................39

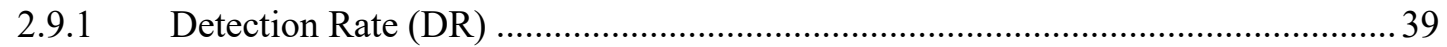

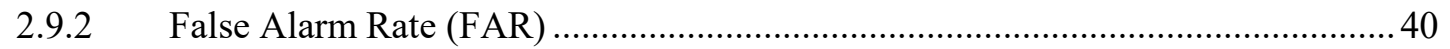

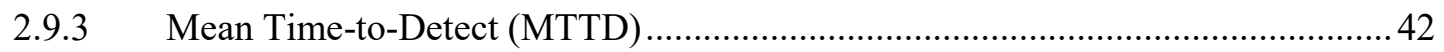




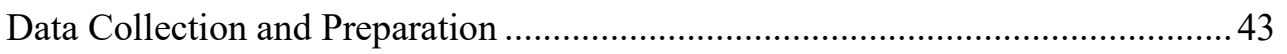

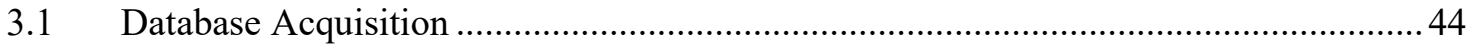

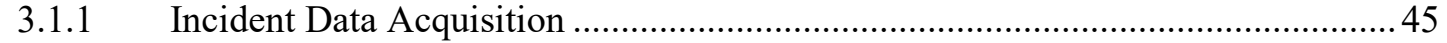

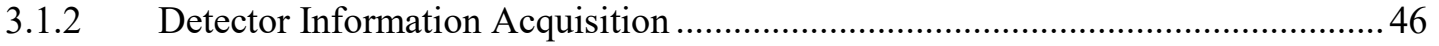

3.1.3 Choice of Factors for Use in the Incident Detection Model .................................. 49

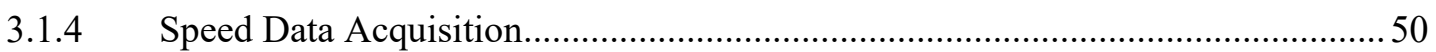

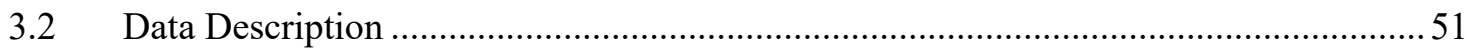

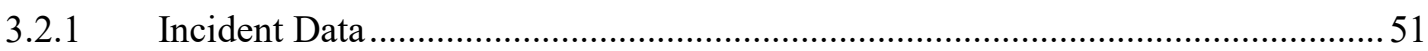

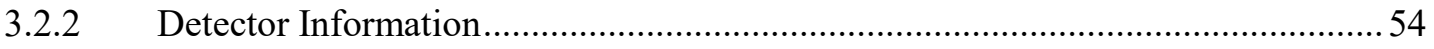

3.2.3 Traffic Flow Variables-Speed-Occupancy-Volume …..........................................56

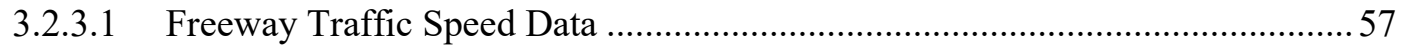

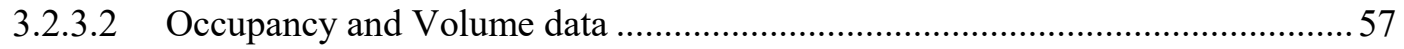

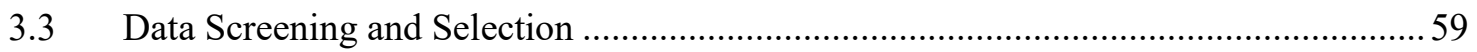

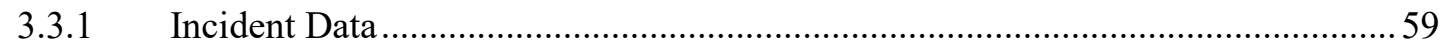

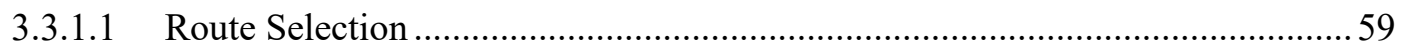

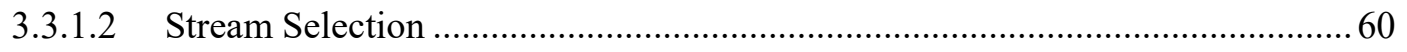

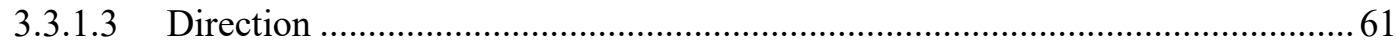

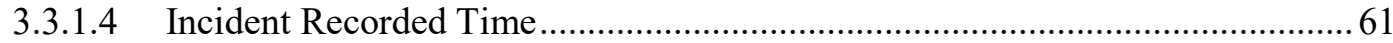

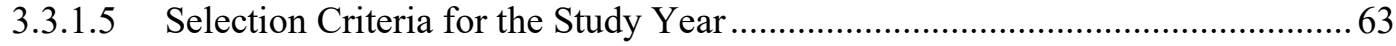

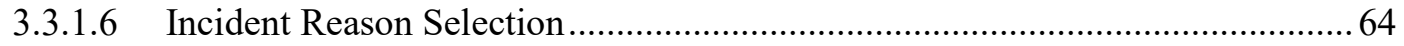

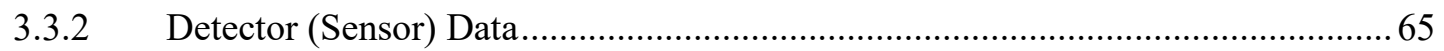

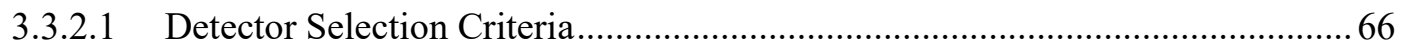

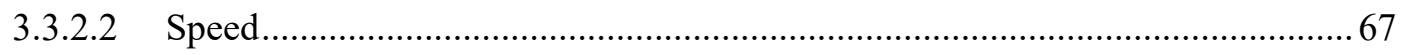

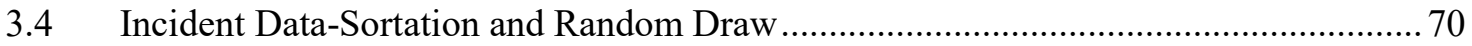

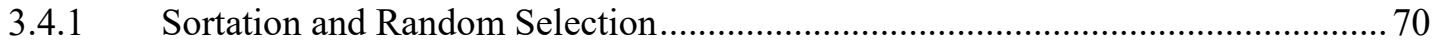

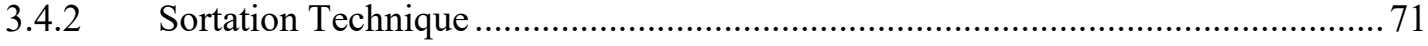

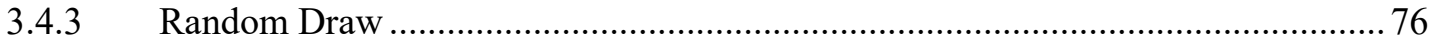

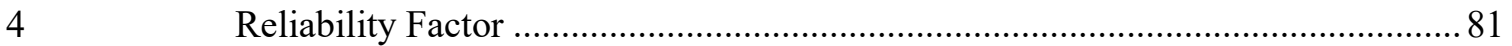

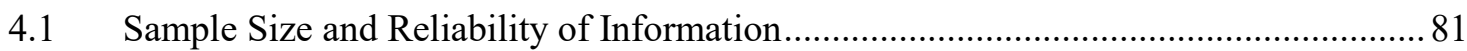

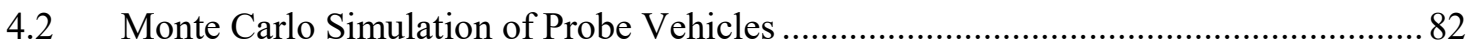

5 Development of the Incident Detection Model ................................................ 103

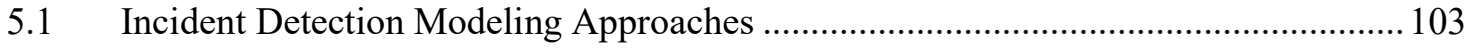

5.1.1 Incident Detection Modelling Philosophy .......................................................... 104 
5.1.2 Travel Facility Cells and Temporal Features of Incidents ................................. 105

5.1.3 Modelling Approaches/ Fundamental Aspects .................................................... 108

5.1.4 Data Fusion for Predictive Speed Modelling ...................................................... 113

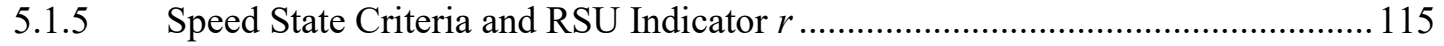

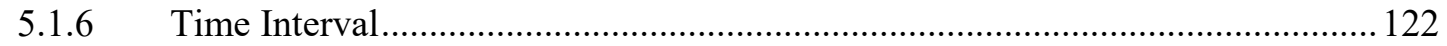

5.2 Modeling Incident Detection and Influencing Factors................................................ 122

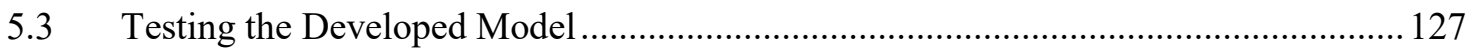

5.3.1 Bayesian Algorithm Application to Incident Data Recorded For Hwy 401 ........ 127

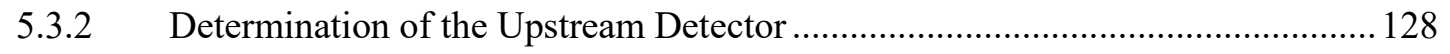

5.3.3 Proposed Criteria for Incident Detection ........................................................ 133

5.3.4 Comprehensive Model Application in Testing the Network ............................... 134

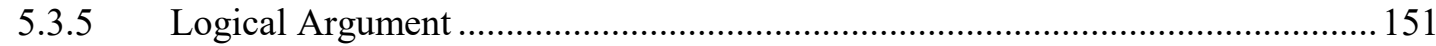

6 Incident Detection Model Development, Calibration, and Validation.................. 158

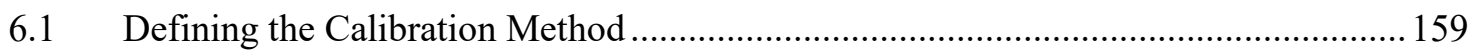

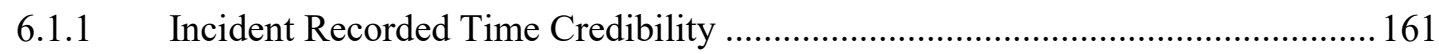

6.1.2 Effect of the Maximum Posterior Probability Level and the Drop in Speed

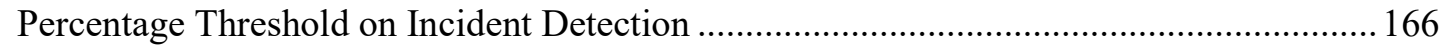

6.1.3 Effect of the Conditional Probability Level on Incident Detection ..................... 168

6.1.4 Effect of the Absolute Speed Range on Incident Detection................................. 172

6.1.5 Consideration of Special Conditions in Calibration Criteria ................................ 174

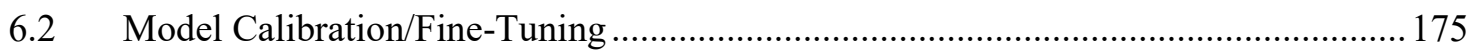

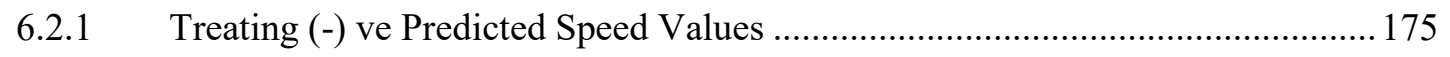

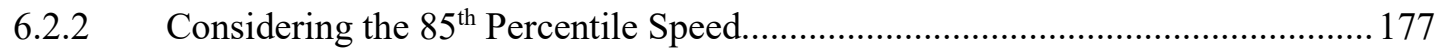

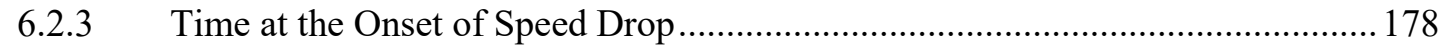

6.3 Methodological Approach for Developing the Model ............................................... 181

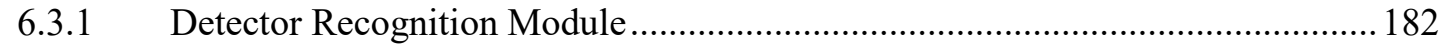

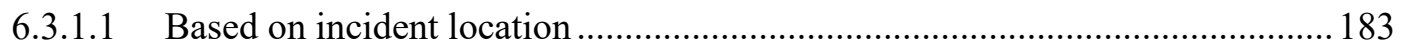

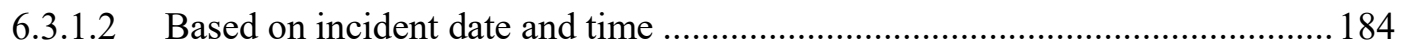

6.3.1.3 Based on distance between detector and incident ....................................... 185

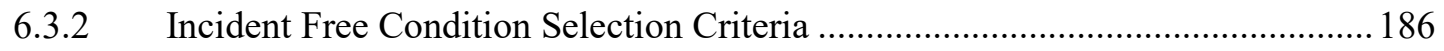

6.3.3 Incident Confirmation Criteria .......................................................................... 189

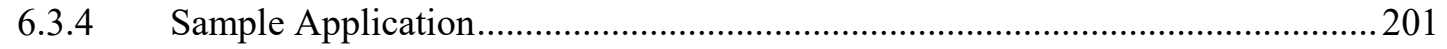

6.4 Computer Program Development (Matlab Language)...............................................2205 


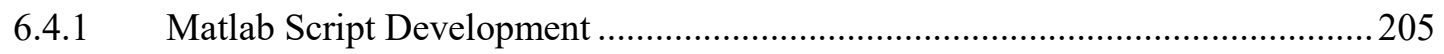

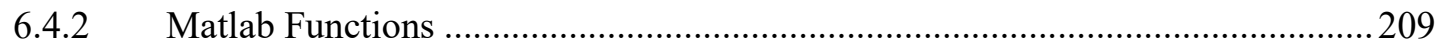

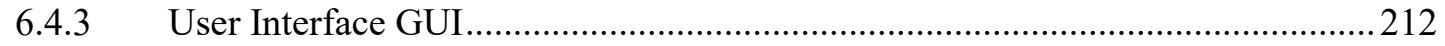

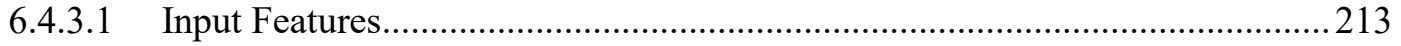

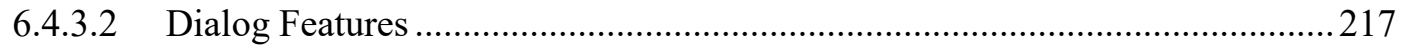

6.4.3.3 Matlab Program Features ........................................................................... 218

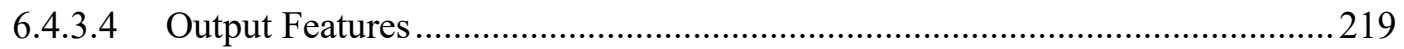

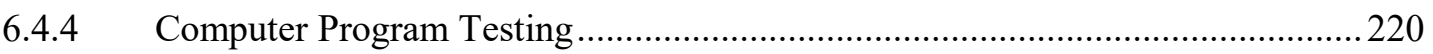

6.5 Algorithm Validation-Utilizing the Developed Matlab Program ...............................232

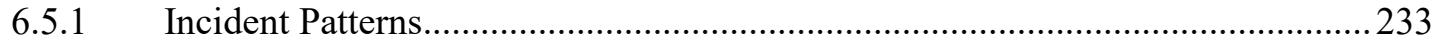

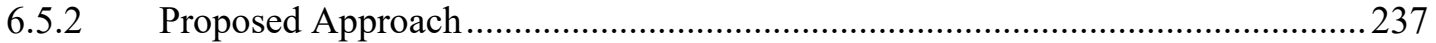

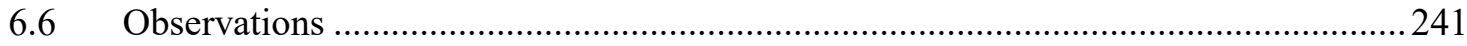

$7 \quad$ Microsimulation as an Aid to Algorithm Testing and Validation.........................242

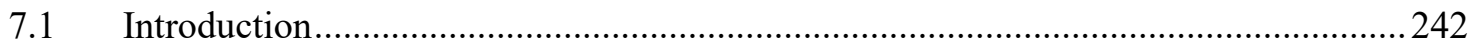

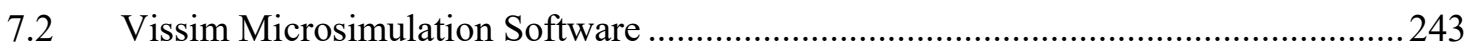

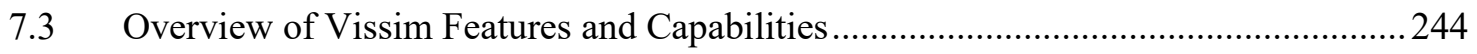

7.4 Objectives and Methodology of Traffic Incident Simulations.....................................245

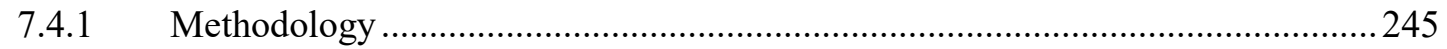

7.5 Traffic Incident Simulation Settings, or Incident Condition Identification .................246

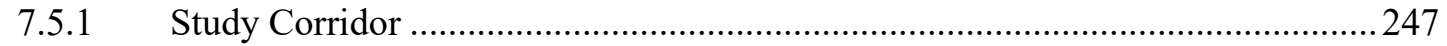

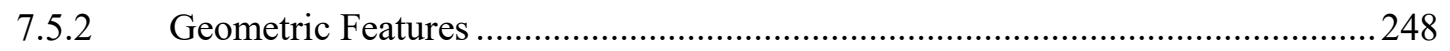

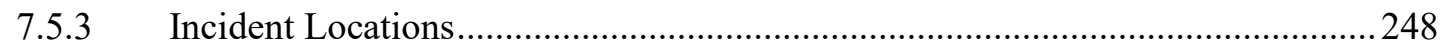

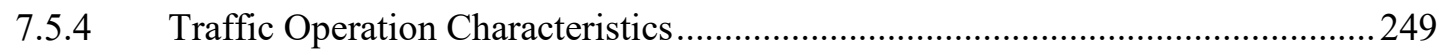

7.5.4.1 Driving Behavior Parameters and Lane Changing.........................................2.

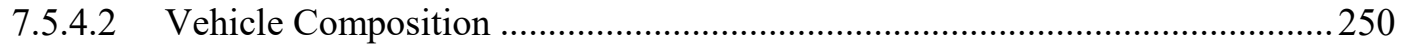

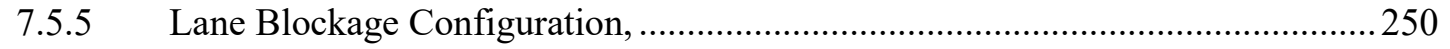

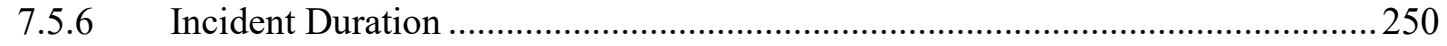

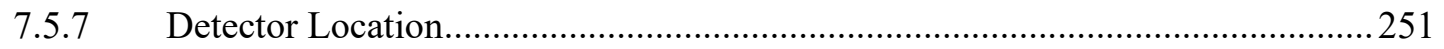

7.5.8 Incident Identification in the Vissim Simulation ..............................................2. 252

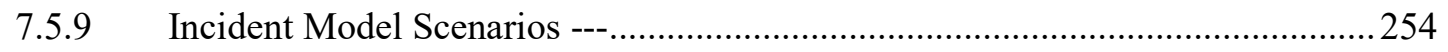

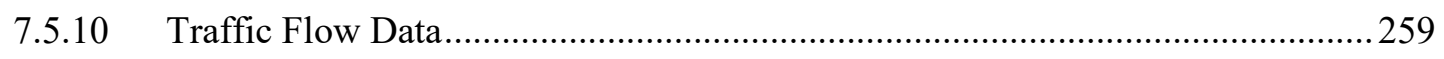

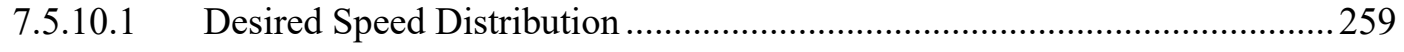

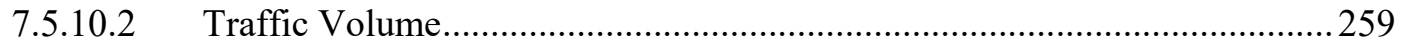




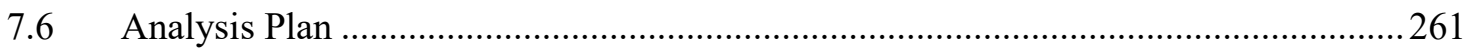

7.6.1 Location of Data Collection Point …...................................................................264

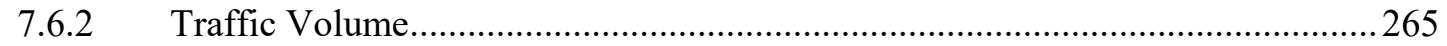

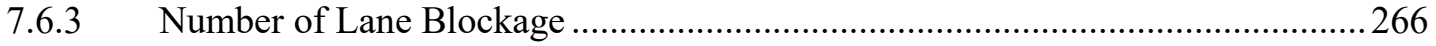

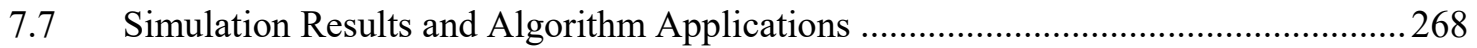

7.8 Discussion on Vissim Model and the Model Output ................................................295

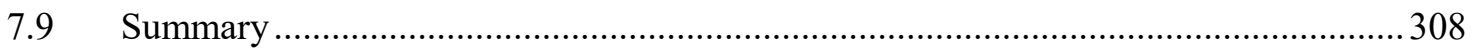

8 Summary, Conclusions, and Future Research Directions ...................................311

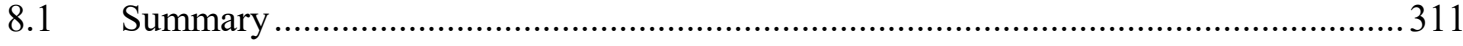

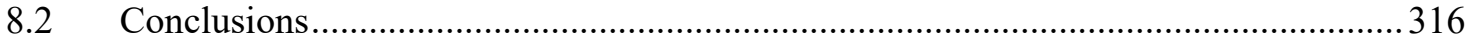

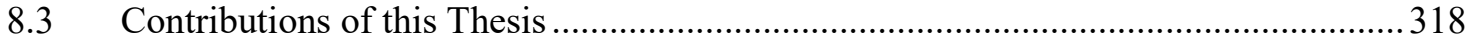

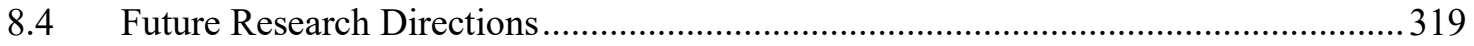

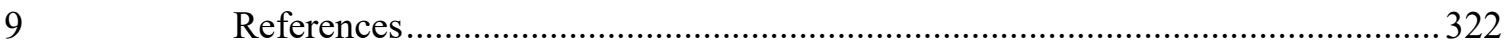

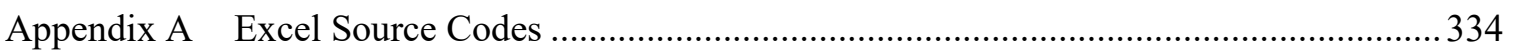

Appendix A.1 Codding Used in Excel for Incident Data Sortation..................................... 334

Appendix B Matlab Source Codes for the Developed Model ................................................. 335

Appendix B.1 Graphical User Interface (GUI) Function …................................................. 338

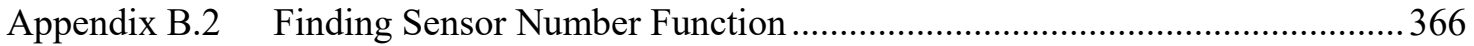

Appendix B.3 Writing Data into csv Files Function .......................................................... 368

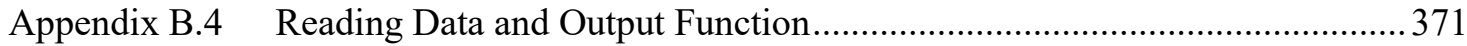

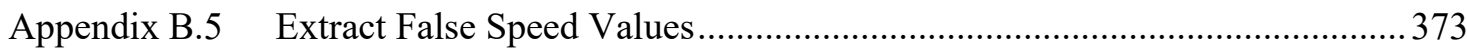

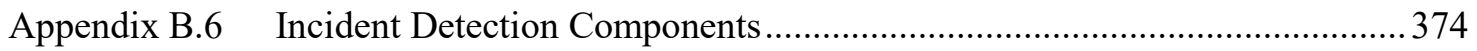

Appendix B.7 Incident Confirmation Procedure Function .............................................. 385

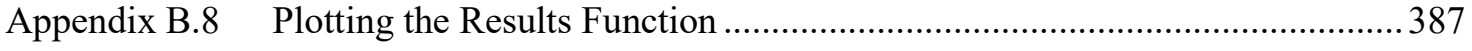

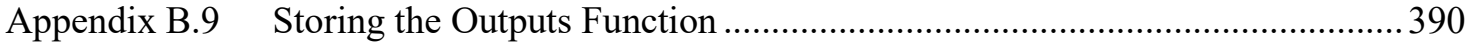

Appendix B.10 Storing the Incident Detection Results Function .........................................392

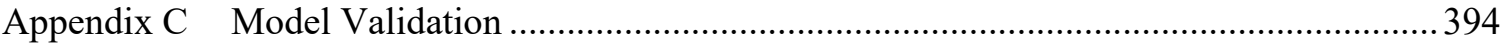

Appendix C.1 Cases with Incident Recorded and Detected ................................................ 394

Appendix C.1.1 Cases under Certain Traffic Condition "Must Detect" -- 38 cases ..........394

Appedix C.1.1.1 Cases with Incident Recorded and Detected -- 32 cases .................... 394

Appedix C.1.1.2 Cases with Incident Recorded and Not Detected -6 cases ................410

Appendix C.2 Cases with Incident Recorded and not Detected.......................................415

Appendix C.2.1 Certain Traffic Condition "Some Degree of Benefit" 30 cases.............. 415 
Appedix C.2.1.1 Cases with Incident Recorded and Detected -- 0 case ...................... 415

Appedix C.2.1.2 Cases with Incident Recorded and Not Detected -- 19 cases ............ 415

Appendix C.3 Cases with Incident Recorded and not Detected........................................ 430

Appendix C.3.1 Cases under Certain Traffic Condition "Not Important." ........................430

Appedix C.3.1.1 Cases with Incident Recorded and Detected...0................................430

Appedix C.3.1.2 Cases with Incident Recorded and Not Detected... 73 cases ............ 430

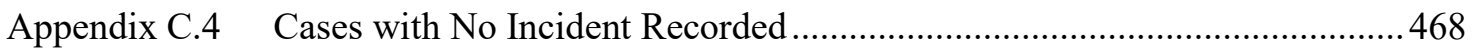

Appendix C.4.1 Cases under Certain Traffic Condition "Must Not Detect" -- 130 cases 468

Appedix C.4.1.1 Cases without Incident Recorded and Detected --- 3 cases ................468

Appedix C.4.1.2 Cases without Incident Recorded and Not Detected----127 cases..... 470

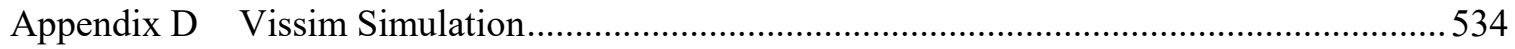

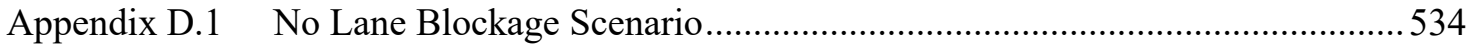

Appendix D.2 Two Lane Blockage Scenario .......................................................................... 554 


\section{List of Figures}

Figure 1.1: Incident detection principle on the freeway …...................................................... 5

Figure 1.2: Traffic Flow characteristics during the incident on the freeway ….............................5

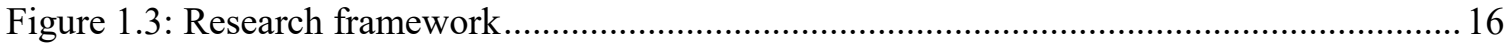

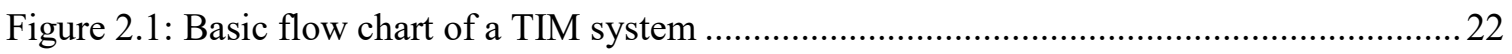

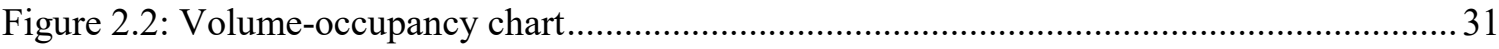

Figure 3.1: Browsing detector information by list from ONE-ITS platform ............................... 47

Figure 3.2: Variables used to set the report parameters (month and year).................................... 47

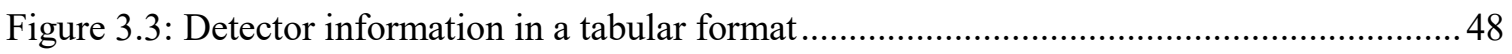

Figure 3.4: Sample section of detectors locations at Hwy 401 ..................................................48

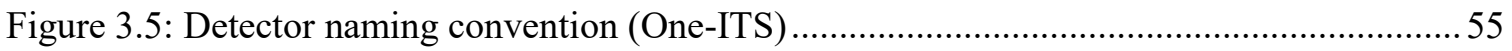

Figure 4.1: P (at least 1 equipped vehicle during one minute) at $500 \mathrm{veh} / \mathrm{h} / \mathrm{lane}$ )...................... 87

Figure 4.2: Cumulative probability, at least 1 equipped vehicle/min at $500 \mathrm{veh} / \mathrm{h} / \mathrm{lane} \ldots \ldots \ldots \ldots \ldots . . . .87$

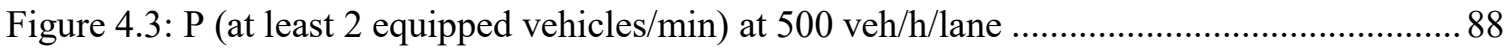

Figure 4.4: Cumulative probability, at least 2 equipped vehicle/min at $500 \mathrm{veh} / \mathrm{h} / \mathrm{lane}$...............8 88

Figure 4.5: P (at least 3 equipped vehicles/min) at $500 \mathrm{veh} / \mathrm{h} / \mathrm{lane}$.......................................... 89

Figure 4.6: Cumulative probability, at least 3 equipped vehicle/min at $500 \mathrm{veh} / \mathrm{h} / \mathrm{lane} \ldots \ldots \ldots \ldots . . . .89$

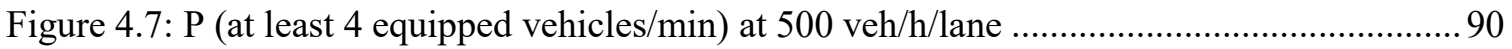

Figure 4.8: Cumulative probability at least 4 equipped vehicle/min at $500 \mathrm{veh} / \mathrm{h} / \mathrm{lane} \ldots \ldots \ldots \ldots \ldots . . . . .90$

Figure 4.9: P (at least 5 equipped vehicles/min) at $500 \mathrm{veh} / \mathrm{h} /$ lane .........................................91

Figure 4.10: Cumulative probability, at least 5 equipped vehicle/min at $500 \mathrm{veh} / \mathrm{h} / \mathrm{lane} \ldots \ldots \ldots \ldots . . .91$

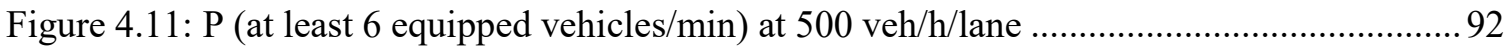

Figure 4.12: Cumulative probability, at least 6 equipped vehicle/min at $500 \mathrm{veh} / \mathrm{h} / \mathrm{lane} \ldots \ldots \ldots \ldots . . . .92$

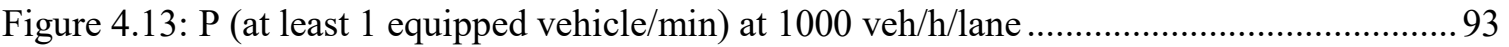

Figure 4.14: Cumulative probability, at least 1 equipped vehicle/min at $1000 \mathrm{veh} / \mathrm{h} / \mathrm{lane} \ldots \ldots \ldots . . .93$

Figure 4.15: P (at least 6 equipped vehicles/min) at $1000 \mathrm{veh} / \mathrm{h} / \mathrm{lane}$....................................... 94

Figure 4.16: Cumulative probability, at least 6 equipped vehicles/min at $1000 \mathrm{veh} / \mathrm{h} /$ lane ..........94

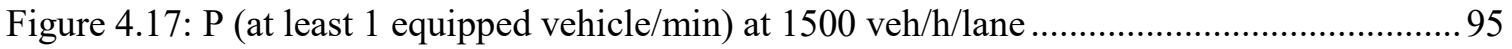

Figure 4.18: Cumulative probability, at least 1 equipped vehicle/min at $1500 \mathrm{veh} / \mathrm{h} / \mathrm{lane} \ldots \ldots \ldots . . . .95$

Figure 4.19: P (at least 6 equipped vehicles/min) at $1500 \mathrm{veh} / \mathrm{h} /$ lane .......................................96 
Figure 4.20: Cumulative probability, at least 6 equipped vehicle/min at $1500 \mathrm{veh} / \mathrm{h} / \mathrm{lane} . \ldots \ldots \ldots . . .97$

Figure 4.21: Cumulative probability, at least 6 equipped vehicle/min at $500 \mathrm{veh} / \mathrm{h} / \mathrm{lane}$............. 99

Figure 4.22: Mean probability, at least 6 equipped vehicle/min at $500 \mathrm{veh} / \mathrm{h} / \mathrm{lane}$..................... 99

Figure 4.23: Cumulative probability, at least 6 equipped vehicle/min at $1000 \mathrm{veh} / \mathrm{h} / \mathrm{lane} \ldots \ldots \ldots . . .100$

Figure 4.24. Mean probability, at least 6 equipped vehicles/min at $1000 \mathrm{veh} / \mathrm{h} / \mathrm{lane} . \ldots \ldots \ldots \ldots \ldots . . . . .100$

Figure 4.25: Cumulative probability, at least 6 equipped vehicle/min .................................... 101

Figure 4.26. Mean probability, at least 6 equipped vehicle/min at $1500 \mathrm{veh} / \mathrm{h} / \mathrm{lane}$.................. 101

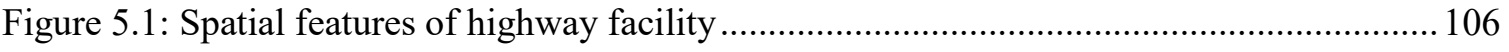

Figure 5.2: Bayesian predictive speed methodology .............................................................. 113

Figure 5.3: The speed states and the corresponding speed ranges .......................................... 117

Figure 5.4: Defining speed at state $\mathrm{s}_{2}$ and the corresponding speed ranges .............................. 118

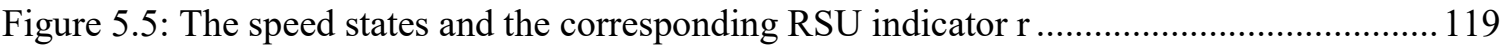

Figure 5.6: Preliminary attempt for computing expected speed ................................................ 124

Figure 5.7: Developed algorithm for incident detection ....................................................... 125

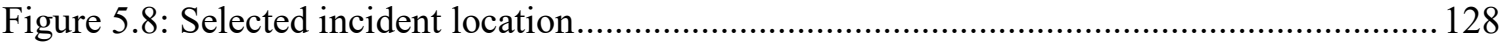

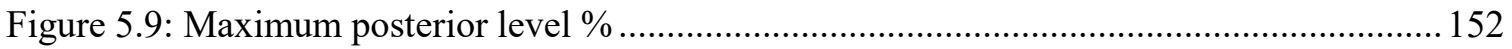

Figure 5.10: Sensor speed data vs. predicted speed data ........................................................ 153

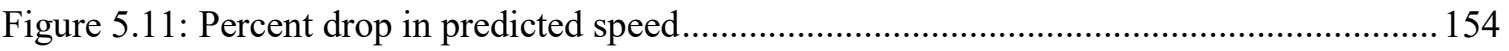

Figure 5.12: Incident detection - developed version of the model .......................................... 155

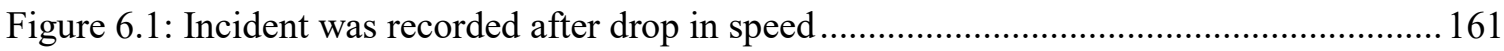

Figure 6.2: Gap between incident recorded time and drop in speed point................................. 162

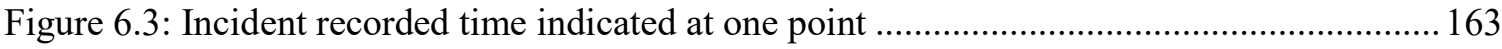

Figure 6.4: Incident recorded time indicated between two points ............................................. 164

Figure 6.5: Free incident condition recorded time indicated at one point................................... 165

Figure 6.6: Free incident condition recorded time indicated between two points ...................... 165

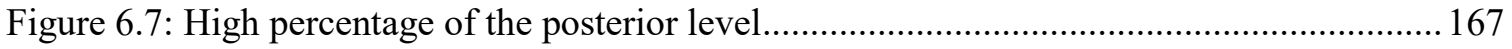

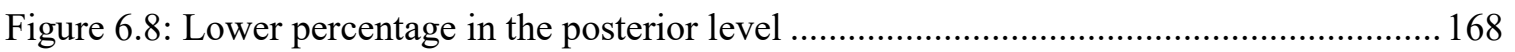

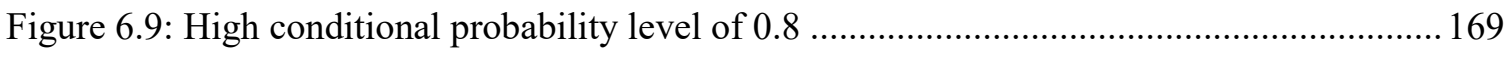

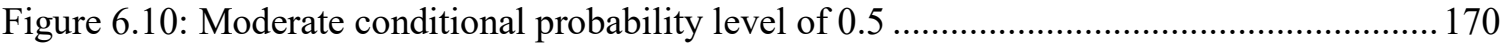

Figure 6.11: High posterior and drop in speed threshold....................................................... 171

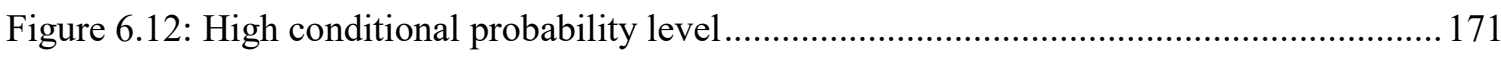




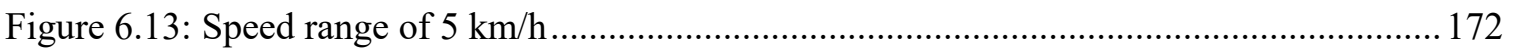

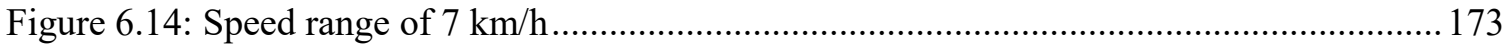

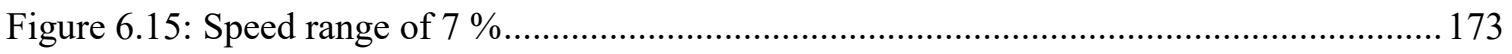

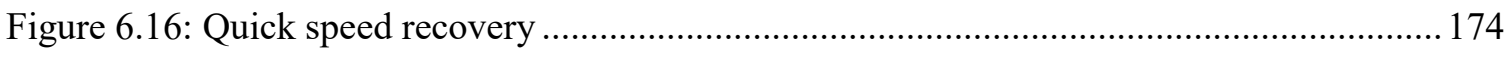

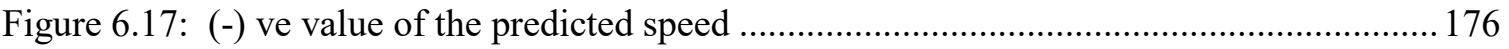

Figure 6.18: (0) speed value replacing any (-) ve predicted speed value................................... 177

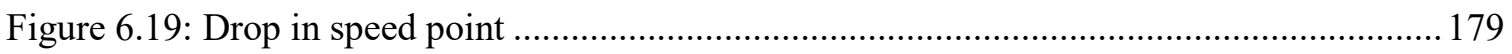

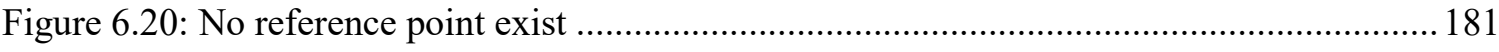

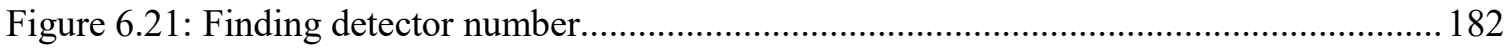

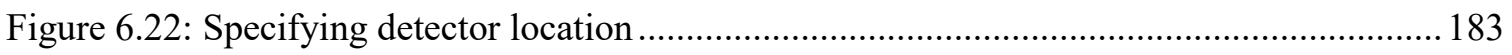

Figure 6.23: Road section from Hwy 401 based on all available incidents data ........................ 184

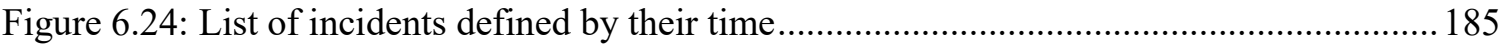

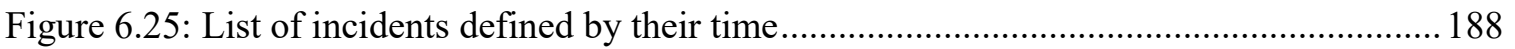

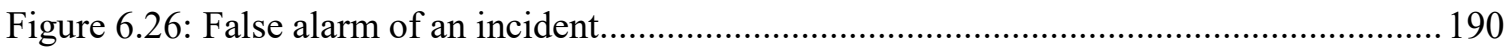

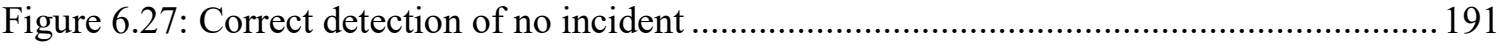

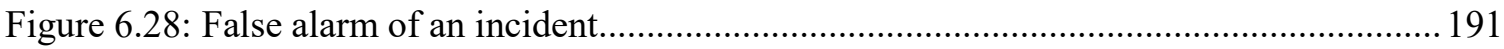

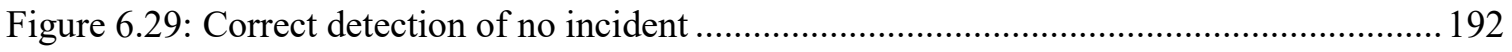

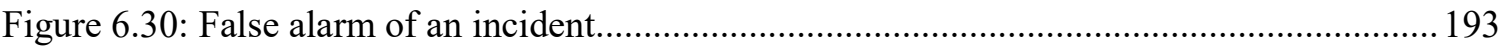

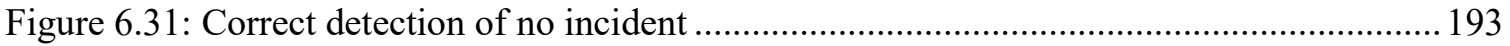

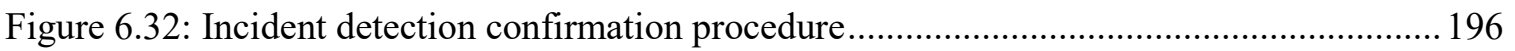

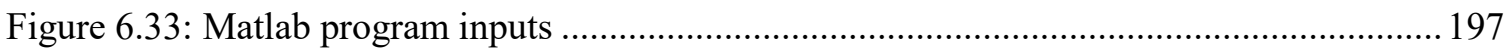

Figure 6.34: False alarm of an incident at the end of the time period........................................ 199

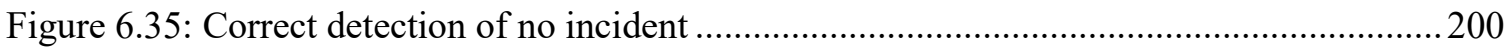

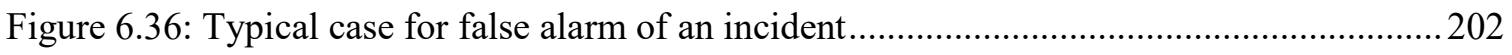

Figure 6.37: Typical case for correct incident detection .......................................................... 202

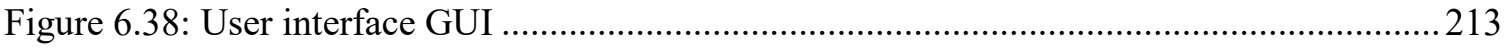

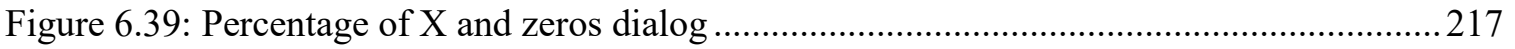

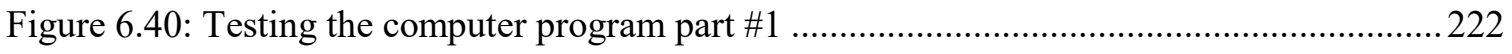

Figure 6.41: Testing the computer program part \#2 …...........................................................223

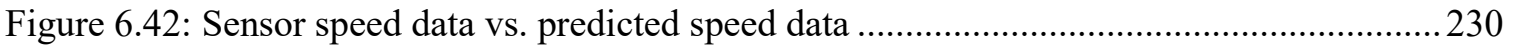

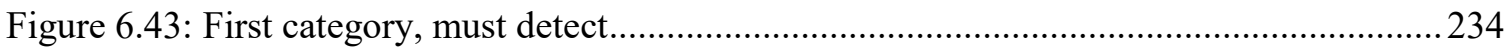




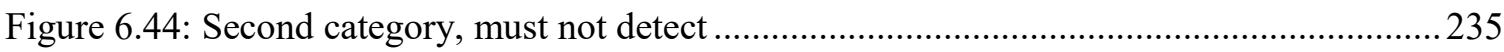

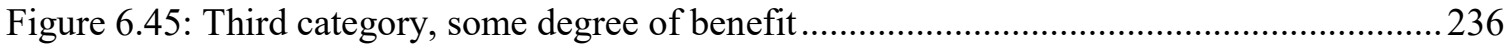

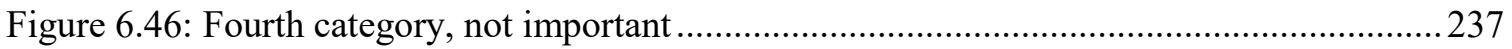

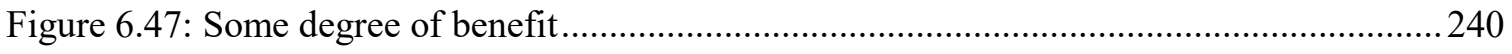

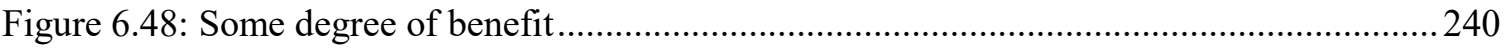

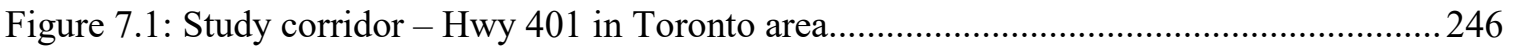

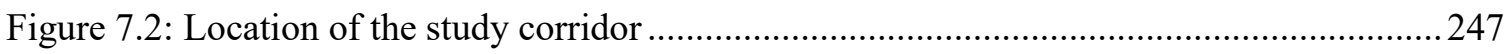

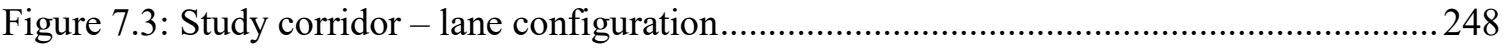

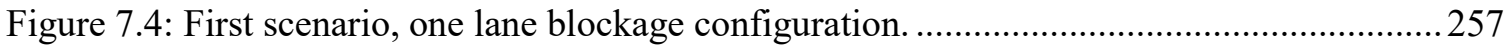

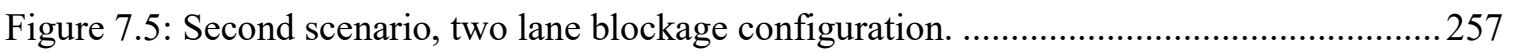

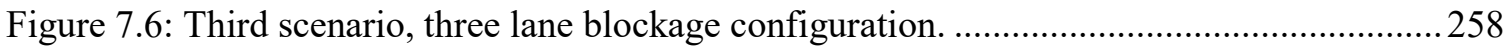

Figure 7.7: Fourth scenario, four lane blockage configuration..............................................258

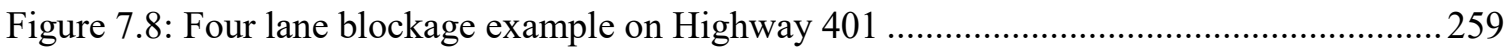

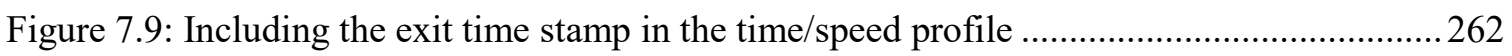

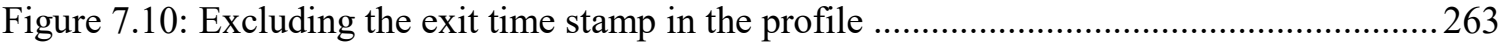

Figure 7.11: Speed values according to each time interval regardless of the location of the data

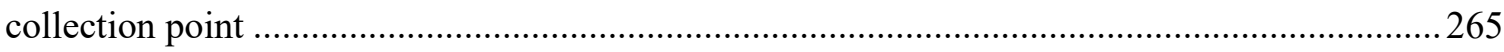

Figure 7.12: Speed data is collected from lane \#1, v=2000 veh/h/lane ....................................269

Figure 7.13: Speed data is collected from lane \#1, Run \#1, v=2000 veh/h/lane........................270

Figure 7.14: Speed data is collected from lane \#1, Run \#1, v=1000 veh/h/lane.........................271

Figure 7.15: Traffic conformation from two lane blockage, $\mathrm{v}=2000 \mathrm{veh} / \mathrm{h} /$ lane ....................... 273

Figure 7.16: Speed data is collected from lane \#1, v=2000 veh/h/lane ...................................2273

Figure 7.17: Speed data is collected from lane \#1, Run \#1, v=2000 veh/h/lane.........................274

Figure 7.18: Speed data is collected from lane \#2, $\mathrm{v}=2000 \mathrm{veh} / \mathrm{h} /$ lane .....................................275

Figure 7.19: Speed data is collected from lane \#2, Run \#1, v=2000 veh/h/lane ........................276

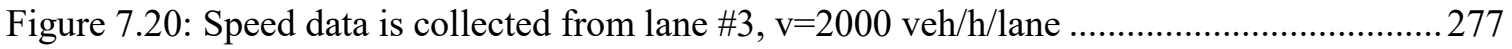

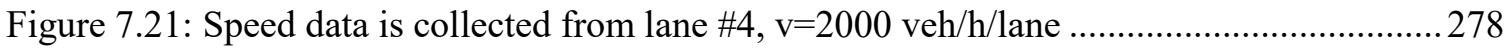

Figure 7.22: Traffic conformation from two lane blockage, $\mathrm{v}=1000 \mathrm{veh} / \mathrm{h} /$ lane .......................279

Figure 7.23: Speed data is collected from lane \#1, Run \#1, v=1000 veh/h/lane........................280

Figure 7.24: Speed data is collected from lane \#2, Run \#1, v=1000 veh/h/lane.......................281

Figure 7.25: Traffic conformation from two lane blockage, $\mathrm{v}=1500 \mathrm{veh} / \mathrm{h} / \mathrm{lane}, 1^{\text {st }}$ seconds of simulation 282 
Figure 7.26: Traffic conformation from two lane blockage, v=1500 veh/h/lane, few min later.. 282

Figure 7.27: Speed data is collected from the lane \#1, Run \#1, v=1500 veh/h/lane ...................283

Figure 7.28: Speed data is collected from lane \#2, Run \#1, v=1500 veh/h/lane..........................284

Figure 7.29: Traffic conformation from two lane blockage, $\mathrm{v}=1250 \mathrm{veh} / \mathrm{h} / \mathrm{lane}, 1^{\text {st }}$ moments.... 285

Figure 7.30: Traffic conformation from two lane blockage, v=1250 veh/h/lane, few min later.. 286

Figure 7.31: Speed data is collected from lane \#1, Run \#1, v=1250 veh/h/lane.......................287

Figure 7.32: Speed data is collected from lane \#1, Run \#2, v=1250 veh/h/lane........................287

Figure 7.33: Speed data is collected from lane \#2, Run \#1, v=1250 veh/h/lane........................288

Figure 7.34: Speed data is collected from lane \#2, Run \#2, v=1250 veh/h/lane........................289

Figure 7.35: Traffic conformation from three lane blockage, $\mathrm{v}=2000 \mathrm{veh} / \mathrm{h} / \mathrm{lane} . . . \ldots \ldots \ldots \ldots \ldots \ldots . . . . . . . .290$

Figure 7.36: Speed data is collected from lane \#1, v=2000 veh/h/lane ....................................290

Figure 7.37: Speed data is collected from lane $\# 2, \mathrm{v}=2000 \mathrm{veh} / \mathrm{h} /$ lane ....................................291

Figure 7.38: Speed data is collected from lane \#3, v=2000 veh/h/lane ...................................291

Figure 7.39: Speed data is collected from lane \#4, $=2000 \mathrm{veh} / \mathrm{h} /$ lane ......................................292

Figure 7.40: Traffic conformation from four lane blockage, $\mathrm{v}=2000 \mathrm{veh} / \mathrm{h} / \mathrm{lane}$......................293

Figure 7.41: Speed data is collected from lane \#1, v=2000 veh/h/lane ....................................293

Figure 7.42: Speed data is collected from lane \#2, $\mathrm{v}=2000 \mathrm{veh} / \mathrm{h} /$ lane ......................................294

Figure 7.43: Speed data is collected from lane \#3, $=2000 \mathrm{veh} / \mathrm{h} /$ lane ......................................294

Figure 7.44: Speed data is collected from lane \#4, v=2000 veh/h/lane ...................................295

Figure 7.45: Speed data is collected from lane \#1, v=2000 veh/h/lane ...................................297

Figure 7.46: Speed data is collected from lane \#2, $\mathrm{v}=2000 \mathrm{veh} / \mathrm{h} /$ lane .....................................297

Figure 7.47: Speed data is collected from lane \#3, v=2000 veh/h/lane ...................................298

Figure 7.48: Speed data is collected from lane \#4, $=2000 \mathrm{veh} / \mathrm{h} /$ lane .....................................299

Figure 7.49: Speed data is collected from lane \#1, v=2000 veh/h/lane .................................... 300

Figure 7.50: Speed data is collected from lane \#2, v=2000 veh/h/lane ...................................301

Figure 7.51: Speed data is collected from lane \#3, v=2000 veh/h/lane .................................... 302

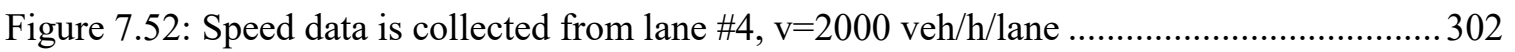

Figure 7.53: Speed data is collected from lane \#1, Run \#1, v=2000 veh/h/lane......................... 303

Figure 7.54: Speed data is collected from lane \#2, Run \#1, v=2000 veh/h/lane.......................... 304

Figure 7.55: Speed data is collected from lane \#1, Run \#1, v=1500 veh/h/lane........................ 305

Figure 7.56: Speed data is collected from lane \#1, Run \#1, v=1250 veh/h/lane........................ 305 
Figure 7.57: Speed data is collected from lane \#1, Run \#2, v=1250 veh/h/lane......................... 306

Figure 7.58: Speed data is collected from lane \#1, Run \#1, v=1000 veh/h/lane......................... 306

Figure 7.59: Speed data is collected from lane \#1, Run \#1, v=1250 veh/h/lane....... 


\section{List of Tables}

Table 1.1: Proportion of freeway segment capacity available under incident condition ................6

Table 2.1: Logic used to determine the cause of congestion ...................................................... 32

Table 3.1: Number of active detectors in each month for all available years ............................... 49

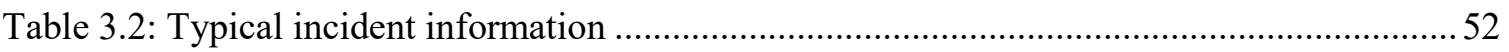

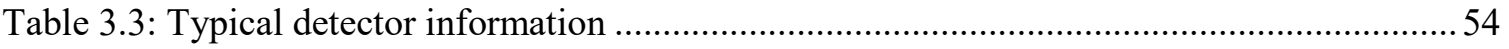

Table 3.4: Qualitative statistical analysis for selecting study year ............................................. 64

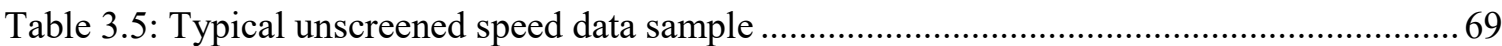

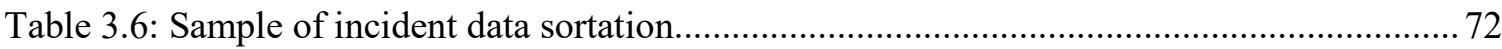

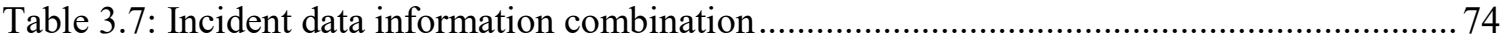

Table 3.8: Incident selection based on lane blockage and incident time ......................................76

Table 3.9: Random number application for selection of incidents ................................................. 77

Table 3.10: Sample of 22 incidents from the randomly drawn group ........................................ 78

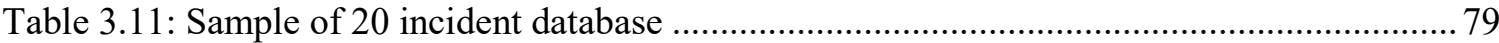

Table 3.12: Sample incident data from group one and two ........................................................... 79

Table 4.1: Results of joint probability and Monte Carlo simulation, 2 lanes/direction ................. 97

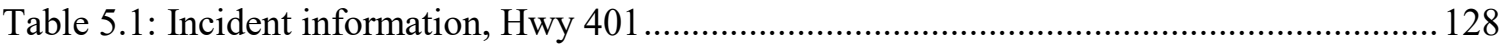

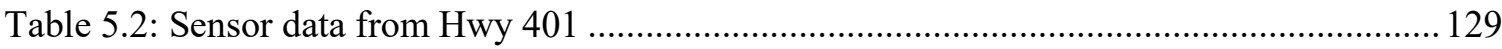

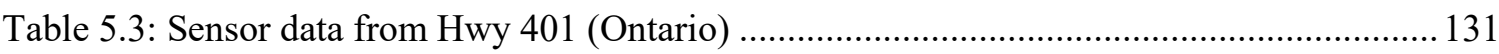

Table 5.4: Screened speed data including the 85th percentile. ................................................... 132

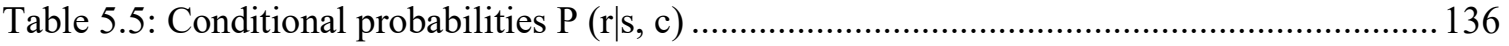

Table 5.6: Predicted speed, adoption of posterior probability \& indication of incident .............. 148

Table 6.1: Hypothetical sensor data for testing the Matlab program (1) ....................................224

Table 6.2: Hypothetical sensor data for testing the Matlab program (2) ...................................225

Table 6.3: Matlab program outputs vs automated excel sheets outputs......................................226

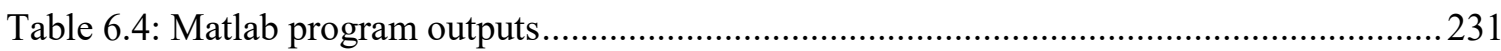

Table 7.1: Commercial vehicle flows by road network ...........................................................260

Table 7.2: Lane count: Number of through lanes on Highway 401 (excludes ongoing or planned

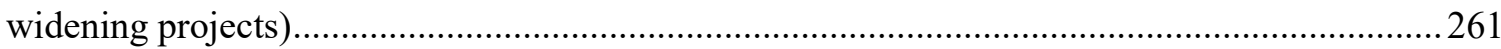




\section{Problem Identification, Definition and Research Approach}

\subsection{Introduction}

Urban freeways are becoming highly congested due to the increasing number of vehicles (including trucks). Due to environmental reasons and constrained resources of land and money to add capacity, Traffic Management Centres (TMCs) are employing a combination of sensing systems and methods to quickly detect and clear traffic incidents in order to relieve congestion (ITS Architecture 2002). As a part of public policy, transportation agencies in developed, as well as developing countries, are eager to find and use measures to improve driving conditions that do not call for expanding capacity.

Non-recurrent congestion that develops on highways due to traffic incidents represents a major portion of congestion on freeways and costs billions of dollars. Traffic incidents, such as accidents and disabled vehicles, cause disruptions to traffic flow. They can induce substantial traffic congestion on freeways, increase fuel consumption, and cause secondary incidents (Lindley 1987). According to the Highway Capacity Manual, (HCM 2010), capacity reduction due to traffic accidents or other incidents lasts from less than 1 hour to as long as 12 hours (in cases of severe accidents, hazardous materials, etc.). According to one study referenced in the HCM (2010), the mean duration of incidence is 37 minutes; more than half last for 30 minutes or less and $82 \%$ of incidents have less than 1 hour duration. Timely and reliable detection and quick clearance of traffic incidents have proven to be crucial and the most important phase to restoring the capacity of freeways.

One of the objectives of incident detection is to provide real-time information to travelers about the occurrence of incidents, their location, and their impacts on travel time. This information is valuable even to travelers familiar with travel times in a route under 
"normal" conditions. Knowledge about incidents enhances the accuracy of predicted link travel times for route-planning purposes and also explains the reason for an unusual increase in travel time. The development of advanced traveler information systems (ATIS) and dynamic route guidance systems is rapidly gaining momentum, and it is envisioned that incident detection will be an essential component of such systems.

A number of methods exist that can be employed to gain knowledge of an incident that has occurred. These are noted below with brief comments on their strengths and weaknesses:

- Detection by video surveillance: highly reliable, but extent of coverage is limited due to cost reasons.

- Detection by receiving information by equipped vehicles that are connected with infrastructure: limited scope for many years into the future due to a very small proportion of vehicles that are expected to subscribe to specialized services for this purpose and also an incident may damage the communication capability of a vehicle.

- Twitters about incidents: coverage and regulatory limitations.

- Detection by inferences drawn from changes in traffic flow: this category of means to detect incidents includes algorithms, probe data, and crowd sourced data. These are viable approaches, but their statistical reliability could be compromised due to insufficient data.

In this research, incident detection algorithms are of interest. Although, algorithms exist and some used by management centres, there is a need to develop new ones with potential to show better performance under applicable conditions. 


\subsection{Background}

\section{Incident Identification}

Several different descriptions of incidents exist in the literature; they depend on the perspective of who interprets them (Stephanedes and Liu 1995; Abdulhai and Ritchie 1995).

From traffic safety viewpoint, an incident is any non-recurring event that could pose a hazard to motorists. Events such as disabled vehicles, spilled loads, collisions, and temporary maintenance and construction activities can be included in this category. Traffic operation personnel have similar views, but they focus on events causing unexpected disruption to traffic flow (Abdulhai and Ritchie 1995 and 1997).

For instance, construction and maintenance activities on the freeway are planned ahead of time and therefore are not unexpected events. These events were excluded in the definition of an incident. Moreover, minor incidents on freeways that do not cause congestion and traffic delays have no effect on the traffic and are excluded from the definition as well. If a broader definition is sought, an 'incident' can be defined as any 'event' taking place in a facility (e.g., a freeway) that causes a reduction in facility capacity.

Automated incident detection (AID) is primarily a traffic management tool. In this context, the incident that is to be detected could be "any unexpected non-recurring event that temporarily disrupts the flow of traffic on a segment of roadway, producing congestion shockwaves, queues, and traffic delays". Typical examples of common freeway and other highway incidents include road collision, stalled vehicle, and spilled debris on the road (Williams \& Guin, 2007). 
Speed and travel time are two factors that are adversely affected by incidents. These provide the basis for many researchers to develop automated incident detection and alarm system for highways. There are many uses of the resulting information in addition to managing the incident. The information is used to alert other drivers regarding speed reduction and to reduce the potential of secondary incidents.

It is useful to note the rational for automated detection of incidents. Despite increasing traffic volume on freeways, for a number of reasons, transportation agencies are not keen on adding capacity and instead are resorting to improved traffic management, including early detection of incidents and their efficient management (ITS Architecture 2002).

\section{Traffic Flow Characteristics during Incident}

In the event of an incident on a freeway, traffic flow features could be classified into three characteristics, as presented in Figure 1.1 and Figure 1.2. Segment A is bounded by the end of the generated shock wave propagation due to the incident and extends beyond that. The traffic flow at this segment is steady with normal speed and density. The reduction in the traffic flow is expected to be observed at both downstream and upstream locations. Segment B is where the shock wave is propagated, and it starts from the point of where the incident took place and extends to the end of the propagation limit of the shock wave. This segment experienced intense traffic and high drop in the speed. Downstream of the incident is characterized by increase in speed and low traffic volume as compared to the normal condition. This segment is shown as $\mathrm{C}$ in Figure 1.2. The flow and speed have recovered in segment $\mathrm{D}$, which is adequately far downstream from the incident (Škorput et al. 2010). 


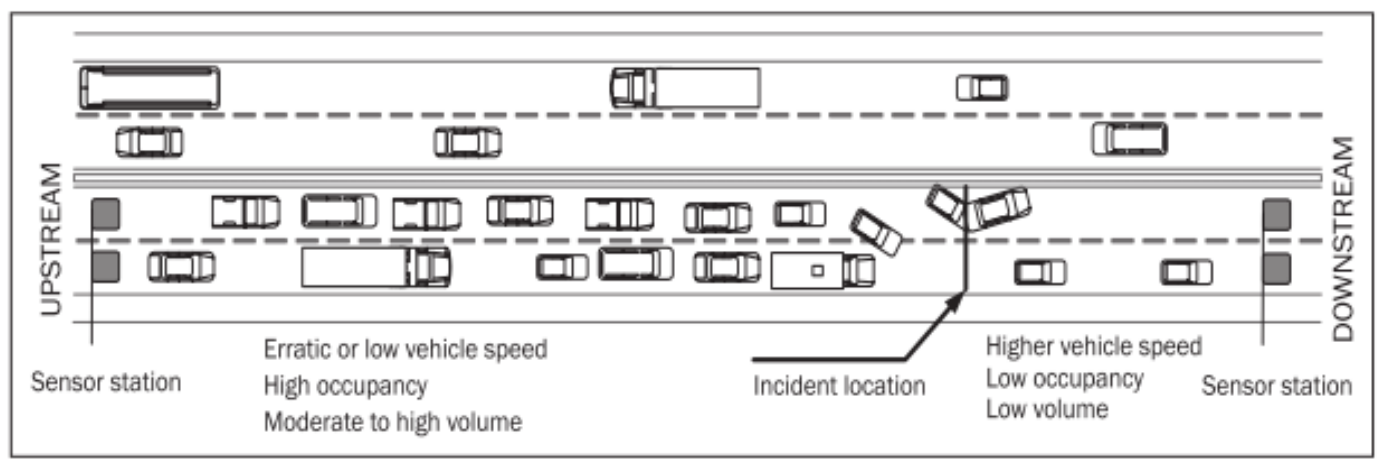

Source: (Škorput et al. 2010)

Figure 1.1: Incident detection principle on the freeway

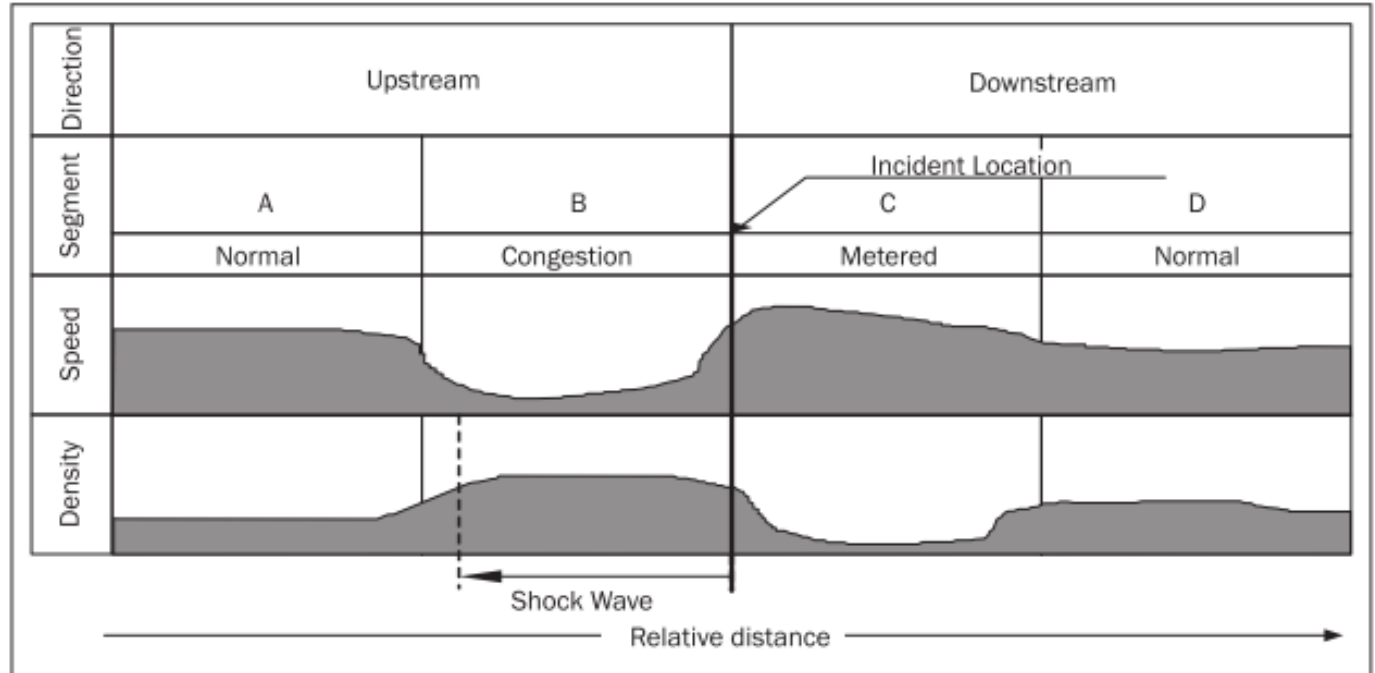

Source: (JHK et al. 1993)

Figure 1.2: Traffic Flow characteristics during the incident on the freeway

\section{Additional Clarification of Types of Incidents}

As noted previously, incidents include vehicle collisions, stalled vehicles on the traveled portion of the freeway, overturned vehicles, and road debris. However, "Stalled vehicles in the shoulder are only counted as incidents if it is perceived, via a review of the video recording or the traffic data, that they are causing a reduction in freeway capacity" 
[MTO-U of T Project]. One can argue that there is a distraction effect of an incident on the traffic of the other side of the freeway. Likewise the case of stalled vehicles in the shoulder, incidents of the traffic of the other side of the freeway are only counted as incidents if they cause a reduction in the capacity of the freeway side under consideration, (e.g. overturned vehicle from one side of the traffic to the other.)

\section{Implications of Incidents}

When incidents take place, additional constraints are generated on the roadway capacity. As a result, queues and delays surge. Incidents have an adverse impact on road safety and are considered major contributors to traffic delay and congestion (Mahmassani et al., 1998). Table 1.1 sourced from the Highway Capacity Manual 2010 shows the effect of incidents on freeway capacity.

Table 1.1: Proportion of freeway segment capacity available under incident condition

\begin{tabular}{|c|c|c|c|c|c|}
\hline $\begin{array}{l}\text { No. of lanes } \\
\text { (one } \\
\text { direction) }\end{array}$ & $\begin{array}{l}\text { Shoulder } \\
\text { disablement }\end{array}$ & $\begin{array}{l}\text { Shoulder } \\
\text { accident }\end{array}$ & $\begin{array}{l}\text { One lane } \\
\text { blocked }\end{array}$ & $\begin{array}{l}\text { Two lanes } \\
\text { blocked }\end{array}$ & $\begin{array}{l}\text { Three lanes } \\
\text { blocked }\end{array}$ \\
\hline 2 & 0.95 & 0.81 & 0.35 & 0.00 & N/A \\
3 & 0.99 & 0.83 & 0.49 & 0.17 & 0.00 \\
4 & 0.99 & 0.85 & 0.58 & 0.25 & 0.13 \\
5 & 0.99 & 0.87 & 0.65 & 0.40 & 0.20 \\
6 & 0.99 & 0.89 & 0.71 & 0.50 & 0.26 \\
7 & 0.99 & 0.91 & 0.75 & 0.57 & 0.36 \\
8 & 0.99 & 0.93 & 0.78 & 0.63 & 0.41 \\
\hline
\end{tabular}

(Based results of two studies), (HCM 2010)

A major source of traffic delay in many large urban areas is usually associated with incidents. In the case of US freeway system, the cost related to incidents has always been 
a concern and hard to avoid. For example, a study shows that the estimated congestion on an urban freeway due to incidents is $60 \%$ of total delay, and this proportion is believed to be increasing (Lindley 1987; Parkany et al. 2005).

Based on knowledge of traffic flow theory, when an incident occurs, lane blockage takes place. It results in a reduction in the occupancy at the downstream station associated with an increase in the traffic volume at the upstream station. A simulation study by Parkany et al. (2005) indicated that lane blockage unrelated to incidents does not cause a significant disruption to traffic flow.

Incidents are random and failure to detect these or late detection of incidents could lead to more secondary incidents on freeways which in turn causes significant disruption to traffic flow. It is well known that incidents have high economic, social, and human impacts. Their early detection is the first step towards effective incident management (Lindley 1987).

\section{Incidents Characterization}

Incidents can be characterized according to:

- Location

- Date, start time, end time, lane blockage, etc.

- The start time of the incident is the time at which the facility capacity is reduced. The end time of the incident is the time at which the capacity-reducing incident is removed.

- In case a video recording is not available, a standardized procedure can be used to find the 'start time.' When an incident occurs, the speed drops sharply and at the same time occupancy surges. In situations where there is no video, or the onset of the incident was 
not recorded, the closest speed data point before the significant drop in speed can be regarded as the onset of the incident.

\subsection{Problem Definition}

At present, fixed sensors (e.g., loop detectors, video cameras, etc.) installed at selected intervals on/along the facility contribute data on speed, occupancy, volume, traffic composition, etc. In association with geometric features of the facility and weather condition data, these information items are used as inputs to incident detection methods in order to automatically detect incidents.

In the case of loop detectors they are limited in numbers, and within the two loop clusters in the freeway, there is no recorded information about the traffic. So, if the distance between the two loop clusters is one or more than one kilometer then, in between this distance the information is not available. Although, induction loop data serve a very useful function and can be regarded as a proxy for data obtainable from a roadside ITS unit, these should be supplemented initially with simulation data and later with real data that can be obtained from probe technologies, crowd sourcing, and connected vehicle systems. Of course, an assumption can be made about space mean speed upstream and downstream a detector cluster based on the speed recorded by the cluster.

Some of the recognized problems in the current incident detection practice include data quality issues, fixed location-specific data, lack of flexibility, and the high cost of moving sensor clusters to another location. According to literature, the cost of installing an inductive loop detector (IDL) could reach up to $\$ 8,200$. Moreover, optical fibers used to 
connect the ILDs cost $\$ 300,000$ per mile. As for reliability, according to official statistics, about $50 \%$ of the installed ILDs are defective (Middleton, Jasek, \& Paker, 1999).

It should be pointed out that the quality of the existing sensor data has improved due to data analysis methods for cleaning up raw data.

In recent years, there has been considerable interest in using probe vehicle technology to gather data on traffic conditions. The latest thrust is to use cell phones as probes and also to track GPS-equipped fleets. Due to proprietary nature of cell phone-based as well as fleet probe data, there is a general absence of research based on these data. Also, the data that could be purchased from vendors do not show the sample size for each segment of the facility. Therefore, reliability of data cannot be inferred.

At the research and development level, currently connected vehicle technology encompasses (1) Vehicle-to-vehicle communication, and (2) vehicle-to-infrastructure (i.e. roadside units). Although the technology is going through a demonstration phase, there is every indication that most vehicles will be connected by 2020-2025.

The vehicle-to-infrastructure connectivity will occur before the vehicle-to-vehicle communications become common place. The vehicle-to-vehicle communications can be relayed to roadside units via the vehicle-infrastructure communication technology. The transportation authorities are optimistic about traffic management in the connected vehicle environment including incident management.

Under present conditions, there is a need to develop an incident detection method that uses induction loop data (as a proxy for roadside unit data) in association with simulated probe vehicle data. The developed method can be modified in the future by using probe vehicle data as well as infrastructure-vehicle connected data. 
A survey was carried out to study the importance of AID algorithm in assisting the decision-making process in traffic management (Williams \& Guin, 2007). The study identified a number of reasons behind the limited implementation of AID algorithms. Also, available incident detection methods based on AID technology were reviewed in terms of perceived performance. The results suggested the need to improve the AID methods and technology.

\section{$1.4 \quad$ Research Objectives}

Reliable automated incident detection methods provide the traffic management personnel an important alert message so that they can verify the incident with video technology (if available) or other available means and quickly initiate the incident management process. Therefore, the primary objective of this research is to develop a predictive model and the associated algorithm for automatically detecting incidents on uninterrupted flow type of facilities by using a combination of induction loop data (as a proxy for roadside unit data) and simulation-based reliability estimates. Modelling incident detection is based on an enhancement of the of the Bayesian method for predictive speed; the specialized enhancement is for the purpose of detecting incidents. The developed model is aimed at detecting incidents at a high degree of reliability under applicable traffic flow conditions.

\subsection{Scope}

The developed model and the algorithm are expected to exhibit the following capabilities: 
(1) Treat the stochastic nature of traffic flow and incident,

(2) Predict the traffic flow pattern over time based on real-time traffic data and reliability estimate, and

(3) Recognize changes in traffic pattern caused by an incident and provide an alert if an incident is detected.

The developed model within the algorithm monitors traffic conditions and recognizes the pattern of the flow on the freeway using two types of data noted above (i.e., fixed location sensor data and reliability estimate). The detector data used in this modelling and simulation study is in essence similar to what is obtainable from a Roadside Unit (RSU) of Vehicle-Infrastructure Integrated (VII) System. These in association with the reliability estimates enabled the calibration and validation of the model/algorithm. The model and the algorithm were refined and validated further by using results of the stochastic microsimulator Vissim. As a part of research, the Matlab platform implemented models with the capability to identify patterns of traffic flow caused by incidents under applicable traffic volume conditions. The code includes a number of criteria that narrow the choice of likely causes of a rapid drop in speed both spatially and temporally.

The model and the associated algorithm work with detector/sensor or Roadside Unit (RSU) data. But, their structure allows minor modifications so that probe and crowdsourced data can be used if such data becomes available and reliability can be assured. The detector could be the commonly used loop type or the new technology-based compact detector placed in the pavement. Also a variety of sensors (e.g. microwave type of sensors) could be placed over the pavement or on the roadside. The RSU is a part of the infrastructure that in addition to data capture has the capability to communicate with 
equipped vehicles. The probe vehicle is equipped with tracking and communication technology so that it can capture and transmit traffic flow condition data. The crowdsourced data is captured by commercial companies by using in vehicle units and cell phones. Following the capture of data, it is processed and sold to users of traffic data. There is a rapid increase in the availability of crowd-source data in the USA (e.g. Waze).

The detector/sensor and RSU data are location-specific. On the other hand the probe data and crowd-sourced data cover cells/segments of the highway. Small modifications can be made to the model and algorithm so as to be able to work with the probe and crowdsourced data.

The role of the incident detection is to provide an early alert to the traffic control centre and it is not a substitute for Autoscope type of video cameras. However, even for camera-equipped highways, the control centre staff may not be pointing a camera at location of an incident.

\subsection{Limitations of the Research Approach and the Product}

There is no claim made that the proposed algorithm will allow the detection of all freeway incidents. The assumption is that detection of all incidents on the freeway by an algorithm is impossible. However, there is a claim that the developed algorithm by treating the stochastic nature of an incident and traffic flow and systematically narrowing the choice of likely causes of the rapid drop in speed both spatially and temporally increases the effectiveness of the developed algorithm. It reduces the mean time to detect (MTTD) in order to find the balance between the false alarm rate (FAR) and the detection rate (DR). 
The algorithm can be applied to any freeway segment that carries traffic with the potential that if an incident occurs it will disrupt traffic flow pattern to an extent that a sensitive model will attempt to recognize it. Such freeway/highway traffic volumes are encountered during high end of "under saturated", "queue-discharge" and "oversaturated" traffic flow regimes.

Consideration is given to conflict points on a freeway upstream the incident location such as on- and off-ramp merge points. Incidents that occurred immediately downstream of these known or expected conflict points are exempted from the analysis so that the incident impact is not influenced by vehicles escaping the scene.

The model is designed to predict one incident at a time. If secondary incidents occur due to distraction effect of an incident (at the same location within a short time period), the model cannot predict the chain of primary and secondary incident.

\subsection{Methodology}

A systematic research framework is advanced that incorporates the tasks required to conduct this thesis research. The approach to the overall study methodology is explained below.

- The causes and impacts of traffic incidents occurring on freeways are studied.

- Reliable and timely incident detection is identified as the most critical phase of incident management.

- A general background search for information on the basic functionality of the basic automated incident detection systems and the prediction algorithms behind them is carried out. 
- The research comprehensively explains the approach for incident and detector (sensor) data collection and analysis.

- Owing to the absence of statistics on reliability of commercially available probe and crowd sourced data, Montecarlo simulation approach was adopted for obtaining reliability estimates.

- The research studies the concept of theoretical foundation of developing the incident detection model.

- The research studies the algorithm formulation and the incident detection criteria.

- A computer programming-based coding environment is used for model development using the Matlab computer language.

- For the purpose of supplementing the calibrating and validating the model with archived data, the stochastic traffic microsimulator Vissim is utilized in association with necessary computer programming.

- Expected results, conclusions, and recommendations are presented.

The developed research methodology noted above was found to be feasible, given the availability of archived detector real-time data and the methodology to generate simulated spatial and temporal data. Advances were made in formulating, developing, and testing Bayesian and Monte Carlo models.

\subsection{Research Framework}

The research framework is presented in Figure 1.3 and its various components are explained and implemented in the following chapters. As a part of the research framework, a literature review was carried out. Findings of previous studies were used as stepping stone 
throughout the thesis research. Please see the thesis organization section for progression of work from this chapter to the concluding part of the thesis.

\subsection{Thesis Organization}

This thesis is organized into eight chapters to cover the main component of this study. Chapter 1 covers problem identification, problem definition, research objectives, methodology, and research framework. Chapter 2 provides background information on incident detection technology in terms of state of knowledge. Data collection and preparation is described in Chapter 3. Chapter 4 is devoted to reliability factor that has shaped the modelling tasks. Chapter 5 covers the model and its parent algorithm. In Chapter 6, a computer programming-based environment using the Matlab computer language is presented and an analysis of the results is provided. Next, the algorithm calibration and validation described in Chapter 6. Generating probe vehicle trajectory data in Vissim Microsimulation software with sensors looped-in under incident conditions for testing and validating the algorithm in detecting incidents is comprehensively discussed in Chapter 7. To close, the conclusions and recommendations are emphasized in Chapter 8 , and the last section provides a list of references used in this thesis. Appendices are presented at the end of this dissertation. 
- Issue identification; problem definition

- Research objectives and methodology

- Literature studies

- Detector (Sensor) and incident data collection

- Sample size \& information reliability issues

- Theoretical foundation of incident detection model(s)

- Example applications

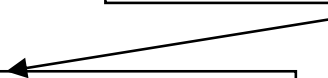

- Algorithm formulation

- Incident detection criteria

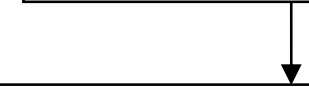

- Simulator application for data generation

- Algorithm validation

- Example applications

Figure 1.3: Research framework 


\section{Literature Studies}

\subsection{Introduction}

This chapter describes how detecting incident in this research differs from the past and current practice. It also describes the basic functionality of the basic automated incident detection systems and the prediction algorithms behind them, along with the performance measures used to evaluate incident detection algorithms.

\subsection{Importance of Prompt Incident Detection}

Transportation has a significant impact in our daily life commuting, and its development has made many of our routines much easy. However, a freeway incident can cause disaster. Nearly 1.3 million people die every year on the world's roads. It was reported in 2008 that traffic injuries ranked fourth among the leading causes of death in the world. About 20 to 50 million people suffer non-fatal injuries that result in disabilities. Road traffic injuries are the leading factor of death among young people aged 15-29 years, and without a proper action to promptly detect road traffic incidents, it is predicted that traffic accidents will be the driving cause of 1.9 million people by 2020 (Wakure, Patkar, Dagale, \& Solanki, 2014).

Reducing congestion on freeways and fatalities is always the main concern of the transportation engineers. Early and efficient automated incident detection is considered one of the main factors to alleviate those concerns. Since the early investigations on AID which date back to 1960 s, there had been numerous studies concerning proposed incident detection algorithms (Ozbay \& Kachroo, 1999). 
Traffic management centres are concerned about clearing the incident in a short period of time as the third step of the incident management system. This step follows detecting the incident and delivering a quick response. The clearance time is measured from the arrival time of the emergency unit and the removal of the incident from the freeway, while the response time is the time that spans from detection of the incident to the arrival of the emergency units. Time to detect (TTD) incident is regarded as an important factor. Its effect on crash fatalities was studied by (Evanco 1996) who conducted a statistical analysis to estimate the impact of the TTD on crash fatalities.

The nationwide incident detection program on urban interstates, freeways, and expressways data from the Fatality Analysis Reporting System in the U.S. was utilized by Evanco to study the effect of average time to detect on road safety. He concluded that a reduction in the average time to detect by $42 \%$ (from 5.2 to 3 minutes) resulted in a reduction in fatalities by $11 \%$ (450 lives/year) and economic benefits amount to about $\$ 931$ million per year. An additional decrease in TTD by $62 \%$ could reduce fatalities by $15.9 \%$ (652 lives/year) and could bring the benefit as high as US\$1.352 billion per year (Evanco, 1997). Results derived from Evanco reveal the significance of prompt incident detection in consideration to reducing number of fatalities as well as increasing the economic benefits associated with rapid incident detection.

\subsection{How Detecting Incidents in this Research differs from Past/ Current Practice?}

As will be mentioned in section 2.8, most of the AID methods take traffic data as input; process the input with an algorithm and present road condition information such as 
the existence of an incident as output. Data needed for an AID method may differ according to selected incident detection algorithm.

This research study differs from past/current practice in the following aspects:

(1) Methods that are based solely on induction loop data do not use any actual or simulated proxy probe data. In this respect, the proposed approach is more advanced than approaches that rely upon induction loop data as the only source of information on traffic flow.

(2) No method was reported that took into account the reliability of data that is used in the incident detection model. This research is going a step beyond by formally taking into account the reliability of data.

(3) Most of the probe-based algorithms make use of travel time to detect significant changes in the traffic in order to judge whether an incident occurred or not. The probe data is sourced from vehicles equipped with toll transponders and GPS receivers. Although their numbers are increasing, these usually do not meet the minimum sample size requirements for statistical reliability.

(4) Most probe-based incident detection systems utilize existing systems such as AVI/ETC systems and due to the limited extent of coverage; they cannot be relied upon on a network basis.

(5) When the probe vehicle is equipped with GPS technology, the positional information is determined from the GPS signals in association with map matching. This information is transmitted to a control centre to display real-time position of the probe vehicle. However, at this point in time, there are errors in map matching and also lost signal due to high buildings in the CBD areas becomes an issue (Parkany and Xie, 2005). 
(6) On the basis of the above information, the approach used in this research is suitable for at least a decade. Specifically, there are only limited numbers of equipped probe vehicles on the road. The widespread adoption of connected vehicles is, at least, a decade away. In the approach used, the fixed-point detector data can be regarded as a proxy for a roadside unit and analysis of simulated probe vehicle data provides the reliability input for model development. Their combination leads to improved model and algorithm than currently available.

\subsection{Traffic Flow Variables}

Main variables used in AID models are noted here. Speed is one of the three traffic flow measures, the others being volume and occupancy or density. The volume and occupancy/density are commonly used terms, but different metrics of speed can be used, depending upon the purpose. These are spot speed, time mean speed, space mean speed.

\subsection{Types of congestion}

Detectors generally are used to detect two types of congestion: recurring and nonrecurring. Non-recurrent congestion that develops on highways due to random or temporary traffic incidents represents the major portion of congestion on freeways and costs billions of dollars. In contrast, recurring congestion is predictable at specific locations and time (Deniz, Celikoglu, \& Gurcanli, 2012). 


\subsection{Freeway Traffic Management Systems}

Due to the limited resources of land and money to build new or expand existing highways, Traffic Management Centers (TMCs) are employing different sensing systems to quickly detect and clear traffic incidents and therefore relieve congestion. Effective traffic incident management systems involve four phases, including detection and verification, information dissemination, response, and incident clearance as shown in Figure 2.1.

The first step of traffic incident management is detecting and verification; incident detection is the process that brings an incident to the attention of the agency or agencies responsible for maintaining traffic flow and safe operation on the facility. This process covers getting information about existence of an incident and its properties such as type, severity, and exact location.

Prompt identification of incidents can be important for conducting the following step in the traffic management system including rapid distribution of information about the existence of an incident. Information may be distributed by existing information technologies such as radio broadcasts, telephone information systems, internet service, etc. Warning other drivers about the existence of an incident, allows motorists to choose alternate routes which mitigate the effects of the incident such as the impact of congestion and possibility of secondary crashes. Rapid dispatch of emergency vehicles is the third step

of a TMCs systems which covers informing responsible agencies or authorities and employing correct personnel and equipment. The last step of a TMCs system is clearance, which covers removal of materials or cars which disrupt traffic flow (Deniz, Celikoglu, and Gurcanli 2012). 
Data Collection

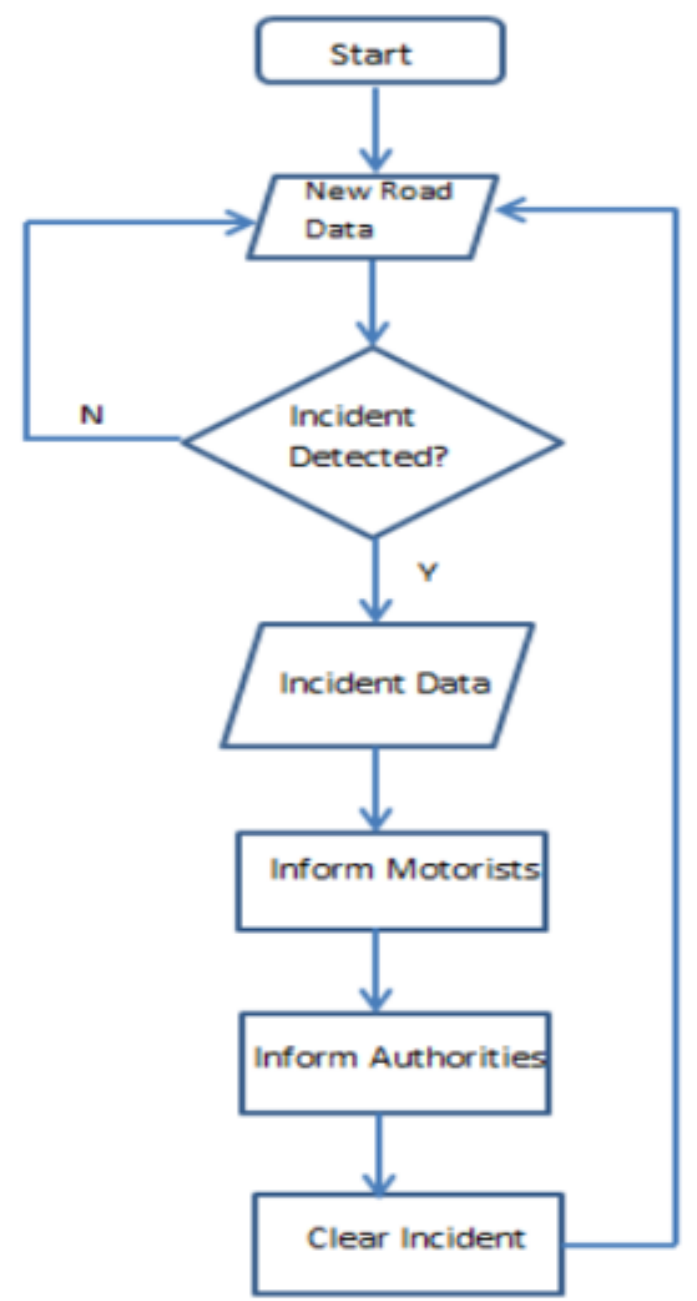

(Deniz, Celikoglu, \& Gurcanli, 2012)

Figure 2.1: Basic flow chart of a TIM system

\subsection{Detector Technologies}

A brief introduction to incident detection was provided in Chapter 1. Further elaboration of technologies is provided here. Freeway/Highway incidents can be detected through a variety of methods including service patrols, and road users' phone calls from the incident location. These are examples of manual incident detection. Modern traffic management systems, however, use various automated incident detection methods to 
ensure that all incidents are found as quickly as possible. Most advanced management systems and technologies in the ITS field rely on real-time traffic data, which reflects current conditions of the traffic network.

A variety of detector technologies and methods are currently available. Selection and performance of detector technologies depend on the types of traffic flow data as well as their reliability, performance in different environmental conditions, consistency, data accuracy, precision and the detector response time. Usually, the reliability of the sensor is carefully evaluated in comparison to other factors. Many traffic surveillance systems are afflicted by low reliability of loop detectors due to frequent malfunctions or breakdowns of sensors which can seriously obstruct the performance of the overall traffic management operations.

Mathematical models and algorithms with appropriate formulation can be used to detect incidents with data obtained by loop detectors (Ozbay K. et al., 2001). Inductive loop detectors (ILDs) collect data from each individual lane. Therefore, almost every lane is equipped with multi-turn of inductive loops that are buried under the pavement. The measured data including volume, presence, occupancy, speed, and classification are transmitted to the traffic management centre at fixed time intervals. Hence, the ILDs are able to measure traffic parameters such as average speed, volume, and occupancy (Klein 1995), (Busch and Fellendorf 1990).

Some other types of sensors do not require disrupting the roadway surface, such as video recording cameras. These are installed in a form of network and contributed video image that capture the presence of vehicle and condition of traffic flow. These images are 
then processed using special software components which enable the conversion of images to data (Gordon and Tighe 2005).

As other stationary sensors video recording cameras have a number of disadvantages that can reduce their use as detectors in monitoring traffic. Among these disadvantages are their installation and maintenance costs. They also usually require highway or lane closure for their repairs, which can induce other societal costs, such as extra congestion or a decrease in local accessibility (Sanwal \& Walrand, 1995). Moreover, video cameras are known to have difficulties serving their intended purpose during severe weather conditions and there is always a need for a periodic calibration to enhance the working abilities of video cameras (Dailey et al., 2000).

The reliability of vehicle detection has improved with the rapid development of electronics technology which added a new feature to the inductive loops based on the digital design features.

Probe vehicle and connected vehicle technologies are not reported here. Interested readers are advised to refer to Khan et al (2014).

\subsection{Automated Incident Detection Algorithms}

Extreme speed differences among individual vehicles, sudden lane changing vehicles, slow-moving vehicles, spilled loads on the roadway, road surface conditions, weather conditions, geometric features of the freeway, etc. are some of the main causes of freeway traffic incidents. Regardless any of these causes, traffic features are always affected by the incident such as traffic volume, speed, and occupancy patterns. Automated 
incident detection methods in association with traffic flow data analyze the pattern change in the traffic feature to declare an incident condition.

However, there was always a challenge to distinguish the change in traffic pattern due to incidents from those caused by common bottlenecks as resonated by lowperformance levels of conventional AID methods. To increase overall performance, many AID methods utilized a 'threshold' concept. A threshold concept can be noted as follows: If a certain parameter of the detection system, estimated from traffic data, is greater than a predetermined threshold value, an incident is declared. These threshold values are predetermined for best performance by trial and error using traffic data with known 'incident present' and 'incident free' traffic flow conditions. Depending on the design of a particular AID method, some detection systems utilize more than one threshold value, such as California algorithm no. 8 which will be discussed later in this chapter (Busch and Fellendorf 1990).

The process of incident detection employs computer algorithms that monitor freeway data from detectors at regular time intervals. Data are examined in terms of one or more of the traffic characteristics in order to evaluate the nature of this traffic characteristic of interest along the facility. Researchers over the years have developed automated incident detection systems that monitor traffic information from freeway facilities and automatically detect such incidents (Ezell S. 2010).

Automated incident detection algorithms on freeways can be characterized in a number of different ways. Most commonly, algorithms are classified according to the type of measurements operation:

- Pattern Recognition Algorithms 
- Traffic Model Algorithms

- Statistical Incident Detection Algorithms

\subsubsection{Pattern Recognition Algorithms}

Pattern recognition algorithms rely on differentiating and recognizing traffic patterns which are typical of incident conditions that differ from incident free flow conditions. This type of algorithm looks at one or more traffic features at the detector stations upstream and downstream of the incident. A feature is selected so that it is sensitive to incident traffic patterns; it can be a single variable such as occupancy or density, or multiple variables such as comparing occupancy at adjacent detector stations. Finding the optimal variable to monitor was always the focus of most of the AID research and the basic principle of these algorithms is that an incident will create a change in this traffic variable levels upstream of the incident as well as downstream the incident (Martin, et al 2001).

Typically, the data from each detector station at each interval is run through a decision tree logic and is then labeled according to the type of traffic conditions they represent. At each node of the decision tree, a data measurement is compared to a present threshold value. Accordingly, an incident is declared when the computed traffic feature exceeds a predetermined threshold value which is calibrated to reflect incident flow conditions (Martin et al., 2001).

As a further classification, pattern recognition algorithms can be categorized as either comparative or single station algorithms. Comparative algorithms are those that compare traffic characteristics at adjacent stations. Single station algorithms are those that perform temporal comparisons at a single station (Martin et al., 2001). 
One drawback of this pattern recognition class of algorithms is that each individual threshold must be set for each detector station for the best incident detection results. So there is a need to calibrate the threshold values, and further improvements may result by varying the thresholds with time of the day. Setting these thresholds is difficult and timeconsuming.

The first promising pattern recognition algorithm - and perhaps the first promising AID method in general - was developed by the California Department of Transportation.

\section{California Algorithm}

The basic California algorithm model is one of the earliest detection algorithms developed in the 1960s on the Los Angeles freeway network to detect freeway incident (Payne \& Tignor 1978). The algorithm is based upon the use of discontinuity in occupancy values between two adjacent loop detector stations from both upstream and downstream locations at the same time interval to identify whether an incident exists between these locations Payne et al. (1976). The algorithm consists of a family of 11 algorithms that detect incidents. It utilizes 60 second average occupancy values from adjacent loop detector station along the freeway, transformed into four different estimates to be used as algorithm inputs. The inputs are in the form of absolute, relative and temporal differences in occupancy values between the two stations.

The algorithm calculates the spatial difference in occupancy, OCCDF, and the relative spatial difference of occupancies, OCCRDF. In addition to these two data, the algorithm uses occupancy values obtained from downstream detectors. A decision tree for this algorithm is structured in a binary base; the decision tree is expressed in three tests 
defined in the following three steps. An incident is signaled when the threshold values in all the three tests are exceeded at the same time interval.

1. The difference between the upstream station occupancy (OCCUS) and the downstream station occupancy (OCCDS) is verified against threshold value T1. If the threshold value is exceeded, then proceed to step two.

2. The ratio of the difference in the upstream and downstream occupancies to the upstream station occupancy $(\mathrm{OCCRDF})=(\mathrm{OCCUS}-\mathrm{OCCDS}) / \mathrm{OCCUS}$ is verified against threshold T2. If this threshold is exceeded, proceed to step three.

3. The ratio of the difference in the upstream and downstream occupancies to the downstream station occupancy (OCCUS - OCCDS)/OCCDS is verified against threshold T3. If this threshold is exceeded, a potential incident is indicated, and step two is repeated. If this threshold is again exceeded, a potential incident alarm is triggered. An incident state is terminated when threshold T2 is no longer exceeded. At every time interval, say 30 seconds, the algorithm checks if each of these three features exceeds its predetermined threshold value. If they all do, an incident alarm is triggered.

New comparative AID algorithms were developed after the development of the basic California algorithm. Ten new algorithms, also based on the California binary decision tree structure, were developed and compared with the California algorithm (Payne, 1976; Payne et al., 1976; Payne and Knobel, 1976; Payne and Tignor, 1978). The details of each algorithm are beyond the scope of this thesis research. The new enhanced California algorithms aimed to reduce false alarm. For instance California algorithm no 8 is one of the better-known algorithms that aims to reduce false alarms due to shock waves approaching from the downstream sectors. In the algorithm no. 8, traffic status is divided 
into 8-different states. Starting from a known traffic status, it uses decision tree logic to determine the change in the traffic status based on changes in occupancy values between two stations. The algorithm compares five threshold values in the logic to define a change in the traffic status. An incident alarm is triggered if a substantial discontinuity in occupancy is detected, where a change in the traffic status is then observed (Yaguang \& Anke, 2006).

Al-Deek et al. (1994) showed that the calibration of California algorithms 7, 8, and 10 was a lengthy process that involved testing as many combinations of threshold values as possible. However, Arceneaux et al. (1990) reported calibration accuracy of algorithm no. 8 as $50-20 \%$ detection rate and 0.125 to $0.003 \%$ false alarm rates using traffic data gathered from Los Angeles freeway system.

Chung and Rosalion (1999) also compared some rule-based algorithms using some incident data from freeways in Melbourne. The California algorithm was found to achieve a DR of $70 \%$ with a FAR of $0.005 \%$.

\subsubsection{Traffic Model Algorithm}

\section{McMaster Algorithm}

The McMaster algorithm uses the model of catastrophe theory to describe the flow lane occupancy relation so as to distinguish between the incident and normal congestion (Gall and Hall, 1989; Persaud and Hall 1989; Persaud and Hall, 1990). It has been found that speed often tends to jump down when the traffic state goes into congestion zone. Niatin (1986) suggested that this discontinuous phenomenon in traffic patterns could be explained by the catastrophe theory. Catastrophe theory can be used in systems where one variable 
changes suddenly while other correlated variables change smoothly. The underlying principle is that a discontinuous event on the freeway will result in a discontinuity in the freeway traffic variables.

The parameters used in traffic incident detection are speed, flow, and occupancy, congestions are created gradually, whereas incidents cause sudden changes which drastically affect the current flow speed. The idea was applied for incident detection based on the fact that the transition from non-congested to congested condition is marked by a sudden drop in speed, accompanied by smoother changes in occupancy and flow.

A limitation of this method is that it depends on accurate speed measurements, which is not always available on TMSs. Besides, even though single-stations algorithms such as the McMaster algorithm may be able to effectively detect congestion, other comparative methods show more potential in differentiating recurrent and non- recurrent congestion.

The McMaster algorithm consists of location specific volume-occupancy plots that classify traffic data points into one of the several areas which are constructed using historical data. Incident congestion is detected if upstream and downstream traffic data points fall into certain areas in the location specific plots. Congestion states are assigned depending on the area in which the volume-occupancy pairs are plotted.

Four major areas on a volume-occupancy chart are represented in two dimensional template which is bounded by three main lines in the template denoted by critical volume (Vcrit), which is the minimum discharge volume, say the minimum discharge downstream of a road during peak incident free condition hour. Critical occupancy (Ocrit), which is the maximum occupancy for uncongested operation, and the most important line which is a curved one that divides the plane into congested and uncongested regions as shown in 
Figure 2.2. This line corresponds to the imaginary edge of the cusp catastrophe plane where the jumps may occur.

The four areas formed from the boundary lines which are corresponding to different traffic flow conditions is represented below:

- Volume-occupancy combination with uncongested traffic flow (area 1)

- Congested flow with volume less than a pre-set critical level (area2)

- Heavily congested flow with volume above a pre-set critical level (area 3)

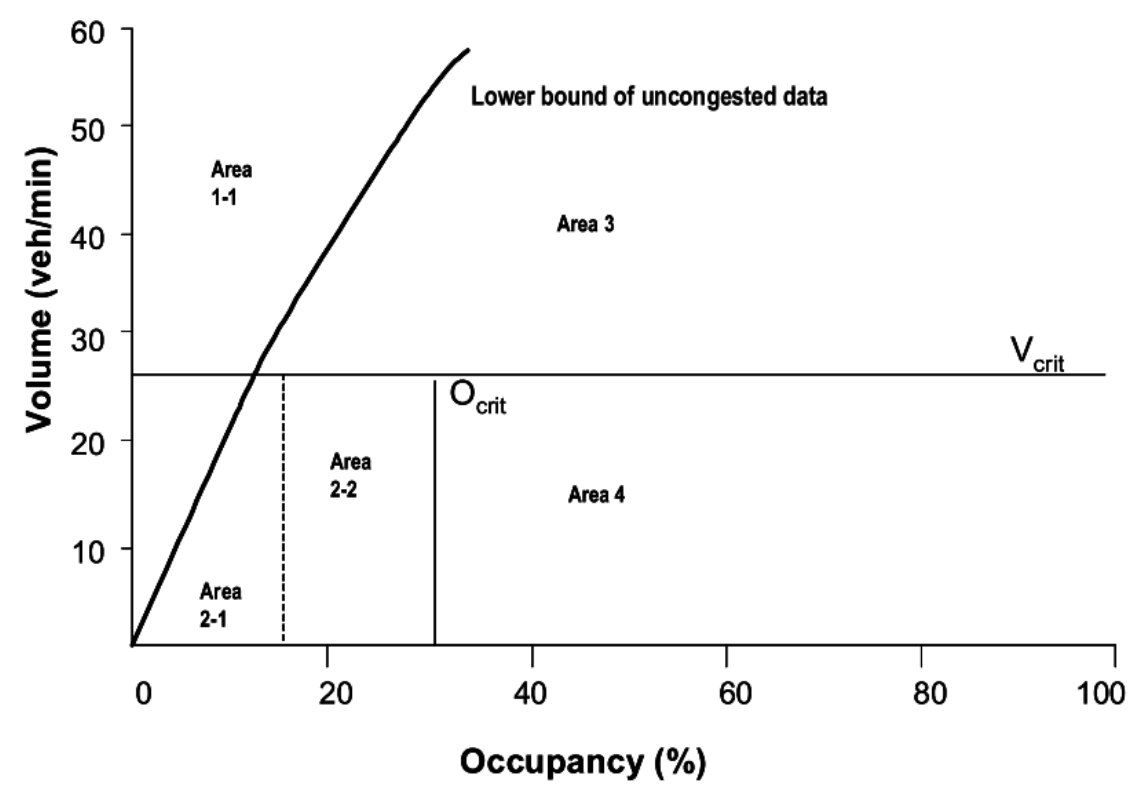

Source: Adapted from Hall et al., (1993)

Figure 2.2: Volume-occupancy chart

- Heavily congested flow with volume below a pre-set critical level and occupancy above a critical level (area 4)

The basis for the implementation of the algorithm is an appropriate template developed for each detector station; an incident alarm is declared if incident congestion 
persisted for three consecutive intervals. The parameters are specific to each site, and thus the algorithm must be calibrated for application at various locations. The calibration of this template has been substantially changed since it was first suggested. The details of the calibration process for old and new templates are given by Persaud et al., (1990) and Hall et al., (1993) respectively.

Simple logic was constructed to identify the existence and cause of the congestion at a freeway segment based on the congestion state of the upstream and downstream locations. First, the traffic state is verified using the template to see whether it is in the congested zone (area 2,3, or 4) or uncongested zone (area 1). The next stage is performed if the traffic stays in the congested zone for some consecutive 30 -seconds intervals. Following that is checking the cause of the congestion logic to distinguish between congestion caused by an incident from one due to other causes.

The downstream station is also verified for traffic state using the same template. The logic is summarized in Table 2.1 (Hall et al., 1993).

\section{Table 2.1: Logic used to determine the cause of congestion}

\begin{tabular}{|c|c|c|c|c|c|c|c|}
\hline & \multirow[b]{2}{*}{ Area } & \multicolumn{6}{|c|}{ Upstream station } \\
\hline & & $1-1$ & $1-2$ & $2-1$ & $2-2$ & 3 & 4 \\
\hline \multirow{6}{*}{ 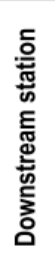 } & $1-1$ & No congestion & No congestion & Congestion & Congestion & Congestion & Congestion \\
\hline & $1-2$ & No congestion & No congestion & Congestion & Incident & Incident & Incident \\
\hline & $2-1$ & No congestion & No congestion & Congestion & Incident & Incident & Incident \\
\hline & $2-2$ & No congestion & No congestion & Congestion & Incident & Incident & Incident \\
\hline & 3 & No congestion & No congestion & Congestion & Congestion & Congestion & Congestion \\
\hline & 4 & No congestion & No congestion & Congestion & Congestion & Congestion & Congestion \\
\hline
\end{tabular}

Source: Hall et al., (1993) 
Hall et al., (1993) conducted an off-line testing of the MacMaster algorithm, 22 detector sites on Queen Elizabeth Highway in Ontario, Canada were selected to obtain traffic data, among which 17 incidents were detectable with a DR of $62.5 \%$ for all detectable incident. The FAR was calculated at $0.0018 \%$, and the MTTD was $3.2 \mathrm{~min}$. On the other hand, an online test took place at the same location, the algorithm attained a DR of $68 \%$, FAR of $0.00078 \%$ and an MTTD of $2.1 \mathrm{~min}$ in the on-line test. The Hall and Shi (1993) paper points out that when tested on-line, the algorithm showed a detection rate of $68 \%$ and the false alarm experience rate was 20 out in the 64 days of test.

\subsubsection{Statistical Incident Detection Algorithms}

The other common type of logic used for detecting incident is the comparison of actual traffic data observed from the freeway section and forecasted traffic variables based on the most recent history of measurements. There is a wide variety of these algorithms that differ in the measurements that are used and the forecasting techniques that are implemented. Basically, these types of algorithms observe the rate at which traffic measurements change rather than their absolute values, as the common practice in the case of pattern recognition algorithms (Yaguang \& Anke, 2006).

Statistical Algorithms are designed to predict traffic characteristics from a set of realtime traffic data. If there is a significant difference between observed detector data and the predicted values, then this change in traffic is classified as an incident (Parkany et al. 2005). Standard statistical techniques are utilized to detect this change (Petre et al. 2001). Logically, to predict incidents, one should compare observed measurements to forecasts based on a set of measured data. The available algorithms differ in terms of the used method 
for measurements and the forecasted techniques implemented. Incidents are detected by the deviations of the measured data from the forecasted values.

Teng et al. (1997) proposed an algorithm that assumes that traffic variables are independently and identically distributed, and then distributes them according to a normal probability density function. The algorithm calculates the log-likelihood of normal and incident conditions based on speed, volume, and occupancy measurements. These algorithms do not limit themselves to the application in incident detection.

Some different algorithms fit into this category, these are noted next. Selected algorithms are described following the list.

1. High Occupancy (HIOCC) algorithm (Collins et al. 1979).

2. Smoothing (DELOS) model (Chassiakos 1993).

3. Auto-Regressive Integrated Moving-Average time series (ARIMA) algorithm (Ahmed and Cook 1982).

4. Filtering models (Chassiakos 1993).

5. Standard Normal Deviates (SND) (Dudek et al. 1974).

6. Bayesian algorithm (Levin and Krause 1978); (Tsai and Case 1979).

\section{Standard Normal Deviate (SND) Algorithm}

The Standard Normal Deviate (SND) algorithm was developed by the Texas Transportation Institute (TTI) in the early 1970s for use in the initial surveillance and control centre in Houston, TX. In this case, the standardized value of the traffic control variable is checked against control limits that are based on the mean and the standard deviation of the data (De Castro and Mendonca 2009). 
The algorithm calculates the mean and the standard deviation of traffic control measures. An incident is detected when a particular value of a traffic variable deviates from the mean of this particular variable. The method is based on the assumption that a sudden change in the traffic will lead to the traffic value to deviate from the mean, and that would define a threshold for detecting incident (Mahmassani et al. 1999). The traffic variables could be a function of volume and speed as was reported by Chassiakos (1993). The most critical aspect of this algorithm is the determination of the thresholds, a process that is not well defined. Further, detector spacing is an issue, since the algorithm relies on shock waves passing over detectors.

\section{Bayesian Algorithm}

Bayesian Algorithms introduced by Levin and Krause (1978) at the Illinois Department of Transportation use stochastic presumption to optimize comparison thresholds. Traffic variables were observed under the existence and absence of incidents and then used to derive optimal thresholds using Bayes' rule. Levin and Krause (1978) found that the application of Bayes' rule in thresholds requires station by station calibration and large incident database which make it to be considered as a complicated application (Mahmassani, Haas, Zhou, \& Peterman, 1999).

The algorithm is based on the assumption that during incident-free conditions, for certain environmental and traffic conditions, the measured values of any selected feature $\mathrm{Z}$ at any detector station will be different. The probability of an incident to occur under certain traffic condition can be derived on the basis of capacity reduction of the selected feature (Levin and Krause 1978; (Mahmassani et al., 1999). 
What sets these algorithms apart from other algorithms is that the probability of an incident to occur can be derived from a percentage result as compared to other algorithms where a yes or no result is provided (Petre et al. 2001).

Some Bayesian algorithms use Bayesian statistic to compute the conditional probability according to the upstream and downstream occupancies. When there is a relative difference in the occupancy, Bayesian statistical techniques are utilized to develop the occupancy distribution during incident and incident free conditions and, therefore, predict the occurrence of an incident (Parkany et al. 2005).

Abuelela and Olariu (2009) in their research developed an automated incident detection technique for non-dense traffic. The Bayesian approach was applied to update a pre-assumed probability of an incident occurrence once it is verified. With detecting high probability of the occurrence of an incident the proposed technique promotes to trigger the alarm. The technique shows better detection rate than some other methods. However, it did not consider driver factors and more consideration still needed in terms of other incident types that might cause multilane blockages or do not even block lanes such as potholes (Abuelela and Olariu 2009).

\section{Requirements for Bayesian Algorithm Application,}

Three records are recognized for satisfying the requirement of the Bayesian algorithm:

- Traffic counts (volume and occupancy) at normal traffic conditions.

- Traffic counts (volume and occupancy) when incident occurs.

- Highway patrol log, which lists the type, location, and severity of incidents (Parkany $\&$ Xie, 2005). 


\section{Use of Bayesian Method}

Bayesian concepts are important to consider when calibrating the threshold for a single feature incident detection system. The basic idea is to choose the threshold for a given traffic feature that minimizes the probability that a true incident is not detected or that incident free condition triggers a false alarm. In another meaning, the optimal threshold for the traffic feature is determined by maximizing the expression:

$\mathrm{P}$ (incident occurring, given an incident signal) + P (no incident occurring, given a nonincident signal).

These require the development of mathematical expressions to represent the probability distributions of a given feature's value for both incident and incident free conditions. The statistical analysis required for this task could be difficult (Levin and Krause 1978).

Levin and Krause (1978) evaluated these purely probabilistic incident detection algorithms and found relative spatial occupancy difference to be the best indicator variable. Additionally, they discovered that the best results were attained when a series of four consecutive incident signals were required before reporting an incident alarm. Their study showed that the single feature Bayesian algorithm compared favorably with the most complicated multi- feature pattern recognition algorithm.

Zhang and Taylor (2005) in their research used Bayesian network technique to develop incident detection algorithms based on the causal probabilistic network. They aimed to imitate traffic operator when traffic data is employed to detect incident. The developed algorithms achieved a unique feature which implies stable detection rate (DR) 
with no significant change in the increase of the false alarm rate (FAR). The results contradict the well-known positive correlation between DR and FAR.

One flexible feature of the Bayesian network approach is its ability to utilize partially available traffic information without a sincere need to update its variables by renewing the state at each detection interval. This flexibility allows the developed algorithm to perform soundly even when there is no continuous supply of detector information at each interval. The performance of the algorithm could be enhanced by utilizing field incident data and considering other traffic data such as travel time (Zhang and Taylor 2005).

\section{The New Bayesian Method}

It should be mentioned here that the Bayesian incident detection model developed and verified in this research is a totally new and original formulation and it does not relate to the above noted models.

The developed Bayesian model differs from the previous Bayesian model in the following accepts:

1- It considers prior probabilities of traffic flow states which represent the stochastic nature of incident and traffic flow pattern

2- It predicts traffic flow condition and recognizes traffic flow pattern that is caused by an incident vs. that under incident free condition.

3- It treats the reliability in predicting traffic flow pattern, by considering the conditional probability factor to convert prior probabilities to posterior probabilities. 
4- It track the drop in traffic speed and the maximum posterior level as an indicators to detect incidents.

For further details, please see methodology section of this thesis.

\subsection{Performance Measures of Incident Detection Algorithms}

One common characteristic that is to be considered when evaluating incident detection algorithms is the consistency between the performance measures. Consistency makes it logical to compare results across regions. They should also be effective and informative.

In this section three measures of effectiveness have been used for evaluating the performance measures of incident detection algorithms, those are false alarm rate (FAR), detection rate (DR), and mean time to detect (MTTD). In order to perform a clear performance measure on any algorithm, these terms are defined.

The following sections identify performance measures for incident detection algorithms.

\subsubsection{Detection Rate (DR)}

The detection rate (DR) is the percentage of a total number of incidents detected during a specified time period. When incidents are detected by developed algorithms, it does not always represent the traffic condition in the freeway. In some cases the detected incident is false. One of the most reliable methods to calculate the number of true incidents is based on the operator log from the traffic management system. The ratio of the number of detected incidents to the total number of actual incidents present in a data stream is 
defined as the Detection Rate (DR) (Min 2012, Kahaki and Nordin 2011). On the other hand, the ratio of the number of undetected incident to the total number of actual incidents is defined as missed detection rate. Only incidents that cause lane blockage are usually regarded for counting the DR. Studies belong to this group generally have reported higher detection rates. On the other hand, regardless of the location, stalled vehicles cause lower detection rate because shoulder incidents often do not have a significant impact on the traffic flow (Petre et al. 2001) and (Martin et al., 2001).

Formula of DR is given as:

$\mathrm{DR}=($ number of detected incident $/$ total number of incident in the data set) $\mathrm{x} 100$.

When DR is close to $100 \%$, the algorithm works well to detect incidents. However, higher DR values may indicate that the algorithm is sensitive, which may result in more false alarms.

\subsubsection{False Alarm Rate (FAR)}

Missed detection rate is not commonly used as an indication of wrongly detected incident, however another evaluation criteria is used for this purpose, called false alarm rate. False alarm rate represents the ratio of wrongly identified incidents to the total number of checks. If the algorithm issues an incident warning for a freeway location at a particular time, but in the absence of an actual incident in the field, this warning is said to be a false alarm. In another meaning, the false alarm rate is the percentage of total false incident alarms occurring during a time period. That is, the detection algorithm detects an incident that does not exist. Since not all the algorithm decisions represent a real incident, any 
triggered alarm on the freeway due to incorrect incident detection is defined as a false alarm (Škorput et al. 2010, Kahaki and Nordin 2011).

Formula of FAR is given as:

$\mathrm{FAR}=($ number of false alarms / total number of algorithm decisions $) \times 100$.

The FAR denominator is the number of detections during a given period. For example, when traffic conditions are scanned once every five minutes for a time period of one hour (12 times per hour), and two of them are false alarms, the FAR is $16.7 \%$. (Asakura, Kusakabe, Long, \& Ushiki, 2015).

According to Min (2012), false alarm rate may be calculated as the number of false alarm per such a period of time within a specified distance. According to Petre et al. (2001), false alarm rate depends on how frequent the decision is made by the algorithm. The FAR is dependent on the number of decisions taken per each detector station for each time interval. There is inconsistency in calculating this value in the literature due to the many ways it can be calculated. Some algorithms make few decisions over the detection time interval which causes a high percentage of reported false alarms. Contrarily, if the number of detectors is high, the false alarm might increase but still will result in low false alarm rate. Low FAR has priority because when drivers are warned a few times without an actual incident, they will ignore the warnings in the future. The FAR was also defined as the ratio of unidentified incidents to the total number of actual present incidents within the data stream (Yaguang \& Anke, 2006).

In general, the FAR is dependent on the DR, reducing the FAR will result in a decrease in the DR. Similarly, if the DR is increased the performance of the algorithm will increase and consequently the FAR will rise. There is also a dependency between the 
MTTD and both the DR and FAR. Where it has been reported that to improve both the DR and FAR it is recommended to allow more time for the algorithm to analyze data, this means by increasing the MTTD. Therefore, reports specify certain limits of DR, FAR, and MTTD in order to better describe the performance of algorithms. These values must be adjusted to provide stability between unacceptable FAR with satisfactory MTTD and DR rate (Martin et al., 2001).

\subsubsection{Mean Time-to-Detect (MTTD)}

The third indicator of the performance of an incident detection algorithm is so called mean time to detect (MTTD). The (MTTD) is the average time an algorithm takes to detect incidents. It is defined by the difference between the reporting times for an incident and the algorithm detection time. The average value for the time to detect might be regarded for a set of data that contain more than one incident at the same time. Some extra time is needed for the incident verification; this period is not usually included when measuring the MTTD (Ritchie and Cheu 1993, Škorput et al. 2010).

It was reported that the relation between the detection rate (DR) and the false alarm rate (FAR) depends on when increasing the DR above the optimum value the FAR will significantly increase. The MTTD is utilized to complement the evaluation criteria (Min, 2012).

Formula of MTTD is given as:

$$
M T T D=\sum_{i=1}^{n}\left(t_{a}-t_{i n c}\right) / n,
$$

Where $n$ is the number of detected incidents, $t_{a}$ is the time that incident was detected, and $t_{\text {inc }}$ is the time that incident occurred. (Deniz et al., 2012). 


\section{Data Collection and Preparation}

There is a general lack of probe vehicle data in Canada and U.S. Probe data although of questionable quality, is proprietary in nature. So, for the development and validation of incident detection model and the corresponding algorithm, sensor data that involve incidents and challenging variation of speed would be most suitable. In order to meet these requirements, a detailed study design for data collection and preparation was developed. It includes data acquisition, data description, and data screening, it also included data selection, data sortation, and random selection of data.

For scientific research, the data preparation has to be done using scientific (statistical) methods. The same applies to initial testing of the model, calibration, and validation. This thesis research falls in this category. Data was acquired from two different sources, namely Ontario traffic data, (ONE-ITS), and Vissim microsimulation. In this chapter Ontario traffic data is discussed in detail and data obtained from Vissim microsimulation is discussed in detail in Chapter 7.

The following is a summary of Greater Toronto Area freeway data (termed in this thesis as Ontario traffic data obtained from ONE-ITS) preparation for analysis, calibration and validation of the developed model.

Step 1. Start with the ONE-ITS data (raw data).

Step 2. Define database acquisition need including incident data, detector information, and speed data.

Step 3. Describe incident database including, location, direction, year, start time, and incident cause.

Step 4. Describe detector information including detector name. 
Step 5. Define traffic flow variables including speed, volume, and occupancy.

Step 6. Define data screening and selection criteria including, year for which data is complete, direction, route, stream, season, and incident recorded time.

Step 7. Define criteria for screening faulty data such as incident recorded time and speed data.

Step 8. Establish criteria for data sortation and a random draw including, sortation techniques and random numbers.

\subsection{Database Acquisition}

Sensor data pertaining to segments of major commuting corridors obtained from ONE-ITS have made a major contribution to this research work. Also, traffic microsimulator (Vissim) was used to generate "equipped vehicle" flow trajectories and simulated probe data for the validation of the methodology.

\section{Ontario traffic data, ONE-ITS system}

The Toronto region freeway traffic data is archived by the ONE-ITS system for the purpose of providing it to researchers free of charge. The database includes incident data, detector information and traffic data, such as speed, occupancy, and volume. Among the traffic data, speed was the most useful for this research. Further comments on the speed data are presented in the following section. Traffic data covered all months from January 2011 to December 2014. This offered the opportunity to observe seasonal effects. 


\subsubsection{Incident Data Acquisition}

As a part of the data acquisition plan, it was intended to obtain a sufficient sample size with $30+$ incidents. Three attempts were made to achieve this target and as a result, a considerable amount of incident data was collected.

\section{Java use, "first attempt."}

The first attempt to obtain incident data was through accessing the ONE-ITS system service. In the ONE-ITS main menu, it was required to download a sample code of how to obtain incidents data and then store these in a csv file. The range of IP addresses that needed to be used to access this service was identified and added to the system. The Java code application on Java JDK 7 was needed to download the incidents data. The URL script was also required for use on the same machine that carried the specified IP address. This attempt was not efficient since it required significant time to write the necessary code for each database download. Moreover, the obtained incident data from this technique did not include the direction of the highway stream where the incidents first occurred. Therefore, a second attempt was made to obtain incident data from University of Toronto (U of T) database archive.

\section{University of Toronto database, missing direction "second attempt."}

In the second attempt, incidents data was obtained from the University of Toronto ( $\mathrm{U}$ of $\mathrm{T}$ ). Although the obtained incident data was comprehensive since it covered an adequate span of time, there was a significant amount of missing information that needed to be identified for data analysis purposes. This missing information was regarding the 
recorded direction of the traffic where the incidents took place. That is, the first obtained database did not identify direction of traffic where incidents took place. Therefore, more detailed incident information became necessary. An in-depth study was implemented to determine the direction by using GIS. But this attempt was not fully satisfactory. It required incident coordinates to be very precise, but such information was not available. Therefore, more detailed incident data was once again pursued.

\section{"Third attempt" to obtain complete incident databases from the University of Toronto archive}

Additional effort was mounted to update the data. This version was intended to include the direction, status, and other information. Eventually, a complete incidents database with all incident details (including the direction) was compiled from $\mathrm{U}$ of $\mathrm{T}$ archive.

Consequently, incidents data became available in csv format for the years 2011, 2012, 2013, and 2014. Incident data for the selected year was converted from csv to the xlsx format for possible further analysis to test the developed model. Excel was utilized as an efficient tool to complete the conversion process. The selected year is discussed in section 3.3.1.5.

\subsubsection{Detector Information Acquisition}

Detector data was downloaded remotely from ONE-ITS website platform. Browsing sensor by list from the website presented detector reports that include detector information as shown in Figure 3.1. It was possible to select the year and month of interest. In our case, 
information pertaining to the year of 2011 was required. The choice of year will be discussed later. The report parameters of interest are shown in Figure 3.2. It was possible to view all detectors for the selected year and month; a sample is shown in Figure 3.3. Following this approach, it was possible to store detector information in a tabular format.

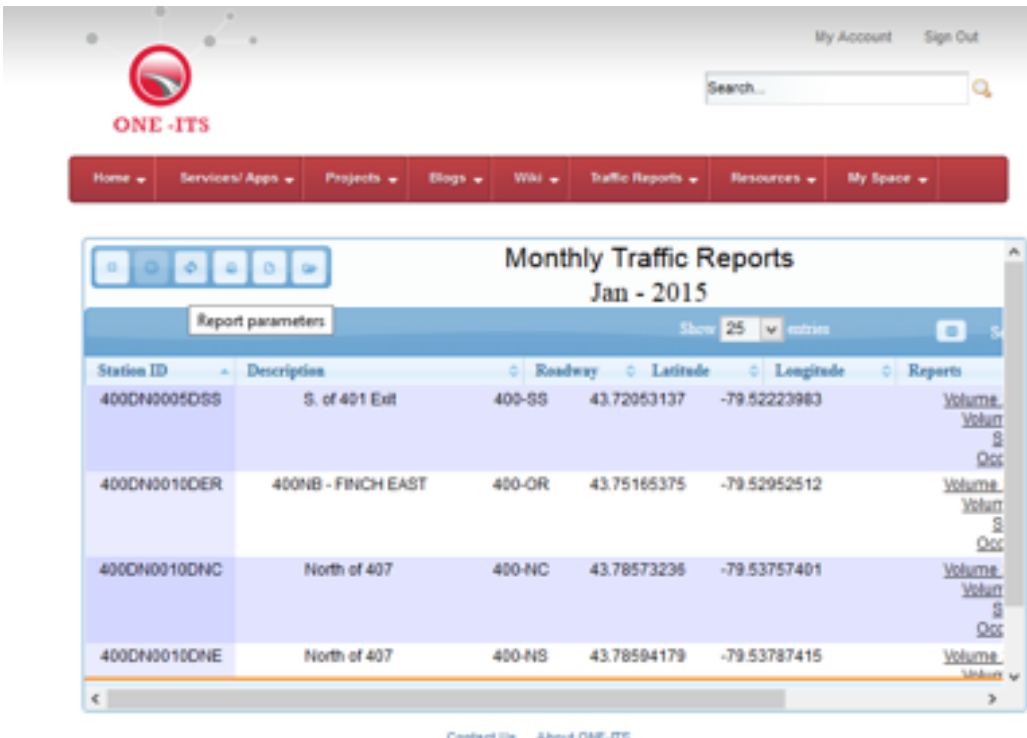

Figure 3.1: Browsing detector information by list from ONE-ITS platform

\begin{tabular}{l} 
Parameters - Traffic Reports \\
\hline (2) Select your report parameters values in the fields below. \\
$\begin{array}{r}\text { Please enter a valid } \\
\text { month number (1-12): }\end{array}$ \\
$\begin{array}{c}\text { Please enter a valid } \\
\text { year number (e.g } \\
2010):\end{array}$ \\
\hline
\end{tabular}

Figure 3.2: Variables used to set the report parameters (month and year) 


\begin{tabular}{|c|c|c|c|c|c|c|c|c|c|c|c|c|}
\hline \multirow[b]{2}{*}{ Device Name } & Dowload a detailed Report & \multicolumn{3}{|c|}{ Show/hide Columns } & \multicolumn{4}{|c|}{ Show 25 entries } & & \multirow{2}{*}{\multicolumn{3}{|c|}{$\hat{v}$}} \\
\hline & Address & $\hat{\imath}$ & Longitude & $\hat{v}$ & Latituc & ude & $\hat{v}$ & & & & & \\
\hline 400DND005DSS & 400-SS - S. of 401 Exit & & -79.52223983 & & & 43.72053137 & & & & View Chart & & $\therefore$ \\
\hline 400DNDO10DER & 400-OR - 400NB - FINCH EAST & & .79 .52962512 & & & 43.75165375 & & & & Viow Chart & & \\
\hline 400DN0010DNC & $400-N C$ - Narth of 407 & & -79.53757 & & & 43.78573 & & & & View Chart & & \\
\hline 400DNDO10DNE & 400 -NS - North of 407 & & -79.53787 & & & 43.78594 & & & & View Chart & & \\
\hline 400DND010DNS & 400-NS - North of Hwry 401 & & -79.52213367 & & & 43.72127932 & & & & View Chart & & \\
\hline 400DND010DSC & 400 -SC - N. of 407 & & .79 .53831368 & & & 43.78506675 & & & & Viow Chart & & \\
\hline 400DN0010DSE & $400-S S$ - N of 407 & & .79 .53815078 & & & 43.78528226 & & & & View Chart & & \\
\hline 400DNDO10DSR & 400-OR - Finch E - $400 \mathrm{~S}$. & & -79.53084454 & & & 43.75264929 & & & & View Chart & & \\
\hline 400DND010DSS & $400-$ SS - N of 401 & & -79.52290066 & & & 43.72191184 & & & & View Chart & & \\
\hline 400DND020DNC & 400-NC - At Hwy 7 & & -79.53751 & & & 43.789 & & & & View Chart & & \\
\hline 400DN0020DNE & 400-NS - At hwy ${ }^{7}$ & & -79.53937 & & & 43.79185 & & & & View Chart & & \\
\hline 400DNDO20DNR & 400-OR - FINCH EAST - $400 \mathrm{~N}$ & & -79.52980952 & & & 43.75345971 & & & & View Chart & & \\
\hline 400DND020DNS & 400-NS - South of Sheppard & & -79.52407 & & & 43.72745 & & & & View Chart & & \\
\hline 400DND020DSC & 400-SC - S. of hwy 7 & & -79.53994547 & & & 43.78775282 & & & & View Chart & & (T) \\
\hline & & & & & First & Previous & 12 & 3 & 4 & 5 Next & Last & \\
\hline
\end{tabular}

Figure 3.3: Detector information in a tabular format

It was of interest to include in the study all detectors mounted along the study corridor (i.e. Highway 401). A sample map view section is shown in Figure 3.4. Therefore, information on all detectors was obtained and from these records, it became possible to select the month that included the largest number of practical records, as shown in Table 3.1 .

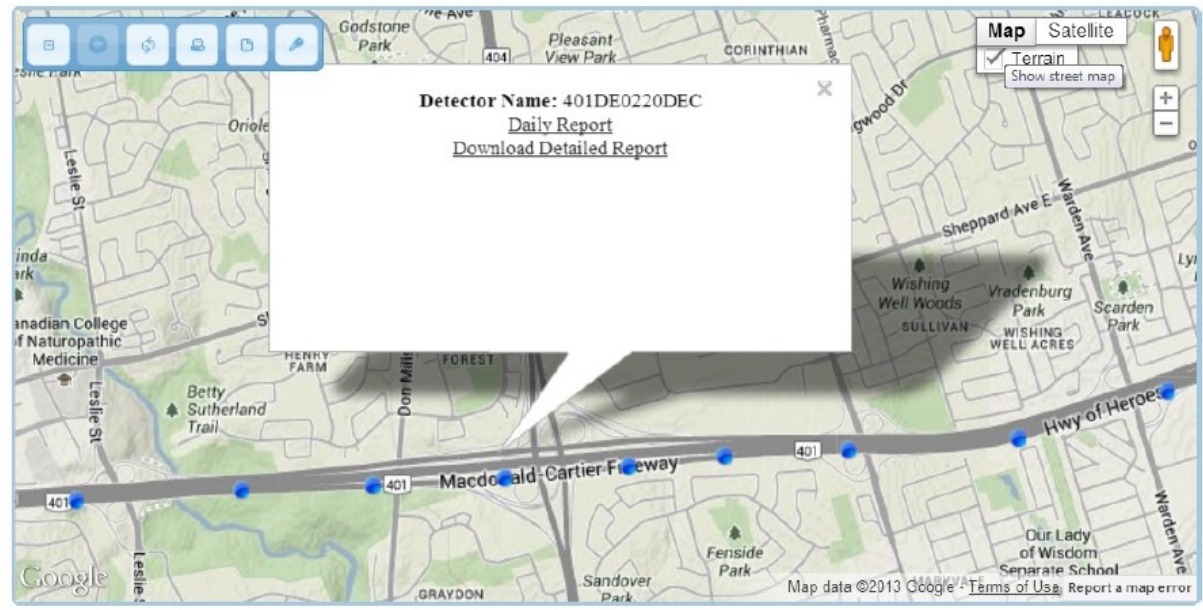

Figure 3.4: Sample section of detectors locations at Hwy 401 
Table 3.1: Number of active detectors in each month for all available years

\begin{tabular}{|c|c|c|c|c|c|c|c|c|c|c|c|c|}
\hline \multirow[t]{2}{*}{ Year } & \multicolumn{12}{|c|}{ Month } \\
\hline & 1 & 2 & 3 & 4 & 5 & 6 & 7 & 8 & 9 & 10 & 11 & 12 \\
\hline 2011 & 762 & 764 & 764 & 764 & 764 & 764 & 764 & 764 & 764 & 764 & 764 & 764 \\
\hline 2012 & 764 & 757 & 764 & 1033 & 1031 & 1026 & 1031 & 1031 & 1031 & 1031 & 1021 & 1028 \\
\hline 2013 & 1031 & 0 & 1031 & 1031 & 1031 & 1031 & 1031 & 0 & 1031 & 1032 & 1032 & 966 \\
\hline 2014 & 1029 & 1029 & 1029 & 1029 & 1029 & 1029 & 1029 & 1029 & 1032 & 1028 & 1034 & 0 \\
\hline
\end{tabular}

Table 3.1 shows that the max number of detectors were recorded for November 2014. Therefore, detector information for that month/year is considered for further analysis.

\subsubsection{Choice of Factors for Use in the Incident Detection Model}

A large number of factors can be used to characterize traffic flow changes caused by incidents. These include speed, density, speed variability among vehicles, and sudden lane changes. Speed and density are the commonly used variables for traffic flow studies. Although density is recognized as a better predictor of the quality of flow, it is difficult to track it along longer stretches of the highway. On the other hand technology and methods are widely available to measure speed.

Although microscopic level data on speed variability and sudden lane changes over short segments of the highway are in theory very useful factors for modelling incident detection, such data are not readily available for many highway facilities.

For the above noted reasons, the developed model and algorithm has focused on projected speed. 


\subsubsection{Speed Data Acquisition}

In order to test the developed model, speed data was acquired at specified locations, defined by a detector's name. It was possible to obtain speed data from the ONE-ITS system archive traffic reports. According to system design, each loop detector station records traffic information which is then included in a monthly report and stored in the ONE-ITS system. For use in this research, it was necessary to obtain traffic data including speed for all detectors mounted along the study freeway section for all the time periods during the study year. A considerable number of files "almost 100,000" were of potential interest.

Speed data files were downloaded from ONE-ITS archives. The procedure required selecting a loop detector name from the ONE-ITS website and then detailed reports were downloaded. These were saved in csv format. Checks were made to reduce the probability of error and the most accurate and updated data version was then used for testing and validating the developed model.

Matlab script was utilized as the operating machine to develop the associated code needed for the model applications. To make the developed Matlab script capable of reading speed data, it was required to convert files from csv format to xlsx format. In order to overcome software limitations, the conversion process was accelerated by utilizing more than one computer to carry out the conversion task. Eventually, speed data was successfully downloaded and ready for further data analysis. 


\subsection{Data Description}

The sortation and random selection of obtained traffic and incident data required a good understanding of the attributes of each variable of the derived database. The sensor and incident data collection process was described in the previous section. A brief description of the derived variables and their implications for incident detection model development are noted in the following subsections.

\subsubsection{Incident Data}

In order to develop a model capable of predicting real life incidents under different incident scenarios such as vehicles collision and disabled vehicles, the obtained incident data should include sufficient information about the incident. Table 3.2 shows a sample of acquired incident information and more details are given below as a typical incident information list.

\section{Typical Incident Information}

A typical incident record would include the following information:

- Location

- Latitude and longitude

- Stream

- Direction

- Recorded-time (when the incident was first recorded)

- Event type or reason (incident or disabled vehicle)

- Affected number of lanes due to the incident 
Table 3.2: Typical incident information

\begin{tabular}{|c|c|c|c|c|c|c|c|}
\hline 旁 & 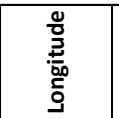 & 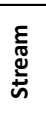 & 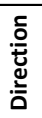 & 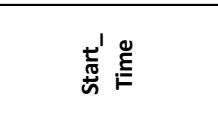 & 등 & 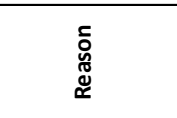 & 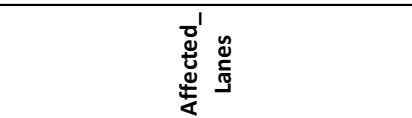 \\
\hline 43.72286 & -79.48148 & $\mathrm{C}$ & W & 2011-01-11 12:18:54 & 401 & Collision & LEFT SHOULDER AND 1 LEFT LANE(S) \\
\hline 43.77496 & -79.28477 & $E$ & W & 2011-01-11 13:16:09 & 401 & Disable Vehicle & 1 RIGHT LANE(S) AND RIGHT SHOULDER \\
\hline 43.72825 & -79.45803 & $\mathrm{C}$ & W & 2011-01-11 15:21:11 & 401 & Collision & 2 RIGHT LANE(S) \\
\hline 43.76316 & -79.38696 & $E$ & $E$ & 2011-01-11 23:29:35 & 401 & Collision & 1 RIGHT LANE(S) \\
\hline 43.71647 & -79.518 & $E$ & $\mathrm{E}$ & 2011-01-12 00:09:47 & 401 & Disable Vehicle & 1 RIGHT LANE(S) \\
\hline 43.70936 & -79.55217 & $\mathrm{E}$ & $\mathrm{E}$ & 2011-01-12 00:24:31 & 401 & Collision & 1 RIGHT LANE(S) \\
\hline 43.72998 & -79.44901 & $\mathrm{E}$ & W & 2011-01-12 06:05:49 & 401 & Collision & 2 RIGHT LANE(S) \\
\hline 43.71656 & -79.51874 & $\mathrm{E}$ & W & 2011-01-12 08:28:24 & 401 & Collision & LEFT SHOULDER AND 1 LEFT LANE(S) \\
\hline 43.74247 & -79.42291 & $E$ & $E$ & 2011-01-12 08:43:30 & 401 & Collision & 1 LEFT LANE(S) \\
\hline 43.72286 & -79.48148 & $\mathrm{C}$ & W & 2011-01-12 09:59:55 & 401 & Collision & 1 RIGHT LANE(S) AND RIGHT SHOULDER \\
\hline
\end{tabular}

- Total number of lanes

A sample of 40 to 50 incidents would be enough to satisfy the statistical requirements.

For use in modelling, key characteristics of incidents should be known. These are described next.

\section{Incident Route Location}

Incident data are recorded for 400 series highways in Ontario, including 400, 401, and 404. Such data are also complied for other highways including the QEW. For instance, incident data recorded for February 2012 includes incidents reported for highways 401, 400, 403, 404, 405, 405, 406, 409, 410, 427, 407 ETR, Hwy11, Hwy 115, Hwy 118, Hwy 12, Hwy 15, Hwy 17, Hwy 26, Hwy 28, Hwy 3, Hwy 35, Hwy 41, Hwy 417, Hwy 58, Hwy 6, Hwy 60, Hwy 62, Hwy 7, Hwy 7/12, Hwy 7A, Hwy 89, Kingston RD, and QEW.

\section{Stream}

The data consists of incidents that occurred in several streams including, express, collectors, express ramp, collector ramp, HOV lane, on ramp, off ramp, and transfer lane. 


\section{Incident Direction}

The data consists of different directions including, eastbound, westbound, northbound, southbound, both directions, Fort Erie bound, Niagara bound, and Toronto bound.

\section{Incident Recorded-Time}

Incident recorded time reported by the transportation control centre often does not provide the exact time when the incident was first recorded. Typically, a single incident is recorded for a given alarm defined by the utilized algorithm; the specific recording will depend on the circumstances (e.g., the location of the incident, and the experience of the staff assigned to keeping records. Incident data obtained from ONE-ITS system indicates that incidents' start times were supplemented manually by traffic operators, and they were stored in the database

\section{Incident Year}

Incident data files were obtained from One-ITS (University of Toronto) for 2010, 2011, 2012, 2013, and 2014. For each year, 12 months of data became available.

\section{Incident Reason}

Many reasons are reported as a cause of incidents in the set of acquired data. In addition to the incidents caused by a collision, other causes such as vehicle fire and spill are also included. For instance, February 2012 incidents data shows several reasons such as collision, disabled vehicle, building fire, daily construction, daily maintenance, debris, 
emergency vehicle, ferry outages, flooding, closure, nightly construction, nightly maintenance closure, one time construction, one time maintenance, police investigation, road conditions, spill, test category, utilities work, vehicle fire, bridge maintenance, ferry outages, and long term construction.

\subsubsection{Detector Information}

The incident data was intended to be used in the developed mode. Also, upstream speed data captured by detectors was of interest. Typical detector information shown in Table 3.3 includes details noted below.

\section{Table 3.3: Typical detector information}

\begin{tabular}{|c|c|c|c|c|c|}
\hline Detector & Address & Status & Longitude & Latitude & Action \\
\hline 400DN0110DSS & 400-SS - At Steeles & inactive & -79.53582856 & 43.77568454 & Volumes (30 mins)SpeedsOccupancyView Chart \\
\hline 400DN0120DNS & 400-NS - At Langstaff & active & -79.54380137 & 43.8106779 & Volumes (30 mins)SpeedsOccupancyView Chart \\
\hline 400DN0120DSS & 400-SS - S. of 407 & active & -79.5368487 & 43.77974263 & Volumes (30 mins)SpeedsOccupancyView Chart \\
\hline 401DE0010DEC & 401-EC - AT KEELE & active & -79.48448851 & 43.72157548 & Volumes (30 mins)SpeedsOccupancyView Chart \\
\hline 401DE0010DEE & 401-EE - W OF KEELE & active & -79.48456517 & 43.72174103 & Volumes (30 mins)SpeedsOccupancyView Chart \\
\hline
\end{tabular}

\section{Typical Detector Information}

- Detector name

- Detector address

- Detector status

- Detector coordinate

- Action

These detector information items are explained in the following subsections. 


\section{Detector Name}

Detectors are identified by their name, which is composed of 12 characters as shown in Figure 3.5. These include highway name, relative location, detector ID, direction, and stream.

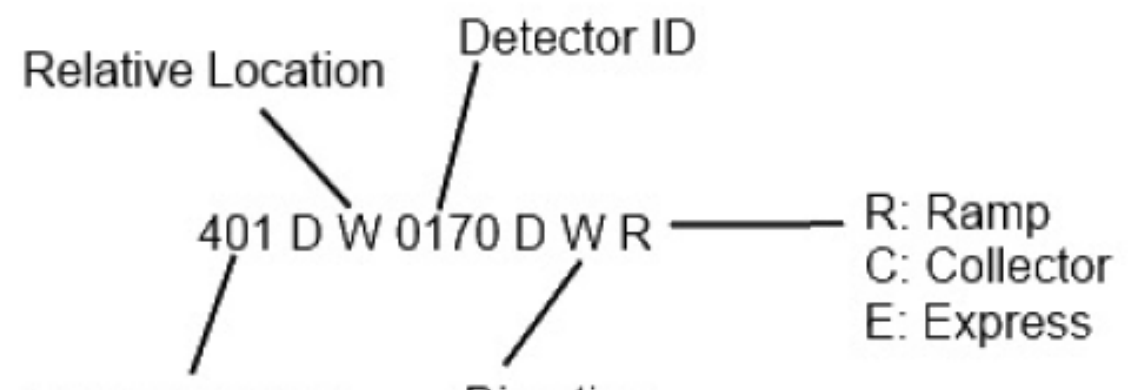

Highway Name Direction

Figure 3.5: Detector naming convention (One-ITS)

Highway name (3 characters): Detectors are located along the Hwy 400 series, and other highways, and the name is given according to the detector location. It can be any of the 400 highway series, but in this study highway 401 was selected for data acquisition purposes.

Character D: is fixed for all detectors.

Detector ID: which is defined by four digits.

Direction (relative location): it indicates the traffic direction at the site of the detector, only the East (E) and West (W) directions were selected in this research.

Detector Type: it indicates which stream the detector is located at; two streams were considered, the express (Es) and the collectors (C). 
In addition, detector information is available for the years 2010, 2011, 2012, 2013, 2014 and few months of the year 2015. This information covers 12 months of each year.

\section{Detector address}

It includes the detector information such as the highway number, direction, stream, and road name.

\section{Detector status}

It indicates whether the detector is active or not active.

\section{Detector coordinates}

It indicates the coordinates of the detector regarding the longitude and latitude.

\section{Action}

It shows the different variables that were recorded by the detector such as speed, occupancy, and volume.

\subsubsection{Traffic Flow Variables-Speed-Occupancy-Volume}

Sensor data obtained from detectors consist of traffic measurements that are recorded every 20 seconds for the 24 hours of every day in the specified study year; this includes speed, volume, and occupancy; also, traffic volume is recorded every 30 minutes. For consistency in illustrating traffic results, the 20 second period was selected to determine the hourly traffic rate and occupancy and then present the three traffic databases. 


\subsubsection{Freeway Traffic Speed Data}

Recorded traffic speed has many applications in traffic engineering, including developing speed patterns, analyzing incident data, and investigating traffic incidents. Traffic speed measurements can have a different interpretation depending upon the condition upstream and downstream of the incident location, as well as the detector location. For example, if there is no congestion in the freeway section (or upstream of the incident location) then the traffic speed is equal to the current operating speed. It could be free flow speed, depending upon volume and density. If on the other hand, queuing exists in the freeway section, then the measured speed reflects the downstream bottleneck capacity caused by the incident.

Four data files were reported by detectors that include traffic measurements. The first file is characterized by the number (2) and represents the speed. Speed is regarded as the most important measurement for the application of the developed incident detection model. Speed expresses the rate at which traffic is moving and, therefore, is a reasonable measure of the current traffic conditions. As would be expected, a freeway incident affects traffic speed. Vehicle speed upstream of an incident is expected to decrease while downstream speed typically increases or remains the same.

\subsubsection{Occupancy and Volume data}

\section{Occupancy}

In this research, occupancy is calculated as a percentage of time the detection zone of a detector is occupied by vehicles.

An incident will typically cause the density and occupancy to increase upstream and to decrease downstream. 


\section{Volume}

The third traffic variable is volume. Volume is typically defined as the number of vehicles passing a point in a given period of time, usually 1 hour.

The obtained volume data is saved in a data file that is characterized by the number (1), and it presents the traffic volume every $30 \mathrm{~min}$; twice this value is the traffic volume per hour. The second volume data file within the database that is not labelled by any number presents traffic volume per 20 seconds; values obtained from this set of data are converted to vehicles per hour by tripling each value and multiplying it by 60 . Furthermore, the volume at each time interval represents the traffic volume at the end of that period.

A test was conducted to validate the accuracy of acquired volume measurements. Two different volume data files were selected for this test. The first data file included volume measured per 20 seconds and the second one presented volume measured per 30 minutes. The results from adding up the 20 seconds volume measurements to a sum of 30 minutes appeared to be approximately equal to the first value of the 30 minutes volume measurement.

\begin{tabular}{|l|l|l|l|l|l|l|}
\hline Time period & $0: 28: 00$ & $0: 28: 20$ & $0: 28: 40$ & $0: 29: 00$ & $0: 29: 20$ & $0: 29: 40$ \\
hh:mm:ss & & & & & & \\
\hline Volume & 5 & 6 & 3 & 4 & 3 & 4 \\
veh/20 sec & & & & & & \\
\hline
\end{tabular}

Total $/ 30 \min =475$ 
It was found that volume data obtained from the summation of 20 seconds data matched with the volume recorded for the 30 minutes period (i.e. 475 vs. 472 vehicles/30 minutes). Therefore, volume data recorded every 20 seconds was selected for data analysis purposes in this research. The implication of this decision was that the vehicles/hour data was computed from vehicles $/ 20$ seconds (i.e. by using the $* 3 * 60$ factor).

\subsection{Data Screening and Selection}

Before developing the incident detection model, it was necessary to define criteria for screening faulty data. For this step, specific screening techniques were applied to the acquired database as described in the following subsections. The screened data was then prepared according to the proposed criteria.

\subsubsection{Incident Data}

When screening the incident data file, to begin with, all incident types were included. The incident data screening and selection process are explained in the following subsections.

\subsubsection{Route Selection}

In this research, data retrieved from Highway 401 was selected. This highway includes two traffic streams, express lanes and collector lanes. This offered the opportunity to study a variety of incidents. Given the expectation of a sufficient number of incidents for Highway 401, all other highways were excluded from the incident database. 
Based on the 400 randomly selected incident cases, which will be discussed later in the following sections of this chapter, a $55 \mathrm{~km}$ length of Hwy 401 was defined as the facility of interest. It consists of 24 lanes and the latitude and longitude for each direction is recorded as follows:

Eastbound- Collector, from 43.681, -79.637 to $43.837,-79.07$

Eastbound- Express, from 43.6406, -79.657 to $43.8306,-79.088$

Westbound- Collector, from 43.6394, -79.661 to 43.8307, -79.089

Westbound- Express, from 43.6391, -79.661 to 43.8306, -79.089

Overall, the 400 selected cases covered the entire length of the selected freeway section.

\subsubsection{Stream Selection}

In accordance with the study design (i.e. as implied by the scope of research), the high occupancy vehicle (HOV) lanes and traffic interchange facilities such as off-ramp, on-ramp, express ramp, collector ramp and transfer lane were not included in this research. It was reported by Asakura et al (2015) that the speed of vehicles varies significantly in sections located near on/off ramps. The explanation offered was that vehicle speed is likely to be affected by car platoons entering from on-ramp or exiting off- ramps.

Also, some data which did not include any stream information were excluded from the study database. Therefore, data pertaining to the express and collector streams of Hwy 401 were considered within the study streams in the incident data analysis. These were assigned the acronyms (Es \& C), respectively. All other streams were excluded from the database, along with incidents for which the traffic stream designation was missing. 


\subsubsection{Direction}

According to incident data obtained for Hwy 401, it was observed that incidents that occurred in the north and south directions form a small percentage of the total number of incidents. For example, for year 2011, the northbound and southbound traffic flow encountered a total of 13 incidents. Since the purpose of this research is to develop incident detection model and algorithm, not exploring the reasons for higher rate of incidents in some directions, it was decided to exclude incident data for the north bound and south bound directions.

The east and west directions are the dominating directions as was observed in almost all the of the recorded incident data. For instance, sorted data for Hwy 401 obtained for the month of February showed that only east and west directions had incidents. As a result, only incident data pertaining to the east-bound and the west-bound directions of Highway 401 were selected for analysis and were named as (Et \& W) respectively. All other directions were excluded from the incident data analysis.

\subsubsection{Incident Recorded Time}

Incidents are recorded by traffic control centres. Commonly, there is some delay prior traffic operators at the control centre can verify that an incident has occurred. This delay includes the time required for an observer to call the first responders (i.e. the police) and/or an alert obtained for an algorithm, and time required to re-point a surveillance camera to investigate if an incident has taken place at the specified time. This process is subject to human error, and there could be some delay before traffic operators ultimately record the 
incident within the incident database (Corens, Verduyn, Schevernels, De Valck, \& Organe, 2011).

Although the incident recorded time is provided within the incident database, it was observed while processing the incident database that in many cases incident recorded time was not accurately recorded. However, in some cases, the incident time was reasonably accurately recorded by the traffic control centres, possibly in cases when a telephone call regarding the time of incident occurrence was correctly recorded. On the other hand, in many other cases, the recorded time seems to be inaccurately recorded. For example, the recorded time was observed to be before the real-time when someone could call about a possible occurrence of an incident. This imprecisely recorded time feature implied that the incident recorded time is unreliable.

Consequently, it was decided to screen unreliable data by not including inaccurate recording of incident time within the analysis process. In order to overcome this data deficiency, it was considered reasonable to employ the incident recorded time as a reference for establishing a range of time within which it was highly likely that an incident occurred without being precise about the exact moment of the incident occurrence.

In chapter (5) incident time stamp included in the incident database is used as an ID to identify the name of an incident during the incident detection process. This is presented as one of the attached annotations with every output chart that shows speed profile during an incident occurrence. This time is also used to set the two boundary lines that border the time frame within which it is certain that an incident occurs. 


\subsubsection{Selection Criteria for the Study Year}

The obtained incident information incorporates the 12-month incident data for the years $2010,2011,2012,2013$, and 2014. Selecting the year depended on the quality of incident data, which was considered of foremost importance in the selection criteria. A qualitative statistical analysis was conducted to choose the year that comprised the largest number of complete incident data. It was found that incident data was missing for most of the months for the year 2010; incidents were noted only for November and December of 2010. Therefore, data for the year 2010 was excluded from the study analysis.

For the next step of the study year selection criteria, the study focused only on the years 2011, 2012, 2013 and 2014. Table 3.4 indicates the number of recorded incidents in each year and provides a simple description of the data quality. As can be observed, some years include a lower number of incidents while other include a large number. Also, some incidents data was transferred from one month to the other during the recording process which created an incomplete incident database for some of those months. Months of the year of 2011 showed a considerable number of incidents that were classified as complete. Therefore, the study year of 2011 was selected for the data analysis in this study. 
Table 3.4: Qualitative statistical analysis for selecting study year

\begin{tabular}{|c|c|c|c|c|c|c|c|c|c|c|c|c|c|}
\hline$\underset{\circlearrowright}{\grave{\Xi}}$ & \multicolumn{3}{|c|}{2011} & \multicolumn{3}{|r|}{2012} & 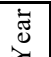 & \multicolumn{3}{|c|}{2013} & \multicolumn{3}{|c|}{2014} \\
\hline 峊 & 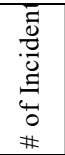 & 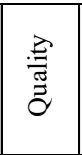 & 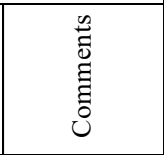 & 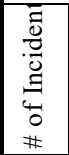 & 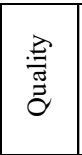 & 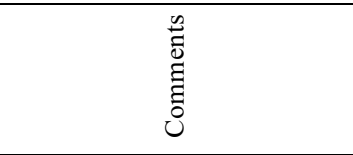 & 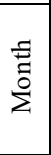 & 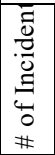 & 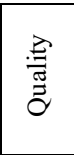 & 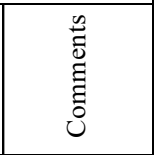 & 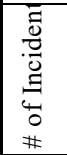 & 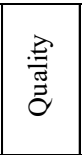 & 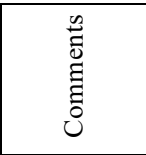 \\
\hline 1 & 708 & $\begin{array}{l}\text { Very } \\
\text { Good } \\
\end{array}$ & No & 1134 & Good & Cleared from null data & 1 & 131 & $\begin{array}{l}\text { Very } \\
\text { Good }\end{array}$ & No & 387 & $\begin{array}{l}\text { Very } \\
\text { Good }\end{array}$ & No \\
\hline 2 & 1227 & $\begin{array}{l}\text { Very } \\
\text { Good }\end{array}$ & No & 1083 & $\begin{array}{l}\text { Very } \\
\text { Good }\end{array}$ & $\begin{array}{l}\text { Cleared from null data. Data } \\
\text { transferred to March }\end{array}$ & 2 & 107 & $\begin{array}{l}\text { Very } \\
\text { Good }\end{array}$ & No & 1517 & $\begin{array}{l}\text { Very } \\
\text { Good }\end{array}$ & No \\
\hline 3 & 1240 & $\begin{array}{l}\text { Very } \\
\text { Good }\end{array}$ & No & 2465 & Good & $\begin{array}{l}\text { Cleared from null data. Data } \\
\text { transferred to April. Quite } \\
\text { Some null in direction }\end{array}$ & 3 & 93 & $\begin{array}{l}\text { Very } \\
\text { Good }\end{array}$ & No & 1486 & $\begin{array}{l}\text { Very } \\
\text { Good }\end{array}$ & No \\
\hline 4 & 1010 & \begin{tabular}{l|} 
Very \\
Good
\end{tabular} & No & 2713 & Good & $\begin{array}{c}\text { Cleared from null data. Data } \\
\text { transferred to May }\end{array}$ & 4 & 19 & $\begin{array}{l}\text { Very } \\
\text { Good }\end{array}$ & $\begin{array}{c}\text { Not enough } \\
\text { incidents }\end{array}$ & 1316 & $\begin{array}{l}\text { Very } \\
\text { Good }\end{array}$ & No \\
\hline 5 & 1669 & $\begin{array}{l}\text { Very } \\
\text { Good }\end{array}$ & No & 2527 & $\begin{array}{l}\text { Very } \\
\text { Good }\end{array}$ & $\begin{array}{l}\text { Cleared from null data. Data } \\
\text { transferred to June. Quite } \\
\text { Some null in direction }\end{array}$ & 5 & 11 & $\begin{array}{l}\text { Very } \\
\text { Good }\end{array}$ & $\begin{array}{c}\text { Not enough } \\
\text { incidents }\end{array}$ & 1049 & $\begin{array}{l}\text { Very } \\
\text { Good }\end{array}$ & No \\
\hline 6 & 3061 & $\begin{array}{l}\text { Very } \\
\text { Good }\end{array}$ & No & 4123 & $\begin{array}{l}\text { Very } \\
\text { Good }\end{array}$ & $\begin{array}{c}\text { Cleared from null data. Data } \\
\text { transferred to July. Some null } \\
\text { in direction }\end{array}$ & 6 & 1 & $\begin{array}{l}\text { Very } \\
\text { Good }\end{array}$ & $\begin{array}{c}\text { Not enough } \\
\text { incidents }\end{array}$ & 795 & $\begin{array}{l}\text { Very } \\
\text { Good }\end{array}$ & No \\
\hline 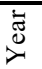 & \multicolumn{3}{|c|}{2011} & \multicolumn{3}{|r|}{2012} & 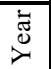 & \multicolumn{3}{|c|}{2013} & \multicolumn{3}{|c|}{2014} \\
\hline 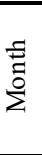 & $\begin{array}{c}\overline{0} \\
: \bar{J} \\
\Xi \\
\Xi \\
0 \\
\# \\
\#\end{array}$ & $\stackrel{\Xi}{\stackrel{\Xi}{\Xi}}$ & 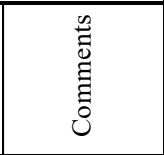 & $\begin{array}{l}\overline{0} \\
\frac{0}{0} \\
0 \\
\Xi \\
0 \\
0 \\
\# \\
\#\end{array}$ & 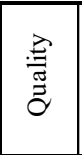 & 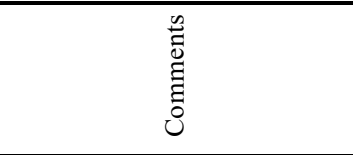 & 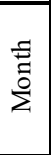 & 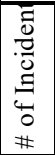 & 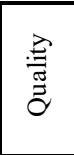 & 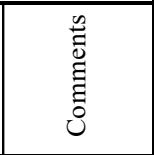 & 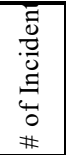 & 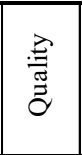 & 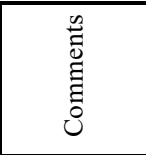 \\
\hline 7 & 2835 & $\begin{array}{l}\text { Very } \\
\text { Good } \\
\end{array}$ & No & 1971 & Poor & $\begin{array}{c}\text { Cleared from null data. } \\
\text { Direction is Null }\end{array}$ & 7 & 1 & $\begin{array}{l}\text { Very } \\
\text { Good }\end{array}$ & $\begin{array}{c}\text { Not enough } \\
\text { incidents }\end{array}$ & 1558 & $\begin{array}{l}\text { Very } \\
\text { Good } \\
\end{array}$ & No \\
\hline 8 & 3189 & $\begin{array}{l}\text { Very } \\
\text { Good }\end{array}$ & $\begin{array}{c}\text { Cleared from } \\
\text { null data }\end{array}$ & 1853 & Poor & $\begin{array}{l}\text { Cleared from null data. } \\
\text { Direction is Null }\end{array}$ & 8 & 1 & $\begin{array}{l}\text { Very } \\
\text { Good }\end{array}$ & $\begin{array}{c}\text { Not enough } \\
\text { incidents }\end{array}$ & 723 & $\begin{array}{l}\text { Very } \\
\text { Good }\end{array}$ & No \\
\hline 9 & 2050 & \begin{tabular}{l|} 
Very \\
Good
\end{tabular} & $\begin{array}{c}\text { Cleared from } \\
\text { null data }\end{array}$ & 821 & Poor & $\begin{array}{c}\text { Cleared from null data. } \\
\text { Direction is Null }\end{array}$ & 9 & 2 & $\begin{array}{l}\text { Very } \\
\text { Good }\end{array}$ & $\begin{array}{c}\text { Not enough } \\
\text { incidents }\end{array}$ & 13 & $\begin{array}{l}\text { Very } \\
\text { Good }\end{array}$ & $\begin{array}{c}\text { Not enough } \\
\text { incidents }\end{array}$ \\
\hline 10 & 1553 & $\begin{array}{l}\text { Very } \\
\text { Good } \\
\end{array}$ & $\begin{array}{c}\text { Cleared from } \\
\text { null data }\end{array}$ & 0 & Poor & & 10 & 5 & $\begin{array}{l}\text { Very } \\
\text { Good }\end{array}$ & $\begin{array}{c}\text { Not enough } \\
\text { incidents }\end{array}$ & 6 & $\begin{array}{l}\text { Very } \\
\text { Good } \\
\end{array}$ & $\begin{array}{c}\text { Not enough } \\
\text { incidents }\end{array}$ \\
\hline 11 & 363 & $\begin{array}{l}\text { Very } \\
\text { Good } \\
\end{array}$ & No & 7 & Poor & & 11 & 812 & $\begin{array}{l}\text { Very } \\
\text { Good }\end{array}$ & No & 1290 & $\begin{array}{l}\text { Very } \\
\text { Good } \\
\end{array}$ & No \\
\hline 12 & 1127 & \begin{tabular}{l|} 
Very \\
Good
\end{tabular} & No & 332 & \begin{tabular}{l|} 
Very \\
Good
\end{tabular} & & 12 & 77 & $\begin{array}{l}\text { Very } \\
\text { Good }\end{array}$ & No & 1386 & $\begin{array}{l}\text { Very } \\
\text { Good }\end{array}$ & No \\
\hline
\end{tabular}

\subsubsection{Incident Reason Selection}

In order to enhance the impact of this research, it was decided to include incidents caused by collisions and incidents caused by disabled vehicles. The database was analyzed in order to separate incidents caused by these reasons from other incidents. 
For the development of a database that was intended to be used for model testing, special care was exercised to ensure that these cases were indeed incident-free and did not show any effect of any incident in adjacent time periods.

As a part of the process of developing the incident detection model, collision and disabled vehicle type of incidents were assigned a reason code of 1 so as to distinguish these from all other incident types. Next code 0 was assigned to all other types of incidents caused by other reasons. This code was applied to correctly distinguish time periods when selecting the time when no incident was recorded. This analysis will be discussed further in the next chapter.

To clarify further, in the user interface (GUI) of the developed model, code 1 incidents are used for testing the model to detect incidents. The code 0 incidents provide a basis for selecting time periods for which no incidents were recorded.

In order to improve the database for developing and testing the incident detection model, it was considered important to select locations and times that were suitable for these purposes. The application of codes as noted above was helpful in achieving objectives.

\subsubsection{Detector (Sensor) Data}

In this part of the thesis document, an explanation is provided of the reasons for giving preference to consider the closest upstream detectors in comparison to the downstream detectors. 


\subsubsection{Detector Selection Criteria}

Detector selection criteria that explain which detector should be considered more important than other detectors when reading traffic data are incident location and direction. Location is defined as the distance from incident and direction implies upstream of incident or downstream of incident.

The distance between the location of an incident and every detector mounted along the freeway is a major factor and is considered the first parameter in the selection criteria. The distance between the detector and the incident was calculated based on the theoretical dimensions without taking into account the exact route length. Traffic that is closest to the incident is high likely to be impacted by the occurrence of the incident. The fact that detectors are commonly installed about $2 \mathrm{~km}$ apart suggests that the maximum possible distance a detector can be away from the incident is almost always less than $2 \mathrm{~km}$.

\section{Upstream and Downstream Traffic}

The second parameter that affects the selection criteria is the location of the detector in relation to the incident. Detectors usually can be upstream or downstream of the incident location. Downstream detectors are not reliable indicators since the choice of speed of vehicles following their passage out of the incident location is highly subjective. On the other hand, it is known that a shock wave of speed reduction travels upstream. Therefore, in this research, in accordance with the prior knowledge in this subject, it was decided to focus only the closest upstream detector from the incident location for traffic data selection. Therefore for model development detectors that are beyond the second upstream detector were not given importance. 
Detectors record traffic data on speed, volume, and occupancy and send the information to the traffic control centre with a time stamp. With well-identified detector information and speed data which includes date and time, it was possible to determine the speed of the upstream traffic. The traffic data is recorded by all the detectors installed in the highway over time (i.e. on an on-going basis) it was important to label the data correctly for each detector.

Another important requirement was that incidents should be identified by their recorded date and time for the specified study year. This enabled the developed model to investigate any recognized drop in speed as the first indication of any potential of incident occurrence.

\subsubsection{Speed}

Speed was regarded as the most important variable for developing and validating the developed model. Therefore, it was necessary to define criteria for screening loop data errors or loop data missing events.

It was observed that for some time periods the recorded speed data was missing. It showed as an $(\mathrm{X})$ in the obtained database or at other time periods or no speed data was recorded. In such cases, the time period with missing or insufficient data was excluded from the analysis and the next time period was considered when screening the speed data. The percentage of missing speed data including (empty or X speed measurements) was calculated. A suggested scale was determined. If the missing speed data was relatively large, the detector under consideration was excluded, and data from the next upstream detector was observed. 
It was noted that at some other time intervals the speed measurement was incorrect, it was wrongly recorded as $2 \mathrm{~km} / \mathrm{h}$ or less, and some cases showed negative speed values as well. In most cases these misleading low-speed values were not following the average free flow speed pattern and therefore, they were considered as skewed values and were omitted from the speed data list. As illustrated in chapter (5), the mechanism of the developed model will first permit to read all speed values from the available speed database within the specified time boundaries. But, time periods that include speed values which are less than or equal to $2 \mathrm{~km} / \mathrm{h}$ will be screened and excluded. Also, all time periods that contain X or empty cell were extracted from the speed database. In model application, all these conditions will become apparent when plotting the time/speed relationship profile.

Table 3.5 presents a typical speed data sample that includes a missing speed value or a speed value that is less than or equal $2 \mathrm{~km} / \mathrm{h}$. The tabulated data was collected from the month of December of 2011; speed values are shown for the first five days of the month.

As can be observed from Table 3.5, on Dec 03, at time 23:58:20, the recorded speed value is $1 \mathrm{~km} / \mathrm{h}$, this is regarded as a false speed recording and was ultimately omitted from the screened speed database.

Another type of data that recorded false speed values was also excluded from the screening process. In these cases, speed values were recorded as a fixed value of $126 \mathrm{~km} / \mathrm{h}$, and the speed values indicated a false recording. Likewise, these time periods were excluded from the screening process. In other cases, speed readings show a sudden drop in the speed followed by a quick speed recovery. If this decline in the speed reaches a certain threshold such as $60 \mathrm{~km} / \mathrm{h}$ (later on this value is considered as an input for model tuning 


\section{Table 3.5: Typical unscreened speed data sample}

\begin{tabular}{|r|r|r|r|r|r|}
\hline Time & 01-Dec-2011 & O2-Dec-2011 & 03-Dec-2011 & 04-Dec-2011 & 05-Dec-2011 \\
\hline $23: 54: 00$ & 67 & 111 & 115 & 120 & 110 \\
\hline $23: 54: 20$ & 133 & 101 & 128 & 102 & \\
\hline $23: 54: 40$ & 102 & 73 & 107 & 128 & 118 \\
\hline $23: 55: 00$ & 93 & 119 & 103 & 127 & 94 \\
\hline $23: 55: 20$ & 119 & 106 & 127 & 112 & 100 \\
\hline $23: 55: 40$ & 128 & 125 & 113 & 114 & 105 \\
\hline $23: 56: 00$ & 106 & $X$ & 114 & 104 & 120 \\
\hline $23: 56: 20$ & 92 & 117 & 111 & 108 & 97 \\
\hline $23: 56: 40$ & 120 & 142 & 126 & 126 & 108 \\
\hline $23: 57: 00$ & 125 & 121 & 104 & 128 & 108 \\
\hline $23: 57: 20$ & 122 & 116 & 114 & 158 & 77 \\
\hline $23: 57: 40$ & 144 & 113 & 109 & 99 & 88 \\
\hline $23: 58: 00$ & 133 & 94 & 95 & 107 & 64 \\
\hline $23: 58: 20$ & 100 & 125 & 117 & 1 & 116 \\
\hline $23: 58: 40$ & 99 & 110 & 102 & 132 & 105 \\
\hline $23: 59: 00$ & 126 & 103 & 124 & 0 & 108 \\
\hline $23: 59: 20$ & 106 & 124 & 111 & 108 & 100 \\
\hline
\end{tabular}

purposes), then this speed reading must be excluded from the data analysis. The same procedure was applied to screen the volume and occupancy data.

The speed, volume, and occupancy data are processed by the developed model and an output file that includes the screened data is generated. This output file will list all the time intervals, and the speed, volume, and occupancy values. At some time intervals where a reasonable volume and occupancy readings exist, and no speed value was recorded, the speed value will appear as zero in the final format of the output file. Therefore, when a time period includes speed value less than or equal to $2 \mathrm{~km} / \mathrm{h}$, empty cell or X value and is considered in the output file, this speed value appears as 0 in the output. 
The above considerations resulted in the data selection criteria for the closest upstream detector. These include speed records with a minimum percentage of false readings such as $(0$, nil, or $\mathrm{x})$ for use by the incident detection application model.

\subsection{Incident Data-Sortation and Random Draw}

On most freeways, traffic speed varies throughout the day and by direction. In addition, freeways exhibit monthly variations in traffic speed that are dependent on the freeway type and location. These variations are a function of different variables such as seasons, peak, off-peak hours, weekdays, weekends, and time of the day. Incidents data sortation should consider various combinations of these variables. These variables will define each incident and are considered as an incident information.

Following the preparation of the incident database that is free of errors, a random selection is to be used to obtain incident data for modelling purposes.

\subsubsection{Sortation and Random Selection}

The following steps are required for the random selection of incidents.

a- Obtain the database that is screened for faults and sorted for comprehensive incident information.

b- The database should include approximately equal number of selected cases that exhibit following incident characteristics: month of the year, east, west, express, collector, weekday, weekend, peak and off-peak periods (a.m. \& p.m.)

c- Use random numbers to select 100 cases. The 100 cases will be randomly selected from the screened database. 
d- Use the random selection method to choose 100 cases those are primarily known to have an incident and also use the random selection method to select 100 cases that are known not to have any incident. In the latter case, incident free condition time period is a requirement.

e- Now, from the 100 case database, select 50 cases for testing the model and fine tuning calibration and use the other 50 cases for model validation purposes.

\subsubsection{Sortation Technique}

Since the year of 2011 was selected for the incident detection application, incidents data from each month of 2011 were added. These amounted to 1469 incidents. In order to bring these incidents data to the final sorting arrangement that is required to apply the random draw, data sortation criteria are needed to simplify this approach.

The following is a description of the sortation criteria.

1- Incident data was sorted based on, month, season, direction, stream, weekday-weekend, peak and off-peak hour, a.m. - p.m., and incident start time. In Table 3.6 a sample of 32 incident cases is presented (out of the 1469 cases). These belong to the month of January as part of the winter season and apply to the collector stream that extends in the east direction. These incidents occurred in WD, WE, peak and off-peak hours. 
Table 3.6: Sample of incident data sortation

\begin{tabular}{|c|c|c|c|c|c|c|c|c|c|c|}
\hline 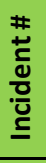 & 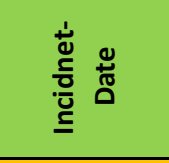 & 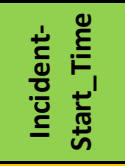 & 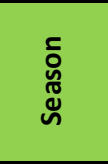 & 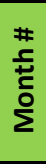 & 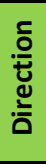 & 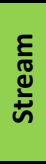 & $\begin{array}{l}\text { س } \\
\text { ò } \\
3\end{array}$ & 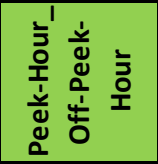 & $\frac{\varepsilon}{\frac{g}{\frac{1}{\delta}}}$ & 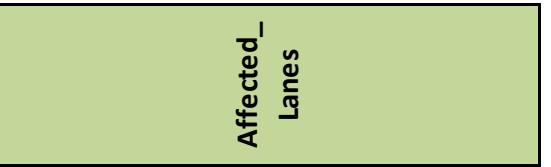 \\
\hline 1 & 10-Jan-2011 & $8: 29: 00$ & Winter & 1 & $E$ & $\mathrm{C}$ & WD & 1-PH am & AM & 1 right lane \\
\hline 2 & 28-Jan-2011 & 6:53:07 & Winter & 1 & $E$ & $\mathrm{C}$ & WD & 1-PH am & AM & 1 LEFT LANE(S) \\
\hline 3 & 28-Jan-2011 & 9:47:51 & Winter & 1 & $E$ & $\mathrm{C}$ & WD & 1-PH am & AM & 1 RIGHT LANE(S) \\
\hline 4 & 5-Jan-2011 & $18: 26: 00$ & Winter & 1 & $E$ & $\mathrm{C}$ & WD & 2-PH pm & PM & 1 right lane \\
\hline 5 & 13-Jan-2011 & 16:00:59 & Winter & 1 & $E$ & $\mathrm{C}$ & WD & 2-PH pm & PM & 1 LEFT LANE(S) \\
\hline 6 & 21-Jan-2011 & $16: 21: 11$ & Winter & 1 & $E$ & $\mathrm{C}$ & WD & 2-PH pm & PM & 1 RIGHT LANE(S) AND RIGHT SHOULDER \\
\hline 7 & 31-Jan-2011 & 17:06:46 & Winter & 1 & $E$ & $\mathrm{C}$ & WD & 2-PH pm & PM & 2 LEFT LANE(S) \\
\hline 8 & 10-Jan-2011 & 6:13:00 & Winter & 1 & $E$ & $\mathrm{C}$ & WD & 3a-OPH am & AM & 1 centre lane \\
\hline 9 & 13-Jan-2011 & 3:19:39 & Winter & 1 & $\mathrm{E}$ & $\mathrm{C}$ & WD & 3a-OPH am & AM & 1 RIGHT LANE(S) \\
\hline 10 & 13-Jan-2011 & 11:49:07 & Winter & 1 & $E$ & $\mathrm{C}$ & WD & $3 \mathrm{~b}-\mathrm{OPH}$ am & AM & LEFT SHOULDER AND 1 LEFT LANE(S) \\
\hline 11 & 20-Jan-2011 & 11:50:08 & Winter & 1 & $E$ & $\mathrm{C}$ & WD & 3b-OPH am & AM & 1 RIGHT LANE(S) \\
\hline 12 & 3-Jan-2011 & $23: 10: 00$ & Winter & 1 & $E$ & $\mathrm{C}$ & WD & $4 \mathrm{~b}-\mathrm{OPH} \mathrm{pm}$ & PM & 1 right lane and right shoulder \\
\hline 13 & 14-Jan-2011 & $22: 26: 12$ & Winter & 1 & $E$ & $\mathrm{C}$ & WD & 4b-OPH pm & PM & 2 RIGHT LANE(S) AND RIGHT SHOULDER \\
\hline 14 & 21-Jan-2011 & 19:10:19 & Winter & 1 & $E$ & $\mathrm{C}$ & WD & 4b-OPH pm & PM & 2 RIGHT LANE(S) AND RIGHT SHOULDER \\
\hline 15 & 21-Jan-2011 & $19: 37: 25$ & Winter & 1 & $E$ & $\mathrm{C}$ & WD & 4b-OPH pm & PM & 1 LEFT LANE(S) \\
\hline 16 & 27-Jan-2011 & $20: 45: 15$ & Winter & 1 & $E$ & $\mathrm{C}$ & WD & 4b-OPH pm & PM & 1 RIGHT LANE(S) AND RIGHT SHOULDER \\
\hline 17 & 31-Jan-2011 & 19:17:06 & Winter & 1 & $\mathrm{E}$ & $\mathrm{C}$ & WD & $4 \mathrm{~b}-\mathrm{OPH} \mathrm{pm}$ & PM & 1 RIGHT LANE(S) \\
\hline 18 & 31-Jan-2011 & 19:27:10 & Winter & 1 & $E$ & $\mathrm{C}$ & WD & $4 \mathrm{~b}-\mathrm{OPH} \mathrm{pm}$ & PM & 2 RIGHT LANE(S) \\
\hline 19 & 15-Jan-2011 & $6: 33: 53$ & Winter & 1 & $E$ & $\mathrm{C}$ & WE & 1-PH am & AM & LEFT SHOULDER AND 1 LEFT LANE(S) \\
\hline 20 & 15-Jan-2011 & 6:52:32 & Winter & 1 & $\mathrm{E}$ & $\mathrm{C}$ & WE & 1-PH am & AM & 2 LEFT LANE(S) \\
\hline 21 & 15-Jan-2011 & $8: 29: 30$ & Winter & 1 & $E$ & $\mathrm{C}$ & WE & 1-PH am & AM & LEFT SHOULDER AND 2 LEFT LANE(S) AND 1 \\
\hline 22 & 15-Jan-2011 & 9:13:40 & Winter & 1 & $\mathrm{E}$ & $\mathrm{C}$ & WE & 1-PH am & AM & 1 LEFT LANE(S) \\
\hline 23 & 15-Jan-2011 & 9:21:35 & Winter & 1 & $\mathrm{E}$ & $\mathrm{C}$ & WE & 1-PH am & AM & 1 RIGHT LANE(S) AND RIGH \\
\hline 24 & 2-Jan-2011 & $15: 37: 00$ & Winter & 1 & $E$ & $\mathrm{C}$ & WE & 2-PH pm & PM & Right shoulder \\
\hline 25 & 2-Jan-2011 & $17: 45: 00$ & Winter & 1 & $E$ & $\mathrm{C}$ & WE & 2-PH pm & PM & 1 left lane \\
\hline 26 & 15-Jan-2011 & $15: 30: 50$ & Winter & 1 & $\mathrm{E}$ & $\mathrm{C}$ & WE & 2-PH pm & PM & 1 RIGHT LANE(S) \\
\hline 27 & 30-Jan-2011 & $15: 32: 30$ & Winter & 1 & $E$ & $\mathrm{C}$ & WE & 2-PH pm & PM & 1 RIGHT LANE(S) AND RIGHT SHOULDER \\
\hline 28 & 1-Jan-2011 & $2: 57: 00$ & Winter & 1 & $E$ & $\mathrm{C}$ & WE & 3a-OPH am & AM & Left shoulder and 2 left lanes \\
\hline 29 & 15-Jan-2011 & $11: 10: 00$ & Winter & 1 & $\mathrm{E}$ & $\mathrm{C}$ & WE & 3b-OPH am & AM & LEFT SHOULDER AND 2 LEFT LANE(S) \\
\hline 30 & 15-Jan-2011 & 11:57:19 & Winter & 1 & $E$ & $\mathrm{C}$ & WE & 3b-OPH am & AM & 2 LEFT LANE(S) \\
\hline 31 & 2-Jan-2011 & $19: 47: 00$ & Winter & 1 & $E$ & $\mathrm{C}$ & WE & 4b-OPH pm & PM & All lanes closed \\
\hline 32 & 16-Jan-2011 & 19:11:10 & Winter & 1 & $E$ & $\mathrm{C}$ & WE & 4b-OPH pm & PM & 1 RIGHT LANE(S) \\
\hline
\end{tabular}

Peak hour traffic was selected for the a.m. and p.m. peak periods. Officially, the morning peak period is from 6:30 a.m. to 10:00 a.m. In the case of the P.M. peak traffic, the following rationale was used to define peak conditions. Based on observations conducted by the Toronto Transit Commission (TTC), demand tops out between 5:00 p.m. and 6:00 p.m., several hours after the highways are most congested from 3:30 p.m. to 4:30 
p.m. Therefore, the evening peak traffic was selected starting from 3:30 p.m. and ending at 7:00 p.m. From the perspective of traffic operation personnel, this period is the most critical for dealing with traffic incidents. This is when the incidents cause the greatest traffic disruption (Corens et al., 2011).

Excel software was used to develop a simple code for incident data selection. The following statements define the method and the code is presented in Appendix A.

- If statement': used to find out peak hours and off-peak hours (PH, OPH)

- If statement': used for specifying the incident seasons

- If statement': used for defining the weekdays and weekends (WD-WE)

- Statement': used for describing the weekdays and weekends regarding numbers (where Sunday $=1$, Monday $=2$, Tuesday $=3$, Wednesday $=4$, Thursday $=5$, Friday $=6$, Saturday $=7$

- Statement': used for converting the date-time to (date value)

2- Out of the 1469 cases, 400 cases were needed for further random draw. The 400 cases were selected based on consistent selection criteria that ultimately included most of the possible available incident data attribute combination as shown in Table 3.7.

3- Data in Table 3.7 reflects a managed combination of 100 incident cases. Moreover, the data show that incidents occurred in the express stream of the east direction for all the 12 months of 2011 (i.e. one out of four other categories). Similarly, the other three categories include 100 incident cases that were selected from the express stream of the west direction. Also, 100 incident cases were selected from each of the collectors of the east and west directions respectively. Overall 400 incident cases were cautiously chosen for the year 2011. 
Table 3.7: Incident data information combination

\begin{tabular}{|c|c|c|c|c|c|c|c|c|c|c|c|c|c|}
\hline \multicolumn{14}{|c|}{2011} \\
\hline \multicolumn{14}{|c|}{ Express-East } \\
\hline WD/WE & Cases & Jan & Feb & Mar & Apr & May & Jun & Jul & Aug & Sep & Oct & Nov & Dec \\
\hline \multirow{4}{*}{ 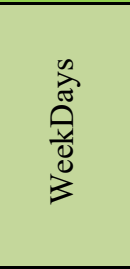 } & 1-PH am & 2 & 2 & 1 & 2 & 2 & 1 & 2 & 2 & 1 & 2 & 2 & 1 \\
\hline & 2-PH pm & 1 & 1 & 2 & 1 & 1 & 2 & 1 & 1 & 2 & 1 & 1 & 2 \\
\hline & 3a-OPH am & 2 & 2 & 1 & 2 & 2 & 1 & 2 & 2 & 1 & 2 & 2 & 1 \\
\hline & 4a-OPH pm & 1 & 1 & 2 & 1 & 1 & 2 & 1 & 1 & 2 & 1 & 1 & 2 \\
\hline \multirow{4}{*}{$\begin{array}{l}\frac{n}{0} \\
\text { 式 } \\
\frac{1}{0} \\
3\end{array}$} & 1-PH am & 1 & 1 & 0 & 1 & 1 & 0 & 1 & 1 & 0 & 1 & 1 & 0 \\
\hline & 2-PH pm & 0 & 1 & 1 & 0 & 1 & 1 & 0 & 1 & 1 & 0 & 1 & 1 \\
\hline & 3a-OPH am & 1 & 1 & 0 & 1 & 1 & 0 & 1 & 1 & 0 & 1 & 1 & 0 \\
\hline & 4a-OPH pm & 0 & 0 & 1 & 0 & 0 & 1 & 0 & 0 & 1 & 0 & 0 & 1 \\
\hline Total & 100 & 8 & 9 & 8 & 8 & 9 & 8 & 8 & 9 & 8 & 8 & 9 & 8 \\
\hline
\end{tabular}

4- Almost equal number of incidents were selected for each month (i.e. eight incident for the month of January and nine incident for the month of February).

5- More weight was given to incidents that occurred on weekdays. In the sortation criteria, two third of the selected incident numbers were assigned to incidents that occurred on weekdays while one-third was allocated to incidents that occurred on weekends.

6- The ratio of the selected number of the incident in the peak hours and off-peak hours was kept the same, $50 \%$ of selected cases fall in peak hours as well as off-peak hours.

7- Morning and evening time was evenly selected among the four categories.

After determining the number of incidents obtainable from each incident information combination, other factors had a significant effect on the selection criteria and needed to be considered. To bring the incident data to the final sorting format that was used to select the 400 cases, it was essential to consider the impact of an incident on the lane blockage configuration and the start time of the incident. More weight was given to incidents that blocked two or more lanes. Also, the incident time was used as an equivalence criterion. 


\section{Manual selection considering lane blockage}

Incident cases were first sorted according to the criteria explained in Table 3.6. The criteria are based on the consideration of the whole landscape of incident data for one entire year, the year of 2011. Incident data were sorted according to the incident attributes such as time of the day, the day of the week, season, direction, and stream.

With substantial consideration of the defined incident data sortation criteria presented in Table 3.7 that took into account each incident attribute, a manual selection was performed on the 1469 incident cases for the selection of the 400 incidents (See Table 3.8 for examples). This is an organized manual selection method which also took into consideration the number of blocked lanes caused by the incident, where more attention was given to incidents causing blockage to two or more lanes.

\section{Manual selection considering incident time}

Another factor that was also taken into consideration was the time when the incident was first reported. One column in the excel sheet was generated to refer to incident time. It was taken into consideration to include all the seasons and the months according to the

criteria explained in Table 3.6. In most cases the number of lanes blocked due to the effect of the incident was crucial when deciding what time, during the specified time range, should be selected. 
Table 3.8: Incident selection based on lane blockage and incident time

\begin{tabular}{|c|c|c|c|c|c|c|c|c|c|c|c|c|}
\hline 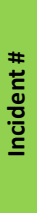 & 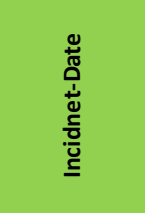 & $\begin{array}{l}5 \\
\text { ర్ } \\
\text { ஸे }\end{array}$ & 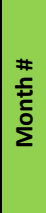 & 을 & $\begin{array}{l}\frac{\varepsilon}{\pi} \\
\text { ஸे } \\
\text { ผे }\end{array}$ & $\begin{array}{l}\text { WD- } \\
\text { WE }\end{array}$ & $\begin{array}{c}400- \\
\text { cases- } \\
\text { selected } \\
\text { based on } \\
\text { incident } \\
\text { time }\end{array}$ & $\begin{array}{c}400- \\
\text { cases- } \\
\text { selected } \\
\text { based on } \\
\text { lane } \\
\text { closure }\end{array}$ & $\begin{array}{l}\text { Incident- } \\
\text { Start_Time }\end{array}$ & Affected_Lanes & $\begin{array}{c}\text { Peek-Hour_ } \\
\text { Off-Peek- } \\
\text { Hour }\end{array}$ & $\begin{array}{l}\text { am- } \\
\text { pm }\end{array}$ \\
\hline 1 & 10-Jan-2011 & Winter & 1 & $\mathrm{E}$ & $\mathrm{C}$ & WD & 1 & 1 & 8:29:00 AM & 1 right lane & 1-PH am & AM \\
\hline 2 & 28-Jan-2011 & Winter & 1 & $E$ & $\mathrm{C}$ & WD & & & 6:53:07 AM & 1 LEFT LANE(S) & 1-PH am & AM \\
\hline 3 & 28-Jan-2011 & Winter & 1 & $\mathrm{E}$ & $\mathrm{C}$ & WD & 1 & 1 & 9:47:51 AM & 1 RIGHT LANE(S) & 1-PH am & AM \\
\hline 4 & 5-Jan-2011 & Winter & 1 & $\mathrm{E}$ & $\mathrm{C}$ & WD & & & 6:26:00 PM & 1 right lane & 2-PH pm & PM \\
\hline 5 & 13-Jan-2011 & Winter & 1 & $E$ & $\mathrm{C}$ & WD & & & 4:00:59 PM & 1 LEFT LANE(S) & 2-PH pm & PM \\
\hline 6 & 21-Jan-2011 & Winter & 1 & $\mathrm{E}$ & $\mathrm{C}$ & WD & & & 4:21:11 PM & 1 RIGHT LANE(S) AND RIGHT SHOULDER & 2-PH pm & PM \\
\hline 7 & 31-Jan-2011 & Winter & 1 & $\mathrm{E}$ & $\mathrm{C}$ & WD & 1 & 1 & 5:06:46 PM & 2 LEFT LANE(S) & 2-PH pm & PM \\
\hline 8 & 10-Jan-2011 & Winter & 1 & $\mathrm{E}$ & $\mathrm{C}$ & WD & & & 6:13:00 AM & 1 centre lane & 3a-OPH am & AM \\
\hline 9 & 13-Jan-2011 & Winter & 1 & $\mathrm{E}$ & $\mathrm{C}$ & WD & 1 & 1 & 3:19:39 AM & 1 RIGHT LANE(S) & 3a-OPH am & AM \\
\hline 10 & 13-Jan-2011 & Winter & 1 & $\mathrm{E}$ & $\mathrm{C}$ & WD & 1 & 1 & 11:49:07 AM & LEFT SHOULDER AND 1 LEFT LANE(S) & 3b-OPH am & AM \\
\hline 11 & 20-Jan-2011 & Winter & 1 & $\mathrm{E}$ & C & WD & & & 11:50:08 AM & 1 RIGHT LANE(S) & 3b-OPH am & AM \\
\hline 12 & 3-Jan-2011 & Winter & 1 & $\mathrm{E}$ & $\mathrm{C}$ & WD & & & 11:10:00 PM & 1 right lane and right shoulder & 4b-OPH pm & PM \\
\hline 13 & 14-Jan-2011 & Winter & 1 & $\mathrm{E}$ & $\mathrm{C}$ & WD & 1 & 1 & 10:26:12 PM & 2 RIGHT LANE(S) AND RIGHT SHOULDER & 4b-OPH pm & PM \\
\hline 14 & 21-Jan-2011 & Winter & 1 & $\mathrm{E}$ & $\mathrm{C}$ & WD & & & 7:10:19 PM & 2 RIGHT LANE(S) AND RIGHT SHOULDER & 4b-OPH pm & PM \\
\hline 15 & 21-Jan-2011 & Winter & 1 & $\mathrm{E}$ & $\mathrm{C}$ & WD & & & 7:37:25 PM & 1 LEFT LANE(S) & 4b-OPH pm & PM \\
\hline 16 & 27-Jan-2011 & Winter & 1 & $\mathrm{E}$ & C & WD & & & 8:45:15 PM & 1 RIGHT LANE(S) AND RIGHT SHOULDER & 4b-OPH pm & PM \\
\hline 17 & 31-Jan-2011 & Winter & 1 & $\mathrm{E}$ & $\mathrm{C}$ & WD & & & 7:17:06 PM & 1 RIGHT LANE(S) & 4b-OPH pm & PM \\
\hline 18 & 31-Jan-2011 & Winter & 1 & $\mathrm{E}$ & $\mathrm{C}$ & WD & & & 7:27:10 PM & 2 RIGHT LANE(S) & 4b-OPH pm & PM \\
\hline 19 & 15-Jan-2011 & Winter & 1 & $\mathrm{E}$ & $\mathrm{C}$ & WE & & & 6:33:53 AM & LEFT SHOULDER AND 1 LEFT LANE(S) & 1-PH am & AM \\
\hline 20 & 15-Jan-2011 & Winter & 1 & $\mathrm{E}$ & $\mathrm{C}$ & WE & & & $6: 52: 32 \mathrm{AM}$ & 2 LEFT LANE(S) & 1-PH am & AM \\
\hline 21 & 15-Jan-2011 & Winter & 1 & $\mathrm{E}$ & $\mathrm{C}$ & WE & & 1 & $8: 29: 30 \mathrm{AM}$ & LEFT SHOULDER AND 2 LEFT LANE(S) AND & 1-PH am & AM \\
\hline 22 & 15-Jan-2011 & Winter & 1 & $\mathrm{E}$ & $\mathrm{C}$ & WE & & & 9:13:40 AM & 1 LEFT LANE(S) & 1-PH am & AM \\
\hline 23 & 15-Jan-2011 & Winter & 1 & $\mathrm{E}$ & $\mathrm{C}$ & WE & 1 & & 9:21:35 AM & 1 RIGHT LANE(S) AND RIGHT SHOULDER & 1-PH am & AM \\
\hline 24 & 2-Jan-2011 & Winter & 1 & $\mathrm{E}$ & $\mathrm{C}$ & WE & & & 3:37:00 PM & Right shoulder & 2-PH pm & PM \\
\hline 25 & 2-Jan-2011 & Winter & 1 & $\mathrm{E}$ & $\mathrm{C}$ & WE & & & 5:45:00 PM & 1 left lane & 2-PH pm & PM \\
\hline 26 & 15-Jan-2011 & Winter & 1 & $\mathrm{E}$ & $\mathrm{C}$ & WE & & & 3:30:50 PM & 1 RIGHT LANE(S) & 2-PH pm & PM \\
\hline 27 & 30-Jan-2011 & Winter & 1 & $\mathrm{E}$ & $\mathrm{C}$ & WE & & & 3:32:30 PM & 1 RIGHT LANE(S) AND RIGHT SHOULDER & 2-PH pm & PM \\
\hline 28 & 1-Jan-2011 & Winter & 1 & $\mathrm{E}$ & $\mathrm{C}$ & WE & 1 & & 2:57:00 AM & Left shoulder and 2 left lanes & 3a-OPH am & AM \\
\hline 29 & 15-Jan-2011 & Winter & 1 & $\mathrm{E}$ & $\mathrm{C}$ & WE & & & 11:10:00 AM & LEFT SHOULDER AND 2 LEFT LANE(S) & 3b-OPH am & AM \\
\hline 30 & 15-Jan-2011 & Winter & 1 & $\mathrm{E}$ & C & WE & & & 11:57:19 AM & 2 LEFT LANE(S) & 3b-OPH am & AM \\
\hline 31 & 2-Jan-2011 & Winter & 1 & $\mathrm{E}$ & $\mathrm{C}$ & WE & & 1 & 7:47:00 PM & All lanes closed & 4b-OPH pm & PM \\
\hline 32 & 16-Jan-2011 & Winter & 1 & $E$ & $\mathrm{C}$ & WE & & & 7:11:10 PM & 1 RIGHT LANE(S) & 4b-OPH pm & PM \\
\hline
\end{tabular}

\subsubsection{Random Draw}

The criteria for selecting 100 cases in a randomly drawn database involved the random number application to 400 selected incident cases. The employed criteria are implied by the following steps. 
1- Incidents were sorted based on the manually selected cases with high consideration given to cases that have a large number of lane blockages, and then a random number was applied for the selection of an incident (Table 3.9).

Table 3.9: Random number application for selection of incidents

\begin{tabular}{|c|c|c|c|c|c|c|c|c|c|c|c|c|}
\hline $\begin{array}{l}\text { Applied } \\
\text { Random } \\
\text { Numbers }\end{array}$ & $\begin{array}{l}\text { Incident \#- } \\
\text { Basic } \\
\text { sortation }\end{array}$ & $\begin{array}{c}\text { Incident- } \\
\text { Date }\end{array}$ & $\begin{array}{l}\text { Incident- } \\
\text { Start_Time }\end{array}$ & 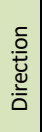 & 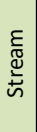 & Season & $\begin{array}{l}\# \\
\text { \# } \\
\stackrel{+}{亡} \\
\sum\end{array}$ & $\begin{array}{l}\text { WD- } \\
\text { WE }\end{array}$ & $\begin{array}{c}\text { 400-cases- } \\
\text { Group } \\
\text { based on } \\
\text { Iane closure }\end{array}$ & Affected_Lanes & $\begin{array}{c}\text { Peek-Hour_ } \\
\text { Off-Peek- } \\
\text { Hour }\end{array}$ & $\begin{array}{l}\text { am- } \\
\text { pm }\end{array}$ \\
\hline 0.86164443 & 1 & $11-01-10$ & $8: 29: 00$ & $E$ & $\mathrm{C}$ & Winter & 1 & WD & 1 & 1 right lane & 1-PH am & AM \\
\hline 0.16480576 & 3 & $11-01-28$ & $9: 47: 51$ & $E$ & C & Winter & 1 & WD & 1 & 1 RIGHT LANE(S) & 1-PH am & AM \\
\hline 0.51180302 & 7 & 11-01-31 & $17: 06: 46$ & $E$ & $C$ & Winter & 1 & WD & 1 & 2 LEFT LANE(S) & 2-PH pm & PM \\
\hline 0.87556239 & 9 & 11-01-13 & $3: 19: 39$ & $E$ & $C$ & Winter & 1 & WD & 1 & 1 RIGHT LANE(S) & 3a-OPH am & AM \\
\hline 0.59060789 & 10 & 11-01-13 & 11:49:07 & $E$ & $C$ & Winter & 1 & WD & 1 & LEFT SHOULDER AND 1 LEFT LANE(S) & $3 \mathrm{~b}-\mathrm{OPH}$ am & AM \\
\hline 0.70832608 & 13 & 11-01-14 & $22: 26: 12$ & $E$ & $\mathrm{C}$ & Winter & 1 & WD & 1 & 2 RIGHT LANE(S) AND RIGHT SHOULDER & 4b-OPH pm & PM \\
\hline 0.13302926 & 21 & 11-01-15 & $8: 29: 30$ & $E$ & $\mathrm{C}$ & Winter & 1 & WE & 1 & LEFT SHOULDER AND 2 LEFT LANE(S) AND & 1-PH am & AM \\
\hline 0.08667704 & 31 & 11-01-02 & 19:47:00 & $E$ & $\mathrm{C}$ & Winter & 1 & WE & 1 & All lanes closed & 4b-OPH pm & PM \\
\hline 0.95481356 & 36 & $11-01-13$ & $9: 41: 20$ & $E$ & $\mathrm{E}$ & Winter & 1 & WD & 1 & 2 RIGHT LANE(S) & 1-PH am & AM \\
\hline 0.3619606 & 38 & 11-01-21 & $8: 12: 56$ & $E$ & $E$ & Winter & 1 & WD & 1 & LEFT SHOULDER AND 2 LEFT LANE(S) & 1-PH am & AM \\
\hline 0.42096797 & 41 & 11-01-19 & $17: 22: 39$ & $\mathrm{E}$ & $E$ & Winter & 1 & WD & 1 & 2 RIGHT LANE(S) & 2-PH pm & PM \\
\hline 0.51735836 & 44 & 11-01-11 & 2:39:00 & $E$ & $E$ & Winter & 1 & WD & 1 & 2 left lanes & 3a-OPH am & AM \\
\hline 0.42671639 & 50 & \begin{tabular}{|l|}
$11-01-12$ \\
\end{tabular} & 10:57:43 & $\mathrm{E}$ & $\mathrm{E}$ & Winter & 1 & WD & 1 & LEFT SHOULDER AND 1 LEFT LANE(S) & $3 \mathrm{~b}-\mathrm{OPH}$ am & AM \\
\hline 0.01191932 & 53 & 11-01-13 & $13: 35: 18$ & $E$ & $E$ & Winter & 1 & WD & 1 & 2 CENTRE LANES(S) & 4a-OPH pm & PM \\
\hline 0.15744316 & 61 & $11-01-16$ & $8: 08: 22$ & $\mathrm{E}$ & $E$ & Winter & 1 & WE & 1 & 1 RIGHT LANE(S) AND RIGHT SHOULDER & 1-PH am & AM \\
\hline 0.52466433 & 63 & 11-01-15 & $12: 51: 50$ & $E$ & $E$ & Winter & 1 & WE & 1 & 1 LEFT LANE(S) & 4a-OPH pm & PM \\
\hline 0.78113315 & 65 & \begin{tabular}{|l|}
$11-01-22$ \\
\end{tabular} & 20:19:04 & $E$ & $E$ & Winter & 1 & WE & 1 & 3 RIGHT LANE(S) & 4b-OPH pm & PM \\
\hline 0.58446657 & 71 & $11-01-12$ & 9:59:55 & W & $\mathrm{C}$ & Winter & 1 & WD & 1 & 1 RIGHT LANE(S) AND RIGHT SHOULDER & 1-PH am & AM \\
\hline 0.87791513 & 72 & 11-01-14 & 9:29:10 & W & $\mathrm{C}$ & Winter & 1 & WD & 1 & 3 RIGHT LANE(S) & 1-PH am & AM \\
\hline 0.5026129 & 77 & \begin{tabular}{|l|}
$11-01-05$ \\
\end{tabular} & $17: 07: 00$ & W & $\mathrm{C}$ & Winter & 1 & WD & 1 & 3 right lanes and right shoulder & 2-PH pm & PM \\
\hline 0.96937778 & 85 & $11-01-28$ & $5: 35: 23$ & W & $\mathrm{C}$ & Winter & 1 & WD & 1 & 1 RIGHT LANE(S) & 3a-OPH am & AM \\
\hline 0.70148031 & 87 & 11-01-17 & 11:06:37 & W & $\mathrm{C}$ & Winter & 1 & WD & 1 & 2 LEFT LANE(S) & 3b-OPH am & AM \\
\hline 0.46083773 & 94 & \begin{tabular}{|l|}
$11-01-17$ \\
\end{tabular} & $20: 35: 25$ & W & $\mathrm{C}$ & Winter & 1 & WD & 1 & 1 RIGHT LANE(S) & 4b-OPH pm & PM \\
\hline 0.80309967 & 95 & 11-01-15 & $7: 29: 31$ & W & $\mathrm{C}$ & Winter & 1 & WE & 1 & 1 RIGHT LANE(S) AND RIGHT SHOULDER & 1-PH am & AM \\
\hline 0.85983127 & 97 & 11-01-09 & $10: 50: 00$ & W & $\mathrm{C}$ & Winter & 1 & WE & 1 & 1 right lane and right shoulder & 3b-OPH am & AM \\
\hline 0.87394429 & 104 & \begin{tabular}{|l|}
$11-01-12$ \\
\end{tabular} & $8: 28: 24$ & W & $E$ & Winter & 1 & WD & 1 & LEFT SHOULDER AND 1 LEFT LANE(S) & 1-PH am & AM \\
\hline 0.19261165 & 105 & $11-01-17$ & $7: 42: 14$ & W & $E$ & Winter & 1 & WD & 1 & 2 RIGHT LANE(S) & 1-PH am & AM \\
\hline 0.74794752 & 108 & $11-01-17$ & 16:33:05 & W & $E$ & Winter & 1 & WD & 1 & 3 RIGHT LANE(S) & 2-PH pm & PM \\
\hline 0.49302053 & 112 & 11-01-12 & 6:05:49 & W & $\mathrm{E}$ & Winter & 1 & WD & 1 & 2 RIGHT LANE(S) & 3a-OPH am & AM \\
\hline
\end{tabular}

2- The 400 incident cases were then sorted based on the random numbers, and grouped in four subsequent groups, each group consisted of 100 randomly classified incident cases which were considered sufficient for model development and validation purposes.

3- Table 3.10 shows a sample of the first 22 incidents from the first group.

4- Finally, incidents were sorted based on the group, direction, stream, date, and incident time. Table 3.11 shows a sample of the first 20 cases of the incident database. 
Table 3.10: Sample of 22 incidents from the randomly drawn group

\begin{tabular}{|c|c|c|c|c|c|c|c|c|c|c|}
\hline $\begin{array}{l}\text { Applied } \\
\text { Random } \\
\text { Numbers }\end{array}$ & $\begin{array}{l}\text { New Case } \\
\text { number- } \\
\text { based on } \\
\text { Random } \\
\text { selection }\end{array}$ & $\begin{array}{c}\text { Incident \#- } \\
\text { Basic } \\
\text { sortation }\end{array}$ & $\begin{array}{l}\text { Selected- } \\
400 \text { cases - } \\
4 \text { groups }\end{array}$ & $\begin{array}{c}100 \text { selected } \\
\text { Cases sorted } \\
\text { based on Dir- } \\
\text { Stream-Date- } \\
\quad \text { Time }\end{array}$ & $\begin{array}{l}\text { Incident- } \\
\text { Date }\end{array}$ & $\begin{array}{l}\text { Incident- } \\
\text { Start_Time }\end{array}$ & 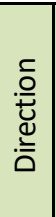 & 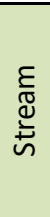 & Season & 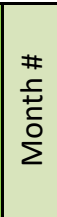 \\
\hline 0.00080833 & 1 & 1077 & 1-Group & 49 & $11-08-12$ & $15: 50: 48$ & $E$ & $E$ & Summer & 8 \\
\hline 0.009654902 & 2 & 403 & 1-Group & 90 & $11-03-16$ & $6: 34: 40$ & $\mathrm{w}$ & $E$ & Spring & 3 \\
\hline 0.009775623 & 3 & 1358 & 1-Group & 28 & $11-12-31$ & $2: 55: 24$ & $\mathrm{E}$ & $C$ & Winter & 12 \\
\hline 0.011919316 & 4 & 53 & 1-Group & 29 & $11-01-13$ & $13: 35: 18$ & $E$ & $E$ & Winter & 1 \\
\hline 0.012035354 & 5 & 796 & 1-Group & 71 & 11-06-28 & $22: 57: 38$ & $\mathrm{w}$ & $C$ & Summer & 6 \\
\hline 0.014204437 & 6 & 457 & 1-Group & 9 & $11-04-11$ & $15: 36: 07$ & $E$ & $C$ & Spring & 4 \\
\hline 0.017735791 & 7 & 192 & 1-Group & 32 & $11-02-27$ & $7: 27: 00$ & $E$ & $E$ & Winter & 2 \\
\hline 0.018424669 & 8 & 1190 & 1-Group & 19 & $11-09-29$ & 10:23:15 & $E$ & $C$ & Fall & 9 \\
\hline 0.018525535 & 9 & 125 & 1-Group & 4 & $11-02-18$ & $9: 18: 13$ & $E$ & C & Winter & 2 \\
\hline 0.027043741 & 10 & 1303 & 1-Group & 78 & 11-10-01 & 1:17:01 & $\mathrm{w}$ & $\mathrm{C}$ & Fall & 10 \\
\hline 0.034127264 & 11 & 1027 & 1-Group & 95 & 11-07-09 & 16:02:50 & $\mathrm{w}$ & $E$ & Summer & 7 \\
\hline 0.037689166 & 12 & 224 & 1-Group & 59 & $11-02-19$ & $15: 47: 22$ & $\mathrm{~W}$ & $C$ & Winter & 2 \\
\hline 0.038361174 & 13 & 839 & 1-Group & 94 & $11-06-12$ & $5: 54: 15$ & $\mathrm{~W}$ & $E$ & Summer & 6 \\
\hline 0.038948664 & 14 & 1090 & 1-Group & 50 & $11-08-22$ & $12: 56: 59$ & $\mathrm{E}$ & $E$ & Summer & 8 \\
\hline 0.042530547 & 15 & 938 & 1-Group & 45 & $11-07-24$ & 10:09:31 & $E$ & $E$ & Summer & 7 \\
\hline 0.042535017 & 16 & 820 & 1-Group & 93 & $11-06-10$ & 11:00:38 & $\mathrm{W}$ & $E$ & Summer & 6 \\
\hline 0.043023518 & 17 & 890 & 1-Group & 16 & $11-07-23$ & $15: 44: 56$ & $E$ & C & Summer & 7 \\
\hline 0.046656303 & 18 & 736 & 1-Group & 42 & $11-06-08$ & $17: 42: 47$ & $E$ & $E$ & Summer & 6 \\
\hline 0.049966398 & 19 & 1082 & 1-Group & 48 & $11-08-08$ & $10: 29: 53$ & $E$ & $E$ & Summer & 8 \\
\hline 0.052840593 & 20 & 1423 & 1-Group & 85 & $11-12-18$ & $3: 00: 48$ & $\mathrm{w}$ & C & Winter & 12 \\
\hline 0.053079671 & 21 & 1327 & 1-Group & 84 & $11-11-30$ & $18: 37: 00$ & $\mathrm{w}$ & C & Fall & 11 \\
\hline 0.055244941 & 22 & 622 & 1-Group & 65 & $11-05-13$ & $18: 20: 13$ & $w$ & $C$ & Spring & 5 \\
\hline
\end{tabular}

5- Each group consisted of 100 randomly selected cases; the first group was selected for the analysis and it was color coded with orange color. This color was taken into account when deciding in certain cases for the data analysis.

6- In addition to group one, the other three groups consisted of 300 incidents and were also obtained based on the same random selection criteria and were given different color codes to distinguish them from each other as can be seen in Table 3.12.

7- Data from group one ends with the number 100 and incident data from the second group starts at 101. 
Table 3.11: Sample of 20 incident database

\begin{tabular}{|c|c|c|c|c|c|c|c|c|}
\hline $\begin{array}{l}\text { Applied } \\
\text { Random } \\
\text { Numbers }\end{array}$ & $\begin{array}{c}\text { New Case } \\
\text { number- } \\
\text { based on } \\
\text { Random } \\
\text { selection } \\
\end{array}$ & $\begin{array}{c}\text { Incident \# } \\
\text { Basic } \\
\text { sortation }\end{array}$ & $\begin{array}{c}\text { Selected- } \\
400 \text { cases - } \\
4 \text { groups }\end{array}$ & $\begin{array}{c}100 \text { selected } \\
\text { Cases sorted } \\
\text { based on Dir- } \\
\text { Stream-Date- } \\
\text { Time } \\
\end{array}$ & $\begin{array}{c}\text { Incident- } \\
\text { Date }\end{array}$ & $\begin{array}{c}\text { Incident- } \\
\text { Start_Time }\end{array}$ & 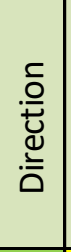 & 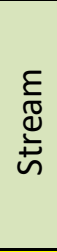 \\
\hline 0.086677044 & 37 & 31 & 1-Group & 1 & 11-01-02 & 19:47:00 & $\mathrm{E}$ & $\mathrm{C}$ \\
\hline 0.133029264 & 55 & 21 & 1-Group & 2 & 11-01-15 & $8: 29: 30$ & $\mathrm{E}$ & $\mathrm{C}$ \\
\hline 0.164805762 & 68 & 3 & 1-Group & 3 & 11-01-28 & 9:47:51 & $E$ & $\mathrm{C}$ \\
\hline 0.018525535 & 9 & 125 & 1-Group & 4 & 11-02-18 & 9:18:13 & $E$ & $\mathrm{C}$ \\
\hline 0.20736729 & 94 & 134 & 1-Group & 5 & $11-02-25$ & 10:11:24 & $E$ & $\mathrm{C}$ \\
\hline 0.188844589 & 83 & 311 & 1-Group & 6 & 11-03-06 & 16:51:04 & $E$ & $\mathrm{C}$ \\
\hline 0.204828535 & 93 & 278 & 1-Group & 7 & 11-03-23 & $8: 35: 24$ & $\mathrm{E}$ & $\mathrm{C}$ \\
\hline 0.186988278 & 80 & 284 & 1-Group & 8 & 11-03-24 & $17: 28: 02$ & $E$ & $\mathrm{C}$ \\
\hline 0.014204437 & 6 & 457 & 1-Group & 9 & 11-04-11 & 15:36:07 & $\mathrm{E}$ & $\mathrm{C}$ \\
\hline 0.213931374 & 97 & 464 & 1-Group & 10 & 11-04-13 & 4:18:18 & $E$ & $\mathrm{C}$ \\
\hline 0.146480984 & 58 & 467 & 1-Group & 11 & 11-04-27 & 10:31:10 & $E$ & $\mathrm{C}$ \\
\hline 0.119156647 & 49 & 579 & 1-Group & 12 & 11-05-01 & 13:47:59 & $\mathrm{E}$ & $\mathrm{C}$ \\
\hline 0.18805408 & 81 & 568 & 1-Group & 13 & $11-05-03$ & 3:45:06 & $E$ & $\mathrm{C}$ \\
\hline 0.156254883 & 63 & 726 & 1-Group & 14 & 11-06-18 & 14:21:30 & $E$ & $\mathrm{C}$ \\
\hline 0.160971842 & 67 & 710 & 1-Group & 15 & 11-06-23 & 4:17:22 & $E$ & $\mathrm{C}$ \\
\hline 0.043023518 & 17 & 890 & 1-Group & 16 & 11-07-23 & $15: 44: 56$ & $E$ & $\mathrm{C}$ \\
\hline 0.104607783 & 43 & 1057 & 1-Group & 17 & 11-08-31 & $22: 15: 15$ & $\mathrm{E}$ & $\mathrm{C}$ \\
\hline 0.067633906 & 29 & 1198 & 1-Group & 18 & 11-09-24 & 2:39:00 & $E$ & $\mathrm{C}$ \\
\hline 0.018424669 & 8 & 1190 & 1-Group & 19 & 11-09-29 & 10:23:15 & $E$ & $\mathrm{C}$ \\
\hline 0.0587727 & 25 & 1264 & 1-Group & 20 & 11-10-05 & 15:59:02 & $E$ & $\mathrm{C}$ \\
\hline
\end{tabular}

Table 3.12: Sample incident data from group one and two

\begin{tabular}{|c|c|c|c|c|c|c|c|c|}
\hline 0.034127264 & 11 & 1027 & 1-Group & 95 & $11-07-09$ & $16: 02: 50$ & W & $\mathrm{E}$ \\
\hline 0.217343279 & 98 & 1247 & 1-Group & 96 & $11-09-15$ & $17: 05: 35$ & W & $\mathrm{E}$ \\
\hline 0.175322892 & 77 & 1250 & 1-Group & 97 & $11-09-26$ & $16: 20: 24$ & W & $\mathrm{E}$ \\
\hline 0.102283237 & 40 & 1318 & 1-Group & 98 & $11-10-22$ & $18: 16: 41$ & W & $\mathrm{E}$ \\
\hline 0.152021937 & 61 & 1439 & 1 -Group & 99 & $11-12-21$ & $0: 25: 23$ & W & $\mathrm{E}$ \\
\hline 0.103150926 & 41 & 1454 & 1 -Group & 100 & $11-12-27$ & $20: 21: 38$ & W & $\mathrm{E}$ \\
\hline 0.511803015 & 187 & 7 & 2-Group & 101 & $11-01-31$ & $17: 06: 46$ & $\mathrm{E}$ & $\mathrm{C}$ \\
\hline 0.371570531 & 142 & 136 & 2-Group & 102 & $11-02-02$ & $21: 53: 45$ & $\mathrm{E}$ & $\mathrm{C}$ \\
\hline 0.422257434 & 163 & 129 & 2-Group & 103 & $11-02-08$ & $17: 24: 39$ & $\mathrm{E}$ & $\mathrm{C}$ \\
\hline 0.421582686 & 162 & 126 & 2-Group & 104 & $11-02-18$ & $9: 30: 41$ & $\mathrm{E}$ & $\mathrm{C}$ \\
\hline 0.431498006 & 165 & 281 & 2-Group & 105 & $11-03-08$ & $17: 48: 57$ & $\mathrm{E}$ & $\mathrm{C}$ \\
\hline
\end{tabular}


8- Incident cases from group one were used to test the model under the consideration that an incident has occurred. Likewise, the same 100 cases were used to test the model for cases that manifested no record of any incident. This could be applied by selecting another time period that was not close to the time when neither the incident nor any other incident type was recorded.

9- If any of the selected cases among the first group showed any error, then the case was excluded from the data analysis list, and the next case in the group replaced that case. For example, case 101 was the first instance to return any case that showed errors while running the code.

10-For statistical purposes, part of the data was used to test/calibrate the model while the other part was utilized for validating the developed model. Following this approach and since the 100 incident cases were randomly selected, the first 50 cases from group one were processed for model calibration purposes. Likewise, the other 50 cases were considered sufficient for model validation.

\section{Summary}

Scientific (statistical) methods were implemented in data preparation in order to obtain a valid database for developing, and testing the incident detection model. In Chapter 4 reliability models are explained. Starting Chapter 5, the process and methods used to develop the incident detection model are described, followed by explanation of computer coded form of the model and its ability to detect incidents. Moreover, a validation method utilizing the obtained incident data is described. 


\section{Reliability Factor}

For incident model development, verification, and application, sufficient reliable data are necessary. Since the model developed in this research (described in the following chapters) is intended to work with detector/roadside unit data as well as probe and crowd sourced data, the reliability factor assumes importance. The reason is that in some sections of the freeway/highway facility under low volume condition there may not be sufficient data available for making reliable predictions.

In the following analyses, for the sake of brevity, references are made only to probe vehicles. However, the models are equally applicable to detector/roadside unit data and crowd-sourced data. The minimum number of probe (or future connected) vehicles required in any part of the freeway for the statistical reliability of reported data depends on the required reliability factor. If the reliability factor is intended to be high, the number of probe vehicles on the road should be high.

\subsection{Sample Size and Reliability of Information}

In this research, sensor clusters which act as proxy for equipped vehicles in association with the roadside unit (RSU) provided spot speed data. However, for the sake

of comprehensiveness, here we explore the case of probe vehicles further. On the assumption that we can estimate (or know) the proportion of vehicles in the traffic stream that are equipped, we can find the probability of an equipped vehicle traveling in the cell during a defined time interval. In a later section of this chapter, further information is provided on the sample size. 


\subsection{Monte Carlo Simulation of Probe Vehicles}

Three traffic levels were considered in this study $(500,1000,1500 \mathrm{veh} / \mathrm{h} / \mathrm{lane})$. The range from $20 \%$ to $100 \%$ of equipped vehicles in the fleet was examined in order to find the significance of both factors (i.e. volume and percent equipped vehicles) in producing a considerable probability level corresponding to the minimum amount of equipped vehicles.

Headway of vehicles arriving in a cell $=f($ traffic volume $q)$, where Headway $=(3600 / q)$ sec/veh.

For example, for 1500 vehicles/h/lane, the average headway can be found as $25 \mathrm{veh} /$ minute or $2.4 \mathrm{sec} / \mathrm{veh}$ average headway.

Poisson probability distribution is usually used in many practical situations when we are interested in measuring how many times a certain event occurs in a particular time interval. It is generally accepted that vehicle arrivals follow Poisson probability distribution.

$\mathrm{P}$ (1 or more vehicles arriving during say, 1 min time interval)

$=1-\mathrm{P}$ (zero arrivals during 1 min time interval)

$=1-\left[(\text { avg. arrival rate })^{0} \cdot \mathrm{e}^{-(\text {avg arrival rate })}\right] / 0$ !

For the $1500 \mathrm{veh} / \mathrm{h} /$ lane case, the avg. arrival rate $=[(1 \mathrm{~h}) / 2.4 \mathrm{sec}] /[60$ time intervals per hour $]=1500 / 60=25$ vehicles $/ 1$ min period

Therefore, $\mathrm{P}$ (one or more vehicles arriving in the cell in one minute interval)

$=1-\left[(25)^{0} \mathrm{e}^{-25}\right] / 0 !=1$ 
For $1000 \mathrm{veh} / \mathrm{h} /$ lane or $16.66 \mathrm{veh} / 1 \mathrm{~min}$ or $=(3600 / 1000)=3.6 \mathrm{sec} / \mathrm{veh}$ headway, $\mathrm{P}($ one or more vehicles arriving in the cell $)=1-\left[(16.66)^{0} \mathrm{e}^{-16.66}\right] / 0 !=1-0.00000097=0.99999$ or 1 .

For $500 \mathrm{veh} / \mathrm{h} /$ lane or $8.33 \mathrm{veh} / 1 \mathrm{~min}$ or $=(3600 / 500)=7.2 \mathrm{sec} / \mathrm{veh}$ headway, $\mathrm{P}($ one or more vehicles arriving in the cell $)=1-\left[(8.33)^{0} \mathrm{e}^{-8.33}\right] / 0 !=1-0.002009=0.99799$ or 1 . Next, we assume that $20 \%$ of vehicle fleet is equipped.

$\mathrm{P}($ equipped vehicles $)=0.2$

Now, we can find the joint probability of one or more vehicles joining the flow in a cell and that they are equipped

$=\mathrm{P}$ (one or more vehicles passing through the cell) $\mathrm{P}$ (of a vehicle to be equipped)

For 20\% fleet equipped scenario:

At 1500 veh/h/lane: $\mathrm{P}=(1.0)(0.2)=0.2$

At 1000 veh/h/lane: $\mathrm{P}=(1.0)(0.2)=0.2$

At 500 veh/h/lane: $\mathrm{P}=$ Approx. (1.0) (0.2) = Approx. 0.2

For $30 \%$ of fleet equipped scenario:

At 1500 veh/h/lane: $\mathrm{P}=(1.0)(0.3)=0.3$

At $1000 \mathrm{veh} / \mathrm{h} /$ lane: $\mathrm{P}=(1.0)(0.3)=0.3$

At $500 \mathrm{veh} / \mathrm{h} /$ lane: $\mathrm{P}=$ Approx. $(1.0)(0.3)=$ Approx. 0.3 
Next step: we find sample size for the estimation of speed. For uninterrupted flow facilities (i.e. freeways, multi-lane highways, etc.), the range of speed could be $25 \mathrm{~km} / \mathrm{h}$ (during incidents) to $105 \mathrm{~km} / \mathrm{h}$ and st. deviation at $30 \%$ of mean could range from 8 to 31.5 $\mathrm{km} / \mathrm{h}$. We can use the lower part of speed range since it is more applicable/critical for incident detection. So, st. deviation of $8 \mathrm{~km} / \mathrm{h}$ is used, and we use the tolerance level of 5 $\mathrm{km} / \mathrm{h}$.

The properties of normal distribution have been used to develop a sample size equation. Where $\mathrm{N}=$ minimum sample size

$$
\begin{aligned}
N=\left(\frac{Z \sigma}{d}\right)^{2} \\
Z=\text { number of standard deviations corresponding to the required confidence level } \\
Z=1.96 \text { for } 95 \% \text { confidence level } \\
Z=1.5 \text { for } 86.6 \% \text { confidence level } \\
\sigma=\text { St. Deviation } \\
\mathrm{d}=\text { tolerance level }=\text { limit of acceptable error in speed estimate }
\end{aligned}
$$

Sample size N for $86.6 \%$ confidence level $=[(1.5 \text { factor for } 86.6 \% \text { conf. level }) *(8)]^{2 /}$ $(5)^{2}=5.76$ or 6 equipped vehicles will be needed during $1 \mathrm{~min}$ interval. This should be acceptable for $30 \%$ equipped level or higher and say $1000 \mathrm{veh} / \mathrm{h} / \mathrm{lane}$ (minimum) where a probability level of 0.85 was reached.

As a very worst case, if $20 \%$ of the fleet is equipped, let us check equipped vehicles at various traffic levels. 
At $500 \mathrm{veh} / \mathrm{h} /$ lane \& $20 \%$ equipped fleet: $(8.33)(0.2)=1.67 \mathrm{veh} / \mathrm{lane}$, then more than 1 equipped vehicle per $1 \mathrm{~min}$. time interval.

For 2 lanes/direction $=1.67 * 2=3.34$, then more than 3 equipped vehicle per 1 min interval For 3 lanes/direction $=1.67 * 3=5.01$ then more than 5 equipped vehicles per 1 min interval. At $1000 \mathrm{veh} / \mathrm{h} / \mathrm{lane} \& 20 \%$ equipped fleet: $(16.66)(0.2)=3.33 \mathrm{veh} / \mathrm{lane}$, then more than 3 equipped vehicle per $1 \mathrm{~min}$. time interval.

For 2 lanes/direction, $=3.33 * 2=6.66$ then more than 6 equipped vehicle per 1 min interval For 3 lanes/direction, $=3.33 * 3=9.99$ then 10 equipped vehicles per $1 \mathrm{~min}$ interval.

At $1500 \mathrm{veh} / \mathrm{h} / \mathrm{lane} \& 20 \%$ equipped fleet: $(25)(0.2)=5.0 \mathrm{veh} / \mathrm{lane}$ are equipped per 1 min. time interval.

For 2 lanes/direction, $=5 * 2=10$ (i.e. 10 equipped vehicles per 1 min interval)

For 3 lanes/direction, $=5 * 3=15$ (i.e. 15 equipped vehicles per 1 min interval).

Now, for $500 \mathrm{veh} / \mathrm{h} / \mathrm{lane} \& 30 \%$ equipped level, on avg. $8.33 \mathrm{veh} / \mathrm{min}$ pass through a lane in the cell. Therefore, equipped fleet is $8.33^{*}(0.3)=2.5$ vehicles/lane (i.e. more than 3 equipped vehicles per 1 min time interval).

For a 2 lanes/direction cell: $2.5 \times 2=5$ equipped vehicles

For a 3 lanes/direction cell: $2.5 \times 3=7.5$ or 8 equipped vehicles

At $1000 \mathrm{veh} / \mathrm{h} / \mathrm{lane} \& 30 \%$ equipped level, on avg. $16.66 \mathrm{veh} / \mathrm{min}$ pass through a lane in the cell, therefore equipped fleet is $16.66 *(0.3)=5$ vehicles/lane i.e. 5 equipped vehicles per 1 min. time interval). 
For a 2 lanes/direction cell: $5 \times 2=10$ equipped vehicles

For a 3 lanes/direction cell: $5 \times 3=15$ equipped vehicles

At $1500 \mathrm{veh} / \mathrm{h} / \mathrm{lane}, 25 \mathrm{veh} / \mathrm{min}$ pass through a lane in the cell. At $30 \%$ equipped level: $25(0.3)=7.5$ (i.e. more than 7 vehicles are equipped per lane).

For 2 lanes/direction, 15 equipped vehicles are equipped.

For 3 lanes/direction, 22.5 or 23 vehicles are equipped.

Now using XLSim, a Monte Carlo Simulation tool, 1000 simulations with normal probability density function were carried out to estimate the Probability (Equipped vehicles $>$ minimum sample size).

Let $\mu$ be avg. equipped vehicles $/ 1$ min period in the cell and $\sigma$ is the standard deviation. Further, set $\sigma=30 \%$ of avg.

We assumed the following. The random variable $\mathrm{X}$ denotes the number of equipped vehicles in the cell in the whole time interval which is 1 minute. $X$ has a Poisson probability distribution and $\mathrm{P}(\mathrm{X}=\mathrm{k})=\mathrm{f}(\mathrm{k})=\left[(\text { avg. equipped vehicles rate })^{\mathrm{k}} \cdot \mathrm{e}^{- \text {(avg equipped vehicle rate) }}\right] / \mathrm{k}$ ! Where: $\mathrm{k}=0 ; 1 ; 2$

For $500 \mathrm{veh} / \mathrm{h} / \mathrm{lane}$, at $30 \%$ equipped level, for 2 lanes, $\mu=5$ and $\sigma=1.5$ or 2 . Following 1000 simulations, the following results were obtained.

$\mathrm{P}($ at least 1 equipped vehicle during one minute time interval $)=\mathrm{P}(\mathrm{X}=1)$ $P(X \geq 1)=1-P(X=0)=0.98$, as illustrated in Figure 4.1 and Figure 4.2. 


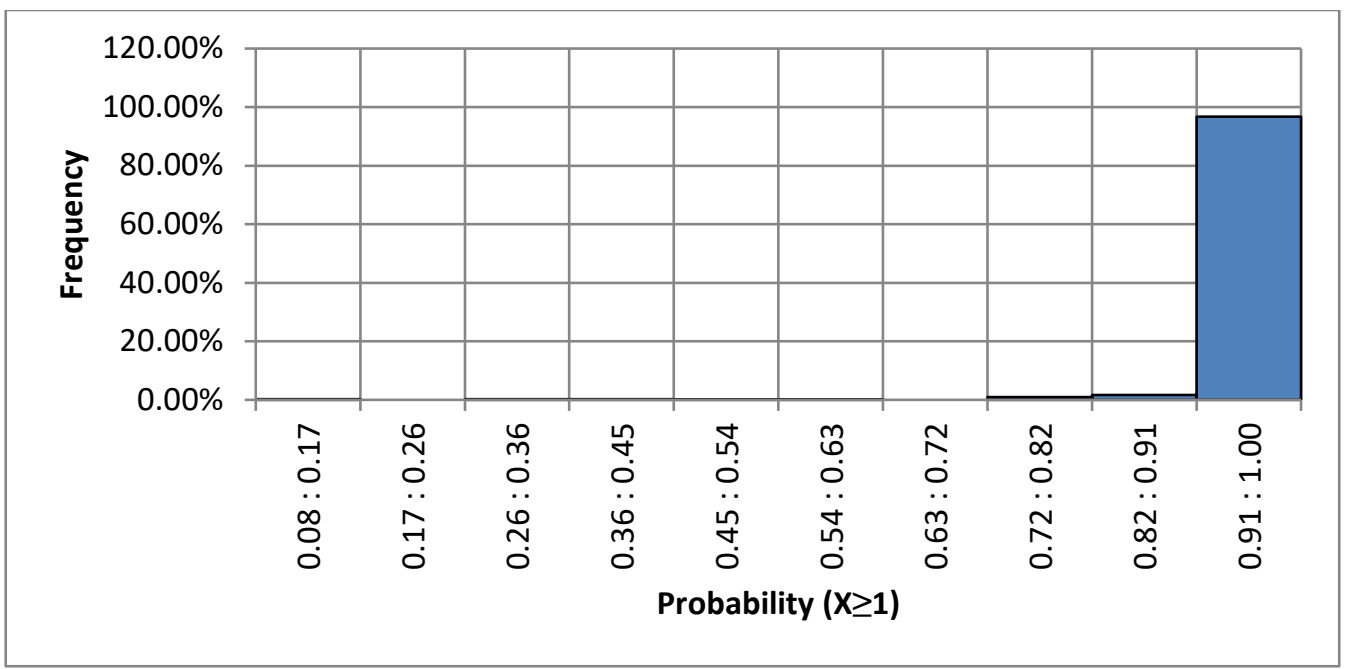

Figure 4.1: $P$ (at least 1 equipped vehicle during one minute) at $500 \mathrm{veh} / \mathrm{h} / \mathrm{lane}$ )

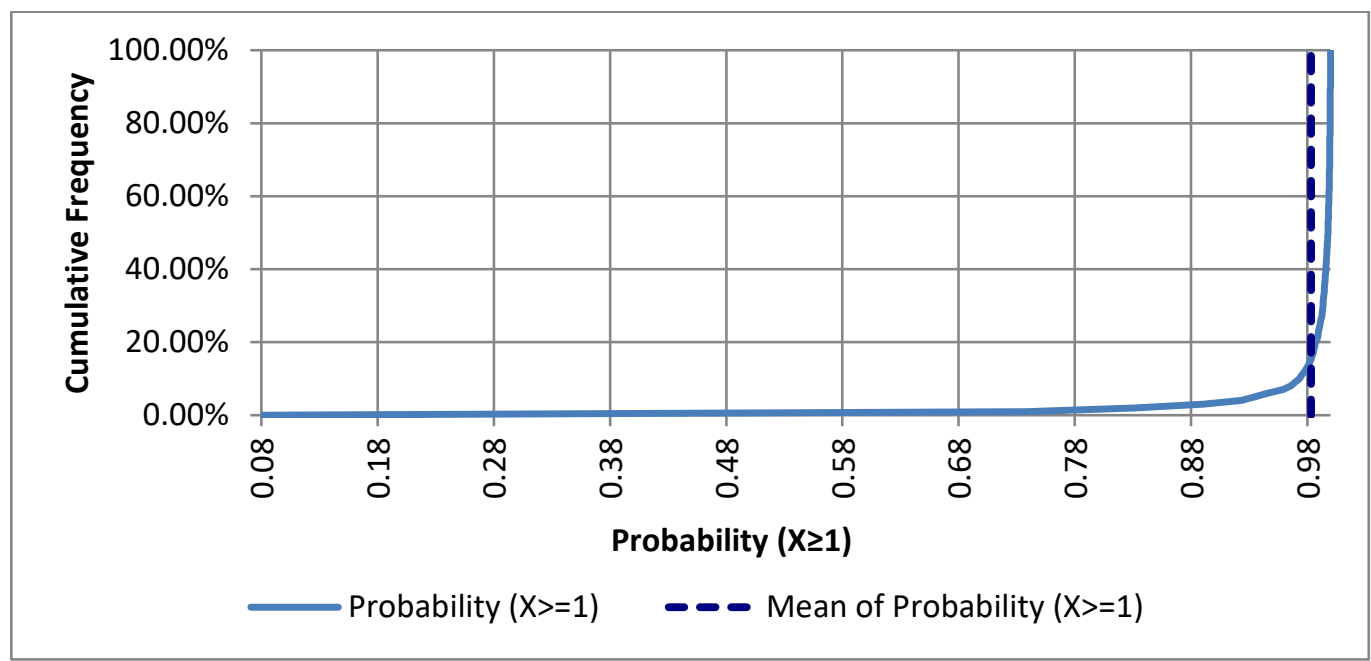

Figure 4.2: Cumulative probability, at least 1 equipped vehicle/min at $500 \mathrm{veh} / \mathrm{h} / \mathrm{lane}$

$\mathrm{P}($ at least 2 equipped vehicles during one minute time interval $)=\mathrm{P}(\mathrm{X} 2)$

$\mathrm{P}(\mathrm{X} 2)=1-(\mathrm{P}(\mathrm{X}=0)+\mathrm{P}(\mathrm{X}=1))=0.94$, as illustrated in Figure 4.3 and Figure 4.4. 


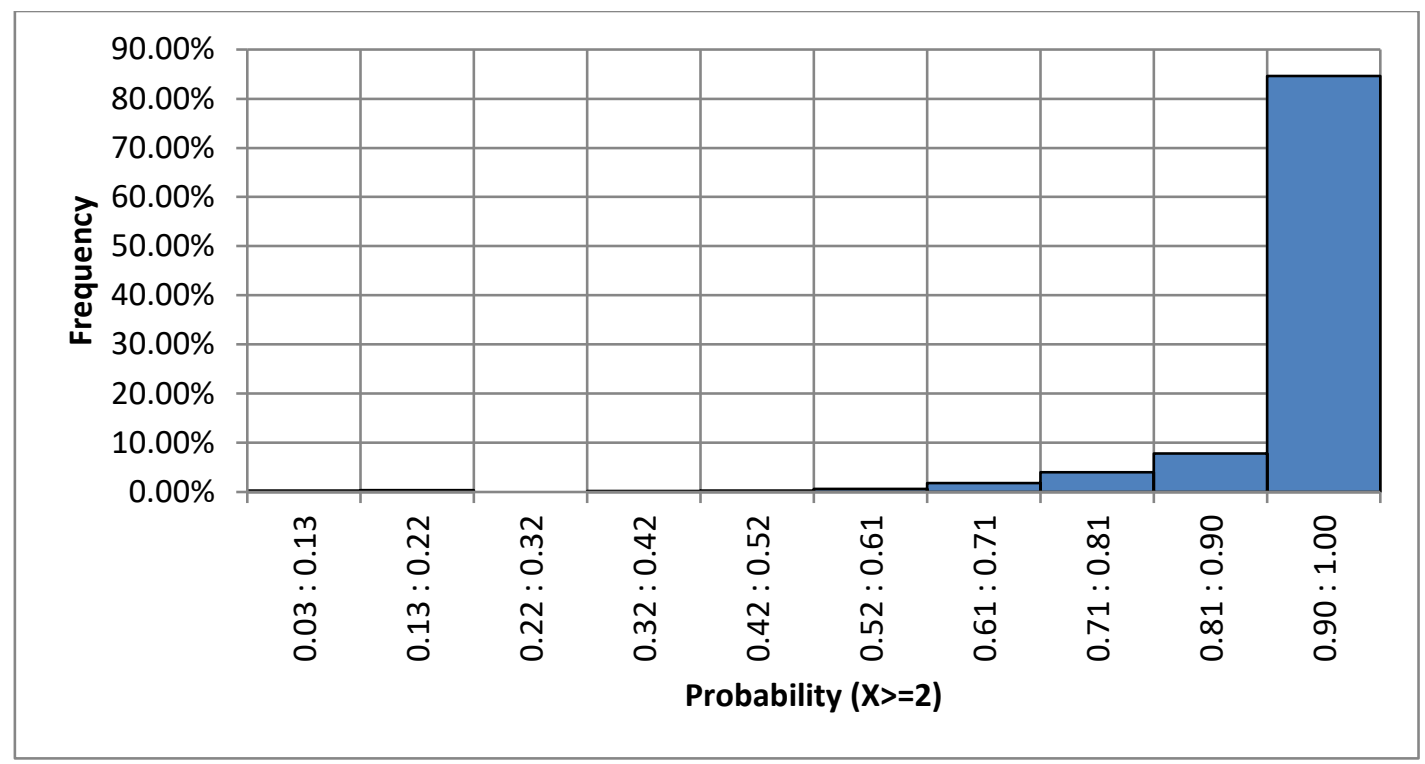

Figure 4.3: P (at least 2 equipped vehicles/min) at $500 \mathrm{veh} / \mathrm{h} / \mathrm{lane}$

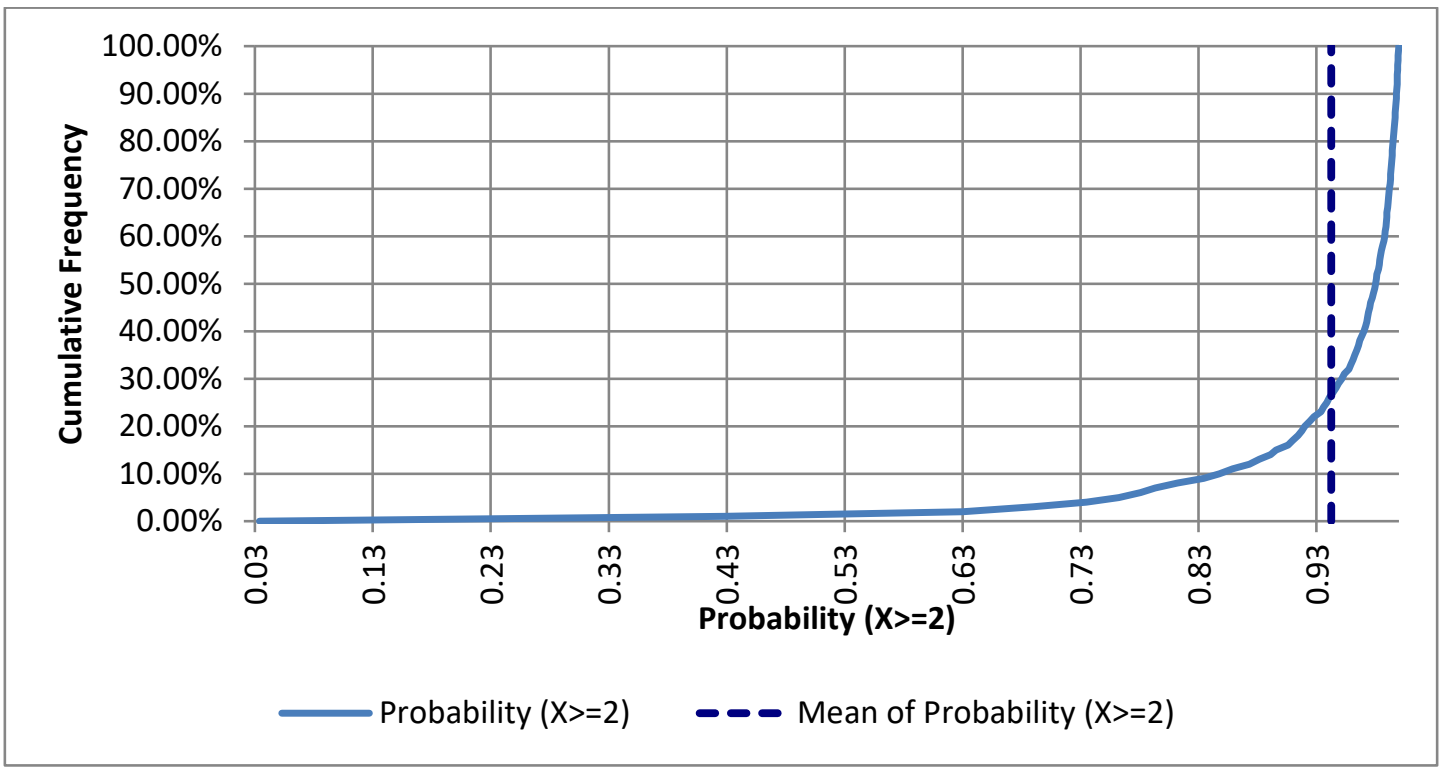

Figure 4.4: Cumulative probability, at least 2 equipped vehicle/min at $500 \mathrm{veh} / \mathrm{h} / \mathrm{lane}$

$\mathrm{P}$ (at least 3 equipped vehicles during one minute time interval $)=\mathrm{P}(\mathrm{X} 3)$

$P(X 3)=1-(P(X=0)+P(X=1)+P(X=2))=0.87$, as illustrated in Figure 4.5 and Figure 4.6. 


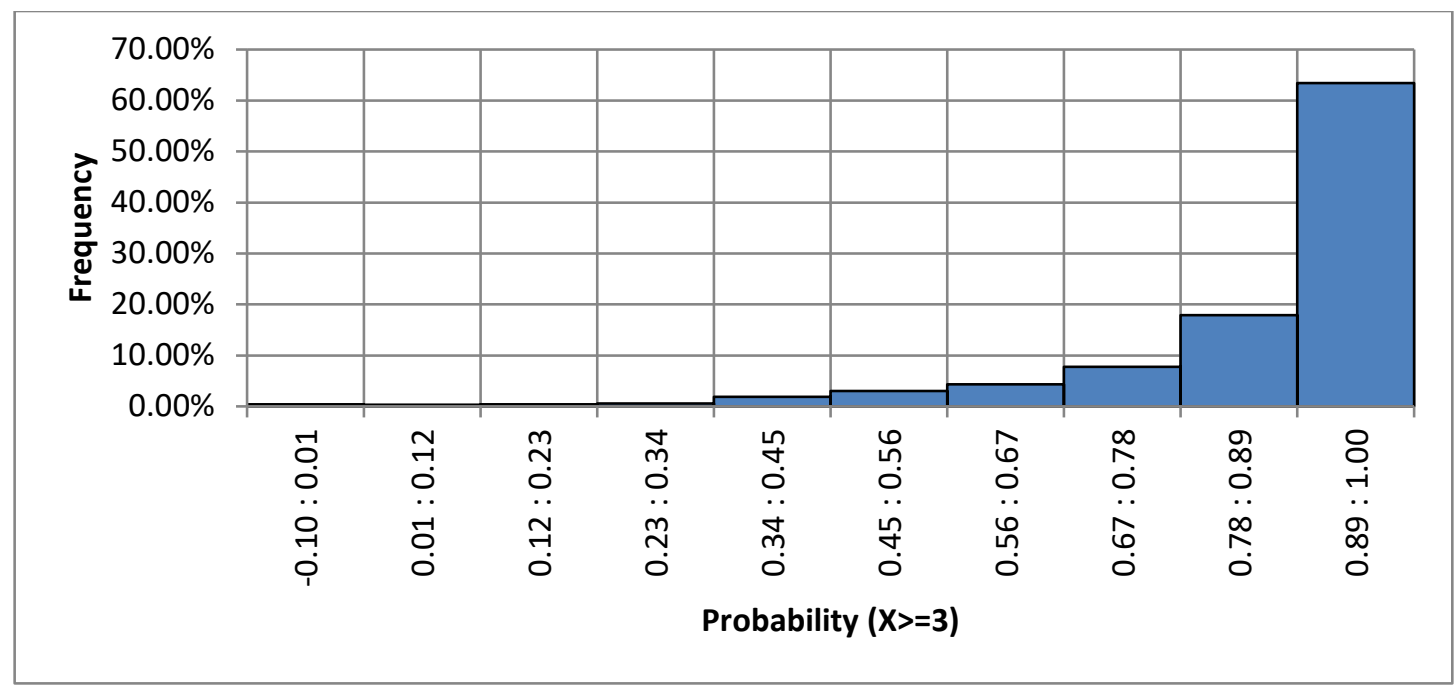

Figure 4.5: $P$ (at least 3 equipped vehicles/min) at $500 \mathrm{veh} / \mathrm{h} / \mathrm{lane}$

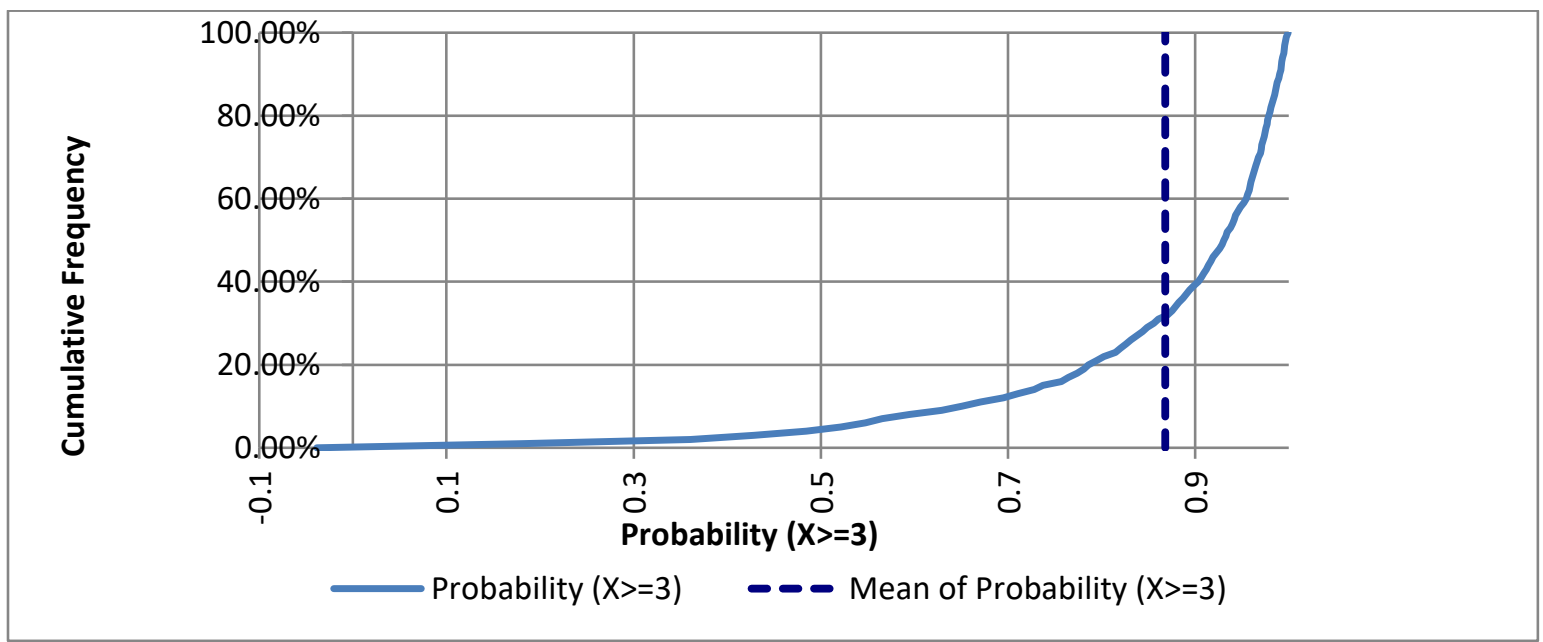

Figure 4.6: Cumulative probability, at least 3 equipped vehicle/min at $500 \mathrm{veh} / \mathrm{h} / \mathrm{lane}$

$\mathrm{P}$ (at least 4 equipped vehicles during one minute time interval $)=\mathrm{P}(\mathrm{X} 4)$

$\mathrm{P}(\mathrm{X} 4)=1-(\mathrm{P}(\mathrm{X}=0)+\mathrm{P}(\mathrm{X}=1)+\mathrm{P}(\mathrm{X}=2)+\mathrm{P}(\mathrm{X}=3))=0.76$, as illustrated in Figure 4.7

and Figure 4.8. 


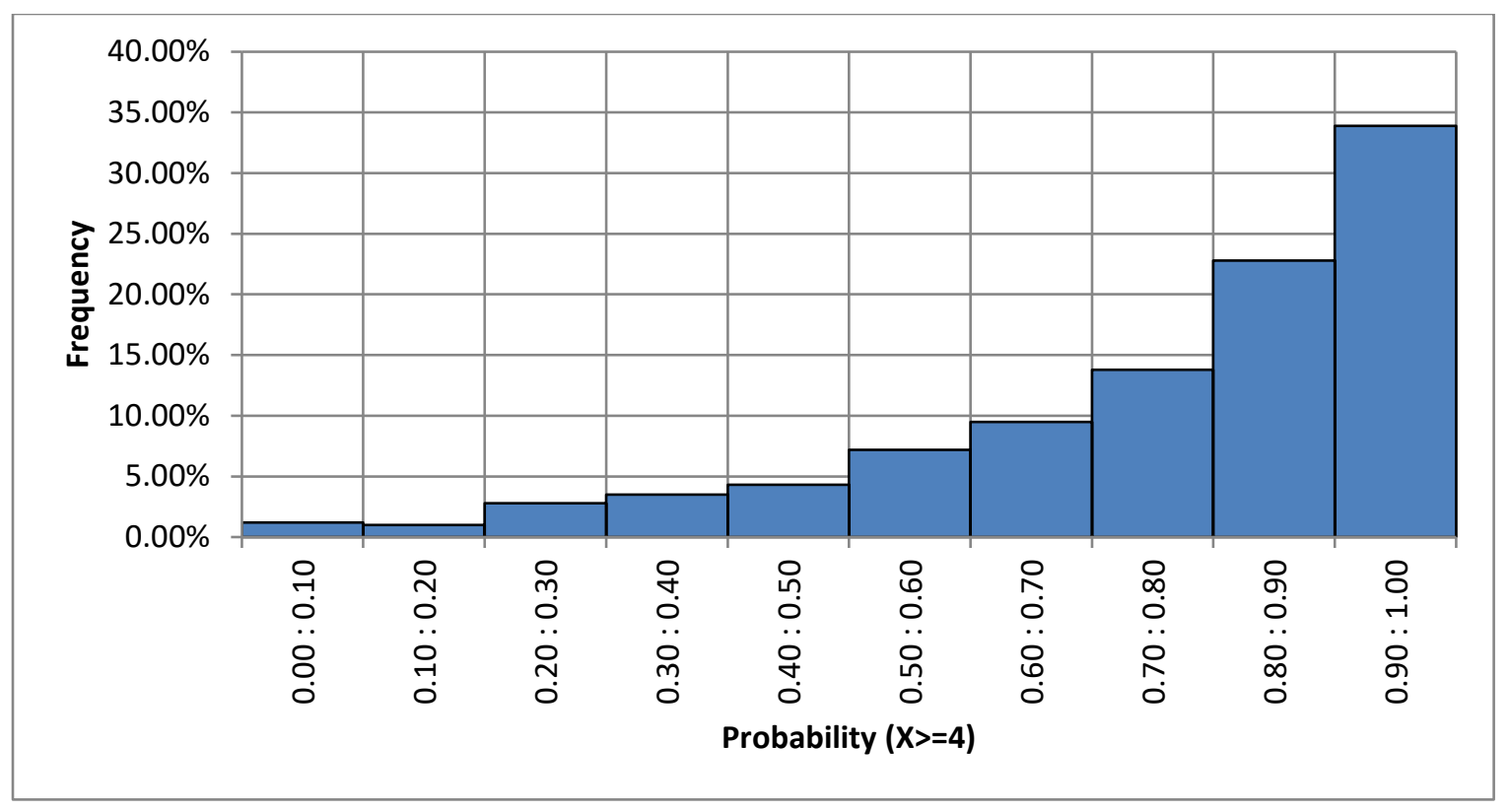

Figure 4.7: P (at least 4 equipped vehicles/min) at $500 \mathrm{veh} / \mathrm{h} / \mathrm{lane}$

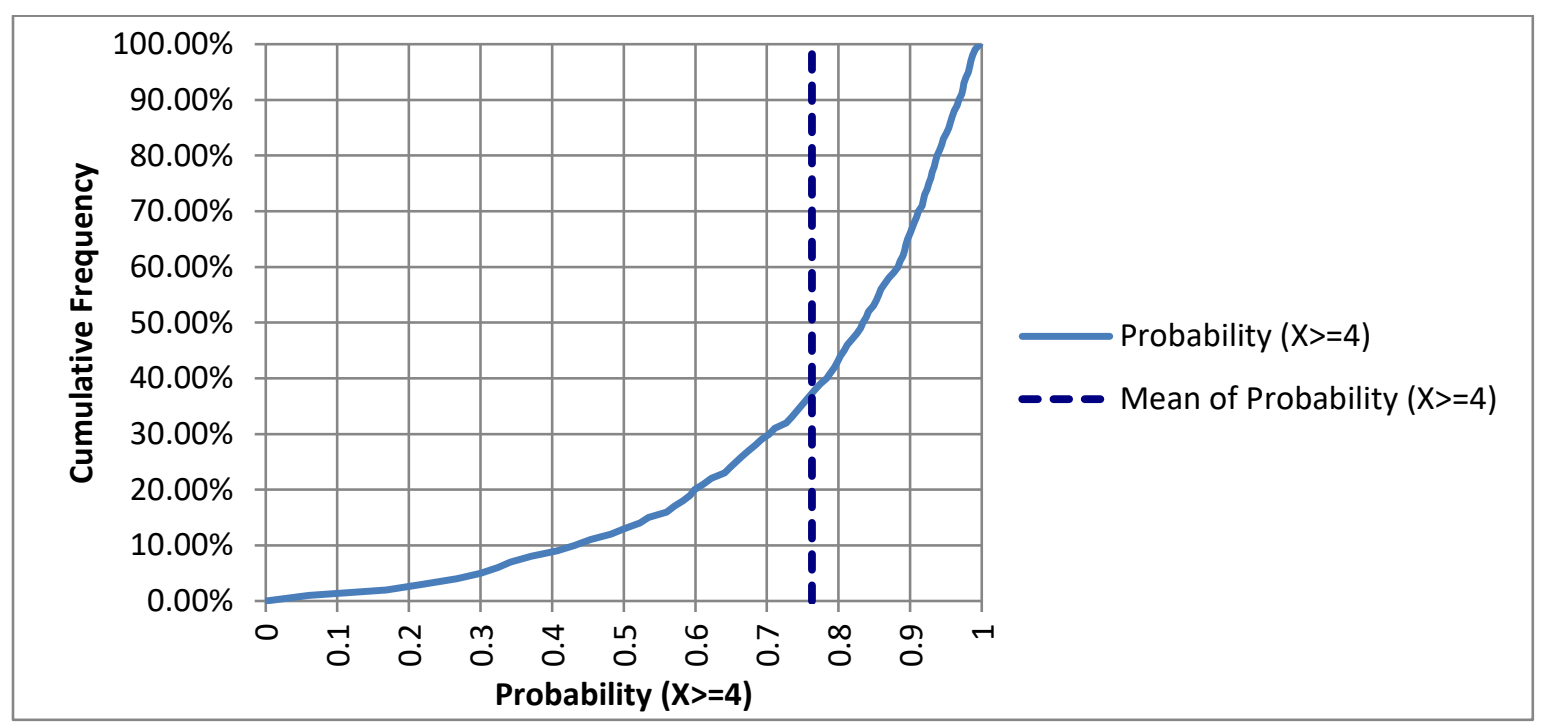

Figure 4.8: Cumulative probability at least 4 equipped vehicle/min at $500 \mathrm{veh} / \mathrm{h} / \mathrm{lane}$

$\mathrm{P}$ (at least 5 equipped vehicles during one minute time interval $)=\mathrm{P}(\mathrm{X} 5)$

$$
\mathrm{P}(\mathrm{X} 5)=1-(\mathrm{P}(\mathrm{X}=0)+\mathrm{P}(\mathrm{X}=1)+\mathrm{P}(\mathrm{X}=2)+\mathrm{P}(\mathrm{X}=3)+\mathrm{P}(\mathrm{X}=4))=0.64 \text {, as illustrated in }
$$

Figure 4.9 and Figure 4.10. 


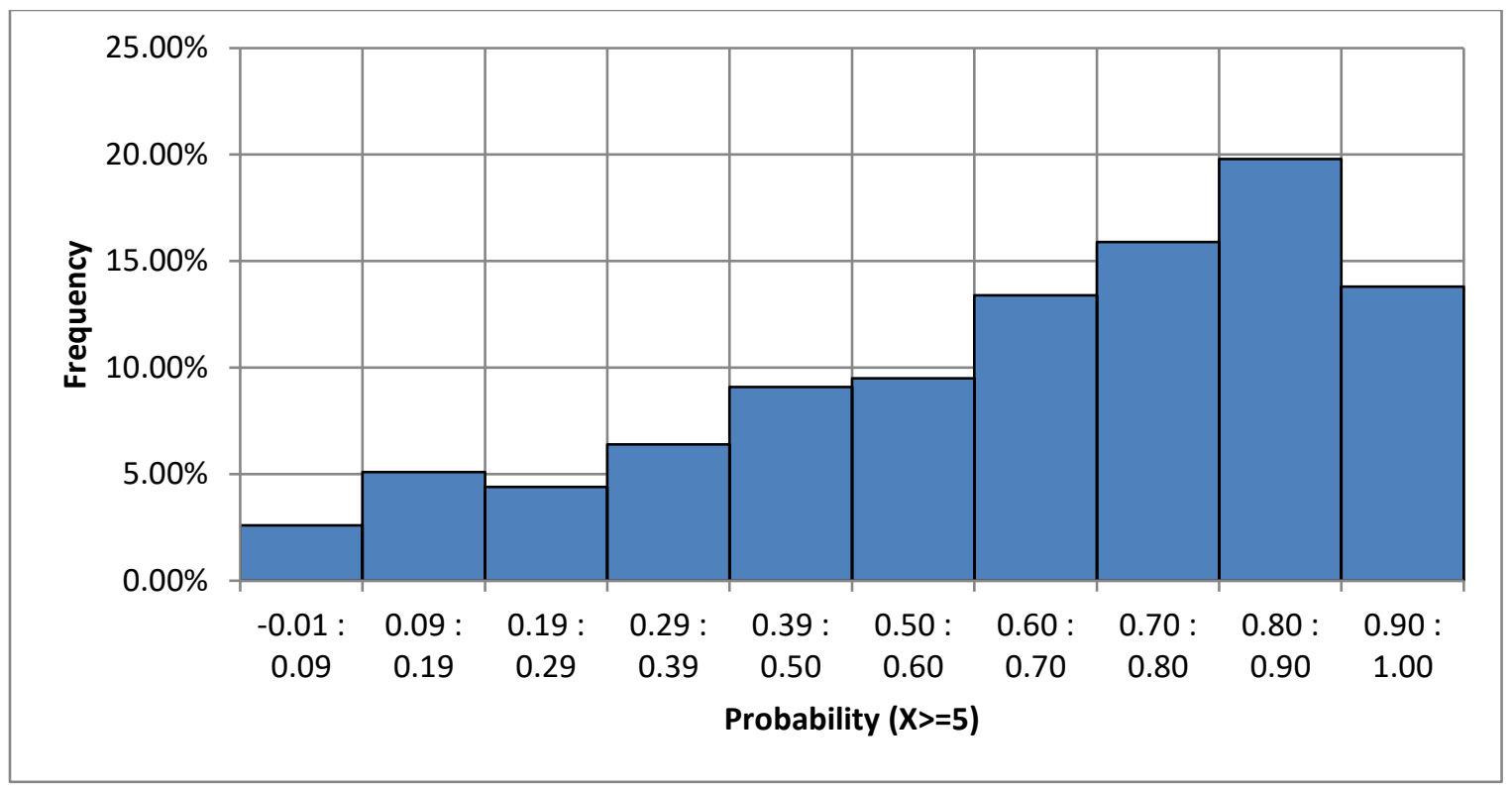

Figure 4.9: $P$ (at least 5 equipped vehicles/min) at $500 \mathrm{veh} / \mathrm{h} /$ lane

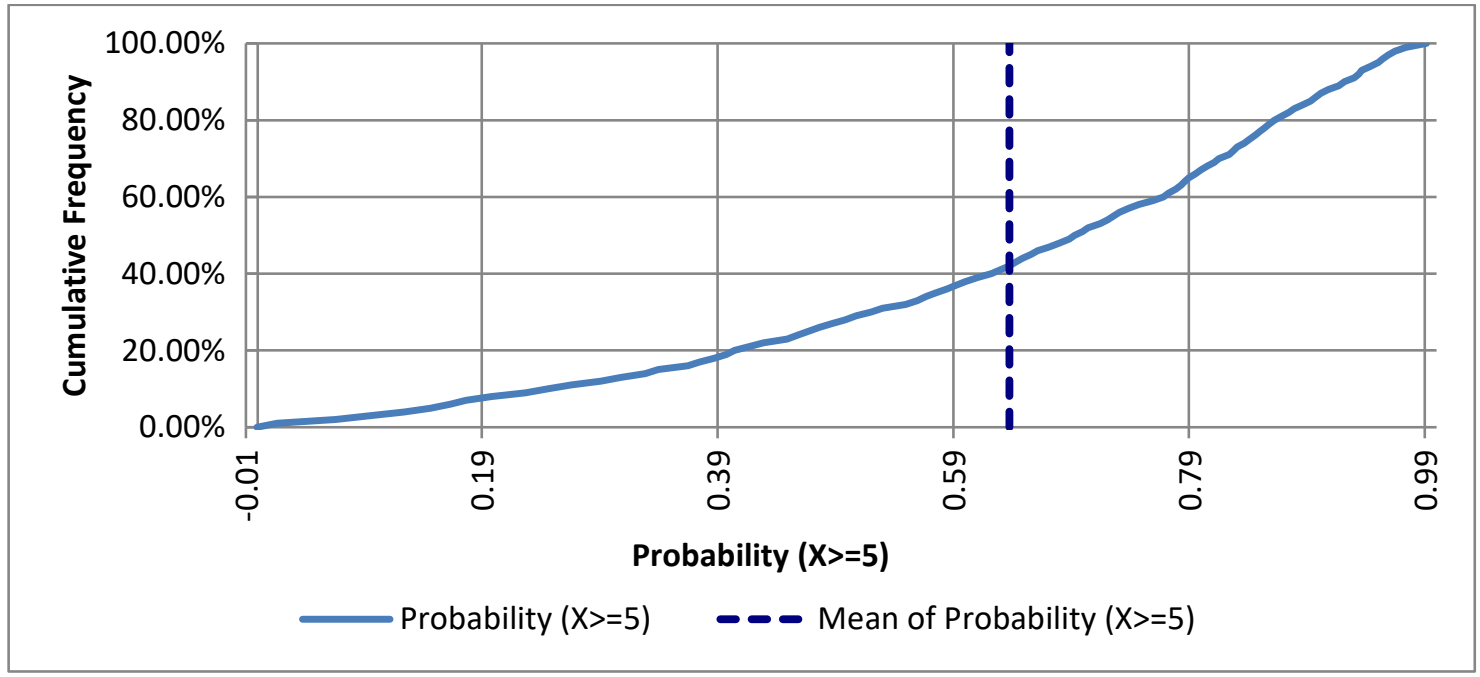

Figure 4.10: Cumulative probability, at least 5 equipped vehicle/min at $500 \mathrm{veh} / \mathrm{h} / \mathrm{lane}$

$\mathrm{P}$ (at least 6 equipped vehicles during one minute time interval $)=\mathrm{P}(\mathrm{X} 6)$

$P(X 6)=1-(P(X=0)+P(X=1)+P(X=2)+P(X=3)+P(X=4)+P(X=6))=0.51$, as

illustrated in Figure 4.11 and Figure 4.12. 


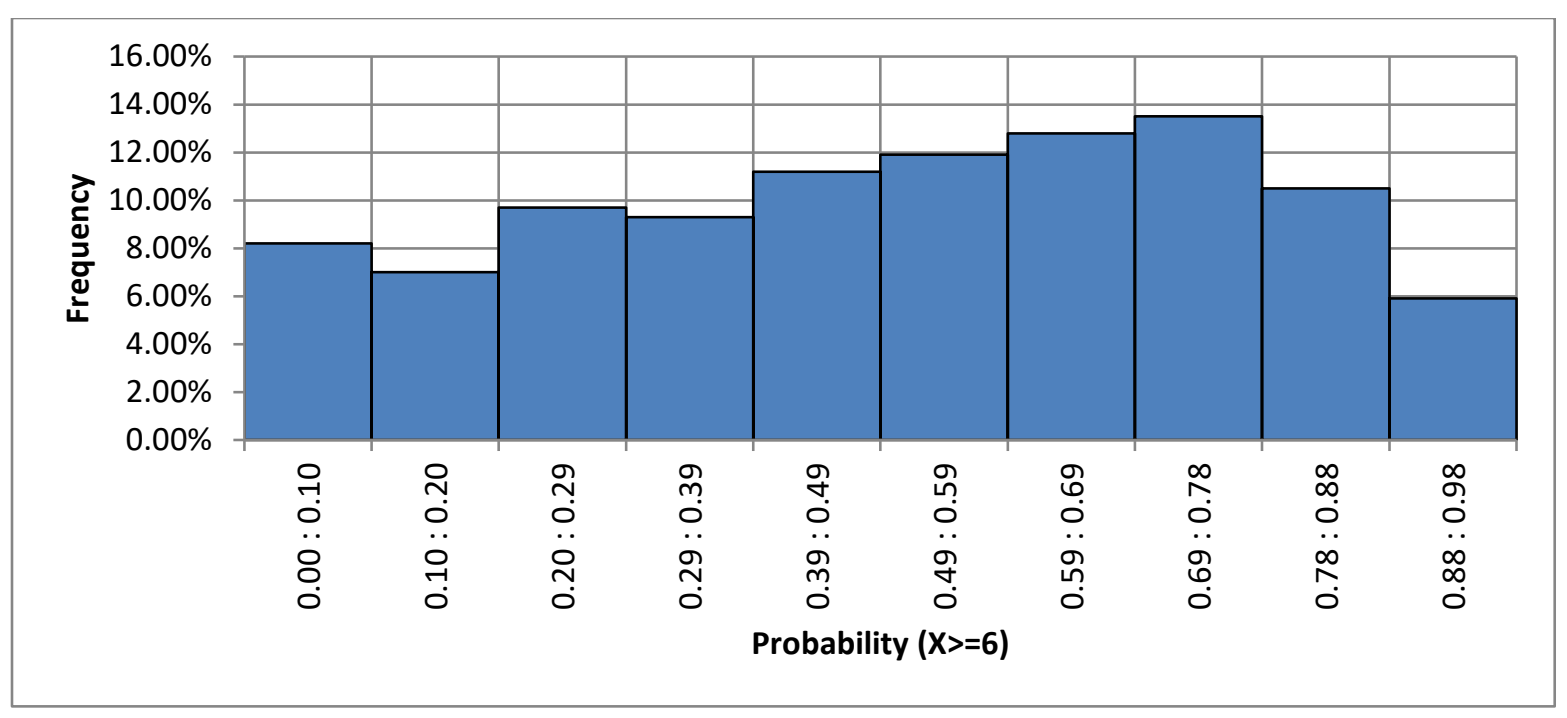

Figure 4.11: P (at least 6 equipped vehicles/min) at $500 \mathrm{veh} / \mathrm{h} / \mathrm{lane}$

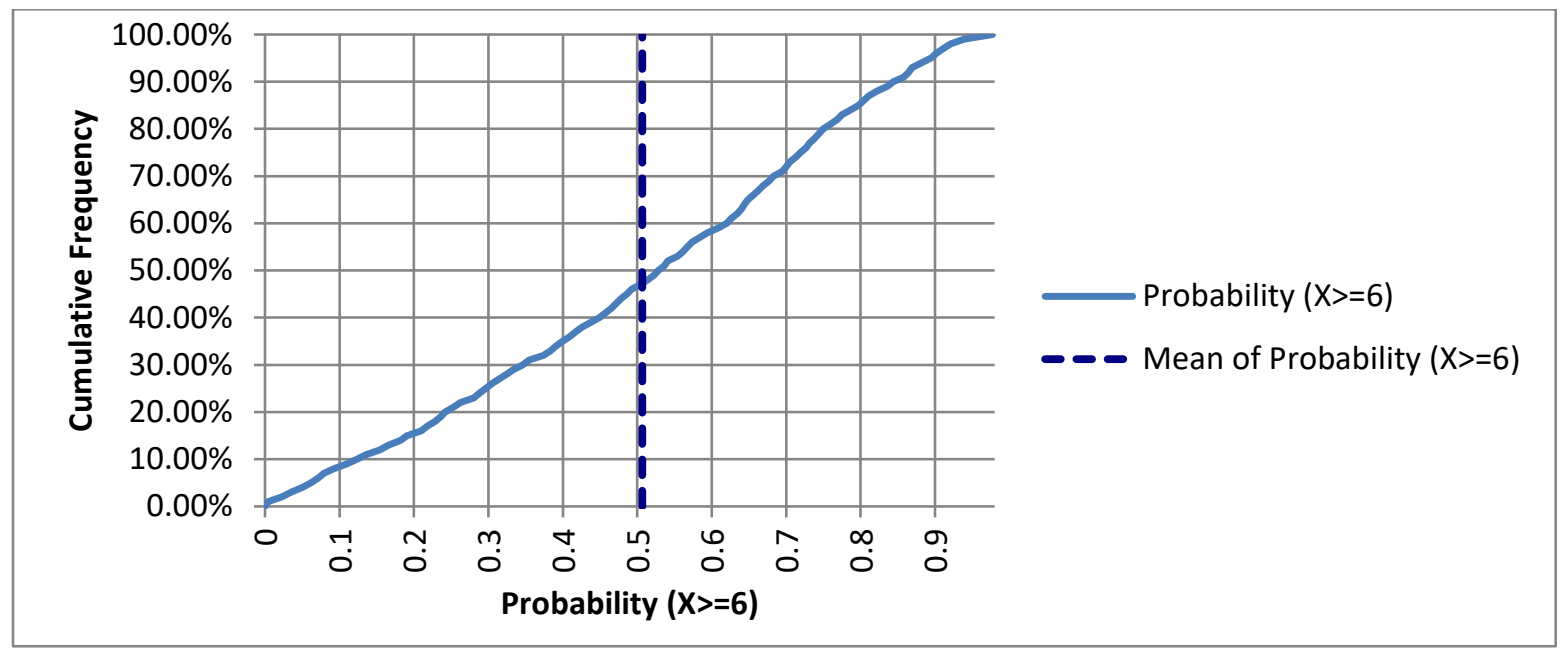

Figure 4.12: Cumulative probability, at least 6 equipped vehicle/min at $500 \mathrm{veh} / \mathrm{h} / \mathrm{lane}$

For $1000 \mathrm{veh} / \mathrm{h} /$ lane, at $30 \%$ equipped level, for 2 lanes, $\mu=10$ and $\sigma=3$. Following 1000 simulations, the following results were obtained.

$\mathrm{P}$ (at least 1 equipped vehicle during one minute time interval $)=\mathrm{P}(\mathrm{X} 1)$ $P(X 1)=1-P(X=0)=0.998$, as illustrated in Figure 4.13 and Figure 4.14. 


\begin{tabular}{|c|c|c|c|c|c|c|c|c|c|c|c|}
\hline \\
\hline & $100.00 \%$ & & & & & & & & & & \\
\hline & $80.00 \%$ & & & & & & & & & & \\
\hline एँّ & $60.00 \%$ & & & & & & & & & & \\
\hline $\bar{\Xi}$ & $4000 \%$ & & & & & & & & & & \\
\hline & 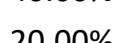 & & & & & & & & & & \\
\hline & \% & & & & & & & & & & \\
\hline & & $\stackrel{n}{\stackrel{n}{0}}$ & $\frac{0}{\stackrel{0}{0}}$ & $\frac{9}{2}$ & $\begin{array}{l}\stackrel{\infty}{\infty} \\
0 \\
0\end{array}$ & $\begin{array}{l}\stackrel{n}{\infty} \\
\infty \\
\infty \\
0\end{array}$ & $\begin{array}{l}\infty \\
\infty \\
\infty \\
0\end{array}$ & ஜூ & ठু & م̆ & $\begin{array}{l}8 \\
- \\
-\end{array}$ \\
\hline & & $\stackrel{\ddot{\circ}}{\stackrel{0}{0}}$ & $\stackrel{\ddot{n}}{\stackrel{0}{0}}$ & $\begin{array}{l}\ddot{0} \\
\stackrel{0}{0}\end{array}$ & $\begin{array}{l}\ddot{\sigma} \\
\stackrel{0}{0}\end{array}$ & $\begin{array}{l}\ddot{\tilde{a}} \\
\infty \\
0\end{array}$ & $\begin{array}{l}\ddot{n} \\
\infty \\
\infty \\
0\end{array}$ & $\begin{array}{l}\ddot{\infty} \\
\infty \\
0 \\
0\end{array}$ & 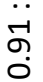 & $\begin{array}{l}\ddot{\sigma} \\
\text { ğ }\end{array}$ & 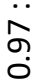 \\
\hline & & & & & & obak & $x>$ & & & & \\
\hline
\end{tabular}

Figure 4.13: $P$ (at least 1 equipped vehicle/min) at $1000 \mathrm{veh} / \mathrm{h} / \mathrm{lane}$

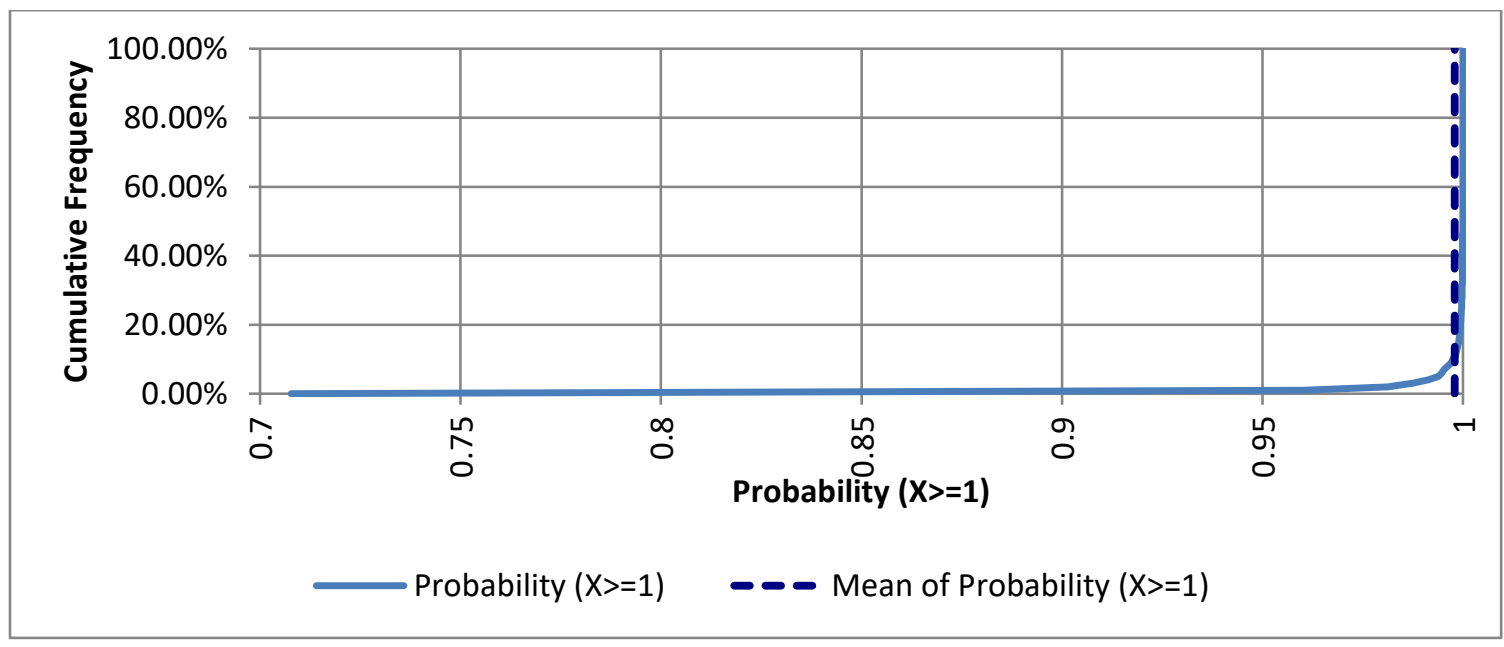

Figure 4.14: Cumulative probability, at least 1 equipped vehicle/min at $1000 \mathrm{veh} / \mathrm{h} / \mathrm{lane}$

$\mathrm{P}$ (at least 2 equipped vehicles during one minute time interval $)=\mathrm{P}(\mathrm{X} 2)$

$\mathrm{P}(\mathrm{X} 2)=1-(\mathrm{P}(\mathrm{X}=0)+\mathrm{P}(\mathrm{X}=1))=0.991$

$\mathrm{P}$ (at least 3 equipped vehicles during one minute time interval $)=\mathrm{P}(\mathrm{X} 3)$

$\mathrm{P}(\mathrm{X} 3)=1-(\mathrm{P}(\mathrm{X}=0)+\mathrm{P}(\mathrm{X}=1)+\mathrm{P}(\mathrm{X}=2))=0.975$

$\mathrm{P}($ at least 4 equipped vehicles during one minute time interval $)=\mathrm{P}(\mathrm{X} 4)$

$P(X 4)=1-(P(X=0)+P(X=1)+P(X=2)+P(X=3))=0.948$ 
$\mathrm{P}$ (at least 5 equipped vehicles during one minute time interval $)=\mathrm{P}(\mathrm{X} 5)$

$P(X 5)=1-(P(X=0)+P(X=1)+P(X=2)+P(X=3)+P(X=4))=0.906$

$\mathrm{P}$ (at least 6 equipped vehicles during one minute time interval $)=\mathrm{P}(\mathrm{X} 6)$

$\mathrm{P}(\mathrm{X} 6)=1-(\mathrm{P}(\mathrm{X}=0)+\mathrm{P}(\mathrm{X}=1)+\mathrm{P}(\mathrm{X}=2)+\mathrm{P}(\mathrm{X}=3)+\mathrm{P}(\mathrm{X}=4)+\mathrm{P}(\mathrm{X}=6))=0.949$, as

illustrated in Figure 4.15 and Figure 4.16.

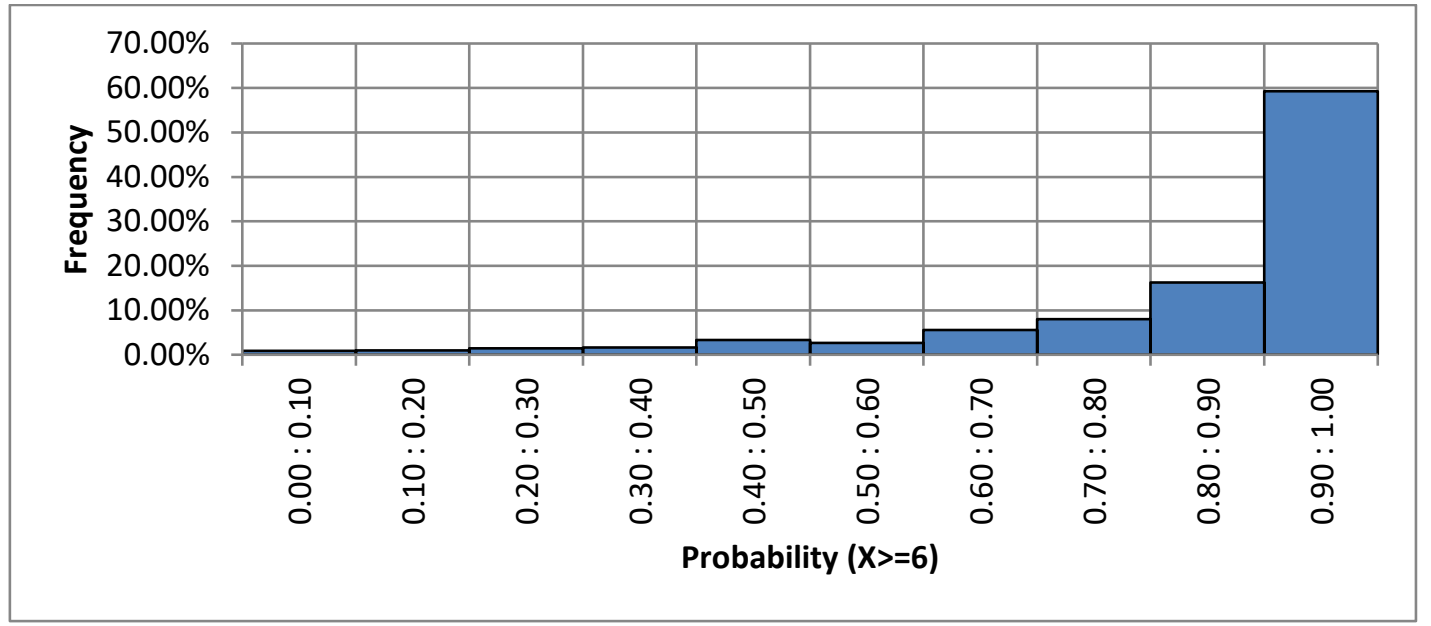

Figure 4.15: $P$ (at least 6 equipped vehicles/min) at $1000 \mathrm{veh} / \mathrm{h} / \mathrm{lane}$

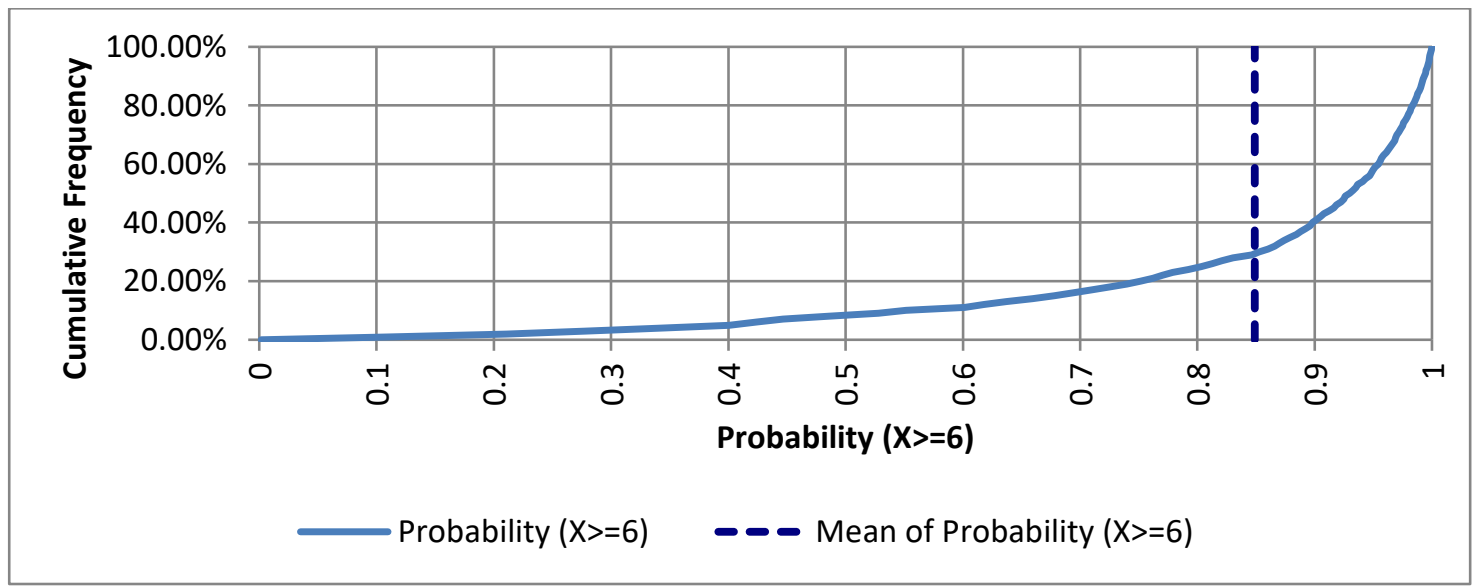

Figure 4.16: Cumulative probability, at least 6 equipped vehicles/min at $1000 \mathrm{veh} / \mathrm{h} / \mathrm{lane}$ 
For $1500 \mathrm{veh} / \mathrm{h} / \mathrm{lane}$, at $30 \%$ equipped level, for 2 lanes, $\mu=15$ and $\sigma=4.5$. Following 1000 simulations, the following results were obtained.

$\mathrm{P}($ at least 1 equipped vehicle during one minute time interval $)=\mathrm{P}(\mathrm{X} 1)$

$P(X 1)=1-P(X=0)=0.995$, as illustrated in Figure 4.17 and Figure 4.18.

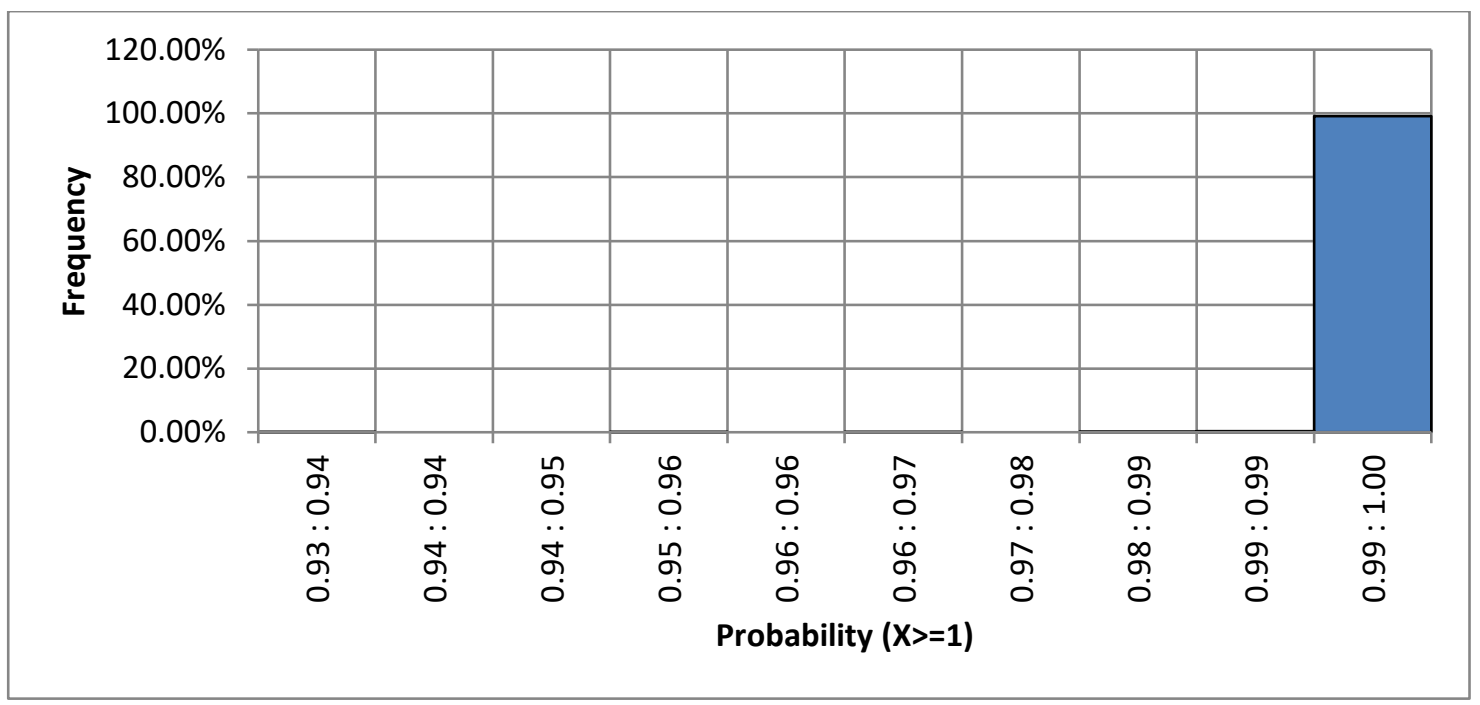

Figure 4.17: P (at least 1 equipped vehicle/min) at $1500 \mathrm{veh} / \mathrm{h} / \mathrm{lane}$

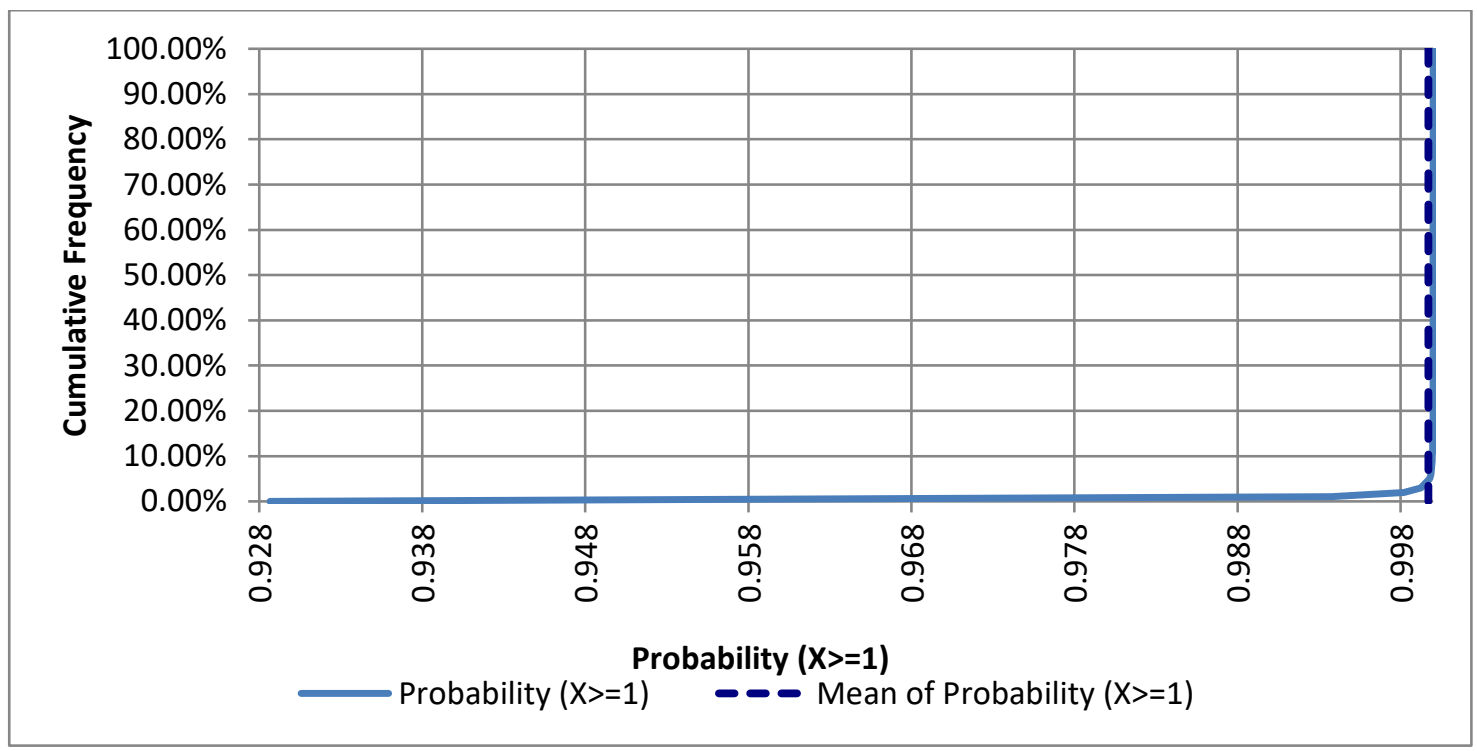

Figure 4.18: Cumulative probability, at least 1 equipped vehicle/min at $1500 \mathrm{veh} / \mathrm{h} / \mathrm{lane}$ 
$\mathrm{P}$ (at least 2 equipped vehicles during one minute time interval $)=\mathrm{P}(\mathrm{X} 2)$

$\mathrm{P}(\mathrm{X} 2)=1-(\mathrm{P}(\mathrm{X}=0)+\mathrm{P}(\mathrm{X}=1))=0.994$

$\mathrm{P}$ (at least 3 equipped vehicles during one minute time interval $)=\mathrm{P}(\mathrm{X} 3)$

$P(X 3)=1-(P(X=0)+P(X=1)+P(X=2))=0.989$

$\mathrm{P}$ (at least 4 equipped vehicles during one minute time interval $)=\mathrm{P}(\mathrm{X} 4)$

$\mathrm{P}(\mathrm{X} 4)=1-(\mathrm{P}(\mathrm{X}=0)+\mathrm{P}(\mathrm{X}=1)+\mathrm{P}(\mathrm{X}=2)+\mathrm{P}(\mathrm{X}=3))=0.981$

$\mathrm{P}$ (at least 5 equipped vehicles during one minute time interval $)=\mathrm{P}(\mathrm{X} 5)$

$\mathrm{P}(\mathrm{X} 5)=1-(\mathrm{P}(\mathrm{X}=0)+\mathrm{P}(\mathrm{X}=1)+\mathrm{P}(\mathrm{X}=2)+\mathrm{P}(\mathrm{X}=3)+\mathrm{P}(\mathrm{X}=4))=0.971$

$\mathrm{P}$ (at least 6 equipped vehicles during one minute time interval $)=\mathrm{P}(\mathrm{X} 6)$

$\mathrm{P}(\mathrm{X} 6)=1-(\mathrm{P}(\mathrm{X}=0)+\mathrm{P}(\mathrm{X}=1)+\mathrm{P}(\mathrm{X}=2)+\mathrm{P}(\mathrm{X}=3)+\mathrm{P}(\mathrm{X}=4)+\mathrm{P}(\mathrm{X}=6))=0.956$, as

illustrated in Figure 4.19 and Figure 4.20.

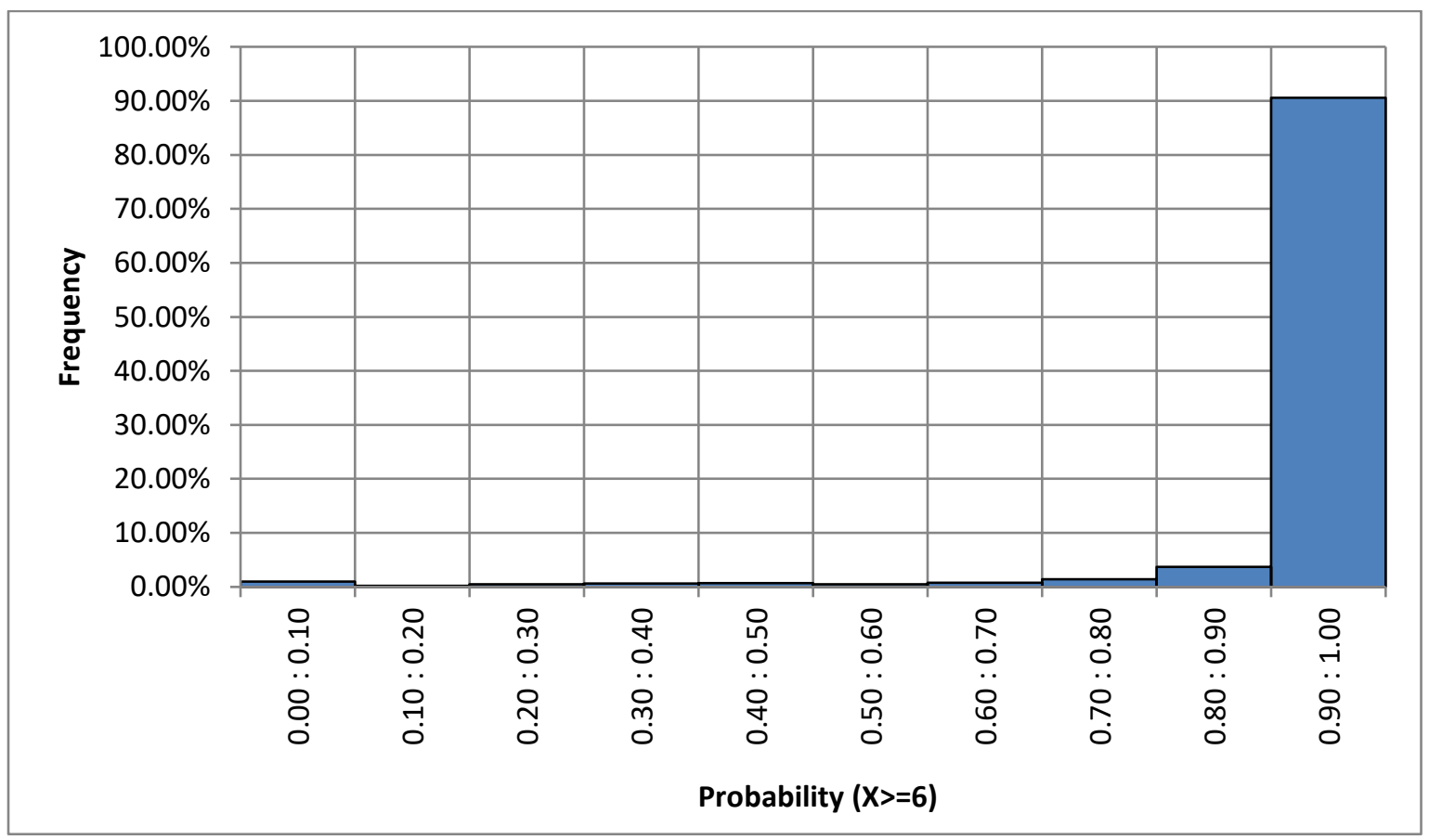

Figure 4.19: $P$ (at least 6 equipped vehicles/min) at $1500 \mathrm{veh} / \mathrm{h} / \mathrm{lane}$ 


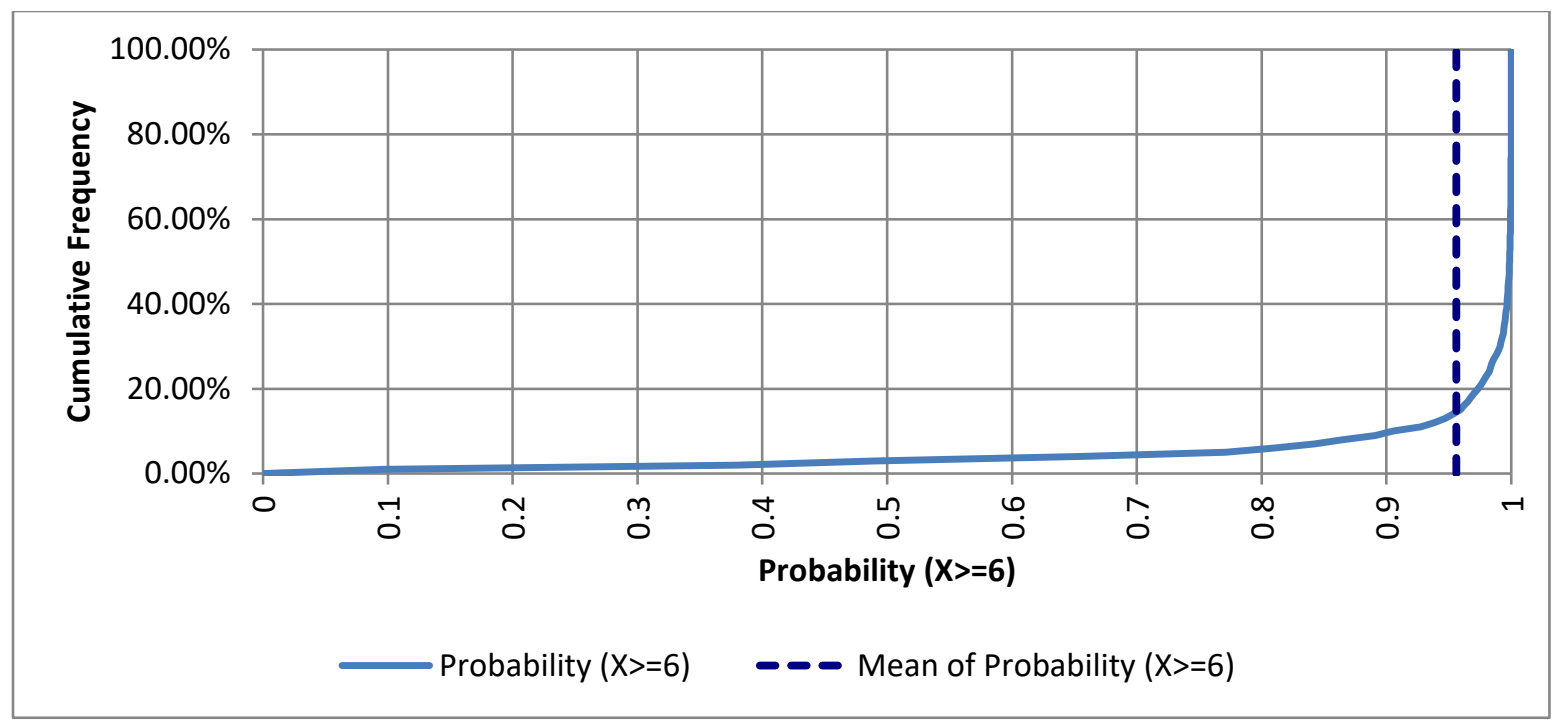

Figure 4.20: Cumulative probability, at least 6 equipped vehicle/min at $1500 \mathrm{veh} / \mathrm{h} / \mathrm{lane}$

Table 4.1 shows the results obtained from the joint probability and Monte Carlo simulation studies.

Table 4.1: Results of joint probability and Monte Carlo simulation, 2 lanes/direction

\begin{tabular}{|c|c|c|c|c|}
\hline $\begin{array}{l}\% \text { fleet } \\
\text { equipped }\end{array}$ & Joint probability analysis & \multicolumn{2}{|c|}{$\begin{array}{l}\text { Monte Carlo Simulation } \\
\text { P(at least } 6 \text { equipped) }\end{array}$} & $P(X>=6)$ \\
\hline & $\underline{500 \mathrm{veh} / \mathrm{h} / \text { lane }}$ & \multicolumn{2}{|c|}{ 500 veh/h/lane } & $\underline{500 \mathrm{veh} / \mathrm{h} / \text { lane }}$ \\
\hline $20 \%$ & $(8.33)(0.2)=1.66$ or $2 \times 2=4 \mathrm{veh} /$ cell & $\mu=4, \sigma=2$ & $\mathrm{P}(\mathrm{X}>=6)=$ & 0.286 \\
\hline $30 \%$ & $(8.33)(0.3)=2.49$ or $3 \times 2=6 \mathrm{veh} /$ cell & $\mu=6, \sigma=2$ & $\mathrm{P}(\mathrm{X}>=6)=$ & 0.538 \\
\hline $40 \%$ & $(8.33)(0.4)=3.33$ or $4 \times 2=8 \mathrm{veh} /$ cell & $\mu=8, \sigma=3$ & $\mathrm{P}(\mathrm{X}>=6)=$ & 0.720 \\
\hline $50 \%$ & $(8.33)(0.5)=4.17$ or $4 \times 2=8 \mathrm{veh} /$ cell & $\mu=8, \sigma=3$ & $P(X>=6)=$ & 0.858 \\
\hline $60 \%$ & $(8.33)(0.6)=4.99$ or $5 \times 2=10 \mathrm{veh} /$ cell & $\mu=10, \sigma=3$ & $\mathrm{P}(\mathrm{X}>=6)=$ & 0.858 \\
\hline $70 \%$ & $(8.33)(0.7)=5.83$ or $6 \times 2=12 \mathrm{veh} /$ cell & $\mu=12, \sigma=4$ & $P(X>=6)=$ & 0.904 \\
\hline $80 \%$ & $(8.33)(0.8)=6.66$ or $7 \times 2=14 \mathrm{veh} /$ cell & $\mu=14, \sigma=4$ & $\mathrm{P}(\mathrm{X}>=6)=$ & 0.928 \\
\hline $90 \%$ & $(8.33)(0.9)=7.49$ or $8 \times 2=16 \mathrm{veh} /$ cell & $\mu=16, \sigma=5$ & $\mathrm{P}(\mathrm{X}>=6)=$ & 0.965 \\
\hline $100 \%$ & $(8.33)(1.0)=8.33$ or $9 \times 2=18 \mathrm{veh} /$ cell & $\mu=18, \sigma=6$ & $P(X>=6)=$ & 0.970 \\
\hline
\end{tabular}




\begin{tabular}{|c|c|c|c|c|}
\hline \multirow[b]{2}{*}{$20 \%$} & \multirow{3}{*}{$\begin{array}{l}1000 \mathrm{veh} / \mathrm{h} / \text { lane } \\
(16.66)(0.2)=3.33 \text { or } 4 \times 2=8 \mathrm{veh} / \text { cell } \\
(16.66)(0.3)=4.99 \text { or } 5 \times 2=10 \mathrm{veh} / \text { cell }\end{array}$} & \multicolumn{2}{|c|}{$1000 \mathrm{veh} / \mathrm{h} /$ lane } & \multirow[b]{2}{*}{0.720} \\
\hline & & $\mu=8, \sigma=3$ & $\mathrm{P}(\mathrm{X}>=6)=$ & \\
\hline $30 \%$ & & $\mu=10, \sigma=3$ & $\mathrm{P}(\mathrm{X}>=6)=$ & 0.858 \\
\hline $40 \%$ & $(16.66)(0.4)=6.66$ or $7 \times 2=14 \mathrm{veh} /$ cell & $\mu=14, \sigma=4$ & $\mathrm{P}(\mathrm{X}>=6)=$ & 0.928 \\
\hline $50 \%$ & $(16.66)(0.5)=8.33$ or $9 \times 2=18 \mathrm{veh} /$ cell & $\mu=18, \sigma=6$ & $\mathrm{P}(\mathrm{X}>=6)=$ & 0.970 \\
\hline $60 \%$ & $(16.66)(0.6)=9.99$ or $10 \times 2=20 \mathrm{veh} /$ cell & $\mu=20, \sigma=6$ & $\mathrm{P}(\mathrm{X}>=6)=$ & 0.985 \\
\hline $70 \%$ & $(16.66)(0.7)=11.66$ or $12 \times 2=24 \mathrm{veh} / \mathrm{cell}$ & $\mu=24, \sigma=7$ & $\mathrm{P}(\mathrm{X}>=6)=$ & 0.985 \\
\hline $80 \%$ & $(16.66)(0.8)=13.33$ or $14 \times 2=28 \mathrm{veh} / \mathrm{cell}$ & $\mu=28, \sigma=9$ & $\mathrm{P}(\mathrm{X}>=6)=$ & 0.992 \\
\hline $90 \%$ & $(16.66)(0.9)=14.99$ or $15 \times 2=30$ veh $/$ cell & $\mu=30, \sigma=9$ & $\mathrm{P}(\mathrm{X}>=6)=$ & 0.996 \\
\hline $100 \%$ & $(16.66)(1.0)=16.66$ or $17 \times 2=34 \mathrm{veh} / \mathrm{cell}$ & $\mu=34, \sigma=11$ & $\mathrm{P}(\mathrm{X}>=6)=$ & 0.994 \\
\hline & $\underline{1500 \mathrm{veh} / \mathrm{h} / \text { lane }}$ & $\underline{1500 \mathrm{veh} / \mathrm{h} / \mathrm{la}}$ & & \\
\hline $20 \%$ & $(25)(0.2)=5$ or $6 \times 2=12 \mathrm{veh} /$ cell & $\mu=12, \sigma=4$ & $\mathrm{P}(\mathrm{X}>=6)=$ & 0.858 \\
\hline $30 \%$ & $(25)(0.3)=7.5$ or $8 \times 2=16 \mathrm{veh} /$ cell & $\mu=16, \sigma=5$ & $\mathrm{P}(\mathrm{X}>=6)=$ & 0.965 \\
\hline $40 \%$ & $(25)(0.4)=10$ or $10 \times 2=20 \mathrm{veh} /$ cell & $\mu=20, \sigma=6$ & $\mathrm{P}(\mathrm{X}>=6)=$ & 0.985 \\
\hline $50 \%$ & $(25)(0.5)=12.5$ or $13 \times 2=26 \mathrm{veh} /$ cell & $\mu=26, \sigma=6$ & $\mathrm{P}(\mathrm{X}>=6)=$ & 0.992 \\
\hline $60 \%$ & $(25)(0.6)=15$ or $15 \times 2=30 \mathrm{veh} /$ cell & $\mu=30, \sigma=8$ & $P(X>=6)=$ & 0.996 \\
\hline $70 \%$ & $(25)(0.7)=17.5$ or $18 \times 2=36 \mathrm{veh} /$ cell & $\mu=36, \sigma=11$ & $\mathrm{P}(\mathrm{X}>=6)=$ & 0.997 \\
\hline $80 \%$ & $(25)(0.8)=20$ or $20 \times 2=40 \mathrm{veh} /$ cell & $\mu=40, \sigma=12$ & $\mathrm{P}(\mathrm{X}>=6)=$ & 0.998 \\
\hline $90 \%$ & $(25)(0.9)=22.5$ or $23 \times 2=46 \mathrm{veh} / \mathrm{cell}$ & $\mu=46, \sigma=14$ & $\mathrm{P}(\mathrm{X}>=6)=$ & 0.999 \\
\hline $100 \%$ & $(25)(1.0)=25$ or $25 \times 2=50 \mathrm{veh} /$ cell & $\mu=50, \sigma=15$ & $\mathrm{P}(\mathrm{X}>=6)=$ & 0.999 \\
\hline
\end{tabular}


For $500 \mathrm{veh} / \mathrm{h} / \mathrm{lane}$, the cumulative probability for the \% fleet equipped vehicles that range from $20 \%$ to $100 \%$ is illustrated in Figure 4.21 , and the mean probability is illustrated in Figure 4.22.

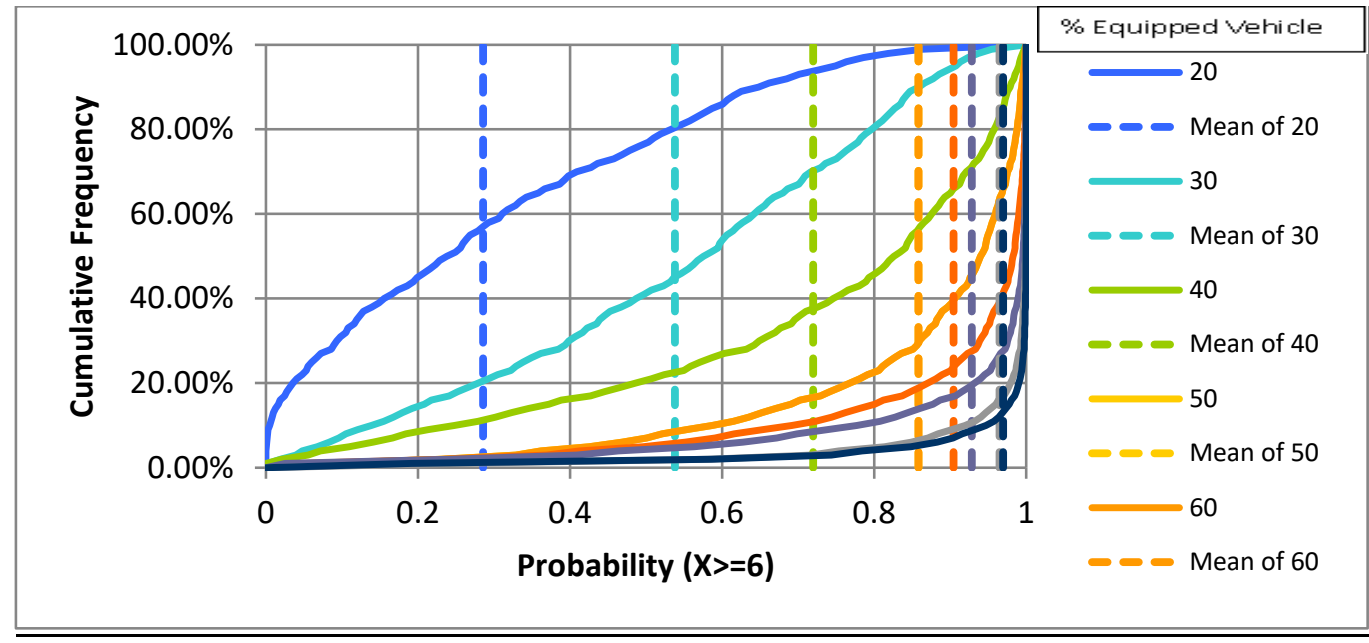

Figure 4.21: Cumulative probability, at least 6 equipped vehicle/min at $500 \mathrm{veh} / \mathrm{h} / \mathrm{lane}$

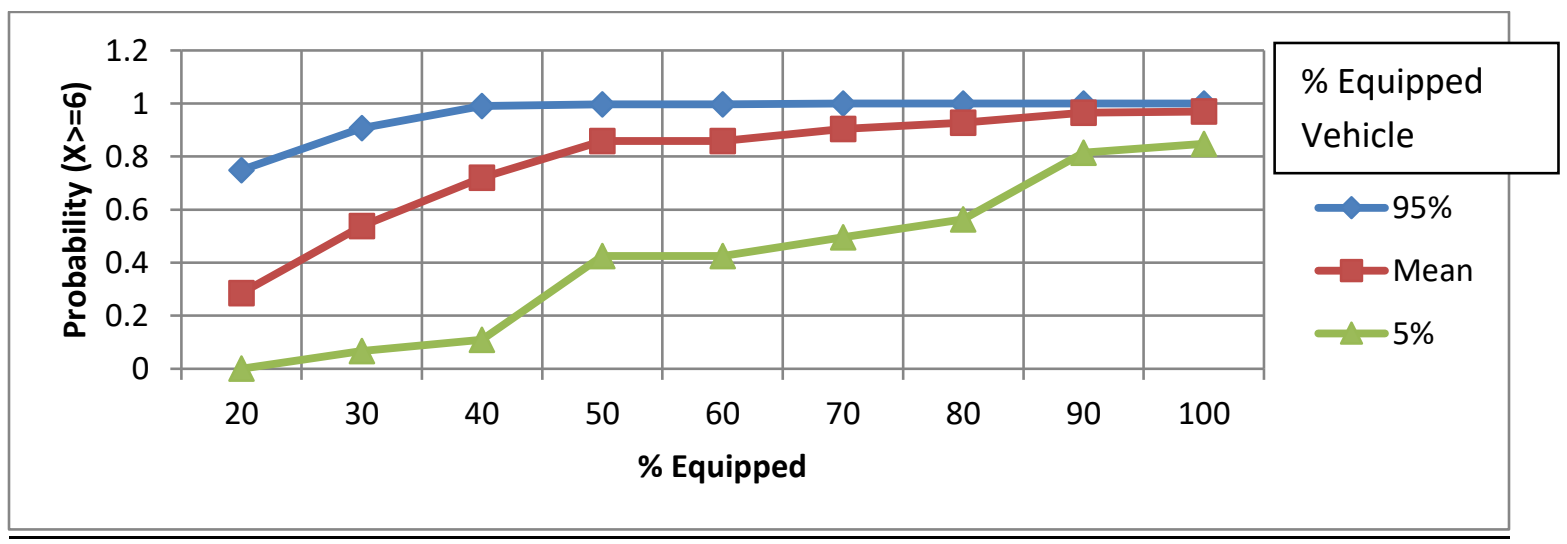

Figure 4.22: Mean probability, at least 6 equipped vehicle/min at $500 \mathrm{veh} / \mathrm{h} / \mathrm{lane}$

For $1000 \mathrm{veh} / \mathrm{h} / \mathrm{lane}$ the cumulative probability for the $\%$ fleet equipped vehicles that range from $20 \%$ to $100 \%$ is illustrated in Figure 4.23 and the mean probability is illustrated in Figure 4.24. 


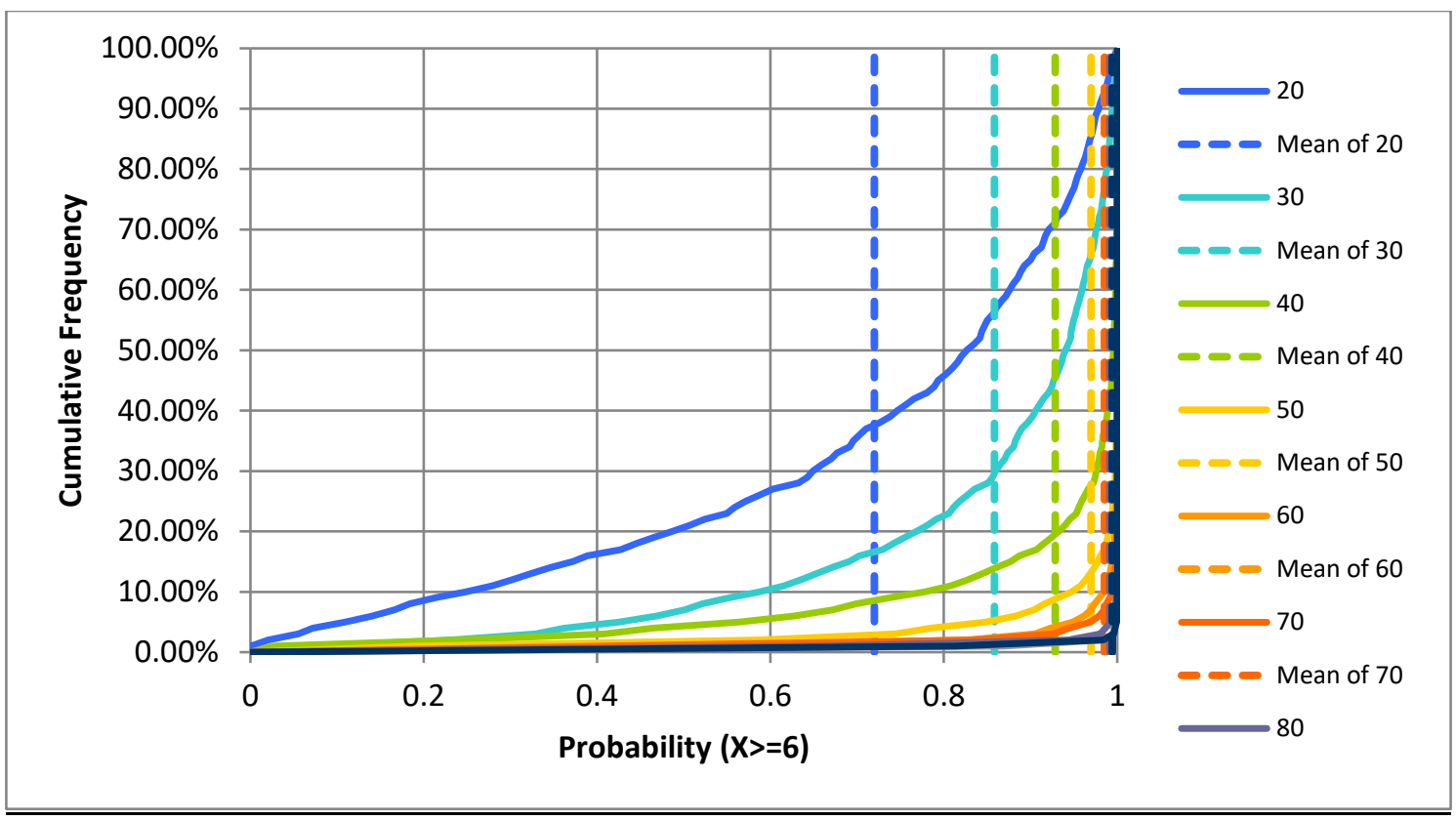

Figure 4.23: Cumulative probability, at least 6 equipped vehicle/min at $1000 \mathrm{veh} / \mathrm{h} / \mathrm{lane}$

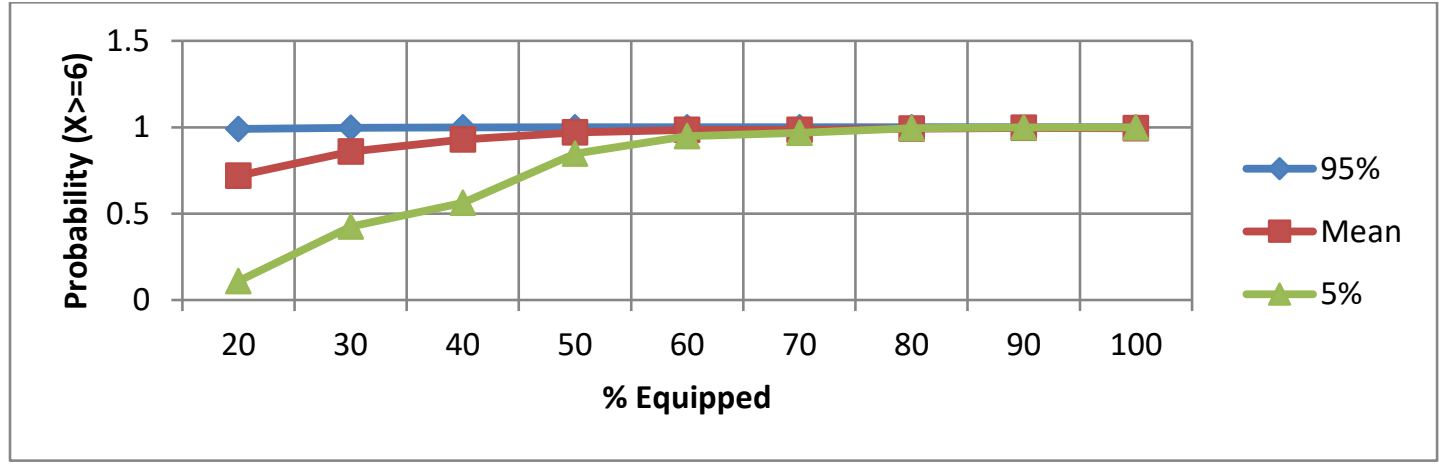

Figure 4.24. Mean probability, at least 6 equipped vehicles/min at $1000 \mathrm{veh} / \mathrm{h} / \mathrm{lane}$

For $1500 \mathrm{veh} / \mathrm{h} /$ lane the cumulative probability for the $\%$ fleet equipped vehicles that range from $20 \%$ to $100 \%$ is illustrated in Figure 4.25 and the mean probability is illustrated in Figure 4.26 . 


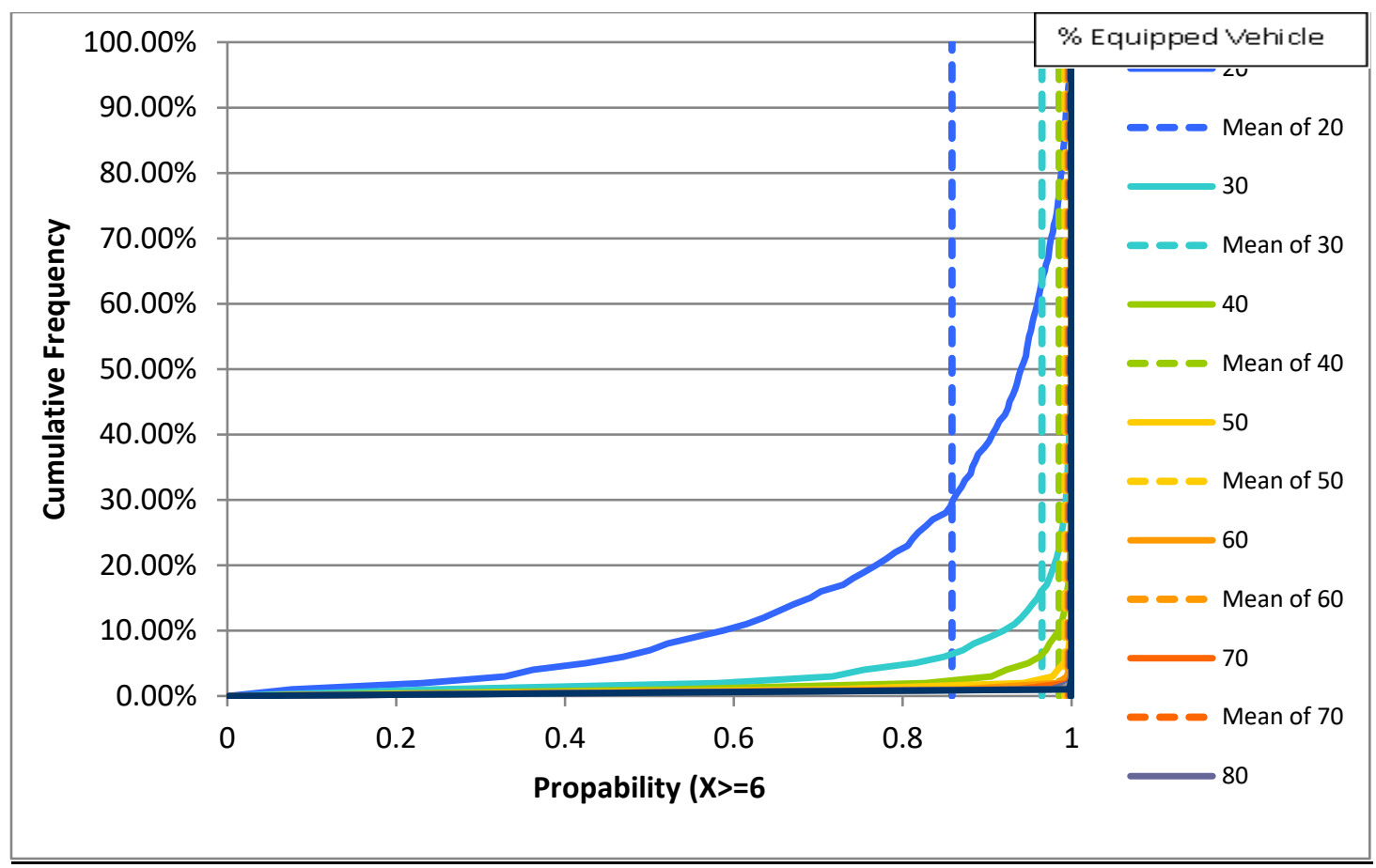

Figure 4.25: Cumulative probability, at least 6 equipped vehicle/min

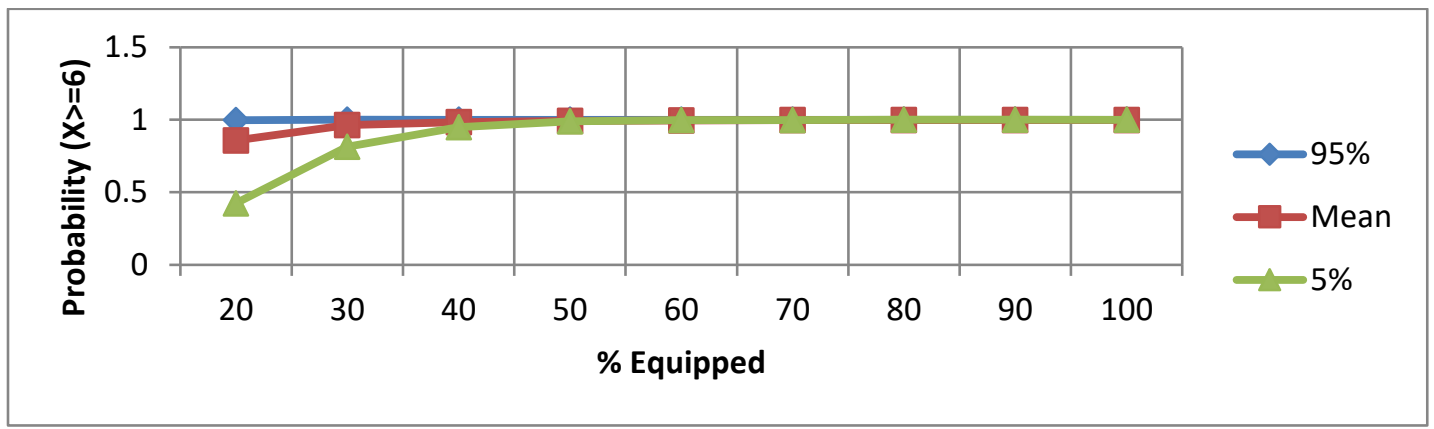

Figure 4.26. Mean probability, at least 6 equipped vehicle/min at $1500 \mathrm{veh} / \mathrm{h} / \mathrm{lane}$

\section{Discussion:}

For $86.6 \%$ confidence level, the minimum sample size of required equipped vehicles during 1 min interval was 6 equipped vehicles. Utilizing XLSim, a Monte Carlo simulation tool and following 1000 simulations, it was concluded that: 
- As a very worst case, if $20 \%$ of the fleet is equipped at a traffic level of $500 \mathrm{veh} / \mathrm{h} / \mathrm{lane}$, 6 equipped vehicles will be in the cell during 1 minute interval did not meet the required probability level. This case was improved to a probability level of 0.86 when the traffic level reached $1500 \mathrm{veh} / \mathrm{h} /$ lane.

- Almost 0.85 probability level for at least 6 equipped vehicles in the cell during $1 \mathrm{~min}$ interval was reached under the assumption that $30 \%$ of the fleet is equipped at a traffic level of $1000 \mathrm{veh} / \mathrm{h} / \mathrm{lane}$.

- This probability level (0.85) was also maintained under the assumption of $20 \%$ of the fleet to be equipped at a traffic level of $1500 \mathrm{veh} / \mathrm{h} / \mathrm{lane}$.

- Increasing the percentage of equipped vehicles in the fleet over $40 \%$ did not result in appreciable change at a traffic level of $1000 \mathrm{veh} / \mathrm{h} / \mathrm{lane}$.

- Increasing the percentage of equipped vehicles in the fleet over 30\% did not result in appreciable change at a traffic level of $1500 \mathrm{veh} / \mathrm{h} / \mathrm{lane}$.

- Considering the case of low traffic level of $500 \mathrm{veh} / \mathrm{h} / \mathrm{lane}$, there was a significant change in the probability level when increasing the percentage of equipped vehicles in the fleet from $20 \%$ to $70 \%$ where the number jumped from 0.28 to 0.90 .

Estimates of reliability level can be inferred from the results presented in this chapter. For example, a reliability level of 0.6 is reached when $30 \%$ of the fleet is equipped at traffic condition of $500 \mathrm{veh} / \mathrm{h} /$ lane. A higher confidence level can be inferred from higher percentages of equipped vehicles in the fleet. These reliability estimates are a required input in the Bayesian incident detection model described in the following chapter. 


\section{Development of the Incident Detection Model}

An introduction to the incident detection model development is presented in this chapter. First, the modeling approach is discussed. Next, it is shown that as a result of an incident, the speed of the traffic flow changes upstream through the incident impact on the traffic condition. A logical approach utilizing the fundamental aspects of the Bayesian method is presented. Based on the discussion that follows, the concept of the developed model is explained. A selected case of the screened data presented in Chapter three is used to illustrate steps and methods used in model development. After testing the general feasibility of the model using its basic version, the final form is presented in the next chapter.

\subsection{Incident Detection Modeling Approaches}

This research advances a model for detecting incidents that can utilize detector/ sensor data or roadside unit data obtainable from future connected infrastructure-vehicle data or probe vehicle data. The developed model is capable of analyzing the speed pattern during a period of time that is well-known to either match an "incident" or "incident free" condition. Therefore this model should be able to detect an incident if one happened or not to detect an incident if none occurred.

The model and the algorithm, which are based on the Bayesian method and is assisted by the Montecarlo method. The Bayesian approach to predict space mean speed and travel time for use in a traveler information system was pioneered by Khan (2010). The advances in the Bayesian approach for the purpose of predicting incidents are one of the many next logical extensions of the predictive travel time Bayesian model. The Bayesian-Monte Carlo 
incident model, in its present stage of development as reported in this thesis helps to detect incidents in an efficient and effective manner. Before proceeding to detailed development work, preliminary results of the initial version of the developed model computed by Excel were examined. These results were assessed to be promising.

The model was developed and validated in an advanced Matlab (Matrix Laboratory) computer programming platform for tracking traffic speed pattern and investigating the fluctuation in the operating speed value obtained from the Highway 401 freeway traffic detectors. Likewise, results of the model validation showed promising results, as explained in the next chapter.

Elements of the research were extended to performance assessment of the developed model for traffic incidents detection. The performance of the developed model and the algorithm were tested by using a number of criteria, namely incident Detection Rate (DR), Time-to-Detect (TTD) incidents, and False Alarm Rate (FAR). These testing criteria are reported in the next chapter utilizing the computational version of the developed model.

\subsubsection{Incident Detection Modelling Philosophy}

In uninterrupted free flow or almost free flow condition, the effect of increasing volume/capacity ratio on operating speed is not well pronounced until approximately the threshold of "queue discharge" region is reached. A freeway facility generally maintains this type of service under incident-free conditions. Due to an increase in the traffic volume, the volume/capacity ratio (and corresponding density) increase, and as the level of service (LOS) starts to drop below D, the speed drops somewhat. Even when LOS is close to falling below E, no sharp decline is experienced in the operating speed. In the absence of incidents, 
even during LOS F operation, there is usually the evidence of recovery of traffic flow from low speed to somewhat higher speed.

If the spot speed shows a decline for a few minutes, it could be due to many factors, out of which are the following two factors:

a- an imbalance of demand and capacity caused by a surge of traffic at on-ramps or at lane drops, resulting in traffic flow breakdown, and

b- due to a serious bottleneck in the freeway usually caused by an incident.

Second factor (b) is of interest in this research. In the case of unexpected capacity loss in an uninterrupted flow type of a highway due to incidents during high traffic periods, the operating speed is expected to suffer a sharp decline in a short period of time. This pattern of speed drop and the evidence of a substantial amount of time taken to recover from low operating speed pattern have been used to infer an incident. That is, such evidence includes the inability of traffic flow to recover quickly within a specified period of time.

The developed model and its algorithm version need to find out if the sharp drop in speed during high traffic volume conditions is due to an incident or if it is due to an imbalance between demand and capacity.

\subsubsection{Travel Facility Cells and Temporal Features of Incidents}

The uninterrupted flow type of a highway facility can be divided into cells as shown in Figure 2.1 and the virtual vehicle-infrastructure integration (VII) roadside units (RSU) can be placed at cell boundaries. In this research, initially, the virtual RSUs have the same capability as the induction loops. That is, these capture data but do not interact with vehicles. These can serve as virtual stations and can communicate to traffic control centres. 
A future generation of the RSU will have the capability to receive and send information to vehicles, and also, these can process data regarding traffic flow condition and hazards. These assumed capabilities are in line with scenarios of technology development.

For modeling traffic states and incidents, we monitor temporal aspect of traffic states within a cell, on a network level. All cells are monitored.

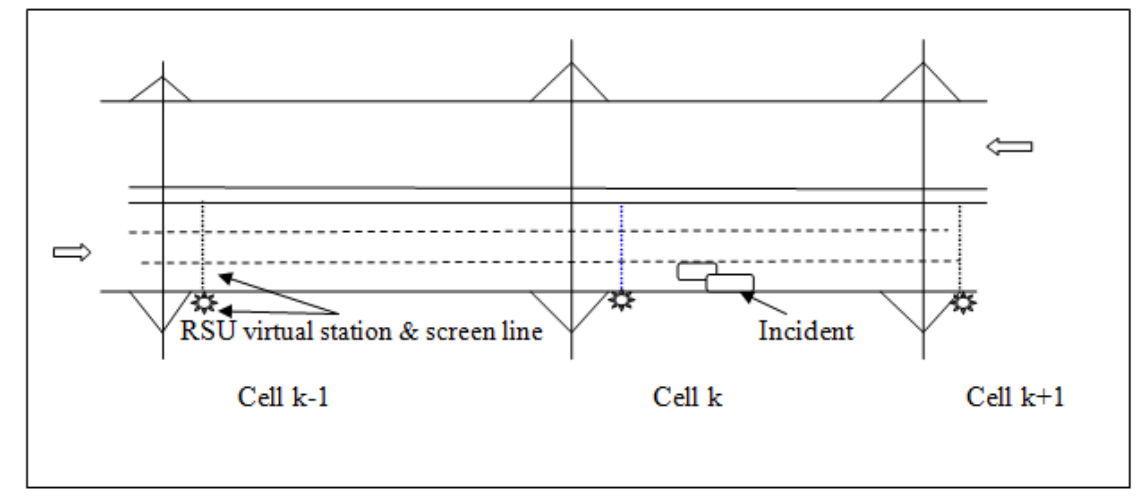

Figure 5.1: Spatial features of highway facility

Since we are interested in modeling the rapidly changing traffic effects of incidents, it is necessary to use small intervals such as 2 minutes or one minute. For now, in this discussion, we use one-minute interval. However, the available data enabled a 20 seconds interval for Model development and validation. Although it can be argued that with the availability of data at very short interval, there is no need to predict the traffic state during the next time period, it should be pointed that the developed algorithm may be used where data refreshment may be at 5 minutes or higher intervals.

At time period $t=0$, traffic flow condition of the previous time period $t=-1$ becomes known from the sensor data. The assumed present RSU technology does not have the 
capability to track on a real-time basis, every vehicle's spatial coordinates, speed, and acceleration/deceleration. Therefore, for now, for each cell, we will work with spot speed as obtainable from a virtual roadside unit (but from its proxy technology of a detector station). We also use the computed space mean speed. It should be noticed that the future RSUs can be programmed to compile speed profile of a vehicle at the specified sensor locations. Therefore, the average spot speed of vehicles at the virtual stations can be obtained. For a pre-defined time interval (e.g. 2 minutes, 1 minute), the average spot speed can be found at the upstream and downstream virtual stations.

It is intended to monitor traffic flow conditions for each time interval in a cell so that incident-related congestion, and therefore, incidents can be detected as quickly as possible. For this purpose, two approaches can be followed.

(1) Use the latest available past (i.e. at $t=-1$ ) sensor data. This approach is valid, provided that traffic flow condition at time interval $\mathrm{t}=0$ will be the same as in the preceding interval (i.e., at $t=-1$ ). If not, traffic flow condition should be predicted at the outset of $\mathrm{t}=0$ and then compared with actual information after it becomes available from the system.

(2) Use the latest sensor data (for $t=-1$ ) as a reading on what is to come next during $t=0$ and use it as a stepping stone in a predictive model to predict the traffic flow condition in the present $\mathrm{t}=0$ period.

Logically, the second approach is preferable since it includes approach 1 and goes beyond it in the form of predictive travel flow condition. 


\subsubsection{Modelling Approaches/ Fundamental Aspects}

The developed algorithm is a predetermined set of rules for conducting computational steps. The developed model is used as a basis for the algorithm and the algorithm serves as the mechanism for model application. The algorithm uses a pre-defined set of rules for predicting speed and applicable probabilities. The incident detection model is the core of the algorithm and in turn the algorithm carries out the computations in an event-driven manner.

\section{Bayesian Method for Predictive Speed Modeling}

Many variations of the Bayesian method exist, depending upon application contexts. In this research, a modified version of the formulation suggested by Raiffa and Schlaifer (1968), Khan (1969), Taylor (2005), and Khan (2010) is used. However, modifications that are explained later in the text leave the basic principles intact.

\section{- Stochastic Traffic State}

In this thesis research, the uncertain state of traffic as characterized by speed is called S. The possible states of nature are: $\mathrm{s}_{1}, \mathrm{~s}_{2}, \ldots \varepsilon \mathrm{S}$. The states of nature are probabilistic. That is, probabilities have to be assigned to each state in the set of states of nature.

\section{- Route Alternatives}

In the usual Bayesian analysis, the decision maker or the decision-making system can select an action alternative $A$ from the set $a$. That is, a set of alternative terminal actions can be defined: $A_{1}, \ldots \varepsilon a$, wherein the decision is to be made by selecting a single act $A$, 
from some domain $a$ of potential acts. In the context of traffic flow, we assume that control system and the driver have options, but that we will predict incidents for one route at a time. So, the comparison of routes is not applicable in this thesis.

\section{- Acquisition of New Information}

A strength of the Bayesian method is to enable additional information acquisition and to use new information to update probabilities of traffic states. The information acquisition activity in the connected vehicle environment (termed experiments in the decision theory terminology) relates to the information system (i.e. virtual RSU or future vehicle-information integration) that can be accessed by the methodology. In the case of incident detection research, only two information acquisition means are definable: $c_{0}$ (no information is obtained from the system and $c_{l}$ (information is obtained from the system). In theory, $c_{0}, c_{1} \varepsilon C$. The incident detection system, in theory, may elect to use one of these from the set $C$ for the purpose of obtaining more information about the route regarding an incident. Here, availability of sensor or RSU data implies that acquiring additional information will not cost money, but its processing may take a few seconds.

\section{- Use of New Information}

The new readings on speed relate to the uncertain states. The sample space or the set of outcomes or results of information acquisition are: $r_{1} \ldots \ldots \varepsilon R$. For each information acquisition activity $c$, there is a space of possible outcomes for that $c$. The space of possible outcomes $R$ is defined to encompass any outcome of any $c$ in $C$. In case no new information 
is obtainable, it is noted as $r_{0}$. If new information is to be acquired, the outcome $r_{1}, r_{2}, \ldots$ cannot be known with certainty and, therefore, is to be treated as uncertain.

\section{- Speed Matrix $\operatorname{SP}(A, s)$}

The speed $S P(c, r, A, s)$ represents the system's estimate for all $c, r, A, S$ combinations. The sequence of a course of action is as follows. The system selects an information acquisition activity $c$, observes a result $r$, selects a particular $A$ (in the case of this research only one $A$ is used), and then a particular state of nature, $s$, occurs. In conceptual terms, the space of all possible combinations of $(c, r, A, s)$ is $C x R \times a x S$.

\section{- Probability Distributions}

The uncertain states of speed $s$ are assigned prior probabilities. That is, these probabilities reflect information available prior to obtaining new data $r$ from the sensors/RSU system. As noticed earlier, the readings $r$ on uncertain states $s$ are obtainable from sensor/RSU data. The prior probabilities are designated as $P^{\prime}(s)$. If continuous probability distributions are applicable, these can be used. However, in the present research, initially, discrete values of $s$ are used and therefore, the probabilities are also discrete. Following analysis of data, continuous prior probability density functions may be used. The $P^{\prime}(s)$ represents the analyst's judgment (on the basis of past data) about the relative likelihood of values of $s$.

A probability measure is required to indicate the likelihood of a reading $r$ representing the true $s$. This conditional probability measure is shown as $P(r \mid s, c)$. This measure can be considered as the reliability of $r$ in representing $s$. As a conditional 
probability, it should read as follows. Given that s is the true state, it is the probability that the reading $r$ will be the result of additional information acquisition $c$. That is, $P(r \mid s, c)$ characterizes information acquisition activity $c$.

A joint probability measure $P(s, r \mid c)$ can be defined over $\boldsymbol{S} x R$ for each information acquisition activity. For Bayesian analysis, it is a requirement to assign, either directly or indirectly, a joint probability $P(s, r \mid c)$ to the joint distribution of $s$ and $r$, over the space $S x$ $R$ for $c$ information acquisition. This implies that the analyst (or the automated system) should define the reliability of each possible information outcome $r$ in predicting the true state of speed $\mathrm{s}$, for $c$.

From the joint probability measure, four other probability measures are defined, as described below.

1. The prior measure $P^{\prime}(s)$ on the states of nature that the decision system would assign to $s$ prior to observing the outcome $r$ of information acquisition $c$.

2. The conditional measure $P(r \mid s, c)$ on the space $R$-- the probability that the outcome $r$ will be observed if the information acquisition activity $c$ is performed and $s$ is the true value of the state of speed.

3. The marginal measure $P(r \mid c)$ on the space $R$ for all $S$ or the probability of observing outcome $r$ from information acquisition $c$. It is computed using the following: $P(r \mid c)=\Sigma P^{\prime}(s) P(r \mid s, c)$

4. The posterior measure $P^{\prime \prime}(s \mid r, c)$ on the space $S$, wherein this is the likelihood of different states $s$, given $r$ and $c$. The analyst or the system assigns this probability measure to the state $s$ after knowing the outcome $r$ of the information acquisition activity $c$. It is computed using the following equation: 


$$
\mathrm{P}^{\prime \prime}(\mathrm{s} \mid \mathrm{r}, \mathrm{c})=\frac{\mathrm{P}^{\prime}(\mathrm{s}) \mathrm{P}(\mathrm{r} \mid \mathrm{s}, \mathrm{c})}{\sum \mathrm{P}^{\prime}(\mathrm{s}) \mathrm{P}(\mathrm{r} \mid \mathrm{s}, \mathrm{c})}
$$

The Bayesian philosophy is that $c$ can be characterized by a conditional probability distribution $P(r \mid s, c)$, such that the relationship between the prior and posterior distribution is given as Bayes Theorem.

\section{- Computing the Expected Speed}

In theory, all sequences of $(c, r, A, s)$ can be analyzed, and the desirability of a course of action (i.e. the sequence) can be found. However, in this research, we are interested in finding the expected speed. This is, we are not using the complete Bayesian formulation and our interest is in using the probabilities to compute expected speed.

The steps are as follows.

1. The likelihood of different states of nature, $s$, is expressed in the form of prior probability distribution $P^{\prime}(s)$.

2. The conditional probability characteristics $P(r \mid s, c)$, is determined for each experiment.

3. Compute the marginal measures $P(r \mid c)$, for each experiment as was noted earlier, where the null is equal to one.

4. Compute the posterior probability distribution $P^{\prime \prime}(s \mid r, c)$ for each combination as defined above wherein for the null, the prior and posterior probabilities are equal.

5. Find speed for each $\operatorname{SP}\left(A_{1}, r, c_{1}\right)$ combination.

6. Find Expected Speed for posterior probability case:

$$
E(S)=P(r \mid c)\left[S P\left(A_{1}, r, c_{1}\right)\right]
$$


Figure 5.2 presents variables (defined above), linkages, and the sequence of steps for computing expected speed.

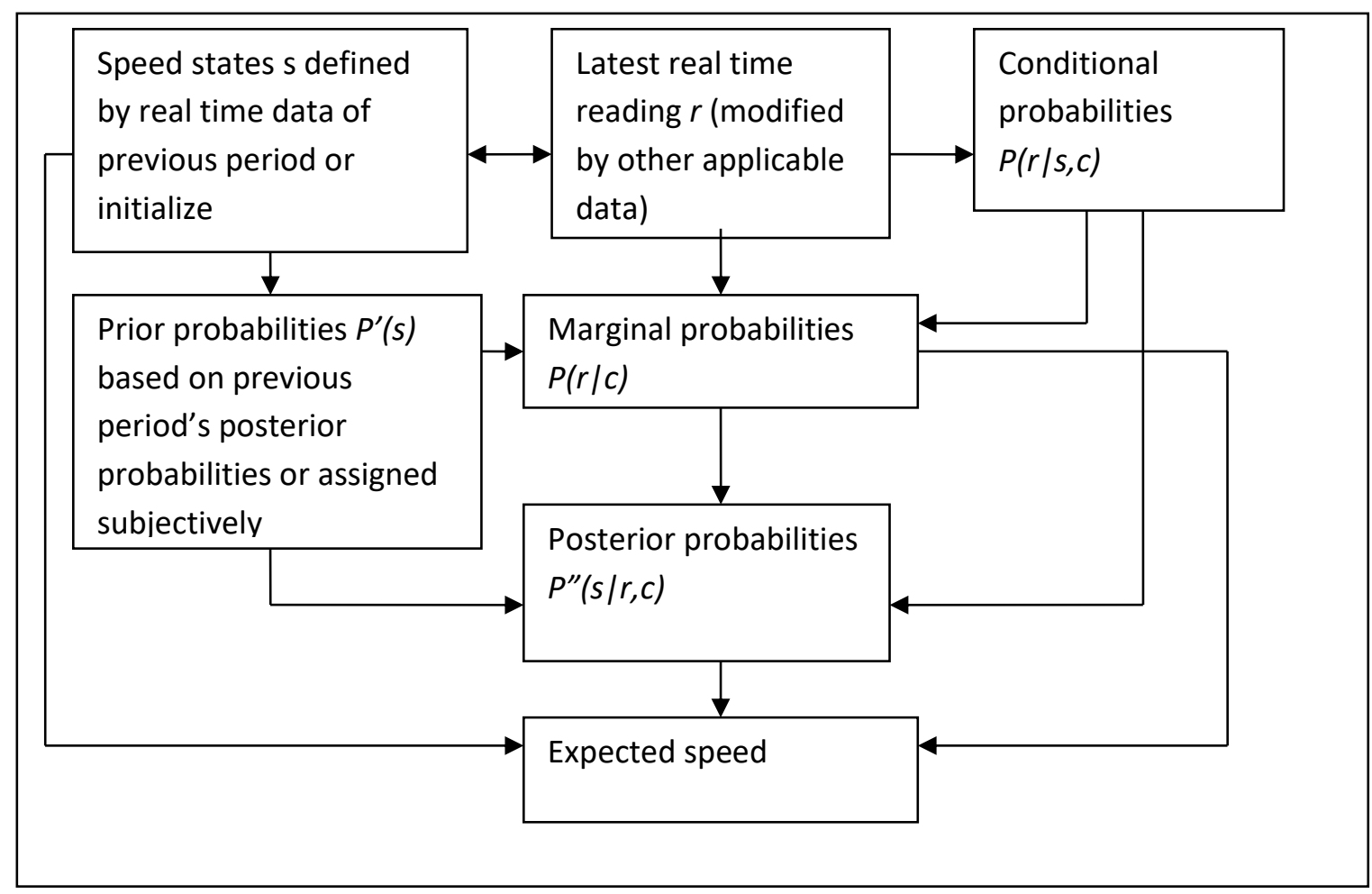

(Adapted from Khan 2010)

Figure 5.2: Bayesian predictive speed methodology

\subsubsection{Data Fusion for Predictive Speed Modelling}

The RSU technology can serve as sources of real-time data on traffic conditions. However, such information by itself becomes obsolete quickly. To make use of this resource, it can serve as a steppingstone for providing predictive speed that can offer an opportunity for early detection of an incident.

As noted earlier, the uncertain speed states are designated by $s$ and at the time of initializing the system, prior probabilities $P^{\prime}(s)$ can be assigned by sourcing these from the 
standard normal density function by using a selected value of standard normal deviate. This is one approach, however, a logit model approach is used in this research to initialize priors.

The $r$ corresponds to $s$ (i.e., for each uncertain $s$, an indicator $r$ can result from the vehicle-infrastructure integration technology, modified by other applicable data (if necessary). The conditional probabilities $P(r \mid s, c)$ and prior probabilities $P^{\prime}(s)$ are used as a means to find marginal probabilities $P(r \mid c)$, which in turn are used to update the probability of speed state by calculating posterior probabilities $P$ " $(s \mid r, c)$ as shown in Figure 5.2. The conditional probability $P(r \mid s, c)$ is read as follows: "Given that $s$ is the true speed state, it is the probability that the additional information acquisition system $c$ (i.e., RSU system) will produce the indicator $r$ that corresponds to $s$ ". That is, if the uncertain speed state implies relatively lower speed due to traffic or other influences on that route, it is the probability that the RSU system will give the same indication.

For a real-time application, speed states $s$ in the subject cell at the selected location and period are defined by using the speed states of the previous period and the result of the RSU systems $r$ based on the latest available technology captured data. For designing a realtime information system, prior probabilities $P^{\prime}(s)$ are based on previous period's posterior probabilities, provided that speed states are not re-defined. If speed states are re-defined due to wide fluctuation of traffic conditions (e.g., under incident condition), the prior probabilities are reset by using the logit method. The RSU indicator $r$ for the current location and period is used as an indicator for selecting the speed state that becomes the most likely speed for the next period as will be discussed in the next subsection. 


\subsubsection{Speed State Criteria and RSU Indicator $r$}

In the Bayesian methodology, speed is considered as uncertain and, therefore, a number of speed states are defined (e.g., low, moderate, high) and their probabilities are used to estimate the expected speed. In conceptual terms, there is no limit on the number of speed states. That is, instead of three states noted above and used later in this thesis, it is valid to use more than three states.

As was discussed in the previous section the uncertain state of traffic as characterized by speed is assigned the letter $\mathrm{S}$, and the possible states of nature are $\mathrm{s}_{1}, \mathrm{~s}_{2}, \mathrm{~s}_{3}, \ldots \varepsilon \mathrm{S}$. In order to determine the speed state for the next period, the RSU indicator $r$ for the current location and period needs to be identified. For the purpose of defining the indicator $r$, criteria are needed to accomplish that. Therefore, criteria are defined by introducing two other intermediate speed points in addition to the major three-speed states defined earlier (i.e. $\mathrm{s}_{1}, \mathrm{~s}_{2}$, and $\mathrm{s}_{3}$ ). These are upper midpoint and lower midpoint. The upper midpoint is at a middle point between the moderate speed state and the high-speed state while the lower midpoint is at a middle point between the moderate speed state and the lower speed state.

So, two speed points are defined in addition to the main three speed states. These are upper-speed state, upper midpoint, moderate speed state, lower midpoint, and lower speed state.

The establishment of the two midpoints (i.e. lower midpoint and the upper midpoint) leads to the formation of four-speed ranges between the speeds values defined above. The first range is defined between speed state $\mathrm{s}_{1}$ and the lower midpoint, and the second range is defined between the lower midpoint and speed state s. $_{2}$ Similarly, the third range is 
defined between speed state $s_{2}$ and the upper midpoint, and finally, the fourth range is defined between the upper midpoint and speed state $\mathrm{s}_{3}$.

Two approaches can be used to define the speed ranges between the five predefined boundaries. The first approach applies a percentage of the speed value that corresponds to the speed state $\mathrm{s}_{2}$ while the second approach uses an absolute speed range. A comparison of these two approaches suggests that the percentage of speed value gives reasonable changes in the speed state at a low-speed level as well as at high-speed level. The change in the speed state found with this approach relates to the speed value under consideration. The change in the speed state at the lower end of speed will be modest as compared to the upper end of speed spectrum. This approach is considered reasonable since it is in accordance with speed profiles of traffic and also it is expected to increase the sensitivity of the model.

The second approach considers a fixed speed range regardless of the speed value under consideration. The speed state is expected to change reasonably at high and intermediate speed levels. However, at the low-speed levels, the change in speed state will be unrealistically drastic. An illustration of the adopted approach is presented later in this chapter.

In summary, using the percentage of speed approach in defining states improves the performance of the model and has an influence in maintaining a consistent difference between the predicted speed and the detected speed at a low-speed level as well as at a high-speed level. 
If the percentage of speed change used for defining speed ranges is defined by ( $\mathrm{x})$ then, the boundaries for the four ranges in relation to the middle speed state $s_{2}$ can be illustrated as shown in Figure 5.3. The relationships are presented below.

- $\mathrm{s}_{1}=(1-(\mathrm{x} / 100)) * \mathrm{~s}_{2}$

- Lower midpoint $=\left(1-(0.5 \mathrm{x} / 100) * \mathrm{~s}_{2}\right.$

- $\mathrm{s}_{2}=\mathrm{s}_{2}$ (i.e. it is anchor point)

- Upper midpoint $=\left(1+(0.5 \mathrm{x} / 100) * \mathrm{~s}_{2}\right.$

- $\mathrm{s}_{3}=(1+(\mathrm{x} / 100)) * \mathrm{~s}_{2}$

Speed States and the Corresponding Speed Ranges

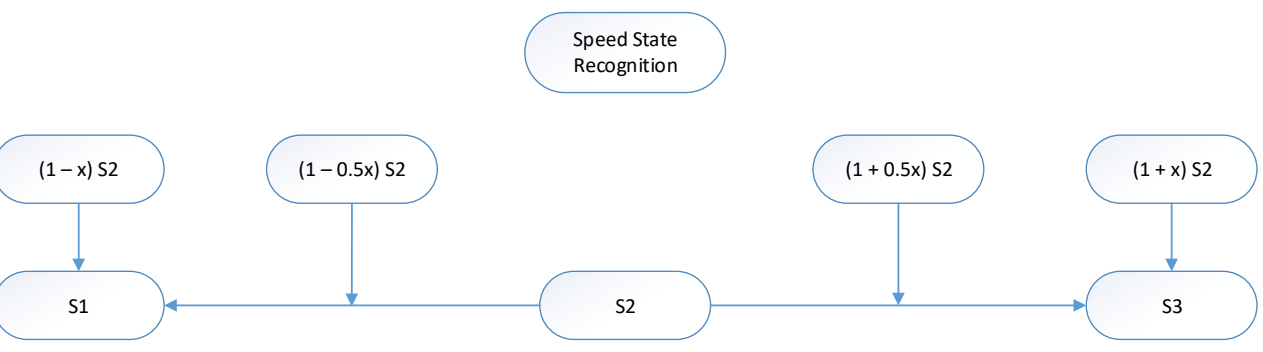

Figure 5.3: The speed states and the corresponding speed ranges

For example, with reference to the moderate speed state $s_{2}$, if the percentage of the predefined speed range is set at $7 \%$, then the speed that corresponds to state $\mathrm{s}_{3}$ is $7 \%$ higher than the speed that corresponds to state $\mathrm{s}_{2}$. Likewise, the speed that corresponds to state $\mathrm{s}_{1}$ is $7 \%$ lower than the speed that corresponds to state $\mathrm{s}_{2}$. The two midpoint speed percentages fall in between. That is, the upper midpoint speed boundary is 3.5\% higher than the speed that corresponds to speed state $\mathrm{s}_{2}$, and the lower midpoint speed boundary is $3.5 \%$ lower 
than the speed that corresponds to speed state $\mathrm{s}_{2}$. Assuming that the speed that corresponds to $s_{2}$ is $100 \mathrm{~km} / \mathrm{h}$, the other speed values that correspond to the other states including the midpoints can be illustrated as shown in Figure 5.4.

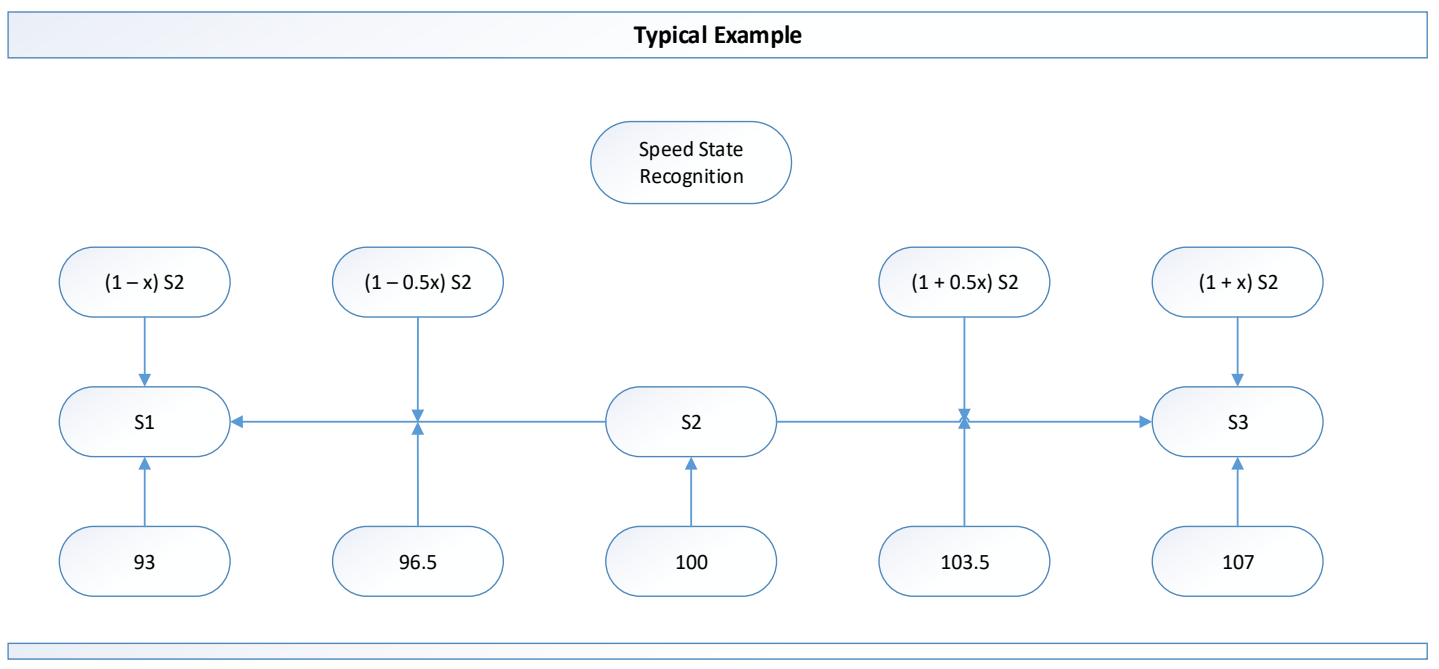

Figure 5.4: Defining speed at state s2 and the corresponding speed ranges

The defined criteria shown above for setting speed states provide the basis for moving forward from the current time period to following time for which a prediction has to be made. The latest speed value that became known from the RSU (i.e. about to be called the speed of the previous period) can fall within the four ranges or in cases of drastic changes in traffic flow, the observed speed can fall beyond a defined range.

Considering the current time period as $(t)$, the current speed (i.e. speed at $t$ ) is represented by (NSP). Using this notation, the speed of the previous time period (t-1) is represented by (OSP). Let (x) represent the selected range between the speed states. To build on the above notations, the following illustration and equations explain the procedure 
to determine the most likely speed state for the next time period $(t+1)$ based on the RSU reading of speed for the current period.

Figure 5.5 illustrates the different speed sates and the corresponding RSU Indicator r.

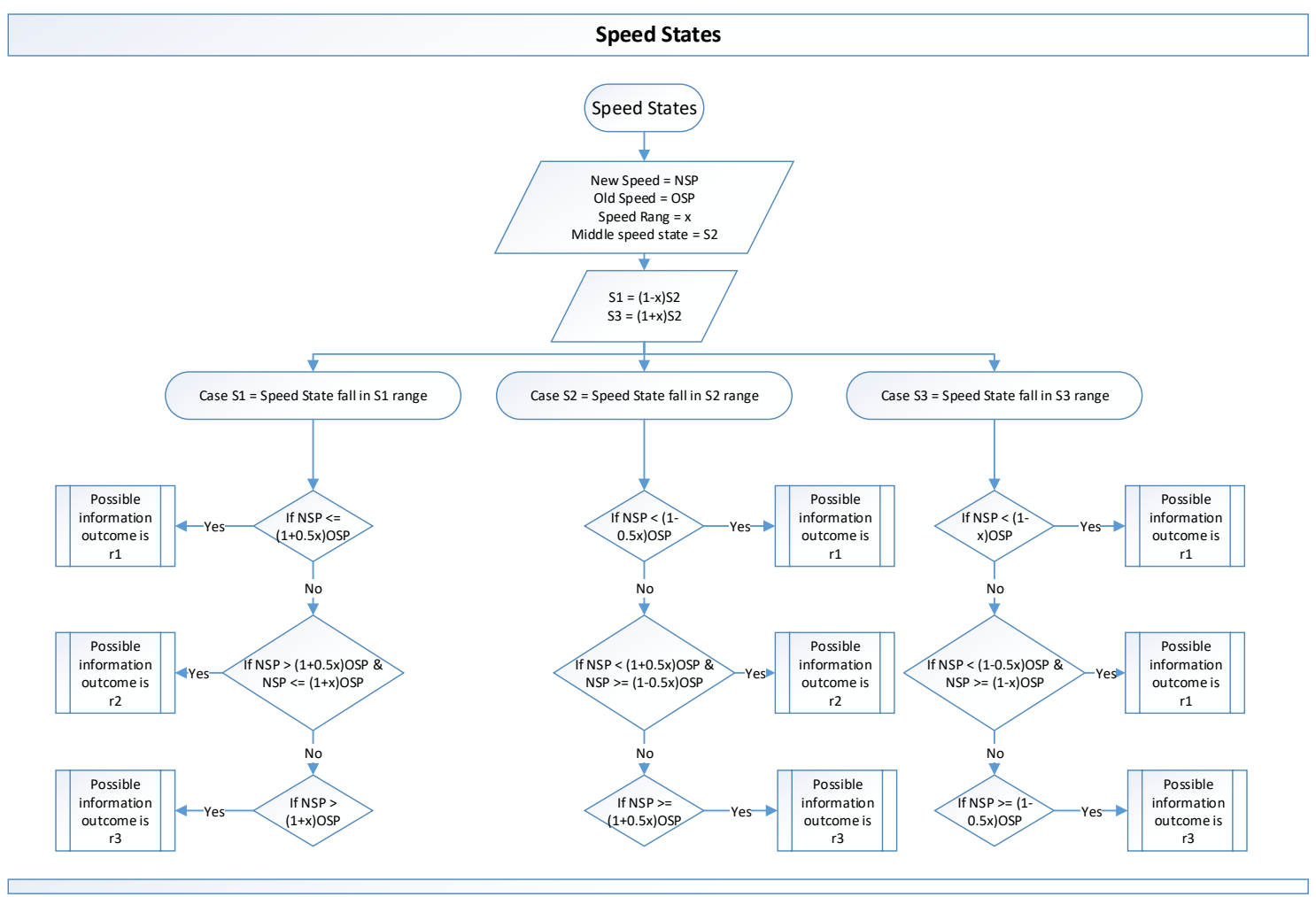

Figure 5.5: The speed states and the corresponding RSU indicator $r$

Considering the three possible speed states from the previous period $\left(s_{1}, s_{2}, s_{3}\right)$, the three possible conditions applied to the current speed are illustrated in the preceding flowchart and discussed next.

- The first possible state from the previous period is s1:

1- If NSP $\leq(1+0.5 \mathrm{x}) *$ OSP then $r=\mathrm{r}_{1}$ and the most likely speed state for the next period is $\mathrm{S}_{1}$. 
The condition mentioned in case one above implies that if the RSU outcome speed is less than or equal the upper midpoint, then the RSU indicator $r$ for the current period is towards $r_{1}$ and the most likely state for the next period is $s_{1}$.

2- If NSP $>(1+0.5 \mathrm{x}) *$ OSP and NSP $\leq(1+\mathrm{x}) *$ OSP then $r=\mathrm{r}_{2}$ and the most likely speed state for the next period is $\mathrm{s}_{2}$.

The condition mentioned in case two above implies that, if the RSU outcome speed is higher than the upper midpoint and less than or equal to the speed value that corresponds to the speed state $\mathrm{s}_{3}$, then the RSU indicator $r$ for the current period is towards $\mathrm{r}_{2}$ and the most likely state for the next period will remain the same $\mathrm{s}_{2}$.

3- If NSP $>(1+\mathrm{x}) *$ OSP then $r=\mathrm{r}_{3}$ and the most likely speed state for the next period is S3.

The condition mentioned in case three above implies that if the RSU outcome speed is higher than the speed value that corresponds to the speed state $\mathrm{s}_{3}$, then the RSU indicator $r$ for the current period is towards $r_{3}$ and the most likely state for the next period is $s_{3}$.

\section{- The second possible state from the previous period is $\mathbf{s}_{2}$ :}

1- If NSP $\geq(1+0.5 \mathrm{x}) *$ OSP then $r=\mathrm{r}_{3}$ and the most likely speed state for the next period is $\mathrm{S}_{3}$.

The condition mentioned in case one above implies that if the RSU outcome speed is higher than or equal the upper midpoint, then the RSU indicator $r$ for the current period is towards $r_{3}$ and the most likely state for the next period is $s_{3}$. 
2- If NSP $<(1+0.5 \mathrm{x}) *$ OSP and NSP $\geq(1-0.5 \mathrm{x}) *$ OSP then $r=\mathrm{r}_{2}$ and most likely speed state for the next period is $\mathrm{s}_{2}$.

The condition mentioned in case two above implies that if the RSU outcome speed is less than the upper midpoint and higher than or equal the lower midpoint, then the RSU indicator $r$ for the current period is towards $\mathrm{r}_{2}$ and the most likely state for the next period will remain the same $\mathrm{s}_{2}$.

3- If NSP $<(1-0.5 \mathrm{x}) *$ OSP then $r=\mathrm{r}_{1}$ and the most likely speed state for the next period is $\mathrm{s}_{1}$.

The condition mentioned in case three above implies that if the RSU outcome speed is less than the lower midpoint, then the RSU indicator $r$ for the current period is towards $\mathrm{r}_{1}$ and the most likely state for the next period is $\mathrm{s}_{1}$.

\section{- The third possible state from the previous period is s3:}

1- If NSP $\geq(1-0.5 \mathrm{x}) *$ OSP then $r=\mathrm{r}_{3}$ and the most likely speed state for the next period is $\mathrm{s}_{3}$.

The condition mentioned in case one above implies that if the RSU outcome speed is higher than or equal the lower midpoint, then the RSU indicator $r$ for the current period is towards $r_{3}$ and the most likely state for the next period is $s_{3}$.

2- If NSP $<(1-0.5 \mathrm{x}) *$ OSP then $r=\mathrm{r}_{1}$ and most likely speed state for the next period is $\mathrm{s}_{1}$.

The condition mentioned in case two above implies that if the RSU outcome speed is less than the lower midpoint, then the RSU indicator $r$ for the current period is towards $\mathrm{r}_{1}$ and the most likely state for the next period is $\mathrm{s}_{1}$. 
For each possible speed state scenario explained above, if there is a change in the next period state, the speed state will be reset and the prior probabilities will be set according to logit formulation. Furthermore, the high probability will be assigned to the speed value of the most likely state.

In all possible speed states, the speed value that corresponds to the middle state $s_{2}$ of the next period should be reset to be equal to the speed value of the current period and other speed states ( $\mathrm{s}_{1}$ and $\mathrm{s}_{2}$ ) should be adjusted accordingly.

\subsubsection{Time Interval}

In this research, to detect incidents quickly, the time interval for updating information should be as short as possible. For now, 20 seconds interval is considered to be appropriate. However, a final decision will be made after initial testing of the methodology. It should be noted that the methodology will be flexible enough to enable the system designer to use another time interval if warranted.

\subsection{Modeling Incident Detection and Influencing Factors}

As discussed earlier in section 5.1.3, the expected speed is obtained by using the uncertain speed states, marginal probabilities and posterior probabilities as shown in Figure 5.2. The steps used in calculations are as follows. (1) Expected speed for various $r$ is found. (2) These are weighted by the marginal probabilities $P(r \mid c)$, where $P(r \mid c)=$ Sum for all $s\left[P^{\prime}(s) P(r \mid s, c)\right]$. The conditional probability can be considered as a measure of the reliability of the sensor/RSU system (assisted by additional sources of information) in providing a reading at the speed that will be manifested in the immediate future. The better 
the quality of the sources of information, the higher will be the $P(r \mid s, c)$. The posterior probability distributions are obtained from the following equation.

$$
\mathrm{P}^{\prime \prime}(\mathrm{s} \mid \mathrm{r}, \mathrm{c})=\frac{\mathrm{P}^{\prime}(\mathrm{s}) \mathrm{P}(\mathrm{r} \mid \mathrm{s}, \mathrm{c})}{\sum \text { for all states }\left[\mathrm{P}^{\prime}(\mathrm{s}) \mathrm{P}(\mathrm{r} \mid \mathrm{s}, \mathrm{c})\right]}
$$

A preliminary attempt at developing the algorithm for automated computations is illustrated in Figure 5.6.

In developing the incident detection model, several factors should be considered for a better incident detection approach. Since the spot speed is an essential factor under consideration in this thesis, the influencing factors should be related to the traffic speed. Two of these factors will be discussed when establishing the preliminary version of the developed model which involve computation utilizing Excel software. The other three factors are also related to the speed and will be discussed in the next chapter as a part of the process of Model refinement. The refinement process considers the establishment, calibration, and validation of the automatic computational version of the model.

The first two factors are considered in the preliminary criteria for establishing the incident detection model; these are the posterior level and the percent drop in the predicted speed. The posterior level specifies the reliability level of continuous change in the speed while maintaining the same speed state. 


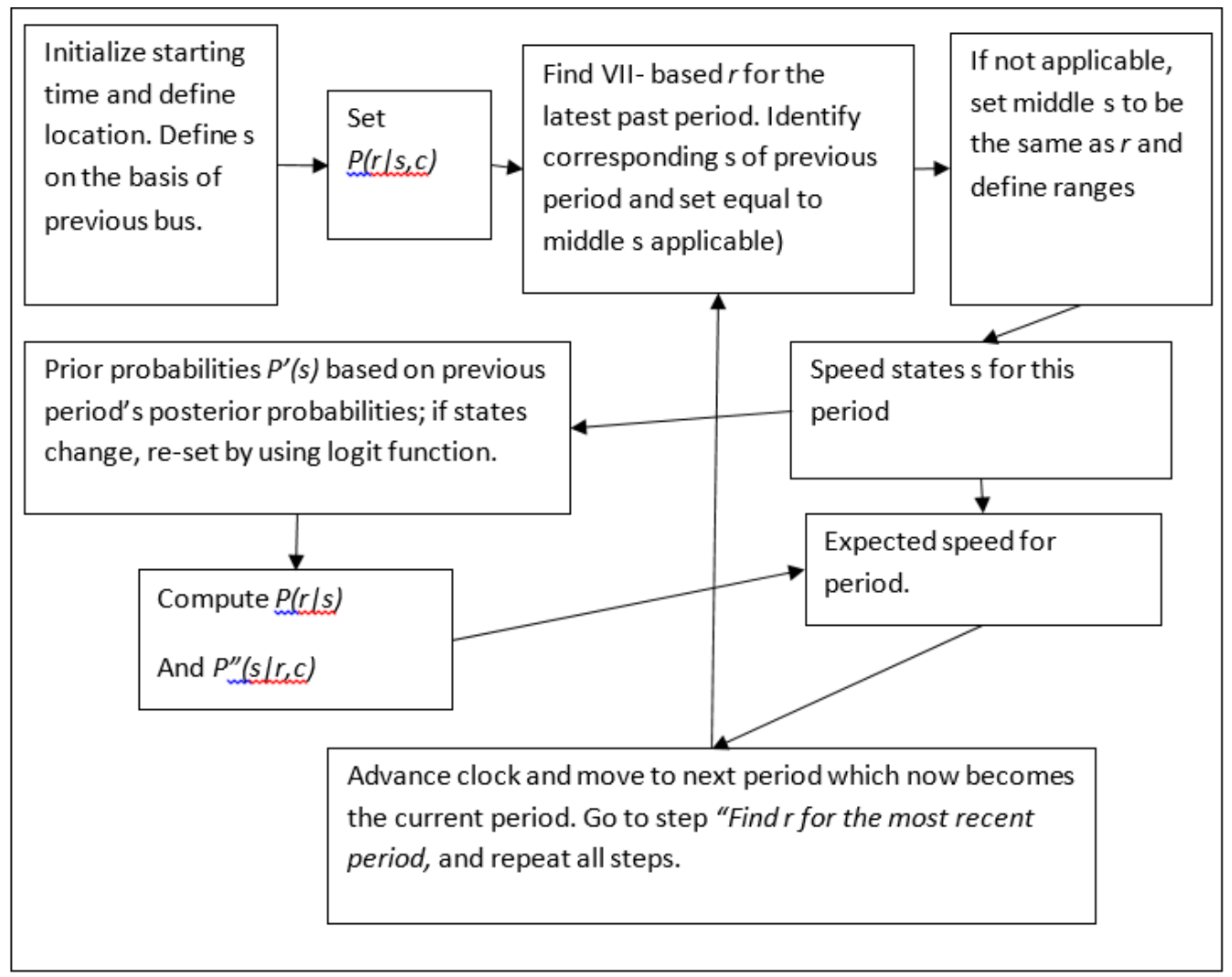

(Adapted from Khan 2010)

Figure 5.6: Preliminary attempt for computing expected speed

To initiate criteria for incident detection, it is becoming essential to investigate these two factors with the intention to examine any indication of a drop in speed followed by a continuous drop towards lower speed values. The observed drop in speed will be subject to incident persistence as will be discussed in the model development part in the next chapter.

The preliminary attempt to detect incidents is represented by the procedure shown in Figure 5.7. The illustrated procedure detects the drop in speed and is subject to incident persistence. Incident persistence will be explained in details in the next chapter. 


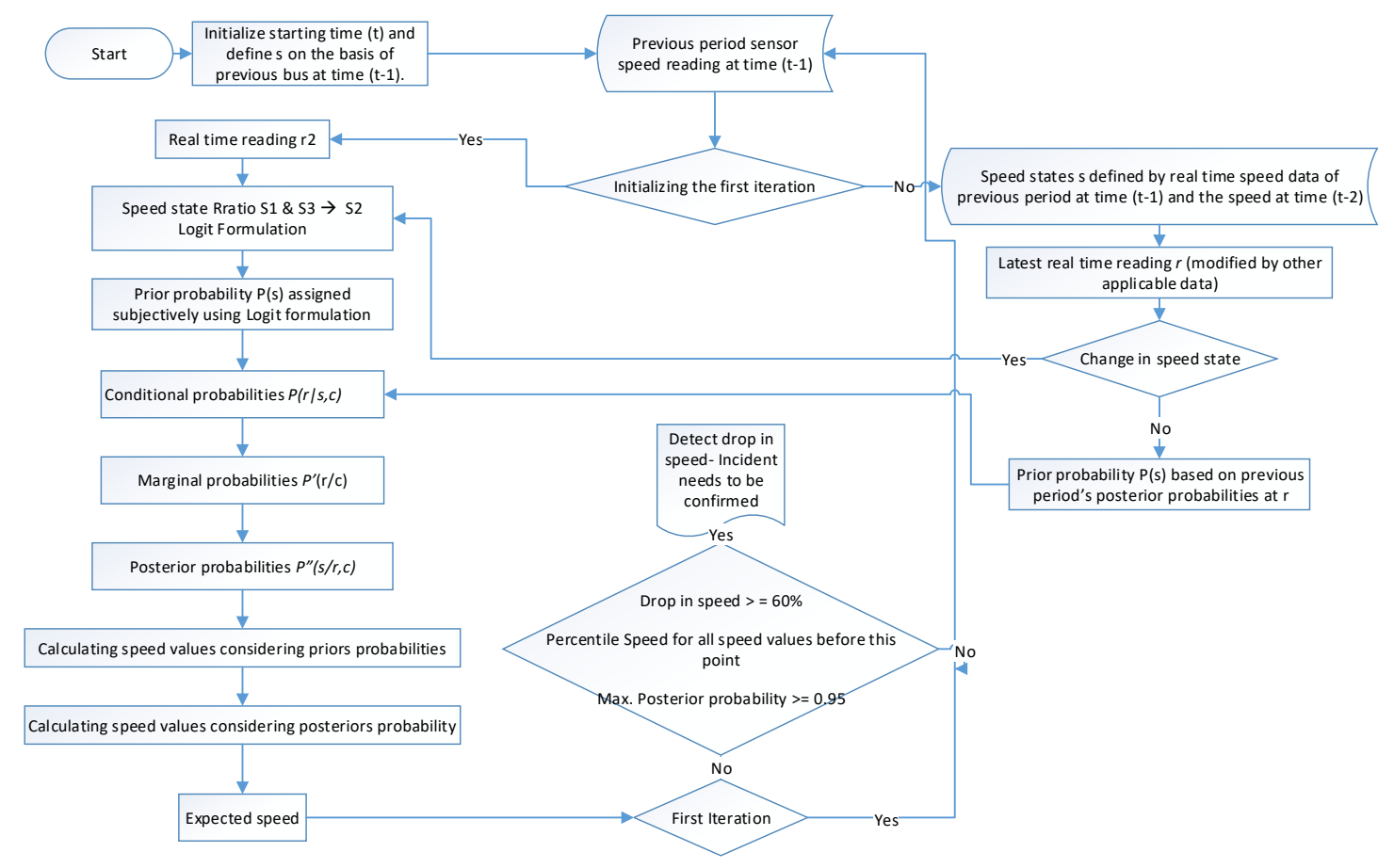

Figure 5.7: Developed algorithm for incident detection

The preliminary attempt can be summarized in the following steps:

1- Find the predicted speed at each time period according to the computations discussed earlier in this chapter.

2- Calculate the percent drop in speed. The percentage of speed drop was initially calculated as a percentage of the first speed reading within the study of speed data. Later on, when developing the model, this percent drop in the speed was modified as a percentage of the $85^{\text {th }}$ percentile of the obtained speed values during the study period. This period should include the time when initial speed values were obtained and continue to the time that corresponds to one last speed reading before the one under consideration. For robustness of the methodology, it was decided to start this period 20 
minutes before the recorded time of incident and it continues till one time period before the $85^{\text {th }}$ percentile speed is calculated.

3- Find the maximum posterior level that corresponds to the high probability speed state at each time period.

4- The criteria used to detect incident suggest that incident trigger will be observed under the following two conditions:

a- When the value of the maximum posterior probability level $(\mathrm{P}$ " $(\mathrm{s} \mid \mathrm{r}))$ exceeds a selected percentage according to the incident detection specification criteria. This percentage is subject to further refinement. Throughout the calibration process, the automated version of the develop model will be used to determine the proper percentage for better incident detection performance. The posterior probability level indicates the reliability of a continuous change in the speed towards one direction of change. In general, the posterior probability level will increase in the case when the speed continues to either increase or decline. This phenomenon requires an examination of changes that occurred in the speed values. For the purpose of detecting an incident, these changes should logically be toward declining speed.

b- The second condition in the incident detection criteria involves examining the drop in the predicted speed. It is when the continuous drop in the predicted speed reaches a certain limit that is very close to a selected percentage level limit specified by the incident detection criteria. This percentage is also subject to further refinement with use of the automated version of the develop model in order to improve the incident detection performance of the model. This percentage should appear with a negative 
sign as an indication of a speed drop rather than any possible increase in the predicted speed.

5- At every time period, the previous two conditions are examined for a simultaneous satisfaction of the specified criteria. The posterior probability level should reach the specified percentage (a very high level of reliability), and the percent drop in the speed should reach the specified percentage as well. The incident trigger will be activated when both conditions are satisfied, and hence, an indication of an incident will be the result.

In the following section, a comprehensive application is conducted on one of the incident cases selected from the incident database described in chapter 3. The proposed criteria are applied to investigate the possible detection of the incident.

\subsection{Testing the Developed Model}

\subsubsection{Bayesian Algorithm Application to Incident Data Recorded For Hwy 401}

For the development and validation of the incident detection model and the algorithm, data that belong to incidents and associated pattern of traffic speed would be most suitable.

The incident for this application was reported on 2011-03-07 at 10:58:31 am. The incident information is presented in Table 5.1. It occurs in the westbound of the collectors of Hwy 401 as shown in Figure 5.8. The reason for the incident was reported as a collision, and it has a serious impact in the corridor, all lanes were closed due to the collision. 
Table 5.1: Incident information, Hwy 401

\begin{tabular}{|c|c|c|c|c|c|c|c|c|c|}
\hline ż & 蔦 & 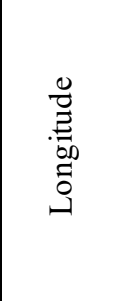 & 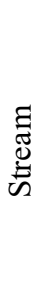 & 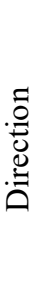 & 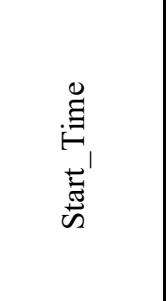 & 苛 & 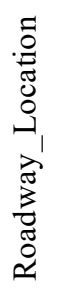 & $\begin{array}{l}\tilde{0} \\
\ddot{\Xi} \\
\stackrel{\Xi}{\simeq}\end{array}$ & 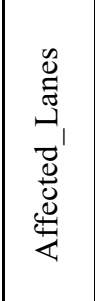 \\
\hline 41 & 43.7748 & $\mid-79.2864$ & $\mathrm{C}$ & W & $\begin{array}{c}2011-03-07 \\
10: 58: 31\end{array}$ & $\begin{array}{c}401 \text { Westbound } \\
\text { Collector(s) } \\
\text { [KENNEDY - ] } \\
\text { Collision }\end{array}$ & 401 & Collision & $\begin{array}{c}\text { All } \\
\text { lanes } \\
\text { closed }\end{array}$ \\
\hline
\end{tabular}

\subsubsection{Determination of the Upstream Detector}

The closest upstream detector to the incident under consideration is named 401DE0290DWC. In looking at the detector information, it is observed that the detector is located in the west direction of the collector stream. Knowing the latitude and longitude of both the detector location and the incident location, it is determined that the detector is located at $434 \mathrm{~m}$ upstream of the incident and is ranked as the first detector that recorded complete traffic data. A summary of the detector information is shown in Table 5.2.

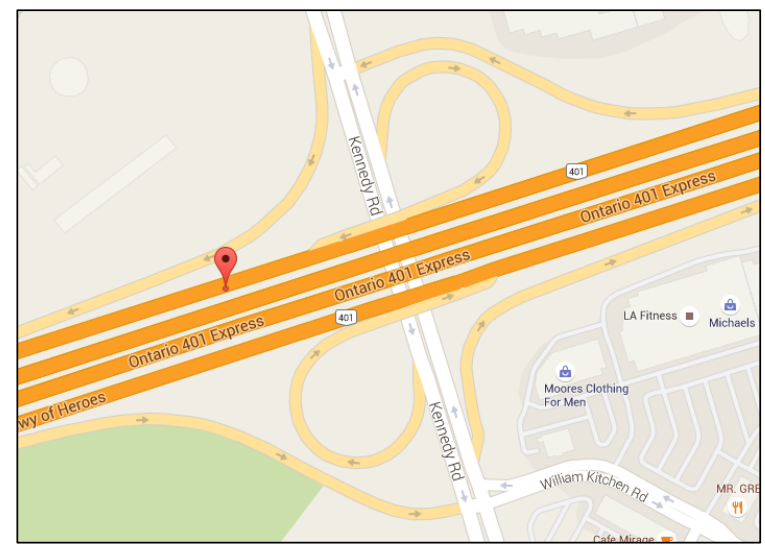

Figure 5.8: Selected incident location 


\section{Table 5.2: Sensor data from Hwy 401}

\begin{tabular}{|c|c|c|c|c|c|c|c|}
\hline$\dot{z}$ & 莺 & 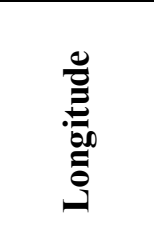 & 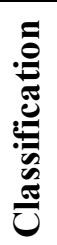 & 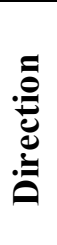 & 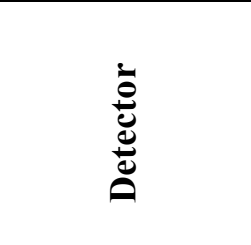 & $\underset{\frac{a}{E}}{\stackrel{n}{E}}$ & $\frac{n}{\tilde{0}}$ \\
\hline 292 & 43.7758 & -79.2822 & $\mathrm{C}$ & W & 401DE0290DWC & active & $\begin{array}{c}\text { 401-WC - AT } \\
\text { KENNEDY }\end{array}$ \\
\hline
\end{tabular}

Since the incident under test was reported on 2011-03-07 at 10:58:31, sensor data pertaining to the same date of the year (2011-03-07) will be of interest. Moreover, a time period within which the incident was reported is investigated. As was discussed in chapter three of this research, incident time is not certain, therefore, a time boundary of $20 \mathrm{~min}$ before and $20 \mathrm{~min}$ after the recorded time of the incident is considered in the final fundamental version of the developed model for testing the network.

Since the incident was recorded at 10:58:31, it is appropriate to start testing the model 20 minutes before this time (i.e. the start time is $10: 38: 40$ and end time is at 11:18:20 for a time interval of 40 minutes). Speed data were acquired from a segment of the major commuting corridor, namely Hwy 401 (Ontario). Table 5.3 presents the data for testing the developed model. The speed data include the time interval at which the spot speed was recorded and the corresponding speed value. As discussed earlier, the suggested time interval is 20 seconds.

From the detector data, it is noticed that for some time intervals, the recorded speed value is reported as (0), (-) or $(\mathrm{X})$. Since these three values are not realistic, they were exempted from the data set subject to study. Detailed criteria for data screening were 
discussed in chapter three of this thesis. So, the resulting screened speed data were the data considered for testing the developed model is show in Table 5.4.

One more variable was introduced in the table, the $85^{\text {th }}$ percentile of the speed data. At each period, the $85^{\text {th }}$ percentile of the predicted speed data was calculated for a range of the speed values.

Since the proportion of the drop in speed is calculated as a percentage of the $85^{\text {th }}$ percentile of the obtained speed values during the study period, when calculating the $85^{\text {th }}$ percentile of the speed data at a time $(t)$, it is necessary to consider all the speed data from the first reading to the time interval $(\mathrm{t})$ under consideration, where $(\mathrm{t})$ is the time when the current speed is to be tested for any possible decline or drop in its values. For instance, at time interval 10:42:00, when applying simple calculation in finding the $85^{\text {th }}$ percentile for the speed values $(94,100,94,92,92,104,99,103,99$, and 83$) \mathrm{km} / \mathrm{h}$, the result is found to be $103.5 \mathrm{~km} / \mathrm{h}$, as observed in Table 5.4 at time interval 10:42:00.

It is important to mention that the first speed value to read is at time interval 10:38:40 and, therefore, the starting time for the model application is considered to be a one-time interval after that, at 10:39:00. 
Table 5.3: Sensor data from Hwy 401 (Ontario)

\begin{tabular}{|c|c|c|c|c|c|}
\hline Time & $\begin{array}{l}\text { Sensor } \\
\text { Speed }\end{array}$ & Time & $\begin{array}{l}\text { Sensor } \\
\text { Speed }\end{array}$ & Time & $\begin{array}{l}\text { Sensor } \\
\text { Speed }\end{array}$ \\
\hline hh:mm:ss & $\mathrm{Km} / \mathrm{h}$ & hh:mm:ss & $\mathrm{Km} / \mathrm{h}$ & hh:mm:ss & $\mathrm{Km} / \mathrm{h}$ \\
\hline 10:38:40' & 94 & 10:52:00' & 105 & 11:05:40' & 3 \\
\hline 10:39:00' & 100 & $10: 52: 20^{\prime}$ & 100 & 11:06:00' & 6 \\
\hline 10:39:20' & 94 & 10:52:40' & 99 & $11: 06: 20^{\prime}$ & 8 \\
\hline 10:39:40' & 92 & 10:53:00' & 97 & 11:06:40' & 7 \\
\hline 10:40:00' & 92 & 10:53:20' & 100 & 11:07:00' & 6 \\
\hline $10: 40: 20^{\prime}$ & 104 & $10: 53: 40^{\prime}$ & 95 & 11:07:20' & 6 \\
\hline $10: 40: 40 '$ & 99 & $10: 54: 00^{\prime}$ & 102 & $11: 07: 40^{\prime}$ & 4 \\
\hline 10:41:00' & 103 & $10: 54: 20^{\prime}$ & 100 & 11:08:00' & 9 \\
\hline 10:41:20' & 99 & $10: 54: 40^{\prime}$ & 108 & $11: 08: 20^{\prime}$ & 7 \\
\hline 10:41:40' & 83 & 10:55:00' & 104 & 11:08:40' & 8 \\
\hline 10:42:00' & 104 & 10:55:20' & 104 & 11:09:00' & 7 \\
\hline 10:42:20' & 100 & 10:55:40' & 101 & 11:09:20' & 3 \\
\hline $10: 42: 40^{\prime}$ & 99 & 10:56:00' & 95 & 11:09:40' & 9 \\
\hline $10: 43: 00^{\prime}$ & 97 & $10: 56: 20^{\prime}$ & 97 & 11:10:00' & 5 \\
\hline 10:43:20' & 107 & 10:56:40' & 100 & $11: 10: 20^{\prime}$ & 4 \\
\hline $10: 43: 40 '$ & 109 & $10: 57: 00^{\prime}$ & 107 & $11: 10: 40^{\prime}$ & 6 \\
\hline 10:44:00' & 111 & $10: 57: 20^{\prime}$ & 93 & 11:11:00' & 4 \\
\hline $10: 44: 20^{\prime}$ & 106 & $10: 57: 40^{\prime}$ & 79 & $11: 11: 20^{\prime}$ & 3 \\
\hline 10:44:40' & 94 & 10:58:20' & 66 & 11:11:40' & 0 \\
\hline $10: 45: 00^{\prime}$ & 93 & $10: 58: 40^{\prime}$ & 54 & $11: 12: 00^{\prime}$ & 0 \\
\hline 10:45:20' & 95 & \begin{tabular}{|l|}
$10: 59: 00 '$ \\
\end{tabular} & 48 & $11: 12: 20^{\prime}$ & 5 \\
\hline 10:45:40' & 104 & 10:59:20' & 30 & $11: 12: 40^{\prime}$ & 6 \\
\hline 10:46:00' & 106 & 10:59:40' & 28 & $11: 13: 00^{\prime}$ & 5 \\
\hline 10:46:20' & 100 & 11:00:00' & 14 & $11: 13: 20^{\prime}$ & 6 \\
\hline $10: 46: 40^{\prime}$ & 102 & 11:00:20' & 11 & $11: 13: 40^{\prime}$ & 5 \\
\hline 10:47:00' & 99 & 11:00:40' & 6 & $11: 14: 00^{\prime}$ & 4 \\
\hline $10: 47: 20^{\prime}$ & 106 & 11:01:00' & 7 & $11: 14: 20^{\prime}$ & 6 \\
\hline $10: 47: 40 '$ & 105 & 11:01:20' & 6 & $11: 14: 40^{\prime}$ & 5 \\
\hline $10: 48: 00^{\prime}$ & 101 & 11:01:40' & 4 & $11: 15: 00^{\prime}$ & 6 \\
\hline 10:48:20' & 102 & 11:02:00' & 6 & $11: 15: 20^{\prime}$ & 5 \\
\hline $10: 48: 40^{\prime}$ & 98 & 11:02:20' & 4 & $11: 15: 40^{\prime}$ & 7 \\
\hline 10:49:00' & 101 & 11:02:40' & 9 & $11: 16: 00^{\prime}$ & 7 \\
\hline 10:49:20' & 99 & 11:03:00' & 11 & $11: 16: 20^{\prime}$ & 4 \\
\hline 10:49:40' & 103 & 11:03:20' & 13 & $11: 16: 40^{\prime}$ & 3 \\
\hline 10:50:00' & 102 & 11:03:40' & 5 & $11: 17: 00^{\prime}$ & 5 \\
\hline 10:50:20' & 100 & 11:04:00' & 3 & $11: 17: 20^{\prime}$ & 0 \\
\hline 10:50:40' & 95 & 11:04:20' & 5 & $11: 17: 40^{\prime}$ & 6 \\
\hline 10:51:00' & 98 & \begin{tabular}{|l|}
$11: 04: 40^{\prime}$ \\
\end{tabular} & 0 & $11: 18: 00^{\prime}$ & 4 \\
\hline 10:51:20' & 102 & 11:05:00' & 7 & 11:18:20' & 4 \\
\hline 10:51:40' & 104 & $11: 05: 20^{\prime}$ & 5 & & \\
\hline
\end{tabular}


Table 5.4: Screened speed data including the 85th percentile.

\begin{tabular}{|c|c|c|c|c|c|c|c|c|}
\hline $\begin{array}{l}\text { Date/ } \\
\text { Time }\end{array}$ & \begin{tabular}{|l|} 
Sensor \\
Speed
\end{tabular} & \begin{tabular}{|c|} 
85th \\
Percentile \\
\end{tabular} & $\begin{array}{l}\text { Date/ } \\
\text { Time }\end{array}$ & $\begin{array}{l}\text { Sensor } \\
\text { Speed }\end{array}$ & \begin{tabular}{|c|} 
85th \\
Percentile
\end{tabular} & $\begin{array}{l}\text { Date/ } \\
\text { Time }\end{array}$ & \begin{tabular}{|l|} 
Sensor \\
Speed \\
\end{tabular} & \begin{tabular}{|c|} 
85th \\
Percentile
\end{tabular} \\
\hline hh:mm:ss & $\mathrm{Km} / \mathrm{h}$ & $\mathrm{Km} / \mathrm{h}$ & hh:mm:ss & $\mathrm{Km} / \mathrm{h}$ & $\mathrm{Km} / \mathrm{h}$ & $\mathrm{hh}: \mathrm{mm}: \mathrm{ss}$ & $\mathrm{Km} / \mathrm{h}$ & $\mathrm{Km} / \mathrm{h}$ \\
\hline 10:38:40' & 94 & 0.0 & 10:51:20' & 102 & 108.4 & 11:04:20' & 5 & 105.7 \\
\hline 10:39:00' & 100 & $\mathrm{~N} / \mathrm{A}$ & 10:51:40' & 104 & 108.1 & 11:05:00' & 7 & 105.5 \\
\hline 10:39:20' & 94 & 94.0 & 10:52:00' & 105 & 107.8 & 11:05:20' & 5 & 105.4 \\
\hline 10:39:40' & 92 & 102.3 & $10: 52: 20^{\prime}$ & 100 & 107.5 & 11:05:40' & 3 & 105.3 \\
\hline 10:40:00' & 92 & 102.3 & 10:52:40' & 99 & 107.2 & 11:06:00' & 6 & 105.3 \\
\hline $10: 40: 20^{\prime}$ & 104 & 101.5 & 10:53:00' & 97 & 106.9 & 11:06:20' & 8 & 105.2 \\
\hline $10: 40: 40^{\prime}$ & 99 & 100.3 & 10:53:20' & 100 & 106.6 & 11:06:40' & 7 & 105.2 \\
\hline 10:41:00' & 103 & 104.8 & 10:53:40' & 95 & 106.4 & 11:07:00' & 6 & 105.1 \\
\hline 10:41:20' & 99 & 104.2 & 10:54:00' & 102 & 106.4 & 11:07:20' & 6 & 105.0 \\
\hline 10:41:40' & 83 & 104.0 & 10:54:20' & 100 & 106.4 & 11:07:40' & 4 & 104.9 \\
\hline 10:42:00' & 104 & 103.5 & 10:54:40' & 108 & 106.4 & 11:08:00' & 9 & 104.8 \\
\hline $10: 42: 20^{\prime}$ & 100 & 103.0 & 10:55:00' & 104 & 106.4 & 11:08:20' & 7 & 104.8 \\
\hline $10: 42: 40^{\prime}$ & 99 & 105.9 & 10:55:20' & 104 & 106.8 & 11:08:40' & 8 & 104.7 \\
\hline 10:43:00' & 97 & 105.4 & 10:55:40' & 101 & 106.4 & 11:09:00' & 7 & 104.6 \\
\hline 10:43:20' & 107 & 104.9 & 10:56:00' & 95 & 106.4 & 11:09:20' & 3 & 104.5 \\
\hline 10:43:40' & 109 & 104.4 & 10:56:20' & 97 & 106.4 & 11:09:40' & 9 & 104.4 \\
\hline $10: 44: 00^{\prime}$ & 111 & 106.4 & 10:56:40' & 100 & 106.4 & 11:10:00' & 5 & 104.3 \\
\hline $10: 44: 20^{\prime}$ & 106 & 106.8 & 10:57:00' & 107 & 106.4 & 11:10:20' & 4 & 104.3 \\
\hline 10:44:40' & 94 & 109.4 & $10: 57: 20^{\prime}$ & 93 & 106.4 & 11:10:40' & 6 & 104.2 \\
\hline $10: 45: 00^{\prime}$ & 93 & 108.9 & 10:57:40' & 79 & 106.5 & 11:11:00' & 4 & 104.2 \\
\hline $10: 45: 20^{\prime}$ & 95 & 108.4 & 10:58:20' & 66 & 106.4 & 11:11:20' & 3 & 104.1 \\
\hline $10: 45: 40^{\prime}$ & 104 & 108.0 & 10:58:40' & 54 & 106.4 & 11:12:20' & 5 & 104.0 \\
\hline 10:46:00' & 106 & 107.5 & 10:59:00' & 48 & 106.4 & 11:12:40' & 6 & 104.0 \\
\hline $10: 46: 20^{\prime}$ & 100 & 107.1 & 10:59:20' & 30 & 106.4 & 11:13:00' & 5 & 103.9 \\
\hline $10: 46: 40^{\prime}$ & 102 & 109.6 & 10:59:40' & 28 & 106.4 & 11:13:20' & 6 & 103.8 \\
\hline 10:47:00' & 99 & 109.2 & 11:00:00' & 14 & 106.4 & 11:13:40' & 5 & 103.8 \\
\hline $10: 47: 20^{\prime}$ & 106 & 108.8 & 11:00:20' & 11 & 106.4 & 11:14:00' & 4 & 103.7 \\
\hline $10: 47: 40^{\prime}$ & 105 & 108.3 & 11:00:40' & 6 & 106.4 & $11: 14: 20^{\prime}$ & 6 & 103.6 \\
\hline 10:48:00' & 101 & 109.0 & 11:01:00' & 7 & 106.4 & 11:14:40' & 5 & 103.5 \\
\hline 10:48:20' & 102 & 109.9 & 11:01:20' & 6 & 106.4 & 11:15:00' & 6 & 103.5 \\
\hline 10:48:40' & 98 & 109.7 & 11:01:40' & 4 & 106.4 & 11:15:20' & 5 & 103.4 \\
\hline 10:49:00' & 101 & 109.5 & 11:02:00' & 6 & 106.4 & 11:15:40' & 7 & 103.3 \\
\hline 10:49:20' & 99 & 109.4 & 11:02:20' & 4 & 106.4 & 11:16:00' & 7 & 103.2 \\
\hline 10:49:40' & 103 & 109.2 & 11:02:40' & 9 & 106.4 & 11:16:20' & 4 & 103.2 \\
\hline 10:50:00' & 102 & 109.1 & 11:03:00' & 11 & 106.3 & $11: 16: 40^{\prime}$ & 3 & 103.1 \\
\hline 10:50:20' & 100 & 108.9 & 11:03:20' & 13 & 106.1 & 11:17:00' & 5 & 103.0 \\
\hline 10:50:40' & 95 & 108.8 & 11:03:40' & 5 & 106.0 & 11:17:40' & 6 & 103.0 \\
\hline \multirow[t]{2}{*}{ 10:51:00' } & 98 & 108.6 & 11:04:00' & 3 & 105.8 & 11:18:00' & 4 & 103.0 \\
\hline & & & & & & 11:18:20' & 4 & 103.0 \\
\hline
\end{tabular}




\subsubsection{Proposed Criteria for Incident Detection}

The criteria used to detect incident suggest that the incident trigger will be observed under the following two conditions:

1- When the value of the maximum posterior probability level $(\mathrm{P} "(\mathrm{~s} \mid \mathrm{r}))$ reaches a selected value. The specified criterion for the posterior probability level to detect an incident in the following application is assumed to be 0.97 (or in commonly understood terms 97\%). This percentage expresses a very high level of reliability. The posterior probability level in the following application is tracked; it is considered satisfying the criterion if it reaches the specified level of $97 \%$. The posterior level will increase in the case the speed continues to either increase or decline which requires examining the changes that occurred in the speed values. For the purpose of detecting any incident, these changes should be toward declining speeds. Therefore, the second condition in the incident detection criteria involves examining the drop in the predicted speed.

2- The second condition that corresponds to incident detection is when the continuous drop in the predicted speed reaches to a certain limit that is very close to a selected percentage limit which satisfies the specified incident detection criteria. The criterion selected to define the percent drop in the predicted speed, calculated as a percentage of the $85^{\text {th }}$ percentile, in the following application is assumed to be $55 \%$. This percentage should be presented with a negative sign in the output as an indication of a speed drop rather than any possible increase in the predicted speed.

Testing the suggested network by implementing the developed model is explained in detail in the following model testing application. The testing of the final automated version will be discussed in the next chapter. 


\subsubsection{Comprehensive Model Application in Testing the Network}

\section{Start time at 10:39:00, 2011-03-07}

From the previous period (that ended at 10:38:40), the sensor reading indicated the average spot speed $=94 \mathrm{~km} / \mathrm{h}$.

\section{- Define the Speed States}

In this thesis, several speed state ranges were examined to effectively represent the available time periods obtained from the sensor data. A range defined by $7 \%$ above and below the middle speed state is considered suitable. Therefore, the speed at state $\mathrm{s}_{1}$ will be assigned a value of $7 \%$ lower than the speed at state $s_{2}$, and the speed at state $s_{3}$ will be assigned a value of $7 \%$ higher than the middle speed value at state $s_{2}$.

Since the speed at state $\mathrm{s}_{2}$ is defined by $94 \mathrm{~km} / \mathrm{h}$, the speed at state $\mathrm{s}_{1}$ represents $87.4 \mathrm{~km} / \mathrm{h}$, and the speed at state $\mathrm{s}_{3}$ represents $100.6 \mathrm{~km} / \mathrm{h}$. These speed values represent a range of speeds of $7 \%$, and the middle level is used for ease of notation and calculations. Since the time interval in all cases is almost fixed, an interval of $20 \mathrm{sec}$ is valid for all cases, so the ratio between the speed values at states $s_{1}$ and $s_{3}$ in relation to $s_{2}$ is considered to be the same for all intervals. Given these values, there will be no need for any future refinement related to ranges between the three speed states.

For the defined time interval, the following relationships between the speed values at the traffic condition states are obtained:

$\mathrm{s}_{1}=0.93 * \mathrm{~s}_{2}, \mathrm{~s}_{2}=1.0 * \mathrm{~s}_{2}, \mathrm{~s}_{3}=1.07 * \mathrm{~s}_{2}$ 
It should be noted here that these multipliers can be modified by analysts. However, it is suggested that the same pattern of ratios should be maintained for reason of consistency and ease of application.

\section{- Define Prior Probabilities P'(s)}

Since we are initializing the system, prior probabilities of traffic states are required. As previously noted, we use a logit formulation so that the most likely state receives the highest probability and the other states are assigned probabilities depending upon how far these are from the most likely state.

Currently, the system is initiated and therefore, the latest available reading obtained from the sensor at the end of 10:38:40 time interval is $r_{2}$. Since $r_{2}$ corresponds to $s_{2}$, therefore $s_{2}$ receives the highest prior probability for the current period. The prior probabilities are calculated according to the following equations.

$\begin{array}{lccc} & \underline{\mathrm{S}} 1 & \underline{\mathrm{s}} 2 & \underline{\mathrm{S}} \underline{3} \\ \text { Speed } & 87.4 & 94 & 100.6 \\ \text { Using } \mathrm{s}_{2} \text { as the most likely state: } \Delta \mathrm{s}+1 & 7.60 & 1 & 7.60 \\ \mathrm{e}^{(1 / \Delta \mathrm{s}+1)} & 1.14 & 2.72 & 1.14 \\ \mathrm{P}(\mathrm{s})=\mathrm{e}^{(1 / \Delta \mathrm{s}+1) / \sum\left[\mathrm{e}^{(1 / \Delta \mathrm{s}+1)}\right]} & 0.23 & 0.54 & 0.23\end{array}$

- Define Conditional Probabilities P(r|s,c)

These reflect the reliability of the available information and are expressed by the proportion of equipped vehicles, traffic volume, and the probability that the minimum 
sample size requirement is encountered. Let us use a lower level of $\mathrm{P}\left(\mathrm{r}_{1} \mid \mathrm{s}_{1}, \mathrm{c}\right)=\mathrm{P}\left(\mathrm{r}_{2} \mid \mathrm{s}_{2}, \mathrm{c}\right)$ $=\mathrm{P}\left(\mathrm{r}_{3} \mid \mathrm{s}_{3}, \mathrm{c}\right)=0.6$ as shown in the Table 5.5. As was discussed in chapter 4, this level of reliability may represent the assumption of having $30 \%$ equipped vehicle in the fleet at traffic condition of 500 vehicle/h/lane.

\section{Table 5.5: Conditional probabilities $P(\mathbf{r} \mid \mathbf{s}, \mathbf{c})$}

\begin{tabular}{|c|c|c|c|}
\hline & $\mathrm{s}_{1}$ & $\mathrm{~s}_{2}$ & $\mathrm{~s}_{3}$ \\
\hline $\mathrm{r}_{1}$ & 0.6 & 0.2 & 0.1 \\
\hline $\mathrm{r}_{2}$ & 0.3 & 0.6 & 0.3 \\
\hline $\mathrm{r}_{3}$ & 0.1 & 0.2 & 0.6 \\
\hline & ------ & ----- & ----- \\
\hline & 1 & 1 & 1 \\
\hline
\end{tabular}

For route $\mathrm{A}_{1}$ (the only route that is under study), the speed states have already been defined.

The Bayesian model application for predicting traffic performance for the applicable time period represented by 10:39:00 is shown below. The following predicted information becomes known at the outset of the current period, and these can be compared with actual data that is reported by the sensor at the end of the current time period (i.e. just before 10:39:20). This will be the reading $r$ that will be used to set the speeds for the various speed states of the next time period. In the following outputs consider the speed range $x=7 \%$. 
10:39:00: The outputs for this period are shown below.

\begin{tabular}{|c|c|c|c|c|c|c|c|c|c|}
\hline \multirow[t]{2}{*}{ Hwy 401} & \multirow[t]{2}{*}{$\begin{array}{l}\text { Speed } \\
\text { State }\end{array}$} & \multirow[t]{2}{*}{2} & \multicolumn{3}{|c|}{ '2011-03-07 10:39:00' } & \multicolumn{2}{|c|}{$\begin{array}{l}\text { Previous period } \\
\text { sens or reading }\end{array}$} & \multirow{2}{*}{$\begin{array}{c}94.00 \\
(1+0.5 x) \\
\text { *PREV }\end{array}$} & \multirow{2}{*}{$\begin{array}{l}\mathrm{km} / \mathrm{h} \\
(1+\mathrm{x}) \\
\text { *PREV }\end{array}$} \\
\hline & & & & & $\begin{array}{l}(1-\mathrm{x}) \\
\text { *PREV }\end{array}$ & $\begin{array}{l}(1-0.5 x) \\
* P R E V\end{array}$ & $\begin{array}{c}\text { PREV at } \\
(\mathrm{t}-1)\end{array}$ & & \\
\hline \multicolumn{4}{|c|}{ Use logit to begin with to find priors } & Speed & 0.00 & 0.00 & 0.0 & 0.00 & 0.00 \\
\hline \multicolumn{4}{|c|}{ Range between speed states $\%=$} & 7 & $\mathrm{~s}_{1}$ & $\mathrm{~S}_{2}$ & $\mathrm{~S}_{3}$ & & \\
\hline \multicolumn{5}{|l|}{ Speed } & 87.42 & 94.00 & 100.58 & & \\
\hline \multicolumn{5}{|l|}{ Delta S-1 } & 7.58 & 1.00 & 7.58 & Sum & \\
\hline \multicolumn{5}{|l|}{$\mathrm{e}^{\wedge}(1 /$ Delta $\mathrm{S}+1)$} & 1.14 & 2.72 & 1.14 & 5.00 & \\
\hline \multicolumn{5}{|l|}{$\mathrm{P}(\mathrm{s})$} & 0.23 & 0.54 & 0.23 & & \\
\hline \multicolumn{2}{|c|}{ Conditional Probability Level } & 0.6 & & & & & & & \\
\hline Prior Probabilities & & & \multicolumn{4}{|c|}{ Conditional Probabilities $\mathrm{P}(\mathrm{r} \mid \mathrm{s}, \mathrm{c})$} & & Marginals & $\mathrm{P}(\mathrm{r} \mid \mathrm{c})$ \\
\hline $\mathrm{P}^{\prime}\left(\mathrm{s}_{1}\right)$ & 0.23 & & & $\mathrm{~s}_{1}$ & $\mathrm{~s}_{2}$ & $\mathrm{~s}_{3}$ & & $\mathrm{r}_{1}$ & 0.27 \\
\hline $\mathrm{P}^{\prime}\left(\mathrm{s}_{2}\right)$ & 0.54 & & $\mathrm{r}_{1}$ & 0.6 & 0.2 & 0.1 & & $\mathrm{r}_{2}$ & 0.46 \\
\hline $\mathrm{P}^{\prime}(\mathrm{s})$ & 0.23 & & $\mathrm{r}_{2}$ & 0.3 & 0.6 & 0.3 & & $\mathrm{r}_{3}$ & 0.27 \\
\hline \multirow[t]{2}{*}{ Sum } & 1 & & $r_{3}$ & 0.1 & 0.2 & 0.6 & & Sum & 1 \\
\hline & & & Sum & 1 & 1 & 1 & & & \\
\hline \multicolumn{2}{|c|}{ Posterior Probabilities P" $(\mathrm{s} \mid \mathrm{r}, \mathrm{c})$} & & & & & \multicolumn{3}{|c|}{$\mathrm{SP}, \mathrm{P}(\mathrm{r} \mid \mathrm{c}) \&$ role of posteriors } & \\
\hline & $\mathrm{s}_{1}$ & $\mathrm{~s}_{2}$ & $\mathrm{~s}_{3}$ & Sum & & & $\mathrm{r}_{1}$ & $\mathrm{r}_{2}$ & $r_{3}$ \\
\hline $\mathrm{r}_{1}$ & 0.510 & 0.405 & 0.085 & 1 & & $\overline{A_{r}}$ & 91.20 & 94.00 & 96.80 \\
\hline $\mathrm{r}_{2}$ & 0.148 & 0.704 & 0.148 & 1 & & $\mathrm{~A}^{\prime}$ & 94.00 & 94.00 & 94.00 \\
\hline $\mathrm{r}_{3}$ & 0.085 & 0.405 & 0.510 & 1 & & $A_{r}-A^{\prime}$ & -2.80 & 0.00 & 2.80 \\
\hline \multicolumn{2}{|l|}{ Base speed } & 94 & & & & $\mathrm{P}(\mathrm{r} \mid \mathrm{e})$ & 0.27 & 0.46 & 0.27 \\
\hline \multicolumn{10}{|l|}{ Speed (SP) Matrix } \\
\hline & $\mathrm{s}_{1}$ & $\mathrm{~s}_{2}$ & $\mathrm{~s}_{3}$ & & & Posteriors & Speed & max speed & \\
\hline Route A1 & 87.42 & 94.00 & 100.58 & & & $\mathrm{SP}\left(\mathrm{A}_{1}, \mathrm{r}_{1}, \mathrm{c}\right)$ & 91.20 & 91.20 & \\
\hline Route $\mathrm{A}_{2}$ & 0 & 0 & 0 & & & $\mathrm{SP}\left(\mathrm{A}_{2}, \mathrm{r}_{1}, \mathrm{c}\right)$ & 0.00 & & \\
\hline \multirow[t]{2}{*}{ Route $\mathrm{A}_{3}$} & 0 & 0 & 0 & & & $\mathrm{SP}\left(\mathrm{A}_{3}, \mathrm{r}_{1}, \mathrm{c}\right)$ & 0.00 & & \\
\hline & & & & & & $\mathrm{SP}\left(\mathrm{A}_{1}, \mathrm{r}_{2}, \mathrm{c}\right)$ & 94.00 & 94.00 & \\
\hline Priors & Speed & max speed & & & & $\mathrm{SP}\left(\mathrm{A}_{2}, \mathrm{r}_{2}, \mathrm{c}\right)$ & 0.00 & & \\
\hline $\mathrm{SP}\left(\mathrm{A}_{1}\right)$ & 94.00 & 94.00 & & & & $\mathrm{SP}\left(\mathrm{A}_{3}, \mathrm{r}_{2}, \mathrm{c}\right)$ & 0.00 & & \\
\hline $\mathrm{SP}\left(\mathrm{A}_{2}\right)$ & 0.00 & & & & & $\mathrm{SP}\left(\mathrm{A}_{1}, \mathrm{r}_{3}, \mathrm{c}\right)$ & 96.80 & 96.80 & \\
\hline \multirow[t]{2}{*}{$\mathrm{SP}\left(\mathrm{A}_{3}\right)$} & 0.00 & & & & & $\mathrm{SP}\left(\mathrm{A}_{2}, \mathrm{r}_{3}, \mathrm{c}\right)$ & 0.00 & & \\
\hline & & & & & & $\mathrm{SP}\left(\mathrm{A}_{3}, \mathrm{r}_{3}, \mathrm{c}\right)$ & 0.00 & & \\
\hline \multicolumn{3}{|l|}{ Expected $($ Speed $)=$} & 94.00 & $\mathrm{~km} / \mathrm{h}$ & & & & & \\
\hline
\end{tabular}

The predicted speed for the period 10:39:00 is $94 \mathrm{~km} / \mathrm{h}$.

Next, wait until the end of 10:39:00 and find out the sensor (actual) speed reading is 100 $\mathrm{km} / \mathrm{h}$. 
In consideration of the speed state, there are four other associated states, the upper speed state is $7 \%$ higher, and the lower speed state is $7 \%$ lower. The two midpoints speed percentages are falling in between, the upper midpoint speed which is $3.5 \%$ higher and the lower midpoint speed which is $3.5 \%$ lower.

Now considering the speed of $94 \mathrm{~km} / \mathrm{h}$ at the middle (moderate) speed state $\mathrm{s}_{2}$ from the previous period 10:38:40, here are the four-speed values that correspond to the other speed states:

Upper speed state is $7 \%$ higher $=100.58 \mathrm{~km} / \mathrm{h}$, Upper midpoint speed is $3.5 \%$ higher $=97.29 \mathrm{~km} / \mathrm{h}$, Lower midpoint speed is $3.5 \%$ lower $=87.42 \mathrm{~km} / \mathrm{h}$, and Lower speed state is $7 \%$ lower $=87.42 \mathrm{~km} / \mathrm{h}$.

Since actual speed of $100 \mathrm{~km} / \mathrm{h}$ for this period 10:39:00 is above the upper midpoint speed of $97.29 \mathrm{~km} / \mathrm{h}$ of the previous period 10:38:40, therefore the trend is towards higher speed and $r_{3}$ is the speed data available at the outset of 10:39:00 period and should be used for the next period of 10:39:20

This means the most likely state is $\mathrm{s}_{3}$ for the next period. Moreover, since there is a change in the states, the speed states will be reset, and the prior probabilities will be set according to logit formulation.

Furthermore, since the most likely state is $\mathrm{s}_{3}$, the high probability will be assigned to the speed state of $\mathrm{s}_{3}$.

So, reset $\mathrm{s}_{2}$ to be equal to the new level of $100 \mathrm{~km} / \mathrm{h}$ and adjust other states as shown in the following output. Where the speed values at the three speed states are $s_{2}=100 \mathrm{~km} / \mathrm{h}$, $\mathrm{s}_{1}=93 \mathrm{~km} / \mathrm{h}$ and $\mathrm{s}_{3}=107 \mathrm{~km} / \mathrm{h}$. These are the outputs of the next period as shown below. 
10:39:20: The outputs for this period are shown below

\begin{tabular}{|c|c|c|c|c|c|c|c|c|c|}
\hline \multirow[t]{2}{*}{ Hwy 401} & \multirow[t]{2}{*}{$\begin{array}{l}\text { Speed } \\
\text { State }\end{array}$} & \multirow[t]{2}{*}{3} & \multicolumn{3}{|c|}{$\underline{2011-03-07 \text { 10:39:20' }}$} & \multicolumn{2}{|c|}{$\begin{array}{c}\text { Previous period } \\
\text { sensor reading }\end{array}$} & \multirow{2}{*}{$\begin{array}{c}100.00 \\
(1+0.5 \mathrm{x}) \\
\text { *PREV }\end{array}$} & \multirow{2}{*}{$\begin{array}{c}\mathrm{km} / \mathrm{h} \\
(1+\mathrm{x}) \\
\text { *PREV }\end{array}$} \\
\hline & & & & & $\begin{array}{l}(1-\mathrm{x}) \\
\text { *PREV } \\
\end{array}$ & $\begin{array}{l}(1-0.5 x) \\
\text { *PREV }\end{array}$ & $\begin{array}{c}\text { PREV at } \\
(\mathrm{t}-1)\end{array}$ & & \\
\hline \multicolumn{4}{|c|}{ r3. Change in states. New r. Use logit } & Speed & 87.42 & 90.71 & 94.0 & 97.29 & 100.58 \\
\hline \multicolumn{4}{|c|}{ Range between speed states $\%=$} & 7 & $\mathrm{~s}_{1}$ & $\mathrm{~s}_{2}$ & $\mathrm{~s}_{3}$ & & \\
\hline \multicolumn{5}{|l|}{ Speed } & 93.00 & 100.00 & 107.00 & & \\
\hline \multicolumn{5}{|l|}{ Delta S-1 } & 15.00 & 8.00 & 1.00 & Sum & \\
\hline \multicolumn{5}{|l|}{$\mathrm{e}^{\wedge}(1 /$ Delta $\mathrm{S}+1)$} & 1.07 & 1.13 & 2.72 & 4.92 & \\
\hline \multicolumn{5}{|l|}{$\mathrm{P}(\mathrm{s})$} & 0.22 & 0.23 & 0.55 & & \\
\hline \multicolumn{2}{|c|}{ Conditional Probability Level } & 0.6 & & & & & & & \\
\hline Prior Probabilities & & & \multicolumn{4}{|c|}{ Conditional Probabilities $\mathrm{P}(\mathrm{r} \mid \mathrm{s}, \mathrm{c})$} & & Marginals & $\mathrm{P}(\mathrm{r} \mid \mathrm{c})$ \\
\hline $\mathrm{P}^{\prime}(\mathrm{s} 1)$ & 0.22 & & & $\mathrm{~s}_{1}$ & $\mathrm{~s}_{2}$ & $\mathrm{~S}_{3}$ & & $\mathrm{r}_{1}$ & 0.23 \\
\hline $\mathrm{P}^{\prime}(\mathrm{s} 2)$ & 0.23 & & $\mathrm{r}_{1}$ & 0.6 & 0.2 & 0.1 & & $\mathrm{r}_{2}$ & 0.37 \\
\hline $\mathrm{P}^{\prime}(\mathrm{s})$ & 0.55 & & $\mathrm{r}_{2}$ & 0.3 & 0.6 & 0.3 & & $\mathrm{r}_{3}$ & 0.40 \\
\hline \multirow[t]{2}{*}{ Sum } & 1 & & $\mathrm{r}_{3}$ & 0.1 & 0.2 & 0.6 & & Sum & 1 \\
\hline & & & Sum & 1 & 1 & 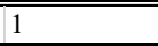 & & & \\
\hline \multicolumn{2}{|c|}{ Posterior Probabilities $\mathrm{P} "(\mathrm{~s} \mid \mathrm{r}, \mathrm{c})$} & & & & & \multicolumn{3}{|c|}{$\mathrm{SP}, \mathrm{P}(\mathrm{r} \mid \mathrm{c}) \&$ role of posteriors } & \\
\hline & $\mathrm{s}_{1}$ & $\mathrm{~s}_{2}$ & $\mathrm{~s}_{3}$ & Sum & & & $\mathrm{r}_{1}$ & $\mathrm{r}_{2}$ & $r_{3}$ \\
\hline $\mathrm{r}_{1}$ & 0.563 & 0.199 & 0.238 & 1 & & $\overline{A_{r}}$ & 97.73 & 101.91 & 105.43 \\
\hline $\mathrm{r}_{2}$ & 0.177 & 0.374 & 0.449 & 1 & & $\mathrm{~A}^{\prime}$ & 102.35 & 102.35 & 102.35 \\
\hline$r_{3}$ & 0.054 & 0.115 & 0.830 & $\mid 1$ & & $A_{r}-A^{\prime}$ & -4.62 & -0.44 & 3.08 \\
\hline \multicolumn{2}{|l|}{ Base speed } & 100 & & & & $\mathrm{P}(\mathrm{r} \mid \mathrm{e})$ & 0.23 & 0.37 & 0.40 \\
\hline \multicolumn{10}{|l|}{ Speed (SP) Matrix } \\
\hline & $\mathrm{s}_{1}$ & $\mathrm{~S}_{2}$ & $\mathrm{~s}_{3}$ & & & Posteriors & Speed & max speed & \\
\hline Route A1 & 93.00 & 100.00 & 107.00 & & & $\mathrm{SP}\left(\mathrm{A}_{1}, \mathrm{r}_{1}, \mathrm{c}\right)$ & 97.73 & 97.73 & \\
\hline Route $\mathrm{A}_{2}$ & 0 & 0 & 0 & & & $\mathrm{SP}\left(\mathrm{A}_{2}, \mathrm{r}_{1}, \mathrm{c}\right)$ & 0.00 & & \\
\hline \multirow[t]{2}{*}{ Route $\mathrm{A}_{3}$} & 0 & 0 & 0 & & & $\mathrm{SP}\left(\mathrm{A}_{3}, \mathrm{r}_{1}, \mathrm{c}\right)$ & 0.00 & & \\
\hline & & & & & & $\mathrm{SP}\left(\mathrm{A}_{1}, \mathrm{r}_{2}, \mathrm{c}\right)$ & 101.91 & 101.91 & \\
\hline Priors & Speed & max speed & & & & $\mathrm{SP}\left(\mathrm{A}_{2}, \mathrm{r}_{2}, \mathrm{c}\right)$ & 0.00 & & \\
\hline $\mathrm{SP}\left(\mathrm{A}_{1}\right)$ & 102.35 & 102.35 & & & & $\mathrm{SP}\left(\mathrm{A}_{3}, \mathrm{r}_{2}, \mathrm{c}\right)$ & 0.00 & & \\
\hline $\mathrm{SP}\left(\mathrm{A}_{2}\right)$ & 0.00 & & & & & $\mathrm{SP}\left(\mathrm{A}_{1}, \mathrm{r}_{3}, \mathrm{c}\right)$ & 105.43 & 105.43 & \\
\hline \multirow[t]{2}{*}{$\mathrm{SP}\left(\mathrm{A}_{3}\right)$} & 0.00 & & & & & $\mathrm{SP}\left(\mathrm{A}_{2}, \mathrm{r}_{3}, \mathrm{c}\right)$ & 0.00 & & \\
\hline & & & & & & $\mathrm{SP}\left(\mathrm{A}_{3}, \mathrm{r}_{3}, \mathrm{c}\right)$ & 0.00 & & \\
\hline \multicolumn{3}{|l|}{ Expected $($ Speed $)=$} & 102.35 & $\mathrm{~km} / \mathrm{h}$ & & & & & \\
\hline
\end{tabular}

The predicted speed for the period 10:39:20 is $102.35 \mathrm{~km} / \mathrm{h}$.

Next, wait until the end of 10:39:20 and find out the sensor actual speed reading is $94 \mathrm{~km} / \mathrm{h}$. 
Considering the speed of $100 \mathrm{~km} / \mathrm{h}$ at the middle (moderate) speed state $\mathrm{s}_{2}$ from the previous period 10:39:00 then, the four-speed values that correspond to the other speed states are:

Upper speed state is $7 \%$ higher $=107 \mathrm{~km} / \mathrm{h}$, Upper midpoint speed is $3.5 \%$ higher $=103.50 \mathrm{~km} / \mathrm{h}$, Lower midpoint speed is $3.5 \%$ lower $=96.50 \mathrm{~km} / \mathrm{h}$, and Lower speed state is $7 \%$ lower $=93 \mathrm{~km} / \mathrm{h}$.

Since actual speed of $94 \mathrm{~km} / \mathrm{h}$ for this period 10:39:20 is below the lower midpoint speed of $96.50 \mathrm{~km} / \mathrm{h}$ of the previous period 10:39:00, therefore the trend is towards lower speed and $\mathrm{r}_{1}$ is the speed data available at the outset of 10:39:20 period, and should be used for the next period of 10:39:40

This means the most likely state is $\mathrm{s}_{1}$ for the next period. And since there is a change in the states, the speed states will be reset, and the prior probabilities will be set according to logit formulation.

Furthermore, since the most likely state is $\mathrm{s}_{1}$, the high probability will be assigned to the speed state of $\mathrm{s}_{1}$.

So, reset $\mathrm{s}_{2}$ to be equal to the new level of $94 \mathrm{~km} / \mathrm{h}$ and adjust other states as shown in the following output. Where the speed values at the three speed states are $\mathrm{s}_{2}=94 \mathrm{~km} / \mathrm{h}$, $\mathrm{s}_{1}=87.42 \mathrm{~km} / \mathrm{h}$ and $\mathrm{s}_{3}=100.58 \mathrm{~km} / \mathrm{h}$

These are the outputs of the next period as shown below. 
10:39:40: The outputs for this period are shown below

\begin{tabular}{|c|c|c|c|c|c|c|c|c|c|}
\hline \multirow[t]{2}{*}{ Hwy 401} & \multirow[t]{2}{*}{$\begin{array}{l}\text { Speed } \\
\text { State }\end{array}$} & \multirow[t]{2}{*}{1} & \multicolumn{3}{|c|}{ '2011-03-07 10:39:40' } & \multicolumn{2}{|c|}{$\begin{array}{c}\text { Previous period } \\
\text { sensor reading }\end{array}$} & \multirow{2}{*}{$\begin{array}{c}94.00 \\
(1+0.5 \mathrm{x}) \\
\text { *PREV } \\
\end{array}$} & \multirow{2}{*}{$\begin{array}{l}\mathrm{km} / \mathrm{h} \\
(1+\mathrm{x}) \\
\text { *PREV } \\
\end{array}$} \\
\hline & & & & & $\begin{array}{l}(1-\mathrm{x}) \\
* \text { PREV }\end{array}$ & $\begin{array}{l}(1-0.5 \mathrm{x}) \\
* \text { PREV }\end{array}$ & $\begin{array}{c}\begin{array}{c}\text { PREV at } \\
(\mathrm{t}-1)\end{array} \\
\end{array}$ & & \\
\hline \multicolumn{4}{|c|}{ r1. Change in states. New r. Use logit } & Speed & 93.00 & 96.50 & 100.0 & 103.50 & 107.00 \\
\hline \multicolumn{4}{|c|}{ Range between speed states $\%=$} & 7 & $\mathrm{~s}_{1}$ & $\mathrm{~s}_{2}$ & $\mathrm{~s}_{3}$ & & \\
\hline \multicolumn{5}{|l|}{ Speed } & 87.42 & 94.00 & 100.58 & & \\
\hline \multicolumn{5}{|l|}{ Delta S-1 } & 1.00 & 7.58 & 14.16 & Sum & \\
\hline \multicolumn{5}{|l|}{$\mathrm{e}^{\wedge}(1 /$ Delta $\mathrm{S}+1)$} & 2.72 & 1.14 & 1.07 & 4.93 & \\
\hline \multicolumn{5}{|l|}{$\mathrm{P}(\mathrm{s})$} & 0.55 & 0.23 & 0.22 & & \\
\hline \multicolumn{2}{|c|}{ Conditional Probability Level } & 0.6 & & & & & & & \\
\hline Prior Probabilities & & & \multicolumn{4}{|c|}{ Conditional Probabilities $\mathrm{P}(\mathrm{r} \mid \mathrm{s}, \mathrm{c})$} & & Marginals & $\mathrm{P}(\mathrm{r} \mid \mathrm{c})$ \\
\hline $\mathrm{P}^{\prime}(\mathrm{s} 1)$ & 0.55 & & & $\mathrm{~s}_{1}$ & $\mathrm{~s}_{2}$ & $\mathrm{~s}_{3}$ & & $\mathrm{r}_{1}$ & 0.40 \\
\hline $\mathrm{P}^{\prime}(\mathrm{s} 2)$ & 0.23 & & $\mathrm{r}_{1}$ & 0.6 & 0.2 & 0.1 & & $\mathrm{r}_{2}$ & 0.37 \\
\hline $\mathrm{P}^{\prime}(\mathrm{s})$ & 0.22 & & $\mathrm{r}_{2}$ & 0.3 & 0.6 & 0.3 & & $\mathrm{r}_{3}$ & 0.23 \\
\hline \multirow[t]{2}{*}{ Sum } & 1 & & $\mathrm{r}_{3}$ & 0.1 & 0.2 & 0.6 & & Sum & 1 \\
\hline & & & Sum & 1 & 1 & 1 & & & \\
\hline \multicolumn{2}{|c|}{ Posterior Probabilities P"(s|r,c) } & & & & & \multicolumn{3}{|c|}{$\mathrm{SP}, \mathrm{P}(\mathrm{r} \mid \mathrm{c}) \&$ role of posteriors } & \\
\hline & $\mathrm{s}_{1}$ & $\mathrm{~s}_{2}$ & $\mathrm{~s}_{3}$ & Sum & & & $\mathrm{r}_{1}$ & $\mathrm{r}_{2}$ & $\mathrm{r}_{3}$ \\
\hline $\mathrm{r}_{1}$ & 0.829 & 0.116 & 0.055 & 1 & & $A_{r}$ & 88.90 & 92.22 & 96.14 \\
\hline $\mathrm{r}_{2}$ & 0.448 & 0.376 & 0.177 & $\mid 1$ & & $\mathrm{~A}^{\prime}$ & 91.81 & 91.81 & 91.81 \\
\hline$r_{3}$ & 0.238 & 0.199 & 0.563 & $\| 1$ & & $A_{r}-A^{\prime}$ & -2.90 & 0.41 & 4.33 \\
\hline \multicolumn{2}{|l|}{ Base speed } & 94 & & & & $\mathrm{P}(\mathrm{r} \mid \mathrm{e})$ & 0.40 & 0.37 & 0.23 \\
\hline \multicolumn{10}{|l|}{ Speed (SP) Matrix } \\
\hline & $\mathrm{s}_{1}$ & $\mathrm{~s}_{2}$ & $\mathrm{~s}_{3}$ & & & Posteriors & Speed & max speed & \\
\hline Route A1 & 87.42 & 94.00 & 100.58 & & & $\mathrm{SP}\left(\mathrm{A}_{1}, \mathrm{r}_{1}, \mathrm{c}\right)$ & 88.90 & 88.90 & \\
\hline Route $\mathrm{A}_{2}$ & 0 & 0 & 0 & & & $\mathrm{SP}\left(\mathrm{A}_{2}, \mathrm{r}_{1}, \mathrm{c}\right)$ & 0.00 & & \\
\hline \multirow[t]{2}{*}{ Route $\mathrm{A}_{3}$} & 0 & 0 & 0 & & & $\mathrm{SP}\left(\mathrm{A}_{3}, \mathrm{r}_{1}, \mathrm{c}\right)$ & 0.00 & & \\
\hline & & & & & & $\mathrm{SP}\left(\mathrm{A}_{1}, \mathrm{r}_{2}, \mathrm{c}\right)$ & 92.22 & 92.22 & \\
\hline Priors & Speed & max speed & & & & $\mathrm{SP}\left(\mathrm{A}_{2}, \mathrm{r}_{2}, \mathrm{c}\right)$ & 0.00 & & \\
\hline $\mathrm{SP}\left(\mathrm{A}_{1}\right)$ & 91.81 & 91.81 & & & & $\mathrm{SP}\left(\mathrm{A}_{3}, \mathrm{r}_{2}, \mathrm{c}\right)$ & 0.00 & & \\
\hline $\mathrm{SP}\left(\mathrm{A}_{2}\right)$ & 0.00 & & & & & $\mathrm{SP}\left(\mathrm{A}_{1}, \mathrm{r}_{3}, \mathrm{c}\right)$ & 96.14 & 96.14 & \\
\hline \multirow[t]{2}{*}{$\mathrm{SP}\left(\mathrm{A}_{3}\right)$} & 0.00 & & & & & $\mathrm{SP}\left(\mathrm{A}_{2}, \mathrm{r}_{3}, \mathrm{c}\right)$ & 0.00 & & \\
\hline & & & & & & $\mathrm{SP}\left(\mathrm{A}_{3}, \mathrm{r}_{3}, \mathrm{c}\right)$ & 0.00 & & \\
\hline \multicolumn{3}{|l|}{ Expected $($ Speed $)=$} & 91.81 & $\mathrm{~km} / \mathrm{h}$ & & & & & \\
\hline
\end{tabular}

The predicted speed for the period 10:39:40 is $91.81 \mathrm{~km} / \mathrm{h}$.

Next, wait until the end of 10:39:40 and find out the sensor actual speed reading is $92 \mathrm{~km} / \mathrm{h}$. 
Considering the speed of $94 \mathrm{~km} / \mathrm{h}$ at the moderate speed state $\mathrm{s}_{2}$ from the previous period 10:39:20 then, the four-speed values that correspond to the other speed states are:

Upper speed state is $7 \%$ higher $=100.58 \mathrm{~km} / \mathrm{h}$, Upper midpoint speed is $3.5 \%$ higher $=97.29 \mathrm{~km} / \mathrm{h}$, Lower midpoint speed is $3.5 \%$ lower $=90.71 \mathrm{~km} / \mathrm{h}$, and Lower speed state is $7 \%$ lower $=87.42 \mathrm{~km} / \mathrm{h}$.

Since actual speed of $92 \mathrm{~km} / \mathrm{h}$ for this period 10:39:40 is below the upper midpoint speed of $97.29 \mathrm{~km} / \mathrm{h}$ of the previous period 10:39:20, the trend is towards lower speed and $r_{1}$ is still the speed data available at the outset of 10:39:40 period and should be used for the next period of 10:40:00

This means that the most likely state is $\mathrm{s}_{1}$ for the next period 10:40:00. Moreover, since there will be no change in the states, the priors will be set equal to posterior probabilities that correspond to $\mathrm{r}_{1}$ from this period 10:39:40. So, reset $\mathrm{s}_{2}$ for the next period to be equal to the new level of $92 \mathrm{~km} / \mathrm{h}$ and adjust other states as shown in the following output.

Where the speed values at the three speed states are $\mathrm{s}_{2}=92 \mathrm{~km} / \mathrm{h}, \mathrm{s}_{1}=85.56 \mathrm{~km} / \mathrm{h}$ and $\mathrm{s}_{3}=98.44 \mathrm{~km} / \mathrm{h}$ 
Skipping the 10:40:00 and a number of following period for the sake of brevity, we reach the following step in this illustration of basic model application.

We have skipped time prior to the following stamp for sake of brevity. However, the logic and computations that are included in this chapter provide full details and therefore there is no loss of information.

10:59:00: The outputs for this period are shown below

\begin{tabular}{|c|c|c|c|c|c|c|c|c|c|}
\hline \multirow[t]{2}{*}{ Hwy 401} & \multirow[t]{2}{*}{$\begin{array}{l}\text { Speed } \\
\text { State }\end{array}$} & \multirow[t]{2}{*}{1} & \multicolumn{3}{|c|}{$\underline{2011-03-0710: 59: 00 '}$} & \multicolumn{2}{|c|}{$\begin{array}{c}\text { Previous period } \\
\text { sensor reading }\end{array}$} & \multirow{2}{*}{$\begin{array}{c}54.00 \\
(1+0.5 x) \\
\text { *PREV }\end{array}$} & \multirow{2}{*}{$\begin{array}{c}\mathrm{km} / \mathrm{h} \\
(1+\mathrm{x}) \\
* \mathrm{PREV} \\
\end{array}$} \\
\hline & & & & & $\begin{array}{l}(1-\mathrm{x}) \\
* \text { PREV }\end{array}$ & $\begin{array}{r}(1-0.5 x) \\
\text { *PREV }\end{array}$ & $\begin{array}{c}\text { PREV at } \\
(\mathrm{t}-1)\end{array}$ & & \\
\hline \multicolumn{4}{|c|}{ r1. No change in states. No change in r. Use posteriors } & Speed & 61.38 & 63.69 & 66.0 & 68.31 & 70.62 \\
\hline \multicolumn{4}{|c|}{ Range between speed states $\%=$} & 7 & $\mathrm{~s}_{1}$ & $\mathrm{~s}_{2}$ & $\mathrm{~s}_{3}$ & & \\
\hline \multicolumn{5}{|c|}{ Speed } & 50.22 & 54.00 & 57.78 & & \\
\hline \multicolumn{5}{|l|}{ Delta S-1 } & 1.00 & 4.78 & 8.56 & Sum & \\
\hline \multicolumn{5}{|l|}{$\mathrm{e}^{\wedge}(1 /$ Delta $\mathrm{S}+1)$} & 2.72 & 1.23 & 1.12 & 5.07 & \\
\hline \multicolumn{5}{|l|}{$\mathrm{P}(\mathrm{s})$} & 0.54 & 0.24 & 0.22 & & \\
\hline & & & & & \multicolumn{3}{|c|}{ Ignore Logit For This Period } & & \\
\hline \multicolumn{2}{|c|}{ Conditional Probability Level } & 0.6 & & & & & & & \\
\hline Prior Probabilities & & & \multicolumn{4}{|c|}{ Conditional Probabilities $\mathrm{P}(\mathrm{r} \mid \mathrm{s}, \mathrm{c})$} & & Marginals & $\mathrm{P}(\mathrm{r} \mid \mathrm{c})$ \\
\hline $\mathrm{P}^{\prime}(\mathrm{s} 1)$ & 0.98 & & & $\mathrm{~s}_{1}$ & $\mathrm{~s}_{2}$ & $\mathrm{~s}_{3}$ & & $\mathrm{r}_{1}$ & 0.59 \\
\hline $\mathrm{P}^{\prime}(\mathrm{s} 2)$ & 0.02 & & $\mathrm{r}_{1}$ & 0.6 & 0.2 & 0.1 & & $\mathrm{r}_{2}$ & 0.30 \\
\hline $\mathrm{P}^{\prime}(\mathrm{s})$ & 0.00 & & $\mathrm{r}_{2}$ & 0.3 & 0.6 & 0.3 & & $\mathrm{r}_{3}$ & 0.10 \\
\hline \multirow[t]{2}{*}{ Sum } & 1 & & $\underline{\mathrm{r}_{3}}$ & 0.1 & 0.2 & 0.6 & & Sum & 1 \\
\hline & & & Sum & 1 & 1 & 1 & & & \\
\hline \multicolumn{2}{|c|}{ Posterior Probabilities $\mathrm{P} "(\mathrm{~s} \mid \mathrm{r}, \mathrm{c})$} & & & & & \multicolumn{3}{|c|}{$\mathrm{SP}, \mathrm{P}(\mathrm{r} \mid \mathrm{c}) \&$ role of posteriors } & \\
\hline & $\mathrm{s}_{1}$ & $\mathrm{~s}_{2}$ & $\mathrm{~s}_{3}$ & Sum & & & $\mathrm{r}_{1}$ & $\mathrm{r}_{2}$ & $\mathrm{r}_{3}$ \\
\hline $\mathrm{r}_{1}$ & 0.995 & 0.005 & 0.000 & 1 & & $\overline{A_{r}}$ & 50.24 & 50.35 & 50.41 \\
\hline $\mathrm{r}_{2}$ & 0.968 & 0.030 & 0.002 & 1 & & $\mathrm{~A}^{\prime}$ & 50.29 & 50.29 & 50.29 \\
\hline $\mathrm{r}_{3}$ & 0.960 & 0.030 & 0.011 & 1 & & $A_{r}-A^{\prime}$ & -0.05 & 0.06 & 0.12 \\
\hline \multicolumn{2}{|l|}{ Base speed } & 54 & & & & $\mathrm{P}(\mathrm{r} \mid \mathrm{e})$ & 0.59 & 0.30 & 0.10 \\
\hline \multicolumn{10}{|l|}{ Speed (SP) Matrix } \\
\hline & $\mathrm{s}_{1}$ & $\mathrm{~s}_{2}$ & $\mathrm{~s}_{3}$ & & & Posteriors & Speed & max speed & \\
\hline Route A1 & 50.22 & 54.00 & 57.78 & & & $\mathrm{SP}\left(\mathrm{A}_{1}, \mathrm{r}_{1}, \mathrm{c}\right)$ & 50.24 & 50.24 & \\
\hline Route $\mathrm{A}_{2}$ & 0 & 0 & 0 & & & $\mathrm{SP}\left(\mathrm{A}_{2}, \mathrm{r}_{1}, \mathrm{c}\right)$ & 0.00 & & \\
\hline \multirow[t]{2}{*}{ Route $\mathrm{A}_{3}$} & 0 & 0 & 0 & & & $\mathrm{SP}\left(\mathrm{A}_{3}, \mathrm{r}_{1}, \mathrm{c}\right)$ & 0.00 & & \\
\hline & & & & & & $\mathrm{SP}\left(\mathrm{A}_{1}, \mathrm{r}_{2}, \mathrm{c}\right)$ & 50.35 & 50.35 & \\
\hline Priors & Speed & max speed & & & & $\mathrm{SP}\left(\mathrm{A}_{2}, \mathrm{r}_{2}, \mathrm{c}\right)$ & 0.00 & & \\
\hline $\mathrm{SP}\left(\mathrm{A}_{1}\right)$ & 50.29 & 50.29 & & & & $\mathrm{SP}\left(\mathrm{A}_{3}, \mathrm{r}_{2}, \mathrm{c}\right)$ & 0.00 & & \\
\hline $\mathrm{SP}\left(\mathrm{A}_{2}\right)$ & 0.00 & & & & & $\mathrm{SP}\left(\mathrm{A}_{1}, \mathrm{r}_{3}, \mathrm{c}\right)$ & 50.41 & 50.41 & \\
\hline \multirow[t]{2}{*}{$\mathrm{SP}\left(\mathrm{A}_{3}\right)$} & 0.00 & & & & & $\mathrm{SP}\left(\mathrm{A}_{2}, \mathrm{r}_{3}, \mathrm{c}\right)$ & 0.00 & & \\
\hline & & & & & & $\mathrm{SP}\left(\mathrm{A}_{3}, \mathrm{r}_{3}, \mathrm{c}\right)$ & 0.00 & & \\
\hline \multicolumn{3}{|l|}{ Expected $($ Speed $)=$} & 50.29 & $\mathrm{~km} / \mathrm{h}$ & & & & & \\
\hline
\end{tabular}


The predicted speed for the period 10:59:00 is $50.29 \mathrm{~km} / \mathrm{h}$.

Next, wait until the end of 10:59:00 and find out that the sensor actual speed reading is 48 $\mathrm{km} / \mathrm{h}$.

Considering the speed of $54 \mathrm{~km} / \mathrm{h}$ at the moderate speed state $\mathrm{s}_{2}$ from the previous period 10:58:40 then, the four speed values correspond to the other speed states are:

Upper speed state is $7 \%$ higher $=57.78 \mathrm{~km} / \mathrm{h}$, Upper midpoint speed is $3.5 \%$ higher $=55.89 \mathrm{~km} / \mathrm{h}$, Lower midpoint speed is $3.5 \%$ lower $=52.11 \mathrm{~km} / \mathrm{h}$, and Lower speed state is $7 \%$ lower $=50.22 \mathrm{~km} / \mathrm{h}$.

Since the actual speed of $48 \mathrm{~km} / \mathrm{h}$ for this period 10:59:00 is below the upper midpoint speed of $55.89 \mathrm{~km} / \mathrm{h}$ of the previous period 10:58:40 therefore, the trend is towards lower speed and $r_{1}$ is still the speed data available at the outset of 10:59:00 period and should be used for the next period of 10:59:20

This means the most likely state is $s_{1}$ for the next period of 10:59:20. Moreover, since there will be no change in the states, the priors will be set equal to posterior probabilities that correspond to $r_{1}$ from this period 10:59:00. So, reset $s_{2}$ for the next period to be equal to the new level of $48 \mathrm{~km} / \mathrm{h}$ and adjust other states as shown in the following output. 
The speed values at the three speed states have become $\mathrm{s}_{2}=48 \mathrm{~km} / \mathrm{h}, \mathrm{s}_{1}=44.64$

$\mathrm{km} / \mathrm{h}$ and $\mathrm{s}_{3}=51.36 \mathrm{~km} / \mathrm{h}$.

These are the outputs of the next period as shown below.

10:59:20: The outputs for this period are shown below

\begin{tabular}{|c|c|c|c|c|c|c|c|c|c|}
\hline \multirow[t]{2}{*}{ Hwy 401} & \multirow[t]{2}{*}{$\begin{array}{l}\text { Speed } \\
\text { State }\end{array}$} & \multirow[t]{2}{*}{1} & \multicolumn{3}{|c|}{ '2011-03-07 10:59:20' } & \multicolumn{2}{|c|}{$\begin{array}{l}\text { Previous period } \\
\text { sensor reading }\end{array}$} & \multirow{2}{*}{$\begin{array}{c}48.00 \\
(1+0.5 \mathrm{x}) \\
\text { *PREV }\end{array}$} & \multirow{2}{*}{$\begin{array}{l}\mathrm{km} / \mathrm{h} \\
(1+\mathrm{x}) \\
\text { *PREV }\end{array}$} \\
\hline & & & & & $\begin{array}{l}(1-\mathrm{x}) \\
* \text { PREV }\end{array}$ & $\begin{array}{l}(1-0.5 \mathrm{x}) \\
* \mathrm{PREV} \\
\end{array}$ & \begin{tabular}{|c|} 
PREV at \\
$(\mathrm{t}-1)$
\end{tabular} & & \\
\hline \multicolumn{4}{|c|}{ r1. No change in states. No change in $\mathrm{r}$. Use posteriors } & Speed & 50.22 & 52.11 & 54.0 & 55.89 & 57.78 \\
\hline \multicolumn{4}{|c|}{ Range between speed states $\%=$} & 7 & $\mathrm{~s}_{1}$ & $\mathrm{~s}_{2}$ & $\mathrm{~s}_{3}$ & & \\
\hline \multicolumn{5}{|l|}{ Speed } & 44.64 & 48.00 & 51.36 & i & \\
\hline \multicolumn{5}{|l|}{ Delta S-1 } & 1.00 & 4.36 & 7.72 & Sum & \\
\hline \multicolumn{5}{|l|}{$\mathrm{e}^{\wedge}(1 /$ Delta $\mathrm{S}+1)$} & 2.72 & 1.26 & 1.14 & 5.11 & \\
\hline \multicolumn{5}{|l|}{$\mathrm{P}(\mathrm{s})$} & 0.53 & 0.25 & 0.22 & & \\
\hline & & & & & \multicolumn{3}{|c|}{ Ignore Logit For This Period } & 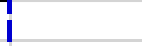 & \\
\hline \multicolumn{2}{|c|}{ Conditional Probability Level } & 0.6 & & & & & & \multirow{2}{*}{ Marginals } & \multirow[b]{2}{*}{$\mathrm{P}(\mathrm{r} \mid \mathrm{c})$} \\
\hline Prior Probabilities & & & \multicolumn{4}{|c|}{ Conditional Probabilities $\mathrm{P}(\mathrm{r} \mid \mathrm{s}, \mathrm{c})$} & & & \\
\hline $\mathrm{P}^{\prime}(\mathrm{s} 1)$ & 0.99 & & & $\mathrm{~s}_{1}$ & $\mathrm{~s}_{2}$ & $\mathrm{~s}_{3}$ & & $r_{1}$ & 0.60 \\
\hline $\mathrm{P}^{\prime}(\mathrm{s} 2)$ & 0.01 & & $\mathrm{r}_{1}$ & 0.6 & 0.2 & 0.1 & & $\mathrm{r}_{2}$ & 0.30 \\
\hline $\mathrm{P}^{\prime}(\mathrm{s})$ & 0.00 & & $\mathrm{r}_{2}$ & 0.3 & 0.6 & 0.3 & & $\mathrm{Ir}_{3}$ & 0.10 \\
\hline \multirow[t]{3}{*}{ Sum } & 1 & & $\mathrm{r}_{3}$ & 0.1 & 0.2 & 0.6 & & Sum & 1 \\
\hline & & & Sum & 1 & 1 & 1 & & & \\
\hline & & & & & & & & i & \\
\hline \multicolumn{2}{|c|}{ Posterior Probabilities P"(s|r,c) } & & & & & \multicolumn{3}{|c|}{$\mathrm{SP}, \mathrm{P}(\mathrm{r} \mid \mathrm{c}) \&$ role of posteriors } & \\
\hline & $\mathrm{s}_{1}$ & $\mathrm{~s}_{2}$ & $\mathrm{~s}_{3}$ & Sum & & & $\mathrm{r}_{1}$ & $\mathrm{r}_{2}$ & $\mathrm{r}_{3}$ \\
\hline $\mathrm{r}_{1}$ & 0.998 & 0.002 & 0.000 & 1 & & $\overline{\mathrm{A}_{\mathrm{r}}}$ & 44.65 & 44.68 & 44.69 \\
\hline $\mathrm{r}_{2}$ & 0.989 & 0.010 & 0.000 & $\mid 1$ & & $\mathrm{~A}^{\prime}$ & 44.66 & 44.66 & 44.66 \\
\hline$r_{3}$ & 0.988 & 0.010 & 0.002 & $\mid 1$ & & $A_{r}-A^{\prime}$ & -0.01 & 0.02 & 0.03 \\
\hline \multicolumn{2}{|l|}{ Base speed } & 48 & & & & $\mathrm{P}(\mathrm{r} \mid \mathrm{e})$ & 0.60 & 0.30 & 0.10 \\
\hline \multicolumn{10}{|l|}{ Speed (SP) Matrix } \\
\hline & $\mathrm{s}_{1}$ & $\mathrm{~s}_{2}$ & $\mathrm{~s}_{3}$ & & & Posteriors & Speed & max speed & \\
\hline Route A1 & 44.64 & 48.00 & 51.36 & & & $\mathrm{SP}\left(\mathrm{A}_{1}, \mathrm{r}_{1}, \mathrm{c}\right)$ & 44.65 & 44.65 & \\
\hline Route $\mathrm{A}_{2}$ & 0 & 0 & 0 & & & $\mathrm{SP}\left(\mathrm{A}_{2}, \mathrm{r}_{1}, \mathrm{c}\right)$ & 0.00 & & \\
\hline \multirow[t]{2}{*}{ Route $\mathrm{A}_{3}$} & 0 & 0 & 0 & & & $\mathrm{SP}\left(\mathrm{A}_{3}, \mathrm{r}_{1}, \mathrm{c}\right)$ & 0.00 & & \\
\hline & & & & & & $\mathrm{SP}\left(\mathrm{A}_{1}, \mathrm{r}_{2}, \mathrm{c}\right)$ & 44.68 & 44.68 & \\
\hline Priors & Speed & max speed & & & & $\mathrm{SP}\left(\mathrm{A}_{2}, \mathrm{r}_{2}, \mathrm{c}\right)$ & 0.00 & & \\
\hline $\operatorname{SP}\left(A_{1}\right)$ & 44.66 & 44.66 & & & & $\mathrm{SP}\left(\mathrm{A}_{3}, \mathrm{r}_{2}, \mathrm{c}\right)$ & 0.00 & & \\
\hline $\mathrm{SP}\left(\mathrm{A}_{2}\right)$ & 0.00 & & & & & $\mathrm{SP}\left(\mathrm{A}_{1}, \mathrm{r}_{3}, \mathrm{c}\right)$ & 44.69 & 44.69 & \\
\hline \multirow[t]{2}{*}{$\mathrm{SP}\left(\mathrm{A}_{3}\right)$} & 0.00 & & & & & $\mathrm{SP}\left(\mathrm{A}_{2}, \mathrm{r}_{3}, \mathrm{c}\right)$ & 0.00 & & \\
\hline & & & & & & $\mathrm{SP}\left(\mathrm{A}_{3}, \mathrm{r}_{3}, \mathrm{c}\right)$ & 0.00 & & \\
\hline \multicolumn{3}{|l|}{ Expected $($ Sp eed $)=$} & 44.66 & $\mathrm{~km} / \mathrm{h}$ & & & & I & \\
\hline
\end{tabular}


The predicted speed for the period 10:59:20 is $44.66 \mathrm{~km} / \mathrm{h}$. Next, wait until the end of 10:59:20 and find out the sensor actual speed reading is now $30 \mathrm{~km} / \mathrm{h}$. Considering the speed of $48 \mathrm{~km} / \mathrm{h}$ at the moderate speed state $\mathrm{s}_{2}$ from the previous period 10:59:00 then, the four-speed values that correspond to the other speed states are:

Upper speed state is $7 \%$ higher $=51.36 \mathrm{~km} / \mathrm{h}$, Upper midpoint speed is $3.5 \%$ higher $=49.68 \mathrm{~km} / \mathrm{h}$, Lower midpoint speed is $3.5 \%$ lower $=46.32 \mathrm{~km} / \mathrm{h}$, and Lower speed state is $7 \%$ lower $=44.64 \mathrm{~km} / \mathrm{h}$.

Since the actual speed of $30 \mathrm{~km} / \mathrm{h}$ for this period 10:59:20 is below the upper midpoint speed of $49.68 \mathrm{~km} / \mathrm{h}$ of the previous period 10:59:00 therefore, the trend is towards lower speed and $r_{1}$ is still the speed data available at the outset of 10:59:20 period and should be used for the next period of 10:59:40

This means the most likely state is $s_{1}$ for the next period of 10:59:4 0. Moreover, since there will be no change in the states, the priors will be set equal to posterior probabilities that correspond to $r_{1}$ from this period 10:59:20. So, reset $s_{2}$ for the next period to be equal to the new level of $30 \mathrm{~km} / \mathrm{h}$ and adjust other states as shown in the following output.

Now the speed values at the three speed states are $s_{2}=30 \mathrm{~km} / \mathrm{h}, \mathrm{s}_{1}=27.9 \mathrm{~km} / \mathrm{h}$ and $\mathrm{s}_{3}=32.1 \mathrm{~km} / \mathrm{h}$.

The criteria proposed to detect incidents in this chapter specifies that incident trigger will be activated under two conditions: (1) when the value of the posterior probability level $(\mathrm{P}$ '( $\mathrm{s} \mid \mathrm{r}))$ exceeds a specified percentage according to the incident detection criteria, and (2) when the continuous drop in the predicted speed reaches a specified percentage. 
In checking the proposed criteria for incident detection, it is noticed that at the end of this period 10:59:20, the maximum posterior probability $\mathrm{P}(\mathrm{r} 2 \mid \mathrm{s} 2)$ level reached 0.998 (a very high level of reliability). This posterior level is an indication of the reliability of a continuous change in the speed towards one direction. For the purpose of detecting any incident, these changes should be toward low-speed level. In general, the observed change in the speed is found to be towards low-speed level. When comparing this value to the threshold defined in the specified criteria, the posterior probability should be higher than a selected value of 0.97 (or 97\%). So, reaching a posterior probability level of $99.8 \%$ is considered satisfying the first condition of detecting an incident.

The second condition in the incident detection criteria involves examining the drop in the predicted speed. It is found that the continuous drop in the predicted speed reaches to a certain limit that is very close to the selected percentage limit; it reaches to a percentage of $-58 \%$ which satisfies the second specified incident detection criterion. The criterion selected to define the percent drop in the speed in the previous application was assumed to be $-55 \%$. This percentage appears with a negative sign as an indication of a speed drop rather than any possible increase in the predicted speed.

The maximum posterior probability level, speed values obtained from the sensor data and the predicted speed using Bayesian model are tabulated in Table 5.6, and a detailed discussion is followed. 
Table 5.6: Predicted speed, adoption of posterior probability $\&$ indication of incident

\begin{tabular}{|c|c|c|c|c|c|c|}
\hline no. & Time period & $\begin{array}{l}\text { Sensor } \\
\text { speed } \\
\text { data } \\
(\mathrm{km} / \mathrm{h})\end{array}$ & $\begin{array}{c}\text { Predicted } \\
\text { speed }\end{array}$ & $\begin{array}{c}\% \text { Drop in } \\
\text { predicted } \\
\text { speed }\end{array}$ & $\begin{array}{l}\text { P"'(s|r) for lower } \\
\text { equipped level } \\
\text { case (i.e., for } \\
\mathrm{P}(\mathrm{r} \mid \mathrm{s})=0.6)\end{array}$ & $\begin{array}{l}\text { Meeting the } \\
\text { criteria }\end{array}$ \\
\hline 2 & '2011-03-07 10:39:00' & 100 & 94.00 & 0 & 0.704 & 'No' \\
\hline 3 & 2011-03-07 10:39:20' & 94 & 102.35 & 9 & 0.83 & 'No' \\
\hline 4 & '2011-03-07 10:39:40' & 92 & 91.81 & -10 & 0.829 & 'No' \\
\hline 5 & '2011-03-07 10:40:00' & 92 & 87.01 & -15 & 0.946 & 'No' \\
\hline 6 & '2011-03-07 10:40:20' & 104 & 85.98 & -15 & 0.983 & 'No' \\
\hline 7 & '2011-03-07 10:40:40' & 99 & 106.45 & 6 & 0.831 & 'No' \\
\hline 8 & '2011-03-07 10:41:00' & 103 & 96.68 & -8 & 0.83 & 'No' \\
\hline 9 & '2011-03-07 10:41:20' & 99 & 103.00 & -1 & 0.706 & 'No' \\
\hline 10 & '2011-03-07 10:41:40' & 83 & 96.68 & -7 & 0.83 & 'No' \\
\hline 11 & '2011-03-07 10:42:00' & 104 & 78.49 & -24 & 0.946 & 'No' \\
\hline 12 & '2011-03-07 10:42:20' & 100 & 106.45 & 3 & 0.831 & 'No' \\
\hline 13 & '2011-03-07 10:42:40' & 99 & 97.65 & -8 & 0.83 & 'No' \\
\hline 14 & '2011-03-07 10:43:00' & 97 & 93.62 & -11 & 0.946 & 'No' \\
\hline 15 & '2011-03-07 10:43:20' & 107 & 90.65 & -14 & 0.983 & 'No' \\
\hline 16 & '2011-03-07 10:43:40' & 109 & 109.52 & 5 & 0.831 & 'No' \\
\hline 17 & '2011-03-07 10:44:00' & 111 & 114.93 & 8 & 0.946 & 'No' \\
\hline 18 & '2011-03-07 10:44:20' & 106 & 118.27 & 11 & 0.983 & 'No' \\
\hline 19 & '2011-03-07 10:44:40' & 94 & 103.50 & -5 & 0.831 & 'No' \\
\hline 20 & '2011-03-07 10:45:00' & 93 & 88.89 & -18 & 0.946 & 'No' \\
\hline 21 & '2011-03-07 10:45:20' & 95 & 86.91 & -20 & 0.983 & 'No' \\
\hline 22 & '2011-03-07 10:45:40' & 104 & 88.47 & -18 & 0.995 & 'No' \\
\hline 23 & '2011-03-07 10:46:00' & 106 & 106.45 & -1 & 0.831 & 'No' \\
\hline 24 & '2011-03-07 10:46:20' & 100 & 111.76 & 4 & 0.946 & 'No' \\
\hline 25 & '2011-03-07 10:46:40' & 102 & 97.65 & -11 & 0.83 & 'No' \\
\hline 26 & '2011-03-07 10:47:00' & 99 & 96.46 & -12 & 0.946 & 'No' \\
\hline 27 & '2011-03-07 10:47:20' & 106 & 92.52 & -15 & 0.983 & 'No' \\
\hline 28 & '2011-03-07 10:47:40' & 105 & 108.50 & 0 & 0.831 & 'No' \\
\hline 29 & '2011-03-07 10:48:00' & 101 & 110.71 & 2 & 0.946 & 'No' \\
\hline 30 & '2011-03-07 10:48:20' & 102 & 98.63 & -10 & 0.83 & 'No' \\
\hline 31 & '2011-03-07 10:48:40' & 98 & 96.46 & -12 & 0.946 & 'No' \\
\hline 32 & '2011-03-07 10:49:00' & 101 & 91.58 & -16 & 0.983 & 'No' \\
\hline
\end{tabular}


Cont.

\begin{tabular}{|c|c|c|c|c|c|c|}
\hline no. & Time period & $\begin{array}{c}\text { Sensor } \\
\text { speed } \\
\text { data } \\
(\mathrm{km} / \mathrm{h}) \\
\end{array}$ & $\begin{array}{l}\text { Predicted } \\
\text { speed }\end{array}$ & $\begin{array}{c}\% \text { Drop in } \\
\text { predicted } \\
\text { speed }\end{array}$ & $\begin{array}{c}\text { P' }(\mathrm{s} \mid \mathrm{r}) \text { for lower } \\
\text { equipped level } \\
\text { case (i.e., for } \\
\mathrm{P}(\mathrm{r} \mid \mathrm{s})=0.6)\end{array}$ & $\begin{array}{c}\text { Meeting the } \\
\text { criteria }\end{array}$ \\
\hline 33 & '2011-03-07 10:49:20' & 99 & 94.06 & -14 & 0.995 & 'No' \\
\hline 34 & '2011-03-07 10:49:40' & 103 & 92.11 & -16 & 0.998 & 'No' \\
\hline 35 & '2011-03-07 10:50:00' & 102 & 103.00 & -6 & 0.706 & 'No' \\
\hline 36 & '2011-03-07 10:50:20' & 100 & 102.00 & -6 & 0.828 & 'No' \\
\hline 37 & '2011-03-07 10:50:40' & 95 & 100.00 & -8 & 0.906 & 'No' \\
\hline 38 & '2011-03-07 10:51:00' & 98 & 92.78 & -15 & 0.83 & 'No' \\
\hline 39 & '2011-03-07 10:51:20' & 102 & 92.68 & -14 & 0.946 & 'No' \\
\hline 40 & '2011-03-07 10:51:40' & 104 & 102.00 & -6 & 0.706 & 'No' \\
\hline 41 & '2011-03-07 10:52:00' & 105 & 104.00 & -4 & 0.828 & 'No' \\
\hline 42 & '2011-03-07 10:52:20' & 100 & 105.00 & -2 & 0.906 & 'No' \\
\hline 43 & '2011-03-07 10:52:40' & 99 & 97.65 & -9 & 0.83 & 'No' \\
\hline 44 & '2011-03-07 10:53:00' & 97 & 93.62 & -12 & 0.946 & 'No' \\
\hline 45 & '2011-03-07 10:53:20' & 100 & 90.65 & -15 & 0.983 & 'No' \\
\hline 46 & '2011-03-07 10:53:40' & 95 & 93.13 & -13 & 0.995 & 'No' \\
\hline 47 & '2011-03-07 10:54:00' & 102 & 88.39 & -17 & 0.998 & 'No' \\
\hline 48 & '2011-03-07 10:54:20' & 100 & 104.40 & -2 & 0.83 & 'No' \\
\hline 49 & '2011-03-07 10:54:40' & 108 & 105.43 & -1 & 0.946 & 'No' \\
\hline 50 & '2011-03-07 10:55:00' & 104 & 115.07 & 8 & 0.983 & 'No' \\
\hline 51 & '2011-03-07 10:55:20' & 104 & 101.55 & -5 & 0.831 & 'No' \\
\hline 52 & '2011-03-07 10:55:40' & 101 & 98.35 & -8 & 0.946 & 'No' \\
\hline 53 & '2011-03-07 10:56:00' & 95 & 94.38 & -11 & 0.983 & 'No' \\
\hline 54 & '2011-03-07 10:56:20' & 97 & 88.47 & -17 & 0.995 & 'No' \\
\hline 55 & '2011-03-07 10:56:40' & 100 & 90.25 & -15 & 0.998 & 'No' \\
\hline 56 & '2011-03-07 10:57:00' & 107 & 93.01 & -13 & 0.999 & 'No' \\
\hline 57 & '2011-03-07 10:57:20' & 93 & 107.00 & 1 & 0.707 & 'No' \\
\hline 58 & '2011-03-07 10:57:40' & 79 & 90.83 & -15 & 0.829 & 'No' \\
\hline 59 & '2011-03-07 10:58:20' & 66 & 74.72 & -30 & 0.945 & 'No' \\
\hline 60 & '2011-03-07 10:58:40' & 54 & 61.68 & -42 & 0.983 & 'No' \\
\hline 61 & '2011-03-07 10:59:00' & 48 & 50.29 & -53 & 0.995 & 'No' \\
\hline 62 & '2011-03-07 10:59:20' & 30 & 44.66 & -58 & 0.998 & Yes \\
\hline
\end{tabular}




\section{Cont.}

\begin{tabular}{|c|c|c|c|c|c|c|}
\hline no. & Time period & $\begin{array}{c}\text { Sensor } \\
\text { speed } \\
\text { data } \\
(\mathrm{km} / \mathrm{h}) \\
\end{array}$ & $\begin{array}{l}\text { Predicted } \\
\text { speed }\end{array}$ & $\begin{array}{c}\% \text { Drop in } \\
\text { predicted } \\
\text { speed }\end{array}$ & $\begin{array}{c}\text { P' }(\mathrm{s} \mid \mathrm{r}) \text { for lower } \\
\text { equipped level } \\
\text { case (i.e., for } \\
\mathrm{P}(\mathrm{r} \mid \mathrm{s})=0.6)\end{array}$ & $\begin{array}{c}\text { Meeting the } \\
\text { criteria }\end{array}$ \\
\hline 63 & '2011-03-07 10:59:40' & 28 & 27.90 & -74 & 0.999 & \\
\hline 64 & '2011-03-07 11:00:00' & 14 & 26.04 & -76 & 1 & \\
\hline 65 & '2011-03-07 11:00:20' & 11 & 13.02 & -88 & 1 & \\
\hline 66 & '2011-03-07 11:00:40' & 6 & 10.23 & -90 & 1 & \\
\hline 67 & '2011-03-07 11:01:00' & 7 & 5.58 & -95 & 1 & \\
\hline 68 & '2011-03-07 11:01:20' & 6 & 7.08 & -93 & 0.745 & \\
\hline 69 & '2011-03-07 11:01:40' & 4 & 5.94 & -94 & 0.739 & \\
\hline 70 & '2011-03-07 11:02:00' & 6 & 3.81 & -96 & 0.909 & \\
\hline 71 & '2011-03-07 11:02:20' & 4 & 6.06 & -94 & 0.739 & \\
\hline 72 & '2011-03-07 11:02:40' & 9 & 3.97 & -96 & 0.722 & \\
\hline 73 & '2011-03-07 11:03:00' & 11 & 9.12 & -91 & 0.756 & \\
\hline 74 & '2011-03-07 11:03:20' & 13 & 11.53 & -89 & 0.916 & \\
\hline 75 & '2011-03-07 11:03:40' & 5 & 13.82 & -87 & 0.973 & \\
\hline 76 & '2011-03-07 11:04:00' & 3 & 4.95 & -95 & 0.731 & \\
\hline 77 & '2011-03-07 11:04:20' & 5 & 2.86 & -97 & 0.906 & \\
\hline 78 & '2011-03-07 11:05:00' & 7 & 5.05 & -95 & 0.731 & \\
\hline 79 & '2011-03-07 11:05:20' & 5 & 7.32 & -93 & 0.906 & \\
\hline 80 & '2011-03-07 11:05:40' & 3 & 4.95 & -95 & 0.731 & \\
\hline 81 & '2011-03-07 11:06:00' & 6 & 2.86 & -97 & 0.906 & \\
\hline 82 & '2011-03-07 11:06:20' & 8 & 6.06 & -94 & 0.739 & \\
\hline 83 & '2011-03-07 11:06:40' & 7 & 8.37 & -92 & 0.909 & \\
\hline 84 & '2011-03-07 11:07:00' & 6 & 6.92 & -93 & 0.745 & \\
\hline 85 & '2011-03-07 11:07:20' & 6 & 5.72 & -95 & 0.912 & \\
\hline 86 & '2011-03-07 11:07:40' & 4 & 5.62 & -95 & 0.971 & \\
\hline 87 & '2011-03-07 11:08:00' & 9 & 3.73 & -96 & 0.991 & \\
\hline 88 & '2011-03-07 11:08:20' & 7 & 9.12 & -91 & 0.756 & \\
\hline 89 & '2011-03-07 11:08:40' & 8 & 6.92 & -93 & 0.745 & \\
\hline 90 & '2011-03-07 11:09:00' & 7 & 8.10 & -92 & 0.751 & \\
\hline
\end{tabular}


Cont.

\begin{tabular}{|c|c|c|c|c|c|c|}
\hline no. & Time period & $\begin{array}{c}\text { Sensor } \\
\text { speed } \\
\text { data } \\
(\mathrm{km} / \mathrm{h})\end{array}$ & $\begin{array}{l}\text { Predicted } \\
\text { speed }\end{array}$ & $\begin{array}{c}\% \text { Drop in } \\
\text { predicted } \\
\text { speed }\end{array}$ & $\begin{array}{c}\text { P' }(\mathrm{s} \mid \mathrm{r}) \text { for lower } \\
\text { equipped level } \\
\text { case (i.e., for } \\
\mathrm{P}(\mathrm{r} \mid \mathrm{s})=0.6)\end{array}$ & $\begin{array}{c}\text { Meeting the } \\
\text { criteria }\end{array}$ \\
\hline 91 & '2011-03-07 11:09:20' & 3 & 6.92 & -93 & 0.745 & \\
\hline 92 & '2011-03-07 11:09:40' & 9 & 2.86 & -97 & 0.912 & \\
\hline 93 & '2011-03-07 11:10:00' & 5 & 9.12 & -91 & 0.756 & \\
\hline 94 & '2011-03-07 11:10:20' & 4 & 4.95 & -95 & 0.731 & \\
\hline \begin{tabular}{|l|}
95 \\
\end{tabular} & '2011-03-07 11:10:40' & 6 & 3.82 & -96 & 0.906 & \\
\hline 96 & '2011-03-07 11:11:00' & 4 & 6.06 & -94 & 0.739 & \\
\hline 97 & '2011-03-07 11:11:20' & 3 & 3.97 & -96 & 0.722 & \\
\hline 98 & '2011-03-07 11:12:20' & 5 & 2.87 & -97 & 0.902 & \\
\hline 99 & '2011-03-07 11:12:40' & 6 & 5.05 & -95 & 0.731 & \\
\hline 100 & '2011-03-07 11:13:00' & 5 & 6.27 & -94 & 0.906 & \\
\hline 101 & '2011-03-07 11:13:20' & 6 & 4.95 & -95 & 0.731 & \\
\hline 102 & '2011-03-07 11:13:40' & 5 & 6.06 & -94 & 0.739 & \\
\hline 103 & '2011-03-07 11:14:00' & 4 & 4.95 & -95 & 0.731 & \\
\hline 104 & '2011-03-07 11:14:20' & 6 & 3.82 & -96 & 0.906 & \\
\hline 105 & '2011-03-07 11:14:40' & 5 & 6.06 & -94 & 0.739 & \\
\hline 106 & '2011-03-07 11:15:00' & 6 & 4.95 & -95 & 0.731 & \\
\hline 107 & '2011-03-07 11:15:20' & 5 & 6.06 & -94 & 0.739 & \\
\hline 108 & '2011-03-07 11:15:40' & 7 & 4.95 & -95 & 0.731 & \\
\hline 109 & '2011-03-07 11:16:00' & 7 & 7.08 & -93 & 0.745 & \\
\hline 110 & '2011-03-07 11:16:20' & 4 & 7.33 & -93 & 0.912 & \\
\hline 111 & '2011-03-07 11:16:40' & 3 & 3.97 & -96 & 0.722 & \\
\hline 112 & '2011-03-07 11:17:00' & 5 & 2.87 & -97 & 0.902 & \\
\hline 113 & '2011-03-07 11:17:40' & 6 & 5.05 & -95 & 0.731 & \\
\hline 114 & '2011-03-07 11:18:00' & 4 & 6.27 & -94 & 0.906 & \\
\hline 115 & '2011-03-07 11:18:20' & 4 & 3.97 & -96 & 0.722 & \\
\hline
\end{tabular}

\subsubsection{Logical Argument}

The developed Bayesian model starts by predicting the speed at each time period and the percent drop in the predicted speed is also calculated. Likewise, the maximum posterior probability level that is associated with each time period is computed. See Table 5.6.

The results of the model reported in this chapter are based on the reliability level (conditional probability) of 0.6. In model application, other applicable values can be used 
as well. A number of observations can be drawn regarding model application for detecting incidents.

The results illustrate that as the speed continues to drop rapidly (possibly due to an incident), the posterior probability will show an increasing trend. If this pattern continues, the posterior probability will rise close to (or equal to) its highest value of 1.0 and will remain at that level for a well-defined time period. Should there be a reversal of the speed trend (due to clearance of the incident), the trend of high posterior probability will be altered due to lower values. This observation can be seen in Table 5.6 and Figure 5.9.

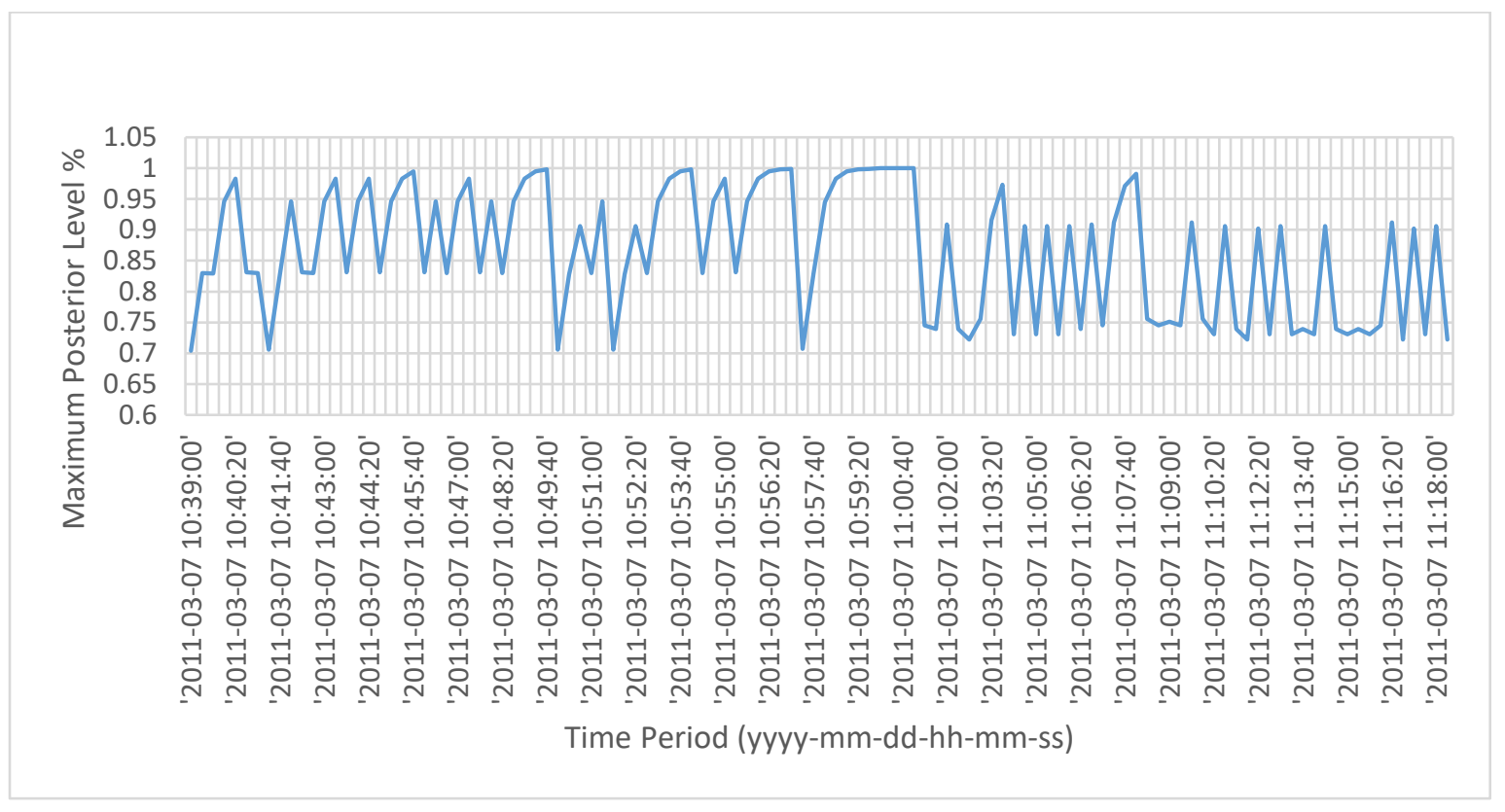

Figure 5.9: Maximum posterior level \%

For the purpose of detecting an incident, the posterior probability should be tracked in order to find out if the pattern of high values exists. The second condition for incident detection is the rapid drop in the predicted speed. 
As illustrated in this section, the application of the model should continue to examine the patterns of posterior probability and rapid speed drop. Should the pre-defined criteria be met, an incident can be declared. Figure 5.9 can be studied for the identification of posterior probability pattern that could be caused by an incident. The pattern of speed drop observable in Figure 5.10 supplements the posterior probability criterion in detecting an incident.

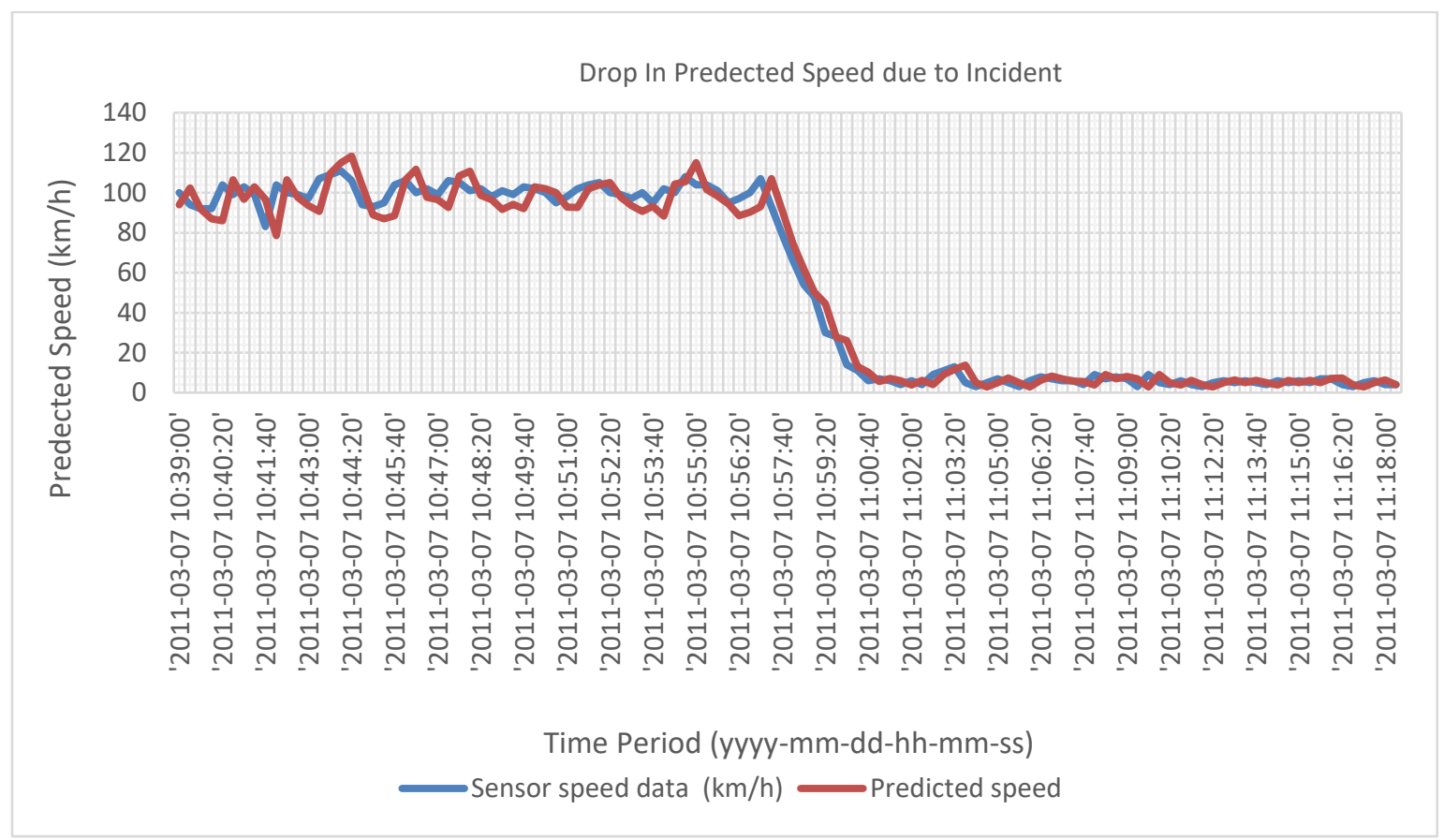

Figure 5.10: Sensor speed data vs. predicted speed data

In Figure 5.10, it can be noticed that at the end of the period 10:59:00 the predicted speed reaches to a low value of $50.29 \mathrm{~km} / \mathrm{h}$. This drop in the predicted speed amounts to $53 \%$ (based on $85^{\text {th }}$ percentile of the obtained spot speed during the study period). The period starts 20 minutes before the incident recorded time and extends till one time period before the time when the incident detection criteria were applied as shown in Figure 5.11. 


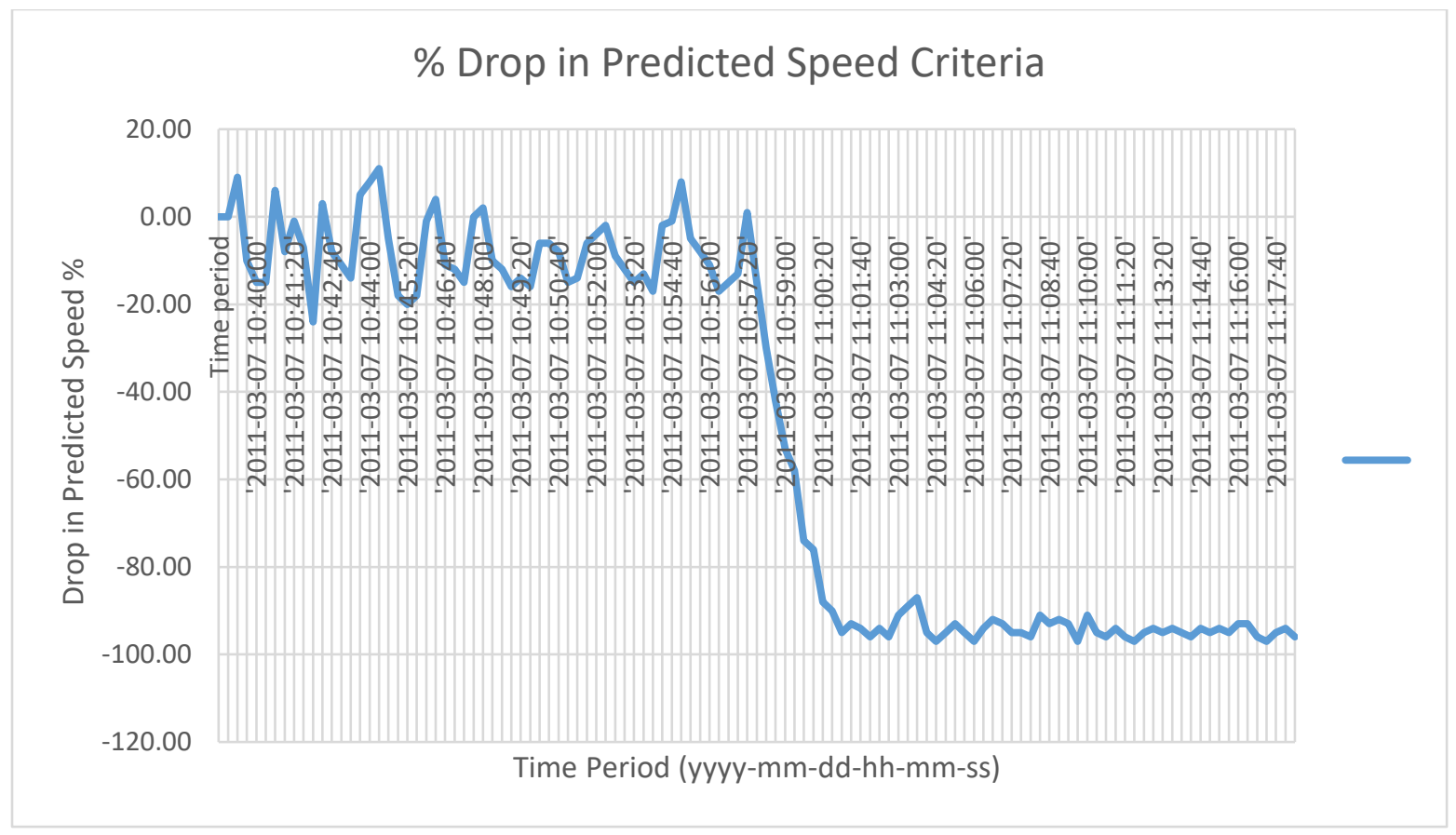

Figure 5.11: Percent drop in predicted speed

Although 53\% drop in speed can be considered as "high" and the posterior probability level shows a high value of 0.995 , the specified criteria are not met. The incident detection criteria state that the second condition is satisfied only when the percent drop in the predicted speed reaches $55 \%$.

So, the search continues by advancing the clock and observing the results for the next time period 10:59:20. Table 5.6 shows that at the end of the period 10:59:20 the predicted speed was $44.66 \mathrm{~km} / \mathrm{h}$, this drop in the predicted speed amounts to $58 \%$. At this point in time, the criteria of high posterior probability value and high speed drop are met and the model triggers the alarm that an incident is detected. Please see Table 5.6 for the notice "yes" regarding an incident. 
To go beyond the type of notice posted in Table 5.6, the next chapter presents additional details. For example, see Figure 5.12 as an illustration of incident detection by the developed model.

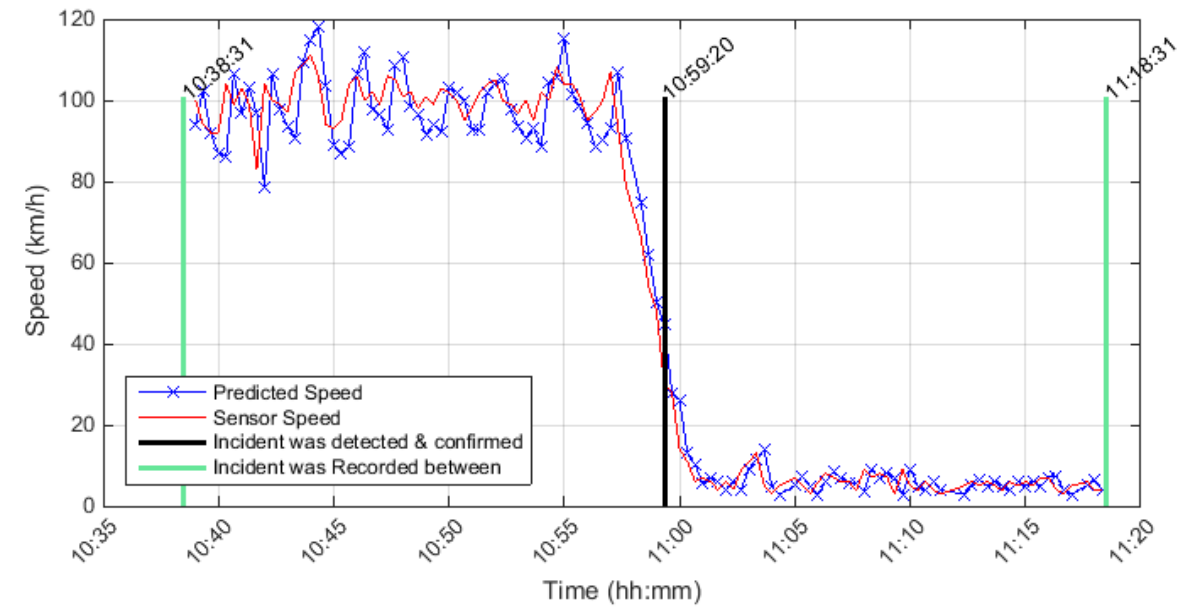

Upstream Detector \# is 401DE0290DWC, Detector Rank = 1, Distance from incident location in $(\mathrm{km})=0.43401$ Season $=$ Spring. Stream $=$ C, Direction $=W$, Date $\&$ Time $=2011-03-0710: 58: 31$ Incident Reason $=$ Collision

Affected Lanes $=$ All lanes closed

Conditional Probability level $=0.6$, Cond.Prob. $r 3 \mathrm{~s} 2=0.2$, Cond.Prob. $r 3 s 1=0.1$, Percent drop in speed $\%=-56$

Max. Posterior Probability level $=0.97$, Percent range between speed states $=7 \%$

Incident was detected at 02:00 minutes from drop in speed point. Incident was confirmed after 5 minutes from the detection time

Figure 5.12: Incident detection - developed version of the model

The drop in speed shown in Figure 5.12 was predicted by the model and verified by the sensor. It can be observed that the model was able to predict speed before the sensor data became available. In spite of this challenge, the differences between these two speed data are minor. That is, the differences between the speed values predicted by the model and reported by the sensor are modest, the highest difference of $5 \mathrm{~km}$ was observed as shown in Figure 5.12. This difference amounts to only $4 \%$ of the predicted speed. 
Another evidence of the capability of the developed model in detecting incidents is its ability to follow any sudden changes in the actual speed reported by the sensor. The developed model proved to be able to predict and follow such speed patterns. For example, see time period from 10:55:20 to 10:57:40 in Table 5.6.

Another favourable feature of the model is the short time taken by the model to detect an incident. In the case of this example incident, about 2:00 minutes following the first severe drop in the predicted speed, the incident was detected. This represents the minimum time to detect an incident and it can be viewed as short. It is expected that this minimum time to detect could become even shorter by increasing the conditional probability which serves as the reliability indicator. A conditional probability level of 0.8 shows less detection time as will be explained in detail in the next chapter. Therefore, it can be stated with confidence that early incident detection is influenced by the higher level of predicted and sensor data reliability.

From the chart in Figure 5.12, it is observed that the speed continues to decline till it reaches low values around $4 \mathrm{~km} / \mathrm{h}$. The defined time boundary under study starts 20 minutes before the recorded time of the incident and continues till 20 minutes after the incident recorded time. The incident report stated that the incident caused blockage to the entire highway section, and all lanes were closed. Consequently, traffic was severely impacted and affected the recovery process as can be observed from low speed values until the end of the study time period.

Under certain incident conditions the incident not causes severe effects (e.g. only one or two lanes may be blocked). In such situations, it is expected that the speed will recover quickly, especially under low volume conditions. There could be other cases even under 
high traffic volume where the speed might decline for a short period of time and then recover back to normal. Further, there could be incidents that result in stop-and-go conditions. These and possibly other incident conditions can be encountered that reflect site-specific geometric and traffic design of a facility and of course traffic characteristics are known to explain effects of incidents.

In order to develop and verify the incident detection model, scenarios mentioned above were considered to extent feasible. After the calibration process, a validation procedure became crucial to bring the developed model to its complete and reliable version. 


\section{Incident Detection Model Development, Calibration, and}

\section{Validation}

One important conclusion to draw from the initial version of the incident detection model application presented in Chapter 5 is that for an incident detection model to work, an incident should cause a rapid drop in speed under prevailing conditions. That is, a drop in the speed is an essential indicator of a disturbance in traffic flow potentially caused by an incident. However, a drop in speed by itself is not sufficient to decide on the occurrence of an incident. The other criterion is the pattern of the increasing value of the posterior probability.

In order to automate the processes of model refinement and validation, the fundamental aspects of the proposed model are utilized to establish a computer program (i.e. an algorithm) using Matlab language. The algorithm reads sensor speed data and the associated time, predicts next period speed, tracks the traffic speed and the posterior probability patterns. A comprehensive discussion about the computer program development is presented in section 6.4 of this chapter.

In this chapter, a calibration method is described which aims to determine the conditions, parameters and threshold values in the algorithm in order to improve model performance. It is common to use applicable indicators, namely detection rate DR, false alarm rate FAR and minimum time to detect incident MTTD, for optimization during the calibration process.

A high DR is appreciated by traffic control and management authorities and of course as expected the minimizing FAR is accorded importance. For enhancing the credibility of the algorithm, minimizing the FAR is used in the calibration process described below. 
Step 1: Prepare the incident data set and divide the data set evenly into calibration and validation sets (as was described in chapter three)

Step 2: Identify the calibration parameters of DR, FAR and MTTD to calibrate the threshold parameters of the developed model and algorithm containing the model. As defined in Chapter 2, the DR and FAR are in percentages and MTTD is in minutes.

Step 3: Run the algorithm on the first portion of data set prepared for calibration purposes (See sections 6.1 to 5.4). It is intended to find a good solution set of threshold values.

Step 4: Test the calibrated algorithm on the second set of data that includes validation dataset, with the same threshold values obtained in Step 3, as presented in section 6.5.

Step 5: If the validation results are satisfactory, the developed conditions and the threshold values are then employed in the algorithm for incident detection of any other incident. Otherwise, the incident data in step 1 needs to be retested, and the calibration process needs to be verified. It may also be necessary to verify and refine the composite objective function in Step 5; repeat steps 2 to 5.

\subsection{Defining the Calibration Method}

\section{Prepare the incident data set (Step 1)}

According to (AP-R364/10 AUSTROADS RESEARCH REPORT Freeway Incident Detection - Technologies and Techniques, 2010), some guidelines for calibration and validation of the developed algorithm should include the following.

In the case incident data is not enough for both calibration and validation, it is recommended to use more or all data for calibration. It is stated that a well-calibrated 
algorithm can be converted into real-life applications, and real-time, on-site validation can be conducted as part of the traffic management centres daily operations.

The availability of incident data is a key to proper incident detection model calibration. The 100 incident cases obtained from the detailed analyses described in chapter three were regarded sufficient as the database of this procedure.

As indicated in chapter three, 100 incident cases obtained from the first sorted group are used to test the model under the consideration that an incident has occurred. Likewise, the same 100 cases are used to test the model for cases that showed no recording of any incident by following certain criteria. This can be applied by selecting another time period that is not close to the time when neither incidents caused by collision or disabled vehicle nor any other incidents type were recorded.

Since there is statistically sufficient data and the 100 incident cases are randomly selected, the first 50 cases were processed for the model calibration purposes. Likewise, statistically sufficient data were available for validating the model. With the 100 randomly selected incident cases, the obtained results are expected to be conclusive due to the adequate amount of incident data.

The algorithm was run on the portion of data set prepared for calibration purposes (Step 3). The output of this step was a set of parameters or threshold values.

Several tests were conducted on the available 50 data cases designated for model calibration. The basic version of the developed Matlab program was used as part of the calibration process. The goal of the tests is to examine the performance of the developed algorithm in detecting and confirming incidents occurrence. Results of these tests enabled decision of further analyses. 
The methodology of the calibration process started by assessing the performance of the developed model in its basic Matlab program version. The following factors were studied: the incident recorded time and assigning threshold values, the conditional probability level, percent drop in speed, maximum posterior probability level, and the ranges of the speed values associated with each speed state.

\subsubsection{Incident Recorded Time Credibility}

Before proceeding with application of these tests, the incident recorded time was examined closely for a better indication of the time when the incident was first recorded.

In some cases, it was noticed that the incident recorded time is some time after the drop in speed occurred, as shown in Figure 6.1. Notice that the incident recorded time is always presented as a vertical green or red color line in the output diagram, while the incident detection indicator is presented in blue or black color line.

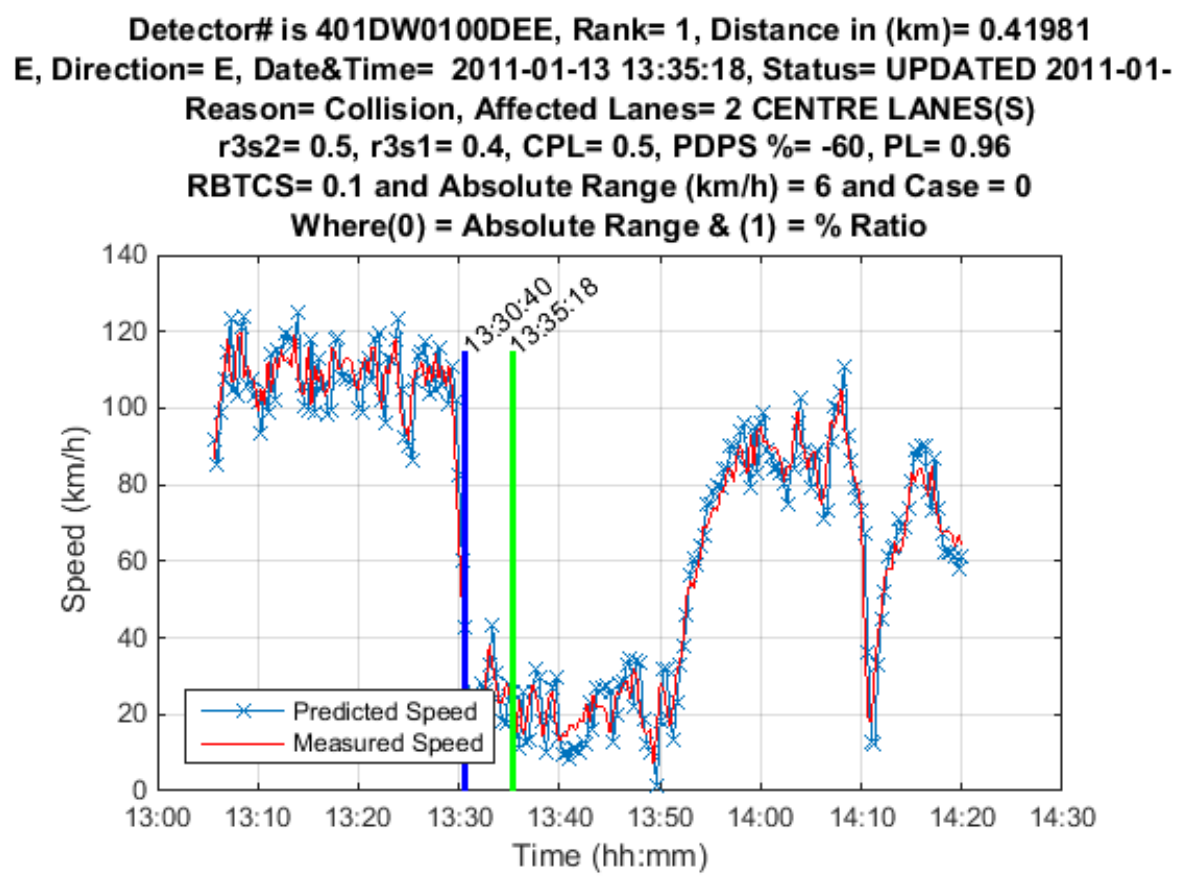

Figure 6.1: Incident was recorded after drop in speed 
Although the detector is located at $410 \mathrm{~m}$ away from the incident, however, the time gap between the moment when the speed started to drop and the moment the incident was recorded is about 4 minutes after the drop in speed. While it is expected to have the incident recorded time falling at some point before any decline in the speed occurs, it is noticed that the incident time was incorrectly reported. This can be interpreted as a delay in the incident reporting process.

In some other cases, the recorded time falls much after the drop in the speed and the time gap between the incident recorded time and the drop in speed point is even larger as can be seen in Figure 6.2.

Detector\# is 401DE0070DEE, Rank= 4, Distance in $(\mathrm{km})=\mathbf{2 . 3 6 7 5}$

$\mathrm{E}$, Direction= E, Date\&Time $=$ 2011-01-12 10:57:43, Status= UPDATED 2011-01Reason= Collision, Affected Lanes= LEFT SHOULDER AND 1 LEFT LANE(S) $\mathrm{r} 3 \mathrm{~s} 2=0.5, \mathrm{r} 3 \mathrm{~s} 1=0.4, \mathrm{CPL}=0.5$, PDPS $\%=-60, \mathrm{PL}=0.96$

RBTCS $=0.1$ and Absolute Range $(\mathrm{km} / \mathrm{h})=2$ and Case $=0$ Where $(0)=$ Absolute Range \& (1) $=\%$ Ratio

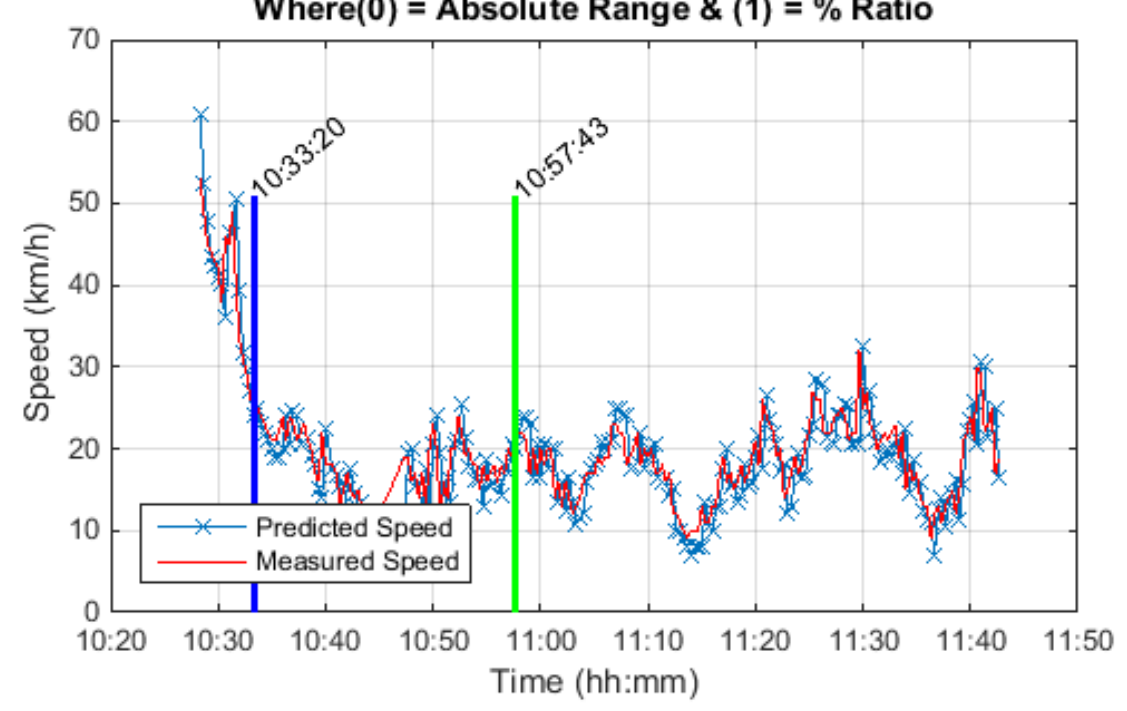

Figure 6.2: Gap between incident recorded time and drop in speed point 
It is noticed that the detector was located far away from the incident due to a lack of any information from the detectors located close to the incident location; the distance is $2.36 \mathrm{~km}$. The data show that the recorded time of the incident is 24 minutes after the onset of speed drop. This can be interpreted as delay in the incident reporting process.

In the output of this basic version of algorithm application, the recorded incident time was plotted in the output diagram as an indicator of the time when the incident was recorded as illustrated in Figure 6.3. Since the recorded time is found to be unreliable, later on in this chapter this indicator is removed from the plotting area. Instead, it was replaced by two boundaries as an indication of a recorded incident within these two boundaries as illustrated in Figure 6.4. Each boundary is proposed to be 20 minutes before and after the recorded time of the incident.

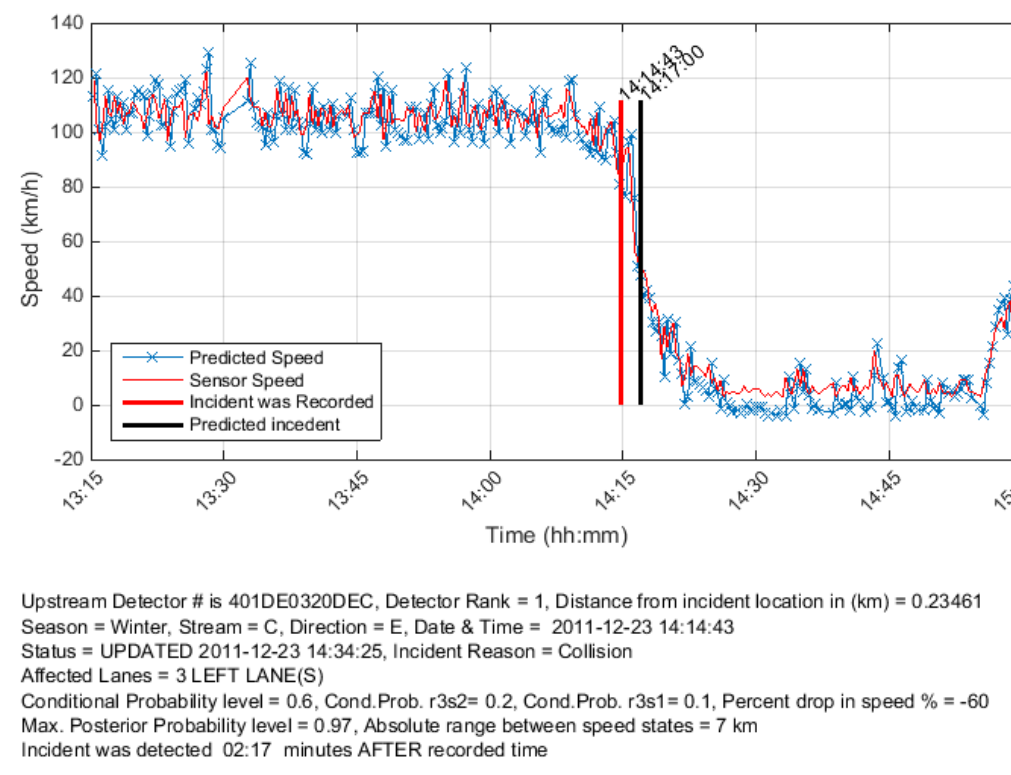

Figure 6.3: Incident recorded time indicated at one point 


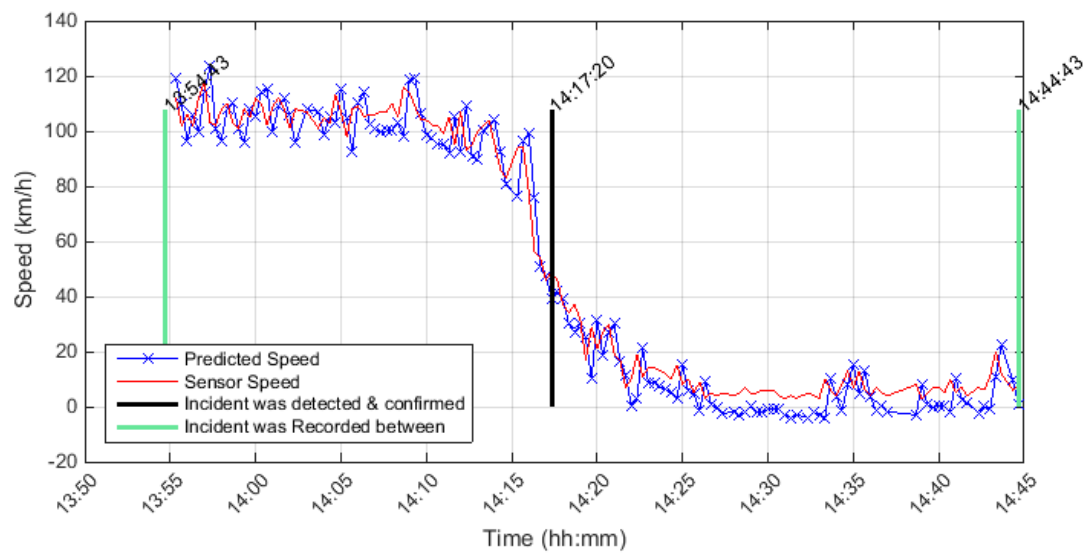

Upstream Detector \# is 401DE0320DEC, Detector Rank $=1$, Distance from incident location in $(\mathrm{km})=0.23461$
Season $=$ Winter, Stream $=\mathrm{C}$, Direction $=\mathrm{E}$, Date $\&$ Time $=2011-12-2314: 14: 43$
Status $=$ UPDATED 2011-12-23 $14: 34: 25$, Incident Reason $=$ Collision
Affected Lanes $=3$ LEFT LANE(S)
Conditional Probability level $=0.6$, Cond.Prob. r $3 \mathrm{~s} 2=0.2$, Cond.Prob. $\mathrm{r} 3 \mathrm{~s} 1=0.1$, Percent drop in speed $\%=-60$
Max. Posterior Probability level $=0.97$, Absolute range between speed states $=7 \mathrm{~km}$
Incident was detected at 01:20 minutes from drop in speed point. Incident was confirmed after 5 minutes from the detection time

Figure 6.4: Incident recorded time indicated between two points

The same observation applies to cases tested under free of incident conditions, where the recorded free of incident condition time was plotted in the output diagram as an indicator of a selected time when no incident was recorded, as illustrated in Figure 6.5. Likewise, since the recorded time is found to be unreliable so, later on in this chapter, this indicator is removed from the plotting area. Instead, it was replaced by two boundaries as an indication of a claimed free of incident condition time within these two boundaries as illustrated in Figure 6.6. 


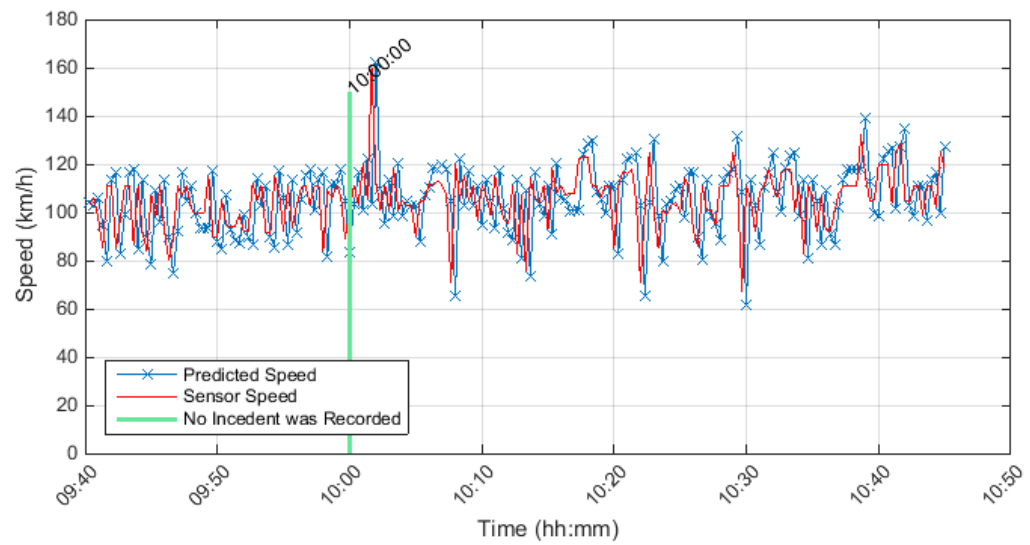

Upstream Detector \# is 401DE0110DEC, Detector Rank $=2$, Distance from incident free location in $(\mathrm{km})=1.3122$ Incident free Season $=$ Spring, Stream $=$ C, Direction $=E$, Date \& Time $=2011-03-06$ 16:51:04 Selected incident free Date/Time $=2011-03-06$ 10:00:00

Conditional Probability level $=0.6$, Cond. Prob. $r 3 \mathrm{~s} 2=0.2$, Cond. Prob. $r 3 \mathrm{~s} 1=0.1$, Percent drop in speed $\%=-60$ Max. Posterior Probability level $=0.97$, Absolute range between speed states $=7 \mathrm{~km}$ (No Incident was Detected)

Figure 6.5: Free incident condition recorded time indicated at one point

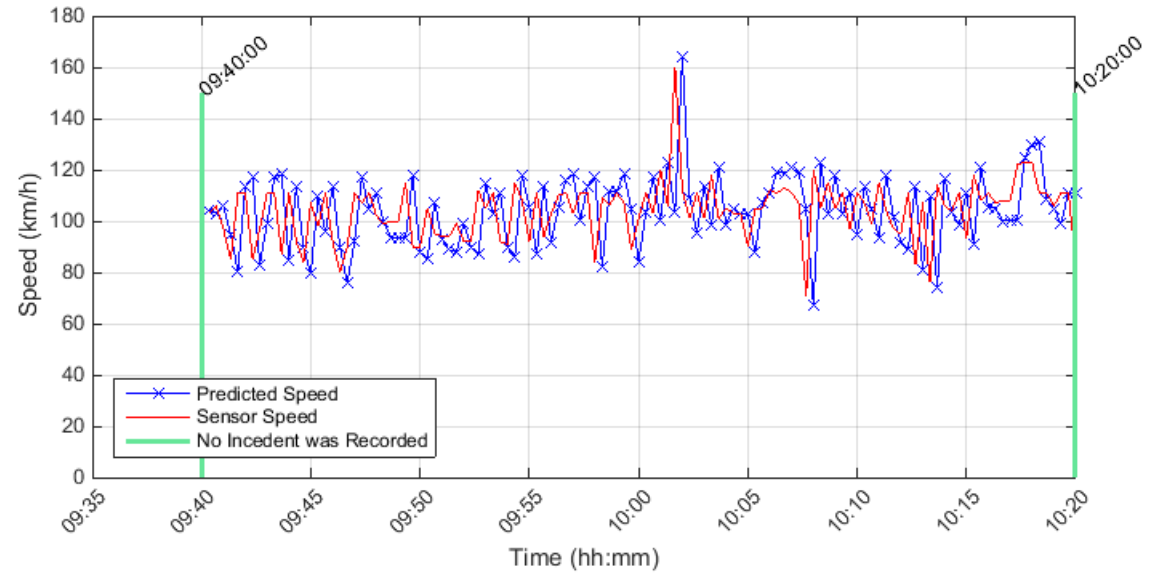

Upstream Detector \# is 401DE0110DEC, Detector Rank $=2$, Distance from incident free location in $(\mathrm{km})=1.3122$ Incident free Season $=$ Spring, Stream $=$ C, Direction $=\mathrm{E}$, Date $\&$ Time $=2011-03-06$ 16:51:04 Selected incident free Date/Time $=2011-03-06$ 10:00:00

Conditional Probability level $=0.6$, Cond.Prob. $r 3 s 2=0.2$, Cond. Prob. $r 3 s 1=0.1$, Percent drop in speed $\%=-56$ Max. Posterior Probability level $=0.97$, Percent range between speed states $=7 \%$ (No Incident was Detected)

Figure 6.6: Free incident condition recorded time indicated between two points 


\subsubsection{Effect of the Maximum Posterior Probability Level and the Drop in Speed Percentage Threshold on Incident Detection}

In the following typical case, the incident was recorded during off-peak hours, and the basic version of the Matlab program was applied to detect the incident. As a preliminary set, the predetermined threshold posterior level for this case is $(0.9)$ or $99 \%$, and the threshold for the percentage of the speed drop is chosen as $70 \%$. The selected conditional probability level is 0.5 .

Although it was off-peak hours, three lanes were affected, which is regarded as a considerable number of blocked lanes. Since the number of lanes in the east direction of Highway 401 at this location are four in total, vehicles backed up in one lane and a slow traffic flow was the expected result.

The detector is located very close to the incident location, almost $140 \mathrm{~m}$. away. This short distance assists in a rapid propagation of the effect of the incident on the upstream traffic in the form of a shock wave and the resulting drop in speed as shown in Figure 6.7. As indicated before the incident recorded time is presented as a line in this case in green color.

From the figure, it is noticed that the incident was not detected, where the green line indicates the unreliable recorded time of the incident. The percent drop in the speed exceeds the defined percentage while it reaches a value of about $94 \%$. However, the maximum posterior level only reaches a value of 0.96 . These two indications do not meet the criteria, and therefore, there was no detection of any incident. 


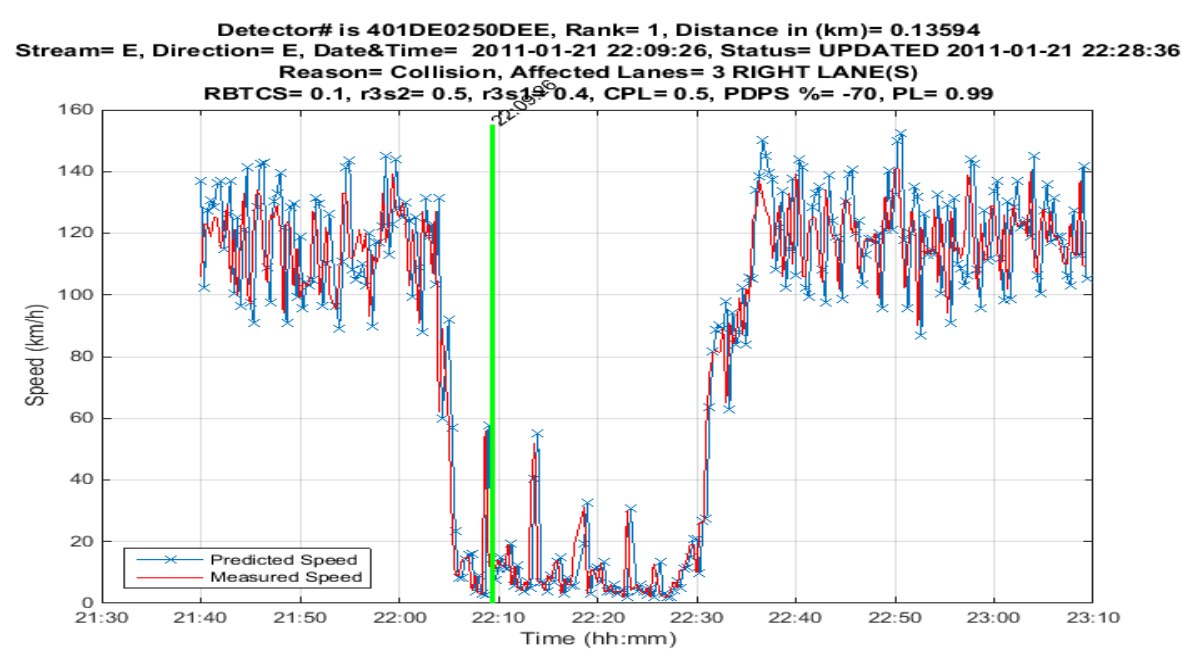

Figure 6.7: High percentage of the posterior level

In this case, the time when the incident took place was almost 22:09 pm. The traffic volume was not too high at that time and therefore, the speed tended to recover to a normal condition within a short period of time. The reason the incident was recorded at a time after the drop in speed could be related to a delay in the reporting process as was discussed earlier in this chapter and in chapter three of this thesis.

To analyze the same case further, the maximum defined posterior probability level was decreased to 0.96 instead of the high level of 0.99 as was indicated in the previous scenario. Also, the total percentage of the speed drop was lowered to $60 \%$ instead of $70 \%$ used earlier. The results showed that the incident was detected as can be seen in Figure 6.8.

From the obtained results it is concluded that the proper selection of both the percentage of the posterior probability level and the drop in speed became significant incident detection criteria. 


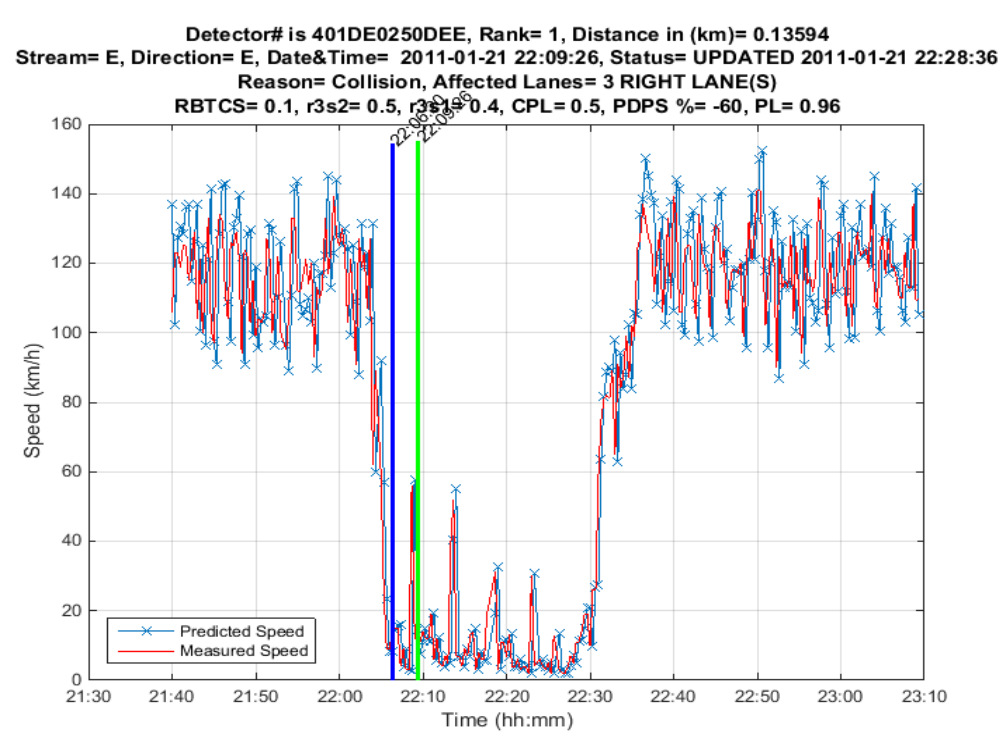

Figure 6.8: Lower percentage in the posterior level

\subsubsection{Effect of the Conditional Probability Level on Incident Detection}

This section provides an explanation of the conditional probability and its effect on the results. As previously explained, this probability reflects the reliability of data and therefore of the overall incident detection algorithm. For comparison purposes, conditional probability levels of 0.5 and 0.8 were tested. However, it should be noted that conditional probability of 0.5 is too coarse for any real world application. Therefore, a higher value of 0.6 or 0.7 is realistic even when the quality of data is not very high.

The conditional probability is $\mathrm{P}(\mathrm{r} / \mathrm{s})$, which means that if the real state is $\mathrm{s}$, it is the probability of obtaining the corresponding $r$ from the system. In other words, the results obtained from the detectors (sensors) that are commonly assumed to represent the traffic condition downstream the detector may not be fully reliable, but their reliability can be expected to be higher than 0.5 out of 1 . In earlier chapters of this thesis, the factors that 
affect reliability were examined (e.g. sensor failure, distance between detectors, in case of RSU equipped vehicles, etc.).

In order to examine the reliability of the model, a high conditional probability level of 0.8 was applied to the previous case shown in Figure 6.7 under the high posterior probability level of 0.99 and percent drop in speed of $70 \%$. This proposed high conditional probability level is representing the assumption of low outages of detectors/sensors or having many equipped connected vehicles on the road, as explained in chapter four. Results showed that the posterior level reached a value of over 0.99 when the drop in speed exceeds the value of $70 \%$. Therefore, the incident was detected in this case as shown in Figure 6.9.

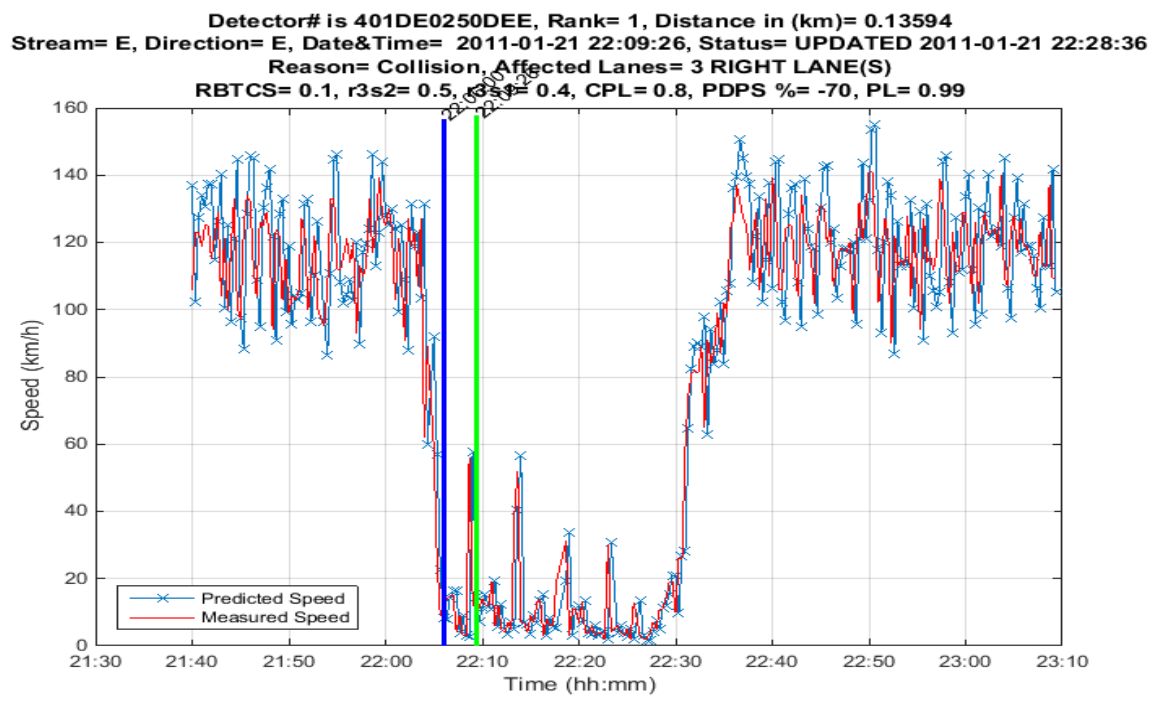

Figure 6.9: High conditional probability level of 0.8

So, for conservative analysis, if the conditional probability level is kept to a lower value of 0.5 , then to drive the algorithm to detect incidents, the posterior threshold level should be set at a lower value in order to enable the algorithm to detect some potential incidents. The reason behind setting a low value of conditional probability is because of the 
assumption that the analyst does not have high confidence in the data that are the inputs to the algorithm.

From the obtained results of several tests, it is concluded that the combination of 0.96 $(96 \%)$ for the posterior probability level along with the drop in speed percentage of $60 \%$ is the properly selected percentage to meet the criteria for incident detection.

A similar typical case is presented in Figure 6.10. It is an attempt to examine the effect of conditional probability level on incident detection. The three threshold values for the posterior probability level, percent drop in speed and the conditional probability are 0.96 , $60 \%$ and 0.5 , respectively.

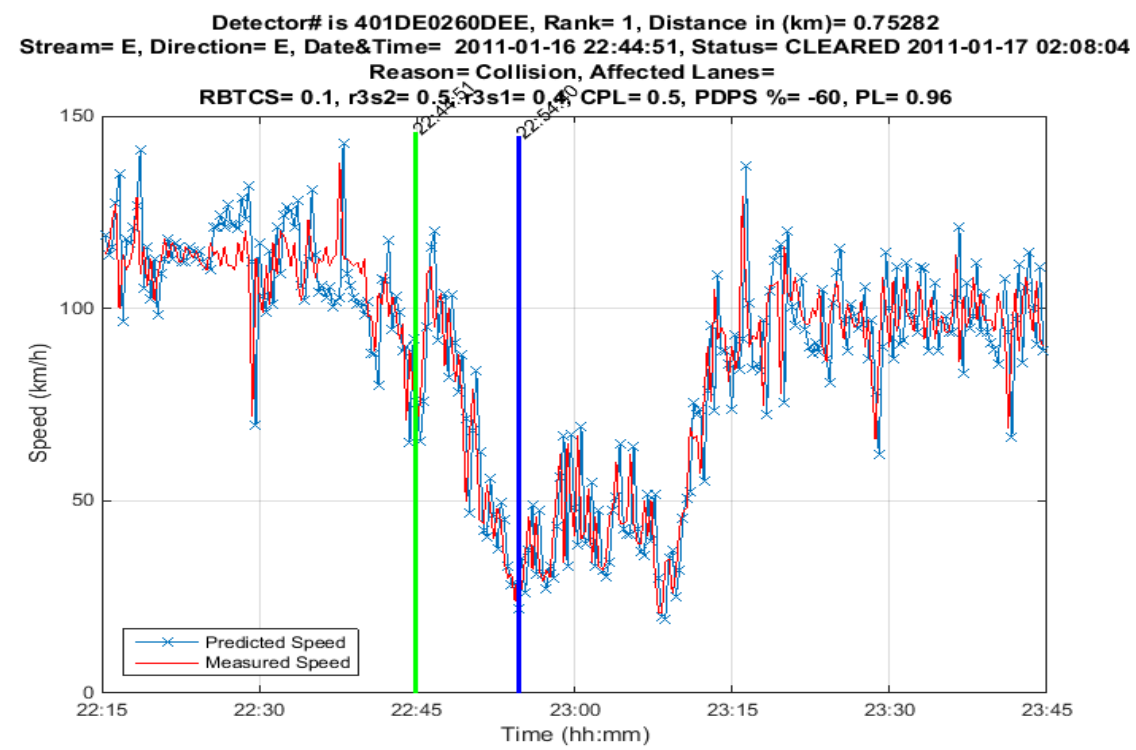

Figure 6.10: Moderate conditional probability level of 0.5

This case needs to be investigated further with some changes in the other factors. Results from the Matlab program were used in evaluating the effectiveness of each factor. 
When increasing both the posterior probability threshold of 0.99 and the drop in speed threshold to $70 \%$, the results showed no indication to any incident as shown in Figure 6.11

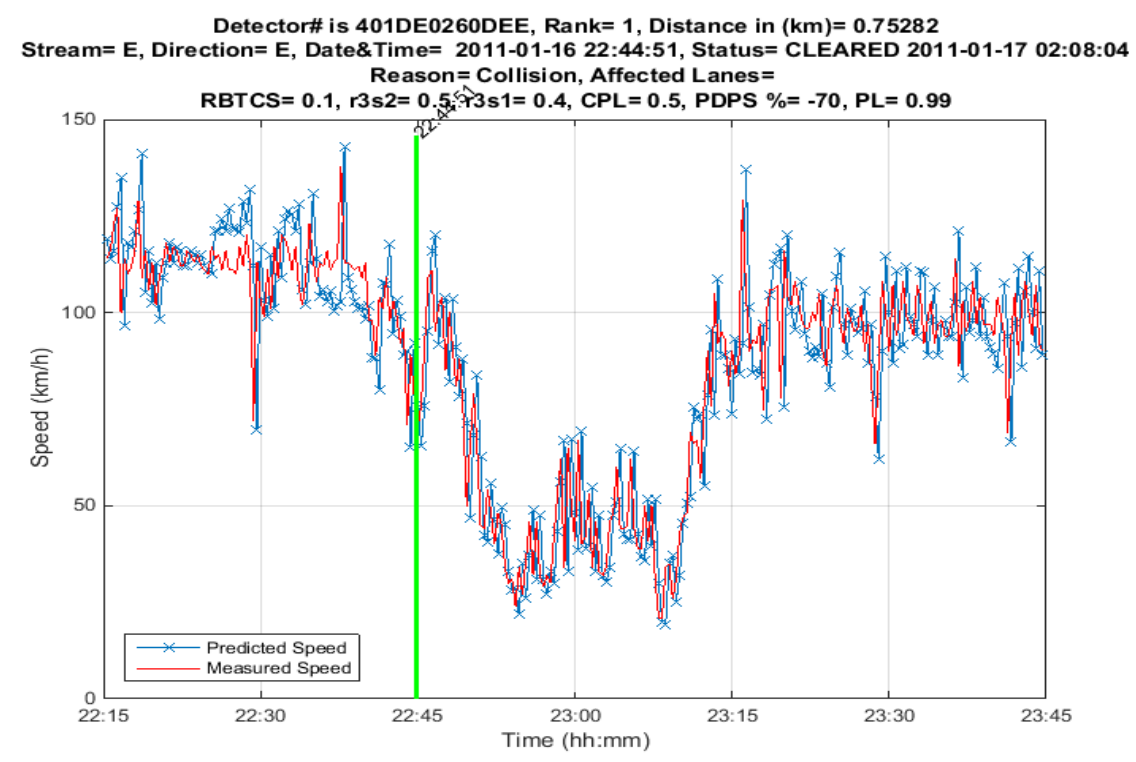

\section{Figure 6.11: High posterior and drop in speed threshold}

However, when the conditional probability level was increased to 0.8 , the incident was detected as can be seen in Figure 6.12.

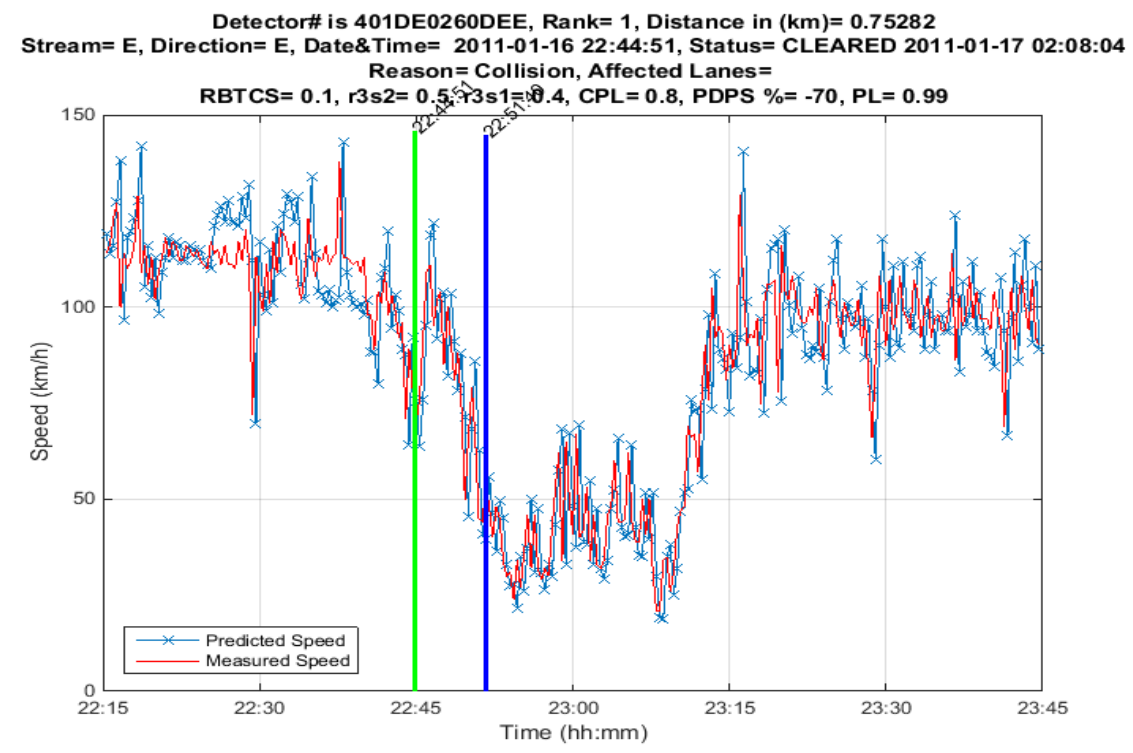

Figure 6.12: High conditional probability level 


\subsubsection{Effect of the Absolute Speed Range on Incident Detection}

In the previous diagram in Figure 6.12, the selected speed range is $2 \mathrm{~km} / \mathrm{h}$, when selecting a higher speed range of $5 \mathrm{~km} / \mathrm{h}$ the same results were obtained. However, it is noticed that for low-speed levels the fixed value of the speed range is still affecting the shape of the predicted speed profile as shown in Figure 6.13. The difference between the recorded speed and the predicted speed is relatively large.

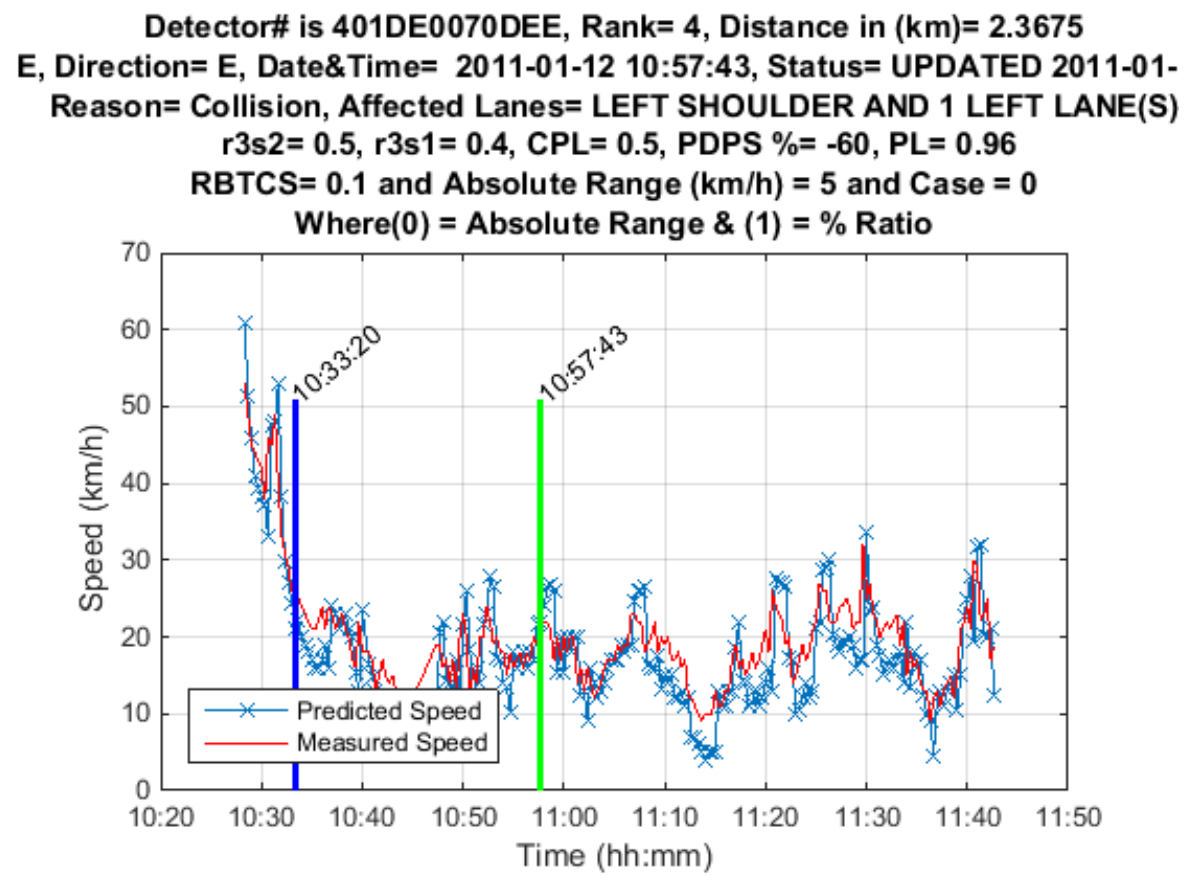

Figure 6.13: Speed range of $5 \mathrm{~km} / \mathrm{h}$

To discuss the effect of the speed range on the final predicted speed, the two cases in

Figure 6.14 and Figure 6.15 are presented. The speed range in both cases is $7 \mathrm{~km} / \mathrm{h}$, and $7 \%$ respectively. It is noticed that, at high-speed level (between $30 \mathrm{~km} / \mathrm{h}$ and $65 \mathrm{~km} / \mathrm{h}$ ), the predicted speed was very close to the recorded speed. However, at low-speed levels (0-30 $\mathrm{km} / \mathrm{h}$ ) it is noticeable that for cases with an absolute range, the predicted speed is always higher than the recorded speed with a value of $7 \mathrm{~km} / \mathrm{h}$. The obtained result indicates a large difference at low-speed levels; this issue is resolved by proposing a percent speed range 
between the speeds for each speed state. In the second case of choosing the percentage format, it is noticeable that the model predicted a very close speed values at low and highspeed levels. Therefore, the percent speed range is proposed for the proceeding analysis.

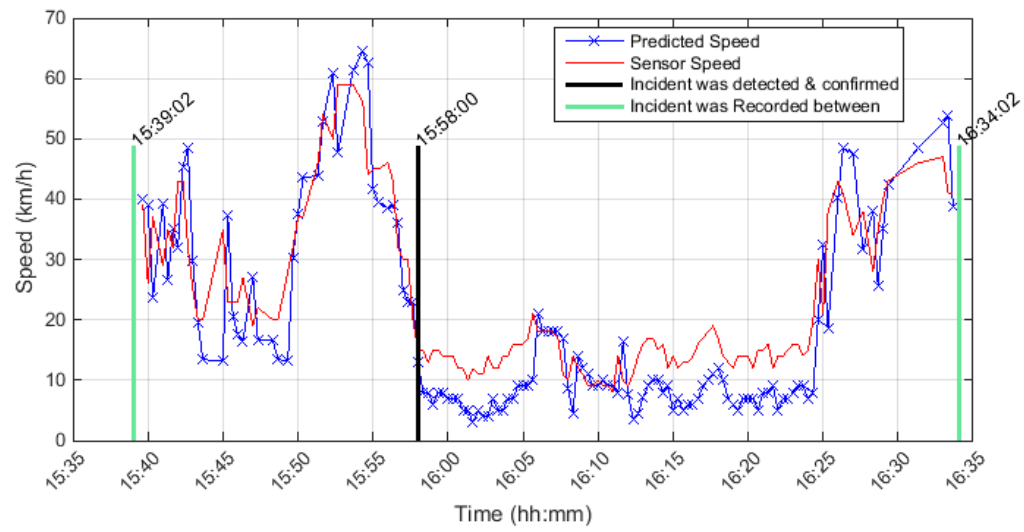

Upstream Detector \# is 401DE0060DEC, Detector Rank $=1$, Distance from incident location in $(\mathrm{km})=0.19316$ Season $=$ Summer, Stream $=$ C, Direction $=E$, Date $\&$ Time $=2011-10-0515: 59: 02$

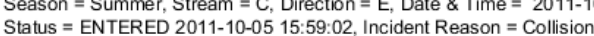
Affected Lanes $=1$ LEFT LANE(S)

Conditional Probability level $=0.6$, Cond.Prob. $r 3 s 2=0.2$, Cond.Prob. $r 3 s 1=0.1$, Percent drop in speed $\%=-60$ Max. Posterior Probability level $=0.97$, Absolute range between speed states $=7 \mathrm{~km}$

Incident was detected at 03:20 minutes from drop in speed point. Incident was confirmed after 5 minutes from the detection time

\section{Figure 6.14: Speed range of $7 \mathrm{~km} / \mathrm{h}$}

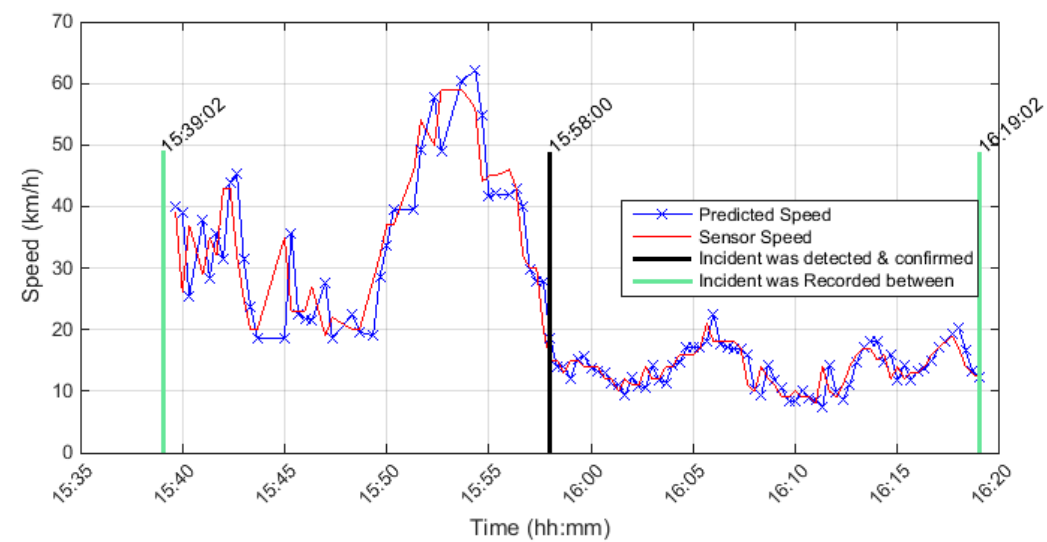

Upstream Detector \# is 401DE0060DEC, Detector Rank $=1$, Distance from incident location in $(\mathrm{km})=0.19316$ Season $=$ Summer, Stream $=C$, Direction $=E$, Date $\&$ Time $=2011-10-0515: 59: 02$ Incident Reason = Collision

Affected Lanes = 1 LEFT LANE(S)

Conditional Probability level $=0.6$, Cond.Prob. $r 3 s 2=0.2$, Cond. Prob. $r 3 s 1=0.1$, Percent drop in speed $\%=-60$

Max. Posterior Probability level $=0.97$, Percent range between speed states $=7 \%$

Incident was detected at 03:40 minutes from drop in speed point. Incident was confirmed after 5 minutes from the detection time

Figure 6.15: Speed range of $7 \%$ 


\subsubsection{Consideration of Special Conditions in Calibration Criteria}

In general, incidents cause a sharp drop in speed and lower speeds continue for a considerable amount of time before recovering back to the normal operating level. However, there are incident cases that do not follow this pattern and of course the incident detection algorithm should be able to handle such cases.

In the following case, the selected posterior probability level was set at a reasonably high level of 0.96, and also the percent drop in speed was set at a somewhat high level of $60 \%$. As shown in Figure 6.16 the drop in speed recovered very quickly back to normal values. That is, although the speed dropped rapidly but it recovered back quickly within a short period of time (i.e. between 7:50h and 8:00h). Although this case differs from the usual incident recovery pattern, the algorithm detected the incident.

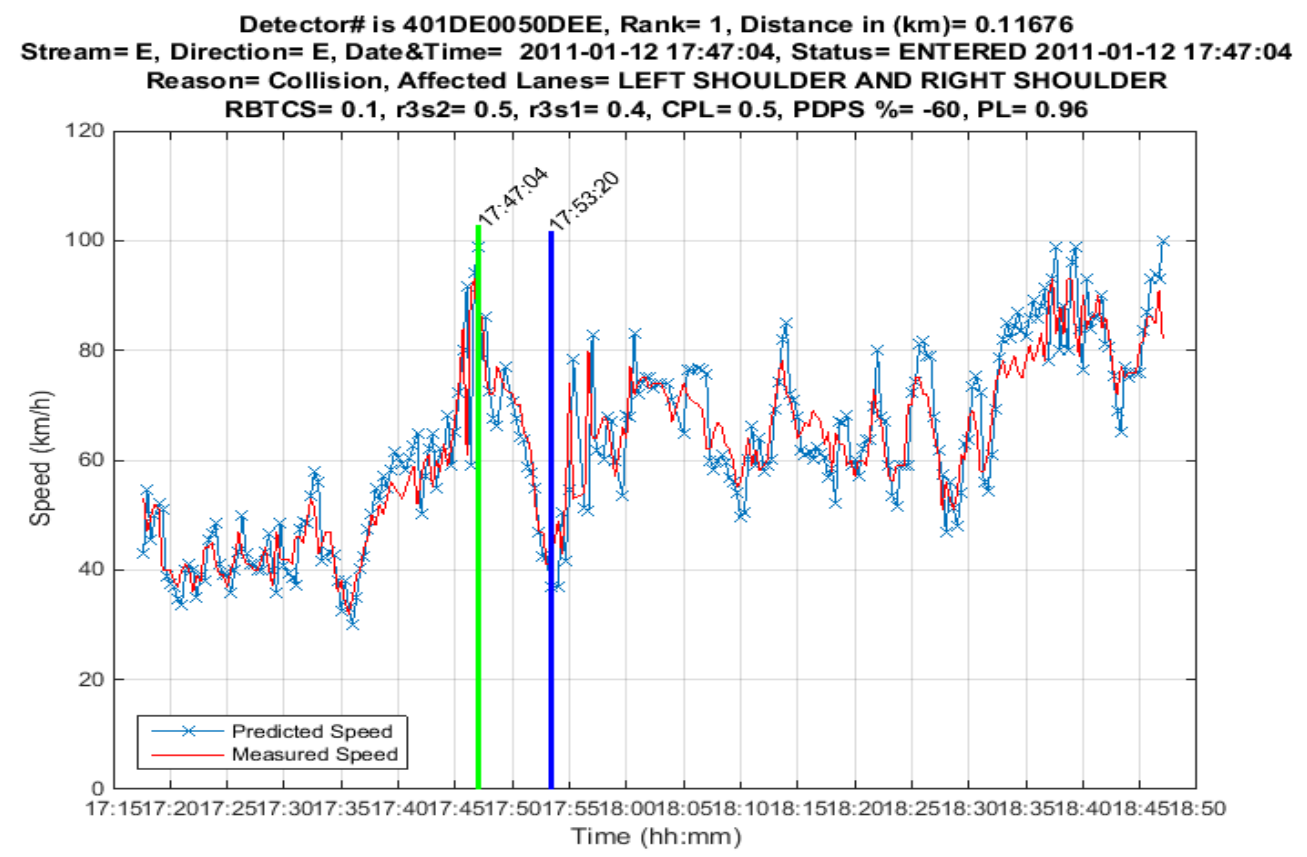

Figure 6.16: Quick speed recovery 
It is observed that there were special circumstances at work that resulted in this unusual incident case. The quick drop in speed after the incident was recorded can be explained due to the short distance between the detector location and the location of the incident. This distance is estimated to be $116 \mathrm{~m}$. The upstream shockwave resulted in a drop in the speed at the detector location.

The quick recovery of speed can also be explained by the site-specific circumstances

of the incident. In this case, only the left and right shoulders were affected and this was the reason for quick recovery of operations back to normal.

In addition to site-specific conditions, another three factors were found to be necessary for incorporation in the developed model in order to improve its ability to detect incidents. These are described in the following sections.

\subsection{Model Calibration/Fine-Tuning}

Before describing the criteria used to calibrate the model, refinements are necessary for obtaining accurate results. These refinements encompass the exclusion of (-) ve predicted speed values, use of the $85^{\text {th }}$ percentile speed, and determining the onset time of speed drop.

\subsubsection{Treating (-) ve Predicted Speed Values}

When applying the computerized version of the developed model using Matlab program, at each time interval the predicted speed for the next period is calculated. In case the speed is declining, and the speed state is moving towards $\mathrm{s}_{1}$, the developed model will predict the speed at the end of the current period that is less than the speed from the previous 
periods. If the prior period speed value is very low, almost close to zero, then the predicted speed will be mathematically calculated and might reach a value that does not exist in real life traffic speed. That is, the value might appear as a negative number in the output. As a result, the predicted speed, in this case, turns out to be a negative number as shown in Figure 6.17.

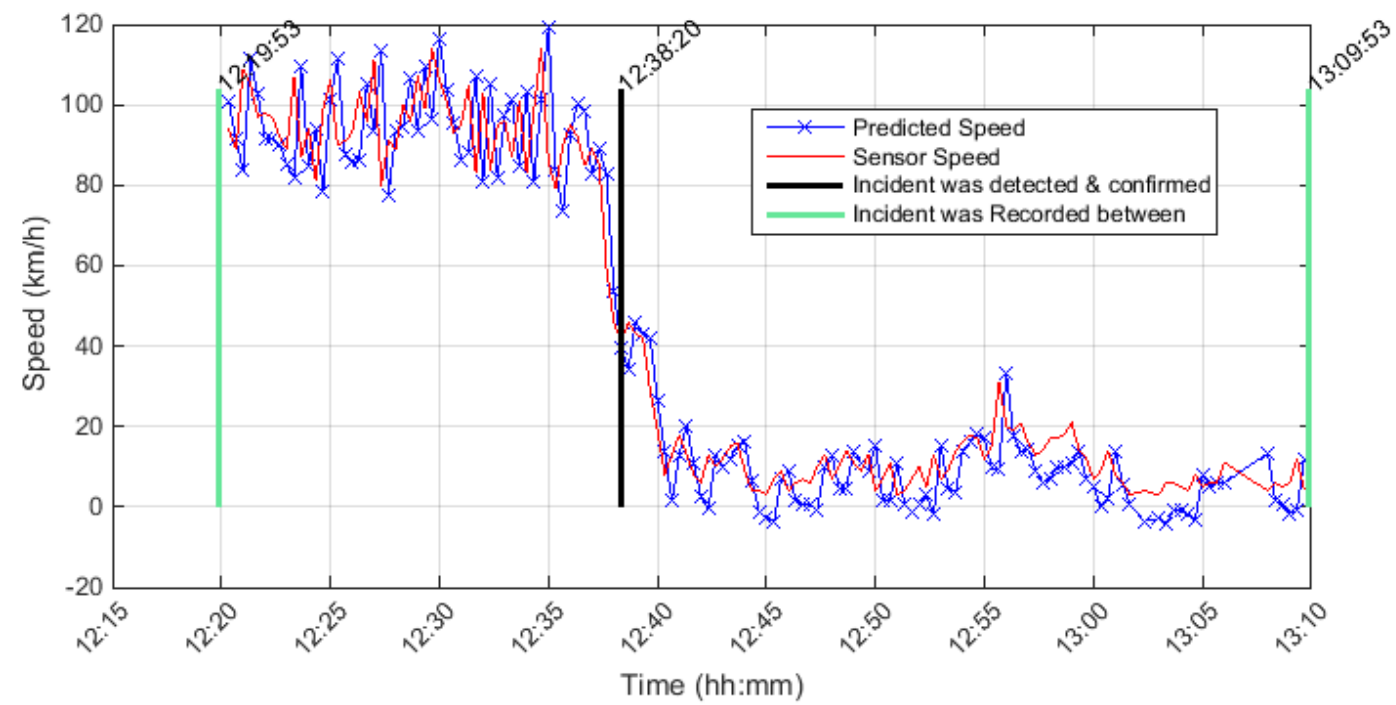

\footnotetext{
Upstream Detector \# is 401DE0330DEC, Detector Rank = 3, Distance from incident location in $(\mathrm{km})=1.5366$ Season $=$ Summer, Stream $=C$, Direction $=E$, Date $\&$ Time $=$ 2011-06-18 12:39:53 Status = ENTERED 2011-06-18 12:39:53, Incident Reason $=$ Collision Affected Lanes $=$ All lanes closed

Conditional Probability level $=0.6$, Cond.Prob. $r 3 s 2=0.2$, Cond.Prob. $r 3 s 1=0.1$, Percent drop in speed $\%=-60$ Max. Posterior Probability level $=0.97$, Absolute range between speed states $=7 \mathrm{~km}$ Incident was detected at 01:40 minutes from drop in speed point. Incident was confirmed after 5 minutes from the detection time
}

Figure 6.17: (-) ve value of the predicted speed

The negative speed value needs to be adjusted to 0 . This step is performed in order to avoid the appearance of any negative speed values in the final output of time/speed charts. Please compare Figure 6.17 and Figure 6.18 in order to appreciate the effect of the 
amendment. Therefore, this refinement states that when the predicted speed turns out to be any value that is less than zero, it is then set to be equal to zero.

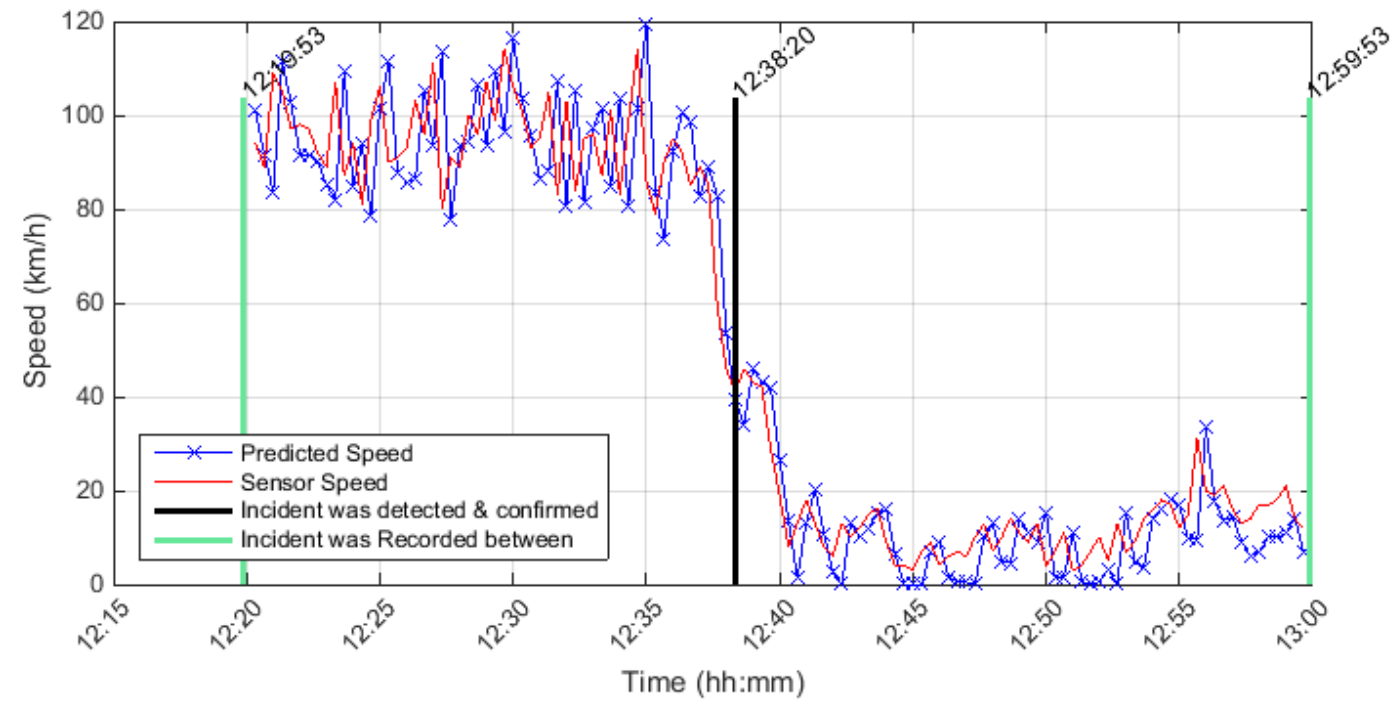

Upstream Detector \# is 401DE0330DEC, Detector Rank = 3, Distance from incident location in $(\mathrm{km})=1.5366$ Season $=$ Summer, Stream $=$ C , Direction $=\mathrm{E}$, Date $\&$ Time $=$ 2011-06-18 12:39:53 Incident Reason $=$ Collision Affected Lanes $=$ All lanes closed

Conditional Probability level $=0.6$, Cond.Prob. $r 3 s 2=0.2$, Cond.Prob. $r 3 s 1=0.1$, Percent drop in speed $\%=-56$ Max. Posterior Probability level $=0.97$, Absolute range between speed states $=7 \mathrm{~km}$

Incident was detected at 01:00 minutes from drop in speed point. Incident was confirmed after 5 minutes from the detection time

Figure 6.18: (0) speed value replacing any (-) ve predicted speed value

\subsubsection{Considering the $85^{\text {th }}$ Percentile Speed}

To satisfy the first condition of the incident detection criteria, the percentage speed drop is to be calculated. The selection of the first speed value (i.e. at the outset of observation) was found to be improper, as it does not represent the speed pattern before the start time. Therefore, the $85^{\text {th }}$ percentile of the speed data within the study time period was considered as a logical choice. 
In order to perform this step, at each time period, the $85^{\text {th }}$ percentile of the predicted speed is calculated for the range of speed values over the study period. When calculating the $85^{\text {th }}$ percentile of the speed data, it is required to consider all the speed data starting from the first reading till one-time interval just prior to the one under consideration (t), where $(t)$ is the time when the current speed is to be investigated for any potential drop in its values. For instance, assuming the speed is to be investigated for a drop at time period of 10:42:20, the $85^{\text {th }}$ percentile speed is calculated based on speed data starting from the beginning of the study time period and ending at 10:42:00.

\subsubsection{Time at the Onset of Speed Drop}

Since the incident recorded time is uncertain, there is a need to define a reference point to relate to the incident detection time. It is a reasonable assumption to consider this reference point as the time when the speed is expected to start dropping to a low-speed value. The criteria used to define this point are illustrated in the flowchart in Figure 6.19, and is explained next.

The criteria can be summarized in the following steps:

1 Find the time at which the incident was detected; at this time the speed state is $\mathrm{s}_{1}$ and the speed value is relatively low.

2 Starting from this point of time and moving the clock one step back, verify the speed state at every time period. When the speed state reaches to $\mathrm{s}_{3}$ then this can be regarded as an indication of a fluctuation in speed point; this point refers to a change in the speed state from $\mathrm{s}_{3}$ to $\mathrm{s}_{1}$. 


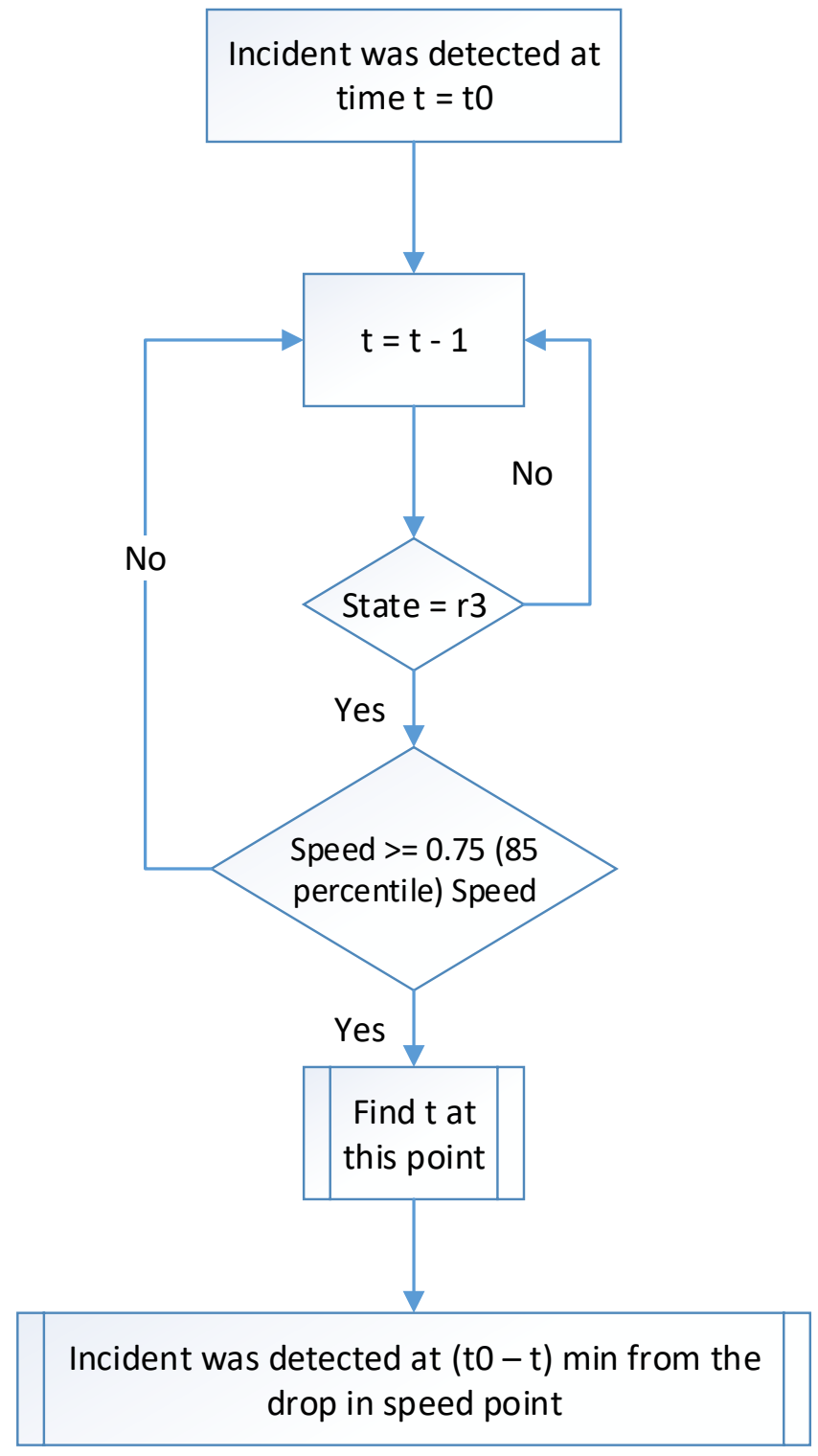

Figure 6.19: Drop in speed point

3 While moving backward the speed is increasing; it is also possible for the speed pattern to change direction, the shift in the speed direction may be towards low speed. Most likely, this scenario occurs at low-speed values where the range between the speed states is small. For that reason, it is required to test the speed value at that particular 
point to assure that the speed state $\mathrm{s}_{3}$ was determined at high-speed value as an expected point where the speed starts to decline.

4 Test the speed value at the same fixed point from the last step regarding its percentage of the $85^{\text {th }}$ percentile speed. A factor is selected to compare this relation; this factor is given as an input to the developed programmable model which will allow better finetuning to the developed model by testing the effect of any selected factor on the accuracy of the detected point. The default selected factor is $75 \%$.

5 The drop in the speed in expected to begin at a point where the speed state is $s_{3}$ and the speed value is bigger than or equal to $75 \%$ of the $85^{\text {th }}$ of the speed values. The $85^{\text {th }}$ percentile is calculated for the speed values that begin from the start time (i.e. $20 \mathrm{~min}$ before the incident recorded time) and continue till one time period before the incident detected time. At this point, the speed can be defined as the last high speed that is recorded when the speed state was $\mathrm{s}_{3}$ and before the speed starts to drop starting from that point.

6 In some cases such as the one shown in Figure 6.20 the reference point does not exist and no point to refer to the incident detection location. A message is provided at the output diagram informing of not detecting any reference point in the case under consideration. The $85^{\text {th }}$ percentile is also needed for other calculations, such as investigating the recovery period, therefore, to meet the speed recovery conditions in such cases, the incident detection point is used as a reference to determine the $85^{\text {th }}$ percentile for this purpose. 


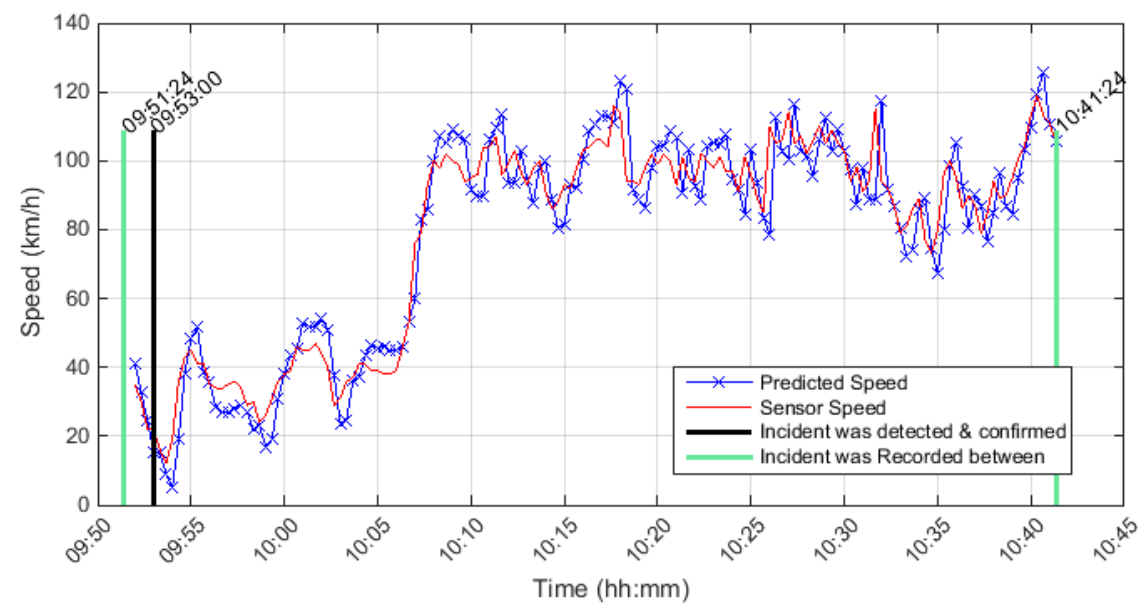

\footnotetext{
Upstream Detector \# is 401DE0010DEC, Detector Rank = 1, Distance from incident location in $(\mathrm{km})=0.35071$ Season $=$ Winter, Stream $=$ C, Direction $=E$, Date $\&$ Time $=2011-02-25$ 10:11:24

Status = UPDATED 2011-02-25 10:23:23, Incident Reason = Collision

Affected Lanes = LEFT SHOULDER AND 2 LEFT LANE(S)

Conditional Probability level $=0.6$, Cond. Prob. $r 3 s 2=0.2$, Cond.Prob. $r 3 s 1=0.1$, Percent drop in speed $\%=-60$

Max. Posterior Probability level $=0.97$, Absolute range between speed states $=7 \mathrm{~km}$

No reference point to any drop in the speed. Incident was confirmed after 5 minutes from the detection time
}

Figure 6.20: No reference point exist

\subsection{Methodological Approach for Developing the Model}

The methodology used to develop the model, and the Matlab program incorporates two essential parts, the first part deals with recognizing the significant detector number to consider for obtaining traffic data. Also, the incident free condition identification is expressed in this part, while the second part is concerned about applying the algorithm for detecting and confirming the incident occurrence.

The first part will be discussed in the following section which defines basic criteria for detector recognition and incident free condition identification. 


\subsubsection{Detector Recognition Module}

The detector is recognized based on three main factors, incident location, the distance between the incident and upstream detector and incident date and time. The procedure of determining the detector number is illustrated in Figure 6.21 while specifying the detector location is illustrated in Figure 6.22. From the two figures, it is shown that the three mentioned factors are controlling the method used to recognize the detector, these factors are described in the following subsections.

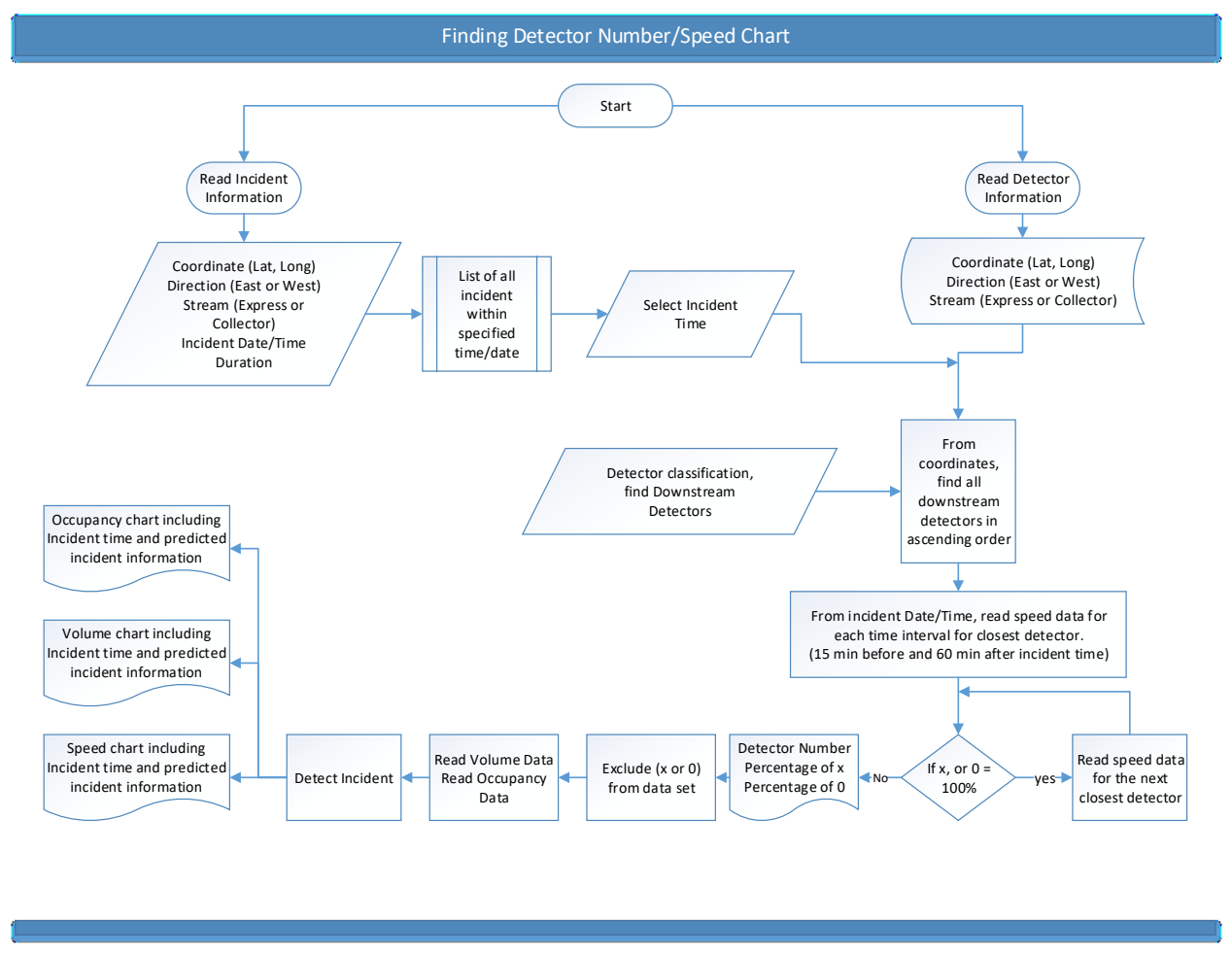

Figure 6.21: Finding detector number 


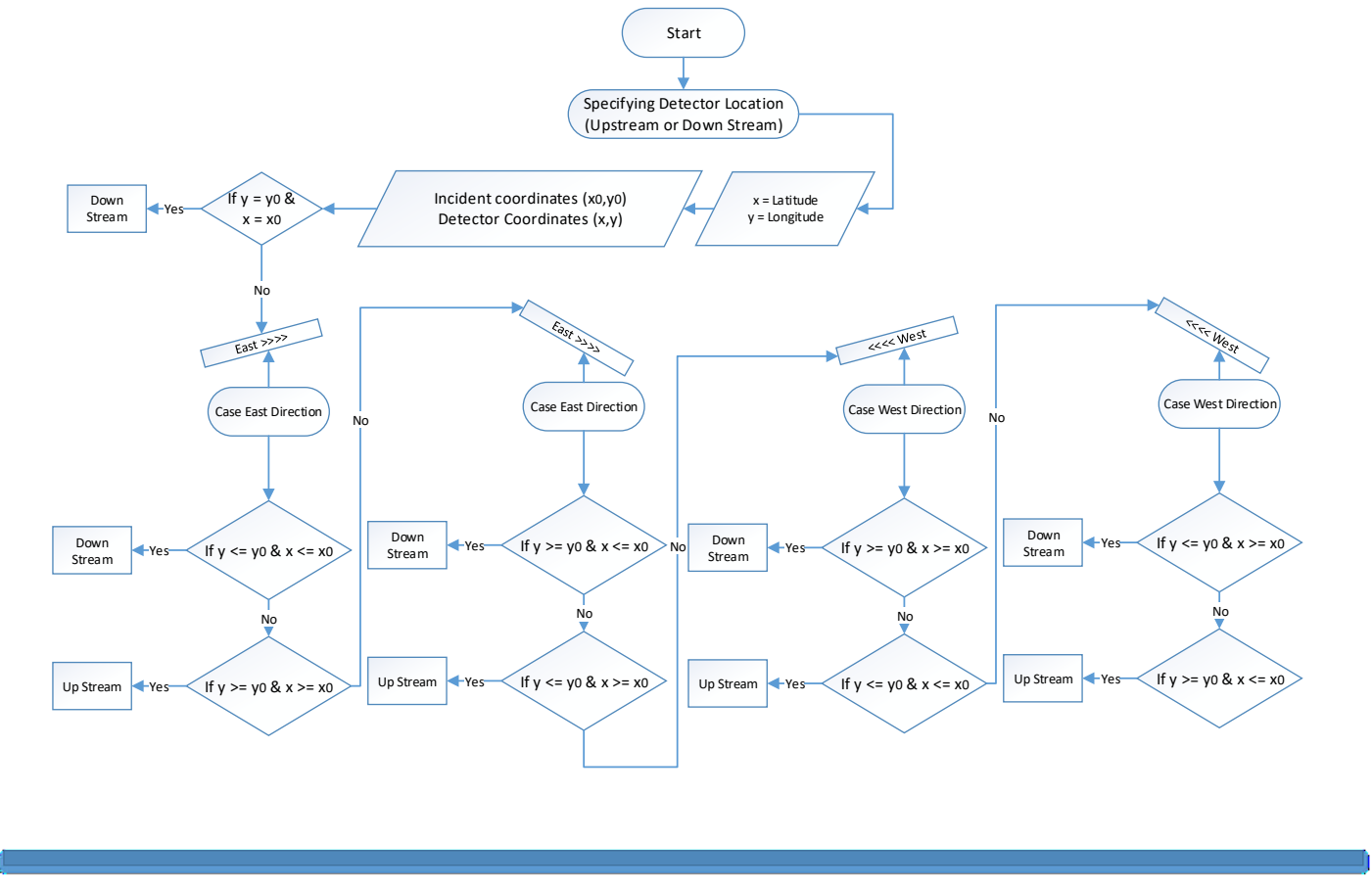

Figure 6.22: Specifying detector location

\subsubsection{Based on incident location}

When an incident occurs in the freeway, the incident is defined by its direction, stream, date, and time. These information items are essential to distinguish each incident from other incidents. Once the incident is selected for the incident detection model applications, its location defined by the latitude and longitude is determined. Following this step is to find the available upstream detectors based on their location information. Since the study corridor of Hwy 401 is a freeway section that extends from the east to the west as shown in Figure 6.23, the relative location of the detector in comparison to the incident location (upstream or downstream) is determined based on the latitude values only. The freeway section selection is based on all available incident data (1452 incident) with consideration to collisions or disabled vehicles. 


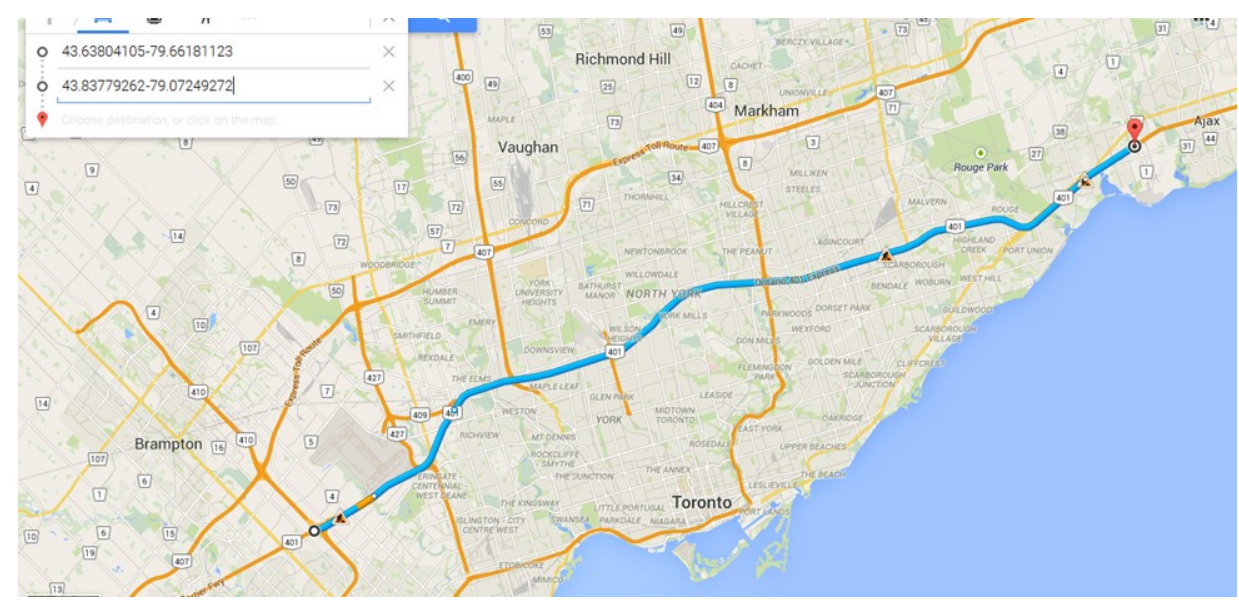

Figure 6.23: Road section from Hwy 401 based on all available incidents data

\subsubsection{Based on incident date and time}

In the developed model the highway direction and stream where it is required to observe and detect incidents is specified as input variables, besides the date and period within which incidents are investigated are also specified. When running the first part of the model, the model examines for possibly recorded incidents within the specified direction, stream, date, and the specified period of time. A list of incidents defined by their time when the incident first took place is provided as shown in Figure 6.24. This information is provided from the screened incident data archive that was obtained from the One ITS database ( $\mathrm{U}$ of $\mathrm{T}$ ) as a core information of all incidents that occurred in each month of the year 2011 . 


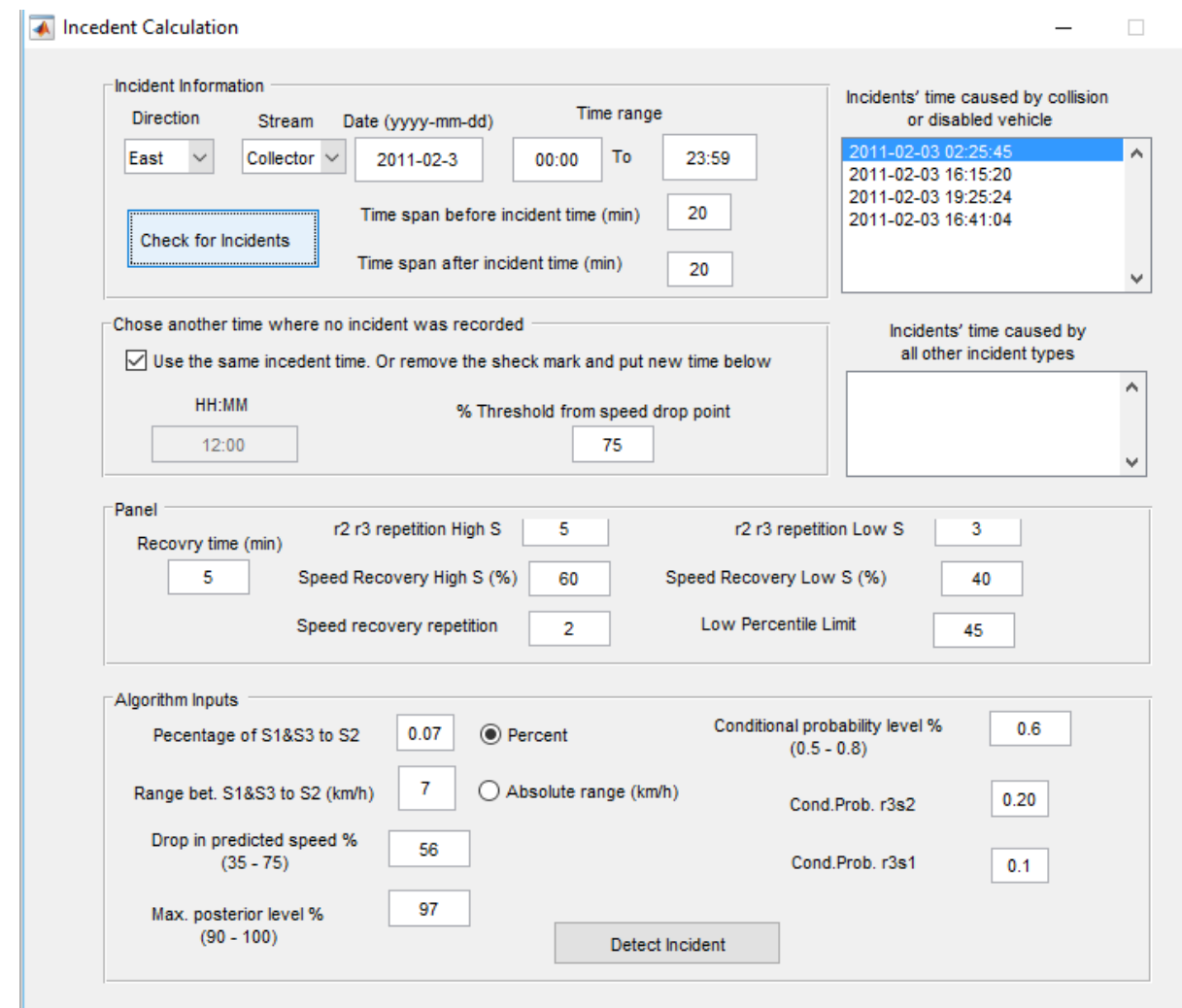

Figure 6.24: List of incidents defined by their time

\subsubsection{Based on distance between detector and incident}

Now, the next step is to select the incident time from the list of available incidents and then run the second part of the model to activate the incident detection process where the model application to detect an incident is applied. After running the incident detection part of the model, the model searches for the closest upstream detector from the incident location as the basic detector to extract traffic information from. Since the study corridor of Hwy 401 is a freeway section that extends from the east to the west, the latitude coordinate was found to be sufficient to determine the distance between the incident location and the upstream detector. 
This detector is taken into consideration for reading traffic information. Although, more than one incident might have been recorded at the same date, however, each incident is recognized as its different location, and therefore, each incident has an impact on its closest upstream detector. Although each incident case has a different upstream detector name that is recognized by reading the traffic information, however, there is still a possibility to have the same detector name for all the incidents that occurred on one specific date. This case is only correct if incidents took place at some location where the closest upstream detector is always the same. In this case, the same detector name will be regarded when reading the traffic data but different incident times will then be applicable.

\subsubsection{Incident Free Condition Selection Criteria}

The process of developing and examining the incident detection model includes examining cases where incident occurrence was confirmed; it also includes examining cases where incident occurrence was not confirmed. Usually, incident time is recorded by the traffic control centre as discussed before in this thesis. On the other hand, all time periods that do not show any incident are regarded as incident free condition periods.

As explained earlier in chapter 3 , incident code 1 refers to an incident by a collision or a disabled vehicle, while incident code 0 refers to an incident that is caused by other reasons.

Since the research domain only focuses on detecting incidents resulting from a collision or disabled vehicle, other types of incidents were not considered under the incident detection model, and incident code 0 were excluded from the incident data selected for the incident detection analysis. 
Since other incident reasons have a certain impact on the upstream traffic flow, therefore time periods that define these incidents is needed to be recognized as well. In order to avoid the effect of other incident types on the traffic follow characteristics when defining new time where it is confirmed that no incident was recorded, here it comes the importunacy to define time periods where all another type of incidents took place. Once the incident free condition periods are defined, it is then possible to examine the developed model in not to detect any incident in cases incident occurrence is not confirmed.

Following this approach, the second part of the incident detection analysis is to select another time where there is no incident recorded. To do that, it is required to choose one of the incidents date/time specified in the first part of the output of the code. This step will define the location of a selected incident and the name of the upstream detector where there is an interest to apply the model. Most important to know about that location is the time periods when any other types of incident occur. After running the first part of the model the time periods became well known.

So, it is important to consider not selecting any time period that is close to these wellknown time periods which refer to the occurrence of any other incident type that occurred on the same date, location, and stream. Considering any other time period which has a considerable gap from the time when it is well known an incident was recorded will be the accurate time that presents the incident free condition.

Incident free condition periods will be identified in the developed model according to the following steps: 
1- A dialog that permits the input of any selected time is incorporated in the developed model; this time is selected based on the criteria defined above.

2- So the selected time will fall in another period different from the presented periods where they show a possibility of any other incident type to occur.

3- When running the first part of the model by identifying the stream, direction, date, and time range, incidents within that range are recognized as shown in Figure 6.25. In the GUI dialog box, there is an option if a new selected time is needed in (hr: mm) with a check box, the default string is always (checked). If unchecked is the selected option, then the updated time is given as new information to be considered when reading the speed, volume, and occupancy data, for instance, time 17:00 hr. is selected as shown in Figure 6.25 .

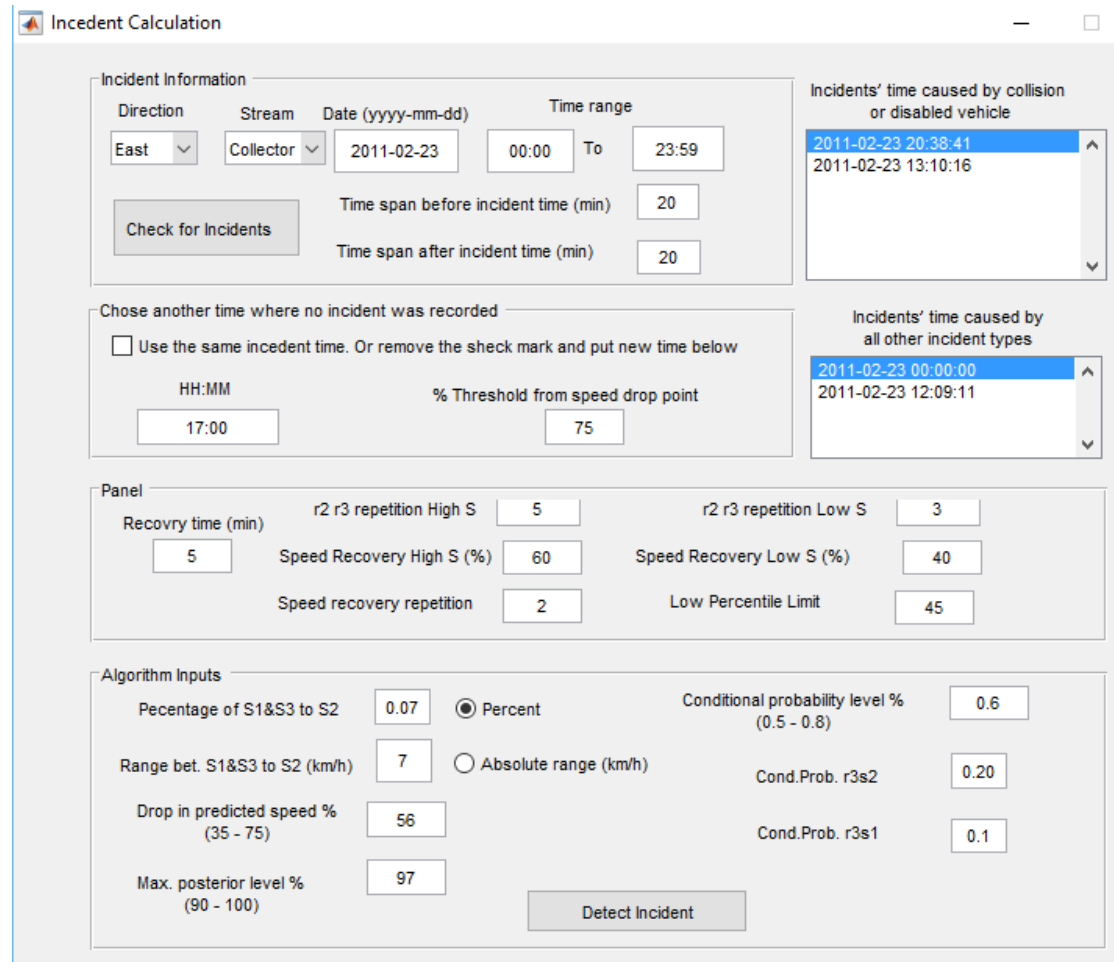

Figure 6.25: List of incidents defined by their time 
4- Using the updated time implies the utilization of the same detector name defined by the necessary basic information that is given in the first part of the model to obtain the speed and other traffic data.

The second part will be discussed in the following section which defines basic criteria for incident detection confirmation.

\subsubsection{Incident Confirmation Criteria}

The incident confirmation criteria were established to further reduce the FAR due to random fluctuations in traffic. Incident confirmation means that an incident alarm is declared only if the incident condition is found to persist.

To confirm whether or not an incident has occurred, another condition must be coupled with both the percent drop in the predicted speed threshold and the high posterior probability level conditions. Accordingly, the algorithm with multiple criteria to detect and confirm the occurrence of an incident was calibrated and developed. The incident should be confirmed by the effect of this additional condition; the condition is set to investigate whether or not the speed recovered to a relatively high-speed value in a short period of time after the drop in speed was detected. If the predicted speed recovers back quickly to a high-speed value according to the specified criteria, then this can be an indication of no incidents and an incident should not be confirmed.

Figure 6.26 illustrates one typical case under consideration, where the incident was not recorded. However, the basic version of the model indicated that an incident occurred at 12:44:00 hr. (the black line in the figure). This indication is considered as a false alarm and the model should be calibrated for better incident detection criteria. 


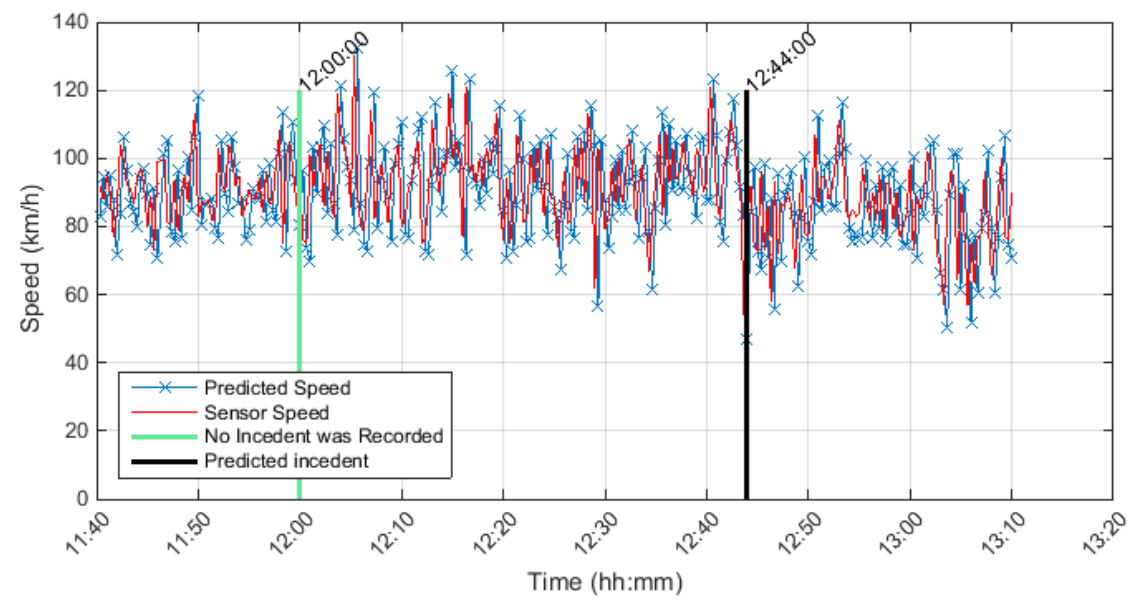

Upstream Detector \# is 401DE0110DEC, Detector Rank = 2, Distance from incident free location in $(\mathrm{km})=1.3122$ Incident free Season $=$ Spring, Stream $=C$, Direction $=E$, Date \& Time $=2011-03-23$ 08:35:24 Selected incident free Date/Time $=2011-03-23$ 12:00:00

Conditional Probability level $=0.6$, Cond.Prob. $r 3 \mathrm{~s} 2=0.2$, Cond. Prob. $r 3 \mathrm{~s} 1=0.1$, Percent drop in speed $\%=-60$

Max. Posterior Probability level $=0.97$, Absolute range between speed states $=7 \mathrm{~km}$

Incident was detected 44:00 minutes AFTER selected incident free time

\section{Figure 6.26: False alarm of an incident}

The indication of an incident as it appears in a vertical black line can be explained as a cause of a rapid drop in the speed at some point which meets the two basic conditions. Since the speed quickly recovered back to normal, then in such a case the model should not detect any incident. Later on in the developed version of the model, the result shows the correct decision of "no incident". This is illustrated in Figure 6.27.

Another typical case that shows false alarm is illustrated in Figure 6.28. The rapid drop in speed occurred at 12:30 hr. resulted in a low percentage in speed drop. The continuous drop in speed also resulted in high posterior level. In this case, the incident was not recorded. However, due to meeting the two basic conditions in the previous incident detection criteria a correct detection of the incident was the logical result at that point. 


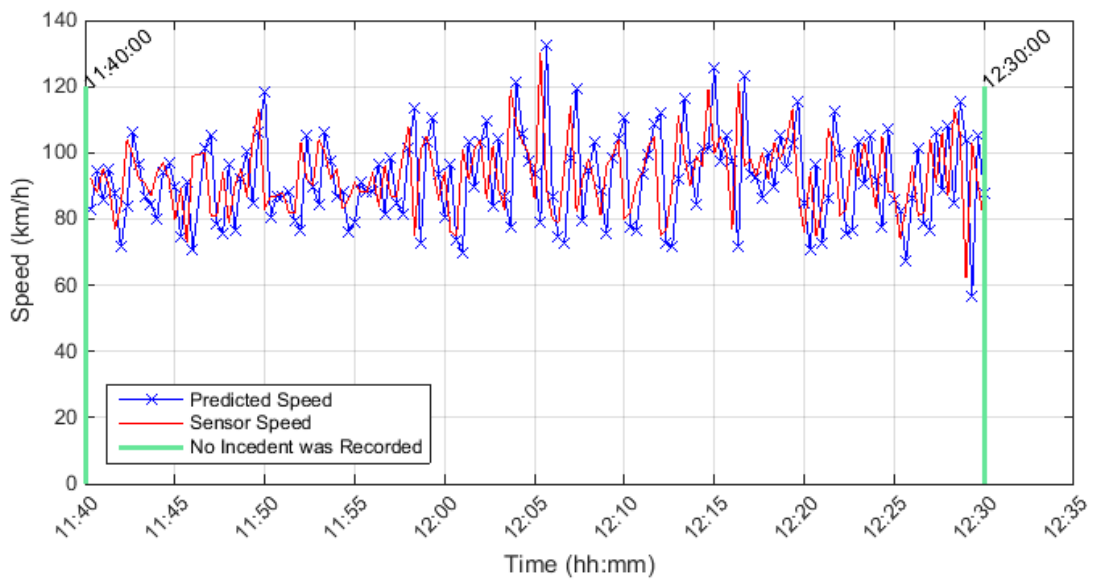

Upstream Detector \# is 401DE0110DEC, Detector Rank $=2$, Distance from incident free location in $(\mathrm{km})=1.3122$ Incident free Season $=$ Spring, Stream $=$ C, Direction $=E$, Date \& Time $=2011-03-23$ 08:35:24 Selected incident free Date/Time $=$ 2011-03-23 12:00:00

Conditional Probability level $=0.6$, Cond. Prob. $r 3 \mathrm{~s} 2=0.2$, Cond. Prob. $\mathrm{r} 3 \mathrm{~s} 1=0.1$, Percent drop in speed $\%=-60$

Max. Posterior Probability level $=0.97$, Absolute range between speed states $=7 \mathrm{~km}$

(No Incident was Detected)

\section{Figure 6.27: Correct detection of no incident}

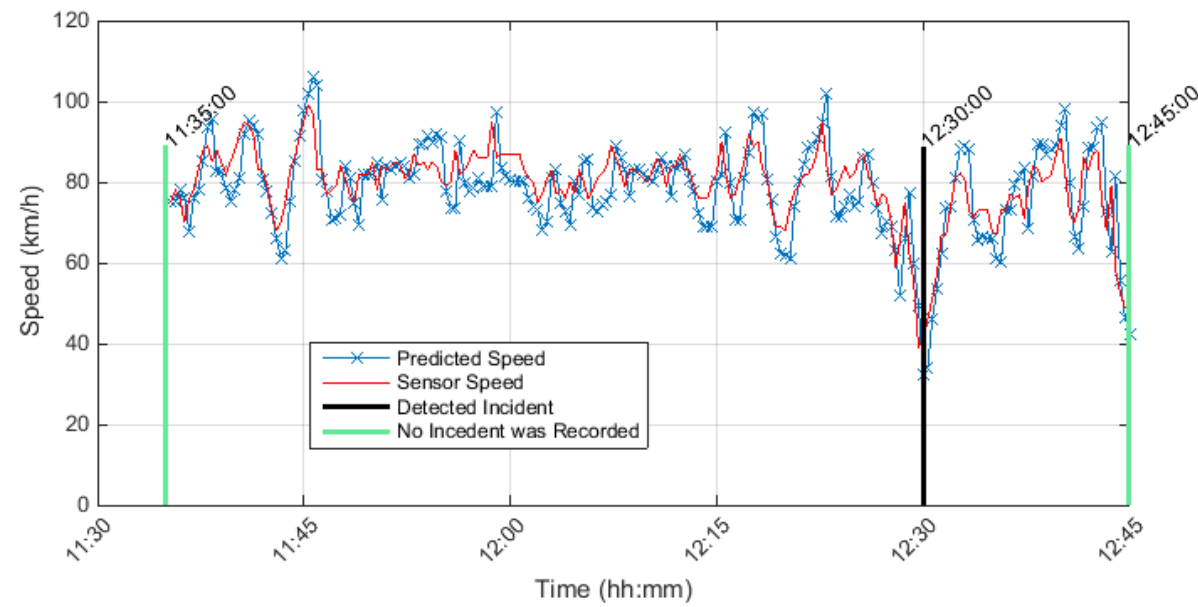

Upstream Detector \# is 401DW0100DEE, Detector Rank = 1, Distance from incident free location in $(\mathrm{km})=0.41981$ Incident free Season $=$ Spring, Stream $=E$, Direction $=E$, Date \& Time $=2011-04-2121: 42: 55$ Selected incident free Date/Time $=2011-04-21$ 12:00:00

Conditional Probability level $=0.6$, Cond. Prob. $r 3 \mathrm{~s} 2=0.2$, Cond.Prob. $r 3 \mathrm{~s} 1=0.1$, Percent drop in speed $\%=-60$

Max. Posterior Probability level $=0.97$, Absolute range between speed states $=7 \mathrm{~km}$

Incident was detected 01:00 minutes AFTER selected incident free time

\section{Figure 6.28: False alarm of an incident}


The developed version of the model which considers the implication of the third condition was capable of eliminating the incident false alarm and the correct decision of no incident was the result as illustrated in Figure 6.29.

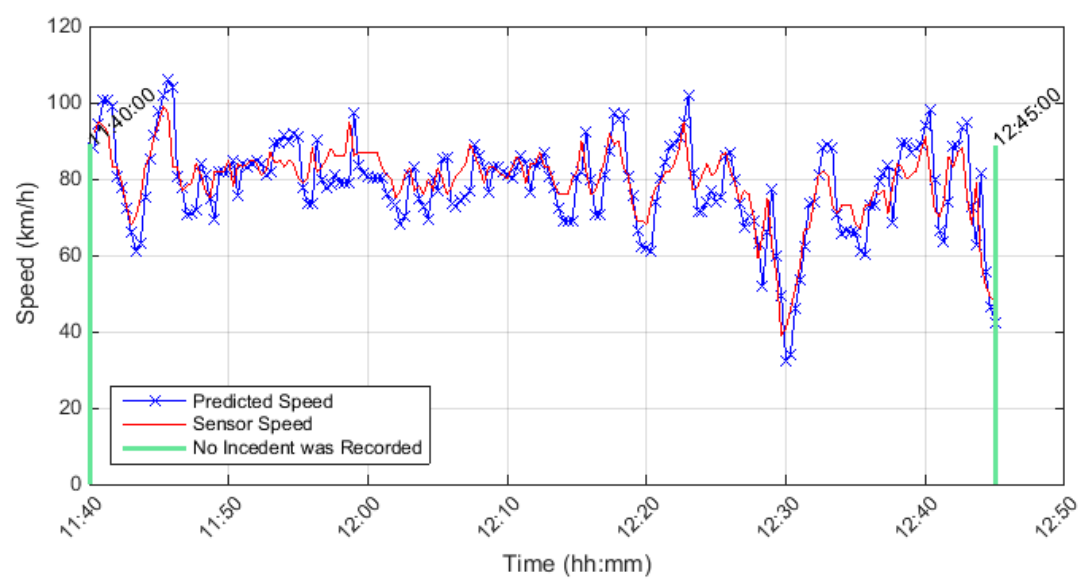

Upstream Detector \# is 401DW0100DEE, Detector Rank = 1, Distance from incident free location in $(\mathrm{km})=0.41981$ Incident free Season $=$ Spring, Stream $=E$, Direction $=E$, Date \& Time $=2011-04-2121: 42: 55$

Selected incident free Date/Time $=2011-04-21$ 12:00:00

Conditional Probability level $=0.6$, Cond.Prob. $r 3 \mathrm{~s} 2=0.2$, Cond. Prob. $\mathrm{r} 3 \mathrm{~s} 1=0.1$, Percent drop in speed $\%=-60$

Max. Posterior Probability level $=0.97$, Absolute range between speed states $=7 \mathrm{~km}$

Drop in speed was detected and No indication of an incident within this period

\section{Figure 6.29: Correct detection of no incident}

Another typical case that shows false alarm is illustrated in Figure 6.30, and the correct decision of "no incident" was the result considering the implication of the third condition, as illustrated in Figure 6.31. 


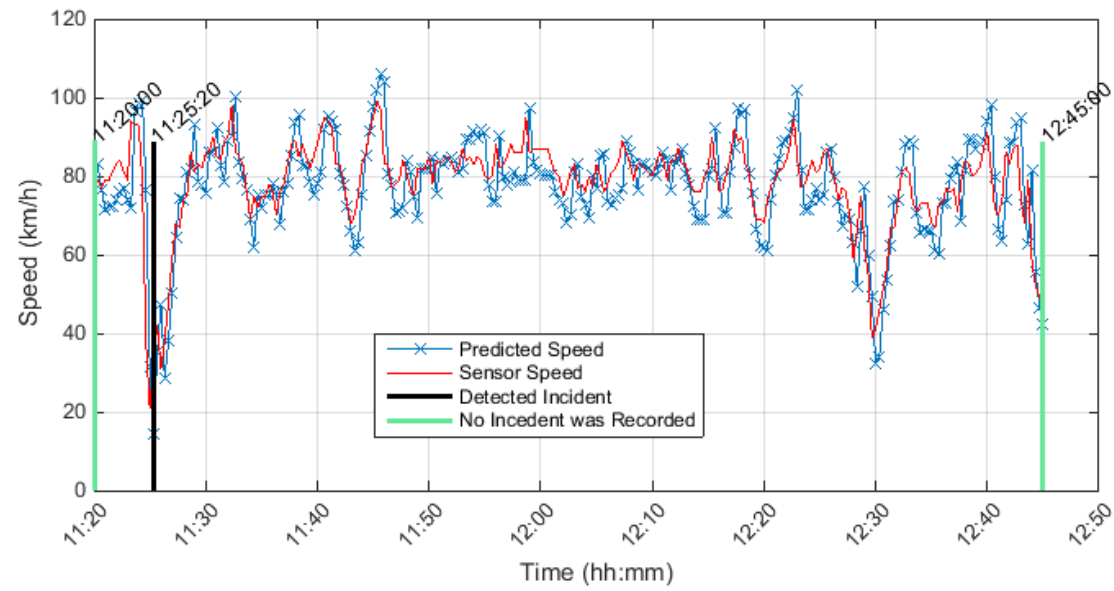

Upstream Detector \# is 401DW0100DEE, Detector Rank = 1, Distance from incident free location in $(\mathrm{km})=0.41981$ Incident free Season $=$ Spring, Stream $=E$, Direction $=E$, Date $\&$ Time $=2011-04-2121: 42: 55$ Selected incident free Date/Time $=2011-04-21$ 12:00:00

Conditional Probability level $=0.6$, Cond.Prob. $r 3 s 2=0.2$, Cond.Prob. $r 3 s 1=0.1$, Percent drop in speed $\%=-60$ Max. Posterior Probability level $=0.97$, Absolute range between speed states $=7 \mathrm{~km}$

Incident was detected 01:00 minutes AFTER selected incident free time

\section{Figure 6.30: False alarm of an incident}

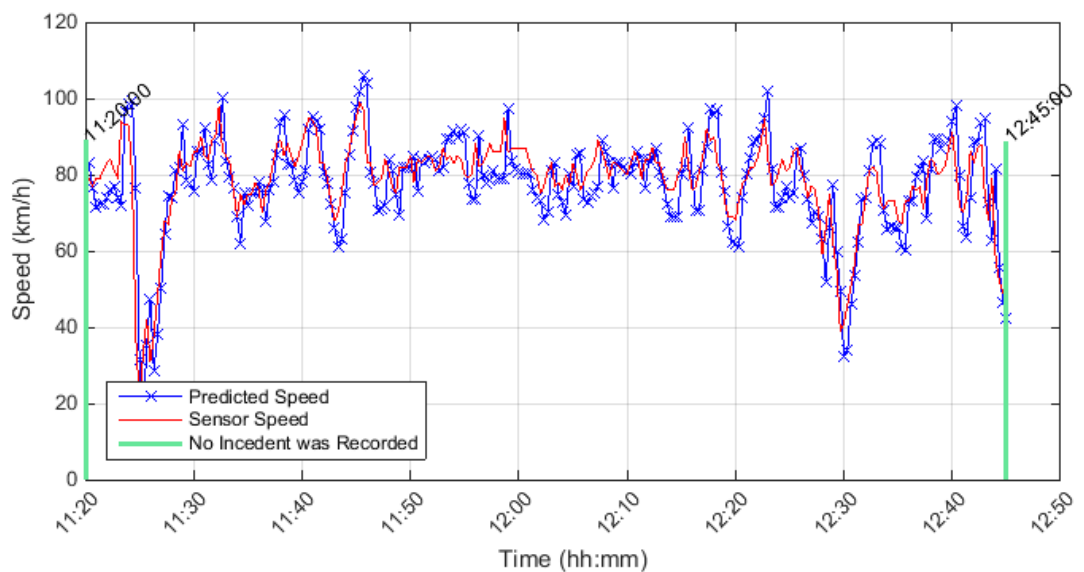

Upstream Detector \# is 401DW0100DEE, Detector Rank = 1, Distance from incident free location in $(\mathrm{km})=0.41981$ Incident free Season $=$ Spring, Stream $=E$, Direction $=E$, Date \& Time $=$ 2011-04-21 21:42:55 Selected incident free Date/Time $=2011-04-21$ 12:00:00

Conditional Probability level $=0.6$, Cond. Prob. $r 3 s 2=0.2$, Cond. Prob. $r 3 s 1=0.1$, Percent drop in speed $\%=-60$ Max. Posterior Probability level $=0.97$, Absolute range between speed states $=7 \mathrm{~km}$

Drop in speed was detected and No indication of an incident within this period

Figure 6.31: Correct detection of no incident 
Although the first condition of the incident detection procedure requires that the percent drop in the predicted speed should meet a specified criterion, but maintaining lowspeed values and assuring that the speed is in a continuous decline will be investigated under the third condition. So, in addition to the two basic conditions (i.e. the posterior probability level and the percent drop in the predicted speed), the third condition is considered as part of the incident detection criteria.

The third condition of the incident detection criteria can be defined as the integration of the predicted speed value and the speed state. This condition requires that the speed value should be in a declining state and should not be in an increasing state. Further, it requires that the speed state should be within the range of the low-speed state $\left(\mathrm{s}_{1}\right)$ for a particular period of time.

The recovery period is to be clarified in the context of the condition for incident confirmation. It is the required time within which the predicted speed will undergo a testing procedure for any potential recovery to a high-speed value. The developed Matlab program will allow one to specify the recovery period in minutes as an input, hence, assisting for a better model calibration.

In order to establish if this new condition is met, speed recovery pattern is to be examined. The number of times the speed repetitively recovers after its first drop, along with the speed value after each speed recovery state, are considered two main factors that need further investigations. Therefore, these two factors were accorded due importance for inclusion in the developed Matlab program.

From the perspective of application, the incident detection confirmation methodology is presented in Figure 6.32. The controlling factors in the flowchart along 
with the sequence of the steps are explained below and several cases are presented for additional clarity.

The methodological steps for the implementation of conditions are noted below.

1- Define the recovery period which is a time period after the incident was detected. A preliminary value of 5 minutes was proposed and was assigned as a default value for this period. This value of 5 minutes was given as an input value to the Matlab program.

2- Within the recovery period, an investigation was conducted on the predicted speed value to examine how frequently it reached to a relatively high-speed value in relation to the $85^{\text {th }}$ percentile speed. This investigation represented the first part of the third condition and is explained in the following two steps:

- Examine the number of times the speed recovers back to a high-speed threshold value which is represented by a speed state reaching $\mathrm{s}_{2}$ or $\mathrm{s}_{3}$. The minimal value for this figure is considered as a factor and is given a default value of (5) when testing high-speed values (when the $85^{\text {th }}$ percentile of the predicted speed is greater than a threshold speed of $45 \mathrm{~km} / \mathrm{h}$ ). This number is given as an input value in the developed Matlab program and termed (repetition for high percentile).

- If the $85^{\text {th }}$ percentile of the predicted speed is less than a low marginal speed of 45 $\mathrm{km} / \mathrm{h}$, then it is considered as a low-speed value. In this case, the threshold number of times the speed recovers back to high speed is decreased by two as a new given input, and a default value of (3) is assigned to it when testing low-speed values. It will also be termed (repetition for low percentile) in the user input interface of the developed Matlab program. 


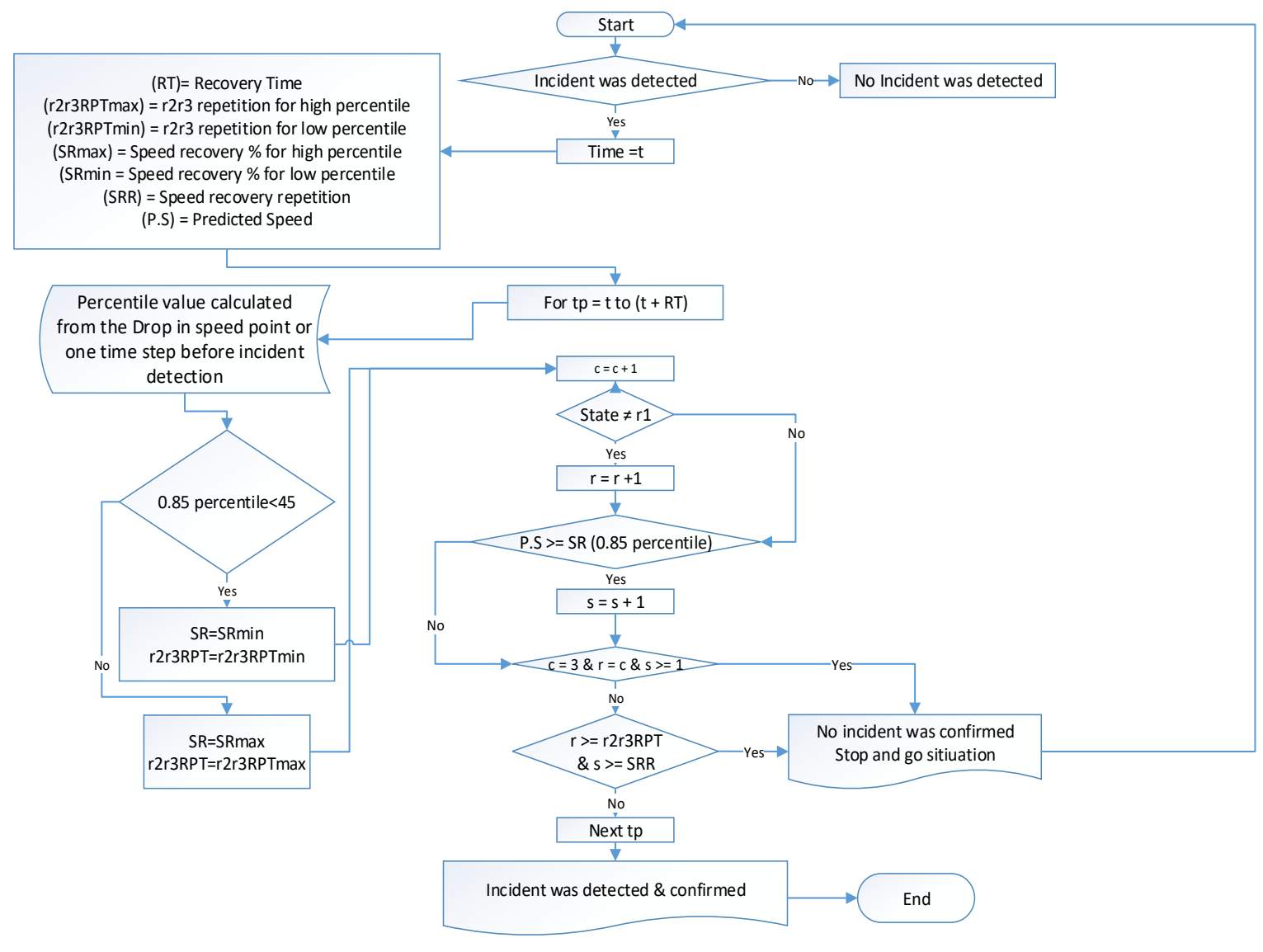

Figure 6.32: Incident detection confirmation procedure

Now, if the number of times the speed recovers back to the threshold speed value never exceeds the specified number of repetition threshold described above within the specified recovery period, then the first part of the third condition is encountered, and the incident is subject to be confirmed after testing the second part of the third condition.

3- The second part of the third condition suggests investigating the predicted speed value during the recovery period to examine its relation to the $85^{\text {th }}$ percentile speed as explained in the following two steps: 
- Examine number of times the predicted speed reaches to a percentage of the $85^{\text {th }}$ percentile of the predicted speed. This percentage is defined by a predetermined threshold level (60\% was proposed as a preferred level for high predicted speed values and $40 \%$ for low predicted speed values). This number of repetitive times is examined with an optimal number given as (2) and is regarded as an input in the Matlab program, termed (Speed recovery repetition).

- To classify high and low-speed level, the $85^{\text {th }}$ percentile of the predicted speed is used as the speed limit under this criteria to define low and high predicted speed levels, where a higher percentile than a threshold speed value of $45 \mathrm{~km} / \mathrm{h}$ is considered as highspeed level and vice versa. The third section of Figure 6.33 shows the arrangements of the different sets of the inputs within the Matlab user interface.

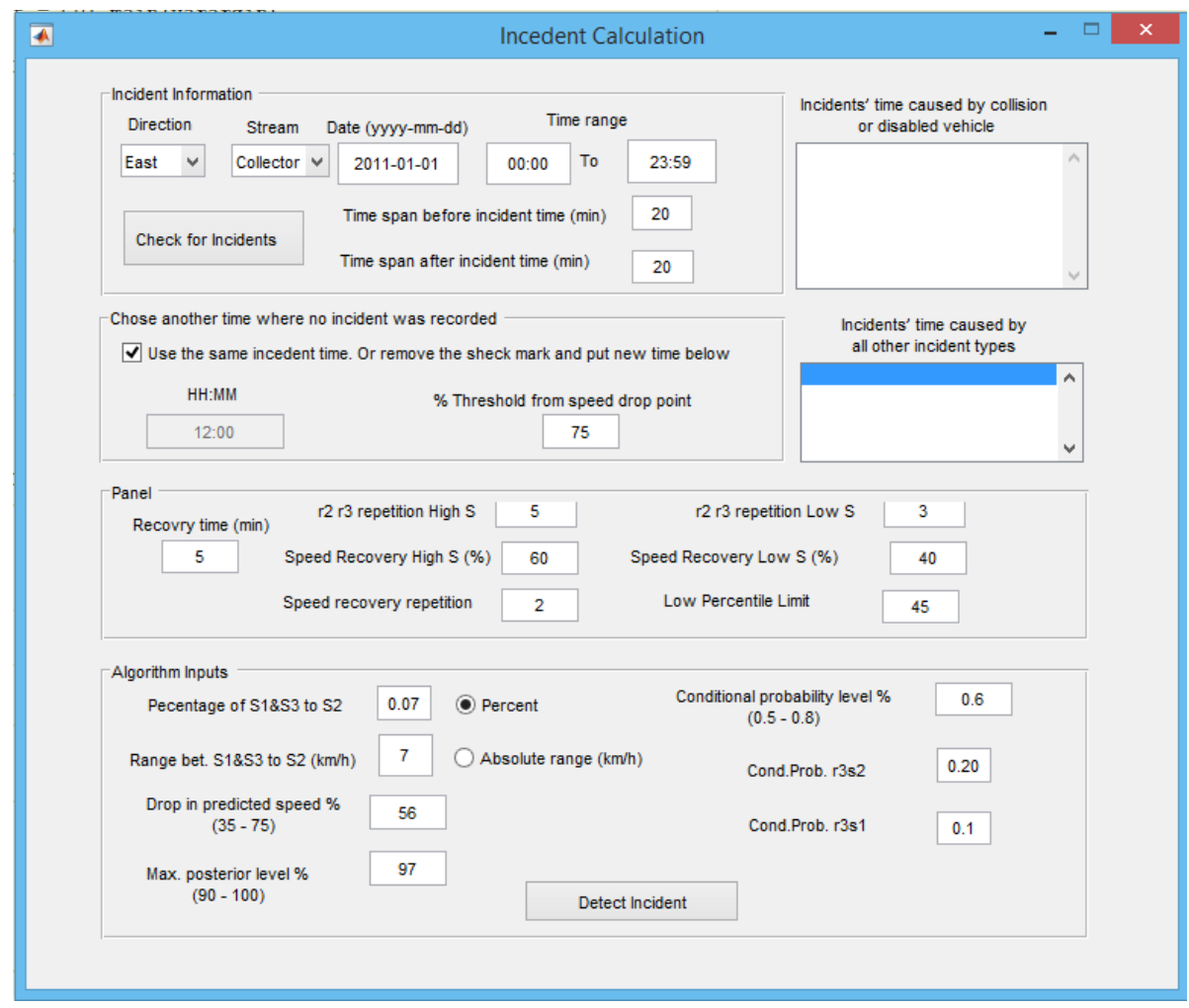

Figure 6.33: Matlab program inputs 
The $85^{\text {th }}$ percentile of the predicted speed is calculated based on the speed values starting from the first time reading until the time when the drop in speed occurs. This is the time that was determined earlier based on calculating the point at which the first drop in speed was observed. In the case that there is no reference point to any decline in the speed, the $85^{\text {th }}$ percentile is determined based on the calculated percentile at one time period before the time when the drop in speed was detected.

If the number of times where the predicted speed value does not exceed the predetermined threshold speed level described above, then the second part of the third condition is encountered, and the incident is subject to be confirmed given that the first part of the third condition is also encountered.

If the third condition was encountered, then, it is most likely that the drop in speed was due to an incident and the detection of the incident is then confirmed. On the other hand, if the third condition does not meet the criteria, then it is a confirmation that the drop in speed was due any other reason such as a stop and go traffic situation caused by high demand, and no incident will be confirmed.

So, after the calibration process is complete, it is expected that the correct detection and confirmation of an incident will only happen if the third condition is encountered along with the basic two conditions of the incident detection criteria.

When applying the calibrated incident detection model, other different scenarios were presented based on whether the incident was detected or not. In the case, the incident was detected more scenarios were also confronted. These scenarios can be explained as the following: 
1- If the drop in speed was detected and no incident was confirmed, then the model will continue to search for the next possible drop in speed within the specified time frame.

2- If the subsequent drop in speed meets the required conditions for detecting a drop in speed, then the speed is tested within the recovery period to either confirm the occurrence of an incident or not. If the incident is confirmed, then a message will be sent confirming the incident detection, if no incident is confirmed another test will be conducted to examine the three conditions in the following time period.

3- The model will continue to test the next time period for any potential incident detection until it reaches the end of the specified time frame limit.

4- In the case, the drop in speed was detected at the end of the time period or even before the end of the time period, so that the whole time span of the recovery period ( 5 minutes) is not applicable as illustrated in Figure 6.34 then the incident should not be confirmed.

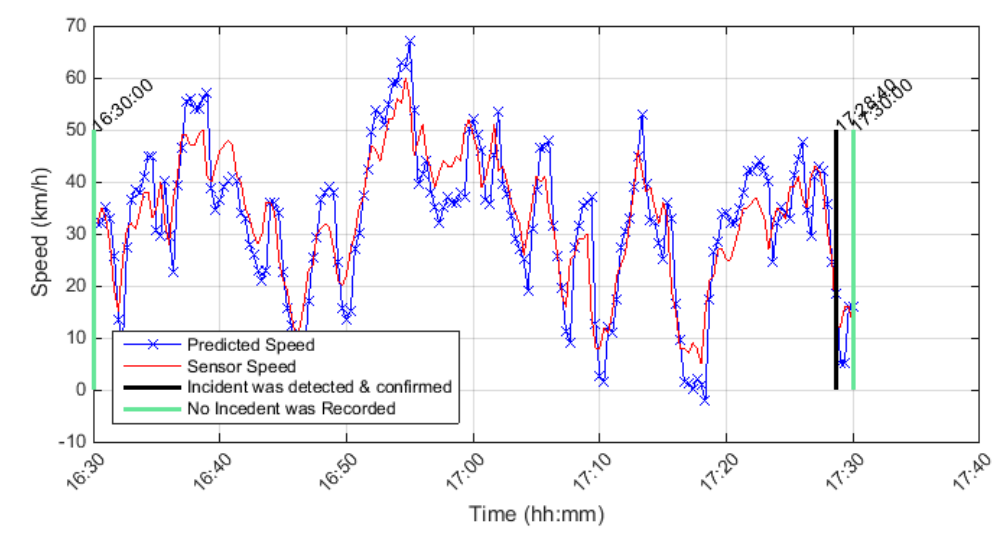

Upstream Detector \# is 401DW0100DEE, Detector Rank = 1, Distance from incident free location in $(\mathrm{km})=0.41981$ Incident free Season $=$ Winter, Stream $=E$, Direction $=E$, Date \& Time $=$ 2011-01-13 13:35:18 Selected incident free Date/Time $=2011-01-13$ 17:00:00

Conditional Probability level $=0.6$, Cond. Prob. $r 3 s 2=0.2$, Cond.Prob. $r 3 s 1=0.1$, Percent drop in speed $\%=-60$

Max. Posterior Probability level $=0.97$, Absolute range between speed states $=7 \mathrm{~km}$

Incident was detected at 02:40 minutes from drop in speed point. Incident was confirmed after 5 minutes from the detection time

Figure 6.34: False alarm of an incident at the end of the time period 
5- Although the results and message indicate that the incident was confirmed after 5 minutes from the detection time, however, it is expected that the incident will not be confirmed if the recovery period is not totally validated. The notice under the figure needs to be amended and the incident indication black line needs to be removed. Also, the legend needs to be corrected. These amendments were applied on the final updated version of the code and in this case, a message is sent notifying that "Recovery period is not enough to confirm the incident, drop in speed was detected and no incident was confirmed," as illustrated in Figure 6.35.
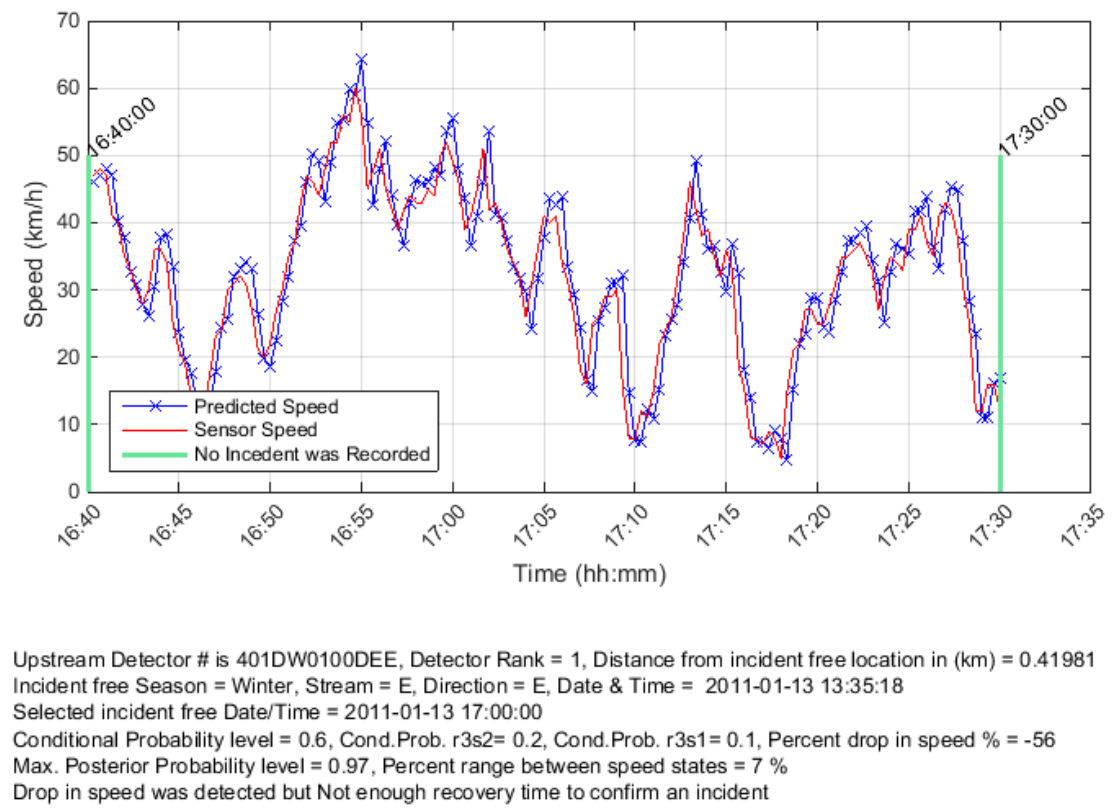

\section{Figure 6.35: Correct detection of no incident}

6- If at the end of the recovery period, no confirmation of any incident happens, another message is presented, as (drop in speed was detected, and no incident was confirmed). 
7- If at the end of the study period, no incident was confirmed, then this is an indication of a drop in speed with no incident persistence as will be displayed in the results "Drop in speed was detected, but no incident was confirmed."

8- On the other hand, if both of the two conditions meet the criteria, then it is a confirmation that the drop in speed was due to an incident.

\subsubsection{Sample Application}

The third condition is applied in several cases. For instance, one typical case is selected to investigate the efficiency and accuracy of the developed model. In this typical case, an incident was recorded between 18:46:22 and 19:51:22 as illustrated in Figure 6.36. The developed model is applied to detect the recorded incident, considering the impact of the third condition. As a preliminary investigation on the factors described in the above subsections, different factors were considered in this application.

When applying the preliminary version of the Matlab computer program, the incident was detected at 8:56:20 as shown in Figure 6.36. When applying the third condition which includes validating the recovery period, the incident was detected at another point which could be more reasonable for an indication of an incident as the speed shows a continuous declining trend. The incident was detected at 19:32:40 and was confirmed as illustrated in Figure 6.37 . 


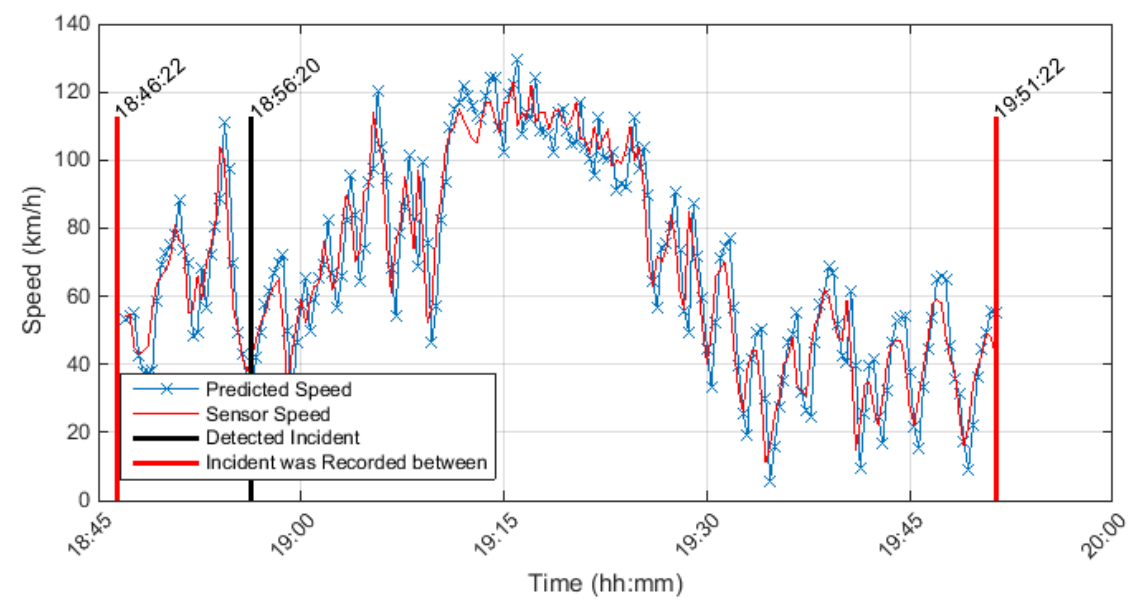

Upstream Detector \# is 401DW0100DEE, Detector Rank = 1, Distance from incident location in $(\mathrm{km})=0.41981$ Season $=$ Spring, Stream $=E$, Direction $=E$, Date $\&$ Time $=2011-04-24$ 19:06:22 Status $=$ ENTERED 2011-04-24 19:06:22, Incident Reason $=$ Collision Affected Lanes $=1$ LEFT LANE(S)

Conditional Probability level $=0.6$, Cond.Prob. $\mathrm{r} 3 \mathrm{~s} 2=0.2$, Cond.Prob. $\mathrm{r} 3 \mathrm{~s} 1=0.1$, Percent drop in speed $\%=-60$ Max. Posterior Probability level $=0.97$, Absolute range between speed states $=7 \mathrm{~km}$

Incident was detected at 02:00 minutes from drop in speed point

Figure 6.36: Typical case for false alarm of an incident

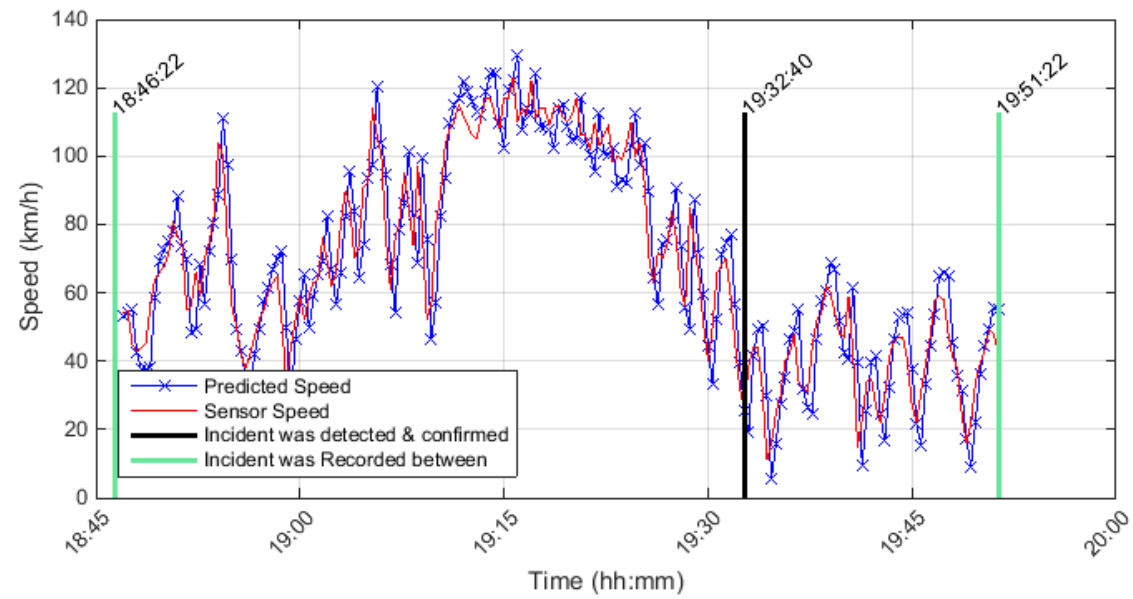

Upstream Detector \# is 401DW0100DEE, Detector Rank $=1$, Distance from incident location in $(\mathrm{km})=0.41981$ Season $=$ Spring, Stream $=E$, Direction $=E$, Date $\&$ Time $=$ 2011-04-24 19:06:22 Status $=$ ENTERED 2011-04-24 19:06:22, Incident Reason = Collision

Affected Lanes $=1 \mathrm{LEFT}$ LANE $(\mathrm{S})$

Conditional Probability level $=0.6$, Cond.Prob. $r 3 s 2=0.2$, Cond. Prob. $r 3 s 1=0.1$, Percent drop in speed $\%=-60$

Max. Posterior Probability level $=0.97$, Absolute range between speed states $=7 \mathrm{~km}$

Incident was detected at 03:40 minutes from drop in speed point. Incident was confirmed after 5 minutes from the detection time

Figure 6.37: Typical case for correct incident detection 
The criteria for applying the incident detection conditions including the third condition for confirming the detected incident is explained in the following steps.

1- After validating the incident detection conditions, the first incident was detected at 18:56:20 and the following investigations are applied.

a- The predicted speed in the following $5 \mathrm{~min}$ of the incident detection time (recovery period) were verified with the $85^{\text {th }}$ percentile speed at the point of the drop in speed.

b- The point of the drop in speed is found at 18:54:20, at this time the first speed state $r 3$ was detected, also the predicted speed at this point is found to be $110.86 \mathrm{~km} / \mathrm{h}$. Since the $85^{\text {th }}$ percentile is found at one time period before the incident detection time of a value of $83.8 \mathrm{~km} / \mathrm{h}$, then the $110.86 \mathrm{~km} / \mathrm{h}$ speed value is considered higher than $\left(0.75^{*}\right.$ 83.83) and therefore, the drop in speed point is determined at 18:54:20.

c- Since the $85^{\text {th }}$ percentile of the speed at the drop in speed point which is $78.45 \mathrm{~km} / \mathrm{h}$ higher than $45 \mathrm{~km} / \mathrm{h}$, so the used percentage for the next step is proposed to be high. A percentage of $65 \%$ in this application will be counted instead of the typical $60 \%$ as mentioned earlier.

d- The $85^{\text {th }}$ percentile speed at the drop in speed point was found to be $78.45 \mathrm{~km} / \mathrm{h}$. Considering the proposed percentage of $65 \%$ of the $85^{\text {th }}$ percentile speed is used to compare with the predicted speed in order to test the requirement of the third condition, this value turns out to be $(0.65 * 78.45=51 \mathrm{~km} / \mathrm{h})$. It is found that the predicted speed reaches to a high-speed value higher than $51 \mathrm{~km} / \mathrm{h}$ for more than the predetermined threshold of repetitive times of ( 2 times) and the speed state continues to be $\mathrm{r} 3$ for more than the threshold repetitive time of (5 times). 
2- Since the two parts of the third condition are not encountered, then the incident is not confirmed.

3- Other incidents were also detected at the following time intervals such as 19:30:00 and 19:30:20, but were not confirmed due to not complying with incident confirmation requirements from the third condition.

4- Within the same study period and moving the clock forward, at the time 19:32:40 another incident was detected and the following investigations are applied:

a- Find the point of drop in speed by first calculating the $85^{\text {th }}$ percentile at one time period prior to the incident detection time $=112.03 \mathrm{~km} / \mathrm{h}$.

b- Apply the $75 \%$ of the obtained percentile $(0.75 * 112.30=84 \mathrm{~km} / \mathrm{h})$.

c- At time 19:29:00 the speed state was $\mathrm{r}_{3}$ and the predicted speed was $87.34 \mathrm{~km} / \mathrm{h}$ which is higher than $84 \mathrm{~km} / \mathrm{h}$, so this time was regarded as the point of drop in speed and the $85^{\text {th }}$ percentile at this point of time is found to be $112.34 \mathrm{~km} / \mathrm{h}$.

- For the speed recovery test, after the time of the incident detection, the predicted speed will be verified whether it exceeds the value of $0.65 *(112.34 \mathrm{~km} / \mathrm{h})=72.8 \mathrm{~km} / \mathrm{h}$. This is applicable for the following $5 \mathrm{~min}$ period after the time when the incident was first detected.

- After conducting the aforementioned check above within the specified recovery period, it is noticed that none of the following predicted speed values exceed $72.8 \mathrm{~km} / \mathrm{h}$ as indicated in Figure 6.37. This satisfies the second condition requirements for confirming the incident occurrence.

- Moreover, the first part of the third condition of not having five time periods, demonstrating $\mathrm{r} 3$ or $\mathrm{r} 2$ state within the recovery period is encountered. So it can be 
concluded that the third condition is encountered and therefore, a correct indication of the incident is confirmed.

\subsection{Computer Program Development (Matlab Language)}

The developed model was coded in Matlab computer programming language (Matrix Laboratory), version 2014b. The computer program consists of several parts, called "functions" by Matlab. Each function contains several sub-functions to make the whole program work efficiently. The development of the computer program, its fundamental version, and the developed versions are defined in section 6.4.1. The source codes for each can be seen in Appendix B of this thesis. The basic methodology including the first two fundamental conditions are included in the first version of the Matlab program; the calibration process continues to evolve through the updated versions of the program. Four other versions are generated from the first version; the last version consists of all the conditions and is the one regarded for validating the developed model.

\subsubsection{Matlab Script Development}

\section{Version-1}

The preliminary version of the Matlab script to detect incident utilizing the proposed algorithm considers applying the basic two conditions of the incident detection methodology. Although the incident recorded time is uncertain, this version presents the recorded time of the incident, it also presents the time span from the incident recorded time to the incident detection time; this time span is shown in (hh: $\mathrm{mm}$ ). The reference marginal speed in this version was the maximum speed, the $85^{\text {th }}$ percentile of the detected speed was 
not included yet. As a preliminary version of the Matlab program, it does not include the third condition that corresponds to the incident confirmation.

This version is used as a stepping stone in the calibration process of the model, the fine-tuning and the inclusion of the third condition was derived from this version.

\section{Version-2}

Since the incident recorded time is uncertain, therefore the incident recorded time was eliminated in this version and replaced by two-time boundaries. These boundaries describe the time frame within which the incident is expected to be recorded. The first boundary is set to be 20 minutes before the incident recorded time, and the second boundary is set to be 20 minutes after the incident recorded time.

When the incident is detected and confirmed, a reference point is included in the second version to express the elapsed time from the point where speed starts to drop until the time when the incident was detected; this time can be considered as the detection time. The onset of dropping speed was defined as the first highest speed before the incident was detected along with high-speed state at $\mathrm{s}_{3}$. The criteria were then amended to include the speed state s2 as well.

In this version the $85^{\text {th }}$ percentile was incorporated as a reference to find the percentage of the drop in speed; it has replaced the maximum speed selection as denoted in the first version. Therefore, the second condition that meets the incident detection criterion was amended; the drop in speed should be examined to meet a percentage of the ( 0.85 percentile speed) instead of the maximum speed. These amendments are expected to have a significant impact on the incident detection criteria; reasonable results are obtained 
after testing this version. The 85 th percentile is calculated for a range of the speed values that start from the first point of the time period under consideration and continues until one time period before the time when the current speed is to be tested for its percentage drop.

This version does not include the third additional condition that investigates the speed recovery test; this condition is incorporated in the third version as part of the calibration process.

\section{Version-3}

In addition to the developed methodology described in version two, this version includes the third condition of the general methodology which focuses on investigating whether the speed has recovered to a high-speed value in a short period of time or the speed maintain a low level after its rapid drop. If this condition is encountered, then, it is confirmed that the drop in speed was due to an incident. On the other hand, if the third condition is not encountered then it is interpreted as any other reason that causes the drop in speed such as a stop \& go situation due to excessive demand, and no incident will be detected.

In the same version, a second criterion to define the ranges of the speed values at each speed state was redefined. The criterion defines an absolute range between speed values at state $s_{1}$ and $s_{3}$ in relation to $s_{2}$; this criterion was included to examine its effect on the speed pattern in comparison to selecting a percentage speed that is defined between the same speed states. This absolute range is given as an input to the Matlab program. 


\section{Version-4}

As part of the calibration process, in addition to version three, this version incorporate the whole principle of the third condition. If at any time period the basic conditions were met, and the drop in speed was detected along with the incident occurrence is not confirmed, then a further investigation is conducted during the study time period for the next potential drop in the speed. Which is a function of the incident recorded time as it might meet the criteria of an occurrence of an incident.

If the second drop in speed meets the required conditions for detecting a drop in speed, then the speed pattern is tested within the recovery period to either confirm the occurrence of an incident or not. If the incident is confirmed, then the message will be delivered confirming the incident occurrence; if no incident is confirmed another test will be conducted, and so on.

\section{Version-5 (Final Version)}

In this version, another factor is added to calibrate the incident confirmation criterion according to the third condition. This factor examines the level of the 85 th percentile of the speed at the point of drop in speed. Accordingly, the number of times the speed recovers back to high speed is decreased by two as a new given input and a default value of (3) are assigned to it.

This final version of the developed Matlab program includes all the criteria mentioned earlier that pertain to the incident detection methodology. 


\subsubsection{Matlab Functions}

The main Matlab program functions are described here briefly. The detailed scripts of the Matlab program that includes all the functions are presented in Appendix B.

\section{MATLAB Function': GUI "Presented in Appendix B.1"}

This feature is considered the primary function and is used to call all other functions and return results to the output files and figures. It passes the inputs defined in the detector information file, incident data file, and the speed-volume-occupancy file that is used by other functions within the incident detection process. Outputs from this function pertaining to speed, occupancy and volume are returned to output folders/files and are plotted in figures.

\section{MATLAB Function': find_sensor_number "Presented in Appendix B.2"}

The main purpose of this function is to find the closest upstream detector from the incident location, this closest detector will be subject to further consideration. Traffic speed data will be obtained from sensors that pertain to this detector. The function serves in selecting the accurate direction and stream where both the incident and the detector are located. It then computes the distance between the incident location and all other detectors' locations. Detectors are then sorted in an ascending order upstream of the incident location based on the computed difference between the latitude of both the incident and the detector coordinates. The latitude coordinate computation shows more accurate measurements, and the closest upstream detector is then determined. The ascending order of downstream 
detectors was also determined by this function, and the numbers of both upstream and downstream detectors are found in ascending order.

\section{MATLAB Function': Writing Data into csv Files "Presented in Appendix B.3"}

This function is used to write the speed database, including speed values into csv files.

\section{MATLAB Function': Reading_data_and_output "Presented in Appendix B.4"}

This function is used to read the speed database, including speed values, date, and time for all obtained data that corresponds to an incident location and direction. The data here includes faulty values of $(0$ and $x)$. It then uses the incident date and time as a reference to generate an index. This index is then used to allocate the date from the speed data file where the speed is of interest, the time boundaries before and after the incident recorded time is also determined using this function. Simply stated, the function is used to read the speed data that corresponds to the same incident time considering the time before and after the incident as specified in the input criteria.

\section{MATLAB Function': extract_the_speed_values "Presented in Appendix B.5"}

This function is used to exclude false data from the speed data file, such as speed data offered as 0 or $\mathrm{x}$ in the speed data file. It then computes the percentage of the cells containing this type of false data. This percentage will then be presented to the Matlab user interface, which will then give the ability to seek the next detector data in the case this percentage is relatively high. 


\section{MATLAB Function': calculate_plot_predicted_speed 'Presented in Appendix}

B.6"

This function is used to apply the incident detection methodology concepts to computing the maximum predicted speed, $85^{\text {th }}$ percentile, point of the first drop in the speed and the percent drop in its value at each time period. It is also used to compute the maximum posteriors' level at every iteration. The main two conditions of the incident detection methodology are incorporated in this function. All messages that are presented in the final output chart pertaining to the final decision of the incident detection are generated in this function.

\section{MATLAB Function': Incident_Confirmation "Presented in Appendix B.7"}

This function is used to apply the criteria defined in the third condition within the incident confirmation process. It is necessary that the incident is detected before this function is applied, as the confirmation processes is an advanced stage after detecting any potential of an incident occurrence.

\section{MATLAB Function': plot_the_results "Presented in Appendix B.8"}

This function is used to plot the speed results, including both the detected speed and the measured speed through the detector; volume and occupancy are also plotted accordingly. Each parameter is plotted on a single chart against time period; the chart also includes information pertaining to the incidents and detectors. 


\section{MATLAB Function': save_output "Presented in Appendix B.9"}

This function is used to store speed data, volume, occupancy, and time that has been read from three separate files to a single one output file called "Volume-SpeedOccupancy." This data is stored for any further investigations and checks.

\section{MATLAB Function': save_predicted_info_output "Presented in Appendix B.10"}

This function is used to store all the results pertaining to the incident detection procedure to a one single initialization file called 'Results_predicted.' These results are stored in a tabulation format; they include the different parameters used to compute the predicted speed such as prior probabilities, posterior probabilities, expected speed, ...etc., the speed state and the incident trigger alarm is also presented in this file. At each time period, variables that correspond to each iteration are listed, the decimal format for each variable is adjusted by this function. Eventually, at the end of the analysis course, if the incident is detected the trigger will be activated and a (Yes) is displayed in the output table.

\subsubsection{User Interface GUI}

The use of user interface enables automatic assignment of the required factors and parameters as illustrated in Figure 6.38. Several input panels were designed to form the whole shape of the main user interface panel; these panels are discussed from top to bottom in the following subsections. 


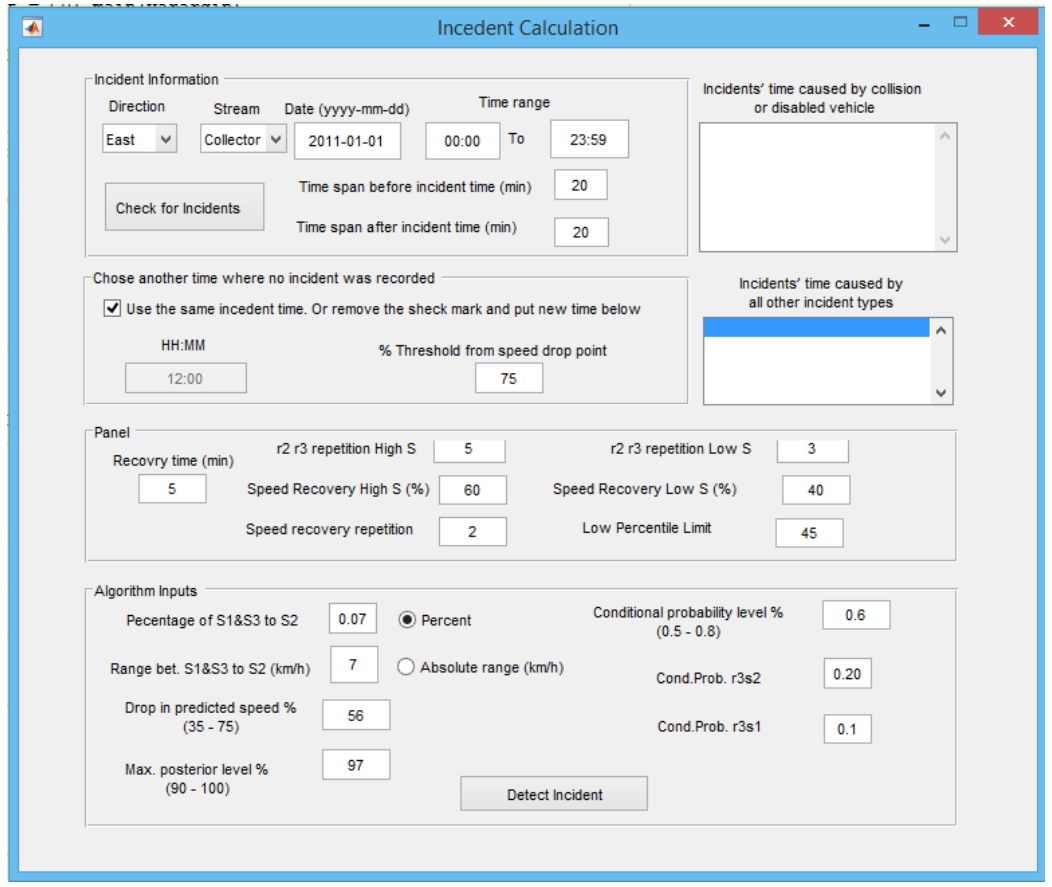

Figure 6.38: User interface GUI

\subsubsection{Input Features}

\section{First upper panel (Incident Information)}

- Drop menu for selecting the direction (East or West)

- Drop menu for selecting the stream (Collector or Express)

- Selection of incident date in (yyyy-mm-dd) format and time range in (mm: ss) format, the selection criteria is based on searching for available incident according to the incident database.

- Selection of the time boundaries defining the limits of the incident recorded time.

- A right side panel that includes sorted incidents caused only by collision or disabled vehicle according to the specified direction, stream, date, and time. The panel is called "Incidents' time caused by collision or disabled vehicle."

- "Check for incidents" push button 


\section{Second middle panel (Identifying incident free condition)}

- A panel that includes a list of the time periods when all other types of incidents occur, called "Incidents' time caused by all other incidents types." When selecting the incident free condition, the selected time should not fall close to any of the listed time periods in the panel, as well as not close to the time periods for the incident times included in the first panel.

- In the dialog box, there is an option if a new selected time is needed in (hr: $\mathrm{mm}$ ) with a check box option. The default string is always verified, no need to enter any new time in this case. If check the box is the option to be selected, then the updated time should be interred as new information to be counted when reading the speed, volume, and occupancy data. Which means that the same selected detector number will be used to extract the speed and other traffic data from. No need to define any other detector as a new input.

- When not focusing on the incident free condition, then a check mark should be applied.

- In this panel, the threshold that identifies the percentage of the speed where the drop point is found, is given.

\section{Third panel (Identifying detection parameters)}

This panel involves the input of many parameters such as:

- $\quad$ Recovery period threshold (min).

- "R2r3 repetition High S," threshold for the number of times the speed retrieves back to a high-speed value (typical is 5). 
- "R2r3 repetition Low S," threshold for the number of times the speed retrieves back to a low-speed value (typical is 3 ).

- "Speed Recovery High S\%," threshold for the percentage of the $85^{\text {th }}$ percentile of the drop in speed point; it is defined by a particular level of $(60 \%$ was selected as a typical level for high predicted speed values)

- "Speed Recovery Low S\%," threshold for the percentage of the $85^{\text {th }}$ percentile of the predicted speed; it is defined by a specified level of ( $40 \%$ was selected as a typical level for low predicted speed values).

- "Speed Recovery Repetition," threshold for the number of times the predicted speed reaches to a selected percentage of the $85^{\text {th }}$ percentile of the predicted speed, this figure is examined with a typical given value of (2).

- The threshold used to define low and high predicted speed levels, when an $85^{\text {th }}$ of the drop in speed point is higher than a typical speed of $45 \mathrm{~km} / \mathrm{h}$ then this is regarded as high-speed level and vice versa.

\section{Fourth panel (Algorithm Inputs)}

- "Percentage of S1\&S3 to S2," is the percentage of the speed range considering the moderate speed state is $\mathrm{s}_{2}$. If selected as a value of 0.07 , then the speed that corresponds to state $\mathrm{s}_{3}$ is $7 \%$ higher than the speed corresponds to state $\mathrm{s}_{2}$ and, the speed corresponds to state $\mathrm{s}_{1}$ is $7 \%$ lower than the speed corresponds to state $\mathrm{s}_{2}$.

- "Range bet. S1\&S3 to S2 $(\mathrm{km} / \mathrm{h})$," is a fixed value of the speed range considering the moderate speed state is $\mathrm{s}_{2}$. If selected as a value of 7 , then the speed that corresponds 
to state $s_{3}$ is $7 \mathrm{~km} / \mathrm{h}$ higher than the speed corresponds to state $s_{2}$ and, the speed corresponds to state $s_{1}$ is $7 \mathrm{~km} / \mathrm{h}$ lower than the speed corresponds to state $\mathrm{s}_{2}$.

- Drop in predicted speed \%( 35-75)", a threshold that defines the second condition that corresponds to incident detection, the continuous drop in the predicted speed computed as a percentage of the $85^{\text {th }}$ percentile speed should reach to this threshold to satisfy the defined criteria. A typical percentage of $55 \%$ was proposed when calibrating and validating the model.

- Max posterior level \% (90-100)", a threshold that defines the second condition that corresponds to incident detection. The maximum posterior level should reach this selected threshold to satisfy the defined criteria. A typical percentage of $97 \%$ was proposed in calibrating and validating the model.

- Conditional probability level \%(0.5-0.8)", a threshold that reflects the reliability of the available information and is expressed by the proportion of equipped vehicles, traffic volume, and the probability that the minimum sample size requirement is encountered. 0.5 is considered a lower level of the conditional probability while 0.8 is considered at a high level.

- Cond Prob. r3s2, it represents the relation between the conditional probability parameters $\mathrm{r} 3$ and $\mathrm{s} 2$, as well as the relation between $\mathrm{r} 1$ and $\mathrm{s} 2$.

- Cond Prob. r3s1, it represents the relation between the conditional probability parameters $\mathrm{r} 3$ and $\mathrm{s} 1$, as well as the relation between $\mathrm{r} 1$ and $\mathrm{s} 3$.

- "Detect Incident" push button to activate the second part of the Matlab application.

- Typical ranges for all the input data are provided as a simple guide to the user. 


\subsubsection{Dialog Features}

- A dialog message is displayed indicating the upstream detector number and the distance between the detector location and the incident location.

- A dialog message is displayed indicating if the detector file does not exist, along with a message prompting the option for selecting the next detector or to terminate the application.

- A dialog message is displayed indicating the percentage of any existence of empty cells, $\mathrm{X}$ or Zero in the speed data file, the percentage is rounded up. Along with a message prompting the option for selecting the next detector or to stop the application as shown in Figure 6.39. Proceeding with the same detector is still a valid option.

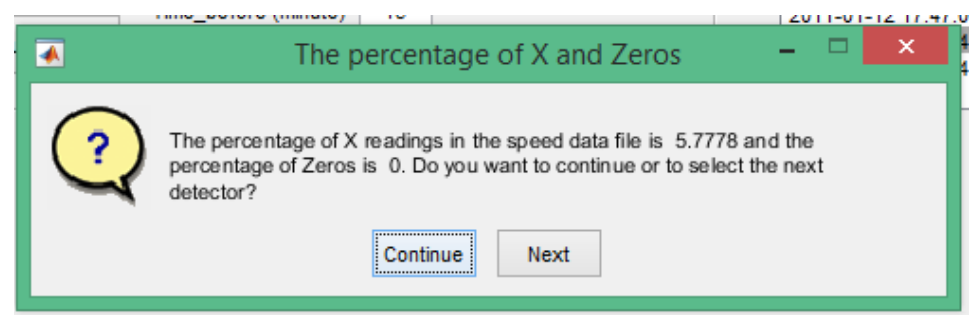

Figure 6.39: Percentage of $X$ and zeros dialog

- A dialog message is displayed indicating the percentage of cells containing $\mathrm{X}$ or 0 in the speed data file when it reaches to a percentage of $100 \%$. Along with a message offering the option for selecting the next detector or to stop the application.

- A dialog message is displayed indicating if the speed data file does not exist within the available data of the study detector.

- A dialog message is displayed indicating that this is the last detector exist within the study section, and no more detectors are available, an icon to terminate the application 
is also offered at this point. This comes to pass when selecting the next detector happened to be the last one, and no more detectors are available.

- To close the dialog box and stop the application, either the close or the stop button is to be clicked, the exit button is not meant for this purpose.

\subsubsection{Matlab Program Features}

- For every incident detection application, when the incident is detected the incident detection process stops. In another word, not more than one incident should be detected for each incident detection application.

- When the first run is conducted after identifying the stream, direction, date, and time ranges, all incidents that happened to occur within that range of time will be determined and displayed in the first panel of the main GUI interface for any further selection.

- To identify the incident free condition, a second dialog is added to the GUI interface. This dialog allows the input for any other selected time; the selection criteria for the free incident condition is based on selecting a time for the observation that is 2 hours before or after a predefined time when incidents are known to take place. So the selected time will fall in another period different from the time period at which incidents were recorded.

- When calculating the distance between the detector and the incident, only the Latitude coordinate was considered. However, when giving the exact distance between the detector and the incident in all other forms of the output, both the longitude and latitude coordinates are used in the computation. 
- In case the drop in speed was detected at the end of the time boundaries or even before the end with a time period less than the recovery period, so that when the recovery test is applied it never covers the proposed recovery period (5min). In this case, a message is displayed indicating that:

"Recovery period is not enough to confirm the incident, drop in speed was detected, and no incident was confirmed."

- After computing the predicted speed, in the case it is found to be less than 0 , then it is set to be equal to a value of 0 . This problem was partially resolved when indicating a speed range of $7 \%$ instead of a fixed speed range of $7 \mathrm{~km} / \mathrm{h}$.

\subsubsection{Output Features}

The final outputs from the final developed Matlab version are arranged in diagrams to include speed, volume, and occupancy profiles. It also involves tables that contain detailed information about the results. Each type of output is stored in a separate file, the diagrams are stored in Matlab format as well as in jpg format, and tables are stored in Matlab format.

The following information is integrated within the output diagrams.

- Diagram name is indicated by the incident date and time, to distinguish it from other diagram names.

- Diagrams are integrated with necessary information; this information provides details about the incident data and critical parameters such as incident reason, season, stream, direction, date, time, and the ratio of speed ranges S1 \& S3 to S2. The diagram also includes the proposed conditional probability level for the analysis, predetermined 
threshold of a percent drop in speed, selected maximum posterior probability threshold level, and the detector number under consideration. The downstream detector distance from the incident location, detector rank as it refers to its position in the order of upstream detectors, and the incident detection time in (hr:mm:ss) format is also indicated by the combined output information.

- Speed profile is displayed first followed by the volume and occupancy profiles.

- Volume and occupancy diagrams consider the same given time period that includes the two proposed time boundaries, in our case $20 \mathrm{~min}$ before and $20 \mathrm{~min}$ after.

- The units used to represent the three traffic data including speed, volume and occupancy are as the following:

- Time ---------- hh: mm

- Volume -------- vehicle/h

- $\quad$ Speed --------- km/h

- Occupancy --- \%

The horizontal alignment represents the time and is rotated 45 degrees in order to have it as a clear printout.

\subsubsection{Computer Program Testing}

For the testing and validation of incident detection Matlab program and the associated algorithm, data that involve incidents and pattern of fluctuating traffic speed would be most suitable. 
The incident under this application was hypothetically reported on 2011-01-12 at 08:04:00 am. The closest upstream detector to the incident under consideration was determined.

Since the incident under test was reported on 2011-01-12 at 08:04:00 am, sensor data pertaining to the same date of the year (2011-01-12) was of interest. Moreover, a time period within which the incident was reported was investigated. As was discussed in chapter three of this thesis, incident time is not certain, therefore, a time boundary of 20 min before and 20 min after the recorded time of the incident was considered for testing the network.

Since the incident was recorded at 08:04:00, then it was considered sound to start the procedure of testing the model application 20 minutes before this time which is 07:44:00 and ends at 08:24:00 for a time interval of 40 minutes. The purpose of this application was to test the Matlab application for certain parameters. Therefore, the analysis was intended to terminate after the detection of the incident, i.e. at 08:10:00 in this application.

The hypothetical speed data was prepared so as to include potential variations in speed changes between speed states. See tables Table 6.1 and Table 6.2. This resulted in suitable data for testing the developed Matlab program. The speed data included all applicable features and the time interval was set at 20 seconds. The final version of the developed Matlab program was validated in all aspects with manually solved application. All results showed identical answers. Speed data of the hypothetical case was incorporated into one of the speed files and was set to fall at the same time when a 
selected incident took place so that the developed Matlab program could treat the data as it is designed to perform.

In this test, it was intended to assume speed values to fluctuate so that it included all possible changes between the speed states as shown in Figure 6.40. and Figure 6.41. The proposed speed values along with the speed states are tabulated in Table 6.1 and extend to Table 6.2. Speed states were determined manually utilizing the developed criteria. They were also found using the Matlab program and results are tabulated in the same two tables mentioned above.

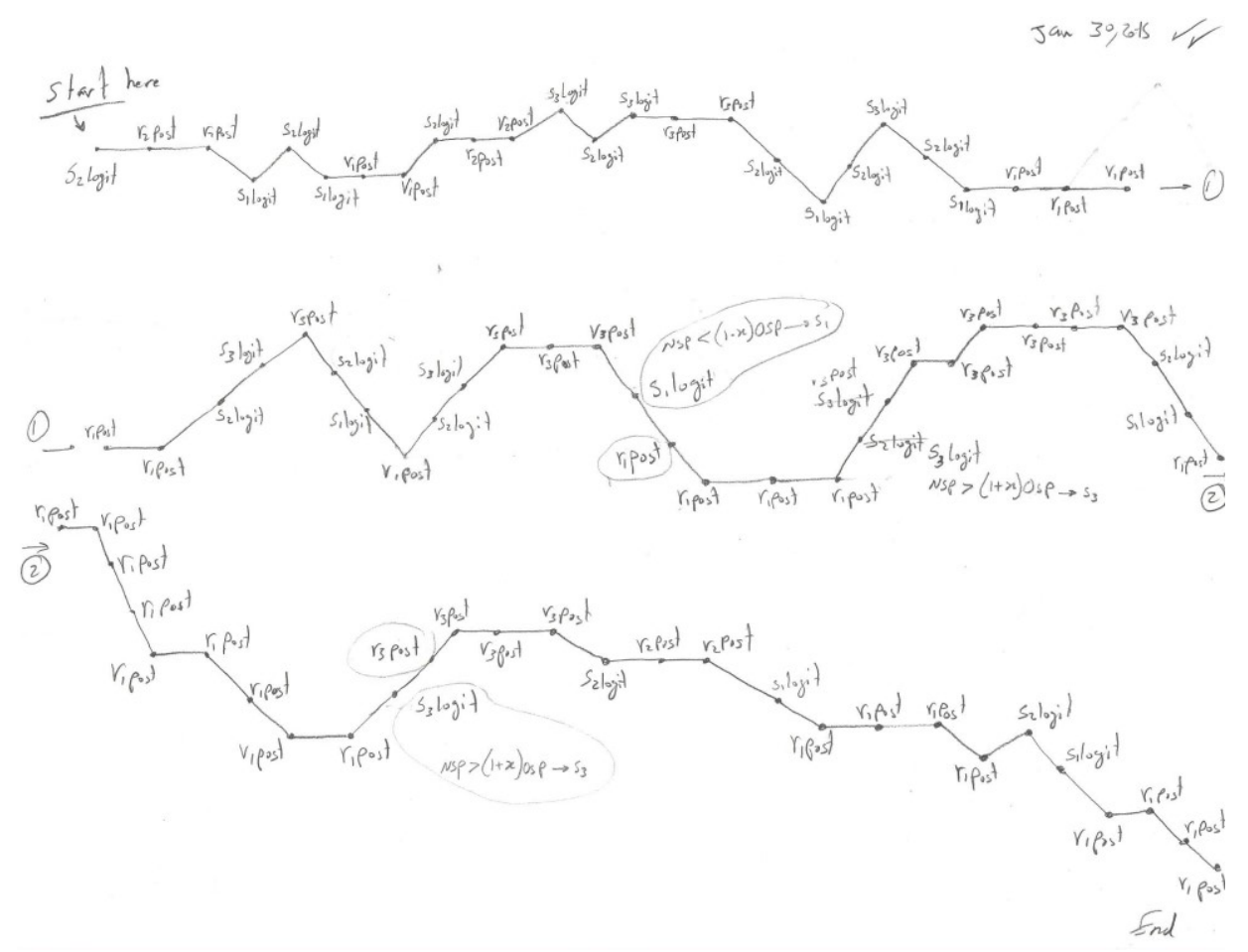

Figure 6.40: Testing the computer program part \#1 


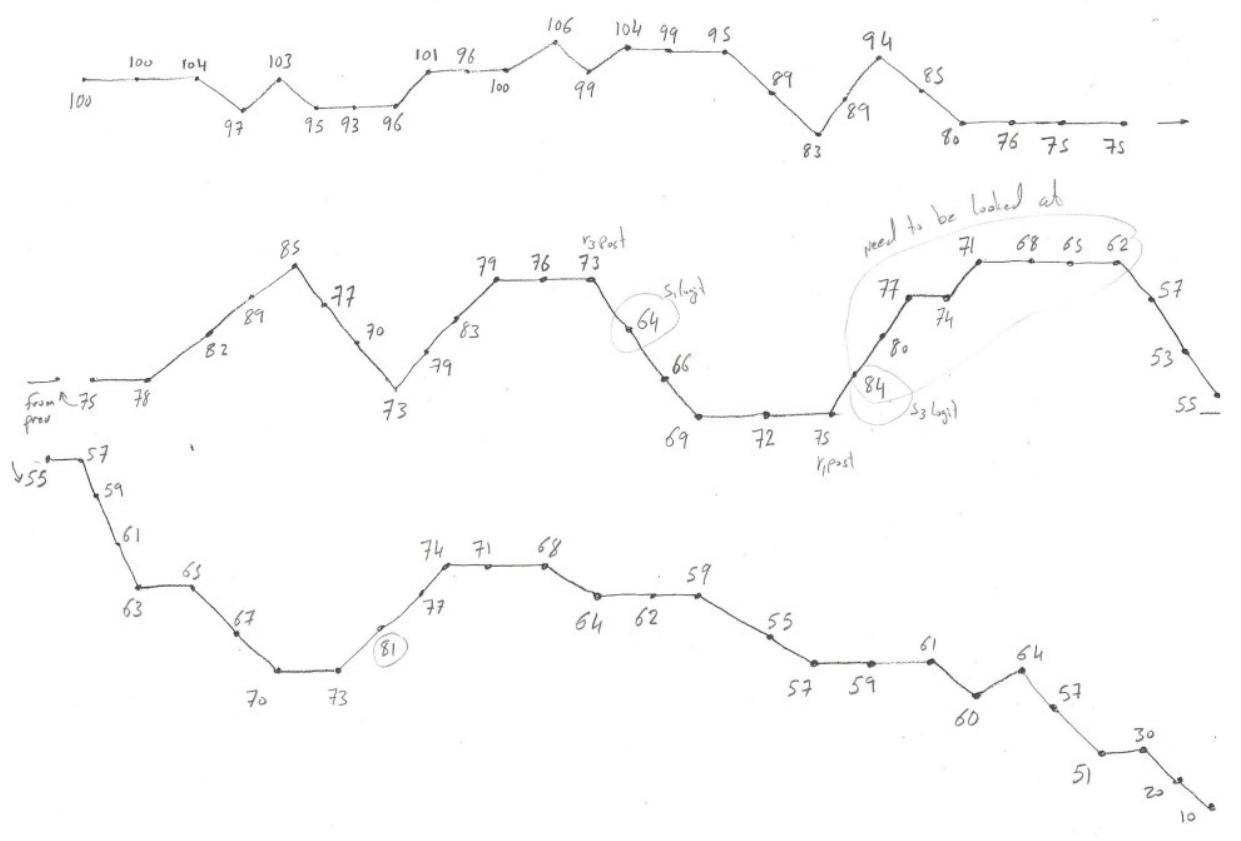

Figure 6.41: Testing the computer program part \#2

The application was carried out by utilizing both the designed automated excel sheets and the developed Matlab program. The developed Matlab program started by predicting the speed at each time period and the percent drop in the predicted speed was also calculated. Likewise, the maximum posterior probability level that was associated with each time period was recorded. Similarly, results obtained from applying the automated excel sheet were used to validate the Matlab results. Results from both methods are presented in Table 6.3, and a detailed discussion is presented following the table. 
Table 6.1: Hypothetical sensor data for testing the Matlab program (1)

\begin{tabular}{|c|c|c|c|c|c|c|}
\hline $0.9 * s 2$ & $0.95 * s 2$ & $\begin{array}{l}\text { Moderate } \\
\text { Speed } \\
\text { State s2 }\end{array}$ & $1.05 * s 2$ & $1.1 * s 2$ & $\begin{array}{c}\text { Speed State } \\
\text { Found } \\
\text { Manually }\end{array}$ & $\begin{array}{c}\text { Speed State } \\
\text { Found from } \\
\text { Code } \\
\text { application } \\
\end{array}$ \\
\hline 90 & 95 & 100 & 105 & 110 & 2 & 2 \\
\hline 90 & 95 & 100 & 105 & 110 & 2 & 2 \\
\hline 93.6 & 98.8 & 104 & 109.2 & 114.4 & 2 & 2 \\
\hline 87.3 & 92.15 & 97 & 101.85 & 106.7 & 1 & 1 \\
\hline 92.7 & 97.85 & 103 & 108.15 & 113.3 & 2 & 2 \\
\hline 85.5 & 90.25 & 95 & 99.75 & 104.5 & 1 & 1 \\
\hline 83.7 & 88.35 & 93 & 97.65 & 102.3 & 1 & 1 \\
\hline 86.4 & 91.2 & 96 & 100.8 & 105.6 & 1 & 1 \\
\hline 90.9 & 95.95 & 101 & 106.05 & 111.1 & 2 & 2 \\
\hline 86.4 & 91.2 & 96 & 100.8 & 105.6 & 2 & 2 \\
\hline 90 & 95 & 100 & 105 & 110 & 2 & 2 \\
\hline 95.4 & 100.7 & 106 & 111.3 & 116.6 & 3 & 3 \\
\hline 89.1 & 94.05 & 99 & 103.95 & 108.9 & 2 & 2 \\
\hline 93.6 & 98.8 & 104 & 109.2 & 114.4 & 3 & 3 \\
\hline 89.1 & 94.05 & 99 & 103.95 & 108.9 & 3 & 3 \\
\hline 85.5 & 90.25 & 95 & 99.75 & 104.5 & 3 & 3 \\
\hline 80.1 & 84.55 & 89 & 93.45 & 97.9 & 2 & 2 \\
\hline 74.7 & 78.85 & 83 & 87.15 & 91.3 & 1 & 1 \\
\hline 80.1 & 84.55 & 89 & 93.45 & 97.9 & 2 & 2 \\
\hline 84.6 & 89.3 & 94 & 98.7 & 103.4 & 3 & 3 \\
\hline 76.5 & 80.75 & 85 & 89.25 & 93.5 & 2 & 2 \\
\hline 72 & 76 & 80 & 84 & 88 & 1 & 1 \\
\hline 64.8 & 68.4 & 72 & 75.6 & 79.2 & 1 & 1 \\
\hline 67.5 & 71.25 & 75 & 78.75 & 82.5 & 1 & 1 \\
\hline 67.5 & 71.25 & 75 & 78.75 & 82.5 & 1 & 1 \\
\hline 70.2 & 74.1 & 78 & 81.9 & 85.8 & 1 & 1 \\
\hline 73.8 & 77.9 & 82 & 86.1 & 90.2 & 2 & 2 \\
\hline 80.1 & 84.55 & 89 & 93.45 & 97.9 & 3 & 3 \\
\hline 76.5 & 80.75 & 85 & 89.25 & 93.5 & 3 & 3 \\
\hline 69.3 & 73.15 & 77 & 80.85 & 84.7 & 2 & 2 \\
\hline 63 & 66.5 & 70 & 73.5 & 77 & 1 & 1 \\
\hline 65.7 & 69.35 & 73 & 76.65 & 80.3 & 1 & 1 \\
\hline 71.1 & 75.05 & 79 & 82.95 & 86.9 & 2 & 2 \\
\hline 74.7 & 78.85 & 83 & 87.15 & 91.3 & 3 & 3 \\
\hline 71.1 & 75.05 & 79 & 82.95 & 86.9 & 3 & 3 \\
\hline 68.4 & 72.2 & 76 & 79.8 & 83.6 & 3 & 3 \\
\hline 65.7 & 69.35 & 73 & 76.65 & 80.3 & 3 & 3 \\
\hline 57.6 & 60.8 & 64 & 67.2 & 70.4 & 1 & 1 \\
\hline 59.4 & 62.7 & 66 & 69.3 & 72.6 & 1 & 1 \\
\hline
\end{tabular}


Table 6.2: Hypothetical sensor data for testing the Matlab program (2)

\begin{tabular}{|c|c|c|c|c|c|c|}
\hline $0.9 *$ s2 & $0.95 * s 2$ & $\begin{array}{c}\text { Moderate } \\
\text { Speed } \\
\text { State s2 }\end{array}$ & $1.05 * s 2$ & $1.1 * s 2$ & $\begin{array}{l}\text { Speed State } \\
\text { Found } \\
\text { Manually }\end{array}$ & $\begin{array}{l}\text { Speed State } \\
\text { Found from } \\
\text { Code } \\
\text { application }\end{array}$ \\
\hline 62.1 & 65.55 & 69 & 72.45 & 75.9 & 1 & 1 \\
\hline 64.8 & 68.4 & 72 & 75.6 & 79.2 & 1 & 1 \\
\hline 67.5 & 71.25 & 75 & 78.75 & 82.5 & 1 & 1 \\
\hline 75.6 & 79.8 & 84 & 88.2 & 92.4 & 3 & 3 \\
\hline 72 & 76 & 80 & 84 & 88 & 3 & 3 \\
\hline 69.3 & 73.15 & 77 & 80.85 & 84.7 & 3 & 3 \\
\hline 66.6 & 70.3 & 74 & 77.7 & 81.4 & 3 & 3 \\
\hline 63.9 & 67.45 & 71 & 74.55 & 78.1 & 3 & 3 \\
\hline 61.2 & 64.6 & 68 & 71.4 & 74.8 & 3 & 3 \\
\hline 58.5 & 61.75 & 65 & 68.25 & 71.5 & 3 & 3 \\
\hline 55.8 & 58.9 & 62 & 65.1 & 68.2 & 3 & 3 \\
\hline 51.3 & 54.15 & 57 & 59.85 & 62.7 & 2 & 2 \\
\hline 47.7 & 50.35 & 53 & 55.65 & 58.3 & 1 & 1 \\
\hline 49.5 & 52.25 & 55 & 57.75 & 60.5 & 1 & 1 \\
\hline 51.3 & 54.15 & 57 & 59.85 & 62.7 & 1 & 1 \\
\hline 53.1 & 56.05 & 59 & 61.95 & 64.9 & 1 & 1 \\
\hline 54.9 & 57.95 & 61 & 64.05 & 67.1 & 1 & 1 \\
\hline 56.7 & 59.85 & 63 & 66.15 & 69.3 & 1 & 1 \\
\hline 58.5 & 61.75 & 65 & 68.25 & 71.5 & 1 & 1 \\
\hline 60.3 & 63.65 & 67 & 70.35 & 73.7 & 1 & 1 \\
\hline 63 & 66.5 & 70 & 73.5 & 77 & 1 & 1 \\
\hline 65.7 & 69.35 & 73 & 76.65 & 80.3 & 1 & 1 \\
\hline 72.9 & 76.95 & 81 & 85.05 & 89.1 & 3 & 3 \\
\hline 69.3 & 73.15 & 77 & 80.85 & 84.7 & 3 & 3 \\
\hline 66.6 & 70.3 & 74 & 77.7 & 81.4 & 3 & 3 \\
\hline 63.9 & 67.45 & 71 & 74.55 & 78.1 & 3 & 3 \\
\hline 61.2 & 64.6 & 68 & 71.4 & 74.8 & 3 & 3 \\
\hline 57.6 & 60.8 & 64 & 67.2 & 70.4 & 2 & 2 \\
\hline 55.8 & 58.9 & 62 & 65.1 & 68.2 & 2 & 2 \\
\hline 53.1 & 56.05 & 59 & 61.95 & 64.9 & 2 & 2 \\
\hline 49.5 & 52.25 & 55 & 57.75 & 60.5 & 1 & 1 \\
\hline 51.3 & 54.15 & 57 & 59.85 & 62.7 & 1 & 1 \\
\hline 53.1 & 56.05 & 59 & 61.95 & 64.9 & 1 & 1 \\
\hline 54.9 & 57.95 & 61 & 64.05 & 67.1 & 1 & 1 \\
\hline 54 & 57 & 60 & 63 & 66 & 1 & 1 \\
\hline 57.6 & 60.8 & 64 & 67.2 & 70.4 & 2 & 2 \\
\hline 51.3 & 54.15 & 57 & 59.85 & 62.7 & 1 & 1 \\
\hline 45.9 & 48.45 & 51 & 53.55 & 56.1 & 1 & 1 \\
\hline 27 & 28.5 & 30 & 31.5 & 33 & 1 & 1 \\
\hline 18 & 19 & 20 & 21 & 22 & 1 & 1 \\
\hline 9 & 9.5 & 10 & 10.5 & 11 & 1 & 1 \\
\hline
\end{tabular}


Table 6.3: Matlab program outputs vs automated excel sheets outputs

\begin{tabular}{|c|c|c|c|c|c|c|c|}
\hline no. & Time period & $\begin{array}{l}\text { Sensor } \\
\text { speed } \\
\text { data } \\
(\mathrm{km} / \mathrm{h})\end{array}$ & $\begin{array}{c}\text { Predicted } \\
\text { speed }\end{array}$ & $\begin{array}{l}\text { Predicted } \\
\text { speed' from } \\
\text { Matlab } \\
\text { Program }\end{array}$ & $\begin{array}{c}\% \text { Drop in } \\
\text { predicted } \\
\text { speed }\end{array}$ & $\begin{array}{c}\mathrm{P} "(\mathrm{~s} \mid \mathrm{r}) \text { for lower } \\
\text { equipped level } \\
\text { case (i.e., for } \\
\mathrm{P}(\mathrm{r} \mid \mathrm{s})=0.5)\end{array}$ & $\begin{array}{l}\text { MAX posterior } \\
\text { from Matlab } \\
\text { Program }\end{array}$ \\
\hline 2 & '2011-01-12 07:44:00' & 100 & 100.00 & 100.00 & 0.00 & 0.67 & 0.67 \\
\hline 3 & '2011-01-12 07:44:20' & 104 & 100.00 & 100.00 & 0.00 & 0.78 & 0.78 \\
\hline 4 & '2011-01-12 07:44:40' & 97 & 104.00 & 104.00 & 4.00 & 0.85 & 0.85 \\
\hline 5 & '2011-01-12 07:45:00' & 103 & 93.68 & 93.68 & -6.32 & 0.74 & 0.74 \\
\hline 6 & '2011-01-12 07:45:20' & 95 & 103.00 & 103.00 & 3.00 & 0.67 & 0.67 \\
\hline 7 & '2011-01-12 07:45:40' & 93 & 91.75 & 91.75 & -8.25 & 0.74 & 0.74 \\
\hline 8 & '2011-01-12 07:46:00' & 96 & 87.21 & 87.21 & -12.79 & 0.86 & 0.86 \\
\hline 9 & '2011-01-12 07:46:20' & 101 & 88.26 & 88.26 & -11.74 & 0.93 & 0.93 \\
\hline 10 & '2011-01-12 07:46:40' & 96 & 101.00 & 101.00 & 1.00 & 0.67 & 0.67 \\
\hline 11 & '2011-01-12 07:47:00' & 100 & 96.00 & 96.00 & -4.00 & 0.78 & 0.78 \\
\hline 12 & '2011-01-12 07:47:20' & 106 & 100.00 & 100.00 & 0.00 & 0.85 & 0.85 \\
\hline 13 & '2011-01-12 07:47:40' & 99 & 109.65 & 109.65 & 9.65 & 0.74 & 0.74 \\
\hline 14 & '2011-01-12 07:48:00' & 104 & 99.00 & 99.00 & -1.00 & 0.67 & 0.67 \\
\hline 15 & '2011-01-12 07:48:20' & 99 & 107.58 & 107.58 & 7.58 & 0.74 & 0.74 \\
\hline 16 & '2011-01-12 07:48:40' & 95 & 105.18 & 105.18 & 5.18 & 0.86 & 0.86 \\
\hline 17 & '2011-01-12 07:49:00' & 89 & 102.67 & 102.67 & 2.67 & 0.93 & 0.93 \\
\hline 18 & '2011-01-12 07:49:20' & 83 & 89.00 & 89.00 & -11.00 & 0.67 & 0.67 \\
\hline 19 & '2011-01-12 07:49:40' & 89 & 80.18 & 80.18 & -19.82 & 0.73 & 0.73 \\
\hline 20 & '2011-01-12 07:50:00' & 94 & 89.00 & 89.00 & -11.00 & 0.67 & 0.67 \\
\hline 21 & '2011-01-12 07:50:20' & 85 & 97.22 & 97.22 & -2.78 & 0.74 & 0.74 \\
\hline 22 & '2011-01-12 07:50:40' & 80 & 85.00 & 85.00 & -15.00 & 0.67 & 0.67 \\
\hline 23 & '2011-01-12 07:51:00' & 72 & 77.29 & 77.29 & -22.71 & 0.73 & 0.73 \\
\hline 24 & '2011-01-12 07:51:20' & 75 & 67.54 & 67.54 & -32.46 & 0.86 & 0.86 \\
\hline 25 & '2011-01-12 07:51:40' & 75 & 68.97 & 68.97 & -31.03 & 0.93 & 0.93 \\
\hline 26 & '2011-01-12 07:52:00' & 78 & 68.21 & 68.21 & -31.79 & 0.97 & 0.97 \\
\hline 27 & '2011-01-12 07:52:20' & 82 & 70.54 & 70.54 & -29.46 & 0.98 & 0.98 \\
\hline 28 & '2011-01-12 07:52:40' & 89 & 82.00 & 82.00 & -18.00 & 0.67 & 0.67 \\
\hline 29 & '2011-01-12 07:53:00' & 85 & 92.03 & 92.03 & -7.97 & 0.74 & 0.74 \\
\hline 30 & '2011-01-12 07:53:20' & 77 & 90.29 & 90.29 & -9.71 & 0.86 & 0.86 \\
\hline 31 & '2011-01-12 07:53:40' & 70 & 77.00 & 77.00 & -23.00 & 0.67 & 0.67 \\
\hline 32 & '2011-01-12 07:54:00' & 73 & 67.65 & 67.65 & -32.35 & 0.73 & 0.73 \\
\hline
\end{tabular}


Cont.

\begin{tabular}{|c|c|c|c|c|c|c|c|}
\hline no. & Time period & $\begin{array}{c}\text { Sensor } \\
\text { speed } \\
\text { data } \\
(\mathrm{km} / \mathrm{h})\end{array}$ & $\begin{array}{l}\text { Predicted } \\
\text { speed }\end{array}$ & $\begin{array}{c}\text { Predicted } \\
\text { speed' from } \\
\text { Matlab } \\
\text { Program }\end{array}$ & $\begin{array}{l}\% \text { Drop in } \\
\text { predicted } \\
\text { speed }\end{array}$ & $\begin{array}{c}\text { P"(s|r) for lower } \\
\text { equipped level } \\
\text { case (i.e., for } \\
\mathrm{P}(\mathrm{r} \mid \mathrm{s})=0.5)\end{array}$ & $\begin{array}{c}\text { MAX posterior' } \\
\text { from Matlab } \\
\text { Program }\end{array}$ \\
\hline 31 & '2011-01-12 07:54:20' & 79 & 68.50 & 68.50 & -31.50 & 0.86 & 0.86 \\
\hline 32 & '2011-01-12 07:54:40' & 83 & 79.00 & 79.00 & -21.00 & 0.67 & 0.67 \\
\hline 33 & '2011-01-12 07:55:00' & 79 & 85.82 & 85.82 & -14.18 & 0.73 & 0.73 \\
\hline 34 & '2011-01-12 07:55:20' & 76 & 83.90 & 83.90 & -16.10 & 0.86 & 0.86 \\
\hline 35 & '2011-01-12 07:55:40' & 73 & 82.12 & 82.12 & -17.88 & 0.93 & 0.93 \\
\hline 35 & '2011-01-12 07:56:00' & 64 & 79.61 & 79.61 & -20.39 & 0.97 & 0.97 \\
\hline 36 & '2011-01-12 07:56:20' & 66 & 61.87 & 61.87 & -38.13 & 0.73 & 0.73 \\
\hline 37 & '2011-01-12 07:56:40' & 69 & 61.94 & 61.94 & -38.06 & 0.86 & 0.86 \\
\hline 38 & '2011-01-12 07:57:00' & 72 & 63.47 & 63.47 & -36.53 & 0.93 & 0.93 \\
\hline 39 & '2011-01-12 07:57:20' & 75 & 65.49 & 65.49 & -34.51 & 0.96 & 0.96 \\
\hline 40 & '2011-01-12 07:57:40' & 84 & 67.84 & 67.84 & -32.16 & 0.98 & 0.98 \\
\hline 41 & '2011-01-12 07:58:00' & 80 & 86.85 & 86.85 & -13.15 & 0.74 & 0.74 \\
\hline 42 & '2011-01-12 07:58:20' & 77 & 84.97 & 84.97 & -15.03 & 0.86 & 0.86 \\
\hline 43 & '2011-01-12 07:58:40' & 74 & 83.20 & 83.20 & -16.80 & 0.93 & 0.93 \\
\hline 44 & '2011-01-12 07:59:00' & 71 & 80.71 & 80.71 & -19.29 & 0.97 & 0.97 \\
\hline 45 & '2011-01-12 07:59:20' & 68 & 77.79 & 77.79 & -22.21 & 0.98 & 0.98 \\
\hline 46 & '2011-01-12 07:59:40' & 65 & 74.66 & 74.66 & -25.34 & 0.99 & 0.99 \\
\hline 47 & '2011-01-12 08:00:00' & 62 & 71.44 & 71.44 & -28.56 & 1.00 & 1.00 \\
\hline 48 & '2011-01-12 08:00:20' & 57 & 68.17 & 68.17 & -31.83 & 1.00 & 1.00 \\
\hline 48 & '2011-01-12 08:00:40' & 53 & 57.00 & 57.00 & -43.00 & 0.66 & 0.66 \\
\hline 49 & '2011-01-12 08:01:00' & 55 & 51.27 & 51.27 & -48.73 & 0.73 & 0.73 \\
\hline 50 & '2011-01-12 08:01:20' & 57 & 51.64 & 51.64 & -48.36 & 0.85 & 0.85 \\
\hline 51 & '2011-01-12 08:01:40' & 59 & 52.45 & 52.45 & -47.55 & 0.93 & 0.93 \\
\hline 52 & '2011-01-12 08:02:00' & 61 & 53.68 & 53.68 & -46.32 & 0.96 & 0.96 \\
\hline 53 & '2011-01-12 08:02:20' & 63 & 55.18 & 55.18 & -44.82 & 0.98 & 0.98 \\
\hline 54 & '2011-01-12 08:02:40' & 65 & 56.83 & 56.83 & -43.17 & 0.99 & 0.99 \\
\hline 55 & '2011-01-12 08:03:00' & 67 & 58.56 & 58.56 & -41.44 & 1.00 & 1.00 \\
\hline 56 & '2011-01-12 08:03:20' & 70 & 60.33 & 60.33 & -39.67 & 1.00 & 1.00 \\
\hline 57 & '2011-01-12 08:03:40' & 73 & 63.02 & 63.02 & -36.98 & 1.00 & 1.00 \\
\hline 58 & '2011-01-12 08:04:00' & 81 & 65.71 & 65.71 & -34.29 & 1.00 & 1.00 \\
\hline
\end{tabular}




\section{Cont.}

\begin{tabular}{|c|c|c|c|c|c|c|c|}
\hline no. & Time period & $\begin{array}{c}\text { Sensor } \\
\text { speed } \\
\text { data } \\
(\mathrm{km} / \mathrm{h})\end{array}$ & $\begin{array}{c}\text { Predicted } \\
\text { speed }\end{array}$ & $\begin{array}{l}\text { Predicted } \\
\text { speed' from } \\
\text { Matlab } \\
\text { Program }\end{array}$ & $\begin{array}{c}\% \text { Drop in } \\
\text { predicted } \\
\text { speed }\end{array}$ & $\begin{array}{c}\mathrm{P} "(\mathrm{~s} \mid \mathrm{r}) \text { for lower } \\
\text { equipped level } \\
\text { case (i.e., for } \\
\mathrm{P}(\mathrm{r} \mid \mathrm{s})=0.5)\end{array}$ & $\begin{array}{l}\text { MAX posterior' } \\
\text { from Matlab } \\
\text { Program }\end{array}$ \\
\hline 59 & '2011-01-12 08:04:20' & 77 & 83.74 & 83.74 & -16.26 & 0.73 & 0.73 \\
\hline 60 & '2011-01-12 08:04:40' & 74 & 81.77 & 81.77 & -18.23 & 0.86 & 0.86 \\
\hline 61 & '2011-01-12 08:05:00' & 71 & 79.96 & 79.96 & -20.04 & 0.93 & 0.93 \\
\hline 62 & '2011-01-12 08:05:20' & 68 & 77.43 & 77.43 & -22.57 & 0.97 & 0.97 \\
\hline 63 & '2011-01-12 08:05:40' & 64 & 74.50 & 74.50 & -25.50 & 0.98 & 0.98 \\
\hline 64 & '2011-01-12 08:06:00' & 62 & 64.00 & 64.00 & -36.00 & 0.66 & 0.66 \\
\hline 65 & '2011-01-12 08:06:20' & 59 & 62.00 & 62.00 & -38.00 & 0.77 & 0.77 \\
\hline 66 & '2011-01-12 08:06:40' & 55 & 59.00 & 59.00 & -41.00 & 0.85 & 0.85 \\
\hline 67 & '2011-01-12 08:07:00' & 57 & 53.20 & 53.20 & -46.80 & 0.73 & 0.73 \\
\hline 68 & '2011-01-12 08:07:20' & 59 & 53.52 & 53.52 & -46.48 & 0.85 & 0.85 \\
\hline 69 & '2011-01-12 08:07:40' & 61 & 54.28 & 54.28 & -45.72 & 0.93 & 0.93 \\
\hline 70 & '2011-01-12 08:08:00' & 60 & 55.49 & 55.49 & -44.51 & 0.96 & 0.96 \\
\hline 71 & '2011-01-12 08:08:20' & 64 & 54.27 & 54.27 & -45.73 & 0.98 & 0.98 \\
\hline 72 & '2011-01-12 08:08:40' & 57 & 64.00 & 64.00 & -36.00 & 0.66 & 0.66 \\
\hline 73 & '2011-01-12 08:09:00' & 51 & 55.12 & 55.12 & -44.88 & 0.73 & 0.73 \\
\hline 74 & '2011-01-12 08:09:20' & 30 & 47.88 & 47.88 & -52.12 & 0.85 & 0.85 \\
\hline 75 & '2011-01-12 08:09:40' & 20 & 27.60 & 27.60 & -72.40 & 0.93 & 0.93 \\
\hline 76 & '2011-01-12 08:10:00' & 10 & 18.19 & 18.19 & -81.81 & 0.96 & 0.96 \\
\hline
\end{tabular}

In this test application, a speed state range was examined to effectively represent the available time period obtained from the sensor data. It was found that a range of $10 \%$ above and below the moderate speed state appeared reasonable. Therefore, the speed at state $\mathrm{s}_{1}$ was assigned a value that was $10 \%$ lower than the speed at state $\mathrm{s}_{2}$, and the speed at state $\mathrm{s}_{3}$ was assigned a $10 \%$ higher speed than the moderate speed value at state $\mathrm{s}_{2}$.

Since the speed at state $\mathrm{s}_{2}$ was defined by a value in $\mathrm{km} / \mathrm{h}$ therefore, the speed at state $\mathrm{s}_{1}$ and $\mathrm{s}_{3}$ represented a range of speeds of $10 \%$, and the middle level was used for ease of notation and calculations. Since the time interval in all cases was almost fixed, an interval 
of $20 \mathrm{sec}$ was valid for all cases. So the ratio between the speed values at states $\mathrm{s}_{1}$ and $\mathrm{s}_{3}$ in relation to $\mathrm{s}_{2}$ was the same for all intervals.

For the time interval that was used, it was decided to keep the following relationships between the speed values at the traffic condition states:

$\mathrm{s}_{1}=0.90 * \mathrm{~s}_{2}, \mathrm{~s}_{2}=1.0 * \mathrm{~s}_{2}, \mathrm{~s}_{3}=1.10 * \mathrm{~s}_{2}$

\section{Logical Argument}

The results of the test application are valid. As noted earlier in chapter five, the posterior level will increase in case the speed continues to increase or continues to decline. As the speed state changes from high to low or the opposite, the posterior level continues to fluctuate between high values. The specified criterion regarding the posterior probability changes was satisfied. It should be noted here that in the context of incident detection, we are interested in declining speed case only.

Until the speed reached the lowest value that would satisfy the criteria the model continued to move the clock to the next time period in an attempt to detect any possible incident. The drop in the predicted speed is presented in Figure 6.42.

Results obtained from the Matlab program are illustrated in Table 6.4. It is noted that same results are obtained as found from manual excel application regarding the maximum posterior level and the speed states. Also, similar predicted speed results were obtained from both approaches. The calculation of the percent drop in speed in the excel calculation sheets was based on the consideration that the first speed reading served as a reference point -- which was the first attempt in model development. The criteria to find the percent drop in speed was then amended to include the condition which states that the percentage 
of the speed drop is to be calculated based on the $85^{\text {th }}$ percentile speed. The first version was only addressing the first speed reading in the speed list.

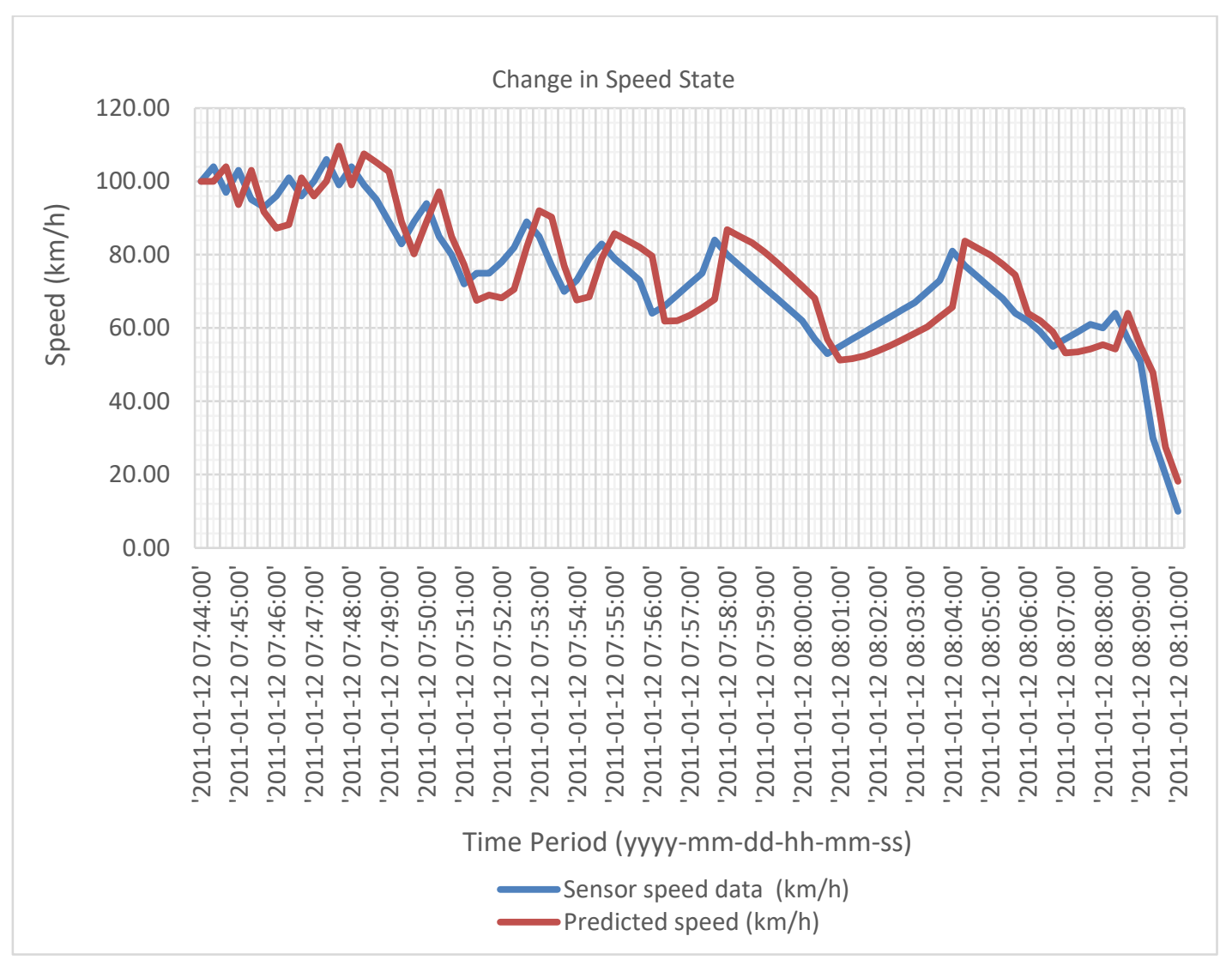

Figure 6.42: Sensor speed data vs. predicted speed data

As a result, there will be some slight difference in the percent drop in the speed when comparing the two approaches. 


\section{Table 6.4: Matlab program outputs}

\begin{tabular}{|c|c|c|c|c|c|c|c|c|c|c|c|c|c|c|c|c|c|c|c|}
\hline 'Date_time' & 'incedent ' & $\left|\begin{array}{c}\text { measured } \\
\text { SP' }\end{array}\right|$ & $\left|\begin{array}{c}\text { Predicted } \\
\text { speed' }\end{array}\right|$ & $\begin{array}{l}\text { 'speed } \\
\text { drop' }\end{array}$ & \begin{tabular}{|c|} 
'MAX \\
posterior \\
' \\
\end{tabular} & 'state' & $\begin{array}{c}\text { 'Speed } \\
\text { s1' }\end{array}$ & 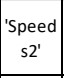 & \begin{tabular}{|c} 
'Speed \\
s3'
\end{tabular} & $\begin{array}{l}\text { 'delta } \\
\text { s1' }\end{array}$ & \begin{tabular}{|c} 
'delta \\
s2'
\end{tabular} & $\begin{array}{c}\text { 'delta } \\
\text { s3' }\end{array}$ & \begin{tabular}{|c|} 
Prior \\
Prob \\
s1' \\
\end{tabular} & \begin{tabular}{|c|} 
'Prior_- \\
Prob \\
s2' \\
\end{tabular} & \begin{tabular}{|c|} 
Prior_- \\
Prob \\
s3' \\
\end{tabular} & $\begin{array}{c}\text { '(1- } \\
\text { RBTCS)* } \\
\text { OSP' } \\
\end{array}$ & \begin{tabular}{|c|} 
\\
\\
$0.5^{*}$ RBTC \\
S)*OSP;' \\
\end{tabular} & $\begin{array}{c}(1+0.5 * \mathrm{R} \\
\text { BTCS)*OS } \\
\mathrm{P}^{\prime}\end{array}$ & $\begin{array}{l}\text { '(1+RBTC } \\
\text { S) })^{*} \text { OSP;' }\end{array}$ \\
\hline '2011-01-12 07:43:40' & 'No' & 100 & & 0 & 0 & 0 & 0 & 0 & 0 & 0 & 0 & 0 & 0 & 0 & 0 & 0 & 0 & 0 & 0 \\
\hline '2011-01-12 07:44:00' & 'No' & 100 & 100.00 & 0 & 0.674096 & 2 & 90 & 100 & 110 & 11 & 1 & 11 & 0.223 & 0.554 & 0.223 & 0 & 0 & 0 & 0 \\
\hline '2011-01-12 07:44:20' & 'No' & 104 & 100.00 & 0 & 0.775145 & 2 & 90 & 100 & 110 & 11 & 1 & 11 & \begin{tabular}{|l|}
0.163 \\
\end{tabular} & \begin{tabular}{|l|l|}
0.674 \\
\end{tabular} & \begin{tabular}{|l|}
0.163 \\
\end{tabular} & 90 & 95 & 105 & 110 \\
\hline '2011-01-12 07:44:40' & 'No' & 97 & 104.00 & 4 & 0.851753 & 2 & 93.6 & 104 & 114.4 & 11.4 & 1 & 11.4 & \begin{tabular}{|l|}
0.112 \\
\end{tabular} & 0.775 & \begin{tabular}{|l|}
0.112 \\
\end{tabular} & 90 & 95 & 105 & 110 \\
\hline '2011-01-12 07:45:00' & 'No' & 103 & 93.68 & \begin{tabular}{|l|}
-9.92767 \\
\end{tabular} & 0.737189 & 1 & 87.3 & 97 & 106.7 & 1 & 10.7 & 20.4 & 0.559 & \begin{tabular}{|l|l|}
0.226 \\
\end{tabular} & 0.216 & 93.6 & 98.8 & 109.2 & 114.4 \\
\hline '2011-01-12 07:45:20' & 'No' & 95 & 103.00 & -0.96154 & 0.674626 & 2 & 92.7 & 103 & 113.3 & 11.3 & 1 & \begin{tabular}{|l|}
11.3 \\
\end{tabular} & 0.223 & \begin{tabular}{|l|}
0.554 \\
\end{tabular} & 0.223 & 87.3 & 92.15 & 101.85 & 106.7 \\
\hline '2011-01-12 07:45:40' & 'No' & 93 & 91.75 & \begin{tabular}{|l|}
-11.781 \\
\end{tabular} & 0.736911 & 1 & 85.5 & 95 & 104.5 & 1 & 10.5 & 20 & \begin{tabular}{|l|}
0.558 \\
\end{tabular} & 0.226 & 0.216 & 92.7 & 97.85 & 108.15 & 113.3 \\
\hline '2011-01-12 07:46:00' & 'No' & 96 & 87.21 & -16.1472 & 0.859817 & 1 & 83.7 & 93 & 102.3 & 1 & 10.3 & 19.6 & \begin{tabular}{|l|l|}
0.737 \\
\end{tabular} & 0.149 & \begin{tabular}{|l|}
0.114 \\
\end{tabular} & 85.5 & 90.25 & 99.75 & 104.5 \\
\hline '2011-01-12 07:46:20' & 'No' & 101 & 88.26 & \begin{tabular}{|l|}
-15.138 \\
\end{tabular} & 0.929946 & 1 & 86.4 & 96 & 105.6 & 1 & 10.6 & 20.2 & 0.86 & \begin{tabular}{|l|}
0.087 \\
\end{tabular} & \begin{tabular}{|l|}
0.053 \\
\end{tabular} & 83.7 & 88.35 & 97.65 & 102.3 \\
\hline '2011-01-12 07:46:40' & 'No' & 96 & 101.00 & -2.88462 & 0.674276 & 2 & 90.9 & 101 & 111.1 & 11.1 & 1 & 11.1 & 0.223 & 0.554 & 0.223 & 86.4 & 91.2 & 100.8 & 105.6 \\
\hline '2011-01-12 07:47:00' & 'No' & 100 & 96.00 & -7.69231 & 0.775288 & 2 & 86.4 & 96 & 105.6 & 10.6 & 1 & 10.6 & \begin{tabular}{|l|}
0.163 \\
\end{tabular} & \begin{tabular}{|l|l|}
0.674 \\
\end{tabular} & 0.163 & 90.9 & 95.95 & 106.05 & 111.1 \\
\hline '2011-01-12 07:47:20' & 'No' & 106 & 100.00 & \begin{tabular}{|l|}
-3.84615 \\
\end{tabular} & 0.851857 & 2 & 90 & 100 & 110 & 11 & 1 & 11 & \begin{tabular}{|l|}
0.112 \\
\end{tabular} & 0.775 & \begin{tabular}{|l|}
0.112 \\
\end{tabular} & 86.4 & 91.2 & 100.8 & 105.6 \\
\hline '2011-01-12 07:47:40' & 'No' & 99 & 109.65 & 5.434054 & 0.738317 & 3 & 95.4 & 106 & 116.6 & 22.2 & 11.6 & 1 & 0.215 & 0.225 & 0.56 & 90 & 95 & 105 & 110 \\
\hline '2011-01-12 07:48:00' & 'No' & 104 & 99.00 & \begin{tabular}{|l|}
-9.71389 \\
\end{tabular} & 0.673913 & 2 & 89.1 & 99 & 108.9 & 10.9 & 1 & 10.9 & 0.223 & \begin{tabular}{|l|l|}
0.554 \\
\end{tabular} & 0.223 & 95.4 & 100.7 & 111.3 & 116.6 \\
\hline '2011-01-12 07:48:20' & 'No' & 99 & 107.58 & \begin{tabular}{|l|}
-1.89017 \\
\end{tabular} & 0.738082 & 3 & 93.6 & 104 & 114.4 & 21.8 & 11.4 & 1 & \begin{tabular}{|l|}
0.216 \\
\end{tabular} & 0.225 & 0.56 & 89.1 & 94.05 & 103.95 & 108.9 \\
\hline '2011-01-12 07:48:40' & 'No' & 95 & 105.18 & -4.07666 & 0.860566 & 3 & 89.1 & 99 & 108.9 & 20.8 & 10.9 & 1 & \begin{tabular}{|l|}
0.114 \\
\end{tabular} & \begin{tabular}{|l|l|}
0.148 \\
\end{tabular} & \begin{tabular}{|l|l|}
0.738 \\
\end{tabular} & 93.6 & 98.8 & 109.2 & 114.4 \\
\hline '2011-01-12 07:49:00' & 'No' & 89 & 102.67 & -6.36547 & 0.930362 & 3 & 85.5 & 95 & 104.5 & 20 & 10.5 & 1 & \begin{tabular}{|l|}
0.053 \\
\end{tabular} & 0.086 & \begin{tabular}{|l|}
0.861 \\
\end{tabular} & 89.1 & 94.05 & 103.95 & 108.9 \\
\hline '2011-01-12 07:49:20' & 'No' & 83 & 89.00 & -18.8337 & 0.671873 & 2 & 80.1 & 89 & 97.9 & 9.9 & 1 & 9.9 & \begin{tabular}{|l|}
0.224 \\
\end{tabular} & 0.551 & 0.224 & 85.5 & 90.25 & 99.75 & 104.5 \\
\hline '2011-01-12 07:49:40' & 'No' & 89 & 80.18 & -26.8747 & 0.734983 & 1 & 74.7 & 83 & 91.3 & 1 & 9.3 & 17.6 & 0.556 & \begin{tabular}{|l|l|}
0.228 \\
\end{tabular} & 0.216 & 80.1 & 84.55 & 93.45 & 97.9 \\
\hline '2011-01-12 07:50:00' & 'No' & 94 & 89.00 & -18.8337 & 0.671873 & 2 & 80.1 & 89 & 97.9 & 9.9 & 1 & 9.9 & \begin{tabular}{|l|}
0.224 \\
\end{tabular} & \begin{tabular}{|l|}
0.551 \\
\end{tabular} & \begin{tabular}{|l|}
0.224 \\
\end{tabular} & 74.7 & 78.85 & 87.15 & 91.3 \\
\hline '2011-01-12 07:50:20' & 'No' & 85 & 97.22 & -11.3409 & 0.736768 & 3 & 84.6 & 94 & 103.4 & 19.8 & 10.4 & 1 & \begin{tabular}{|l|}
0.216 \\
\end{tabular} & \begin{tabular}{|l|l|}
0.226 \\
\end{tabular} & \begin{tabular}{|l|}
0.558 \\
\end{tabular} & 80.1 & 84.55 & 93.45 & 97.9 \\
\hline '2011-01-12 07:50:40' & 'No' & 80 & 85.00 & -22.4816 & 0.670935 & 2 & 76.5 & 85 & 93.5 & 9.5 & 1 & 9.5 & \begin{tabular}{|l|}
0.225 \\
\end{tabular} & 0.55 & 0.225 & 84.6 & 89.3 & 98.7 & 103.4 \\
\hline '2011-01-12 07:51:00' & 'No' & 72 & 77.29 & -29.5116 & 0.734417 & 1 & 72 & 80 & 88 & 1 & 9 & 17 & \begin{tabular}{|l|}
0.555 \\
\end{tabular} & \begin{tabular}{|l|}
0.228 \\
\end{tabular} & \begin{tabular}{|l|}
0.217 \\
\end{tabular} & 76.5 & 80.75 & 89.25 & 93.5 \\
\hline '2011-01-12 07:51:20' & 'No' & 75 & 67.54 & -38.4071 & 0.858218 & 1 & 64.8 & 72 & 79.2 & 1 & 8.2 & \begin{tabular}{|l|}
15.4 \\
\end{tabular} & \begin{tabular}{|l|l|}
0.734 \\
\end{tabular} & 0.151 & 0.115 & 72 & 76 & 84 & 88 \\
\hline '2011-01-12 07:51:40' & 'No' & 75 & 68.97 & -37.1051 & 0.929057 & 1 & 67.5 & 75 & 82.5 & 1 & 8.5 & 16 & \begin{tabular}{|l|}
0.858 \\
\end{tabular} & \begin{tabular}{|l|l|}
0.088 \\
\end{tabular} & \begin{tabular}{|l|}
0.054 \\
\end{tabular} & 64.8 & 68.4 & 75.6 & 79.2 \\
\hline '2011-01-12 07:52:00' & 'No' & 78 & 68.21 & -37.7974 & 0.965547 & 1 & 67.5 & 75 & 82.5 & 1 & 8.5 & 16 & \begin{tabular}{|l|}
0.929 \\
\end{tabular} & 0.048 & \begin{tabular}{|l|}
0.023 \\
\end{tabular} & 67.5 & 71.25 & 78.75 & 82.5 \\
\hline '2011-01-12 07:52:20' & 'No' & 82 & 70.54 & -35.6653 & 0.983436 & 1 & 70.2 & 78 & 85.8 & 1 & 8.8 & 16.6 & 0.966 & 0.025 & 0.01 & 67.5 & 71.25 & 78.75 & 82.5 \\
\hline '2011-01-12 07:52:40' & 'No' & 89 & 82.00 & -25.2176 & 0.670176 & 2 & 73.8 & 82 & 90.2 & 9.2 & 1 & 9.2 & 0.225 & 0.549 & 0.225 & 70.2 & 74.1 & 81.9 & 85.8 \\
\hline '2011-01-12 07:53:00' & 'No' & 85 & 92.03 & -16.0662 & 0.736008 & 3 & 80.1 & 89 & 97.9 & 18.8 & 9.9 & 1 & \begin{tabular}{|l|}
0.216 \\
\end{tabular} & 0.227 & \begin{tabular}{|l|}
0.557 \\
\end{tabular} & 73.8 & 77.9 & 86.1 & 90.2 \\
\hline '2011-01-12 07:53:20' & 'No' & 77 & 90.29 & -17.6616 & 0.859238 & 3 & 76.5 & 85 & 93.5 & 18 & 9.5 & 1 & \begin{tabular}{|l|}
0.114 \\
\end{tabular} & \begin{tabular}{|l|}
0.15 \\
\end{tabular} & 0.736 & 80.1 & 84.55 & 93.45 & 97.9 \\
\hline '2011-01-12 07:53:40' & 'No' & 70 & 77.00 & -29.7775 & 0.668794 & 2 & 69.3 & 77 & 84.7 & 8.7 & 1 & 8.7 & 0.226 & \begin{tabular}{|l|l|}
0.548 \\
\end{tabular} & 0.226 & 76.5 & 80.75 & 89.25 & 93.5 \\
\hline '2011-01-12 07:54:00' & 'No' & 73 & 67.65 & -38.3013 & 0.732211 & 1 & 63 & 70 & 77 & 1 & 8 & 15 & 0.552 & 0.23 & 0.217 & 69.3 & 73.15 & 80.85 & 84.7 \\
\hline '2011-01-12 07:54:20' & 'No' & 79 & 68.50 & -37.5333 & 0.8568 & 1 & 65.7 & 73 & 80.3 & 1 & 8.3 & 15.6 & \begin{tabular}{|l|l|}
0.732 \\
\end{tabular} & 0.153 & 0.115 & 63 & 66.5 & 73.5 & 77 \\
\hline '2011-01-12 07:54:40' & 'No' & 83 & 79.00 & -27.9535 & 0.669366 & 2 & 71.1 & 79 & 86.9 & 8.9 & 1 & 8.9 & 0.226 & \begin{tabular}{|l|l|}
0.548 \\
\end{tabular} & 0.226 & 65.7 & 69.35 & 76.65 & 80.3 \\
\hline '2011-01-12 07:55:00' & 'No' & 79 & 85.82 & -21.7364 & 0.734983 & 3 & 74.7 & 83 & 91.3 & 17.6 & 9.3 & 1 & \begin{tabular}{|l|}
0.216 \\
\end{tabular} & 0.228 & 0.556 & 71.1 & 75.05 & 82.95 & 86.9 \\
\hline '2011-01-12 07:55:20' & 'No' & 76 & 83.90 & \begin{tabular}{|l|}
-23.483 \\
\end{tabular} & 0.858581 & 3 & 71.1 & 79 & 86.9 & 16.8 & 8.9 & 1 & \begin{tabular}{|l|}
0.114 \\
\end{tabular} & 0.151 & 0.735 & 74.7 & 78.85 & 87.15 & 91.3 \\
\hline '2011-01-12 07:55:40' & 'No' & 73 & 82.12 & -25.1093 & 0.929259 & 3 & 68.4 & 76 & 83.6 & 16.2 & 8.6 & 1 & \begin{tabular}{|l|}
0.053 \\
\end{tabular} & \begin{tabular}{|l|l|}
0.088 \\
\end{tabular} & 0.859 & 71.1 & 75.05 & 82.95 & 86.9 \\
\hline '2011-01-12 07:56:00' & 'No' & 64 & 79.61 & -27.3931 & 0.965651 & 3 & 65.7 & 73 & 80.3 & 15.6 & 8.3 & 1 & \begin{tabular}{|l|}
0.023 \\
\end{tabular} & \begin{tabular}{|l|l|}
0.048 \\
\end{tabular} & \begin{tabular}{|l|}
0.929 \\
\end{tabular} & 68.4 & 72.2 & 79.8 & 83.6 \\
\hline '2011-01-12 07:56:20' & 'No' & 66 & 61.87 & -43.5753 & 0.730586 & 1 & 57.6 & 64 & 70.4 & 1 & 7.4 & 13.8 & 0.55 & \begin{tabular}{|l|}
0.232 \\
\end{tabular} & 0.218 & 65.7 & 69.35 & 76.65 & 80.3 \\
\hline '2011-01-12 07:56:40' & 'No' & 69 & 61.94 & \begin{tabular}{|l|}
-43.511 \\
\end{tabular} & 0.855753 & 1 & 59.4 & 66 & 72.6 & 1 & 7.6 & 14.2 & \begin{tabular}{|l|l|}
0.731 \\
\end{tabular} & 0.154 & 0.116 & 57.6 & 60.8 & 67.2 & 70.4 \\
\hline
\end{tabular}


Cont.

\begin{tabular}{|c|c|c|c|c|c|c|c|c|c|c|c|c|c|c|c|c|c|c|c|}
\hline 'Date_time' & 'incedent ' & $\left|\begin{array}{c}\text { 'measured } \\
\text { SP' }\end{array}\right|$ & $\left|\begin{array}{c}\text { 'Predicted } \\
\text { speed' }\end{array}\right|$ & $\begin{array}{l}\text { 'speed } \\
\text { drop' }\end{array}$ & \begin{tabular}{|c|} 
'MAX \\
posterior \\
\end{tabular} & 'state' & $\begin{array}{c}\text { 'Speed } \\
\text { s1' }\end{array}$ & $\begin{array}{c}\text { 'Speed } \\
\text { s2' }\end{array}$ & $\left|\begin{array}{c}\text { 'Speed } \\
\text { s3' }\end{array}\right|$ & $\mid \begin{array}{c}\text { 'delta } \\
\text { s1' }\end{array}$ & $\begin{array}{c}\text { 'delta } \\
\text { s2' }\end{array}$ & $\mid \begin{array}{c}\text { 'delta } \\
\text { s3' }\end{array}$ & \begin{tabular}{|c|} 
Prior \\
Prob \\
s1' \\
\end{tabular} & \begin{tabular}{|c|} 
'Prior_- \\
Prob \\
s2'
\end{tabular} & \begin{tabular}{|c|} 
'Prior_- \\
Prob \\
s3'
\end{tabular} & \begin{tabular}{|c|} 
'(1- \\
RBTCS)* \\
OSP' \\
\end{tabular} & $\begin{array}{c}\text { '(1- } \\
0.5^{*} \text { RBTC } \\
\text { S) }{ }^{*} \text { OSP;' }\end{array}$ & $\begin{array}{c}\text { '(1+0.5*R } \\
\text { BTCS }) * O S \\
\mathrm{P}^{\prime}\end{array}$ & $\begin{array}{l}\text { '(1+RBTC } \\
\text { S)*OSP;' }\end{array}$ \\
\hline '2011-01-12 07:57:00' & 'No' & 72 & 63.47 & -42.1175 & 0.927685 & 1 & \begin{tabular}{ll|}
62.1 \\
\end{tabular} & 69 & 75.9 & 1 & 7.9 & 14.8 & 0.856 & \begin{tabular}{|l|}
0.09 \\
\end{tabular} & 0.054 & 59.4 & 62.7 & 69.3 & 72.6 \\
\hline '2011-01-12 07:57:20' & 'No' & 75 & 65.49 & -40.2746 & 0.964837 & 1 & \begin{tabular}{ll|}
64.8 \\
\end{tabular} & 72 & 79.2 & 1 & 8.2 & 15.4 & 0.928 & \begin{tabular}{|l|}
0.049 \\
\end{tabular} & \begin{tabular}{|l|}
0.023 \\
\end{tabular} & 62.1 & 65.55 & 72.45 & 75.9 \\
\hline '2011-01-12 07:57:40' & 'No' & 84 & 67.84 & -38.134 & 0.983081 & 1 & 67.5 & 75 & 82.5 & 1 & 8.5 & 16 & 0.965 & \begin{tabular}{|l|}
0.025 \\
\end{tabular} & 0.01 & 64.8 & 68.4 & 75.6 & 79.2 \\
\hline '2011-01-12 07:58:00' & 'No' & 80 & 86.85 & -20.7914 & 0.735163 & 3 & 75.6 & 84 & 92.4 & 17.8 & 9.4 & 1 & 0.216 & 0.228 & 0.556 & 67.5 & 71.25 & 78.75 & 82.5 \\
\hline '2011-01-12 07:58:20' & 'No' & 77 & 84.97 & -22.5128 & 0.858697 & 3 & 72 & 80 & 88 & 17 & 9 & 1 & \begin{tabular}{|l|}
0.114 \\
\end{tabular} & 0.15 & 0.735 & 75.6 & 79.8 & 88.2 & 92.4 \\
\hline '2011-01-12 07:58:40' & 'No' & 74 & 83.20 & -24.1229 & 0.929324 & 3 & 69.3 & 77 & 84.7 & 16.4 & 8.7 & 1 & 0.053 & \begin{tabular}{|l|}
0.088 \\
\end{tabular} & 0.859 & 72 & 76 & 84 & 88 \\
\hline '2011-01-12 07:59:00' & 'No' & 71 & 80.71 & -26.3979 & 0.965684 & 3 & 66.6 & 74 & 81.4 & 15.8 & 8.4 & 1 & 0.023 & 0.048 & \begin{tabular}{|l|}
0.929 \\
\end{tabular} & 69.3 & 73.15 & 80.85 & 84.7 \\
\hline '2011-01-12 07:59:20' & 'No' & 68 & 77.79 & \begin{tabular}{|l|}
-29.0588 \\
\end{tabular} & 0.983505 & 3 & 63.9 & 71 & 78.1 & \begin{tabular}{|l|}
15.2 \\
\end{tabular} & 8.1 & 1 & \begin{tabular}{|l|}
0.01 \\
\end{tabular} & 0.025 & 0.966 & 66.6 & 70.3 & 77.7 & 81.4 \\
\hline '2011-01-12 07:59:40' & 'No' & 65 & 74.66 & -31.9104 & 0.992076 & 3 & 61.2 & 68 & 74.8 & 14.6 & 7.8 & 1 & 0.004 & \begin{tabular}{|l|}
0.013 \\
\end{tabular} & 0.984 & 63.9 & 67.45 & 74.55 & 78.1 \\
\hline '2011-01-12 08:00:00' & 'No' & 62 & 71.44 & \begin{tabular}{|l|}
-34.8497 \\
\end{tabular} & 0.99618 & 3 & 58.5 & 65 & 71.5 & 14 & 7.5 & 1 & \begin{tabular}{|l|}
0.002 \\
\end{tabular} & \begin{tabular}{|l|}
0.006 \\
\end{tabular} & 0.992 & 61.2 & 64.6 & 71.4 & 74.8 \\
\hline '2011-01-12 08:00:20' & 'No' & 57 & 68.17 & -37.8281 & 0.99815 & 3 & 55.8 & 62 & 68.2 & 13.4 & 7.2 & 1 & $6 \mathrm{E}-04$ & 0.003 & 0.996 & 58.5 & 61.75 & 68.25 & 71.5 \\
\hline '2011-01-12 08:00:40' & 'No' & 53 & 57.00 & -48.0171 & 0.66115 & 2 & 51.3 & 57 & 62.7 & 6.7 & 1 & 6.7 & 0.23 & \begin{tabular}{|l|}
0.539 \\
\end{tabular} & 0.23 & 55.8 & 58.9 & 65.1 & 68.2 \\
\hline '2011-01-12 08:01:00' & 'No' & 55 & 51.27 & -53.2453 & 0.726754 & 1 & 47.7 & 53 & 58.3 & 1 & 6.3 & 11.6 & 0.546 & 0.235 & \begin{tabular}{|l|}
0.219 \\
\end{tabular} & 51.3 & 54.15 & 59.85 & 62.7 \\
\hline '2011-01-12 08:01:20' & 'No' & 57 & 51.64 & -52.9017 & 0.853278 & 1 & 49.5 & 55 & 60.5 & 1 & 6.5 & 12 & 0.727 & 0.157 & \begin{tabular}{|l|}
0.117 \\
\end{tabular} & 47.7 & 50.35 & 55.65 & 58.3 \\
\hline '2011-01-12 08:01:40' & 'Yes' & 59 & 52.45 & $\mid-52.1681$ & \begin{tabular}{|l|}
0.926304 \\
\end{tabular} & 1 & 51.3 & 57 & 62.7 & 1 & 6.7 & 12.4 & \begin{tabular}{|l|}
0.853 \\
\end{tabular} & \begin{tabular}{|l|}
0.092 \\
\end{tabular} & \begin{tabular}{|l|}
0.055 \\
\end{tabular} & 49.5 & 52.25 & 57.75 & 60.5 \\
\hline '2011-01-12 08:02:00' & 'No' & 61 & 53.68 & -51.0494 & 0.964122 & 1 & 53.1 & 59 & 64.9 & 1 & 6.9 & 12.8 & \begin{tabular}{|l|}
0.926 \\
\end{tabular} & \begin{tabular}{|l|}
0.05 \\
\end{tabular} & \begin{tabular}{|l|}
0.024 \\
\end{tabular} & 51.3 & 54.15 & 59.85 & 62.7 \\
\hline '2011-01-12 08:02:20' & 'No' & 63 & 55.18 & -49.6776 & 0.982724 & 1 & 54.9 & 61 & 67.1 & 1 & 7.1 & 13.2 & 0.964 & \begin{tabular}{|l|}
0.026 \\
\end{tabular} & 0.01 & 53.1 & 56.05 & 61.95 & 64.9 \\
\hline '2011-01-12 08:02:40' & 'No' & 65 & 56.83 & -48.1682 & 0.99169 & 1 & 56.7 & 63 & 69.3 & 1 & 7.3 & 13.6 & \begin{tabular}{|l|}
0.983 \\
\end{tabular} & \begin{tabular}{|l|}
0.013 \\
\end{tabular} & 0.004 & 54.9 & 57.95 & 64.05 & 67.1 \\
\hline '2011-01-12 08:03:00' & 'No' & 67 & 58.56 & -46.5902 & 0.995991 & 1 & 58.5 & 65 & 71.5 & 1 & 7.5 & 14 & 0.992 & 0.007 & 0.002 & 56.7 & 59.85 & 66.15 & 69.3 \\
\hline '2011-01-12 08:03:20' & 'No' & 70 & 60.33 & -44.9791 & |0.998057 & 1 & 60.3 & 67 & 73.7 & 1 & 7.7 & 14.4 & 0.996 & \begin{tabular}{|l|}
0.003 \\
\end{tabular} & 7E-04 & 58.5 & 61.75 & 68.25 & 71.5 \\
\hline '2011-01-12 08:03:40' & 'No' & 73 & 63.02 & -42.5311 & 0.999054 & 1 & 63 & 70 & 77 & 1 & 8 & 15 & \begin{tabular}{|l|}
0.998 \\
\end{tabular} & \begin{tabular}{|l|}
0.002 \\
\end{tabular} & \begin{tabular}{|l|}
$3 E-04$ \\
\end{tabular} & 60.3 & 63.65 & 70.35 & 73.7 \\
\hline '2011-01-12 08:04:00' & 'No' & 81 & 65.71 & -40.0759 & 0.999537 & 1 & 65.7 & 73 & 80.3 & 1 & 8.3 & 15.6 & \begin{tabular}{|l|}
0.999 \\
\end{tabular} & $8 \mathrm{E}-04$ & 1E-04 & 63 & 66.5 & 73.5 & 77 \\
\hline '2011-01-12 08:04:20' & 'No' & 77 & 83.74 & -23.6265 & \begin{tabular}{|l|}
0.73461 \\
\end{tabular} & 3 & 72.9 & 81 & 89.1 & 17.2 & 9.1 & 1 & \begin{tabular}{|l|}
0.217 \\
\end{tabular} & \begin{tabular}{|l|}
0.228 \\
\end{tabular} & 0.555 & 65.7 & 69.35 & 76.65 & 80.3 \\
\hline '2011-01-12 08:04:40' & 'No' & 74 & 81.77 & -25.4234 & 0.858342 & 3 & 69.3 & 77 & 84.7 & 16.4 & 8.7 & 1 & \begin{tabular}{|l|}
0.115 \\
\end{tabular} & 0.151 & 0.735 & 72.9 & 76.95 & 85.05 & 89.1 \\
\hline '2011-01-12 08:05:00' & 'No' & 71 & 79.96 & -27.0821 & 0.929126 & 3 & 66.6 & 74 & 81.4 & 15.8 & 8.4 & 1 & 0.054 & \begin{tabular}{|l|}
0.088 \\
\end{tabular} & 0.858 & 69.3 & 73.15 & 80.85 & 84.7 \\
\hline '2011-01-12 08:05:20' & 'No' & 68 & 77.43 & -29.3833 & 0.965582 & 3 & 63.9 & 71 & 78.1 & 15.2 & 8.1 & 1 & 0.023 & \begin{tabular}{|l|}
0.048 \\
\end{tabular} & 0.929 & 66.6 & 70.3 & 77.7 & 81.4 \\
\hline '2011-01-12 08:05:40' & 'No' & 64 & 74.50 & \begin{tabular}{|l|}
-32.057 \\
\end{tabular} & 0.983454 & 3 & 61.2 & 68 & 74.8 & 14.6 & 7.8 & 1 & \begin{tabular}{|l|}
0.01 \\
\end{tabular} & 0.025 & 0.966 & 63.9 & 67.45 & 74.55 & 78.1 \\
\hline '2011-01-12 08:06:00' & 'No' & 62 & 64.00 & -41.6332 & 0.664306 & 2 & 57.6 & 64 & 70.4 & 7.4 & 1 & 7.4 & \begin{tabular}{|l|}
0.229 \\
\end{tabular} & \begin{tabular}{|l|}
0.543 \\
\end{tabular} & 0.229 & 61.2 & 64.6 & 71.4 & 74.8 \\
\hline '2011-01-12 08:06:20' & 'No' & 59 & 62.00 & -43.4572 & 0.767343 & 2 & 55.8 & 62 & 68.2 & 7.2 & 1 & 7.2 & 0.168 & \begin{tabular}{|l|}
0.664 \\
\end{tabular} & \begin{tabular}{|l|}
0.168 \\
\end{tabular} & 57.6 & 60.8 & 67.2 & 70.4 \\
\hline '2011-01-12 08:06:40' & 'No' & 55 & 59.00 & |-46.1931 & 0.846082 & 2 & 53.1 & 59 & 64.9 & 6.9 & 1 & 6.9 & 0.116 & \begin{tabular}{|l|}
0.767 \\
\end{tabular} & \begin{tabular}{|l|}
0.116 \\
\end{tabular} & 55.8 & 58.9 & 65.1 & 68.2 \\
\hline '2011-01-12 08:07:00' & 'No' & 57 & 53.20 & -51.487 & 0.727553 & 1 & 49.5 & 55 & 60.5 & 1 & 6.5 & 12 & \begin{tabular}{|l|}
0.547 \\
\end{tabular} & 0.235 & \begin{tabular}{|l|}
0.219 \\
\end{tabular} & 53.1 & 56.05 & 61.95 & 64.9 \\
\hline '2011-01-12 08:07:20' & 'No' & 59 & 53.52 & -51.1942 & 0.853795 & 1 & 51.3 & 57 & 62.7 & 1 & 6.7 & 12.4 & \begin{tabular}{|l|}
0.728 \\
\end{tabular} & 0.156 & 0.116 & 49.5 & 52.25 & 57.75 & 60.5 \\
\hline '2011-01-12 08:07:40' & 'No' & 61 & 54.28 & -50.4932 & 0.926592 & 1 & 53.1 & 59 & 64.9 & 1 & 6.9 & 12.8 & \begin{tabular}{|l|}
0.854 \\
\end{tabular} & 0.092 & \begin{tabular}{|l|}
0.055 \\
\end{tabular} & 51.3 & 54.15 & 59.85 & 62.7 \\
\hline '2011-01-12 08:08:00' & 'No' & 60 & 55.49 & \begin{tabular}{|l|}
-49.392 \\
\end{tabular} & 0.964271 & 1 & 54.9 & 61 & 67.1 & 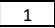 & 7.1 & 13.2 & \begin{tabular}{|l|}
0.927 \\
\end{tabular} & 0.05 & \begin{tabular}{|l|}
0.024 \\
\end{tabular} & 53.1 & 56.05 & 61.95 & 64.9 \\
\hline '2011-01-12 08:08:20' & 'No' & 64 & 54.27 & -50.5035 & 0.982798 & 1 & 54 & 60 & 66 & 1 & 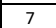 & 13 & 0.964 & \begin{tabular}{|l|}
0.026 \\
\end{tabular} & 0.01 & 54.9 & 57.95 & 64.05 & 67.1 \\
\hline '2011-01-12 08:08:40' & 'No' & 57 & 64.00 & -41.6332 & 0.664306 & 2 & 57.6 & 64 & 70.4 & 7.4 & 1 & 7.4 & 0.229 & \begin{tabular}{|l|}
0.543 \\
\end{tabular} & \begin{tabular}{|l|}
0.229 \\
\end{tabular} & 54 & 57 & 63 & 66 \\
\hline '2011-01-12 08:09:00' & 'No' & 51 & 55.12 & -49.7288 & 0.728301 & 1 & 51.3 & 57 & 62.7 & 1 & 6.7 & 12.4 & \begin{tabular}{|l|}
0.548 \\
\end{tabular} & \begin{tabular}{|l|}
0.234 \\
\end{tabular} & \begin{tabular}{|l|}
0.218 \\
\end{tabular} & 57.6 & 60.8 & 67.2 & 70.4 \\
\hline '2011-01-12 08:09:20' & 'No' & 30 & 47.88 & -56.336 & 0.854279 & 1 & 45.9 & 51 & 56.1 & 1 & 6.1 & 11.2 & \begin{tabular}{|l|}
0.728 \\
\end{tabular} & 0.156 & 0.116 & 51.3 & 54.15 & 59.85 & 62.7 \\
\hline '2011-01-12 08:09:40' & 'No' & 20 & 27.60 & \begin{tabular}{|l|}
-74.8287 \\
\end{tabular} & 0.926862 & 1 & 27 & 30 & 33 & 1 & 4 & 7 & 0.854 & \begin{tabular}{|l|}
0.091 \\
\end{tabular} & 0.055 & 45.9 & 48.45 & 53.55 & 56.1 \\
\hline '2011-01-12 08:10:00' & 'No' & 10 & 18.19 & \begin{tabular}{|l|}
-83.4078 \\
\end{tabular} & 0.964411 & 1 & 18 & 20 & 22 & 1 & 3 & 5 & \begin{tabular}{|l|}
0.927 \\
\end{tabular} & \begin{tabular}{|l|}
0.049 \\
\end{tabular} & \begin{tabular}{|l|}
0.024 \\
\end{tabular} & 27 & 28.5 & 31.5 & 33 \\
\hline
\end{tabular}

\subsection{Algorithm Validation-Utilizing the Developed Matlab Program}

The validation method was applied to the available verified database that included 260 incidents and non-incident data set. The developed calibrated algorithm programmed in Matlab language was used. Among the 260 cases, there were 130 cases where incidents were recorded while in the other 130 cases no incidents were recorded. The results are shown in Table 6.3, which confirmed that the developed algorithm achieves satisfactory results. After finer calibration (i.e. with the deployment of the third condition and the fine tuning), it was observed that the developed model performed much better than the results that were previously reported in chapter 5 . 
It also appears that the developed model has benefited from a high level of calibration tests to screen out false alarms. It was shown that the FAR was reduced during the calibration test.

The next step (Step 4) was to test the calibrated algorithm on the second set of data that included validation dataset, with the threshold values obtained in Step 3.

Results from the analysis showed that out of the 130 cases under recorded incident consideration, $29 \%$ of the cases showed a significant drop in speed and the incident was subject to further detection investigation by using the algorithm. Although $56 \%$ of the cases exhibited an incident recording, however, these cases did not show any drop in speed and were not subject to any further incident detection investigation. At the same time, $15 \%$ of the cases showed some degree of drop in speed and was subject to further study.

Moreover, analysis results show that out of 130 cases under "no recorded incident consideration", $3 \%$ of the cases showed a significant drop in speed and the incident was subject to further detection investigation by using the algorithm. In addition, 97\% of the cases exhibited no incident recording and were subject to further incident detection investigations.

As a result, it was decided to classify the cases into three categories according to the possible expected traffic condition. Please see the following subsection for further discussions.

\subsubsection{Incident Patterns}

In order to bring these results forward for additional study, three categories describing the traffic conditions were proposed to further reduce the false alarm rate. 
The proposed categories with the associated traffic conditions are as follows:

1- Must Detect: The first part of this category considered cases that showed recorded incidents and had high potential for the detection of an incident. In these cases, a drop in speed occurred and low-speed values were maintained. One of these cases is illustrated in Figure 6.43, and other similar cases are presented in Appendix C.1. In this category of cases, traffic condition had much potential to point to an incident. It was expected that the algorithm must detect incidents in this category. Any incident case that was not successfully detected in this category adversely impacted the detection rate of the algorithm.

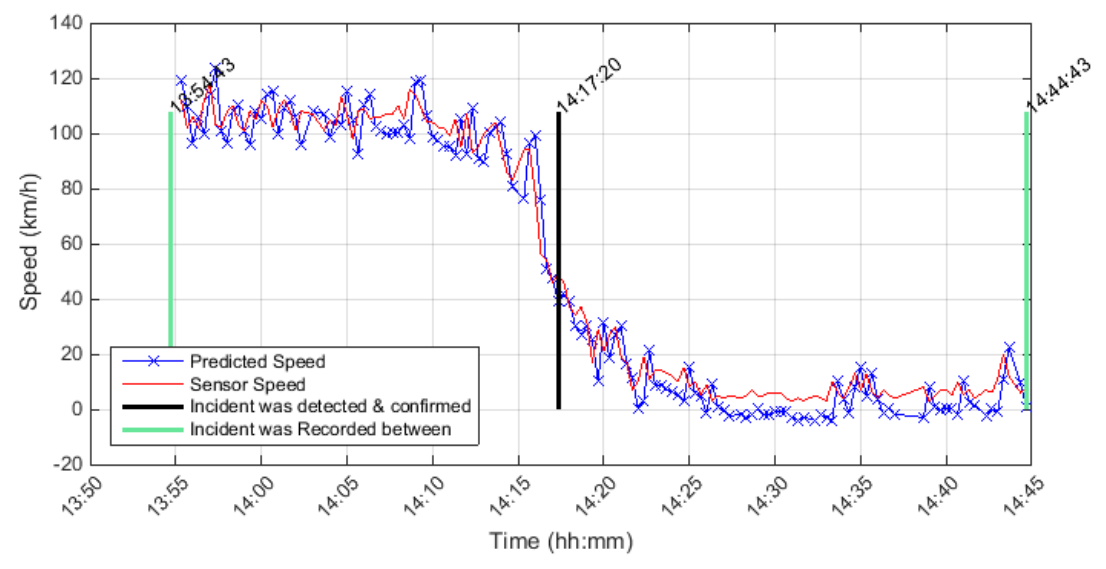

Upstream Detector \# is 401DE0320DEC, Detector Rank $=1$, Distance from incident location in $(\mathrm{km})=0.23461$ Season $=$ Winter, Stream $=$ C, Direction $=E$, Date $\&$ Time $=2011-12-2314: 14: 43$ Status = UPDATED 2011-12-23 14:34:25, Incident Reason = Collision Affected Lanes $=3$ LEFT LANE(S)

Conditional Probability level $=0.6$, Cond. Prob. $r 3 s 2=0.2$, Cond.Prob. $r 3 s 1=0.1$, Percent drop in speed $\%=-60$

Max. Posterior Probability level $=0.97$, Absolute range between speed states $=7 \mathrm{~km}$

Incident was detected at 01:20 minutes from drop in speed point. Incident was confirmed after 5 minutes from the detection time

\section{Figure 6.43: First category, must detect}

2- Must Not Detect: The second part of this category involves cases with no incidents and offered high potential for being recognized as "incident-free". One of these cases 
is illustrated in Figure 6.44, other similar cases are presented in Appendix C.4. In this category, traffic condition was representing a low possibility of incident occurrence, and it was expected that the algorithm must not detect any incident. Any incident detection within these cases adversely impacted the "no detection rate" of the algorithm.

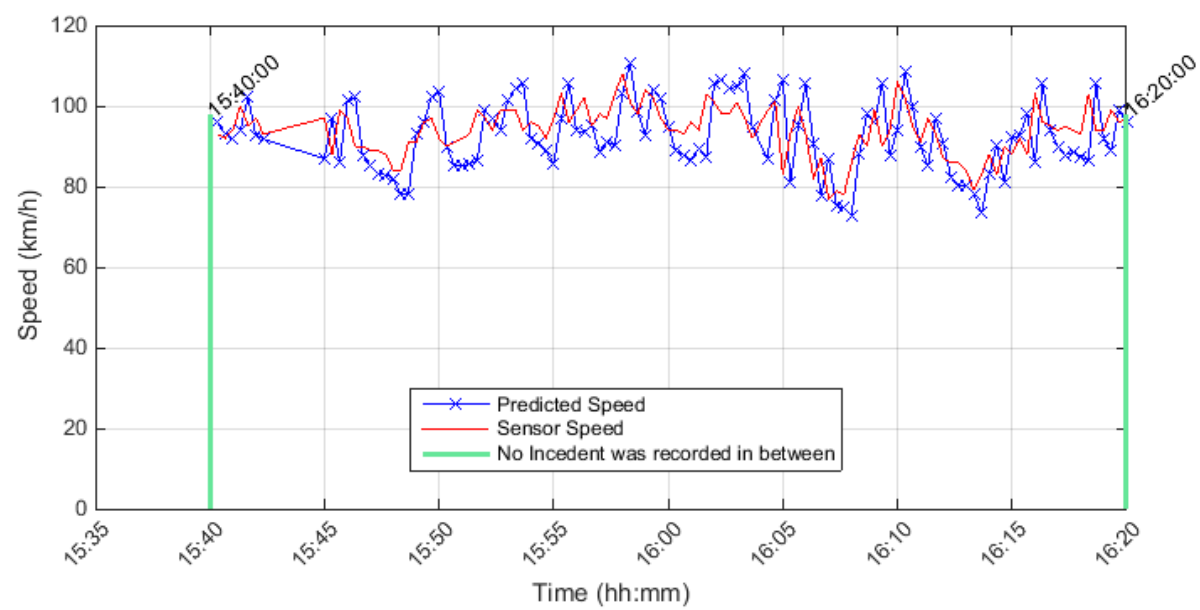

Upstream Detector \# is 401DW0160DWC, Detector Rank = 1, Distance from incident free location in $(\mathrm{km})=0.1854$ Incident free Season $=$ Spring, Stream $=$ C, Direction $=W$, Date \& Time $=2011-05-04$ 06:59:13 Selected incident free Date/Time $=2011-05-04$ 16:00:00

Conditional Probability level $=0.6$, Cond.Prob. $r 3 s 2=0.2$, Cond.Prob. $r 3 s 1=0.1$, Percent drop in speed $\%=-56$ Max. Posterior Probability level $=0.97$, Percent range between speed states $=7 \%$

(No Incident was Detected)

Figure 6.44: Second category, must not detect

3- Some Degree of Benefit: The third part of this category considered cases that showed recorded incidents and offered some degree of incident detection potential. This category considered cases where a number of lanes were affected by the incident. Traffic volume was also expected to be low during off-peak hours or weekends. In these cases, some drop in speed occurred and also speed fluctuated rapidly as illustrated in Figure 6.45. Other similar cases are presented in Appendix C.2. 


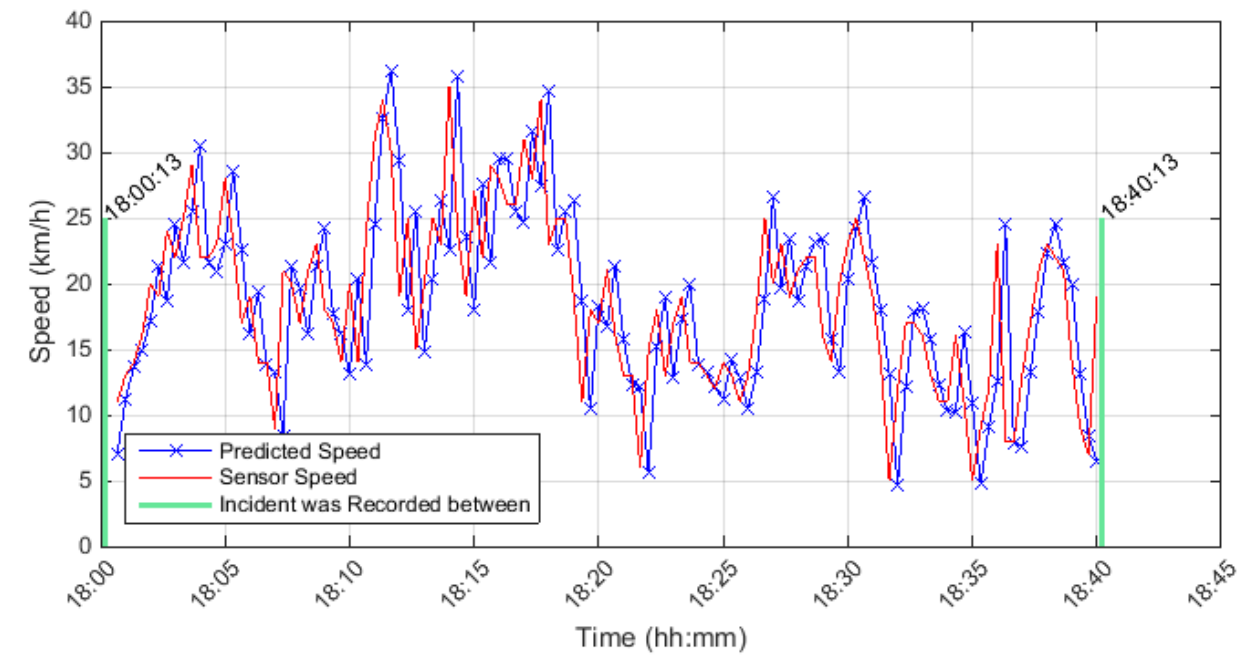

\footnotetext{
Upstream Detector \# is 401DE0160DWC, Detector Rank = 1, Distance from incident location in $(\mathrm{km})=0.12541$

Season $=$ Spring, Stream $=$ C, Direction $=$ W, Date $\&$ Time $=$ 2011-05-13 18:20:13

Incident Reason $=$ Collision

Affected Lanes $=2$ RIGHT LANE(S) AND RIGHT SHOULDER

Conditional Probability level $=0.6$, Cond.Prob. $r 3 s 2=0.2$, Cond. Prob. $r 3 s 1=0.1$, Percent drop in speed $\%=-56$

Max. Posterior Probability level $=0.97$. Percent range between speed states $=7 \%$

Drop in speed was detected but Not enough recovery time to confirm an incident
}

\section{Figure 6.45: Third category, some degree of benefit}

In the third category, there are cases where speed drop did not occur and the state of low speed could not be observed. Figure 6.46 presents such a case and other similar cases are presented in Appendix C.3. 

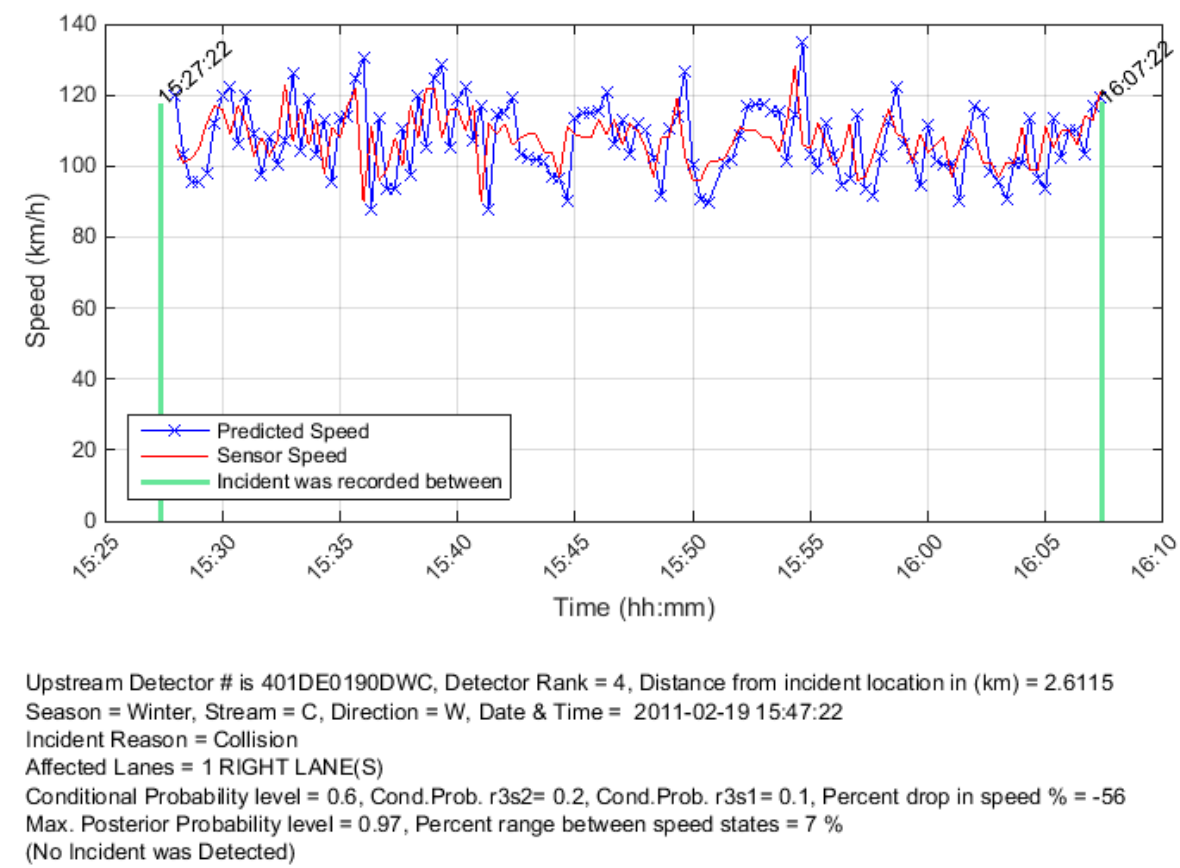

\section{Figure 6.46: Fourth category, not important}

\subsubsection{Proposed Approach}

- The first 130 cases out of the 400 randomly selected available sample were tested using the algorithm. The test was carried out during time periods with a recorded incident as well as during "incident-free time" periods. These test cases fall into the following categories. Total number of cases with an incident recorded 130. Out of these, 38 cases had traffic flow pattern that is commonly associated with the occurrence of an incident. Under these traffic condition, detection of an incident is a must and algorithms are commonly aimed at the detection of such incidents. There were 19 cases that if correctly detected could offer some degree of benefit. There were 73 cases that had no appreciable effect on traffic flow and therefore detection of these is not important. As 
in common in incident detection algorithm research as well as in real world traffic management field, detecting incidents that have potential to cause traffic disruptions is accorded much importance.

- Although detecting incidents with potential to cause traffic flow disruptions is important, traffic control centre personnel find false alarms to be annoying and much importance is assigned to minimization of the false alarm rate (FAR). The algorithm was tested by using 130 cases with no record of incidents.

\section{Outcomes}

In this research, the developed algorithm was used (a) to detect incidents in cases that are known to have incidents (DR), and (b) to find out the false alarm rate in cases that do not have recorded incidents. In this test, detection of an incident that did not happen becomes a "false alarm" and the rate of such alarms is termed the false alarm rate (FAR).

The following are the observations on the detection rate (DR)

(a) For category of cases with traffic flow pattern that offers much potential for incident detection, the algorithm achieved $84 \%$ detection rate (DR) and the remaining $16 \%$ of incidents were not detected, these were considered as missed detections.

As noted previously, many incidents do not significantly affect operating speed and therefore these cannot be detected with an algorithm that is calibrated to detect incidents that disrupt traffic conditions on the highway. If the traffic managers wish to learn about such incidents with very little impact potential, some other measures must be taken in these cases. Although these cases are representing $56 \%$ of the total selected cases under this 
category, it is proposed that these cases are beyond the scope of this research and cannot be included in evaluating the incident detection algorithm.

There is still a low percentage of the selected cases which offer some benefit if detected. Although in these cases there were some changes in the speed, but it was not enough for a complete incident indication and confirmation. Therefore, in $15 \%$ of cases, no incident was detected. If the algorithm is to be calibrated to give better results in such cases, the false alarm rate will significantly be affected. Two of these case are illustrated in Figure 6.47 and Figure 6.48 and other similar cases are presented in Appendix C.2. In these cases, changes in the traffic operating speed were not enough to allow to meet the incident detection criteria, as can be appreciated from the figures, the only available sign is a slight decline in the speed with big fluctuation in some cases. These are not enough for an incident occurrence indication. Therefore, the algorithm should not be applied in these cases with major benefit potential and run the risk of high false alarm rate.

(b) When the algorithm was applied to 130 cases with no record of an incident, only 3 false alarms were issued. This gives a false alarm rate of $0.038 \%$.

This rate is calculated based on the number of decisions the algorithm makes within the study period which is proposed to be 40 minutes for each case. Let us assume that the average time period required by the algorithm to trigger the alarm is 20 minutes. So, the average number of decisions for each case is $(20 * 3)$, since the decision is conducted each 20 seconds. Now, the total number of decisions for the 130 cases is $7800(130 * 20 * 3)$. This will give a false alarm rate of $(3 / 7800) * 100=0.038 \%$ 


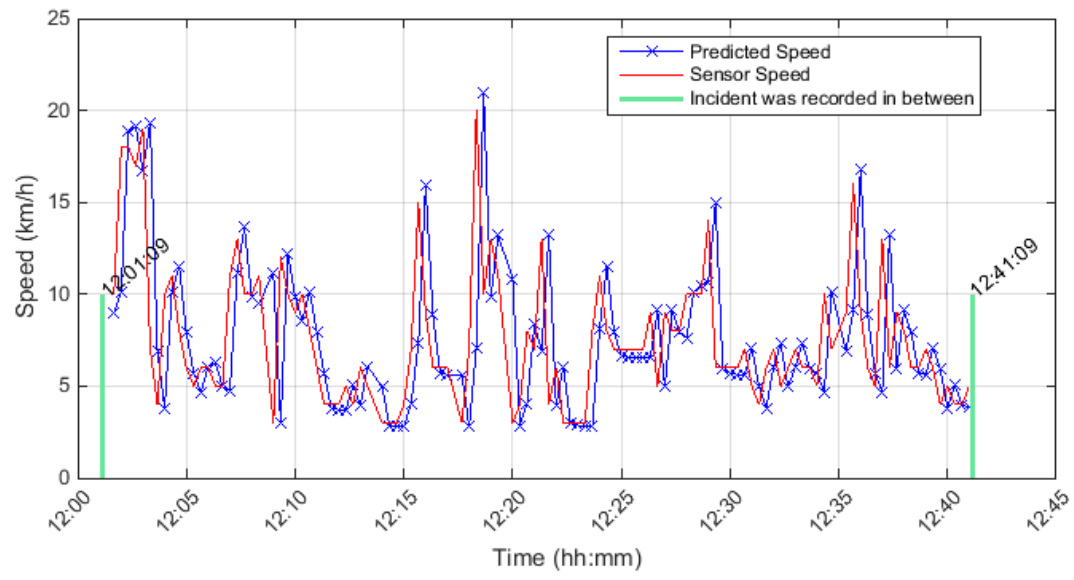

Upstream Detector \# is 401DW0060DWC, Detector Rank = 1, Distance from incident location in $(\mathrm{km})=0.43697$ Season $=$ Summer, Stream $=$ C, Direction $=$ W, Date $\&$ Time $=2011-10-21$ 12:21:09 Incident Reason $=$ Collision

Affected Lanes $=$ LEFT SHOULDER AND 1 LEFT LANE(S)

Conditional Probability level $=0.6$, Cond.Prob. $r 3 s 2=0.2$, Cond. Prob. $r 3 s 1=0.1$, Percent drop in speed $\%=-56$

Max. Posterior Probability level $=0.97$, Percent range between speed states $=7 \%$

Drop in speed was detected and No indication of an incident within this period

Figure 6.47: Some degree of benefit

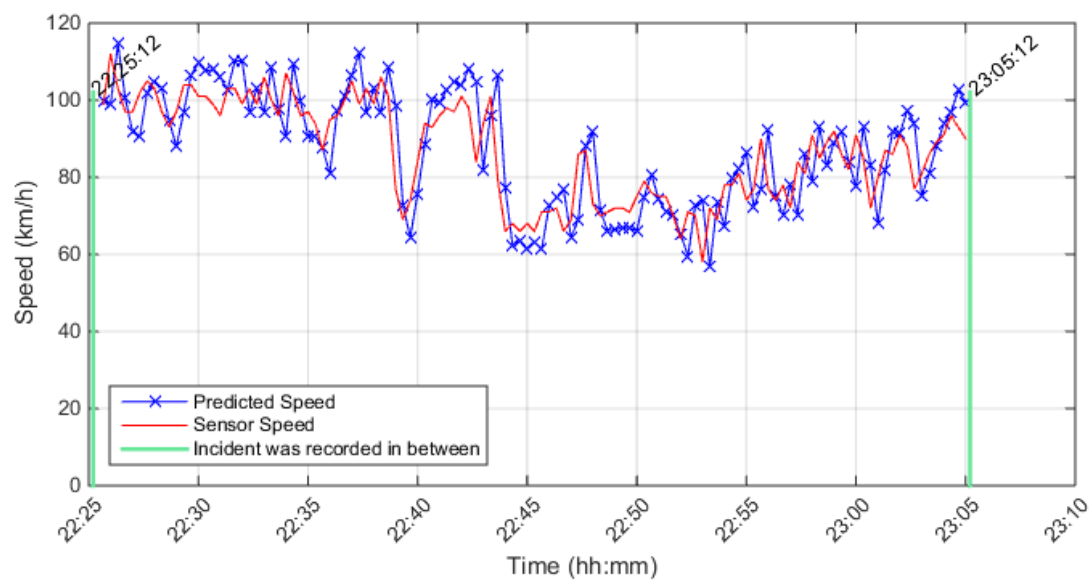

Upstream Detector \# is 401DE0100DWC, Detector Rank = 1, Distance from incident location in $(\mathrm{km})=0.11847$ Season $=$ Winter, Stream $=$ C, Direction $=$ W, Date $\&$ Time $=2011-12-24$ 22:45:12

Incident Reason $=$ Collision

Affected Lanes = 1 LEFT LANE(S)

Conditional Probability level $=0.6$, Cond.Prob. $r 3 s 2=0.2$, Cond.Prob. $r 3 s 1=0.1$, Percent drop in speed $\%=-40$ Max. Posterior Probability level $=0.97$, Percent range between speed states $=7 \%$

Drop in speed was detected and No indication of an incident within this period

\section{Figure 6.48: Some degree of benefit}




\subsection{Observations}

The incident detection analysis provides valuable information so that traffic control authorities can take timely action to reduce the impacts of incidents. The developed algorithm was calibrated and verified with the intent to detect those incidents that have high potential to disrupt traffic. When applied to a statistically significant sample of such cases, the detection rate was $84 \%$ and therefore $16 \%$ of known incidents were not detected. The false alarm rate was less than $3 \%$.

Analyses carried out on cases with a record of an incident, and the incident was correctly detected, the response can be quick, 5 minutes or less. The time when the incident was detected can be used as a reference. It was observed that the minimum time to detect (MTTD) is very small in most cases; it can be up to 2:00 min. If the drop in speed was not rapid, detection of the incident took more time. The point of the first drop in speed can be used only as a reference in this case.

For cases with a record of incident and if the incident was not detected by the algorithm, it is because the operating speed pattern does not give the required indication of dropping speed.

For cases without an incident, the algorithm correctly does not detect any incident in most cases, but three incidents were incorrectly detected by the algorithm. In these cases operating speed shows a rapid drop. Although there was no record of the incident in the archived data, the design of the algorithm resulted in incident detection. 


\section{Microsimulation as an Aid to Algorithm Testing and}

\section{Validation}

\subsection{Introduction}

A good and reliable incident detection model is an essential element of an effective traffic incident detection system that can potentially detect incidents quickly (Tian \& Dong, 2012).This research is aimed at the enhancement of the state of knowledge in incident detection model development. In addition to the use of actual archived data, it was decided to use traffic stochastic simulation technology to enhance the scope of the research. Traffic microsimulation can be used as a tool to develop, calibrate and test incident detection models and associated algorithms. This tool is used as a supplementary approach to actual archived data-based modelling approach described in previous chapters of this thesis.

Two different scenarios were considered in the simulation part of the study. In the first scenario, an incident occurred at a certain location and was recorded by the traffic control centre at some time of the day while in the second scenario an incident never occurred at any location and no indication of any incident at that time of the day was recorded. The developed incident detection model was applied to both scenarios to determine whether there was any incident within the specified period of time at that specified location. It was found that the developed model achieved the intended goals indicated earlier in this thesis.

When an incident occurs, a change in the traffic flow state is expected, and the information is usually provided by detectors placed at different locations in the highway as described earlier in this thesis. Traffic flow parameters defined by road detectors are 
transmitted to the traffic control centre where models are installed to detect incidents (Cheng, Lin, Liu, \& Gu, 2010).

Microsimulation with fine time resolution is a well-accepted tool for modelling traffic flow under a variety of conditions (Corens et al., 2011). In this research, data sources from Highway 401 were adopted to serve in microsimulation for incident detection model and algorithm testing tasks. The microsimulation software was used as a useful tool to generate traffic data. The obtained throughout data from the microsimulation software was used as an input for implementing and validating the incident detection model.

This chapter covers research in traffic incident detection under the belief that traffic parameters are obtainable from traffic simulation software. Results from Simulation software proved to represent the real world when imposing traffic incident on a freeway section.

\subsection{Vissim Microsimulation Software}

A number of traffic microsimulation packages are available to simulate and analyze the effect of an incident on highway networks. One of the most comprehensive microsimulation software is Vissim (Corens et al., 2011). It was developed and is marketed by the PTV Company. It can address changes in the traffic parameters that could happen after an incident is imposed in the planned freeway section (Pulugurtha, 2002). Therefore, Vissim was used to simulate a freeway section and generate real-time data. This simulator provides detailed and focused outputs, in tabular format and via animated graphics. (“9_State-of-the-Art Detector Technologies," 2001). 
Although some Highway 401 features were used, it was the intent of this part of research to work with a generic case study so that the results can be of general use and not just to freeways in the Toronto Region. The data generated was used for testing the algorithm and additional data was used for validation.

\subsection{Overview of Vissim Features and Capabilities}

Vissim, as a microsimulation software, provides detailed outputs on a fraction of a second basis which in turn makes more detailed analysis possible. It enables a user to graphically build a network including its attributes such as links, connectors, lane widths, speed zones, etc. Such a model of the highway can be constructed over a pre-defined graphical map. The user can analyze traffic operations including vehicle composition, lane allocation, etc. (Bloomberg, Hill, Box, \& Dale, 2000).

Another element of Vissim is the signal state generator (SSG) which is a standalone part and was not utilized in this research since we are not simulating interrupted flow facilities Interested readers can refer to (Martin, 2011) and (Bloomberg et al., 2000) for information on the SSG part of Vissim. Following a preliminary study, it was determined that Vissim is capable of simulating traffic incidents on the freeway. Vissim produces several types of output data, including travel time and speed. Furthermore, speed can be measured at different locations on the freeway section. However, it should be noted that Vissim can only measure the speed at each individual lane which might be one of the limitations that need to be considered while implementing incident traffic simulation. 


\subsection{Objectives and Methodology of Traffic Incident Simulations}

The objective of the simulation part of the research was to use Vissim to simulate and analyze the impact of incidents on traffic flow and to use the obtained throughputs to further validate the incident detection model. It should be noted that the real-world data on traffic flow under incident conditions reflect a wide variety of disruption features (e.g. location within the highway section, traffic volume in various lanes, etc.). On the other hand in Vissim, the incident is simulated by imposing sudden stops of vehicles.

To address the objective of incident simulation, a series of simulation runs were conducted by applying pre-defined freeway and traffic conditions (e.g. volume, road characteristics, and lane blockage configurations). After obtaining the before and after incident data, the developed algorithm that incorporates the incident detection model. The model parameters were updated in order to enhance performance in terms of high detection rate, low false alarm rate and short detection time.

\subsubsection{Methodology}

A section of a freeway (e.g. Highway 401 in the Greater Toronto Area) was built in the simulator.

Figure 7.1 illustrates the selected freeway section from Highway 401 used for simulation studies. 


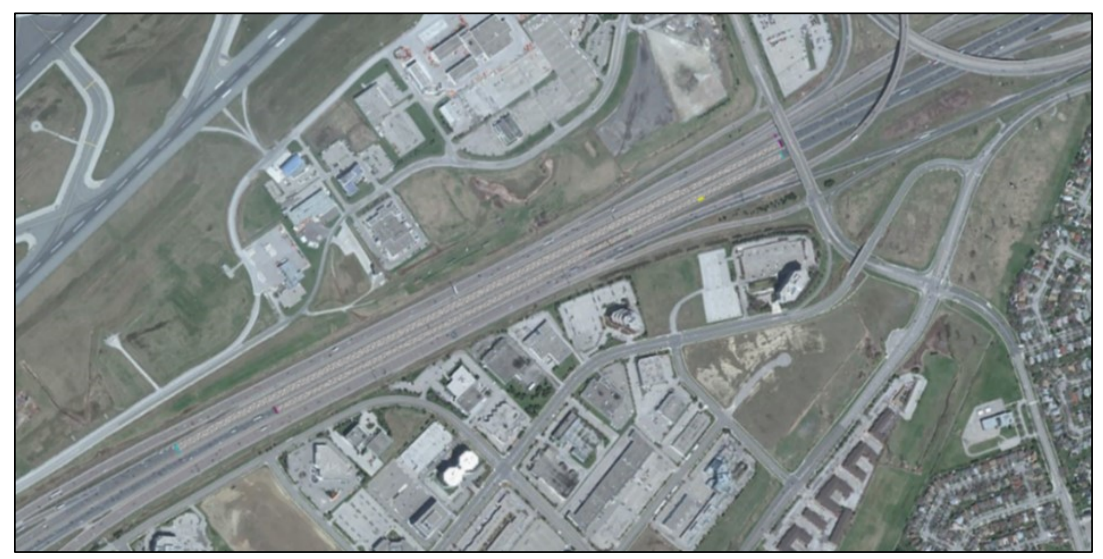

Figure 7.1: Study corridor - Hwy 401 in Toronto area

(Google Earth)

Real data obtained from detectors installed on Highway 401 was used to assist in constructing the microsimulation model.

\subsection{Traffic Incident Simulation Settings, or Incident Condition}

\section{Identification}

In the Vissim model each incident condition was defined by ten parameters:

Study corridor, geometric features, incident location, traffic operation characteristics, vehicle composition, lane blockage configuration, incident duration, detector location, incident identification in the Vissim simulation, incident model scenarios, and traffic flow data.

In the modeling process, these parameters were treated as variables, and changes of these variables were reflected in altered incident conditions. These variables are discussed in the following sections. 


\subsubsection{Study Corridor}

The first step was to define the study corridor of a freeway. For this purpose, Highway 401 was proposed as a good example of a study corridor for incident modeling. The HW401 section that was considered suitable as an example is located between Highway 427 and Highway 403/410, starting from a point located between Shuttle Dr. and Explorer Dr. to the overpass Renforth Dr. This section extends for $1140 \mathrm{~m}$ in the east direction of the express lanes of Highway 401. The section extends approximately between the coordinates $43.663754,-79.603615$ to $43.668410,-79.591494$ east.

Figure 7.2 shows the location of the study corridor/link used in the simulation study and Figure 7.3 presents lane configuration. The incident is assumed to take place at a distance of $950 \mathrm{~m}$ from the proposed starting point.

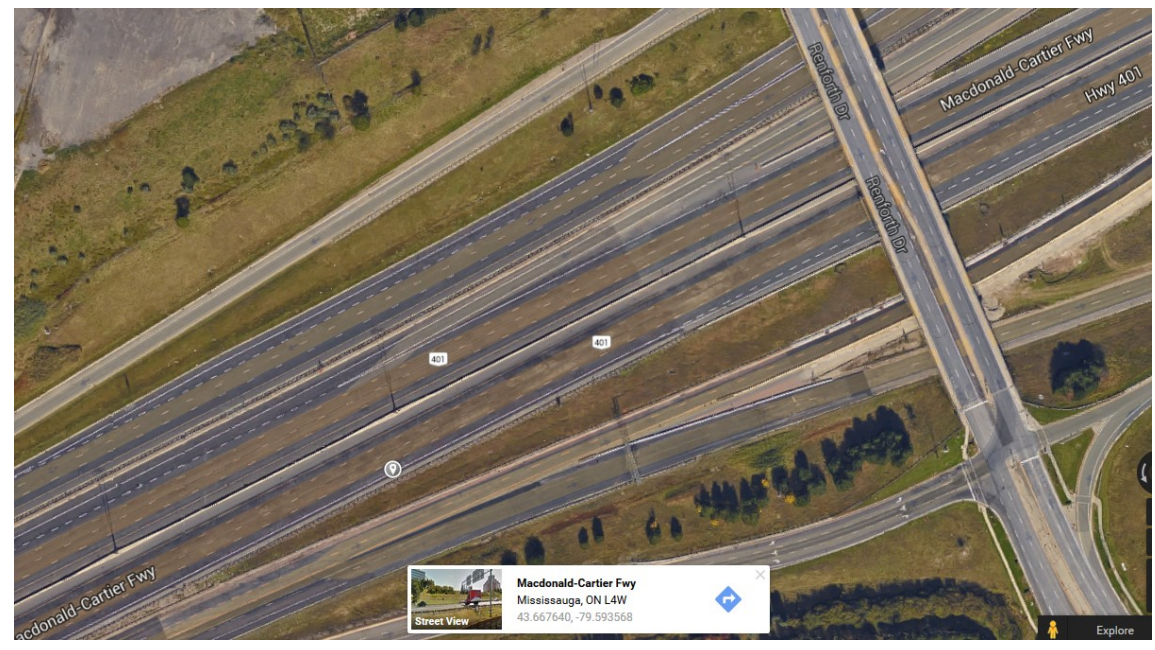

Figure 7.2: Location of the study corridor

(Google Earth) 


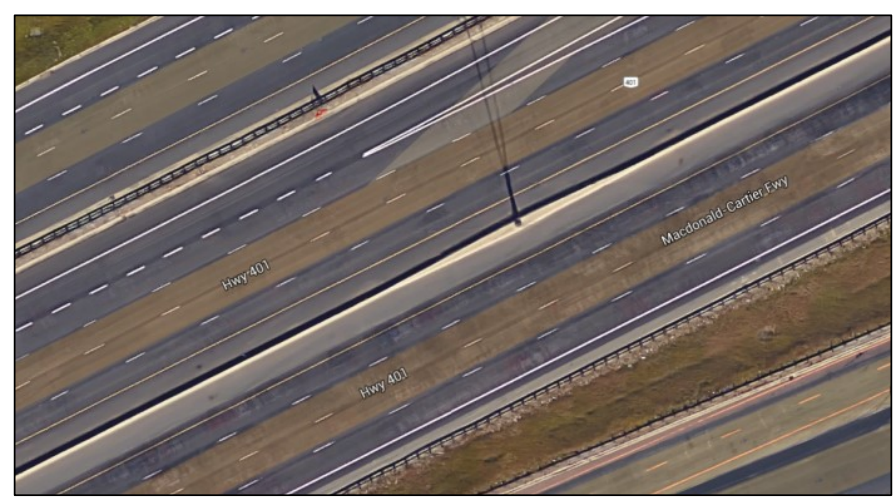

Figure 7.3: Study corridor - lane configuration

(Google Earth)

\subsubsection{Geometric Features}

The geometric features of the simulated freeway section includes relevant data regarding the length of the freeway section, number of lanes, lane widths. These parameters of the freeway section were defined as follows: a straight freeway section of $1140 \mathrm{~m}$ in length; four lanes with two shoulders, one on the right and the other on the left, traffic in the right lane with free lane changing behavior, and $3.5 \mathrm{~m}$ width of lane. The traffic is assumed to be moving in the eastbound and no existence for any on-ramp or off-ramp within the freeway section.

\subsubsection{Incident Locations}

According to one of the primary conditions in this research, incident location could be any location from the defined set of non-critical locations on the study corridor. This type of data was important because this research is not solely focused on the situations revealing high collision occurrence. The location of the incident was allocated so that the 
option for rerouting does not exist. It was assumed that incident took place at $950 \mathrm{~m}$ from the starting point of the simulated section.

\subsubsection{Traffic Operation Characteristics}

\subsubsection{Driving Behavior Parameters and Lane Changing}

In Vissim, driving behaviour was coded so as to simulate the real-world characteristics of traffic. Therefore, this enabled the possibility for any vehicle to apply lane change to the left or to the right (Abuelela, 2011).

In Vissim, vehicles change lanes to either perform necessary lane changes or free lane changes. Necessary lane changes are implemented to reach the next connector or route while free lane changes are permitted to vehicles to overtake slower leading traffic if higher speed is required (Abuelela, 2011).

In the current simulation approach, vehicles performed free lane changes. When an

incident occurred, vehicles slowed down to maintain the suitable gap with the leading vehicle and attempted to overtake to recover the required speed. Passing the leading vehicle required a change in lanes so that vehicles changed lanes according to the available safety distance in the direction of travel. This, in turn, depended on the speed of the vehicle changing lane and the speed of the vehicle approaching from behind on the lane to be switched to for free lane change. 


\subsubsection{Vehicle Composition}

To generate vehicles in Vissim, vehicle composition was defined. In addition to cars, low relative volume of trucks were also added to the traffic. This resulted in representative vehicle composition for the freeway facility.

\subsubsection{Lane Blockage Configuration,}

One of the important variables to define incident conditions is the number of lanes blocked for through traffic due to the incident. The impact of lane blockage is analyzed in details in the following section, from no lane blockage to only one lane blockage to full freeway blockage for the affected direction. Since the capacity reduction due to incidents is always proportional to the number of lanes blocked, lane blockage parameter has a significant impact on freeway capacity reduction and hence on vehicles' speed pattern traveling along the freeway section.

\subsubsection{Incident Duration}

Incident Duration is one more variable that defines incident conditions in Vissim microsimulation model. Incident duration is usually known as the time needed to detect incident through incident verification, emergency teams' response, scene clearance till normal traffic is retrieved (Brackstone, Fisher, \& McDonald, 2001). Many factors could have an impact on incident duration, including traffic volume, lane blockage configuration and other factors about the efficiency of the responding teams. These make it sometimes challenging to highway police patrol to give a precise prediction of incident duration. 
In this chapter, the incident duration is the period from the moment when the simulation starts to the moment when the simulation ends. One simulation period is proposed for the Vissim model, 10 minutes was found to be a suitable time frame to examine the implications of the simulated incident on the existing traffic flow. In reality, this would be treated as part of the clearance time, the time required to clear the lanes after the incident, which might vary from few minutes to more than an hour (Brackstone et al., 2001).

\subsubsection{Detector Location}

In Vissim, setting a detector is only associated with traffic signals, and since it is one of the characteristics of a freeway not to involve traffic signals, it was decided to seek other alternatives. Another feature in Vissim called Data Collection Point was utilized to collect traffic data as an alternative to road detectors. The data collection points were placed at a particular location to collect traffic data before and after the incident occurrence. One Data Collection Point was positioned in each lane $200 \mathrm{~m}$ upstream of the incident location and $750 \mathrm{~m}$ from the starting point of simulation.

The selected freeway section consists of four lanes; therefore, four data collection points were positioned at each lane to collect traffic data. Vehicles were generated at the starting point of the designated link in the study corridor, at speed fluctuating around the desired speed. According to the design of simulation, the generated traffic continued to travel along the link. Each lane demonstrated a number of vehicles traveling on it, and each data collection point recorded traffic information data. Some of the necessary traffic data pertained to vehicle entry time and vehicle speed. Other collected data included vehicle number in the network, vehicle type, vehicle length, occupancy, and vehicle acceleration. 
Vissim allowed the measurement of time a vehicle would take to travel between two specified points. Since the distance between these two points is known, therefore it was possible to calculate vehicle speed between the two specified points. Of course, it was necessary to use a common segment of the freeway to collect data on entry time of each vehicle at the boundary point and its associated speed. This method proved to be more complicated than the previous method of collecting traffic data described earlier. Besides, the results were very close to the results obtained from the data collection method at any point in the simulated Freeway segment. Therefore, traffic data obtained from the data collection point method were used.

\subsubsection{Incident Identification in the Vissim Simulation}

Even though Vissim model was not specially designed to analyze non-recurrent congestion including freeway incident replications, previous authors have successfully coded incident-conditions in Vissim (Deniz et al. 2012). For instance, the reduced speed distribution with a low value of $0.001 \mathrm{~km} / \mathrm{h}$ in Vissim was used to specify the location of an incident in the freeway section. A simulation time of 10 minutes was set to imitate the time of an accident. The simulation parameter tool was utilized to define the simulation time which in turn simulates the time period the incident is expected to keep on blocking the lane in the study freeway section.

Defining the incident in the Vissim simulation can be done by defining changes to

the desired speed (i.e. using reduced speed area). It is possible to use a reduced speed distribution that simulates the vehicle speed in the event of an incident, where vehicles tend 
to decelerate to manage their speed reduction. Each vehicle in the traffic stream maintains the safety headway.

In the event of an incident, the disabled vehicle stops all of a sudden, and its speed drops dramatically to reach a zero value within a few seconds. The following vehicle will be having two choices so as to keep a safety distance away from the stopped vehicle. The first choice is to slow down and make a full stop. The second choice is to overtake the leading vehicle and recover speed. In both cases, a drop in the speed of the following vehicles is expected.

A reduced speed area was defined between two very low-speed values. A range between of 0.001 and $0.1 \mathrm{~km} / \mathrm{h}$ was defined to imitate vehicle speed in the event of an incident. The normal distribution was utilized to identify the lower and higher speed values in the defined reduced speed area which in turn simulated the sudden stop of the first vehicle when it reached the specified zone, hence causing a complete blockage of the lane. Since a very low reduced speed value was selected, the leading vehicle stopped moving and the time period the incident was expected to keep blocking the lane in the study lane during the simulation time. In order to control the driving behavior in the event of an incident, it was possible to edit the already defined driving behavior by changing the required attributes to comply with the incident condition.

\section{Summary:}

Incident Location determines the position of very low reduced speed area; almost zero speed reduction was used to model the incident. Lane blockage determined the number of reduced speed areas, with one per each lane blocked at the incident location. 
Incident duration matched the simulation time. Reduced speed areas were identified to make sure that vehicles comply with incident conditions and stopped in the area of lane blockage.

\subsubsection{Incident Model Scenarios ---}

The simulation model took into account the following guidelines:

- Vehicle composition included Cars and HGV (Trucks),

- Freeway section length was $1140 \mathrm{~m}$,

- Lane width was $3.5 \mathrm{~m}$,

- Incident was located at $950 \mathrm{~m}$,

- Incident occurred after approximately $35 \mathrm{sec}$ from simulation starting time,

- Desired speed was $100 \mathrm{~km} / \mathrm{h}$,

- Volume: four volume levels were selected for simulation purposes including $1000,1250,1500$, and $2000 \mathrm{veh} / \mathrm{h} / \mathrm{lane}$,

- Data Collection Points were set at $750 \mathrm{~m}$

- Travel Time segment was set at $700-800 \mathrm{~m}$

- Node Results segment was set at $700-800 \mathrm{~m}$

Five incident scenarios in the freeway section were considered to simulate incident and generate traffic data for testing the developed incident detection model. Each incident scenario was developed to simulate different incident conditions based on the number of blocked lanes caused by an incident occurrence and the potential response to each incident situation. 
To study the impact of an incident that occurred in a freeway section, the five different scenarios were considered for Vissim modeling. Each scenario represented a particular lane blockage configuration. The first scenario represented the four-lane freeway section with the incident blocking the rightmost lane of the freeway section. The second scenario represented the freeway section with incident blocking the first and second rightmost lanes. The third scenario represented the freeway section with incident blocking the rightmost three lanes. The fourth scenario represented the freeway section with incident blocking the entire direction of the freeway section including the four lanes. The last scenario represented the basic free flow condition that did not include any incidents.

Since Highway 401 was used only to represent a typical freeway section that could be found anywhere around the world, there was no need to calibrate the Vissim model with the basic non-incident scenario for a specific Highway 401 segment, and model default values were not altered.

The default factors are commonly used for North American and European freeways. That is, if a specific facility is not to be modelled, defaults are to be used. In the present research, the purpose of VISSIM use is to model an uninterrupted flow facility/Freeway (it could be any real-life facility in any city) for the purpose of testing the developed incident detection model/algorithm. This modelling and simulation is not specific to HW 401.

Weidmann 99 Car Following Parameters that are commonly used in the USA and Europe for general applications such as simulation of a Basic Segment and Merging/Weaving operations were used. Please refer to VISSIM manual or Mai et al (2011) for a description of car-following parameters and values. Parameters for a section of HW401 are not of interest because it could limit the simulator to be fit for application to 
that section only. The used Weidmann parameters served the purpose of testing the incident detection algorithm since the algorithm is intended for applications anywhere.

Weidmana parameter values are available for vehicle types and classes as well as for driving and lane change behavior. These parameters of motorized traffic originate from research work done by the University of Karlsruhe. The VISSIM driver behavior logic is based on the continued research of Wiedemann. In the USA, DOTs such as Oregon DOT recommend the use of these parameters and the Weidmann default values fall within the range of values suggested by US DOTs such as Oregon.

For each incident scenario, all incident condition parameters mentioned earlier at the beginning of section 8.5 were kept identical to non-incident scenario during the simulation time from 0 second to 600 seconds. The incident location was chosen to be at a distance of $950 \mathrm{~m}$ away from the starting point. Since the desired freeway speed was $100 \mathrm{~km} / \mathrm{h}$, therefore at a simulation time of 35 seconds, an incident was assumed to occur at the furthest downstream part of the study section, blocking one or more lanes. Figure 7.4 to Figure 7.8 show the five incident scenarios including their applicable lane blockage configurations.

Figure 7.4 shows the first scenario which involves one lane blockage configuration, Figure 7.5 shows the second scenario which involves two lane blockage configuration, Figure 7.6 shows the third scenario which involves three lane blockage configuration, and Figure 7.7 shows the fourth scenario which involves four-lane blockage configuration. The incident lasted 600 seconds which is the total simulation time. 


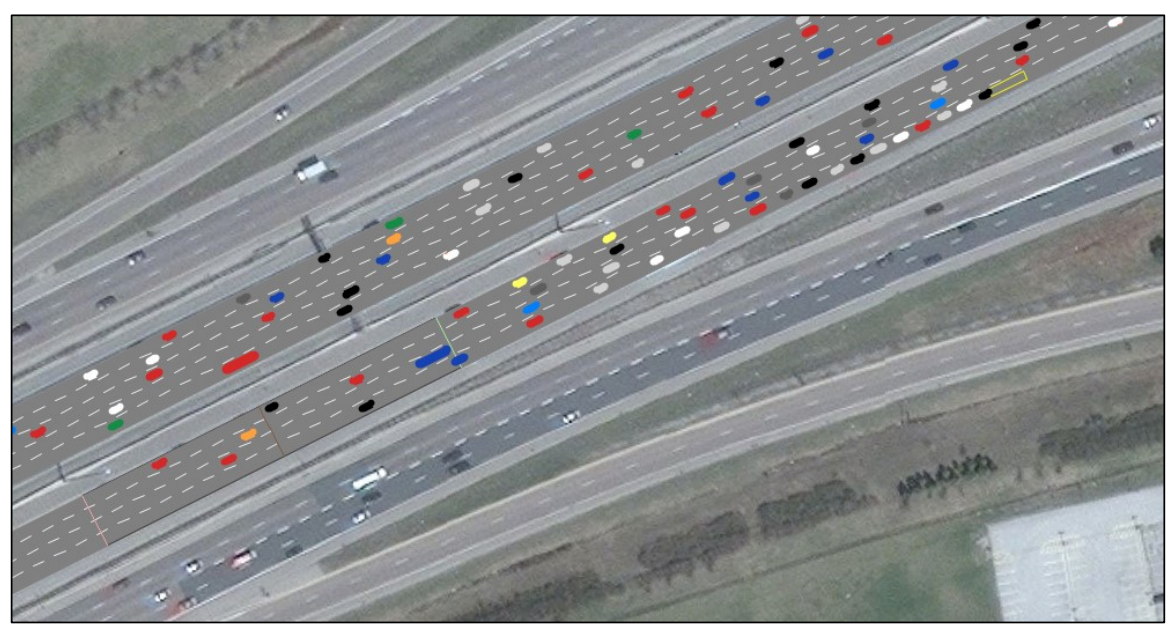

Figure 7.4: First scenario, one lane blockage configuration.

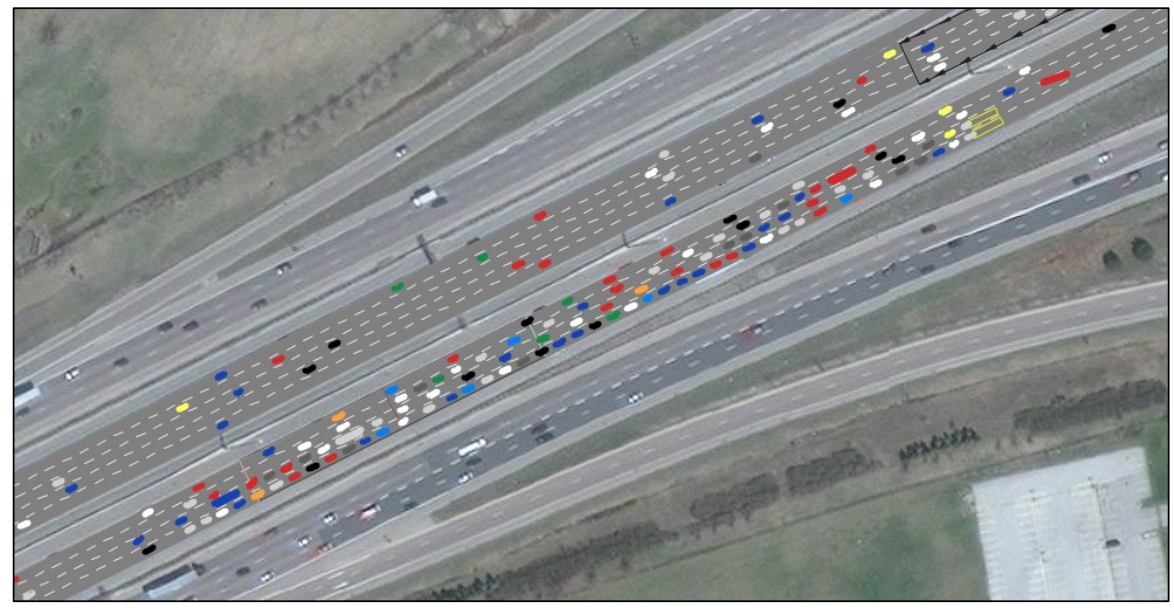

Figure 7.5: Second scenario, two lane blockage configuration.

Notable observations made in incident scenarios are the variability of vehicle speed at each time interval. One of the approaches to address this issue is by making multiple runs. 


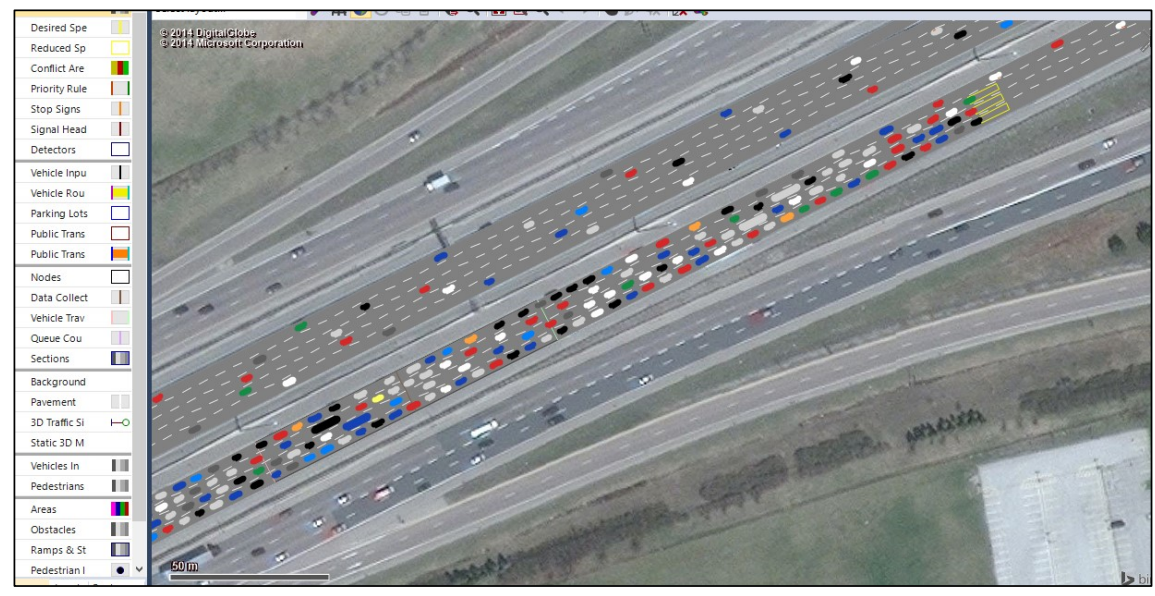

Figure 7.6: Third scenario, three lane blockage configuration.

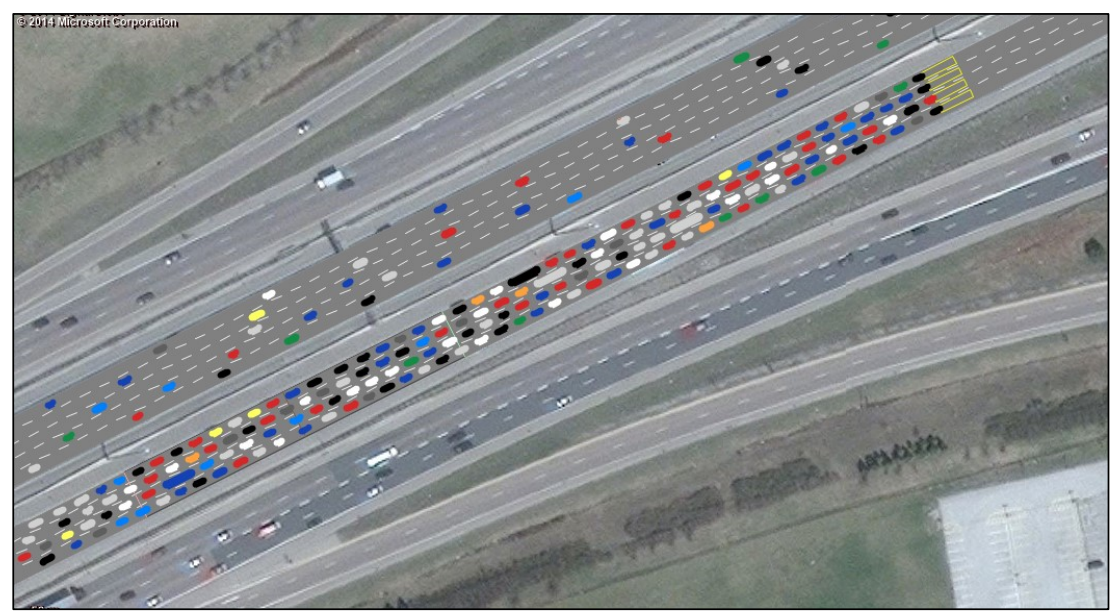

Figure 7.7: Fourth scenario, four lane blockage configuration.

To view a real incident event that caused four lane blockage is a collision involving at least 50 vehicles that occurred at the eastbound section of Highway 401 just one minute after midnight. This resulted in a complete closure of Highway 401 as shown in Figure 7.8. 


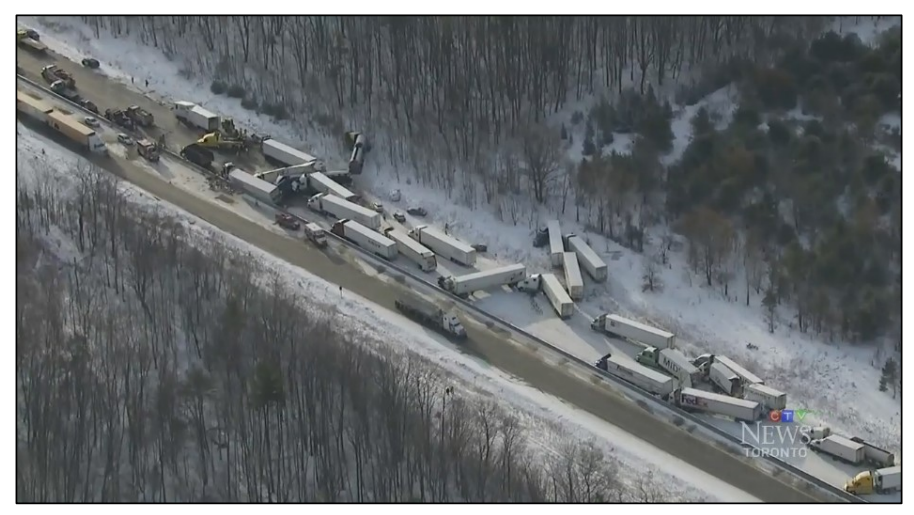

Figure 7.8: Four lane blockage example on Highway 401

\subsubsection{Traffic Flow Data}

Another two factors that would change the incident condition are the desired speed and the traffic volume generated at the beginning of the freeway section.

\subsubsection{Desired Speed Distribution}

The desired speed distribution is assumed to be the same as the posted speed limit on Ontario freeways which is $100 \mathrm{~km} / \mathrm{h}$.

\subsubsection{Traffic Volume}

Traffic volume data were derived from the Ministry of Transportation's (MTO) inventory of annual traffic data for the 400 series freeways for the year 2010 . The volumes were first calculated using the given AADT. Due to the fluctuations in the AADT between segments, the values were adjusted so as to consider wide variation in traffic volumes that included different levels. It was also noted that the MTO volume counts were per segment, including all streams in the two directions. So in the case of collectors and express 
configuration, the total volume was divided into four equal portions and assigned to each stream.

For instance, the proposed freeway section on Highway 401 was located between Highway 427 and Highway 403/410. According to the MTO counts, this section consists of three segments. Traffic flow data of all lanes upstream and downstream in the same segment is shown in Table 7.1. Since there is variation in the traffic volumes between the segments, the average value was assumed as a guide for generating traffic volume at the beginning of the simulation line. From Table 7.1 the following three volumes were observed $(384,900 ; 381,400 ; 353,100)$ which is averaged as $373,000 \mathrm{veh} /$ day/segment.

According to the segment streams configuration shown in Table 7.2, the freeway consists of 18 lanes. This enables the calculation of number of vehicles per hour for each lane. The result is an average volume of $864 \mathrm{veh} / \mathrm{h} / \mathrm{lane}$.

Table 7.1: Commercial vehicle flows by road network

\begin{tabular}{|c|c|c|c|c|}
\hline Highway & Location Description - From & Location Description - To & Dist. (km) & \begin{tabular}{|c|}
2010 \\
AADT \\
\end{tabular} \\
\hline 401 & ISLINGTON AVE IC -ETOBICOKE & DIXON RD IC -ETOBICOKE & 2.5 & 342,700 \\
\hline 401 & DIXONRDIC-ETOBICOKE & HWr 427352 & 2.4 & 236,500 \\
\hline 401 & HWY 427352 & RENFORTH DR IC & 0.8 & 384,900 \\
\hline 401 & RENFORTH DR IC & DIXIE RD IC-PEEL RD 4 & 4.3 & 381,400 \\
\hline 401 & DIXIE RD IC-PEEL RD 4 & $\mathrm{HWY} 403 / 410 \mathrm{IC}$ & 1.5 & 353,100 \\
\hline 401 & HWY $403 / 410 \mathrm{IC}$ & HWY 10/HURONTARIO ST IC-MISS. & 2.8 & 187,700 \\
\hline 401 & HWY 10/HURONTARIO ST IC -MISS. & MAVIS RD IC & 2.1 & 187,100 \\
\hline 401 & MAVIS RDIC & MISSISSAUGA RD IC & 3.5 & 160,200 \\
\hline 401 & MISSISSAUGA RD IC & WINSTON CHURCHILL BLVD IC & 3.4 & 126,600 \\
\hline 401 & WINSTON CHURCHILL BLVD IC & HWY 407 IC & 2.3 & 116,100 \\
\hline 401 & HWY $407 \mathrm{ICC}$ & TRAFALGAR RD IC-MLITON & 2.4 & 138,900 \\
\hline 401 & TRAFALGAR RD IC -MLLTON & JAMES SNOW PKWY IC & 4.2 & 130,700 \\
\hline 401 & JAMES SNOW PKWY IC & HWY 25 IC-MLLTON & 3.7 & 119,800 \\
\hline 401 & HWY 25 IC-MLLTON & GUELPH LINE IC -MLTON & 8.2 & 103,600 \\
\hline 401 & GUELPH LINE IC -MLTON & MLTON WLTS & 4.5 & 107,300 \\
\hline 401 & MILTON WLTS & E JCT HWY G/BROCK RD IC & 7.3 & 107,300 \\
\hline 401 & E JCT HWY G/BROCK RD IC & WJCT HWY G/HANLON EXPY IC & 4.4 & 107,500 \\
\hline 401 & W JCT HWY GIHANLON EXPYIC & REG RD33-TOWNLINE RD & 9.2 & 97,100 \\
\hline 401 & REG RD33-TOWNLINE RD & FrankiiN Blvd & 2.3 & 112,000 \\
\hline 401 & Frankin Blvd & HWY 24-HESPELER RD IC-CAMBRIDGE & 1.7 & 100,400 \\
\hline 401 & HWY 24-HESPELER RD IC-CAMBRIDGE & HWY 8 IC-KITCHENER & 4.6 & 125,600 \\
\hline 401 & HWY 8 IC-KITCHENER & WATERLOO RD 28-HOMER WATSON IC & 2.9 & 71,900 \\
\hline 401 & WATERLOO RD 28-HOMER WATSON IC & WATERLOO RD 97 IC & 7.1 & 53,000 \\
\hline 401 & WATERLOO RD 97 IC & WATERLOO REG/OXFORD CTY BDY & 4.9 & 46,000 \\
\hline 401 & WATERLOO REG/OXFORD CTY BDY & OXFORD RD 29 IC & 12.9 & 46,000 \\
\hline 401 & OXFORD RD 29 IC & OXFORD HWY 2 IC & 12.2 & 43,600 \\
\hline 401 & OXFORD HWY 2 IC & TOWERLINE RD IC-OXFORD RD 15 & 1.6 & 45,700 \\
\hline 401 & TOWERLINE RD IC-OXFORD RD 15 & $\mathrm{HWY} 403 \mathrm{IC}$ & 1.0 & 45,700 \\
\hline 401 & HWr $403 \mathrm{IC}$ & OXFORD ROAD 59 IC-WOODSTOCK & 3.4 & 59,900 \\
\hline 401 & OXFORD ROAD 59 IC -WOODSTOCK & SWEABURG RD IC-OXFORD RD 12 & 2.1 & 68,400 \\
\hline 401 & SWEABURG RD IC-OXFORD RD 12 & OXFORD RD 6 -FOLDENS LINE IC & 7.6 & 66,600 \\
\hline 401 & OXFORD RD 6 -FOLDENS LINE IC & HWY $19 \mathrm{IC}$-INGERSOLL & 3.7 & 63,100 \\
\hline 401 & HWY 19 IC -INGERSOLL & CULLODEN RD IC-OXFORD RD 10 & 2.5 & 65,700 \\
\hline
\end{tabular}

(AP-R364/10 AUSTROADS RESEARCH REPORT Freeway Incident Detection - Technologies and Techniques, 2010) 
Table 7.2: Lane count: Number of through lanes on Highway 401 (excludes ongoing or planned widening projects)

\begin{tabular}{|c|c|c|c|c|c|c|c|c|c|c|c|c|}
\hline Location & $\begin{array}{l}\text { E C Row } \\
\text { Expressway } \\
\text { to Essex } \\
\text { County } \\
\text { Road } 42\end{array}$ & $\begin{array}{l}\text { Essex } \\
\text { County Road } \\
42 \text { to } \\
\text { Highway } 402\end{array}$ & $\begin{array}{l}\text { Highway } 402 \\
\text { to } \\
\text { Highway } 403 \text { / } \\
410\end{array}$ & $\begin{array}{l}\text { Highway } 403 \text { / } \\
410 \text { to } \\
\text { Highway } 427\end{array}$ & $\begin{array}{l}\text { Highway } 427 \\
\text { to } \\
\text { Highway } 27\end{array}$ & $\begin{array}{l}\text { Highway } 27 \\
\text { to } \\
\text { Highway } 409\end{array}$ & $\begin{array}{l}\text { Highway } 409 \\
\text { to Brock } \\
\text { Road }\end{array}$ & $\begin{array}{l}\text { Brock } \\
\text { Road to } \\
\text { Salem } \\
\text { Road }\end{array}$ & $\begin{array}{l}\text { Salem } \\
\text { Road to } \\
\text { Burnham } \\
\text { Street }\end{array}$ & $\begin{array}{l}\text { Burnham } \\
\text { Street to } \\
\text { Frontenac } \\
\text { County } \\
\text { Road } 38\end{array}$ & \begin{tabular}{|l} 
Frontenac \\
County \\
Road 38 \\
to \\
Montreal \\
Street
\end{tabular} & $\begin{array}{l}\text { Montreal } \\
\text { Street to } \\
\text { Quebec } \\
\text { border }\end{array}$ \\
\hline $\begin{array}{l}\text { Lane } \\
\text { count }\end{array}$ & 6 lanes ${ }^{[16]}$ & 4 lanes ${ }^{[28]}$ & 6 lanes ${ }^{[16]}$ & $\begin{array}{l}\text { 18-lane } \\
\text { collector- } \\
\text { express } \\
\text { system[7] }\end{array}$ & 8 lanes $^{[0]}$ & 10 lanes $^{[35]}$ & $\begin{array}{l}\text { 12-16-lane } \\
\text { collector- } \\
\text { express } \\
\text { system[44] }\end{array}$ & 10 lanes $^{[44]}$ & 6 lanes ${ }^{[48]}$ & 4 lanes ${ }^{[12]}$ & 6 lanes $^{[58]}$ & 4 lanes $^{[58]}$ \\
\hline Distance $^{[2]}$ & $\begin{array}{l}55.7 \mathrm{~km} \\
(34.6 \mathrm{mi})\end{array}$ & $\begin{array}{l}127.5 \mathrm{~km} \\
(79.2 \mathrm{mi})\end{array}$ & $\begin{array}{l}161.3 \mathrm{~km} \\
(100.2 \mathrm{mi})\end{array}$ & $\begin{array}{l}5.8 \mathrm{~km} \\
(3.6 \mathrm{mi})\end{array}$ & $\begin{array}{l}0.8 \mathrm{~km} \\
(0.50 \mathrm{mi})\end{array}$ & $\begin{array}{l}3.9 \mathrm{~km} \\
(2.4 \mathrm{mi})\end{array}$ & $\begin{array}{l}43.3 \mathrm{~km} \\
(26.9 \mathrm{mi})\end{array}$ & $\begin{array}{l}6.0 \mathrm{~km} \\
(3.7 \mathrm{mi})\end{array}$ & $\begin{array}{l}68.3 \mathrm{~km} \\
(42.4 \mathrm{mi})\end{array}$ & $\begin{array}{l}138.2 \mathrm{~km} \\
(85.9 \mathrm{mi})\end{array}$ & $\begin{array}{l}8.2 \mathrm{~km} \\
(5.1 \mathrm{mi})\end{array}$ & $\begin{array}{l}209.0 \mathrm{~km} \\
(129.9 \mathrm{mi})\end{array}$ \\
\hline
\end{tabular}

(AP-R364/10 AUSTROADS RESEARCH REPORT Freeway Incident Detection - Technologies and Techniques, 2010)

\subsection{Analysis Plan}

The main goals of this section are to examine the capability of the developed model to detect incident by examining simulated traffic parameters for a particular lane blockage level at a specified location and the traffic volume level. Also, it is targeted to examine the effects of different traffic volume levels on the change in vehicle speed that is expected to drop dramatically from high-speed value to a low value for a particular lane blockage configuration in the event of an incident.

The planned analysis procedures are considering some factors that are controlling data collection plan; these factors are as follows:

- Compare results for different locations of Data Collection Point to identify the best location that represents traffic speed data.

- Compare simulation runs with different volume levels to see the improvements in the model capabilities in detecting incident after reaching to considerable volume level and whether the number of undetected cases is statistically significant (confidence level). 
- Compare results for a different number of lane blockages to identify the most conventional lane blockage configuration in a real life incident.

Vehicle speed can be easily transferred to time/speed chart using MS-Excel. Moreover, time/speed data was treated and rearranged for the developed algorithm applications.

Values of two variables were obtained from Vissim simulations; the first one is termed "Time enter" and the second one is termed "Time exit." It is noticed that there is a slight difference between the two time stamps with some difference in the corresponding speed at each time stamp. This difference is related to the time when the vehicle first enters the data point and the time when the vehicle leaves the data point. Therefore, the two-time stamps were integrated together to give more continuity in the Time-Speed profile. A test was conducted to see the effect of including the exit time stamp as illustrated in Figure 7.9 in comparison to excluding it as illustrated in Figure 7.10.

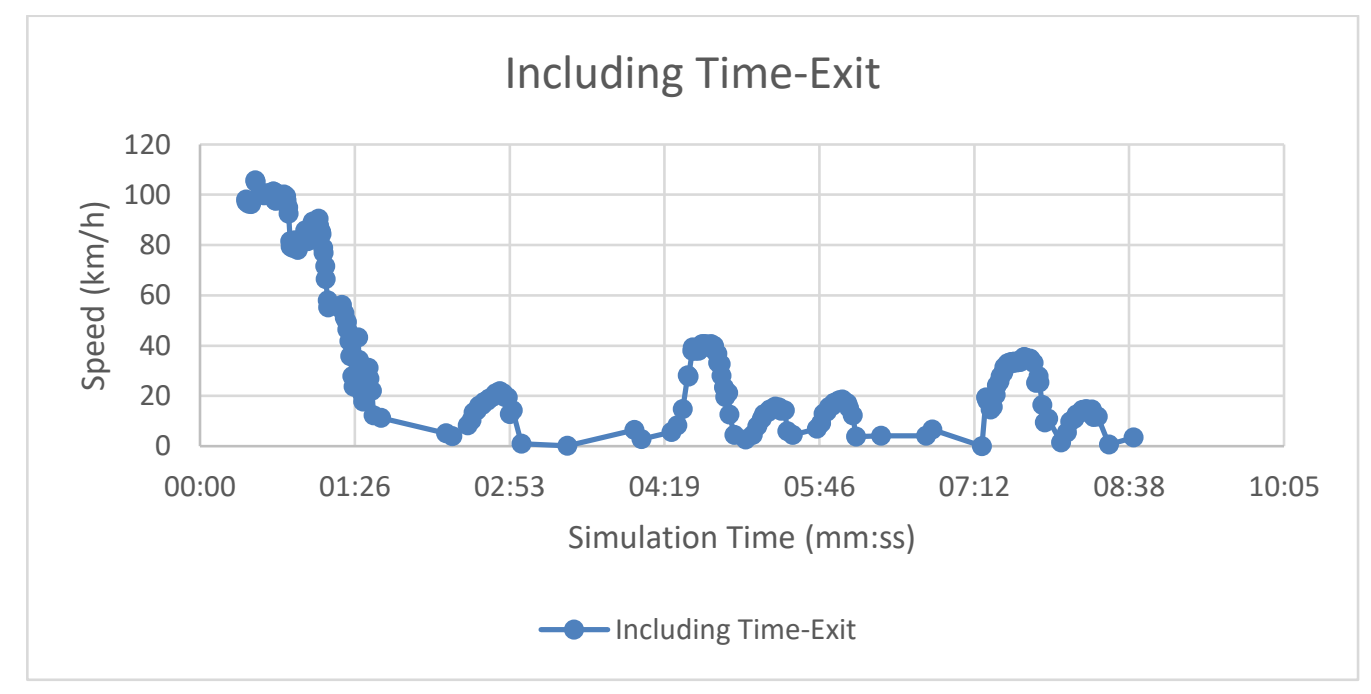

Figure 7.9: Including the exit time stamp in the time/speed profile 


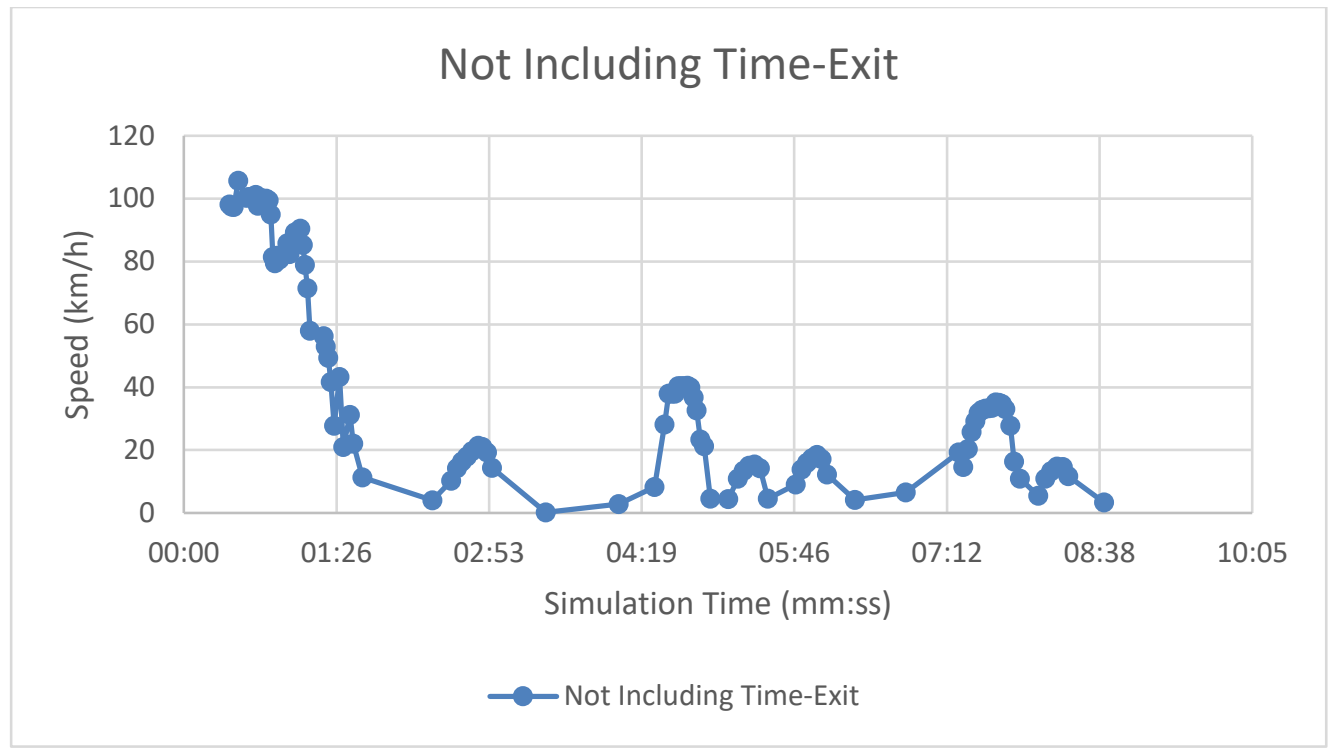

Figure 7.10: Excluding the exit time stamp in the profile

For the purpose of getting a smoother curve in the relationship between time and speed, it was decided to consider speed values obtained from both time stamps as it is expected to incorporate more time interval and hence the corresponding speed.

To generate traffic data for testing the developed model, the five scenarios discussed in section 7.5.9 were considered to simulate incident in the defined freeway section. Each scenario considers different lane blockage configuration in addition to the non-lane blockage where no incident occurred. Each lane blockage configuration was examined by finding the relationship between time and traffic speed; one data collection point was set at each lane to obtain the speed values for each vehicle in the stream at each simulation time interval. The simulation output from the analysis plan should primarily provide the speed measures for each response and thus illustrates the relation between the simulation time and the speed values for each stated factor. 


\subsubsection{Location of Data Collection Point}

Before running the simulation, some factors should be examined to get the most proper time/speed values that are mostly typical of the simulated traffic condition. The first factor among these factors that appears here to be of considerable importance is the location of data collection point, where time and speed measurements are of extreme interest. There are four possible locations available to obtain time/speed data outputs, each data point fixed at each lane is considered as one source of data collection. Primarily, it is necessary to examine the time/speed relationship from each lane of the four lanes of the freeway section of each tested scenario.

One can propose an approach for taking the average speed recorded in the four lanes that correspond to one particular time period such as 30 seconds. However, doing so might not be the best method of obtaining the speed values. The fact that vehicles traveling along the blocked lane are much slower than those traveling in the second, third and fourth lanes suggest that the average speed is something in the middle and not representing the real impact of the incident on the traffic flow. This conclusion led to not proposing this approach at a very early time in the process of obtaining the speed values of traffic.

Therefore, the first attempt was to consider the time and the corresponding speed of each vehicle passing the data collection point; this approach does not show reasonable results for one, two and three lane blockage configurations. However, in the four-lane blockage and no lane blockage configurations, the results were sound.

Figure 7.11 illustrates the relationship between the time and vehicle speed in the case of one lane blockage under the consideration that speed values are recorded according to each time interval regardless of the location of the data collection point. 


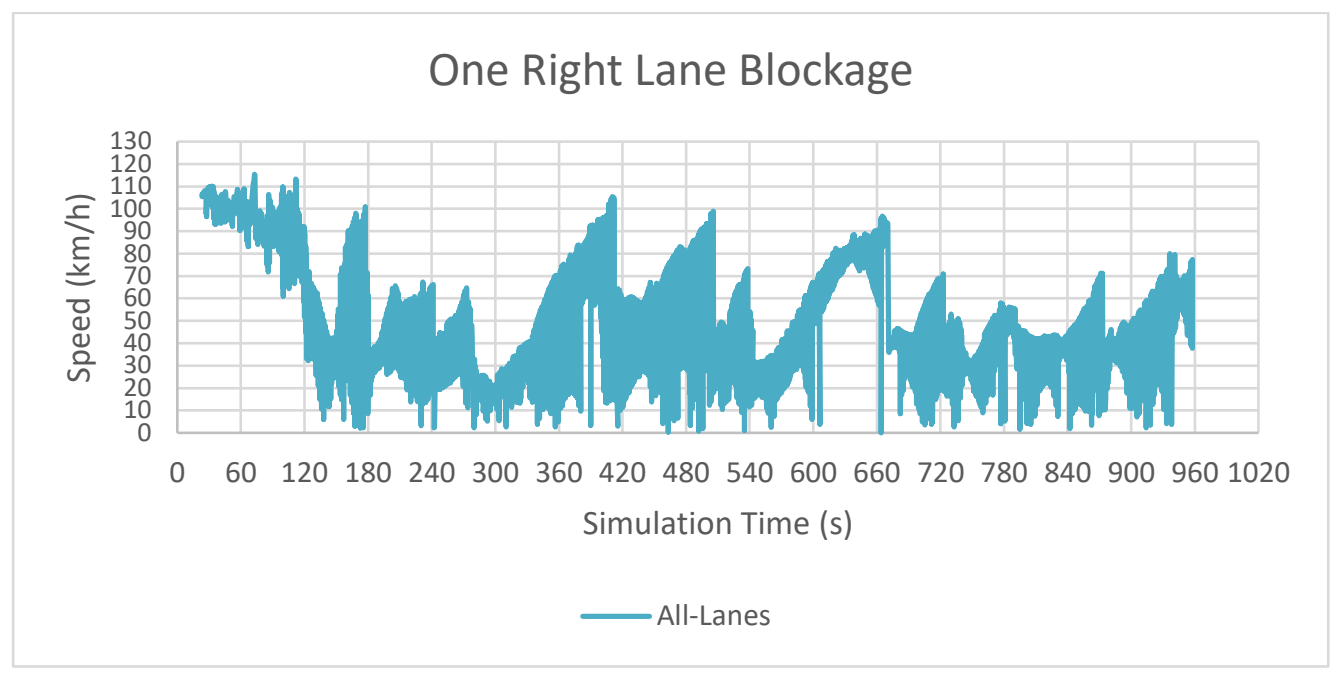

Figure 7.11: Speed values according to each time interval regardless of the location of the data collection point

It was observed from Figure 7.11 that there is a major variation in the vehicle speed values. This variation could be interpreted as follows. Here, the following vehicles traveling in the blocked lane tended to slow down to maintain safe distance headways from the leading slow vehicles. Another observation is that following vehicles attempted to overtake the slow leading vehicles whenever gaps in the second lane allowed to do so. This was not the case for vehicles traveling in the third and the fourth lane, where vehicles managed to maintain relatively high speed.

\subsubsection{Traffic Volume}

When using Vissim for the micro-simulation under the same traffic condition, one of the major inputs is traffic volume. It is the second factor that is as important as the first two factors which could change the traffic flow condition in the event of an incident. Traffic 
volume should be generated at the beginning of the freeway section. In order to generate different traffic conditions, road section was loaded with 4 different traffic volume conditions (which are $1000 \mathrm{veh} / \mathrm{h} / \mathrm{lane}, 1250 \mathrm{veh} / \mathrm{h} / \mathrm{lane}, 1500 \mathrm{veh} / \mathrm{h} / \mathrm{lane}$, and 2000 veh/h/lane). The simulation was tested under the four different traffic volume conditions.

\subsubsection{Number of Lane Blockage}

The third factor can be the lane blockage configuration as stated previously. Four scenarios reflected lane blockage configuration as noted earlier. There is also the no lane blockage configuration to consider. At least one simulation run was conducted for each scenario to examine the relationship between time and speed.

The focus should be on two lane blockage configuration where results showed that two lane blockage was more representative, and is the most likely scenario in the case of freeway incident. This conclusion led to give more consideration to the scenario of having two lane blockage when obtaining time/speed data. However, impacts of the other three scenarios were studied as well. Special attention was also given to the scenario of having no incident in the freeway and, therefore, no lanes were affected or closed due to any incident.

Simulations were run for every possible lane blockage configuration, a selected level of traffic volume, and 10 minutes incident duration. To reduce stochastic variability, multiple runs were conducted for each scenario. For statistical reasons, simulations were repeated 30 times for the most likely scenarios, such as two-lane blockage level and no lane blockage with a traffic volume of $2000 \mathrm{veh} / \mathrm{h} / \mathrm{lane}$. In this scenario, the time/speed reading was obtained from the data collection point located in the first lane. Simulations 
were also repeated ten times for the same two-lane blockage scenarios when the time/speed reading was obtained from the second lane.

Other traffic volumes were also examined, and a number of runs were repeated. Ten runs were conducted on cases involving 1000,1250 , and $1500 \mathrm{veh} / \mathrm{h} /$ lane under the condition that time/speed readings were obtained from the first lane, as well as, cases when reading, was obtained from the second lane.

In case no lane was blocked, time/speed data were obtained from the first lane. 30 runs were conducted when traffic volume reached $2000 \mathrm{veh} / \mathrm{h} / \mathrm{lane}$. However, ten simulation runs were performed when traffic volume reached a lower level of 1000 veh/h/lane.

The primary focus of the simulation runs was on aggregate freeway speed. Therefore, in the following sections, each lane blockage scenario was examined and the four traffic volume levels were also included in the investigation. Each chosen scenario was characterized by a certain number of blocked lanes and defined traffic volume.

An example of obtained results for each scenario is shown in figures illustrated within the text part of the thesis while the results for all other runs are shown in Appendix D. Each figure shows the relationship between time and traffic speed for each scenario, drop in speed, and possible incident detection of each case. Incident is reported as detected if the provided time/speed information satisfies the incident detection criteria defined in the developed model (usually when the three conditions set for the incident detection criteria are met). Furthermore, a brief description of the used variables in the devolved algorithm for detecting incident is shown in each figure. 
It should be noted that some information presented is not necessary because it is not reflecting real information related to the simulation environment (e.g. detector number, detector rank, distance from the incident, and season). Such information appears in the figures due to the reason that it is standard information built in the developed model when examining real life incident data.

\subsection{Simulation Results and Algorithm Applications}

For each simulation run, data were saved in specific data files which were employed to generate required output data. In turn, output data were treated and rearranged to give more focus to the simulation time and speed. These throughputs were saved in another set of separate data files for the purpose of using these to test the developed model. According to lane blockage configurations, results from each scenario are discussed in the following sub-sections.

\section{No Lane Blockage Scenario}

No lane blockage is considered to be the basic lane configuration. Traffic generated in Vissim simulation travels in all four lanes following the desired speed variable. When there is no lane blockage, free flow movement is the general traffic flow trend dominating the movement in each lane.

At free flow condition, vehicles are driving at the desired speed and not affected by traffic volumes applied. However, to generalize the application of the developed model it was decided to examine two traffic volume levels when testing the no lane blockage 
scenario. Therefore, in this scenario, two traffic volume levels were regarded for the simulation purpose, high traffic volume level, and low traffic volume level.

Figure 7.12 illustrates the free flow speed condition in the freeway under high traffic volume level of $2000 \mathrm{veh} / \mathrm{h} / \mathrm{lane}$ as the primary case for no incident scenario. In this scenario, data was collected from data collection point located in the first lane. Other cases when the data collection point is located in the second, third and fourth lane is given in Appendix D.1.

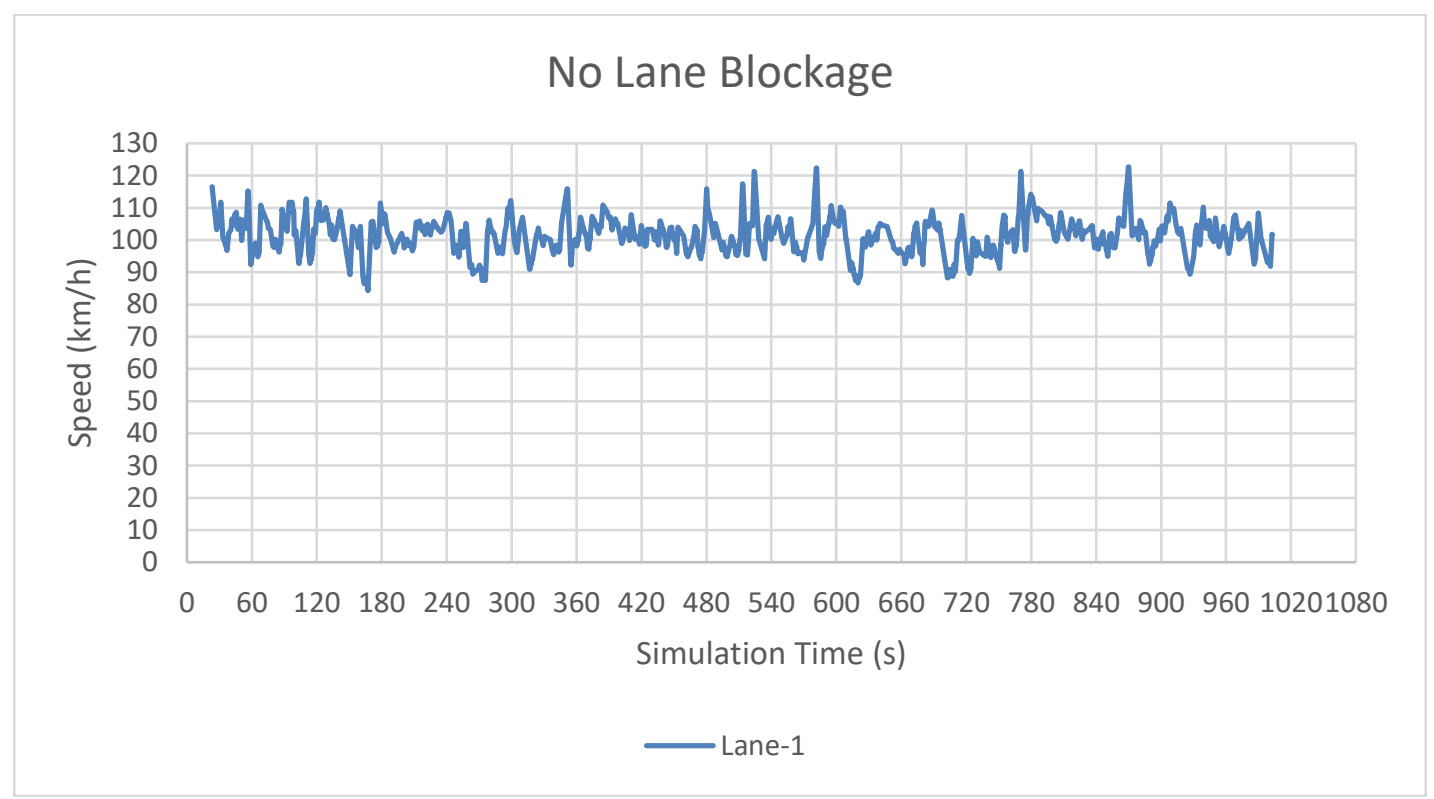

Figure 7.12: Speed data is collected from lane \#1, v=2000 veh/h/lane

Results obtained from the simulation runs including speed data for free flow traffic in the freeway section was utilized to test the developed model. Considering data is collected from the first lane, 30 simulation runs were conducted, and the results were examined by the algorithm. The necessary model input data files including, incident data files, detector information, and speed files were adopted to test the newly generated speed 
values obtained from the Vissim simulation results. Figure 7.13 illustrates the incident detection charts for predefined no incident case. From the figure, it was shown that no incident was detected which mainly reflects the assumption of having no incident in the Vissim simulation network.

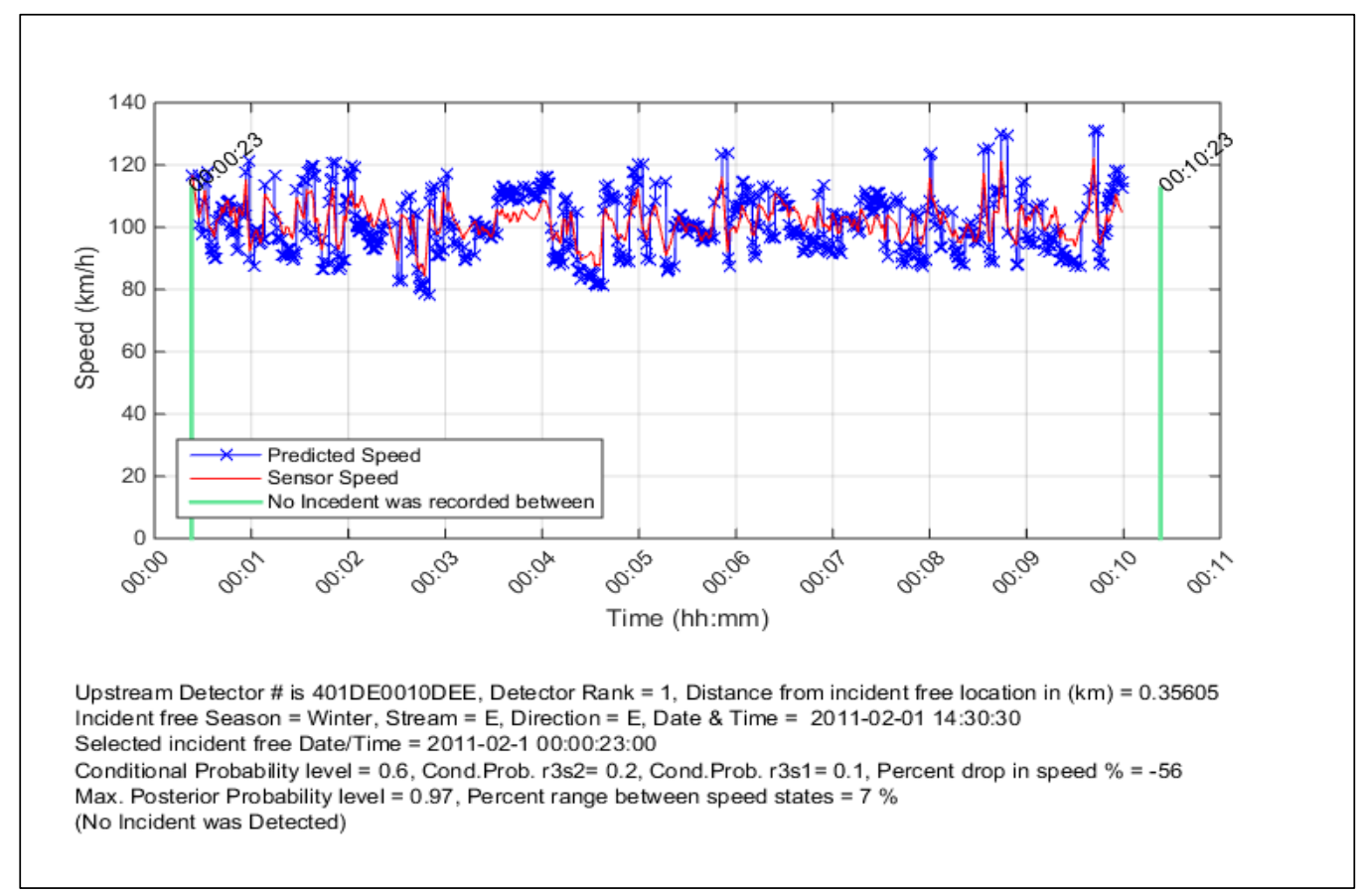

Figure 7.13: Speed data is collected from lane \#1, Run \#1, v=2000 veh/h/lane

For the statistical purposes, another 29 simulation runs were tested by the developed model and incident detection charts are given in Appendix D.1. Overall, the algorithm results show no detection of any incidents which is reflecting the valid assumption in the simulation model.

Under the same conditions but with low traffic volume level of $1000 \mathrm{veh} / \mathrm{h} / \mathrm{lane}$, Vissim simulation was conducted for the same free flow section. Ten runs were conducted 
for this scenario. Time/speed data collected from the results were tested by the developed model which in turn shows an indication of no incident as illustrated in Figure 7.14.

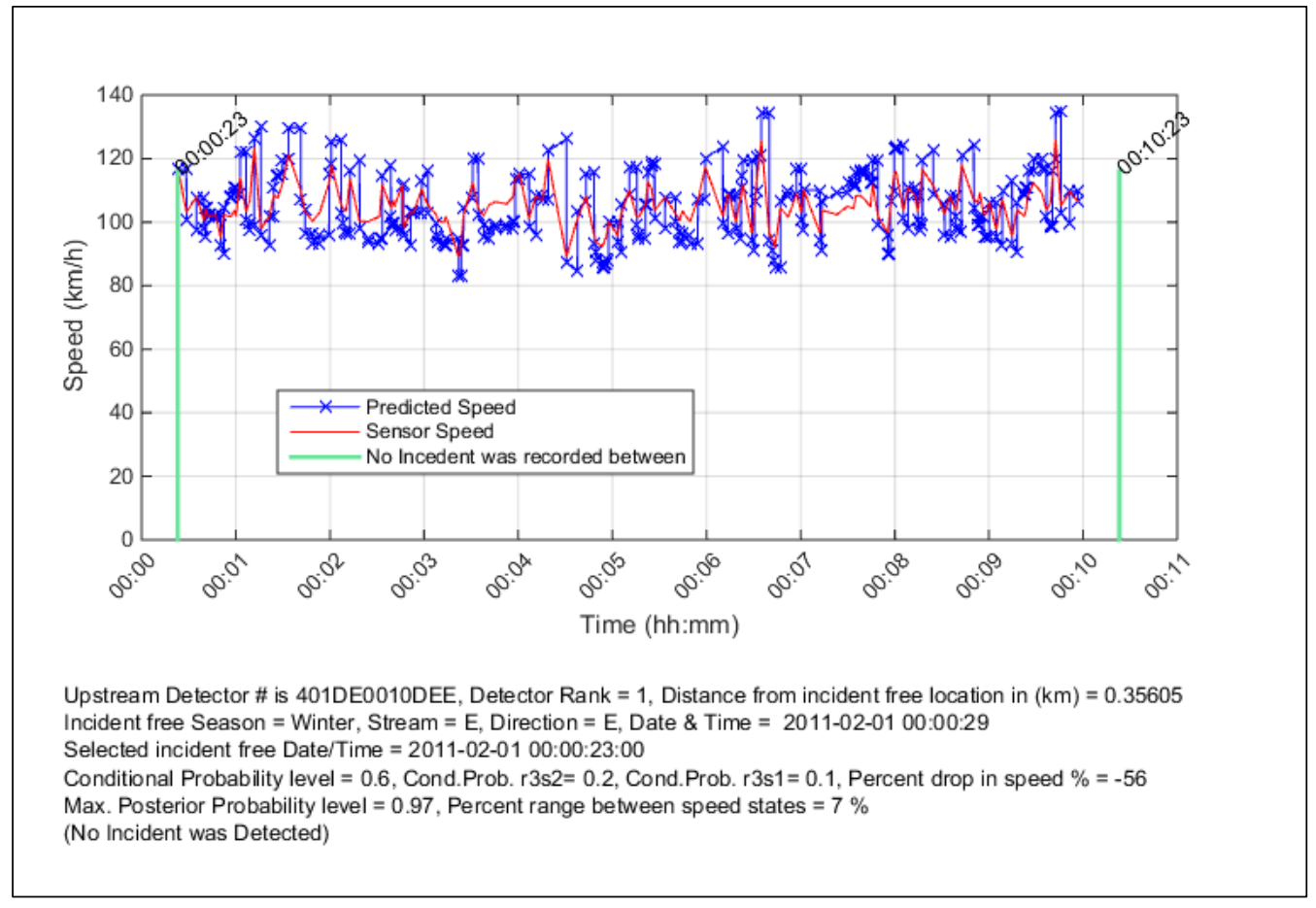

Figure 7.14: Speed data is collected from lane \#1, Run \#1, v=1000 veh/h/lane

Similar to the previous high volume level scenario, statistical purposes require a considerable number of runs for the same traffic conditions, which was selected to be ten runs this time. Therefore, another 9 case were tested by the developed model and incident detection tested cases are given in Appendix D.1. Overall, the algorithm results show no indication of any incidents in the nine tested cases which is reflecting the real situation in the simulation assumption as there was no lane blockage in this scenario. 
Given that almost $100 \%$ of the Vissim simulation results coincide with the developed model results, it can be inferred that the developed model was sound in not detecting any incident in cases traffic is simulated under no lane blockage configuration.

In the next section, two-lane blockage scenario is examined.

\section{Two Lane Blockage Scenario}

The second possible scenario in the event of traffic incident is that two lanes in the freeway section are blocked by the stopped vehicles. Blocking two lanes will decrease capacity to almost half so that traffic volume will have a significant effect on the free flow speed. Therefore, it was essential to examine the impact of different traffic volume levels on the traffic flow speed in the simulated freeway section. As indicated earlier, the three traffic volume levels selected for analysis were 2000, 1500, 1250 and $1000 \mathrm{veh} / \mathrm{h} / \mathrm{lane}$. And as discussed earlier, it was decided to obtain traffic data including time and speed from data collection points located in the rightmost first lane.

\section{Two Lane Blockage Scenario with Traffic volume level of $2000 \mathrm{veh} / \mathrm{h} / \mathrm{lane}$ Collecting data from the first rightmost lane}

The first simulation run considered a traffic volume level of $2000 \mathrm{veh} / \mathrm{h} / \mathrm{lane}$ as the peak flow condition. Figure 7.15 shows the impact on traffic flow condition for increased number of the upstream vehicles caused by the incident which resulted in two lane blockage. The main focus of the analysis in this scenario was traffic speed in case of an incident. 
Data obtained from the first lane were organized for better illustration of the relation between time and speed for this particular lane blockage configuration and traffic volume. The results were demonstrated in Figure 7.16 which illustrates the potential drop in speed due to the incident occurrence in case two lanes were blocked.

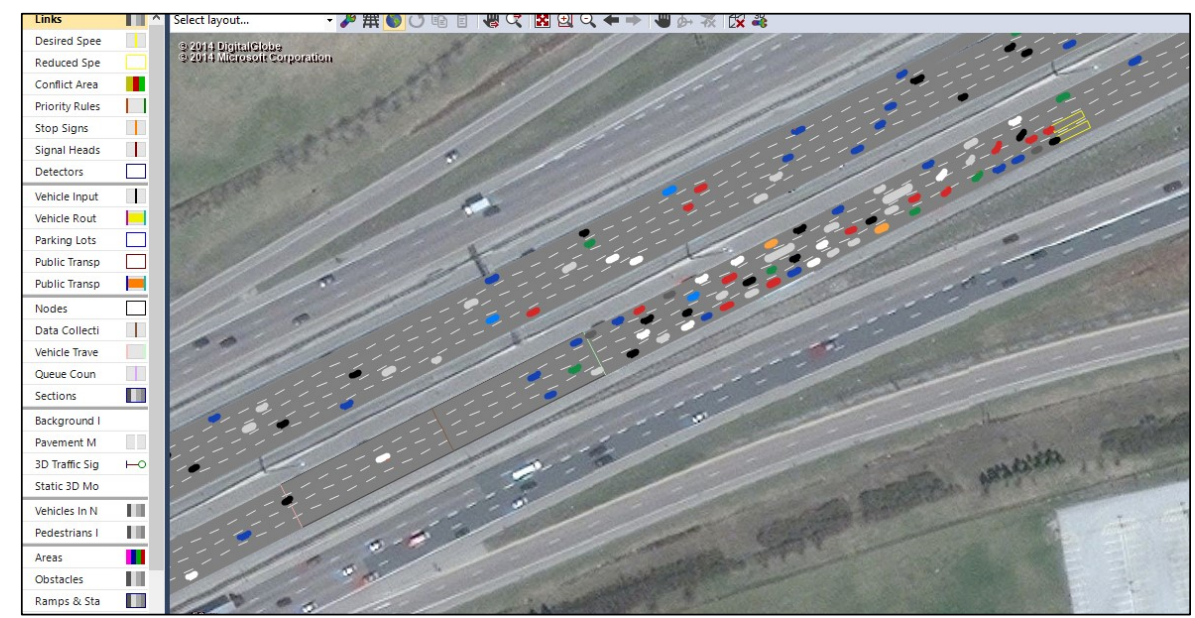

Figure 7.15: Traffic conformation from two lane blockage, $v=2000 \mathrm{veh} / \mathrm{h} / \mathrm{lane}$

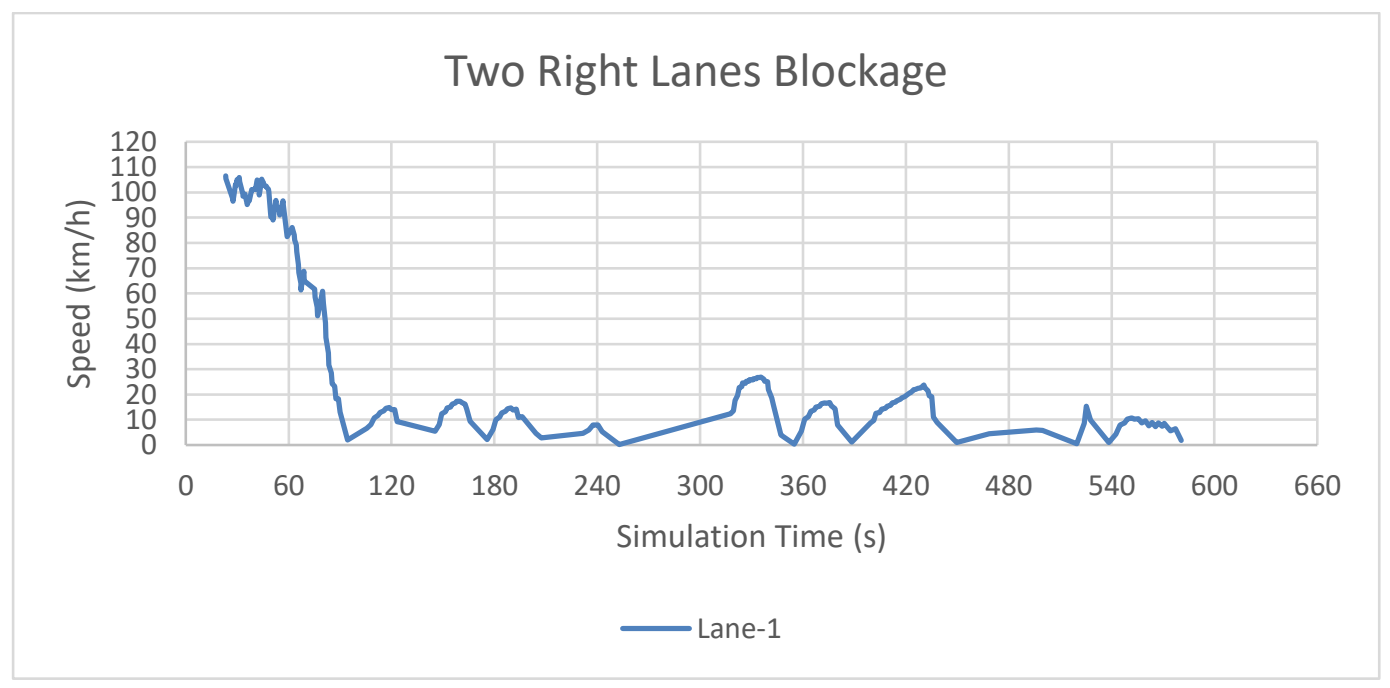

Figure 7.16: Speed data is collected from lane $\# 1, \mathrm{v}=2000 \mathrm{veh} / \mathrm{h} /$ lane 
To reduce stochastic variability, multiple runs must be conducted for this scenario as well. Results from each single run were used in the developed model, the number of simulation runs selected for this purpose were 30 runs.

Under the specified traffic volume and given lane blockage configuration, incident detection analysis was performed using the developed model. The output showed encouraging results. $100 \%$ of the tested runs showed an indication of an incident and the developed algorithm was capable of detecting the incident in each run. Figure 7.17 illustrates incident detection results utilizing the developed model and the other 29 incident detection tested cases are included in Appendix D.2

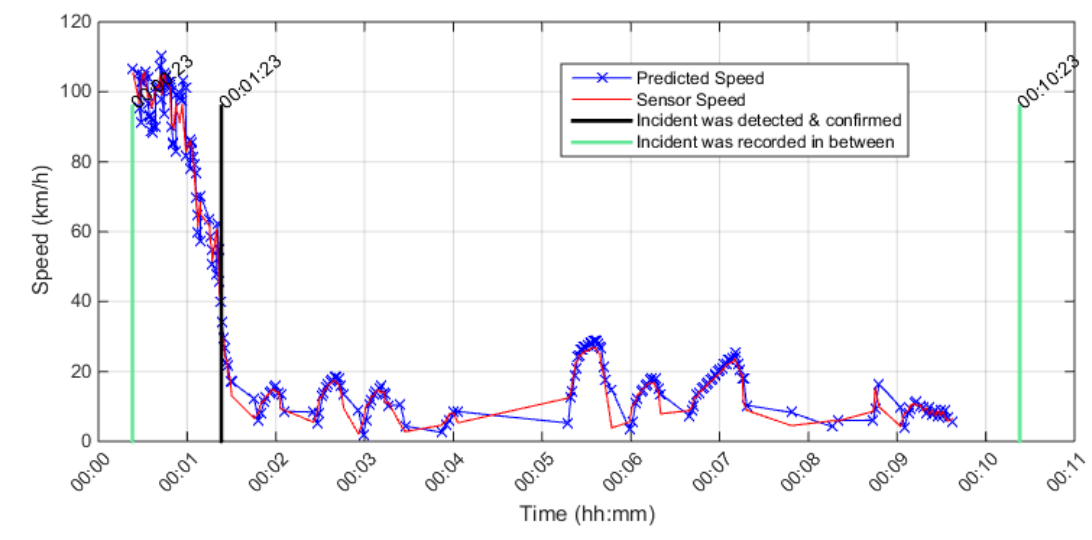

Upstream Detector \# is 401DE0010DEE, Detector Rank $=1$, Distance from incident location in $(\mathrm{km})=0.35605$ Season $=$ Winter, Stream $=E$, Direction $=E$, Date \& Time $=2011-02-0100: 00: 23$ Incident Reason $=$ Collision Affected Lanes $=2$ RIGHT LANE(S)

Conditional Probability level $=0.6$, Cond. Prob. $r 3 s 2=0.2$, Cond. Prob. $r 3 s 1=0.1$, Percent drop in speed $\%=-56$ Max. Posterior Probability level $=0.97$, Percent range between speed states $=7 \%$

Incident was detected at 00:18 minutes from drop in speed point. Incident was confirmed after 5 minutes from the detection time

Figure 7.17: Speed data is collected from lane \#1, Run \#1, v=2000 veh/h/lane 


\section{Two Lane Blockage Scenario with Traffic volume level of 2000 veh/h/lane}

\section{Collecting data from the second rightmost lane}

The effect of the incident on adjacent lanes was necessary to compare results obtained from both data collection points. Therefore, there was a need to examine the location of the data collection point. As mentioned in the previous discussion, traffic data were obtained from the first rightmost lane. It is a relevant question whether retrieving data from the second rightmost lane would be as promising as data obtained from the first rightmost lane. The simulation was conducted under the same traffic conditions of 2000 $\mathrm{veh} / \mathrm{h} /$ lane and same lane blockage configuration, but data was collected from the second rightmost lane. Ten simulation runs were selected for this case and the outputs obtained from all runs showed positive results as was expected from the planned test. The developed model was successfully able to detect incident for all ten different speed profiles obtained from each run. Figure 7.18 illustrates the drop in speed observed from each simulation run.

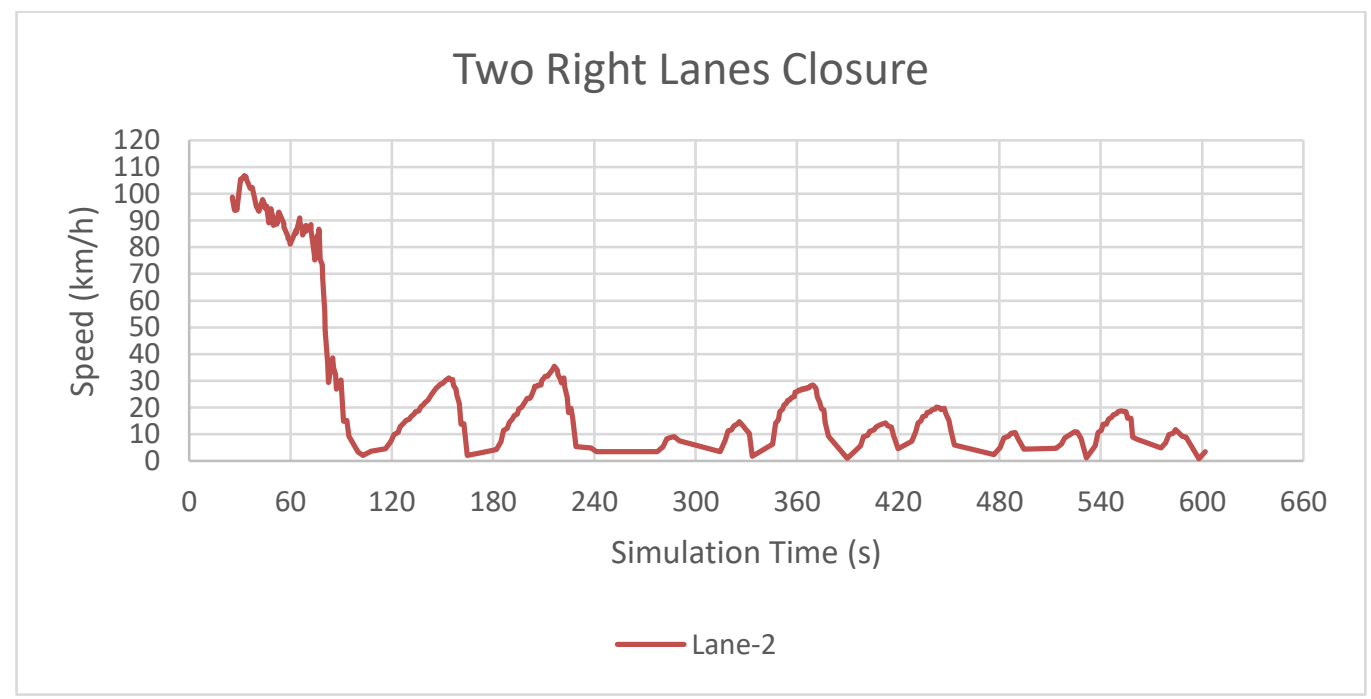

Figure 7.18: Speed data is collected from lane \#2, v=2000 veh/h/lane 
Under the specified traffic volume and given lane blockage configuration and considering that data are collected from the second rightmost lane the algorithm showed results as positive as in the previous case where data were collected from the first rightmost lane. $100 \%$ of the tested simulation runs when examined by the developed model showed an indication of an incident and the developed model was successfully able to detect the incident in every simulation run. Figure 7.19 illustrates incident detection utilizing the developed model and the other nine incident detection tested cases are given in Appendix D.2

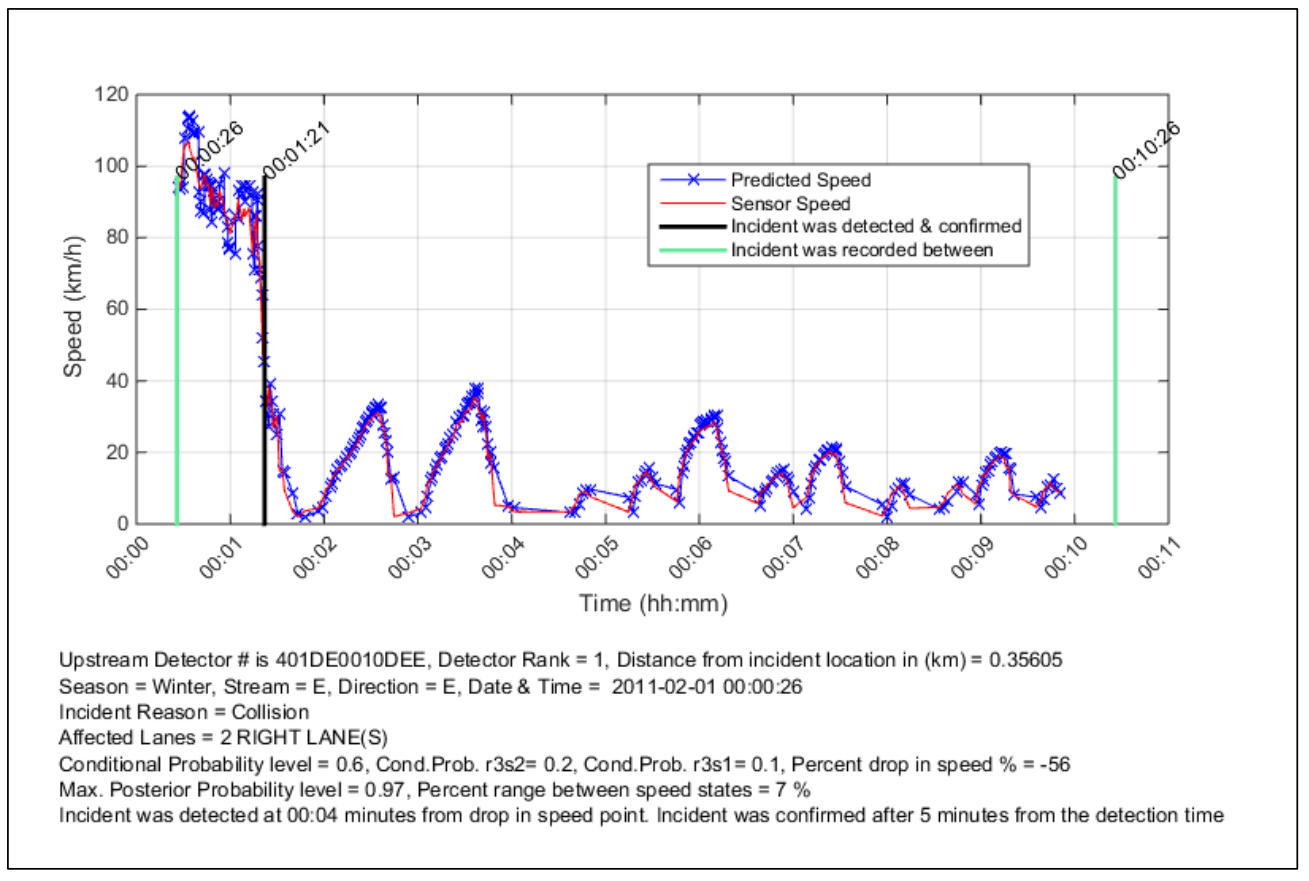

Figure 7.19: Speed data is collected from lane \#2, Run \#1, v=2000 veh/h/lane

In examining the traffic data collected from the third lane, under the same traffic condition ( $\mathrm{v}=2000 \mathrm{veh} / \mathrm{h} / \mathrm{lane})$ and same lane blockage configuration, obtained results showed a reasonable relation between time and speed. This relation is representative of showing speed reduction in the freeway section that occurred due to the incident. These 
implications can be interpreted as the effect of vehicle changing lanes and therefore interrupting traffic. However, low-speed values do not remain at the same low level. The speed recovers back to high values. Vehicles traveling in the third lane attempt to discourage lane changes by drivers from lanes one and two and consequently recover higher speed. As a result, speed recovers to high values that could reach $50 \%$ of the free flow speed for a short period of time and then back to low-speed value as illustrated in Figure 7.20.

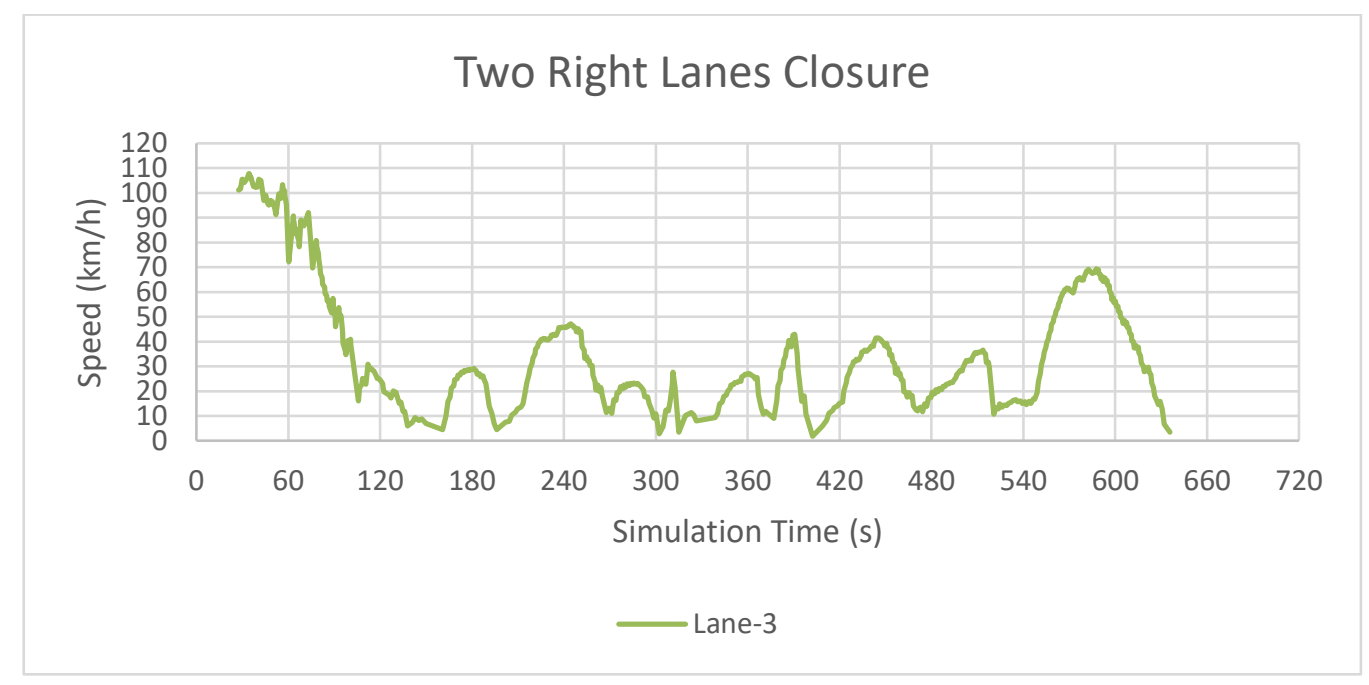

Figure 7.20: Speed data is collected from lane $\# 3, \mathrm{v}=2000 \mathrm{veh} / \mathrm{h} / \mathrm{lane}$

The relatively high-speed values do not last for long period of time, once a certain number of vehicles traveling in the first and second right lanes start the overtaking maneuver, the speed drops back again and so on. This traffic behaviour shows some escalation in the speed values, and it is clearer when recording the traffic data from the fourth leftmost lane as shown in Figure 7.21. 
The previous two scenarios (obtaining data from the third and fourth lanes) are shown to be not representative of the occurrence of an incident and might not be the optimum set of data to be used for incident detection purposes. Therefore, it was decided that data recorded from the first lane was the best representative scenario of recording time/speed data in the freeway in the event an incident took place and caused blockages to the two rightmost lanes in the freeway section.

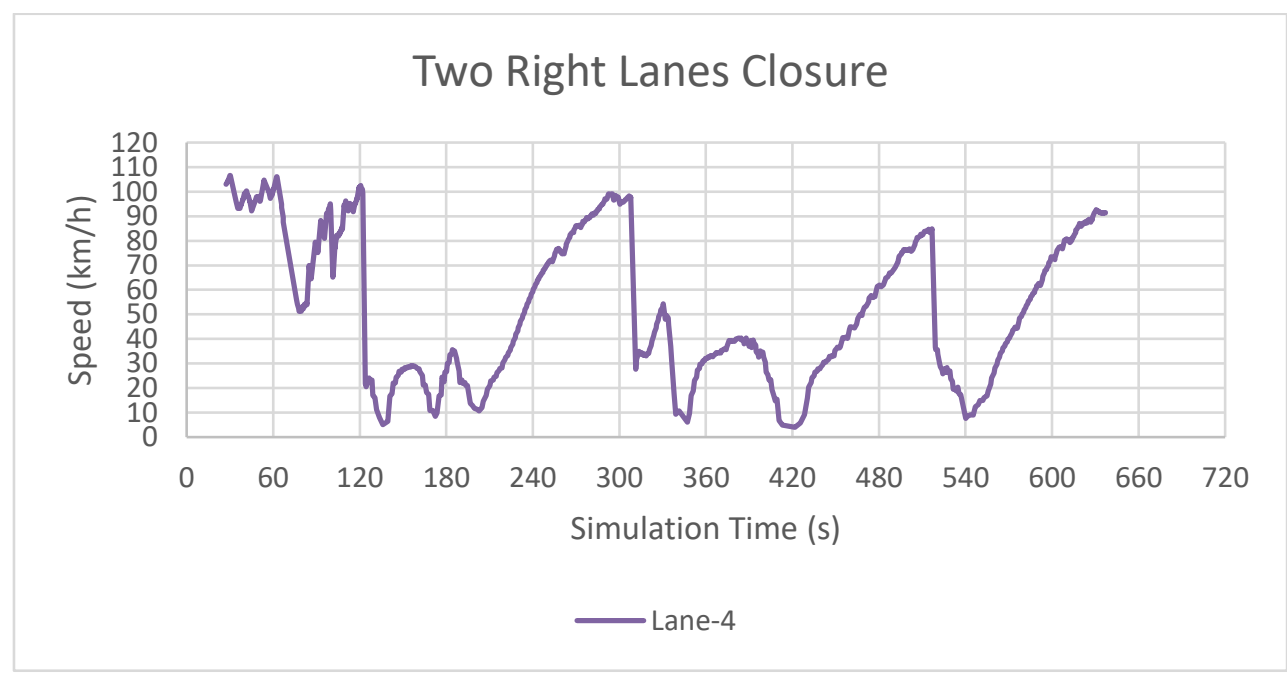

Figure 7.21: Speed data is collected from lane \#4, v=2000 veh/h/lane

\section{Two Lane Blockage Scenario with Traffic volume level of $1000 \mathrm{veh} / \mathrm{h} / \mathrm{lane}$ Collecting data from the first rightmost lane}

The second traffic volume level that is as important as the first selected volume level was $1000 \mathrm{veh} / \mathrm{h} / \mathrm{lane}$. This volume demonstrates a very low traffic volume which is the case for non-peak hour traffic. In such cases, vehicles are traveling at a free flow speed level and vehicle speed is not usually influenced by leading traffic. It is also expected that 
traffic volume is low enough for a clear indication of any drop in the speed that might occur due to any traffic incident. This traffic condition scenario is shown in Figure 7.22.

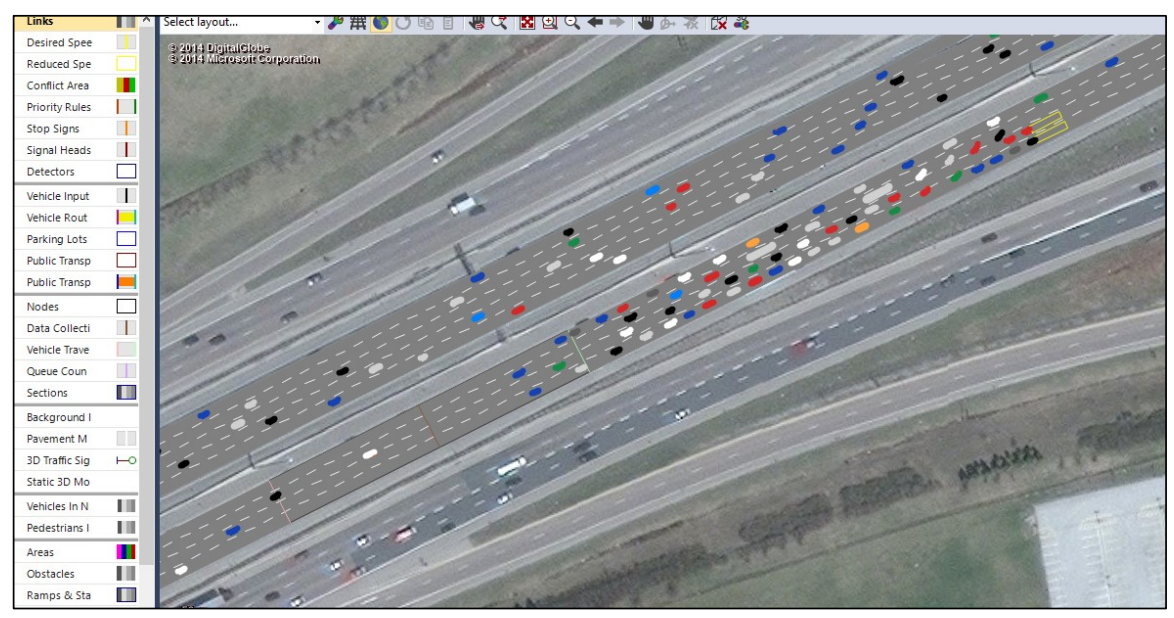

Figure 7.22: Traffic conformation from two lane blockage, $v=1000 \mathrm{veh} / \mathrm{h} /$ lane

As can be observed from the figure, vehicle batch up upstream is very short and that data collection point is not influenced by any significant slow speed movement. The quick backup could be explained as low traffic traveling on the freeway allows following vehicles to change lane without causing any significant interruption to the traffic flow movement. Therefore, a drop in speed was not the typical speed pattern.

Application of the developed model under the specified traffic volume of 1000 $\mathrm{veh} / \mathrm{h} /$ lane and a given lane blockage configuration and also considering that data are collected from the first lane, did not produce results required for meeting incident detection criteria. Therefore, the incident was not detected. Moreover, another 9 data sets obtained from 9 simulation runs using the developed model did not detect incidents for the same reason. Figure 7.23 illustrates incident detection results utilizing the developed model and the other nine incident detection tested cases are given in Appendix D.2. 


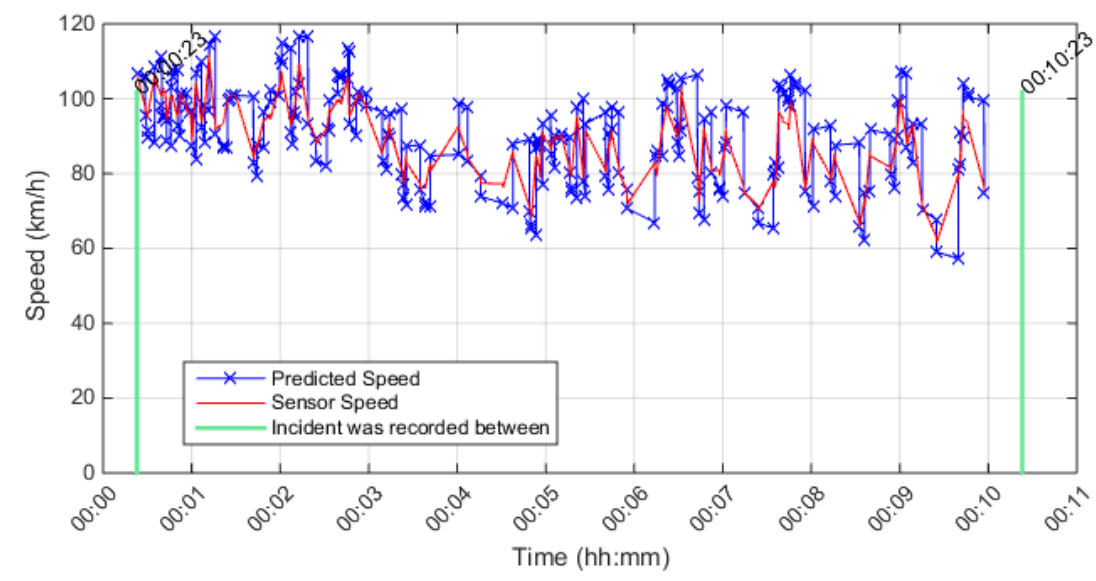

Upstream Detector \# is 401DE0010DEE, Detector Rank = 1, Distance from incident location in $(\mathrm{km})=0.35605$ Season $=$ Winter, Stream $=E$, Direction $=E$, Date $\&$ Time $=2011-02-0100: 00: 23$ Incident Reason $=$ Collision

Affected Lanes = 2 RIGHT LANE(S)

Conditional Probability level $=0.6$, Cond.Prob. $\mathrm{r} 3 \mathrm{~s} 2=0.2$, Cond.Prob. $\mathrm{r} 3 \mathrm{~s} 1=0.1$, Percent drop in speed $\%=-56$

Max. Posterior Probability level $=0.97$, Percent range between speed states $=7 \%$

(No Incident was Detected)

Figure 7.23: Speed data is collected from lane \#1, Run \#1, v=1000 veh/h/lane

\section{Two Lane Blockage Scenario with Traffic volume level of $1000 \mathrm{veh} / \mathrm{h} / \mathrm{lane}$ \\ Collecting data from the second rightmost lane}

As in the previous scenario, it was necessary to examine the location of the data collection point. Traffic data were obtained from the first lane in the previous discussions. In this, part data were obtained from the second lane, and the difference in obtained results was observed. The simulation run was conducted under the same traffic conditions of 1000 $\mathrm{veh} / \mathrm{h} /$ lane and same lane blockage configuration. Ten runs were selected for this case and results obtained from all runs fall in the expectations planned for this test. It was found that the developed model did not detect any incident under low traffic volume condition. Figure 7.24 illustrates incident detection results utilizing the developed model and the other nine incident detection tested cases are given in Appendix D.2. 


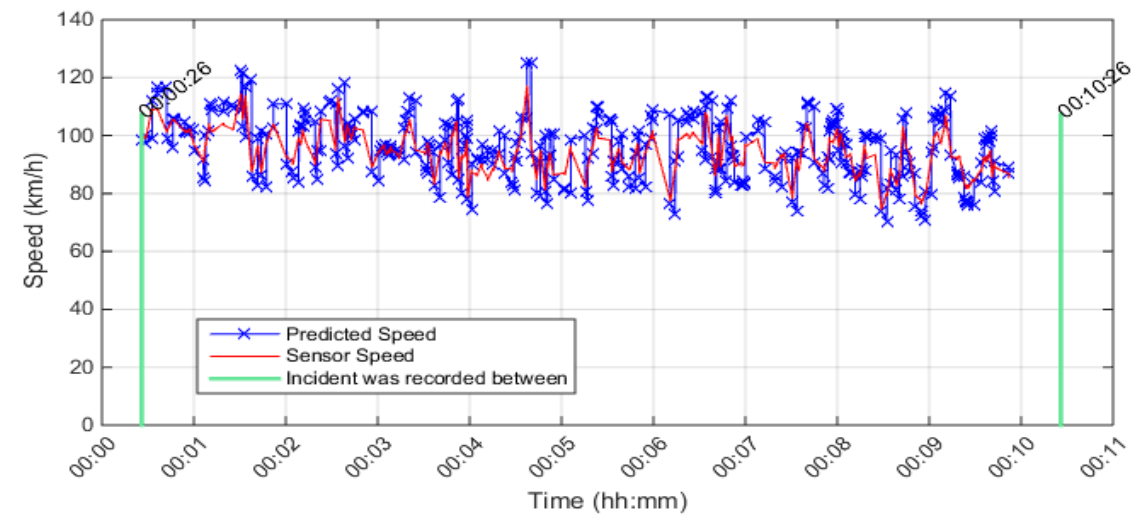

Upstream Detector \# is 401DE0010DEE, Detector Rank = 1, Distance from incident location in $(\mathrm{km})=0.35605$ Season $=$ Winter, Stream $=E$, Direction $=E$, Date \& Time $=2011-02-0100: 00: 26$ Incident Reason $=$ Collision

Affected Lanes $=2$ RIGHT LANE(S)

Conditional Probability level $=0.6$, Cond.Prob. $r 3 s 2=0.2$, Cond.Prob. $r 3 s 1=0.1$, Percent drop in speed $\%=-56$ Max. Posterior Probability level $=0.97$, Percent range between speed states $=7 \%$ (No Incident was Detected)

Figure 7.24: Speed data is collected from lane \#2, Run \#1, v=1000 veh/h/lane

\section{Two Lane Blockage Scenario with Traffic volume level of $1500 \mathrm{veh} / \mathrm{h} / \mathrm{lane}$ \\ Collecting data from the first rightmost lane}

The third traffic volume level was $1500 \mathrm{veh} / \mathrm{h} / \mathrm{lane}$. This is a medium traffic volume which is the case of the freeway traffic at some hours in the day where the traffic is not too high as well as not too low. In such cases, speed is somehow influenced by leading traffic. It is expected that traffic volume is high enough for a clear indication of any drop in the speed that might occur due to a traffic incident. This traffic condition scenario is shown in Figure 7.25 in the first simulation seconds and as time passes traffic is backing up as shown in Figure 7.26. 


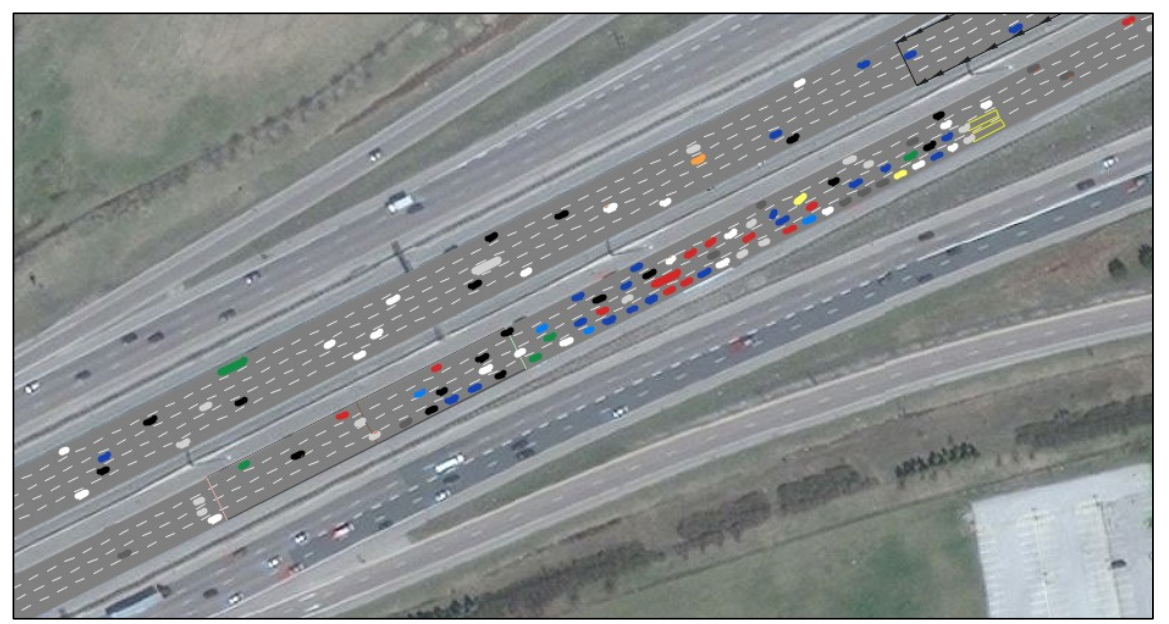

Figure 7.25: Traffic conformation from two lane blockage, $v=1500 \mathrm{veh} / \mathrm{h} / \mathrm{lane}, 1^{\text {st }}$ seconds of simulation

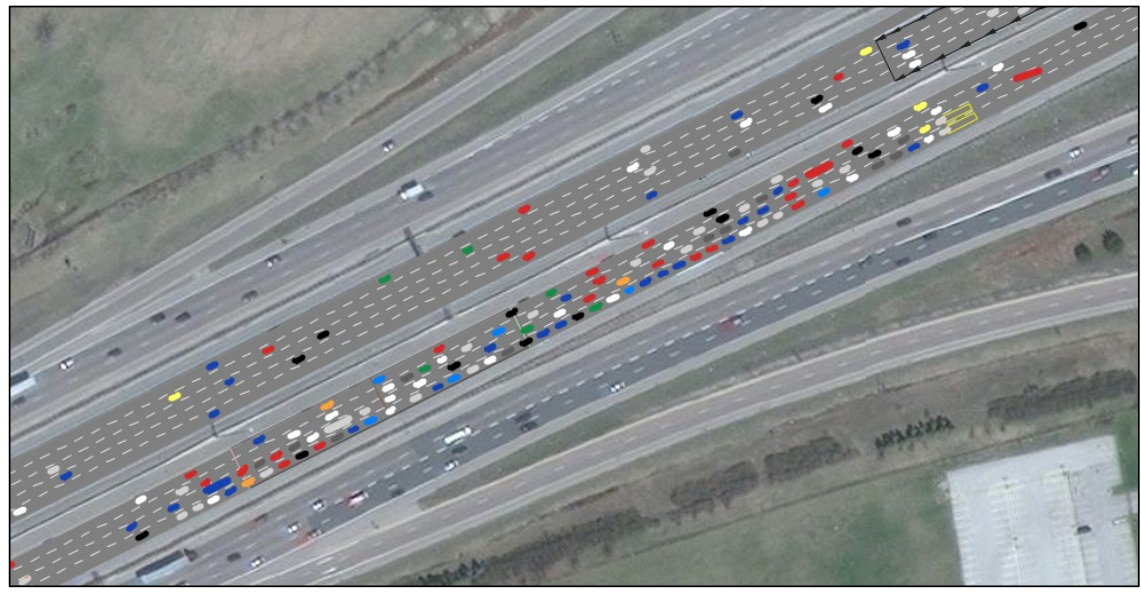

Figure 7.26: Traffic conformation from two lane blockage, v=1500 veh/h/lane, few min later

As can be observed from the above figure, due to the relatively high traffic volume incident causes some congestion upstream the stopped vehicle. The backup continues to build up causing a significant drop in the traffic speed movement. The significant decline could be explained by the high traffic traveling on the freeway, preventing following 
vehicles to change lanes and successful attempts result in traffic interrupts. Therefore, a drop in speed was the typical speed pattern.

Under the specified traffic volume of $1500 \mathrm{veh} / \mathrm{h} / \mathrm{lane}$ and the given two lane blockage configuration and considering data was collected from the first lane, the model was applied to test this case. Results indicate an incident detection. Examining 10 data sets obtained from 10 simulation runs using the developed model, it was found that all cases showed a significant drop in speed which is the cause the developed model was successful in detecting the incident under the specified traffic volume condition. Figure 7.27 illustrates incident detection results utilizing the developed model and the other nine incident detection tested cases are presented in Appendix D.2.

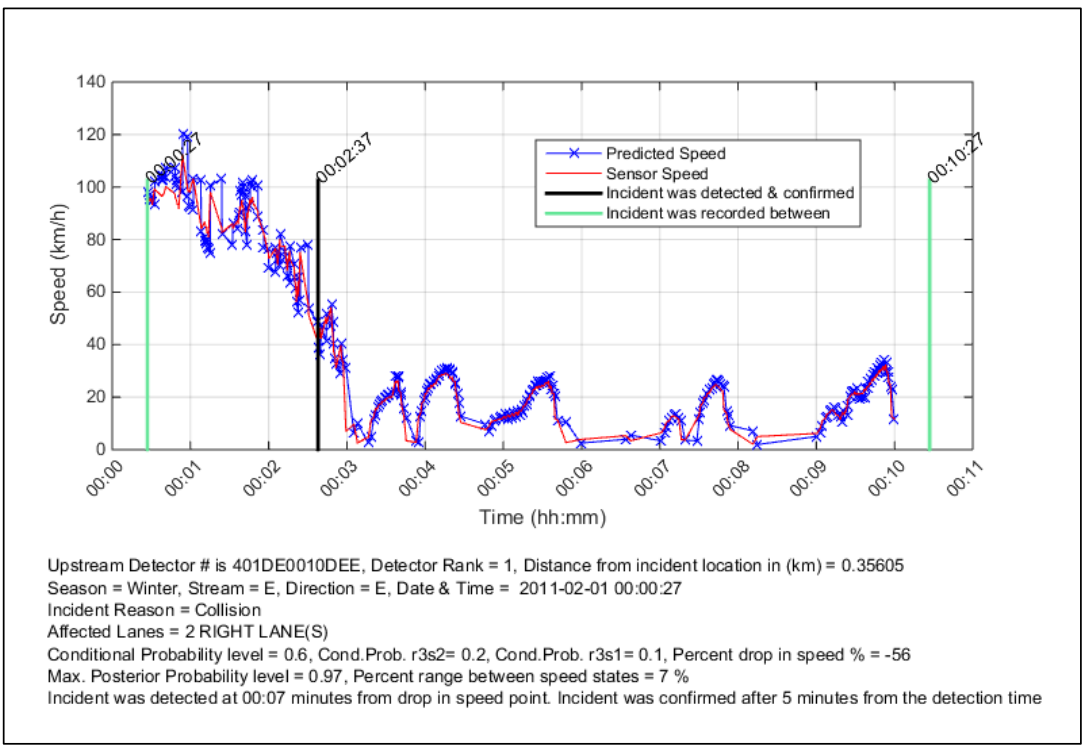

Figure 7.27: Speed data is collected from the lane \#1, Run \#1, v=1500 veh/h/lane 


\section{Two Lane Blockage Scenario with Traffic volume level of $1500 \mathrm{veh} / \mathrm{h} / \mathrm{lane}$}

\section{Collecting data from the second rightmost lane}

The simulation was conducted under the same traffic conditions of $1500 \mathrm{veh} / \mathrm{h} / \mathrm{lane}$ and same lane blockage configuration as used in the previous scenario. Ten runs were selected for this case and results obtained from all runs were found to match the expectations planned for this test. The developed model detected incidents under the present relatively high traffic volume condition. Figure 7.28 illustrates incident detection results utilizing the developed model and the other nine incident detection test cases are given in Appendix D.2.

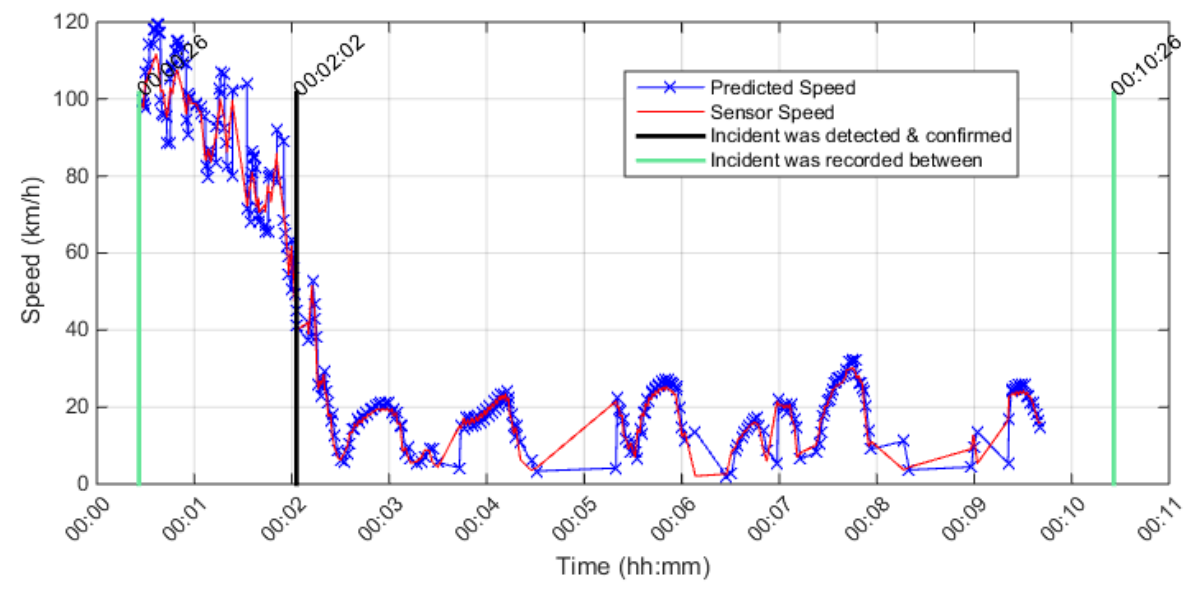

Upstream Detector \# is 401DE0010DEE, Detector Rank = 1, Distance from incident location in $(\mathrm{km})=0.35605$ Season $=$ Winter, Stream $=E$, Direction $=E$, Date \& Time $=2011-02-0100: 00: 26$ Incident Reason $=$ Collision

Affected Lanes $=2$ RIGHT LANE(S)

Conditional Probability level $=0.6$, Cond.Prob. $r 3 \mathrm{~s} 2=0.2$, Cond.Prob. $r 3 \mathrm{~s} 1=0.1$. Percent drop in speed $\%=-56$

Max. Posterior Probability level $=0.97$, Percent range between speed states $=7 \%$

Incident was detected at 00:07 minutes from drop in speed point. Incident was confirmed after 5 minutes from the detection time

Figure 7.28: Speed data is collected from lane \#2, Run \#1, v=1500 veh/h/lane 


\section{Two Lane Blockage Scenario with Traffic volume level of $1250 \mathrm{veh} / \mathrm{h} / \mathrm{lane}$}

\section{Collecting data from the first rightmost lane}

Observations from the previous two different scenarios including the 1000 and the $1500 \mathrm{veh} / \mathrm{h} /$ lane suggest the need to investigate one more traffic volume level. Incident detection results obtained from low traffic volume of $1000 \mathrm{veh} / \mathrm{h} / \mathrm{lane}$ do not show a drop in speed and hence no incident was detected. On the other hand, incident detection results obtained from high traffic flow level of $2000 \mathrm{veh} / \mathrm{h} /$ lane show a significant drop in speed and incident was detected for all tested cases.

Next, $1250 \mathrm{veh} / \mathrm{h} /$ lane case was investigated. This volume level is considered below medium level and much closer to non-peak traffic condition. Figure 7.29 illustrates traffic flow condition in the first moments of the simulation while Figure 7.30 shows traffic flow conditions after some minutes from the beginning of the simulation.

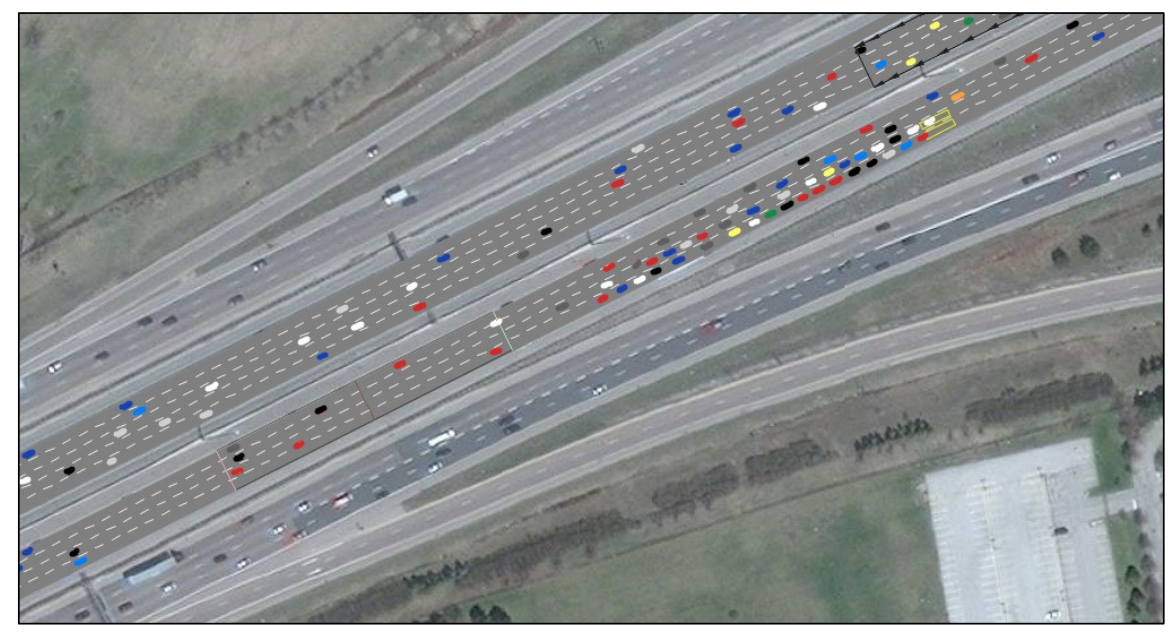

Figure 7.29: Traffic conformation from two lane blockage, $v=1250 \mathrm{veh} / \mathrm{h} / \mathrm{lane}, \mathbf{1}^{\text {st }}$ moments 


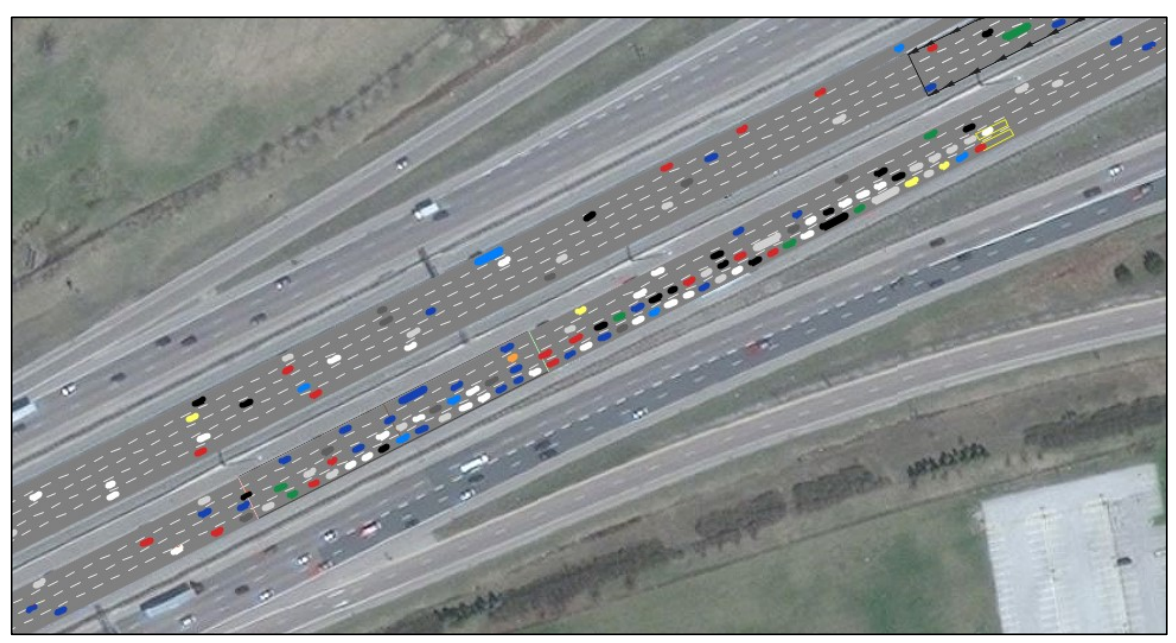

Figure 7.30: Traffic conformation from two lane blockage, $v=1250 \mathrm{veh} / \mathrm{h} / \mathrm{lane}$, few min later

In such cases, traffic speed is somewhat affected by traffic volume. However, it was found that this level of traffic was high enough for a clear indication of speed drop that might occur due to a traffic incident. Figure 7.31 shows a significant drop in speed that made it possible to detect the incident using the developed model. But, the developed model was not able to detect the incident in all cases, as illustrated in Figure 7.32. In such cases, the drop in speed did not satisfy the criteria built into the model to detect incidents. The other nine incident detection tested cases are included in Appendix D.2.

On the basis of ten simulation runs, it was found that in $80 \%$ of the tested cases, the incident was detected using the developed model. 


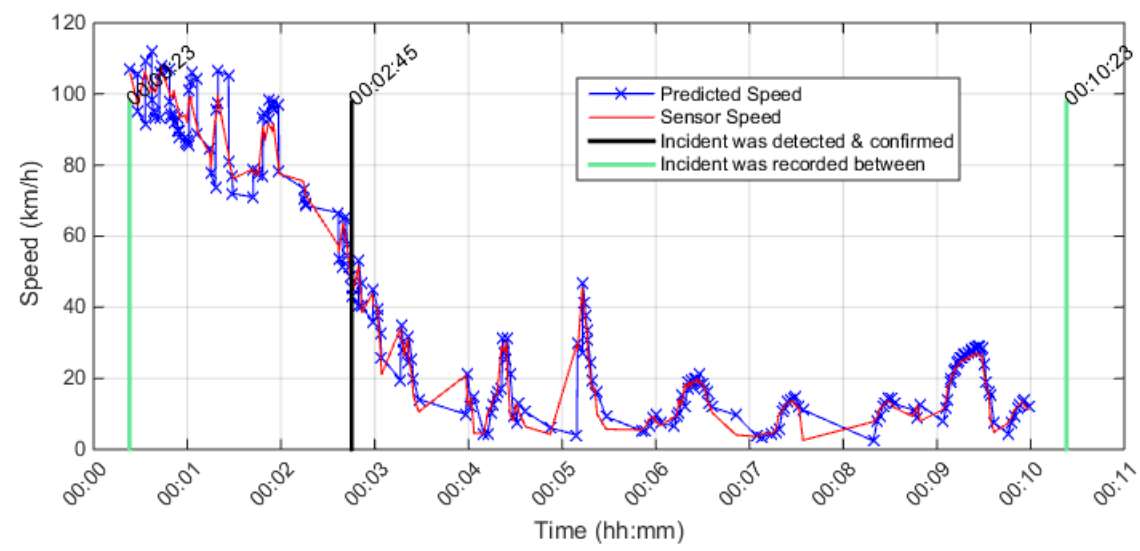

Upstream Detector \# is 401DE0010DEE, Detector Rank $=1$, Distance from incident location in $(\mathrm{km})=0.35605$ Season $=$ Winter, Stream $=E$, Direction $=E$, Date \& Time $=2011-02-0100: 00: 23$

Incident Reason $=$ Collision

Affected Lanes $=2$ RIGHT LANE(S)

Conditional Probability level $=0.6$, Cond.Prob. $r 3 s 2=0.2$, Cond.Prob. $r 3 s 1=0.1$, Percent drop in speed $\%=-56$

Max. Posterior Probability level $=0.97$, Percent range between speed states $=7 \%$

Incident was detected at 00:46 minutes from drop in speed point. Incident was confirmed after 5 minutes from the detection time

Figure 7.31: Speed data is collected from lane \#1, Run \#1, v=1250 veh/h/lane

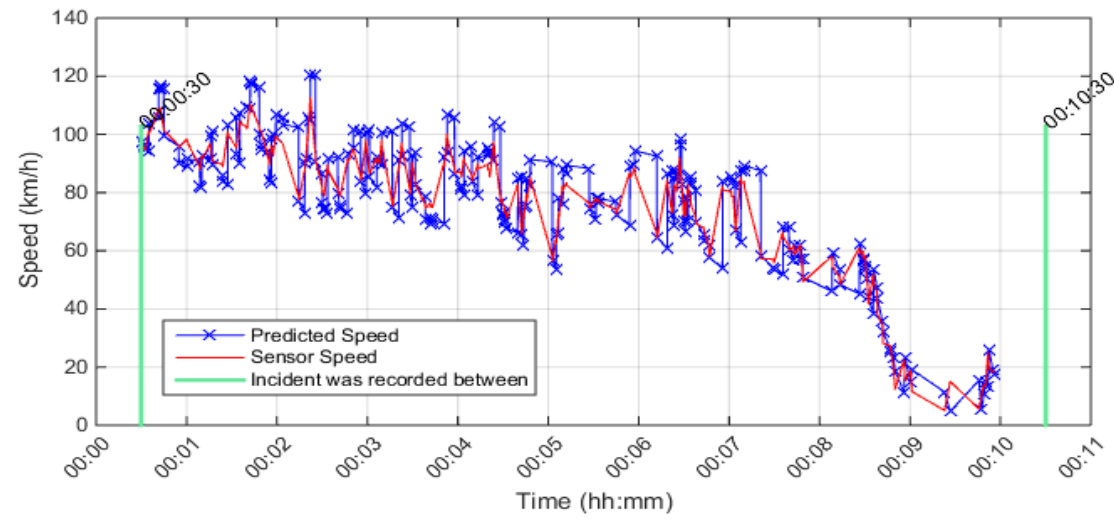

Upstream Detector \# is 401DE0010DEE, Detector Rank = 1, Distance from incident location in $(\mathrm{km})=0.35605$ Season $=$ Winter, Stream $=E$, Direction $=E$, Date \& Time $=$ 2011-02-01 00:00:30 Incident Reason $=$ Collision

Affected Lanes $=2$ RIGHT LANE(S)

Conditional Probability level $=0.6$, Cond.Prob. $r 3 s 2=0.2$, Cond.Prob. $r 3 s 1=0.1$, Percent drop in speed $\%=-56$

Max. Posterior Probability level $=0.97$, Percent range between speed states $=7 \%$

Drop in speed was detected but Not enough recovery time to confirm an incident

Figure 7.32: Speed data is collected from lane \#1, Run \#2, v=1250 veh/h/lane 


\section{Two Lane Blockage Scenario with Traffic volume level of $1250 \mathrm{veh} / \mathrm{h} / \mathrm{lane}$}

\section{Collecting data from the second rightmost lane}

Here, data were obtained from the second lane, and results were compared with the case when data were obtained from lane 1. Ten runs were carried out. It was found that in $70 \%$ of this relatively low traffic volume simulation cases, the developed model detected the incidents. Figure 7.33 illustrates incident detection results utilizing the developed model for the case when the incident was detected, and Figure 7.34 illustrates one of the cases when no incident was detected. The other eight incident detection tested cases are given in Appendix D.2.

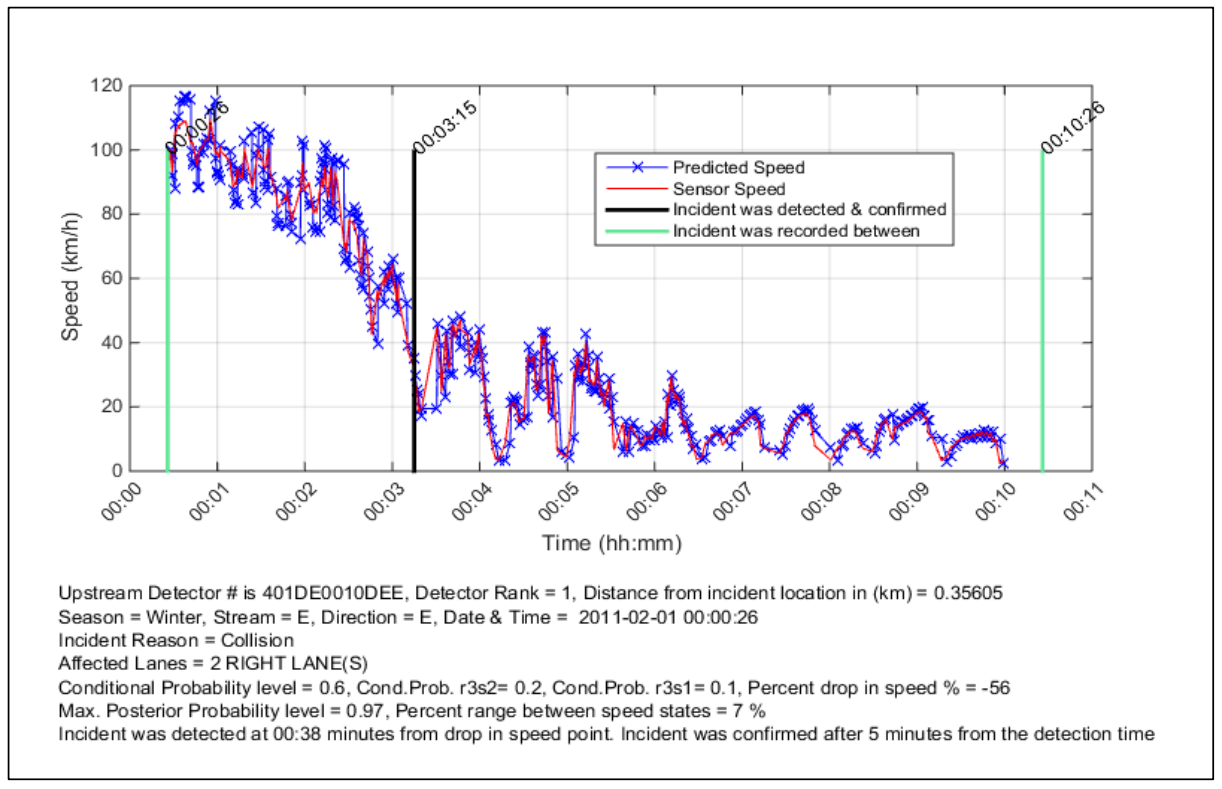

Figure 7.33: Speed data is collected from lane \#2, Run \#1, v=1250 veh/h/lane 


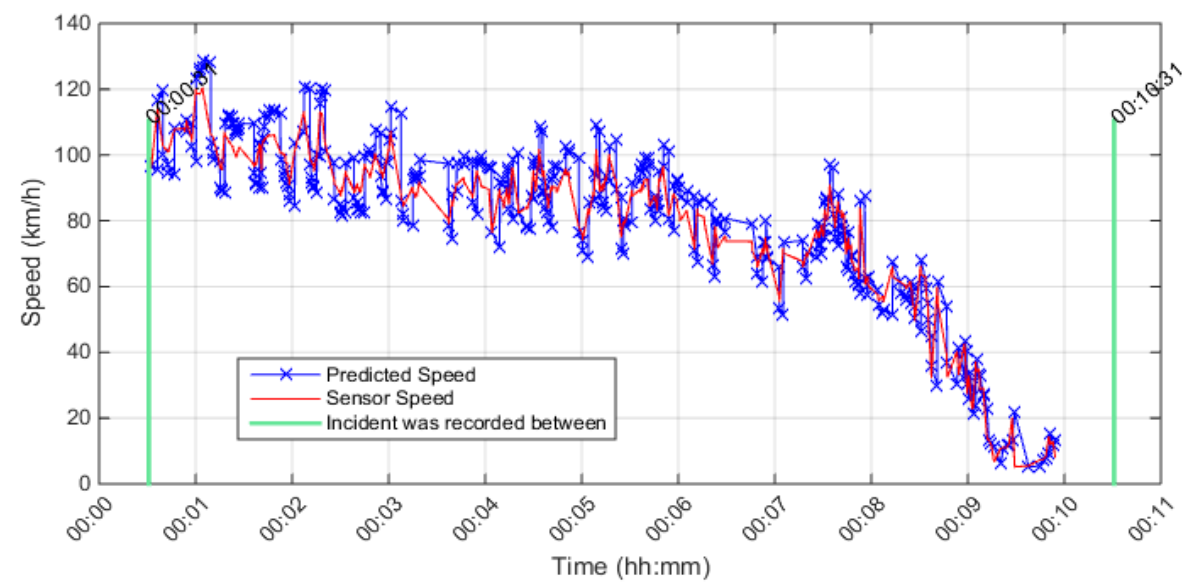

Upstream Detector \# is 401DE0010DEE, Detector Rank $=1$, Distance from incident location in $(\mathrm{km})=0.35605$ Season $=$ Winter, Stream $=E$, Direction $=E$, Date \& Time $=$ 2011-02-01 00:00:31 Incident Reason $=$ Collision

Affected Lanes $=2$ RIGHT LANE(S)

Conditional Probability level $=0.6$, Cond.Prob. $r 3 s 2=0.2$, Cond.Prob. $r 3 s 1=0.1$, Percent drop in speed $\%=-56$

Max. Posterior Probability level $=0.97$, Percent range between speed states $=7 \%$

Drop in speed was detected but Not enough recovery time to confirm an incident

Figure 7.34: Speed data is collected from lane \#2, Run \#2, v=1250 veh/h/lane

\section{Three Lane Blockage Scenario with Traffic volume level of $2000 \mathrm{veh} / \mathrm{h} / \mathrm{lane}$}

\section{Collecting data from the first, second, third and fourth lanes}

Other scenarios such as three lane blockage configuration and four-lane blockage configuration were also tested, and the drop in traffic speed was examined. Figure 7.35 shows the traffic flow condition under three lane blockage configuration along with the associated upstream traffic jam. Traffic volume in this scenario was selected to be 2000 $\mathrm{veh} / \mathrm{h} / \mathrm{lane}$ and data were obtained from data collection point located in the first lane. Three lane blockage showed a significant impact on the freeway capacity and therefore, a rapid drop in the speed occurred as shown in Figure 7.36. If the model is to be tested for detecting the incident in this case, it is expected that the model will detect the incident. 


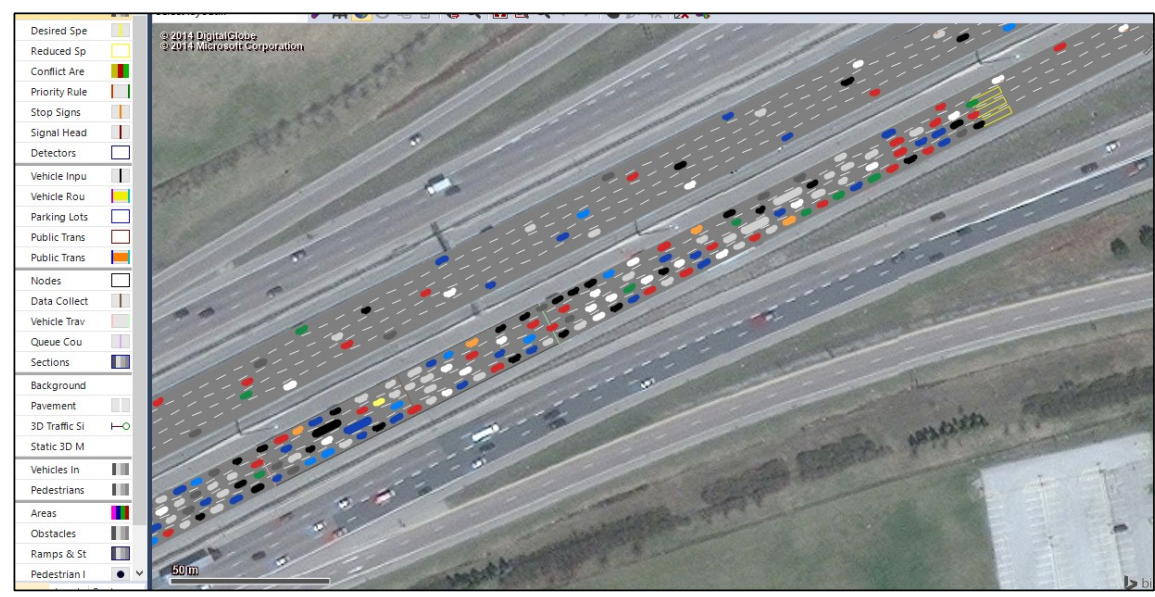

Figure 7.35: Traffic conformation from three lane blockage, $v=2000 \mathrm{veh} / \mathrm{h} / \mathrm{lane}$

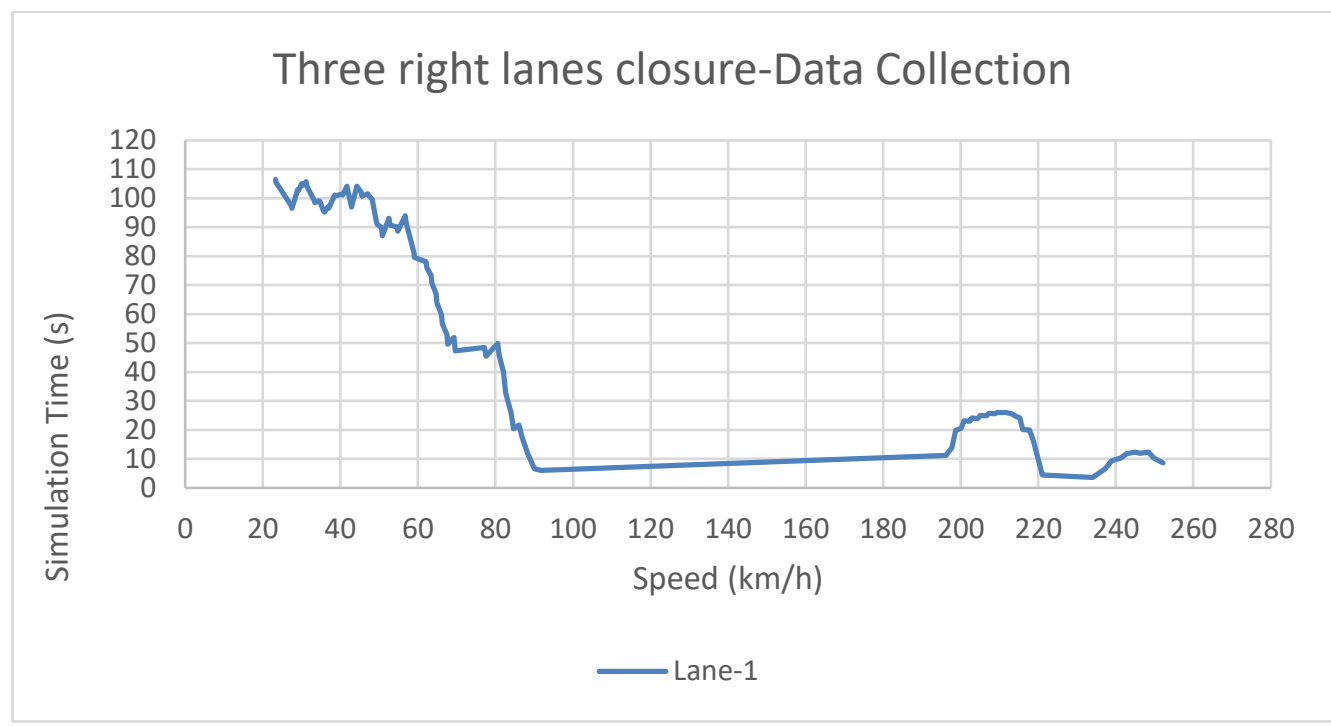

Figure 7.36: Speed data is collected from lane \#1, v=2000 veh/h/lane

When data collection point was located at either the second or the third lane, similar rapid drop in speed was observed. This is deemed an ideal scenario for offering high incident detection potential. See Figure 7.37 and Figure 7.38. 


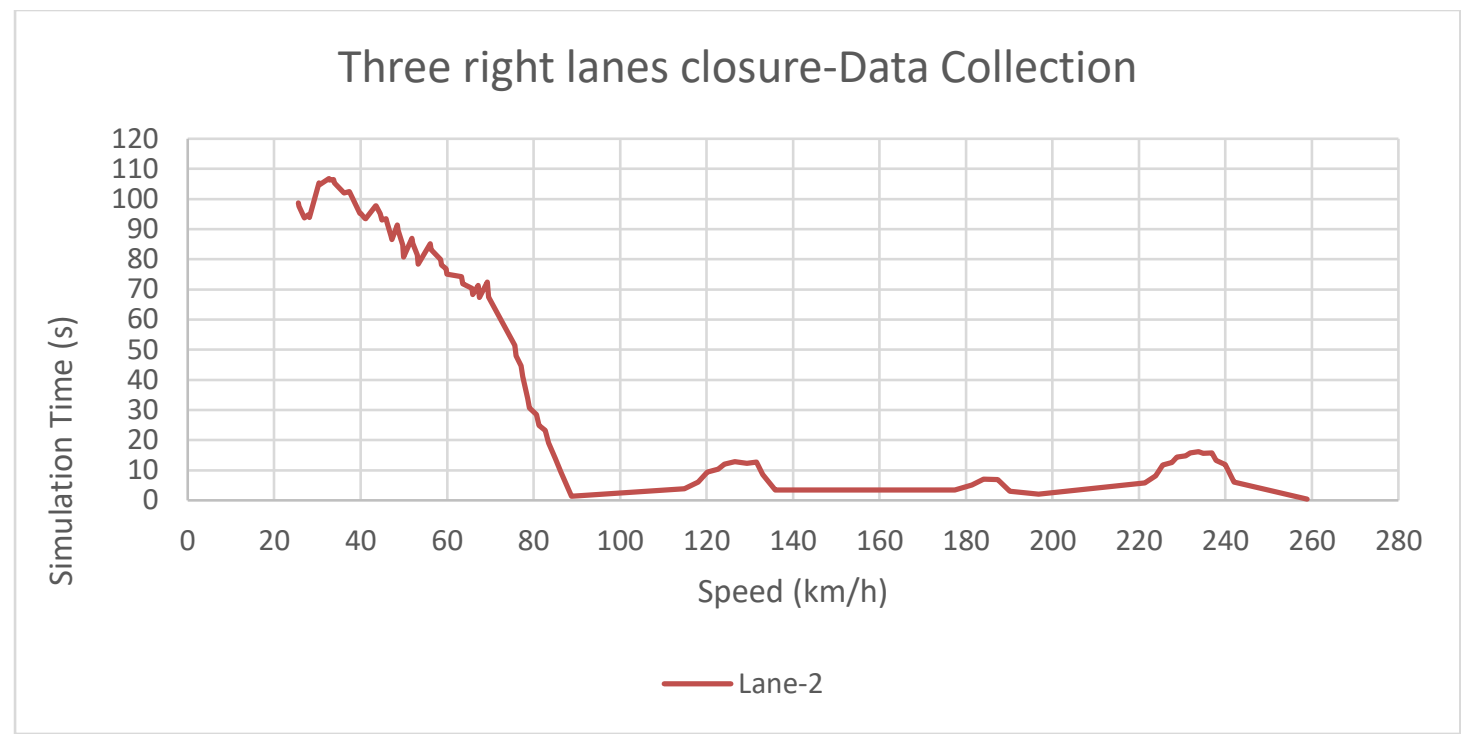

Figure 7.37: Speed data is collected from lane \#2, v=2000 veh/h/lane

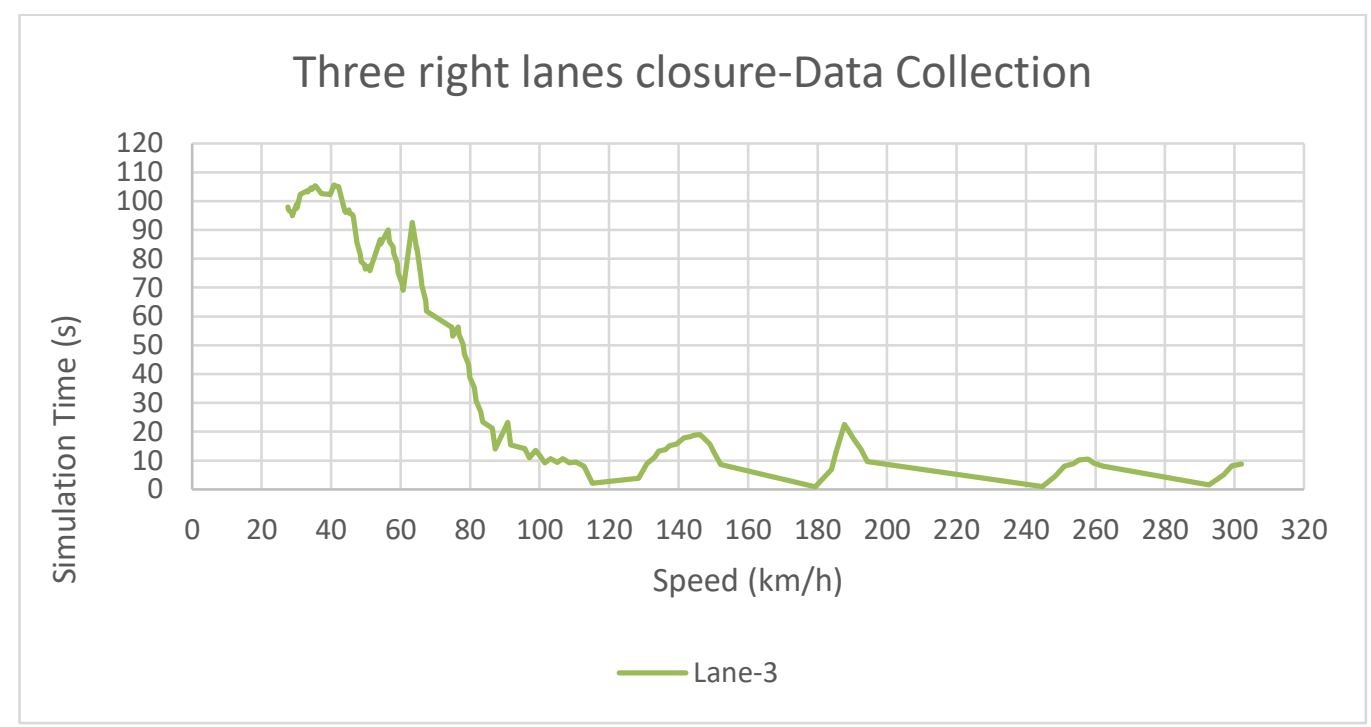

Figure 7.38: Speed data is collected from lane \#3, v=2000 veh/h/lane

When the data were collected from the fourth lane, speed values indicated that at some time interval some vehicles managed to recover high speed but a rapid drop in speed occurred right after as illustrated in Figure 7.39. 


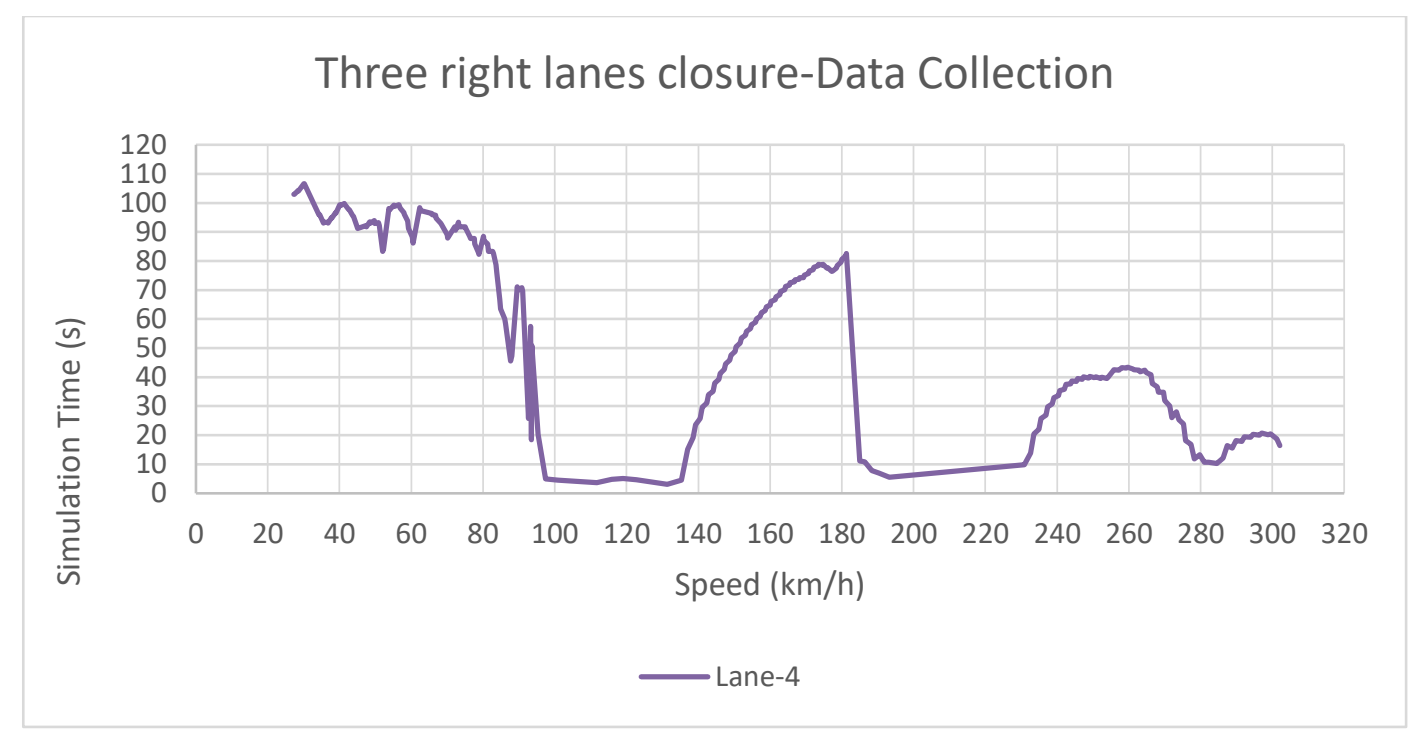

Figure 7.39: Speed data is collected from lane \#4, v=2000 veh/h/lane

\section{Four Lane Blockage Scenario with Traffic volume level of $2000 \mathrm{veh} / \mathrm{h} / \mathrm{lane}$}

\section{Collecting data from the first, second, third and fourth lanes}

Like the three-lane blockage case, the four-lane blockage scenario also showed a significant drop in speed and therefore offered the potential to detect the incident. Figure 7.40 shows the four-lane blockage configuration. When data collection point was located at the first, second, third and fourth lanes, a drop in the speed was observed. Results shown in Figure 7.41, Figure 7.42, Figure 7.43 and Figure 7.44 do not suggest high potential for incident detection. The speed drop is not significant since it is similar to lanes closure or maintenance scenario. 


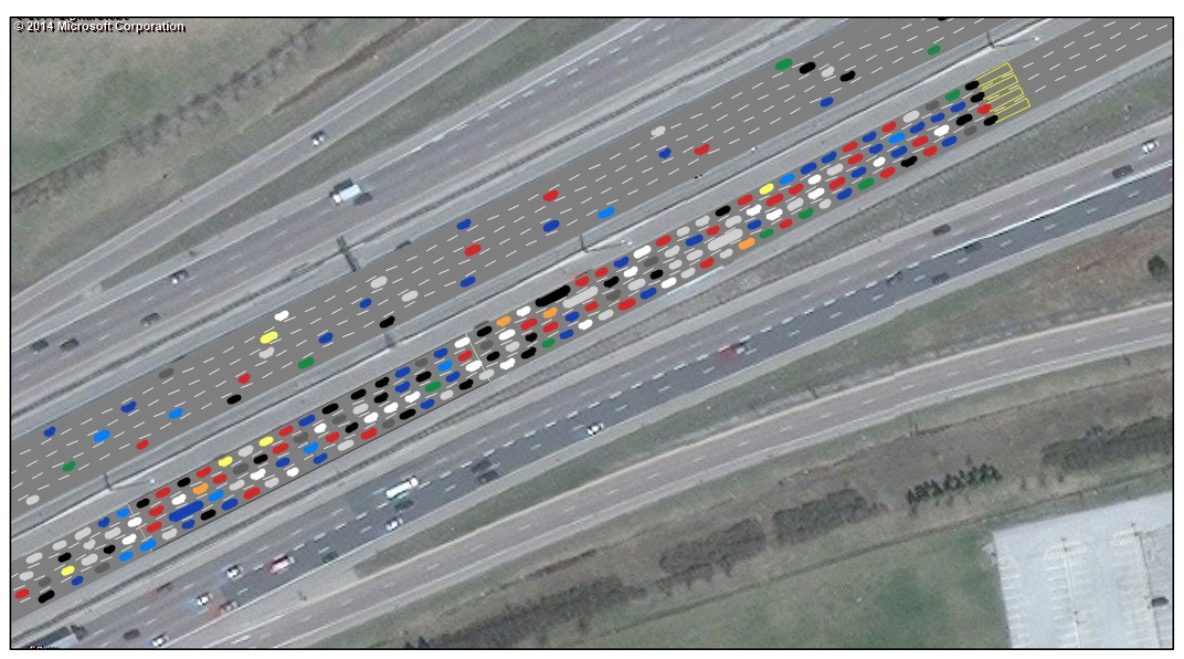

Figure 7.40: Traffic conformation from four lane blockage, $v=2000 \mathrm{veh} / \mathrm{h} / \mathrm{lane}$

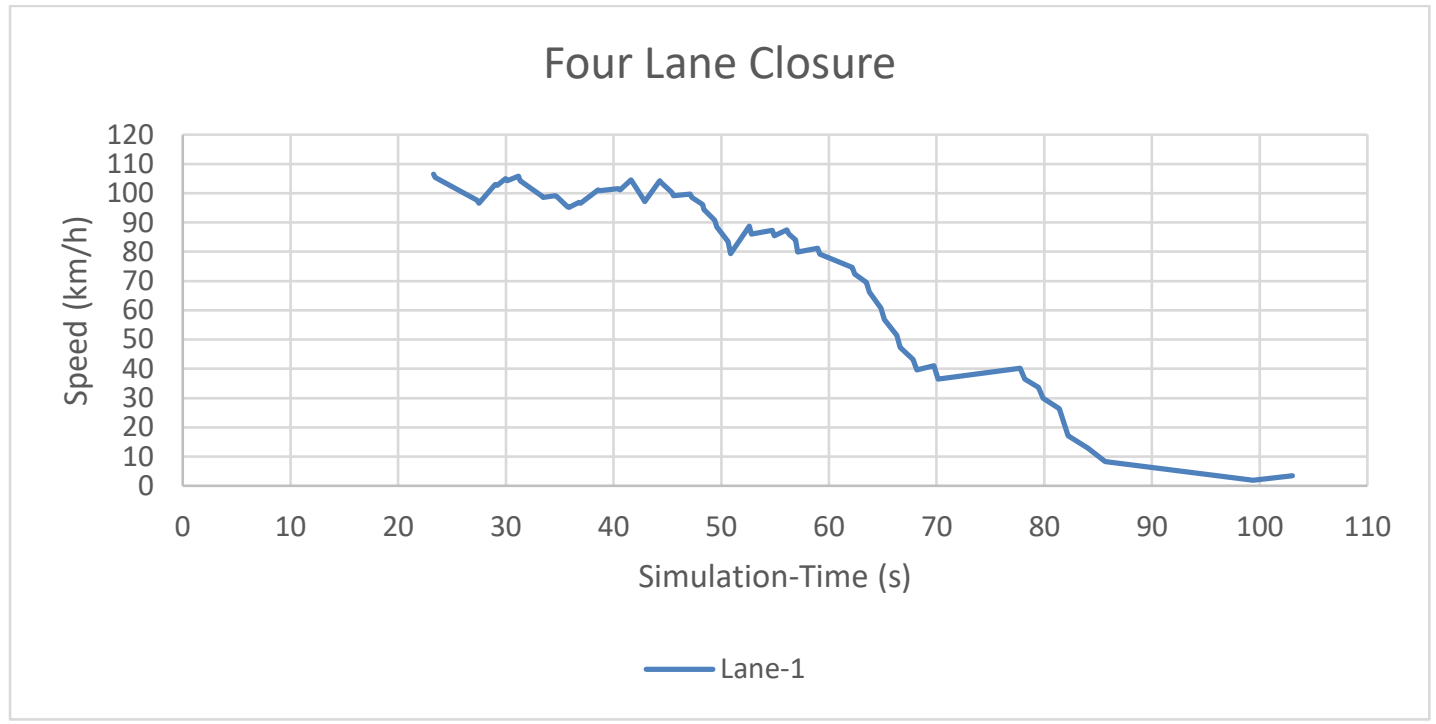

Figure 7.41: Speed data is collected from lane \#1, v=2000 veh/h/lane 


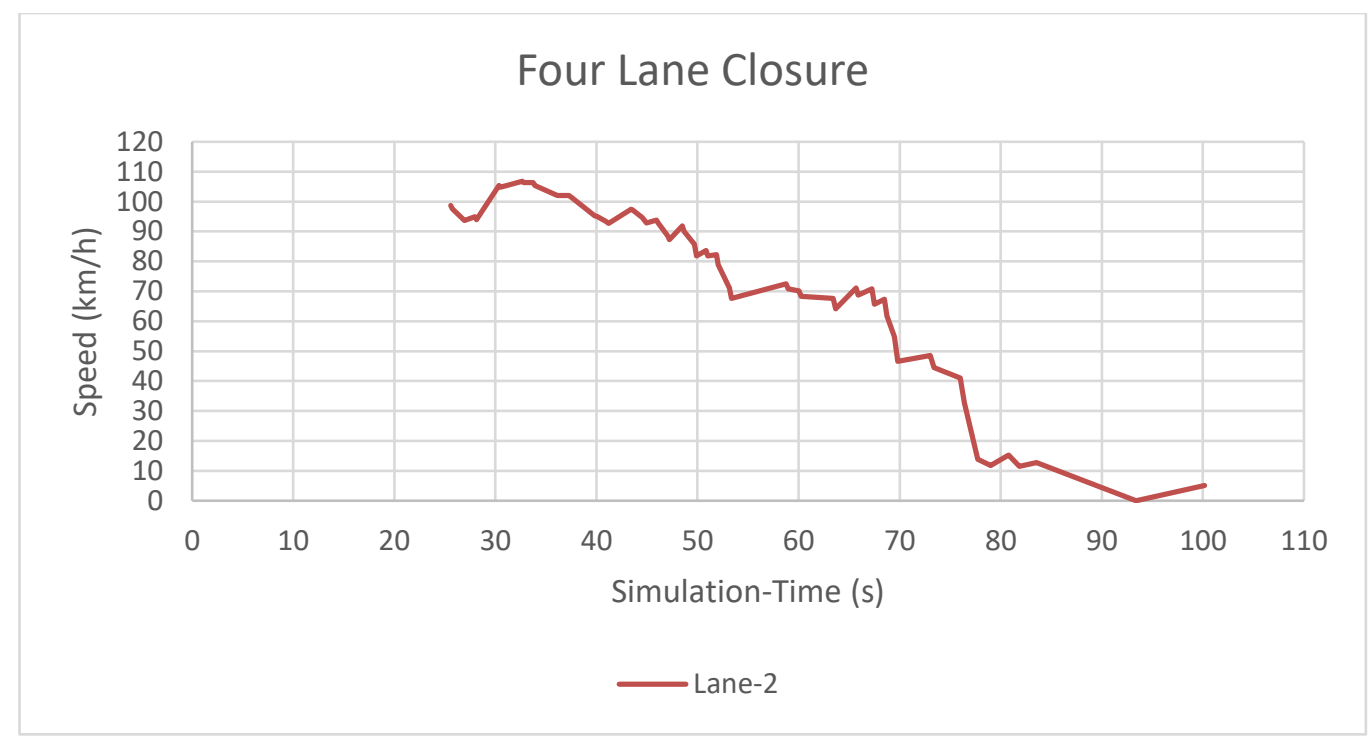

Figure 7.42: Speed data is collected from lane \#2, v=2000 veh/h/lane

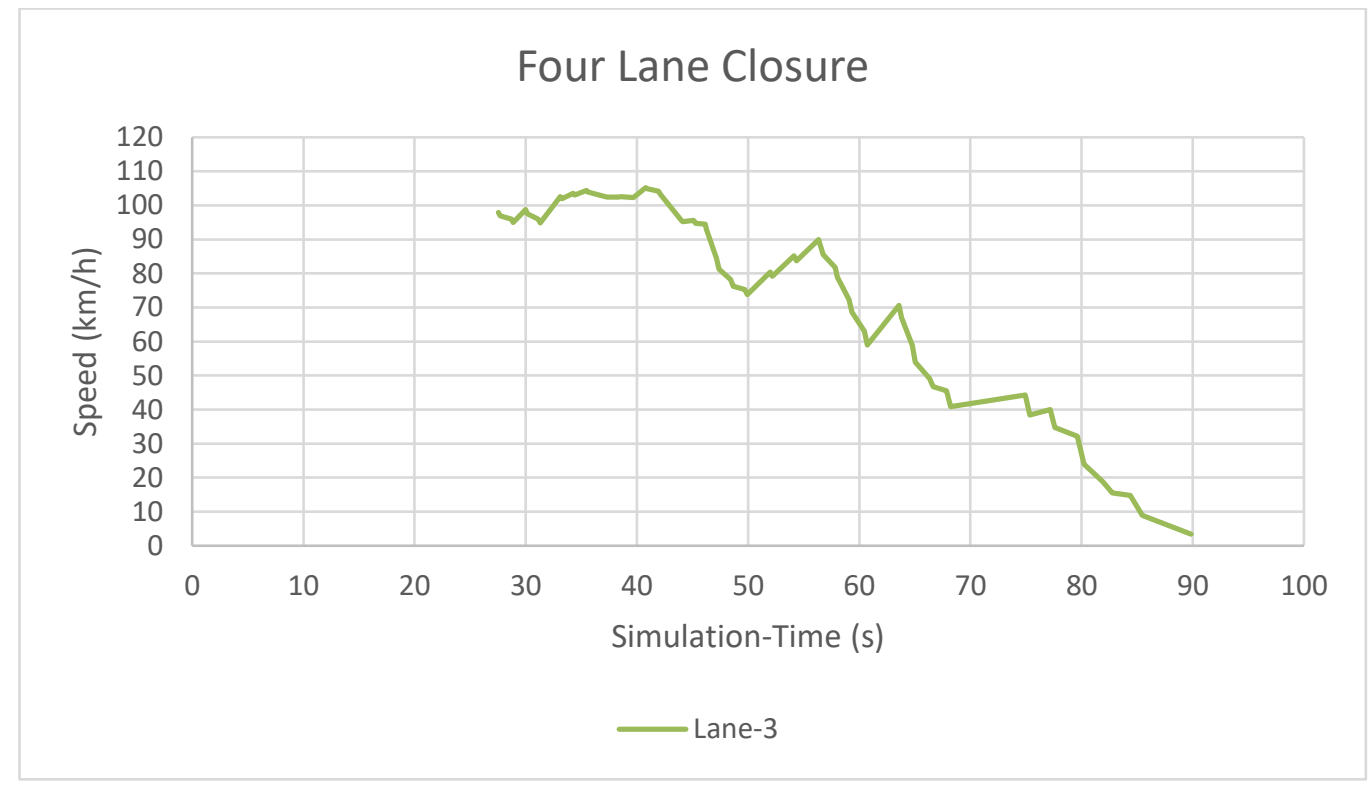

Figure 7.43: Speed data is collected from lane \#3, v=2000 veh/h/lane 


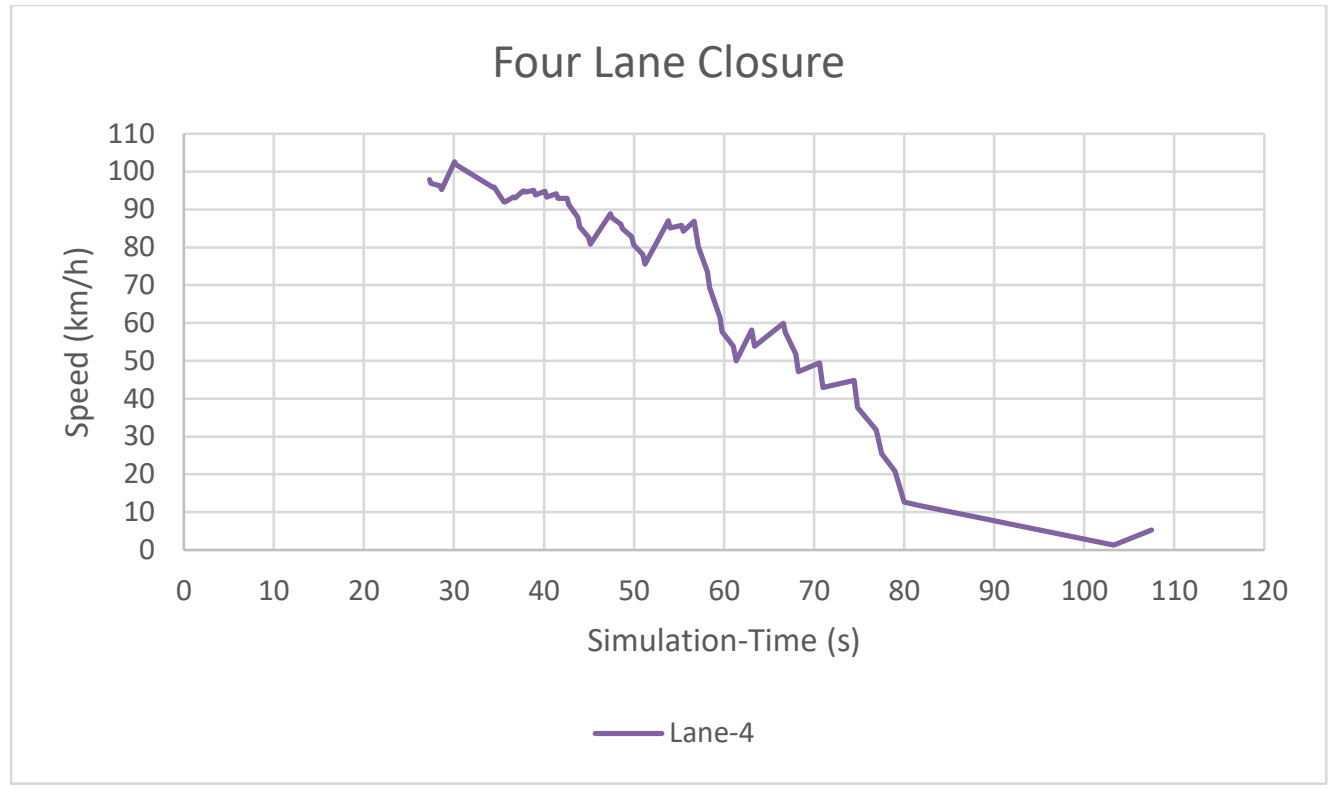

Figure 7.44: Speed data is collected from lane \#4, v=2000 veh/h/lane

Results obtained from simulation of incidents are discussed in the following subsections. Incident detection accuracy of the developed model is noted to be due to the availability of real-world field data that was used earlier to calibrate and validate the model before its application to Vissim-generated outputs.

\subsection{Discussion on Vissim Model and the Model Output}

Vissim microsimulation was used to simulate predefined freeway incidents for four typical different scenarios, in addition to simulating traffic flow when there was no incident in the freeway section.

As expected, speed is the most easily understood term and measure. The drop in speed that was observed from the simulation results when the incident was introduced in the simulated traffic at the freeway section showed a consistent pattern produced by an incident. Obtained results from the predefined scenarios were used for testing the 
developed incident detection model. In the following subsections, each result set is discussed.

Scenario 2a - Incident occurred in the rightmost lane of the freeway section:

In this scenario, an incident was simulated in the rightmost lane of the freeway section. The incident was located at 950 meters from the starting point, and the data collection point was located $200 \mathrm{~m}$ (upstream) from the incident location, the desired speed on the link was $100 \mathrm{~km} / \mathrm{h}$. Regarding the speed pattern the following was observed:

1- When reading data from data collection point located in the first lane, it was noticed that rapid drop in the speed started in the second minute of simulation time. This rapid drop was interpreted as of the significant impact of the incident on upstream traffic traveling in the first lane. As well, the speed dropped until it reached almost to a stopped condition and continued to oscillate between few $\mathrm{km} / \mathrm{h}$ above $40 \mathrm{~km} / \mathrm{h}$ and zero speed. This pattern continued to be the same until the end of the simulation period of ten minutes as illustrated in Figure 7.45.

2- The speed profile obtained from data collection point located in the second lane showed a dramatic drop in speed after 2 minutes as well. However, speed continued to oscillate until the end of the simulation time but reached high values up to $80 \mathrm{~km} / \mathrm{h}$ during some time intervals. The oscillation in speed was interpreted as vehicles traveling in the second lane were interrupted by lane changes by vehicles from lane 1. As shown in Figure 7.46. 


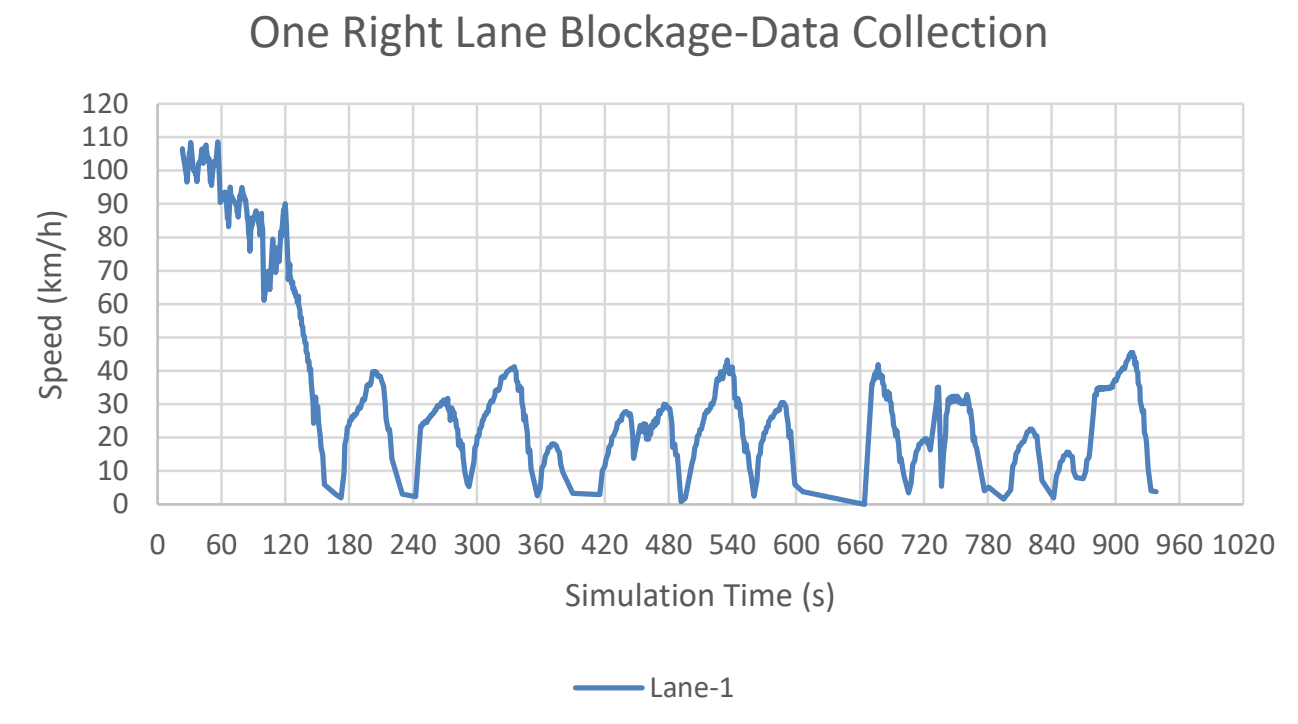

Figure 7.45: Speed data is collected from lane \#1, v=2000 veh/h/lane

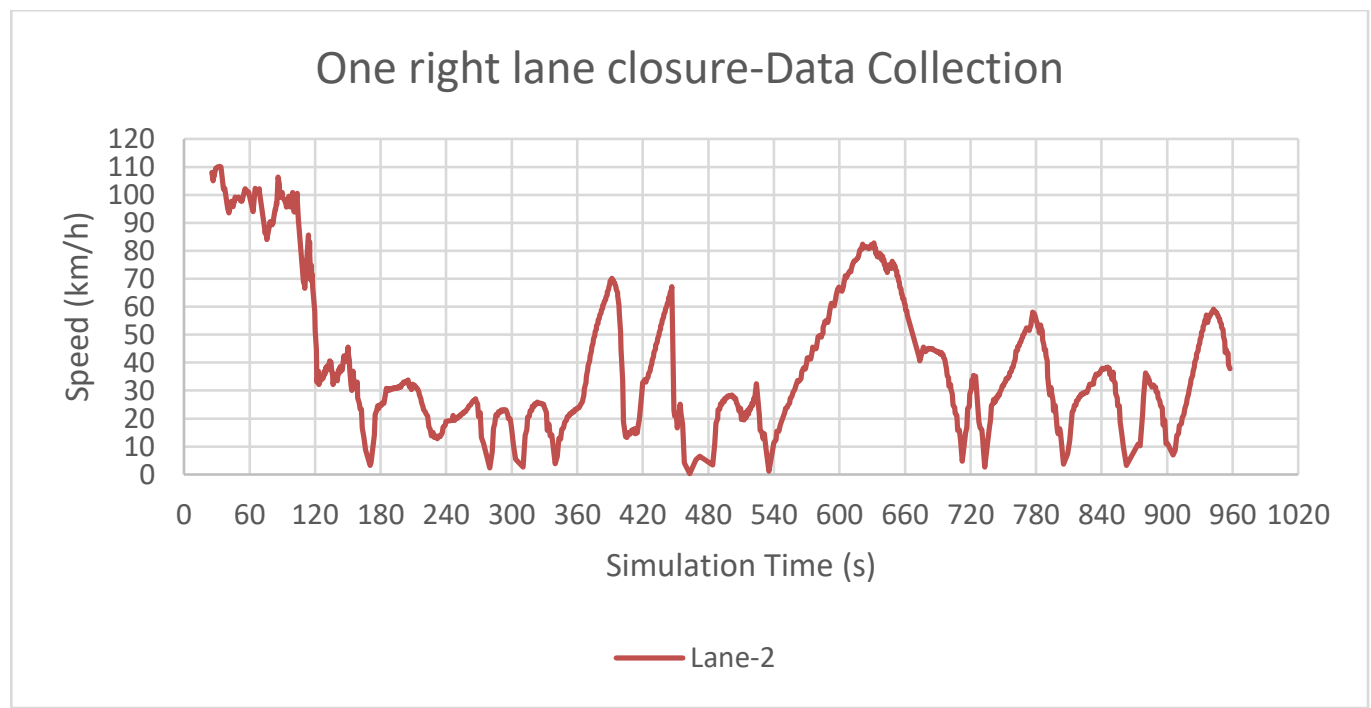

Figure 7.46: Speed data is collected from lane \#2, v=2000 veh/h/lane

3- Another two scenarios included incident simulated in the first rightmost lane and traffic speed on the link showed frequent oscillation from very high to very low values. In this case, data were obtained from data collection point located in either the third or fourth 
lane. These two scenarios showed that traffic moving in the third and fourth lane was not significantly affected by the traffic jam caused by an incident in the first lane. Rather, traffic was interrupted every time an overtaking process happened as a regular action from traffic in the first lane as illustrated in Figure 7.47 and Figure 7.48.

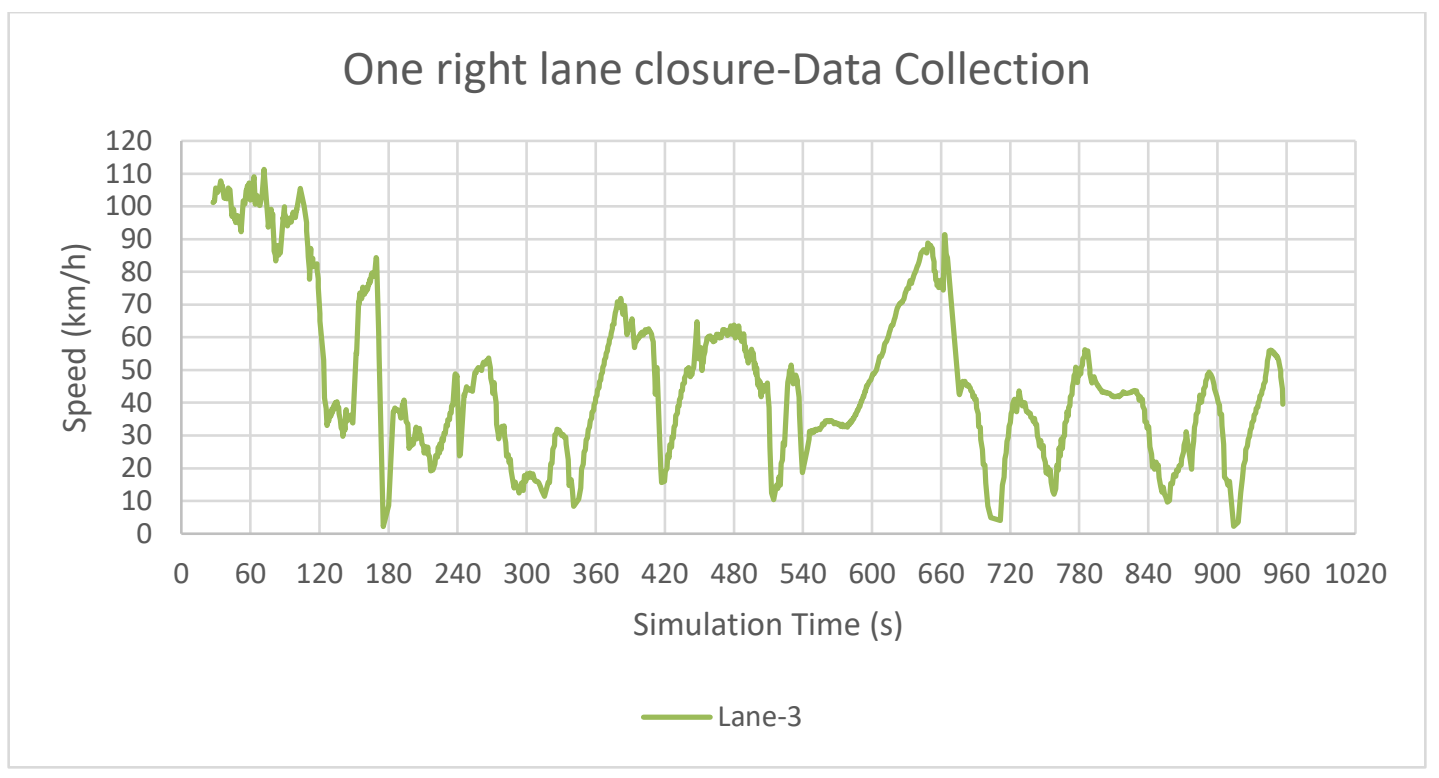

Figure 7.47: Speed data is collected from lane $\# 3, \mathbf{v}=\mathbf{2 0 0 0} \mathrm{veh} / \mathrm{h} / \mathrm{lane}$

Scenario $2 \mathbf{b}$ - Incident blocked the rightmost two lanes of the freeway section:

In this scenario, an incident was simulated in the two rightmost lanes of the freeway. The incident was located at 950 meters from the starting point, and the data collection point was located at $200 \mathrm{~m}$ from the incident location, the selected desired speed on the link was $100 \mathrm{~km} / \mathrm{h}$ which required about 35 seconds to arrive at the stopped vehicle. Regarding the speed pattern the following was observed: 


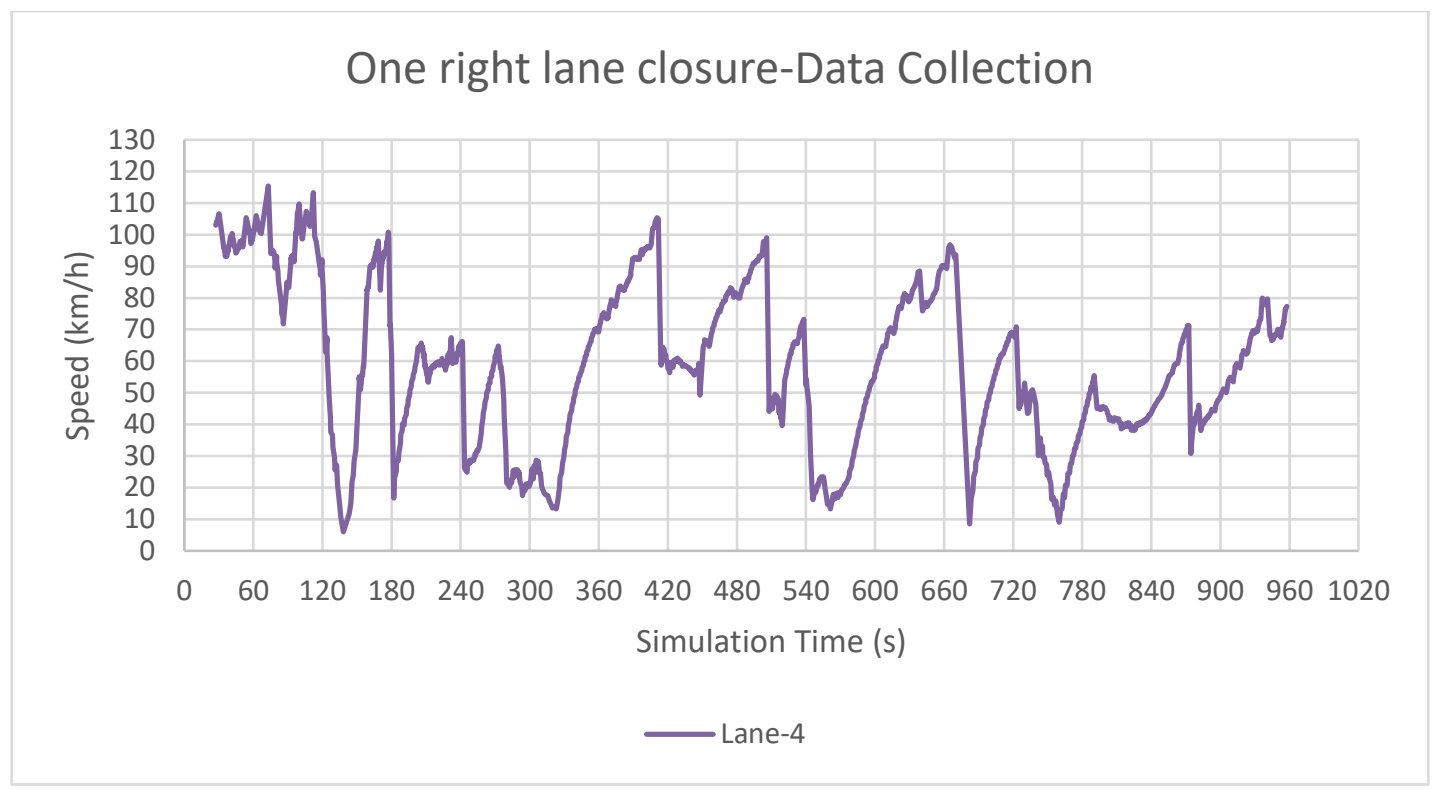

Figure 7.48: Speed data is collected from lane \#4, v=2000 veh/h/lane

1- When reading data from data collection point located in the first lane, it was noticed that dramatic drop in the speed started at the 90th second of simulation time. This was interpreted as a significant impact of the incident on upstream traffic traveling in the first lane. As well, the speed dropped until it reached almost to a stopping speed and continue to oscillate between few $\mathrm{km} / \mathrm{h}$ below $30 \mathrm{~km} / \mathrm{h}$ and zero speed. This pattern continued until the end of the simulation period of 10 minutes as illustrated in Figure 7.49 . 


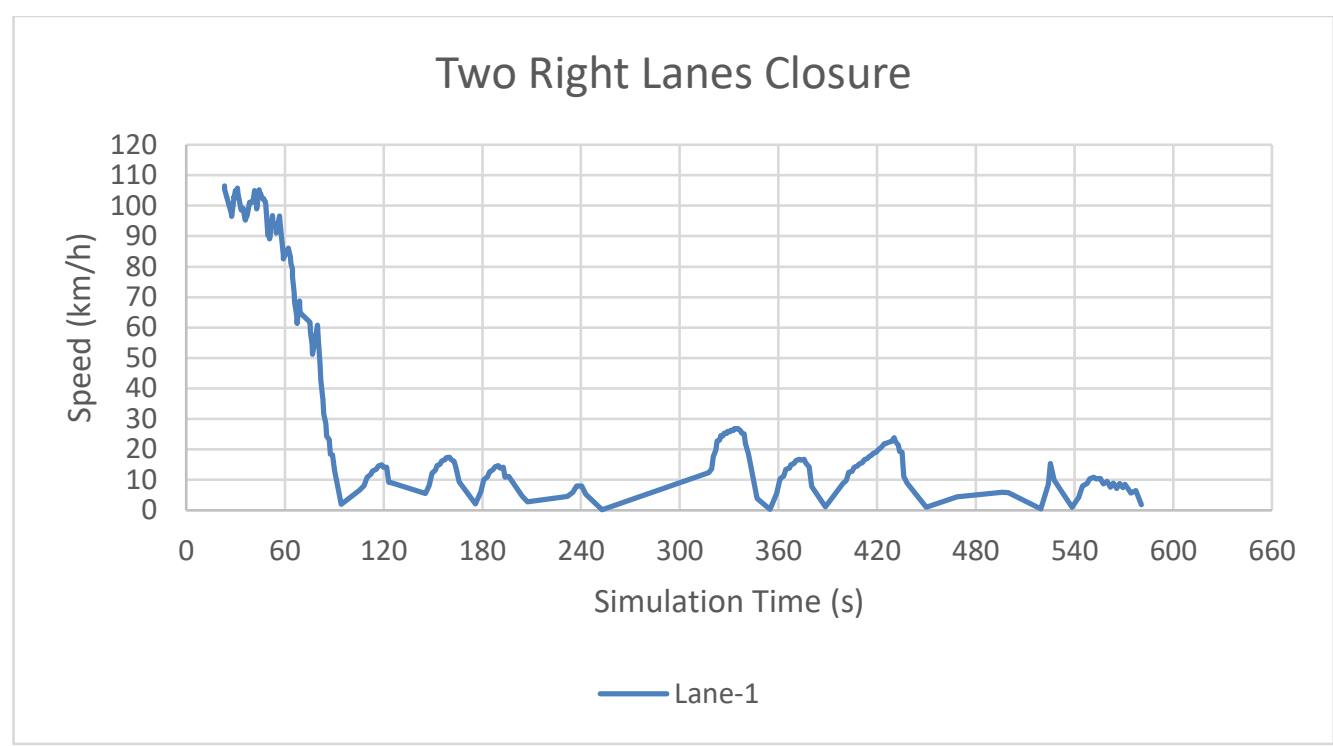

Figure 7.49: Speed data is collected from lane \#1, v=2000 veh/h/lane

2- On the other hand, the speed profile obtained from data collection point located on the second lane shows a dramatic drop in speed after 90 seconds. However, speed continued to oscillate until the end of the simulation time but reached high values around $30 \mathrm{~km} / \mathrm{h}$ for more than once at some time intervals. The low-speed value was interpreted as vehicles traveling in the second lane were interrupted by slowing leading vehicles in the second lane but somehow managed to overtake leading traffic at some safe time. Therefore, speed recovered to approximately $30 \mathrm{~km} / \mathrm{h}$ but then dropped back again due to the continuous jam in the two right lanes as illustrated in Figure 7.50. 


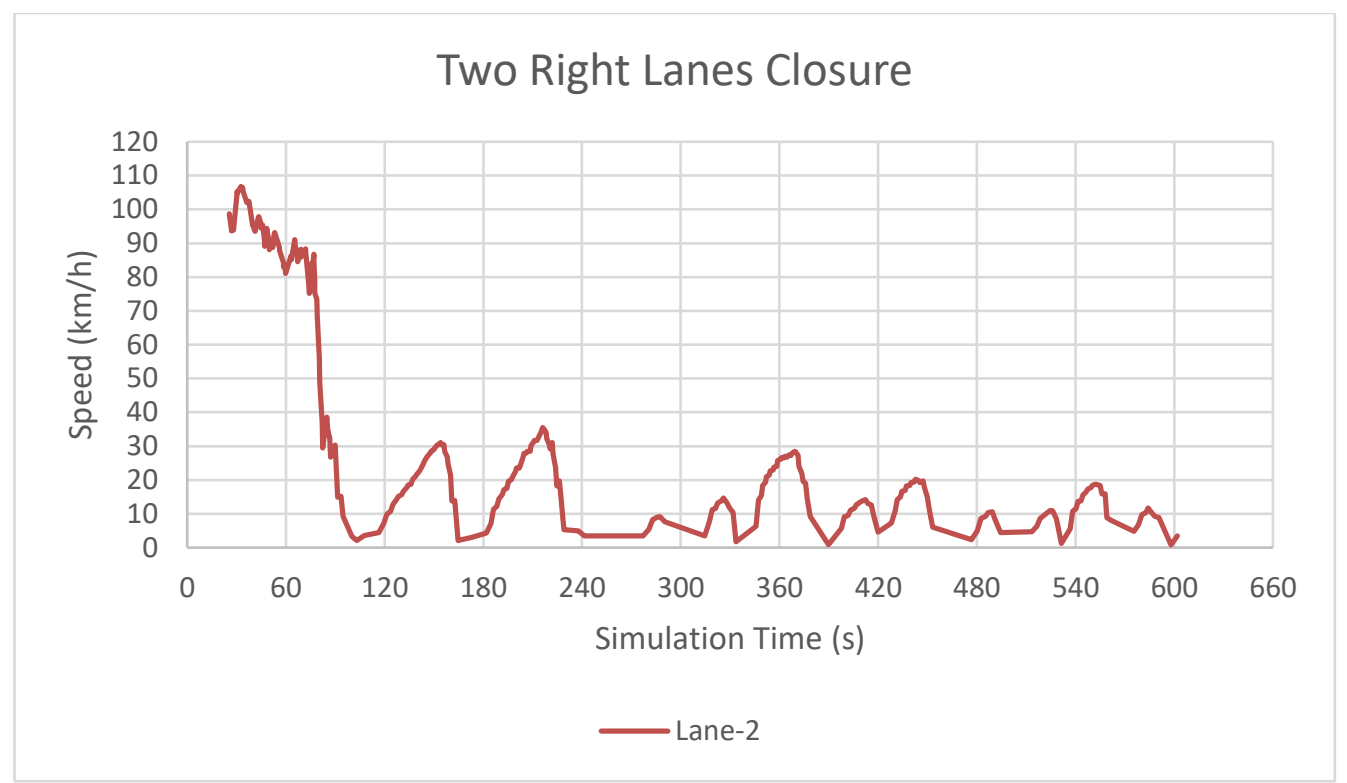

Figure 7.50: Speed data is collected from lane \#2, v=2000 veh/h/lane

3- Another two scenarios showed frequent speed oscillations from very high to very low with data obtained from data collection point located in the third or fourth lane. Incident was simulated on the two rightmost lanes. In these two scenarios, traffic in the third and fourth lane was not significantly affected by the traffic jam due to the incident occurrence in the first two rightmost lanes. Rather, traffic was interrupted every time an overtaking process happened from traffic traveling in the first and second lanes as illustrated in Figure 7.51 and Figure 7.52. 


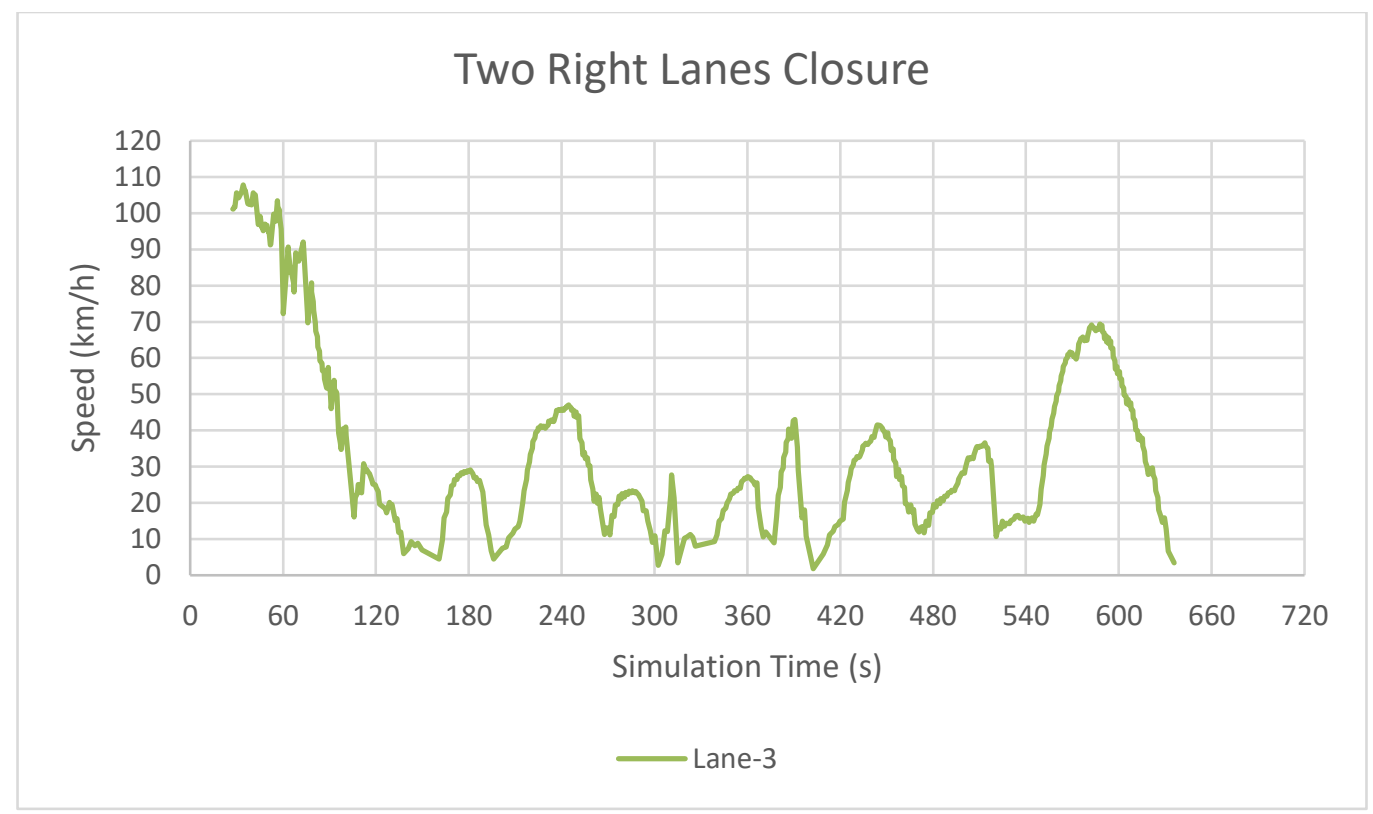

Figure 7.51: Speed data is collected from lane \#3, v=2000 veh/h/lane

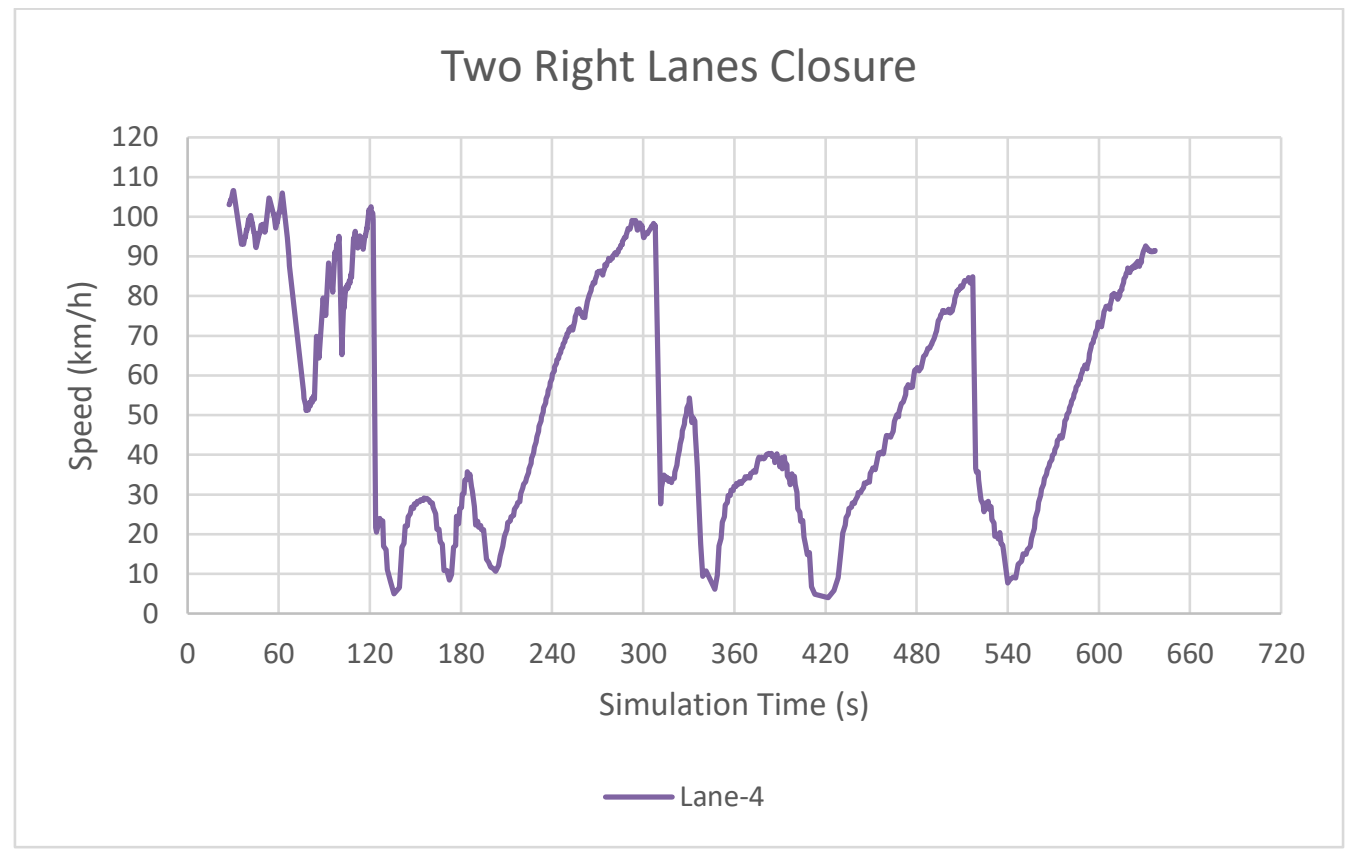

Figure 7.52: Speed data is collected from lane \#4, v=2000 veh/h/lane

4- Data collected from the first and second lane reflect the impact of an incident that occurred downstream, blocking the two right lanes. It is clear that the speed dropped as 
expected and resembles real life incidents that usually occur on freeways. This gives an incentive to use these cases for testing the developed incident detection model.

5- Cases presented in Figure 7.53 and Figure 7.54 were selected among a number of simulated runs to illustrate incident detection. Observations showed that reduction in speed due to the simulated incident along with other factors fall in the detection criteria specified in the devolved model, and therefore incident was successfully detected. All other tested simulation runs provided similar results. These are included in Appendix D.2.

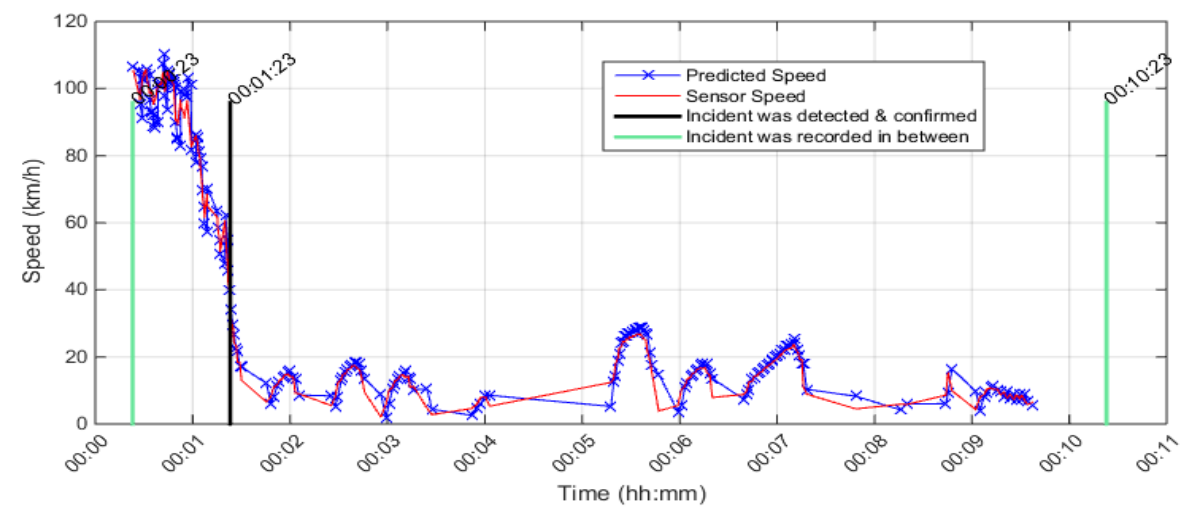

Upstream Detector \# is 401DE0010DEE, Detector Rank = 1, Distance from incident location in $(\mathrm{km})=0.35605$ Season $=$ Winter, Stream $=E$, Direction $=E$, Date $\&$ Time $=2011-02-0100: 00: 23$ Incident Reaso $=$ Collision

Affected Lanes = 2 RIGHT LANE(S)

Conditional Probability level $=0.6$, Cond.Prob. $r 3 s 2=0.2$, Cond. Prob. $r 3 s 1=0.1$, Percent drop in speed $\%=-56$

Max. Posterior Probability level $=0.97$, Percent range between speed states $=7 \%$

Incident was detected at 00:18 minutes from drop in speed point. Incident was confirmed after 5 minutes from the detection time

Figure 7.53: Speed data is collected from lane \#1, Run \#1, v=2000 veh/h/lane 


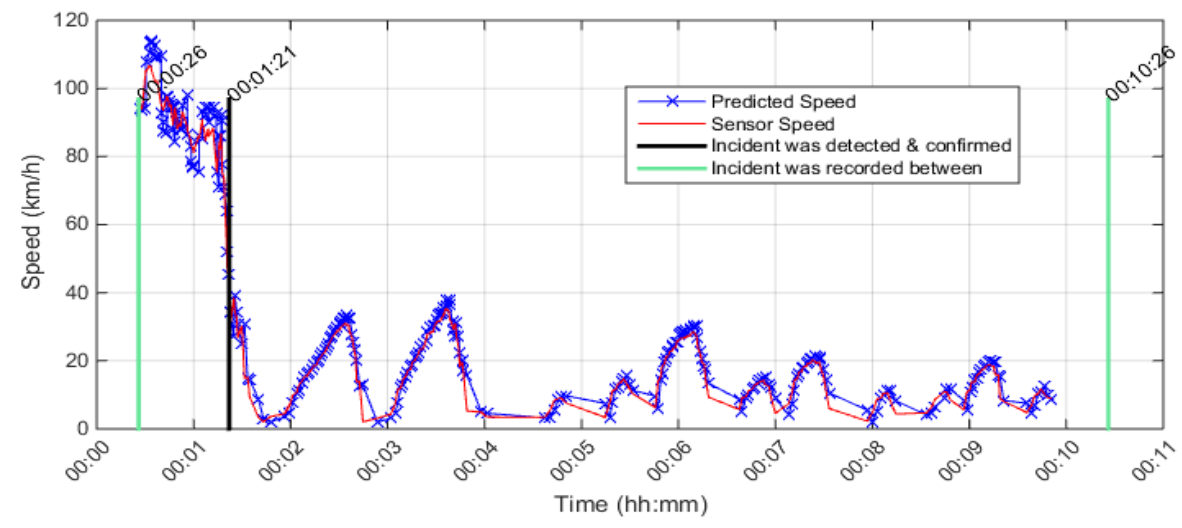

Upstream Detector \# is 401DE0010DEE, Detector Rank = 1, Distance from incident location in $(\mathrm{km})=0.35605$

Season $=$ Winter, Stream $=E$, Direction $=E$, Date $\&$ Time $=2011-02-0100: 00: 26$

Incident Reason $=$ Collision

Incident Reason $=$ Collision
Affected Lanes $=2$ RIGHT LANE(S)

Conditional Probability level $=0.6$, Cond.Prob. $r 3 s 2=0.2$, Cond. Prob. $r 3 s 1=0.1$, Percent drop in speed $\%=-56$

Max. Posterior Probability level $=0.97$, Percent range between speed states $=7 \%$

Incident was detected at 00:04 minutes from drop in speed point. Incident was confirmed after 5 minutes from the detection time

Figure 7.54: Speed data is collected from lane \#2, Run \#1, v=2000 veh/h/lane

Further study of the impact of traffic volume level on incident detection was required.

Therefore, four traffic volume levels were applied in this simulation analysis, including $1000,1250,1500 \mathrm{veh} / \mathrm{h} /$ lane. From the previous results obtained in this study, it was anticipated that traffic volume level has a significant impact on vehicle speed in the freeway. Simulations were carried out using the same lane blockage configuration and same desired speed with data recorded from data collection point located in the first lane. Results obtained for $1500,1250,1000 \mathrm{veh} / \mathrm{h} /$ lane volume levels are demonstrated in Figure 7.55, Figure 7.56, Figure 7.57, Figure 7.58, respectively. 


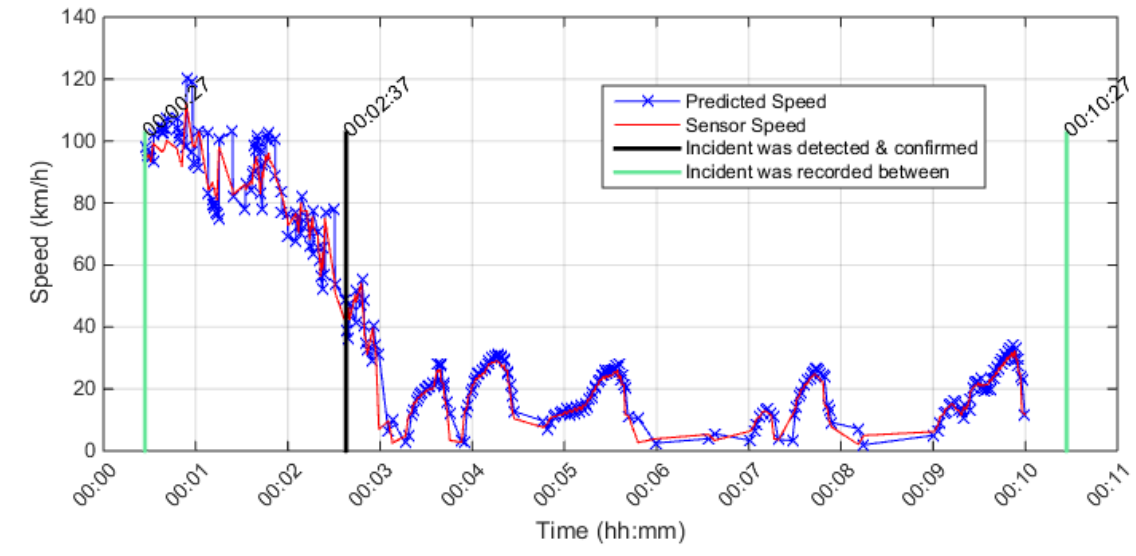

Upstream Detector \# is 401DE0010DEE, Detector Rank = 1, Distance from incident location in $(\mathrm{km})=0.35605$ Season $=$ Winter, Stream $=E$, Direction $=E$, Date \& Time $=2011-02-0100: 00: 27$ Incident Reason $=$ Collision

Affected Lanes $=2$ RIGHT LANE(S)

Conditional Probability level $=0.6$, Cond.Prob. $r 3 s 2=0.2$, Cond.Prob. $r 3 s 1=0.1$, Percent drop in speed $\%=-56$

Max. Posterior Probability level $=0.97$, Percent range between speed states $=7 \%$

Incident was detected at 00:07 minutes from drop in speed point. Incident was confirmed after 5 minutes from the detection time

Figure 7.55: Speed data is collected from lane \#1, Run \#1, v=1500 veh/h/lane

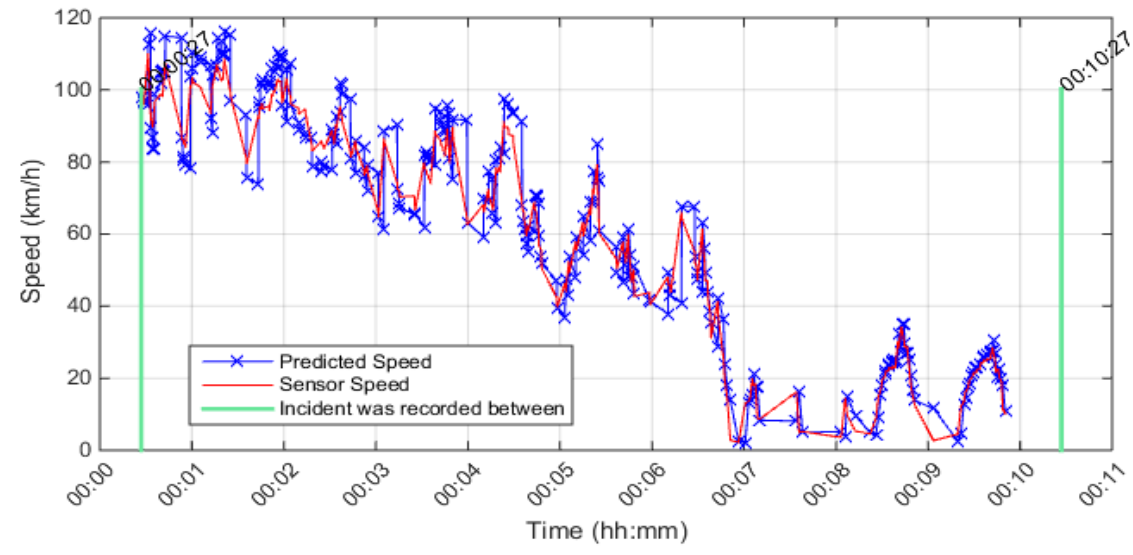

Upstream Detector \# is 401DE0010DEE, Detector Rank $=1$, Distance from incident location in $(\mathrm{km})=0.35605$ Season $=$ Winter, Stream $=E$, Direction $=E$, Date \& Time $=$ 2011-02-01 00:00:27 Incident Reaso $=$ Collision

Affected Lanes = 2 RIGHT LANE(S)

Conditional Probability level $=0.6$, Cond.Prob. $r 3 s 2=0.2$, Cond.Prob. $r 3 s 1=0.1$, Percent drop in speed $\%=-56$ Max. Posterior Probability level $=0.97$, Percent range between speed states $=7 \%$ Drop in speed was detected but Not enough recovery time to confirm an incident

Figure 7.56: Speed data is collected from lane \#1, Run \#1, v=1250 veh/h/lane 


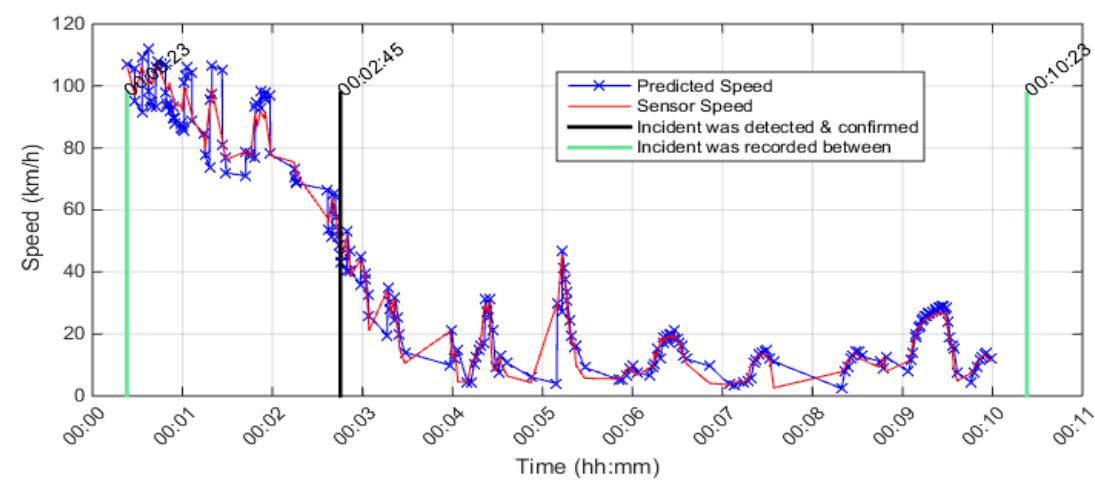

Upstream Detector \# is 401DE0010DEE, Detector Rank $=1$, Distance from incident location in $(\mathrm{km})=0.35605$ Season $=$ Winter, Stream $=E$, Direction $=E$, Date $\&$ Time $=2011-02-0100: 00: 23$ Incident Reason $=$ Collision

Affected Lanes $=2$ RIGHT LANE(S)

Conditional Probability level $=0.6$, Cond.Prob. $r 3 \mathrm{~s} 2=0.2$, Cond. Prob. $r 3 \mathrm{~s} 1=0.1$, Percent drop in speed $\%=-56$

Max. Posterior Probability level $=0.97$, Percent range between speed states $=7 \%$

Incident was detected at 00:46 minutes from drop in speed point. Incident was confirmed after 5 minutes from the detection time

Figure 7.57: Speed data is collected from lane \#1, Run \#2, v=1250 veh/h/lane

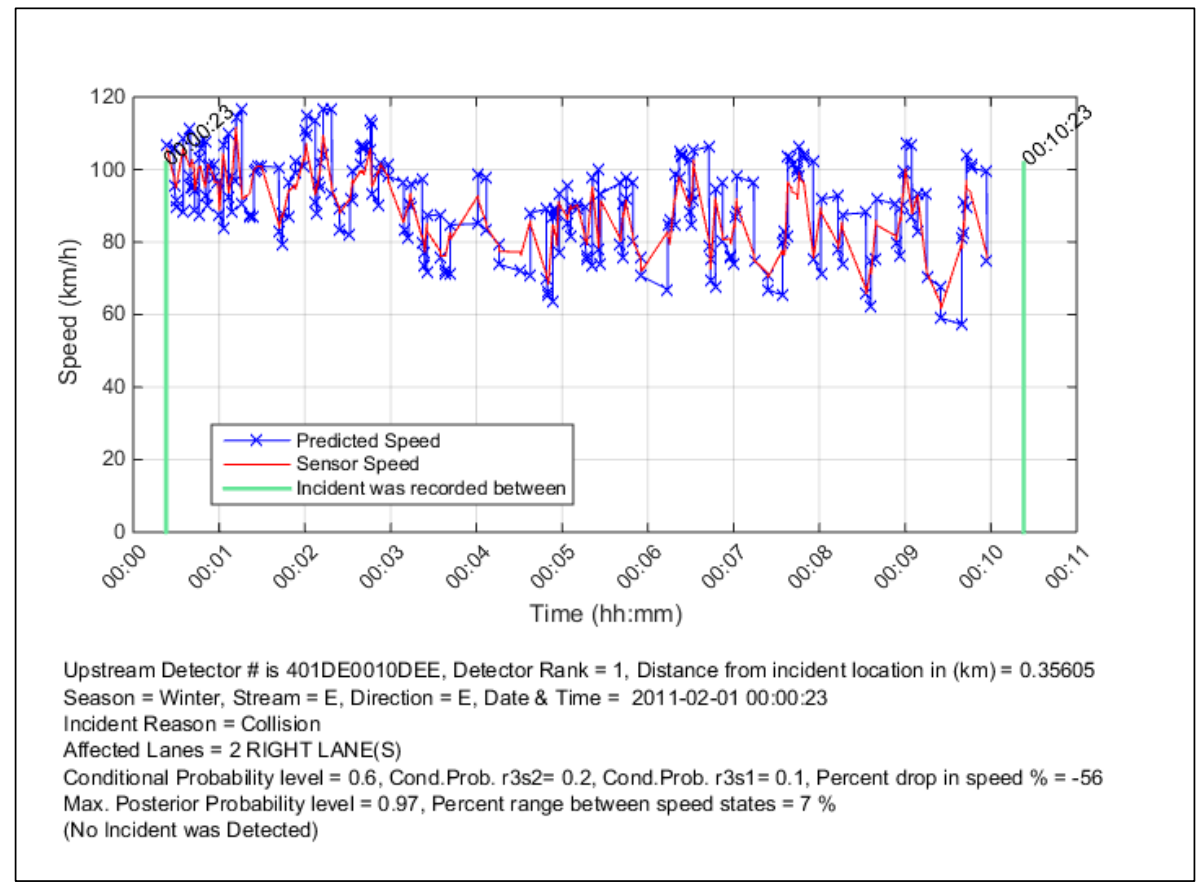

Figure 7.58: Speed data is collected from lane \#1, Run \#1, v=1000 veh/h/lane

The reduction in speed drop is demonstrated in the previous figures. When speed was plotted for each traffic volume level against the simulation minutes, it was found that every 
case showed some level of speed drop after the incident occurred. The rapid speed drop was found to be after around 90 seconds and was recorded at a volume level of 2000 veh/h/lane (high traffic volume during peak hours).

The decrease in speed was tested by the model to investigate the possibility of detecting any incident. Results showed that incident was detected by the developed model. Likewise, a less severe drop was found to be after around 160 seconds and was recorded at a volume level of $1500 \mathrm{veh} / \mathrm{h} / \mathrm{lane}$. Using the same model factors, the drop in speed was also tested by the model and results indicated the detection of an incident.

Further study revealed that at a low volume level of $1000 \mathrm{veh} / \mathrm{h} / \mathrm{lane}$ when freeway section was not seriously impacted by the incident in the two-lane blockage incident scenario. Figure 7.58 demonstrates model findings in an attempt to detect any incident, but since the speed drop does not fully satisfy the criteria defined in the developed model, the incident was not detected. It was found that at traffic volume level of $1000 \mathrm{veh} / \mathrm{h} / \mathrm{lane}$, there is a low probability of detecting an incident if the model is calibrated for keeping false alarms to be low.

Increasing the volume to a level between the intermediate and low level recorded gradual decline in the speed drops from 100 to almost zero $\mathrm{km} / \mathrm{h}$ in the first 120 seconds at some simulation runs. In other simulation runs the speed pattern osculated gradually towards low speed. Clearly, higher traffic volume had more severe impact on the drop in speed when the incident occurred. Further, the sensitivity level of the developed model was enough to serve in detecting incidents at low traffic volume, but it did not serve very well at some simulation runs when speed was osculating gradually as a reflection of the stop and go condition. This case is always challenging when it comes to incident detection as 
shown in Figure 7.59. In general, more than $80 \%$ of the simulation runs show results that agree well with the fact that incident was introduced to the two right-most lanes on the freeway.

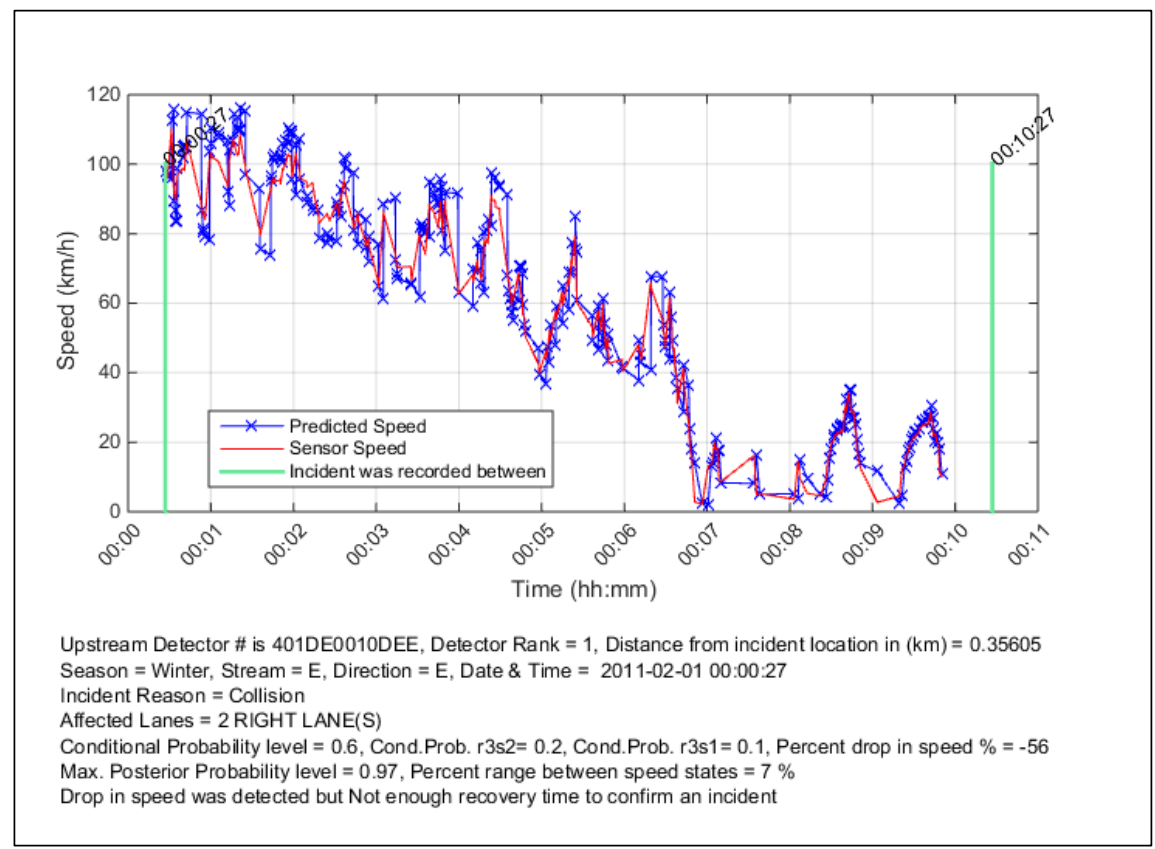

Figure 7.59: Speed data is collected from lane \#1, Run \#1, v=1250 veh/h/lane

\subsection{Summary}

Vissim microsimulation was used to simulate freeway incidents in four different scenarios; each scenario represented a particular lane blockage configuration. Moreover, in each scenario different traffic volume levels were applied. In the case when two rightmost lanes were blocked due to an incident and data collection points were located either in the first or second lane, the following are the observations regarding drop in vehicle speed: 
1- Scenario \#2 with two rightmost lanes blocked was found to be the most general lane blockage configuration in the event of an incident on the freeway section.

2- At high traffic volume, all simulation runs showed a rapid drop in speed induced by an incident on the freeway. Developed model was able to detect incidents in all 40 simulation runs, including reading data from the first or second lane.

3- If the traffic volume was decreased by $25 \%$, provided that two lane blockage configuration applied and $100 \mathrm{~km} / \mathrm{h}$ desired speed was maintained, all simulation runs showed a rapid drop in speed, and the developed model was able to detect incident for all 20 simulation runs including collecting data from the first or second lane.

4- For the same lane blockage configuration and when traffic volume was reduced by about $37 \%$, which is almost close to intermediate level, there was no indication of a significant drop in traffic speed due to an incident. However, the developed algorithm was able to detect an incident in $80 \%$ of tested cases. For the other $20 \%$ of cases, the speed drop was not significant, and it was very hard to tell about any possibility of an occurrence of an incident.

5- It was not intended to increase the developed model sensitivity for allowing it to detect more incidents within the $20 \%$ portion; doing so might increase the false alarm rate in cases no incident was recorded.

6- Low traffic volume of $1000 \mathrm{veh} / \mathrm{h} / \mathrm{lane}$ did not offer indication of any drop in traffic speed in the event of freeway incident that blocked the two rightmost lanes. Therefore, in the case of low traffic volume, the incident was not detected in most cases.

7- For the same low traffic volume scenario, the speed drop due to an incident was about $20 \%$, which was not enough to satisfy the criteria specified in the developed model. 
8- As expected, volumes between 1250 and $2000 \mathrm{veh} / \mathrm{h} /$ lane offered a higher drop in speed due to the incident and hence the developed model was capable of detecting an incident in almost all cases.

9- The incident induced a drop in speed in cases where data collection points were located in either third or fourth lanes under high traffic volume of $2000 \mathrm{veh} / \mathrm{h} / \mathrm{lane}$.

10-For data collection point located in the third or fourth lane, providing that an incident caused two rightmost lane blockage, the drop in speed was not representative of any indication of the incident. Speed fluctuated up and down, indicating a slow and go movement.

11- For data collection point located in the third or fourth lane, data should only be recorded when an incident causes blockage to the three rightmost lanes. 


\section{Summary, Conclusions, and Future Research Directions}

\subsection{Summary}

\section{Developed Bayesian Model}

- A Bayesian model was developed for recognizing traffic flow patterns both when such a pattern is caused by an incident and when the pattern reflects incident-free operating conditions.

- Starting from the predictive speed capability, in the context of incident prediction, the Bayesian model produces a profile of speed drop over time and tracks probabilities of traffic states that are associated with incidents.

- The Bayesian model enables the conversion of prior probabilities into posterior probabilities of traffic states. For this purpose, conditional probabilities are applied that reflect the reliability of the data that the model uses to convert prior probabilities into posterior probabilities.

- The Bayesian model for incident detection is calibrated and verified with detector and incident archived data. However, the design of the model permits the use of the data obtainable from the roadside unit (RSU) of a future connected vehicle system as well as probe vehicle and crowd-sourced data.

- For use in the incident detection model, the data obtained from the field should be reliable. However, due to a number of reasons, reliability of data can be compromised. In the case of RSU, probe vehicle, and crowd sourced data, the required sample size may be compromised due to low traffic volume and low proportion of equipped vehicles within the traffic stream in a given part of the highway. In the case of detectors (sensors) whether built into pavement or installed on the roadside or mounted above 
the pavement, outages can occur. Also, the spacing of such detectors becomes a reliability issue. Of course, the traffic volume in association with working detectors should meet the minimum sample size requirements.

- In order to obtain a clear appreciation of data sources and reliability issues, this thesis document presents Monte Carlo simulation and statistical methods before the Bayesian model for incident detection is presented.

- In discussing the reliability of the Bayesian incident model (as characterized by the conditional probability), frequent references are made to the reliability variable that connects the early chapters of this thesis with the later chapters.

- The developed Bayesian model starts by predicting the speed at each time period and the percent drop in the predicted speed is also calculated. Likewise, the maximum posterior probability level that is associated with each time period is recorded.

- In the context of incident detection, at the conditional probability level (i.e. reliability level) of 0.6 , the posterior probability increases as the operating speed continues to decline. This reflects the phenomenon that declining speed reflects a disruption in traffic flow caused for example by an incident and in turn strengthens the posterior probability of traffic states. If the pattern of a sharp drop in speed continues over a number of time periods, and if the pre-defined threshold values of posterior probability and percent drop in speed are reached, the model indicates an incident.

- The model has the capability to predict the operating speed for the next period by incorporating the latest available sensor (detector) reading in the logic of the model. The developed model follows any sudden changes in the operating speed reported by 
the sensor. The developed model proved to be able to predict and follow the speed pattern.

- On the basis of information examined in the thesis and reasons that are explained, it was decided to consider the recorded time of incidents (as these appear in the database), as unreliable. Therefore, it was decided to measure the time to detect an incident by using the time when the first severe drop in the predicted speed was encountered. In most cases this time was found to be close to 2:00 minutes before the incident is detected. This is representing the minimum time to detect which is an indicator of the effectiveness of the model.

- At conditional probability (i.e. reliability) level of 0.8 , the incident was detected faster than at 0.6 (i.e. the "time to detect" was shorter). Therefore, early incident detection is a reflection of the higher level of reliability of the model.

- Under certain incident conditions, the incident causes a blockage of all lanes in the highway section. This event has a severe impact on the traffic condition and the speed recovery. As a result, it is believed that within the study period such an effect of the incident does not allow quick recovery and the speed remains low until the end of the study time period.

- Under other incident conditions the severity of the incident may be less than that noted above (i.e. only one or two blocked lanes impact traffic). In such situations, it is expected that the speed will recover to normal operating speed values in a relatively short period of time, especially under low volume travel condition.

- In some other cases even under high traffic volume, the speed might decline for a short period of time and then recovers back again to normal operation. Also, there can be 
cases that are characterized by stop and go conditions. These observations suggest that not all incidents have the same or similar pattern of speed drop and recovery. Therefore the incident detection model faces many challenges and is designed to cope with a variety of speed drop and recovery patterns.

- The model reported in this thesis is calibrated to accommodate the possible scenarios described above. After the calibration process, a validation procedure was applied and the developed model was advanced to its complete and reliable version.

- For automating the computations and for eventual on-line implementation, the incident detection model was incorporated into an algorithm and Matlab was used to code the algorithm.

\section{Outcomes}

Four possible outcomes are the result from the traffic incident detection algorithm: correct detection of the incident, correct detection of no incident, a false alarm of an incident and missing of the incident.

For model calibration and validation purposes, the developed algorithm was used (a) to detect an incident when it was known that there was an incident, and (b) not to detect an incident when it was known that there was no incident. The following are the results and associated observations on model capability.

- The detection rate reached a high level of $84 \%$ under applicable traffic conditions and therefore the remaining $16 \%$ were not detected. As noted below, under applicable traffic conditions, the false alarm rate was $0.038 \%$. 
- The false alarm rate was found to be very low. As noted earlier, a false alarm implies that the algorithm detected an incident in a situation where there was no incident.

- From the results obtained in this research using the Bayesian method, the algorithm provides satisfactory performance when applied to cases that do not involve recorded incidents. Almost 97\% of the cases show the correct indication of "no incident". In the case of the remaining 3\%, a rapid drop in speed was the reason for the algorithm to provide an alert even though there was no incident.

- The algorithm should be applied only to traffic volume conditions when an incident can potentially disrupt traffic flow and cause highly adverse impacts. Therefore, cases with low traffic volume are deemed to be not important when evaluating the developed model. For example, there were incident cases that did not show a drop in speed. Keeping in mind the broad definition of an incident, such cases represent $56 \%$ of total cases under consideration and were therefore considered as "not significant" for the application of any evaluation criteria.

- For some cases, a low level of benefit was obtainable from the model application. In these cases, minor changes in speed were observed, but this pattern was not enough to meet the criteria for incident indication. For $15 \%$ of such selected cases, no incident was detected. If the algorithm is to be calibrated to give better results in such cases, the false alarm rate is expected to increase significantly. So, in the context of this thesis, minor speed fluctuation alone is not enough as a sufficient alert to the incident detection model. The algorithm was not applicable in such cases to detect an incident and therefore some other means of detection could produce benefits (e.g. automated 
notification of vehicle disability or deployment of airbags or maybe a twitter if allowed by the traffic law of the jurisdiction).

\section{Vissim Simulation}

- Computer simulation was found to be satisfactory in representing the real world conditions. The data sourced from Highway 401 were adopted to serve in microsimulations with the use of a well-known software (i.e. Vissim marketed by the PTV Company). The microsimulation model was applied as a useful tool to generate traffic flow conditions under incident scenarios as well as scenarios without incidents. The obtained traffic and performance data from the microsimulation software was used as an input for implementing and validating the incident detection model.

- In the Basic scenario, freeway traffic was simulated with no incident. Both high and low traffic volume gave similar results under steady flow condition. Data collected from each lane showed same output. Use of the Vissim data in the incident detection algorithm resulted in correct answer (i.e. no incident). Therefore, no false alarms were a result of any of the model application.

- In the incident scenario, the results were generally similar to those obtained with the use of actual archived data.

\subsection{Conclusions}

- A freeway traffic management system that quickly detects and removes incidents from the freeway increases the safety of the travelers and decreases overall traffic delay. This gives incentives to traffic management centres to focus on prompt incident detection 
and dispatching incident management teams as quickly as possible. For this reason, an automated incident detection algorithm based on the developed Bayesian model was designed to enhance the process of detecting freeway incidents and expediting the dispatching of first responders (i.e. the emergency management teams) to the scene.

- This research study was conducted with data covering 365 days of a year. All seasons were covered and most of the applicable day/night time periods were included. A total of 400 incident cases were randomly drawn out of 1600 valid detector/sensor database cases. The research produced a sizeable database of 260 cases "with incident" and "with no incident".

- In general, the results were consistent, and the end product was a set of clear, defensible, and well-supported indicators of the performance of the new developed model.

- The recommended incident performance indicators are: detection rate (DR), false alarm rate (FAR) and mean time-to-detect (MTTD). Based on the literature review and data analysis in this study, the recommended target values for calibration and validation are DR $84 \%$, and therefore the rate of not detecting incidents $16 \%$.

- The false alarm rate for the developed model was found to be very low. That is, the Bayesian method-based algorithm did not detect an incident in most cases where there was no incident. Almost $97 \%$ of the cases show the correct indication of no incident. In the case of the remaining 3\%, a rapid drop in speed was the reason for the algorithm to provide an alert even though there was no incident. 
- Application of the incident detection model/algorithm to Vissim simulated data produced results that were generally in line with those obtained from actual archived incident and detector-based traffic flow data.

\subsection{Contributions of this Thesis}

- This thesis research makes a significant contribution to knowledge in incident detection with the use of algorithms.

- The approach used to detect an incident in this research is based on original Bayesian formulation and is designed to apply to data from:

Present generation of detectors/sensors placed in the pavement or on the roadside or above the pavement,

Future roadside unit (RSU) of the connected vehicle system

$>$ Probe vehicle data

$>$ Crowd-sourced data.

- The incident detection criteria are enhanced by tracking the level of speed drop at various speed levels. Applicable factors for model effectiveness were advanced.

- In this research, the Bayesian predictive speed model was adapted and extended for the purpose of detecting incidents.

- In addition to the Bayesian model calibrated and validated with actual GTA freeway data, a microsimulation approach was applied to obtain data for testing the algorithm.

- Statistical methods and the Monte Carlo simulation technique were applied to develop estimates of the reliability of data for use in the Bayesian model for incident detection. The Monte Carlo simulation application is useful for estimating the proportion of the 
vehicles in the traffic stream that are equipped, and the probability of an equipped vehicle traveling in the highway cell of interest during a defined time interval. These factors enable the use of data obtained from sources noted above and provide a basis to estimate the conditional probability values used in the Bayesian incident detection model.

- Refinement of Monte Carlo simulation was applied to improve functionality, including the required reliability factor. This factor defined the minimum number of probe (or future connected) vehicles required in any part of the freeway for the statistical reliability of captured data. If the reliability factor is specified to be high, the number of probe/equipped vehicles on the road should be high.

- The highway model in Microsimulation software Vissim with sensors looped-in for the purpose of generating equipped vehicle trajectory data under incident condition was developed. This enabled the study of scenarios of traffic incidents. Vissim was found to be useful for testing and validating the algorithm in detecting incidents. This information is a contribution to knowledge.

- Matlab platform and code enabled the development of the algorithm for automatically detecting incidents. The algorithm is based on the calibrated and validated the Bayesian model and can be used for on-line application with minor modifications.

\subsection{Future Research Directions}

- The developed model and the algorithm can be advanced further in order to take these to the level of on-line implementation for detecting incidents automatically. However, 
it is beyond the scope of this thesis research to take the algorithm to the on-line retail level.

- Future research can aim at further refinement of multi-criteria and investigate criteria weights in order to detect almost all incidents within the study period.

- Future research can investigate the installation of the algorithm at each roadside unit or detector cluster location. Following the reading of speed data, a quick search for indications of an incident can be carried out.

- Approaches for altering or resetting detection criteria dynamically in response to changes in traffic flow pattern can be investigated as a part of future research.

- The study of the graduated performance of the developed algorithm under different traffic flow conditions will add to the knowledge base for traffic managers. This research has illustrated high performance for high traffic flow conditions. To go beyond these findings, there is a need to produce performance information for other traffic flow conditions.

- This research can serve as a foundation for more advanced future models intended to estimate traffic states and detect incidents based on connected and autonomous vehicle technology. The statistical methods in association with the Monte Carlo simulation technique can be adapted in order to develop formal reliability specifications.

- Although new generation advanced technology detectors and roadside units (RSU) are expected to contribute data in the future, additional data will become widely available from crowd-sources such as Waze. The incident detection model and the algorithm should be adapted so that these can work with crowd-sourced data. 
- In general, modeling incident detection based on data obtained from the roadside unit (sensor) and equipped vehicle communications is an area of considerable challenge. Given the current interest in connected and autonomous vehicles, modeling incident detection in these environments can benefit from the findings of this research. For example, the design of the future roadside unit (RSU) for vehicle-infrastructure data exchange can be made more comprehensive by adding the incident detection capability. 


\section{References}

Abdulhai, B., and Ritchie, S. G. (1995). "Performance of Artificial Neural Networks for Incident Detection in ITS.” Transportation Congress: Civil Engineers-Key to the World Infrastructure, New York ASCE, Vol. 1, pp. 227-238.

Abdulhai, B., and Ritchie, S. G. (1997). "Development of Universally Transferable Freeway Incident Detection Framework.” Transportation Research Board, 76th Annual Meeting, Paper no. 971121, January 12-16, Washington, D.C.

Abdulhai, B., and Ritchie, S. (1998). "Preprocessor Feature Extractor and Postprocessor Probabilistic Output Interpreter for Improved Freeway Incident Detection.” Transportation Research Record no. 1678, 277-286.

Abuelela, M. (2011). “A Framework For Incident Detection And Notification In Vehicular Ad-Hoc By Abstract A Framework For Incident Detection And Notification In Vehicular Ad-Hoc.”

Abuelela, M., and Olariu, S. (2009). “Automatic Incident Detection in VANETs: A Bayesian Approach,” Department of Computer Science, Old Dominion University, Norfolk, VA 23529-0162.

Ahmad, S. A., and Cook A. R. (1980) "Time-Series for Freeway Incident Detection," ASCE J. Transportation Engineering, Vol. 106, No. 6, pp. 731-745. 
Ahmed, S.A., Cook, A.R. (1982). “Application of Time-Series Analysis Techniques to Freeway Incident Detection.” Transportation Research Record 841, TRB, National Research Council, Washington D.C.

Al-Deek, H., Garib A., and Radwan, A. E. (1994). "New Method for Estimating Freeway Incident Congestion.” Transportation Research Record No. 1494, pp. 30-39.

Anon (2001). State-of-the-Art Detector Technologies.

Anon (2010). "Freeway Incident Detection - Technologies and Techniques," Austroads Research Report AP-R364/10.

Arceneaux, J., Smith, J., Dunnett, A., and Payne H. (1990). "Calibration of Incident Detection Algorithms for Operational Use." Traffic Control Methods, Proceedings of The Fifth Engineering Foundation Conference on Traffic Control Methods, Engineering Foundation, Santa Barbara, California, pp. 17-32.

Asakura, Y., Kusakabe, T., Long, N. X. and Ushiki, T. (2015) “Incident Detection Methods Using Probe Vehicles with On-board GPS Equipment," Transportation Research Procedia. Elsevier B.V., 6(June 2014), pp. 17-27. doi: 10.1016/j.trpro.2015.03.003.

Basis Neural Networks" presentation at 78th Transportation Research Record Journal, Washington D.C., 1999.

Bloomberg, L. et al. (2000). "A Comparison of the Vissim and CORSIM Traffic Simulation Models." 98104 (August). 
Brackstone, M., Fisher, G. \& McDonald, M. (2001). "The Use of Probe Vehicles on Motorways, Some Empirical Observations." Proceedings of the World Congress on Intelligent Transport Systems, pp.1-12.

Busch, F., and Fellendorf, M. (1990). "Automatic Incident Detection on Motorways.” Traffic Engineering and Control, Vol. 31, No. 4, April, pp. 221-227.

Chassiakos, Athanasios P., Stephanedes, and Yorgos J. (1993). "Smoothing Algorithms for Incident Detection.” Transportation Research Record 1394 (8-16) TRB, National Research Council, Washington D.C.

Cheng, X. et al. (2010). "Procedia Engineering Highway Traffic Incident Detection," Based on BPNN. Procedia Engineering, 7, pp.482-489.

Chung, E \& Rosalion, N (1999). "An evaluation of freeway incident detection algorithms using field data," Australian Transport Research Forum, 23rd, 1999, Perth, Western Australia, Department of Transport, Western Australia, vol.23, part 1, pp.459-76.

Collins, J.F., Hopkins, C.M., and Martin, J. A. (1979). “Automatic Incident Detection TRRL Algorithms HIOCC and PATREG.” Supplementary Report No. 526, Transport and Road Research Laboratory.

Corens, P. et al. (2011). "Using microsimulation to evaluate traffic safety." Traffic Engineering and Control, 52(3), pp.136-139. 
Dailey, D.J., Cathey, F.W., and Pumrin, S. (2000) "An Algorithm to Estimate Mean Traffic Speed Using Uncalibrated Cameras." IEEE Transactions on Intelligent Transportation Systems: Volume 1, no. 2, pp. 98 - 107.

De Castro-Neto, Mendonca, M. (2009). "Towards Universality in Automatic Freeway Incident Detection: A Calibration-Free Algorithm." Ph.D. diss., University of Tennessee.

Deniz, O., Celikoglu, H. B. and Gurcanli, G. E. (2012) “Overview to Some Incident Detection Algorithms : a Comparative Evaluation with Istanbul Freeway Data," (October), pp. 274-284.

Dowling, R., Skabardonis A., and Alexiadis V., (2004). “Guidelines for Applying Traffic Simulation Software," Traffic Analysis Toolbox-Volume 3, Report FHWA-HRT-04-040.

Dudek, C.L., Messer, C.J., and Nuckles, N.B (1974). "Incident detection on urban freeway." Transportation Research Record, No. 495, TRB, National Research Council, pp. 12-24, 1974.

Evanco, W. M. (1996) “The Impact of Rapid Incident Detection on Freeway Accident Fatalities.” Mitretek, Center for Information Systems.

Evanco, W. M. (1997) "Reducing urban freeway accident fatalities with rapid incident detection.”

Ezell, S. (2010). "Explaining international IT application leadership: Intelligent transportation systems.” The Information Technology \& Innovation Foundation 
Fangming, Tian; Han, Dong, (2012). "Simulation of traffic incident detection based on Vissim and neural network," in Computer Science and Automation Engineering (CSAE), IEEE International Conference on, vol.2, no., pp.51-55, 25-27 May

Gall, A.I., and Hall, F.L. (1989). “Distinguishing between incident congestion and recurrent congestion: a proposed logic." Transportation Research Record, No. 1232, TRB, National Research Council, pp. 1-8.

Gordon, R.L. and Tighe, W.,”Traffic Control Systems Handbook “, Washington: Dunn Engineering Associates, 2005.

Guin, A. (2004). “An Incident Detection Algorithm Based On a Discrete State Propagation Model of Traffic Flow," Georgia Institute of Technology.

Hall, FL., Shi, Y. \& Atala, G (1993). "On-line testing of the McMaster algorithm under recurrent congestion," Transportation Research Record, no. 1394, pp.1-7.

Highway Capacity Manual, 2010.

JHK and Associates (1993). "Intelligent Vehicle Highway Systems," The State of the Art, New York, NY, March.

Kahaki, S., M., and Nordin, M., J. (2011). "Vision-based Automatic Incident Detection System using Image Sequences for Intersections,” International Conference on Pattern Analysis and Intelligent Robotics 28-29, Putrajaya, Malaysia, June.

Khan, Ata M. (1969). "Regional Transport System Plan Selection Problem: A Decision Theoretic Framework for Policy Decisions" Ph.D. diss., University of Waterloo. 
Khan, Ata M. (2010). "Bayesian predictive travel time methodology for advanced traveller information system," Journal of Advanced Transportation, Wiley-Blackwell, ID: ATR-10-0064.R3 (Accepted on August 7, 2010), early view online 4 OCT 2010|DOI:10.1002/atr.147.

Khan, A.M., Lutz, K., Kolechkina, T., Hammoud, A. (2014). Cost-Effective Transition from Loops to Probe Data for MTO's Traffic Management. Prepared for the Ministry of Transportation, Ontario. Prepared by the Transportation Research Centre, Carleton University, Ottawa.

Klein, a. (1995) Publication Number “Detection Technology for IVHS” FHWA-RD-96100, July.

Klein, L. A. (2001). "Sensor Technologies and Data Requirements for ITS, Norwood, MA: Artech House, pp. 549.

Levin, M., and Krause, G.M. (1978). "Incident detection: a Bayesian approach.” Transportation Research Record, No. 682, TRB, National Research Council, pp. 52-58.

Lindley, J.A. (1987). "Urban freeway congestion: quantification of the problem and effectiveness of potential solutions." ITE Journal, Vol. 57, No. 1, pp. 27-32.

Liu, Y., Lei, W. Jianquan, Q. Yi and W. Huimin, (2008) "Back- Propagation Neural Network for Traffic Incident Detection Based on Fusion of Loop Detector and Probe Vehicle Data. Natural Computation", ICNC '08. Fourth International Conference on, hlm. $116-120$. 
Mahmassani, H. S., Haas, C., Zhou, S. and Peterman, J. (1999) "Evaluation of Incident Detection Methodologies," Center for Transportation Research, University of Texas, Report No. FHWA/TX-00/1795-1, October.

Mahmassani, H.S., Haas, C., Zhou, S. and Peterman, J (1999). "Evaluation of incident detection methodologies.”, Research Report 1795-1, Center for Transportation Research, University of Texas at Austin, Austin, TX, October.

Mai, C., Maciejewski, C., Bauer, T. (2011). "Protocol for Vissim Simulation", Oregon Department of Transportation.

Mak C. L., Fan, H. S. L. (2005). “Transferability of Express Incident Detection Algorithms to Singapore and Melbourne.” In Journal of Transportation Engineering, vol. 131, no. 2, pp. 101-111.

Martin, P. T., Perrin, J., Hansen, B., Kump, R., and Moore, D. (2001) “Incident Detection Algorithm Evaluation,” Utah Department of Transportation, (March).

Martin, P.T. (2011). "Freeway Incidents : Simulation and Analysis”. (September).

Middleton, D., Jasek, D., \& Paker, R. (1999). "Evaluation of some existing technologies for vehicle detection.” College Station, TX: Texas: Transportation Institute.

Min Liu (2012). “Incorporating General Incident Knowledge into Automatic Incident Detection: A Markov Logic Network Method”. Master Thesis, Civil and Environmental Engineering, University of Wisconsin-Madison. 
Neter J., Wasserman W., and Kutner M. (1985). “Applied Linear Statistical Models,” Second Edition, Richard D.IrWIN, Inc.

Ozbay, K., and Kachroo, P. (1999). "Incident management in intelligent transportation systems Repository Citation \& quot;" Incident management in intelligent transportation systems," pp. 1-248. Available at: http://digitalscholarship.unlv.edu/ece_fac_articles

Parkany, E., and Xie, C. (2005). “A Complete Review of Incident Detection Algorithms \& Their Deployment: What Works and What Doesn't," Prepared for The New England Transportation Consortium, NETCR37 Project No. 00-7, February 7.

Payne H. J. and Tignor, S.C. (1978). "Freeway incident-detection algorithms based on decision trees with states," Transportation Research Record, (682): $30\{37\}$.

Payne, H. J., (1976). "Development and Testing of Incident Detection Algorithms. Vol. 1: Summary of results. FHWA report no. FHWA-RD-7619.

Payne, H. J., Helfenbein, E. D., Knobel H. C. (1976). "Development and Testing of Incident Detection Algorithms." Vol. 2: Research methodology and results. FHWA report no. FHWA-RD-7620.

Payne, H. J., Tignor S. C. (1978). "Freeway Incident-Detection Algorithms Based on Decision Trees with States.” Transportation Research Record 682, 30-37

Peeta, S., Das, D. (1998.) “Continuous Learning Framework for Freeway Incident Detection.” In Transportation Research Record 1644, pp. 124-131. 
Payne, H.J., Knobel H. C. (1976). “Development and Testing of Incident Detection Algorithms. Vol. 3: User guidelines.” FHWA report no. FHWA-RD-7621

Persaud. N, Hall. F. L., and Hall L. M., (1990) “Congestion Identification Aspects of the McMaster Incident Detection Algorithm,” Transportation Research Record, tech. rep. 1287, pp. 167-75.

Persaud. B. N., and Hall. F. L. (1989) "Catastrophe Theory and Patterns in 30-Second Freeway Traffic Data Implications for Incident Detection". Transportation Research Pant A General. Vol. 23A. No. 2, pp. 103-1 13.

Petre T. Martin, Joseph Perrin, Blake Hansen, (2001). "Incident Detection Algorithm Evaluation," University of Utah, Prepared for Utah Department of Transportation.

PTV America (2004). “Vissim User Manual,” PTV Planning Transport Verkehr AG.

Pulugurtha, S.S., (2002). "Simulating and Analyzing Incidents Using CORSIM and Vissim Traffic Simulation Software.”, (702).

Pushkar, A., Hall, F., and Acha-daza, J. A. (1994). "Estimation of Speeds from SingleLoop Freeway Flow and Occupancy Data Using Cusp Catastrophe Theory Model,” Transportation Research Record 1457, p149-157, Washington D.C.

Raiffa, H., and Schlaifer, R. (1968). “Applied Statistical Decision Theory,” The M. I. T. Pre Cambridge. 356 S., 2 Tab., Preis 37. Ref: http://ntl.bts.gov/DOCS/96100/ch02/ ch02_03.html 
Rilett, L. R., and Park, D., "Direct Forecasting of Freeway Corridor Travel Times Using Spectral.

Ritchie, S. G., and Cheu, R. L. (1993). "Simulation of Freeway Incident Detection Using Artificial Neural Networks," Institute of Transportation Studies and Department of Civil Engineering, University of California, Transpn. Res.-C, Vol. I, No.3. pp. 203-217.

Sanwal, K.K., and Walrand, J. (1995) "Vehicles as Probes. California PATH Working Paper." California Path Program: Institute of Transportation Studies. University of California, Berkeley.

Škorput P., Mandžuka S., and Jelušić N. (2010). "Real-time Detection of Road Traffic Incidents," Promet - Traffic \& Transportation, Vol. 22, No. 4, 273-283 273.

Stephanedes, Y. J, and Chassiakos, A. P. (1993). “Application of Filtering Techniques for Incident Detection. Journal of Transportation Engineering” vol. 119, no.1, pp. 13-26

Stephanedes, Y., Hourdakis, J. (1996). “Transferability of Freeway Incident Detection Algorithms. In Transportation Research Record 1554, pp. 184-195.

Taylor, Sarah J. (1969) "Development of a Bayesian Decision Theory Framework to Enhance the Design of Rear-End Collision Warning Systems" Ph.D. diss., Carleton University, 1969.

Tian, F. \& Dong, H. (2012). "Simulation of traffic incident detection based on Vissim and neural network". In CSAE 2012 - Proceedings, 2012 IEEE International Conference 
on Computer Science and Automation Engineering. IEEE, pp. 51-55. Available at: http://ieeexplore.ieee.org/lpdocs/epic03/wrapper.htm?arnumber=6272726.

Tsai, J. and Case, E.R. (1979). "Development of freeway incident detection algorithms by using pattern recognition techniques.”, Transportation Research Record, No. 722, TRB, National Research Council, pp. 113-116.

Wakure, A. R., Patkar, A. R., Dagale, M. V. and Solanki, P. P. (2014) "Vehicle Accident Detection and Reporting System Using GPS and GSM,” International Journal of Engineering Research and Development, 10(4), pp. 25-28. Available at: http://www.ijerd.com/paper/vol10-issue4/Version_4/B1042528.pdf.

William, B. M., Guin, A. (2007). “Traffic Management Center Use of Incident Detection Algorithms: Findings of a Nationwide Survey.” IEEE Transactions on Intelligent Transportation Systems. Short papers, vol. 8, no.2, pp. 351-358.

Williams, B. M., \& Guin, A. (2007). "Traffic management center use of incident detection algorithms: Findings of a nationwide survey." IEEE Transactions on Intelligent Transportation Systems, 8(2), 351-358. http://doi.org/10.1109/TITS.2007.894193

Yaguang, K. and Anke, X. (2006) “Urban Traffic Incident Detection Based On Fuzzy Logic,” IECON 2006 - 32nd Annual Conference on IEEE Industrial Electronics. IEEE, pp. 772-775. doi: 10.1109/IECON.2006.347245.

Yang Zhaosheng. (2003). "Introduction to Intelligent Transportation Systems," Beijing: People's Communications Press, pp.130-132. 
Zhang Jinglei, Wang original. (2005). "Traffic incident detection algorithm research". Wuhan University of Technology (Transportation Science and Engineering Edition), vol.29, pp. 30-3.

Zhang, K. and Taylor, M. A. P. (2004). "Incident detection on freeways: a Bayesian network approach," 27th Australasian Transport Research Forum, Adelaide, Transport Systems Centre, University of South Australia October.

Zhang, K., and Taylor, M., A., (2005). “Towards Transferable Incident Detection Algorithms," Journal of the Eastern Asia Society for Transportation Studies, Vol. 6, pp. $2263-2274$.

Zhu response, Wang Hong, the Hongyan. (2008). "Traffic flow parameters and dynamic prediction method of traffic events.” Nanjing: Southeast University Press, pp.45-60. 


\section{Appendix A Excel Source Codes}

This Appendix presents the Excel source codes for the developed model. It includes the source code for incident data sortation.

\section{Appendix A.1 Codding Used in Excel for Incident Data}

\section{Sortation}

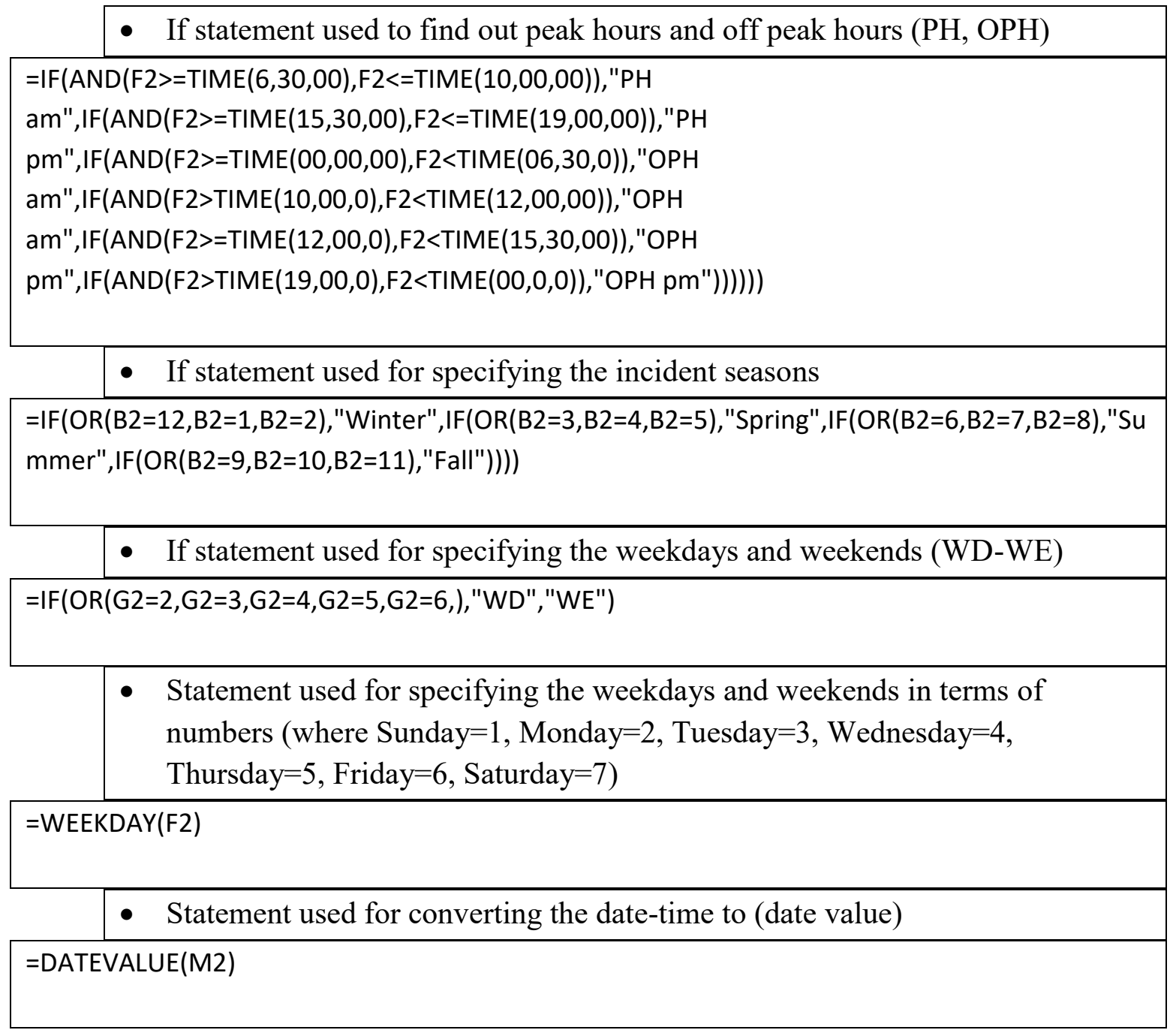




\title{
Appendix B Matlab Source Codes for the Developed
}

\begin{abstract}
Model
This Appendix presents the Matlab source codes for the developed model. It includes the source code for the main Graphical User Interface GUI, which is the primary function and is used to call for all other functions and return results to the output files and figures
\end{abstract}

\section{Code Script Abbreviations}

- Calculate_plot_predicted_speed function

- $\quad$ Predicted_SP, $==$ Predicted Speed

- $\quad$ PIT, == Predicted Incident Time

- PIT_max, == Maximum Predicted Incident Time

- $\quad$ Trigger, $==$ an indication of incident based on - drop in speed and posterior level ( $1=$ incident detected, $0=$ no incident detected $)$

- Inci_Test, == an indication of incident confirmation ( $1=$ incident was detected and confirmed, $0=$ incident was detected and not confirmed)

- calculate_plot_predicted_speed, == function

- $\quad$ RBTCS, is Relationship between Traffic Condition State (S1 --> S2 \& S3 --> S2, it ranges between 0 and 1)

- $\quad \mathrm{r} 3 \mathrm{~s} 2=0.25 ; \quad \%$ Conditional Probability percentage out of 1

- $\quad \mathrm{r} 3 \mathrm{~s} 1=0.2 ; \quad \%$ Conditional Probability percentage out of $1, \mathrm{r} 1 \mathrm{~s} 1=(1-(\mathrm{CPL}+\mathrm{r} 3 \mathrm{~s} 1))$

- $\quad \mathrm{CPL},==\%$ Conditional probability level, the range could be between 0.3-0.9 
- PDPS, $==\%$ Percent drop in predicted speed in percentage, the range is between 0 and $100 \%$

- $\mathrm{PL},==\%$ Maximum posterior level at which incident will be detected

- Threshold, $==$ is the percentage from the 0.85 percentile below which the second condition to find the point of drop in speed is satisfied

- Recovery_Time, $=$ the suggested time period within which the two conditions for incident persistence will be tested.

- $\quad$ 22r3_Repetition, $==$ no of repetition of either $\mathrm{r} 2$ or $\mathrm{r} 3$ within the recovery time as one of the conditions for the incident confirmation conditions

- Speed_Recovery, $==$ the percentage from the 0.85 percentile of the speeds till the point of drop in speed that needs to be met as the second condition of the incident confirmation

- Speed_Recovery_repetiotion, $==$ no of repetition that the speed recovery will meet the conditions of the incident confirmation part

- ChPSP, $==$ Check Predicted Speed

- ChPL, $==$ Check Posterior Level

- Time_Duration_Index, $=$ it is the time period starting from the time when incident was first detected and till the end of the speed recovery period

- state_Duration, $==$ it indicates the state vector for all time period during the recovery time

- IsThereDrop, $==$ it has two values $(1=$ an indication of drop in speed, $0=$ no indication of any drop in speed) 
- if IsThereDrop $==0$, Use_this_prctile_SP $=($ Speed_Recovery $/ 100) * \operatorname{prctile} \_S P ; \%$ when there is no reference to any drop in speed the percentile is calculated at the time when the incident was detected

- else if IsThereDrop $==1, \quad$ Use_this_prctile_SP $=$ (Speed_Recovery/100)*Percentile(index); \%when there is reference to any drop in speed the percentile is calculated at the time of the point where there is a drop in speed

- Incident_Confirmation function

- Inci_Test $=1 ;===$ the default value is that there is a confirmation of an incident every time unless the following three conditions are met

- Time_index $=0 ;==$ it is the time at which there is no confirmation to any detection of an incident 


\section{Appendix B.1 Graphical User Interface (GUI) Function}

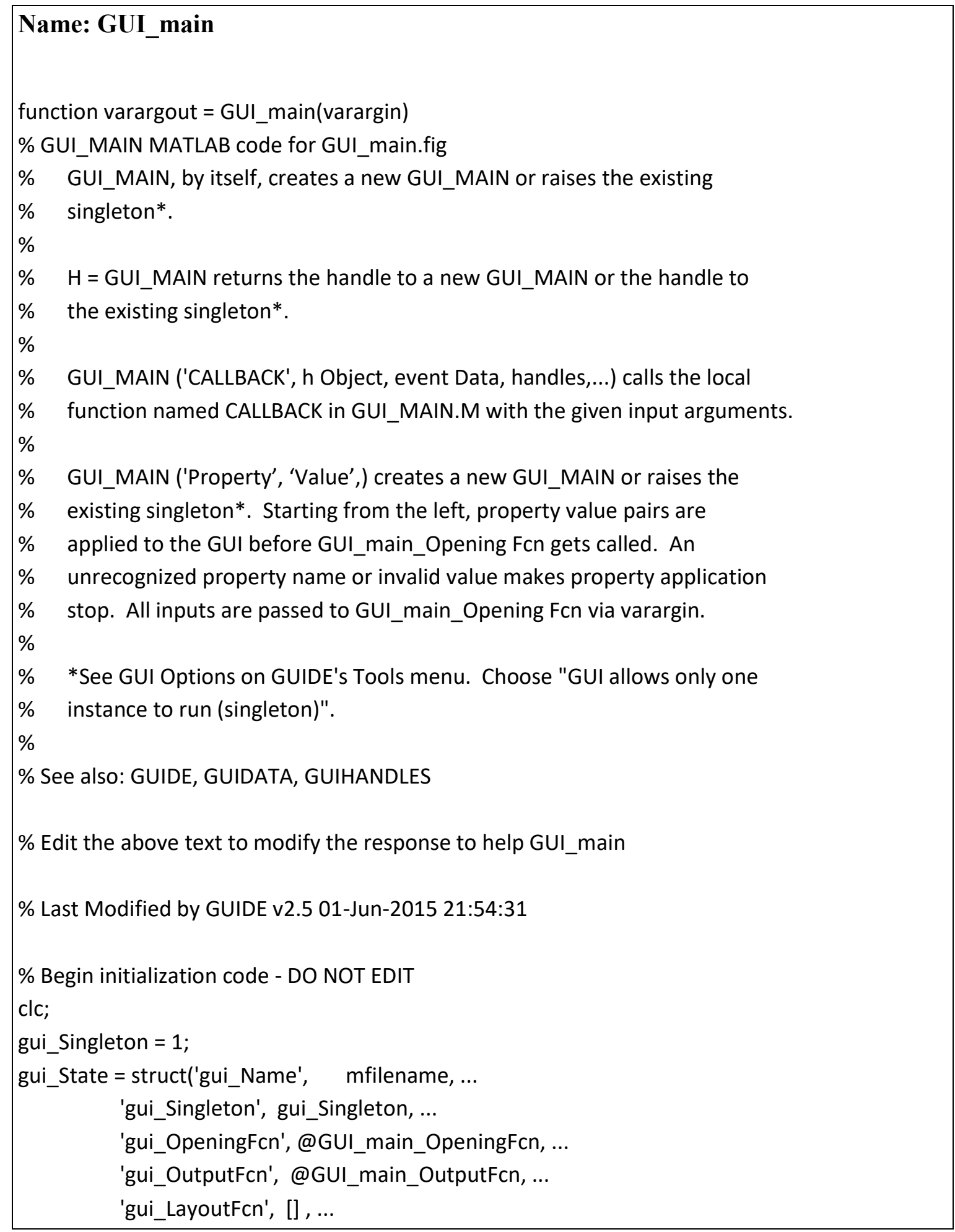




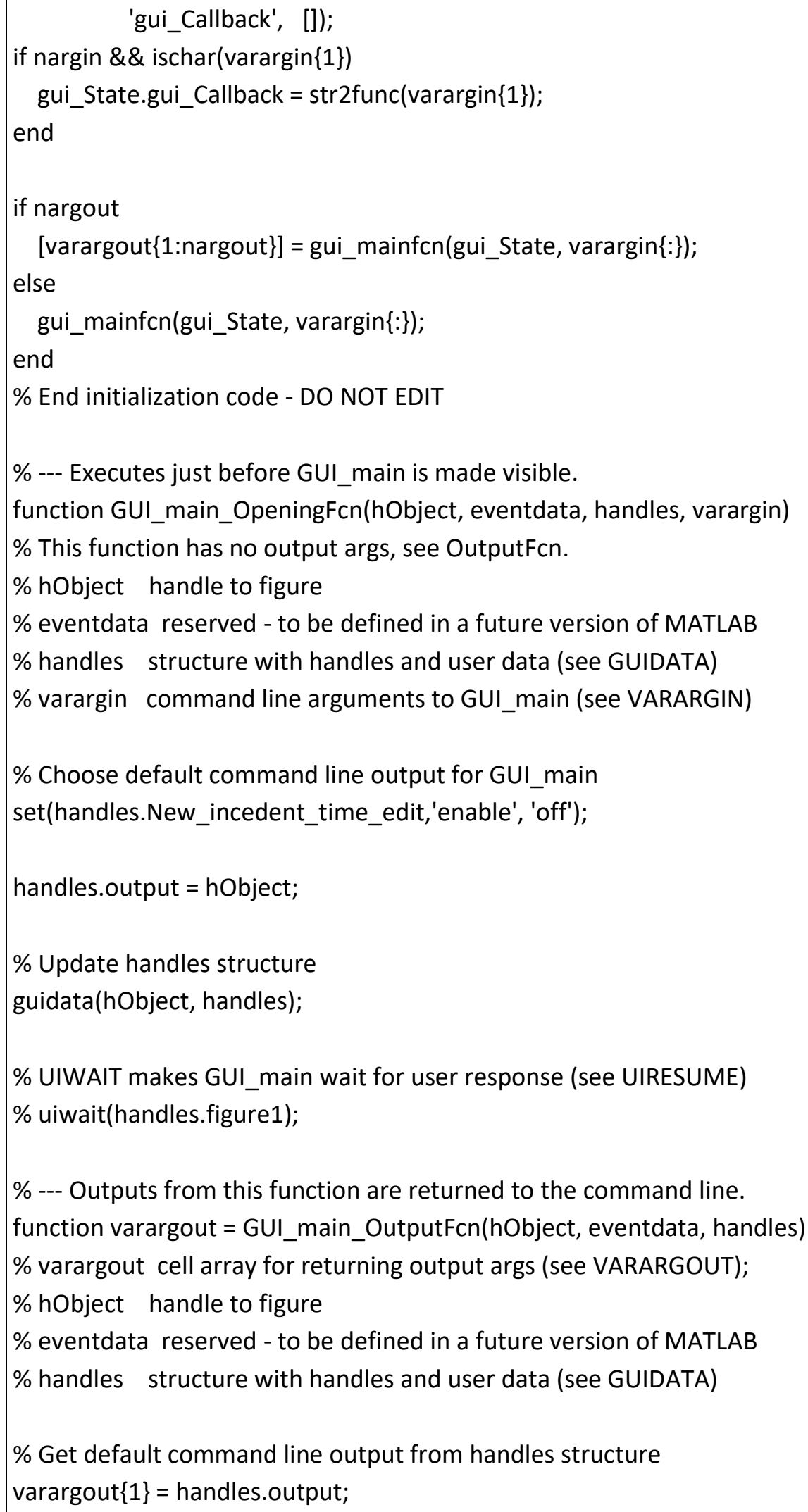




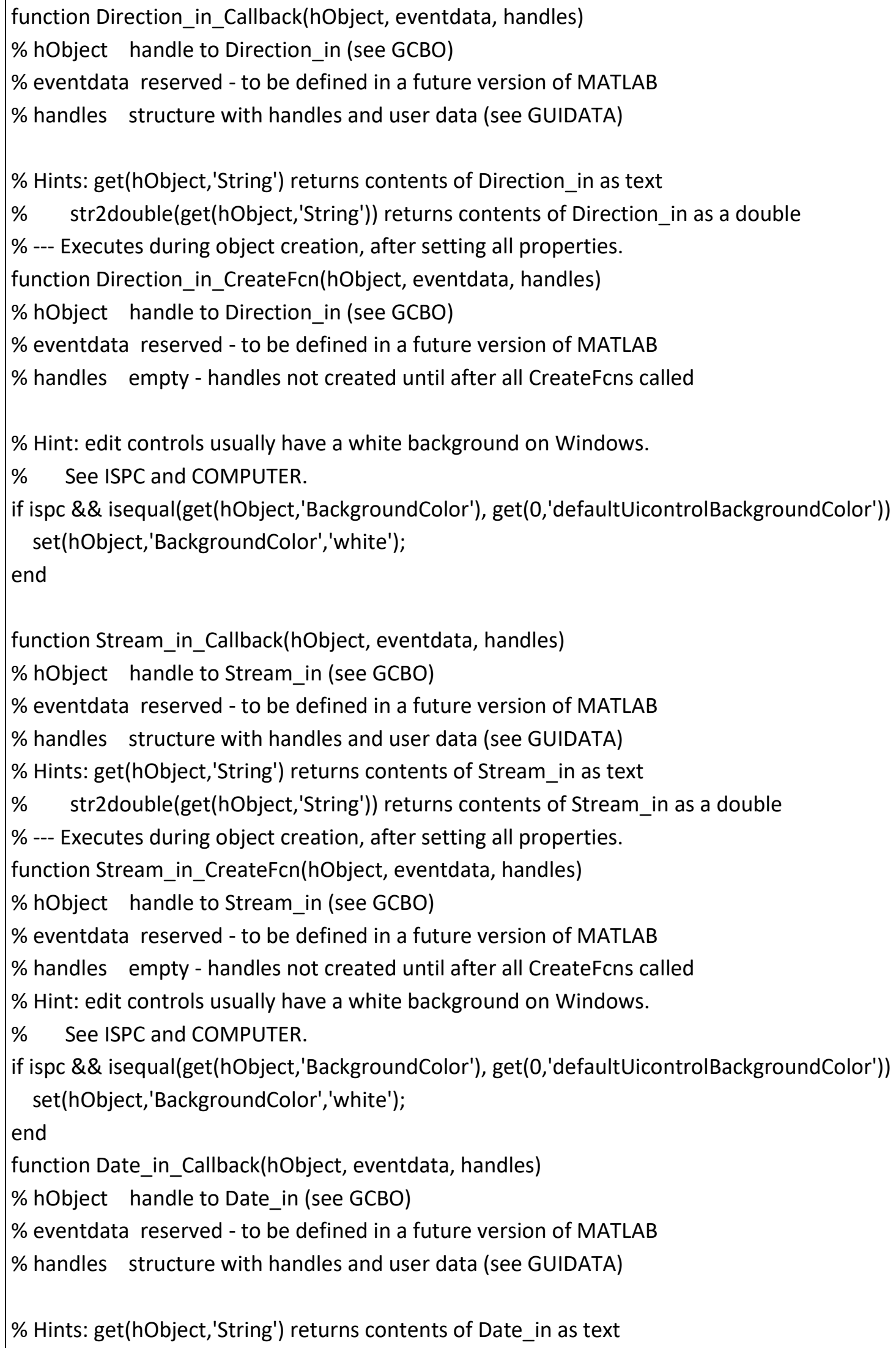




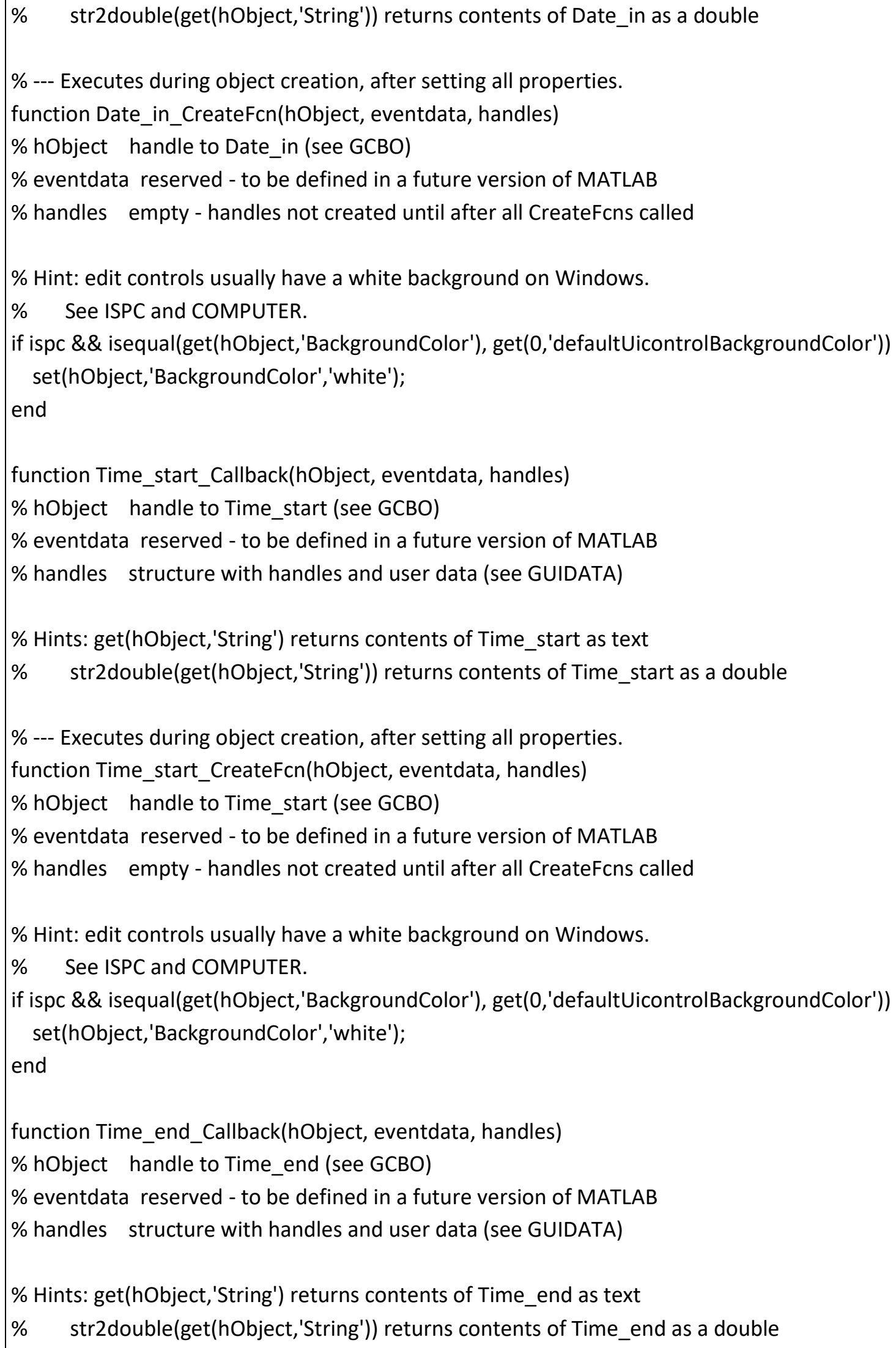


$\%$--- Executes during object creation, after setting all properties.

function Time_end_CreateFcn(hObject, eventdata, handles)

$\%$ hObject handle to Time_end (see GCBO)

$\%$ eventdata reserved - to be defined in a future version of MATLAB

$\%$ handles empty - handles not created until after all CreateFcns called

\% Hint: edit controls usually have a white background on Windows.

$\% \quad$ See ISPC and COMPUTER.

if ispc \&\& isequal(get(hObject,'BackgroundColor'), get(0,'defaultUicontrolBackgroundColor')) set(hObject,'BackgroundColor','white');

end

$\%$--- Executes on selection change in Incedent_list.

function Incedent_list_Callback(hObject, eventdata, handles)

$\%$ hObject handle to Incedent_list (see GCBO)

$\%$ eventdata reserved - to be defined in a future version of MATLAB

$\%$ handles structure with handles and user data (see GUIDATA)

$\%$ Hints: contents = cellstr(get(hObject,'String')) returns Incedent_list contents as cell array

$\% \quad$ contents\{get(hObject,'Value')\} returns selected item from Incedent_list

$\%$ Index = get(handles.Incedent_list,'value')

guidata(hObject, handles); \% We should use this line to update all the handles

$\%$--- Executes during object creation, after setting all properties.

function Incedent_list_CreateFcn(hObject, eventdata, handles)

$\%$ hObject handle to Incedent_list (see GCBO)

$\%$ eventdata reserved - to be defined in a future version of MATLAB

$\%$ handles empty - handles not created until after all CreateFcns called

\% Hint: listbox controls usually have a white background on Windows.

$\%$ See ISPC and COMPUTER.

if ispc \&\& isequal(get(hObject,'BackgroundColor'), get(0,'defaultUicontrolBackgroundColor')) set(hObject,'BackgroundColor','white');

end

$\%$--- Executes on button press in Run_program.

function Run_program_Callback(hObject, eventdata, handles)

$\%$ hObject handle to Run_program (see GCBO)

$\%$ eventdata reserved - to be defined in a future version of MATLAB

$\%$ handles structure with handles and user data (see GUIDATA) 


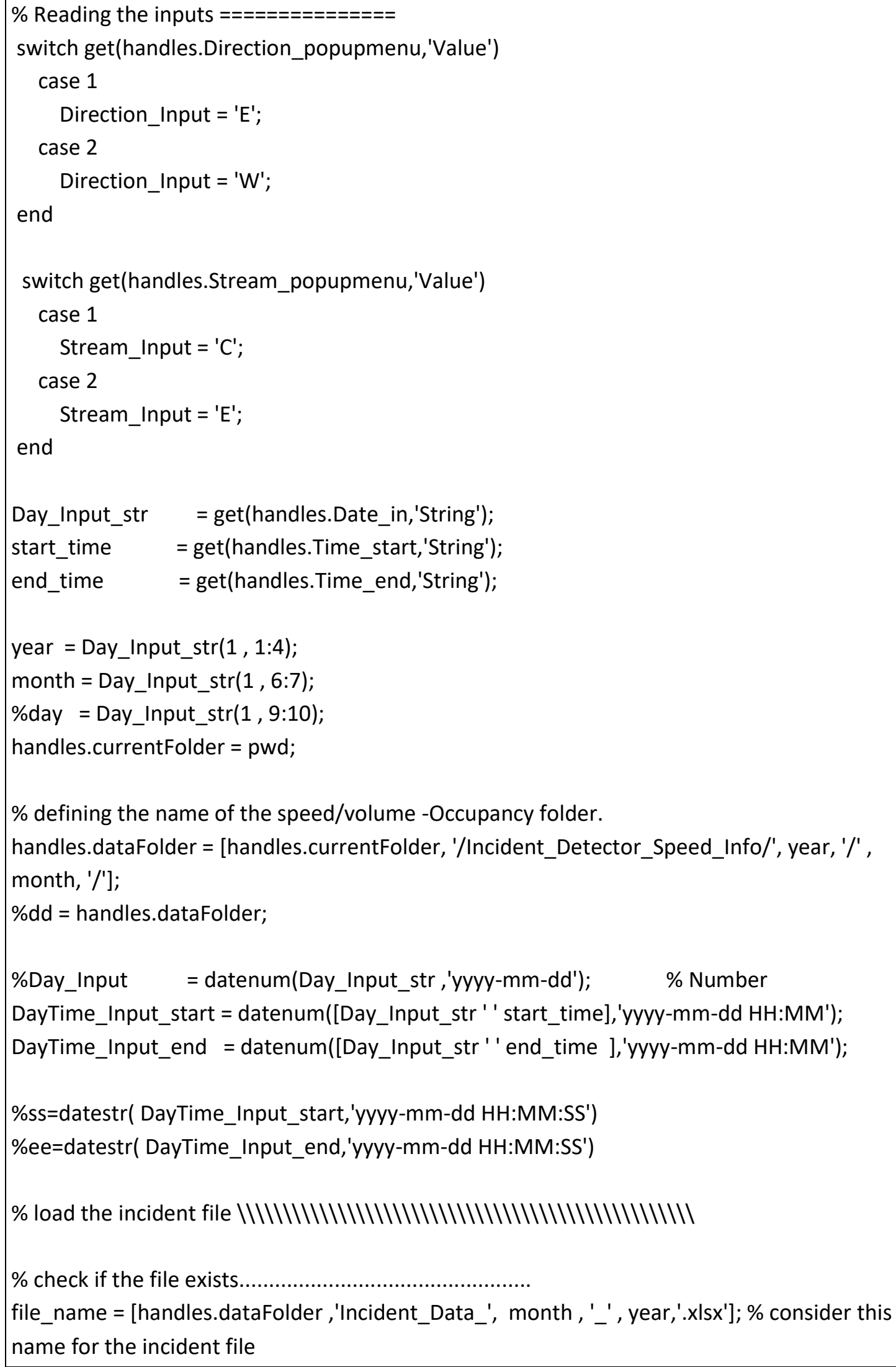




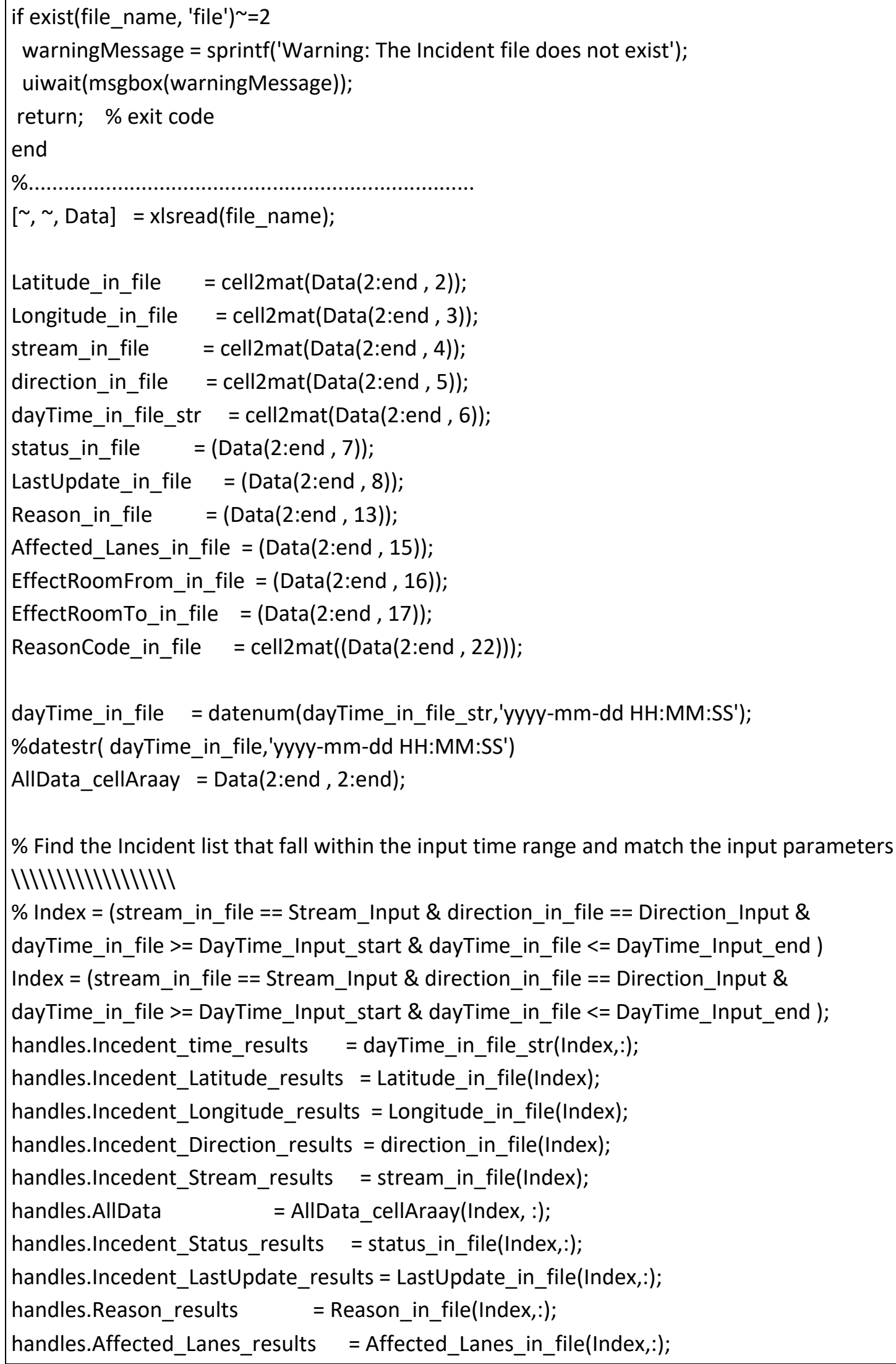




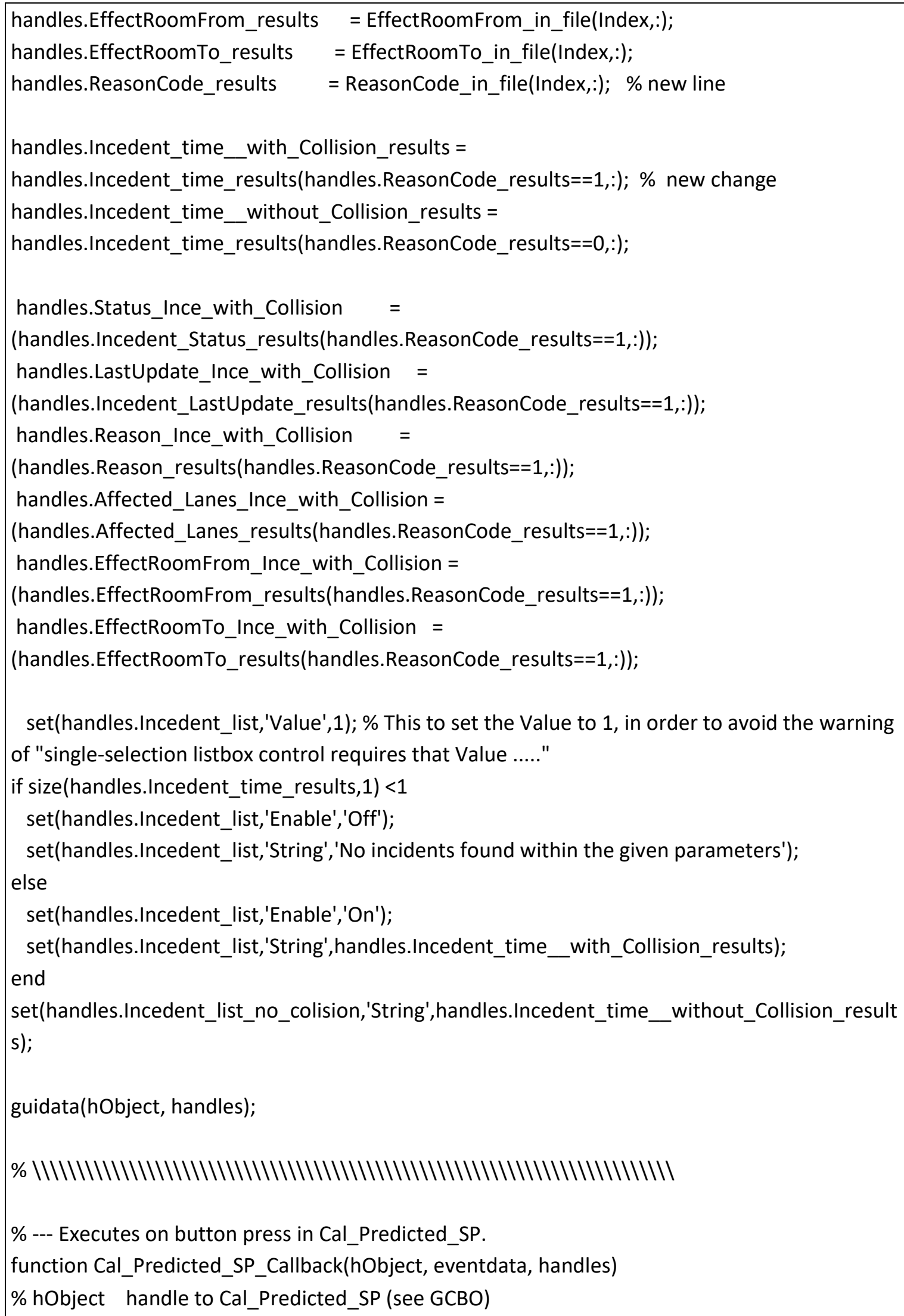




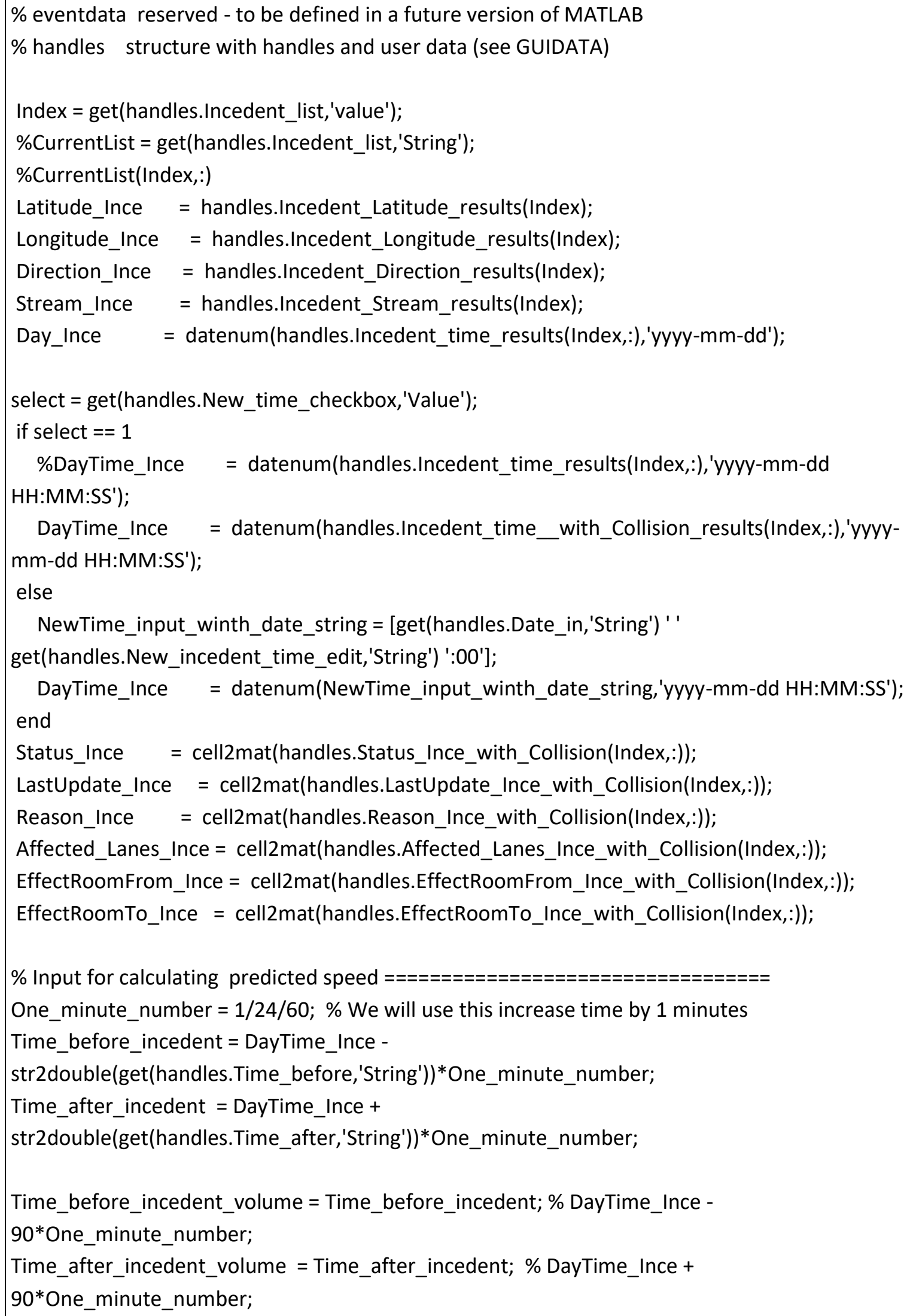




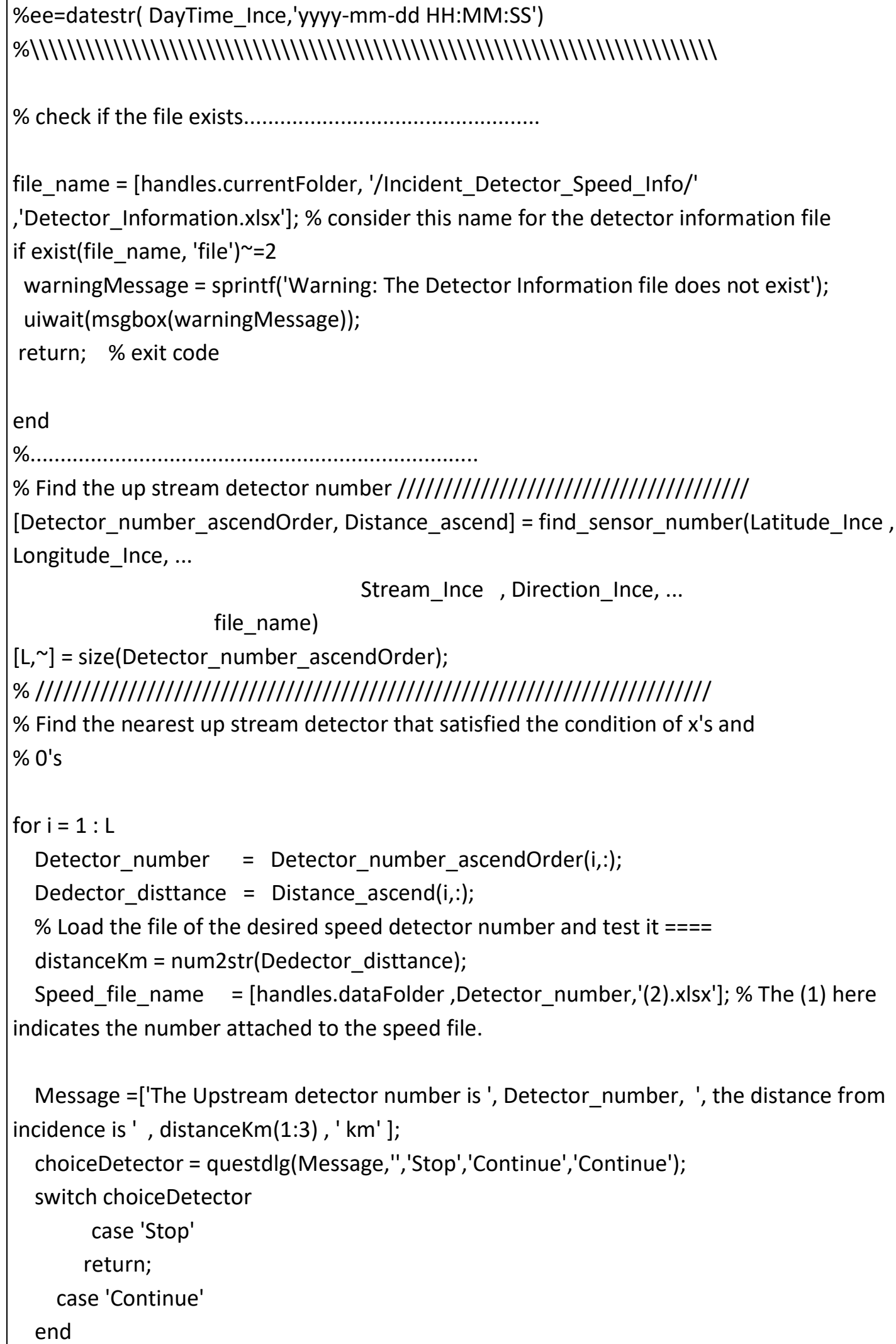


Detector_number_str = num2str(Detector_number);

MessageDF =['The Speed file for Detector \# ', Detector_number_str, ' does not exist. Do you want to stop or proceed with the next detector?'];

if exist(Speed_file_name, 'file') $=2$

$\%$ Construct a questdlg with three options

choiceFD = questdlg(MessageDF, 'Warning', 'Stop','Next','Stop');

$\%$ Handle response

switch choiceFD

case 'Stop'

return;

case 'Next'

if $\mathrm{i}==\mathrm{L}$

message_last_detector $=$ 'This is the last detector and no more detectors

available';

chose_detector_end = questdlg(message_last_detector, 'Warning', 'End','End'); switch chose_detector_end

case 'End'

return;

end

else

continue;

end

end

end

[SMDATI1, Time_interval1, percentage_of_x, percentage_of_zeros] =

Reading_data_and_output (Speed_file_name, Time_after_incedent,Time_before_incedent, Day_Ince);

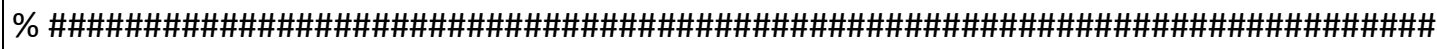

$\operatorname{str} X=$ num $2 \operatorname{str}($ int16(percentage_of_x));

strZ = num2str(int16(percentage_of_zeros));

Message $=[$ 'The percentage of $X$ readings in the speed data file is ',strX, ' $\%$ and the percentage of Zeros is ',strZ ,'\%. Do you want to continue or to select the next detector?'];

Message100 $=$ 'The percentage of $X$ or Zeros in the speed data is $100 \%$ : Do you want to select the next detector or Stop?';

$\%$ PopUP dailog to to let you know the percent of $X$ and zeros

if percentage_of_ $x<100 \& \&$ percentage_of_zeros $<100$ \& \&

percentage_of_x+percentage_of_zeros $\sim 100$ 


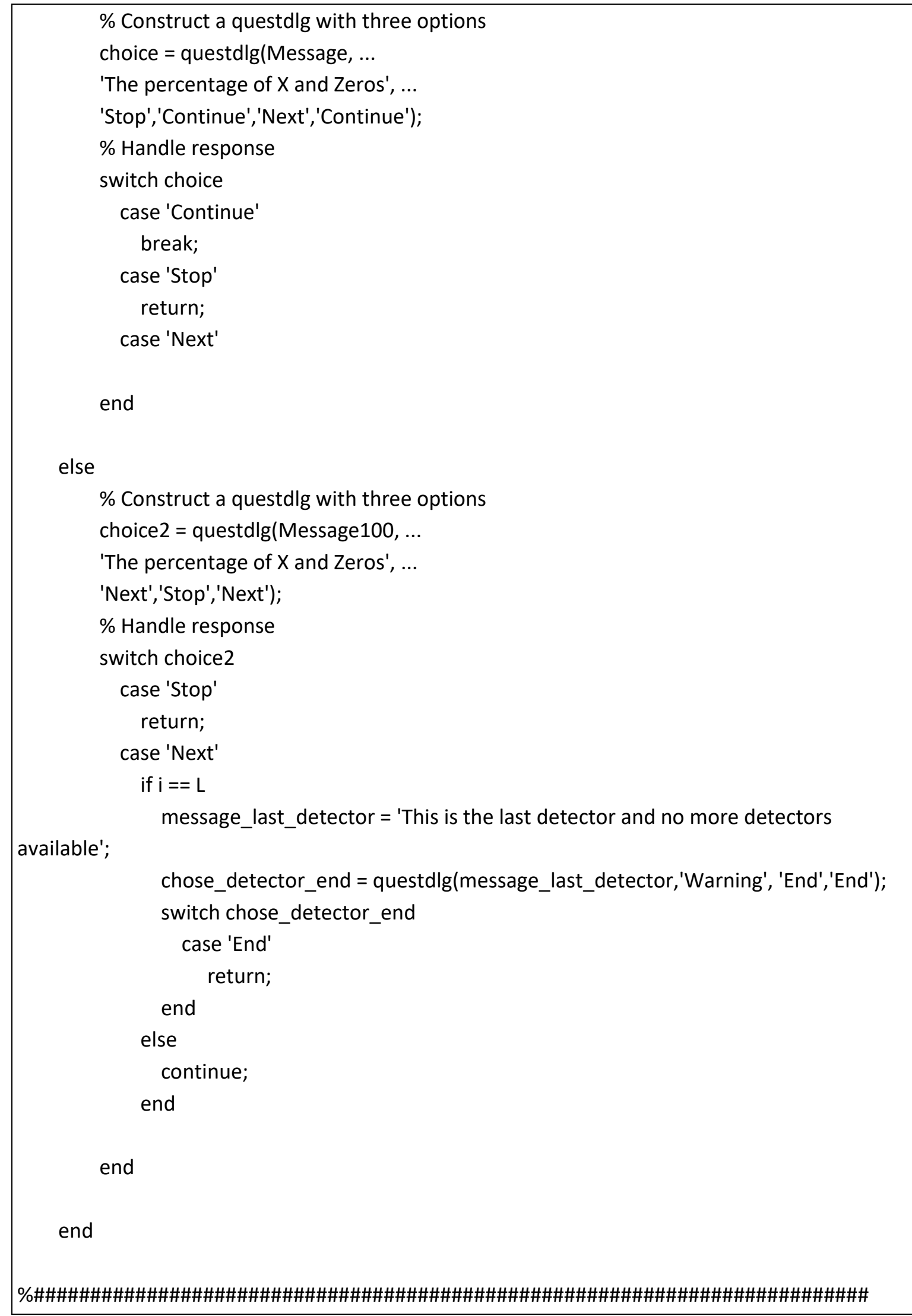




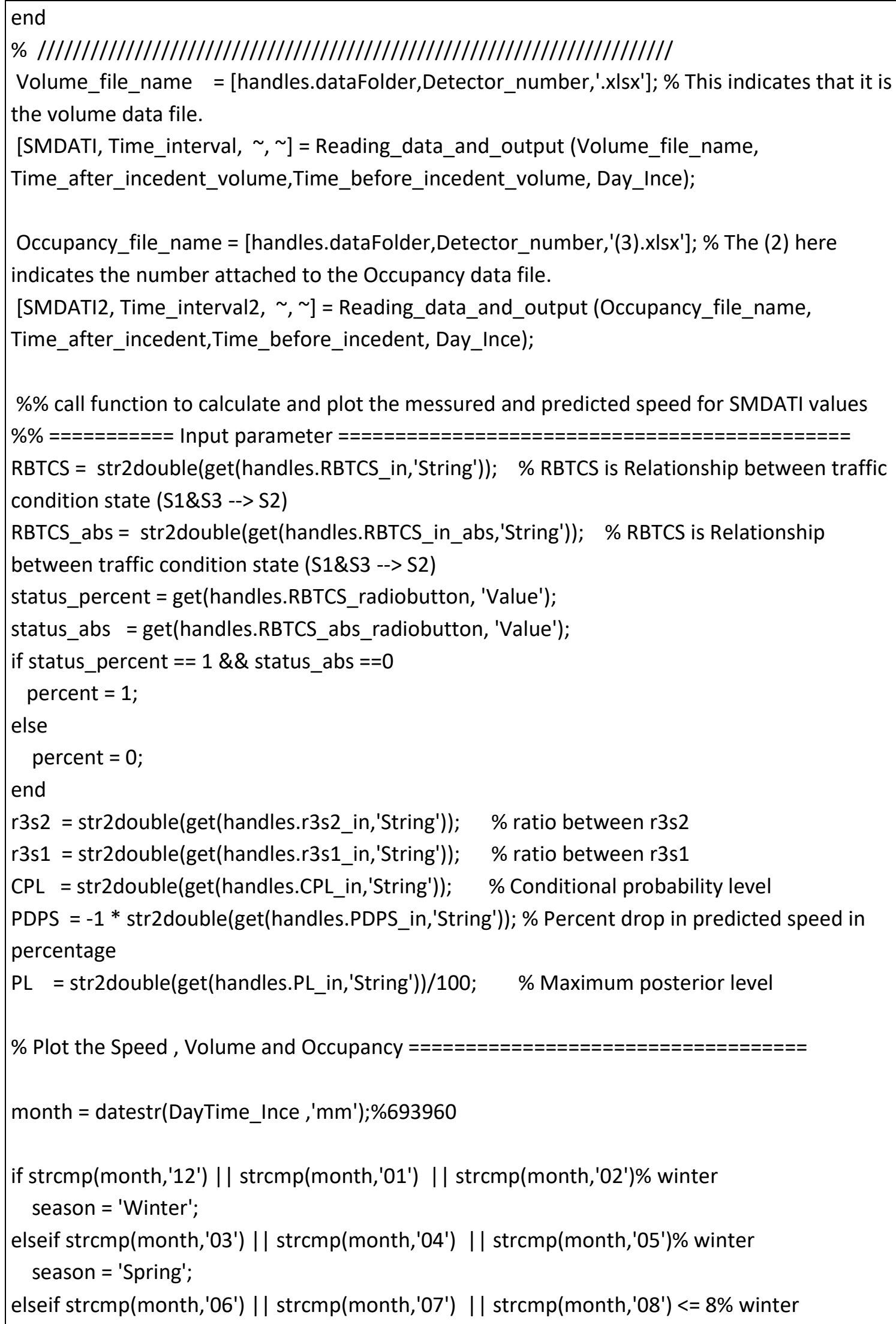




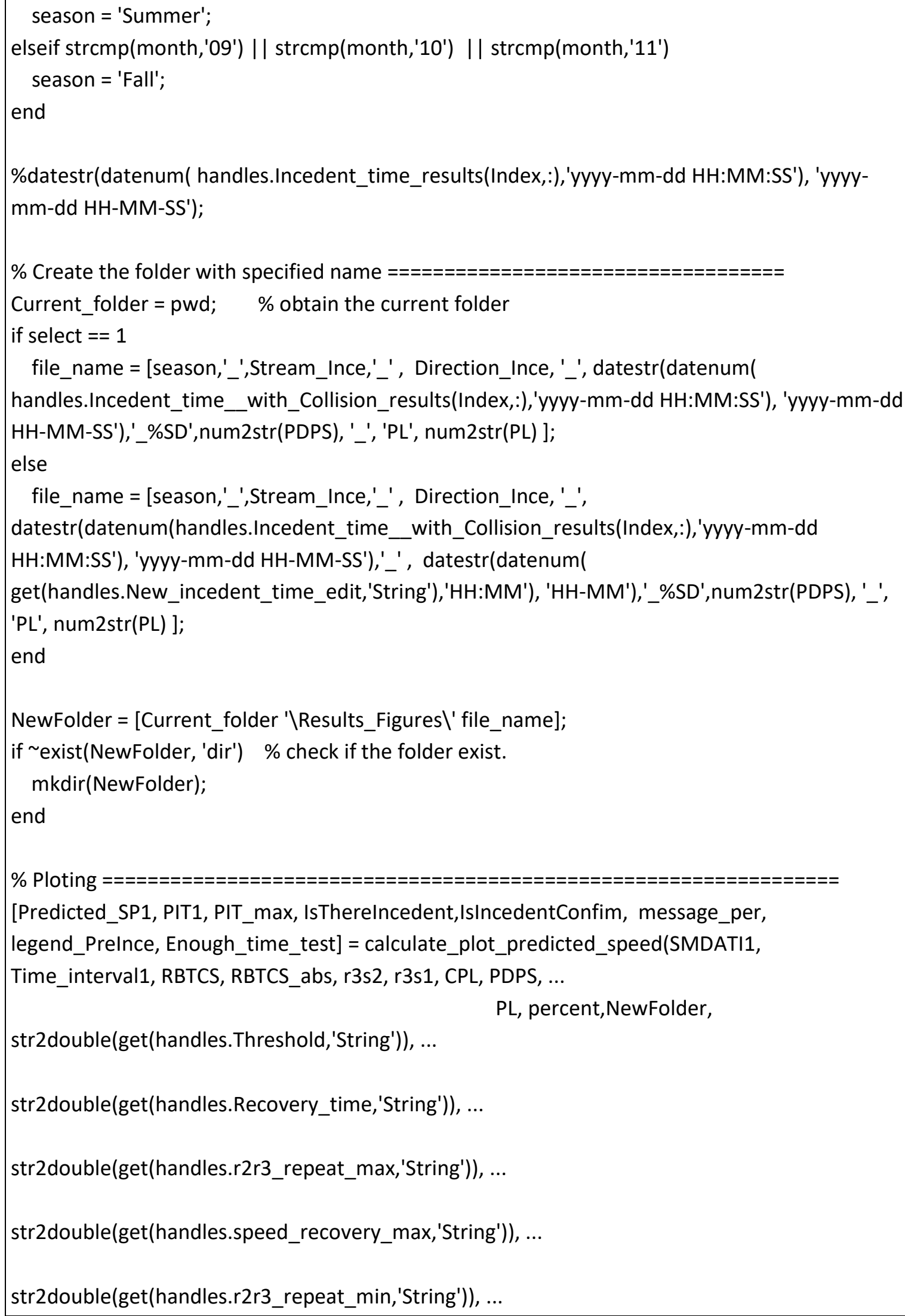


str2double(get(handles.speed_recovery_min,'String')), ...

str2double(get(handles.min_threshold,'String')), ...

str2double(get(handles.Speed_recov_repetition,'String')), ...

Time_after_incedent);

$\% \%$ We moved the following code to the calculate_plot_predected_speed.m function ---->

$\%$ if IsTherelncedent $==1$

$\%$ if IsIncedentConfim == 1

$\% \quad$ legend_Prelnce = 'Incident was detected \& confirmed';

$\%$ else

$\% \quad$ legend_Prelnce = 'Incident was detected \& NOT confirmed';

$\%$ end

$\%$ else

$\% \quad$ legend_Prelnce = 'Incident was NOT detected';

$\%$ end

Det_info = ['Upstream Detector \# is ',Detector_number, ', Detector Rank = ' num2str(i)];

Output1 = [ 'Season = ', season, ', Stream = ', Stream_Ince , ', Direction = ', Direction_Ince, ',

Date $\&$ Time $=$ ', handles.Incedent_time_with_Collision_results(Index,:)];

$\%$ Output2 = [ 'Status =', Status_Ince , ', Incident Reason =', Reason_Ince];

\%Output1 = [ 'Season = ',season, ', Stream = ', Stream_Ince , ', Direction = ', Direction_Ince];

Output2 = [ 'Incident Reason =', Reason_Ince];

output21 = ['Affected Lanes =' ,Affected_Lanes_Ince];

Input3 = ['Conditional Probability level $={ }^{\prime}$, num2str(CPL), ', Cond.Prob. r3s2 = ', num2str(r3s2)

, ', Cond.Prob. r3s1= ' , num2str(r3s1), ', Percent drop in speed \% = ' , num2str(PDPS) ];

if percent $==1$

Input4 = ['Max. Posterior Probability level = ',num2str(PL) , ', Percent range between speed states $=$ ' , num2str(RBTCS*100) , ' \%';];

else

Input4 = ['Max. Posterior Probability level = ',num2str(PL) , ', Absolute range between speed states = ' , num2str(RBTCS_abs) , ' km';];

end

if select $==1$

Det_info $=\left[\right.$ Det_info ', Distance from incident location in $(\mathrm{km})={ }^{\prime}$ ,num2str(Dedector_disttance) ];

boxInfo $=\{$ Det_info,Output1,Output2,output21, Input3, Input4 $\}$; 


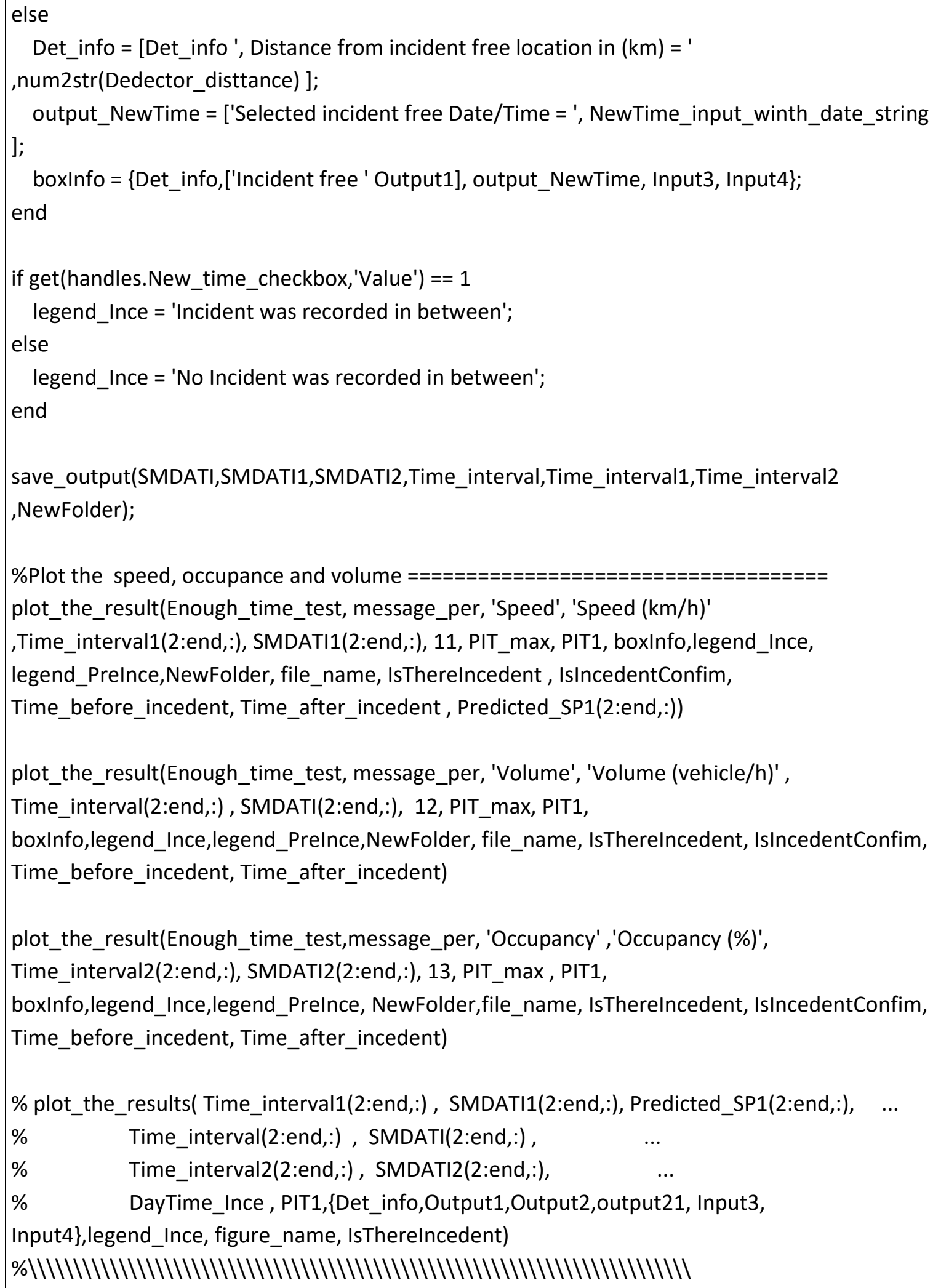




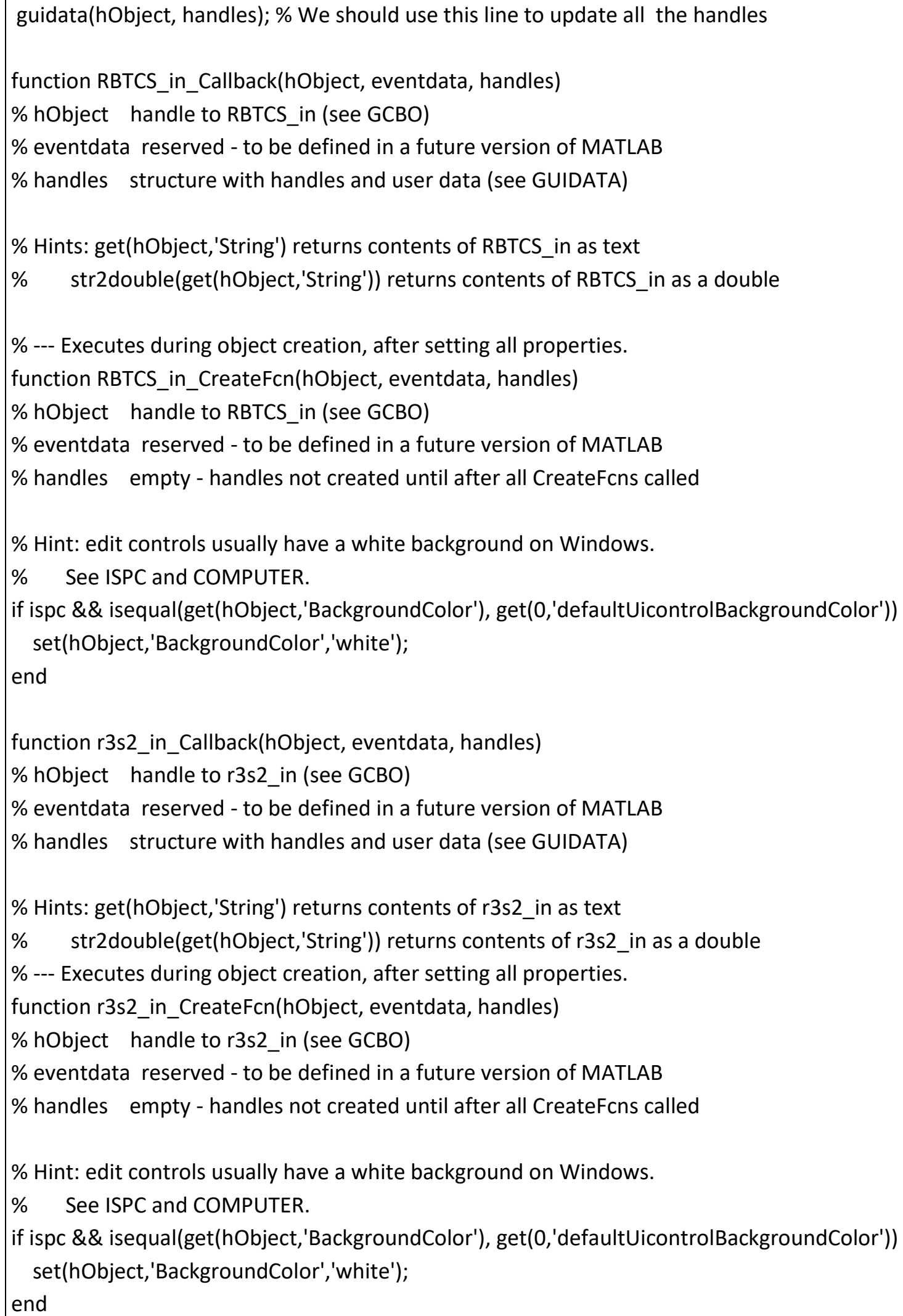




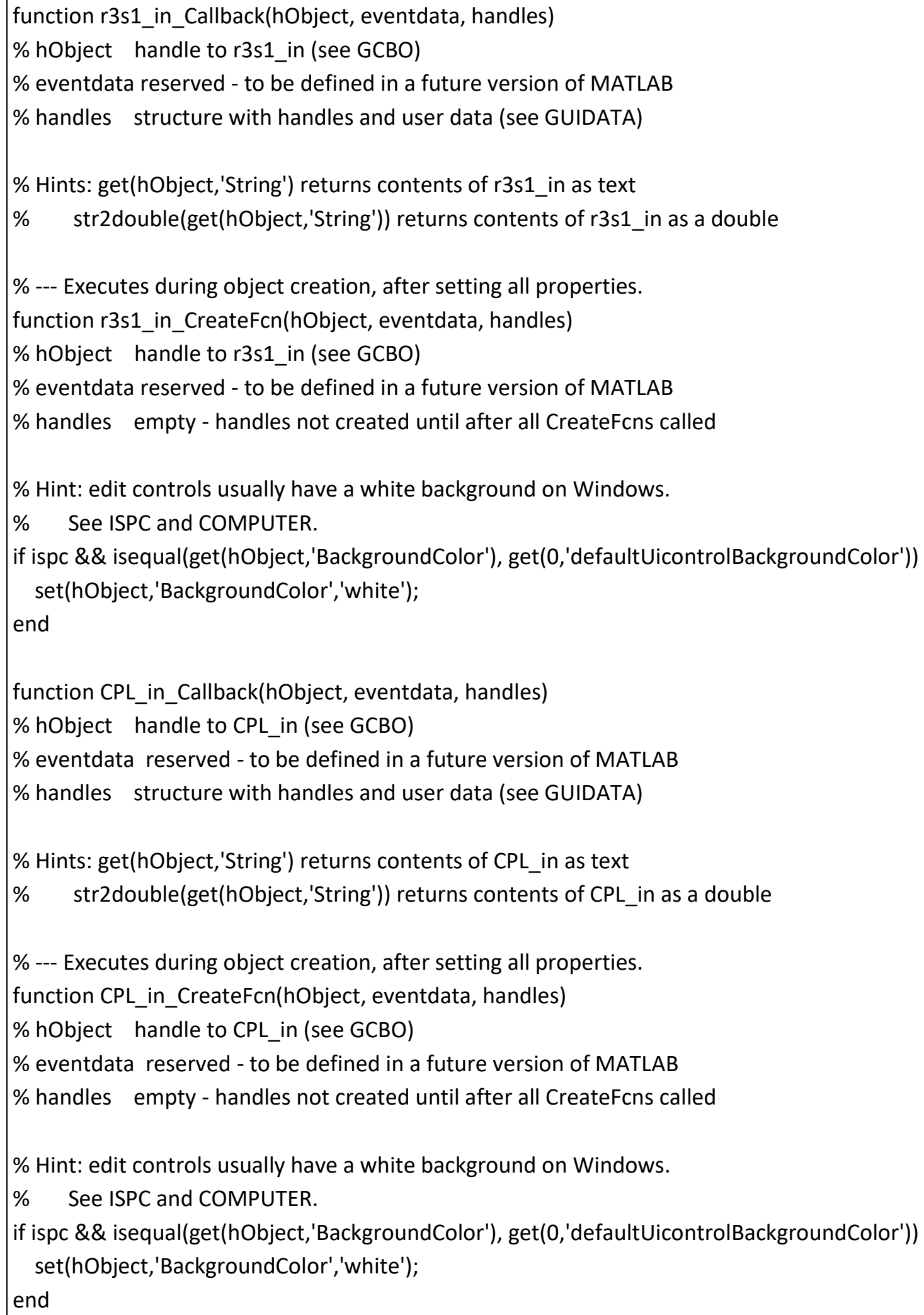




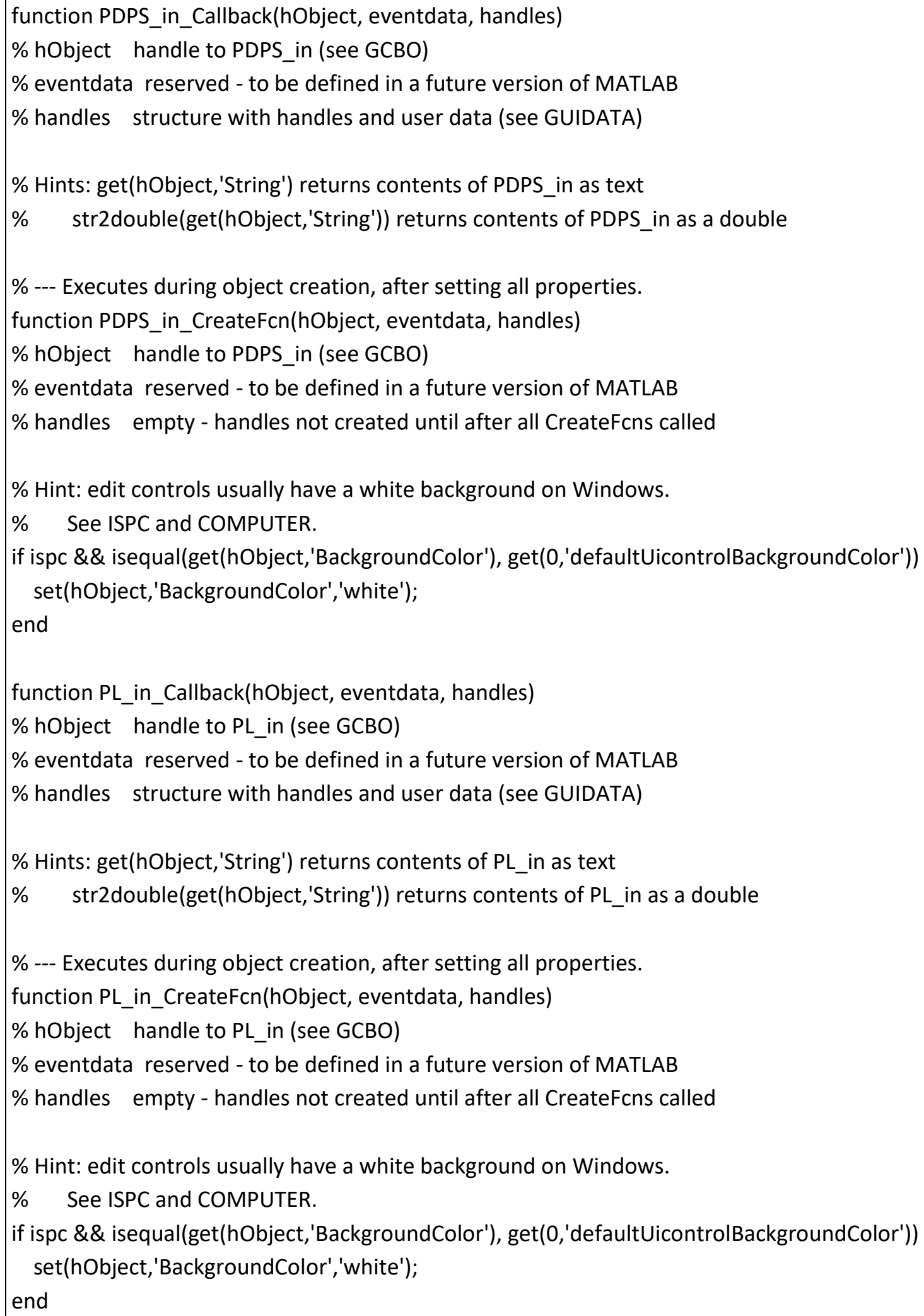




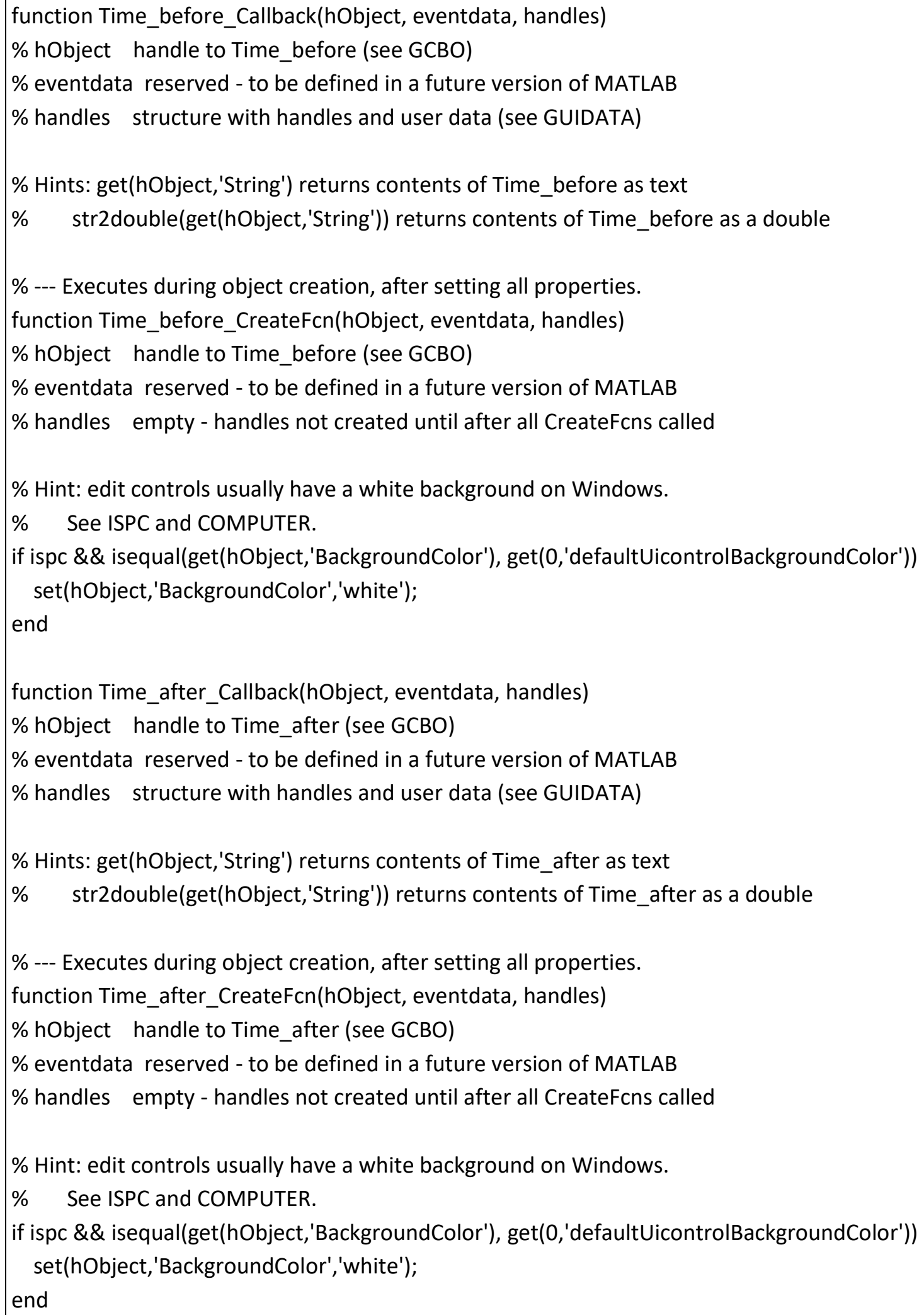


$\%$--- Executes on selection change in Direction_popupmenu.

function Direction_popupmenu_Callback(hObject, eventdata, handles)

$\%$ hObject handle to Direction_popupmenu (see GCBO)

$\%$ eventdata reserved - to be defined in a future version of MATLAB

$\%$ handles structure with handles and user data (see GUIDATA)

$\%$ Hints: contents $=$ cellstr(get(hObject,'String')) returns Direction_popupmenu contents as cell array

$\% \quad$ contents $\{$ get(hObject,'Value')\} returns selected item from Direction_popupmenu

$\%$--- Executes during object creation, after setting all properties.

function Direction_popupmenu_CreateFcn(hObject, eventdata, handles)

$\%$ hObject handle to Direction_popupmenu (see GCBO)

$\%$ eventdata reserved - to be defined in a future version of MATLAB

$\%$ handles empty - handles not created until after all CreateFcns called

\% Hint: popupmenu controls usually have a white background on Windows.

$\% \quad$ See ISPC and COMPUTER.

if ispc \&\& isequal(get(hObject,'BackgroundColor'), get(0,'defaultUicontrolBackgroundColor')) set(hObject,''BackgroundColor','white');

end

$\%$--- Executes on selection change in Stream_popupmenu.

function Stream_popupmenu_Callback(hObject, eventdata, handles)

$\%$ hObject handle to Stream_popupmenu (see GCBO)

$\%$ eventdata reserved - to be defined in a future version of MATLAB

$\%$ handles structure with handles and user data (see GUIDATA)

\% Hints: contents = cellstr(get(hObject,'String')) returns Stream_popupmenu contents as cell array

$\% \quad$ contents $\{$ get(hObject,'Value')\} returns selected item from Stream_popupmenu

$\%$--- Executes during object creation, after setting all properties.

function Stream_popupmenu_CreateFcn(hObject, eventdata, handles)

$\%$ hObject handle to Stream_popupmenu (see GCBO)

$\%$ eventdata reserved - to be defined in a future version of MATLAB

$\%$ handles empty - handles not created until after all CreateFcns called

\% Hint: popupmenu controls usually have a white background on Windows.

$\% \quad$ See ISPC and COMPUTER.

if ispc \&\& isequal(get(hObject,'BackgroundColor'), get(0,'defaultUicontrolBackgroundColor')) 


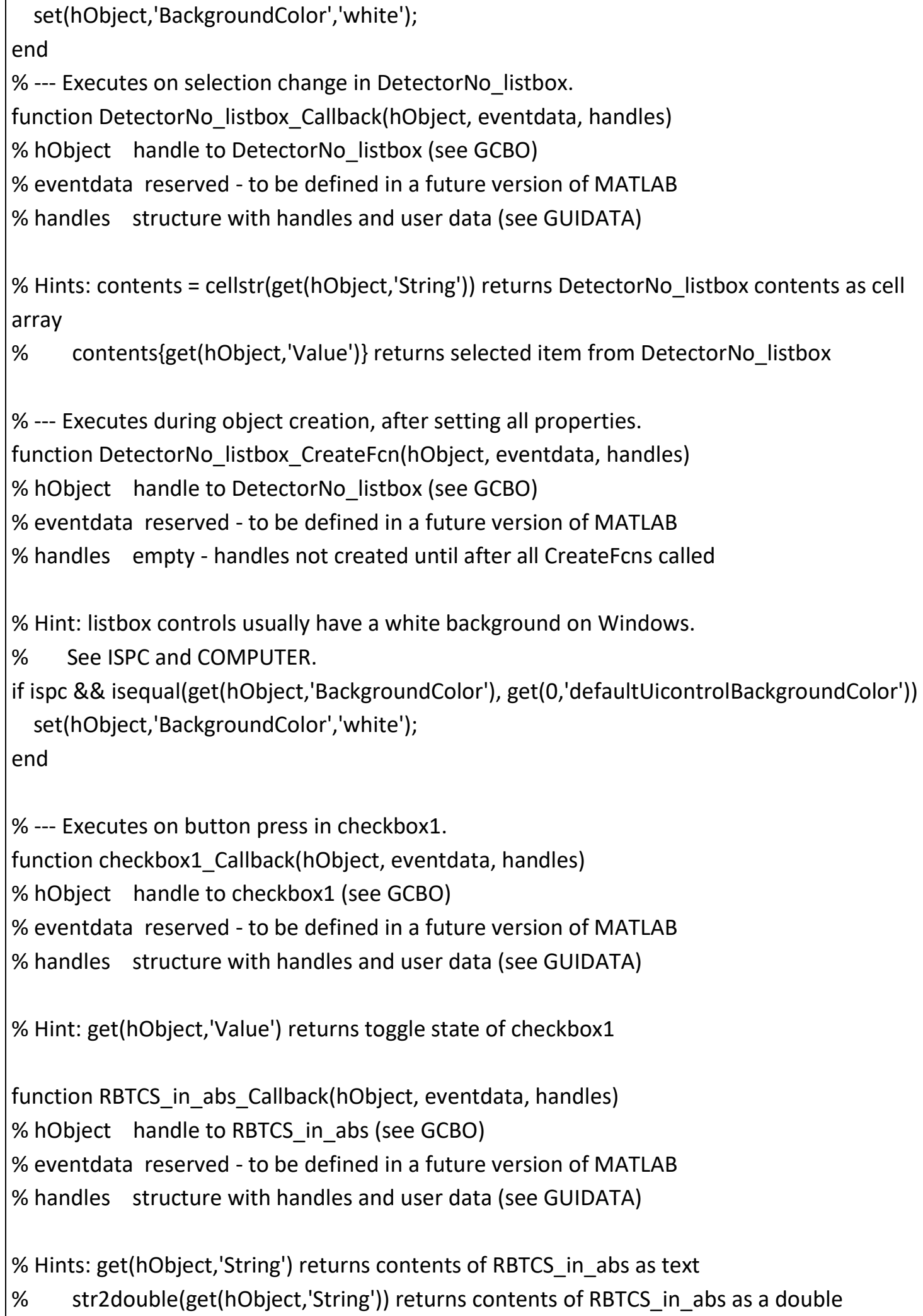




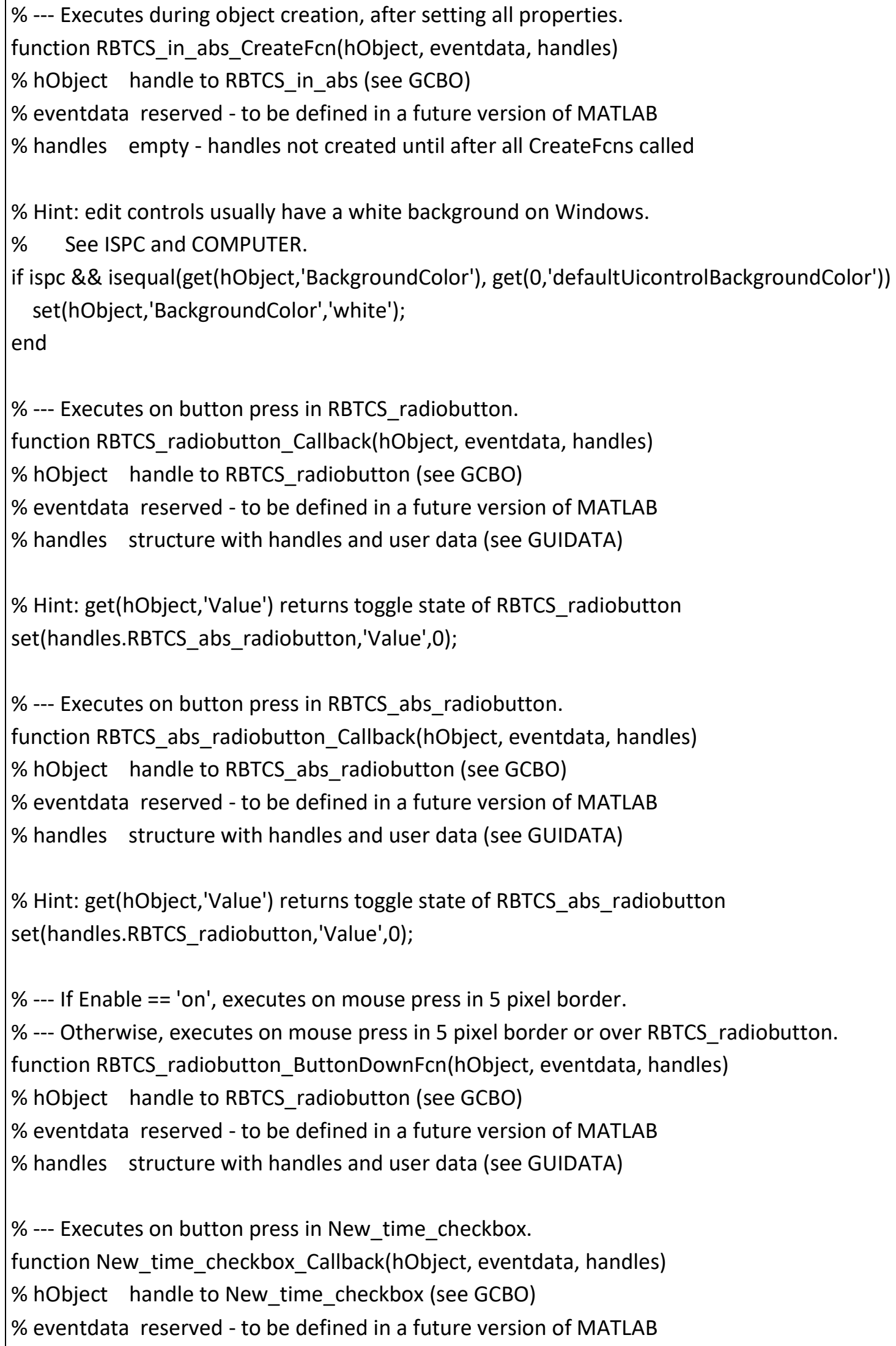




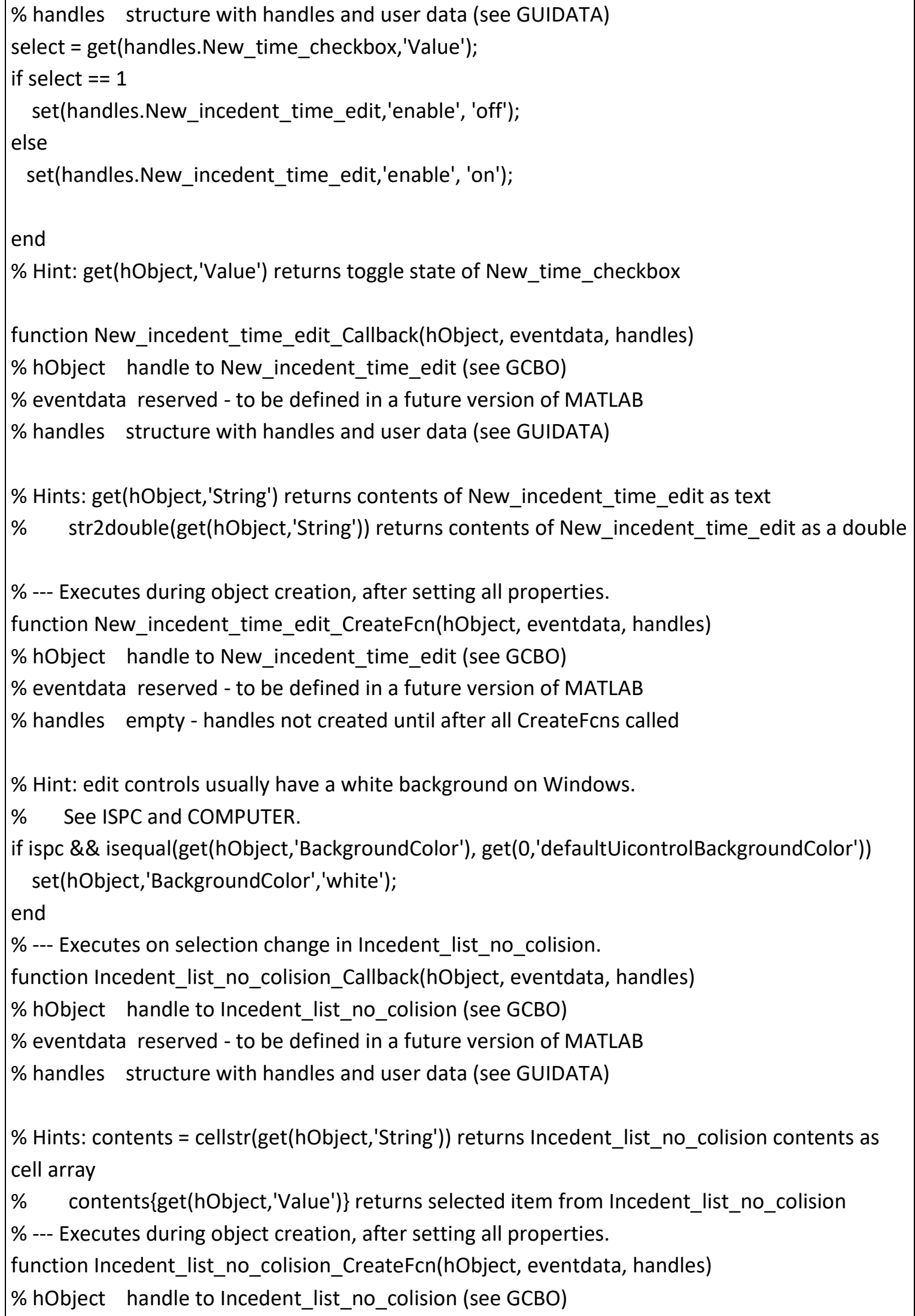




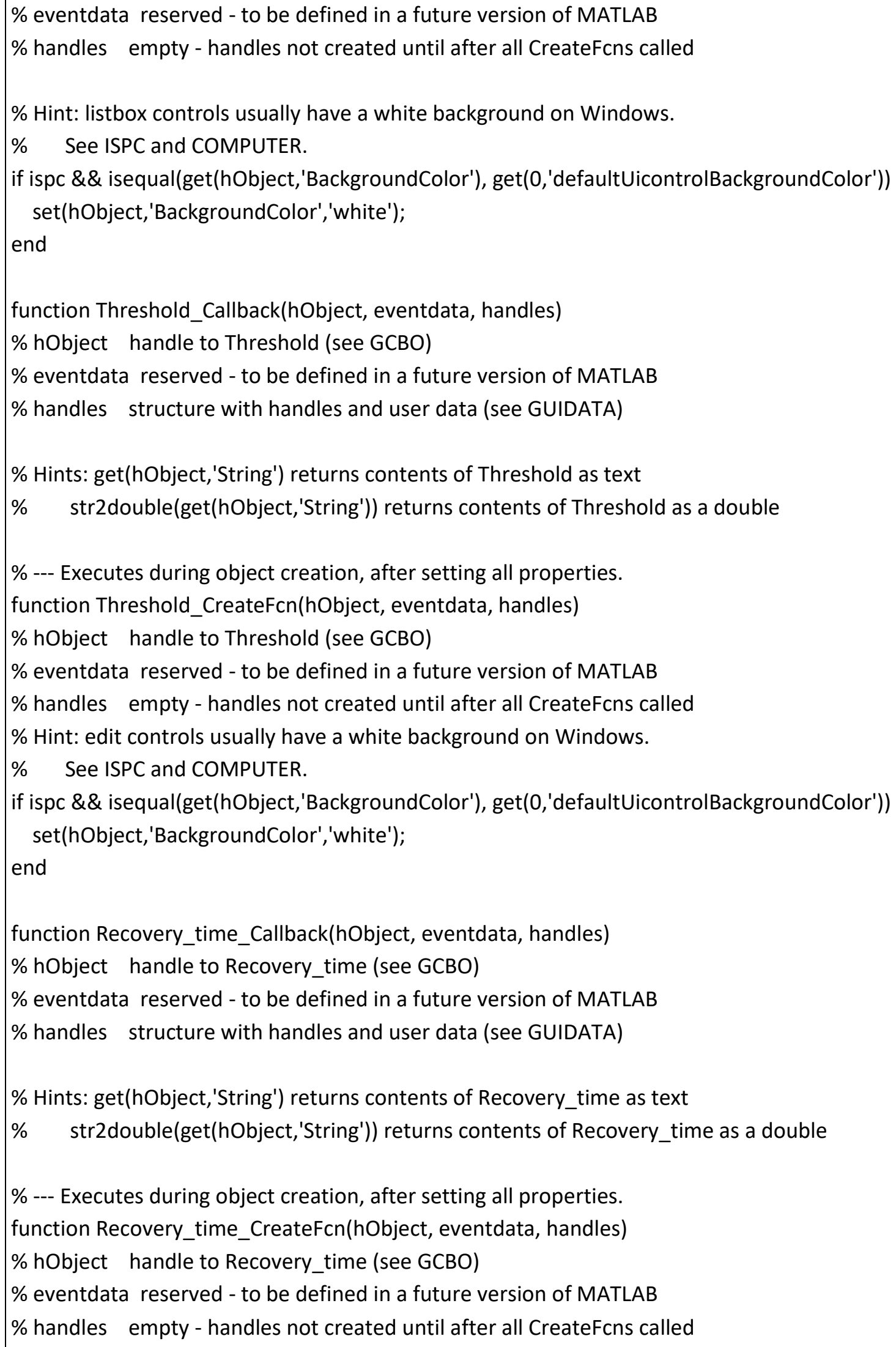




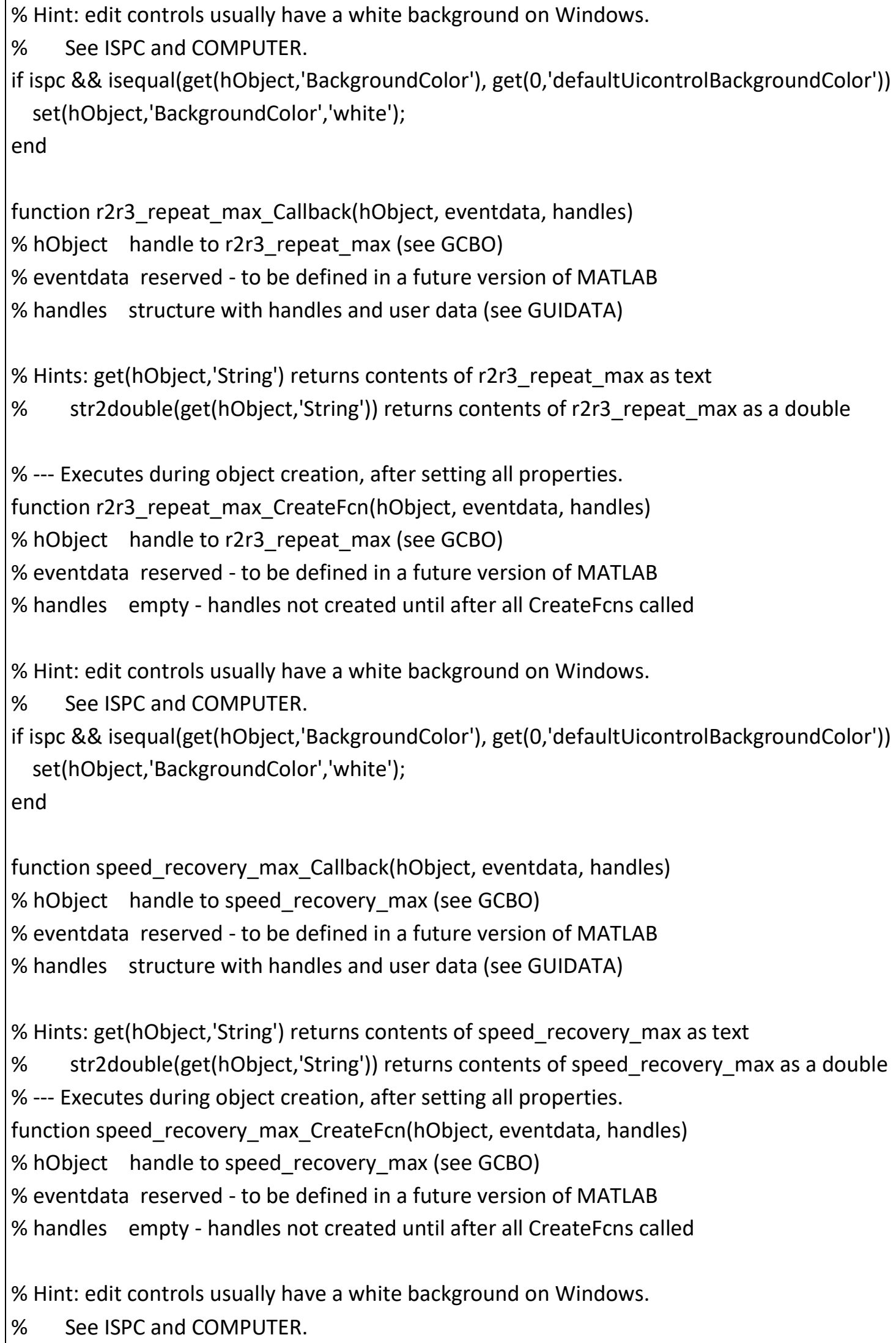




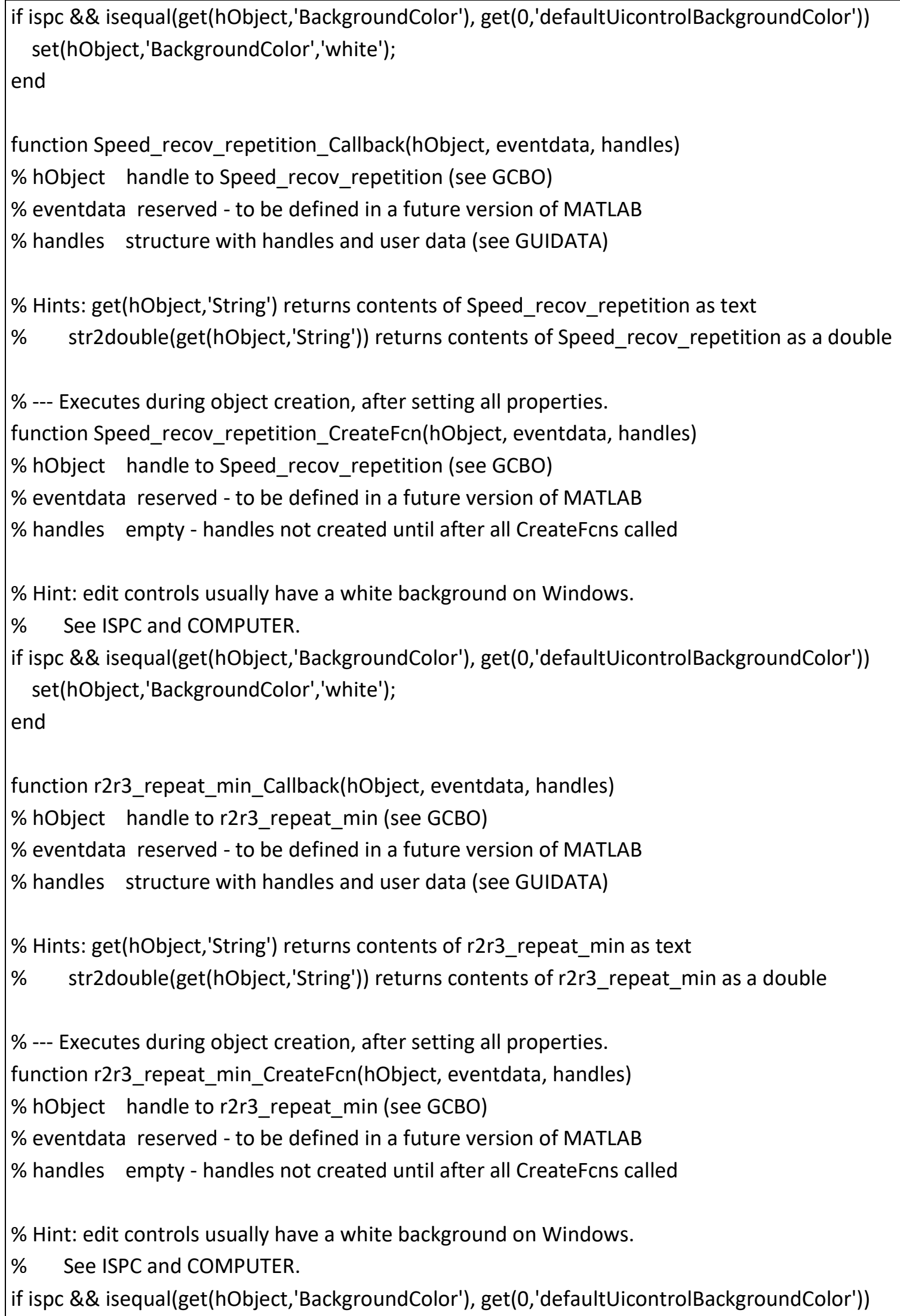




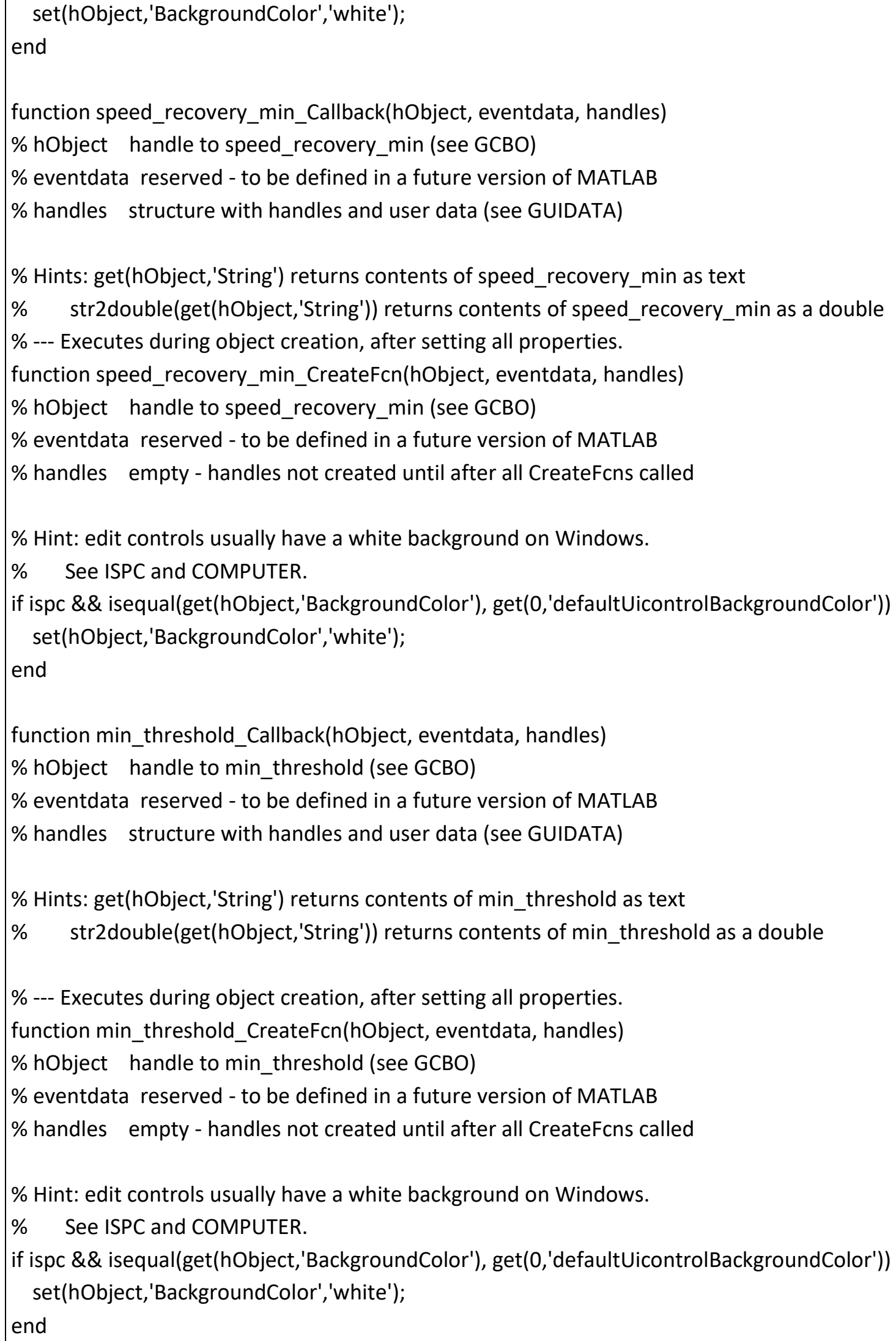




\section{Appendix B.2 Finding Sensor Number Function}

This Appendix presents the Matlab source codes for the developed model. It includes the source code for finding the closest upstream detector from the incident location.

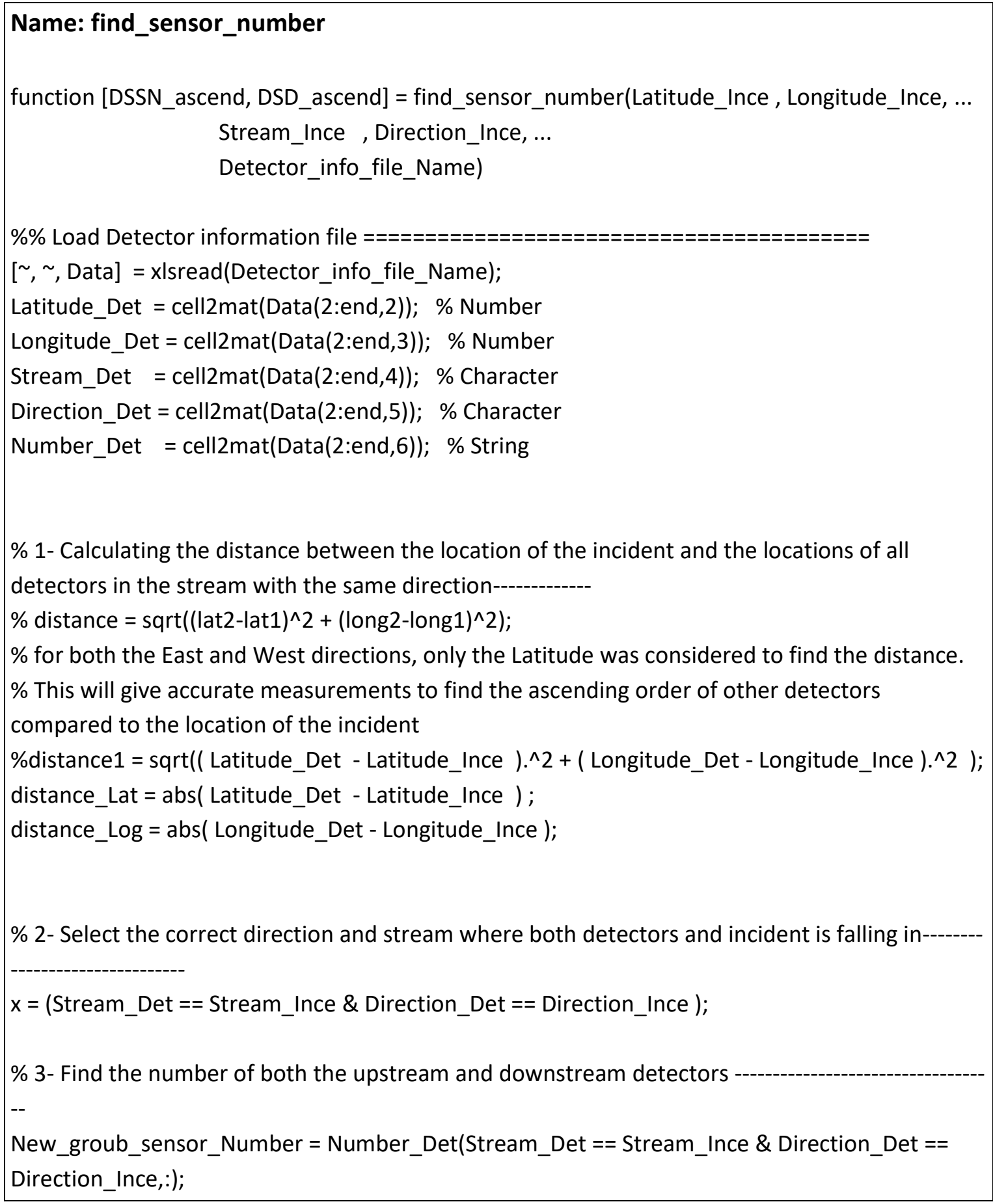




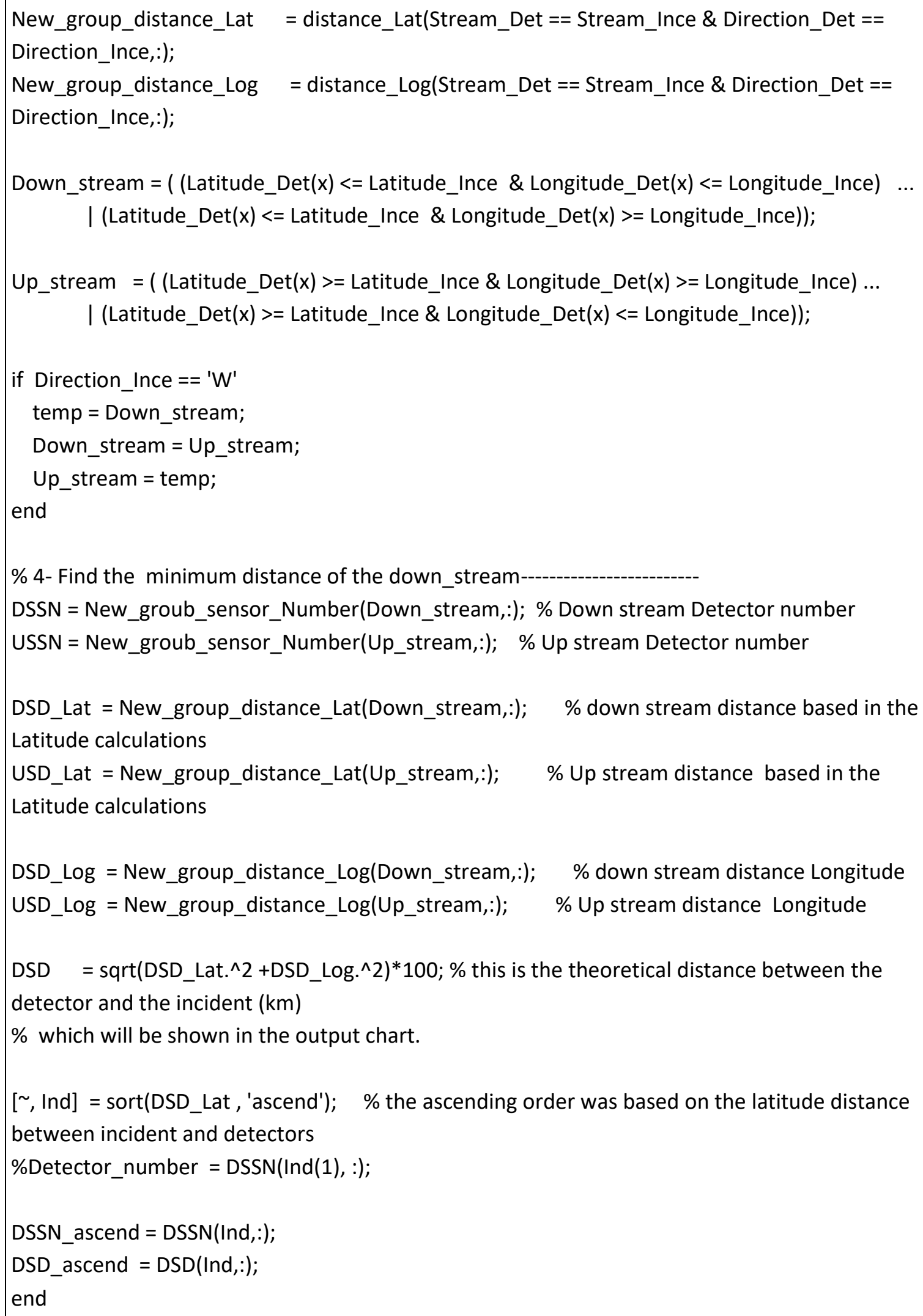




\section{Appendix B.3 Writing Data into csv Files Function}

This Appendix presents the Matlab source codes for the developed model. It includes the source code for the writing data into csv files.

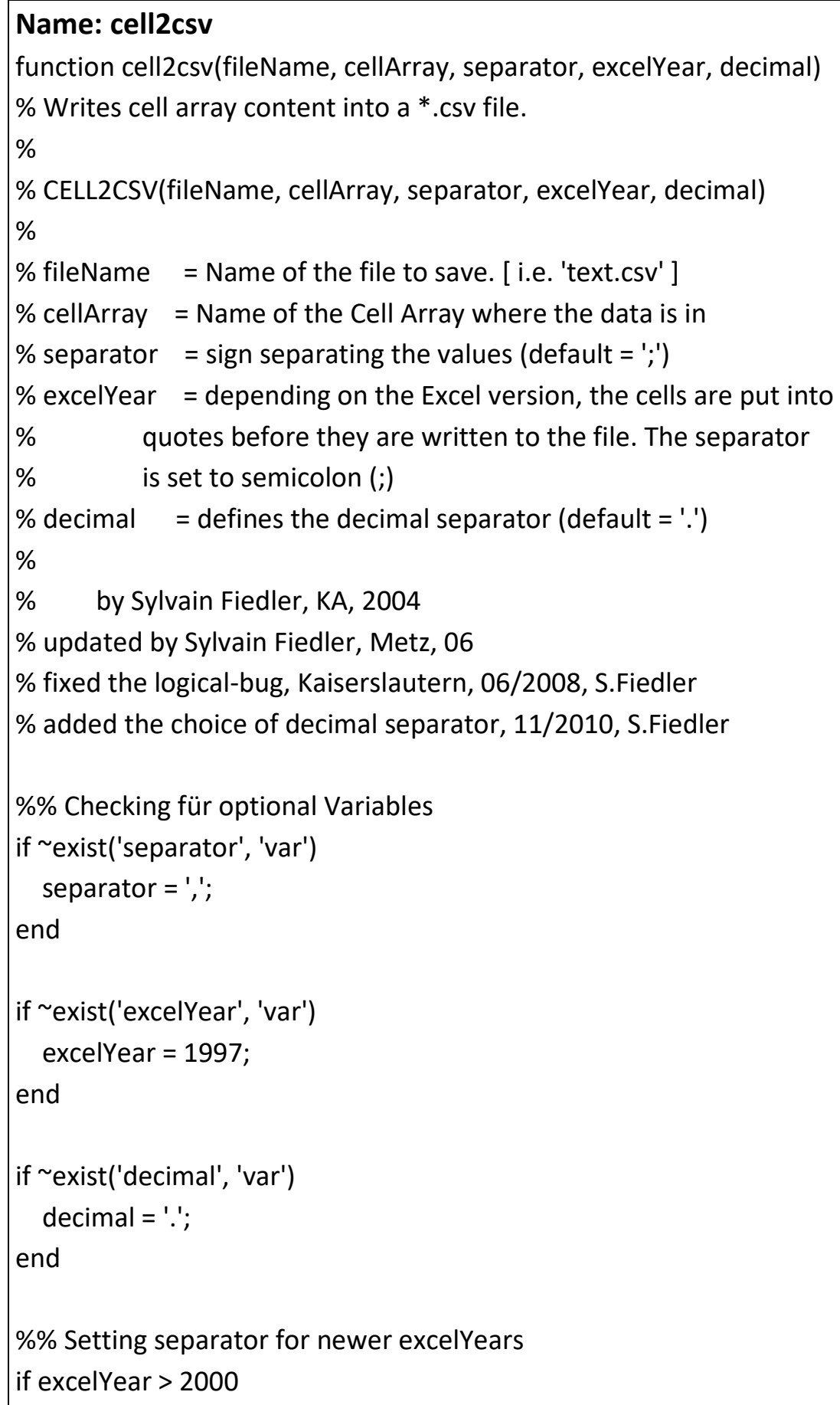




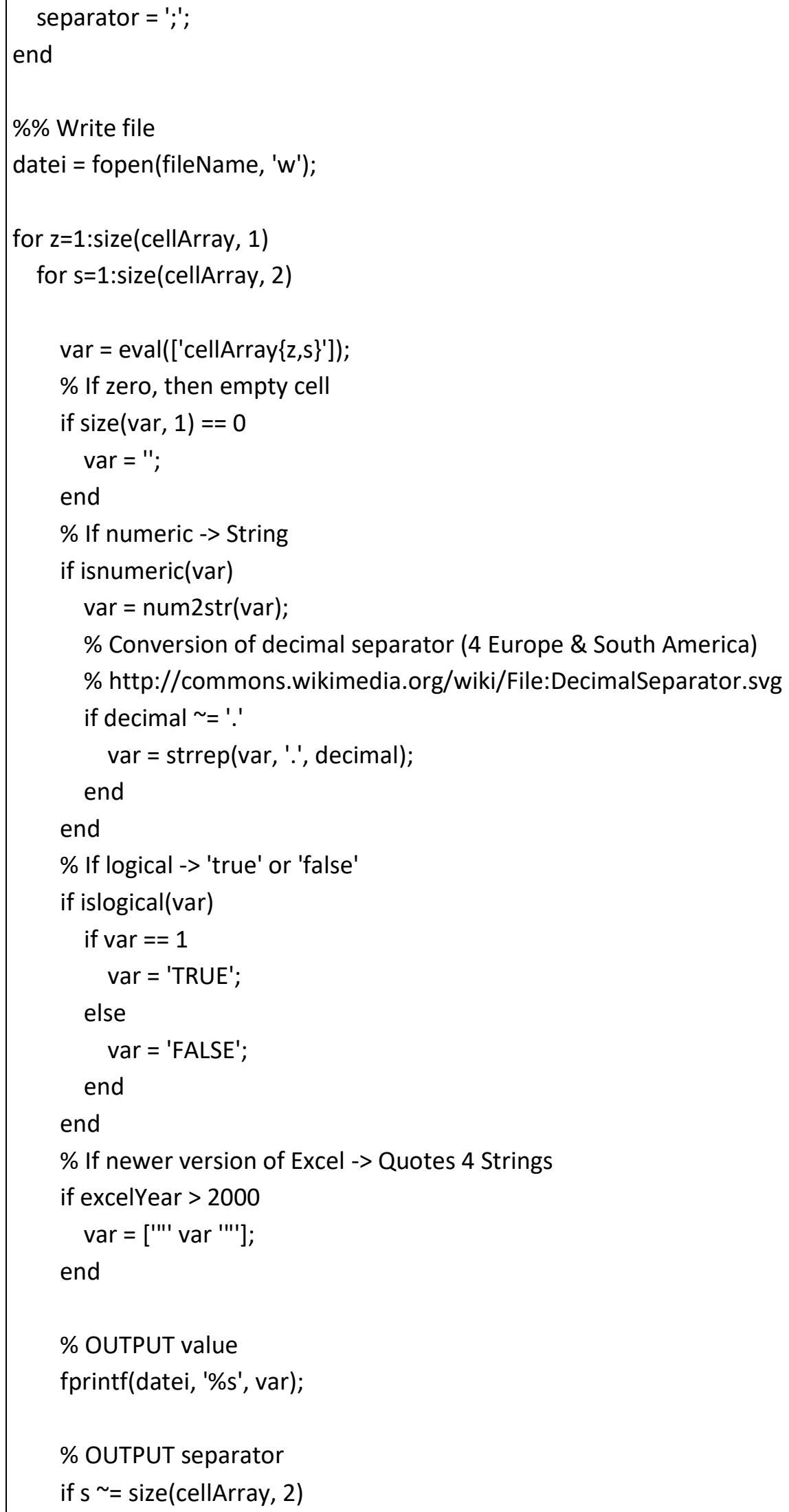




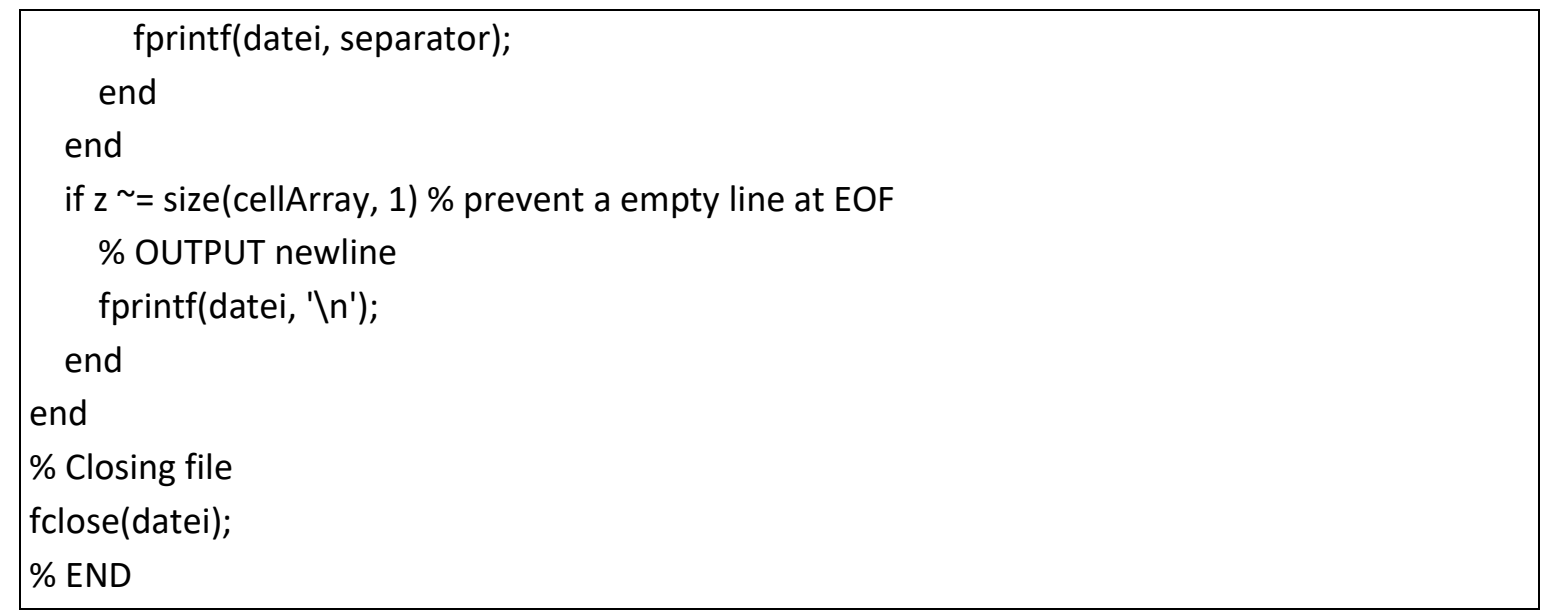




\section{Appendix B.4 Reading Data and Output Function}

This Appendix presents the Matlab source codes for the developed model. It includes the source code for the reading the data. It is used to read the speed database, including speed values, date, and time for all obtained data that corresponds to an incident location and direction.

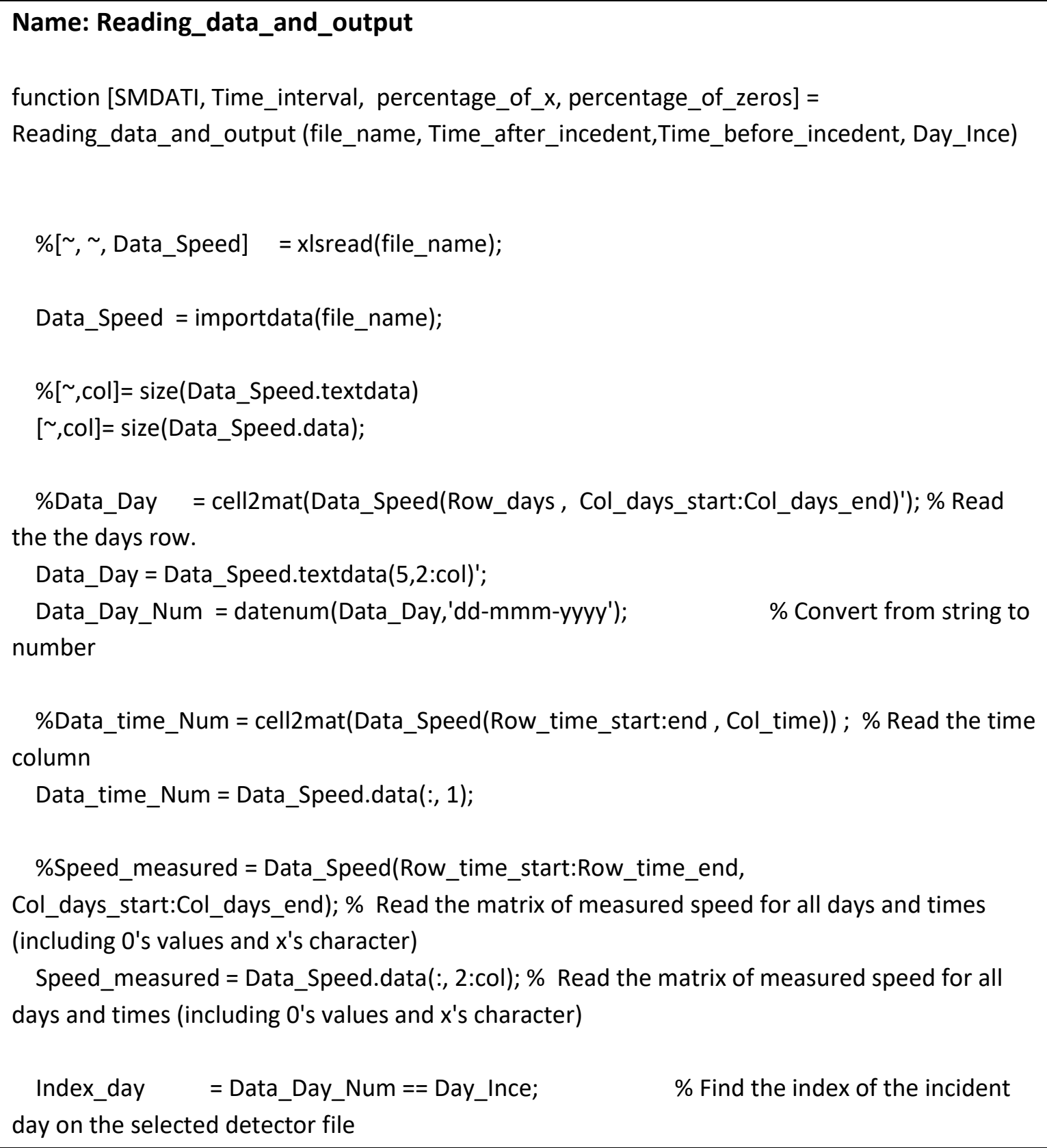




DateTime_Speed_Num = Data_Day_Num(Index_day) + Data_time_Num; \% Add day to
the time column
\%datestr( DateTime_Speed_Num,'yyyy-mm-dd HH:MM:SS')
Index_time_interval = DateTime_Speed_Num <= Time_after_incedent ...
\[ \begin{array}{l}\text { \& DateTime_Speed_Num >=Time_before_incedent; } \\ \text { the time interval }\end{array} \]
SMDATI_CellArray = Speed_measured(Index_time_interval , Index_day); \%
SMDATI_CellArray is a Cell Array contains the speed Measured at day of incident day and time
interval
[SMDATI, index_SP, percentage_of_x, percentage_of_zeros] =
extract_the_speed_values(SMDATI_CellArray);
Time_interval = DateTime_Speed_Num(Index_time_interval);
Time_interval = Time_interval(index_SP);




\section{Appendix B.5 Extract False Speed Values}

This Appendix presents the Matlab source codes for the developed model. It includes the source code for excluding false data from the speed data file.

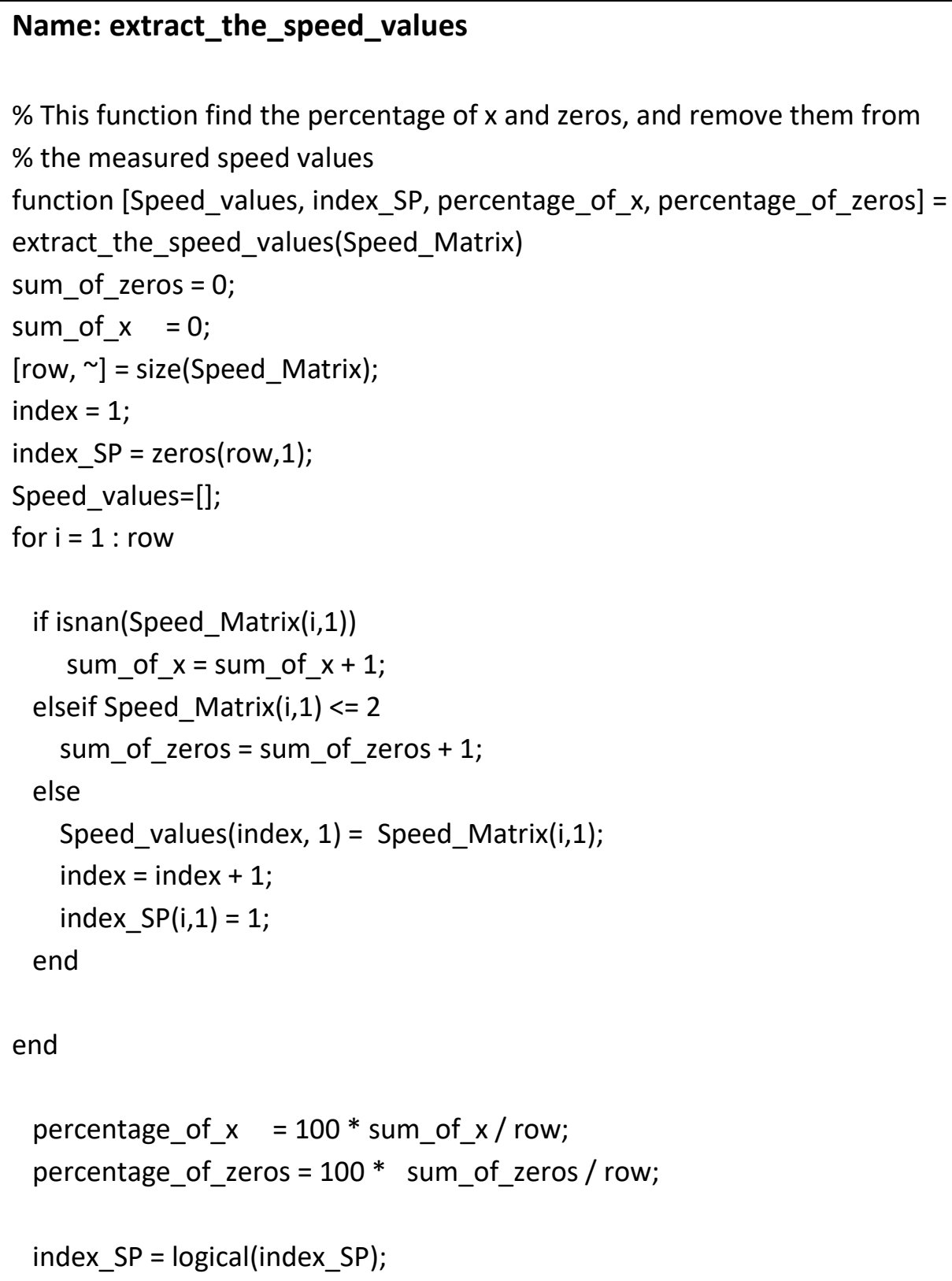




\section{Appendix B.6 Incident Detection Components}

This Appendix presents the Matlab source codes for the developed model. It includes the source code for the incident detection components.

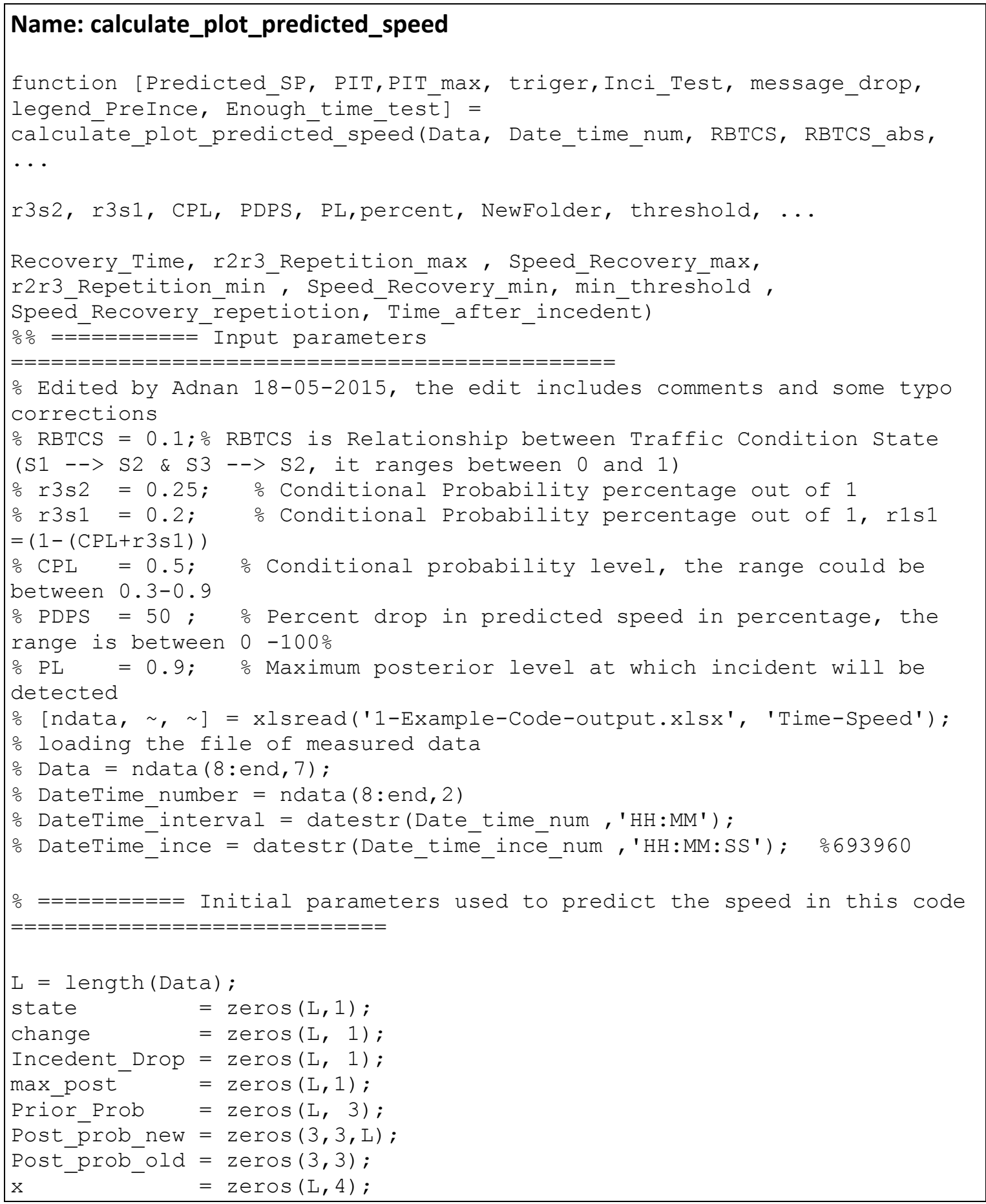




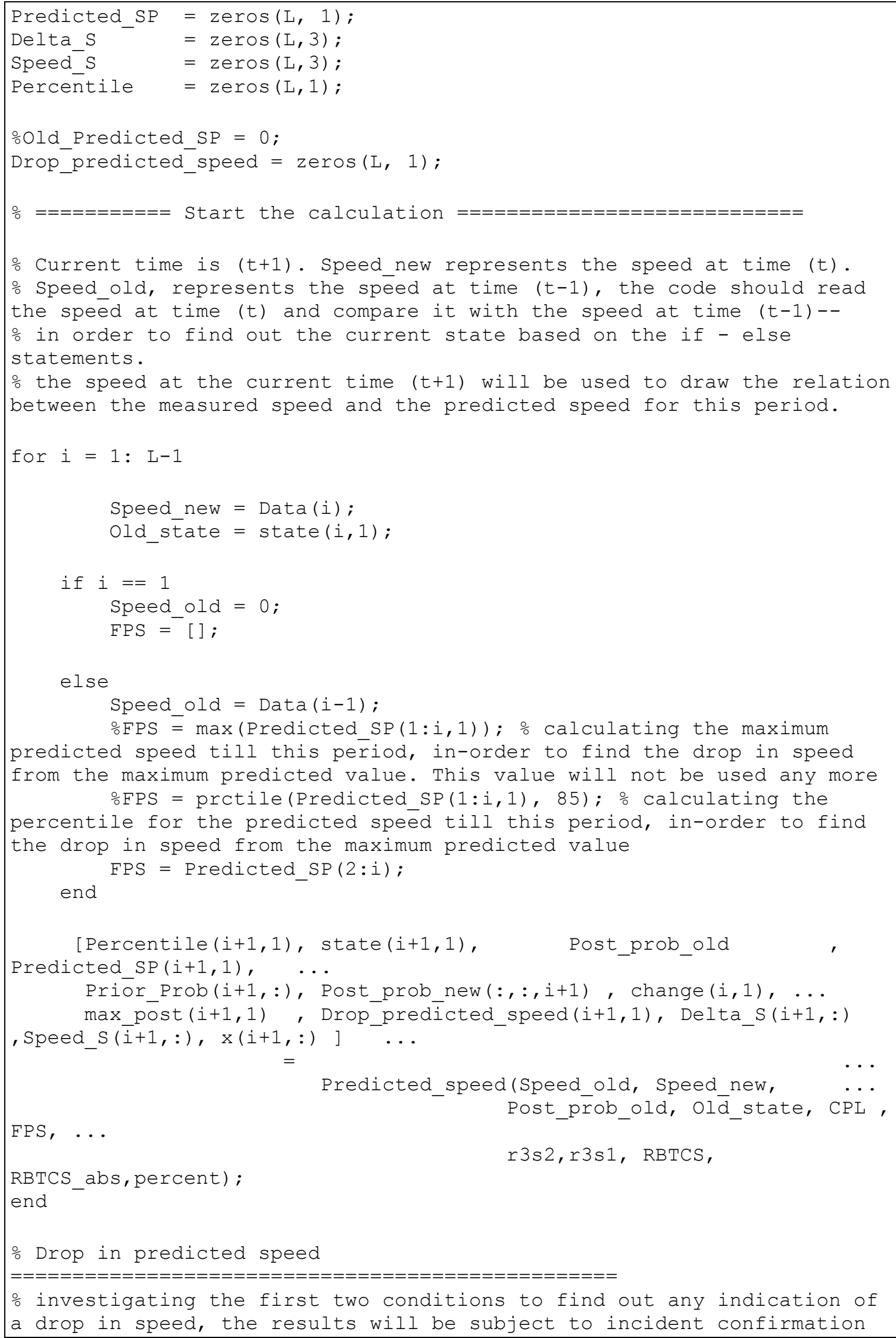




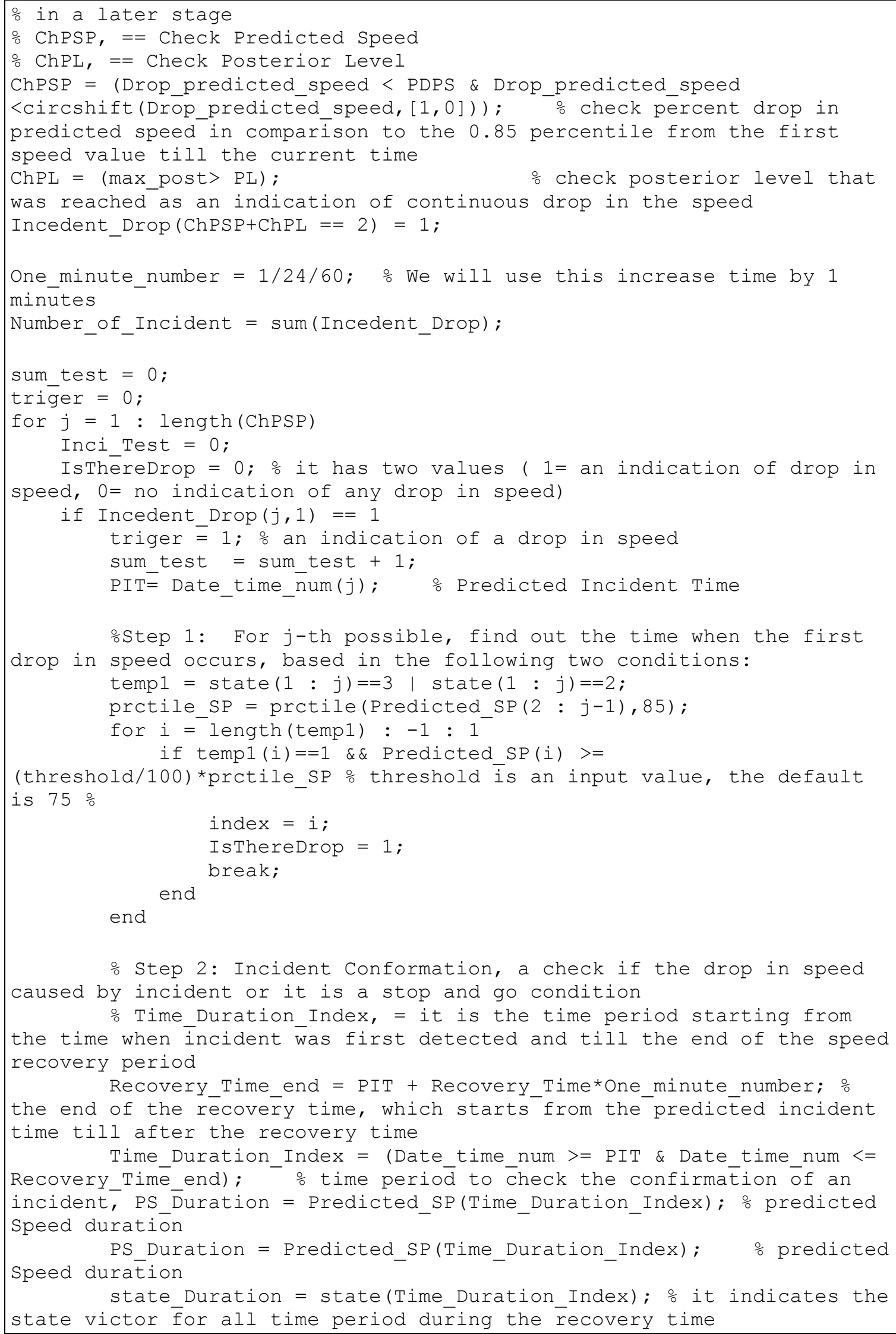

o Step 2: Incident Conformation, a check if the drop in speed caused by incident or it is a stop and go condition

\% Time Duration Index, = it is the time period starting from the time when incident was first detected and till the end of the speed recovery period 
\% $\quad$ the following steps are the two extra conditions in v5. for low percentile less than a specified value the number of the

o repetition of $r 2 r 3$ and the selected percent of the percentile is decreased to lower values to increase the sensitivity of

algorithm when detecting incident

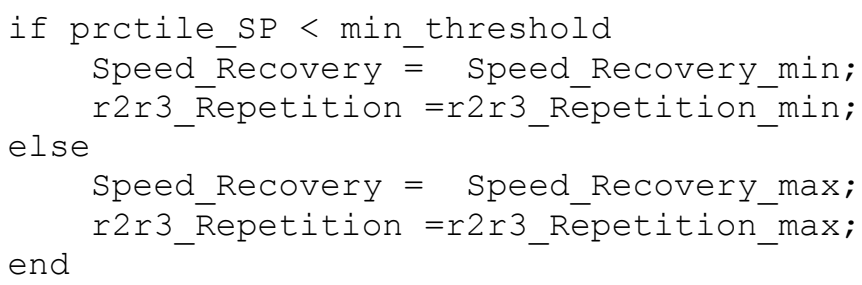




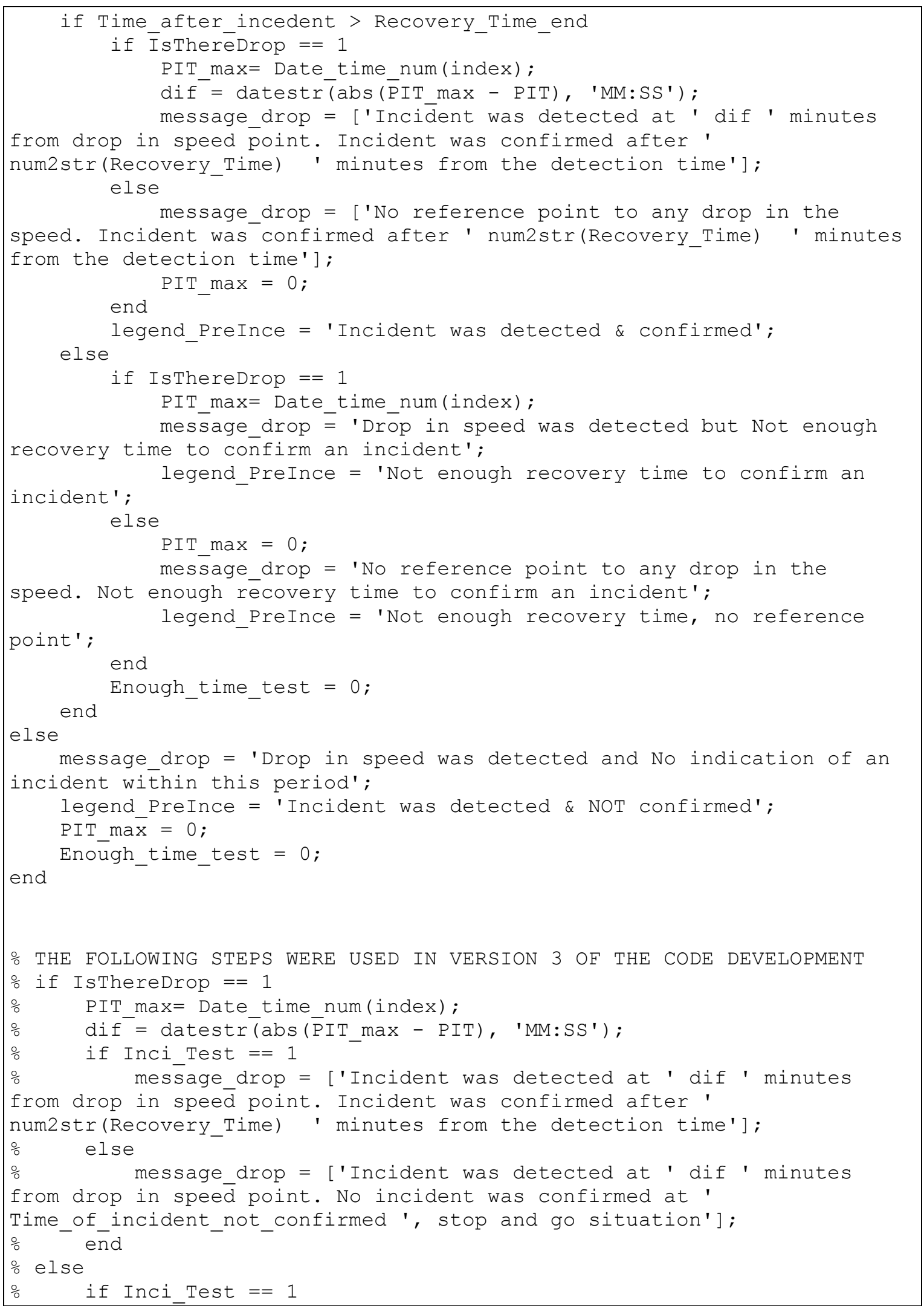




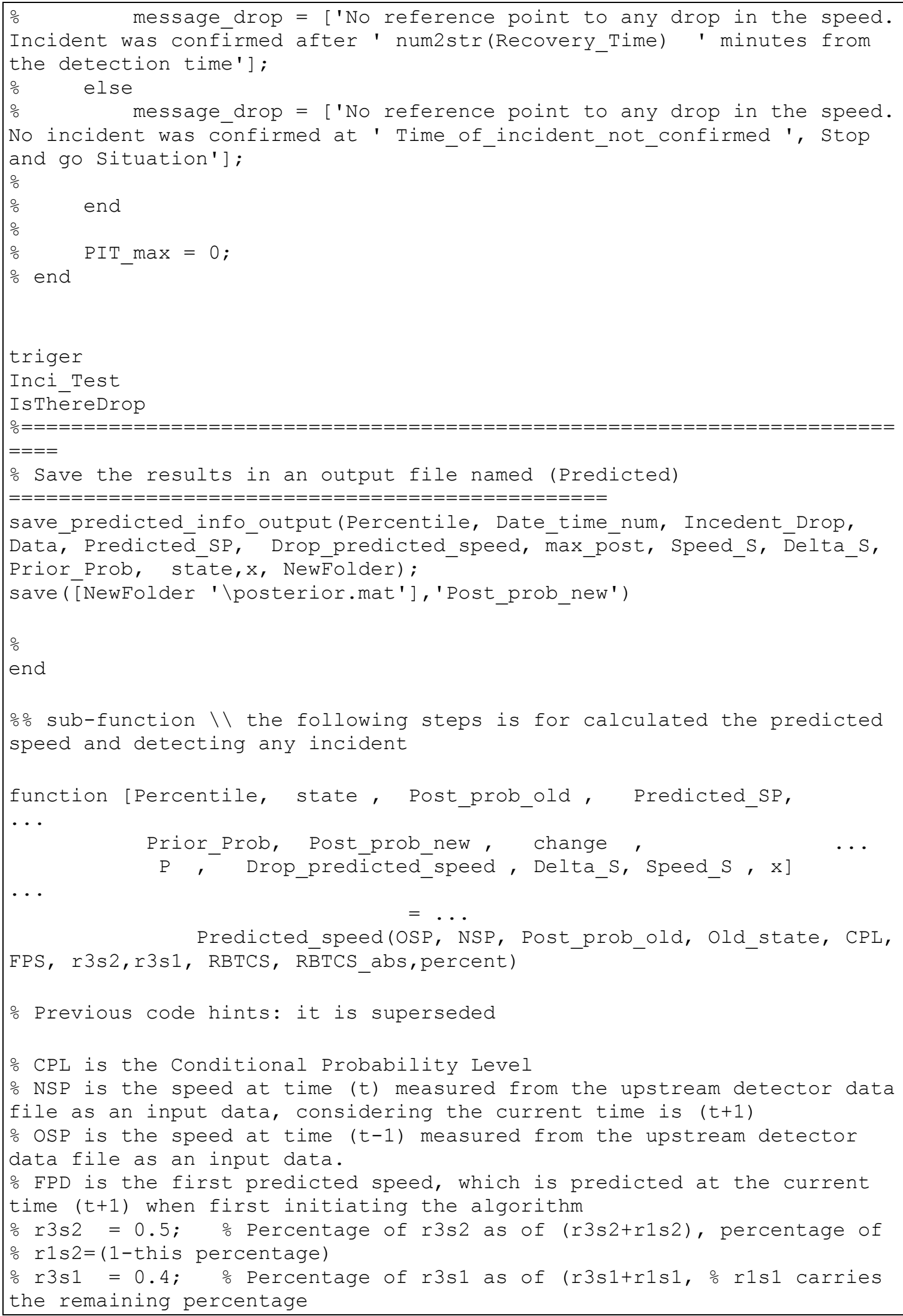




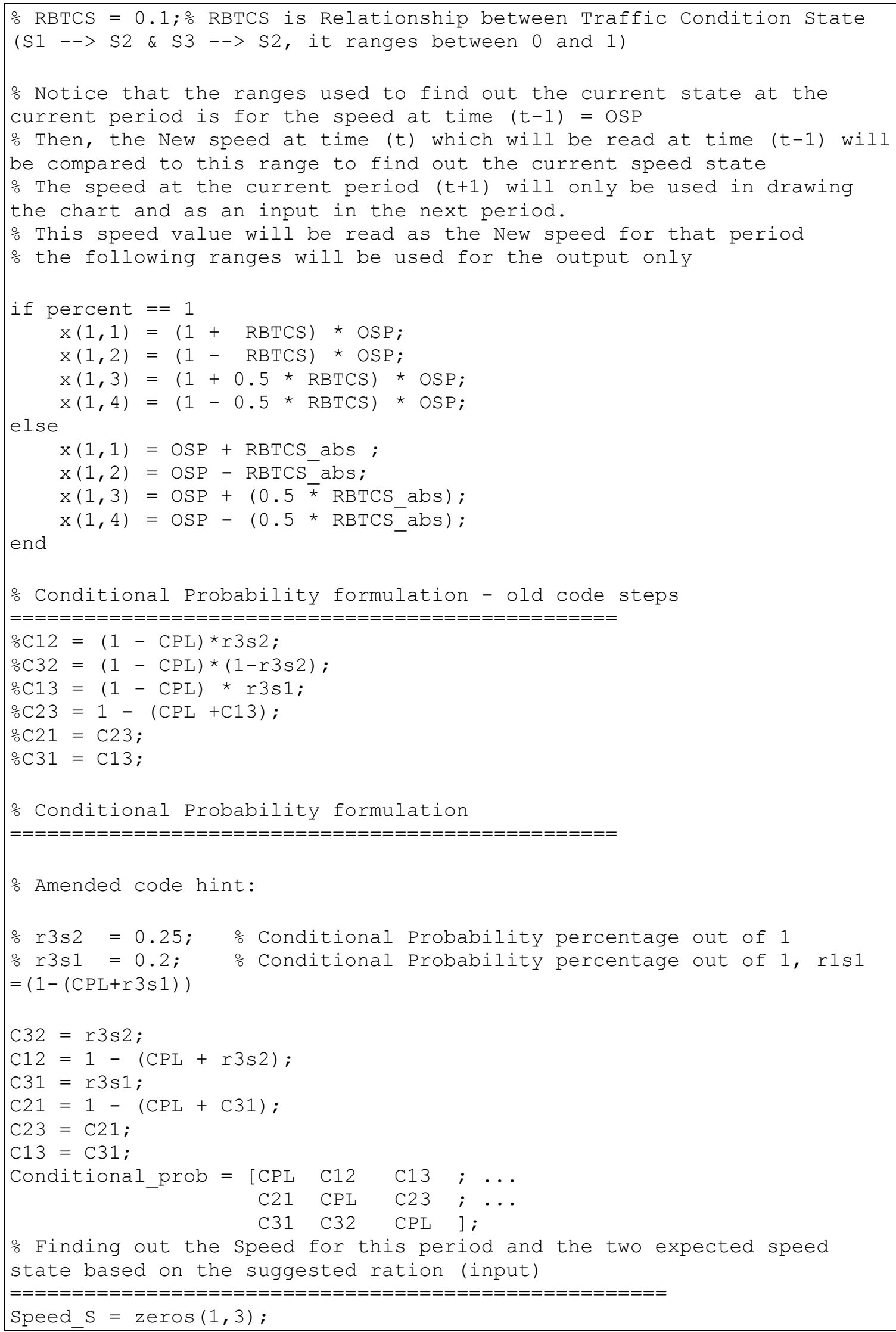




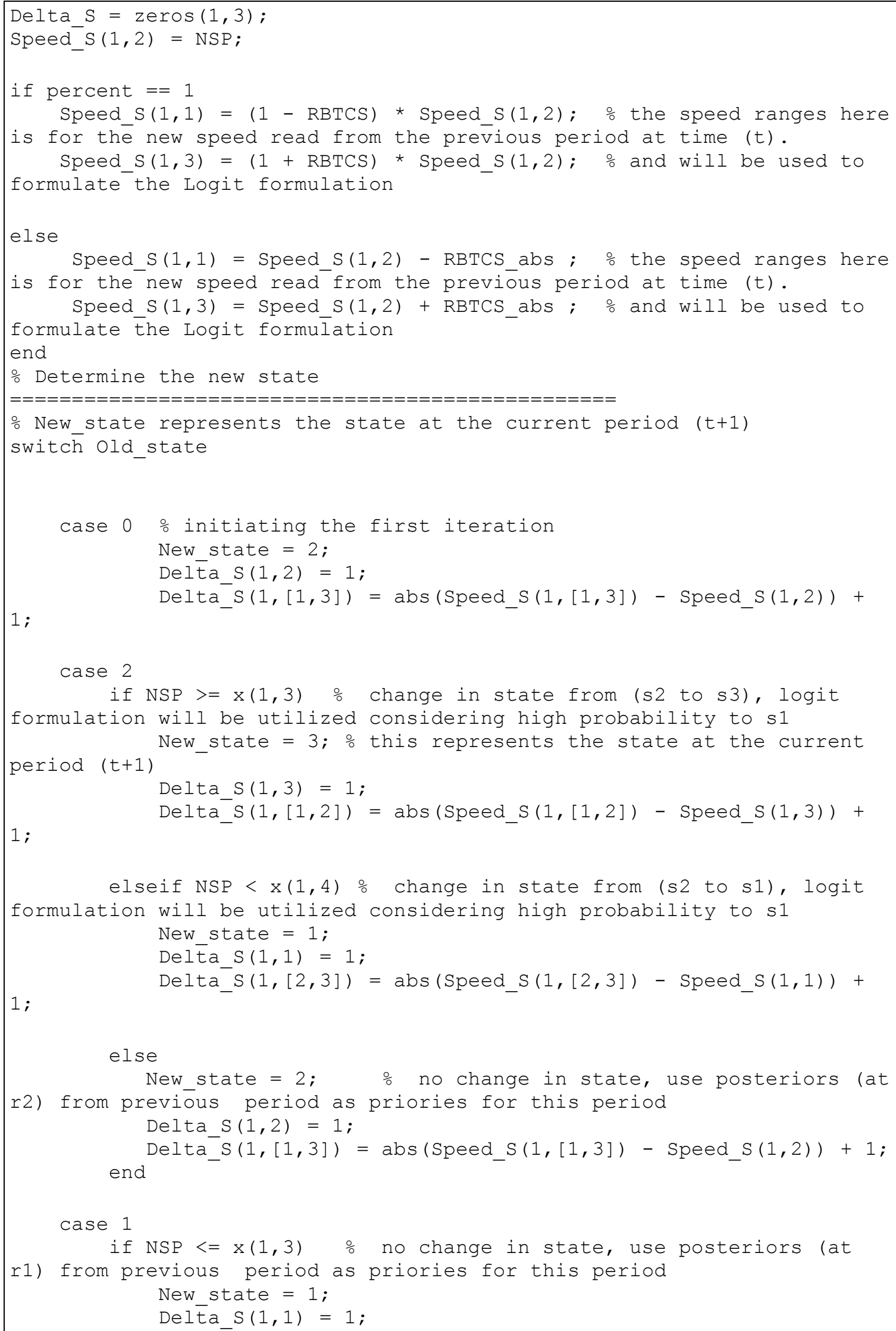




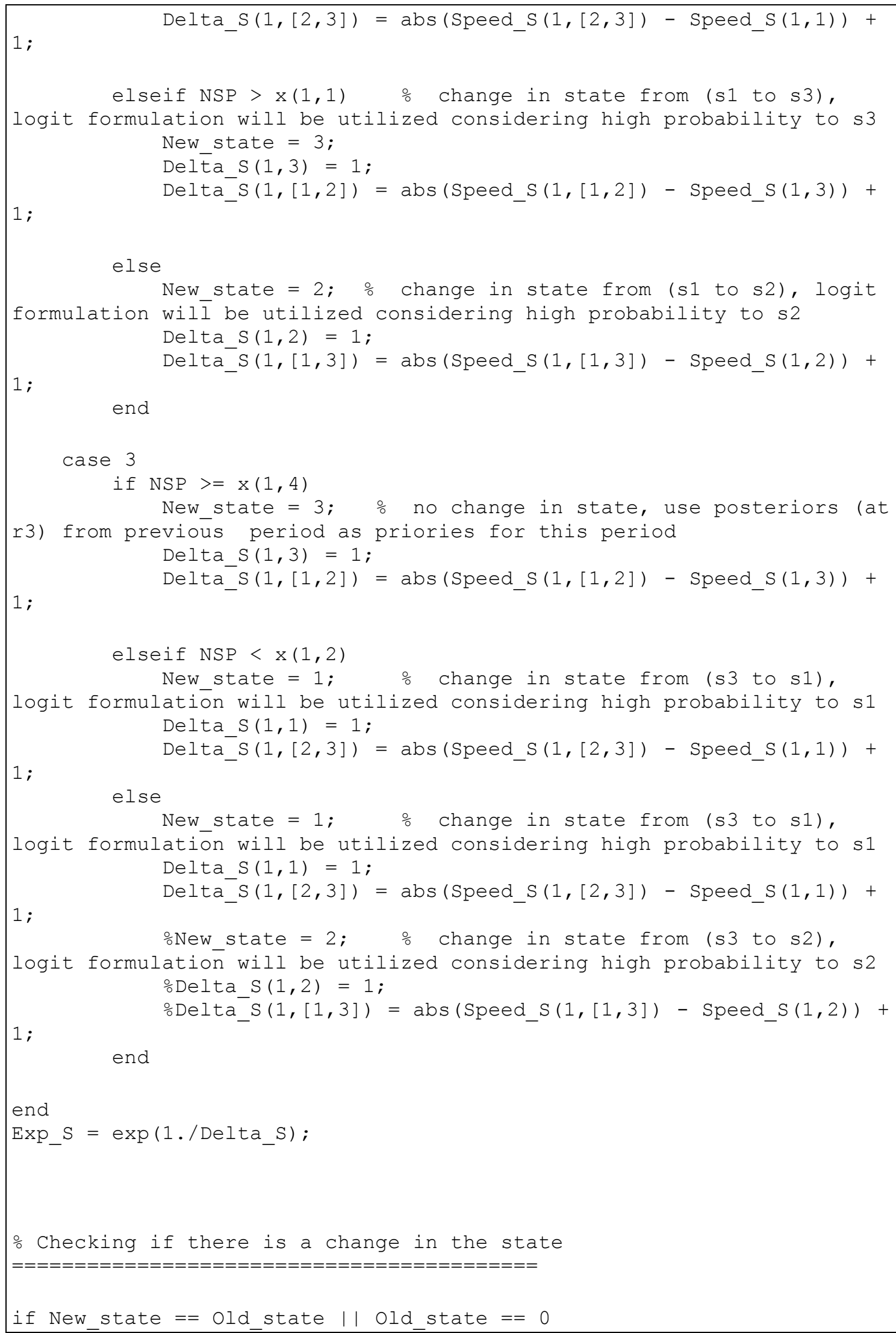




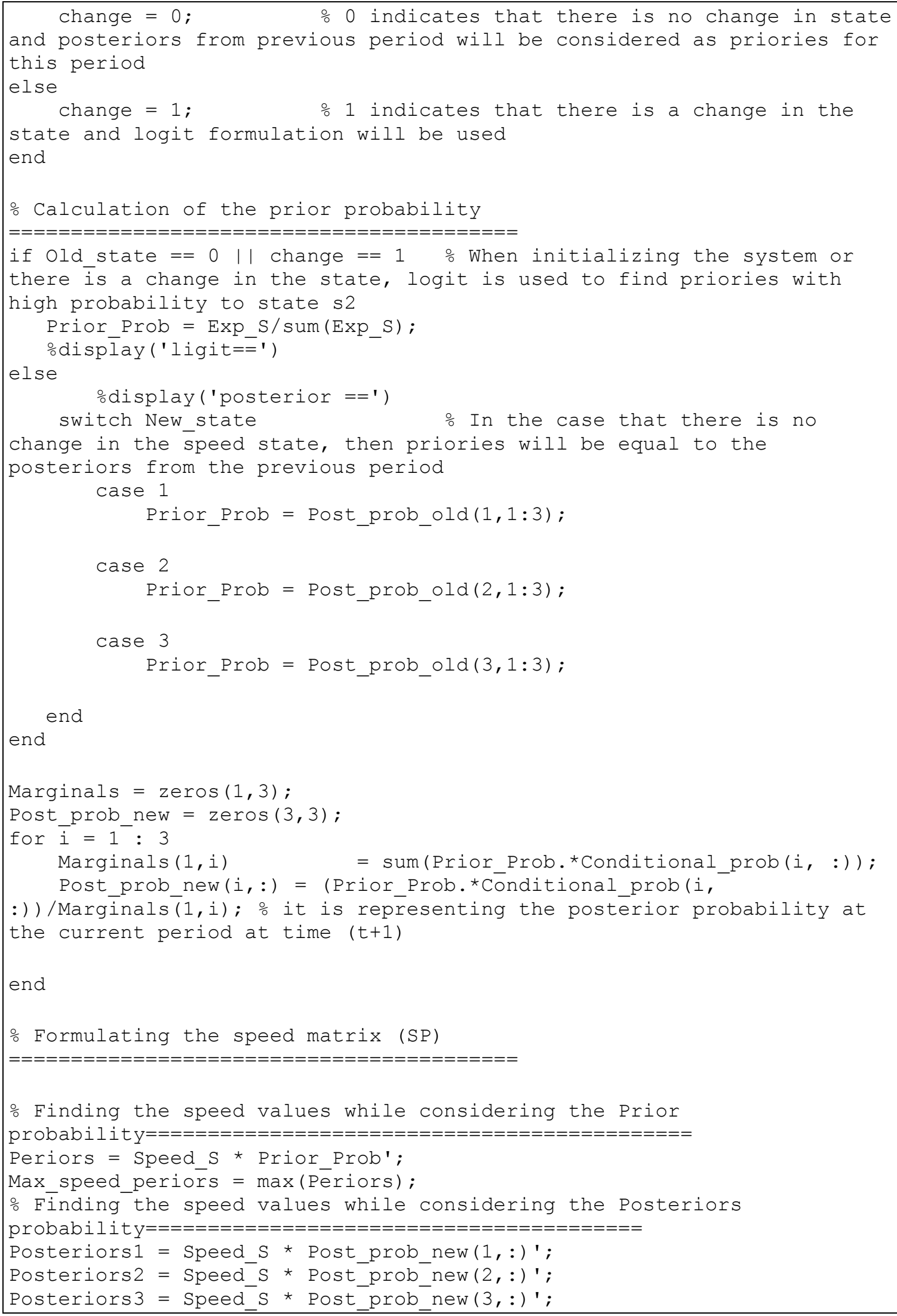




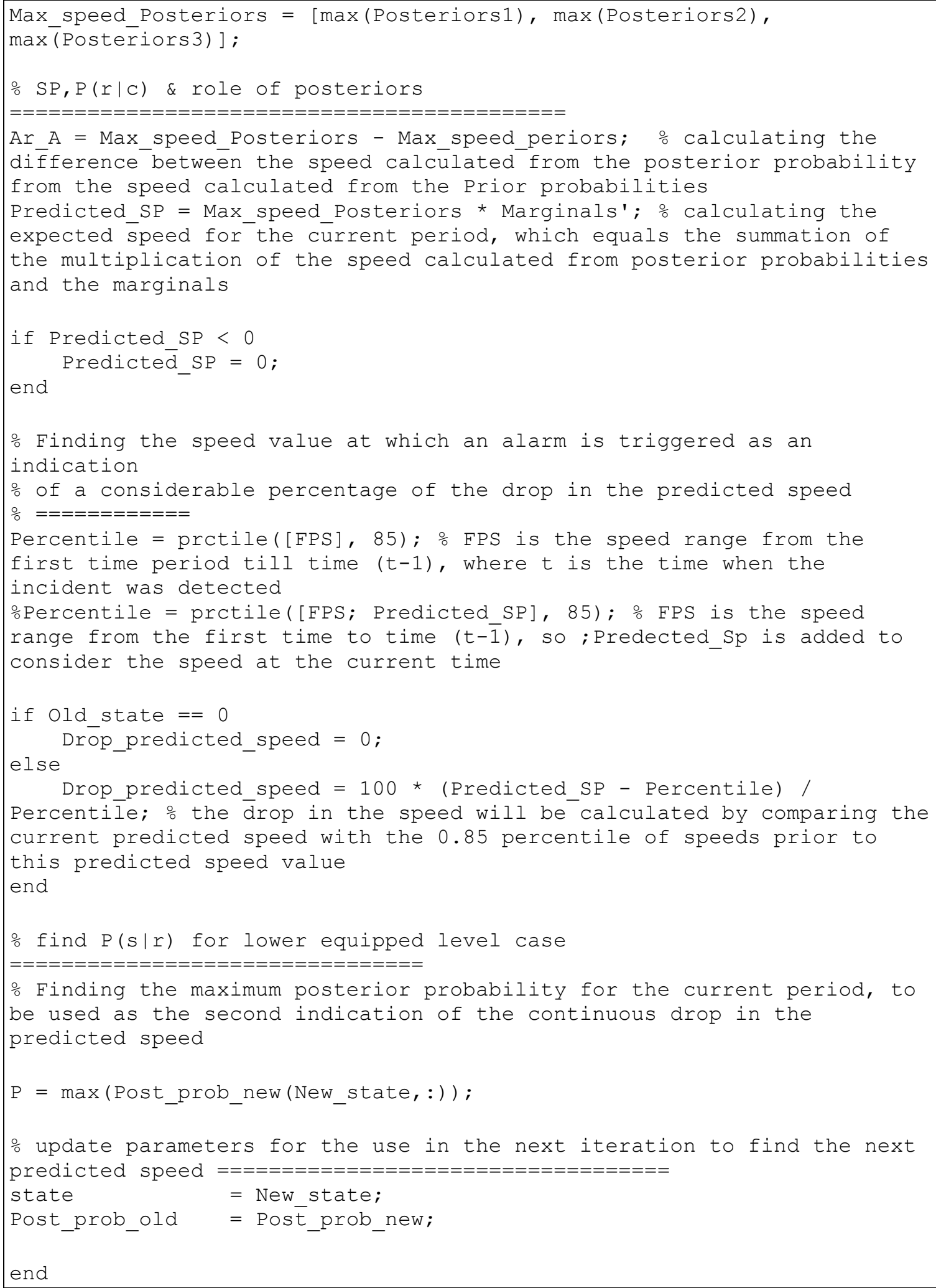




\section{Appendix B.7 Incident Confirmation Procedure Function}

This Appendix presents the Matlab source codes for the developed model. It includes the source code for incident confirmation, which is used to apply the criteria defined in the third condition within the incident confirmation process.

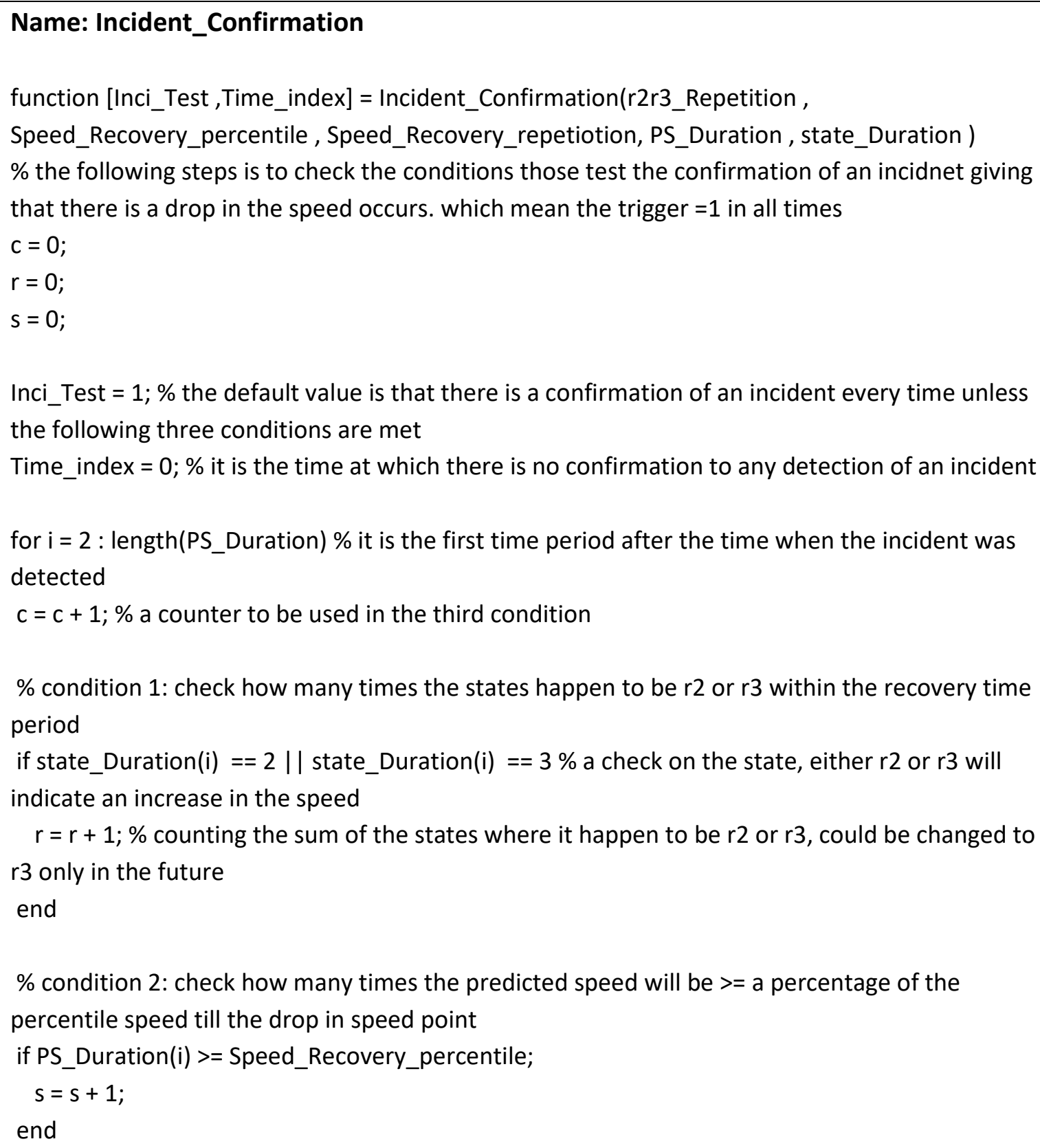




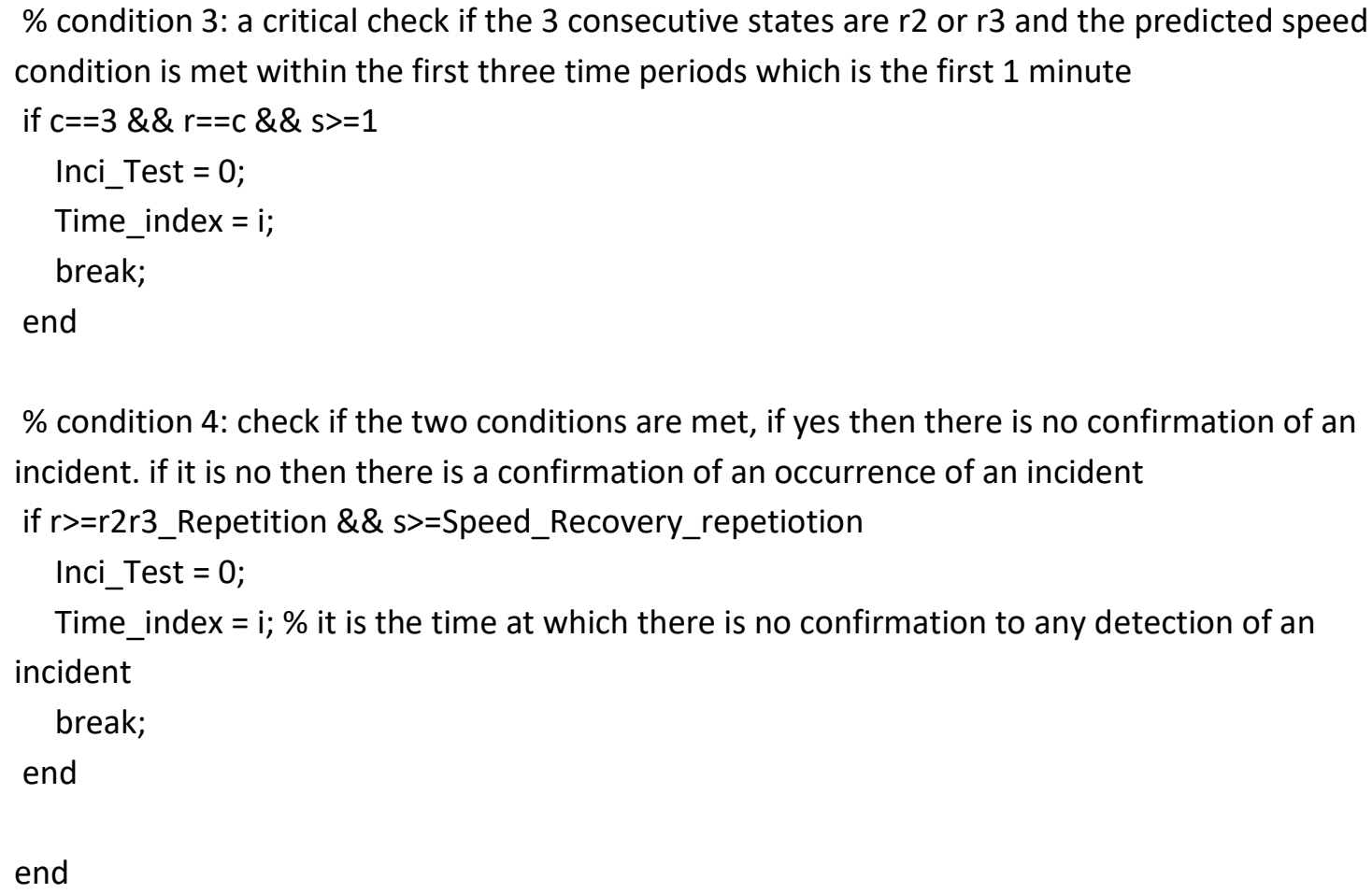




\section{Appendix B.8 Plotting the Results Function}

This Appendix presents the Matlab source codes for the developed model. It includes the source code for plotting the results. It is used to plot the speed results, including both the detected speed and the measured speed through the detector, volume and occupancy are also plotted accordingly.

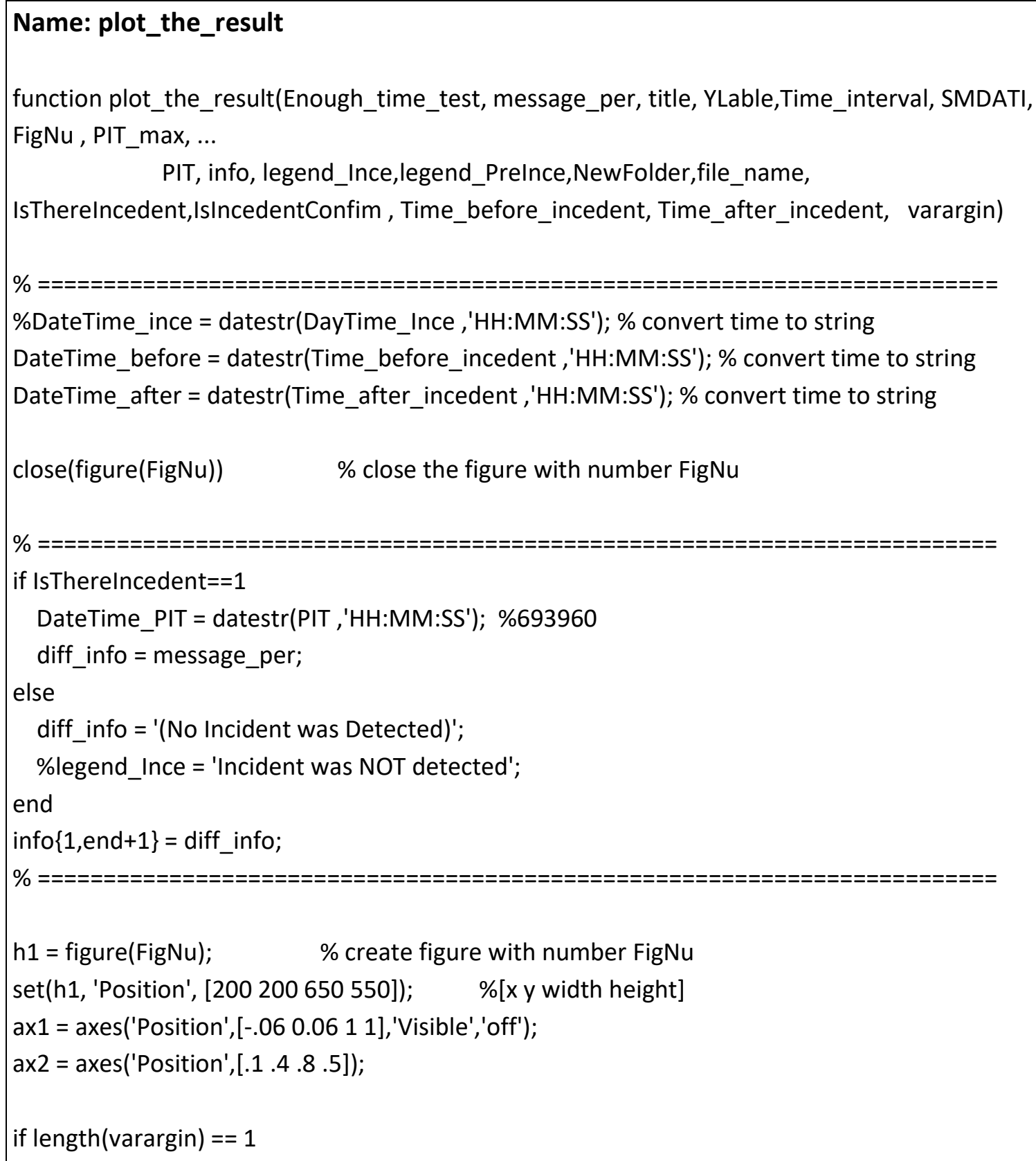




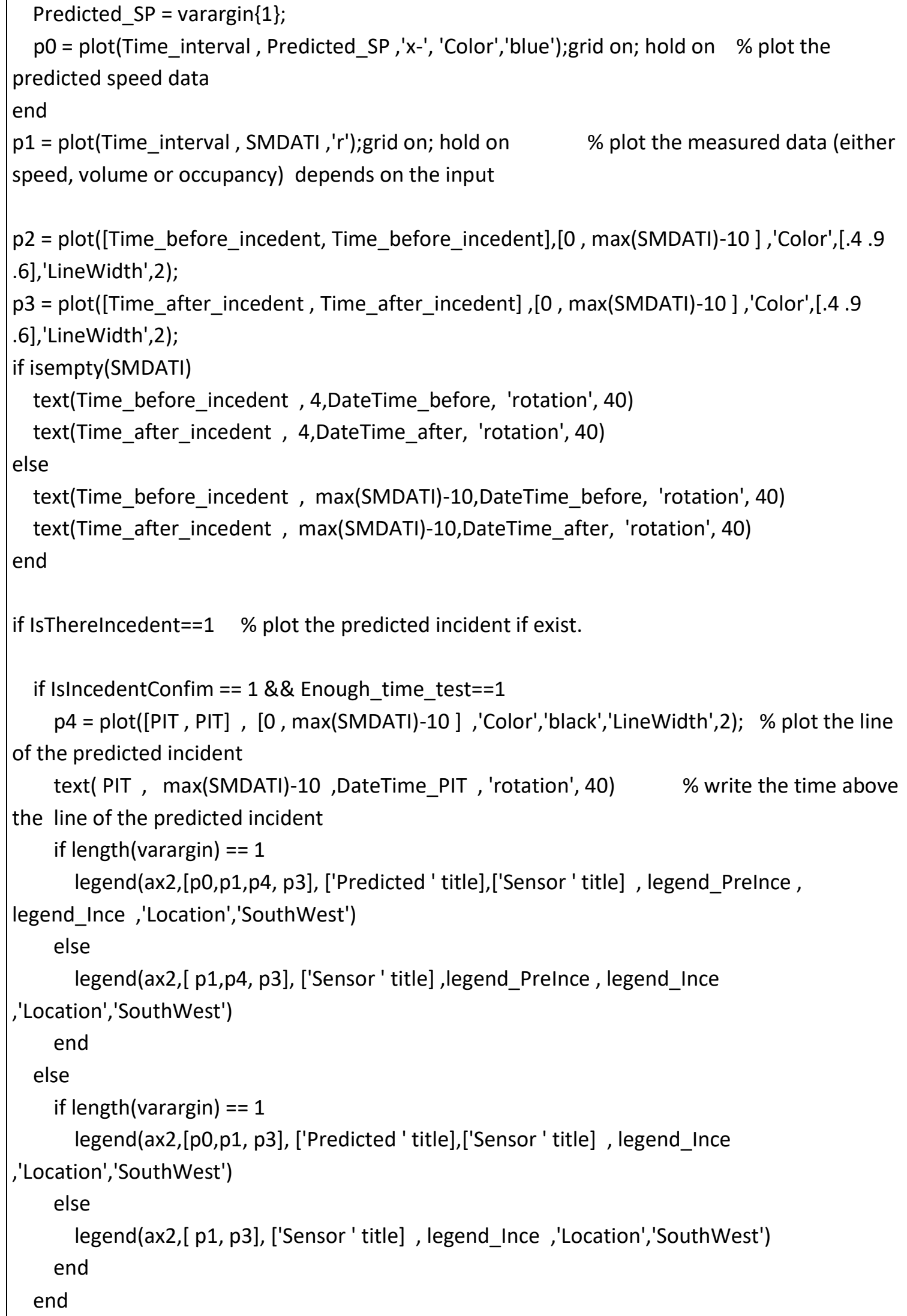




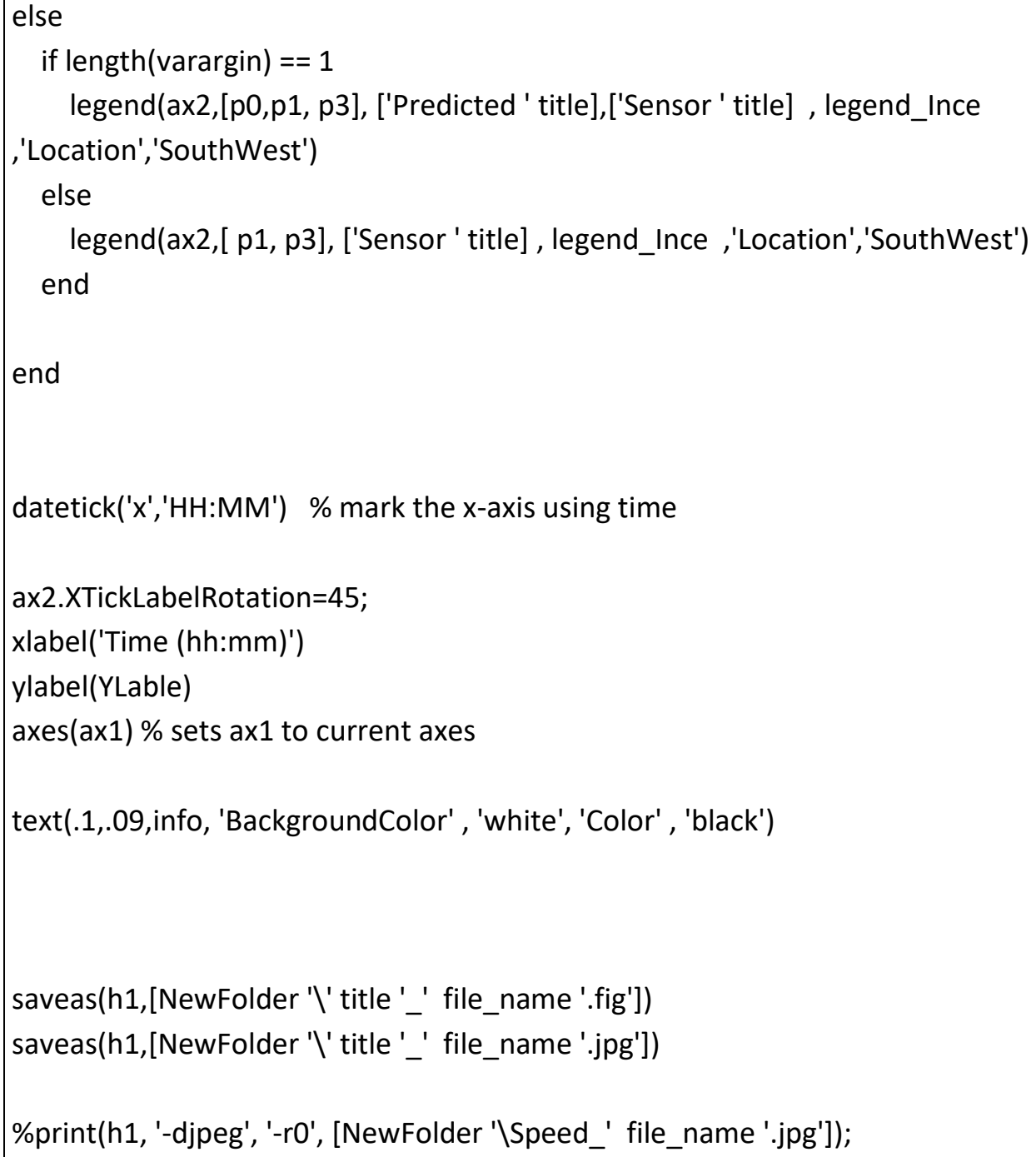




\section{Appendix B.9 Storing the Outputs Function}

This Appendix presents the Matlab source codes for the developed model. It includes the source code for storing the outputs, which is used to store speed data, time, volume, and occupancy.

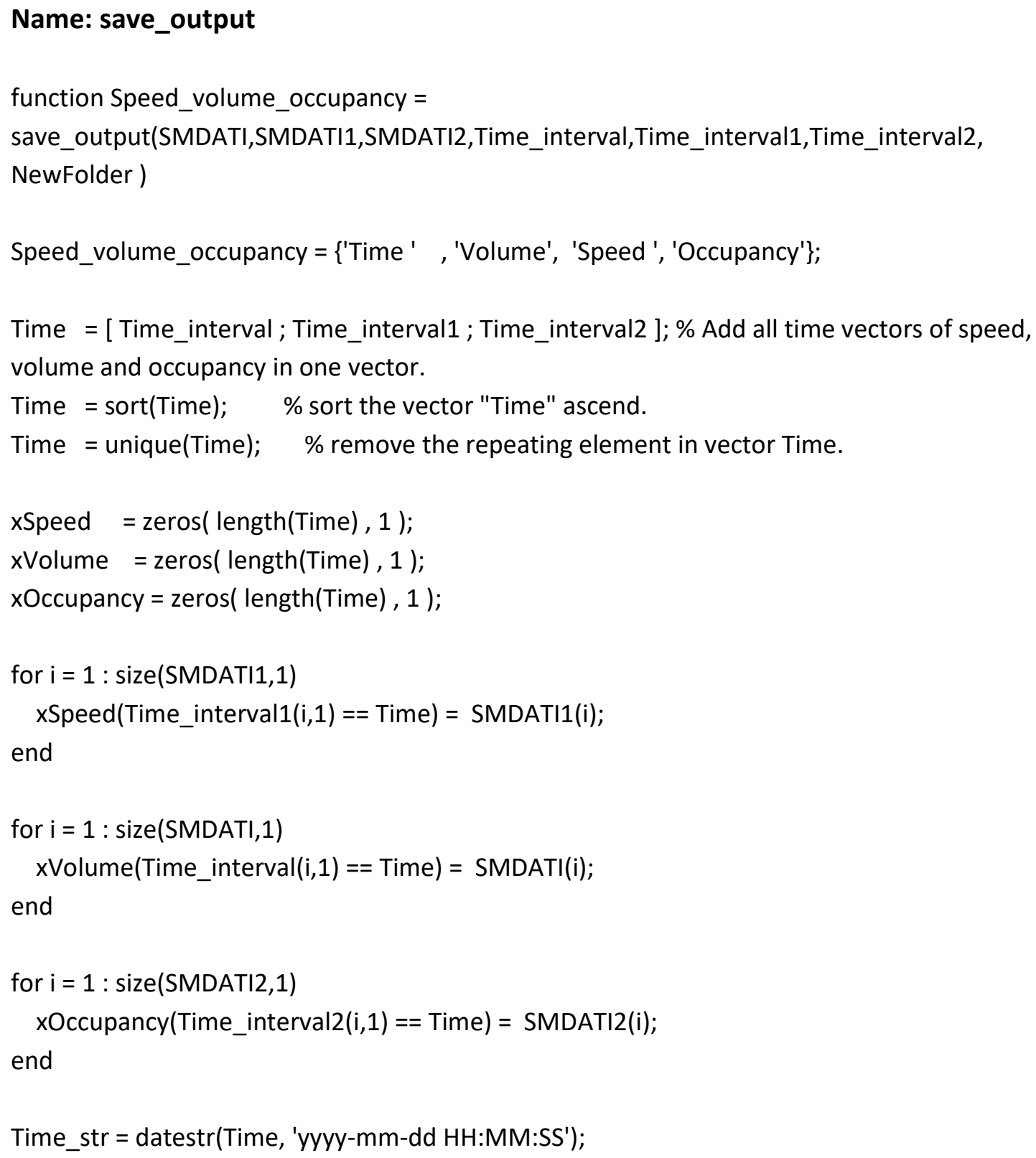




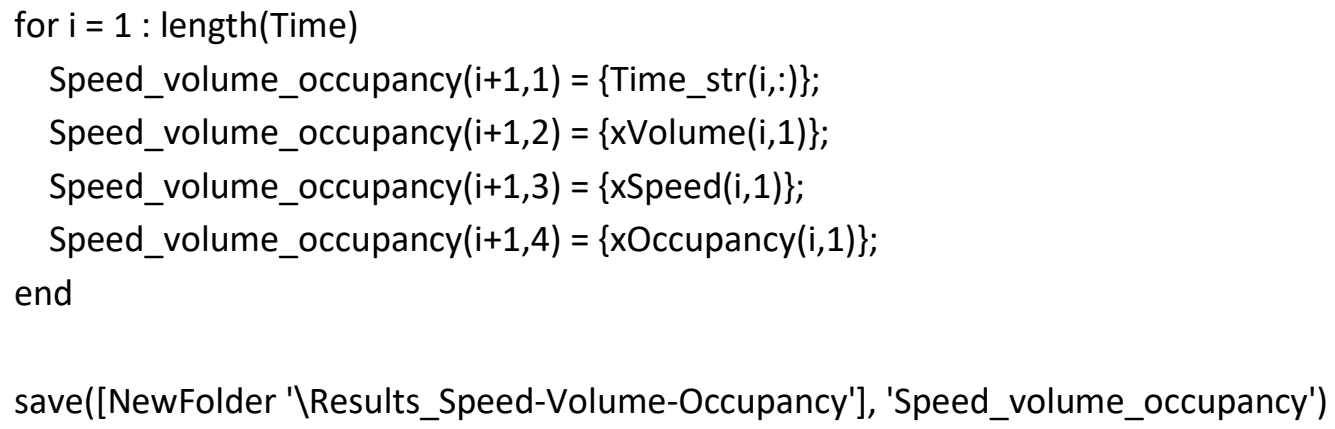




\section{Appendix B.10 Storing the Incident Detection Results Function}

This Appendix presents the Matlab source codes for the developed model. It includes the source code for storing the results, which is used to store all the results pertain to the incident detection procedure.

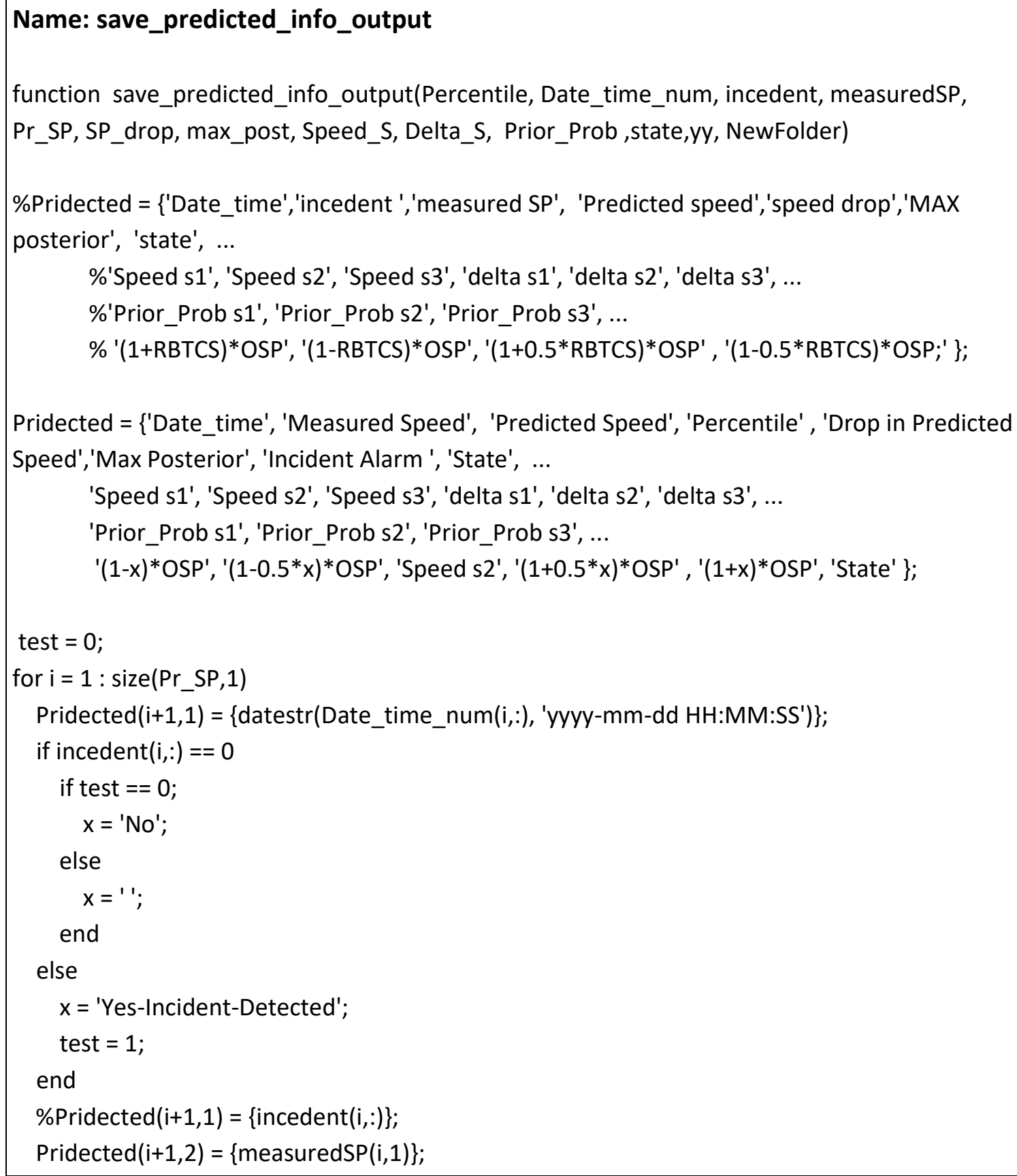




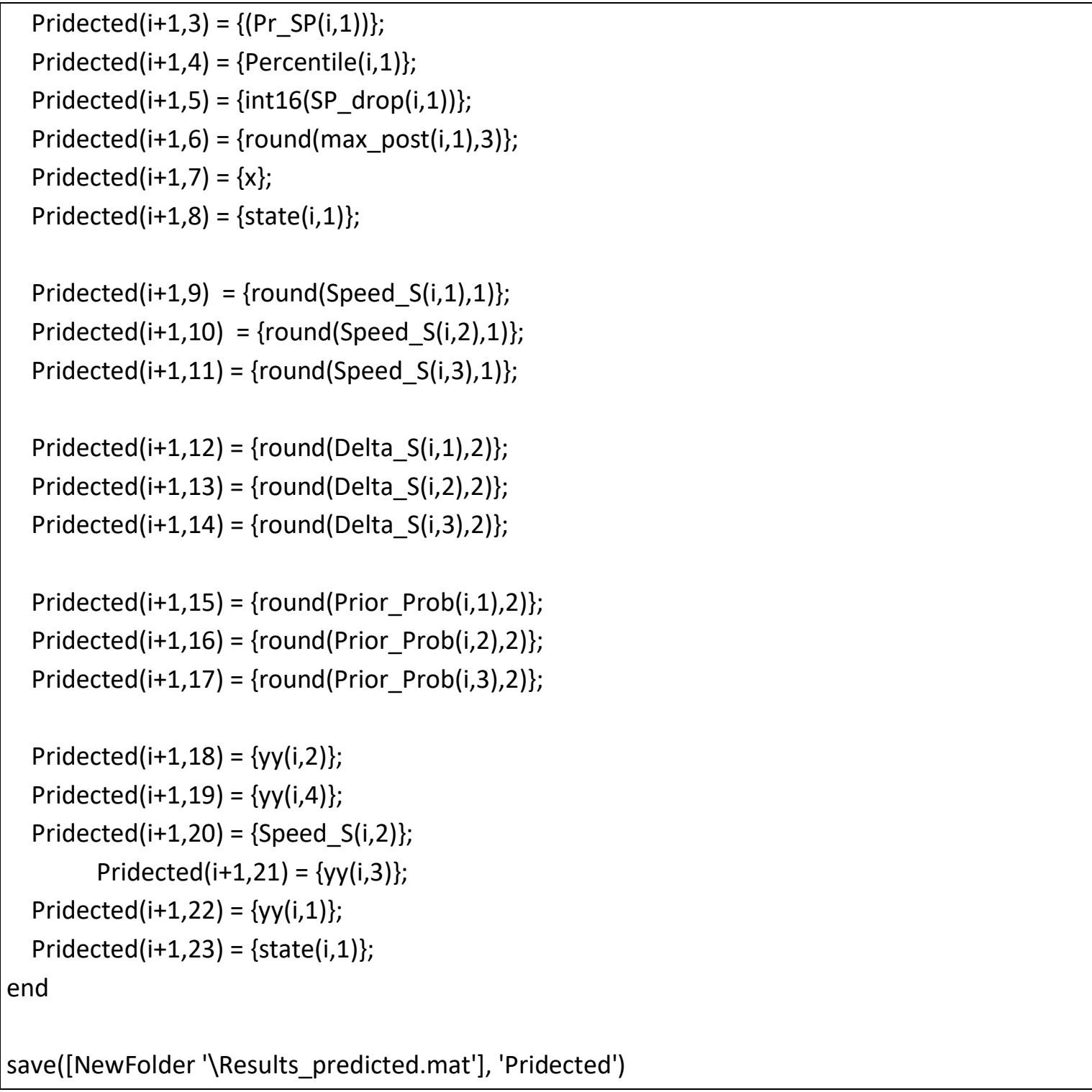




\section{Appendix C Model Validation}

This appendix presents the validation outcomes that resulted from the model application on 38 cases as described in chapter 6. Cases presented in this part are under certain traffic condition "Must Detect," by holding the parameters resulted from the calibration process. The model was applied to cases that are well known to have incident recorded.

\section{Appendix C.1 Cases with Incident Recorded and Detected}

\section{Appendix C.1.1 Cases under Certain Traffic Condition "Must Detect"} -- 38 cases

\section{Appedix C.1.1.1 Cases with Incident Recorded and Detected -- 32 cases}

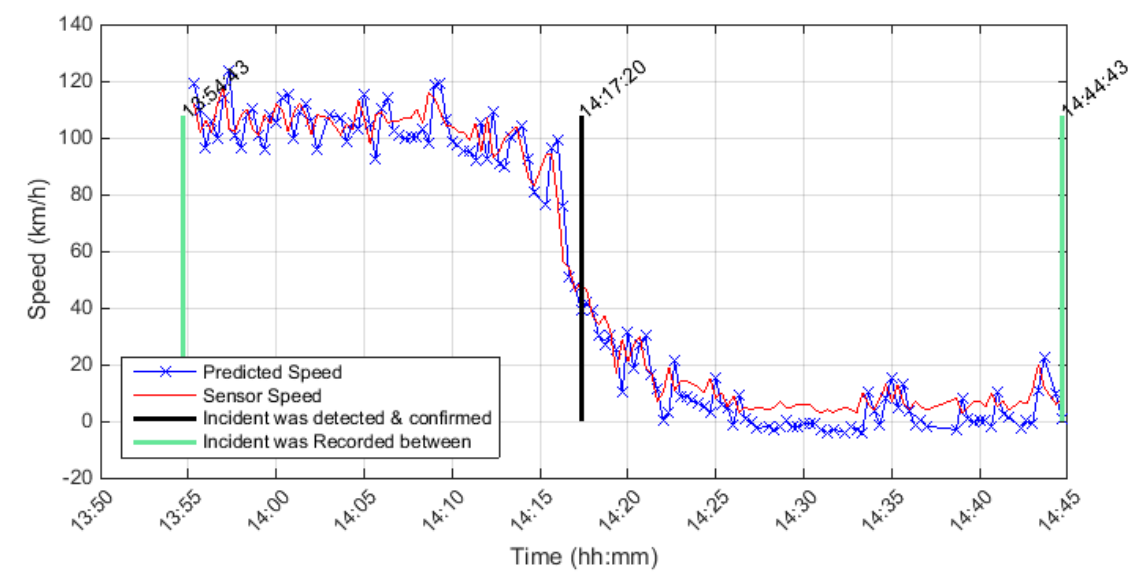

Upstream Detector \# is 401DE0320DEC, Detector Rank = 1, Distance from incident location in $(\mathrm{km})=0.23461$

Season $=$ Winter, Stream $=$ C, Direction $=\mathrm{E}$, Date $\&$ Time $=2011-12-2314: 14: 43$

Status = UPDATED 2011-12-23 14:34:25, Incident Reason = Collision

Affected Lanes $=3$ LEFT LANE $(S)$

Conditional Probability level $=0.6$, Cond.Prob. $r 3 \mathrm{~s} 2=0.2$, Cond.Prob. $r 3 \mathrm{~s} 1=0.1$, Percent drop in speed $\%=-60$

Max. Posterior Probability level $=0.97$, Absolute range between speed states $=7 \mathrm{~km}$

Incident was detected at 01:20 minutes from drop in speed point. Incident was confirmed after 5 minutes from the detection time 


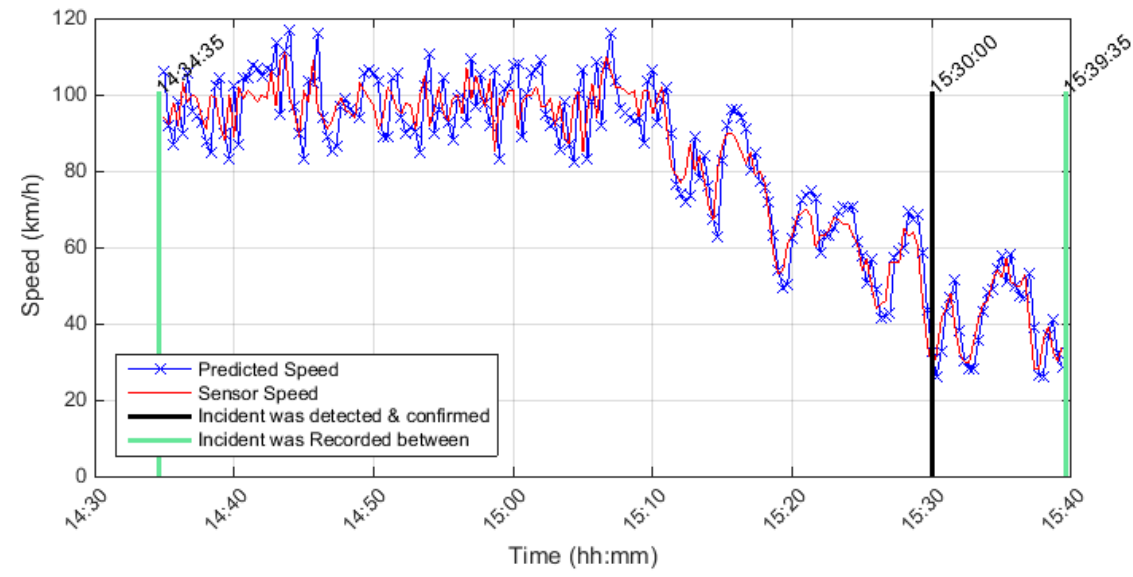

Upstream Detector \# is 401DW0100DEE, Detector Rank = 1, Distance from incident location in $(\mathrm{km})=0.059954$ Season $=$ Winter, Stream $=E$, Direction $=E$, Date $\&$ Time $=2011-02-0314: 54: 35$ Incident Reason $=$ Disable Vehicle

Affected Lanes = 1 RIGHT LANE(S)

Conditional Probability level $=0.6$, Cond.Prob. $r 3 \mathrm{~s} 2=0.2$, Cond. Prob. $r 3 \mathrm{~s} 1=0.1$, Percent drop in speed $\%=-60$

Max. Posterior Probability level $=0.97$, Percent range between speed states $=7 \%$

Incident was detected at 12:40 minutes from drop in speed point. Incident was confirmed after 5 minutes from the detection time

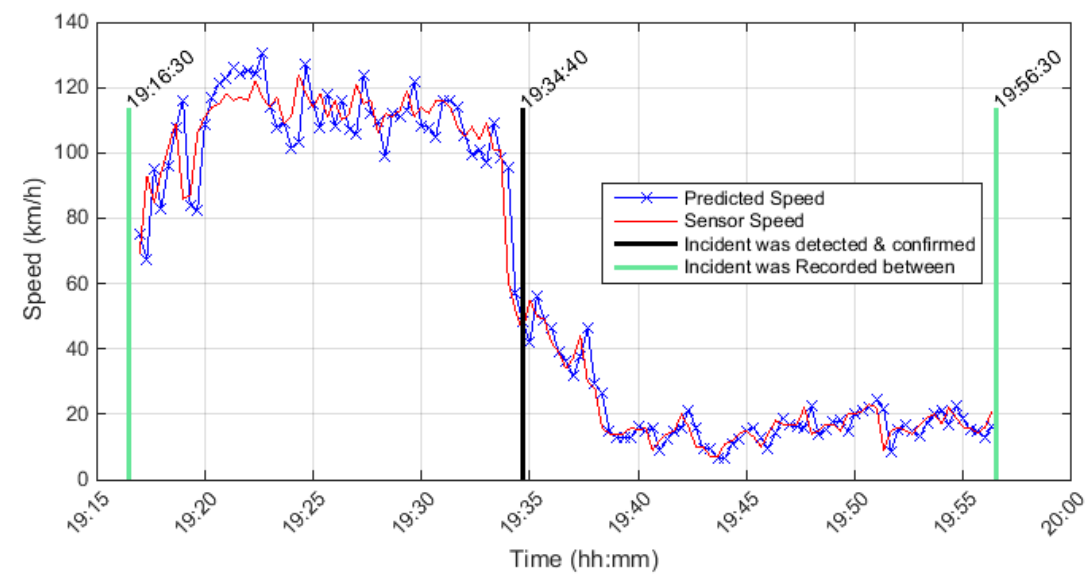

Upstream Detector \# is 401DE0590DEE, Detector Rank = 1, Distance from incident location in $(\mathrm{km})=0.15896$ Season $=$ Summer, Stream $=E$, Direction $=E$, Date \& Time $=2011-10-2019: 36: 30$

Incident Reason $=$ Collision

Affected Lanes = 1 RIGHT LANE(S) AND RIGHT SHOULDER

Conditional Probability level $=0.6$, Cond.Prob. $r 3 \mathrm{~s} 2=0.2$, Cond.Prob. $r 3 \mathrm{~s} 1=0.1$, Percent drop in speed $\%=-56$

Max. Posterior Probability level $=0.97$, Percent range between speed states $=7 \%$

Incident was detected at 01:20 minutes from drop in speed point. Incident was confirmed after 5 minutes from the detection time 


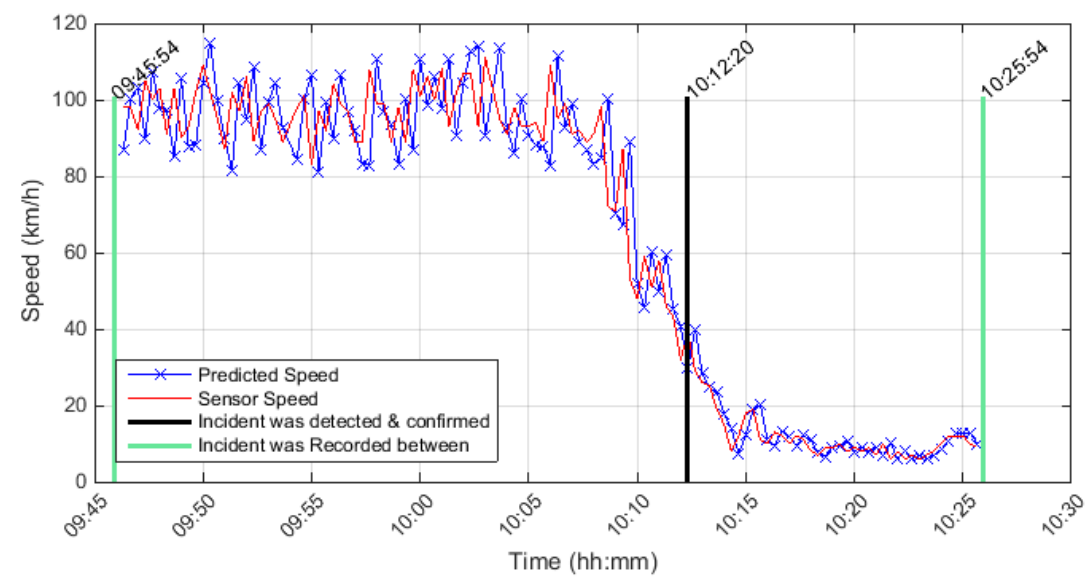

Upstream Detector \# is 401DW0270DEE, Detector Rank = 1, Distance from incident location in $(\mathrm{km})=0.24432$ Season $=$ Winter, Stream $=E$, Direction $=E$, Date $\&$ Time $=2011-12-30$ 10:05:54 Incident Reason $=$ Collision

Affected Lanes $=3$ CENTRE LANE(S)

Conditional Probability level $=0.6$, Cond. Prob. $r 3 s 2=0.2$, Cond. Prob. $r 3 s 1=0.1$, Percent drop in speed $\%=-56$

Max. Posterior Probability level $=0.97$, Percent range between speed states $=7 \%$

Incident was detected at 02:40 minutes from drop in speed point. Incident was confirmed after 5 minutes from the detection time

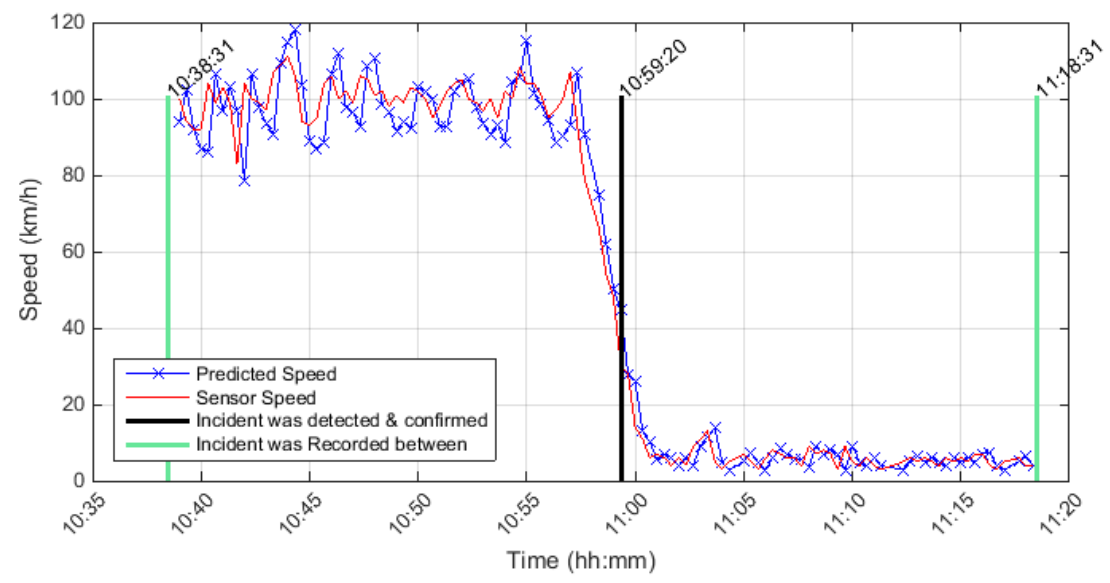

Upstream Detector \# is 401DE0290DWC, Detector Rank = 1, Distance from incident location in $(\mathrm{km})=0.43401$ Season $=$ Spring, Stream $=$ C, Direction $=W$, Date $\&$ Time $=2011-03-0710: 58: 31$ Incident Reaso $=$ Collision

Affected Lanes $=$ All lanes closed

Conditional Probability level $=0.6$, Cond.Prob. $r 3 s 2=0.2$, Cond.Prob. $r 3 s 1=0.1$, Percent drop in speed $\%=-56$ Max. Posterior Probability level $=0.97$, Percent range between speed states $=7 \%$

Incident was detected at 02:00 minutes from drop in speed point. Incident was confirmed after 5 minutes from the detection time 


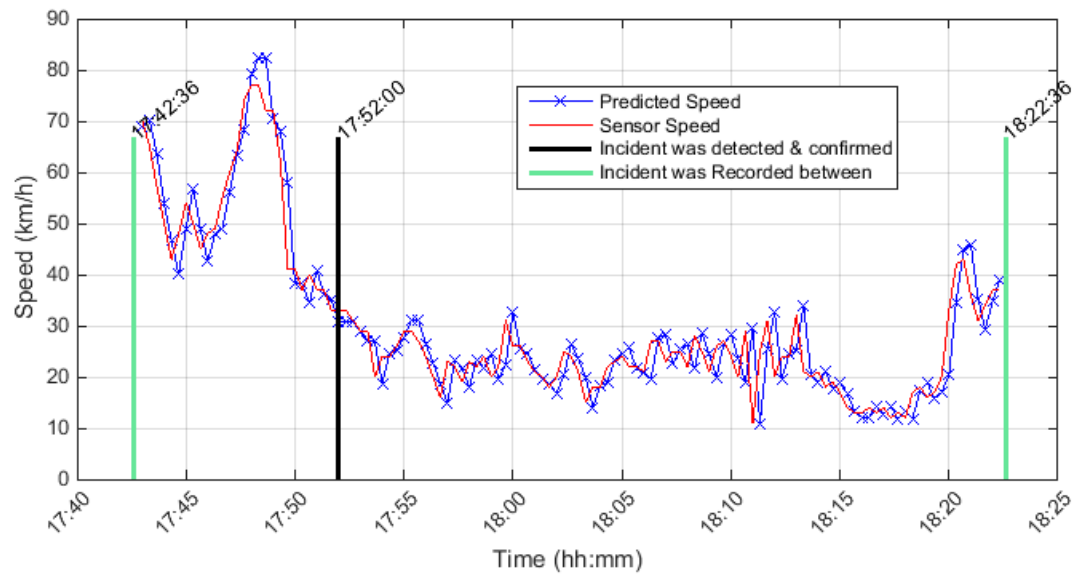

Upstream Detector \# is 401DW0060DWC, Detector Rank = 1, Distance from incident location in $(\mathrm{km})=0.43697$ Season $=$ Spring, Stream $=$ C, Direction $=W$, Date $\&$ Time $=$ 2011-03-19 18:02:36 Incident Reason $=$ Collision

Affected Lanes $=2$ LEFT LANE(S)

Conditional Probability level $=0.6$, Cond. Prob. $r 3 s 2=0.2$, Cond. Prob. $r 3 s 1=0.1$, Percent drop in speed $\%=-56$

Max. Posterior Probability level $=0.97$, Percent range between speed states $=7 \%$

Incident was detected at 03:20 minutes from drop in speed point. Incident was confirmed after 5 minutes from the detection time

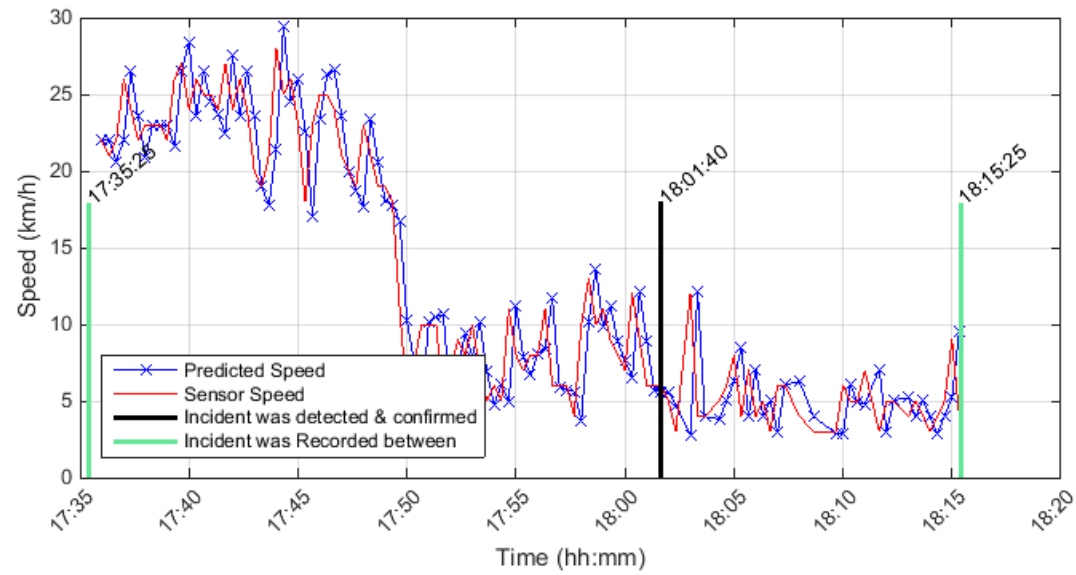

Upstream Detector \# is 401DE0150DWC, Detector Rank $=3$, Distance from incident location in $(\mathrm{km})=1.5503$ Season $=$ Spring, Stream $=$ C, Direction $=W$, Date $\&$ Time $=2011-05-1417: 55: 25$ Incident Reason $=$ Collision

Affected Lanes $=2$ LEFT LANE(S)

Conditional Probability level $=0.6$, Cond. Prob. $r 3 s 2=0.2$, Cond. Prob. $r 3 s 1=0.1$, Percent drop in speed $\%=-56$

Max. Posterior Probability level $=0.97$, Percent range between speed states $=7 \%$

Incident was detected at 13:20 minutes from drop in speed point. Incident was confirmed after 5 minutes from the detection time 


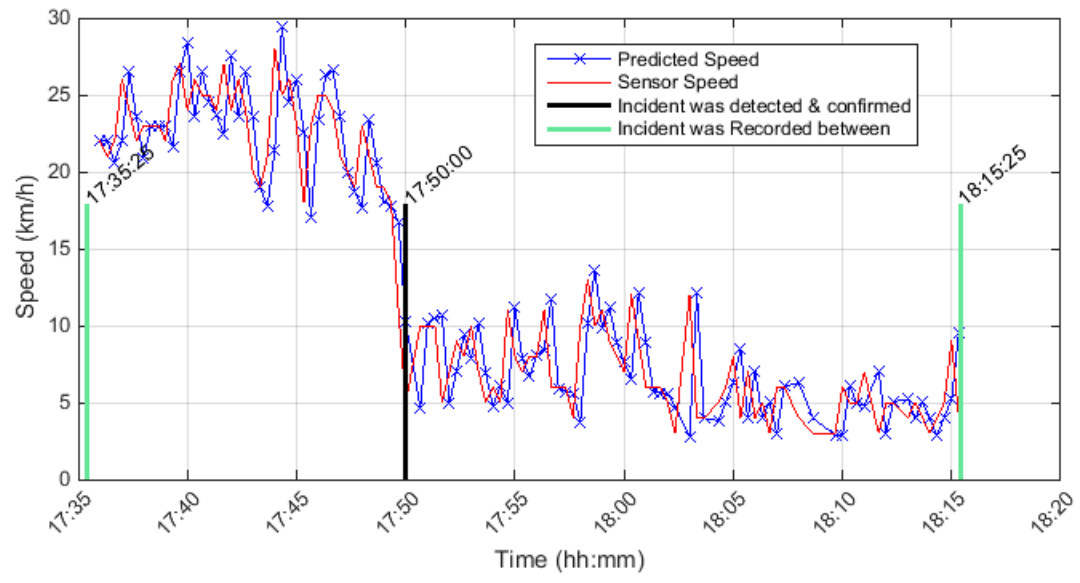

Upstream Detector \# is 401DE0150DWC, Detector Rank = 3, Distance from incident location in $(\mathrm{km})=1.5503$ Season $=$ Spring, Stream $=$ C, Direction $=$ W, Date $\&$ Time $=$ 2011-05-14 17:55:25

Incident Reason $=$ Collision

Affected Lanes $=2$ LEFT LANE $(\mathrm{S})$

Conditional Probability level $=0.6$, Cond.Prob. $r 3 s 2=0.2$, Cond. Prob. $r 3 s 1=0.1$, Percent drop in speed $\%=-56$

Max. Posterior Probability level $=0.97$, Percent range between speed states $=7 \%$

Incident was detected at 01:40 minutes from drop in speed point. Incident was confirmed after 5 minutes from the detection time

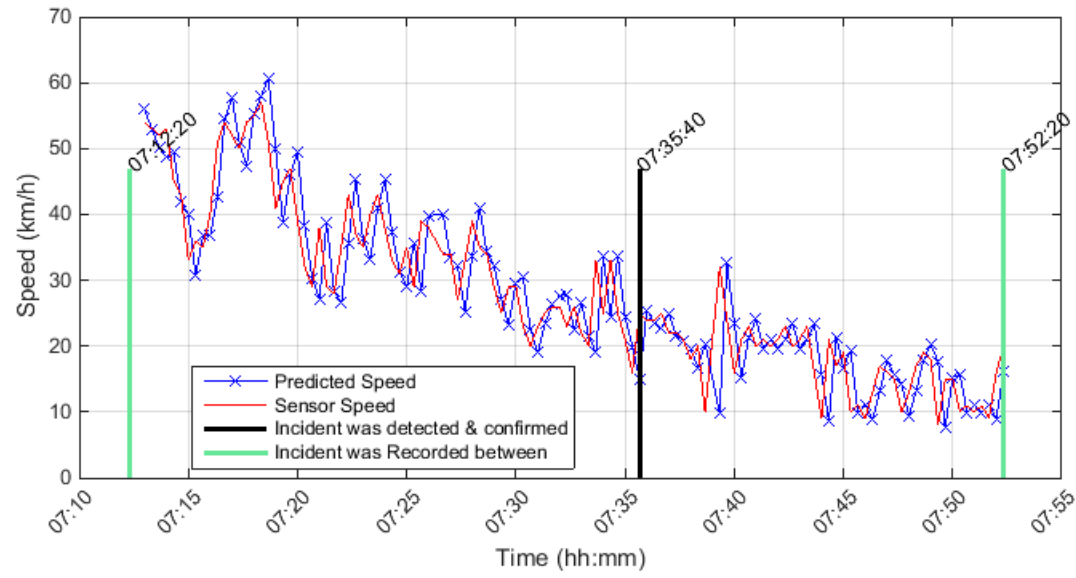

Upstream Detector \# is 401DE0380DWC, Detector Rank = 1, Distance from incident location in $(\mathrm{km})=0.71086$ Season $=$ Summer, Stream $=$ C, Direction $=W$, Date \& Time $=2011-06-3007: 32: 20$ Incident Reason $=$ Collision

Affected Lanes = 2 RIGHT LANE(S)

Conditional Probability level $=0.6$, Cond.Prob. $r 3 s 2=0.2$, Cond. Prob. $r 3 s 1=0.1$, Percent drop in speed $\%=-56$

Max. Posterior Probability level $=0.97$, Percent range between speed states $=7 \%$

Incident was detected at 07:20 minutes from drop in speed point. Incident was confirmed after 5 minutes from the detection time 


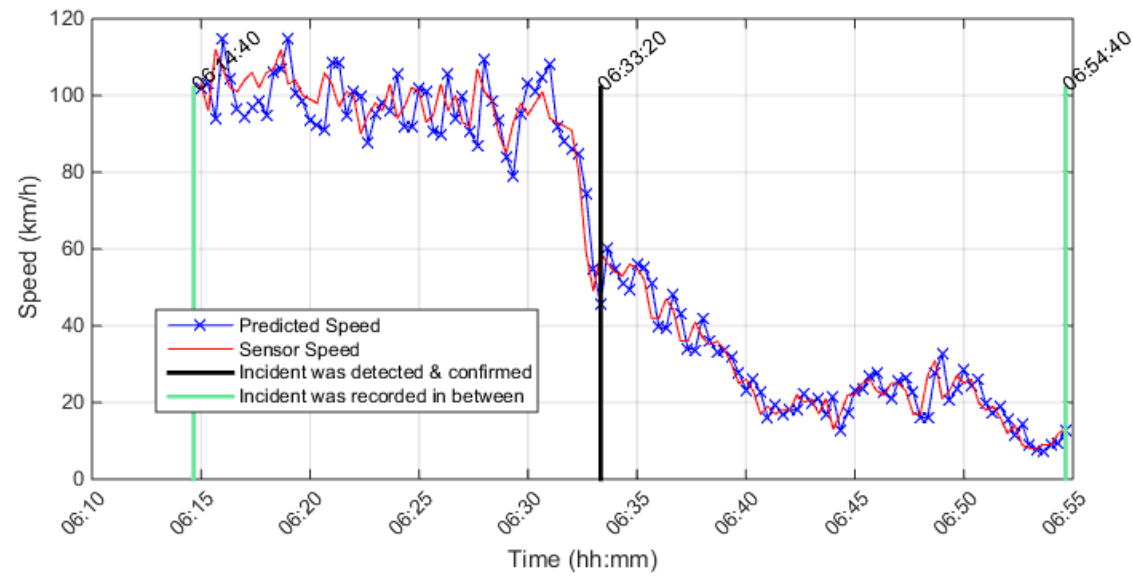

Upstream Detector \# is 401DW0020DWE, Detector Rank = 1, Distance from incident location in $(\mathrm{km})=0.13067$ Season $=$ Spring, Stream $=E$, Direction $=W$, Date $\&$ Time $=2011-03-16$ 06:34:40 Incident Reason $=$ Collision

Affected Lanes $=2$ RIGHT LANE(S)

Conditional Probability level $=0.6$, Cond.Prob. $r 3 s 2=0.2$, Cond.Prob. $r 3 s 1=0.1$, Percent drop in speed $\%=-56$

Max. Posterior Probability level $=0.97$, Percent range between speed states $=7 \%$

Incident was detected at 02:20 minutes from drop in speed point. Incident was confirmed within 5 minutes from the detection tim

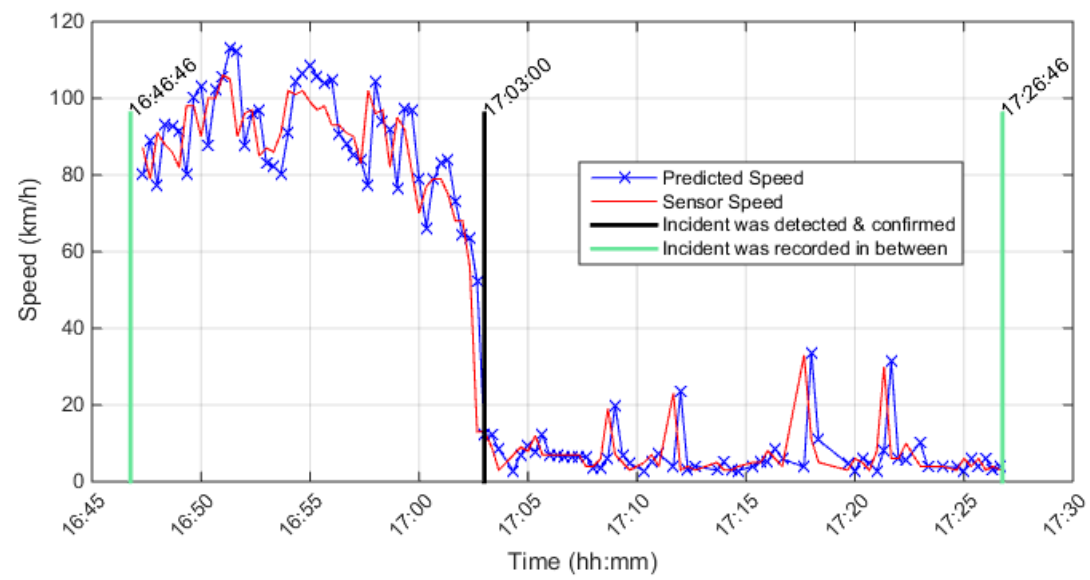

Upstream Detector \# is 401DE0160DEC, Detector Rank = 1, Distance from incident location in $(\mathrm{km})=0.2396$ Season $=$ Winter, Stream $=$ C, Direction $=E$, Date $\&$ Time $=2011-01-3117: 06: 46$

Incident Reason $=$ Collision

Affected Lanes $=2$ LEFT LANE $(\mathrm{S})$

Conditional Probability level $=0.6$, Cond.Prob. $r 3 s 2=0.2$, Cond.Prob. $r 3 s 1=0.1$, Percent drop in speed $\%=-56$

Max. Posterior Probability level $=0.97$, Percent range between speed states $=7 \%$

Incident was detected at 01:40 minutes from drop in speed point. Incident was confirmed within 5 minutes from the detection tim 


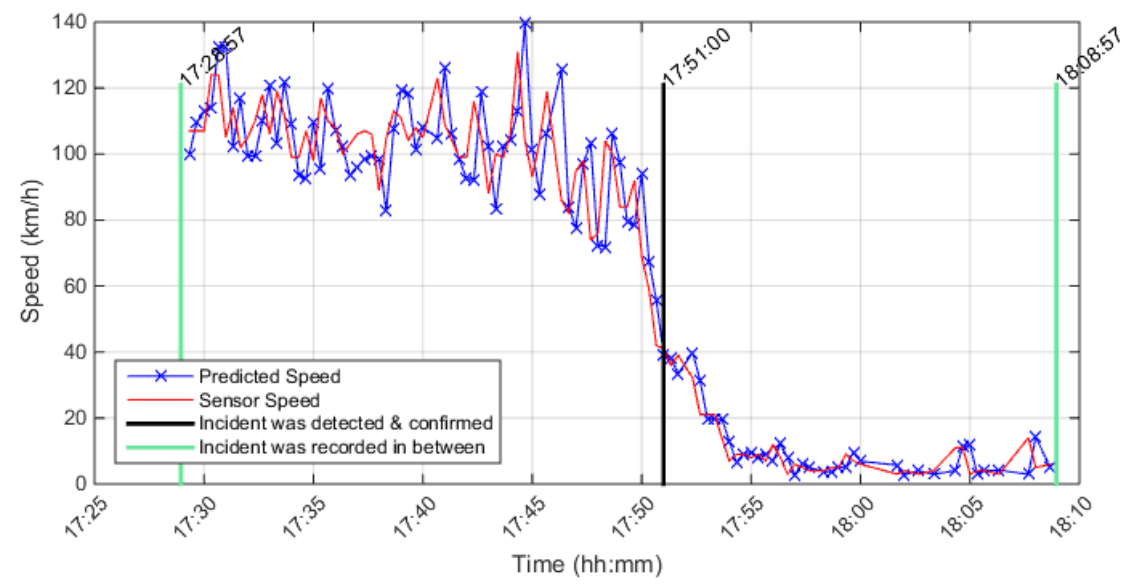

Upstream Detector \# is 401DW0180DEC, Detector Rank = 1, Distance from incident location in $(\mathrm{km})=0.25258$ Season $=$ Spring, Stream $=$ C, Direction $=E$, Date $\&$ Time $=2011-03-08$ 17:48:57 Incident Reason $=$ Collision

Affected Lanes $=3$ RIGHT LANE(S)

Conditional Probability level $=0.6$, Cond.Prob. $r 3 s 2=0.2$, Cond. Prob. $r 3 s 1=0.1$, Percent drop in speed $\%=-56$

Max. Posterior Probability level $=0.97$, Percent range between speed states $=7 \%$

Incident was detected at 01:00 minutes from drop in speed point. Incident was confirmed within 5 minutes from the detection timı

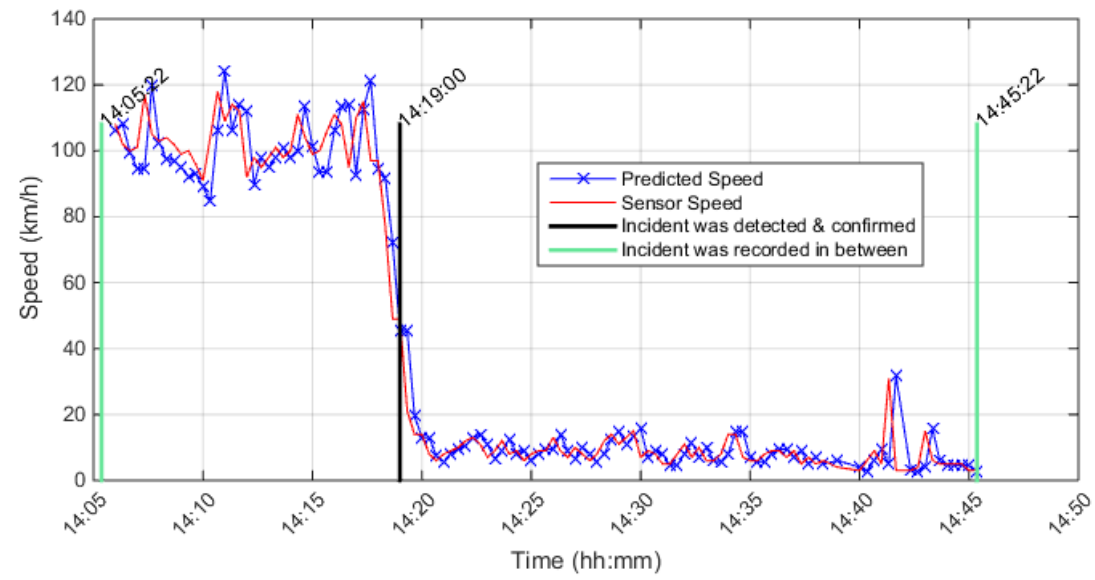

Upstream Detector \# is 401DW0160DEC, Detector Rank = 1, Distance from incident location in $(\mathrm{km})=0.33732$ Season $=$ Spring, Stream $=$ C, Direction $=$ E, Date $\&$ Time $=$ 2011-03-16 14:25:22 Incident Reason $=$ Collision

Affected Lanes $=$ LEFT SHOULDER AND 3 LEFT LANE(S)

Conditional Probability level $=0.6$, Cond.Prob. $r 3 s 2=0.2$, Cond. Prob. $r 3 s 1=0.1$, Percent drop in speed $\%=-56$

Max. Posterior Probability level $=0.97$, Percent range between speed states $=7 \%$

Incident was detected at 01:20 minutes from drop in speed point. Incident was confirmed within 5 minutes from the detection tim 


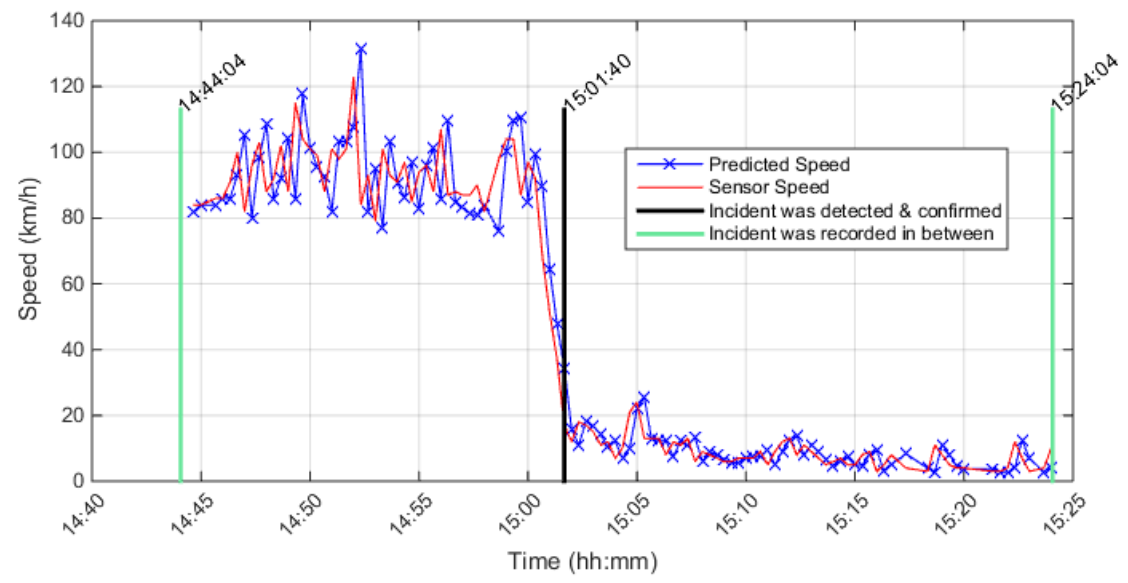

Upstream Detector \# is 401DE0330DEC, Detector Rank = 1, Distance from incident location in $(\mathrm{km})=0.68588$ Season $=$ Spring, Stream $=$ C, Direction $=E$, Date $\&$ Time $=2011-05-20$ 15:04:04 Incident Reason $=$ Collision

Affected Lanes $=3$ RIGHT LANE(S) AND RIGHT SHOULDER

Conditional Probability level $=0.6$, Cond. Prob. $r 3 s 2=0.2$, Cond. Prob. $r 3 s 1=0.1$, Percent drop in speed $\%=-56$

Max. Posterior Probability level $=0.97$, Percent range between speed states $=7 \%$

Incident was detected at 01:20 minutes from drop in speed point. Incident was confirmed within 5 minutes from the detection tim

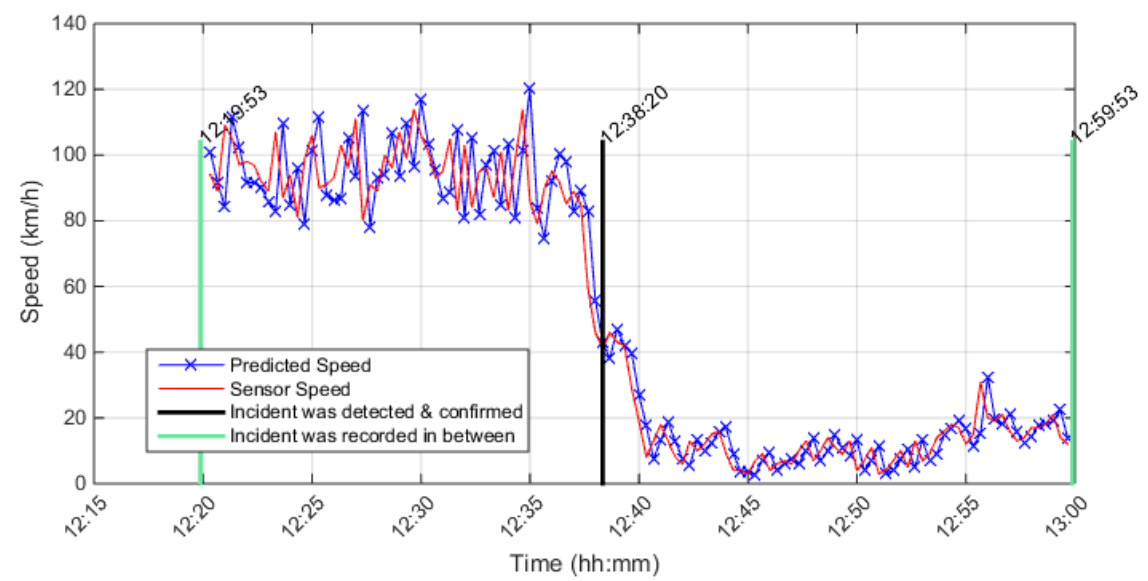

Upstream Detector \# is 401DE0330DEC, Detector Rank = 3, Distance from incident location in $(\mathrm{km})=1.5366$ Season $=$ Summer, Stream $=$ C, Direction $=E$, Date $\&$ Time $=2011-06-1812: 39: 53$ Incident Reason $=$ Collision

Affected Lanes = All lanes closed

Conditional Probability level $=0.6$, Cond. Prob. $r 3 \mathrm{~s} 2=0.2$, Cond.Prob. $r 3 \mathrm{~s} 1=0.1$, Percent drop in speed $\%=-56$

Max. Posterior Probability level $=0.97$, Percent range between speed states $=7 \%$

Incident was detected at 01:00 minutes from drop in speed point. Incident was confirmed within 5 minutes from the detection tim 


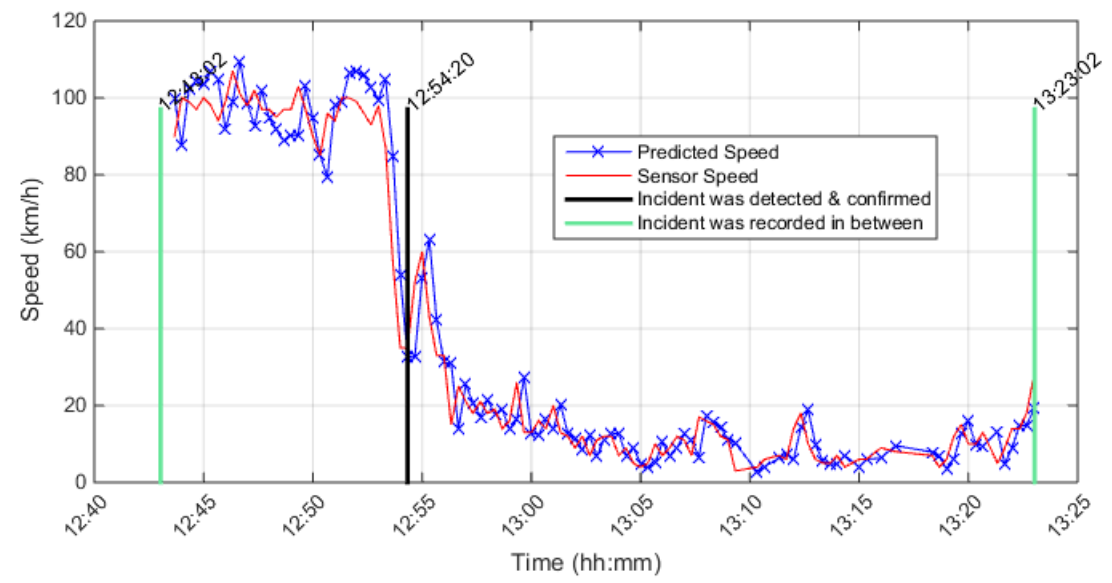

Upstream Detector \# is 401DW0050DEC, Detector Rank = 1, Distance from incident location in $(\mathrm{km})=0.4472$ Season $=$ Summer, Stream $=$ C, Direction $=E$, Date $\&$ Time $=2011-06-2913: 03: 02$ Incident Reason $=$ Collision

Affected Lanes = LEFT SHOULDER AND 2 LEFT LANE(S)

Conditional Probability level $=0.6$, Cond. Prob. $r 3 s 2=0.2$, Cond. Prob. $r 3 s 1=0.1$, Percent drop in speed $\%=-56$

Max. Posterior Probability level $=0.97$, Percent range between speed states $=7 \%$

Incident was detected at 01:00 minutes from drop in speed point. Incident was confirmed within 5 minutes from the detection timı

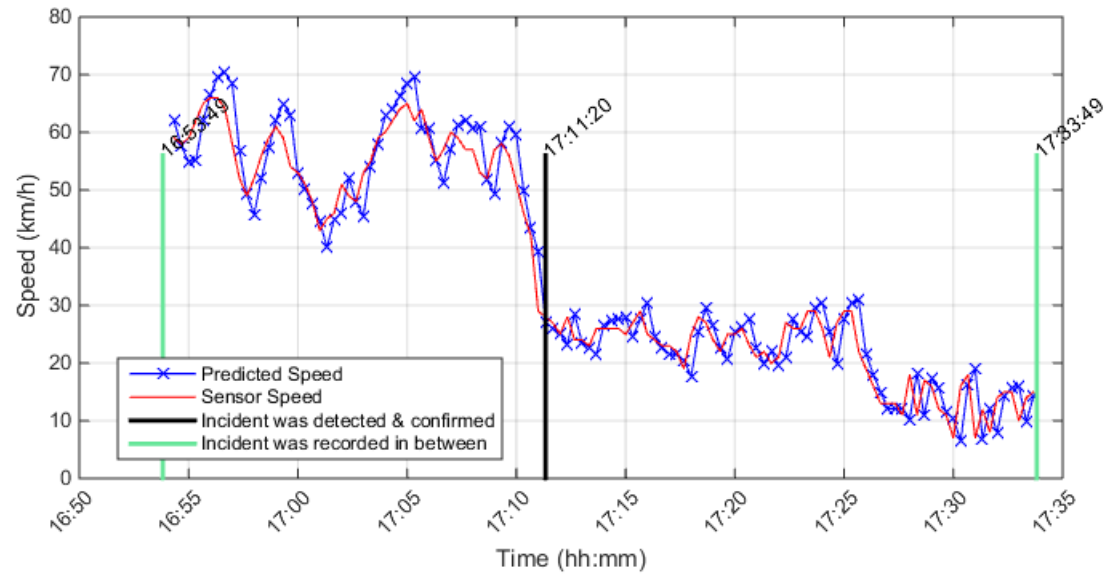

Upstream Detector \# is 401DE0100DEC, Detector Rank = 1, Distance from incident location in $(\mathrm{km})=0.26025$ Season $=$ Summer, Stream $=$ C, Direction $=E$, Date $\&$ Time $=2011-09-2017: 13: 49$ Incident Reason $=$ Collision

Affected Lanes $=2$ LEFT LANE(S)

Conditional Probability level $=0.6$, Cond.Prob. $r 3 \mathrm{~s} 2=0.2$, Cond. Prob. $r 3 \mathrm{~s} 1=0.1$, Percent drop in speed $\%=-56$

Max. Posterior Probability level $=0.97$, Percent range between speed states $=7 \%$

Incident was detected at 01:20 minutes from drop in speed point. Incident was confirmed within 5 minutes from the detection tim! 


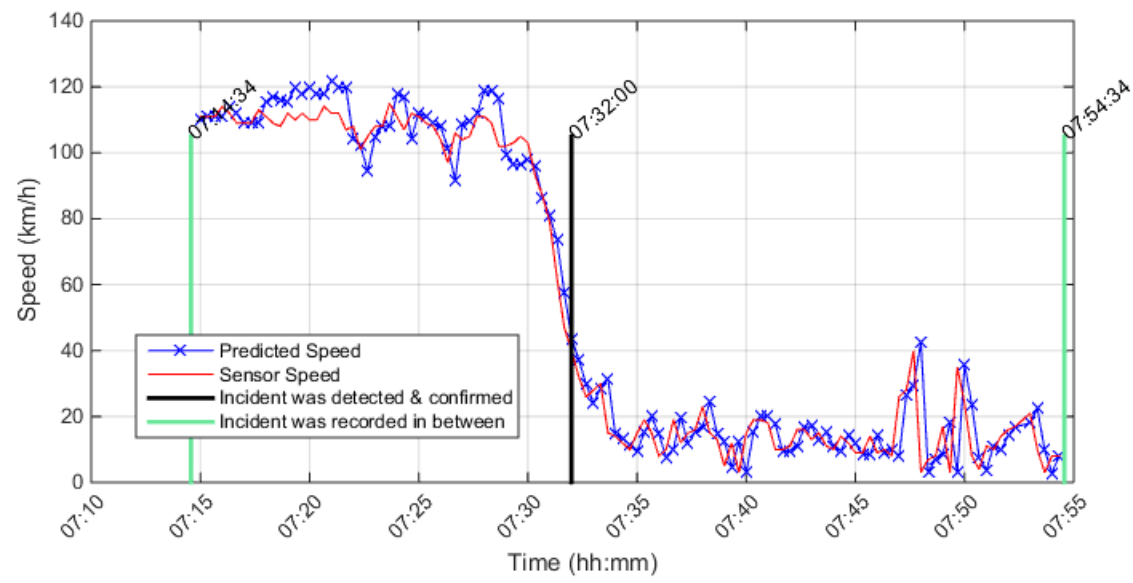

Upstream Detector \# is 401DE0270DEC, Detector Rank = 1, Distance from incident location in $(\mathrm{km})=0.85553$ Season $=$ Summer, Stream $=$ C, Direction $=E$, Date $\&$ Time $=2011-09-3007: 34: 34$ Incident Reason $=$ Collision

Affected Lanes = LEFT SHOULDER AND 2 LEFT LANE(S) AND RIGHT SHOULDER

Conditional Probability level $=0.6$, Cond.Prob. $r 3 s 2=0.2$, Cond. Prob. $r 3 s 1=0.1$, Percent drop in speed $\%=-56$

Max. Posterior Probability level $=0.97$, Percent range between speed states $=7 \%$

Incident was detected at 03:20 minutes from drop in speed point. Incident was confirmed within 5 minutes from the detection tim

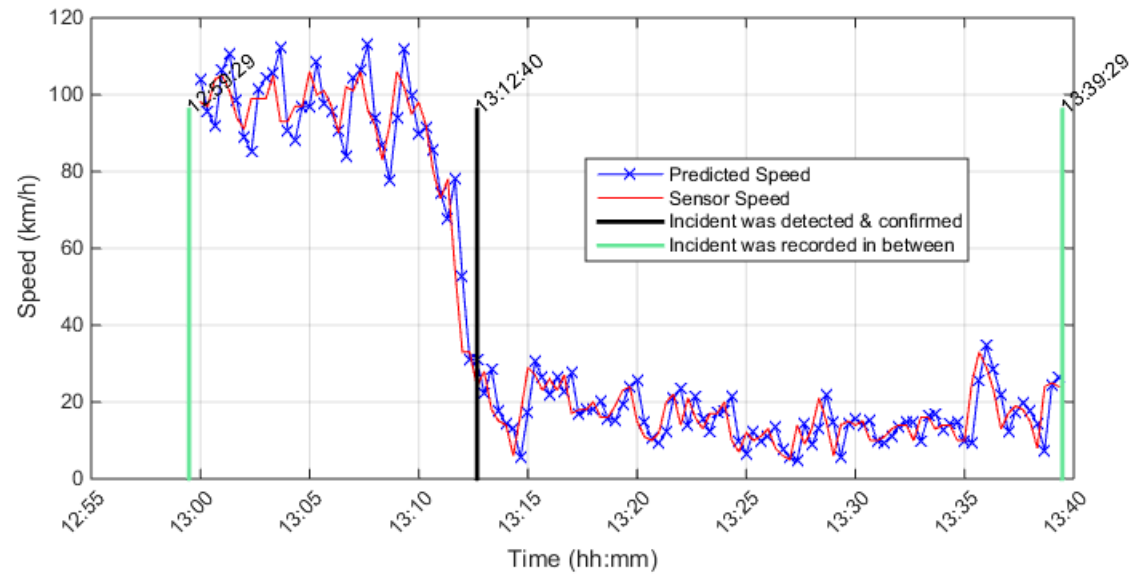

Upstream Detector \# is 401DE0170DEC, Detector Rank $=3$, Distance from incident location in $(\mathrm{km})=1.9227$ Season $=$ Summer, Stream $=$ C, Direction $=E$, Date $\&$ Time $=2011-10-2313: 19: 29$ Incident Reason $=$ Collision

Affected Lanes = 1 RIGHT LANE(S)

Conditional Probability level $=0.6$, Cond. Prob. $r 3 s 2=0.2$, Cond. Prob. $r 3 s 1=0.1$, Percent drop in speed $\%=-56$

Max. Posterior Probability level $=0.97$, Percent range between speed states $=7 \%$

Incident was detected at 03:20 minutes from drop in speed point. Incident was confirmed within 5 minutes from the detection tim! 


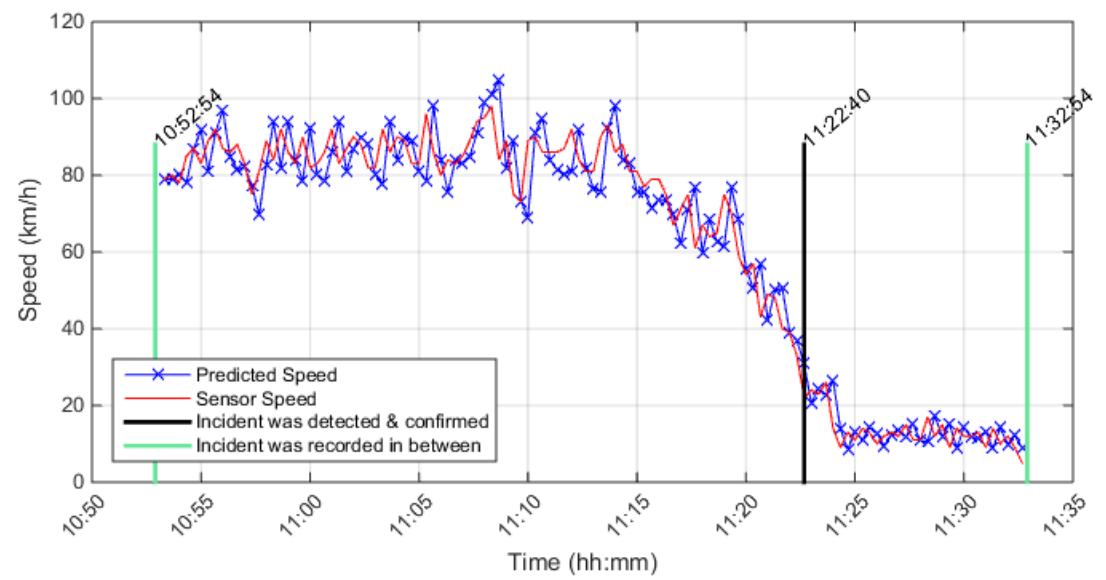

Upstream Detector \# is 401DE0130DEC, Detector Rank = 2, Distance from incident location in $(\mathrm{km})=1.1356$ Season $=$ Summer, Stream $=$ C, Direction $=E$, Date $\&$ Time $=2011-11-2911: 12: 54$ Incident Reason $=$ Collision

Affected Lanes $=2$ LEFT LANE(S)

Conditional Probability level $=0.6$, Cond.Prob. $r 3 s 2=0.2$, Cond.Prob. $r 3 s 1=0.1$, Percent drop in speed $\%=-56$

Max. Posterior Probability level $=0.97$, Percent range between speed states $=7 \%$

Incident was detected at 03:20 minutes from drop in speed point. Incident was confirmed within 5 minutes from the detection tim!

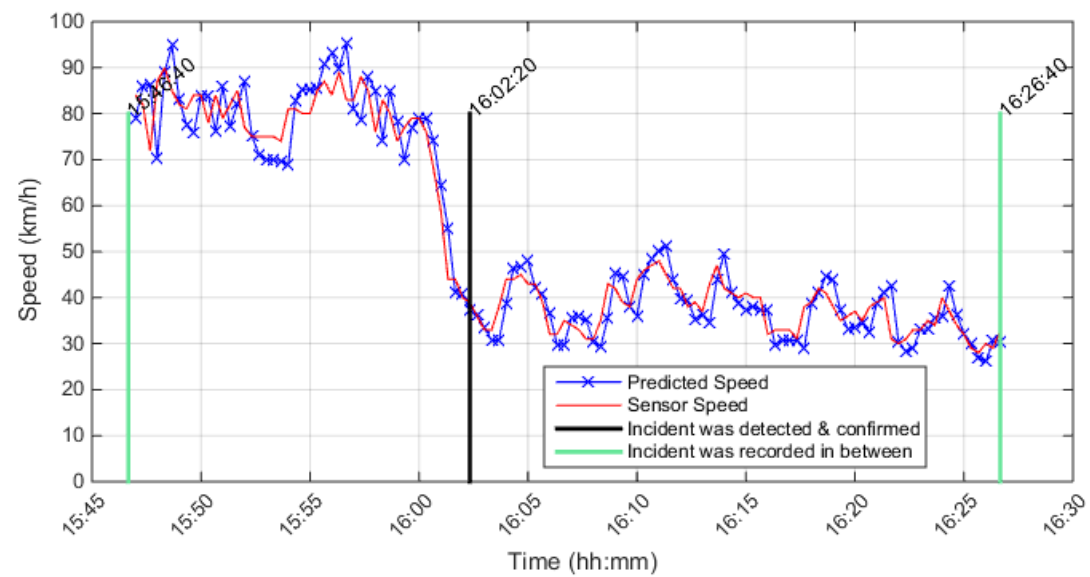

Upstream Detector \# is 401DE0190DEE, Detector Rank = 1, Distance from incident location in $(\mathrm{km})=0.31753$

Season $=$ Winter, Stream $=E$, Direction $=E$, Date \& Time $=$ 2011-02-24 16:06:40

Incident Reason $=$ Disable Vehicle

Affected Lanes = LEFT SHOULDER AND 3 LEFT LANE(S)

Conditional Probability level $=0.6$, Cond.Prob. $r 3 \mathrm{~s} 2=0.2$, Cond.Prob. $r 3 \mathrm{~s} 1=0.1$, Percent drop in speed $\%=-56$

Max. Posterior Probability level $=0.97$. Percent range between speed states $=7 \%$

Incident was detected at 02:00 minutes from drop in speed point. Incident was confirmed within 5 minutes from the detection tim 


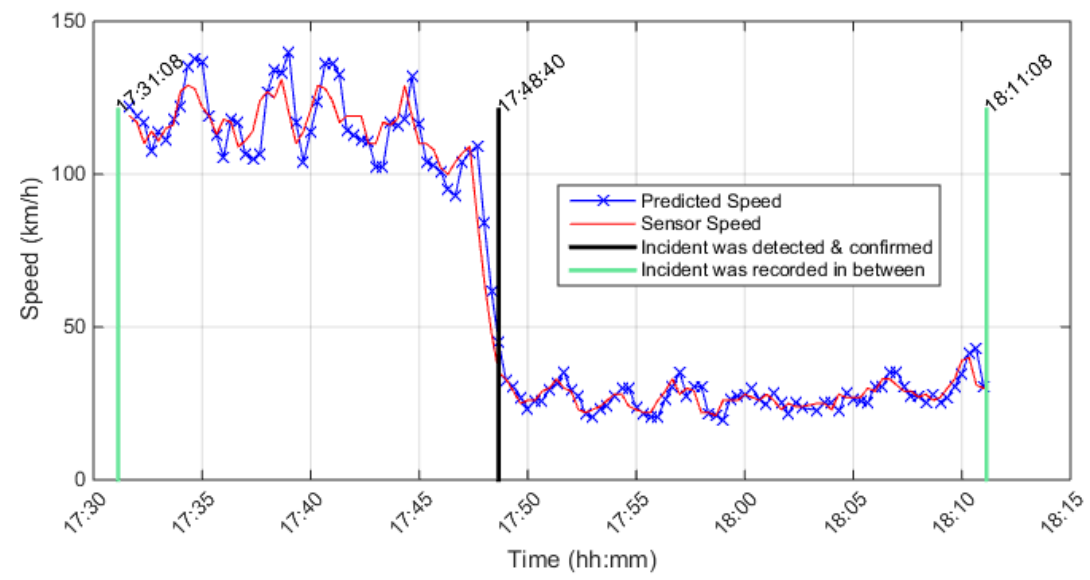

Upstream Detector \# is 401DW0210DEE, Detector Rank = 1, Distance from incident location in $(\mathrm{km})=0.02872$ Season $=$ Spring, Stream $=E$, Direction $=E$, Date \& Time $=2011-05-2117: 51: 08$ Incident Reason $=$ Collision

Affected Lanes = 2 RIGHT LANE(S)

Conditional Probability level $=0.6$, Cond. Prob. $r 3 s 2=0.2$, Cond. Prob. $r 3 s 1=0.1$, Percent drop in speed $\%=-56$ Max. Posterior Probability level $=0.97$, Percent range between speed states $=7 \%$

Incident was detected at 01:00 minutes from drop in speed point. Incident was confirmed within 5 minutes from the detection tim

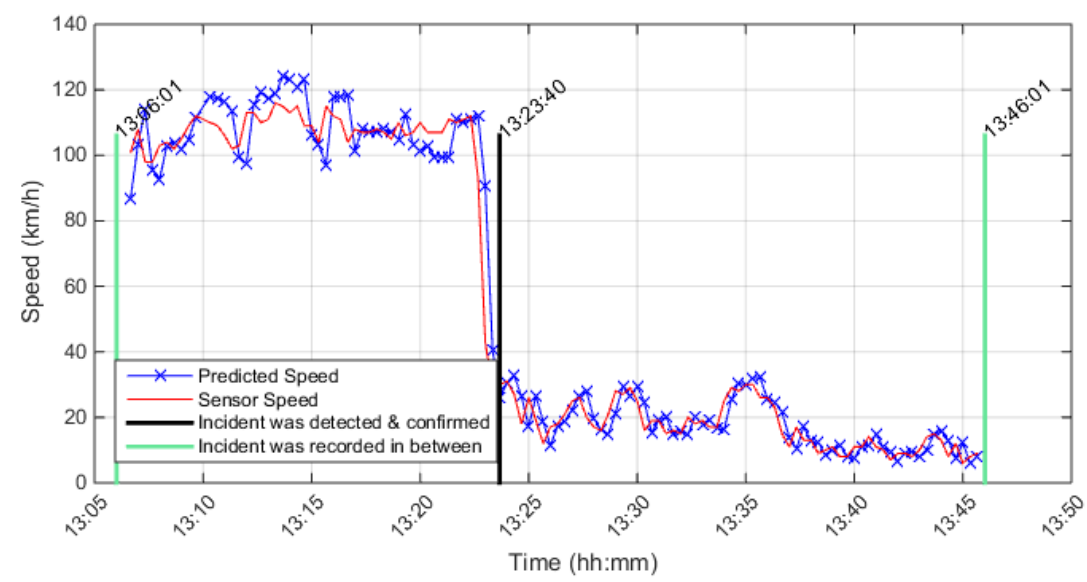

Upstream Detector \# is 401DW0100DEE, Detector Rank = 1, Distance from incident location in $(\mathrm{km})=0.41981$ Season $=$ Summer, Stream $=E$, Direction $=E$, Date $\&$ Time $=2011-09-18$ 13:26:01 Incident Reason $=$ Collision

Affected Lanes $=2$ CENTRE LANES(S)

Conditional Probability level $=0.6$, Cond.Prob. $r 3 s 2=0.2$, Cond.Prob. $r 3 s 1=0.1$, Percent drop in speed $\%=-56$ Max. Posterior Probability level $=0.97$, Percent range between speed states $=7 \%$

Incident was detected at 01:00 minutes from drop in speed point. Incident was confirmed within 5 minutes from the detection timı 


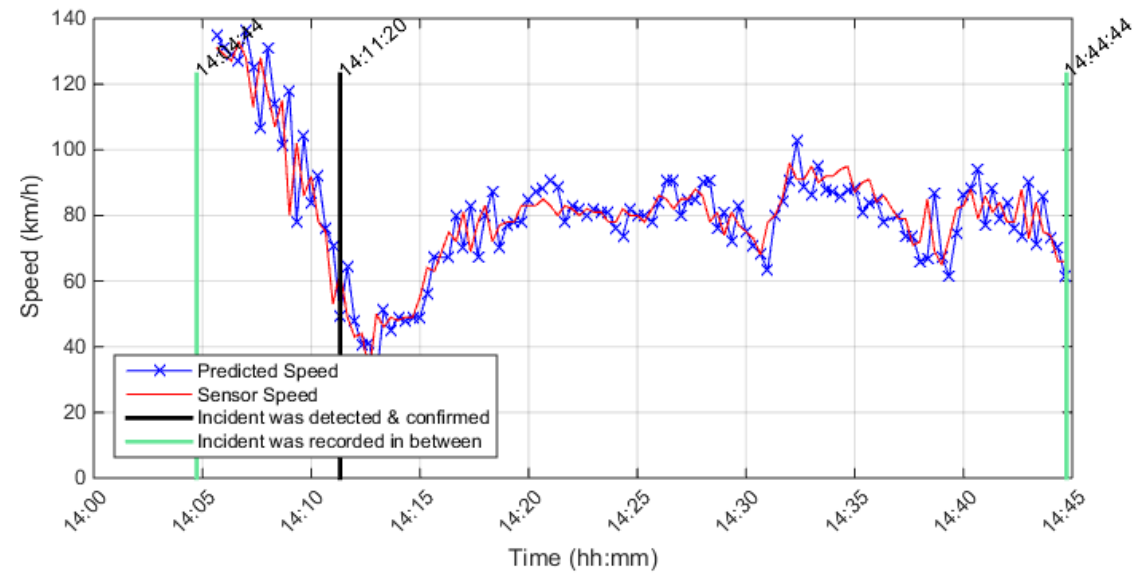

Upstream Detector \# is 401DE0060DEE, Detector Rank = 1, Distance from incident location in $(\mathrm{km})=0.19919$ Season $=$ Winter, Stream $=E$, Direction $=E$, Date $\&$ Time $=2011-12-1714: 24: 44$ Incident Reason $=$ Collision

Affected Lanes $=$ LEFT SHOULDER AND 1 LEFT LANE(S)

Conditional Probability level $=0.6$, Cond.Prob. $r 3 s 2=0.2$, Cond.Prob. $r 3 s 1=0.1$, Percent drop in speed $\%=-56$

Max. Posterior Probability level $=0.97$, Percent range between speed states $=7 \%$

Incident was detected at 01:40 minutes from drop in speed point. Incident was confirmed within 5 minutes from the detection tim

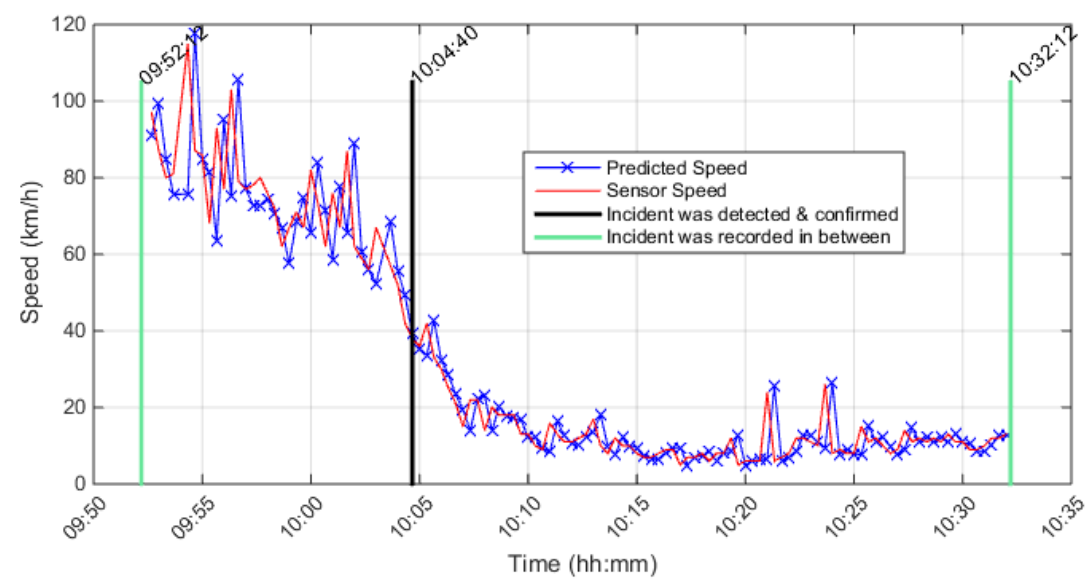

Upstream Detector \# is 401DW0260DEE, Detector Rank = 1, Distance from incident location in $(\mathrm{km})=0.36837$ Season $=$ Winter, Stream $=E$, Direction $=E$, Date \& Time $=2011-12-3010: 12: 12$ Incident Reason $=$ Collision

Affected Lanes $=2$ CENTRE LANE(S)

Conditional Probability level $=0.6$, Cond.Prob. $r 3 s 2=0.2$, Cond.Prob. $r 3 s 1=0.1$, Percent drop in speed $\%=-56$

Max. Posterior Probability level $=0.97$, Percent range between speed states $=7 \%$

Incident was detected at 01:00 minutes from drop in speed point. Incident was confirmed within 5 minutes from the detection timı 


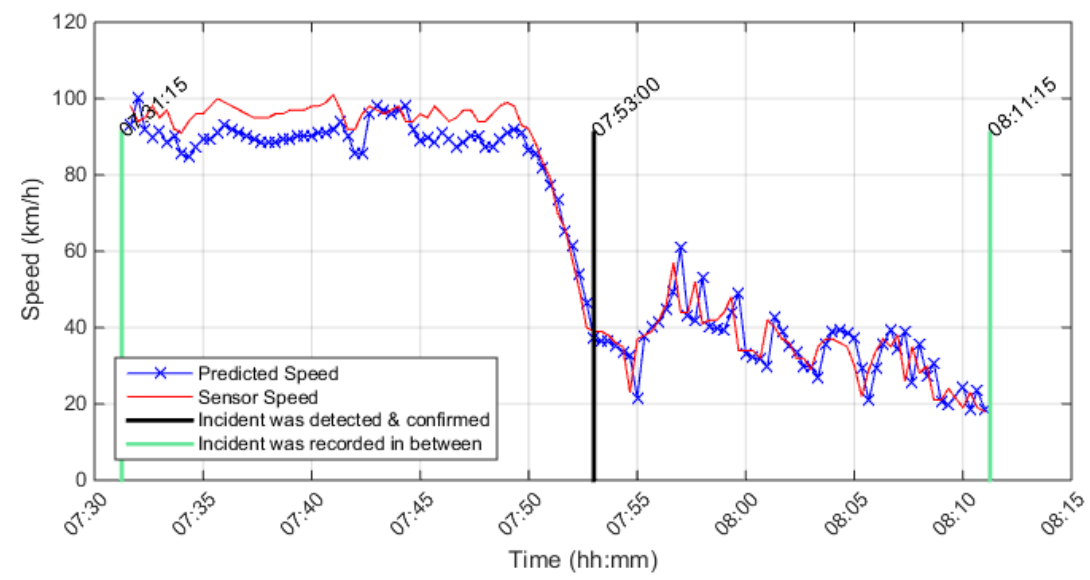

Upstream Detector \# is 401DE0270DWC, Detector Rank = 1, Distance from incident location in $(\mathrm{km})=0.5857$ Season $=$ Spring, Stream $=$ C, Direction $=$ W, Date $\&$ Time $=$ 2011-03-25 07:51:15 Incident Reason $=$ Collision

Affected Lanes $=3$ RIGHT LANE(S)

Conditional Probability level $=0.6$, Cond. Prob. $r 3 s 2=0.2$, Cond. Prob. $r 3 s 1=0.1$, Percent drop in speed $\%=-56$

Max. Posterior Probability level $=0.97$, Percent range between speed states $=7 \%$

Incident was detected at 08:40 minutes from drop in speed point. Incident was confirmed within 5 minutes from the detection tim

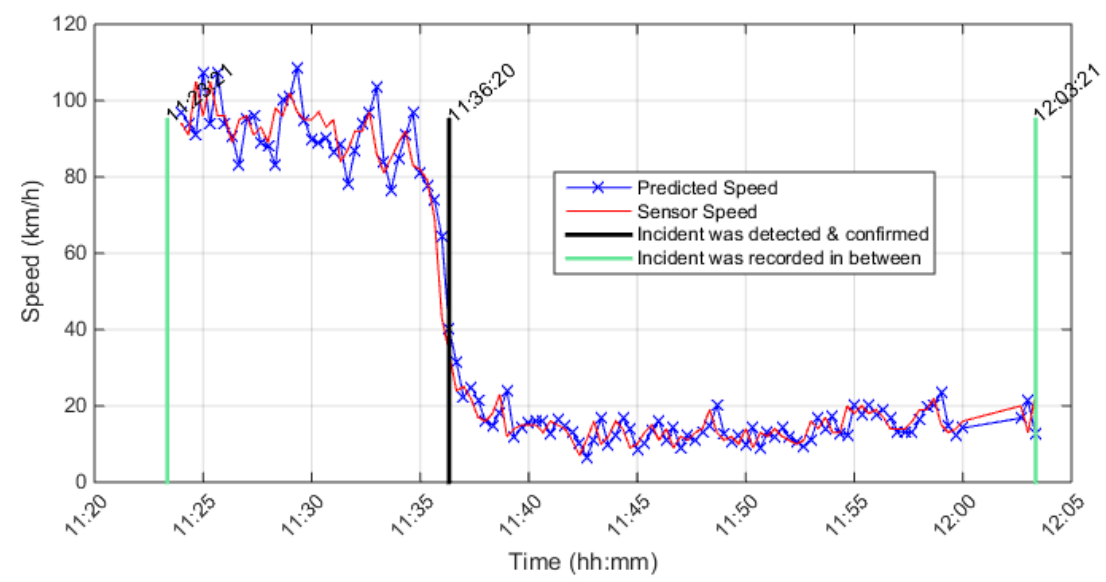

Upstream Detector \# is 401DE0100DWC, Detector Rank = 1, Distance from incident location in $(\mathrm{km})=0.11847$ Season $=$ Winter, Stream $=$ C, Direction $=$ W, Date $\&$ Time $=$ 2011-12-2611:43:21

Incident Reason $=$ Collision

Affected Lanes $=2$ CENTRE LANES(S)

Conditional Probability level $=0.6$, Cond.Prob. $r 3 s 2=0.2$, Cond.Prob. $r 3 s 1=0.1$, Percent drop in speed $\%=-56$

Max. Posterior Probability level $=0.97$, Percent range between speed states $=7 \%$

Incident was detected at 01:40 minutes from drop in speed point. Incident was confirmed within 5 minutes from the detection timı 


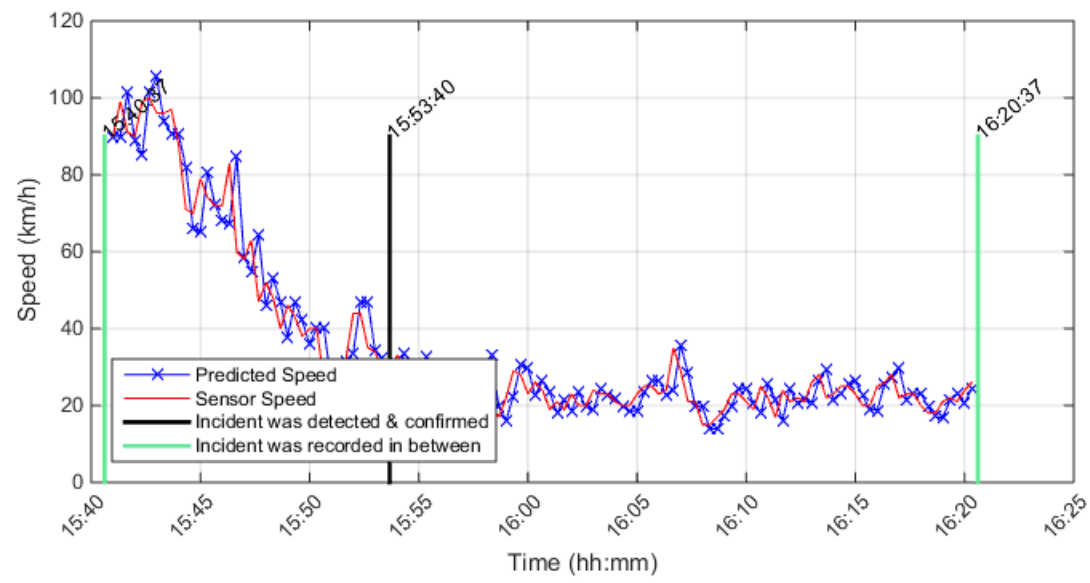

Upstream Detector \# is 401DE0160DWE, Detector Rank = 1, Distance from incident location in $(\mathrm{km})=0.12782$ Season $=$ Spring, Stream $=E$, Direction $=W$, Date $\&$ Time $=2011-05-2116: 00: 37$ Incident Reason $=$ Collision

Affected Lanes = 1 RIGHT LANE(S) AND RIGHT SHOULDER

Conditional Probability level $=0.6$, Cond.Prob. $r 3 s 2=0.2$, Cond. Prob. $r 3 s 1=0.1$, Percent drop in speed $\%=-56$

Max. Posterior Probability level $=0.97$, Percent range between speed states $=7 \%$

Incident was detected at 07:00 minutes from drop in speed point. Incident was confirmed within 5 minutes from the detection tim

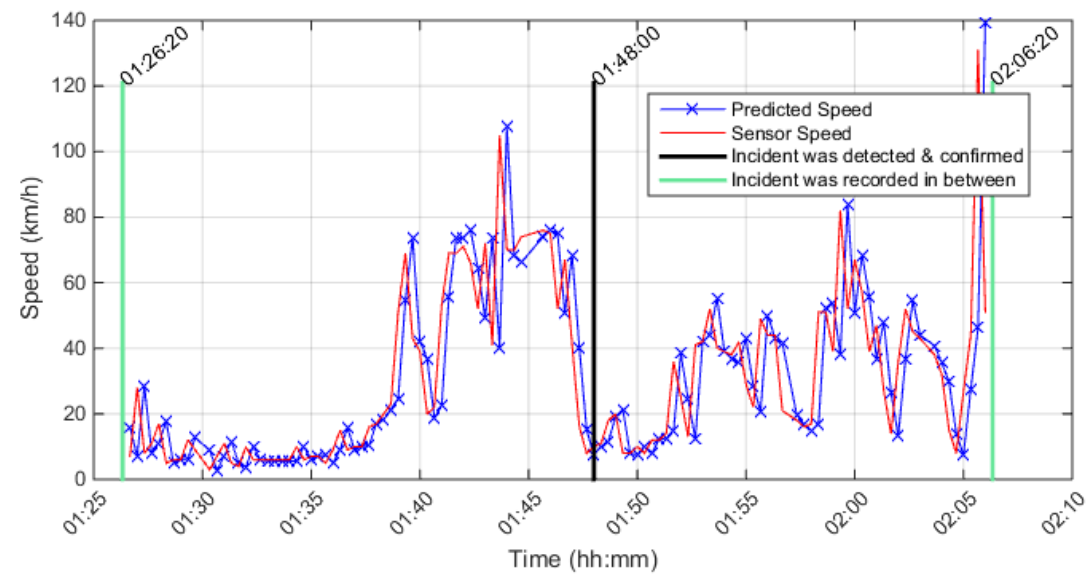

Upstream Detector \# is 401DW0270DWE, Detector Rank = 3, Distance from incident location in $(\mathrm{km})=1.7666$ Season $=$ Spring, Stream $=E$, Direction $=W$, Date $\&$ Time $=2011-05-28$ 01:46:20

Incident Reason $=$ Collision

Affected Lanes = All lanes closed

Conditional Probability level $=0.6$, Cond.Prob. $r 3 \mathrm{~s} 2=0.2$, Cond.Prob. $r 3 \mathrm{~s} 1=0.1$, Percent drop in speed $\%=-56$

Max. Posterior Probability level $=0.97$, Percent range between speed states $=7 \%$

Incident was detected at 01:00 minutes from drop in speed point. Incident was confirmed within 5 minutes from the detection tim

After investigating the reason for the low speed before 1:40, it appears that there was another incident which took place at 1:14. While the first incident was recovering at 1:40 
another incident occurred and caused another drop in speed and was detected at 1:48. This investigation is illustrated in the next figure.

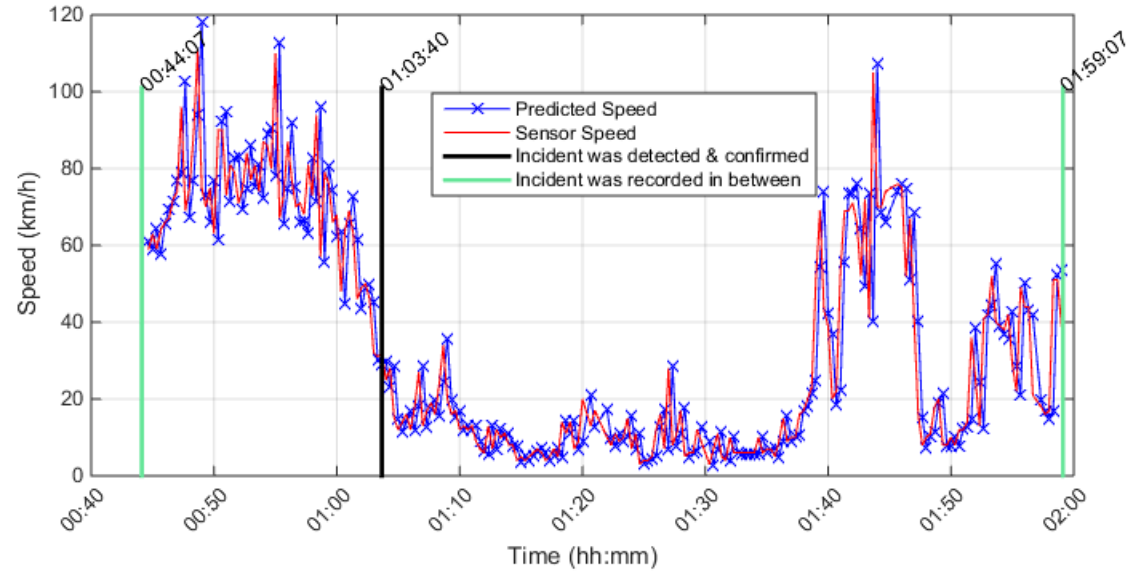

Upstream Detector \# is 401DW0270DWE, Detector Rank $=3$, Distance from incident location in $(\mathrm{km})=1.7666$ Season $=$ Spring, Stream $=E$, Direction $=$ W, Date $\&$ Time $=2011-05-28$ 01:14:07 Incident Reason $=$ Collision

Affected Lanes $=$ All lanes closed

Conditional Probability level $=0.6$, Cond.Prob. $r 3 s 2=0.2$, Cond. Prob. $r 3 s 1=0.1$, Percent drop in speed $\%=-56$

Max. Posterior Probability level $=0.97$, Percent range between speed states $=7 \%$

Incident was detected at 02:20 minutes from drop in speed point. Incident was confirmed within 5 minutes from the detection tim

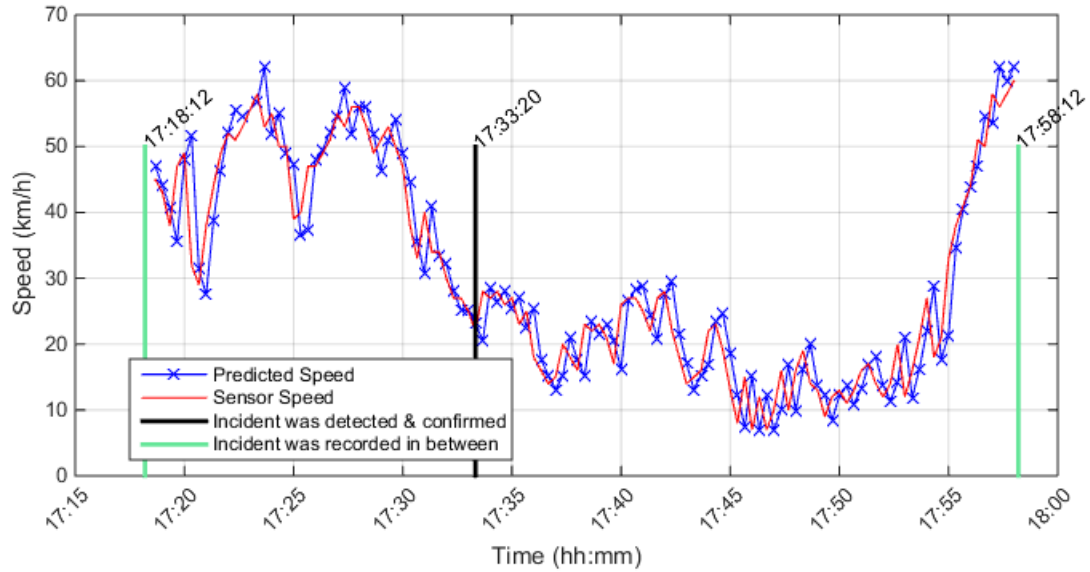

Upstream Detector \# is 401DE0160DWE, Detector Rank $=1$, Distance from incident location in $(\mathrm{km})=0.12782$ Season $=$ Summer, Stream $=E$, Direction $=$ W, Date $\&$ Time $=$ 2011-09-17 17:38:12 Incident Reason $=$ Collision

Affected Lanes $=2$ LEFT LANE $(S)$

Conditional Probability level $=0.6$, Cond. Prob. $r 3 s 2=0.2$, Cond. Prob. $r 3 s 1=0.1$, Percent drop in speed $\%=-56$

Max. Posterior Probability level $=0.97$, Percent range between speed states $=7 \%$

Incident was detected at 03:40 minutes from drop in speed point. Incident was confirmed within 5 minutes from the detection tim! 


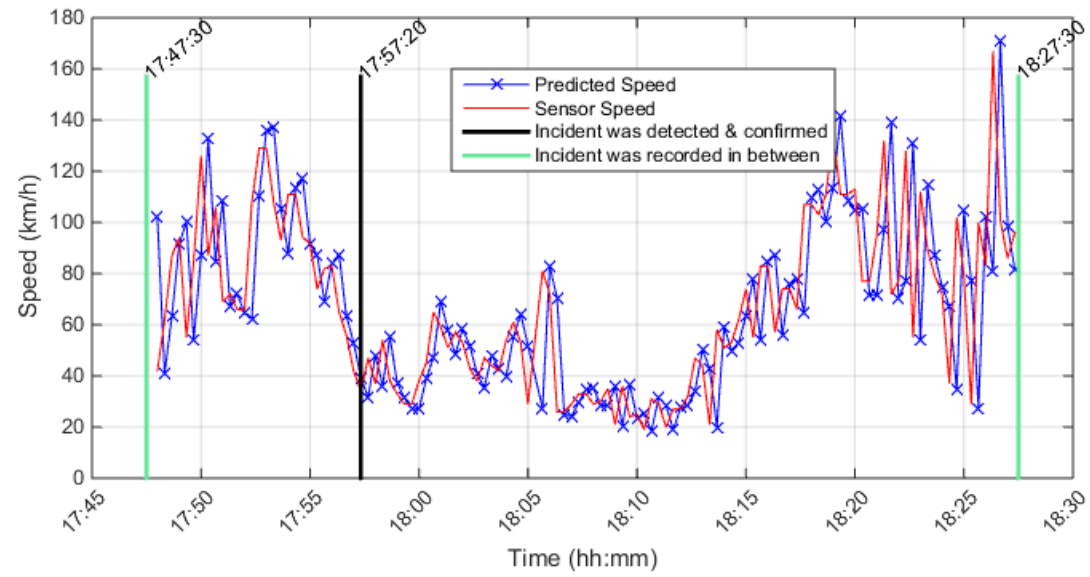

Upstream Detector \# is 401DE0270DWE, Detector Rank = 1, Distance from incident location in $(\mathrm{km})=0.59072$ Season $=$ Summer, Stream $=E$, Direction $=W$, Date $\&$ Time $=2011-11-29$ 18:07:30 Incident Reason $=$ Disable Vehicle

Affected Lanes $=1$ CENTRE LANES(S)

Conditional Probability level $=0.6$, Cond.Prob. $r 3 s 2=0.2$, Cond. Prob. $r 3 s 1=0.1$, Percent drop in speed $\%=-56$

Max. Posterior Probability level $=0.97$, Percent range between speed states $=7 \%$

Incident was detected at 01:00 minutes from drop in speed point. Incident was confirmed within 5 minutes from the detection tim

\section{Appedix C.1.1.2 Cases with Incident Recorded and Not Detected - 6 cases}

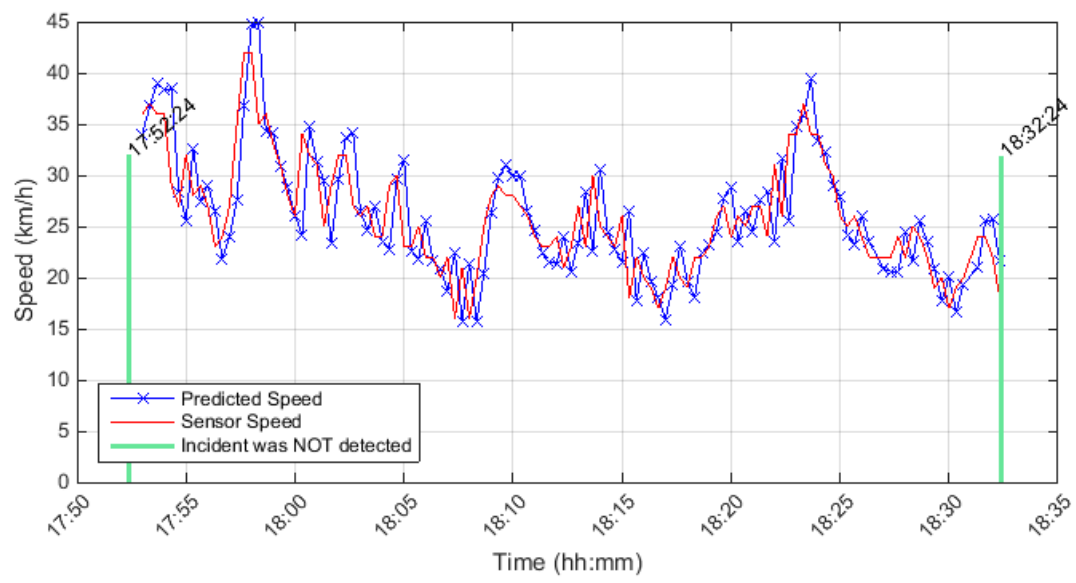

Upstream Detector \# is 401DW0020DEE, Detector Rank = 1, Distance from incident location in $(\mathrm{km})=0.035848$ Season $=$ Winter, Stream $=E$, Direction $=E$, Date \& Time $=2011-12-15$ 18:12:24 Incident Reason $=$ Collision

Affected Lanes $=2$ RIGHT LANE(S)

Conditional Probability level $=0.6$, Cond. Prob. $r 3 \mathrm{~s} 2=0.2$, Cond. Prob. $r 3 \mathrm{~s} 1=0.1$, Percent drop in speed $\%=-56$ Max. Posterior Probability level $=0.97$, Percent range between speed states $=7 \%$ (No Incident was Detected) 


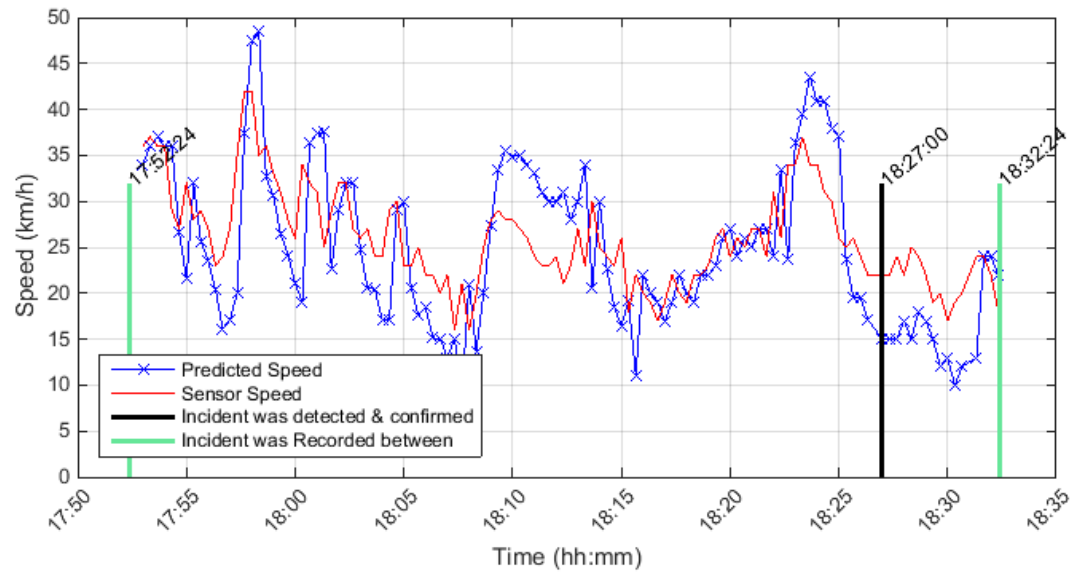

Upstream Detector \# is 401DW0020DEE, Detector Rank = 1, Distance from incident location in $(\mathrm{km})=0.035848$ Season $=$ Winter, Stream $=E$, Direction $=E$, Date $\&$ Time $=2011-12-15$ 18:12:24 Incident Reason $=$ Collision

Affected Lanes = 2RIGHT LANE(S)

Conditional Probability level $=0.6$, Cond. Prob. $r 3 s 2=0.2$, Cond. Prob. $r 3 s 1=0.1$, Percent drop in speed $\%=-56$

Max. Posterior Probability level $=0.97$, Absolute range between speed states $=7 \mathrm{~km}$

Incident was detected at 02:00 minutes from drop in speed point. Incident was confirmed after 5 minutes from the detection time

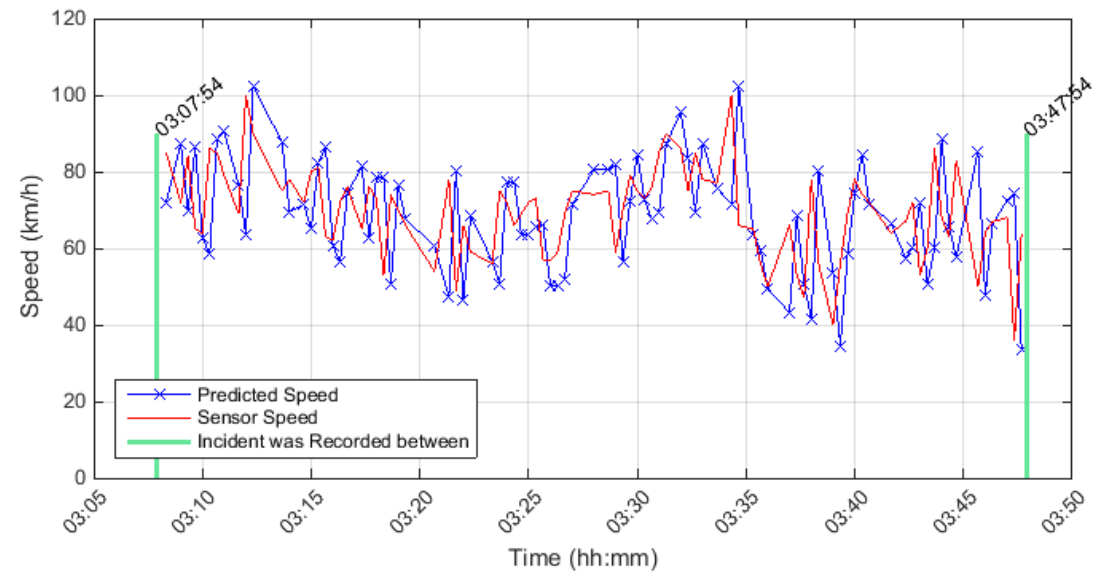

Upstream Detector \# is 401DE0090DWC, Detector Rank $=1$, Distance from incident location in $(\mathrm{km})=0.59485$ Season $=$ Winter, Stream $=$ C , Direction $=W$, Date $\&$ Time $=2011-02-02$ 03:27:54 Incident Reason $=$ Disable Vehicle

Affected Lanes = 1 RIGHT LANE(S) AND RIGHT SHOULDER

Conditional Probability level $=0.6$, Cond. Prob. $r 3 \mathrm{~s} 2=0.2$, Cond.Prob. $r 3 \mathrm{~s} 1=0.1$, Percent drop in speed $\%=-56$

Max. Posterior Probability level $=0.97$, Absolute range between speed states $=7 \mathrm{~km}$

(No Incident was Detected) 


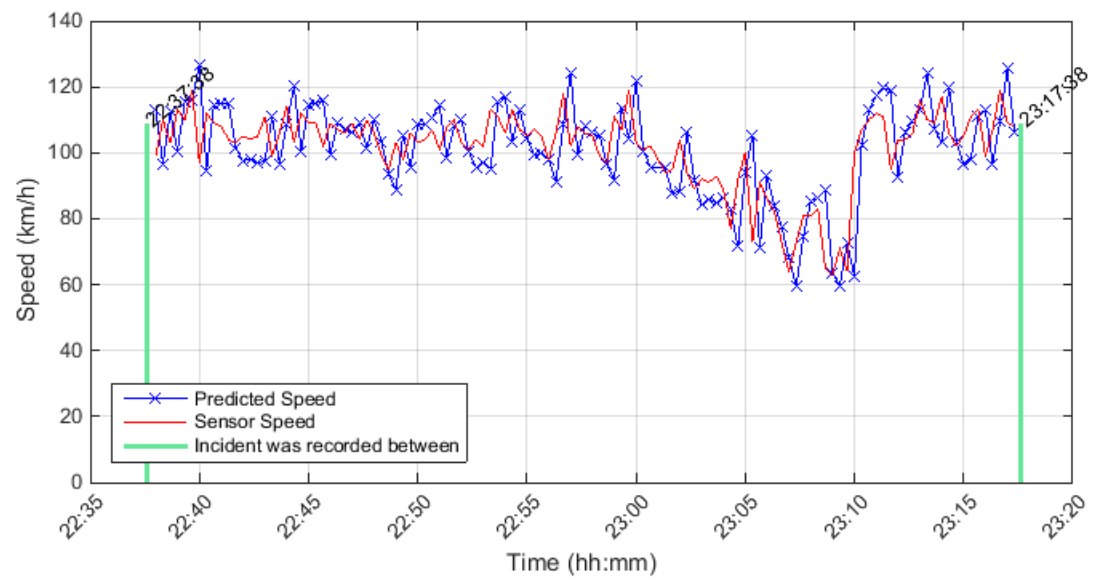

Upstream Detector \# is 401DE0210DWC, Detector Rank = 1, Distance from incident location in $(\mathrm{km})=0.35779$ Season $=$ Summer, Stream $=$ C, Direction $=W$, Date $\&$ Time $=2011-06-28$ 22:57:38

Incident Reason $=$ Collision

Affected Lanes $=3$ LEFT LANE(S)

Conditional Probability level $=0.6$, Cond.Prob. $r 3 s 2=0.2$, Cond.Prob. $r 3 s 1=0.1$, Percent drop in speed $\%=-56$

Max. Posterior Probability level $=0.97$, Percent range between speed states $=7 \%$

(No Incident was Detected)

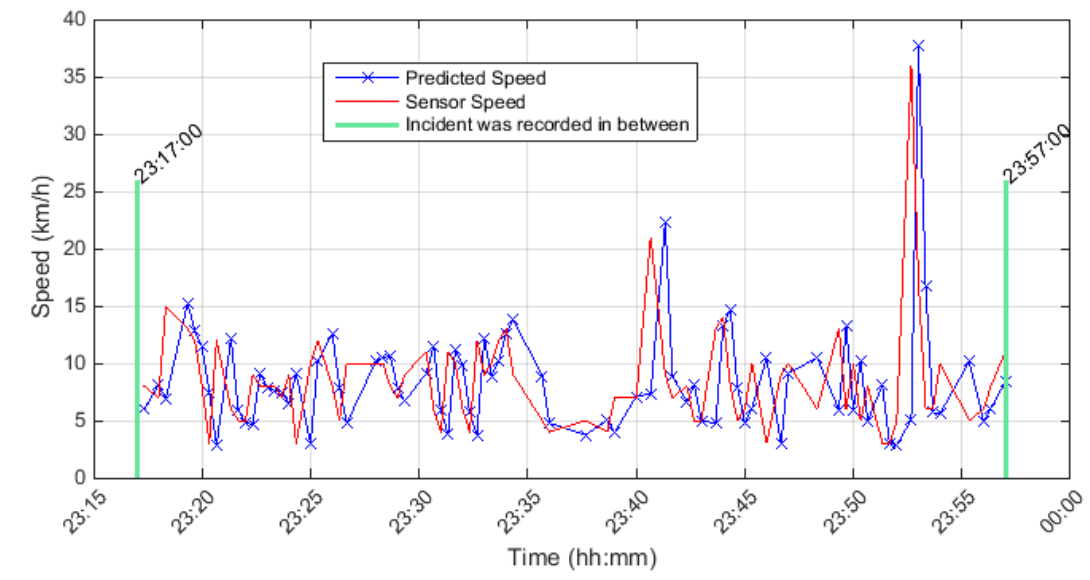

Upstream Detector \# is 401DW0020DWC, Detector Rank = 3, Distance from incident location in $(\mathrm{km})=1.9461$ Season $=$ Summer, Stream $=$ C, Direction $=$ W, Date $\&$ Time $=$ 2011-09-16 23:37:00 Incident Reason $=$ Collision

Affected Lanes $=3$ CENTRE LANE(S)

Conditional Probability level $=0.6$, Cond.Prob. $r 3 s 2=0.2$, Cond.Prob. $r 3 s 1=0.1$, Percent drop in speed $\%=-56$

Max. Posterior Probability level $=0.97$, Percent range between speed states $=7 \%$

Drop in speed was detected and No indication of an incident within this period 


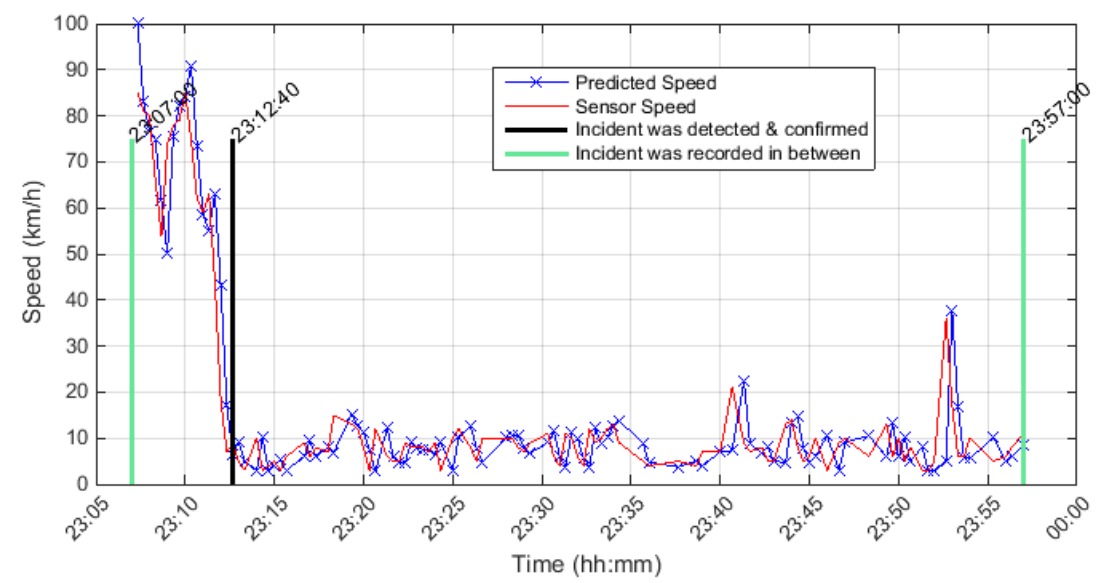

Upstream Detector \# is 401DW0020DWC, Detector Rank = 3, Distance from incident location in $(\mathrm{km})=1.9461$ Season $=$ Summer, Stream $=C$, Direction $=W$, Date \& Time $=2011-09-16$ 23:37:00 Incident Reason $=$ Collision

Affected Lanes $=3$ CENTRE LANE(S)

Conditional Probability level $=0.6$, Cond.Prob. $r 3 s 2=0.2$, Cond.Prob. $r 3 s 1=0.1$, Percent drop in speed $\%=-56$

Max. Posterior Probability level $=0.97$, Percent range between speed states $=7 \%$

Incident was detected at 02:20 minutes from drop in speed point. Incident was confirmed after 5 minutes from the detection time

Same as previous case with more time frame before incident recorded time

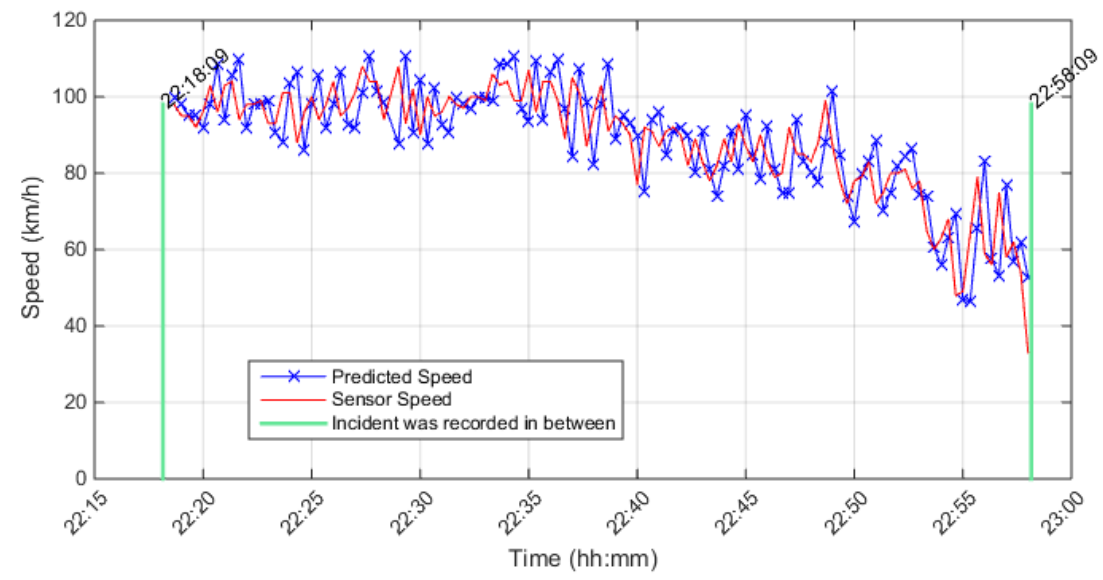

Upstream Detector \# is 401DE0050DWC, Detector Rank = 1, Distance from incident location in $(\mathrm{km})=0.45946$ Season $=$ Winter, Stream $=$ C, Direction $=$ W, Date \& Time $=2011-12-2622: 38: 09$ Incident Reason $=$ Collision

Affected Lanes = 1 LEFT LANE(S) AND 2 RIGHT LANE(S) AND 0 CENTRE LANE(S)

Conditional Probability level $=0.6$, Cond. Prob. $r 3 s 2=0.2$, Cond. Prob. $r 3 s 1=0.1$, Percent drop in speed $\%=-56$ Max. Posterior Probability level $=0.97$, Percent range between speed states $=7 \%$ (No Incident was Detected) 
Same as the previous case if $5 \mathrm{~min}$ before and $50 \mathrm{~min}$ after the incident recorded time was selected.

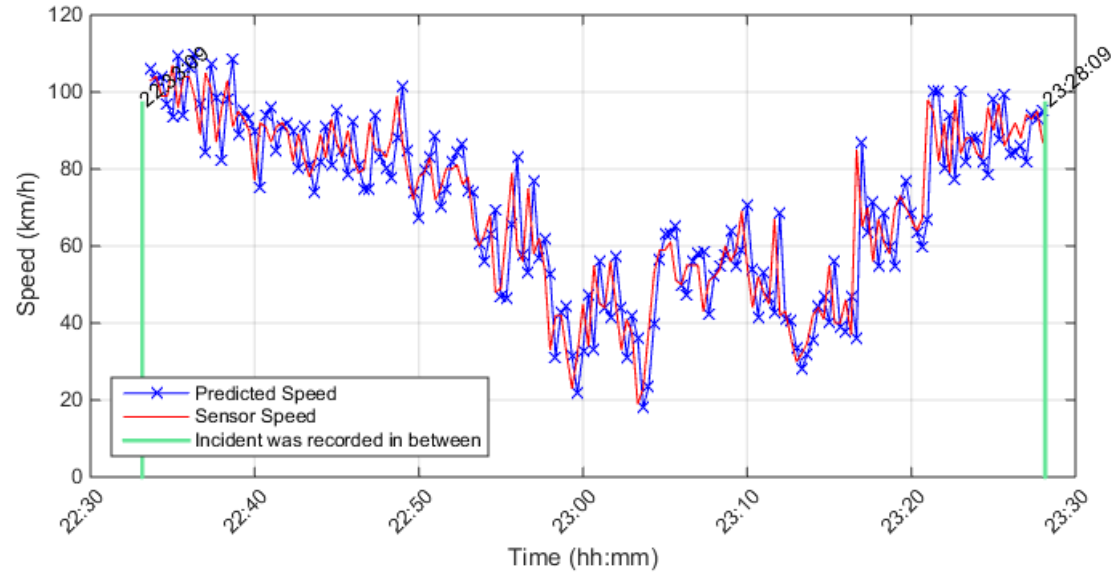

Upstream Detector \# is 401DE0050DWC, Detector Rank = 1, Distance from incident location in $(\mathrm{km})=0.45946$ Season $=$ Winter, Stream $=$ C, Direction $=$ W, Date \& Time $=$ 2011-12-26 22:38:09 Incident Reason $=$ Collision

Affected Lanes $=1$ LEFT LANE(S) AND 2 RIGHT LANE(S) AND 0 CENTRE LANE(S)

Conditional Probability level $=0.6$, Cond. Prob. $r 3 s 2=0.2$, Cond. Prob. $r 3 s 1=0.1$, Percent drop in speed $\%=-56$

Max. Posterior Probability level $=0.97$, Percent range between speed states $=7 \%$

Drop in speed was detected and No indication of an incident within this period

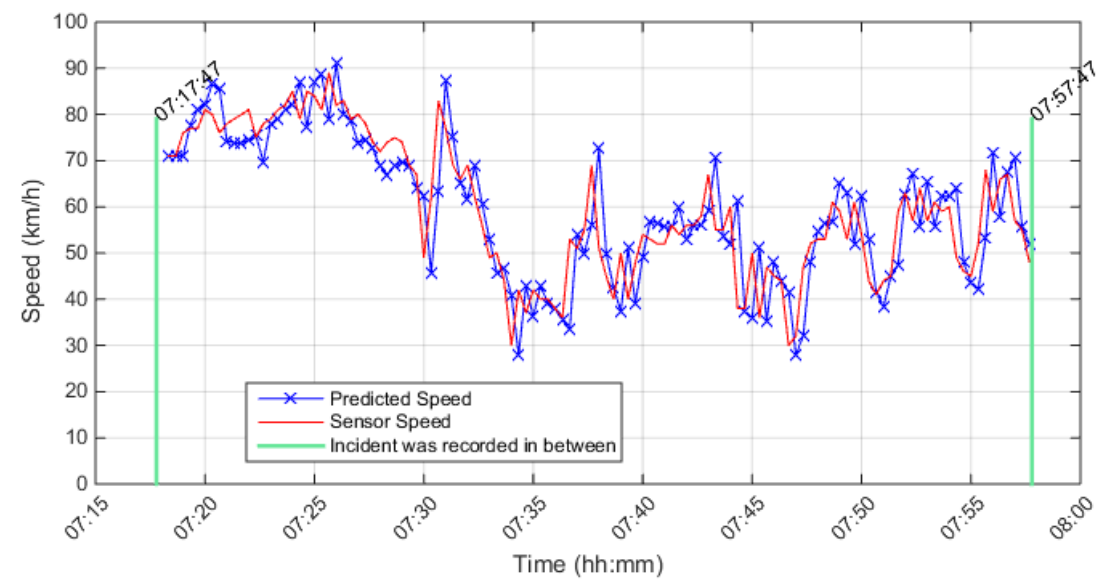

Upstream Detector \# is 401DE0020DWE, Detector Rank = 1, Distance from incident location in $(\mathrm{km})=0.28759$ Season $=$ Summer, Stream $=E$, Direction $=W$, Date $\&$ Time $=2011-09-20$ 07:37:47 Incident Reason $=$ Collision

Affected Lanes = LEFT SHOULDER AND 1 LEFT LANE(S)

Conditional Probability level $=0.6$, Cond.Prob. $r 3 s 2=0.2$, Cond. Prob. $r 3 s 1=0.1$, Percent drop in speed $\%=-56$

Max. Posterior Probability level $=0.97$, Percent range between speed states $=7 \%$

Drop in speed was detected and No indication of an incident within this period 


\section{Appendix C.2 Cases with Incident Recorded and not Detected}

This appendix presents the validation outcomes that resulted from the model application on 19 cases as described in chapter 6 . Cases presented in this part are under certain traffic condition "Some Degree of Benefit", by holding the parameters that resulted from the calibration process. The model was applied to cases with incidents.

\section{Appendix C.2.1 Certain Traffic Condition "Some Degree of Benefit" 30 cases}

Appedix C.2.1.1 Cases with Incident Recorded and Detected -- 0 case Appedix C.2.1.2 Cases with Incident Recorded and Not Detected -- 19 cases

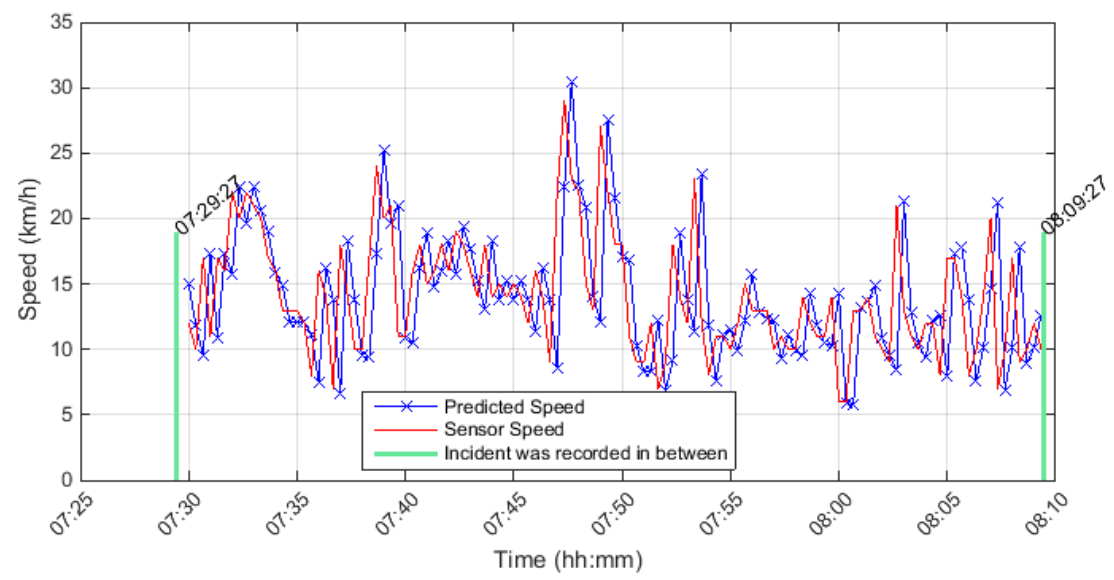

Upstream Detector \# is 401DE0020DWC, Detector Rank = 1, Distance from incident location in $(\mathrm{km})=0.63426$ Season $=$ Summer, Stream $=$ C, Direction $=$ W, Date $\&$ Time $=2011-10-19$ 07:49:27 Incident Reason $=$ Collision

Affected Lanes $=1$ CENTRE LANES(S)

Conditional Probability level $=0.6$, Cond. Prob. $r 3 s 2=0.2$, Cond. Prob. $r 3 s 1=0.1$, Percent drop in speed $\%=-56$

Max. Posterior Probability level $=0.97$, Percent range between speed states $=7 \%$

Drop in speed was detected and No indication of an incident within this period 


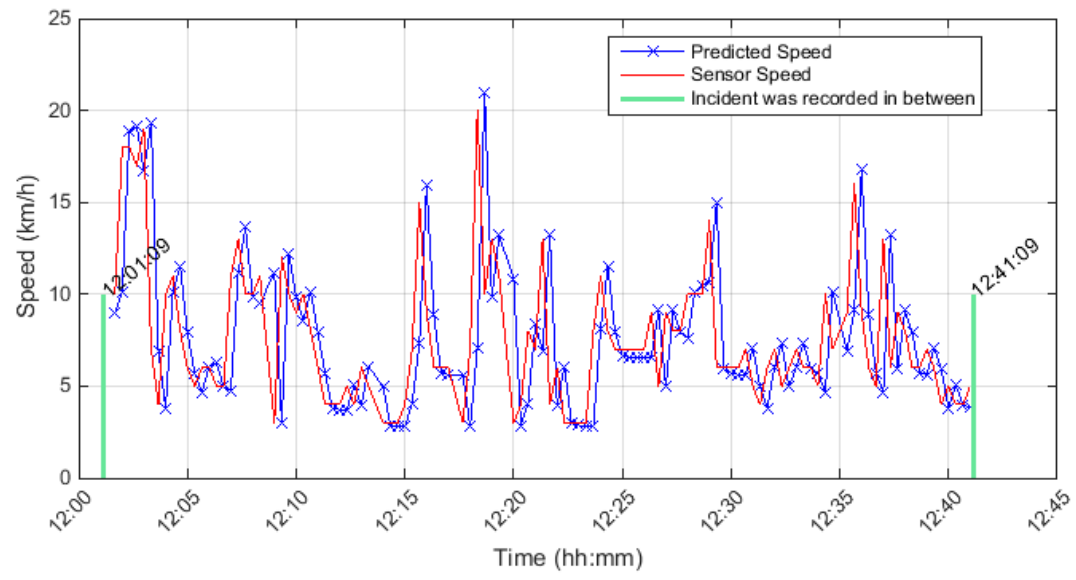

Upstream Detector \# is 401DW0060DWC, Detector Rank = 1, Distance from incident location in $(\mathrm{km})=0.43697$ Season $=$ Summer, Stream $=$ C, Direction $=$ W, Date $\&$ Time $=2011-10-21$ 12:21:09 Incident Reason $=$ Collision

Affected Lanes $=$ LEFT SHOULDER AND 1 LEFT LANE(S)

Conditional Probability level $=0.6$, Cond. Prob. $r 3 s 2=0.2$, Cond. Prob. $r 3 s 1=0.1$, Percent drop in speed $\%=-56$

Max. Posterior Probability level $=0.97$, Percent range between speed states $=7 \%$

Drop in speed was detected and No indication of an incident within this period

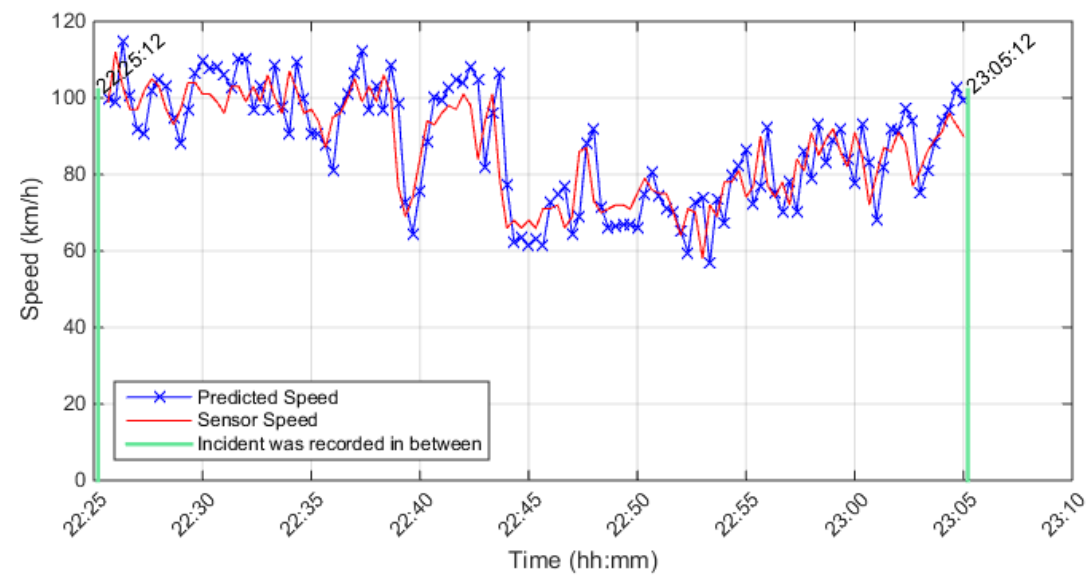

Upstream Detector \# is 401DE0100DWC, Detector Rank $=1$, Distance from incident location in $(\mathrm{km})=0.11847$ Season $=$ Winter, Stream $=C$, Direction $=W$, Date \& Time $=2011-12-24$ 22:45:12 Incident Reason $=$ Collision

Affected Lanes = 1 LEFT LANE(S)

Conditional Probability level $=0.6$, Cond.Prob. $r 3 s 2=0.2$, Cond. Prob. $r 3 s 1=0.1$. Percent drop in speed $\%=-56$

Max. Posterior Probability level $=0.97$, Percent range between speed states $=7 \%$

(No Incident was Detected)

The drop in speed never reached $56 \%$ of the percentile speed, so the incident was not detected. Even when the percentage of the drop in speed was lowered to $40 \%$, the incident 
was not confirmed. That is because speed recovered quickly within $5 \mathrm{~min}$ as in the next

\section{figure}

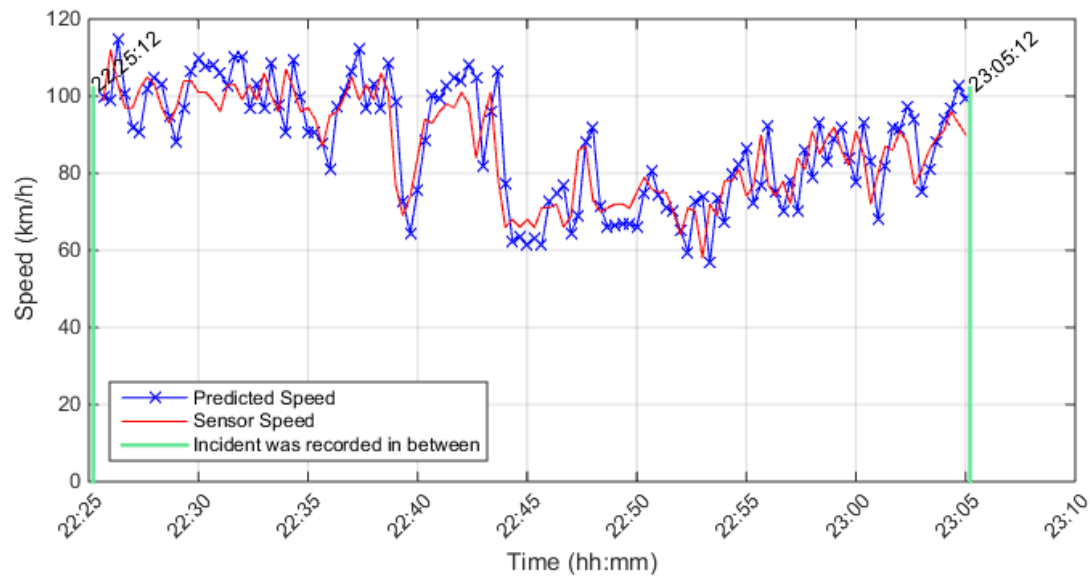

Upstream Detector \# is 401DE0100DWC, Detector Rank = 1, Distance from incident location in $(\mathrm{km})=0.11847$ Season $=$ Winter, Stream $=$ C, Direction $=$ W, Date $\&$ Time $=2011-12-24$ 22:45:12 Incident Reason $=$ Collision

Affected Lanes $=1$ LEFT LANE(S)

Conditional Probability level $=0.6$, Cond.Prob. $r 3 s 2=0.2$, Cond. Prob. $r 3 s 1=0.1$, Percent drop in speed $\%=-40$ Max. Posterior Probability level $=0.97$, Percent range between speed states $=7 \%$ Drop in speed was detected and No indication of an incident within this period

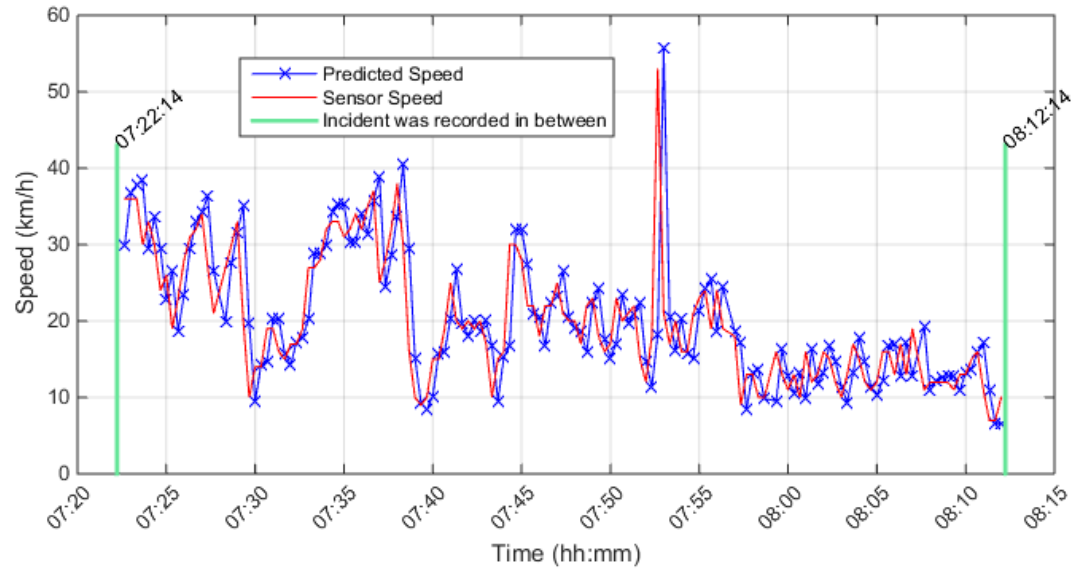

Upstream Detector \# is 401DE0210DWE, Detector Rank = 1, Distance from incident location in $(\mathrm{km})=0.30762$ Season $=$ Winter, Stream $=E$, Direction $=W$, Date $\&$ Time $=$ 2011-01-17 07:42:14 Incident Reason $=$ Disable Vehicle Affected Lanes $=2$ RIGHT LANE(S)

Conditional Probability level $=0.6$, Cond. Prob. $r 3 s 2=0.2$, Cond. Prob. $r 3 s 1=0.1$, Percent drop in speed $\%=-56$

Max. Posterior Probability level $=0.97$, Percent range between speed states $=7 \%$

Drop in speed was detected but Not enough recovery time to confirm an incident 


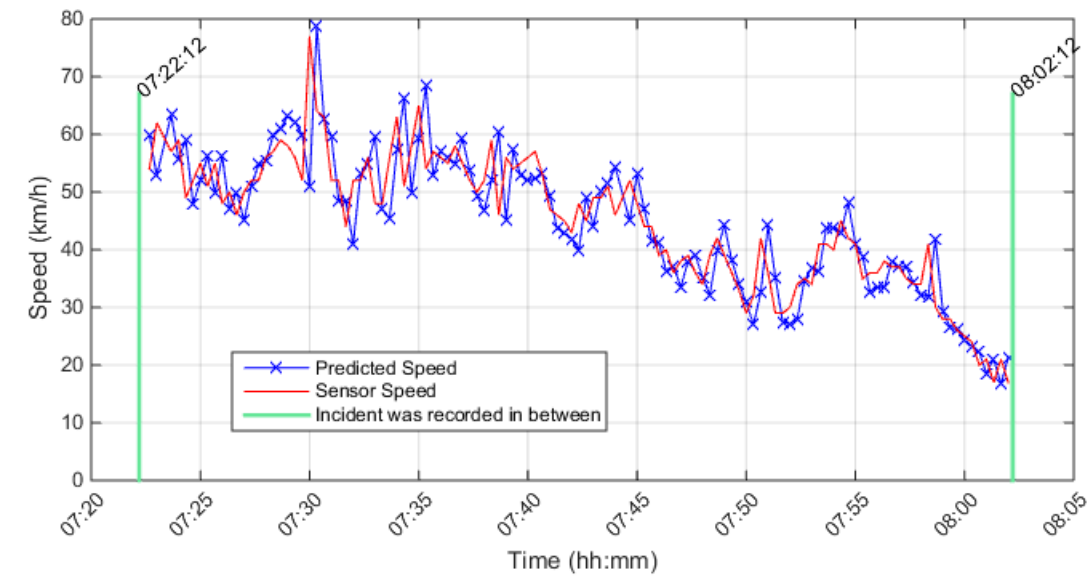

Upstream Detector \# is 401DE0190DWE, Detector Rank = 1, Distance from incident location in $(\mathrm{km})=0.15621$ Season $=$ Winter, Stream $=$ E, Direction $=$ W, Date $\&$ Time $=2011-01-15$ 07:42:12

Incident Reason $=$ Collision

Affected Lanes = 2 RIGHT LANE(S) AND RIGHT SHOULDER

Conditional Probability level $=0.6$, Cond.Prob. $r 3 s 2=0.2$, Cond.Prob. $r 3 s 1=0.1$, Percent drop in speed $\%=-56$

Max. Posterior Probability level $=0.97$, Percent range between speed states $=7 \%$

Drop in speed was detected but Not enough recovery time to confirm an incident

According to the given time frame, 20 min before and $20 \mathrm{~min}$ after the recovery time does not pass to confirm the incident.

If $10 \mathrm{~min}$ is added to the time after, in an attempt to investigate more about any possibility of an incident, the results will be different as presented in the next slide 


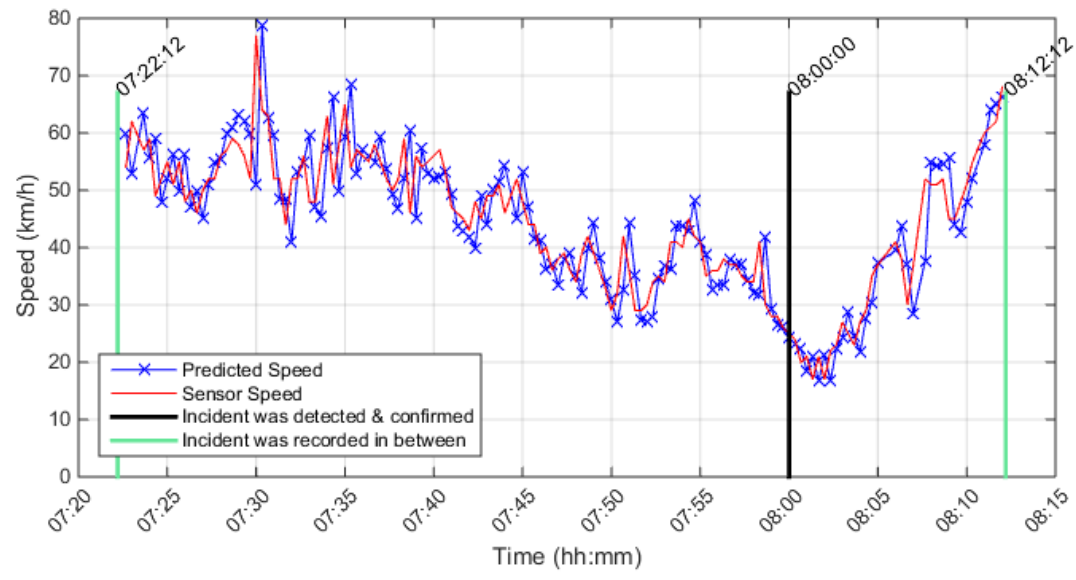

Upstream Detector \# is 401DE0190DWE, Detector Rank = 1, Distance from incident location in $(\mathrm{km})=0.15621$ Season $=$ Winter, Stream $=E$, Direction $=W$, Date $\&$ Time $=2011-01-15$ 07:42:12 Incident Reason $=$ Collision

Affected Lanes $=2$ RIGHT LANE(S) AND RIGHT SHOULDER

Conditional Probability level $=0.6$, Cond.Prob. $r 3 \mathrm{~s} 2=0.2$, Cond. Prob. $r 3 \mathrm{~s} 1=0.1$, Percent drop in speed $\%=-56$

Max. Posterior Probability level $=0.97$, Percent range between speed states $=7 \%$

Incident was detected at 05:20 minutes from drop in speed point. Incident was confirmed after 5 minutes from the detection time

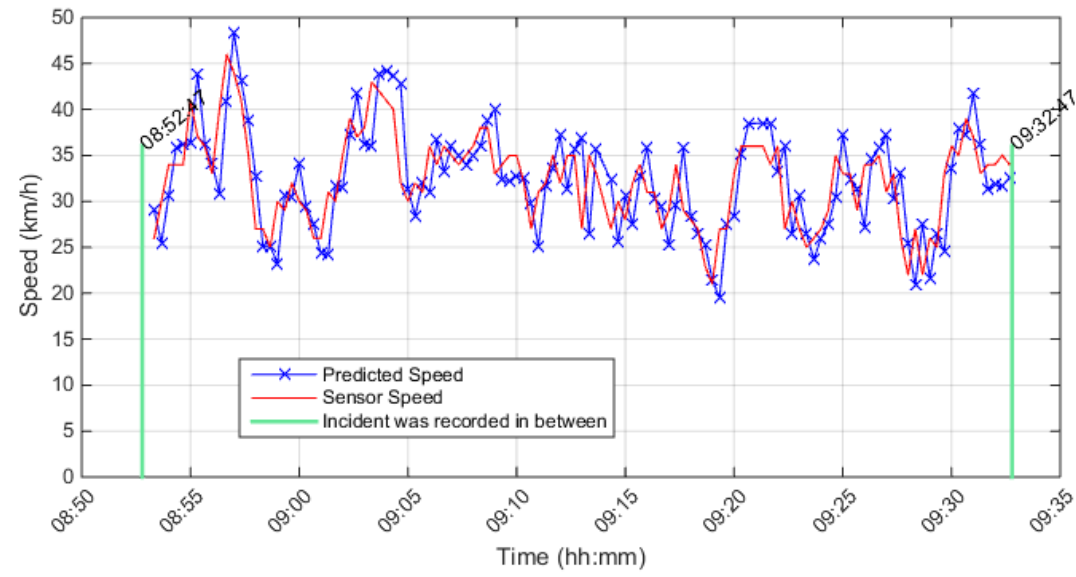

Upstream Detector \# is 401DE0360DWE, Detector Rank $=2$, Distance from incident location in $(\mathrm{km})=0.97011$ Season $=$ Spring. Stream $=$ E, Direction $=$ W, Date $\&$ Time $=2011-05-26$ 09:12:47 Incident Reason $=$ Collision

Affected Lanes $=2$ LEFT LANE(S)

Conditional Probability level $=0.6$, Cond.Prob. $r 3 s 2=0.2$, Cond. Prob. $r 3 s 1=0.1$, Percent drop in speed $\%=-56$ Max. Posterior Probability level $=0.97$, Percent range between speed states $=7 \%$

(No Incident was Detected) 


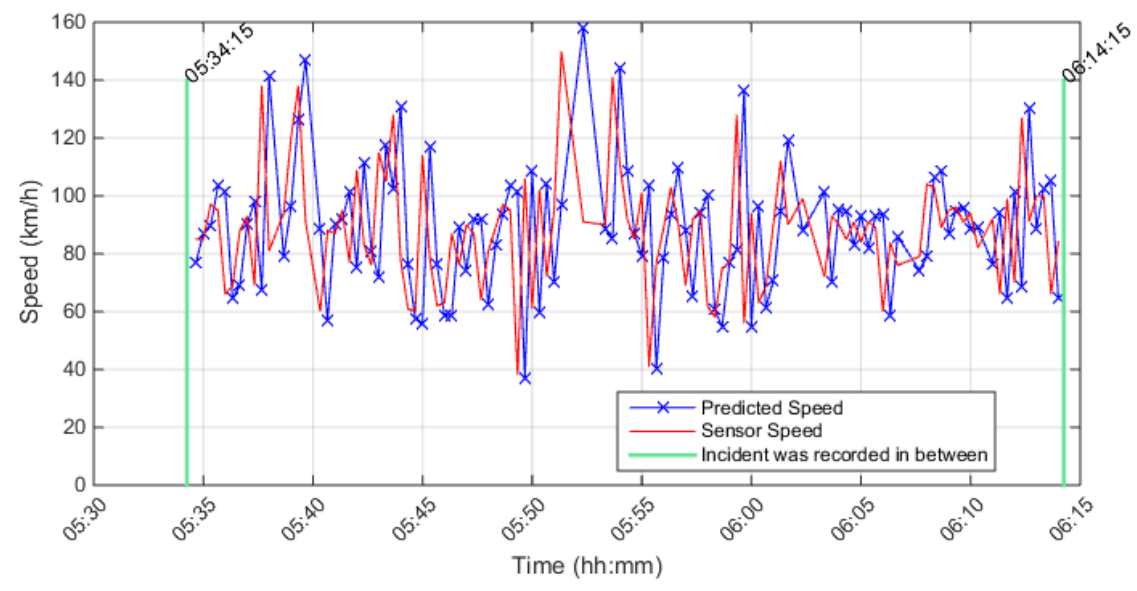

Upstream Detector \# is 401DE0340DWE, Detector Rank = 3, Distance from incident location in $(\mathrm{km})=1.6024$ Season $=$ Summer, Stream $=E$, Direction $=W$, Date $\&$ Time $=$ 2011-06-12 05:54:15

Incident Reason $=$ Collision

Affected Lanes = 2 RIGHT LANE(S) AND RIGHT SHOULDER

Conditional Probability level $=0.6$, Cond.Prob. $r 3 s 2=0.2$, Cond. Prob. $r 3 s 1=0.1$, Percent drop in speed $\%=-56$ Max. Posterior Probability level $=0.97$, Percent range between speed states $=7 \%$ (No Incident was Detected)

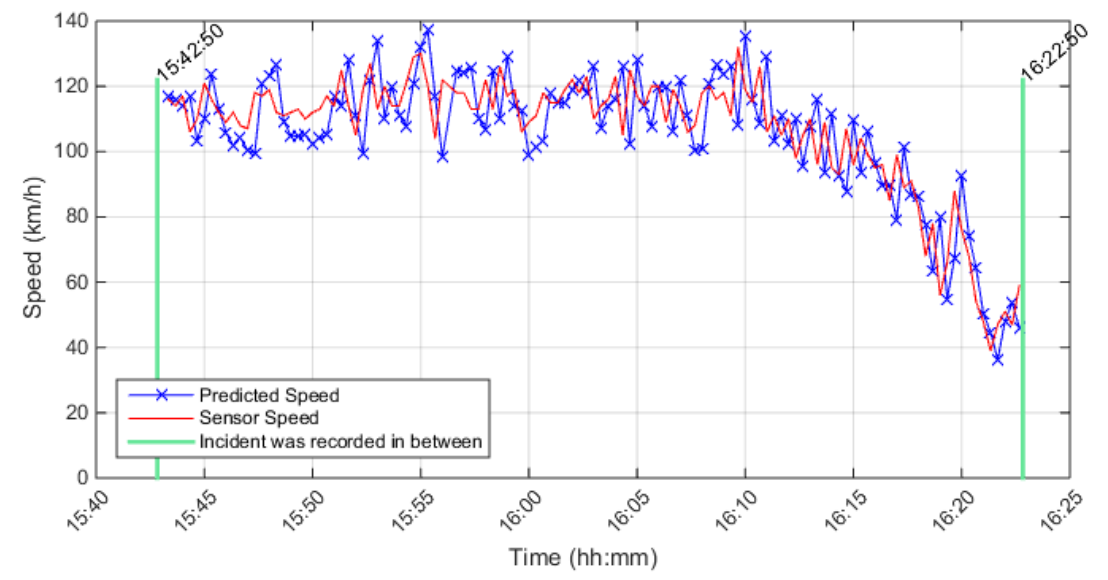

Upstream Detector \# is 401DE0150DWE, Detector Rank = 2, Distance from incident location in $(\mathrm{km})=1.3152$ Season $=$ Summer, Stream $=E$, Direction $=W$, Date $\&$ Time $=2011-07-0916: 02: 50$ Incident Reason $=$ Collision

Affected Lanes = LEFT SHOULDER AND 1 LEFT LANE(S)

Conditional Probability level $=0.6$, Cond.Prob. $r 3 \mathrm{~s} 2=0.2$, Cond.Prob. $r 3 \mathrm{~s} 1=0.1$, Percent drop in speed $\%=-56$

Max. Posterior Probability level $=0.97$, Percent range between speed states $=7 \%$

Drop in speed was detected but Not enough recovery time to confirm an incident

\section{Considering the time after is $30 \mathrm{~min}$}




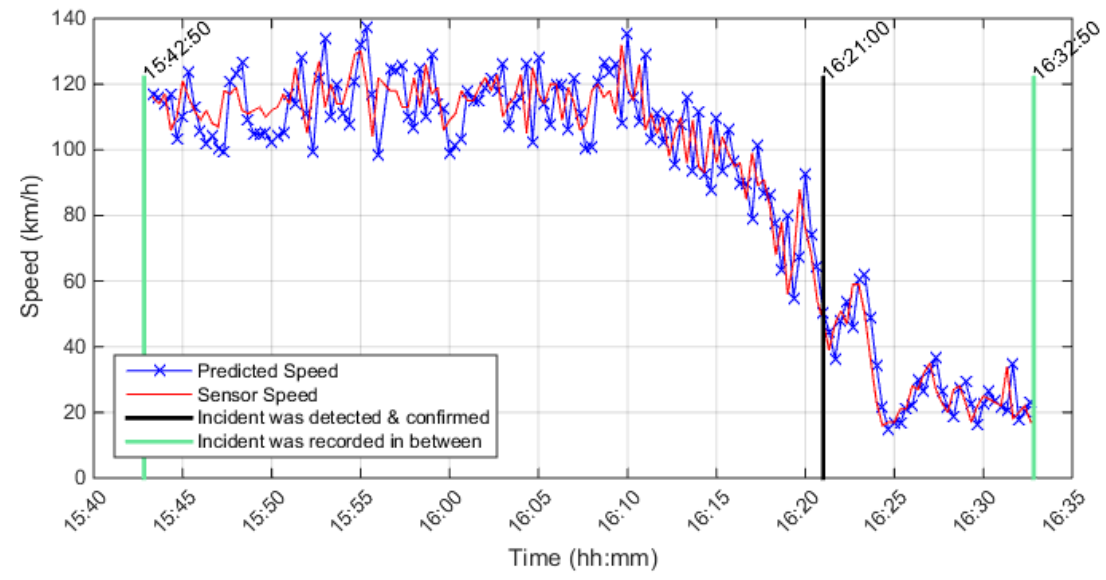

Upstream Detector \# is 401DE0150DWE, Detector Rank = 2, Distance from incident location in $(\mathrm{km})=1.3152$ Season $=$ Summer, Stream $=E$, Direction $=W$, Date $\&$ Time $=$ 2011-07-09 16:02:50 Incident Reason $=$ Collision

Affected Lanes $=$ LEFT SHOULDER AND 1 LEFT LANE(S)

Conditional Probability level $=0.6$, Cond.Prob. $r 3 \mathrm{~s} 2=0.2$, Cond.Prob. $\mathrm{r} 3 \mathrm{~s} 1=0.1$, Percent drop in speed $\%=-56$

Max. Posterior Probability level $=0.97$, Percent range between speed states $=7 \%$

Incident was detected at 03:40 minutes from drop in speed point. Incident was confirmed within 5 minutes from the detection timı

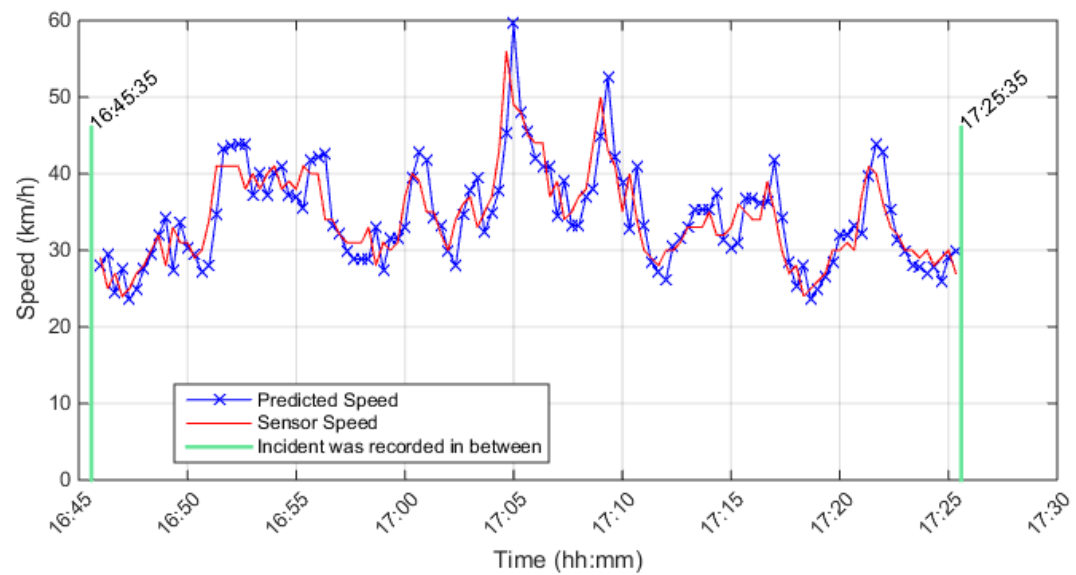

Upstream Detector \# is 401DE0060DWE, Detector Rank = 1, Distance from incident location in $(\mathrm{km})=0.17254$ Season $=$ Summer, Stream $=E$, Direction $=W$, Date $\&$ Time $=2011-09-15$ 17:05:35

Incident Reason $=$ Disable Vehicle

Affected Lanes = 2 RIGHT LANE(S)

Conditional Probability level $=0.6$, Cond. Prob. $r 3 s 2=0.2$, Cond. Prob. $r 3 s 1=0.1$, Percent drop in speed $\%=-56$

Max. Posterior Probability level $=0.97$, Percent range between speed states $=7 \%$

(No Incident was Detected) 


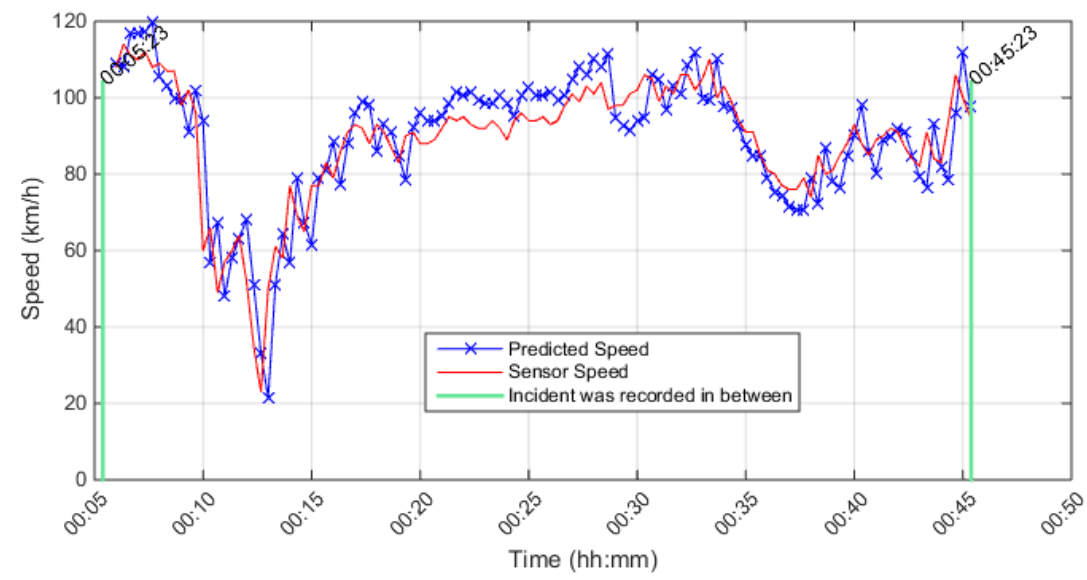

Upstream Detector \# is 401DW0040DWE, Detector Rank $=1$, Distance from incident location in $(\mathrm{km})=0.22683$ Season $=$ Winter, Stream $=E$, Direction $=$ W, Date \& Time $=$ 2011-12-21 00:25:23 Incident Reason $=$ Collision

Affected Lanes $=$ LEFT SHOULDER AND 2 LEFT LANE(S)

Conditional Probability level $=0.6$, Cond. Prob. $r 3 s 2=0.2$, Cond. Prob. $r 3 s 1=0.1$, Percent drop in speed $\%=-56$

Max. Posterior Probability level $=0.97$, Percent range between speed states $=7 \%$

Drop in speed was detected and No indication of an incident within this period

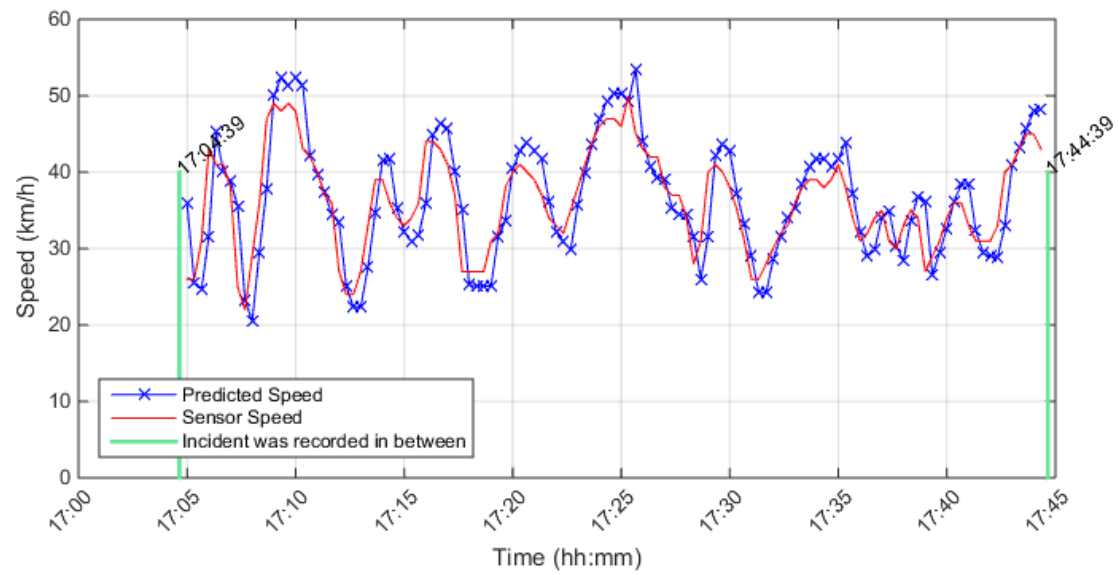

Upstream Detector \# is 401DE0100DEC, Detector Rank $=1$, Distance from incident location in $(\mathrm{km})=0.26025$ Season $=$ Winter, Stream $=$ C , Direction $=E$, Date $\&$ Time $=2011-02-08$ 17:24:39

Incident Reason $=$ Disable Vehicle

Affected Lanes $=2$ RIGHT LANE(S) AND RIGHT SHOULDER

Conditional Probability level $=0.6$, Cond.Prob. $r 3 s 2=0.2$, Cond.Prob. $r 3 s 1=0.1$, Percent drop in speed $\%=-56$

Max. Posterior Probability level $=0.97$, Percent range between speed states $=7 \%$

Drop in speed was detected and No indication of an incident within this period 


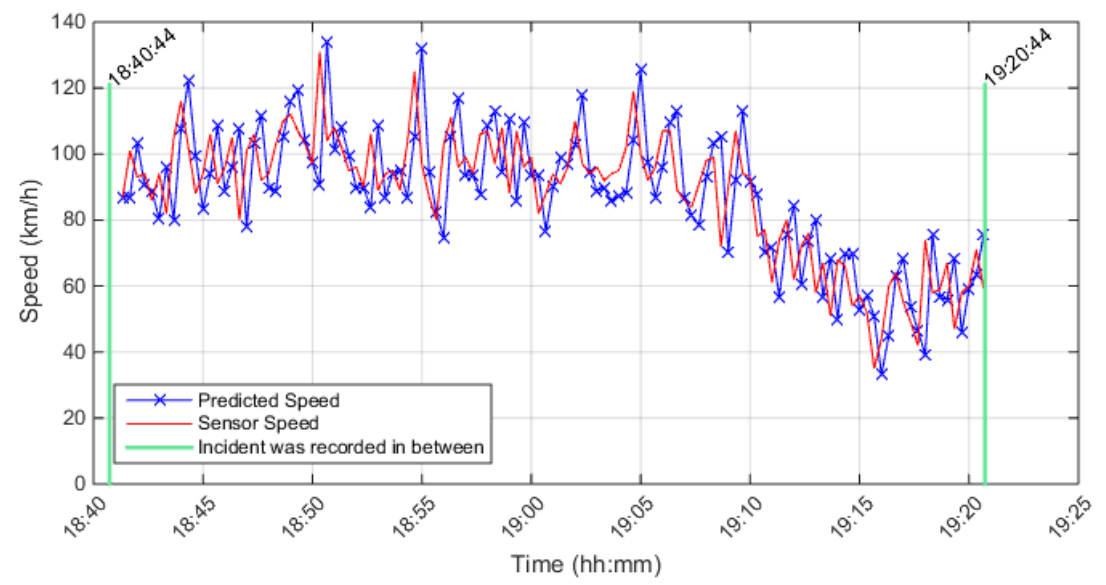

Upstream Detector \# is 401DE0330DEC, Detector Rank = 3, Distance from incident location in $(\mathrm{km})=1.5366$ Season $=$ Summer, Stream $=$ C, Direction $=E$, Date $\&$ Time $=2011-06-24$ 19:00:44 Incident Reason $=$ Collision

Affected Lanes $=$ All lanes closed

Conditional Probability level $=0.6$, Cond.Prob. $\mathrm{r} 3 \mathrm{~s} 2=0.2$, Cond. Prob. $\mathrm{r} 3 \mathrm{~s} 1=0.1$, Percent drop in speed $\%=-56$

Max. Posterior Probability level $=0.97$, Percent range between speed states $=7 \%$

Drop in speed was detected and No indication of an incident within this period

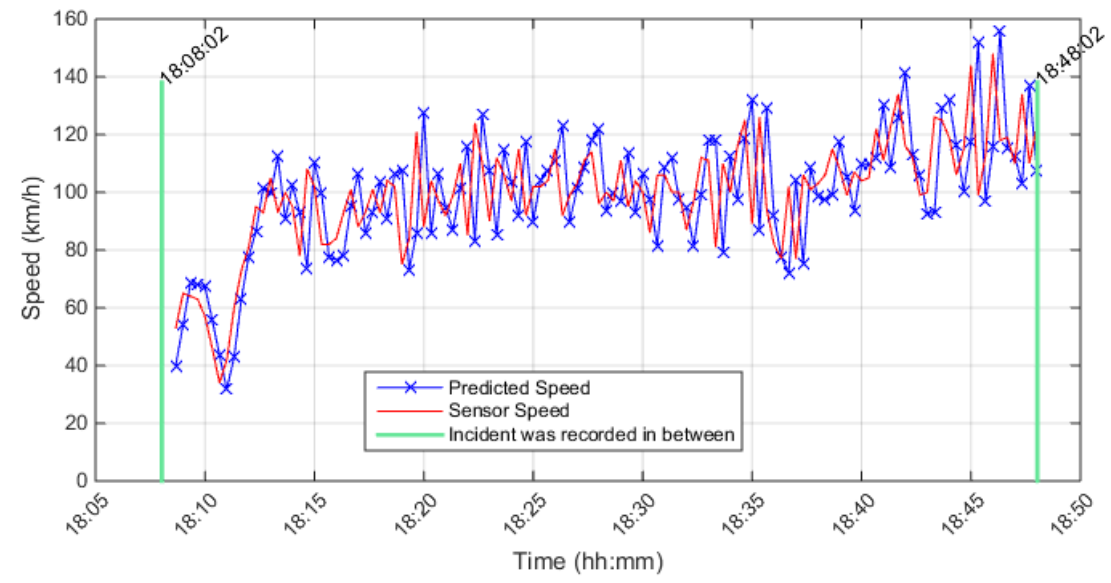

Upstream Detector \# is 401DE0110DEC, Detector Rank = 2, Distance from incident location in $(\mathrm{km})=0.78244$ Season $=$ Summer, Stream $=C$, Direction $=E$, Date $\&$ Time $=2011-07-1918: 28: 02$ Incident Reason $=$ Collision

Affected Lanes $=$ LEFT SHOULDER AND 3 LEFT LANE(S)

Conditional Probability level $=0.6$, Cond.Prob. $r 3 s 2=0.2$, Cond. Prob. $r 3 s 1=0.1$, Percent drop in speed $\%=-56$

Max. Posterior Probability level $=0.97$, Percent range between speed states $=7 \%$

(No Incident was Detected) 


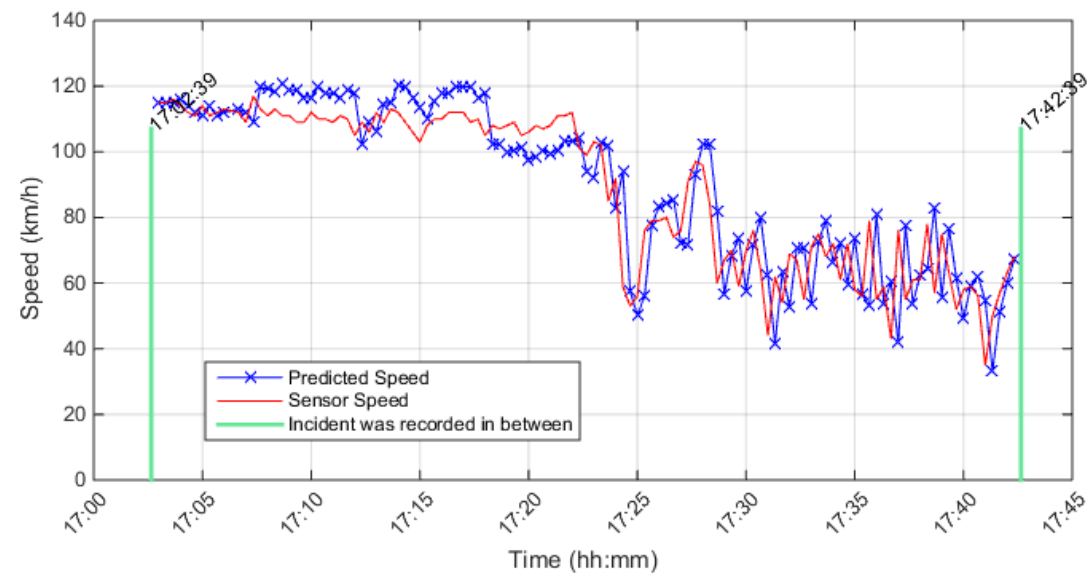

Upstream Detector \# is 401DE0320DEE, Detector Rank $=2$, Distance from incident location in $(\mathrm{km})=0.87834$ Season $=$ Winter, Stream $=E$, Direction $=E$, Date $\&$ Time $=2011-01-1917: 22: 39$ Incident Reason $=$ Collision

Affected Lanes = 2 RIGHT LANE(S)

Conditional Probability level $=0.6$, Cond. Prob. $r 3 s 2=0.2$, Cond. Prob. $r 3 s 1=0.1$, Percent drop in speed $\%=-56$ Max. Posterior Probability level $=0.97$, Percent range between speed states $=7 \%$ (No Incident was Detected)

Same as previous case if time frame is selected to be 40 min before and 40 min after

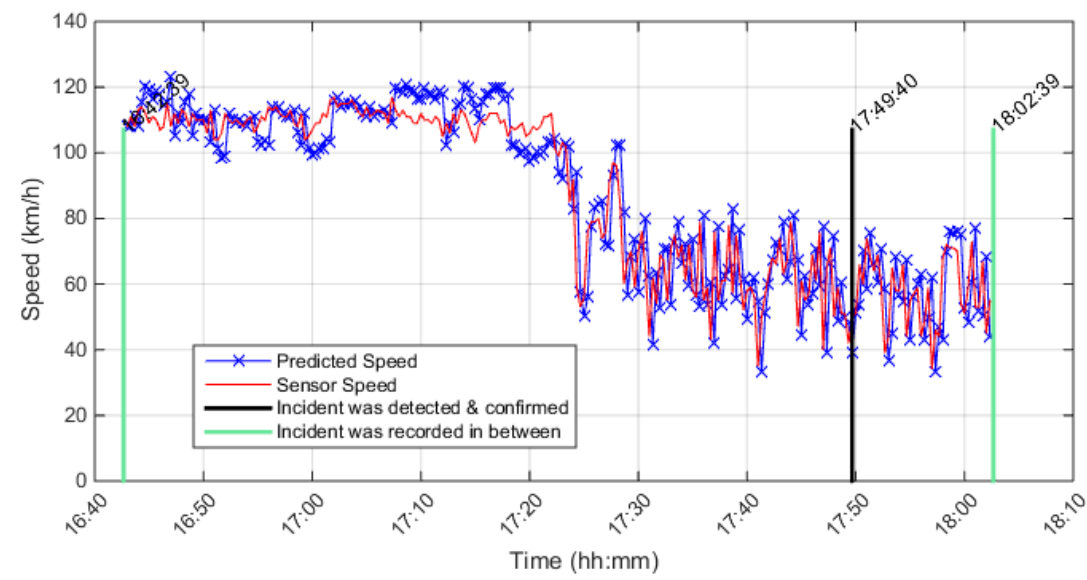

Upstream Detector \# is 401DE0320DEE, Detector Rank $=2$, Distance from incident location in $(\mathrm{km})=0.87834$ Season $=$ Winter, Stream $=E$, Direction $=E$, Date \& Time $=2011-01-19$ 17:22:39 Incident Reason $=$ Collision

Affected Lanes = 2 RIGHT LANE(S)

Conditional Probability level $=0.6$, Cond.Prob. $r 3 s 2=0.2$, Cond.Prob. $r 3 s 1=0.1$, Percent drop in speed $\%=-56$

Max. Posterior Probability level $=0.97$, Percent range between speed states $=7 \%$

Incident was detected at 21:20 minutes from drop in speed point. Incident was confirmed within 5 minutes from the detection tim 


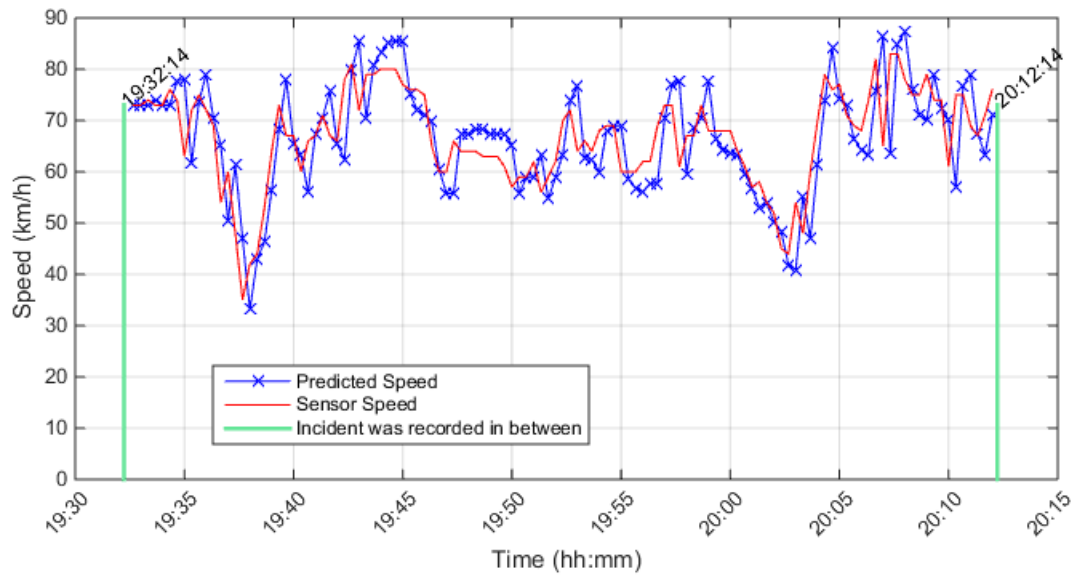

Upstream Detector \# is 401DW0060DWC, Detector Rank = 1, Distance from incident location in $(\mathrm{km})=0.43697$ Season $=$ Winter, Stream $=$ C, Direction $=$ W, Date $\&$ Time $=$ 2011-02-18 19:52:14 Incident Reason $=$ Collision

Affected Lanes = LEFT SHOULDER AND 2 LEFT LANE(S)

Conditional Probability level $=0.6$, Cond.Prob. $r 3 s 2=0.2$, Cond. Prob. $r 3 s 1=0.1$, Percent drop in speed $\%=-56$ Max. Posterior Probability level $=0.97$, Percent range between speed states $=7 \%$ (No Incident was Detected)

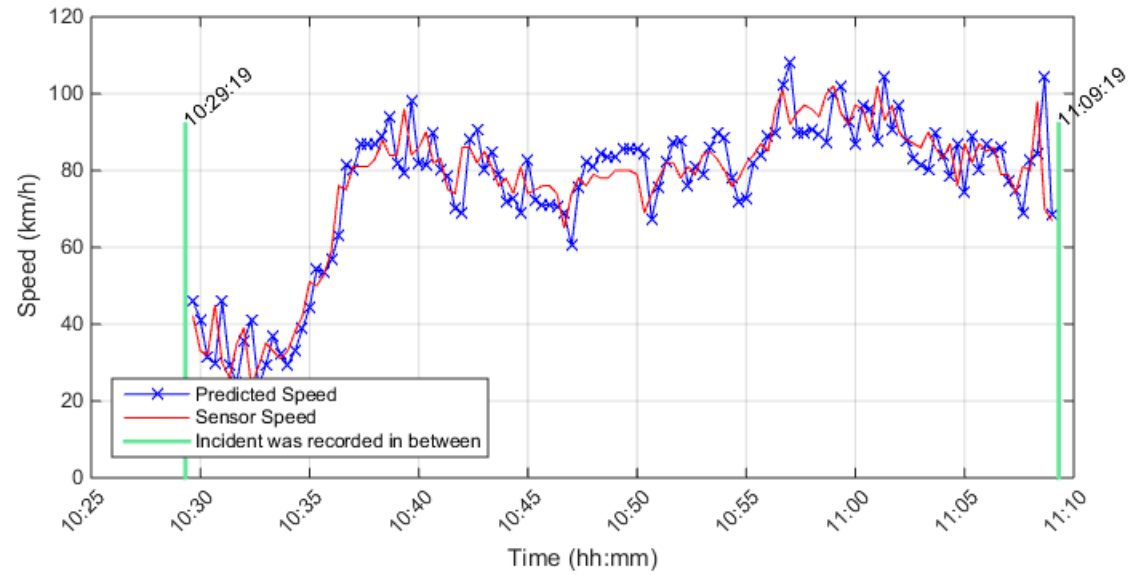

Upstream Detector \# is 401DE0090DWC, Detector Rank $=1$, Distance from incident location in $(\mathrm{km})=0.59485$ Season $=$ Summer, Stream $=$ C, Direction $=W$, Date $\&$ Time $=2011-06-06$ 10:49:19 ncident Reason $=$ Collision

Affected Lanes $=$ LEFT SHOULDER AND 2 LEFT LANE(S)

Conditional Probability level $=0.6$, Cond. Prob. $r 3 \mathrm{~s} 2=0.2$, Cond. Prob. $r 3 \mathrm{~s} 1=0.1$, Percent drop in speed $\%=-56$

Max. Posterior Probability level $=0.97$, Percent range between speed states $=7 \%$

(No Incident was Detected)

\section{Same as previous case if 60 min before incident recorded time is selected}




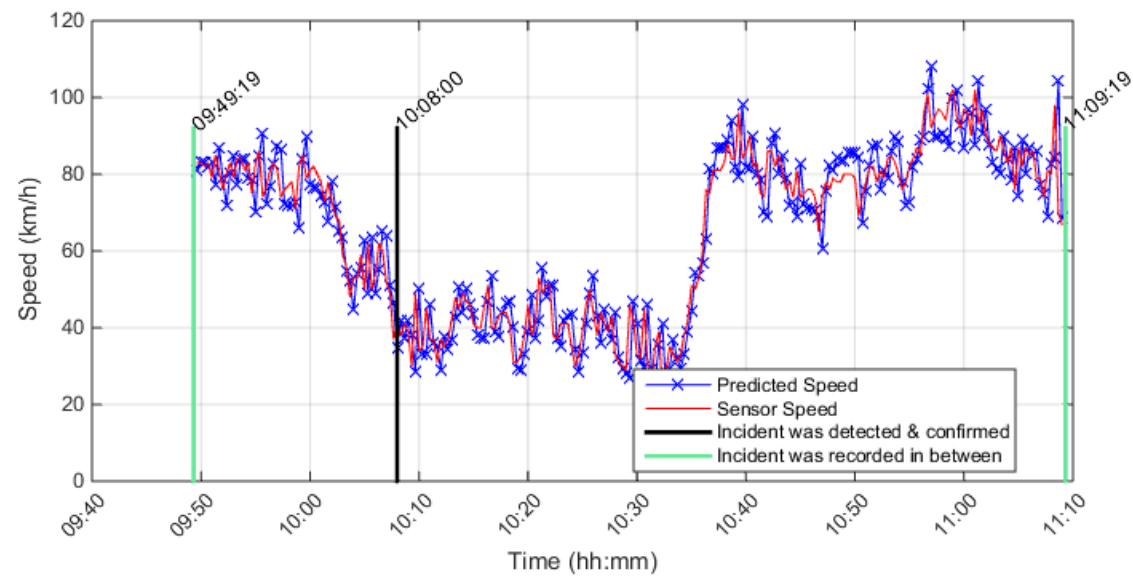

Upstream Detector \# is 401DE0090DWC, Detector Rank = 1, Distance from incident location in $(\mathrm{km})=0.59485$ Season $=$ Summer, Stream $=$ C, Direction $=W$, Date \& Time $=$ 2011-06-06 10:49:19 Incident Reason $=$ Collision

Affected Lanes = LEFT SHOULDER AND 2 LEFT LANE(S)

Conditional Probability level $=0.6$, Cond.Prob. $r 3 s 2=0.2$, Cond.Prob. $r 3 s 1=0.1$, Percent drop in speed $\%=-56$

Max. Posterior Probability level $=0.97$, Percent range between speed states $=7 \%$

Incident was detected at 01:00 minutes from drop in speed point. Incident was confirmed within 5 minutes from the detection tim

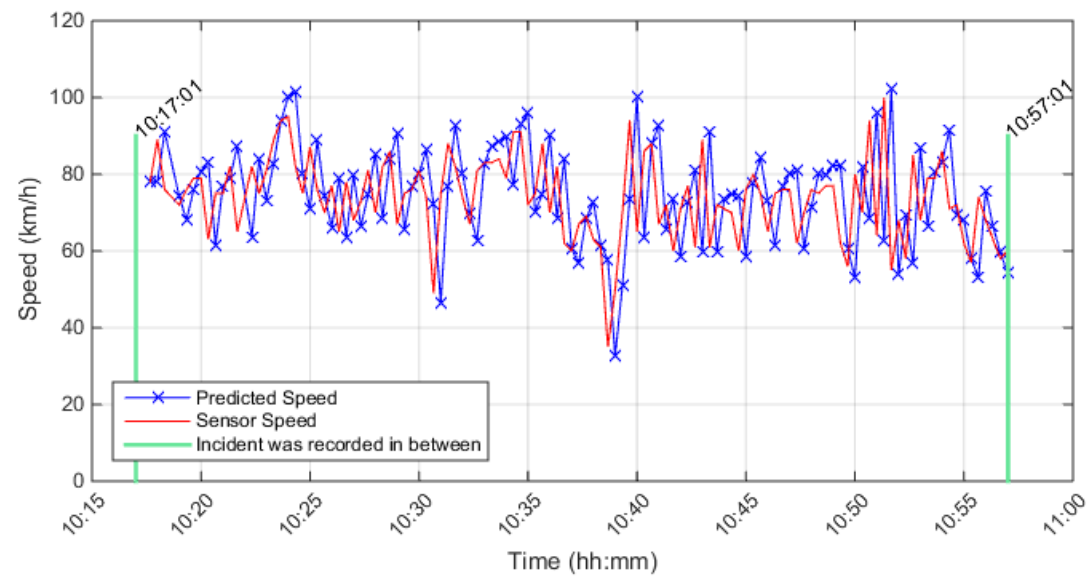

Upstream Detector \# is 401DE0430DWC, Detector Rank = 1, Distance from incident location in $(\mathrm{km})=0.5003$ Season $=$ Summer, Stream $=$ C, Direction $=$ W, Date $\&$ Time $=2011-09-15$ 10:37:01 Incident Reason $=$ Collision

Affected Lanes = LEFT SHOULDER AND 4 LEFT LANE(S)

Conditional Probability level $=0.6$, Cond. Prob. $r 3 s 2=0.2$, Cond. Prob. $r 3 s 1=0.1$, Percent drop in speed $\%=-56$

Max. Posterior Probability level $=0.97$, Percent range between speed states $=7 \%$

Drop in speed was detected and No indication of an incident within this period

For the previous case, even if $120 \mathrm{~min}$ before and after is considered, no indication of any drop in speed is shown. 


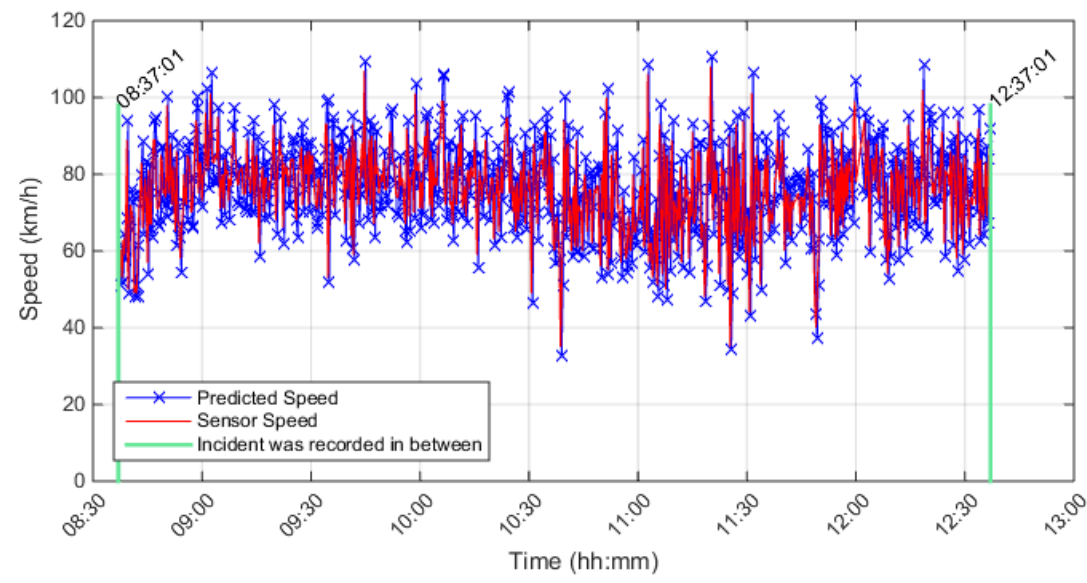

Upstream Detector \# is 401DE0430DWC, Detector Rank = 1, Distance from incident location in $(\mathrm{km})=0.5003$ Season $=$ Summer, Stream $=$ C, Direction $=$ W, Date \& Time $=$ 2011-09-15 10:37:01 Incident Reason $=$ Collision

Affected Lanes $=$ LEFT SHOULDER AND 4 LEFT LANE(S)

Conditional Probability level $=0.6$, Cond. Prob. $r 3 \mathrm{~s} 2=0.2$, Cond. Prob. $\mathrm{r} 3 \mathrm{~s} 1=0.1$, Percent drop in speed $\%=-56$

Max. Posterior Probability level $=0.97$, Percent range between speed states $=7 \%$

Drop in speed was detected and No indication of an incident within this period

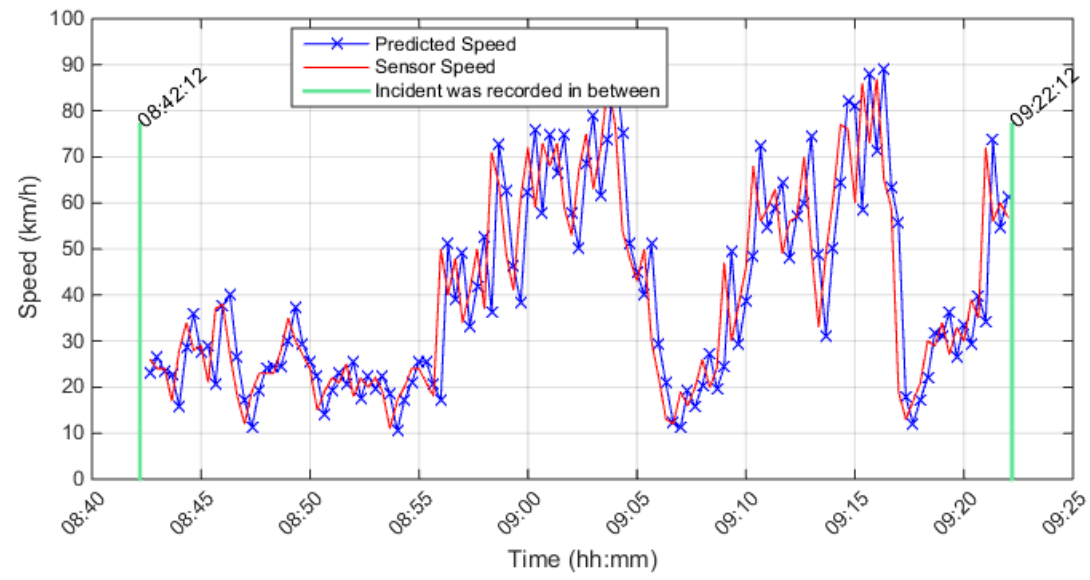

Upstream Detector \# is 401DE0090DWE, Detector Rank = 1, Distance from incident location in $(\mathrm{km})=0.6034$ Season $=$ Summer, Stream $=E$, Direction $=$ W, Date $\&$ Time $=$ 2011-08-02 09:02:12 Incident Reason $=$ Collision

Affected Lanes $=2$ RIGHT LANE(S)

Conditional Probability level $=0.6$, Cond. Prob. $r 3 s 2=0.2$, Cond. Prob. $r 3 s 1=0.1$. Percent drop in speed $\%=-56$

Max. Posterior Probability level $=0.97$, Percent range between speed states $=7 \%$

Drop in speed was detected and No indication of an incident within this period

Same as previous case if $60 \mathrm{~min}$ before and after is selected 


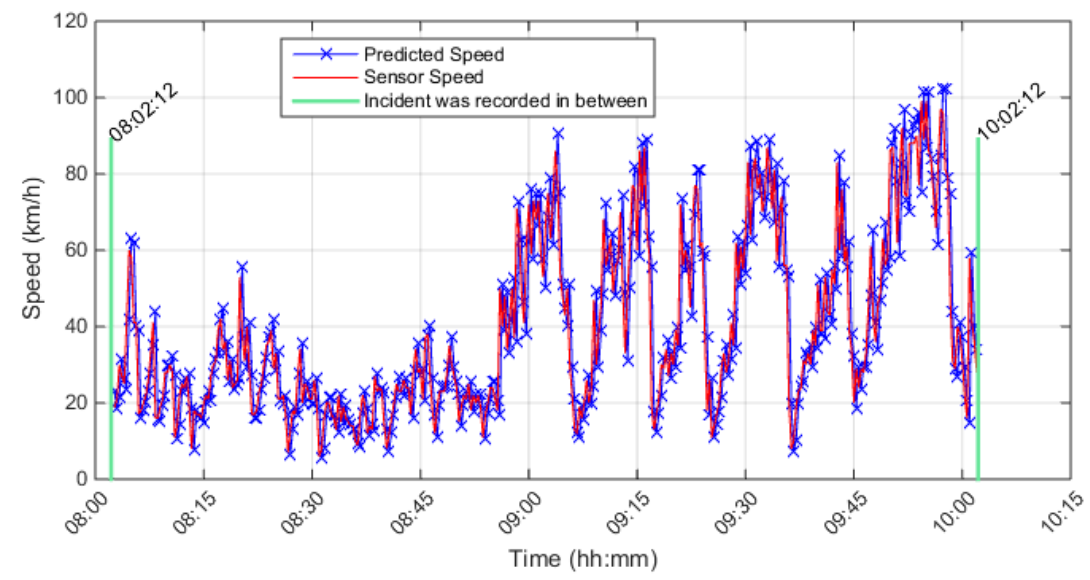

Upstream Detector \# is 401DE0090DWE, Detector Rank = 1, Distance from incident location in $(\mathrm{km})=0.6034$ Season $=$ Summer, Stream $=E$, Direction $=W$, Date $\&$ Time $=$ 2011-08-02 09:02:12 Incident Reason $=$ Collision

Affected Lanes $=2$ RIGHT LANE(S)

Conditional Probability level $=0.6$, Cond.Prob. $r 3 \mathrm{~s} 2=0.2$, Cond. Prob. $r 3 \mathrm{~s} 1=0.1$, Percent drop in speed $\%=-56$ Max. Posterior Probability level $=0.97$, Percent range between speed states $=7 \%$

Drop in speed was detected but Not enough recovery time to confirm an incident

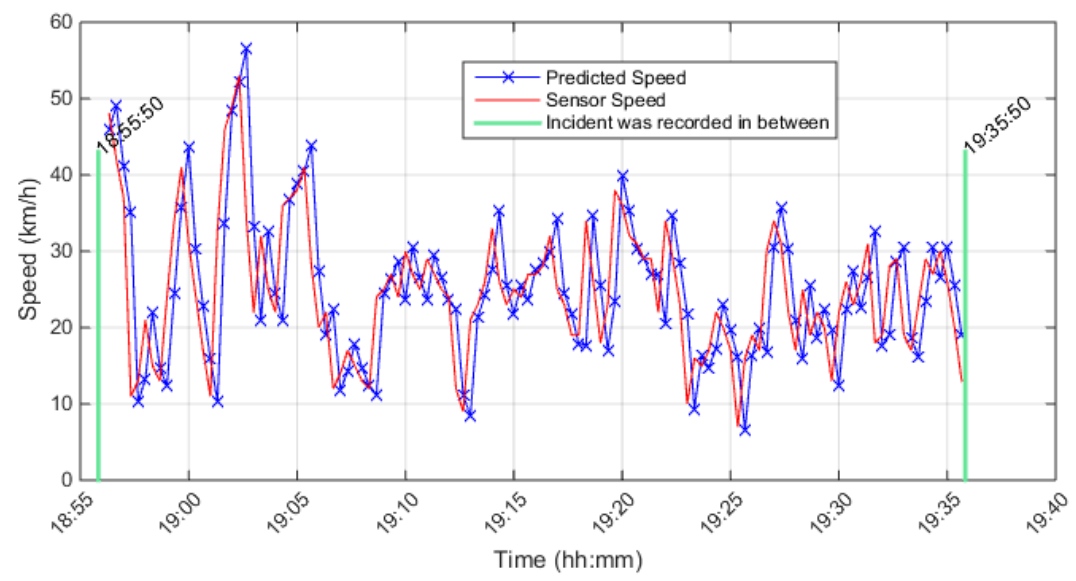

Upstream Detector \# is 401DE0050DWE, Detector Rank $=1$, Distance from incident location in $(\mathrm{km})=0.36941$ Season $=$ Summer, Stream $=E$, Direction $=W$, Date \& Time $=$ 2011-09-17 19:15:50 Incident Reason $=$ Collision

Affected Lanes = 1 RIGHT LANE(S) AND RIGHT SHOULDER

Conditional Probability level $=0.6$, Cond.Prob. $r 3 \mathrm{~s} 2=0.2$, Cond.Prob. $r 3 \mathrm{~s} 1=0.1$, Percent drop in speed $\%=-56$ Max. Posterior Probability level $=0.97$, Percent range between speed states $=7 \%$

Drop in speed was detected and No indication of an incident within this period 


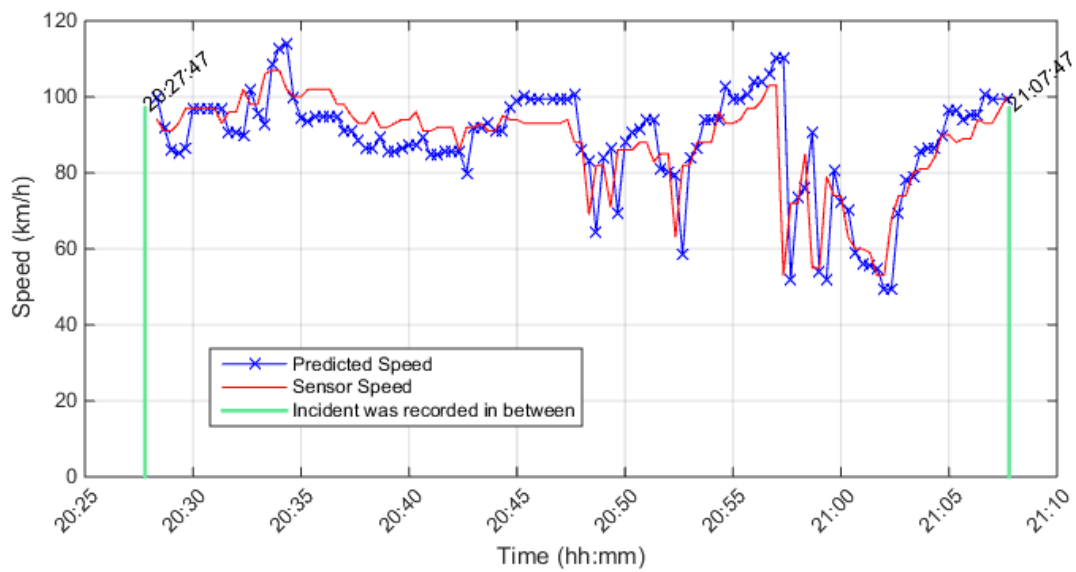

Upstream Detector \# is 401DE0140DWE, Detector Rank = 1, Distance from incident location in $(\mathrm{km})=0.93575$ Season $=$ Winter, Stream $=E$, Direction $=W$, Date $\&$ Time $=$ 2011-12-27 20:47:47

Incident Reason $=$ Collision

Affected Lanes = All lanes closed

Conditional Probability level $=0.6$, Cond.Prob. $r 3 \mathrm{~s} 2=0.2$ Cond. Prob. $r 3 \mathrm{~s} 1=0.1$, Percent drop in speed $\%=-56$

Max. Posterior Probability level $=0.97$, Percent range between speed states $=7 \%$

(No Incident was Detected) 


\section{Appendix C.3 Cases with Incident Recorded and not Detected}

This appendix presents the validation outcomes that resulted from the model application on 73 cases as described in chapter 6. Cases presented in this part are under certain traffic condition "Not Important", by holding the parameters resulted from the calibration process. The model was applied to cases with the incident.

\section{Appendix C.3.1 Cases under Certain Traffic Condition "Not Important."}

Appedix C.3.1.1 Cases with Incident Recorded and Detected...0

Appedix C.3.1.2 Cases with Incident Recorded and Not Detected... 73 cases

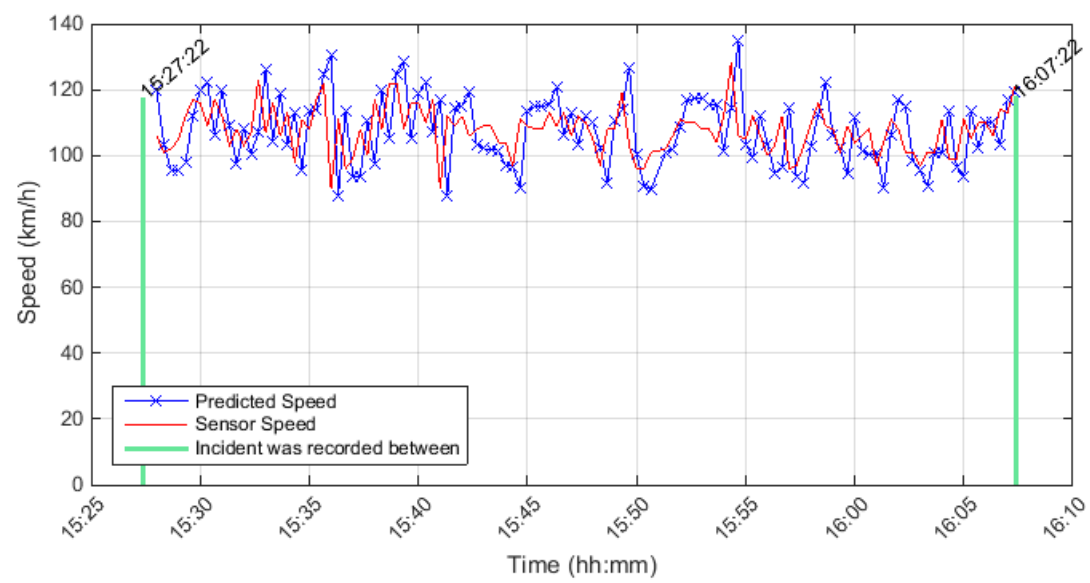

Upstream Detector \# is 401DE0190DWC, Detector Rank = 4, Distance from incident location in $(\mathrm{km})=2.6115$ Season $=$ Winter, Stream $=$ C, Direction $=$ W, Date \& Time $=$ 2011-02-19 15:47:22 Incident Reason $=$ Collision Affected Lanes = 1 RIGHT LANE(S)

Conditional Probability level $=0.6$, Cond.Prob. $r 3 s 2=0.2$, Cond. Prob. $r 3 s 1=0.1$, Percent drop in speed $\%=-56$ Max. Posterior Probability level $=0.97$, Percent range between speed states $=7 \%$ (No Incident was Detected) 
It is noticed that the detector is far away from the incident location which gave wrong readings.

Also, from this case the distance between detectors was found to be $800 \mathrm{~m}$.

Such a case might not be counted because it is not reliable since data for the speed is not available for first three upstream detectors

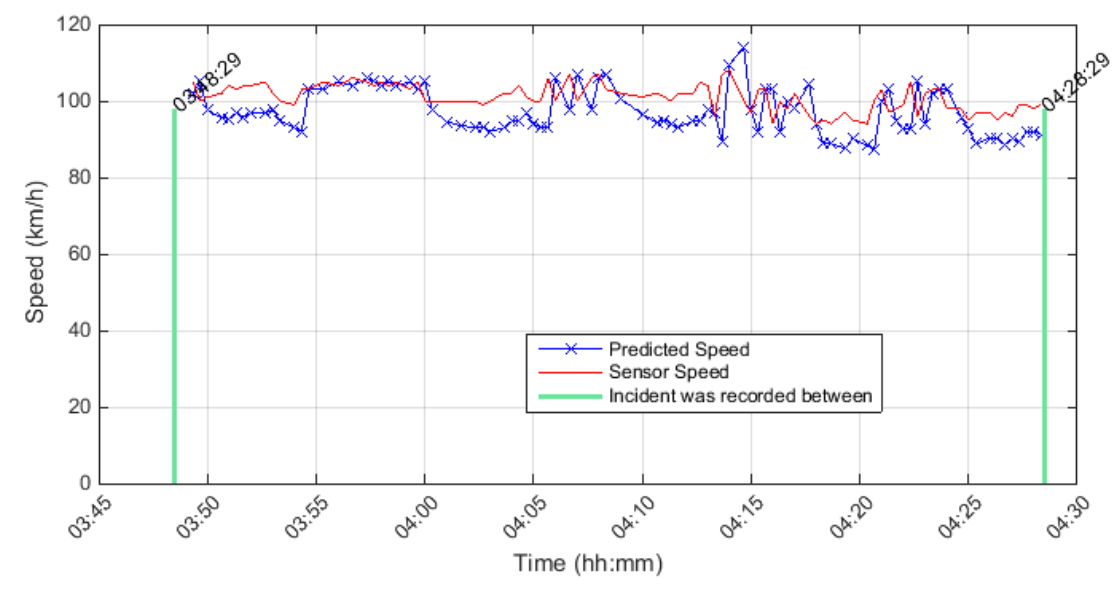

Upstream Detector \# is 401DW0040DWC, Detector Rank = 1, Distance from incident location in $(\mathrm{km})=0.20609$ Season $=$ Spring, Stream $=$ C, Direction $=W$, Date $\&$ Time $=2011-03-18$ 04:08:29 Incident Reason $=$ Disable Vehicle Affected Lanes = 1 RIGHT LANE(S)

Conditional Probability level $=0.6$, Cond. Prob. $r 3 s 2=0.2$, Cond. Prob. $r 3 s 1=0.1$, Percent drop in speed $\%=-56$ Max. Posterior Probability level $=0.97$, Percent range between speed states $=7 \%$ (No Incident was Detected)

It is observed that the time is morning time and only one right lane was closed. Maybe this is the reason behind not experiencing any drop in the speed, and therefore incident was not detected 


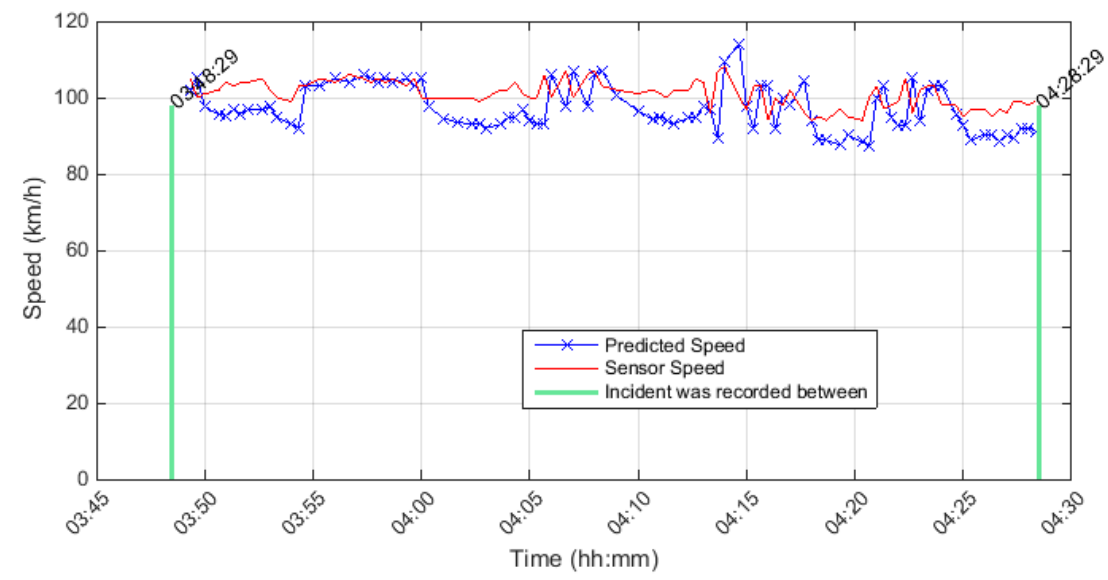

Upstream Detector \# is 401DW0040DWC, Detector Rank = 1, Distance from incident location in $(\mathrm{km})=0.20609$ Season $=$ Spring, Stream $=$ C, Direction $=W$, Date $\&$ Time $=2011-03-18$ 04:08:29 Incident Reason $=$ Disable Vehicle Affected Lanes = 1 RIGHT LANE(S)

Conditional Probability level $=0.6$, Cond.Prob. $r 3 s 2=0.2$. Cond. Prob. $r 3 s 1=0.1$, Percent drop in speed $\%=-56$ Max. Posterior Probability level $=0.97$. Percent range between speed states $=7 \%$ (No Incident was Detected)

It is observed that the time is morning time and only one right lane was closed. Maybe this is the reason behind not experiencing any drop in the speed, and therefore incident was not detected

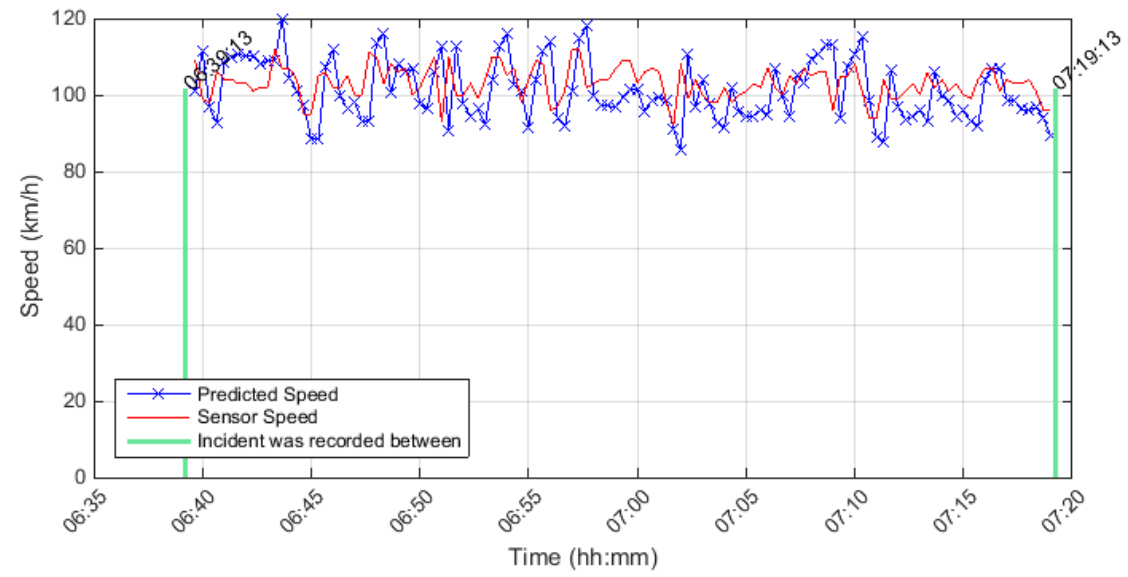

Upstream Detector \# is 401DW0160DWC, Detector Rank = 1, Distance from incident location in $(\mathrm{km})=0.1854$ Season $=$ Spring, Stream $=$ C, Direction $=W$, Date $\&$ Time $=2011-05-04$ 06:59:13 Incident Reason $=$ Collision Affected Lanes $=2$ LEFT LANE(S)

Conditional Probability level $=0.6$, Cond. Prob. $r 3 \mathrm{~s} 2=0.2$, Cond.Prob. $r 3 \mathrm{~s} 1=0.1$, Percent drop in speed $\%=-56$ Max. Posterior Probability level $=0.97$, Percent range between speed states $=7 \%$ (No Incident was Detected) 


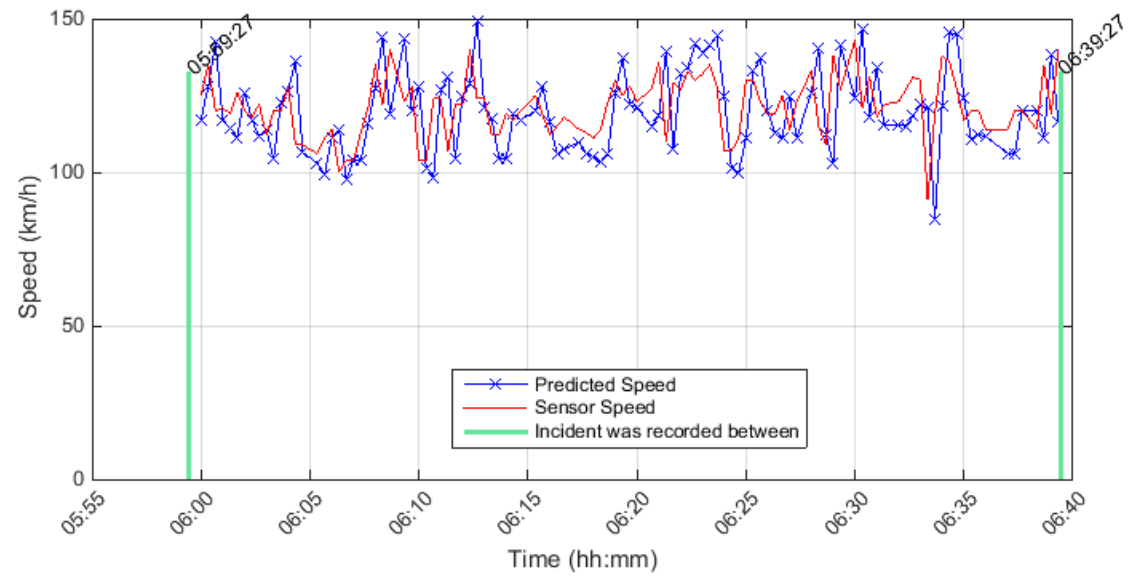

Upstream Detector \# is 401DE0190DWC, Detector Rank = 1, Distance from incident location in $(\mathrm{km})=0.16001$ Season $=$ Spring, Stream $=$ C, Direction $=W$, Date $\&$ Time $=$ 2011-05-14 06:19:27 Incident Reason $=$ Collision

Affected Lanes $=$ LEFT SHOULDER AND 3 LEFT LANE(S)

Conditional Probability level $=0.6$, Cond.Prob. $r 3 s 2=0.2$, Cond.Prob. $r 3 s 1=0.1$, Percent drop in speed $\%=-56$

Max. Posterior Probability level $=0.97$, Percent range between speed states $=7 \%$

(No Incident was Detected)

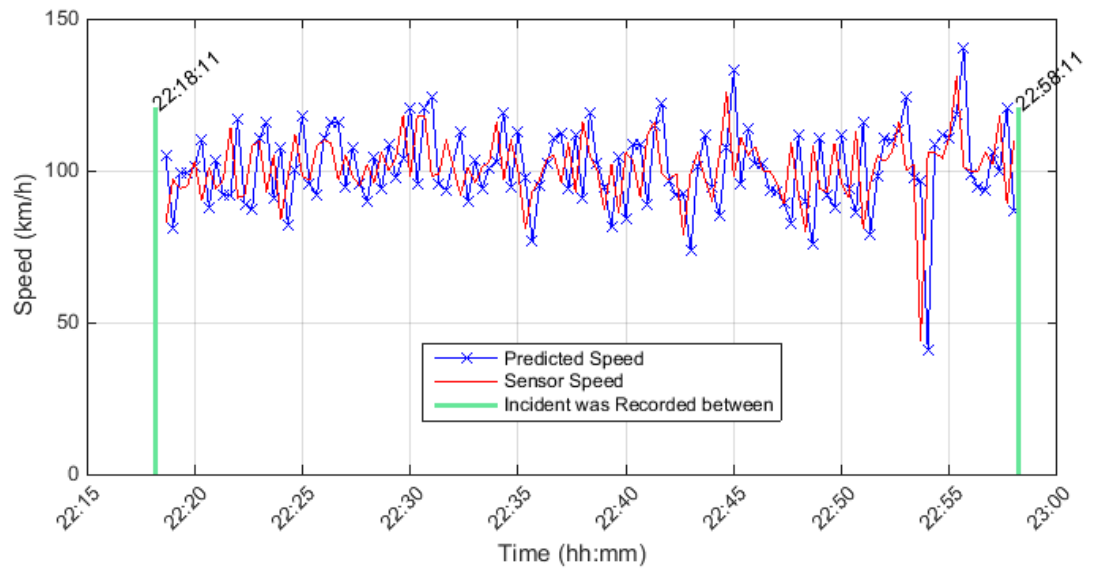

Upstream Detector \# is 401DE0370DWC, Detector Rank $=3$, Distance from incident location in $(\mathrm{km})=2.0297$ Season $=$ Spring, Stream $=$ C, Direction $=$ W, Date $\&$ Time $=$ 2011-05-24 22:38:11 Incident Reason $=$ Collision

Affected Lanes = 2 RIGHT LANE(S)

Conditional Probability level $=0.6$, Cond.Prob. $r 3 s 2=0.2$, Cond.Prob. $r 3 s 1=0.1$, Percent drop in speed $\%=-56$ Max. Posterior Probability level $=0.97$, Percent range between speed states $=7 \%$

Drop in speed was detected and No indication of an incident within this period 


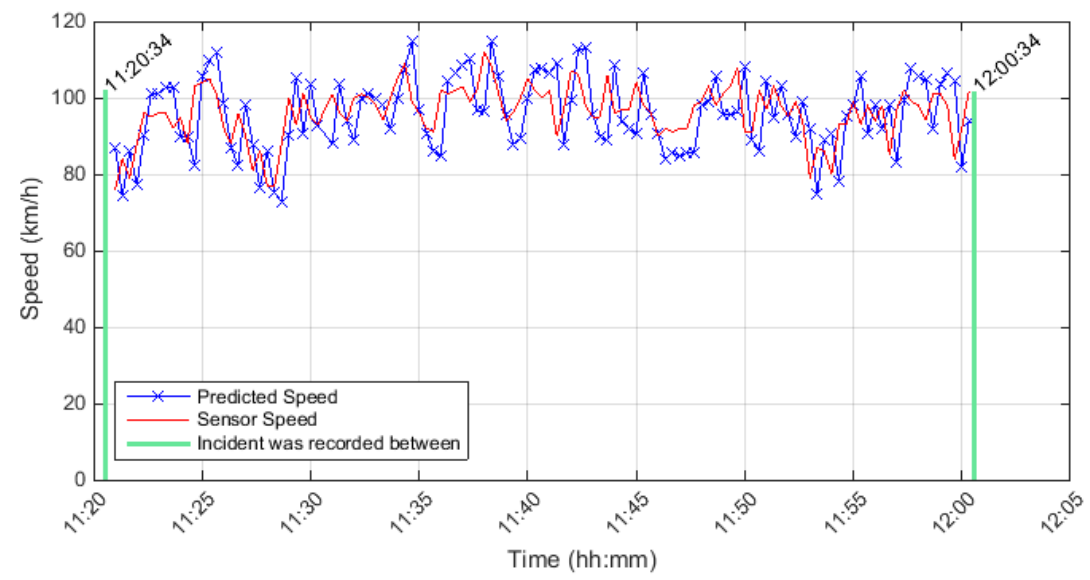

Upstream Detector \# is 401DE0290DWC, Detector Rank = 1, Distance from incident location in $(\mathrm{km})=0.27312$ Season $=$ Spring, Stream $=$ C, Direction $=W$, Date $\&$ Time $=$ 2011-05-17 11:40:34 Incident Reason $=$ Collision

Affected Lanes = 2 RIGHT LANE(S)

Conditional Probability level $=0.6$, Cond.Prob. $r 3 \mathrm{~s} 2=0.2$, Cond. Prob. $r 3 \mathrm{~s} 1=0.1$, Percent drop in speed $\%=-56$ Max. Posterior Probability level $=0.97$, Percent range between speed states $=7 \%$ (No Incident was Detected)

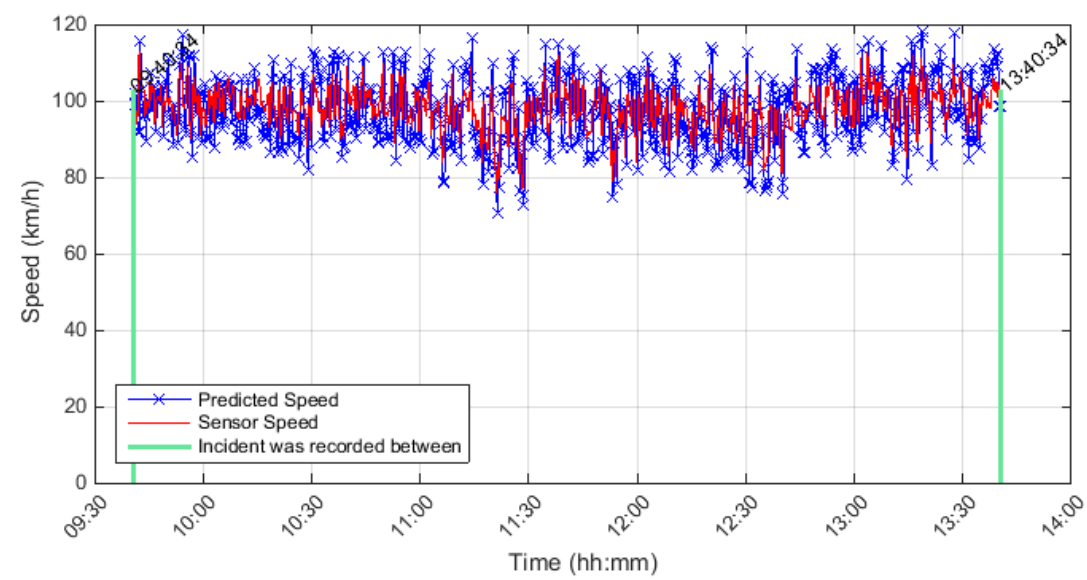

Upstream Detector \# is 401DE0290DWC, Detector Rank = 1, Distance from incident location in $(\mathrm{km})=0.27312$ Season $=$ Spring, Stream $=$ C , Direction $=$ W, Date $\&$ Time $=$ 2011-05-1711:40:34 Incident Reason $=$ Collision

Affected Lanes = 2 RIGHT LANE(S)

Conditional Probability level $=0.6$, Cond. Prob. $r 3 s 2=0.2$, Cond.Prob. $r 3 s 1=0.1$, Percent drop in speed $\%=-56$ Max. Posterior Probability level $=0.97$, Percent range between speed states $=7 \%$

(No Incident was Detected)

Same as the previous case if a large range is selected. 


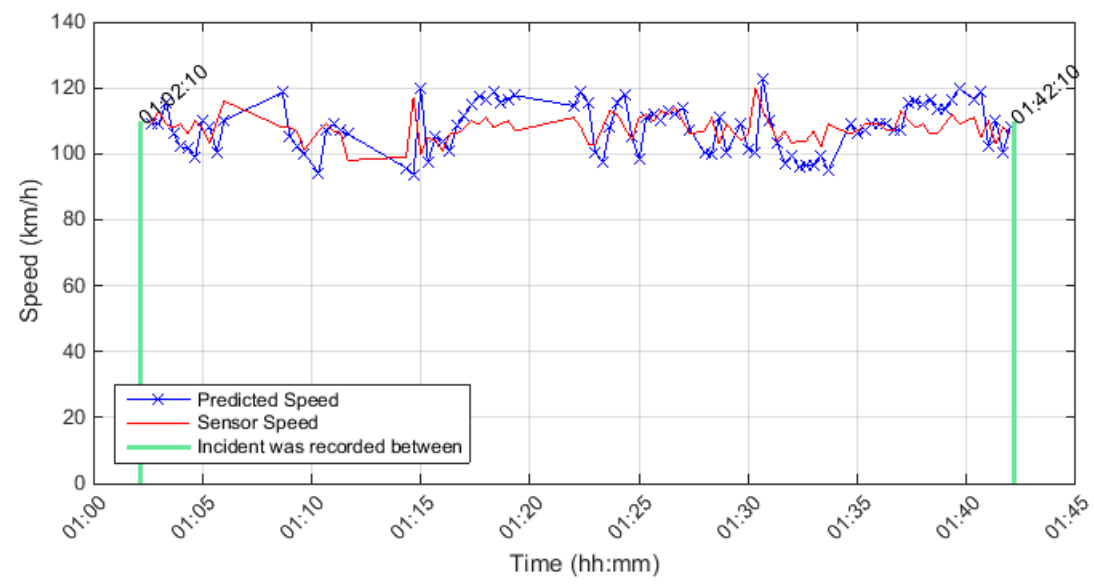

Upstream Detector \# is 401DE0020DWC, Detector Rank = 1, Distance from incident location in $(\mathrm{km})=0.39159$ Season $=$ Summer, Stream $=$ C, Direction $=W$, Date $\&$ Time $=2011-07-08$ 01:22:10 Incident Reason $=$ Collision

Affected Lanes = 2RIGHT LANE(S)

Conditional Probability level $=0.6$, Cond.Prob. $r 3 s 2=0.2$, Cond.Prob. $r 3 s 1=0.1$, Percent drop in speed $\%=-56$ Max. Posterior Probability level $=0.97$, Percent range between speed states $=7 \%$ (No Incident was Detected)

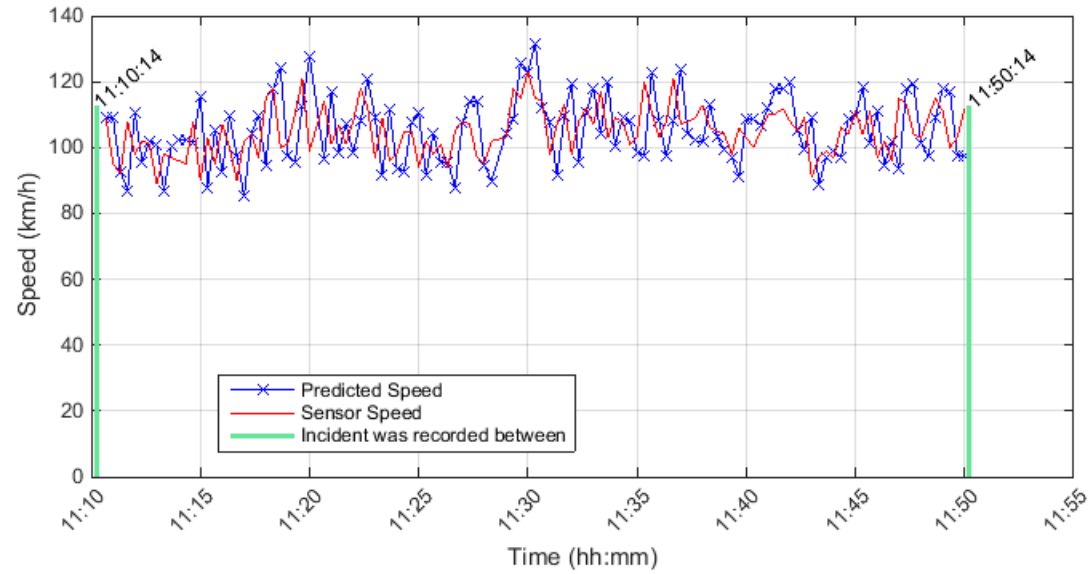

Upstream Detector \# is 401DE0350DWC, Detector Rank $=1$, Distance from incident location in $(\mathrm{km})=0.24334$ Season $=$ Summer, Stream $=$ C, Direction $=$ W, Date \& Time $=2011-08-0711: 30: 14$ Incident Reason $=$ Collision

Affected Lanes $=2$ RIGHT LANE(S)

Conditional Probability level $=0.6$, Cond.Prob. $r 3 s 2=0.2$, Cond. Prob. $r 3 s 1=0.1$, Percent drop in speed $\%=-56$

Max. Posterior Probability level $=0.97$. Percent range between speed states $=7 \%$

(No Incident was Detected) 


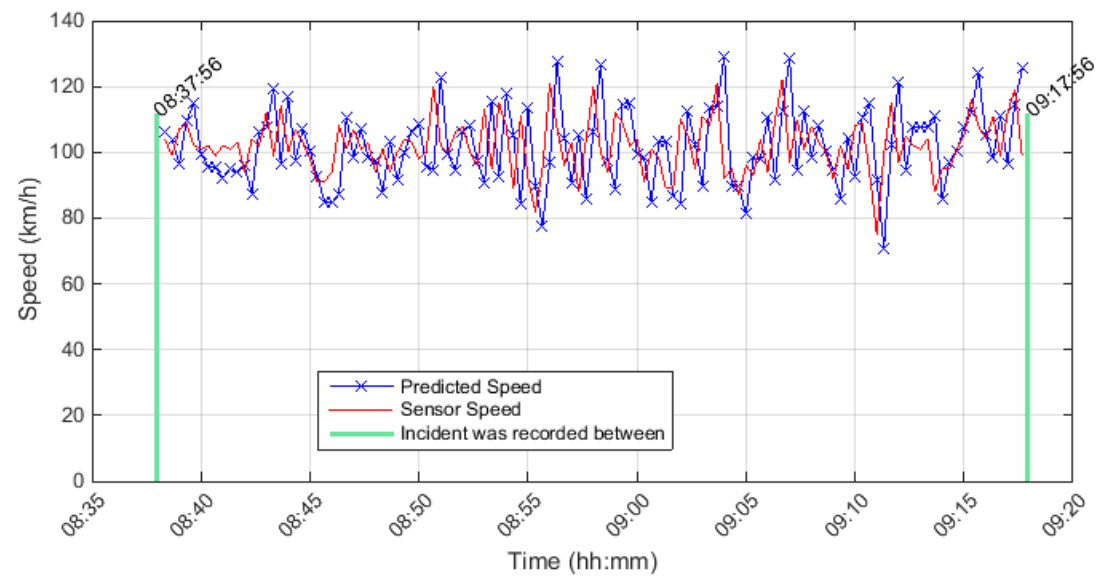

Upstream Detector \# is 401DW0150DWC, Detector Rank = 2, Distance from incident location in $(\mathrm{km})=0.87619$ Season $=$ Summer, Stream $=$ C, Direction $=$ W, Date $\&$ Time $=2011-09-15$ 08:57:56 Incident Reason $=$ Collision

Affected Lanes $=$ LEFT SHOULDER AND 3 LEFT LANE(S)

Conditional Probability level $=0.6$, Cond.Prob. $r 3 s 2=0.2$, Cond.Prob. $r 3 s 1=0.1$, Percent drop in speed $\%=-56$

Max. Posterior Probability level $=0.97$, Percent range between speed states $=7 \%$

(No Incident was Detected)

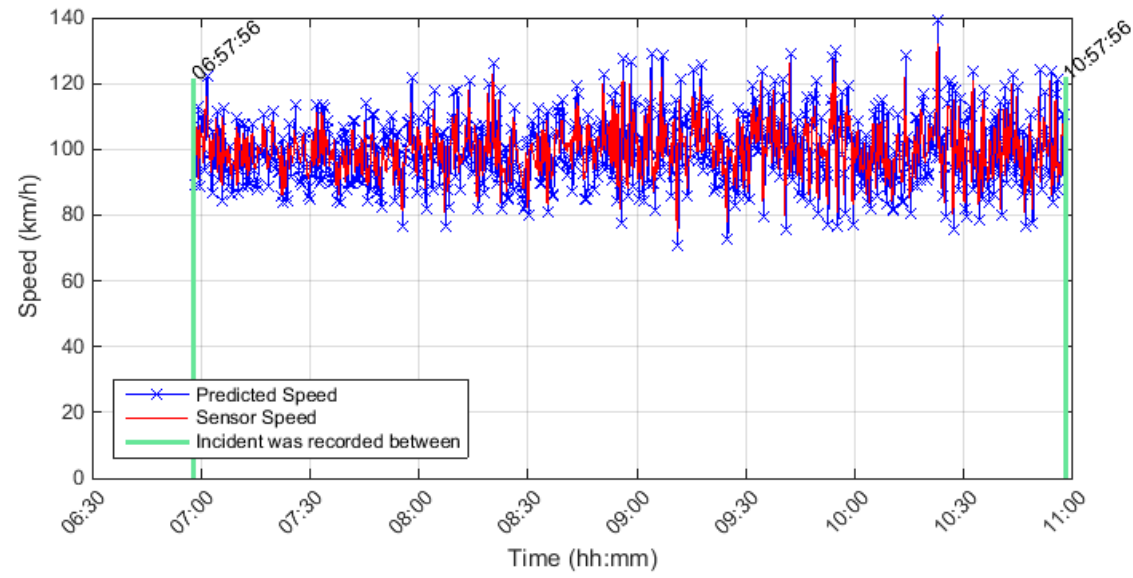

Upstream Detector \# is 401DW0150DWC, Detector Rank = 2, Distance from incident location in $(\mathrm{km})=0.87619$ Season $=$ Summer, Stream $=$ C, Direction $=$ W, Date \& Time $=2011-09-15$ 08:57:56 Incident Reason $=$ Collision

Affected Lanes = LEFT SHOULDER AND 3 LEFT LANE(S)

Conditional Probability level $=0.6$, Cond. Prob. $r 3 s 2=0.2$, Cond. Prob. $r 3 s 1=0.1$, Percent drop in speed $\%=-56$

Max. Posterior Probability level $=0.97$, Percent range between speed states $=7 \%$

(No Incident was Detected)

Same as the previous case with large time frame 


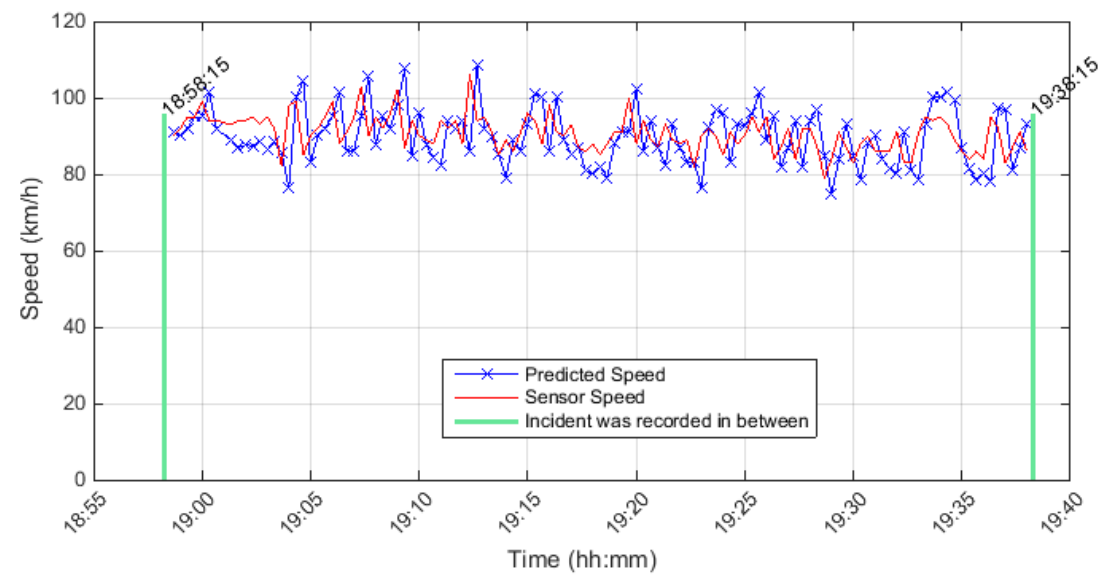

Upstream Detector \# is 401DE0160DWC, Detector Rank = 1, Distance from incident location in $(\mathrm{km})=0.12541$ Season $=$ Summer, Stream $=$ C, Direction $=$ W, Date \& Time $=2011-09-25$ 19:18:15 Incident Reason $=$ Disable Vehicle

Affected Lanes $=1$ RIGHT LANE(S)

Conditional Probability level $=0.6$, Cond.Prob. $r 3 s 2=0.2$, Cond. Prob. $r 3 s 1=0.1$, Percent drop in speed $\%=-56$ Max. Posterior Probability level $=0.97$, Percent range between speed states $=7 \%$ (No Incident was Detected)

Same as the previous case if a disabled vehicle and one lane was closed is the case.

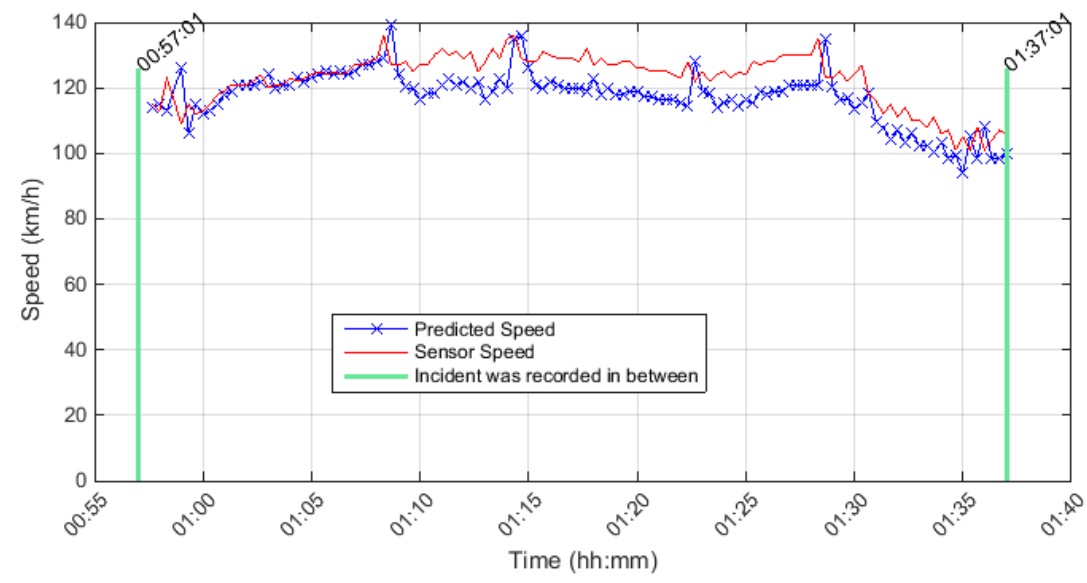

Upstream Detector \# is 401DW0070DWC, Detector Rank = 1, Distance from incident location in $(\mathrm{km})=0.52634$ Season $=$ Summer, Stream $=$ C, Direction $=W$, Date $\&$ Time $=2011-10-01$ 01:17:01 Incident Reason $=$ Collision Affected Lanes $=$ All lanes closed

Conditional Probability level $=0.6$, Cond.Prob. $r 3 s 2=0.2$, Cond.Prob. $r 3 s 1=0.1$, Percent drop in speed $\%=-56$ Max. Posterior Probability level $=0.97$, Percent range between speed states $=7 \%$ (No Incident was Detected)

In this case the volume was low, the average volume was about $7 \mathrm{v} / \mathrm{h}$ 


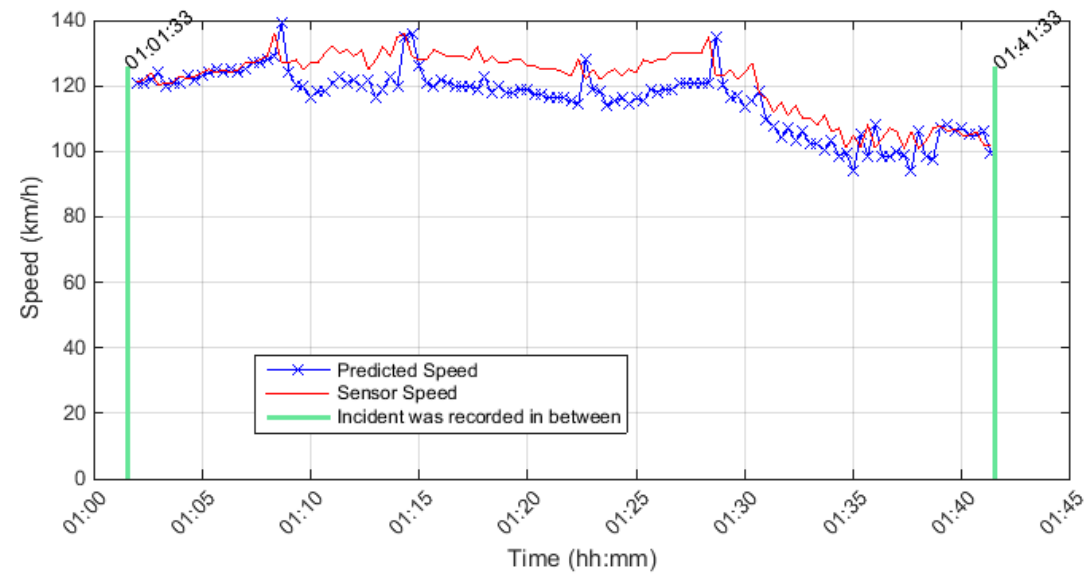

Upstream Detector \# is 401DW0070DWC, Detector Rank = 1, Distance from incident location in $(\mathrm{km})=0.52634$ Season $=$ Summer, Stream $=$ C, Direction $=$ W, Date $\&$ Time $=2011-10-0101: 21: 33$ Incident Reason $=$ Collision

Affected Lanes $=$ All lanes closed

Conditional Probability level $=0.6$, Cond. Prob. $r 3 s 2=0.2$, Cond. Prob. $r 3 s 1=0.1$, Percent drop in speed $\%=-56$ Max. Posterior Probability level $=0.97$, Percent range between speed states $=7 \%$ (No Incident was Detected)

In this case the volume was low, the average volume was about $7 \mathrm{v} / \mathrm{h}$

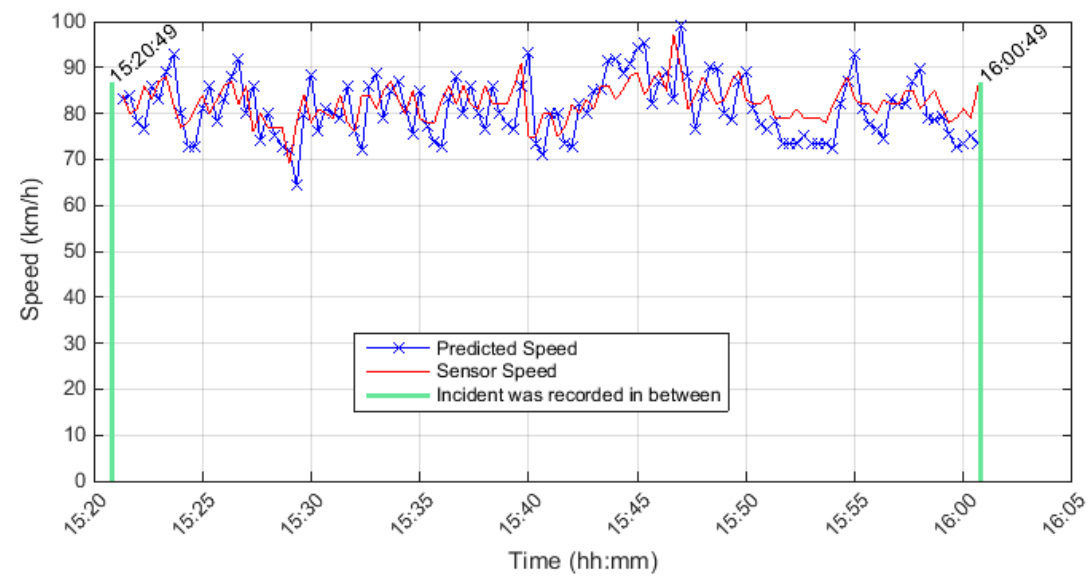

Upstream Detector \# is 401DE0090DWC, Detector Rank = 1, Distance from incident location in $(\mathrm{km})=0.59485$ Season $=$ Summer, Stream $=$ C, Direction $=$ W, Date $\&$ Time $=2011-10-23$ 15:40:49 Incident Reason $=$ Collision

Affected Lanes $=$ LEFT SHOULDER AND 2 RIGHT LANE(S)

Conditional Probability level $=0.6$, Cond. Prob. $r 3 s 2=0.2$, Cond. Prob. $r 3 s 1=0.1$, Percent drop in speed $\%=-56$ Max. Posterior Probability level $=0.97$, Percent range between speed states $=7 \%$ (No Incident was Detected) 


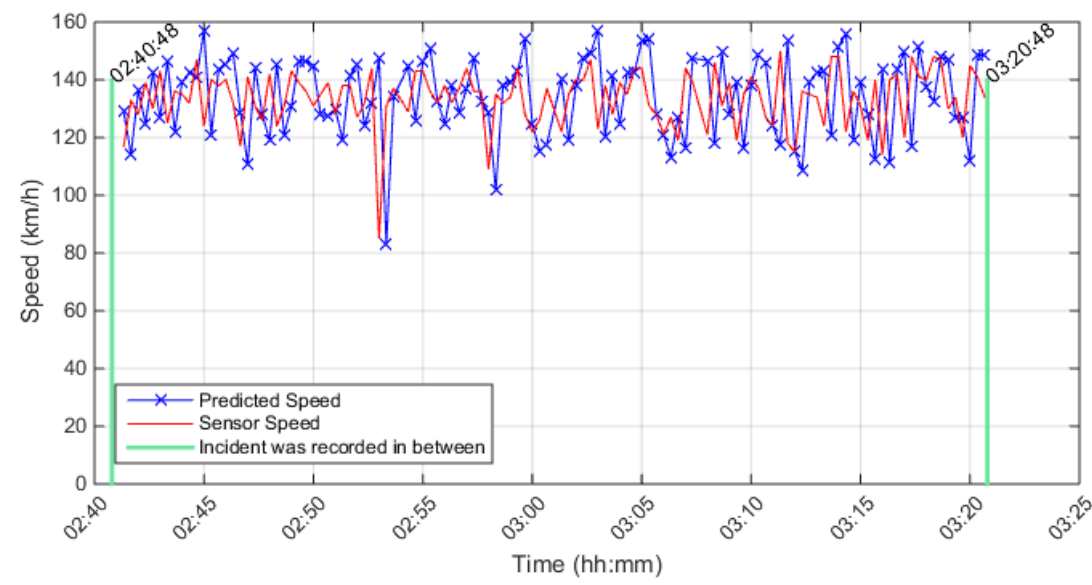

Upstream Detector \# is 401DE0020DWC, Detector Rank = 1, Distance from incident location in $(\mathrm{km})=0.39159$ Season $=$ Winter, Stream $=$ C, Direction $=W$, Date $\&$ Time $=2011-12-18$ 03:00:48 Incident Reason $=$ Collision Affected Lanes $=1$ RIGHT LANE(S)

Conditional Probability level $=0.6$, Cond.Prob. $r 3 s 2=0.2$, Cond. Prob. $r 3 s 1=0.1$, Percent drop in speed $\%=-56$ Max. Posterior Probability level $=0.97$, Percent range between speed states $=7 \%$ (No Incident was Detected)

\section{In this case it is AM time and only one right lane was affected}

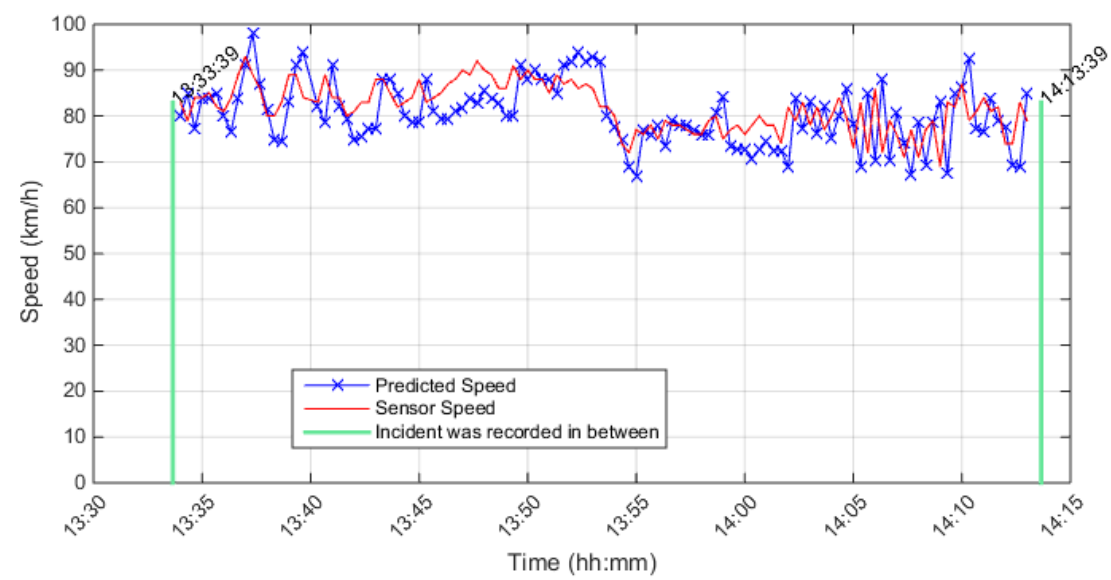

Upstream Detector \# is 401DE0240DWE, Detector Rank $=1$, Distance from incident location in $(\mathrm{km})=0.13998$ Season $=$ Spring, Stream $=E$, Direction $=W$, Date $\&$ Time $=2011-03-17$ 13:53:39 Incident Reason $=$ Collision

Affected Lanes = 2 RIGHT LANE(S) AND RIGHT SHOULDER

Conditional Probability level $=0.6$, Cond.Prob. $r 3 s 2=0.2$, Cond.Prob. $r 3 s 1=0.1$, Percent drop in speed $\%=-56$ Max. Posterior Probability level $=0.97$, Percent range between speed states $=7 \%$

(No Incident was Detected) 
Even if the time frame is extended to $60 \mathrm{~min}$ before and $60 \mathrm{~min}$ after the incident recorded time, still no detection of any incident

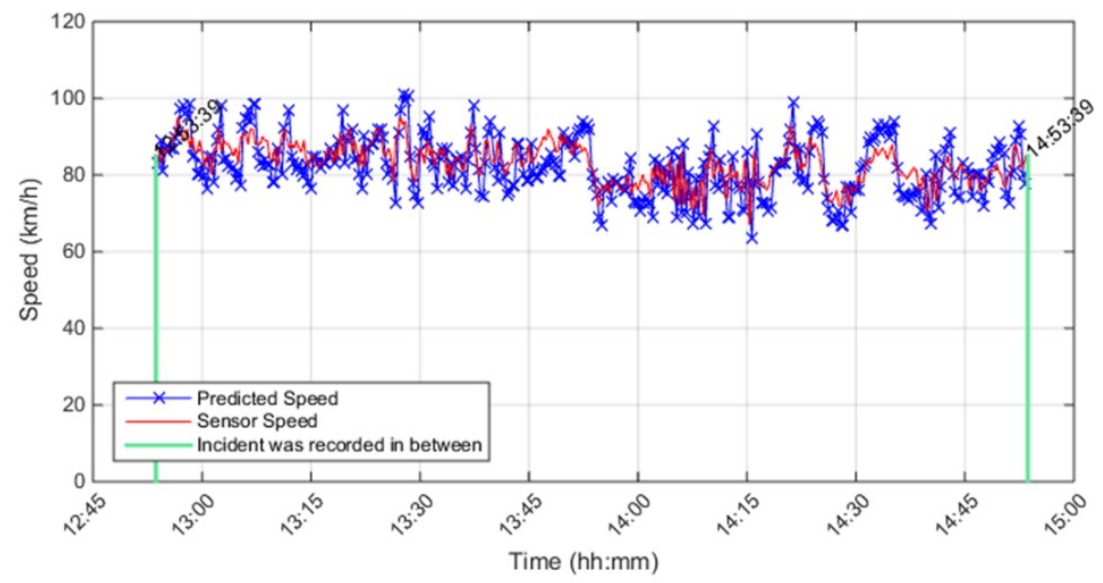

Upstream Detector \# is 401DE0240DWE, Detector Rank = 1, Distance from incident location in $(\mathrm{km})=0.13998$ Season $=$ Spring, Stream $=$ E, Direction $=$ W, Date $\&$ Time $=$ 2011-03-17 13:53:39

Incident Reason $=$ Collision
Affected Lanes $=2$ RIGHT LANE(S) AND RIGHT SHOULDER

Conditional Probability level $=0.6$, Cond.Prob. $r 3 s 2=0.2$, Cond. Prob. $r 3 s 1=0.1$, Percent drop in speed $\%=-56$ Max. Posterior Probability level $=0.97$, Percent range between speed states $=7 \%$ (No Incident was Detected)

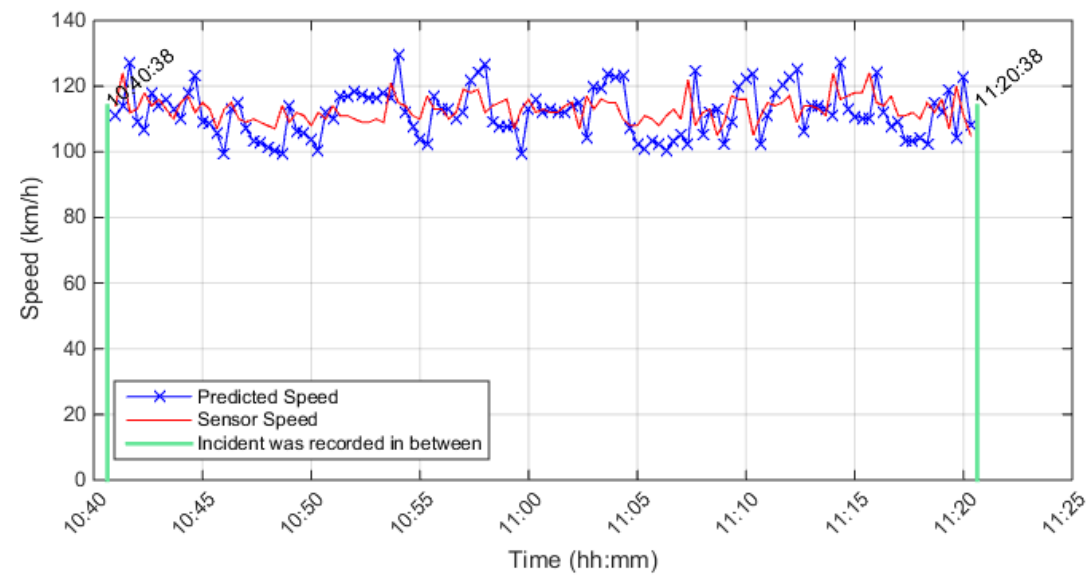

Upstream Detector \# is 401DE0410DWE, Detector Rank = 1, Distance from incident location in $(\mathrm{km})=4.2027$ Season $=$ Summer, Stream $=E$, Direction $=W$, Date $\&$ Time $=2011-06-1011: 00: 38$ Incident Reason $=$ Collision

Affected Lanes $=$ LEFT SHOULDER AND 2 LEFT LANE(S)

Conditional Probability level $=0.6$, Cond. Prob. $r 3 \mathrm{~s} 2=0.2$, Cond. Prob. $r 3 \mathrm{~s} 1=0.1$, Percent drop in speed $\%=-56$ Max. Posterior Probability level $=0.97$, Percent range between speed states $=7 \%$ (No Incident was Detected) 


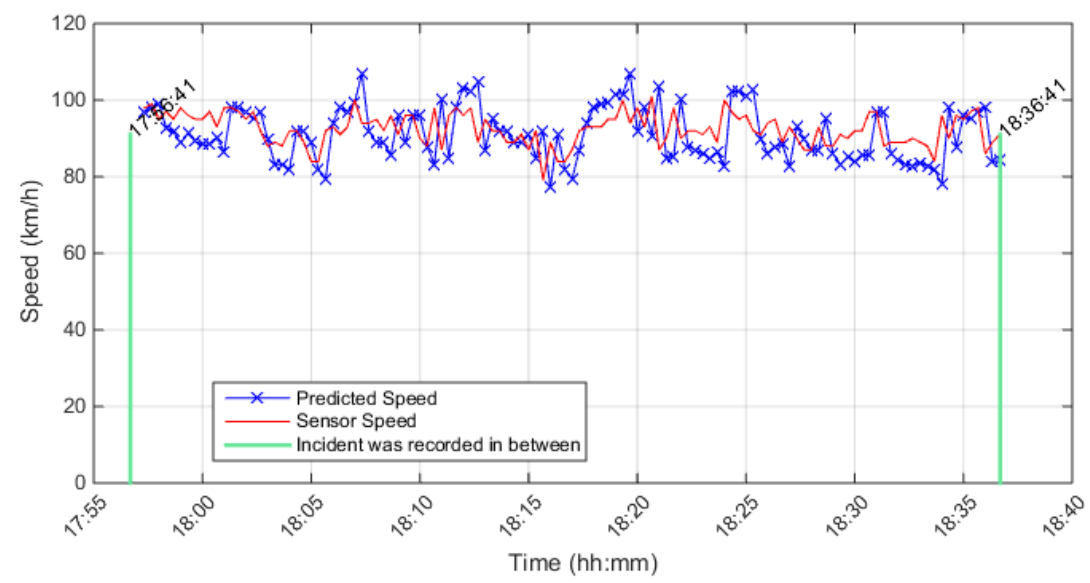

Upstream Detector \# is 401DE0220DWE, Detector Rank = 1, Distance from incident location in $(\mathrm{km})=0.30243$ Season $=$ Summer, Stream $=E$, Direction $=W$, Date $\&$ Time $=2011-10-22$ 18:16:41 Incident Reason $=$ Collision

Affected Lanes $=$ LEFT SHOULDER AND 1 LEFT LANE(S)

Conditional Probability level $=0.6$, Cond. Prob. $r 3 \mathrm{~s} 2=0.2$, Cond. Prob. $\mathrm{r} 3 \mathrm{~s} 1=0.1$, Percent drop in speed $\%=-56$ Max. Posterior Probability level $=0.97$, Percent range between speed states $=7 \%$ (No Incident was Detected)

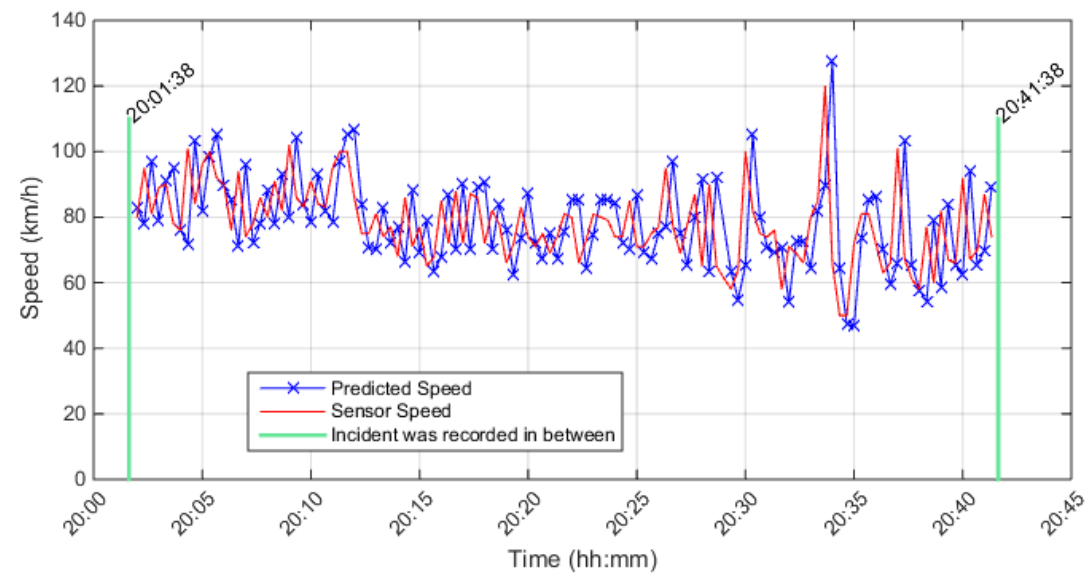

Upstream Detector \# is 401DE0100DWE, Detector Rank = 1, Distance from incident location in $(\mathrm{km})=0.12114$ Season $=$ Winter, Stream $=$ E, Direction $=$ W, Date $\&$ Time $=2011-12-27$ 20:21:38 Incident Reason $=$ Collision

Affected Lanes = 2 RIGHT LANE(S) AND RIGHT SHOULDER

Conditional Probability level $=0.6$, Cond.Prob. $r 3 s 2=0.2$, Cond. Prob. $r 3 s 1=0.1$, Percent drop in speed $\%=-56$ Max. Posterior Probability level $=0.97$, Percent range between speed states $=7 \%$

(No Incident was Detected) 


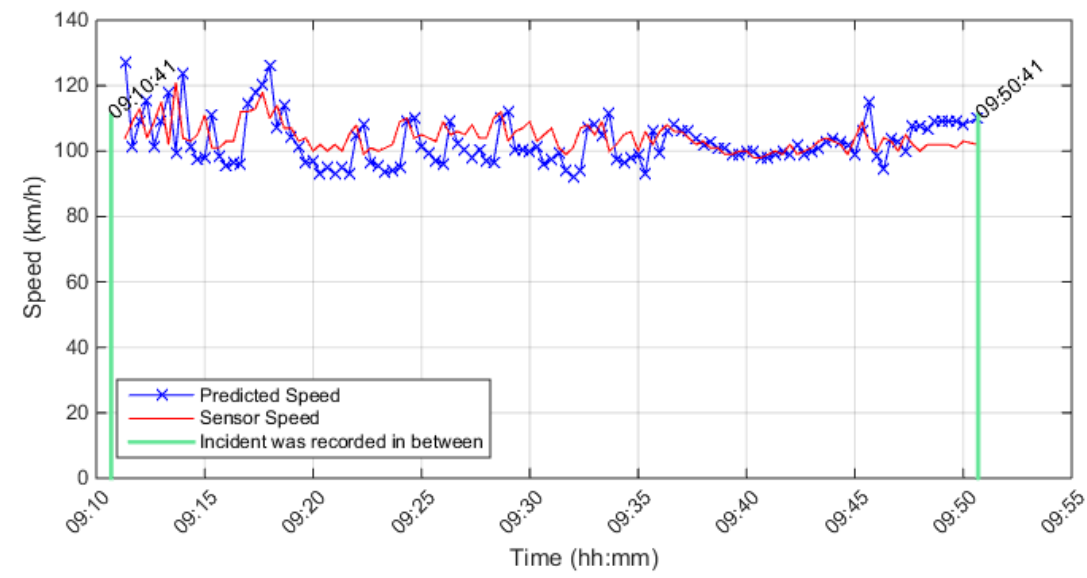

Upstream Detector \# is 401DE0490DEC, Detector Rank $=1$, Distance from incident location in $(\mathrm{km})=0.10164$ Season $=$ Winter, Stream $=$ C, Direction $=E$, Date $\&$ Time $=2011-02-18$ 09:30:41 Incident Reason $=$ Collision

Affected Lanes = LEFT SHOULDER AND 2 LEFT LANE(S)

Conditional Probability level $=0.6$, Cond.Prob. $r 3 \mathrm{~s} 2=0.2$, Cond. Prob. $r 3 \mathrm{~s} 1=0.1$, Percent drop in speed $\%=-56$

Max. Posterior Probability level $=0.97$, Percent range between speed states $=7 \%$

(No Incident was Detected)

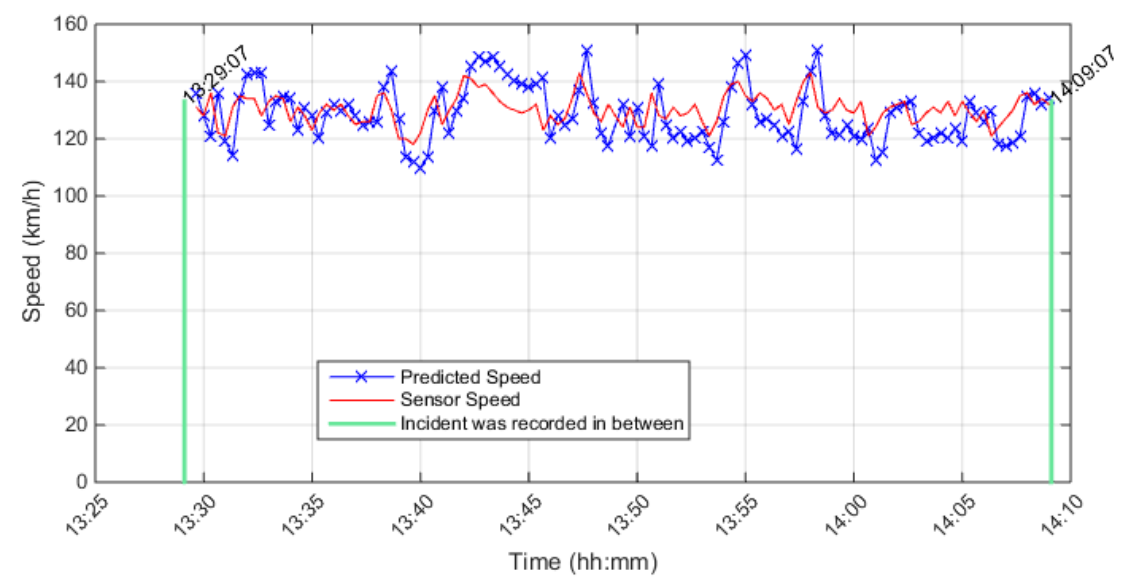

Upstream Detector \# is 401DE0530DEC, Detector Rank $=1$, Distance from incident location in $(\mathrm{km})=0.43916$ Season $=$ Spring, Stream $=$ C, Direction $=E$, Date $\&$ Time $=2011-03-26$ 13:49:07

Incident Reason $=$ Disable Vehicle

Affected Lanes = 1 RIGHT LANE(S) AND RIGHT SHOULDER

Conditional Probability level $=0.6$, Cond.Prob. $\mathrm{r} 3 \mathrm{~s} 2=0.2$, Cond.Prob. $\mathrm{r} 3 \mathrm{~s} 1=0.1$, Percent drop in speed $\%=-56$ Max. Posterior Probability level $=0.97$, Percent range between speed states $=7 \%$

(No Incident was Detected) 


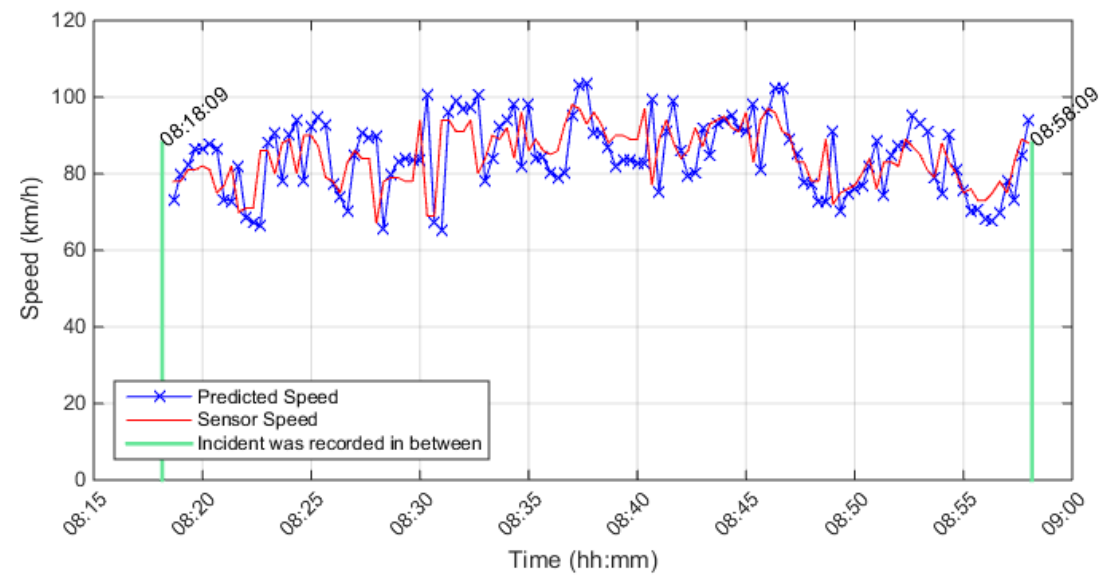

Upstream Detector \# is 401DW0100DEC, Detector Rank = 1, Distance from incident location in $(\mathrm{km})=0.021101$ Season $=$ Summer, Stream $=C$, Direction $=E$, Date $\&$ Time $=2011-08-16$ 08:38:09 Incident Reason $=$ Collision

Affected Lanes = 2 RIGHT LANE(S)

Conditional Probability level $=0.6$, Cond. Prob. $r 3 s 2=0.2$, Cond. Prob. $r 3 s 1=0.1$, Percent drop in speed $\%=-56$ Max. Posterior Probability level $=0.97$, Percent range between speed states $=7 \%$ (No Incident was Detected)

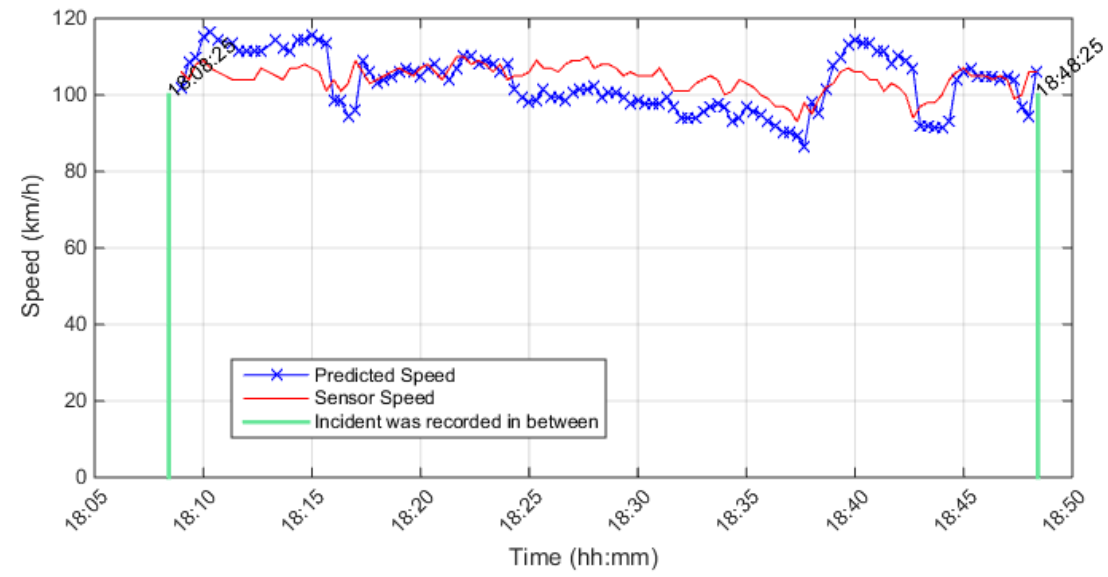

Upstream Detector \# is 401DE0210DEC, Detector Rank = 1, Distance from incident location in $(\mathrm{km})=0.20489$ Season $=$ Summer, Stream $=$ C, Direction $=E$, Date $\&$ Time $=2011-09-2518: 28: 25$ Incident Reason $=$ Disable Vehicle

Affected Lanes = 1 CENTRE LANE(S)

Conditional Probability level $=0.6$, Cond.Prob. $r 3 s 2=0.2$, Cond. Prob. $r 3 s 1=0.1$, Percent drop in speed $\%=-56$ Max. Posterior Probability level $=0.97$, Percent range between speed states $=7 \%$

(No Incident was Detected) 


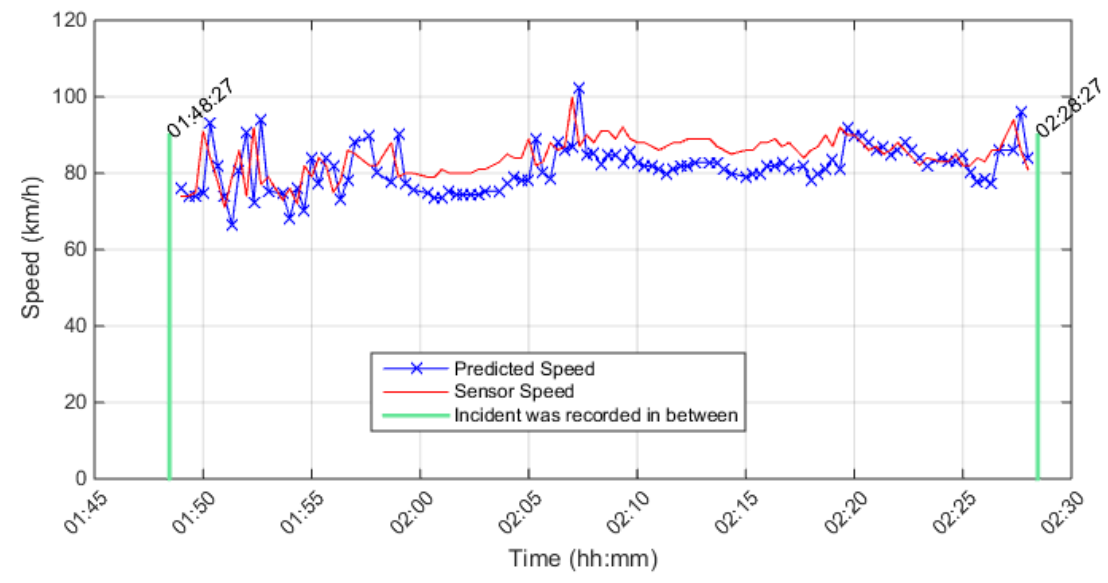

Upstream Detector \# is 401DE0350DEC, Detector Rank = 1, Distance from incident location in $(\mathrm{km})=0.14426$ Season $=$ Summer, Stream $=$ C, Direction $=E$, Date $\&$ Time $=2011-09-30$ 02:08:27 Incident Reason $=$ Collision

Affected Lanes = 1 RIGHT LANE(S)

Conditional Probability level $=0.6$, Cond. Prob. $r 3 s 2=0.2$, Cond. Prob. $r 3 s 1=0.1$, Percent drop in speed $\%=-56$ Max. Posterior Probability level $=0.97$, Percent range between speed states $=7 \%$ (No Incident was Detected)

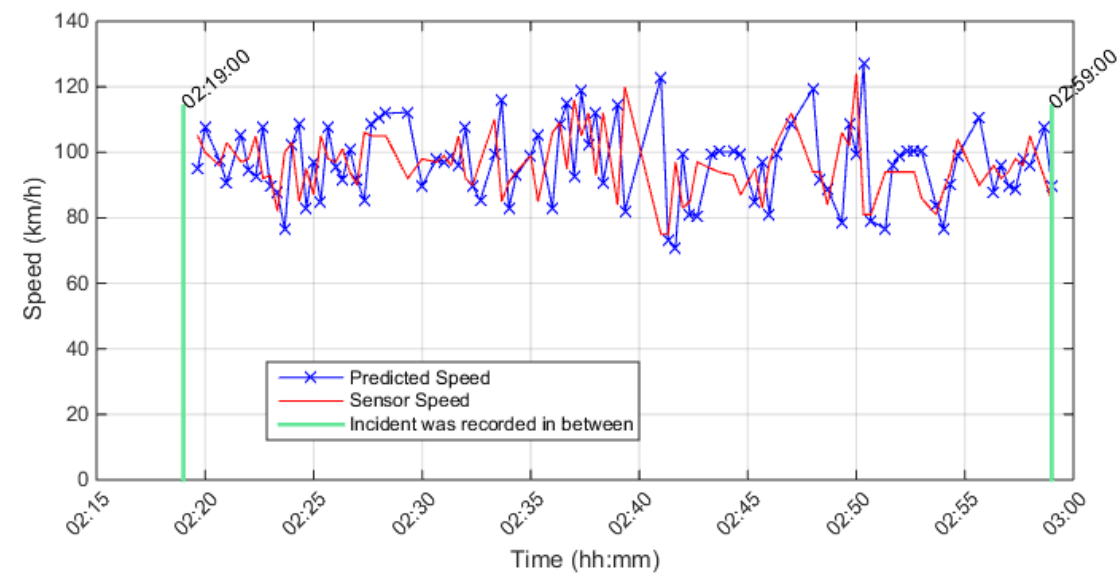

Upstream Detector \# is 401DW0110DEE, Detector Rank = 1, Distance from incident location in $(\mathrm{km})=0.28861$ Season $=$ Winter, Stream $=E$, Direction $=E$, Date \& Time $=$ 2011-01-11 02:39:00 Incident Reason $=$ Collision

Affected Lanes $=2$ left lanes

Conditional Probability level $=0.6$, Cond.Prob. $r 3 s 2=0.2$, Cond.Prob. $r 3 s 1=0.1$, Percent drop in speed $\%=-56$ Max. Posterior Probability level $=0.97$, Percent range between speed states $=7 \%$

(No Incident was Detected) 


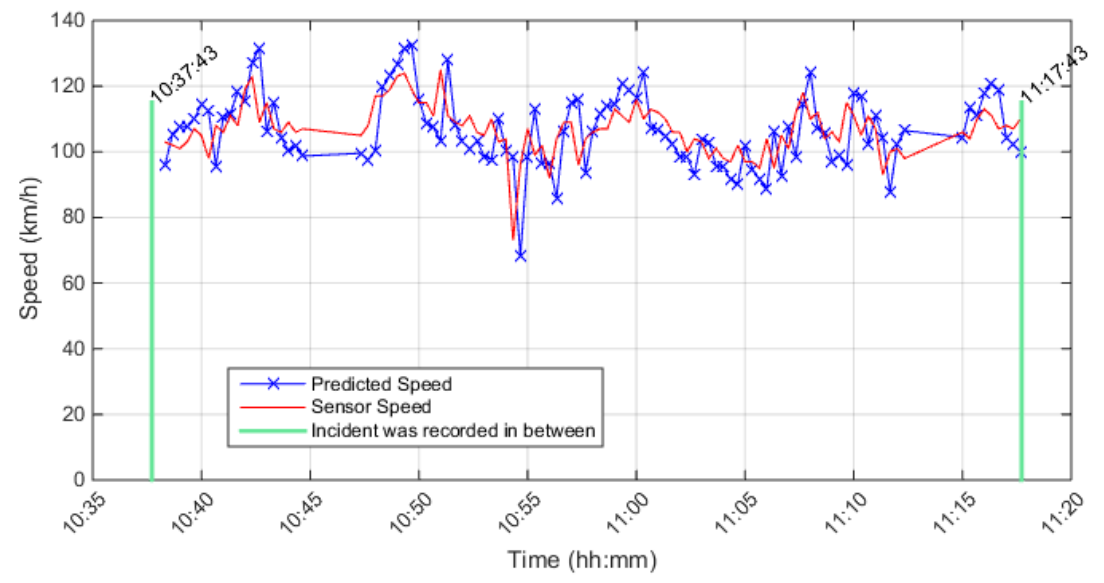

Upstream Detector \# is 401DE0170DEE, Detector Rank $=3$, Distance from incident location in $(\mathrm{km})=1.9295$ Season $=$ Winter, Stream $=E$, Direction $=E$, Date $\&$ Time $=2011-01-1210: 57: 43$ Incident Reason $=$ Collision

Affected Lanes = LEFT SHOULDER AND 1 LEFT LANE(S)

Conditional Probability level $=0.6$, Cond. Prob. $r 3 s 2=0.2$, Cond. Prob. $r 3 s 1=0.1$, Percent drop in speed $\%=-56$ Max. Posterior Probability level $=0.97$, Percent range between speed states $=7 \%$ (No Incident was Detected)

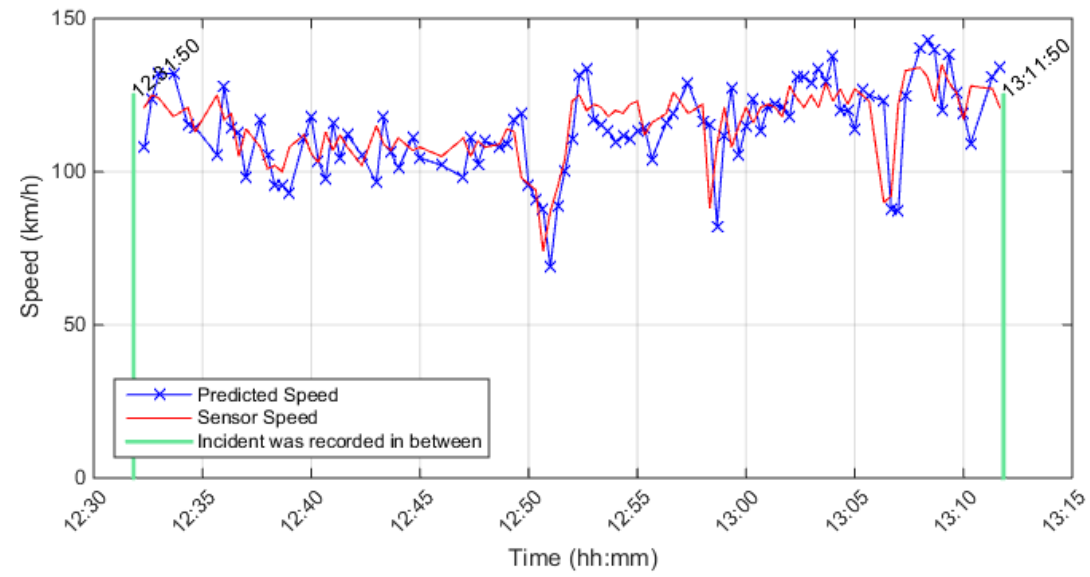

Upstream Detector \# is 401DE0120DEE, Detector Rank $=1$, Distance from incident location in $(\mathrm{km})=0.83799$ Season $=$ Winter, Stream $=E$, Direction $=E$, Date $\&$ Time $=2011-01-1512: 51: 50$

Incident Reason $=$ Disable Vehicle

Affected Lanes $=1$ LEFT LANE(S)

Conditional Probability level $=0.6$, Cond.Prob. $r 3 \mathrm{~s} 2=0.2$, Cond.Prob. $r 3 \mathrm{~s} 1=0.1$, Percent drop in speed $\%=-56$

Max. Posterior Probability level $=0.97$, Percent range between speed states $=7 \%$

(No Incident was Detected) 


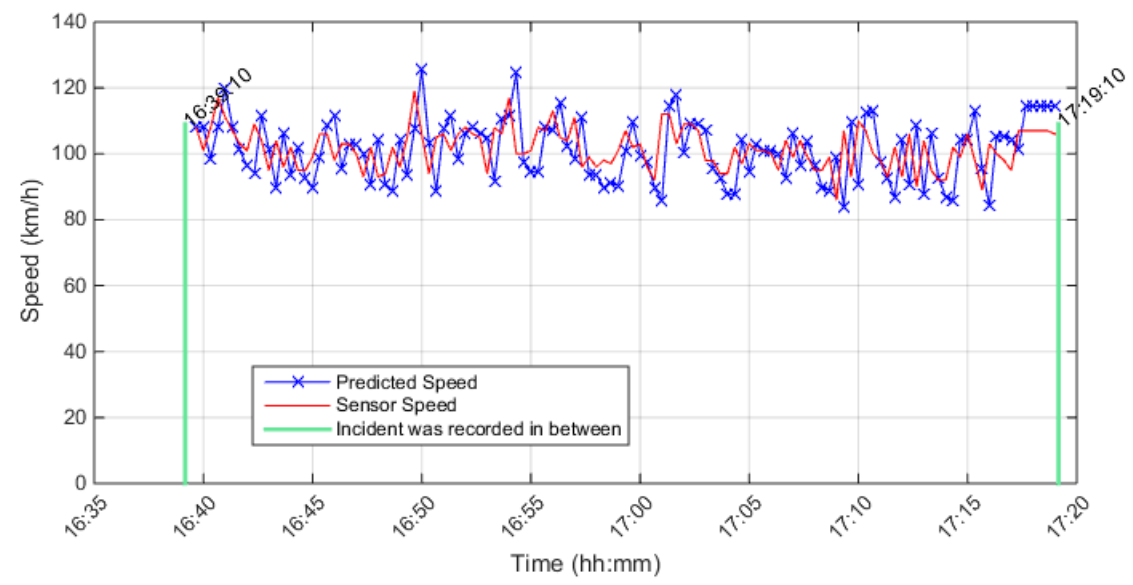

Upstream Detector \# is 401DW0260DEE, Detector Rank = 1, Distance from incident location in $(\mathrm{km})=0.36837$ Season $=$ Spring, Stream $=E$, Direction $=E$, Date \& Time $=2011-03-16$ 16:59:10 Incident Reason $=$ Collision

Affected Lanes $=$ All lanes closed

Conditional Probability level $=0.6$, Cond.Prob. $r 3 s 2=0.2$, Cond.Prob. $r 3 s 1=0.1$, Percent drop in speed $\%=-56$

Max. Posterior Probability level $=0.97$, Percent range between speed states $=7 \%$

(No Incident was Detected)

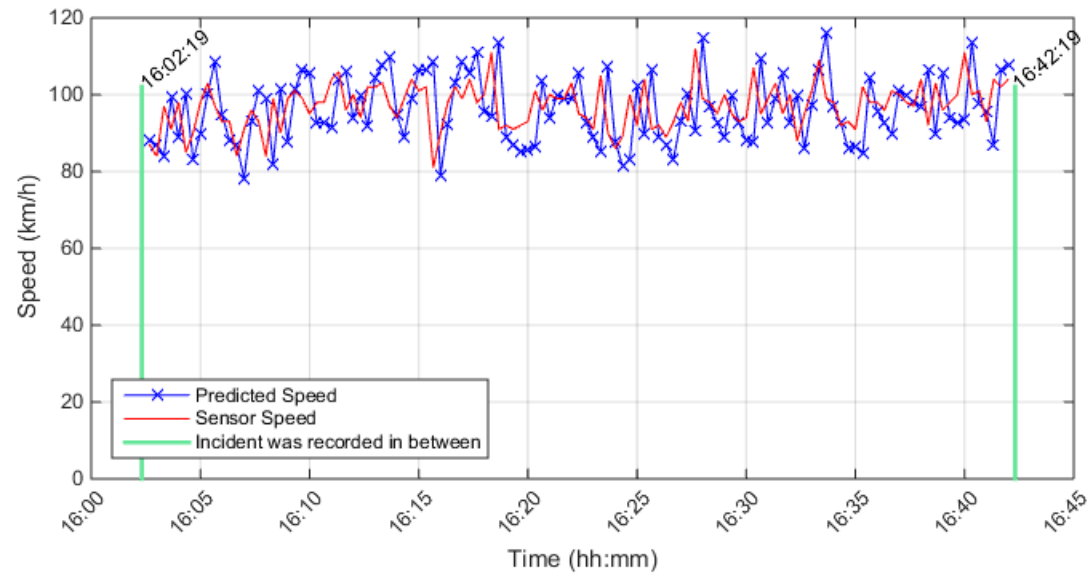

Upstream Detector \# is 401DW0300DEE, Detector Rank = 1, Distance from incident location in $(\mathrm{km})=0.63014$ Season $=$ Spring, Stream $=E$, Direction $=E$, Date $\&$ Time $=2011-05-0316: 22: 19$ Incident Reason $=$ Disable Vehicle

Affected Lanes $=1$ CENTRE LANES(S)

Conditional Probability level $=0.6$, Cond.Prob. $r 3 s 2=0.2$, Cond. Prob. $r 3 s 1=0.1$, Percent drop in speed $\%=-56$

Max. Posterior Probability level $=0.97$. Percent range between speed states $=7 \%$

(No Incident was Detected) 


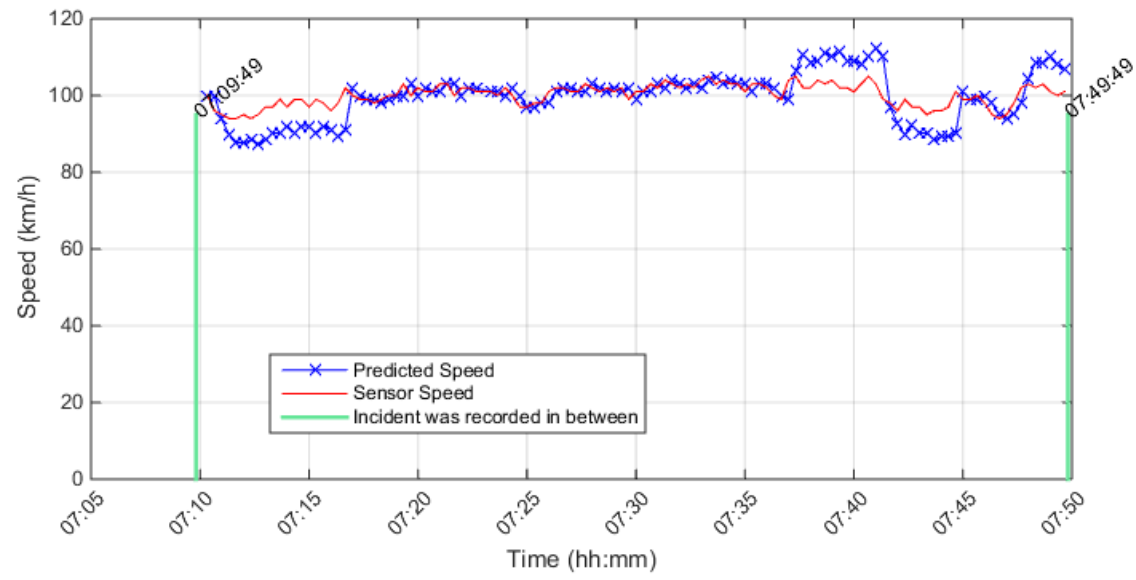

Upstream Detector \# is 401DE0160DEE, Detector Rank = 1, Distance from incident location in $(\mathrm{km})=0.24324$ Season $=$ Spring, Stream $=E$, Direction $=E$, Date $\&$ Time $=$ 2011-05-04 07:29:49 Incident Reason $=$ Collision

Affected Lanes = 1 RIGHT LANE(S)

Conditional Probability level $=0.6$, Cond.Prob. $r 3 s 2=0.2$, Cond. Prob. $r 3 s 1=0.1$, Percent drop in speed $\%=-56$

Max. Posterior Probability level $=0.97$, Percent range between speed states $=7 \%$

(No Incident was Detected)

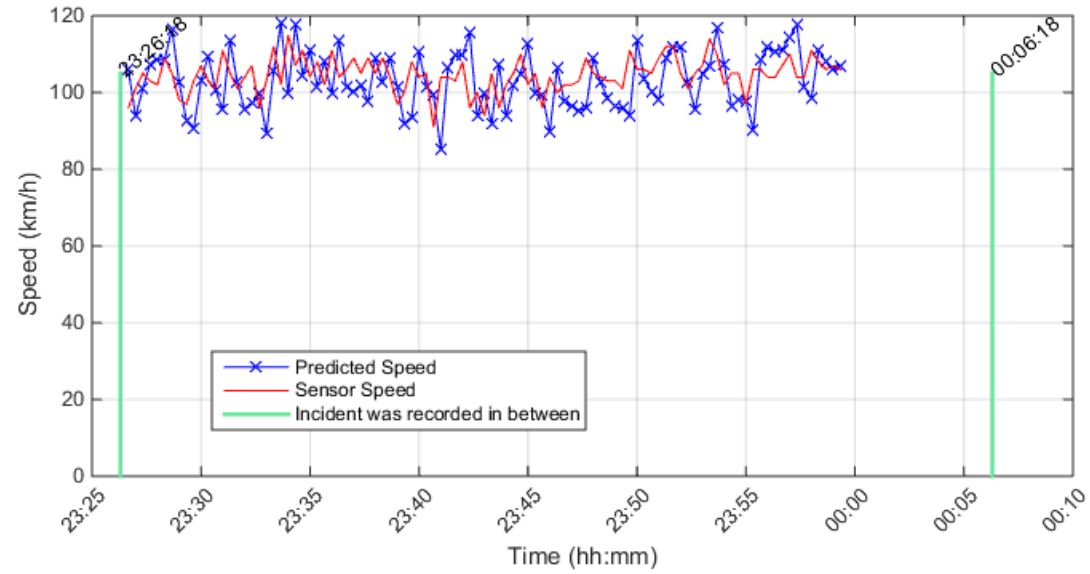

Upstream Detector \# is 401DE0590DEE, Detector Rank = 1, Distance from incident location in $(\mathrm{km})=0.15896$ Season $=$ Spring, Stream $=E$, Direction $=E$, Date $\&$ Time $=$ 2011-05-14 23:46:18 Incident Reason $=$ Collision

Affected Lanes $=2$ LEFT LANE(S)

Conditional Probability level $=0.6$, Cond.Prob. $r 3 s 2=0.2$, Cond.Prob. $r 3 s 1=0.1$, Percent drop in speed $\%=-56$

Max. Posterior Probability level $=0.97$, Percent range between speed states $=7 \%$

(No Incident was Detected) 


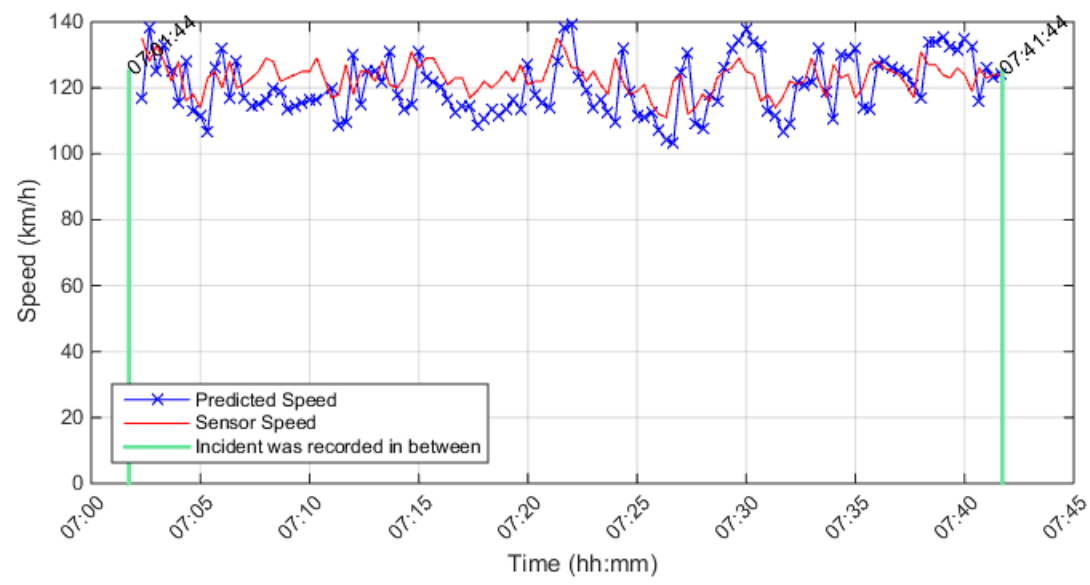

Upstream Detector \# is 401DE0440DEE, Detector Rank = 1, Distance from incident location in $(\mathrm{km})=4.444$ Season $=$ Summer, Stream $=E$, Direction $=E$, Date $\&$ Time $=2011-06-22$ 07:21:44 Incident Reason $=$ Collision

Affected Lanes $=2$ RIGHT LANE(S) AND RIGHT SHOULDER

Conditional Probability level $=0.6$, Cond. Prob. $r 3 s 2=0.2$, Cond. Prob. $r 3 s 1=0.1$, Percent drop in speed $\%=-56$

Max. Posterior Probability level $=0.97$, Percent range between speed states $=7 \%$

(No Incident was Detected)

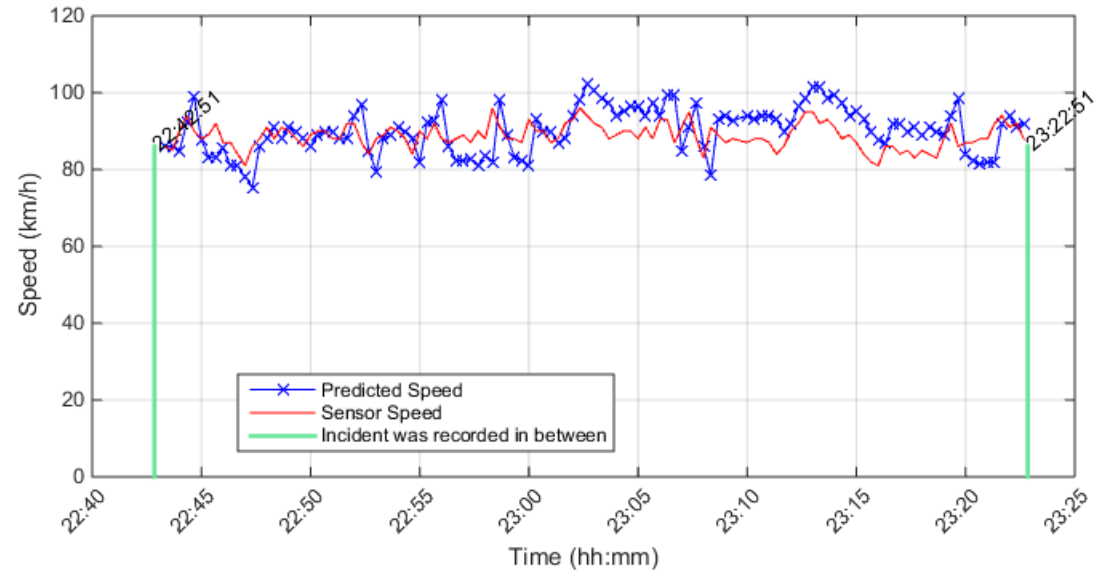

Upstream Detector \# is 401DE0340DEE, Detector Rank $=3$, Distance from incident location in $(\mathrm{km})=1.43$ Season $=$ Summer, Stream $=E$, Direction $=E$, Date $\&$ Time $=2011-07-29$ 23:02:51 Incident Reason $=$ Collision

Affected Lanes $=3$ LEFT LANE(S)

Conditional Probability level $=0.6$, Cond.Prob. $r 3 s 2=0.2$, Cond. Prob. $r 3 s 1=0.1$, Percent drop in speed $\%=-56$

Max. Posterior Probability level $=0.97$. Percent range between speed states $=7 \%$

(No Incident was Detected) 


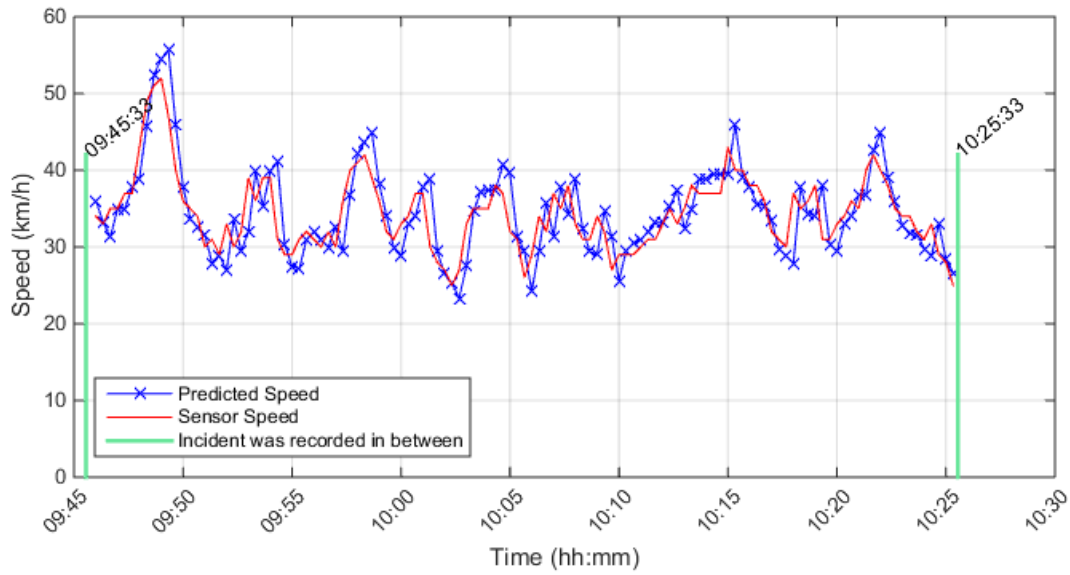

Upstream Detector \# is 401DW0010DEE, Detector Rank = 2, Distance from incident location in $(\mathrm{km})=1.1139$ Season $=$ Summer, Stream $=E$, Direction $=E$, Date $\&$ Time $=2011-08-05$ 10:05:33 Incident Reason $=$ Disable Vehicle

Affected Lanes $=2$ RIGHT LANE(S) AND RIGHT SHOULDER

Conditional Probability level $=0.6$, Cond.Prob. $r 3 \mathrm{~s} 2=0.2$, Cond. Prob. $r 3 \mathrm{~s} 1=0.1$, Percent drop in speed $\%=-56$

Max. Posterior Probability level $=0.97$, Percent range between speed states $=7 \%$

(No Incident was Detected)

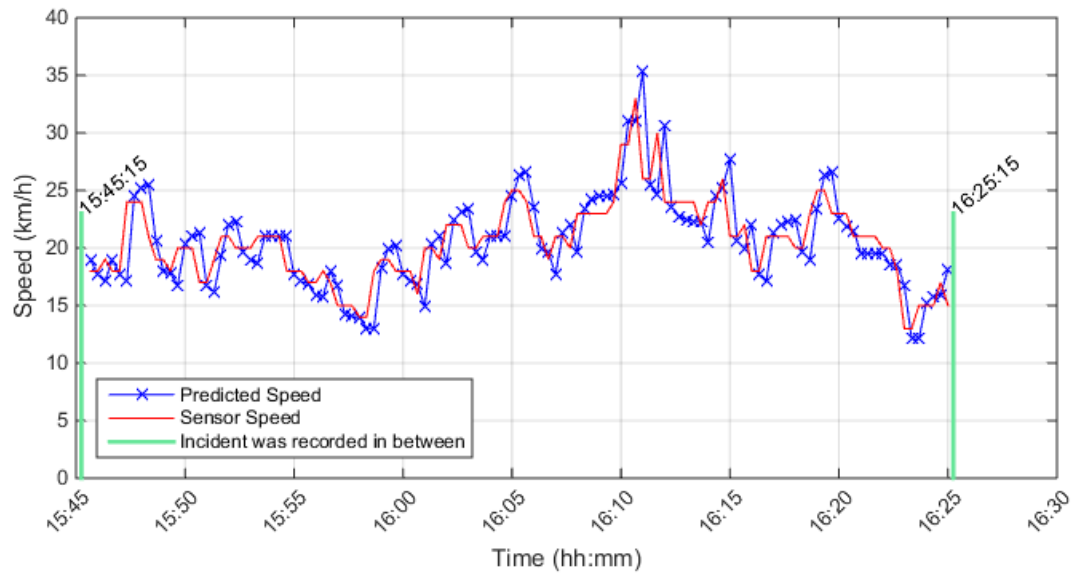

Upstream Detector \# is 401DE0050DEE, Detector Rank = 2, Distance from incident location in $(\mathrm{km})=1.0253$ Season $=$ Summer, Stream $=E$, Direction $=E$, Date \& Time $=2011-09-24$ 16:05:15 Incident Reason $=$ Collision

Affected Lanes $=2$ RIGHT LANE(S) AND RIGHT SHOULDER

Conditional Probability level $=0.6$, Cond.Prob. $r 3 \mathrm{~s} 2=0.2$, Cond. Prob. $r 3 \mathrm{~s} 1=0.1$, Percent drop in speed $\%=-56$

Max. Posterior Probability level $=0.97$, Percent range between speed states $=7 \%$

(No Incident was Detected)

\section{With 120 min before}




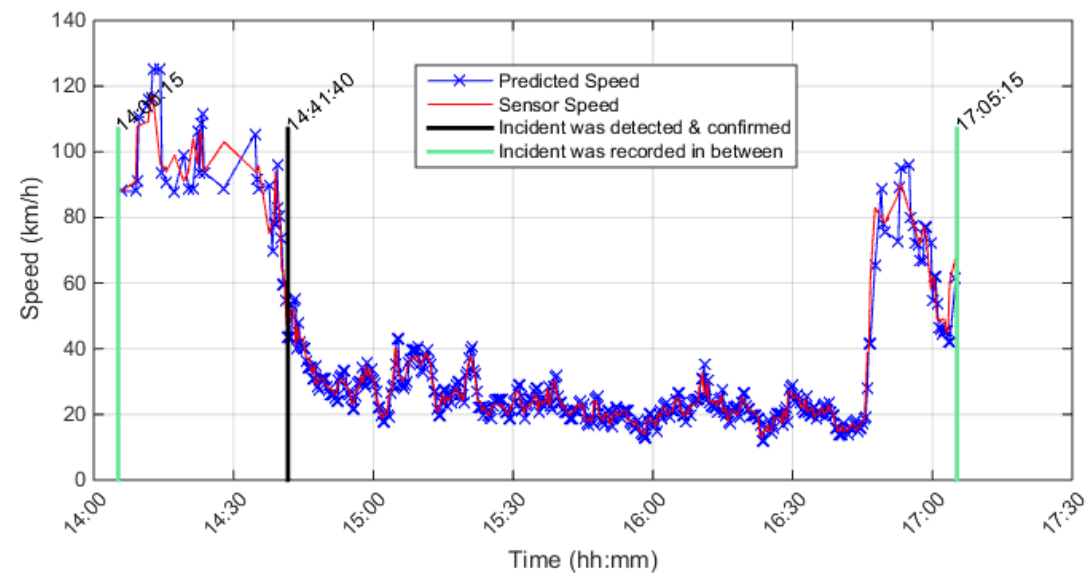

Upstream Detector \# is 401DE0050DEE, Detector Rank = 2, Distance from incident location in $(\mathrm{km})=1.0253$ Season $=$ Summer, Stream $=E$, Direction $=E$, Date $\&$ Time $=2011-09-24$ 16:05:15 Incident Reason $=$ Collision

Affected Lanes = 2RIGHT LANE(S) AND RIGHT SHOULDER

Conditional Probability level $=0.6$, Cond. Prob. $r 3 s 2=0.2$, Cond. Prob. $r 3 s 1=0.1$, Percent drop in speed $\%=-56$

Max. Posterior Probability level $=0.97$, Percent range between speed states $=7 \%$

Incident was detected at 02:20 minutes from drop in speed point. Incident was confirmed within 5 minutes from the detection timı

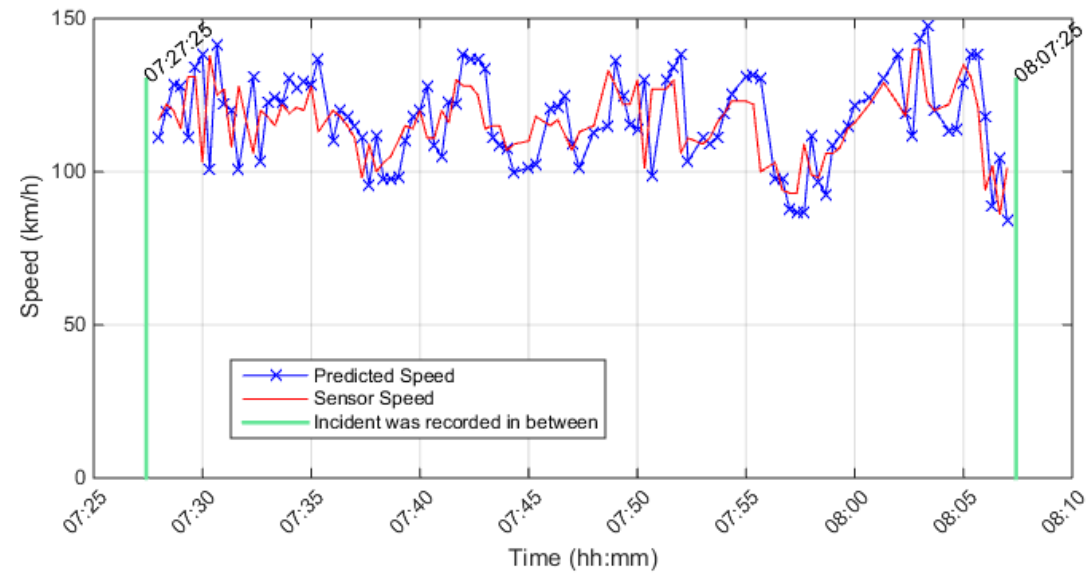

Upstream Detector \# is 401DE0080DEE, Detector Rank $=3$, Distance from incident location in $(\mathrm{km})=1.7056$ Season $=$ Summer, Stream $=E$, Direction $=E$, Date \& Time $=2011-10-01$ 07:47:25 Incident Reason $=$ Collision

Affected Lanes = 1 RIGHT LANE(S)

Conditional Probability level $=0.6$, Cond. Prob. $r 3 \mathrm{~s} 2=0.2$, Cond.Prob. $r 3 \mathrm{~s} 1=0.1$, Percent drop in speed $\%=-56$ Max. Posterior Probability level $=0.97$, Percent range between speed states $=7 \%$

(No Incident was Detected) 


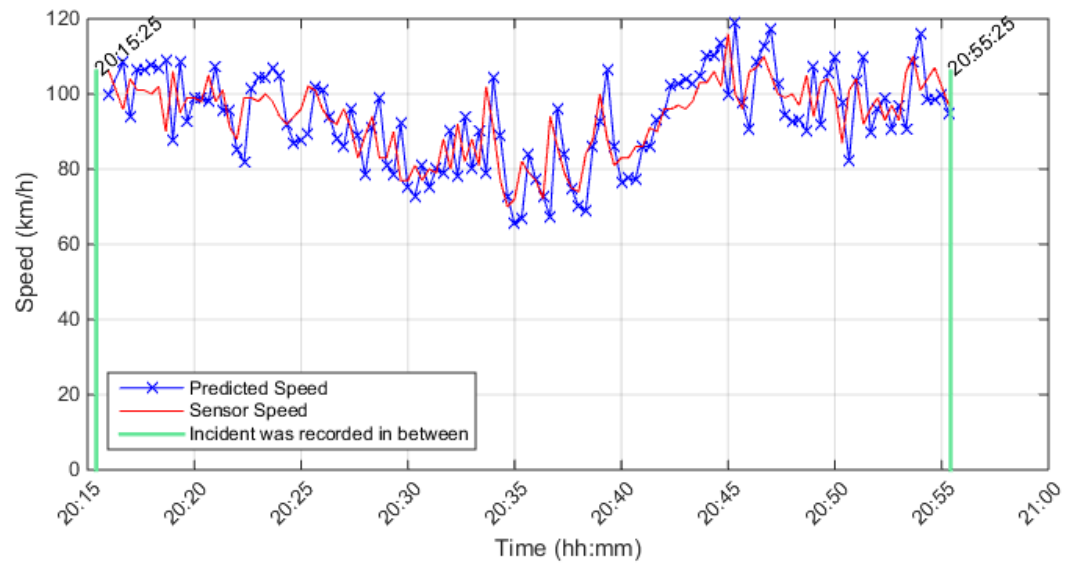

Upstream Detector \# is 401DW0160DWC, Detector Rank = 1, Distance from incident location in $(\mathrm{km})=0.1854$ Season $=$ Winter, Stream $=$ C, Direction $=$ W, Date $\&$ Time $=$ 2011-01-17 20:35:25

Incident Reason $=$ Collision

Affected Lanes = 1 RIGHT LANE(S)

Conditional Probability level $=0.6$, Cond. Prob. $r 3 s 2=0.2$, Cond. Prob. $r 3 s 1=0.1$, Percent drop in speed $\%=-56$

Max. Posterior Probability level $=0.97$, Percent range between speed states $=7 \%$

(No Incident was Detected)

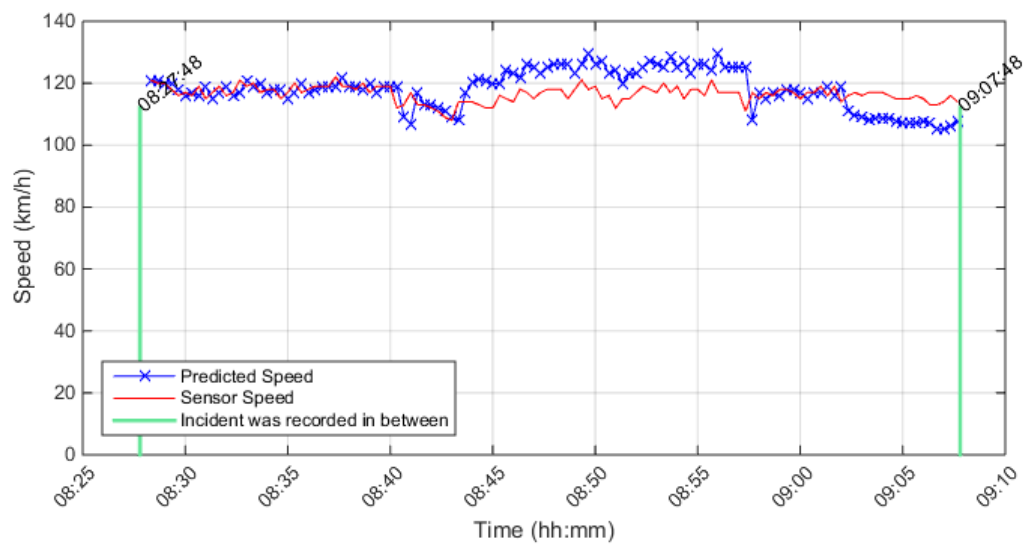

Upstream Detector \# is 401DE0530DWC, Detector Rank = 3, Distance from incident location in $(\mathrm{km})=1.6372$ Season $=$ Spring, Stream $=C$, Direction $=W$, Date $\&$ Time $=2011-04-1608: 47: 48$

Incident Reason $=$ Collision

Affected Lanes $=$ All lanes closed

Conditional Probability level $=0.6$, Cond. Prob. $r 3 s 2=0.2$, Cond. Prob. $r 3 s 1=0.1$, Percent drop in speed $\%=-56$

Max. Posterior Probability level $=0.97$, Percent range between speed states $=7 \%$

(No Incident was Detected) 


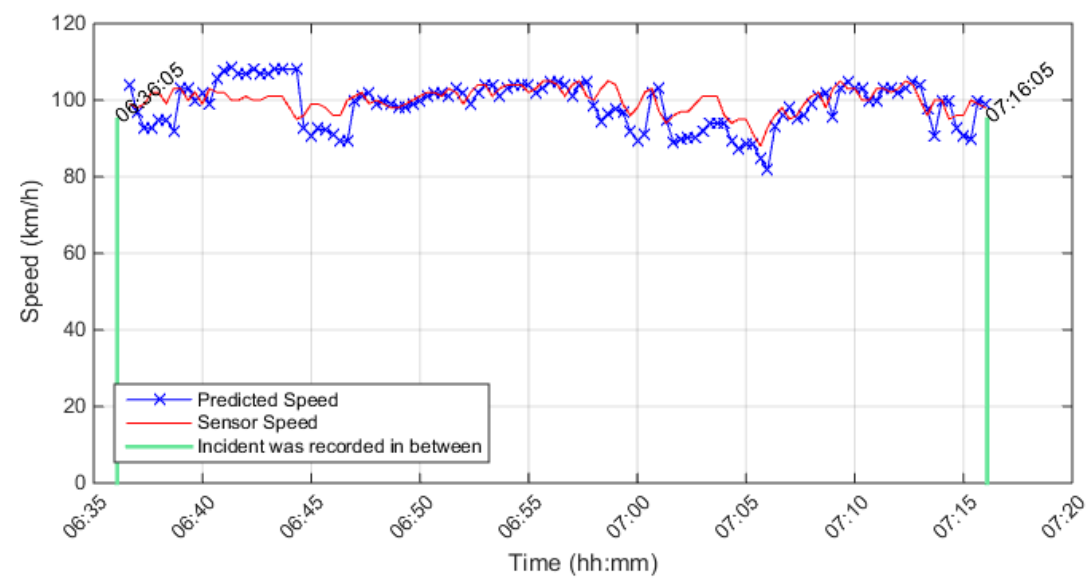

Upstream Detector \# is 401DE0500DWC, Detector Rank = 3, Distance from incident location in $(\mathrm{km})=1.4372$ Season $=$ Spring, Stream $=$ C , Direction $=W$, Date $\&$ Time $=2011-04-08$ 06:56:05 Incident Reason $=$ Disable Vehicle

Affected Lanes = 1 RIGHT LANE(S)

Conditional Probability level $=0.6$, Cond.Prob. $r 3 s 2=0.2$, Cond. Prob. $r 3 s 1=0.1$, Percent drop in speed $\%=-56$ Max. Posterior Probability level $=0.97$, Percent range between speed states $=7 \%$ (No Incident was Detected)

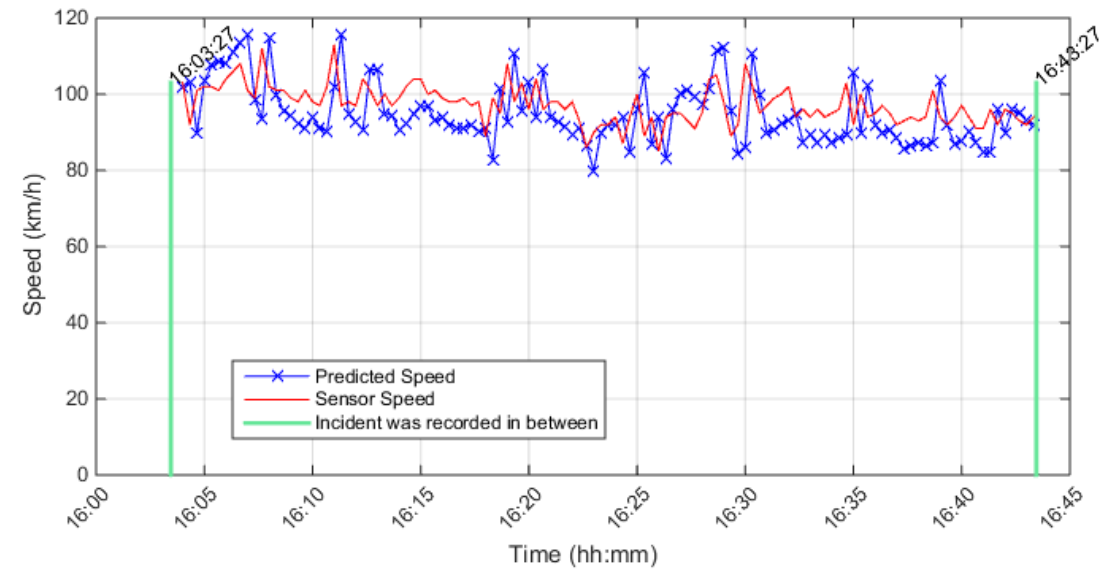

Upstream Detector \# is 401DE0060DWC, Detector Rank $=1$, Distance from incident location in $(\mathrm{km})=0.16757$ Season $=$ Winter, Stream $=$ C, Direction $=$ W, Date $\&$ Time $=2011-02-0516: 23: 27$

Incident Reason $=$ Disable Vehicle

Affected Lanes = 1 RIGHT LANE(S) AND RIGHT SHOULDER

Conditional Probability level $=0.6$, Cond.Prob. $r 3 s 2=0.2$, Cond.Prob. $r 3 s 1=0.1$, Percent drop in speed $\%=-56$ Max. Posterior Probability level $=0.97$, Percent range between speed states $=7 \%$

(No Incident was Detected) 


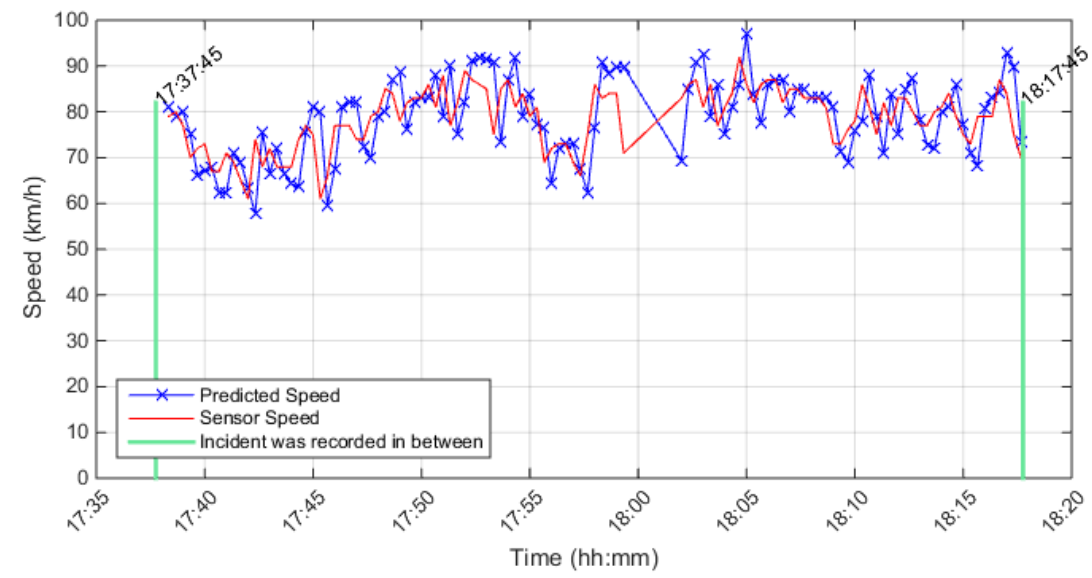

Upstream Detector \# is 401DW0020DWC, Detector Rank = 1, Distance from incident location in $(\mathrm{km})=0.88006$ Season $=$ Summer, Stream $=$ C, Direction $=W$, Date $\&$ Time $=2011-06-1017: 57: 45$ Incident Reason $=$ Collision

Affected Lanes $=2$ RIGHT LANE(S) AND RIGHT SHOULDER

Conditional Probability level $=0.6$, Cond.Prob. $r 3 \mathrm{~s} 2=0.2$, Cond.Prob. $r 3 \mathrm{~s} 1=0.1$, Percent drop in speed $\%=-56$

Max. Posterior Probability level $=0.97$, Percent range between speed states $=7 \%$

(No Incident was Detected)

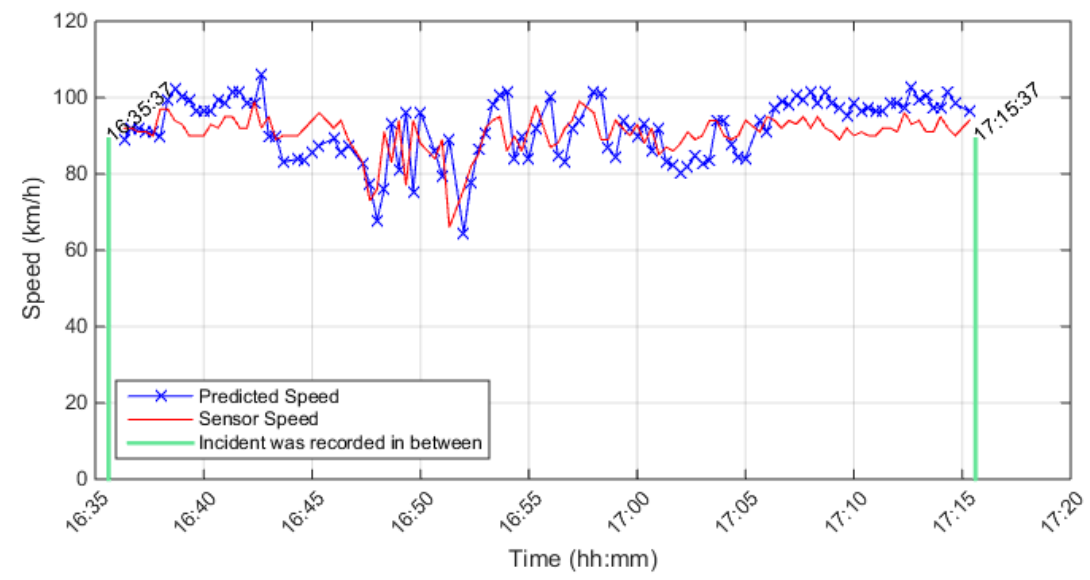

Upstream Detector \# is 401DE0220DWC, Detector Rank = 1, Distance from incident location in $(\mathrm{km})=0.29463$ Season $=$ Spring, Stream $=$ C, Direction $=$ W, Date $\&$ Time $=$ 2011-04-26 16:55:37

Incident Reason $=$ Collision

Affected Lanes = 2 RIGHT LANE(S) AND RIGHT SHOULDER

Conditional Probability level $=0.6$, Cond.Prob. $r 3 \mathrm{~s} 2=0.2$, Cond.Prob. $r 3 \mathrm{~s} 1=0.1$, Percent drop in speed $\%=-56$ Max. Posterior Probability level $=0.97$, Percent range between speed states $=7 \%$

(No Incident was Detected) 


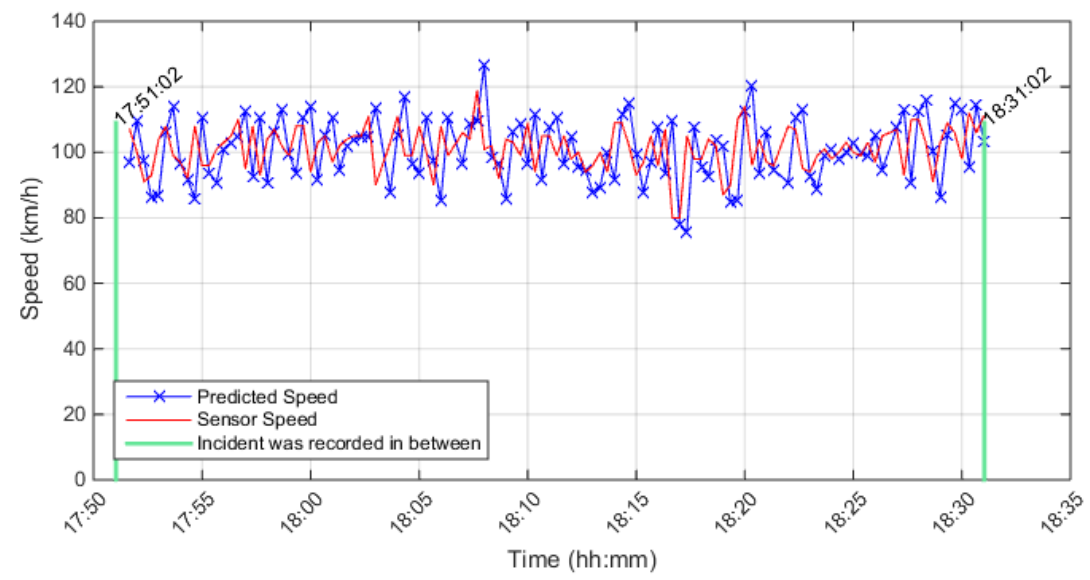

Upstream Detector \# is 401DE0370DWC, Detector Rank = 3, Distance from incident location in $(\mathrm{km})=2.0297$ Season $=$ Summer, Stream $=$ C, Direction $=$ W, Date $\&$ Time $=2011-06-05$ 18:11:02 Incident Reason $=$ Collision

Affected Lanes $=$ LEFT SHOULDER AND 2 LEFT LANE(S)

Conditional Probability level $=0.6$, Cond. Prob. $r 3 s 2=0.2$, Cond. Prob. $r 3 s 1=0.1$, Percent drop in speed $\%=-56$ Max. Posterior Probability level $=0.97$, Percent range between speed states $=7 \%$ (No Incident was Detected)

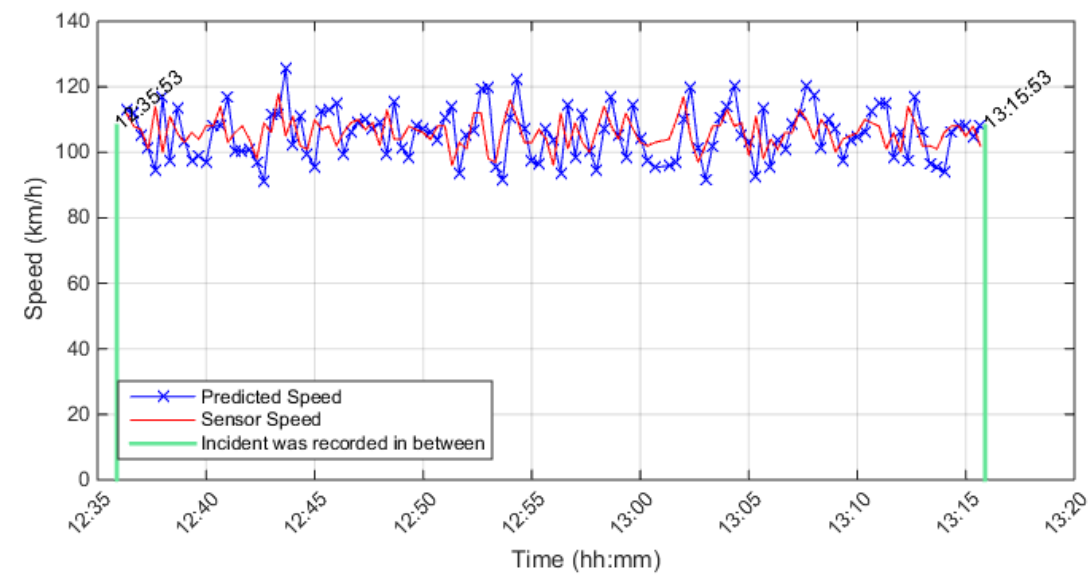

Upstream Detector \# is 401DE0320DWC, Detector Rank = 1, Distance from incident location in $(\mathrm{km})=0.13977$ Season $=$ Summer, Stream $=$ C, Direction $=W$, Date $\&$ Time $=2011-06-12$ 12:55:53

Incident Reason $=$ Collision
Affected Lanes $=$ LEFT SHOULDER AND 1 LEFT LANE(S)

Conditional Probability level $=0.6$, Cond.Prob. $r 3 s 2=0.2$, Cond. Prob. $r 3 s 1=0.1$, Percent drop in speed $\%=-56$ Max. Posterior Probability level $=0.97$, Percent range between speed states $=7 \%$ (No Incident was Detected) 


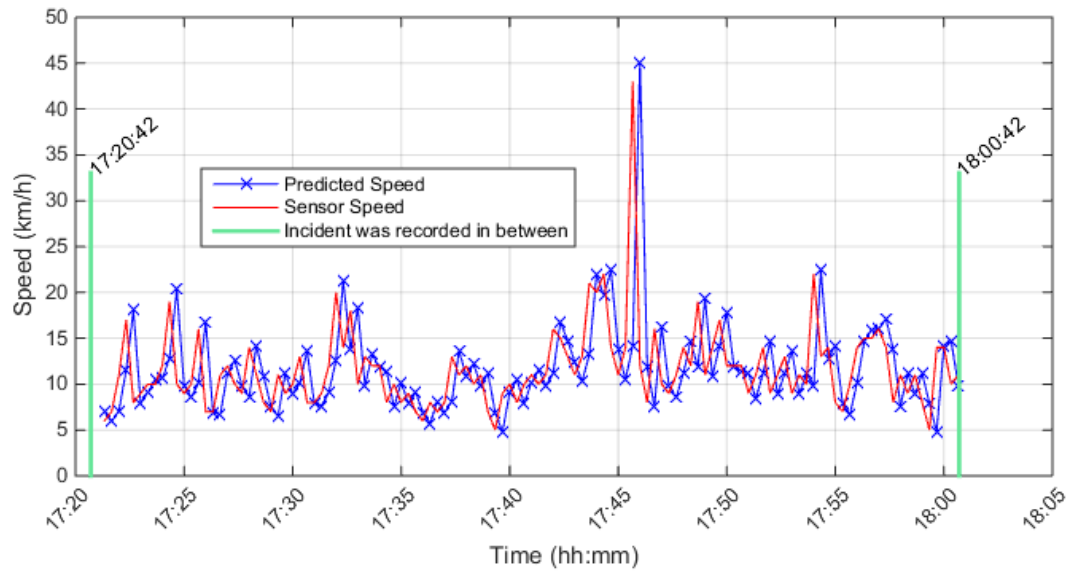

Upstream Detector \# is 401DE0050DWC, Detector Rank $=1$, Distance from incident location in $(\mathrm{km})=0.45946$ Season $=$ Summer, Stream $=$ C , Direction $=$ W, Date $\&$ Time $=2011-07-0517: 40: 42$ Incident Reason $=$ Collision

Affected Lanes $=2$ CENTRE LANES(S)

Conditional Probability level $=0.6$, Cond. Prob. $r 3 s 2=0.2$, Cond. Prob. $r 3 s 1=0.1$, Percent drop in speed $\%=-56$ Max. Posterior Probability level $=0.97$, Percent range between speed states $=7 \%$ (No Incident was Detected)

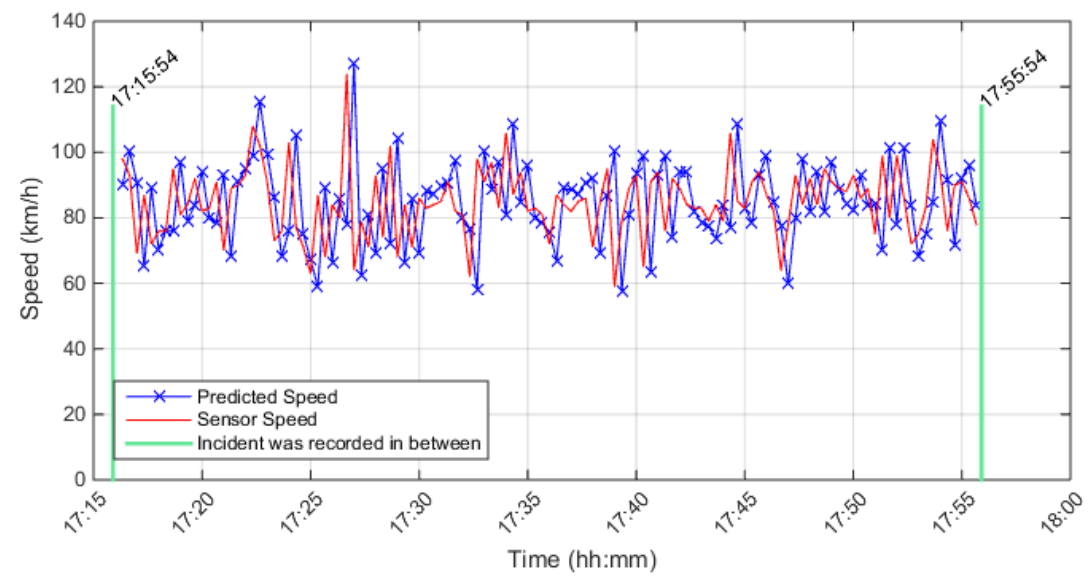

Upstream Detector \# is 401DE0150DWC, Detector Rank = 3, Distance from incident location in $(\mathrm{km})=1.5503$ Season $=$ Summer, Stream $=$ C, Direction $=$ W, Date $\&$ Time $=2011-08-07$ 17:35:54 Incident Reason $=$ Collision Affected Lanes $=2$ LEFT LANE(S)

Conditional Probability level $=0.6$, Cond. Prob. $r 3 \mathrm{~s} 2=0.2$, Cond. Prob. $\mathrm{r} 3 \mathrm{~s} 1=0.1$, Percent drop in speed $\%=-56$ Max. Posterior Probability level $=0.97$. Percent range between speed states $=7 \%$ (No Incident was Detected) 


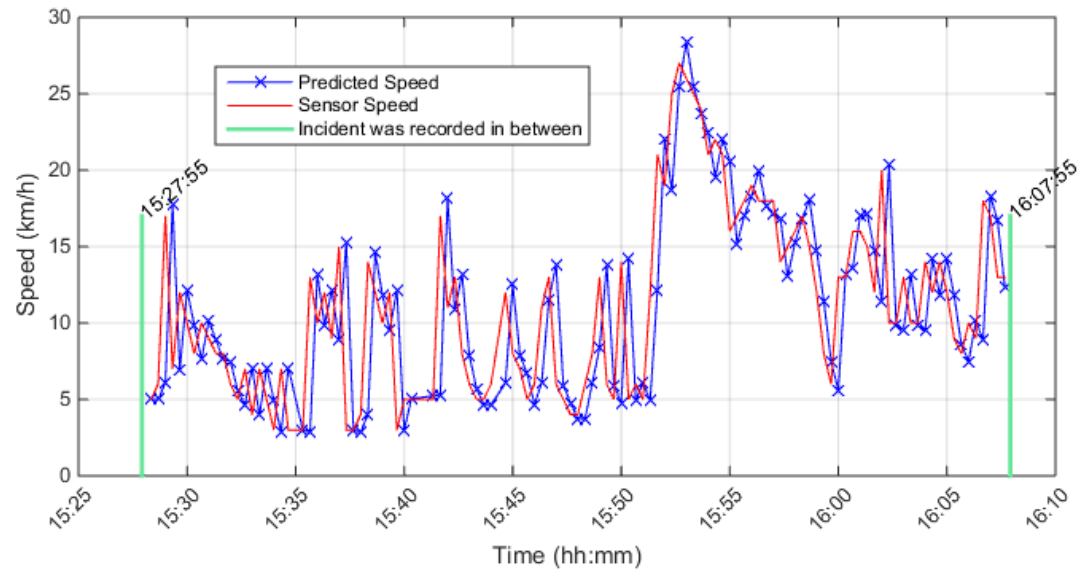

Upstream Detector \# is 401DW0060DWC, Detector Rank = 1, Distance from incident location in $(\mathrm{km})=0.26756$ Season $=$ Summer, Stream $=$ C , Direction $=$ W, Date $\&$ Time $=2011-09-15$ 15:47:55 Incident Reason $=$ Collision

Affected Lanes = 1 RIGHT LANE(S)

Conditional Probability level $=0.6$, Cond. Prob. $r 3 s 2=0.2$, Cond. Prob. $r 3 s 1=0.1$, Percent drop in speed $\%=-56$

Max. Posterior Probability level $=0.97$, Percent range between speed states $=7 \%$

Drop in speed was detected but Not enough recovery time to confirm an incident

For the previous case, even if 120 min before and after is taken, no indication of any drop in speed

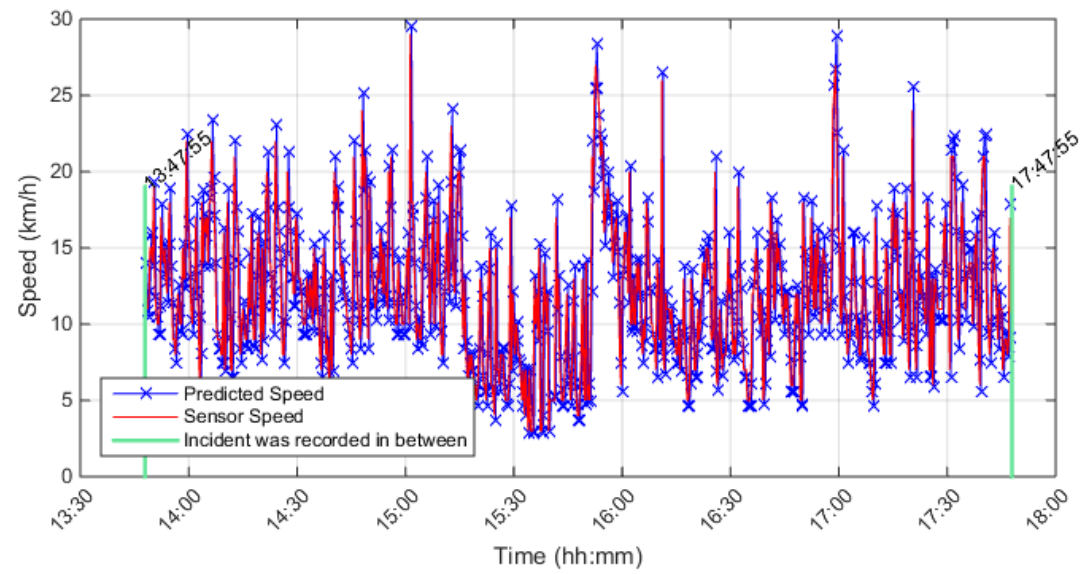

Upstream Detector \# is 401DW0060DWC, Detector Rank $=1$, Distance from incident location in $(\mathrm{km})=0.26756$ Season $=$ Summer, Stream $=C$, Direction $=W$, Date $\&$ Time $=2011-09-15$ 15:47:55

Incident Reason $=$ Collision

Affected Lanes = 1 RIGHT LANE(S)

Conditional Probability level $=0.6$, Cond.Prob. $r 3 \mathrm{~s} 2=0.2$, Cond.Prob. $r 3 \mathrm{~s} 1=0.1$, Percent drop in speed $\%=-56$

Max. Posterior Probability level $=0.97$, Percent range between speed states $=7 \%$

Drop in speed was detected and No indication of an incident within this period 


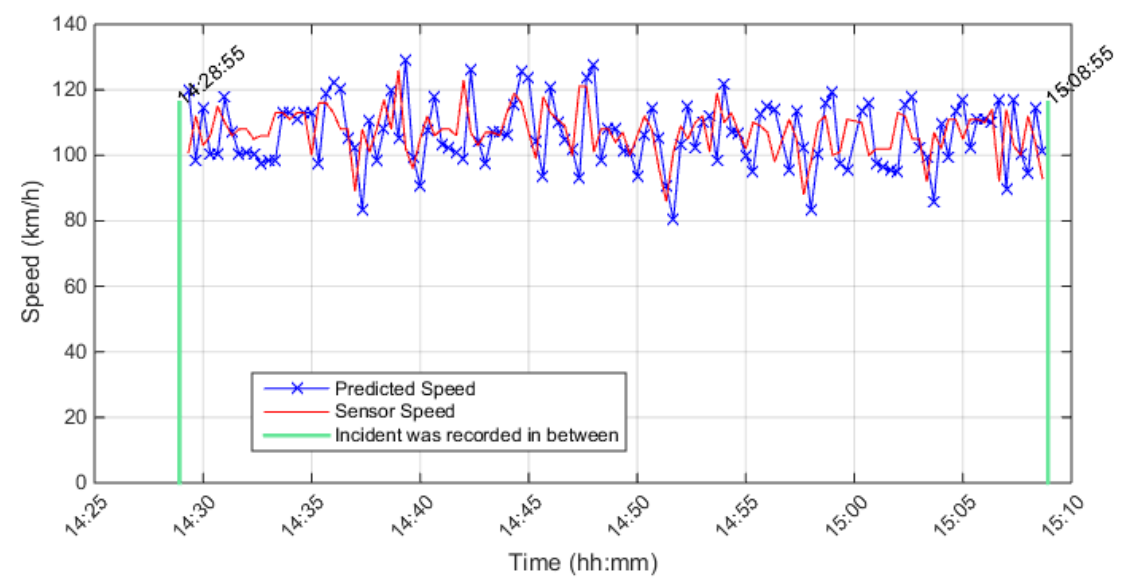

Upstream Detector \# is 401DE0350DWC, Detector Rank = 1 , Distance from incident location in $(\mathrm{km})=0.24334$ Season $=$ Winter, Stream $=$ C, Direction $=$ W, Date $\&$ Time $=2011-12-0214: 48: 55$ Incident Reason $=$ Collision

Affected Lanes $=2$ RIGHT LANE(S) AND RIGHT SHOULDER

Conditional Probability level $=0.6$, Cond. Prob. $r 3 s 2=0.2$, Cond.Prob. $r 3 s 1=0.1$, Percent drop in speed $\%=-56$ Max. Posterior Probability level $=0.97$, Percent range between speed states $=7 \%$ (No Incident was Detected)

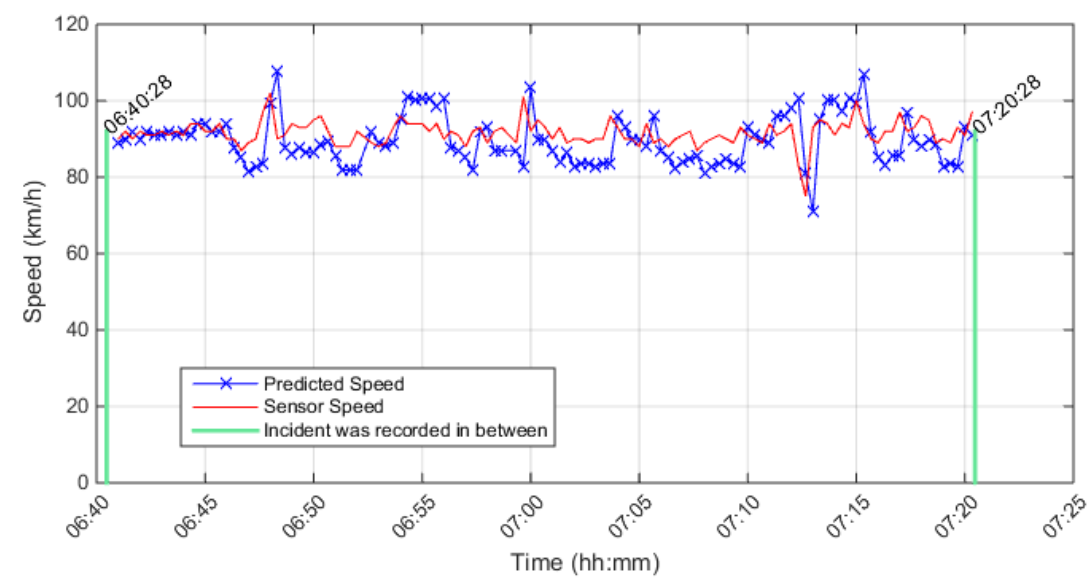

Upstream Detector \# is 401DE0220DWC, Detector Rank = 2, Distance from incident location in $(\mathrm{km})=1.1626$ Season $=$ Winter, Stream $=$ C, Direction $=$ W, Date $\&$ Time $=2011-12-15$ 07:00:28 Incident Reason $=$ Collision

Affected Lanes $=1$ LEFT LANE(S)

Conditional Probability level $=0.6$, Cond.Prob. $r 3 s 2=0.2$, Cond.Prob. $r 3 s 1=0.1$, Percent drop in speed $\%=-56$ Max. Posterior Probability level $=0.97$, Percent range between speed states $=7 \%$ (No Incident was Detected) 


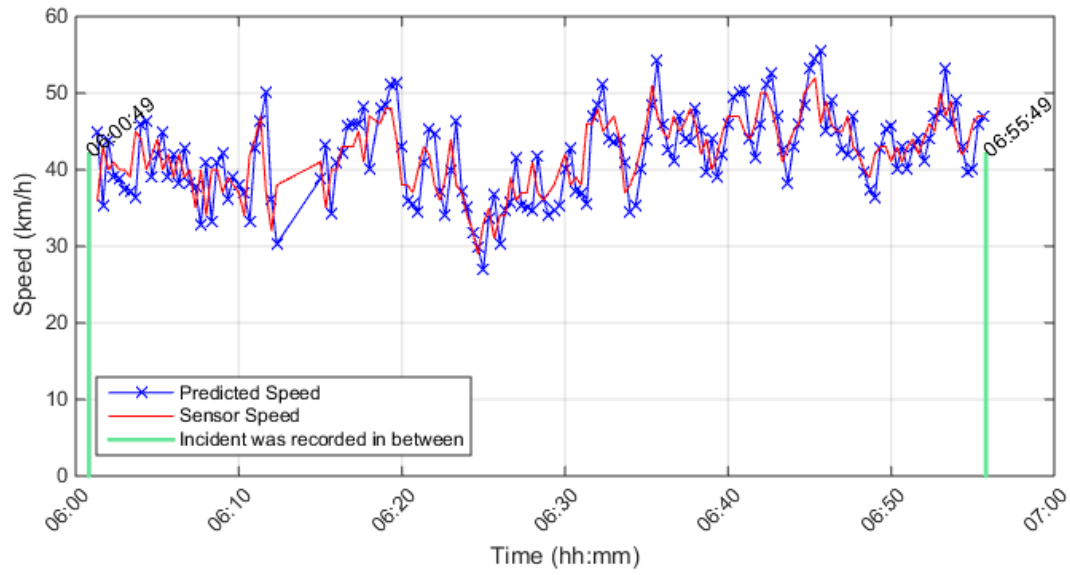

Upstream Detector \# is 401DE0060DWE, Detector Rank = 1, Distance from incident location in $(\mathrm{km})=0.17254$ Season $=$ Winter, Stream $=$ E, Direction $=$ W, Date $\&$ Time $=$ 2011-01-12 06:05:49 Incident Reason $=$ Collision

Affected Lanes = 2 RIGHT LANE(S)

Conditional Probability level $=0.6$, Cond. Prob. $r 3 \mathrm{~s} 2=0.2$, Cond. Prob. $r 3 \mathrm{~s} 1=0.1$, Percent drop in speed $\%=-56$ Max. Posterior Probability level $=0.97$, Percent range between speed states $=7 \%$ (No Incident was Detected)

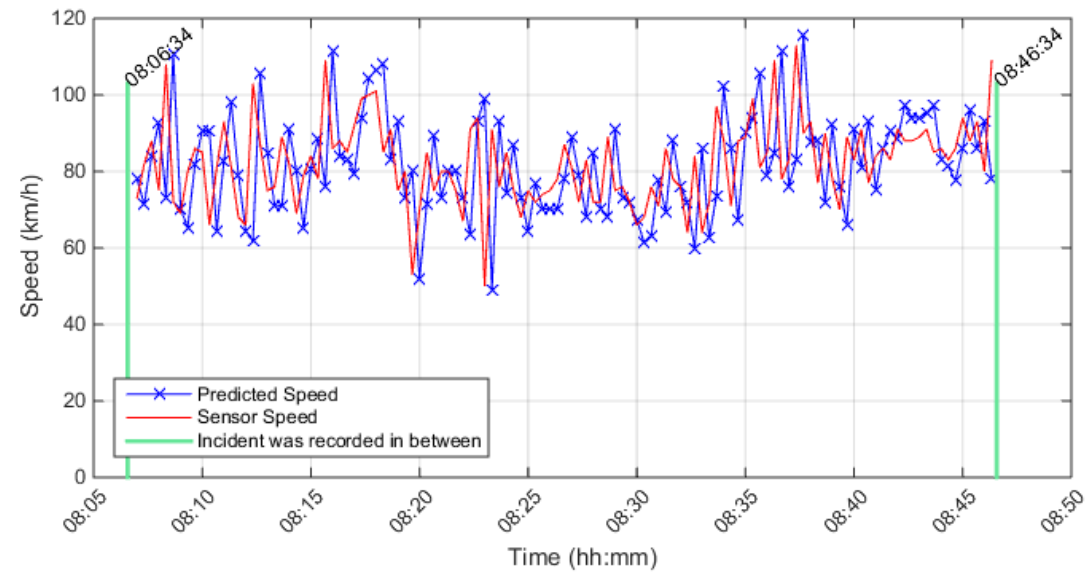

Upstream Detector \# is 401DE0050DWE, Detector Rank = 1, Distance from incident location in $(\mathrm{km})=0.36941$ Season $=$ Winter, Stream $=$ E, Direction $=$ W, Date $\&$ Time $=2011-02-02$ 08:26:34 Incident Reason $=$ Collision

Affected Lanes = 3 RIGHT LANE(S)

Conditional Probability level $=0.6$, Cond.Prob. $r 3 s 2=0.2$, Cond.Prob. $r 3 s 1=0.1$, Percent drop in speed $\%=-56$ Max. Posterior Probability level $=0.97$. Percent range between speed states $=7 \%$

(No Incident was Detected) 


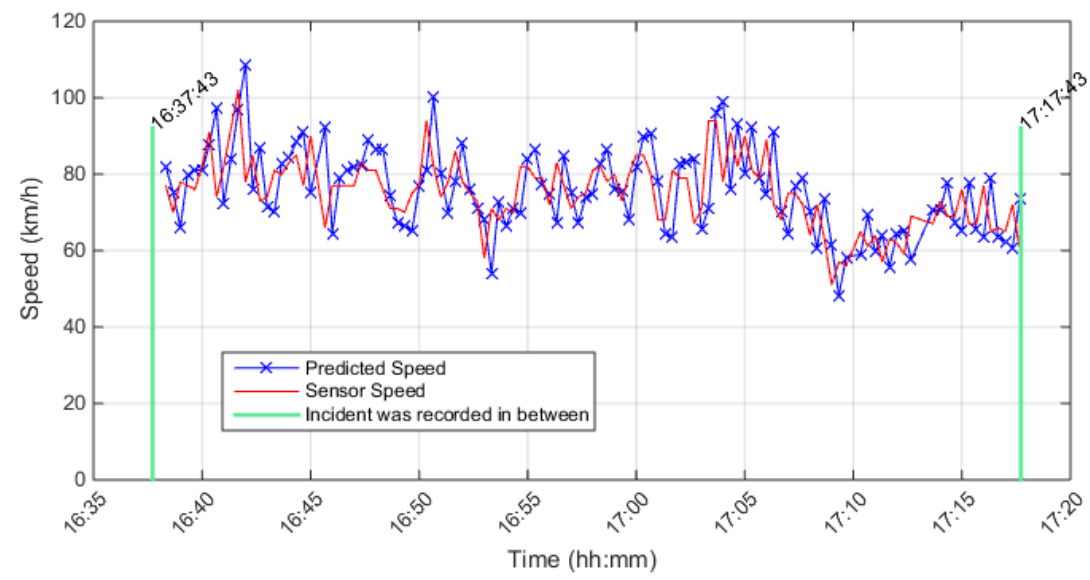

Upstream Detector \# is 401DE0290DWE, Detector Rank = 1, Distance from incident location in $(\mathrm{km})=0.27431$ Season $=$ Winter, Stream $=$ E, Direction $=$ W, Date $\&$ Time $=$ 2011-02-02 16:57:43 Incident Reason $=$ Collision

Affected Lanes $=1$ RIGHT LANE(S) AND RIGHT SHOULDER

Conditional Probability level $=0.6$, Cond.Prob. $r 3 \mathrm{~s} 2=0.2$, Cond. Prob. $r 3 \mathrm{~s} 1=0.1$, Percent drop in speed $\%=-56$ Max. Posterior Probability level $=0.97$, Percent range between speed states $=7 \%$ (No Incident was Detected)

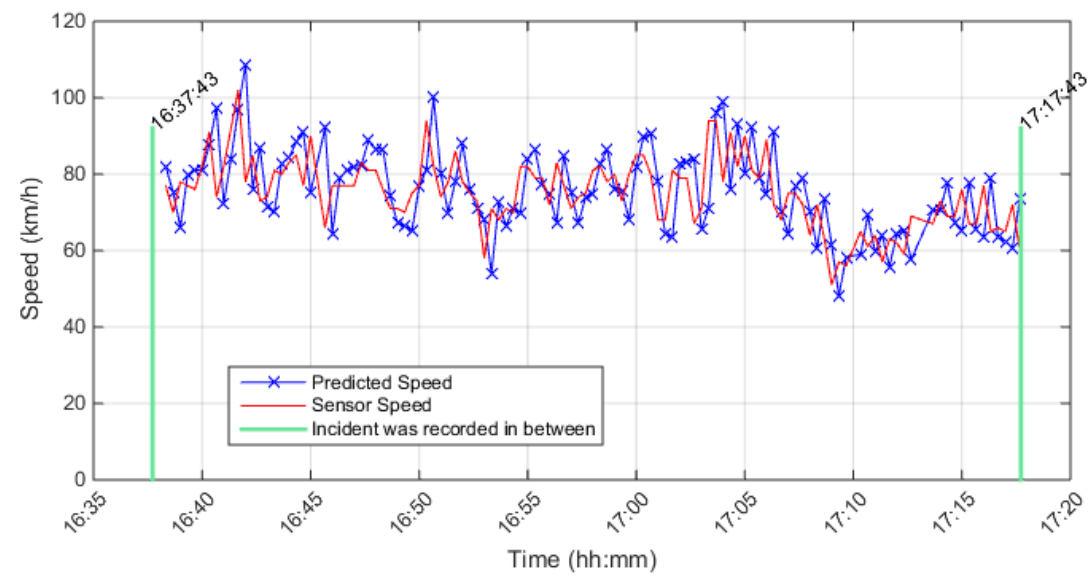

Upstream Detector \# is 401DE0290DWE, Detector Rank = 1, Distance from incident location in $(\mathrm{km})=0.27431$ Season $=$ Winter, Stream $=E$, Direction $=W$, Date $\&$ Time $=$ 2011-02-02 16:57:43 Incident Reason $=$ Collision

Affected Lanes $=1$ RIGHT LANE(S) AND RIGHT SHOULDER

Conditional Probability level $=0.6$, Cond.Prob. $r 3 \mathrm{~s} 2=0.2$, Cond. Prob. $r 3 \mathrm{~s} 1=0.1$, Percent drop in speed $\%=-56$

Max. Posterior Probability level $=0.97$. Percent range between speed states $=7 \%$

(No Incident was Detected) 


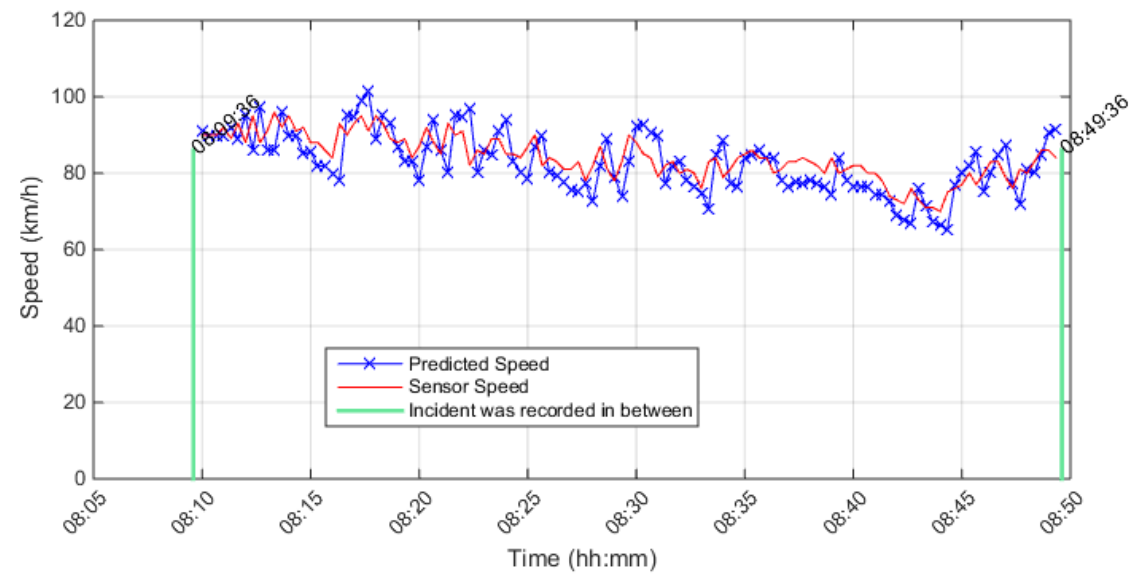

Upstream Detector \# is 401DE0020DWE, Detector Rank = 1, Distance from incident location in $(\mathrm{km})=0.39496$ Season $=$ Spring, Stream $=E$, Direction $=W$, Date $\&$ Time $=$ 2011-03-06 08:29:36 Incident Reason $=$ Collision

Affected Lanes = 2 RIGHT LANE(S)

Conditional Probability level $=0.6$, Cond. Prob. $r 3 s 2=0.2$, Cond. Prob. $r 3 s 1=0.1$, Percent drop in speed $\%=-56$ Max. Posterior Probability level $=0.97$, Percent range between speed states $=7 \%$ (No Incident was Detected)

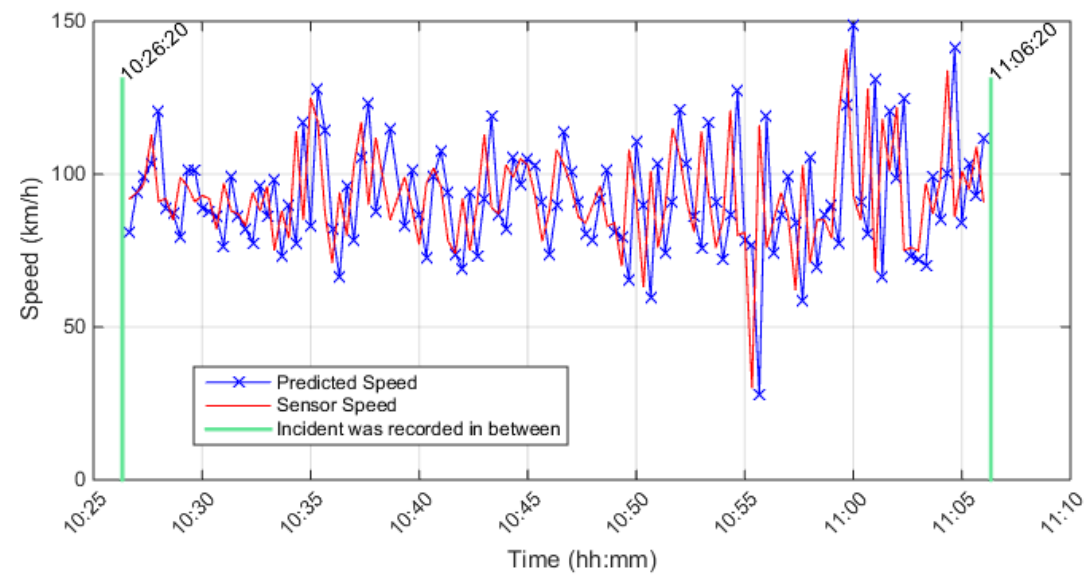

Upstream Detector \# is 401DW0260DWE, Detector Rank = 1, Distance from incident location in $(\mathrm{km})=0.17943$ Season $=$ Spring, Stream $=\mathrm{E}$, Direction $=\mathrm{W}$, Date $\&$ Time $=2011-03-23$ 10:46:20 Incident Reason $=$ Collision

Affected Lanes $=3$ LEFT LANE(S)

Conditional Probability level $=0.6$, Cond.Prob. $r 3 s 2=0.2$, Cond.Prob. $r 3 s 1=0.1$, Percent drop in speed $\%=-56$ Max. Posterior Probability level $=0.97$, Percent range between speed states $=7 \%$

Drop in speed was detected and No indication of an incident within this period 


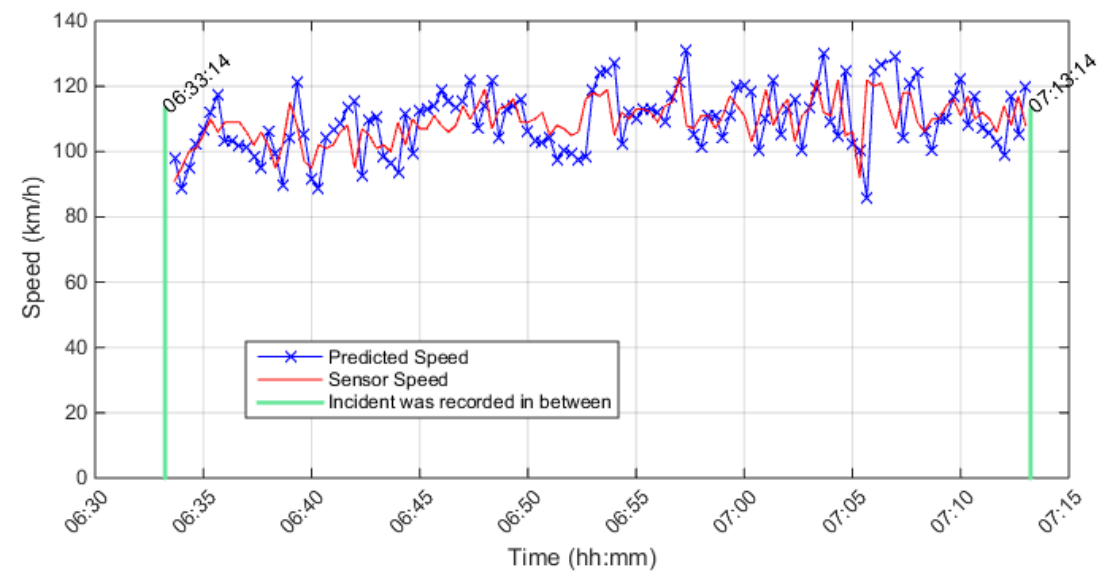

Upstream Detector \# is 401DW0020DWE, Detector Rank $=1$, Distance from incident location in $(\mathrm{km})=0.13067$ Season $=$ Spring, Stream $=E$, Direction $=$ W, Date \& Time $=$ 2011-04-09 06:53:14 Incident Reason $=$ Collision

Affected Lanes = LEFT SHOULDER AND 1 LEFT LANE(S)

Conditional Probability level $=0.6$, Cond. Prob. $r 3 \mathrm{~s} 2=0.2$, Cond.Prob. $r 3 \mathrm{~s} 1=0.1$, Percent drop in speed $\%=-56$

Max. Posterior Probability level $=0.97$. Percent range between speed states $=7 \%$

(No Incident was Detected)

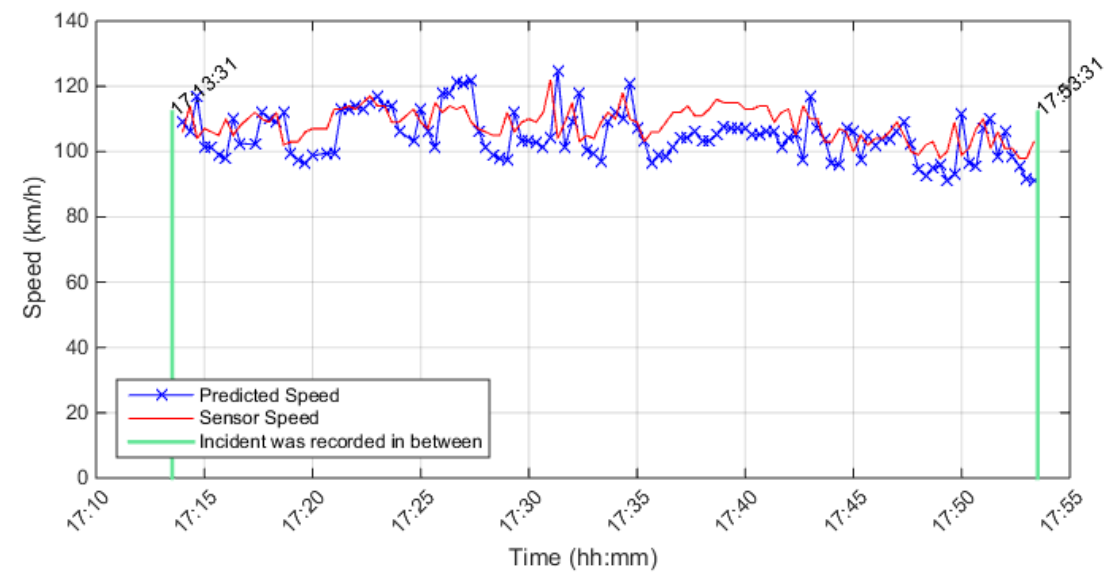

Upstream Detector \# is 401DE0020DWE, Detector Rank = 1, Distance from incident location in $(\mathrm{km})=0.39496$ Season $=$ Summer, Stream $=E$, Direction $=$ W. Date \& Time $=2011-06-0517: 33: 31$

Incident Reason $=$ Collision

Affected Lanes = 1 CENTRE LANES(S)

Conditional Probability level $=0.6$, Cond.Prob. $r 3 s 2=0.2$, Cond. Prob. $r 3 s 1=0.1$, Percent drop in speed $\%=-56$

Max. Posterior Probability level $=0.97$, Percent range between speed states $=7 \%$

(No Incident was Detected) 


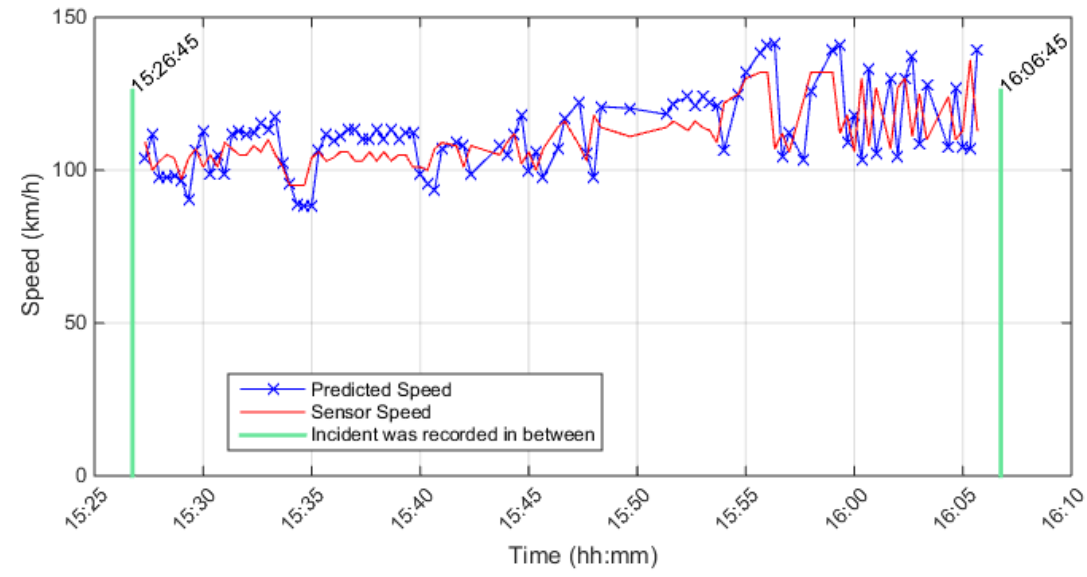

Upstream Detector \# is 401DE0020DWE, Detector Rank = 1, Distance from incident location in $(\mathrm{km})=0.39496$ Season $=$ Summer, Stream $=E$, Direction $=W$, Date $\&$ Time $=2011-06-2015: 46: 45$ Incident Reason $=$ Collision

Affected Lanes $=$ LEFT SHOULDER AND 2 LEFT LANE(S)

Conditional Probability level $=0.6$, Cond. Prob. $r 3 s 2=0.2$, Cond. Prob. $r 3 s 1=0.1$, Percent drop in speed $\%=-56$ Max. Posterior Probability level $=0.97$, Percent range between speed states $=7 \%$ (No Incident was Detected)

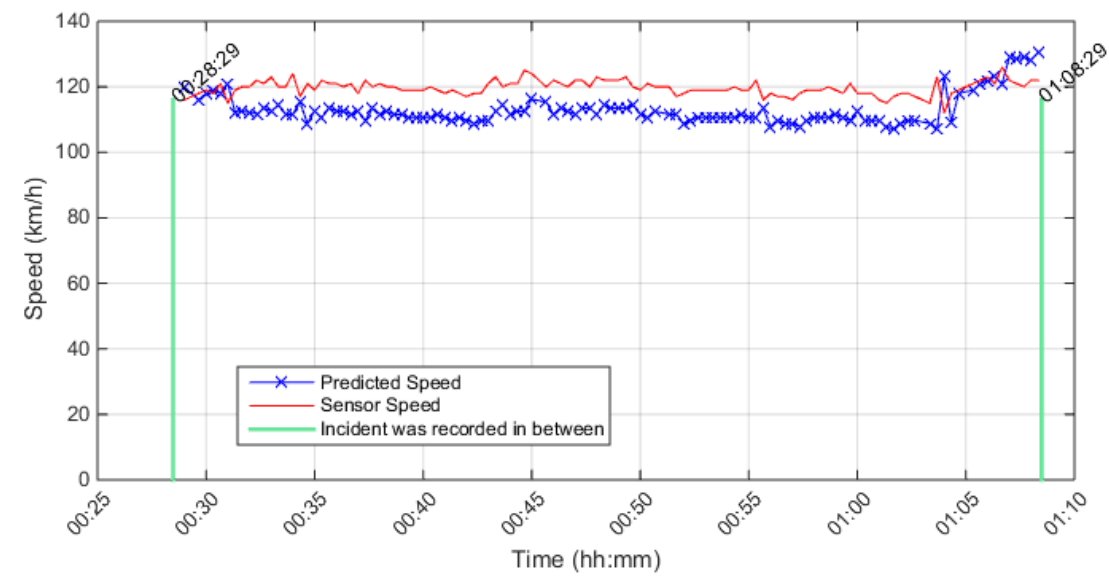

Upstream Detector \# is 401DE0130DWE, Detector Rank = 1, Distance from incident location in $(\mathrm{km})=0.14797$ Season $=$ Summer, Stream $=E$, Direction $=$ W, Date \& Time $=$ 2011-07-05 00:48:29 Incident Reason $=$ Collision

Affected Lanes = 2RIGHT LANE(S)

Conditional Probability level $=0.6$, Cond.Prob. $r 3 s 2=0.2$, Cond.Prob. $r 3 s 1=0.1$, Percent drop in speed $\%=-56$ Max. Posterior Probability level $=0.97$, Percent range between speed states $=7 \%$

(No Incident was Detected) 


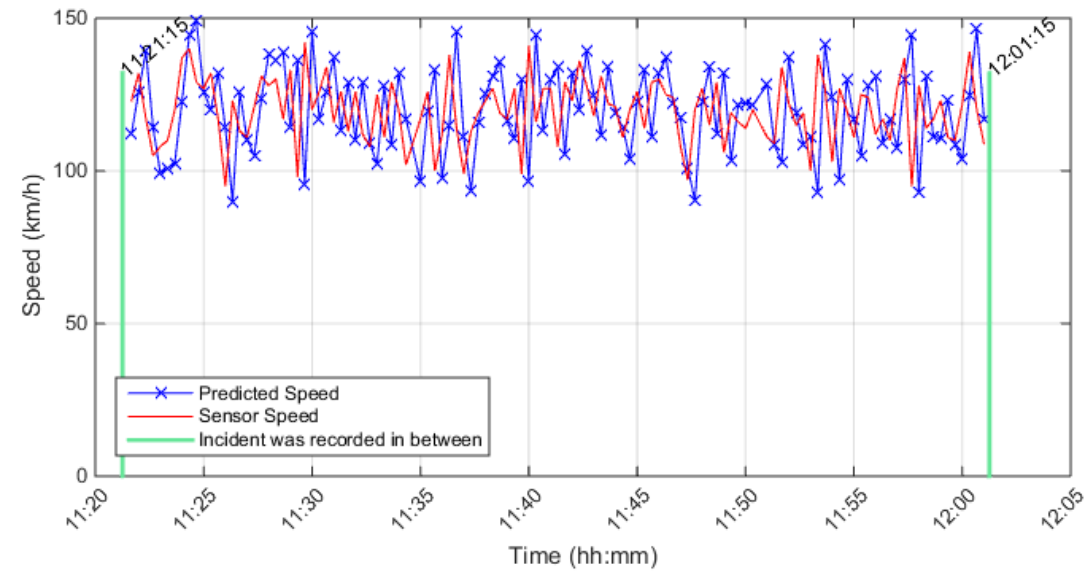

Upstream Detector \# is 401DW0280DWE, Detector Rank $=2$, Distance from incident location in $(\mathrm{km})=1.0705$ Season $=$ Summer, Stream $=E$, Direction $=W$, Date $\&$ Time $=2011-07-26$ 11:41:15 Incident Reason $=$ Collision

Affected Lanes $=2$ RIGHT LANE(S)

Conditional Probability level $=0.6$, Cond.Prob. $r 3 s 2=0.2$, Cond. Prob. $r 3 s 1=0.1$. Percent drop in speed $\%=-56$

Max. Posterior Probability level $=0.97$, Percent range between speed states $=7 \%$

(No Incident was Detected)

Same as previous case if $60 \mathrm{~min}$ before and $60 \mathrm{~min}$ after was considered

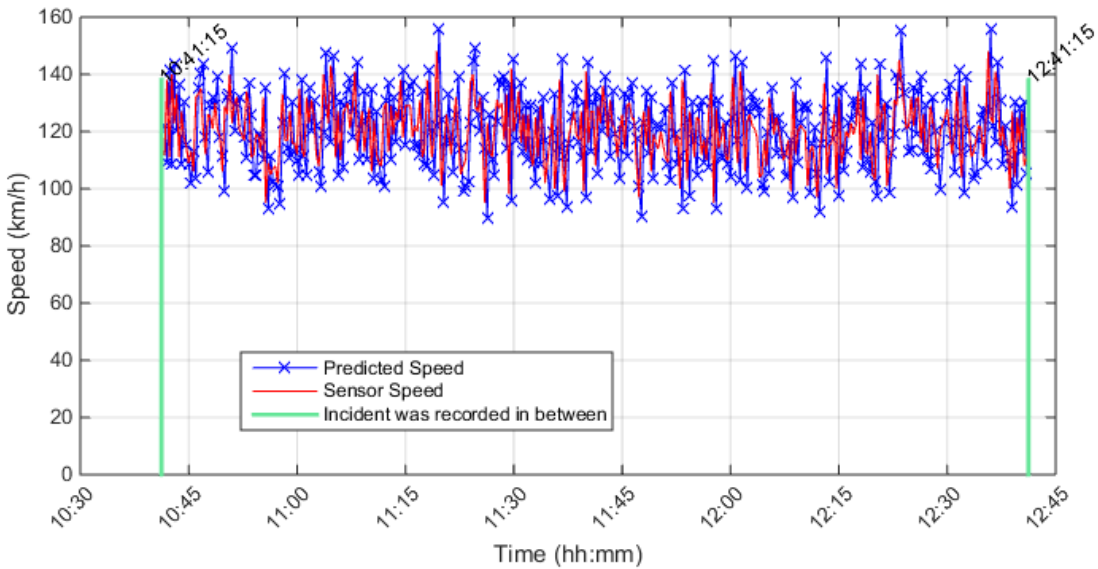

Upstream Detector \# is 401DW0280DWE, Detector Rank = 2, Distance from incident location in $(\mathrm{km})=1.0705$ Season $=$ Summer, Stream $=E$, Direction $=W$, Date $\&$ Time $=2011-07-2611: 41: 15$ Incident Reason $=$ Collision

Affected Lanes $=2$ RIGHT LANE(S)

Conditional Probability level $=0.6$, Cond. Prob. $r 3 s 2=0.2$, Cond. Prob. $r 3 s 1=0.1$. Percent drop in speed $\%=-56$

Max. Posterior Probability level $=0.97$, Percent range between speed states $=7 \%$

(No Incident was Detected) 


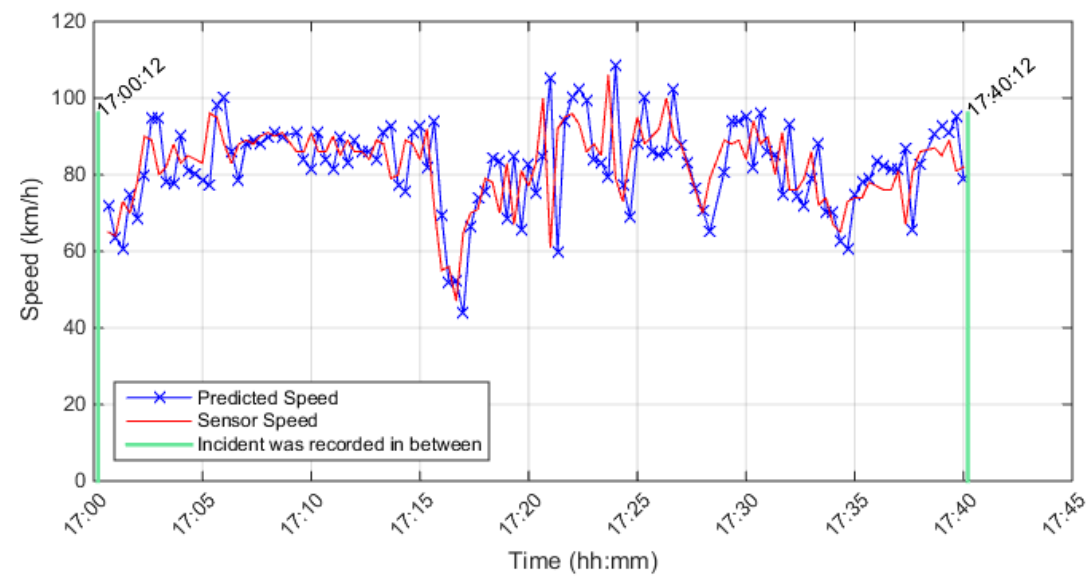

Upstream Detector \# is 401DE0020DWE, Detector Rank = 1, Distance from incident location in $(\mathrm{km})=0.39496$ Season $=$ Summer, Stream $=E$, Direction $=W$, Date $\&$ Time $=$ 2011-07-29 17:20:12 Incident Reason $=$ Collision

Affected Lanes $=3$ RIGHT LANE(S)

Conditional Probability level $=0.6$, Cond. Prob. $r 3 s 2=0.2$, Cond. Prob. $r 3 s 1=0.1$, Percent drop in speed $\%=-56$ Max. Posterior Probability level $=0.97$, Percent range between speed states $=7 \%$ (No Incident was Detected)

\section{Same as previous case if 60 min before and after was considered}

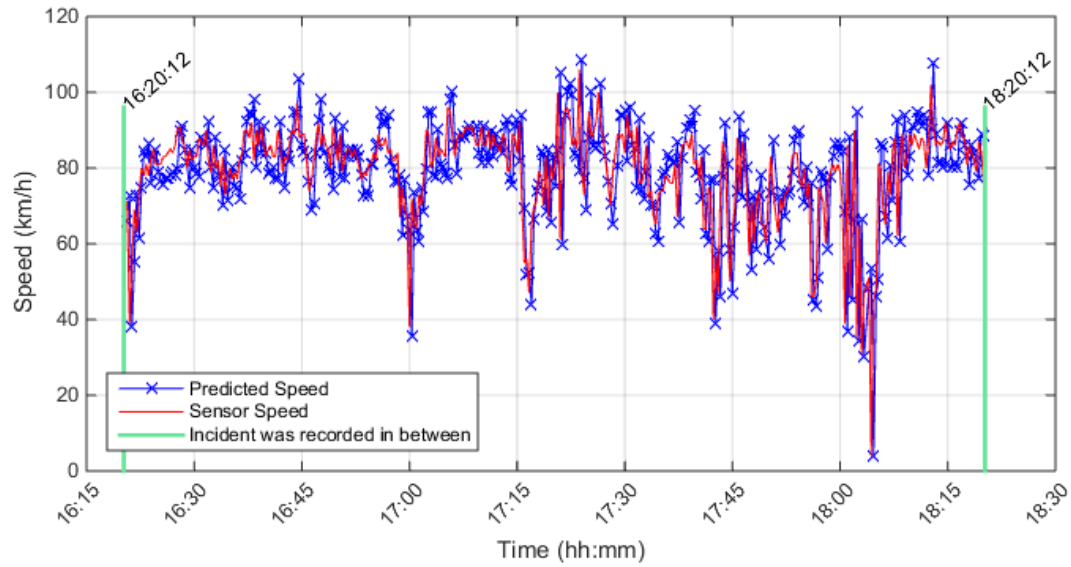

Upstream Detector \# is 401DE0020DWE, Detector Rank $=1$, Distance from incident location in $(\mathrm{km})=0.39496$ Season $=$ Summer, Stream $=E$, Direction $=W$, Date $\&$ Time $=2011-07-2917: 20: 12$ Incident Reason $=$ Collision

Affected Lanes $=3$ RIGHT LANE(S)

Conditional Probability level $=0.6$, Cond.Prob. $r 3 \mathrm{~s} 2=0.2$, Cond.Prob. $r 3 \mathrm{~s} 1=0.1$, Percent drop in speed $\%=-56$

Max. Posterior Probability level $=0.97$, Percent range between speed states $=7 \%$

Drop in speed was detected and No indication of an incident within this period 


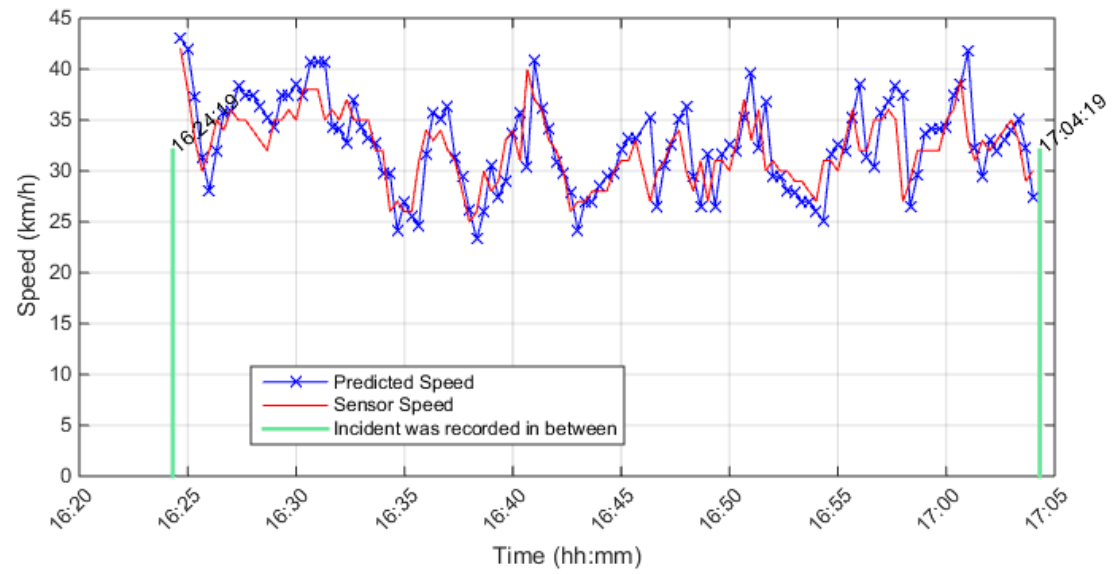

Upstream Detector \# is 401DE0060DWE, Detector Rank $=1$, Distance from incident location in $(\mathrm{km})=0.17254$ Season $=$ Summer, Stream $=E$, Direction $=W$, Date $\&$ Time $=2011-08-03$ 16:44:19 Incident Reason $=$ Collision

Affected Lanes $=$ LEFT SHOULDER AND 2 LEFT LANE(S)

Conditional Probability level $=0.6$, Cond.Prob. $r 3 \mathrm{~s} 2=0.2$, Cond. Prob. $r 3 \mathrm{~s} 1=0.1$, Percent drop in speed $\%=-56$

Max. Posterior Probability level $=0.97$, Percent range between speed states $=7 \%$

(No Incident was Detected)

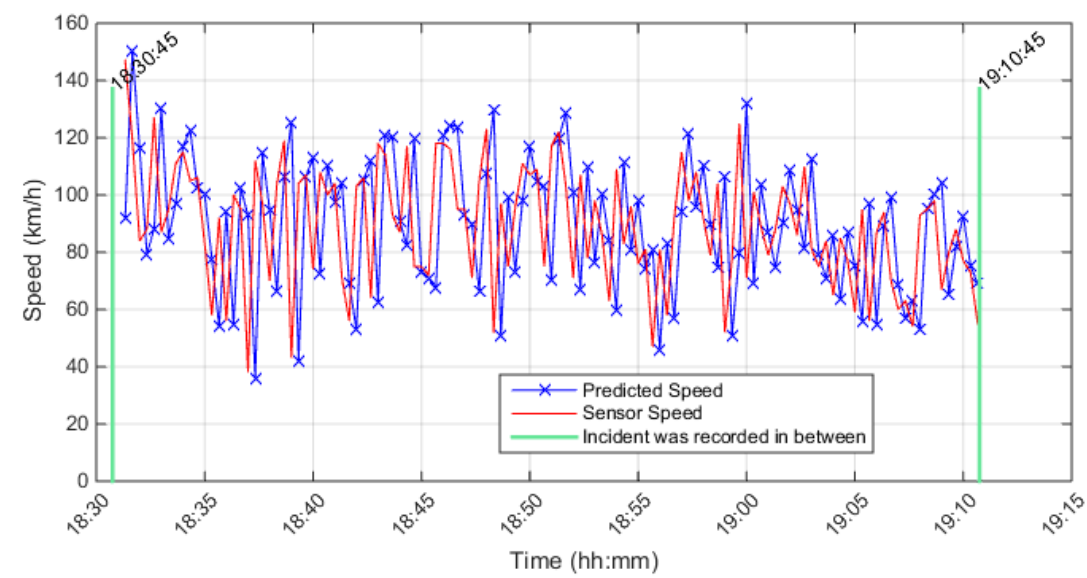

Upstream Detector \# is 401DE0340DWE, Detector Rank = 3, Distance from incident location in $(\mathrm{km})=1.6024$ Season $=$ Summer, Stream $=E$, Direction $=W$, Date $\&$ Time $=$ 2011-08-06 18:50:45 Incident Reason $=$ Collision

Affected Lanes = 1 RIGHT LANE(S)

Conditional Probability level $=0.6$, Cond.Prob. $r 3 s 2=0.2$, Cond.Prob. $r 3 s 1=0.1$, Percent drop in speed $\%=-56$ Max. Posterior Probability level $=0.97$, Percent range between speed states $=7 \%$

Drop in speed was detected and No indication of an incident within this period

Same as the previous case if 60 min before and after was considered 


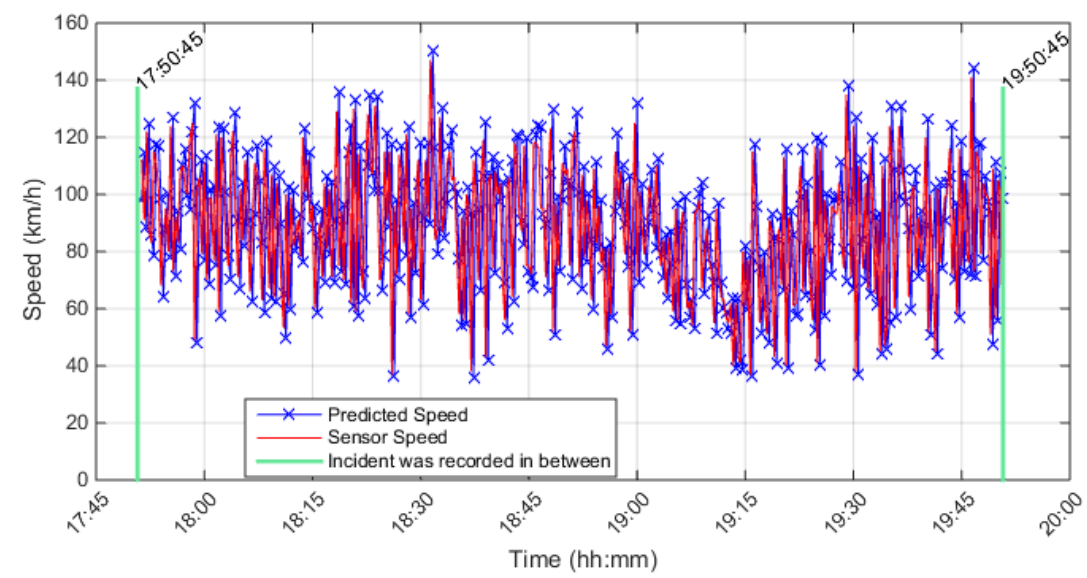

Upstream Detector \# is 401DE0340DWE, Detector Rank $=3$, Distance from incident location in $(\mathrm{km})=1.6024$ Season $=$ Summer, Stream $=E$, Direction $=W$, Date $\&$ Time $=$ 2011-08-06 18:50:45 Incident Reason $=$ Collision

Affected Lanes = 1 RIGHT LANE(S)

Conditional Probability level $=0.6$, Cond.Prob. $r 3 \mathrm{~s} 2=0.2$, Cond.Prob. $r 3 \mathrm{~s} 1=0.1$, Percent drop in speed $\%=-56$

Max. Posterior Probability level $=0.97$, Percent range between speed states $=7 \%$

Drop in speed was detected but Not enough recovery time to confirm an incident

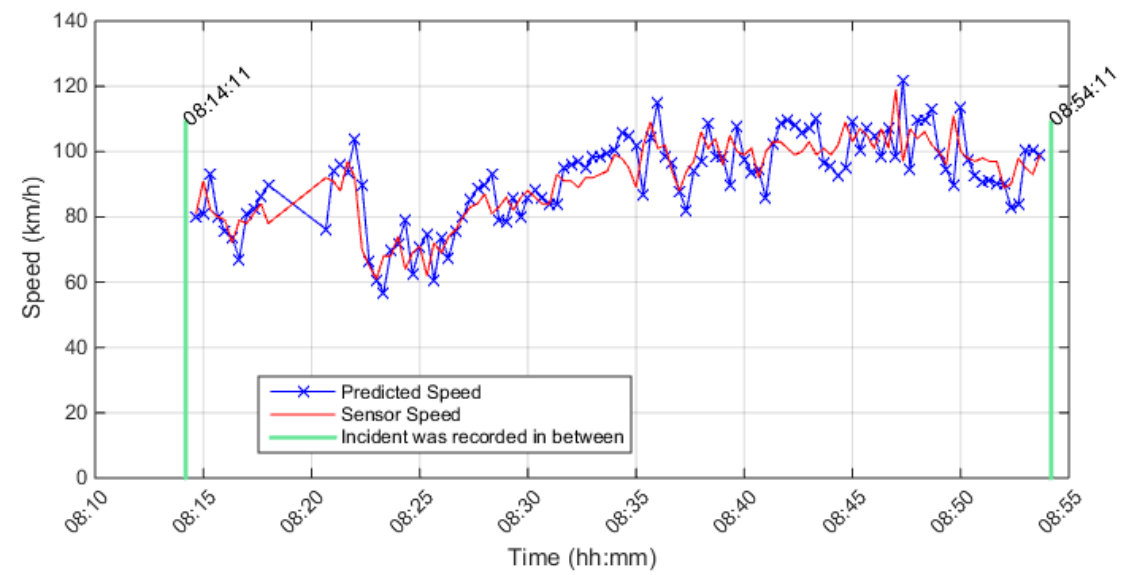

Upstream Detector \# is 401DE0020DWE, Detector Rank = 1, Distance from incident location in $(\mathrm{km})=0.39496$ Season $=$ Summer, Stream $=E$, Direction $=W$, Date \& Time $=2011-08-08$ 08:34:11 Incident Reason $=$ Collision

Affected Lanes = 2 RIGHT LANE(S)

Conditional Probability level $=0.6$, Cond. Prob. $r 3 s 2=0.2$, Cond.Prob. $r 3 s 1=0.1$, Percent drop in speed $\%=-56$ Max. Posterior Probability level $=0.97$, Percent range between speed states $=7 \%$

(No Incident was Detected) 


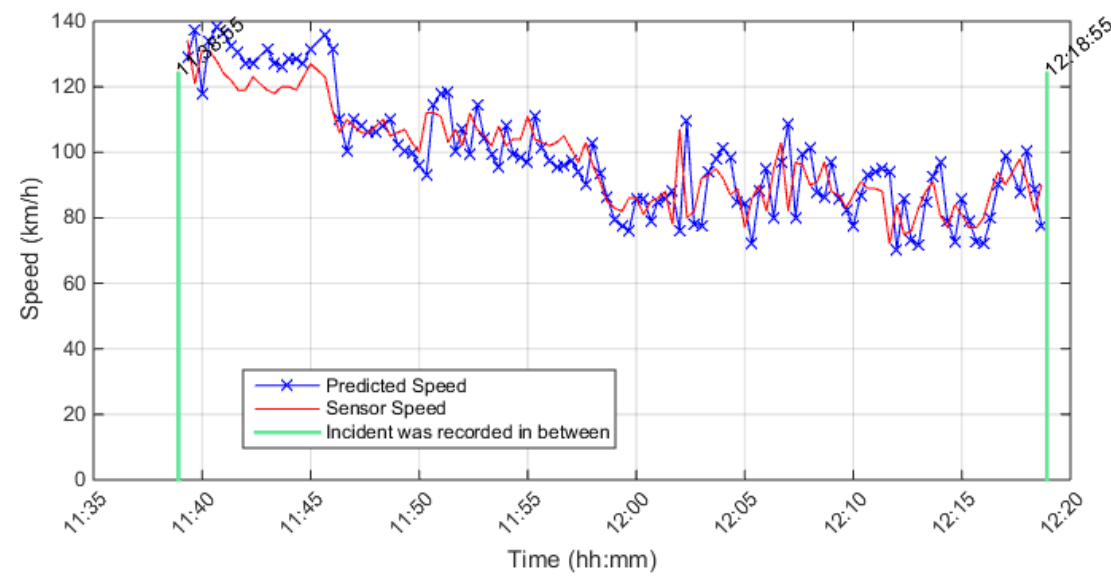

Upstream Detector \# is 401DE0020DWE, Detector Rank = 1, Distance from incident location in $(\mathrm{km})=0.39496$ Season $=$ Summer, Stream $=E$, Direction $=W$, Date $\&$ Time $=2011-08-28$ 11:58:55 Incident Reason $=$ Collision

Affected Lanes $=1$ LEFT LANE(S)

Conditional Probability level $=0.6$, Cond. Prob. $r 3 s 2=0.2$, Cond. Prob. $r 3 s 1=0.1$, Percent drop in speed $\%=-56$ Max. Posterior Probability level $=0.97$, Percent range between speed states $=7 \%$

(No Incident was Detected) 


\section{Appendix C.4 Cases with No Incident Recorded}

This appendix presents the validation outcomes that resulted from the model application on 130 cases as described in chapter 6. Cases presented in this part are under certain traffic condition "Must Not Detect." By holding the parameters these resulted from the calibration process. The model was applied to cases with no incident.

\section{Appendix C.4.1 Cases under Certain Traffic Condition "Must Not Detect" -- 130 cases}

\section{Appedix C.4.1.1 Cases without Incident Recorded and Detected --- 3 cases}

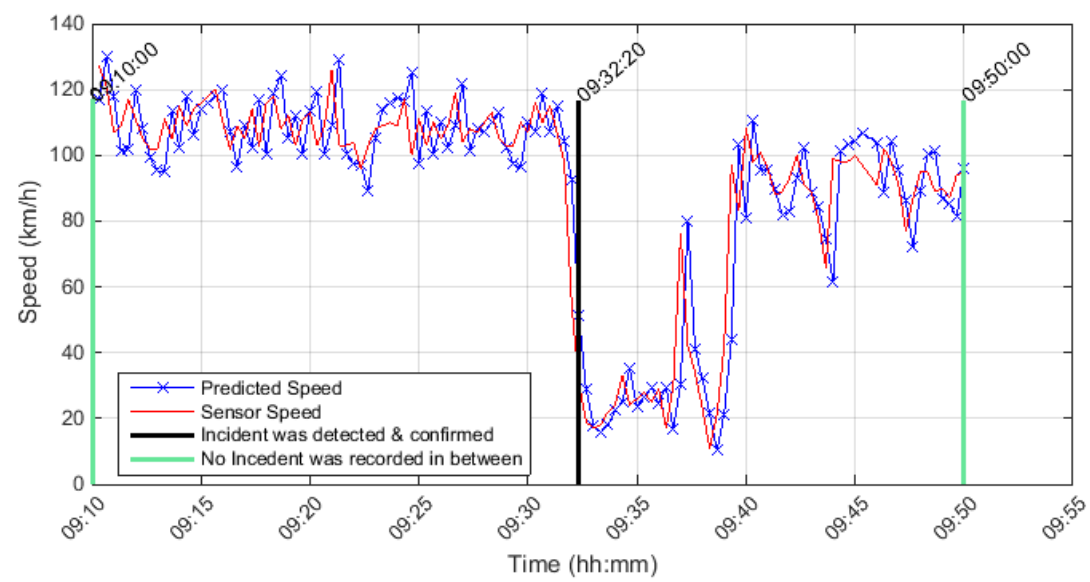

Upstream Detector \# is 401DE0350DWC, Detector Rank = 1, Distance from incident free location in $(\mathrm{km})=0.24334$ Incident free Season $=$ Summer, Stream $=C$, Direction $=W$, Date $\&$ Time $=2011-08-07$ 11:30:14 Selected incident free Date/Time $=2011-08-07$ 09:30:00

Conditional Probability level $=0.6$, Cond. Prob. $r 3 s 2=0.2$, Cond. Prob. $r 3 s 1=0.1$, Percent drop in speed $\%=-56$ Max. Posterior Probability level $=0.97$, Percent range between speed states $=7 \%$ 


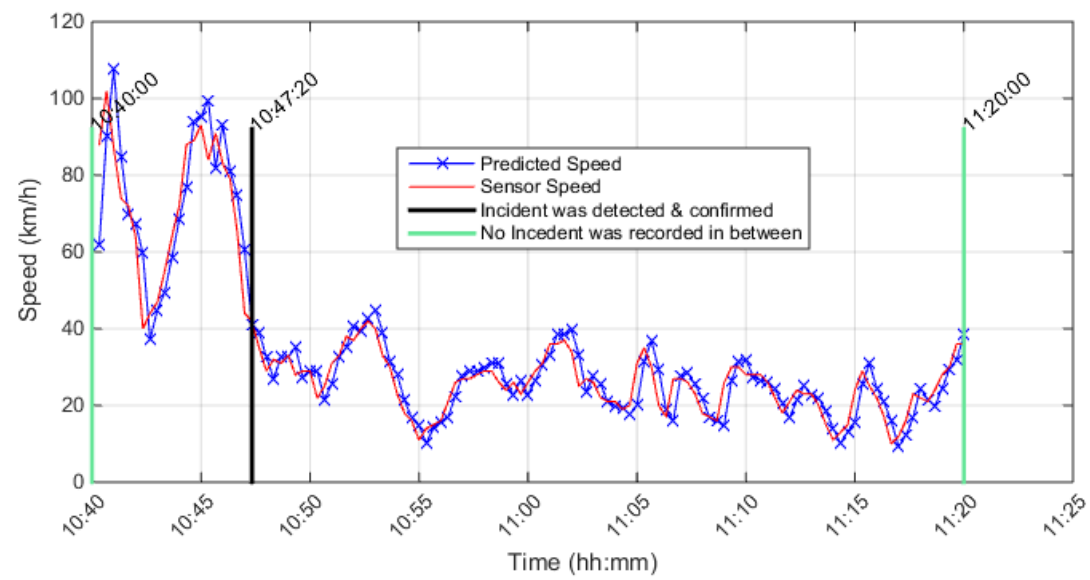

Upstream Detector \# is 401DE0150DWE, Detector Rank = 2, Distance from incident free location in $(\mathrm{km})=1.3152$ Incident free Season $=$ Summer, Stream $=\mathrm{E}$, Direction $=\mathrm{W}$, Date \& Time $=$ 2011-07-09 16:02:50 Selected incident free Date/Time $=2011-07-09$ 11:00:00

Conditional Probability level $=0.6$, Cond.Prob. $r 3 \mathrm{~s} 2=0.2$, Cond.Prob. $r 3 \mathrm{~s} 1=0.1$, Percent drop in speed $\%=-56$ Max. Posterior Probability level $=0.97$, Percent range between speed states $=7 \%$

Incident was detected at 01:20 minutes from drop in speed point. Incident was confirmed within 5 minutes from the detection tim

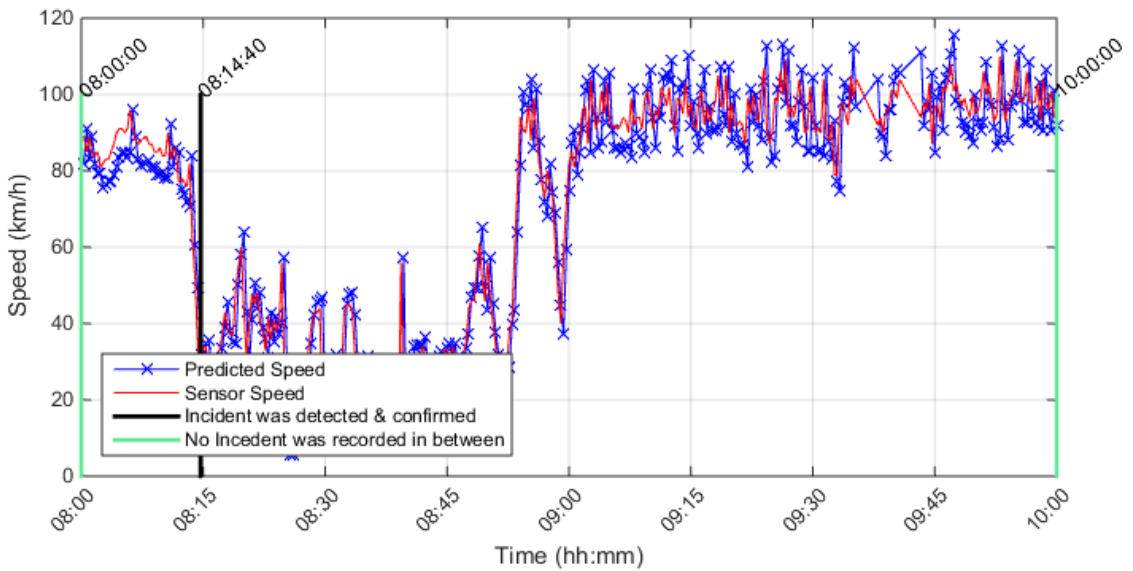

Upstream Detector \# is 401DE0050DWC, Detector Rank = 1, Distance from incident free location in $(\mathrm{km})=0.45946$ Incident free Season $=$ Summer, Stream $=$ C , Direction $=$ W, Date $\&$ Time $=$ 2011-07-05 17:40:42 Selected incident free Date/Time $=$ 2011-07-05 09:00:00

Conditional Probability level $=0.6$, Cond. Prob. $r 3 s 2=0.2$, Cond. Prob. $r 3 s 1=0.1$, Percent drop in speed $\%=-56$

Max. Posterior Probability level $=0.97$, Percent range between speed states $=7 \%$

Incident was detected at 01:00 minutes from drop in speed point. Incident was confirmed within 5 minutes from the detection tim 


\section{Appedix C.4.1.2 Cases without Incident Recorded and Not Detected----127 cases}

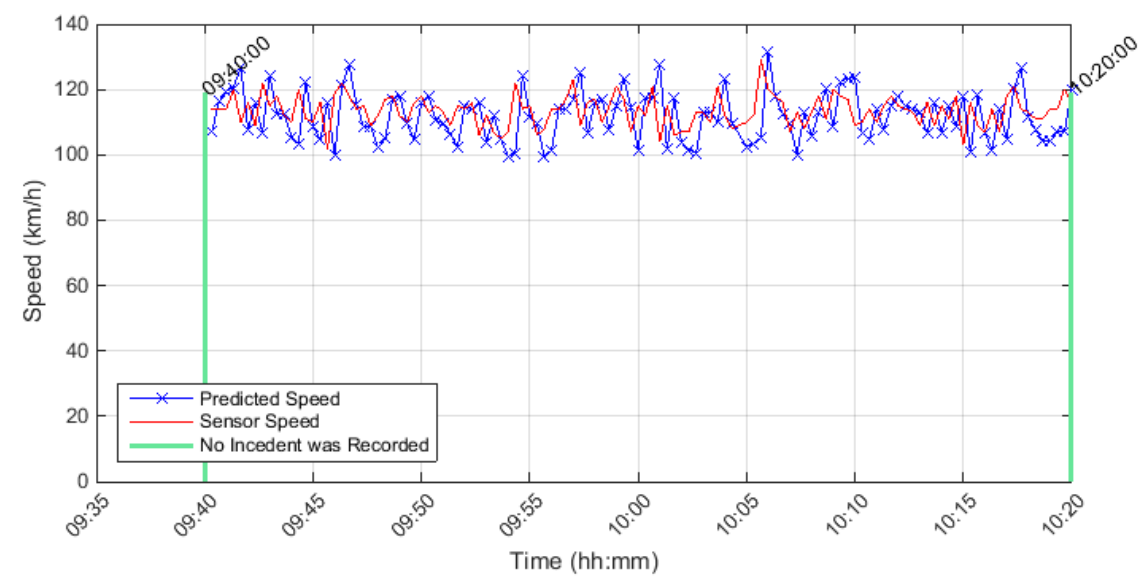

Upstream Detector \# is 401DE0590DEE, Detector Rank = 1, Distance from incident free location in $(\mathrm{km})=0.15896$ Incident free Season $=$ Summer, Stream $=E$, Direction $=E$, Date \& Time $=2011-10-20$ 19:36:30 Selected incident free Date/Time $=2011-10-20$ 10:00:00

Conditional Probability level $=0.6$, Cond.Prob. $r 3 \mathrm{~s} 2=0.2$, Cond.Prob. $r 3 \mathrm{~s} 1=0.1$, Percent drop in speed $\%=-60$

Max. Posterior Probability level $=0.97$, Absolute range between speed states $=7 \mathrm{~km}$

(No Incident was Detected)

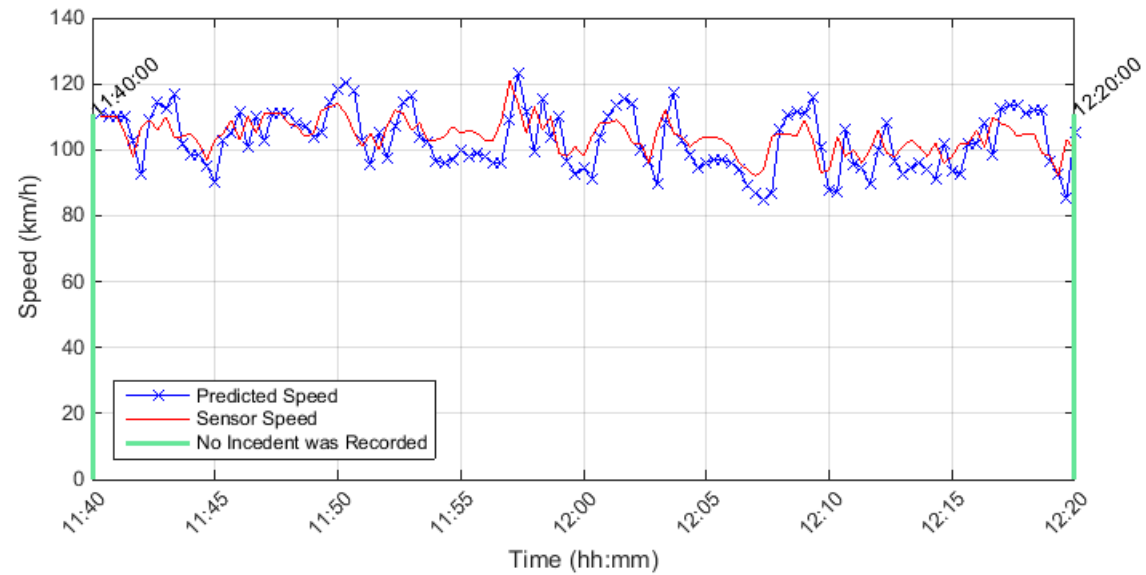

Upstream Detector \# is 401DW0020DEE, Detector Rank = 1, Distance from incident free location in $(\mathrm{km})=0.035848$ Incident free Season $=$ Winter, Stream $=E$, Direction $=E$, Date \& Time $=2011-12-15$ 18:12:24

Selected incident free Date/Time $=2011-12-15$ 12:00:00

Conditional Probability level $=0.6$, Cond.Prob. $r 3 \mathrm{~s} 2=0.2$, Cond.Prob. $r 3 \mathrm{~s} 1=0.1$, Percent drop in $\mathrm{speed} \%=-60$ Max. Posterior Probability level $=0.97$, Absolute range between speed states $=7 \mathrm{~km}$

(No Incident was Detected) 


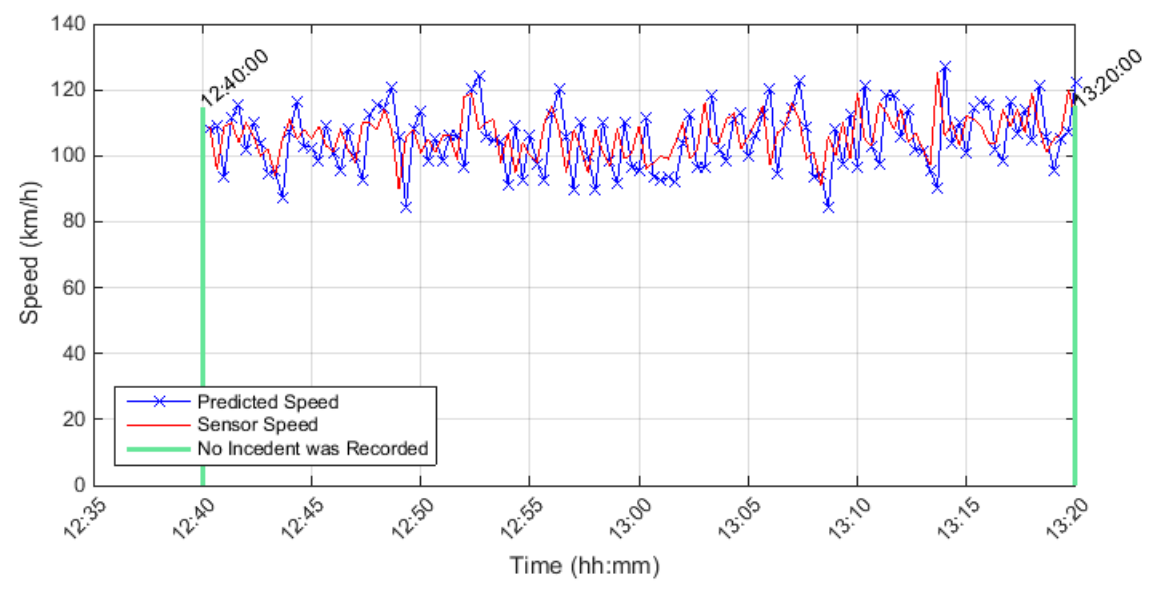

Upstream Detector \# is 401DW0270DEE, Detector Rank = 1, Distance from incident free location in $(\mathrm{km})=0.24432$ Incident free Season $=$ Winter, Stream $=E$, Direction $=E$, Date \& Time $=$ 2011-12-30 10:05:54

Selected incident free Date/Time $=2011-12-30$ 13:00:00

Conditional Probability level $=0.6$, Cond.Prob. $r 3 \mathrm{~s} 2=0.2$, Cond.Prob. $r 3 \mathrm{~s} 1=0.1$, Percent drop in speed $\%=-60$

Max. Posterior Probability level $=0.97$, Absolute range between speed states $=7 \mathrm{~km}$

(No Incident was Detected)

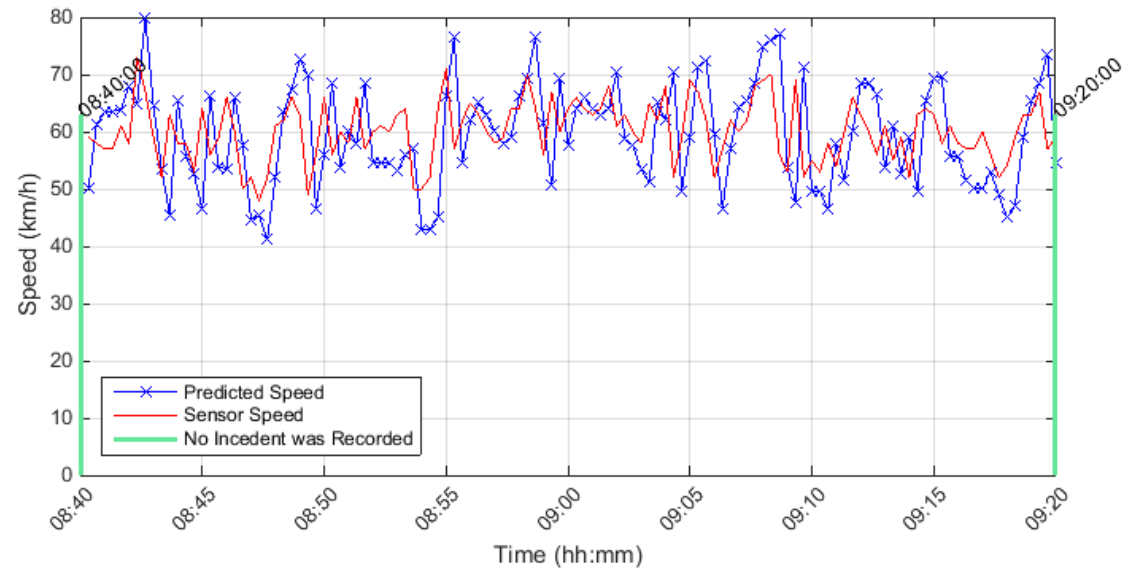

Upstream Detector \# is 401DE0090DWC, Detector Rank = 1, Distance from incident free location in $(\mathrm{km})=0.59485$ Incident free Season $=$ Winter, Stream $=$ C, Direction $=$ W, Date \& Time $=$ 2011-02-02 03:27:54

Selected incident free Date/Time $=$ 2011-02-02 09:00:00

Conditional Probability level $=0.6$, Cond. Prob. $r 3 s 2=0.2$, Cond. Prob. $r 3 s 1=0.1$, Percent drop in speed $\%=-60$

Max. Posterior Probability level $=0.97$, Absolute range between speed states $=7 \mathrm{~km}$

(No Incident was Detected) 


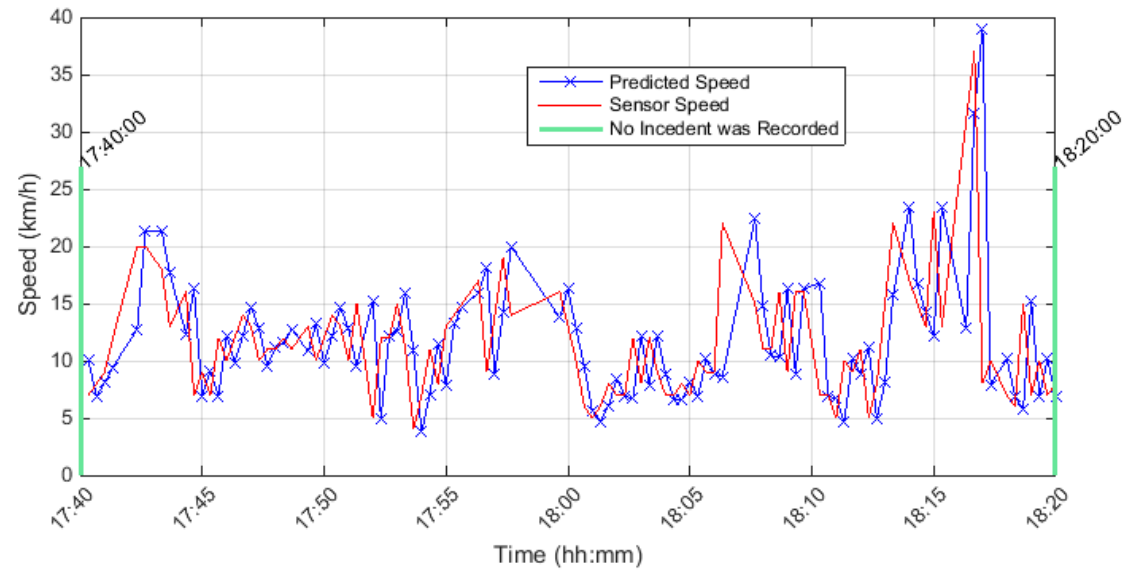

Upstream Detector \# is 401DW0190DWC, Detector Rank = 1, Distance from incident free location in $(\mathrm{km})=0.44011$ Incident free Season $=$ Winter, Stream $=C$, Direction $=W$, Date $\&$ Time $=2011-02-08$ 11:19:11 Selected incident free Date/Time $=2011-02-08$ 18:00:00

Conditional Probability level $=0.6$, Cond.Prob. $r 3 s 2=0.2$, Cond.Prob. $r 3 s 1=0.1$, Percent drop in speed $\%=-56$ Max. Posterior Probability level $=0.97$, Percent range between speed states $=7 \%$ Drop in speed was detected and No indication of an incident within this period

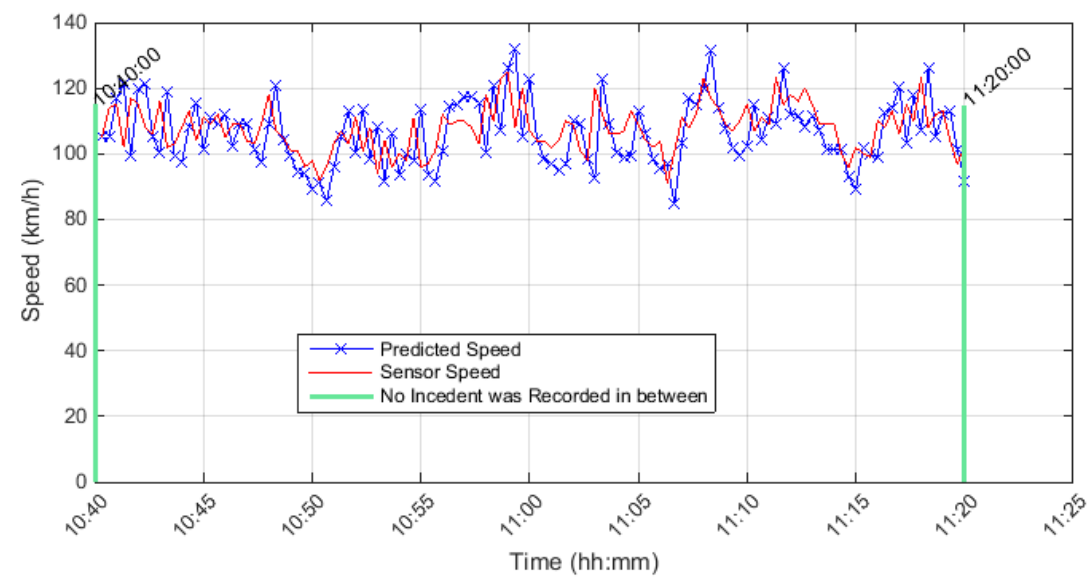

Upstream Detector \# is 401DE0190DWC, Detector Rank = 4, Distance from incident free location in $(\mathrm{km})=2.6115$ Incident free Season $=$ Winter, Stream $=C$, Direction $=W$, Date \& Time $=2011-02-19$ 15:47:22 Selected incident free Date/Time $=2011-02-19$ 11:00:00

Conditional Probability level $=0.6$, Cond. Prob. $r 3 \mathrm{~s} 2=0.2$, Cond. Prob. $r 3 \mathrm{~s} 1=0.1$, Percent drop in speed $\%=-56$

Max. Posterior Probability level $=0.97$, Percent range between speed states $=7 \%$

(No Incident was Detected) 


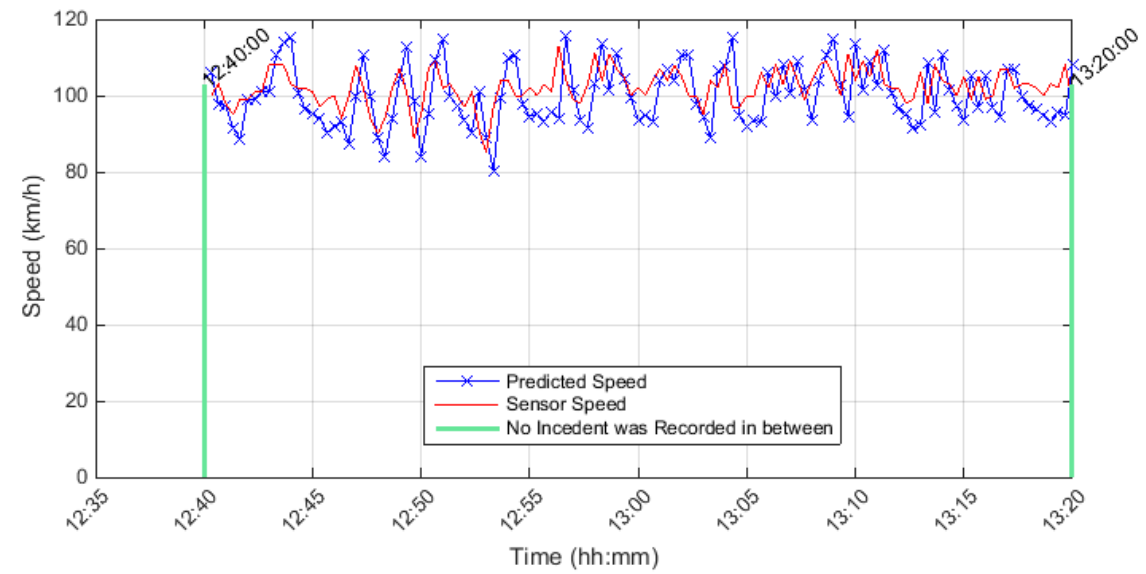

Upstream Detector \# is 401DE0290DWC, Detector Rank $=1$, Distance from incident free location in $(\mathrm{km})=0.43401$ Incident free Season $=$ Spring. Stream $=$ C, Direction $=$ W, Date \& Time $=2011-03-07$ 10:58:31 Selected incident free Date/Time $=2011-03-07$ 13:00:00

Conditional Probability level $=0.6$, Cond.Prob. $r 3 \mathrm{~s} 2=0.2$, Cond.Prob. $r 3 \mathrm{~s} 1=0.1$, Percent drop in speed $\%=-56$ Max. Posterior Probability level $=0.97$, Percent range between speed states $=7 \%$ (No Incident was Detected)

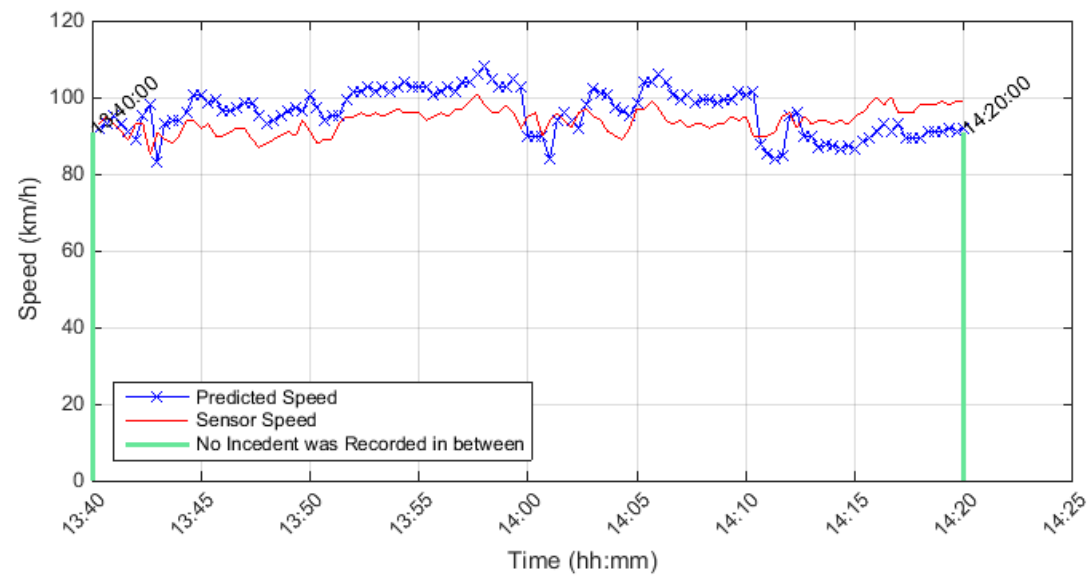

Upstream Detector \# is 401DW0040DWC, Detector Rank = 1, Distance from incident free location in $(\mathrm{km})=0.20609$ Incident free Season $=$ Spring, Stream $=$ C, Direction $=W$, Date \& Time $=$ 2011-03-18 04:08:29 Selected incident free Date/Time $=$ 2011-03-18 14:00:00

Conditional Probability level $=0.6$, Cond.Prob. $r 3 \mathrm{~s} 2=0.2$, Cond.Prob. $\mathrm{r} 3 \mathrm{~s} 1=0.1$, Percent drop in speed $\%=-56$ Max. Posterior Probability level $=0.97$, Percent range between speed states $=7 \%$ (No Incident was Detected) 


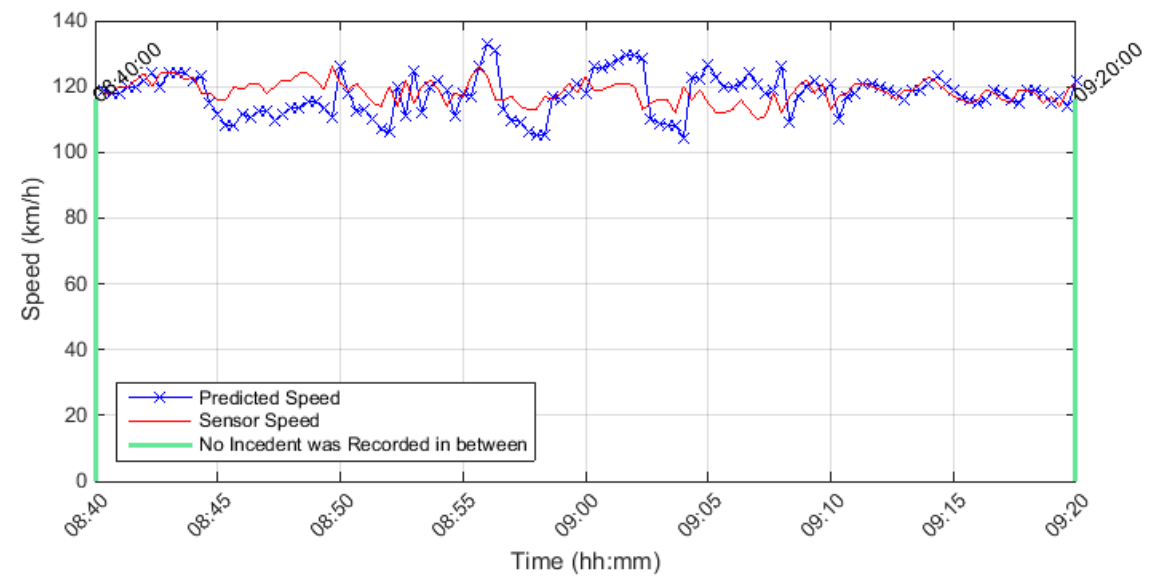

Upstream Detector \# is 401DW0060DWC, Detector Rank = 1, Distance from incident free location in $(\mathrm{km})=0.43697$ Incident free Season $=$ Spring, Stream $=$ C, Direction $=$ W, Date \& Time $=2011-03-19$ 18:02:36

Selected incident free Date/Time $=2$ 2011-03-19 09:00:00

Conditional Probability level $=0.6$, Cond.Prob. $r 3 \mathrm{~s} 2=0.2$, Cond. Prob. $r 3 \mathrm{~s} 1=0.1$, Percent drop in speed $\%=-56$ Max. Posterior Probability level $=0.97$, Percent range between speed states $=7 \%$

(No Incident was Detected)

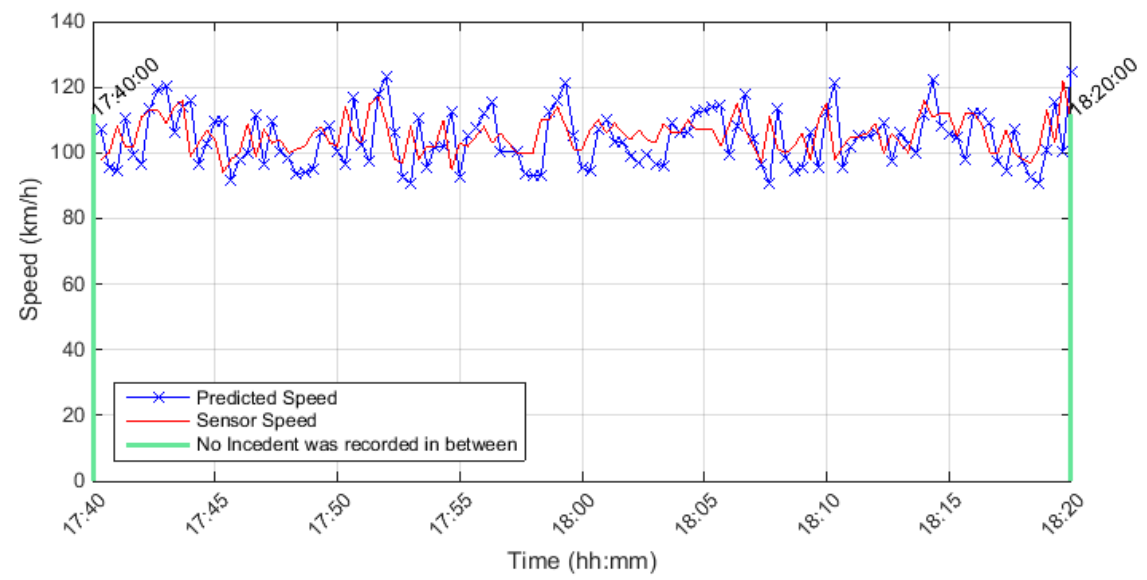

Upstream Detector \# is 401DE0050DWC, Detector Rank = 1, Distance from incident free location in $(\mathrm{km})=0.45946$ Incident free Season $=$ Spring, Stream $=$ C, Direction $=W$, Date \& Time $=2011-04-17$ 04:15:09

Selected incident free Date/Time $=2011-04-17$ 18:00:00

Conditional Probability level $=0.6$, Cond. Prob. $r 3 \mathrm{~s} 2=0.2$, Cond. Prob. $\mathrm{r} 3 \mathrm{~s} 1=0.1$, Percent drop in speed $\%=-56$ Max. Posterior Probability level $=0.97$, Percent range between speed states $=7 \%$

(No Incident was Detected) 


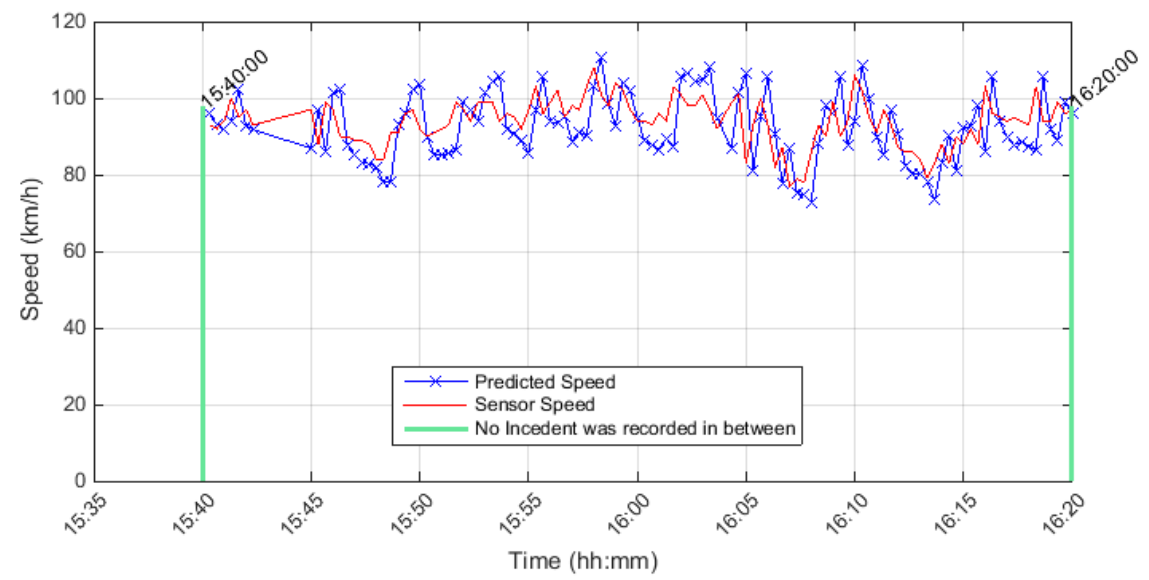

Upstream Detector \# is 401DW0160DWC, Detector Rank = 1, Distance from incident free location in $(\mathrm{km})=0.1854$ Incident free Season $=$ Spring, Stream $=$ C, Direction $=$ W, Date \& Time $=$ 2011-05-04 06:59:13

Selected incident free Date/Time $=2011-05-04$ 16:00:00

Conditional Probability level $=0.6$, Cond. Prob. $r 3 s 2=0.2$, Cond. Prob. $r 3 s 1=0.1$, Percent drop in speed $\%=-56$ Max. Posterior Probability level $=0.97$, Percent range between speed states $=7 \%$

(No Incident was Detected)

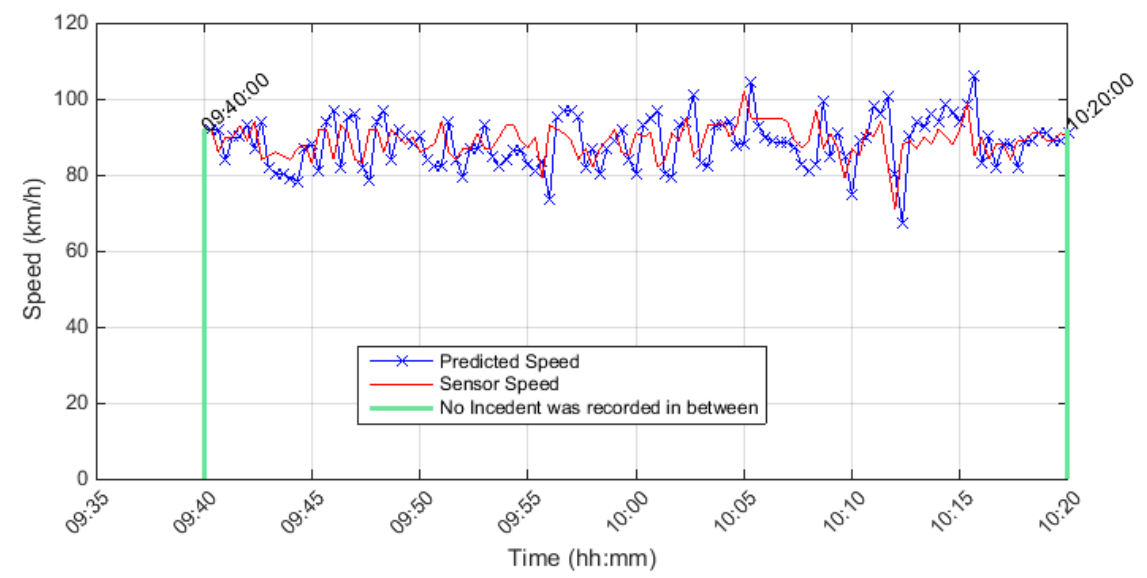

Upstream Detector \# is 401DE0160DWC, Detector Rank = 1, Distance from incident free location in $(\mathrm{km})=0.12541$ Incident free Season $=$ Spring, Stream $=C$, Direction $=$ W, Date \& Time $=$ 2011-05-13 18:20:13 Selected incident free Date/Time $=2011-05-13$ 10:00:00

Conditional Probability level $=0.6$, Cond.Prob. $r 3 \mathrm{~s} 2=0.2$, Cond.Prob. $r 3 \mathrm{~s} 1=0.1$, Percent drop in speed $\%=-56$

Max. Posterior Probability level $=0.97$, Percent range between speed states $=7 \%$

(No Incident was Detected) 


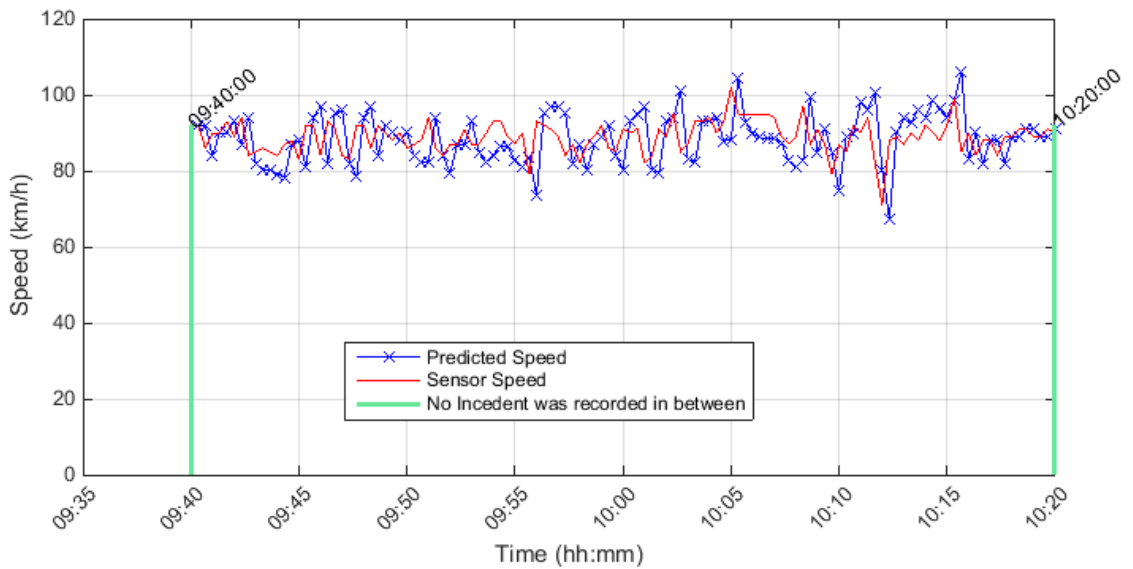

Upstream Detector \# is 401DE0160DWC, Detector Rank $=1$, Distance from incident free location in $(\mathrm{km})=0.12541$ Incident free Season $=$ Spring, Stream $=C$, Direction $=W$, Date $\&$ Time $=2011-05-13$ 18:20:13 Selected incident free Date/Time $=2011-05-13$ 10:00:00

Conditional Probability level $=0.6$, Cond. Prob. $r 3 \mathrm{~s} 2=0.2$, Cond.Prob. $r 3 \mathrm{~s} 1=0.1$, Percent drop in speed $\%=-56$ Max. Posterior Probability level $=0.97$, Percent range between speed states $=7 \%$ (No Incident was Detected)

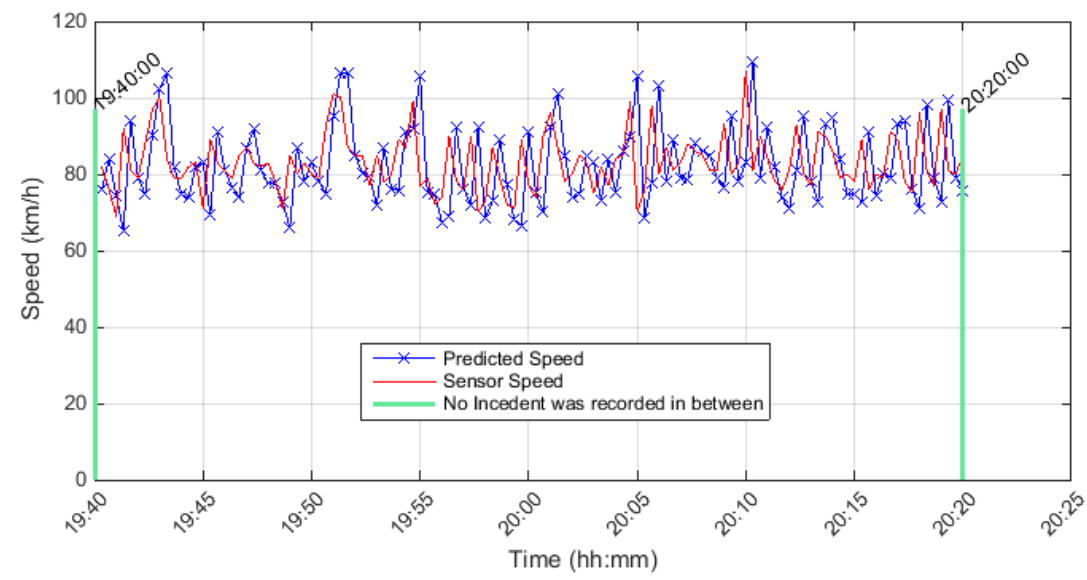

Upstream Detector \# is 401DE0150DWC, Detector Rank $=3$, Distance from incident free location in $(\mathrm{km})=1.5503$ Incident free Season $=$ Spring. Stream $=$ C, Direction $=$ W. Date \& Time $=2011-05-14$ 17:55:25

Selected incident free Date/Time $=$ 2011-05-14 20:00:00

Conditional Probability level $=0.6$, Cond.Prob. $r 3 s 2=0.2$, Cond.Prob. $r 3 s 1=0.1$, Percent drop in speed $\%=-56$ Max. Posterior Probability level $=0.97$, Percent range between speed states $=7 \%$

(No Incident was Detected) 


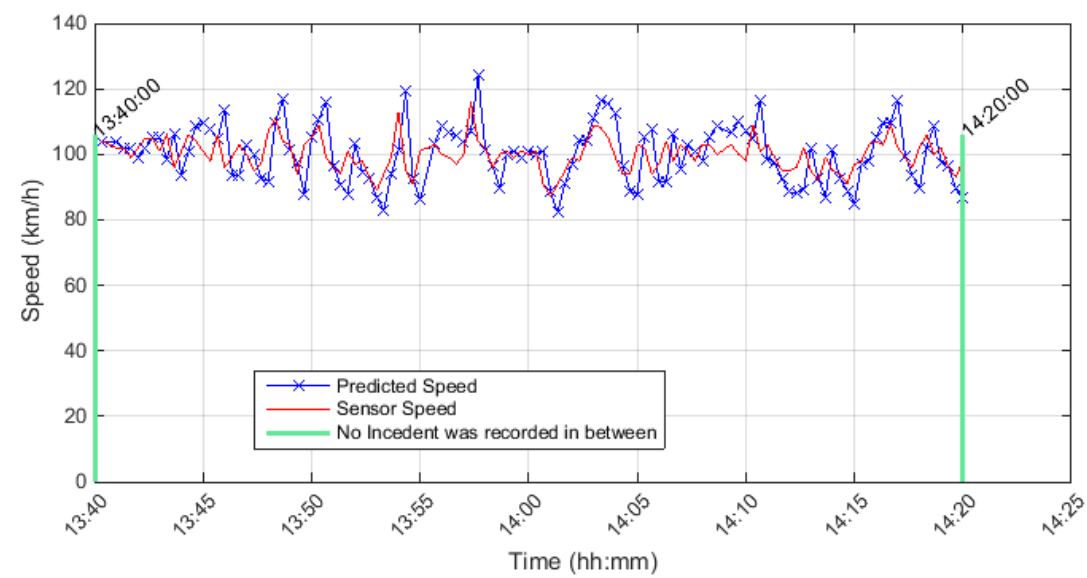

Upstream Detector \# is 401DE0290DWC, Detector Rank = 1, Distance from incident free location in $(\mathrm{km})=0.27312$ Incident free Season $=$ Spring, Stream $=C$, Direction $=W$, Date \& Time $=2011-05-17$ 11:40:34 Selected incident free Date/Time $=2011-05-17$ 14:00:00

Conditional Probability level $=0.6$, Cond.Prob. $r 3 s 2=0.2$, Cond.Prob. $r 3 s 1=0.1$, Percent drop in speed $\%=-56$ Max. Posterior Probability level $=0.97$, Percent range between speed states $=7 \%$ (No Incident was Detected)

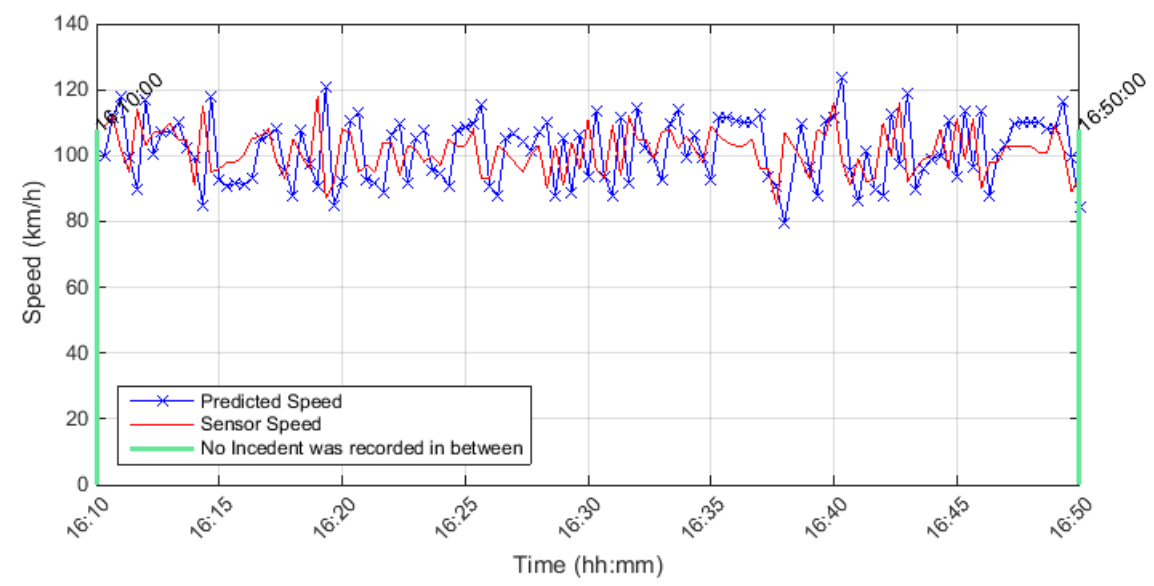

Upstream Detector \# is 401DE0370DWC, Detector Rank = 3, Distance from incident free location in $(\mathrm{km})=2.0297$ Incident free Season $=$ Spring, Stream $=$ C, Direction $=$ W, Date \& Time $=2011-05-24$ 22:38:11

Selected incident free Date/Time $=2011-05-24$ 16:30:00

Conditional Probability level $=0.6$, Cond. Prob. $r 3 \mathrm{~s} 2=0.2$, Cond. Prob. $r 3 \mathrm{~s} 1=0.1$, Percent drop in speed $\%=-56$

Max. Posterior Probability level $=0.97$, Percent range between speed states $=7 \%$

(No Incident was Detected) 


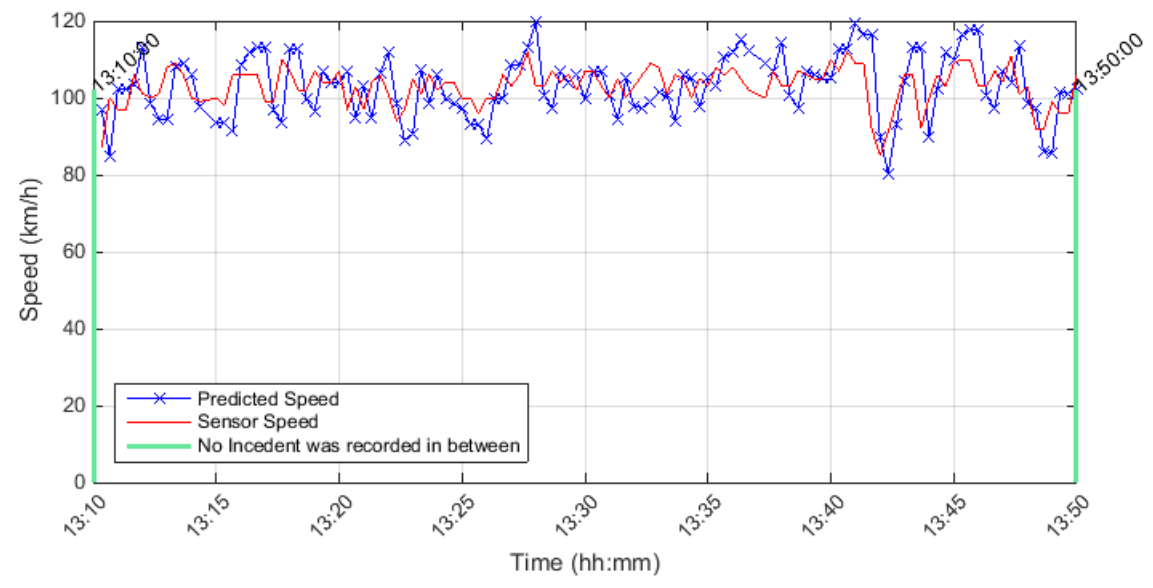

Upstream Detector \# is 401DE0210DWC, Detector Rank = 1, Distance from incident free location in $(\mathrm{km})=0.35779$ Incident free Season $=$ Summer, Stream $=C$, Direction $=W$, Date \& Time $=2011-06-28$ 22:57:38

Selected incident free Date/Time $=2011-06-28$ 13:30:00

Conditional Probability level $=0.6$, Cond.Prob. $r 3 s 2=0.2$, Cond. Prob. $r 3 s 1=0.1$, Percent drop in speed $\%=-56$

Max. Posterior Probability level $=0.97$, Percent range between speed states $=7 \%$

(No Incident was Detected)

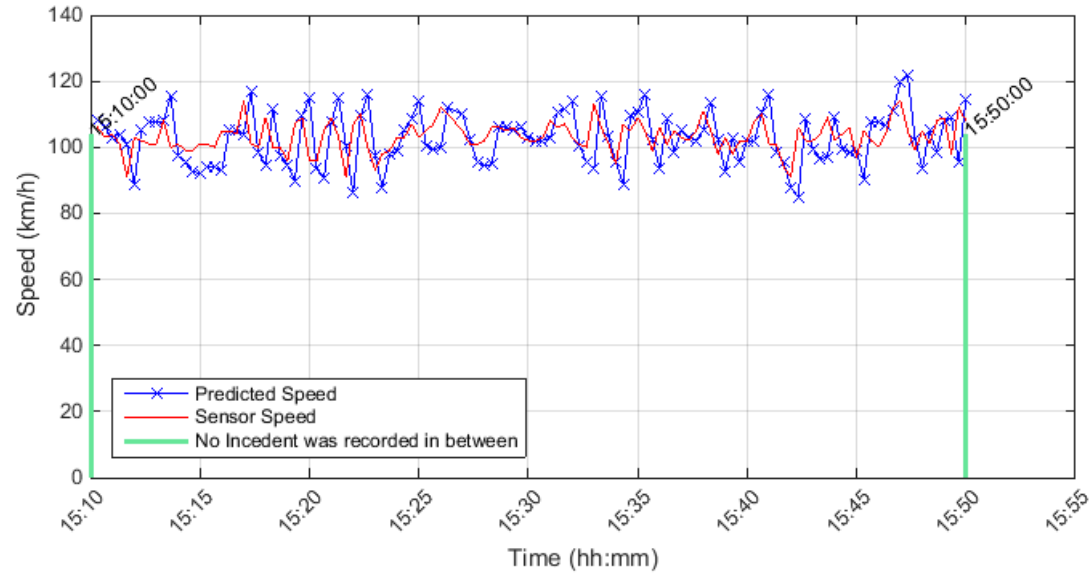

Upstream Detector \# is 401DE0380DWC, Detector Rank $=1$, Distance from incident free location in $(\mathrm{km})=0.71086$ Incident free Season $=$ Summer, Stream $=$ C , Direction $=$ W, Date \& Time $=2011-06-30$ 07:32:20 Selected incident free Date/Time $=2011-06-3015: 30: 00$

Conditional Probability level $=0.6$, Cond.Prob. $r 3 \mathrm{~s} 2=0.2$, Cond. Prob. $r 3 \mathrm{~s} 1=0.1$, Percent drop in speed $\%=-56$ Max. Posterior Probability level $=0.97$, Percent range between speed states $=7 \%$

(No Incident was Detected) 


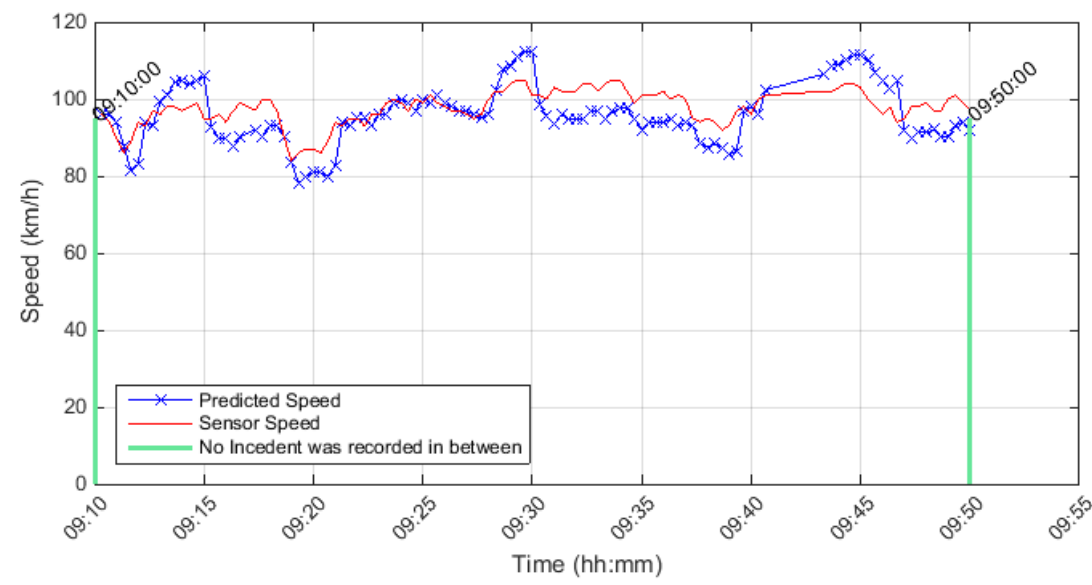

Upstream Detector \# is 401DE0020DWC, Detector Rank $=1$, Distance from incident free location in $(\mathrm{km})=0.39159$ Incident free Season $=$ Summer, Stream $=C$, Direction $=W$, Date \& Time $=2011-07-08$ 01:22:10 Selected incident free Date/Time $=$ 2011-07-08 09:30:00

Conditional Probability level $=0.6$, Cond.Prob. $r 3 \mathrm{~s} 2=0.2$, Cond.Prob. $r 3 \mathrm{~s} 1=0.1$, Percent drop in speed $\%=-56$

Max. Posterior Probability level $=0.97$, Percent range between speed states $=7 \%$

(No Incident was Detected)

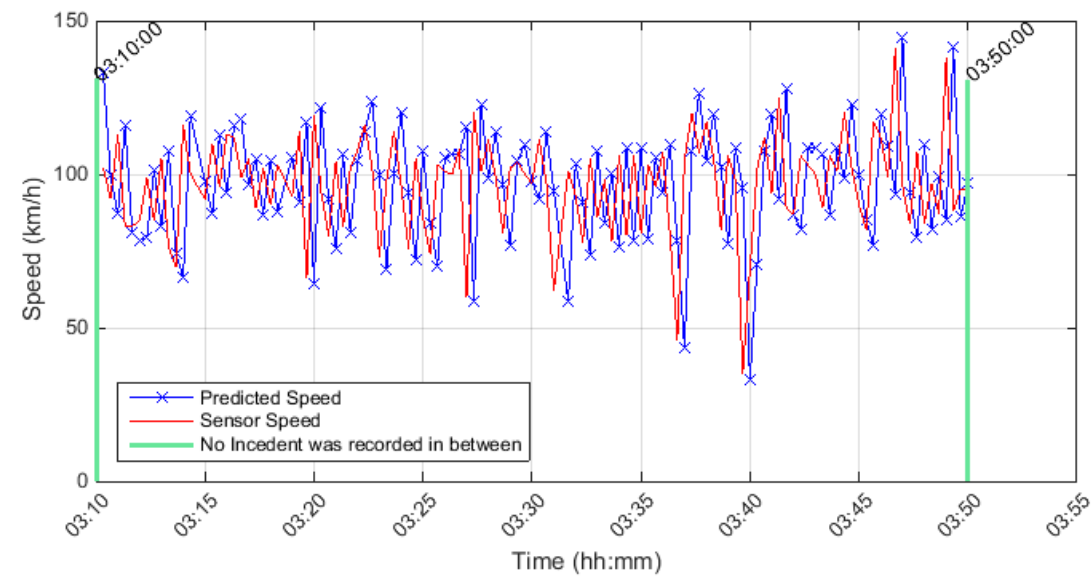

Upstream Detector \# is 401DW0150DWC, Detector Rank = 2, Distance from incident free location in $(\mathrm{km})=0.87619$ Incident free Season $=$ Summer, Stream $=$ C, Direction $=$ W, Date \& Time $=2011-09-15$ 08:57:56

Selected incident free Date/Time $=2011-09-15$ 03:30:00

Conditional Probability level $=0.6$, Cond.Prob. $r 3 s 2=0.2$, Cond. Prob. $r 3 s 1=0.1$, Percent drop in speed $\%=-56$

Max. Posterior Probability level $=0.97$, Percent range between speed states $=7 \%$

(No Incident was Detected) 


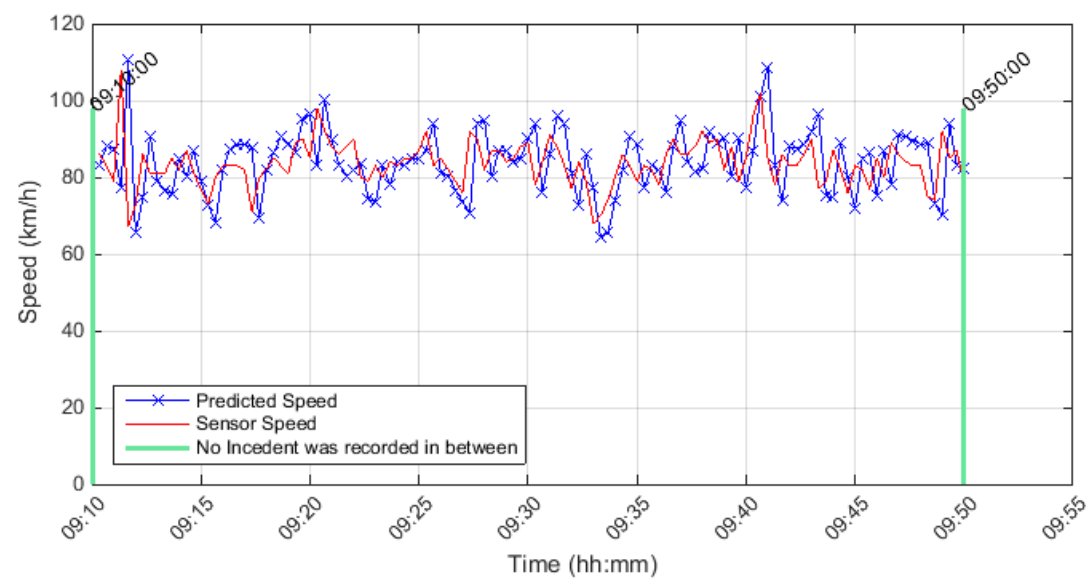

Upstream Detector \# is 401DW0020DWC, Detector Rank = 3, Distance from incident free location in $(\mathrm{km})=1.9461$ Incident free Season $=$ Summer, Stream $=$ C , Direction $=$ W, Date \& Time $=$ 2011-09-16 23:37:00

Selected incident free Date/Time $=2011-09-16$ 09:30:00

Conditional Probability level $=0.6$, Cond.Prob. $r 3 \mathrm{~s} 2=0.2$, Cond. Prob. $r 3 \mathrm{~s} 1=0.1$, Percent drop in speed $\%=-56$ Max. Posterior Probability level $=0.97$, Percent range between speed states $=7 \%$

(No Incident was Detected)

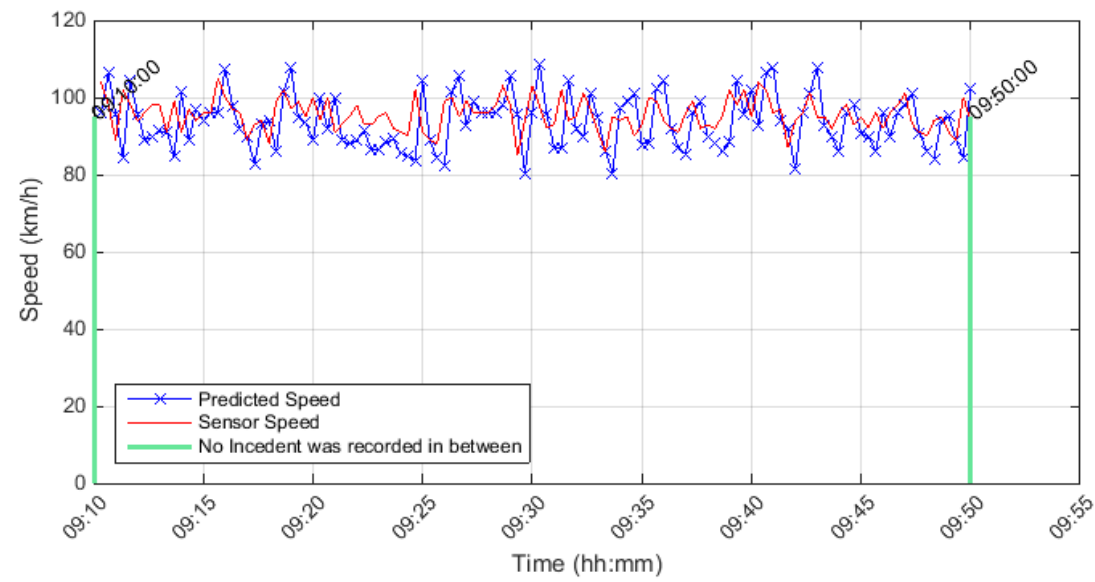

Upstream Detector \# is 401DE0160DWC, Detector Rank = 1, Distance from incident free location in $(\mathrm{km})=0.12541$ Incident free Season $=$ Summer, Stream $=$ C, Direction $=$ W, Date \& Time $=2011-09-25$ 19:18:15 Selected incident free Date/Time $=2011-09-2509: 30: 00$

Conditional Probability level $=0.6$, Cond.Prob. r3s2 $=0.2$, Cond.Prob. $r 3 s 1=0.1$, Percent drop in speed $\%=-56$

Max. Posterior Probability level $=0.97$, Percent range between speed states $=7 \%$

(No Incident was Detected) 


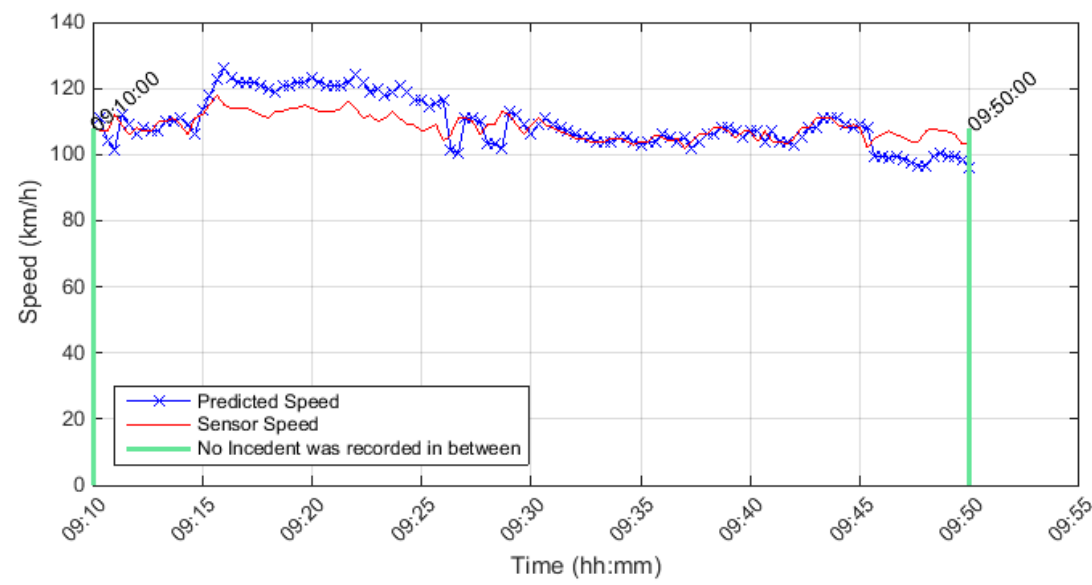

Upstream Detector \# is 401DW0070DWC, Detector Rank = 1, Distance from incident free location in $(\mathrm{km})=0.52634$ Incident free Season $=$ Summer, Stream $=$ C, Direction $=$ W, Date \& Time $=$ 2011-10-01 01:17:01 Selected incident free Date/Time $=2011-10-0109: 30: 00$

Conditional Probability level $=0.6$, Cond.Prob. $r 3 s 2=0.2$, Cond. Prob. $r 3 s 1=0.1$, Percent drop in speed $\%=-56$

Max. Posterior Probability level $=0.97$, Percent range between speed states $=7 \%$

(No Incident was Detected)

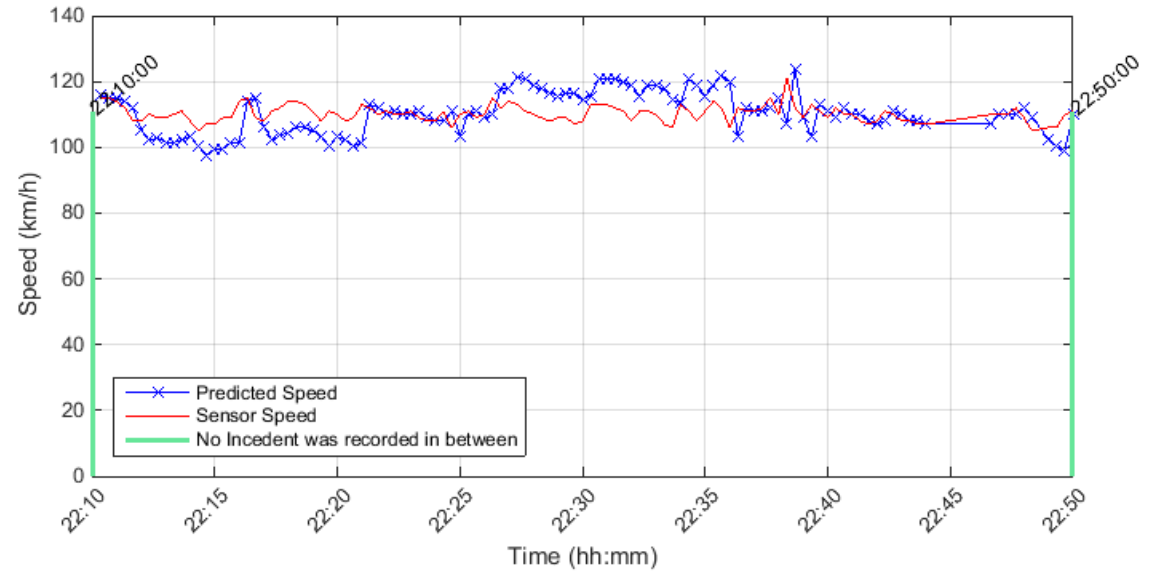

Upstream Detector \# is 401DW0070DWC, Detector Rank = 1, Distance from incident free location in $(\mathrm{km})=0.52634$ Incident free Season $=$ Summer, Stream $=$ C, Direction $=W$, Date \& Time $=2011-10-01$ 01:21:33

Selected incident free Date/Time $=2011-10-0122: 30: 00$

Conditional Probability level $=0.6$, Cond.Prob. $r 3 \mathrm{~s} 2=0.2$, Cond.Prob. $r 3 \mathrm{~s} 1=0.1$, Percent drop in speed $\%=-56$ Max. Posterior Probability level $=0.97$, Percent range between speed states $=7 \%$

(No Incident was Detected) 


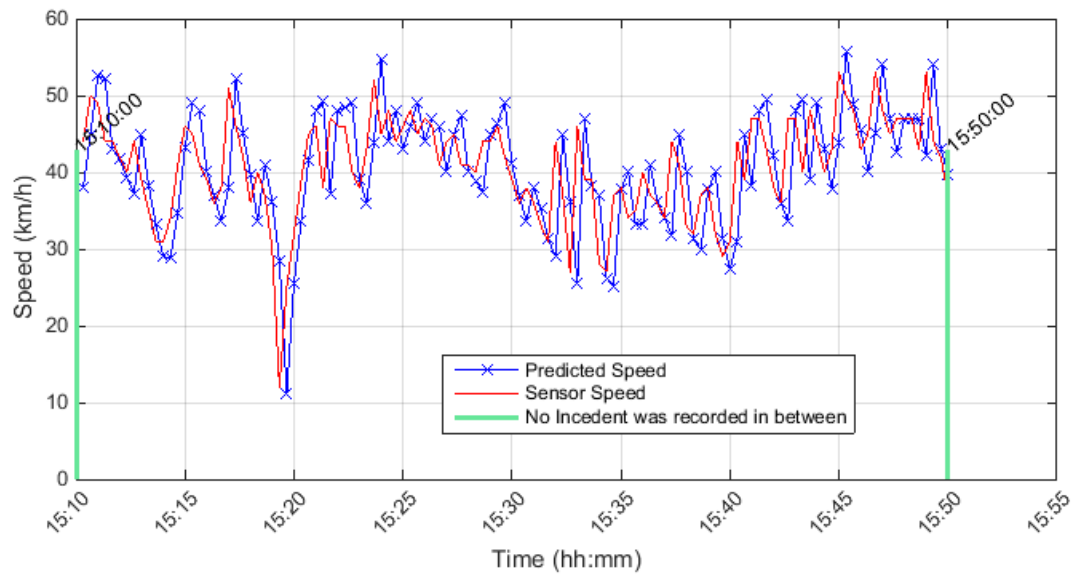

Upstream Detector \# is 401DE0020DWC, Detector Rank $=1$, Distance from incident free location in $(\mathrm{km})=0.63426$ Incident free Season $=$ Summer, Stream $=C$, Direction $=W$, Date \& Time $=2011-10-19$ 07:49:27

Selected incident free Date/Time $=2011-10-19$ 15:30:00

Conditional Probability level $=0.6$, Cond.Prob. $r 3 \mathrm{~s} 2=0.2$, Cond. Prob. $r 3 \mathrm{~s} 1=0.1$, Percent drop in speed $\%=-56$

Max. Posterior Probability level $=0.97$, Percent range between speed states $=7 \%$

Drop in speed was detected and No indication of an incident within this period

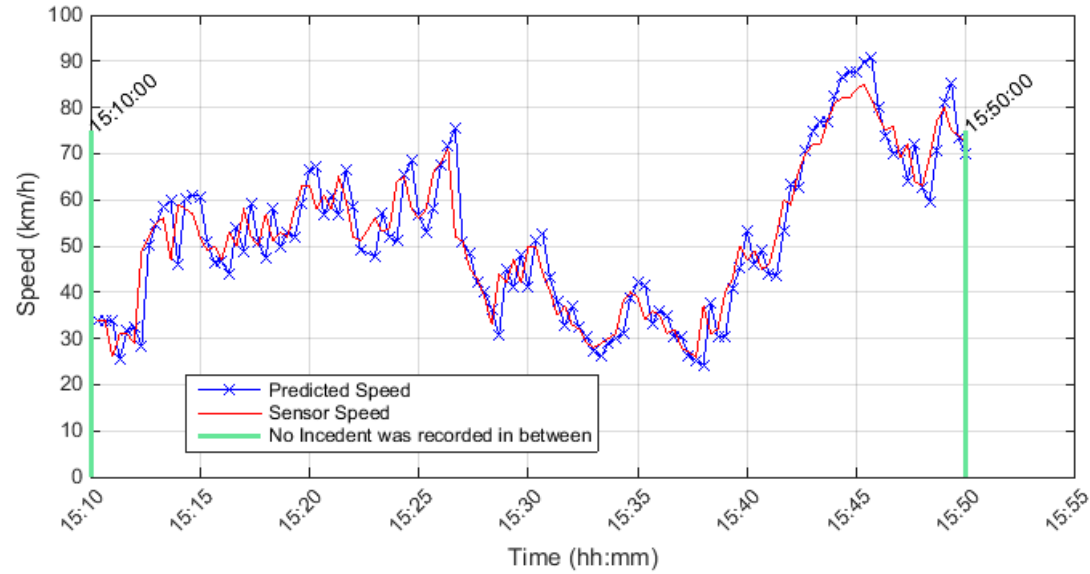

Upstream Detector \# is 401DE0240DWC, Detector Rank $=1$, Distance from incident free location in $(\mathrm{km})=0.14429$ Incident free Season $=$ Summer, Stream $=C$, Direction $=W$, Date \& Time $=2011-10-1923: 45: 47$

Selected incident free Date/Time $=2011-10-19$ 15:30:00

Conditional Probability level $=0.6$, Cond.Prob. $r 3 \mathrm{~s} 2=0.2$, Cond. Prob. $r 3 \mathrm{~s} 1=0.1$, Percent drop in speed $\%=-56$

Max. Posterior Probability level $=0.97$, Percent range between speed states $=7 \%$

Drop in speed was detected and No indication of an incident within this period 


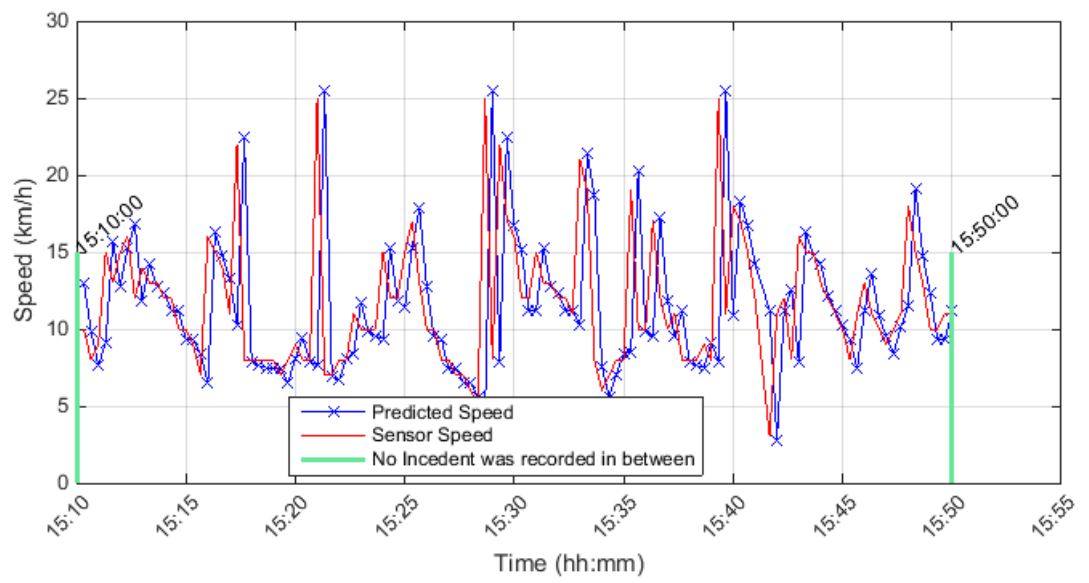

Upstream Detector \# is 401DW0060DWC, Detector Rank = 1, Distance from incident free location in $(\mathrm{km})=0.43697$ Incident free Season $=$ Summer, Stream $=$ C, Direction $=$ W, Date \& Time $=2011-10-2112: 21: 09$ Selected incident free Date/Time $=2011-10-21$ 15:30:00

Conditional Probability level $=0.6$, Cond.Prob. $r 3 \mathrm{~s} 2=0.2$, Cond.Prob. $r 3 \mathrm{~s} 1=0.1$, Percent drop in speed $\%=-56$

Max. Posterior Probability level $=0.97$, Percent range between speed states $=7 \%$

Drop in speed was detected and No indication of an incident within this period

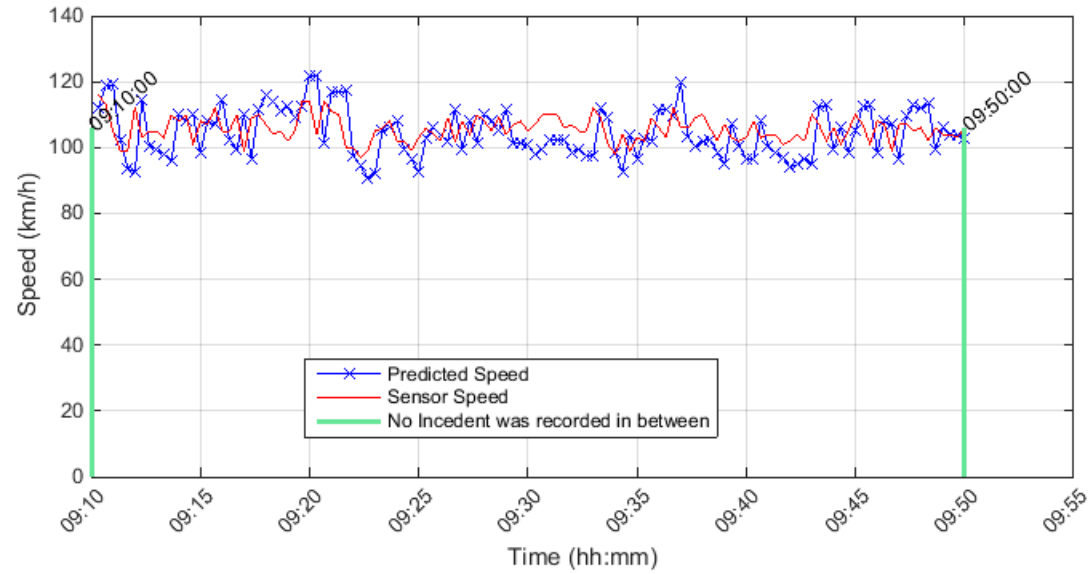

Upstream Detector \# is 401DE0090DWC, Detector Rank = 1, Distance from incident free location in $(\mathrm{km})=0.59485$ Incident free Season $=$ Summer, Stream $=$ C , Direction $=$ W, Date \& Time $=2011-10-23$ 15:40:49 Selected incident free Date/Time $=2011-10-23$ 09:30:00

Conditional Probability level $=0.6$, Cond.Prob. $\mathrm{r} 3 \mathrm{~s} 2=0.2$, Cond.Prob. $\mathrm{r} 3 \mathrm{~s} 1=0.1$, Percent drop in speed $\%=-56$ Max. Posterior Probability level $=0.97$, Percent range between speed states $=7 \%$

(No Incident was Detected) 


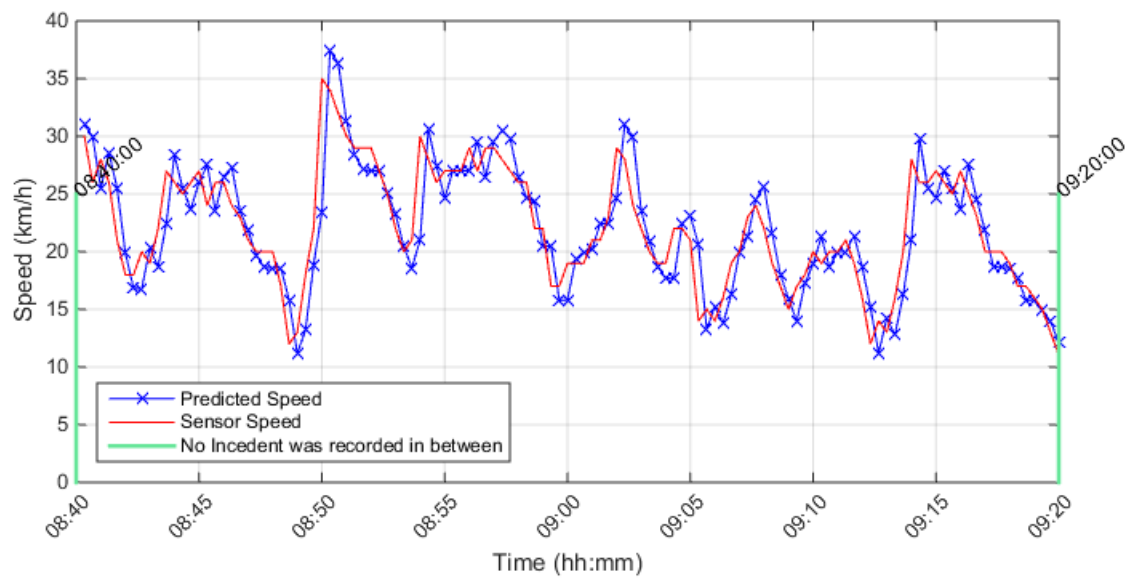

Upstream Detector \# is 401DW0060DWC, Detector Rank = 1, Distance from incident free location in $(\mathrm{km})=0.43697$ Incident free Season $=$ Summer, Stream $=$ C , Direction $=W$, Date \& Time $=2011-11-30$ 18:37:00

Selected incident free Date/Time $=$ 2011-11-30 09:00:00

Conditional Probability level $=0.6$, Cond.Prob. $r 3 \mathrm{~s} 2=0.2$, Cond.Prob. $r 3 \mathrm{~s} 1=0.1$, Percent drop in speed $\%=-56$

Max. Posterior Probability level $=0.97$, Percent range between speed states $=7 \%$

Drop in speed was detected but Not enough recovery time to confirm an incident

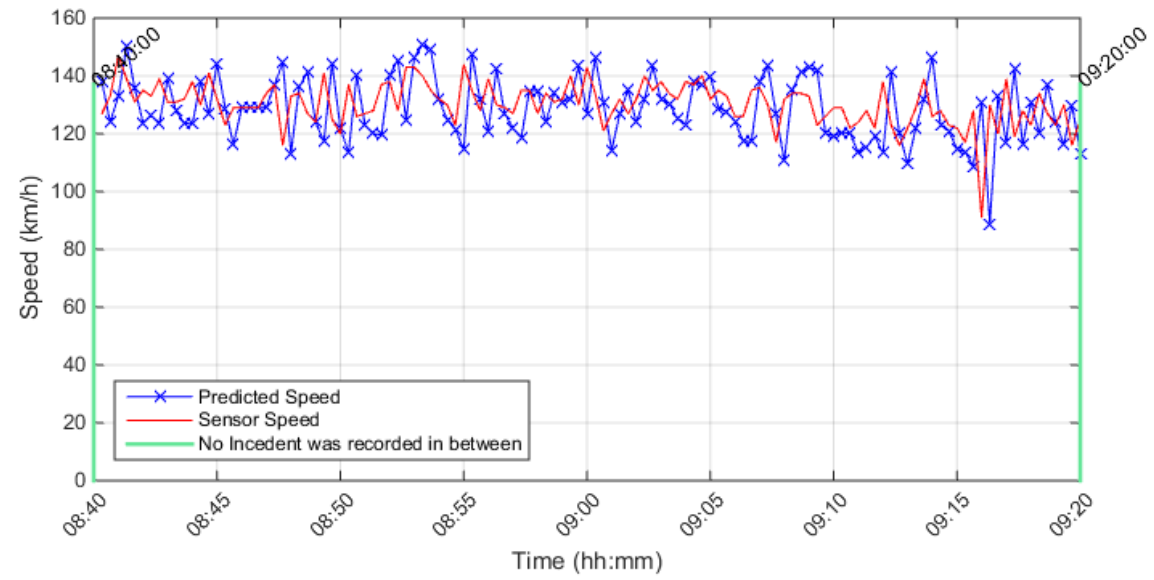

Upstream Detector \# is 401DE0020DWC, Detector Rank = 1, Distance from incident free location in $(\mathrm{km})=0.39159$ Incident free Season $=$ Winter, Stream $=$ C, Direction $=W$, Date \& Time $=2011-12-18$ 03:00:48 Selected incident free Date/Time $=2011-12-18$ 09:00:00

Conditional Probability level $=0.6$, Cond.Prob. $r 3 s 2=0.2$, Cond.Prob. $r 3 s 1=0.1$, Percent drop in speed $\%=-56$ Max. Posterior Probability level $=0.97$, Percent range between speed states $=7 \%$

(No Incident was Detected) 


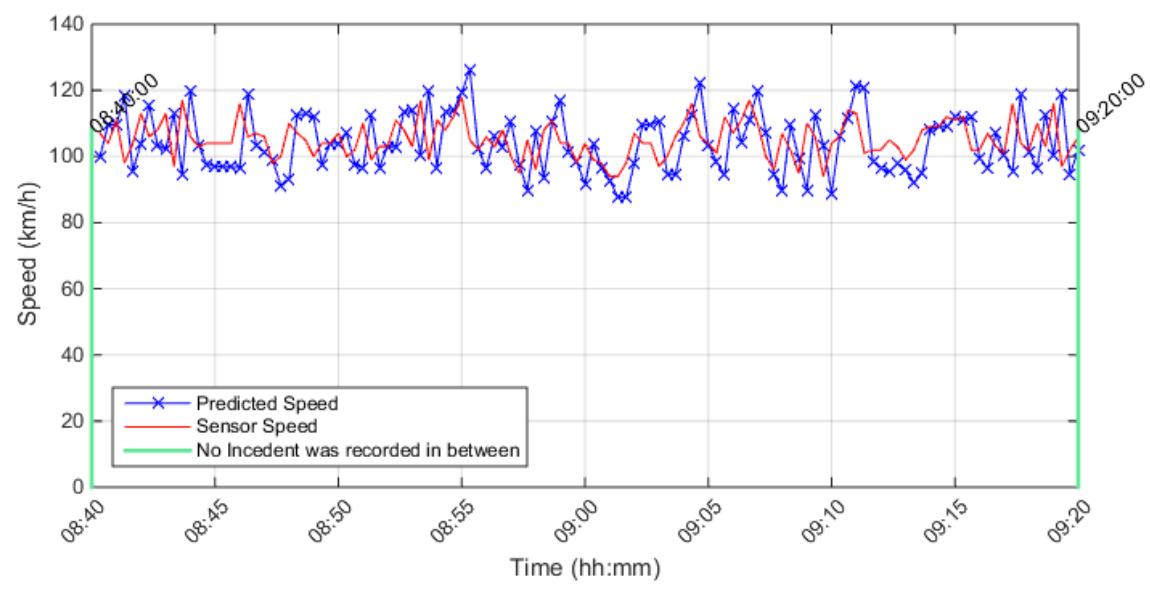

Upstream Detector \# is 401DE0100DWC, Detector Rank = 1, Distance from incident free location in $(\mathrm{km})=0.11847$ Incident free Season $=$ Winter, Stream $=$ C, Direction $=$ W, Date \& Time $=2011-12-24$ 22:45:12 Selected incident free Date/Time $=2011-12-24$ 09:00:00

Conditional Probability level $=0.6$, Cond.Prob. $r 3 s 2=0.2$, Cond. Prob. $r 3 s 1=0.1$, Percent drop in speed $\%=-56$

Max. Posterior Probability level $=0.97$, Percent range between speed states $=7 \%$

(No Incident was Detected)

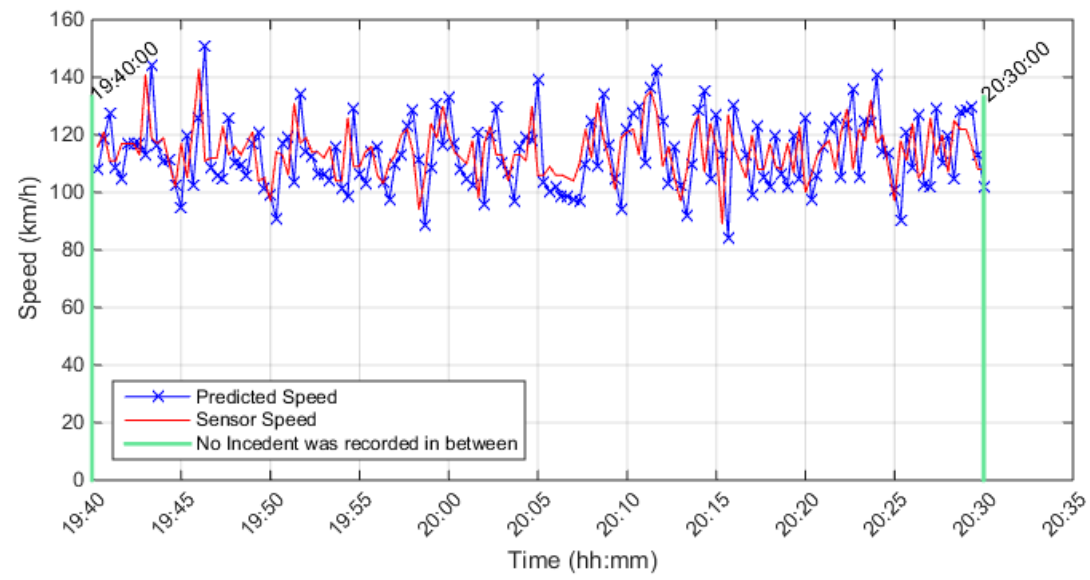

Upstream Detector \# is 401DE0210DWE, Detector Rank = 1, Distance from incident free location in $(\mathrm{km})=0.30762$ Incident free Season $=$ Winter, Stream $=E$, Direction $=$ W, Date \& Time $=2011-01-17$ 07:42:14 Selected incident free Date/Time $=$ 2011-01-17 20:00:00

Conditional Probability level $=0.6$, Cond. Prob. $\mathrm{r} 3 \mathrm{~s} 2=0.2$, Cond.Prob. $\mathrm{r} 3 \mathrm{~s} 1=0.1$, Percent drop in speed $\%=-56$ Max. Posterior Probability level $=0.97$, Percent range between speed states $=7 \%$

(No Incident was Detected) 


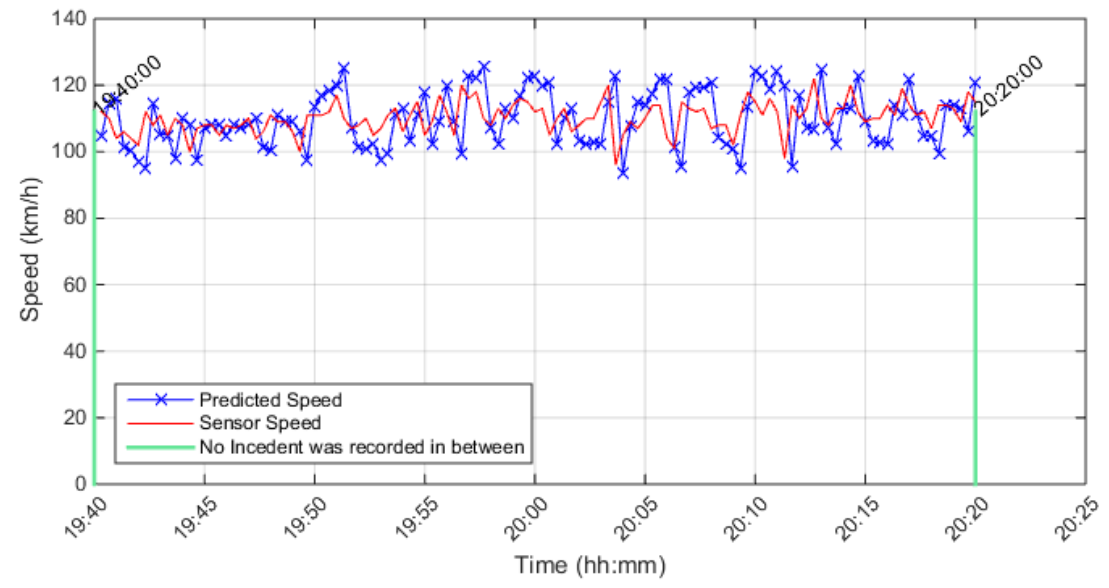

Upstream Detector \# is 401DW0020DWE, Detector Rank = 1, Distance from incident free location in $(\mathrm{km})=0.13067$ Incident free Season $=$ Spring, Stream $=E$, Direction $=W$, Date $\&$ Time $=2011-03-16$ 06:34:40 Selected incident free Date $/$ Time $=$ 2011-03-16 20:00:00

Conditional Probability level $=0.6$, Cond. Prob. $r 3 s 2=0.2$, Cond. Prob. $r 3 s 1=0.1$, Percent drop in speed $\%=-56$ Max. Posterior Probability level $=0.97$, Percent range between speed states $=7 \%$ (No Incident was Detected)

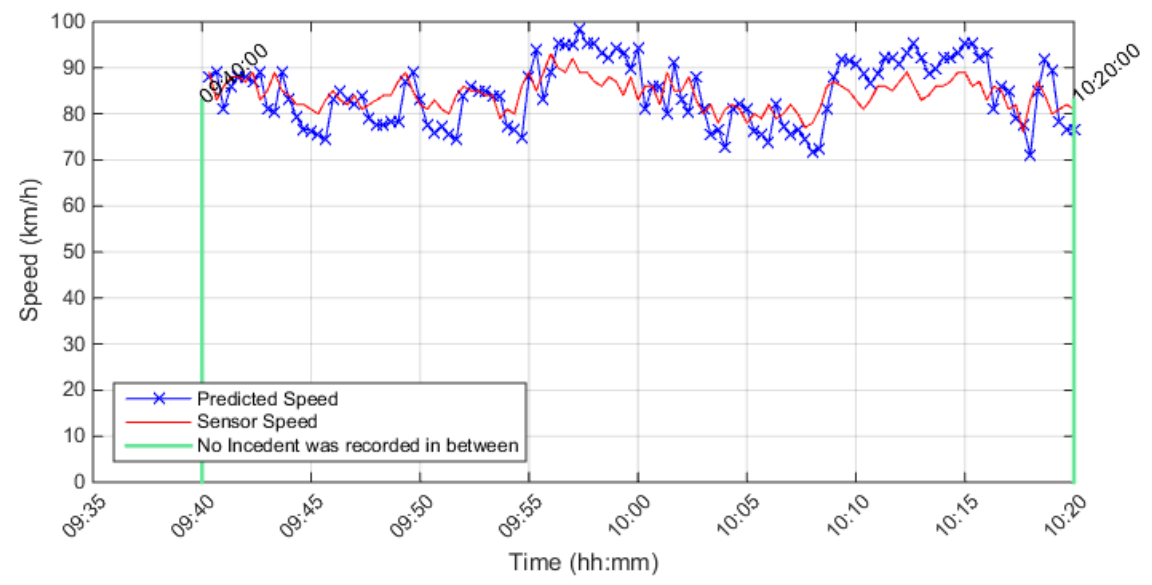

Upstream Detector \# is 401DE0240DWE, Detector Rank $=1$, Distance from incident free location in $(\mathrm{km})=0.13998$ Incident free Season $=$ Spring, Stream $=E$, Direction $=W$, Date \& Time $=2011-03-17$ 13:53:39

Selected incident free Date/Time $=2011-03-17$ 10:00:00

Conditional Probability level $=0.6$, Cond.Prob. $r 3 s 2=0.2$, Cond. Prob. $r 3 s 1=0.1$, Percent drop in speed $\%=-56$ Max. Posterior Probability level $=0.97$, Percent range between speed states $=7 \%$

(No Incident was Detected) 


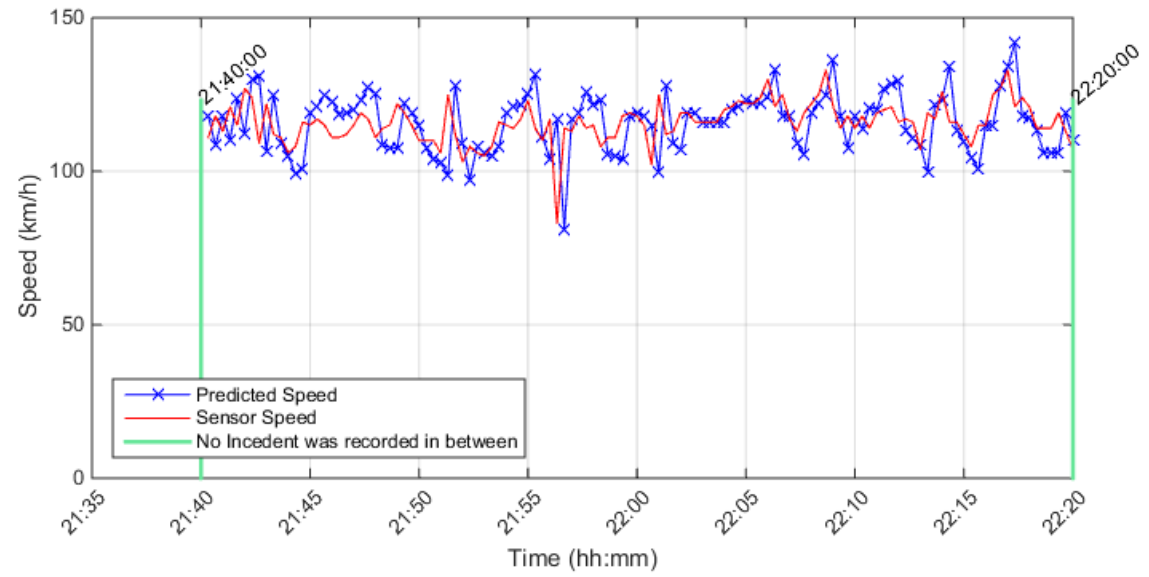

Upstream Detector \# is 401DE0360DWE, Detector Rank $=2$, Distance from incident free location in $(\mathrm{km})=0.97011$ Incident free Season $=$ Spring. Stream $=$ E, Direction $=$ W, Date \& Time $=2011-05-26$ 09:12:47

Selected incident free Date/Time $=2011-05-26$ 22:00:00

Conditional Probability level $=0.6$, Cond.Prob. $r 3 \mathrm{~s} 2=0.2$, Cond. Prob. $r 3 \mathrm{~s} 1=0.1$, Percent drop in speed $\%=-56$

Max. Posterior Probability level $=0.97$. Percent range between speed states $=7 \%$

(No Incident was Detected)

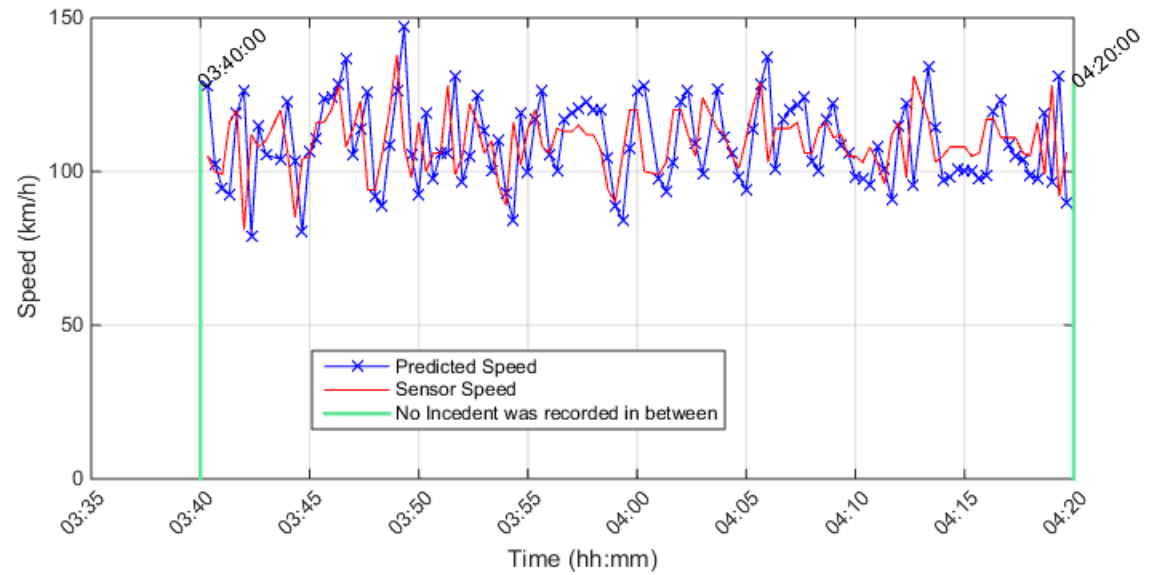

Upstream Detector \# is 401DE0410DWE, Detector Rank = 1, Distance from incident free location in $(\mathrm{km})=4.2027$ Incident free Season $=$ Summer, Stream $=E$, Direction $=$ W, Date \& Time $=$ 2011-06-10 11:00:38

Selected incident free Date/Time $=2011-06-10$ 04:00:00

Conditional Probability level $=0.6$, Cond.Prob. $r 3 s 2=0.2$, Cond.Prob. $r 3 s 1=0.1$, Percent drop in speed $\%=-56$

Max. Posterior Probability level $=0.97$, Percent range between speed states $=7 \%$

(No Incident was Detected) 


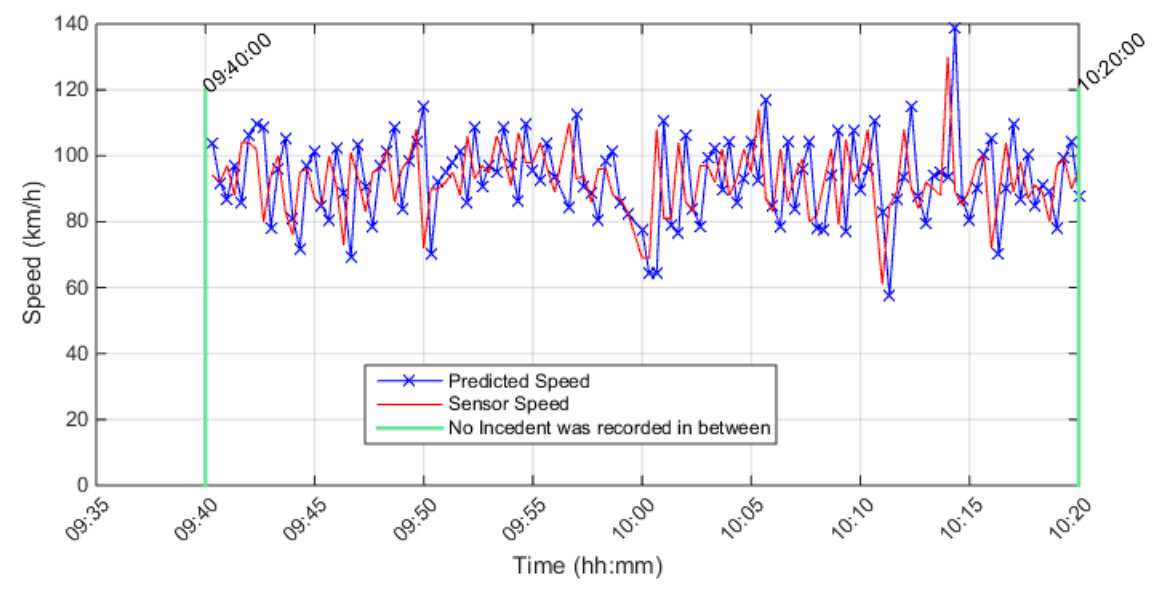

Upstream Detector \# is 401DE0340DWE, Detector Rank = 3, Distance from incident free location in $(\mathrm{km})=1.6024$ Incident free Season $=$ Summer, Stream $=E$, Direction $=W$, Date \& Time $=2011-06-12$ 05:54:15 Selected incident free Date/Time $=$ 2011-06-12 10:00:00

Conditional Probability level $=0.6$, Cond.Prob. $r 3 \mathrm{~s} 2=0.2$, Cond. Prob. $\mathrm{r} 3 \mathrm{~s} 1=0.1$, Percent drop in speed $\%=-56$ Max. Posterior Probability level $=0.97$, Percent range between speed states $=7 \%$

(No Incident was Detected)

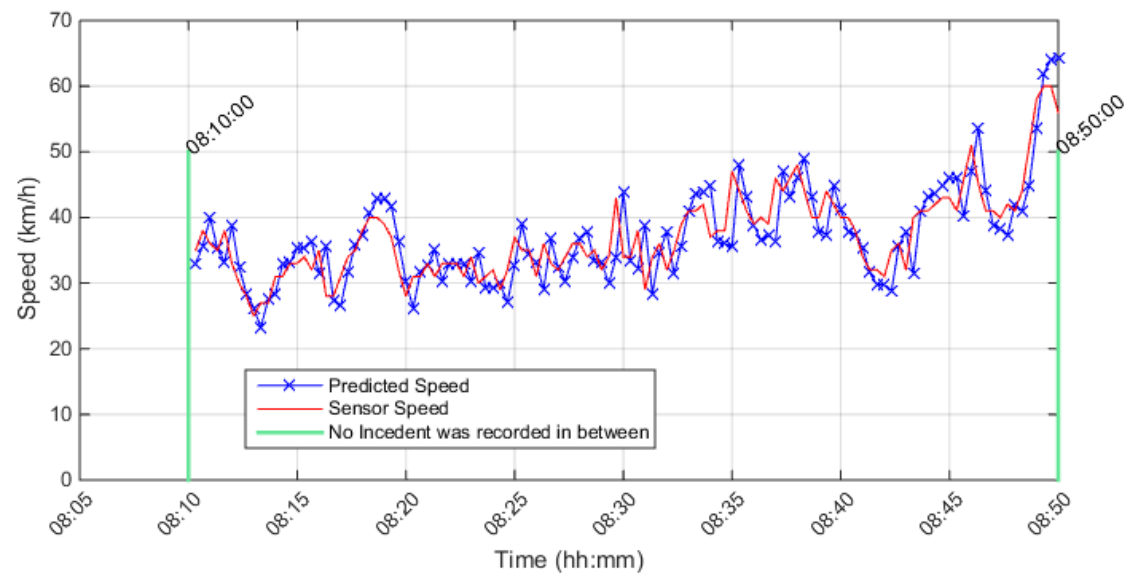

Upstream Detector \# is 401DE0060DWE, Detector Rank = 1, Distance from incident free location in $(\mathrm{km})=0.17254$ Incident free Season $=$ Summer, Stream $=\mathrm{E}$, Direction $=\mathrm{W}$, Date \& Time $=2011-09-1517: 05: 35$

Selected incident free Date/Time $=2011-09-15$ 08:30:00

Conditional Probability level $=0.6$, Cond.Prob. $r 3 \mathrm{~s} 2=0.2$, Cond.Prob. $r 3 \mathrm{~s} 1=0.1$, Percent drop in speed $\%=-56$

Max. Posterior Probability level $=0.97$, Percent range between speed states $=7 \%$

(No Incident was Detected) 


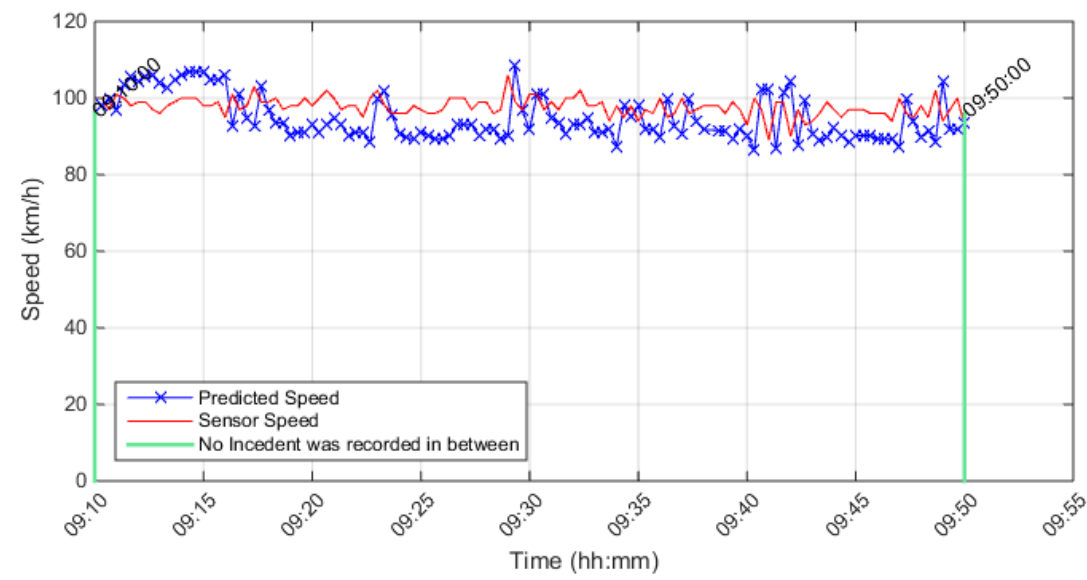

Upstream Detector \# is 401DE0220DWE, Detector Rank = 1, Distance from incident free location in $(\mathrm{km})=0.30243$ Incident free Season $=$ Summer, Stream $=E$, Direction $=W$, Date $\&$ Time $=2011-10-2218: 16: 41$

Selected incident free Date/Time $=$ 2011-10-22 09:30:00

Conditional Probability level $=0.6$, Cond. Prob. $r 3 \mathrm{~s} 2=0.2$, Cond.Prob. $r 3 \mathrm{~s} 1=0.1$, Percent drop in speed $\%=-56$ Max. Posterior Probability level $=0.97$, Percent range between speed states $=7 \%$

(No Incident was Detected)

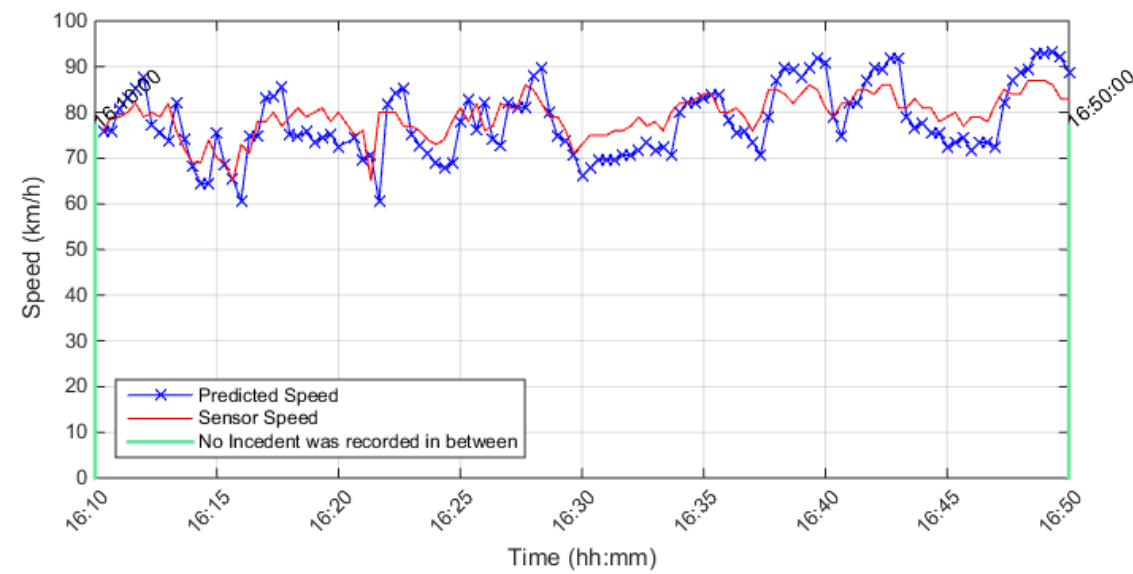

Upstream Detector \# is 401DW0040DWE, Detector Rank = 1, Distance from incident free location in $(\mathrm{km})=0.22683$ Incident free Season $=$ Winter, Stream $=E$, Direction $=W$, Date \& Time $=2011-12-21$ 00:25:23 Selected incident free Date/Time $=2011-12-2116: 30: 00$

Conditional Probability level $=0.6$, Cond.Prob. $r 3 \mathrm{~s} 2=0.2$, Cond.Prob. $r 3 \mathrm{~s} 1=0.1$, Percent drop in speed $\%=-56$

Max. Posterior Probability level $=0.97$, Percent range between speed states $=7 \%$

(No Incident was Detected) 


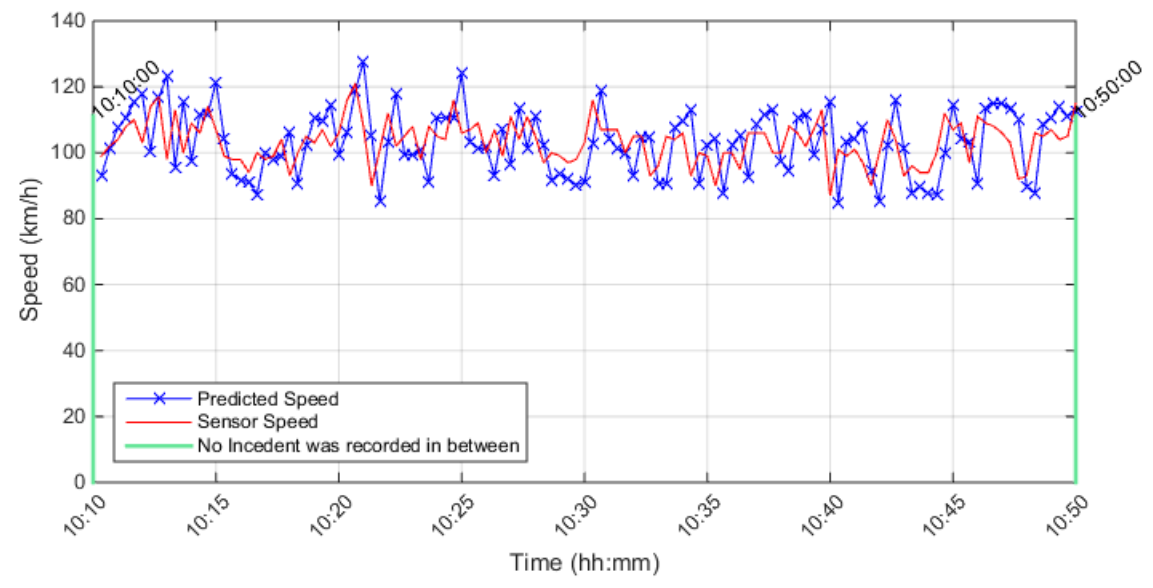

Upstream Detector \# is 401DE0100DWE, Detector Rank = 1, Distance from incident free location in $(\mathrm{km})=0.12114$ Incident free Season $=$ Winter, Stream $=E$, Direction $=W$, Date \& Time $=2011-12-27$ 20:21:38

Selected incident free Date/Time $=2011-12-27$ 10:30:00

Conditional Probability level $=0.6$, Cond.Prob. $r 3 \mathrm{~s} 2=0.2$, Cond.Prob. $r 3 \mathrm{~s} 1=0.1$, Percent drop in speed $\%=-56$

Max. Posterior Probability level $=0.97$, Percent range between speed states $=7 \%$

(No Incident was Detected)

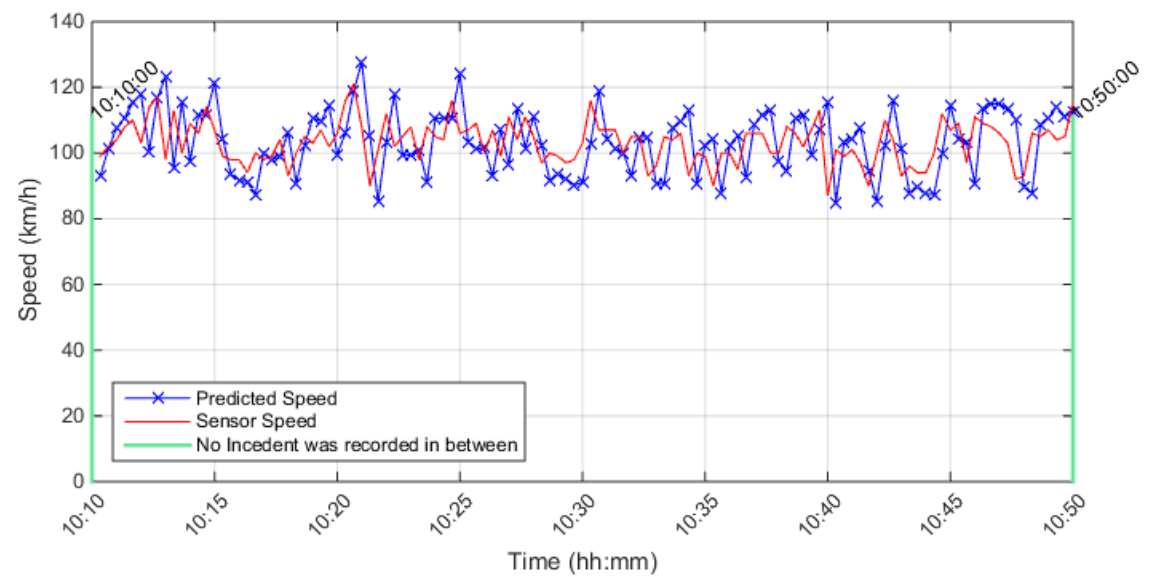

Upstream Detector \# is 401DE0100DWE, Detector Rank = 1, Distance from incident free location in $(\mathrm{km})=0.12114$ Incident free Season $=$ Winter, Stream $=\mathrm{E}$, Direction $=\mathrm{W}$, Date \& Time $=$ 2011-12-27 20:21:38

Selected incident free Date/Time $=2011-12-27$ 10:30:00

Conditional Probability level $=0.6$, Cond.Prob. $r 3 s 2=0.2$, Cond.Prob. $r 3 s 1=0.1$, Percent drop in speed $\%=-56$

Max. Posterior Probability level $=0.97$, Percent range between speed states $=7 \%$

(No Incident was Detected) 


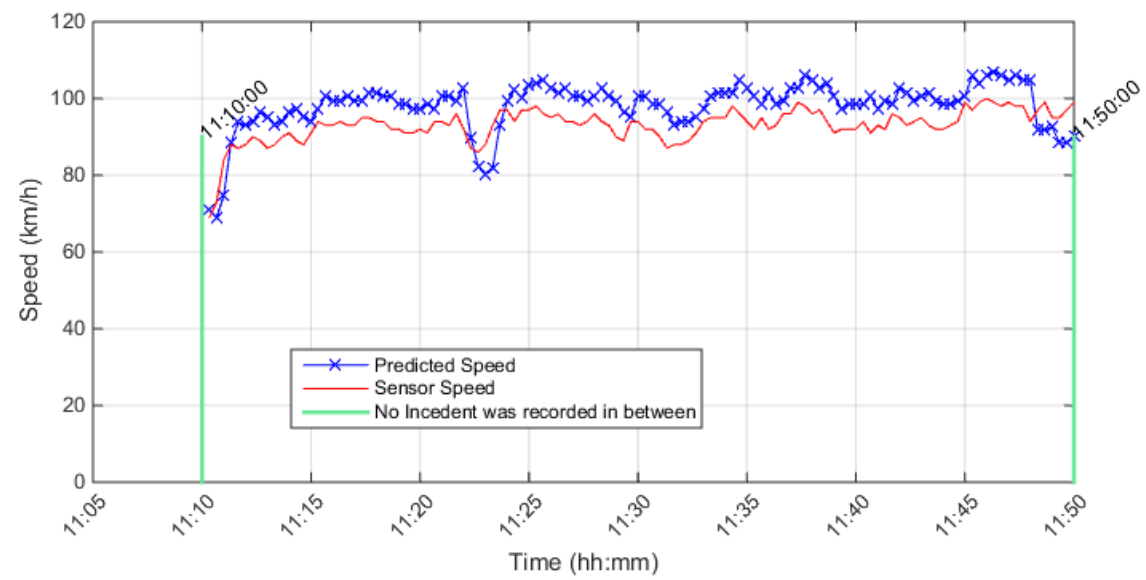

Upstream Detector \# is 401DE0100DEC, Detector Rank =1, Distance from incident free location in $(\mathrm{km})=0.26025$ Incident free Season $=$ Winter, Stream $=$ C, Direction $=E$, Date \& Time $=$ 2011-02-08 17:24:39

Selected incident free Date/Time $=2011-02-08$ 11:30:00

Conditional Probability level $=0.6$, Cond.Prob. $r 3 s 2=0.2$, Cond.Prob. $r 3 s 1=0.1$, Percent drop in speed $\%=-56$ Max. Posterior Probability level $=0.97$, Percent range between speed states $=7 \%$

(No Incident was Detected)

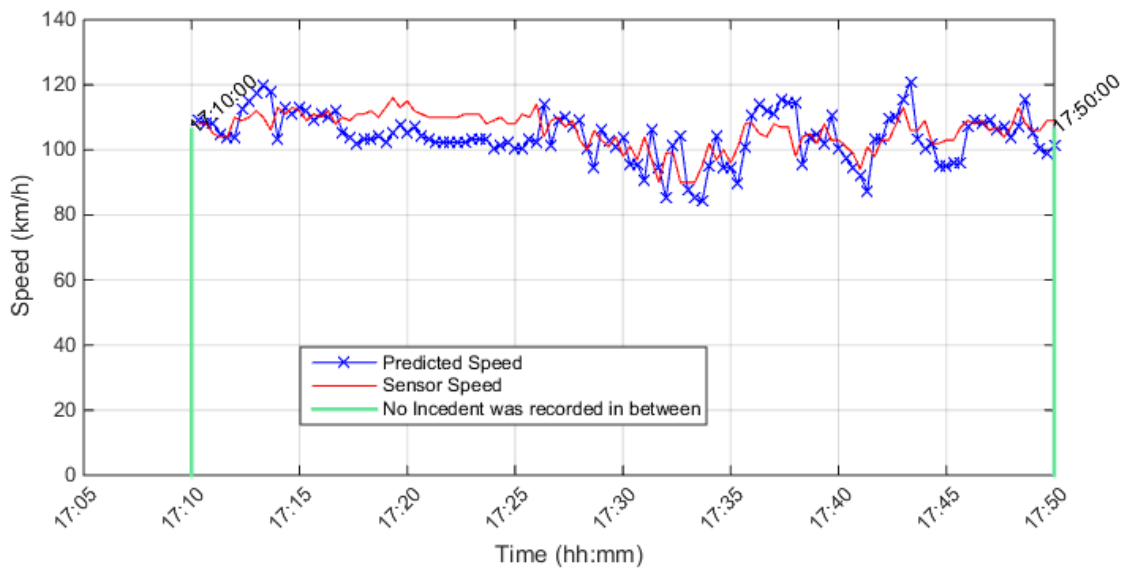

Upstream Detector \# is 401DE0490DEC, Detector Rank = 1, Distance from incident free location in $(\mathrm{km})=0.10164$ Incident free Season $=$ Winter, Stream $=C$, Direction $=E$, Date \& Time $=$ 2011-02-18 09:30:41 Selected incident free Date/Time $=$ 2011-02-18 17:30:00

Conditional Probability level $=0.6$, Cond.Prob. $r 3 \mathrm{~s} 2=0.2$, Cond.Prob. $r 3 \mathrm{~s} 1=0.1$, Percent drop in speed $\%=-56$

Max. Posterior Probability level $=0.97$, Percent range between speed states $=7 \%$

(No Incident was Detected) 


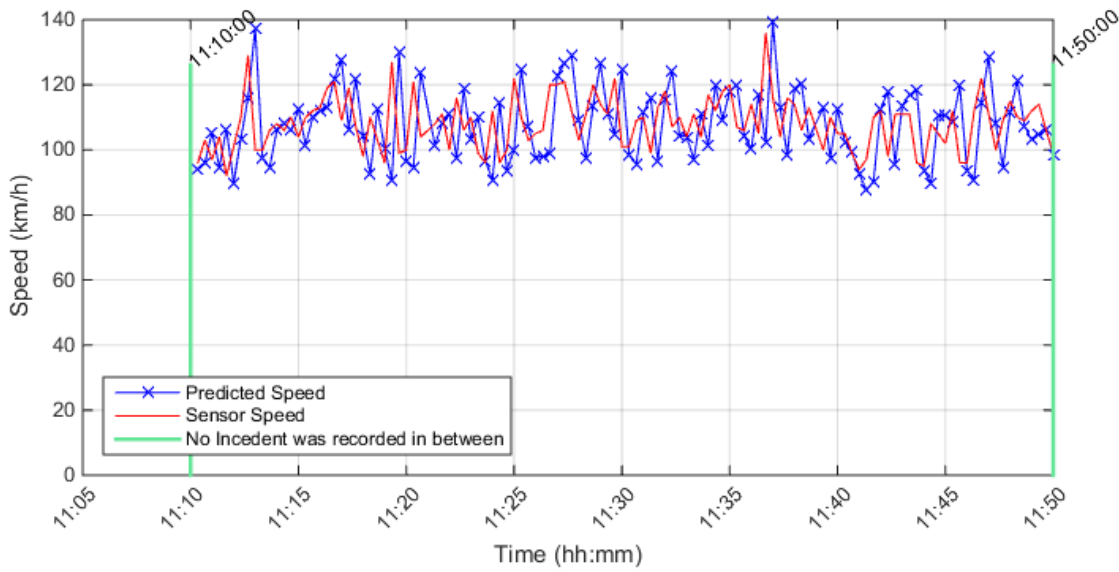

Upstream Detector \# is 401DW0180DEC, Detector Rank = 1, Distance from incident free location in $(\mathrm{km})=0.25258$ Incident free Season $=$ Spring, Stream $=$ C, Direction $=E$, Date \& Time $=$ 2011-03-08 17:48:57

Selected incident free Date/Time $=$ 2011-03-08 11:30:00

Conditional Probability level $=0.6$, Cond. Prob. $r 3 \mathrm{~s} 2=0.2$, Cond. Prob. $r 3 \mathrm{~s} 1=0.1$, Percent drop in speed $\%=-56$

Max. Posterior Probability level $=0.97$, Percent range between speed states $=7 \%$

(No Incident was Detected)

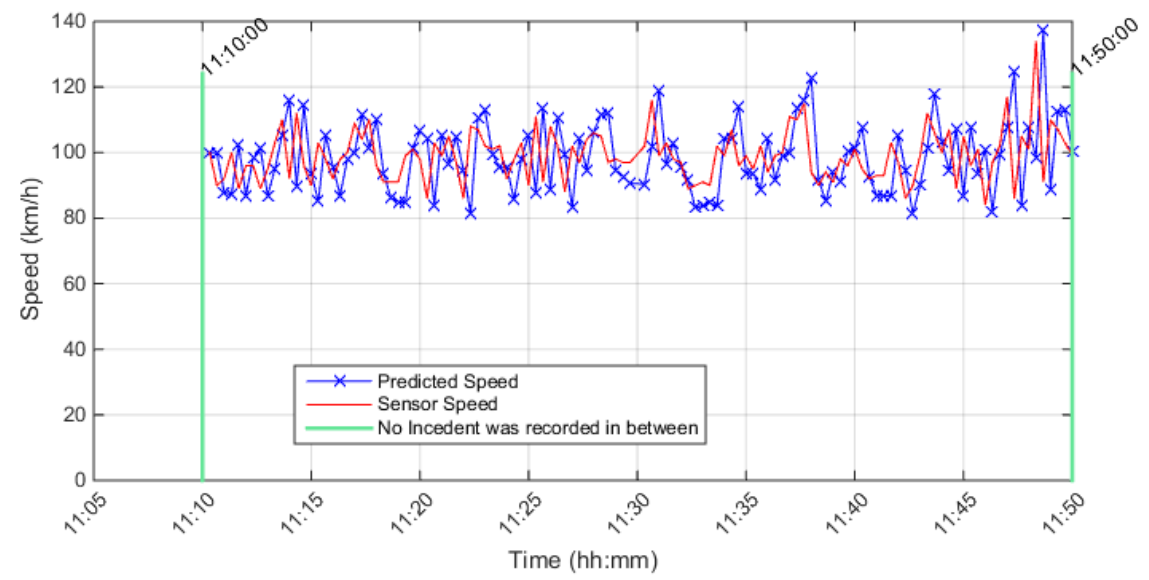

Upstream Detector \# is 401DW0160DEC, Detector Rank = 1, Distance from incident free location in $(\mathrm{km})=0.33732$ Incident free Season $=$ Spring, Stream $=$ C, Direction $=E$, Date \& Time $=2011-03-16$ 14:25:22

Selected incident free Date/Time $=2011-03-16$ 11:30:00

Conditional Probability level $=0.6$, Cond.Prob. $r 3 \mathrm{~s} 2=0.2$, Cond.Prob. $\mathrm{r} 3 \mathrm{~s} 1=0.1$, Percent drop in speed $\%=-56$

Max. Posterior Probability level $=0.97$, Percent range between speed states $=7 \%$

(No Incident was Detected) 


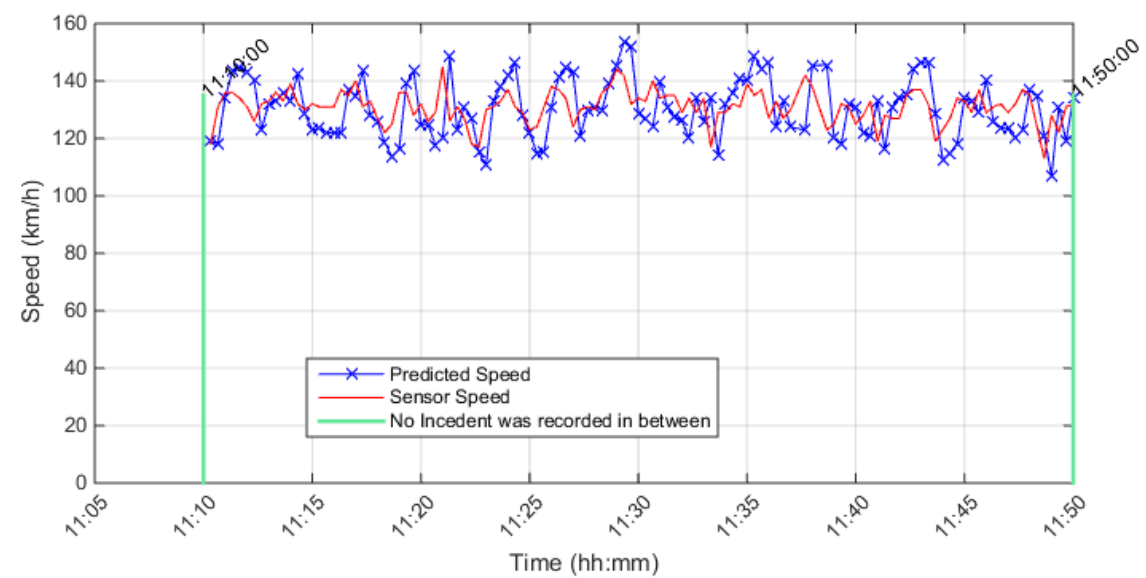

Upstream Detector \# is 401DE0530DEC, Detector Rank = 1, Distance from incident free location in $(\mathrm{km})=0.43916$ Incident free Season $=$ Spring, Stream $=$ C, Direction $=$ E, Date \& Time $=2011-03-26$ 13:49:07

Selected incident free Date/Time $=2011-03-26$ 11:30:00

Conditional Probability level $=0.6$, Cond.Prob. $r 3 s 2=0.2$, Cond.Prob. $r 3 s 1=0.1$, Percent drop in speed $\%=-56$ Max. Posterior Probability level $=0.97$, Percent range between speed states $=7 \%$

(No Incident was Detected)

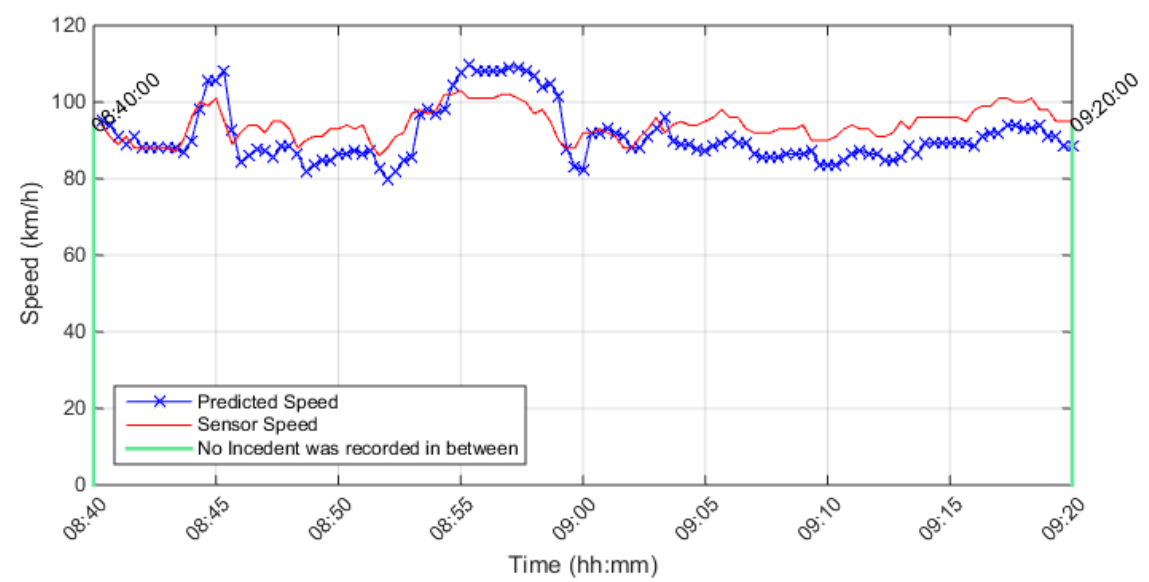

Upstream Detector \# is 401DE0100DEC, Detector Rank = 1, Distance from incident free location in $(\mathrm{km})=0.26025$ Incident free Season $=$ Spring, Stream $=\mathrm{C}$, Direction $=\mathrm{E}$, Date $\&$ Time $=2011-05-13$ 19:08:25 Selected incident free Date/Time $=$ 2011-05-13 09:00:00

Conditional Probability level $=0.6$, Cond. Prob. $r 3 s 2=0.2$, Cond. Prob. $r 3 s 1=0.1$, Percent drop in speed $\%=-56$

Max. Posterior Probability level $=0.97$, Percent range between speed states $=7 \%$

(No Incident was Detected) 


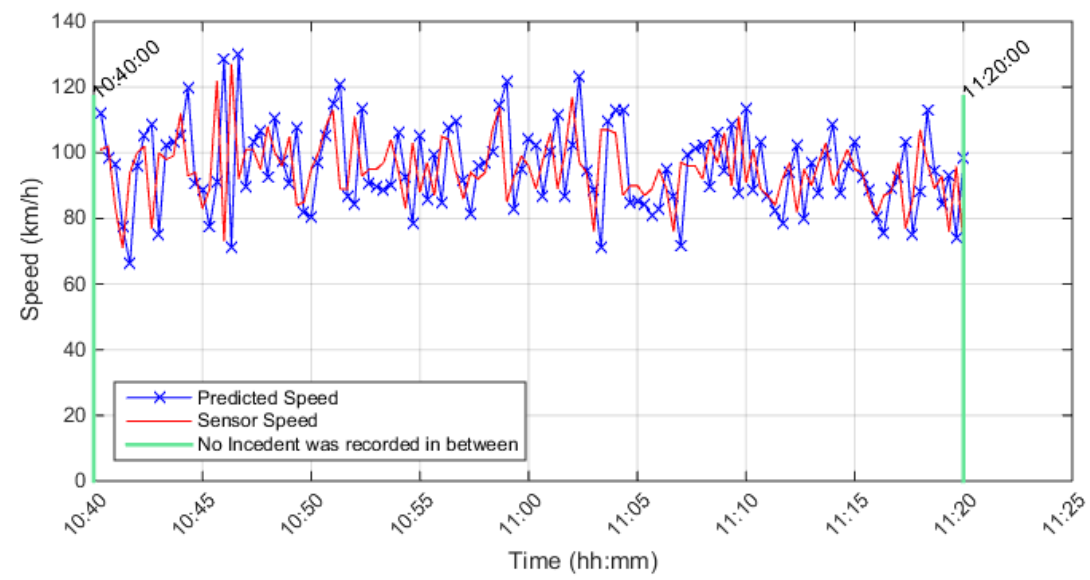

Upstream Detector \# is 401DE0330DEC, Detector Rank $=1$, Distance from incident free location in $(\mathrm{km})=0.68588$ Incident free Season $=$ Spring, Stream $=$ C, Direction $=E$, Date $\&$ Time $=2011-05-20$ 15:04:04

Selected incident free Date/Time $=2011-05-2011: 00: 00$

Conditional Probability level $=0.6$, Cond. Prob. $r 3 \mathrm{~s} 2=0.2$, Cond.Prob. $r 3 \mathrm{~s} 1=0.1$, Percent drop in speed $\%=-56$ Max. Posterior Probability level $=0.97$, Percent range between speed states $=7 \%$

(No Incident was Detected)

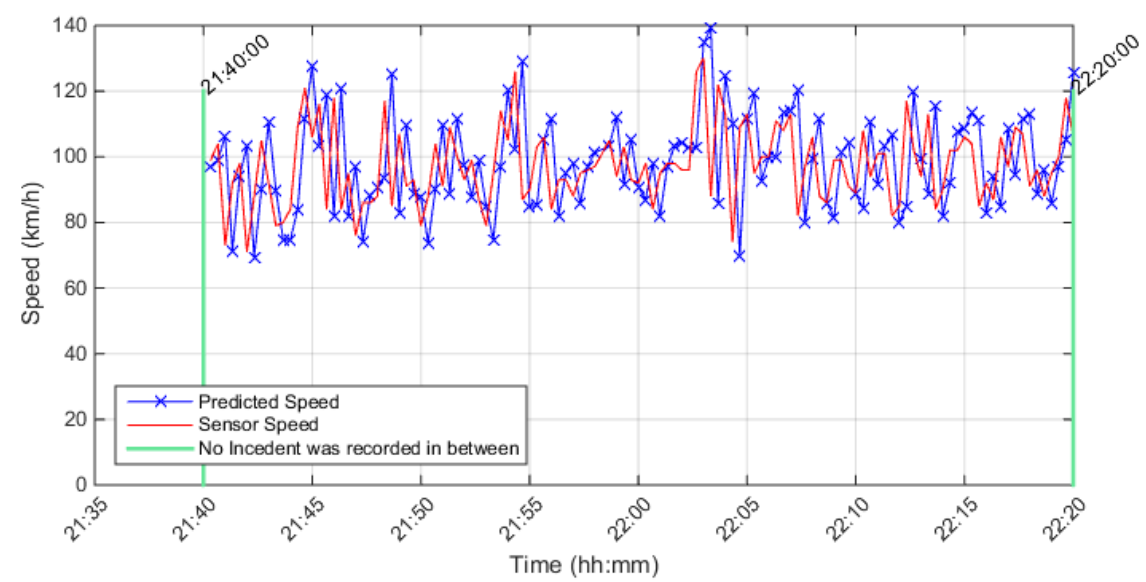

Upstream Detector \# is 401DE0330DEC, Detector Rank = 3, Distance from incident free location in $(\mathrm{km})=1.5366$ Incident free Season $=$ Summer, Stream $=$ C, Direction $=E$, Date $\&$ Time $=2011-06-18$ 12:39:53 Selected incident free Date/Time $=$ 2011-06-18 22:00:00

Conditional Probability level $=0.6$, Cond. Prob. $r 3 s 2=0.2$, Cond. Prob. $r 3 s 1=0.1$, Percent drop in speed $\%=-56$ Max. Posterior Probability level $=0.97$, Percent range between speed states $=7 \%$ (No Incident was Detected) 


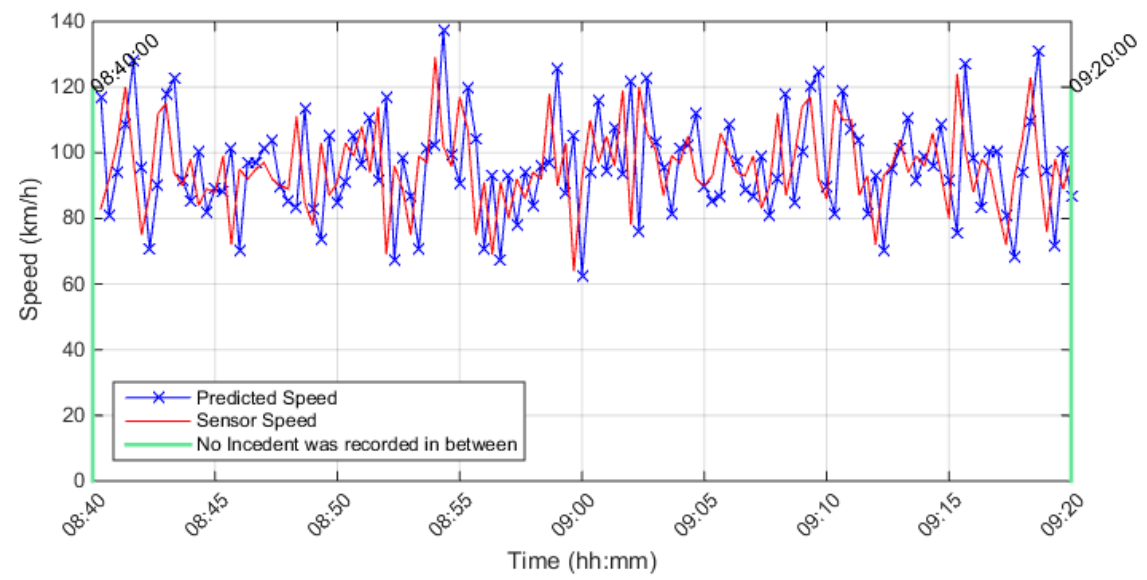

Upstream Detector \# is 401DE0330DEC, Detector Rank = 3, Distance from incident free location in $(\mathrm{km})=1.5366$ Incident free Season $=$ Summer, Stream $=C$, Direction $=E$, Date \& Time $=2011-06-24$ 19:00:44 Selected incident free Date/Time $=2011-06-24$ 09:00:00

Conditional Probability level $=0.6$, Cond.Prob. $r 3 \mathrm{~s} 2=0.2$, Cond.Prob. $r 3 \mathrm{~s} 1=0.1$, Percent drop in speed $\%=-56$ Max. Posterior Probability level $=0.97$, Percent range between speed states $=7 \%$

(No Incident was Detected)

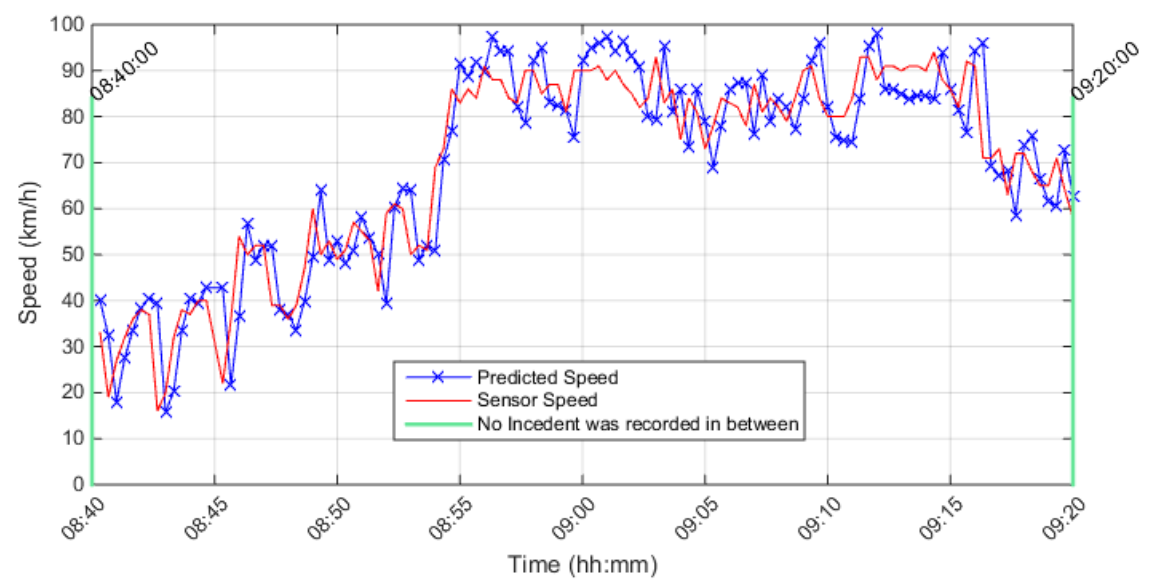

Upstream Detector \# is 401DW0050DEC, Detector Rank $=1$, Distance from incident free location in $(\mathrm{km})=0.4472$ Incident free Season $=$ Summer, Stream $=$ C, Direction $=E$, Date \& Time $=2011-06-29$ 13:03:02 Selected incident free Date/Time $=2011-06-29$ 09:00:00

Conditional Probability level $=0.6$, Cond. Prob. $r 3 s 2=0.2$, Cond. Prob. $r 3 s 1=0.1$, Percent drop in speed $\%=-56$ Max. Posterior Probability level $=0.97$, Percent range between speed states $=7 \%$ (No Incident was Detected) 


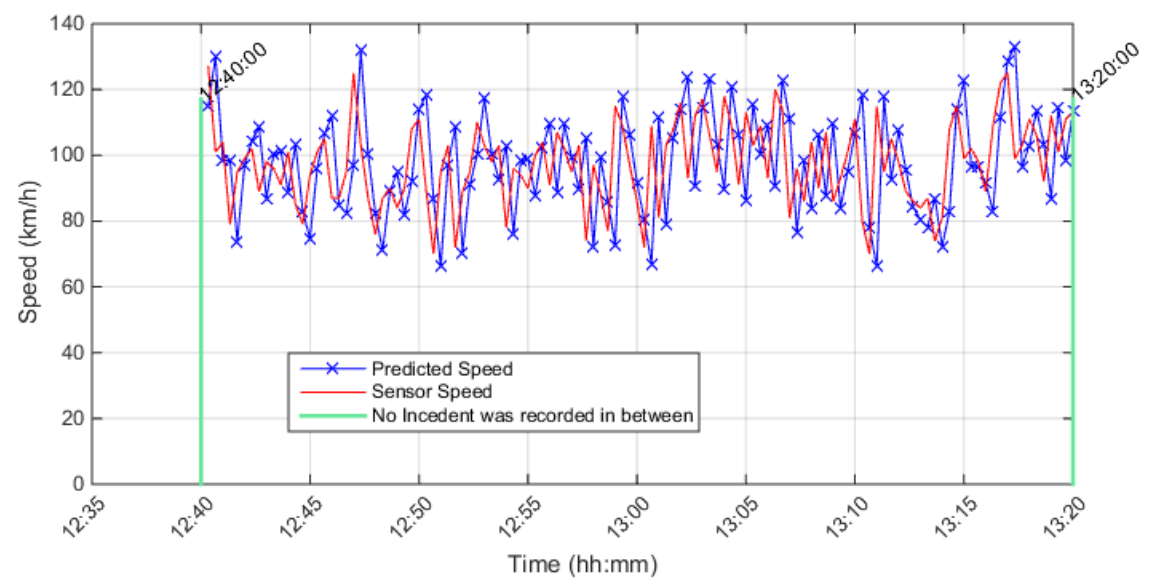

Upstream Detector \# is 401DE0110DEC, Detector Rank $=2$, Distance from incident free location in $(\mathrm{km})=0.78244$ Incident free Season $=$ Summer, Stream $=C$, Direction $=E$, Date $\&$ Time $=2011-07-19$ 18:28:02 Selected incident free Date/Time $=2011-07-19$ 13:00:00

Conditional Probability level $=0.6$, Cond.Prob. $r 3 s 2=0.2$, Cond. Prob. $r 3 s 1=0.1$, Percent drop in speed $\%=-56$ Max. Posterior Probability level $=0.97$, Percent range between speed states $=7 \%$

(No Incident was Detected)

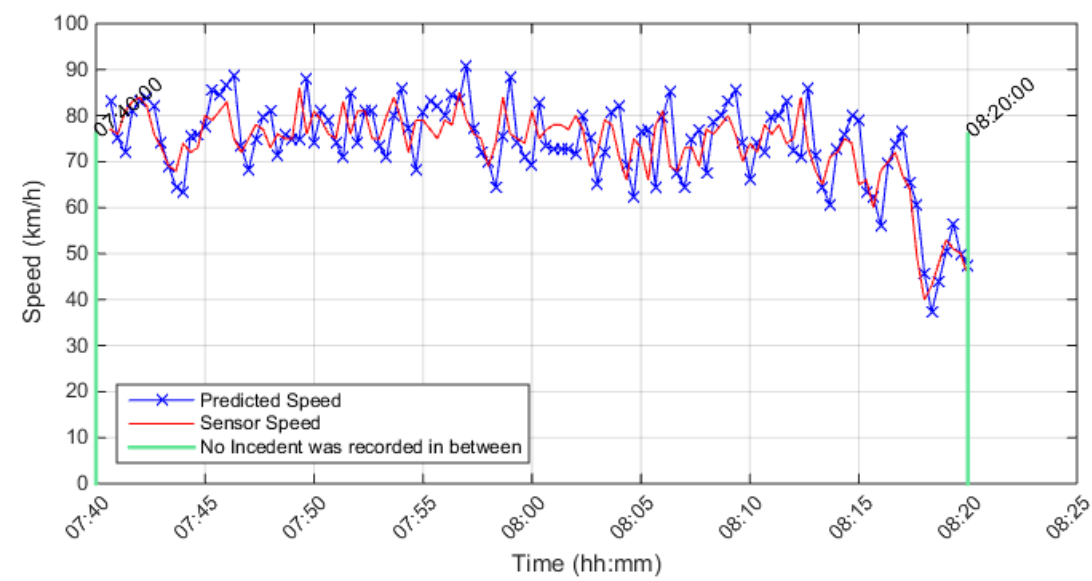

Upstream Detector \# is 401DE0130DEC, Detector Rank = 2, Distance from incident free location in $(\mathrm{km})=1.1356$ Incident free Season $=$ Summer, Stream $=$ C , Direction $=\mathrm{E}$, Date $\&$ Time $=2011-11-29$ 11:12:54 Selected incident free Date/Time $=2011-11-29$ 08:00:00

Conditional Probability level $=0.6$, Cond.Prob. $r 3 s 2=0.2$, Cond.Prob. $r 3 s 1=0.1$, Percent drop in speed $\%=-56$ Max. Posterior Probability level $=0.97$, Percent range between speed states $=7 \%$ (No Incident was Detected) 


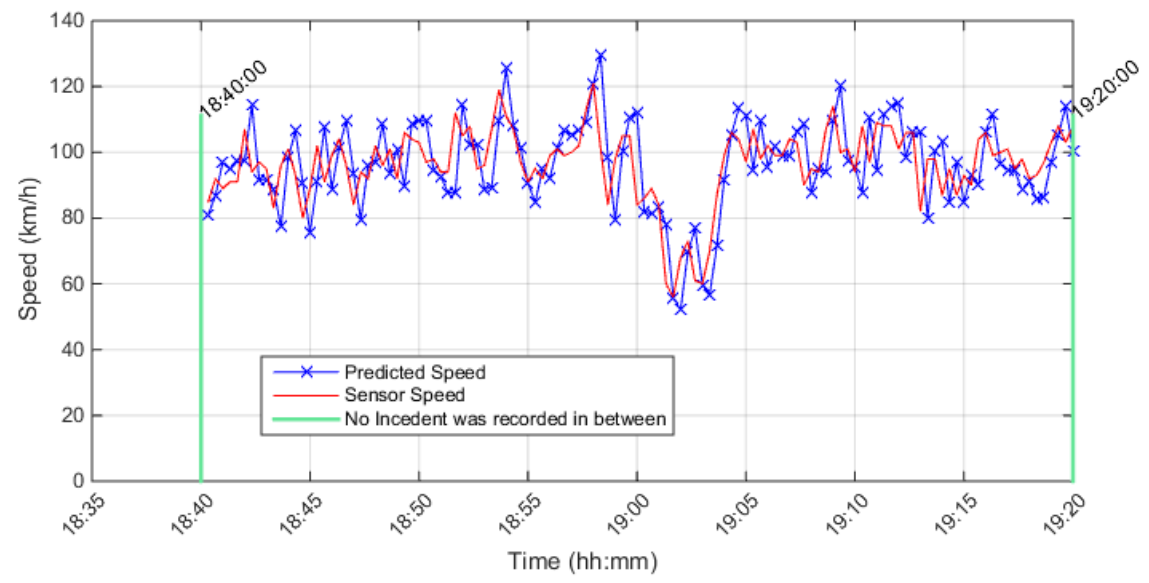

Upstream Detector \# is 401DW0010DEC, Detector Rank $=2$, Distance from incident free location in $(\mathrm{km})=1.1101$ Incident free Season $=$ Winter, Stream $=$ C, Direction $=$ E, Date \& Time $=2011-12-02$ 15:15:37

Selected incident free Date/Time $=$ 2011-12-02 19:00:00

Conditional Probability level $=0.6$, Cond.Prob. $r 3 \mathrm{~s} 2=0.2$, Cond. Prob. $r 3 \mathrm{~s} 1=0.1$, Percent drop in speed $\%=-56$ Max. Posterior Probability level $=0.97$, Percent range between speed states $=7 \%$

(No Incident was Detected)

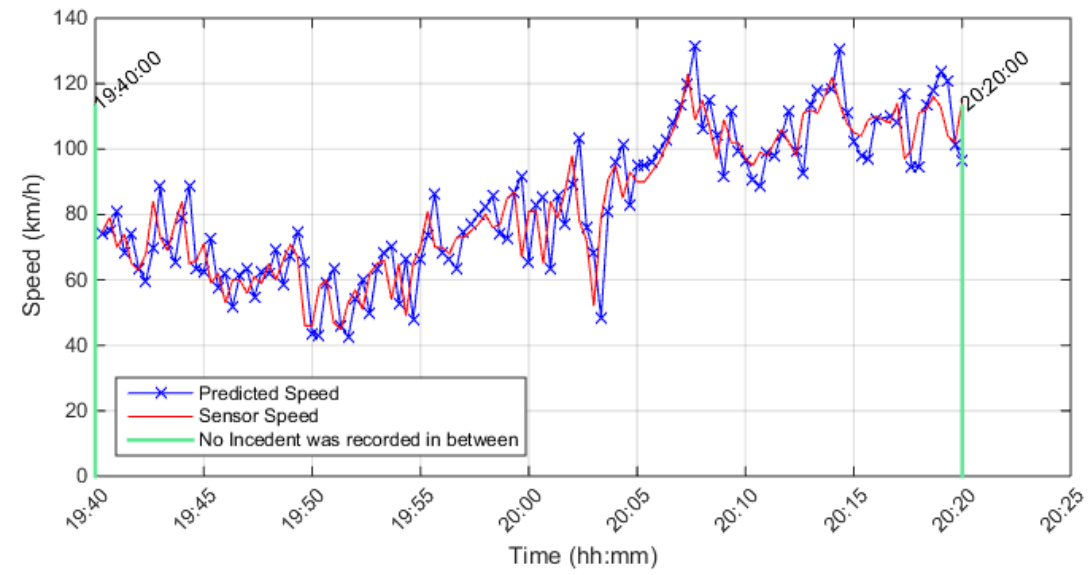

Upstream Detector \# is 401DE0170DEE, Detector Rank = 3, Distance from incident free location in $(\mathrm{km})=1.9295$ Incident free Season $=$ Winter, Stream $=E$, Direction $=E$, Date \& Time $=$ 2011-01-12 10:57:43 Selected incident free Date/Time $=2011-01-12$ 20:00:00

Conditional Probability level $=0.6$, Cond.Prob. $r 3 \mathrm{~s} 2=0.2$, Cond. Prob. $r 3 \mathrm{~s} 1=0.1$, Percent drop in speed $\%=-56$ Max. Posterior Probability level $=0.97$, Percent range between speed states $=7 \%$ (No Incident was Detected) 


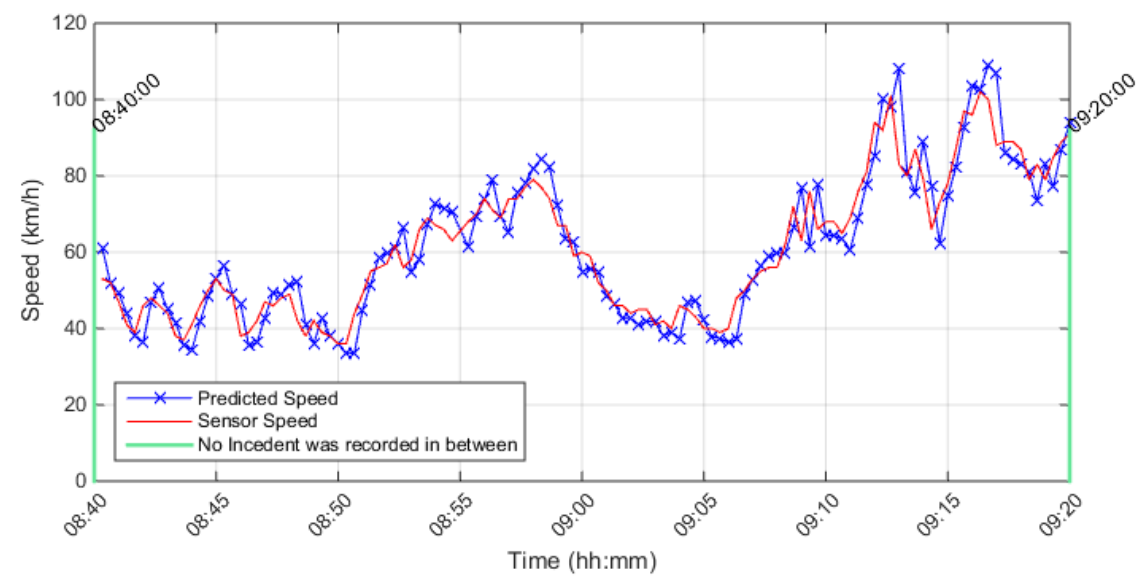

Upstream Detector \# is 401DE0190DEE, Detector Rank = 1, Distance from incident free location in $(\mathrm{km})=0.31753$ Incident free Season $=$ Winter, Stream $=E$, Direction $=E$, Date \& Time $=$ 2011-02-24 16:06:40 Selected incident free Date/Time $=2011-02-24$ 09:00:00

Conditional Probability level $=0.6$, Cond. Prob. $r 3 \mathrm{~s} 2=0.2$, Cond.Prob. $r 3 \mathrm{~s} 1=0.1$, Percent drop in speed $\%=-56$ Max. Posterior Probability level $=0.97$, Percent range between speed states $=7 \%$ (No Incident was Detected)

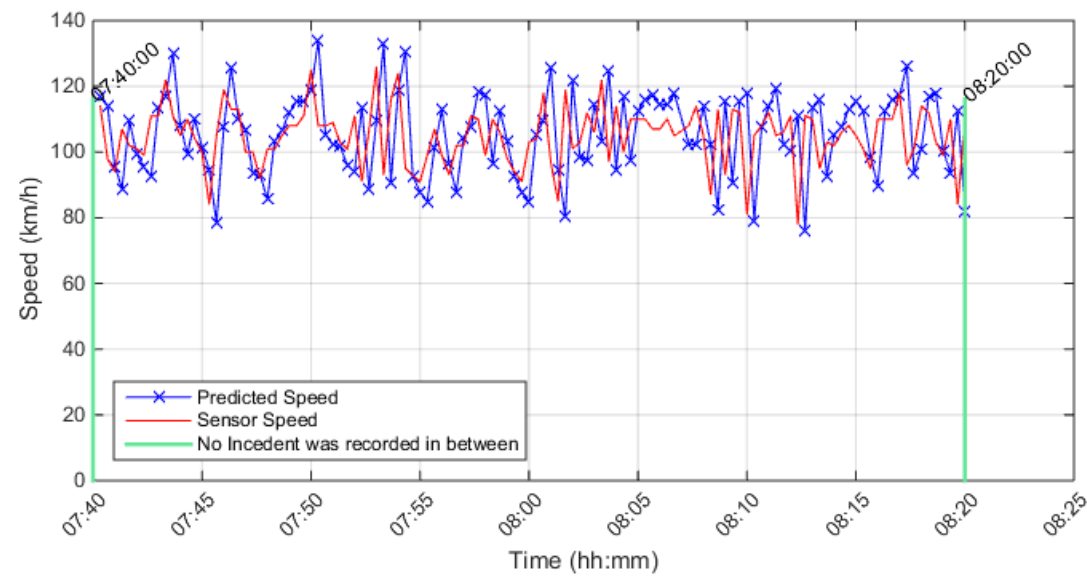

Upstream Detector \# is 401DE0490DEE, Detector Rank = 1, Distance from incident free location in $(\mathrm{km})=4.1527$ Incident free Season $=$ Spring, Stream $=E$, Direction $=E$, Date \& Time $=$ 2011-04-11 18:06:31 Selected incident free Date/Time $=2011-04-11$ 08:00:00

Conditional Probability level $=0.6$, Cond.Prob. $r 3 s 2=0.2$, Cond. Prob. $r 3 s 1=0.1$, Percent drop in speed $\%=-56$ Max. Posterior Probability level $=0.97$, Percent range between speed states $=7 \%$ (No Incident was Detected) 


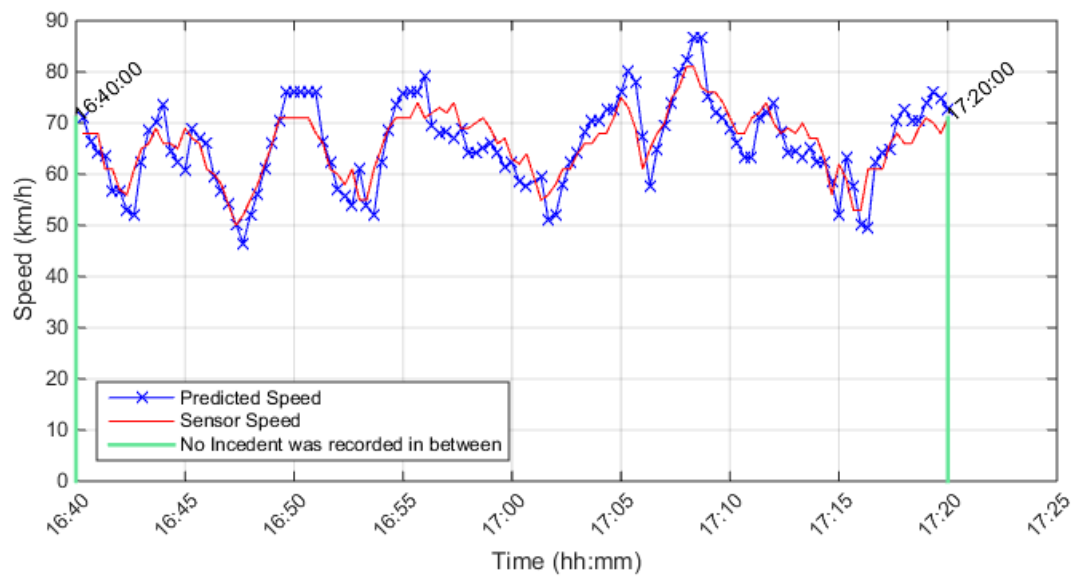

Upstream Detector \# is 401DE0160DEE, Detector Rank $=1$, Distance from incident free location in $(\mathrm{km})=0.24324$ Incident free Season $=$ Spring. Stream $=$ E, Direction $=E$, Date \& Time $=$ 2011-05-04 07:29:49

Selected incident free Date/Time $=2011-05-04$ 17:00:00

Conditional Probability level $=0.6$, Cond.Prob. $r 3 \mathrm{~s} 2=0.2$, Cond. Prob. $r 3 \mathrm{~s} 1=0.1$, Percent drop in speed $\%=-56$ Max. Posterior Probability level $=0.97$, Percent range between speed states $=7 \%$

(No Incident was Detected)

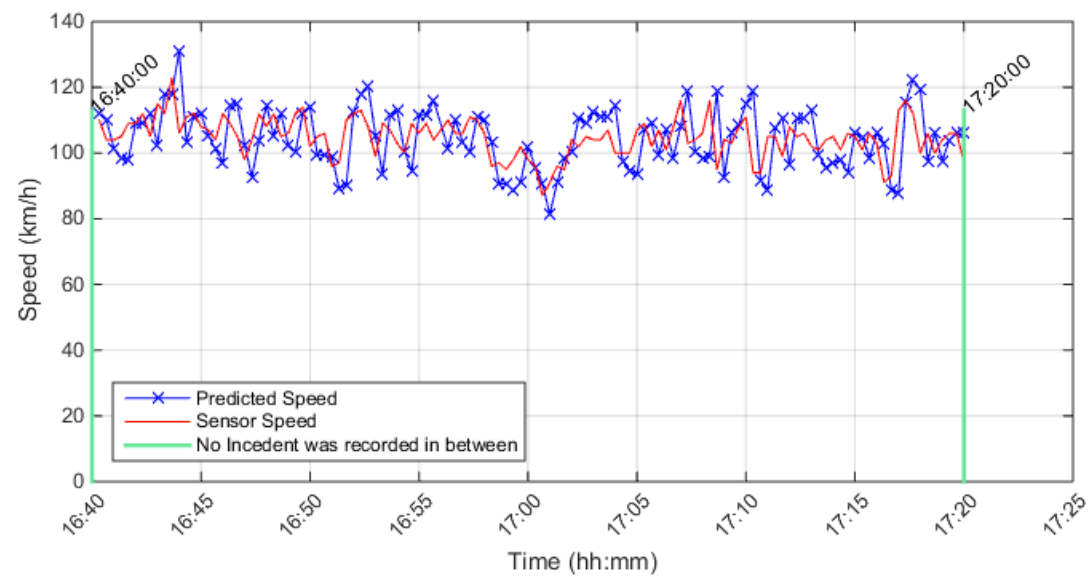

Upstream Detector \# is 401DE0590DEE, Detector Rank = 1, Distance from incident free location in $(\mathrm{km})=0.15896$ Incident free Season $=$ Spring, Stream $=E$, Direction $=E$, Date \& Time $=$ 2011-05-14 23:46:18

Selected incident free Date/Time $=2011-05-14$ 17:00:00

Conditional Probability level $=0.6$, Cond. Prob. $r 3 \mathrm{~s} 2=0.2$, Cond. Prob. $r 3 \mathrm{~s} 1=0.1$, Percent drop in speed $\%=-56$

Max. Posterior Probability level $=0.97$, Percent range between speed states $=7 \%$

(No Incident was Detected) 


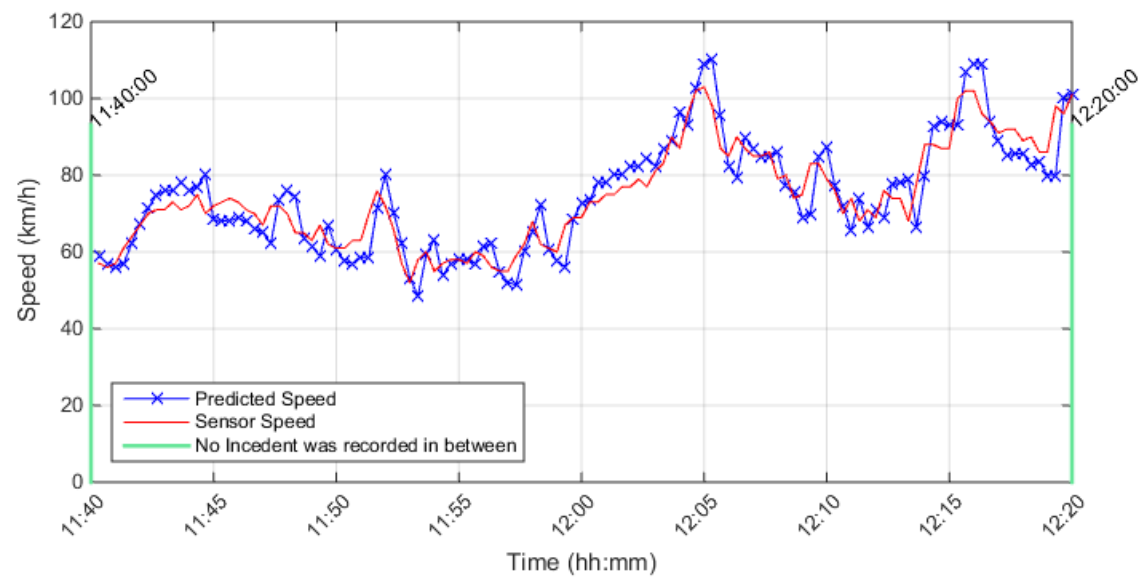

Upstream Detector \# is 401DW0010DEE, Detector Rank $=2$, Distance from incident free location in $(\mathrm{km})=1.1139$ Incident free Season $=$ Summer, Stream $=E$, Direction $=E$, Date \& Time $=2011-08-05$ 10:05:33 Selected incident free Date/Time $=2011-08-05$ 12:00:00

Conditional Probability level $=0.6$, Cond.Prob. $r 3 \mathrm{~s} 2=0.2$, Cond. Prob. $r 3 \mathrm{~s} 1=0.1$, Percent drop in speed $\%=-56$ Max. Posterior Probability level $=0.97$, Percent range between speed states $=7 \%$

(No Incident was Detected)

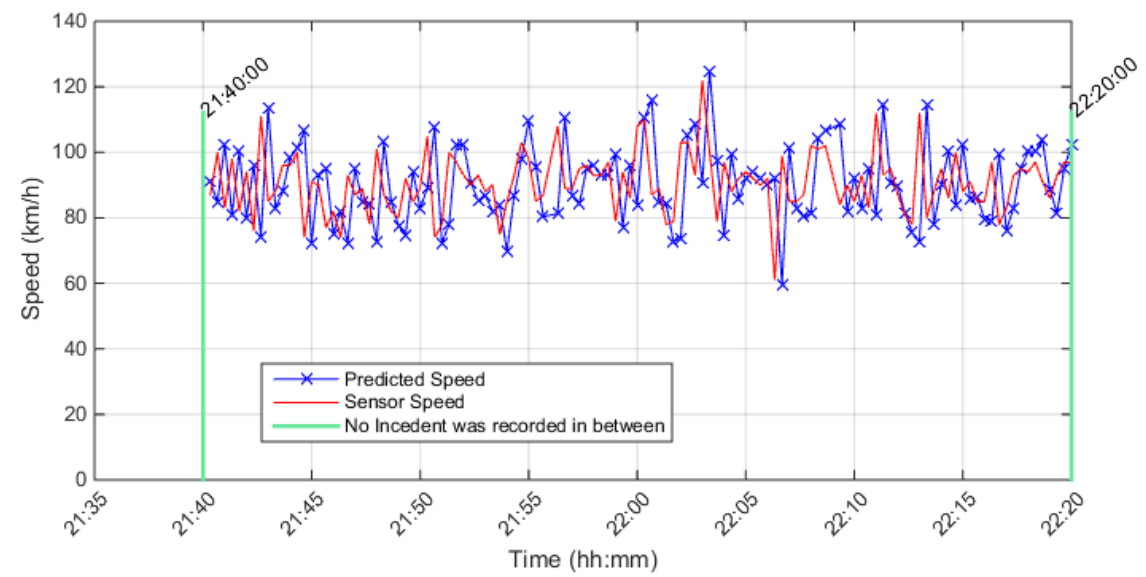

Upstream Detector \# is 401DW0260DEE, Detector Rank = 1, Distance from incident free location in $(\mathrm{km})=0.36837$ Incident free Season $=$ Winter, Stream $=E$, Direction $=E$, Date \& Time $=$ 2011-12-30 10:12:12

Selected incident free Date/Time $=2011-12-30$ 22:00:00

Conditional Probability level $=0.6$, Cond.Prob. $\mathrm{r} 3 \mathrm{~s} 2=0.2$, Cond. Prob. $\mathrm{r} 3 \mathrm{~s} 1=0.1$, Percent drop in speed $\%=-56$

Max. Posterior Probability level $=0.97$, Percent range between speed states $=7 \%$

(No Incident was Detected) 


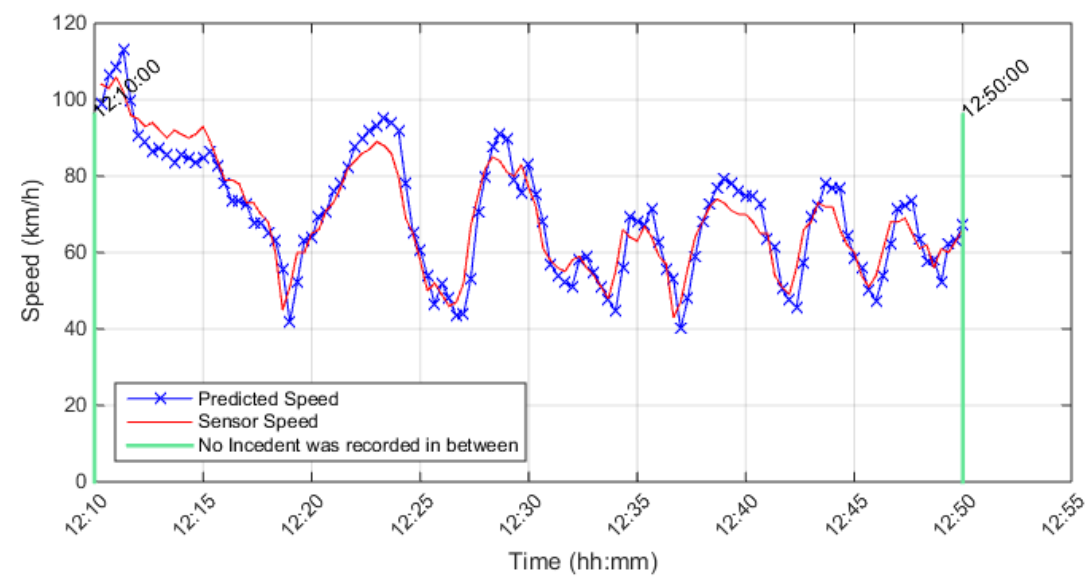

Upstream Detector \# is 401DW0060DWC, Detector Rank = 1, Distance from incident free location in $(\mathrm{km})=0.43697$ Incident free Season $=$ Winter, Stream $=$ C, Direction $=$ W, Date \& Time $=2011-02-18$ 19:52:14 Selected incident free Date/Time $=2011-02-18$ 12:30:00

Conditional Probability level $=0.6$, Cond.Prob. $r 3 s 2=0.2$, Cond.Prob. $r 3 s 1=0.1$, Percent drop in speed $\%=-56$ Max. Posterior Probability level $=0.97$, Percent range between speed states $=7 \%$

Drop in speed was detected and No indication of an incident within this period

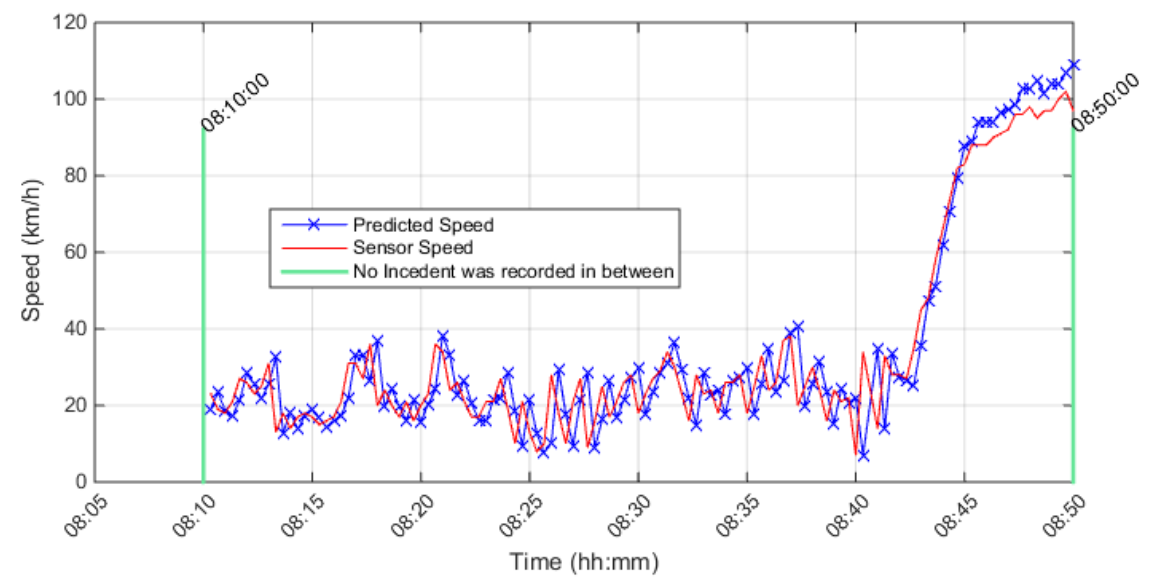

Upstream Detector \# is 401DE0270DWC, Detector Rank = 1, Distance from incident free location in $(\mathrm{km})=0.5857$ Incident free Season $=$ Spring, Stream $=C$, Direction $=W$, Date $\&$ Time $=2011-03-25$ 07:51:15 Selected incident free Date/Time $=2011-03-25$ 08:30:00

Conditional Probability level $=0.6$, Cond. Prob. $r 3 \mathrm{~s} 2=0.2$, Cond. Prob. $\mathrm{r} 3 \mathrm{~s} 1=0.1$, Percent drop in speed $\%=-56$

Max. Posterior Probability level $=0.97$, Percent range between speed states $=7 \%$

(No Incident was Detected) 


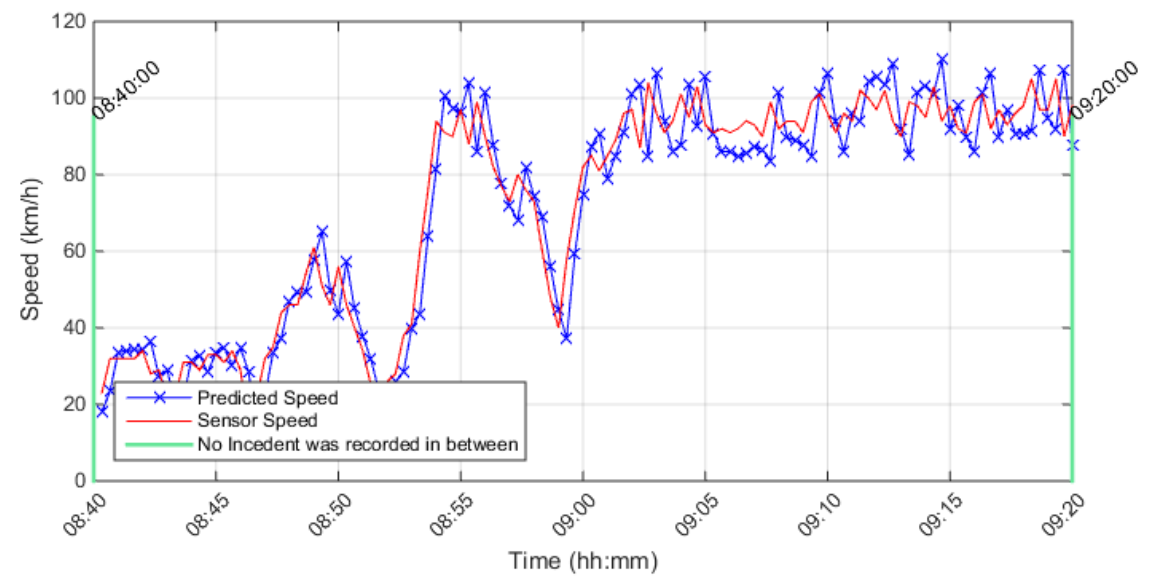

Upstream Detector \# is 401DE0050DWC, Detector Rank = 1, Distance from incident free location in $(\mathrm{km})=0.45946$ Incident free Season $=$ Summer, Stream $=C$, Direction $=W$, Date \& Time $=2011-07-0517: 40: 42$

Selected incident free Date/Time $=$ 2011-07-05 09:00:00

Conditional Probability level $=0.6$, Cond. Prob. $r 3 \mathrm{~s} 2=0.2$, Cond.Prob. $r 3 \mathrm{~s} 1=0.1$, Percent drop in speed $\%=-56$ Max. Posterior Probability level $=0.97$, Percent range between speed states $=7 \%$

(No Incident was Detected)

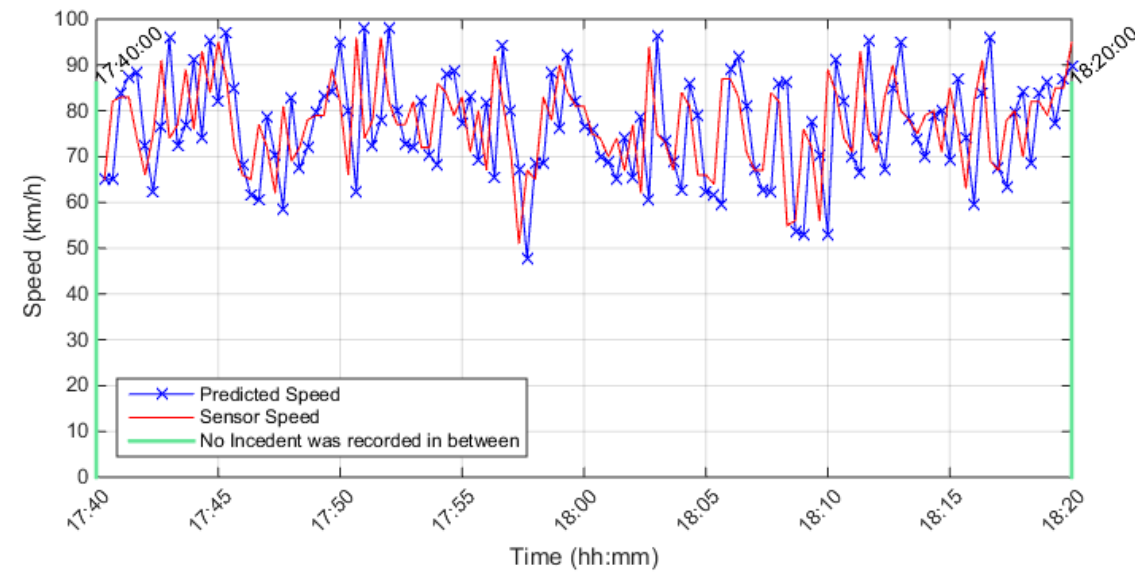

Upstream Detector \# is 401DE0430DWC, Detector Rank = 1, Distance from incident free location in $(\mathrm{km})=0.5003$ Incident free Season $=$ Summer, Stream $=$ C, , Direction $=W$, Date \& Time $=$ 2011-09-15 10:37:01

Selected incident free Date/Time $=2011-09-15$ 18:00:00

Conditional Probability level $=0.6$, Cond.Prob. $r 3 \mathrm{~s} 2=0.2$, Cond. Prob. $\mathrm{r} 3 \mathrm{~s} 1=0.1$, Percent drop in speed $\%=-56$ Max. Posterior Probability level $=0.97$, Percent range between speed states $=7 \%$

(No Incident was Detected) 


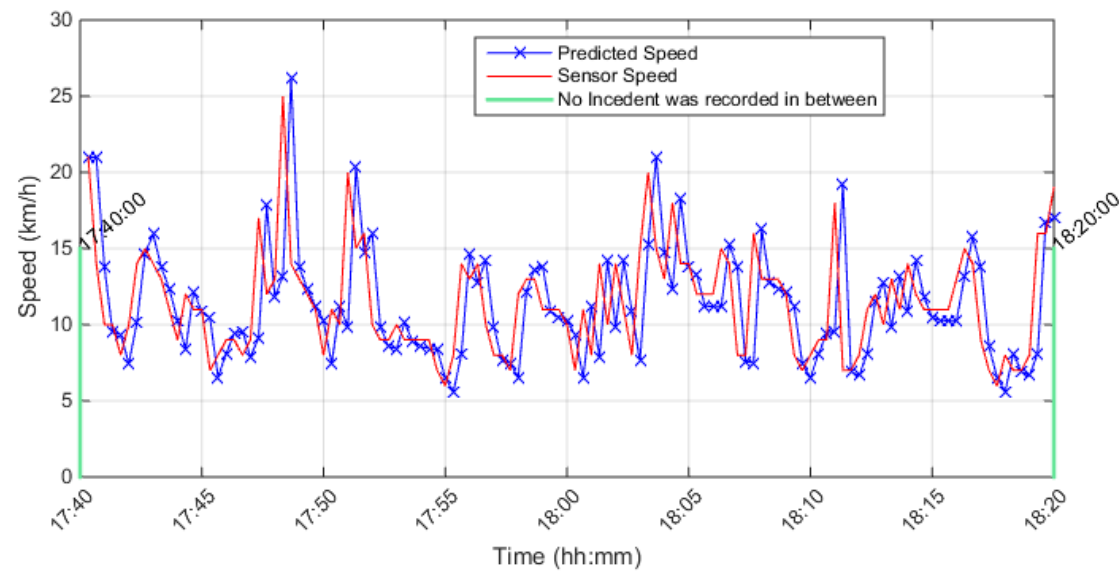

Upstream Detector \# is 401DW0060DWC, Detector Rank = 1, Distance from incident free location in $(\mathrm{km})=0.26756$ Incident free Season $=$ Summer, Stream $=$ C, Direction $=W$, Date \& Time $=$ 2011-09-15 15:47:55 Selected incident free Date/Time $=2011-09-15$ 18:00:00

Conditional Probability level $=0.6$, Cond. Prob. $r 3 \mathrm{~s} 2=0.2$, Cond.Prob. $r 3 \mathrm{~s} 1=0.1$, Percent drop in speed $\%=-56$ Max. Posterior Probability level $=0.97$, Percent range between speed states $=7 \%$ Drop in speed was detected and No indication of an incident within this period

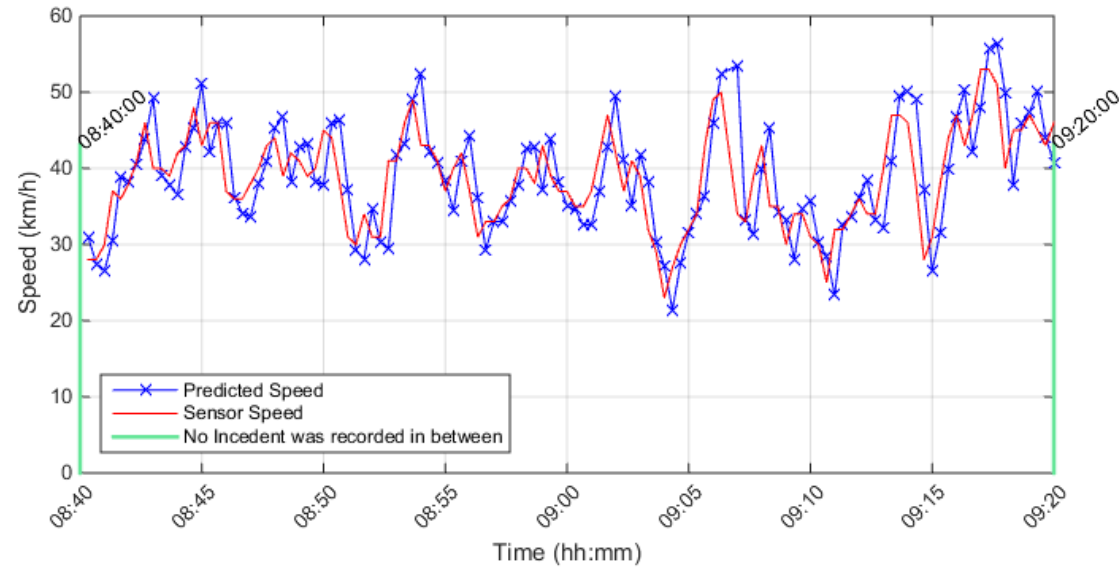

Upstream Detector \# is 401DE0350DWC, Detector Rank = 1, Distance from incident free location in $(\mathrm{km})=0.24334$ Incident free Season $=$ Winter, Stream $=C$, Direction $=W$, Date \& Time $=2011-12-02$ 14:48:55 Selected incident free Date/Time $=2011-12-02$ 09:00:00

Conditional Probability level $=0.6$, Cond.Prob. $\mathrm{r} 3 \mathrm{~s} 2=0.2$, Cond.Prob. $\mathrm{r} 3 \mathrm{~s} 1=0.1$, Percent drop in speed $\%=-56$

Max. Posterior Probability level $=0.97$, Percent range between speed states $=7 \%$

(No Incident was Detected) 


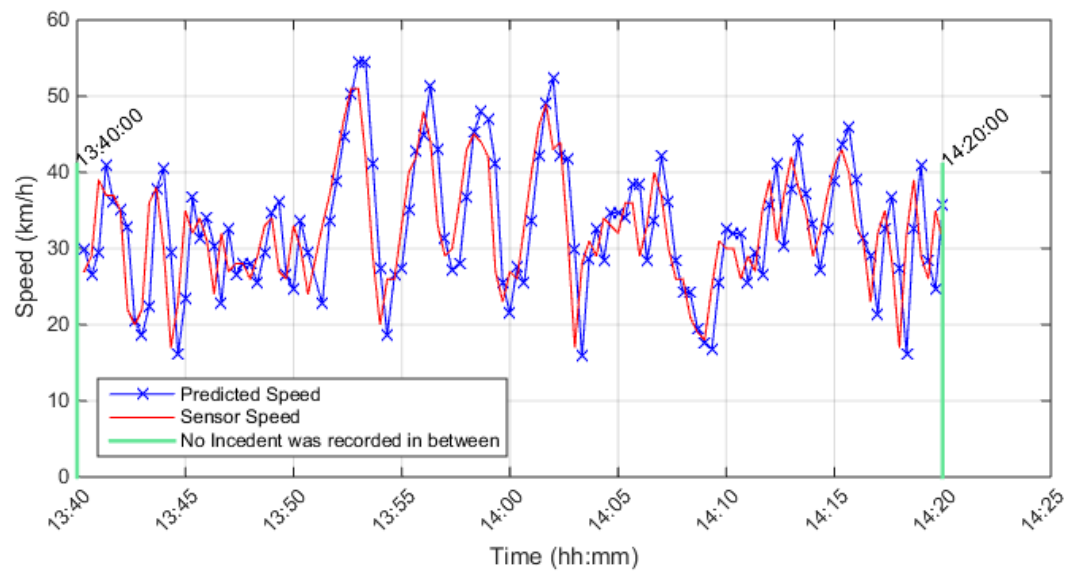

Upstream Detector \# is 401DE0100DWC, Detector Rank = 1, Distance from incident free location in $(\mathrm{km})=0.11847$ Incident free Season $=$ Winter, Stream $=$ C, Direction $=W$, Date \& Time $=2011-12-26$ 11:43:21 Selected incident free Date/Time $=2011-12-26$ 14:00:00

Conditional Probability level $=0.6$, Cond. Prob. $r 3 \mathrm{~s} 2=0.2$, Cond.Prob. $r 3 \mathrm{~s} 1=0.1$, Percent drop in speed $\%=-56$ Max. Posterior Probability level $=0.97$, Percent range between speed states $=7 \%$ Drop in speed was detected and No indication of an incident within this period

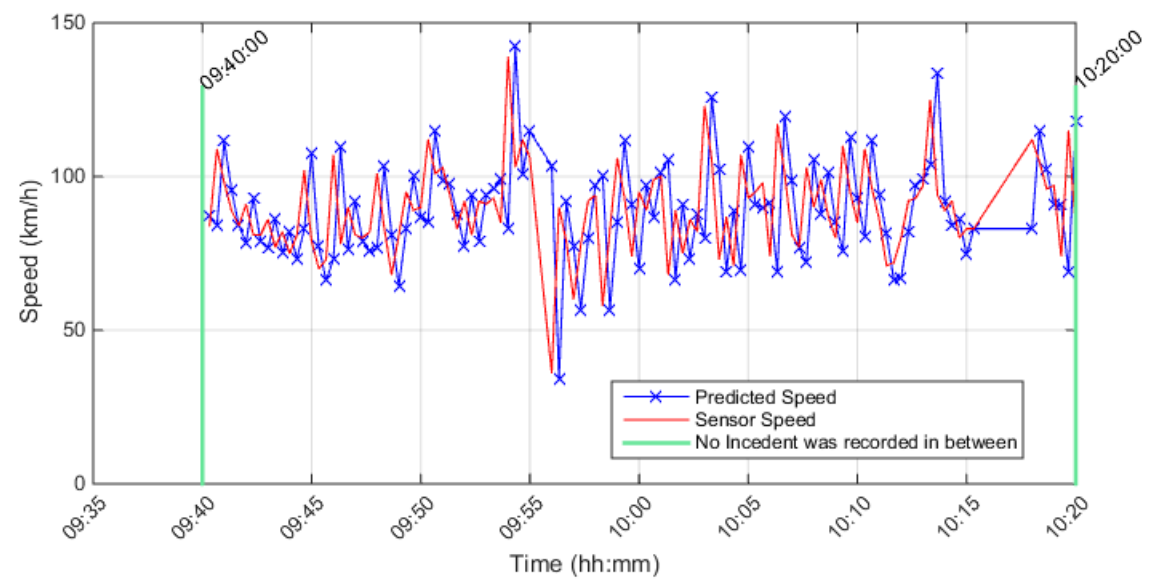

Upstream Detector \# is 401DE0050DWE, Detector Rank = 1, Distance from incident free location in $(\mathrm{km})=0.36941$ Incident free Season $=$ Winter, Stream $=E$, Direction $=W$, Date \& Time $=2011-02-02$ 08:26:34 Selected incident free Date/Time $=2011-02-02$ 10:00:00

Conditional Probability level $=0.6$, Cond.Prob. $r 3 s 2=0.2$, Cond. Prob. $r 3 s 1=0.1$, Percent drop in speed $\%=-56$ Max. Posterior Probability level $=0.97$, Percent range between speed states $=7 \%$ (No Incident was Detected) 


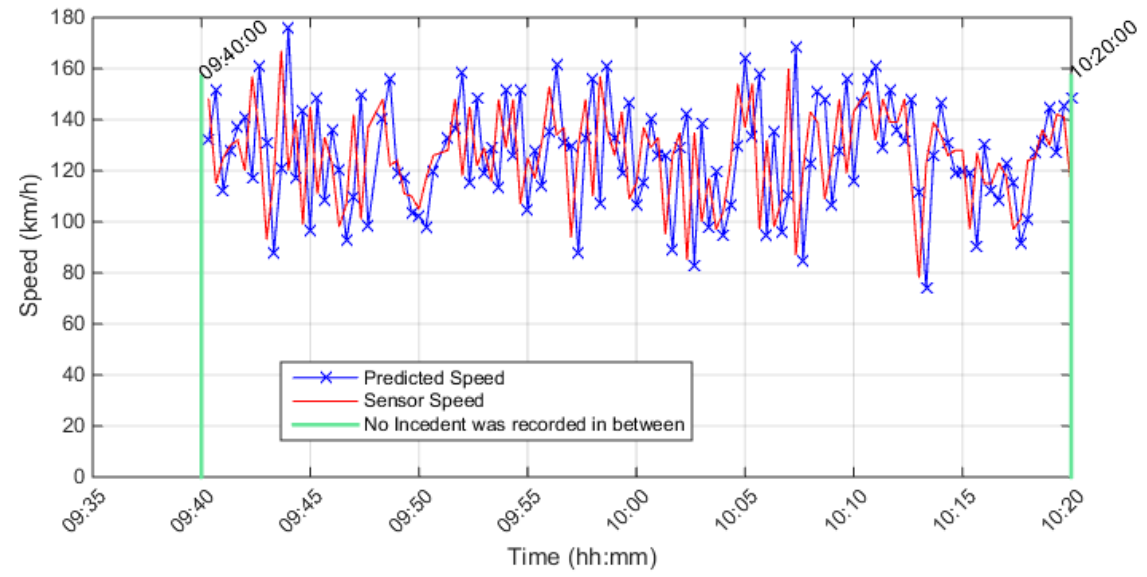

Upstream Detector \# is 401DE0270DWE, Detector Rank = 1, Distance from incident free location in $(\mathrm{km})=0.59072$ Incident free Season $=$ Spring, Stream $=E$, Direction $=W$, Date \& Time $=2011-04-10$ 04:26:53

Selected incident free Date/Time $=2011-04-10$ 10:00:00

Conditional Probability level $=0.6$, Cond. Prob. $r 3 \mathrm{~s} 2=0.2$, Cond.Prob. $r 3 \mathrm{~s} 1=0.1$, Percent drop in speed $\%=-56$ Max. Posterior Probability level $=0.97$, Percent range between speed states $=7 \%$

(No Incident was Detected)

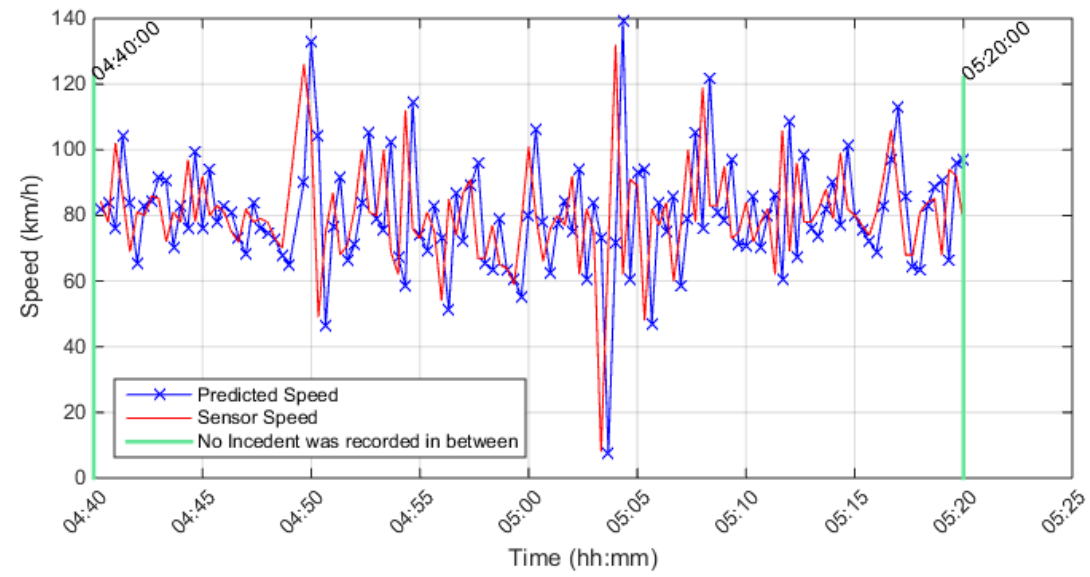

Upstream Detector \# is 401DE0090DWE, Detector Rank = 1, Distance from incident free location in $(\mathrm{km})=0.6034$ Incident free Season $=$ Summer, Stream $=\mathrm{E}$, Direction $=\mathrm{W}$, Date $\&$ Time $=$ 2011-08-02 09:02:12 Selected incident free Date/Time $=$ 2011-08-02 05:00:00

Conditional Probability level $=0.6$, Cond. Prob. $r 3 s 2=0.2$, Cond.Prob. $r 3 s 1=0.1$, Percent drop in speed $\%=-56$ Max. Posterior Probability level $=0.97$, Percent range between speed states $=7 \%$ (No Incident was Detected) 


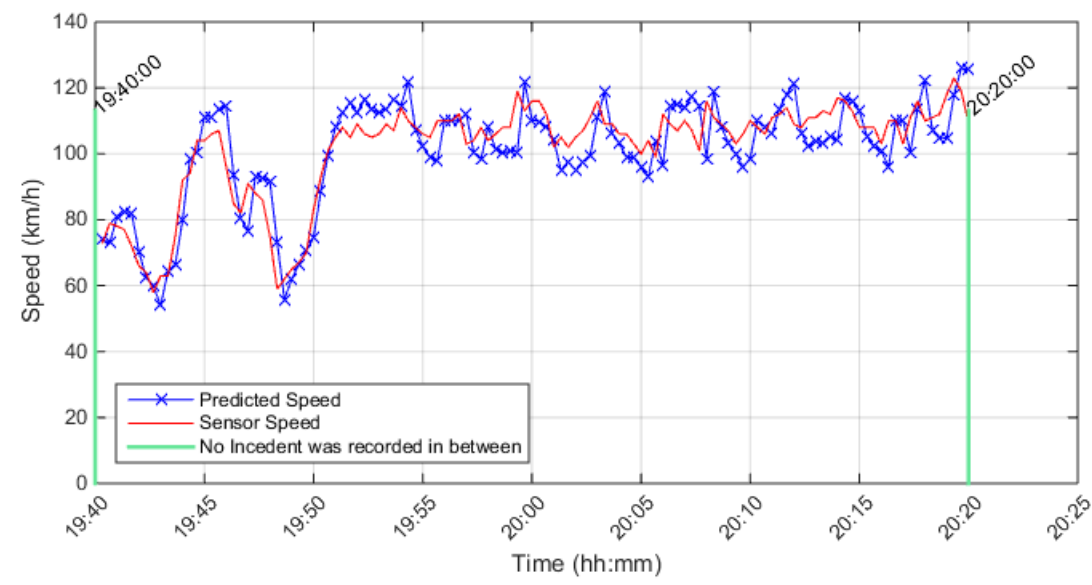

Upstream Detector \# is 401DE0060DWE, Detector Rank = 1, Distance from incident free location in $(\mathrm{km})=0.17254$ Incident free Season $=$ Summer, Stream $=E$, Direction $=W$, Date $\&$ Time $=2011-08-0316: 44: 19$

Selected incident free Date/Time $=$ 2011-08-03 20:00:00

Conditional Probability level $=0.6$, Cond.Prob. $r 3 \mathrm{~s} 2=0.2$, Cond.Prob. $r 3 \mathrm{~s} 1=0.1$, Percent drop in speed $\%=-56$

Max. Posterior Probability level $=0.97$, Percent range between speed states $=7 \%$

(No Incident was Detected)

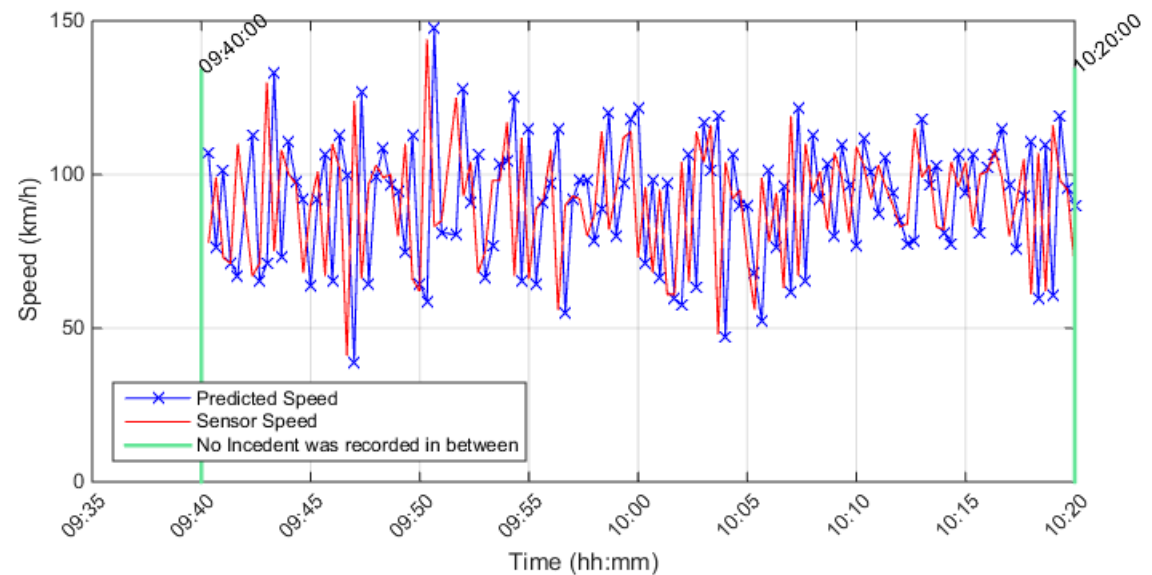

Upstream Detector \# is 401DE0340DWE, Detector Rank = 3, Distance from incident free location in $(\mathrm{km})=1.6024$ Incident free Season $=$ Summer, Stream $=E$, Direction $=W$, Date \& Time $=$ 2011-08-06 18:50:45 Selected incident free Date/Time $=2011-08-06$ 10:00:00

Conditional Probability level $=0.6$, Cond.Prob. $r 3 \mathrm{~s} 2=0.2$, Cond. Prob. $r 3 \mathrm{~s} 1=0.1$, Percent drop in speed $\%=-56$ Max. Posterior Probability level $=0.97$, Percent range between speed states $=7 \%$ (No Incident was Detected) 


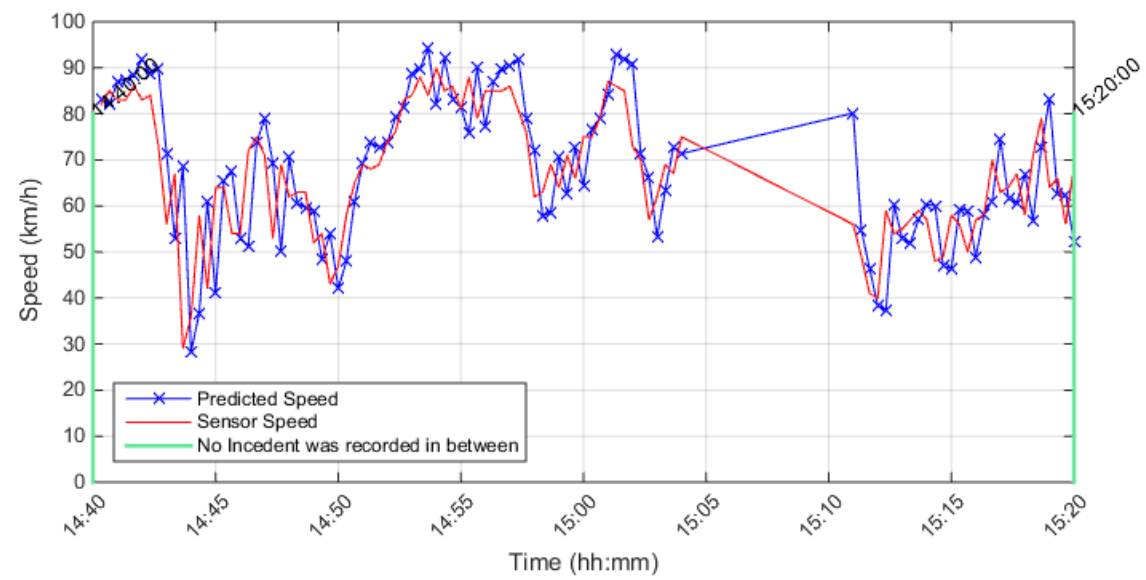

Upstream Detector \# is 401DE0020DWE, Detector Rank = 1, Distance from incident free location in $(\mathrm{km})=0.39496$ Incident free Season $=$ Summer, Stream $=\mathrm{E}$, Direction $=\mathrm{W}$, Date $\&$ Time $=2011-08-08$ 08:34:11

Selected incident free Date/Time $=2011-08-08$ 15:00:00

Conditional Probability level $=0.6$, Cond.Prob. $r 3 \mathrm{~s} 2=0.2$, Cond. Prob. $r 3 \mathrm{~s} 1=0.1$, Percent drop in speed $\%=-56$

Max. Posterior Probability level $=0.97$, Percent range between speed states $=7 \%$

Drop in speed was detected and No indication of an incident within this period

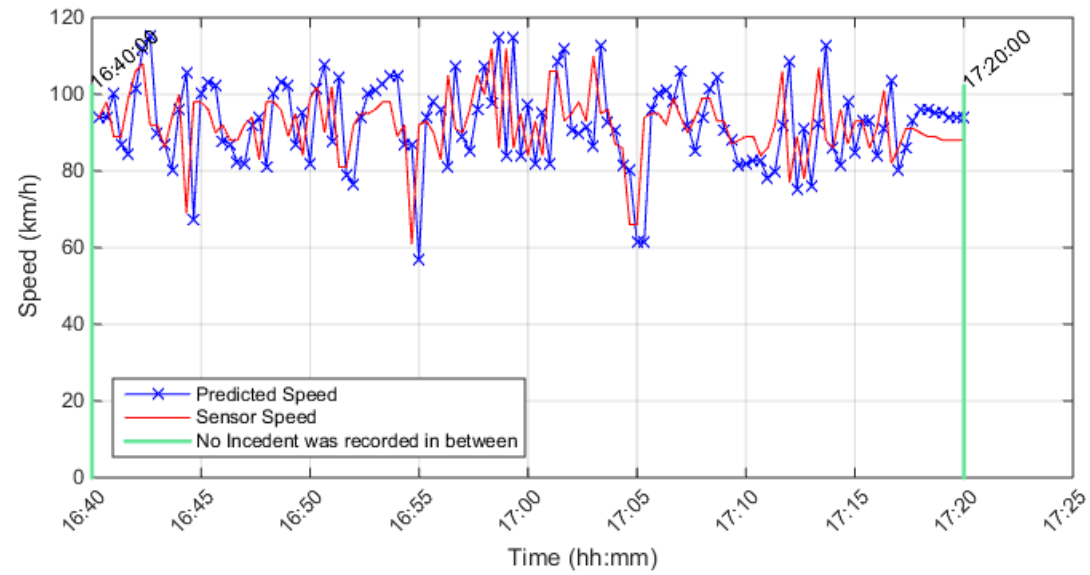

Upstream Detector \# is 401DW0080DWE, Detector Rank = 2, Distance from incident free location in $(\mathrm{km})=6.1426$ ncident free Season $=$ Summer, Stream $=E$, Direction $=W$, Date \& Time $=2011-10-2413: 40: 15$ Selected incident free Date/Time $=2011-10-24$ 17:00:00

Conditional Probability level $=0.6$, Cond. Prob. $r 3 \mathrm{~s} 2=0.2$, Cond. Prob. $r 3 \mathrm{~s} 1=0.1$, Percent drop in speed $\%=-56$ Max. Posterior Probability level $=0.97$, Percent range between speed states $=7 \%$ (No Incident was Detected) 


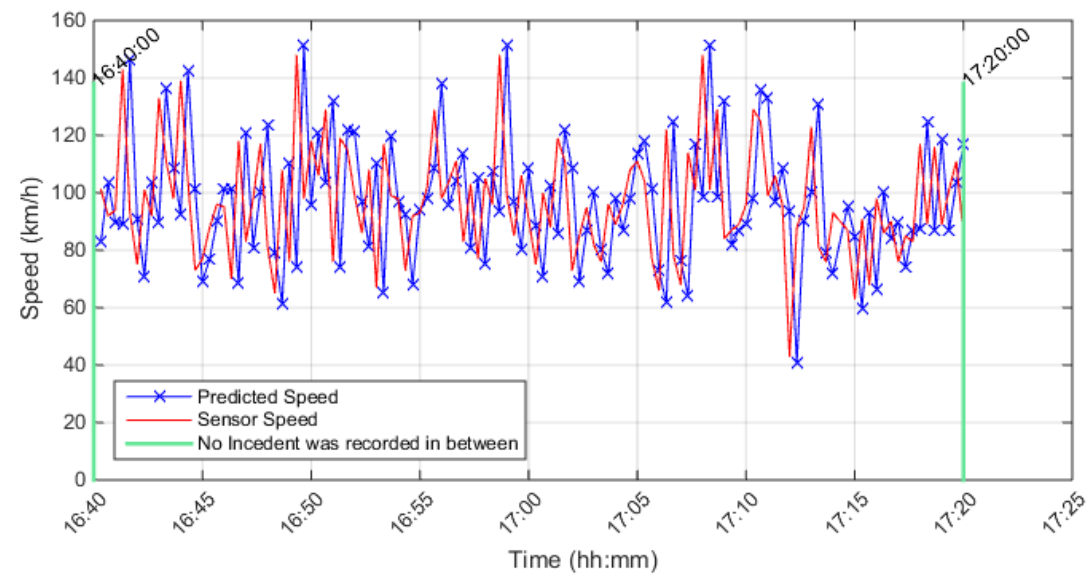

Upstream Detector \# is 401DE0270DWE, Detector Rank = 1, Distance from incident free location in $(\mathrm{km})=0.59072$ Incident free Season $=$ Summer, Stream $=E$, Direction $=W$, Date \& Time $=$ 2011-11-29 18:07:30

Selected incident free Date/Time $=2011-11-29$ 17:00:00

Conditional Probability level $=0.6$, Cond. Prob. $r 3 \mathrm{~s} 2=0.2$, Cond.Prob. $r 3 \mathrm{~s} 1=0.1$, Percent drop in speed $\%=-56$ Max. Posterior Probability level $=0.97$, Percent range between speed states $=7 \%$

(No Incident was Detected)

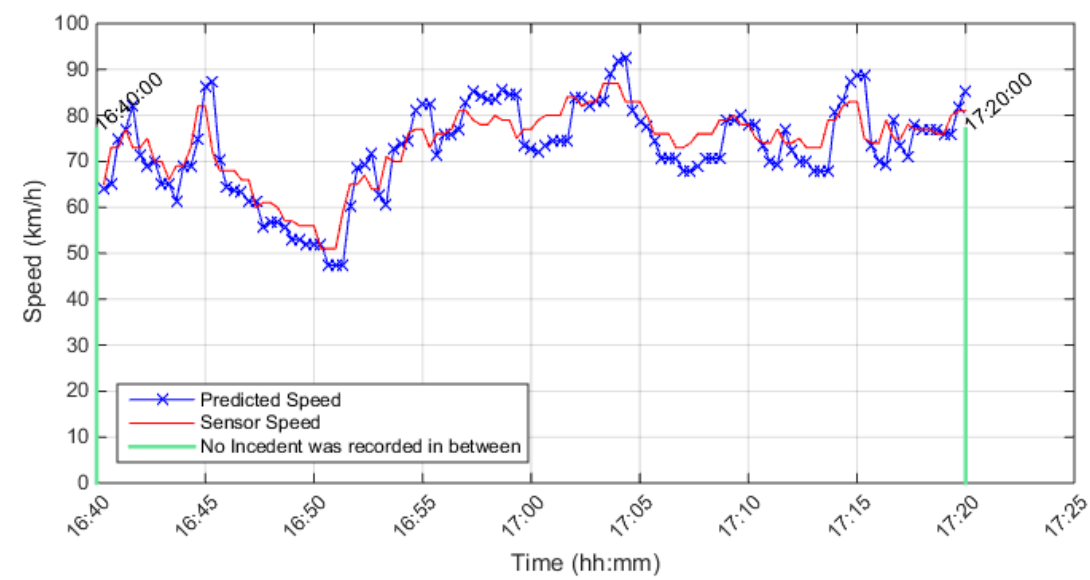

Upstream Detector \# is 401DE0140DWE, Detector Rank = 1, Distance from incident free location in $(\mathrm{km})=0.93575$ Incident free Season $=$ Winter, Stream $=E$, Direction $=W$, Date $\&$ Time $=2011-12-27$ 20:47:47 Selected incident free Date/Time $=2011-12-27$ 17:00:00

Conditional Probability level $=0.6$, Cond.Prob. $r 3 \mathrm{~s} 2=0.2$, Cond.Prob. $r 3 \mathrm{~s} 1=0.1$, Percent drop in speed $\%=-56$

Max. Posterior Probability level $=0.97$. Percent range between speed states $=7 \%$

(No Incident was Detected) 


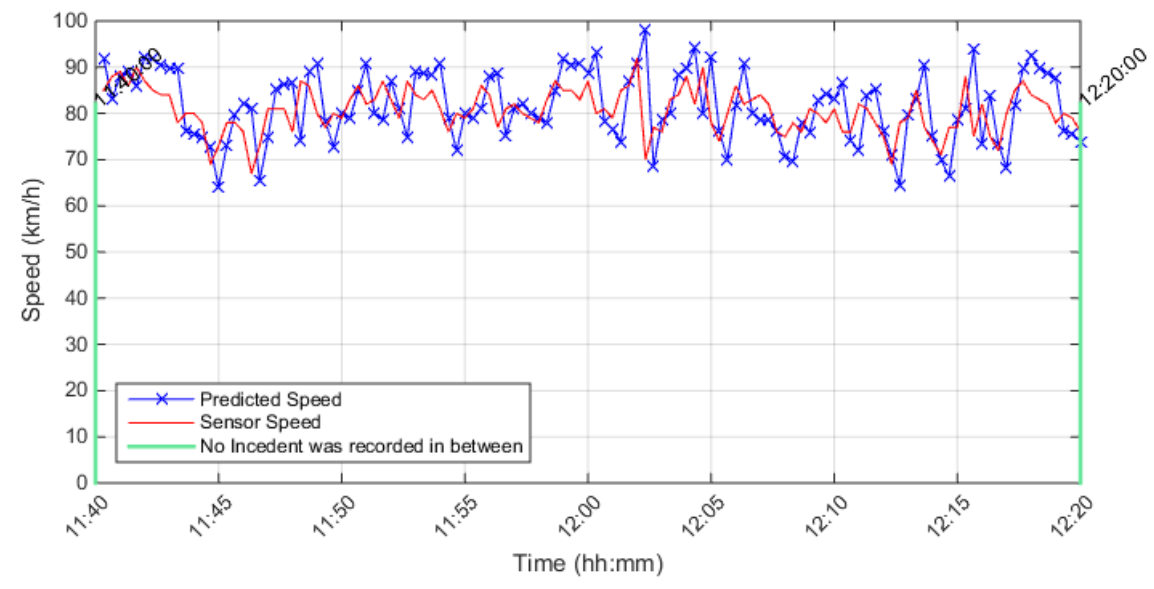

Upstream Detector \# is 401DW0100DEC, Detector Rank $=1$, Distance from incident free location in $(\mathrm{km})=0.021101$ Incident free Season $=$ Summer, Stream $=C$, Direction $=E$, Date \& Time $=2011-08-16$ 08:38:09 Selected incident free Date/Time $=2011-08-16$ 12:00:00

Conditional Probability level $=0.6$, Cond.Prob. $r 3 \mathrm{~s} 2=0.2$, Cond.Prob. $r 3 \mathrm{~s} 1=0.1$, Percent drop in speed $\%=-56$

Max. Posterior Probability level $=0.97$, Percent range between speed states $=7 \%$

(No Incident was Detected)

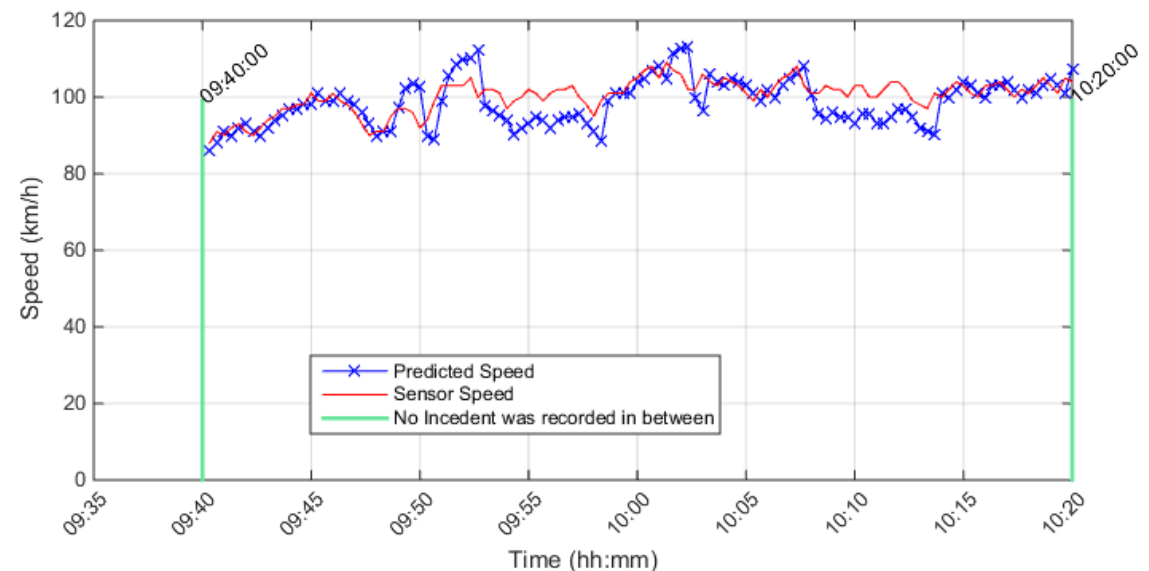

Upstream Detector \# is 401DE0100DEC, Detector Rank = 1, Distance from incident free location in $(\mathrm{km})=0.26025$ Incident free Season $=$ Summer, Stream $=$ C, Direction $=\mathrm{E}$, Date \& Time $=2011-09-2017: 13: 49$

Selected incident free Date/Time $=2011-09-20$ 10:00:00

Conditional Probability level $=0.6$, Cond. Prob. $r 3 s 2=0.2$, Cond.Prob. $r 3 s 1=0.1$, Percent drop in speed $\%=-56$

Max. Posterior Probability level $=0.97$, Percent range between speed states $=7 \%$

(No Incident was Detected) 


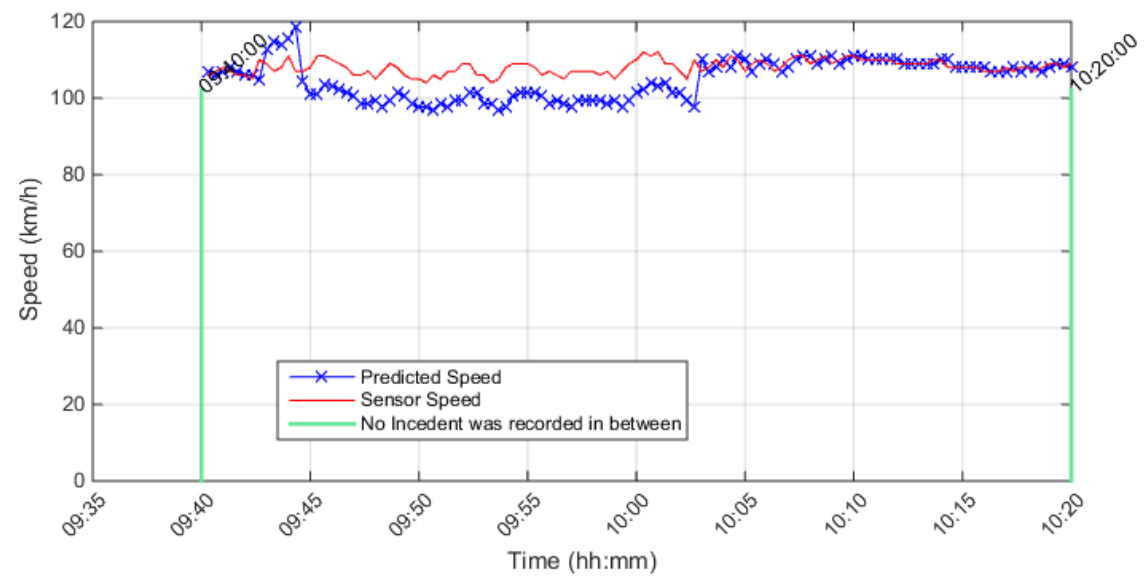

Upstream Detector \# is 401DE0210DEC, Detector Rank $=1$, Distance from incident free location in $(\mathrm{km})=0.20489$ Incident free Season $=$ Summer, Stream $=C$, Direction $=E$, Date \& Time $=2011-09-25$ 18:28:25 Selected incident free Date/Time $=2011-09-25$ 10:00:00

Conditional Probability level $=0.6$, Cond. Prob. $r 3 \mathrm{~s} 2=0.2$, Cond. Prob. $r 3 \mathrm{~s} 1=0.1$, Percent drop in speed $\%=-56$ Max. Posterior Probability level $=0.97$, Percent range between speed states $=7 \%$

(No Incident was Detected)

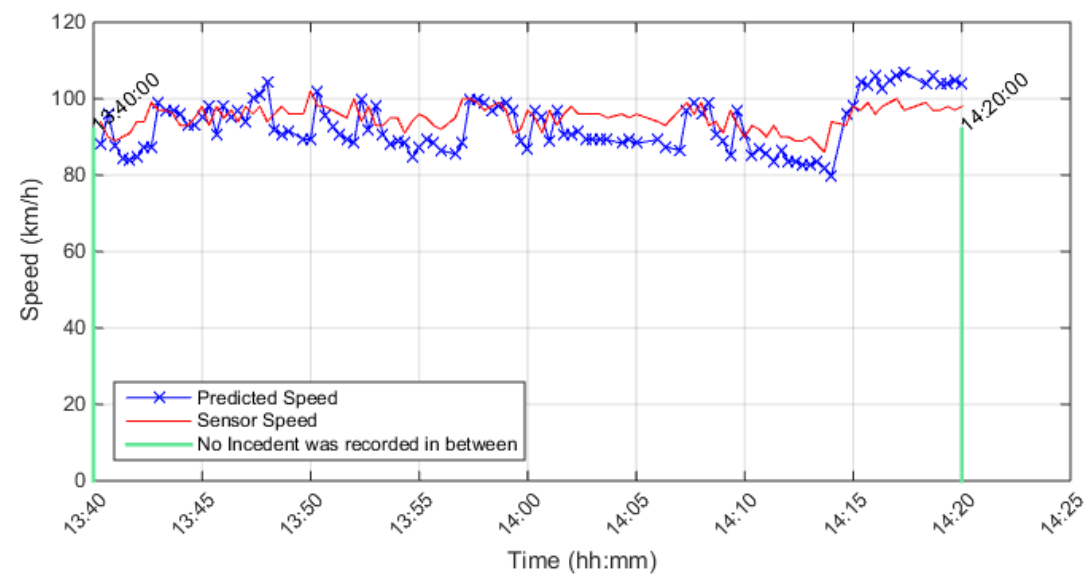

Upstream Detector \# is 401DE0350DEC, Detector Rank = 1, Distance from incident free location in $(\mathrm{km})=0.14426$ Incident free Season $=$ Summer, Stream $=\mathrm{C}$, Direction $=\mathrm{E}$, Date $\&$ Time $=2011-09-30$ 02:08:27 Selected incident free Date/Time $=2011-09-3014: 00: 00$

Conditional Probability level $=0.6$, Cond.Prob. $r 3 \mathrm{~s} 2=0.2$, Cond.Prob. $\mathrm{r} 3 \mathrm{~s} 1=0.1$, Percent drop in speed $\%=-56$

Max. Posterior Probability level $=0.97$, Percent range between speed states $=7 \%$

(No Incident was Detected) 


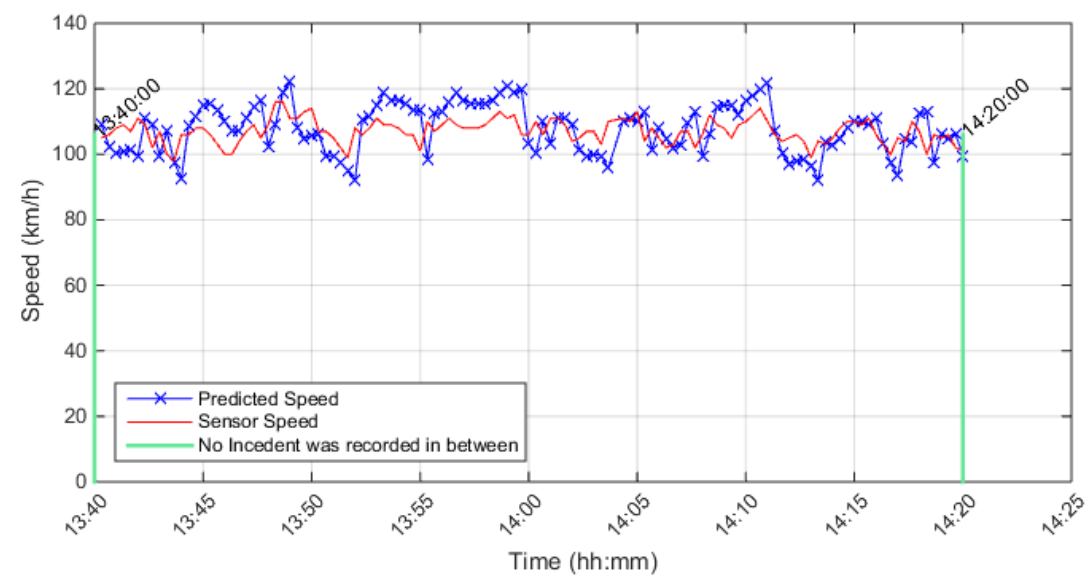

Upstream Detector \# is 401DE0270DEC, Detector Rank = 1, Distance from incident free location in $(\mathrm{km})=0.85553$ Incident free Season $=$ Summer, Stream $=\mathrm{C}$, Direction $=\mathrm{E}$, Date $\&$ Time $=2011-09-30$ 07:34:34 Selected incident free Date/Time $=2011-09-30$ 14:00:00

Conditional Probability level $=0.6$, Cond.Prob. $r 3 s 2=0.2$, Cond.Prob. $r 3 s 1=0.1$, Percent drop in speed $\%=-56$ Max. Posterior Probability level $=0.97$, Percent range between speed states $=7 \%$ (No Incident was Detected)

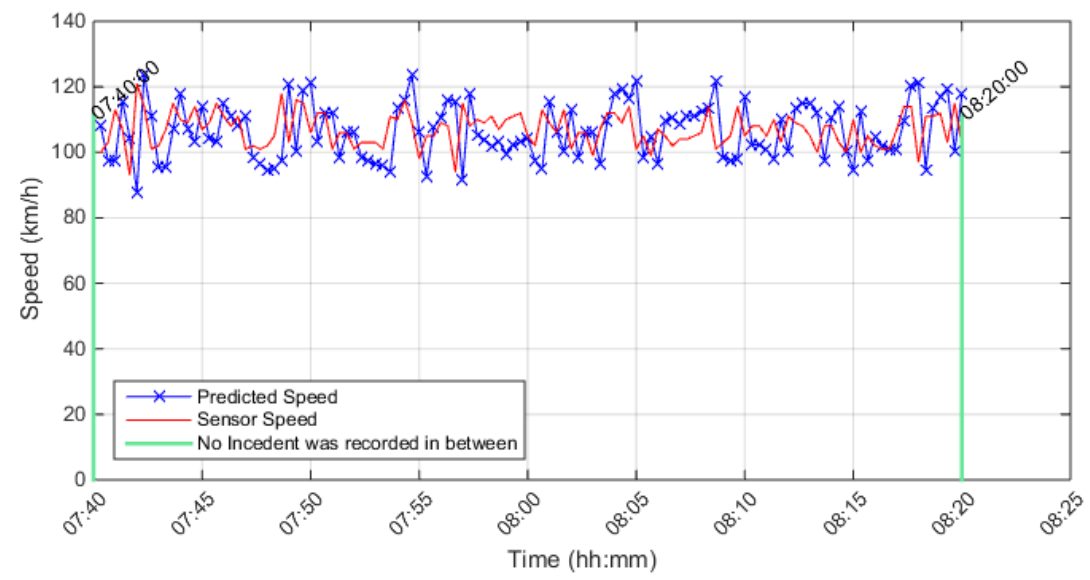

Upstream Detector \# is 401DE0170DEC, Detector Rank = 3, Distance from incident free location in $(\mathrm{km})=1.9227$ Incident free Season $=$ Summer, Stream $=C$, Direction $=E$, Date $\&$ Time $=2011-10-23$ 13:19:29 Selected incident free Date/Time $=2011-10-23$ 08:00:00

Conditional Probability level $=0.6$, Cond. Prob. $r 3 \mathrm{~s} 2=0.2$, Cond.Prob. $r 3 \mathrm{~s} 1=0.1$, Percent drop in speed $\%=-56$

Max. Posterior Probability level $=0.97$. Percent range between speed states $=7 \%$

(No Incident was Detected) 


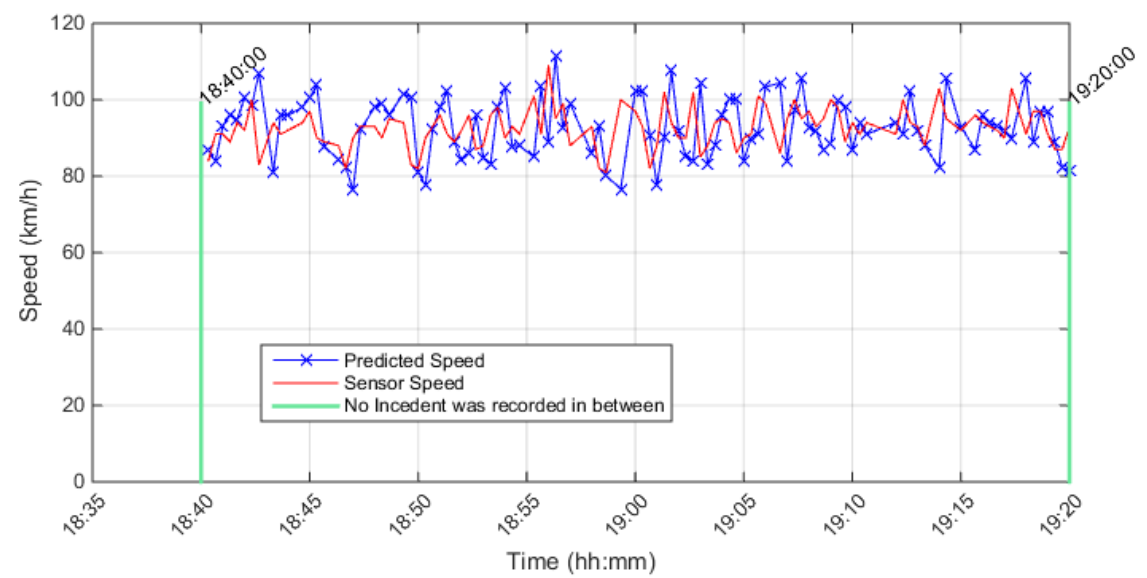

Upstream Detector \# is 401DW0110DEE, Detector Rank = 1, Distance from incident free location in $(\mathrm{km})=0.28861$ Incident free Season $=$ Winter, Stream $=E$, Direction $=E$, Date \& Time $=$ 2011-01-11 02:39:00 Selected incident free Date/Time $=2011-01-11$ 19:00:00

Conditional Probability level $=0.6$, Cond.Prob. $r 3 s 2=0.2$, Cond.Prob. $r 3 s 1=0.1$, Percent drop in speed $\%=-56$ Max. Posterior Probability level $=0.97$, Percent range between speed states $=7 \%$ (No Incident was Detected)

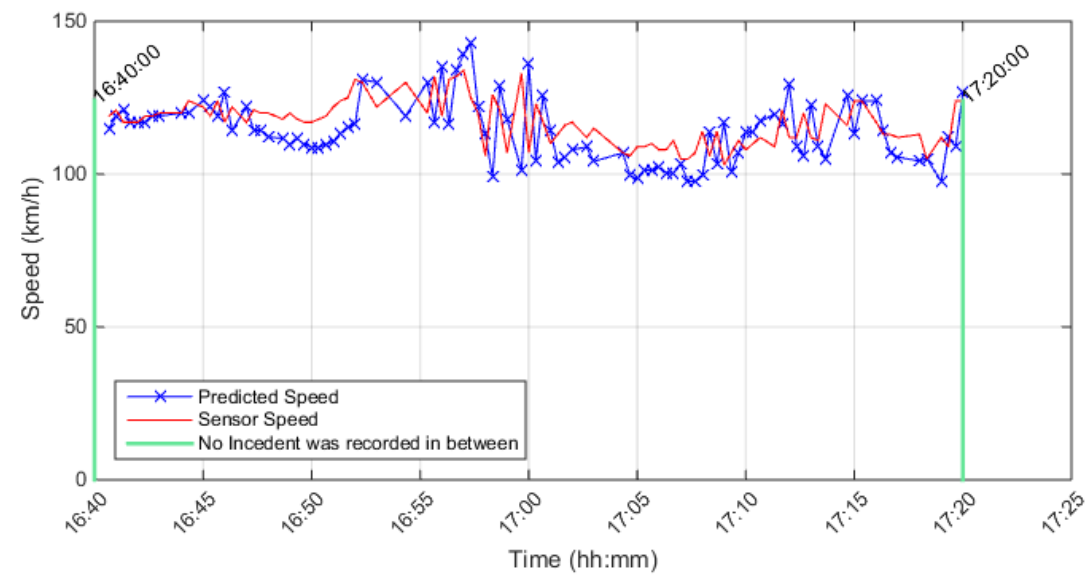

Upstream Detector \# is 401DE0120DEE, Detector Rank = 1, Distance from incident free location in $(\mathrm{km})=0.83799$ Incident free Season $=$ Winter, Stream $=E$, Direction $=E$, Date \& Time $=2011-01-15$ 12:51:50 Selected incident free Date/Time $=$ 2011-01-15 17:00:00

Conditional Probability level $=0.6$, Cond. Prob. $r 3 \mathrm{~s} 2=0.2$, Cond.Prob. $r 3 \mathrm{~s} 1=0.1$, Percent drop in speed $\%=-56$

Max. Posterior Probability level $=0.97$, Percent range between speed states $=7 \%$

(No Incident was Detected) 


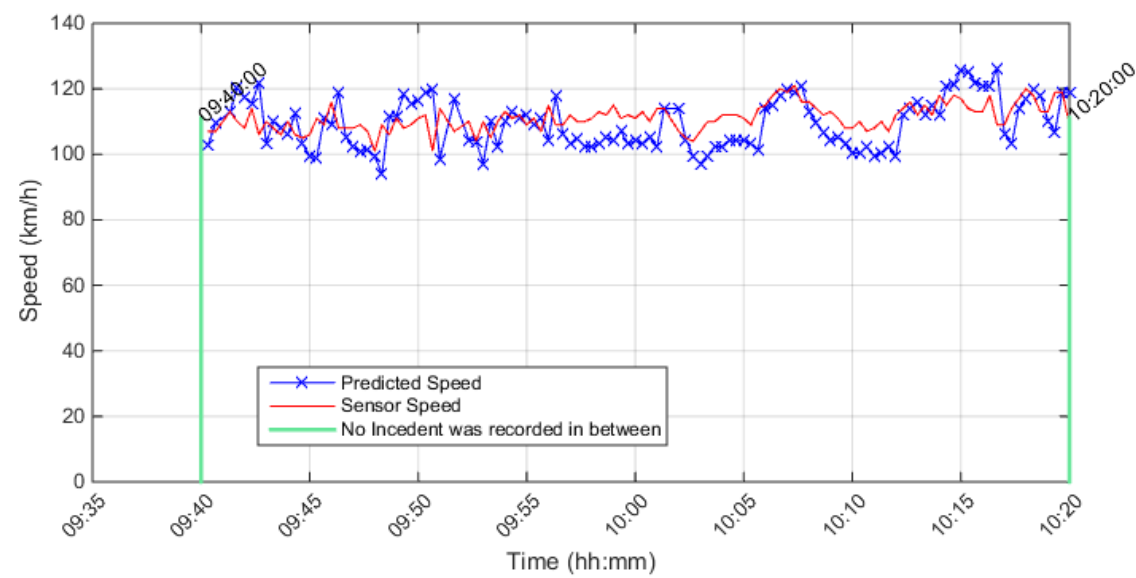

Upstream Detector \# is 401DE0320DEE, Detector Rank = 2, Distance from incident free location in $(\mathrm{km})=0.87834$ Incident free Season $=$ Winter, Stream $=E$, Direction $=E$, Date $\&$ Time $=2011-01-19$ 17:22:39

Selected incident free Date/Time $=2011-01-19$ 10:00:00

Conditional Probability level $=0.6$, Cond.Prob. $r 3 s 2=0.2$, Cond.Prob. $r 3 s 1=0.1$, Percent drop in speed $\%=-56$ Max. Posterior Probability level $=0.97$, Percent range between speed states $=7 \%$ (No Incident was Detected)

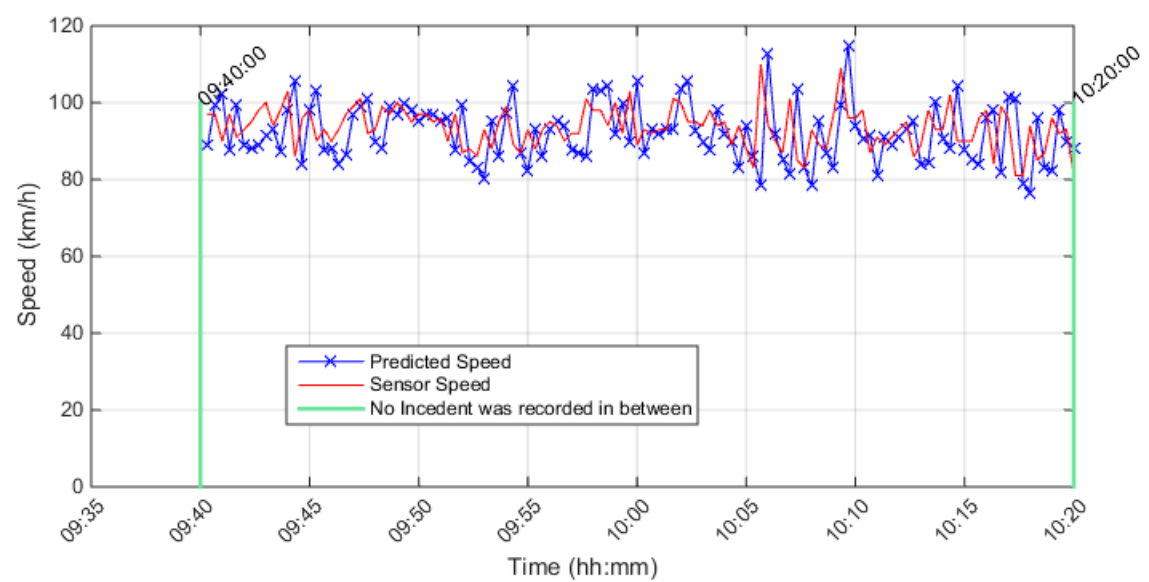

Upstream Detector \# is 401DW0260DEE, Detector Rank = 1, Distance from incident free location in $(\mathrm{km})=0.36837$ Incident free Season $=$ Spring, Stream $=E$, Direction $=E$, Date \& Time $=$ 2011-03-16 16:59:10

Selected incident free Date/Time $=2011-03-16$ 10:00:00

Conditional Probability level $=0.6$, Cond.Prob. $r 3 s 2=0.2$, Cond. Prob. $r 3 s 1=0.1$, Percent drop in speed $\%=-56$

Max. Posterior Probability level $=0.97$, Percent range between speed states $=7 \%$

(No Incident was Detected) 


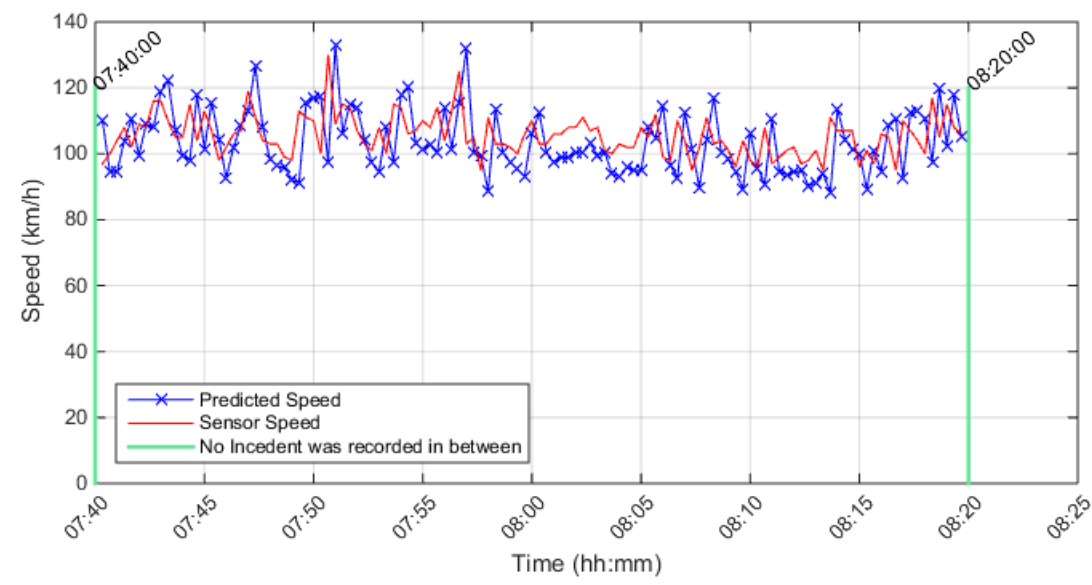

Upstream Detector \# is 401DW0300DEE, Detector Rank = 1, Distance from incident free location in $(\mathrm{km})=0.63014$ Incident free Season $=$ Spring, Stream $=E$, Direction $=E$, Date $\&$ Time $=2011-05-03$ 16:22:19

Selected incident free Date/Time $=2011-05-03$ 08:00:00

Conditional Probability level $=0.6$, Cond.Prob. $r 3 \mathrm{~s} 2=0.2$, Cond.Prob. $r 3 \mathrm{~s} 1=0.1$, Percent drop in speed $\%=-56$

Max. Posterior Probability level $=0.97$, Percent range between speed states $=7 \%$

(No Incident was Detected)

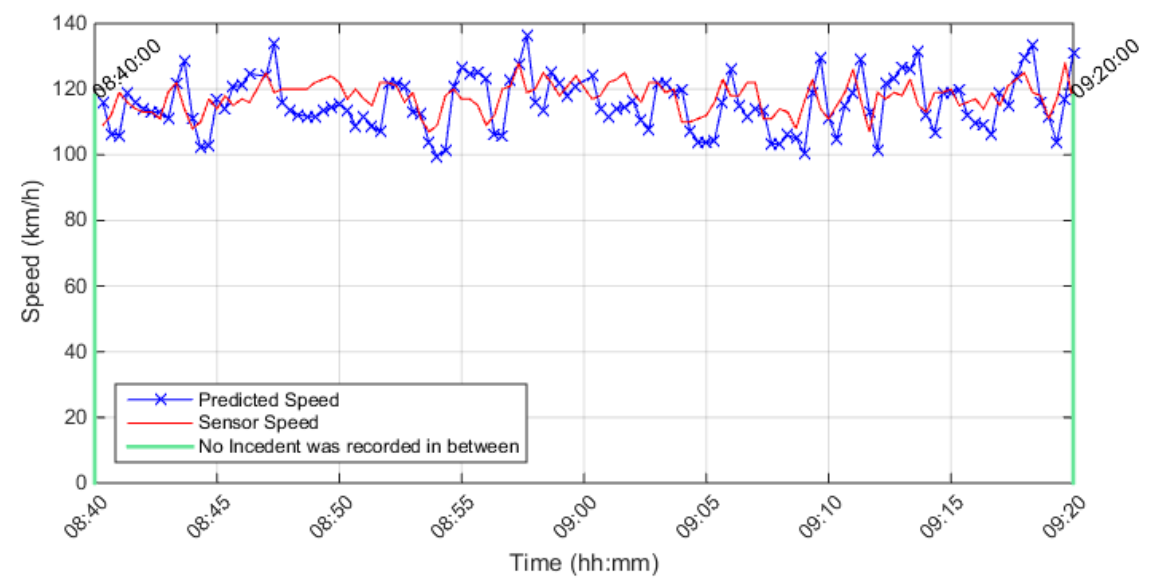

Upstream Detector \# is 401DW0210DEE, Detector Rank = 1, Distance from incident free location in $(\mathrm{km})=0.02872$ Incident free Season $=$ Spring, Stream $=E$, Direction $=E$, Date $\&$ Time $=2011-05-2117: 51: 08$

Selected incident free Date/Time $=2011-05-21$ 09:00:00

Conditional Probability level $=0.6$, Cond.Prob. $r 3 s 2=0.2$, Cond.Prob. $r 3 s 1=0.1$, Percent drop in speed $\%=-56$

Max. Posterior Probability level $=0.97$, Percent range between speed states $=7 \%$

(No Incident was Detected) 


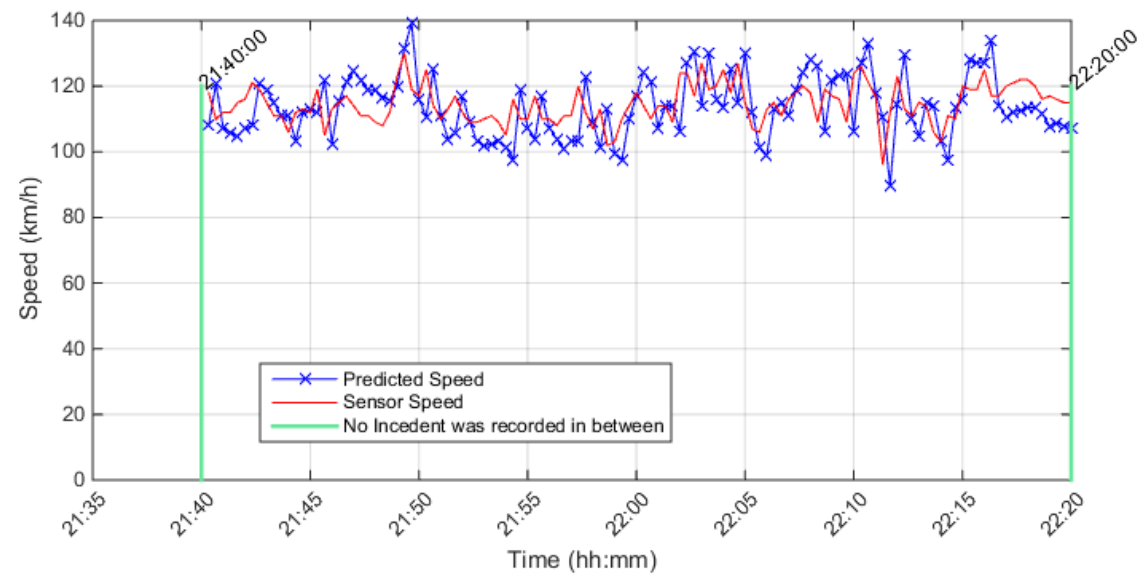

Upstream Detector \# is 401DE0440DEE, Detector Rank = 1, Distance from incident free location in $(\mathrm{km})=4.444$ Incident free Season $=$ Summer, Stream $=E$, Direction $=E$, Date \& Time $=2011-06-22$ 07:21:44 Selected incident free Date/Time $=$ 2011-06-22 22:00:00

Conditional Probability level $=0.6$, Cond.Prob. $r 3 s 2=0.2$, Cond.Prob. $r 3 s 1=0.1$, Percent drop in speed $\%=-56$ Max. Posterior Probability level $=0.97$, Percent range between speed states $=7 \%$

(No Incident was Detected)

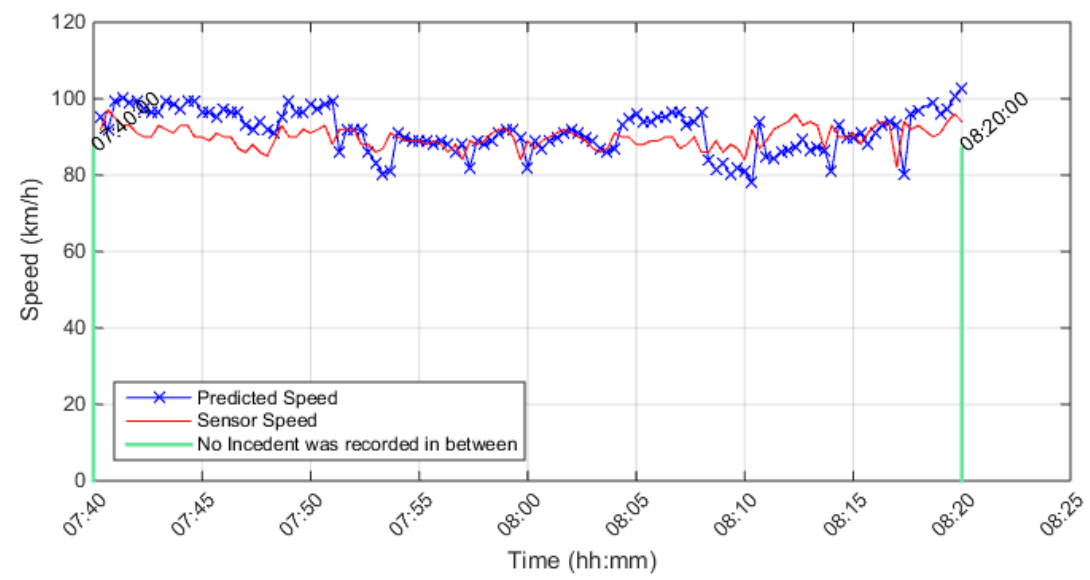

Upstream Detector \# is 401DE0340DEE, Detector Rank = 3, Distance from incident free location in $(\mathrm{km})=1.43$ Incident free Season $=$ Summer, Stream $=E$, Direction $=E$, Date \& Time $=$ 2011-07-29 23:02:51 Selected incident free Date/Time $=2011-07-29$ 08:00:00

Conditional Probability level $=0.6$, Cond.Prob. $r 3 \mathrm{~s} 2=0.2$, Cond.Prob. $\mathrm{r} 3 \mathrm{~s} 1=0.1$, Percent drop in speed $\%=-56$

Max. Posterior Probability level $=0.97$, Percent range between speed states $=7 \%$

(No Incident was Detected) 


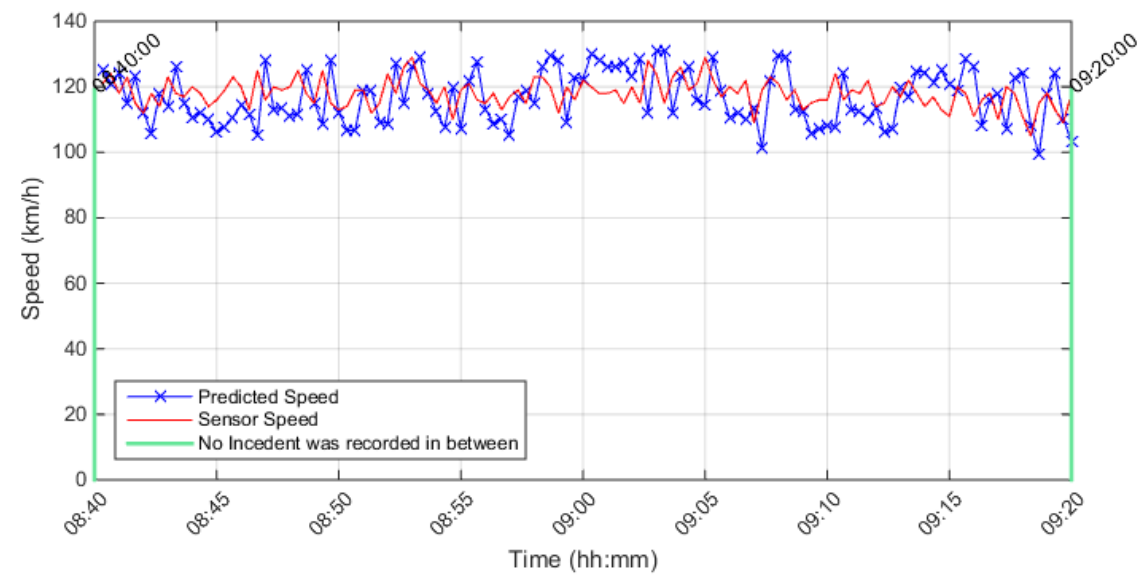

Upstream Detector \# is 401DW0100DEE, Detector Rank = 1, Distance from incident free location in $(\mathrm{km})=0.41981$ Incident free Season $=$ Summer, Stream $=E$, Direction $=E$, Date \& Time $=2011-09-18$ 13:26:01 Selected incident free Date/Time $=2011-09-18$ 09:00:00

Conditional Probability level $=0.6$, Cond.Prob. $r 3 s 2=0.2$, Cond.Prob. $r 3 s 1=0.1$, Percent drop in speed $\%=-56$ Max. Posterior Probability level $=0.97$, Percent range between speed states $=7 \%$ (No Incident was Detected)

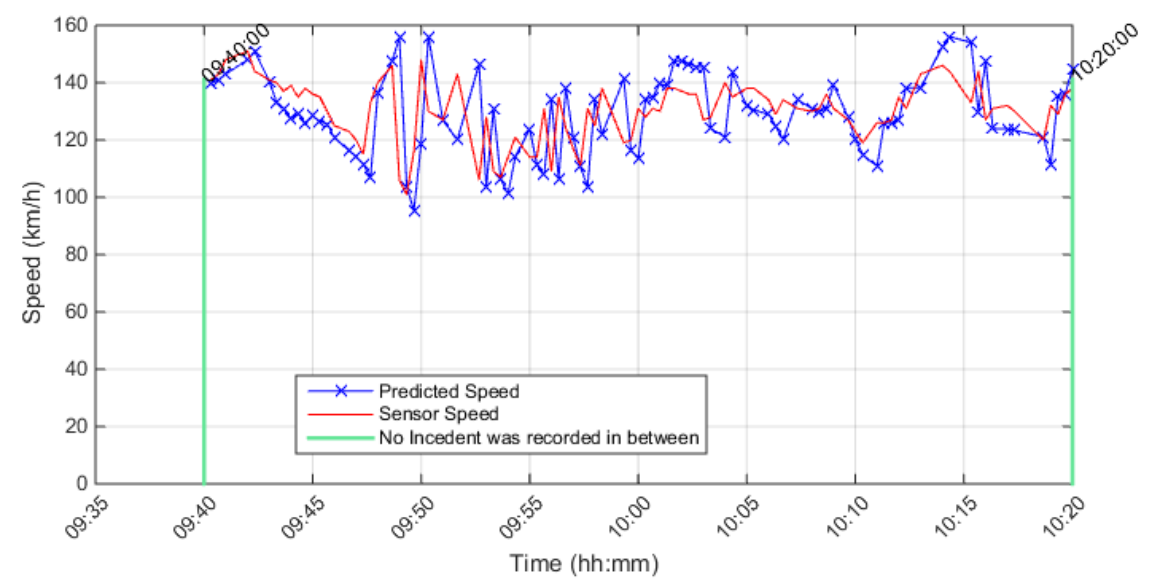

Upstream Detector \# is 401DE0060DEE, Detector Rank = 1, Distance from incident free location in $(\mathrm{km})=0.19919$ Incident free Season $=$ Summer, Stream $=E$, Direction $=E$, Date $\&$ Time $=$ 2011-09-24 16:05:15 Selected incident free Date/Time $=2011-09-24$ 10:00:00

Conditional Probability level $=0.6$, Cond. Prob. $r 3 \mathrm{~s} 2=0.2$, Cond.Prob. $r 3 \mathrm{~s} 1=0.1$, Percent drop in speed $\%=-56$

Max. Posterior Probability level $=0.97$. Percent range between speed states $=7 \%$

(No Incident was Detected) 


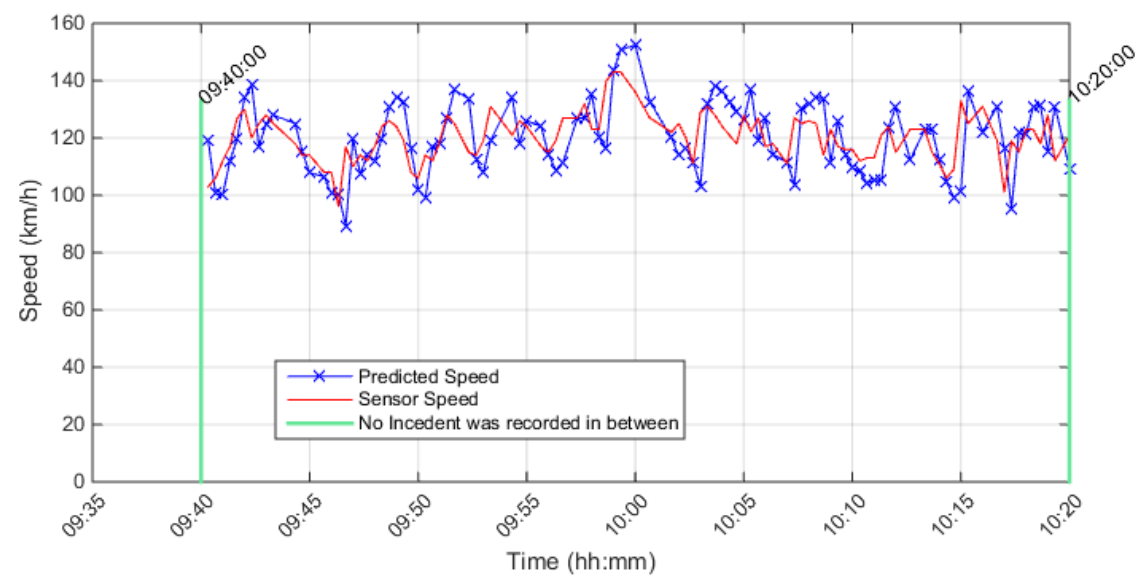

Upstream Detector \# is 401DE0080DEE, Detector Rank = 3, Distance from incident free location in $(\mathrm{km})=1.7056$ Incident free Season $=$ Summer, Stream $=E$, Direction $=E$, Date \& Time $=2011-10-01$ 07:47:25 Selected incident free Date/Time $=2011-10-01$ 10:00:00

Conditional Probability level $=0.6$, Cond.Prob. $r 3 s 2=0.2$, Cond.Prob. $r 3 s 1=0.1$, Percent drop in speed $\%=-56$ Max. Posterior Probability level $=0.97$, Percent range between speed states $=7 \%$ (No Incident was Detected)

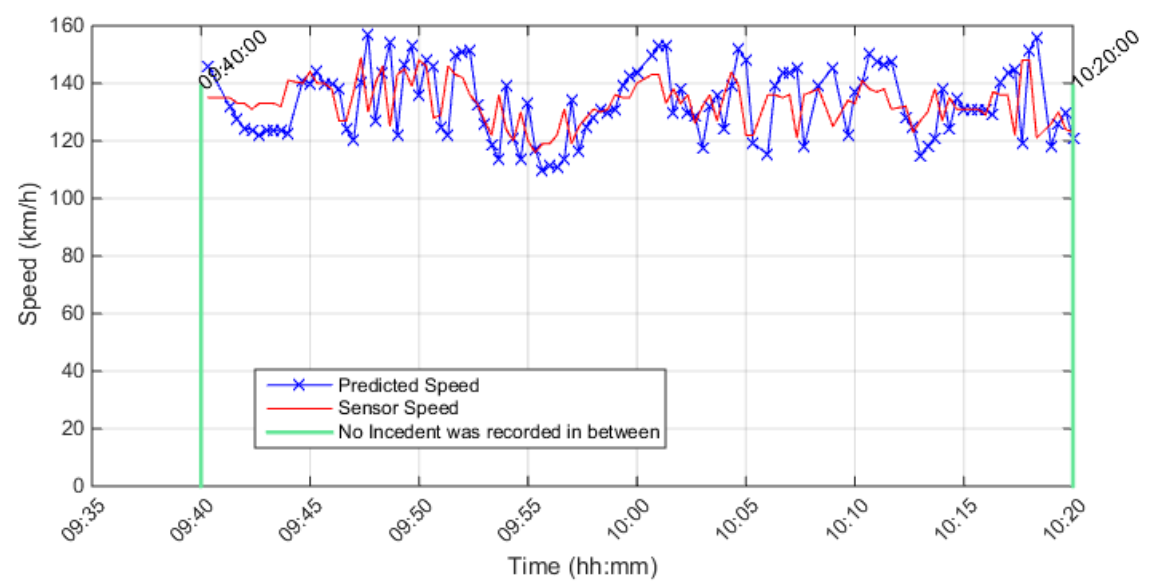

Upstream Detector \# is 401DE0060DEE, Detector Rank = 1, Distance from incident free location in $(\mathrm{km})=0.19919$ Incident free Season $=$ Winter, Stream $=\mathrm{E}$, Direction $=\mathrm{E}$, Date \& Time $=$ 2011-12-17 14:24:44 Selected incident free Date/Time $=2011-12-17$ 10:00:00

Conditional Probability level $=0.6$, Cond.Prob. $r 3 \mathrm{~s} 2=0.2$, Cond. Prob. $r 3 \mathrm{~s} 1=0.1$, Percent drop in speed $\%=-56$ Max. Posterior Probability level $=0.97$, Percent range between speed states $=7 \%$ (No Incident was Detected) 


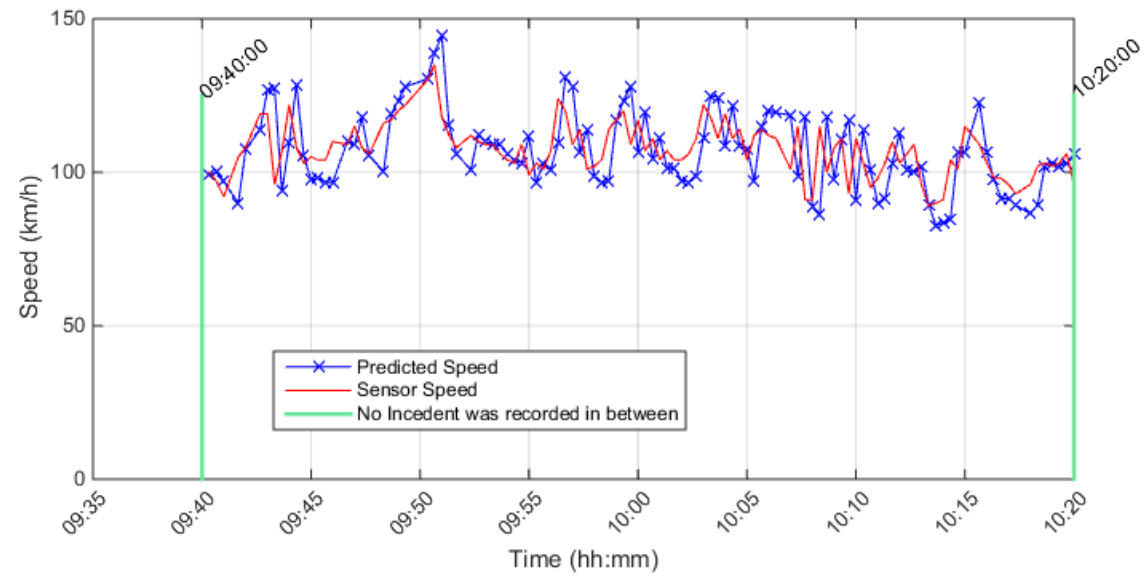

Upstream Detector \# is 401DW0090DWC, Detector Rank = 1, Distance from incident free location in $(\mathrm{km})=5.4451$ Incident free Season $=$ Winter, Stream $=C$, Direction $=$ W, Date \& Time $=$ 2011-01-05 17:07:00

Selected incident free Date/Time $=2011-01-05$ 10:00:00

Conditional Probability level $=0.6$, Cond.Prob. $r 3 \mathrm{~s} 2=0.2$, Cond. Prob. $r 3 \mathrm{~s} 1=0.1$, Percent drop in speed $\%=-56$ Max. Posterior Probability level $=0.97$, Percent range between speed states $=7 \%$

(No Incident was Detected)

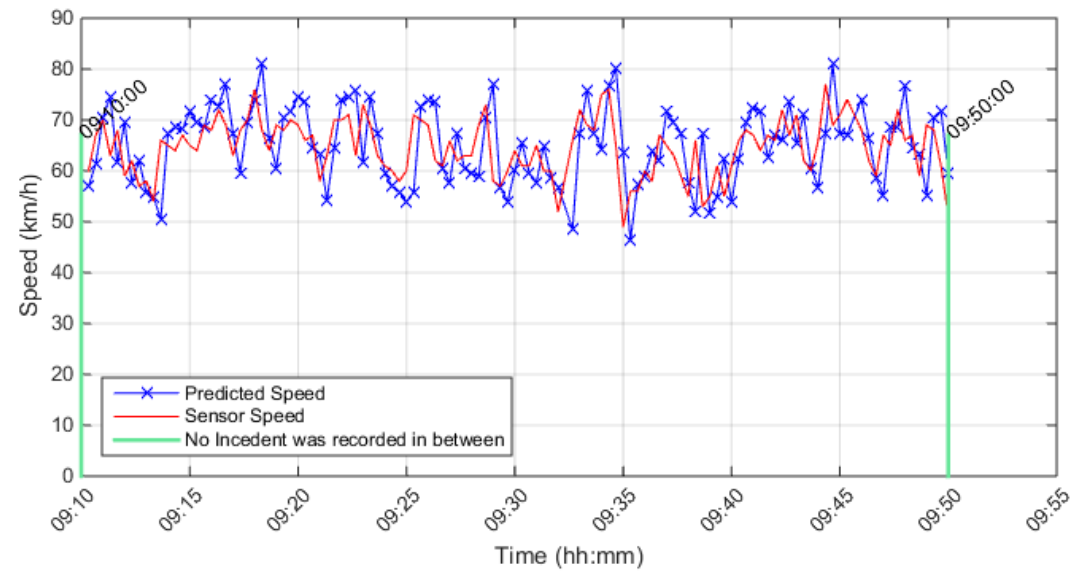

Upstream Detector \# is 401DW0160DWC, Detector Rank = 1, Distance from incident free location in $(\mathrm{km})=0.1854$ Incident free Season $=$ Winter, Stream $=C$, Direction $=$ W, Date \& Time $=$ 2011-01-17 20:35:25

Selected incident free Date $/$ Time $=2011-01-17$ 09:30:00

Conditional Probability level $=0.6$, Cond.Prob. $r 3 s 2=0.2$, Cond.Prob. $r 3 s 1=0.1$, Percent drop in speed $\%=-56$

Max. Posterior Probability level $=0.97$, Percent range between speed states $=7 \%$

(No Incident was Detected) 


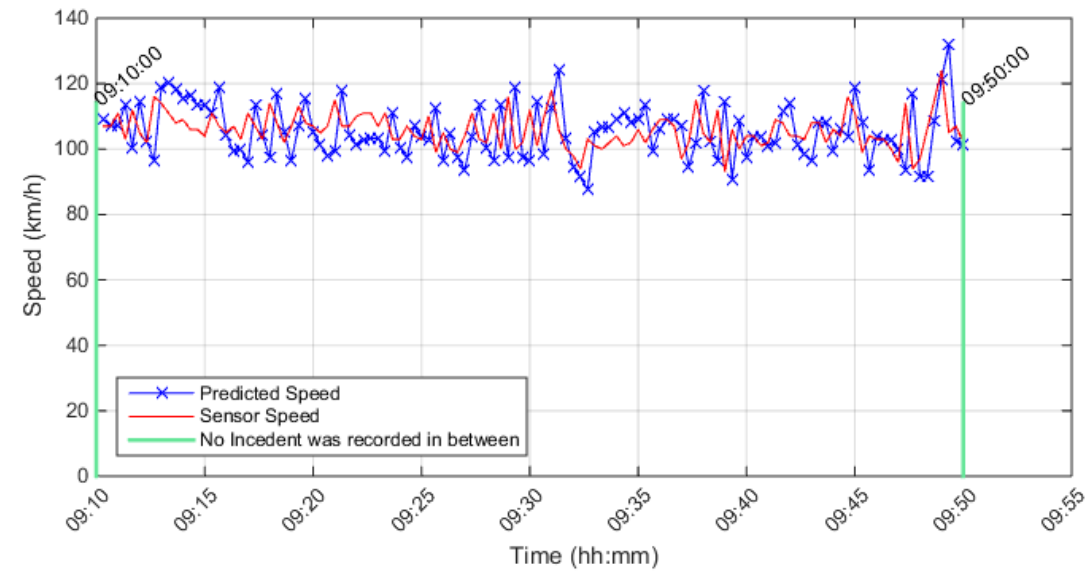

Upstream Detector \# is 401DE0060DWC, Detector Rank = 1, Distance from incident free location in $(\mathrm{km})=0.16757$ Incident free Season $=$ Winter, Stream $=$ C, Direction $=W$, Date \& Time $=2011-02-05$ 16:23:27

Selected incident free Date/Time $=$ 2011-02-05 09:30:00

Conditional Probability level $=0.6$, Cond. Prob. $r 3 s 2=0.2$, Cond. Prob. $r 3 s 1=0.1$, Percent drop in speed $\%=-56$ Max. Posterior Probability level $=0.97$, Percent range between speed states $=7 \%$ (No Incident was Detected)

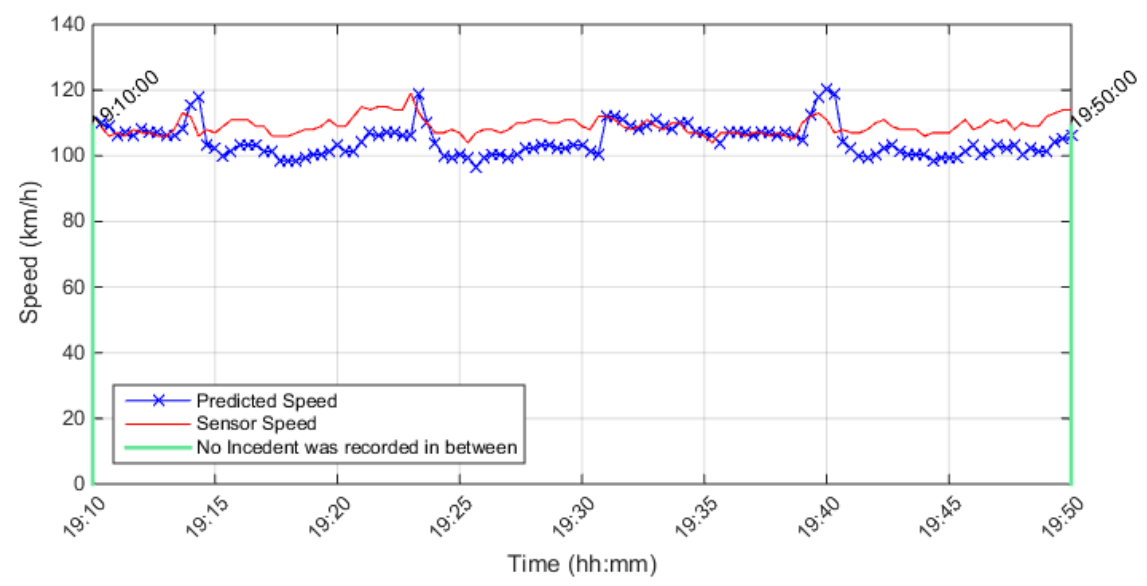

Upstream Detector \# is 401DE0480DWC, Detector Rank = 1, Distance from incident free location in $(\mathrm{km})=0.16297$ Incident free Season $=$ Spring, Stream $=$ C, Direction $=$ W, Date $\&$ Time $=2011-04-08$ 06:56:05 Selected incident free Date/Time $=$ 2011-04-08 19:30:00

Conditional Probability level $=0.6$, Cond. Prob. $r 3 s 2=0.2$, Cond. Prob. $r 3 s 1=0.1$, Percent drop in speed $\%=-56$

Max. Posterior Probability level $=0.97$, Percent range between speed states $=7 \%$

(No Incident was Detected) 


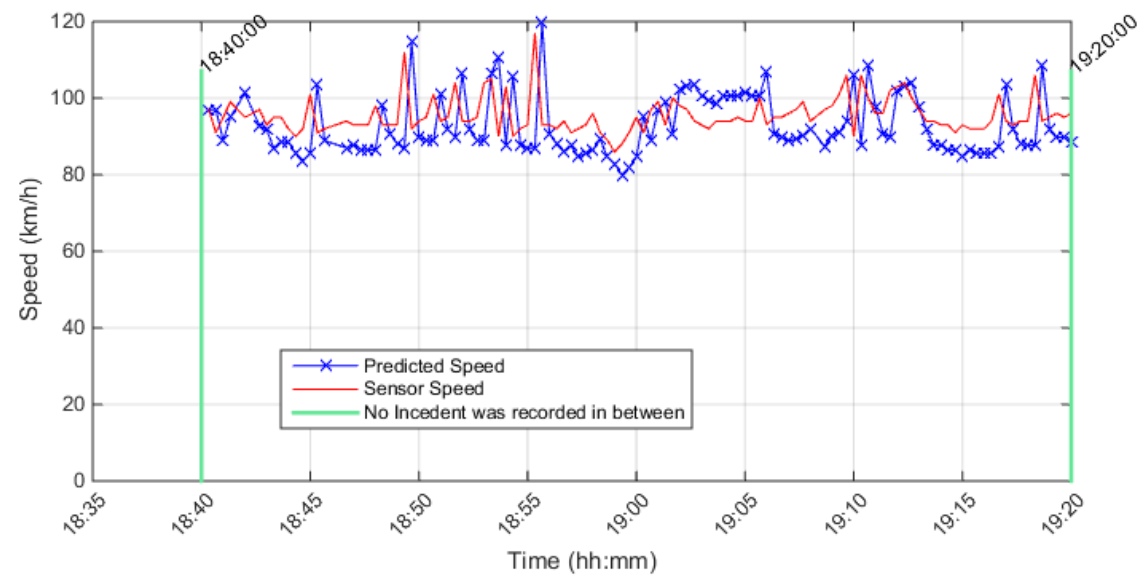

Upstream Detector \# is 401DE0220DWC, Detector Rank = 1, Distance from incident free location in $(\mathrm{km})=0.29463$ Incident free Season $=$ Spring, Stream $=$ C, Direction $=$ W, Date \& Time $=2011-04-26$ 16:55:37

Selected incident free Date/Time $=2011-04-26$ 19:00:00

Conditional Probability level $=0.6$, Cond.Prob. $r 3 s 2=0.2$, Cond.Prob. $r 3 s 1=0.1$, Percent drop in speed $\%=-56$ Max. Posterior Probability level $=0.97$, Percent range between speed states $=7 \%$

(No Incident was Detected)

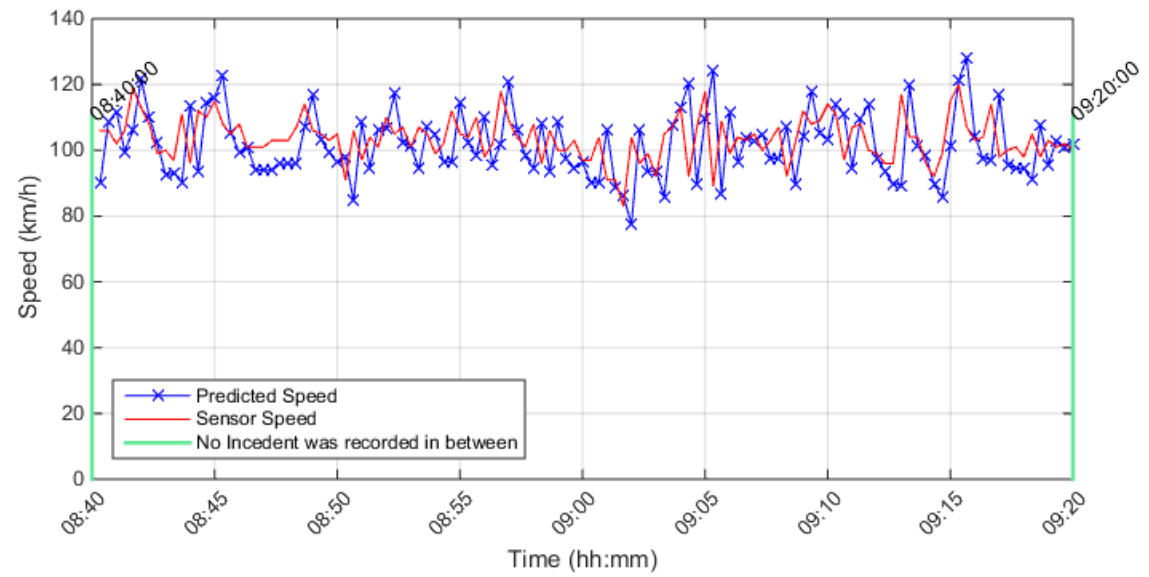

Upstream Detector \# is 401DE0370DWC, Detector Rank $=3$, Distance from incident free location in $(\mathrm{km})=2.0297$ Incident free Season $=$ Summer, Stream $=$ C , Direction $=$ W, Date \& Time $=$ 2011-06-05 18:11:02 Selected incident free Date/Time $=$ 2011-06-05 9:00:00

Conditional Probability level $=0.6$, Cond. Prob. $r 3 s 2=0.2$, Cond. Prob. $r 3 s 1=0.1$, Percent drop in speed $\%=-56$

Max. Posterior Probability level $=0.97$, Percent range between speed states $=7 \%$

(No Incident was Detected) 


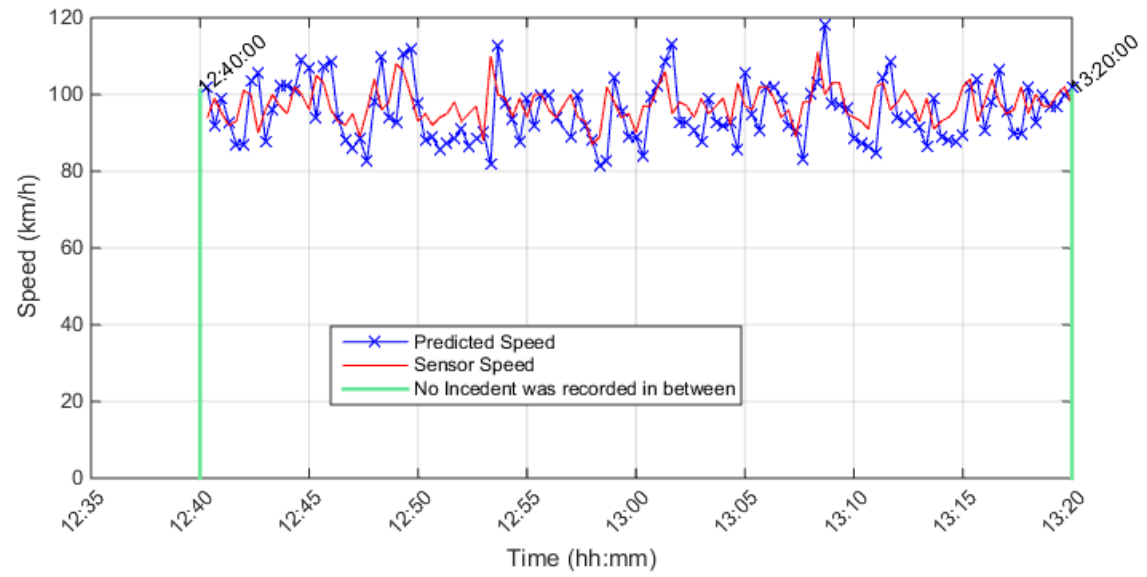

Upstream Detector \# is 401DE0090DWC, Detector Rank $=1$, Distance from incident free location in $(\mathrm{km})=0.59485$ Incident free Season $=$ Summer, Stream $=$ C , Direction $=W$, Date \& Time $=2011-06-06$ 10:49:19 Selected incident free Date/Time $=2011-06-06$ 13:00:00

Conditional Probability level $=0.6$, Cond.Prob. $r 3 \mathrm{~s} 2=0.2$, Cond. Prob. $r 3 \mathrm{~s} 1=0.1$, Percent drop in speed $\%=-56$ Max. Posterior Probability level $=0.97$, Percent range between speed states $=7 \%$

(No Incident was Detected)

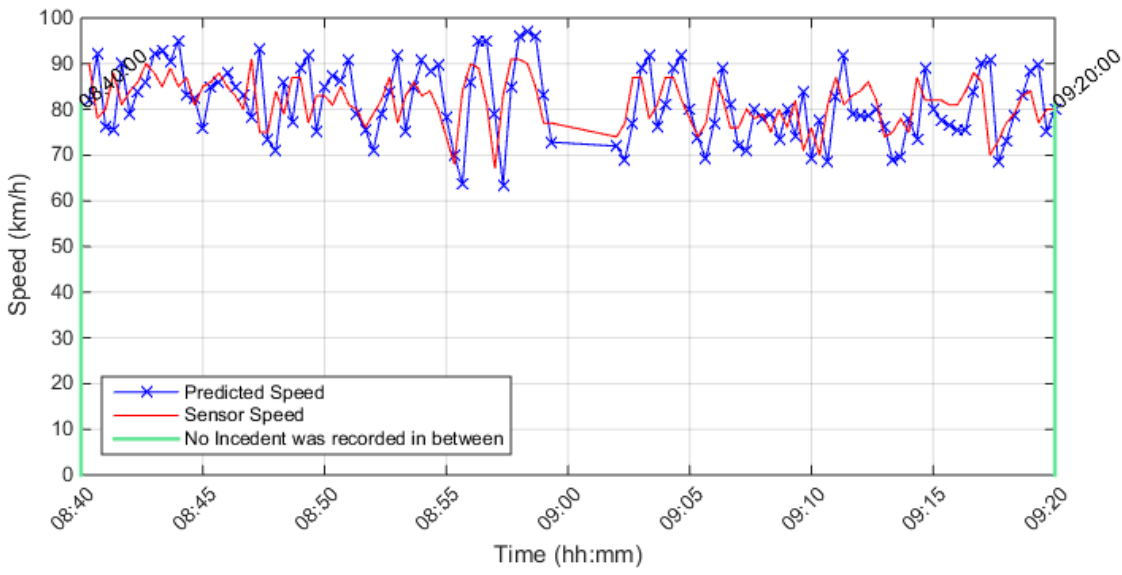

Upstream Detector \# is 401DW0020DWC, Detector Rank = 1, Distance from incident free location in $(\mathrm{km})=0.88006$ Incident free Season $=$ Summer, Stream $=$ C , Direction $=$ W, Date \& Time $=$ 2011-06-10 17:57:45 Selected incident free Date/Time $=2011-06-10$ 09:00:00

Conditional Probability level $=0.6$, Cond.Prob. $r 3 \mathrm{~s} 2=0.2$, Cond.Prob. $r 3 \mathrm{~s} 1=0.1$, Percent drop in speed $\%=-56$

Max. Posterior Probability level $=0.97$, Percent range between speed states $=7 \%$

(No Incident was Detected) 


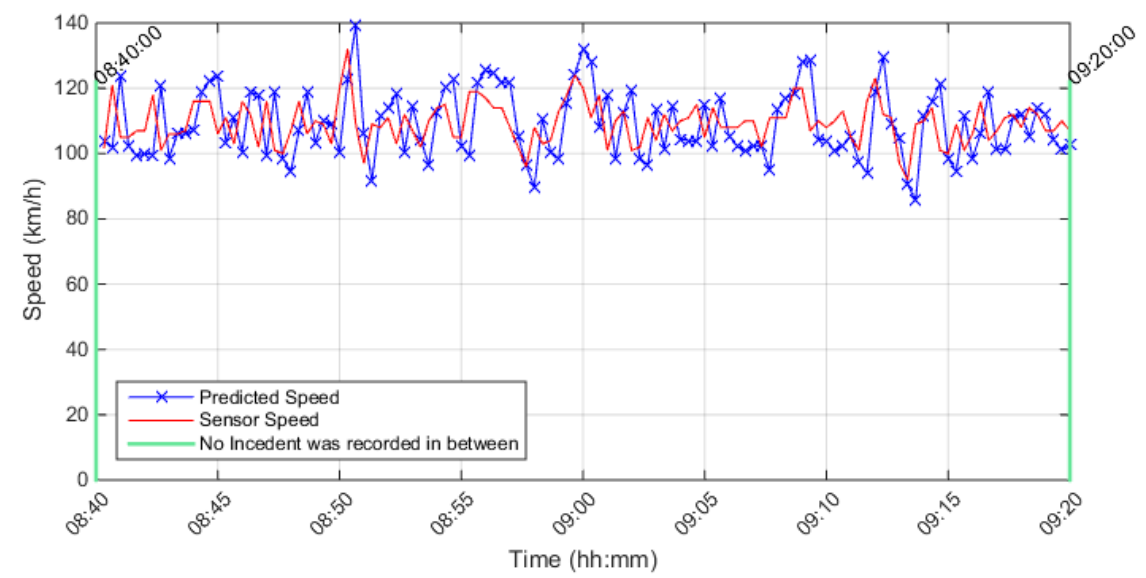

Upstream Detector \# is 401DE0320DWC, Detector Rank = 1, Distance from incident free location in $(\mathrm{km})=0.13977$ Incident free Season $=$ Summer, Stream $=$ C, Direction $=$ W, Date \& Time $=$ 2011-06-12 12:55:53 Selected incident free Date/Time $=2011-06-12$ 09:00:00

Conditional Probability level $=0.6$, Cond. Prob. $r 3 \mathrm{~s} 2=0.2$, Cond.Prob. $r 3 \mathrm{~s} 1=0.1$, Percent drop in speed $\%=-56$ Max. Posterior Probability level $=0.97$, Percent range between speed states $=7 \%$ (No Incident was Detected)

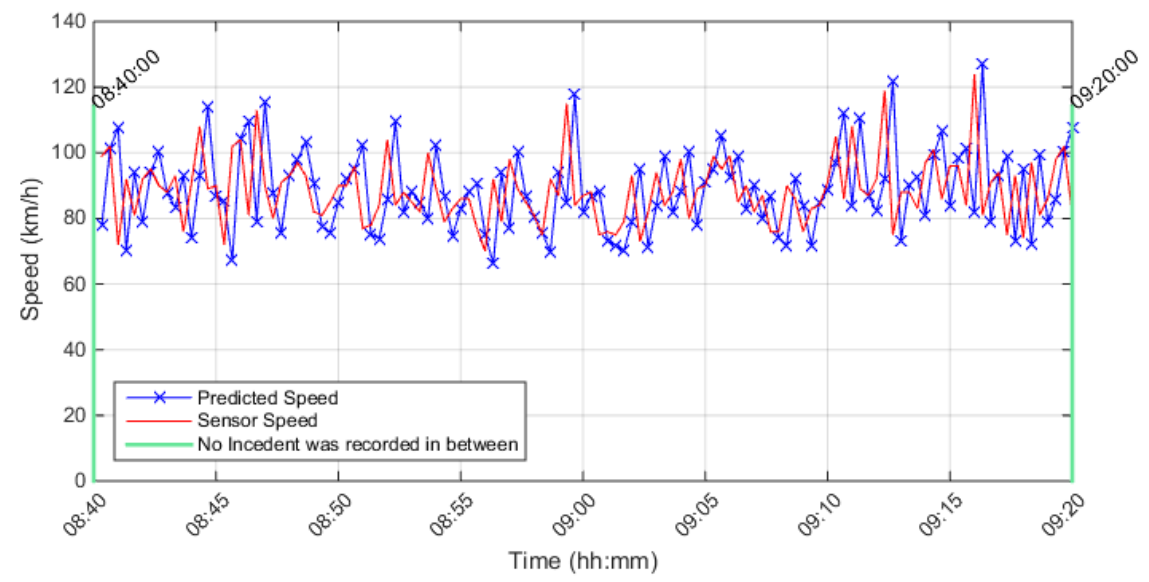

Upstream Detector \# is 401DE0150DWC, Detector Rank $=3$, Distance from incident free location in $(\mathrm{km})=1.5503$ Incident free Season $=$ Summer, Stream $=C$, Direction $=W$, Date \& Time $=2011-08-07$ 17:35:54 Selected incident free Date/Time $=2011-08-07$ 09:00:00

Conditional Probability level $=0.6$, Cond.Prob. $r 3 \mathrm{~s} 2=0.2$, Cond. Prob. $r 3 \mathrm{~s} 1=0.1$, Percent drop in speed $\%=-56$

Max. Posterior Probability level $=0.97$, Percent range between speed states $=7 \%$

(No Incident was Detected) 


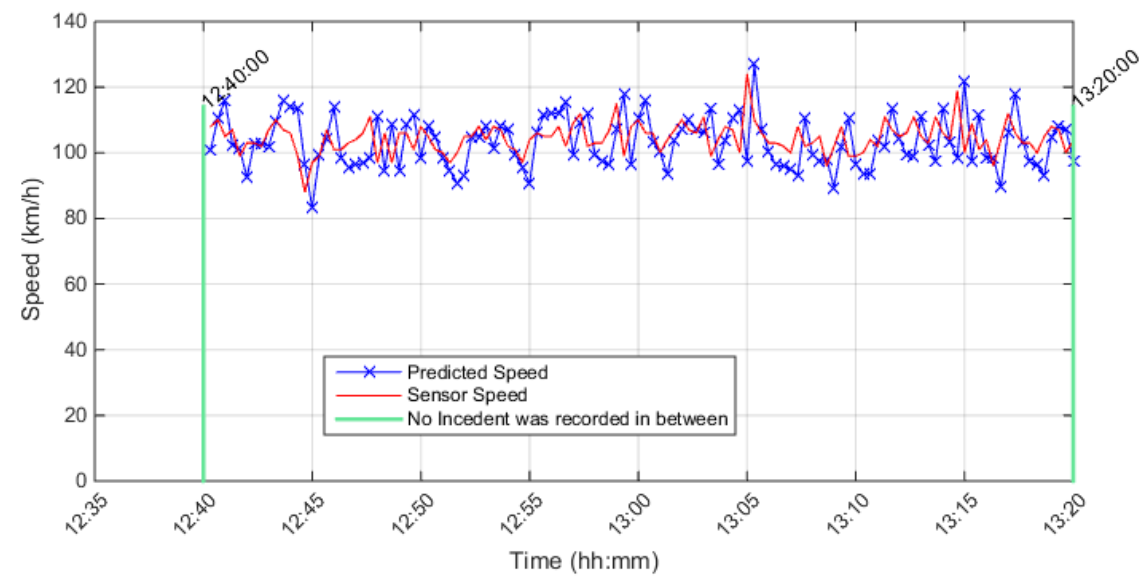

Upstream Detector \# is 401DE0060DWC, Detector Rank $=1$, Distance from incident free location in $(\mathrm{km})=0.16757$ Incident free Season $=$ Summer, Stream $=C$, Direction $=W$, Date \& Time $=2011-10-0603: 41: 21$

Selected incident free Date/Time $=2011-10-06$ 13:00:00

Conditional Probability level $=0.6$, Cond.Prob. $r 3 \mathrm{~s} 2=0.2$, Cond.Prob. $r 3 \mathrm{~s} 1=0.1$, Percent drop in speed $\%=-56$ Max. Posterior Probability level $=0.97$, Percent range between speed states $=7 \%$

(No Incident was Detected)

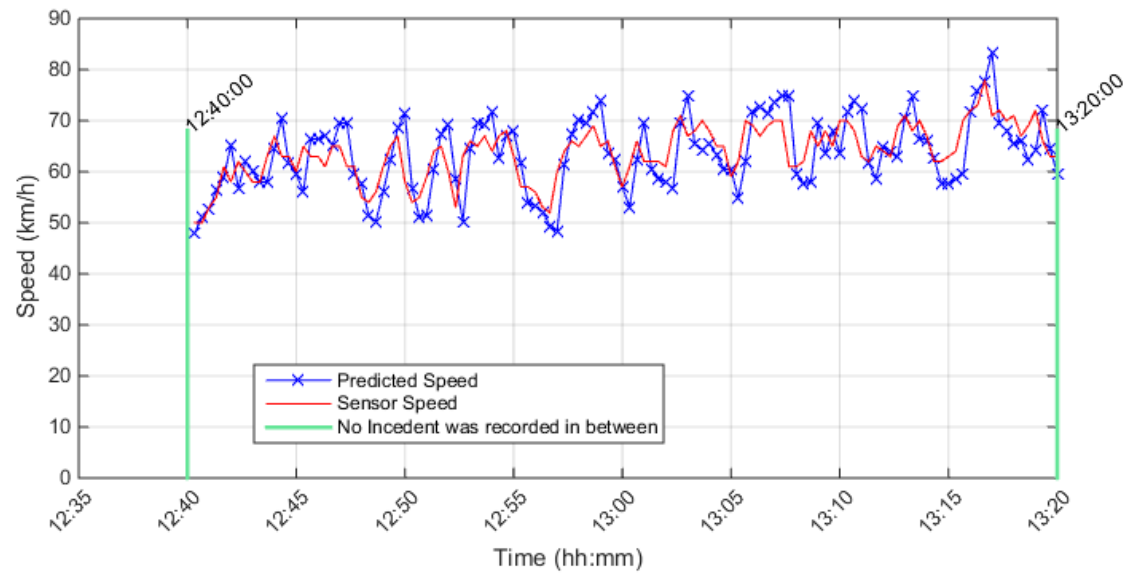

Upstream Detector \# is 401DE0020DWC, Detector Rank = 1, Distance from incident free location in $(\mathrm{km})=0.39159$ Incident free Season $=$ Summer, Stream $=$ C , Direction $=$ W, Date $\&$ Time $=$ 2011-11-30 16:49:51 Selected incident free Date/Time $=2011-11-30$ 13:00:00

Conditional Probability level $=0.6$, Cond. Prob. $r 3 s 2=0.2$, Cond. Prob. $r 3 s 1=0.1$, Percent drop in speed $\%=-56$

Max. Posterior Probability level $=0.97$, Percent range between speed states $=7 \%$

(No Incident was Detected) 


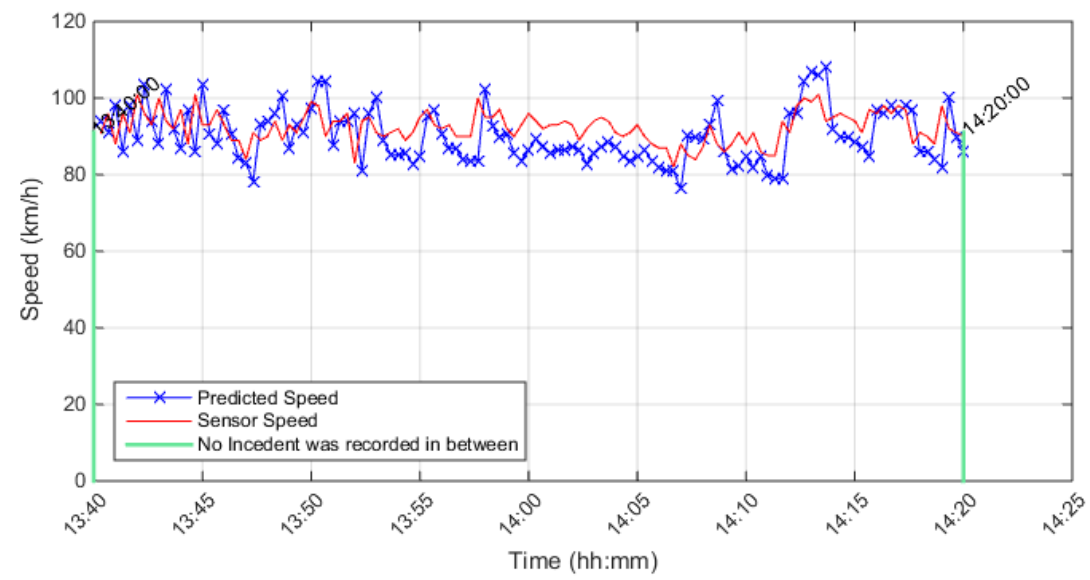

Upstream Detector \# is 401DE0220DWC, Detector Rank = 2, Distance from incident free location in $(\mathrm{km})=1.1626$ Incident free Season $=$ Winter, Stream $=$ C, Direction $=$ W, Date \& Time $=2011-12-15$ 07:00:28

Selected incident free Date/Time $=2011-12-15$ 14:00:00

Conditional Probability level $=0.6$, Cond.Prob. $r 3 s 2=0.2$, Cond.Prob. $r 3 s 1=0.1$, Percent drop in speed $\%=-56$ Max. Posterior Probability level $=0.97$, Percent range between speed states $=7 \%$

(No Incident was Detected)

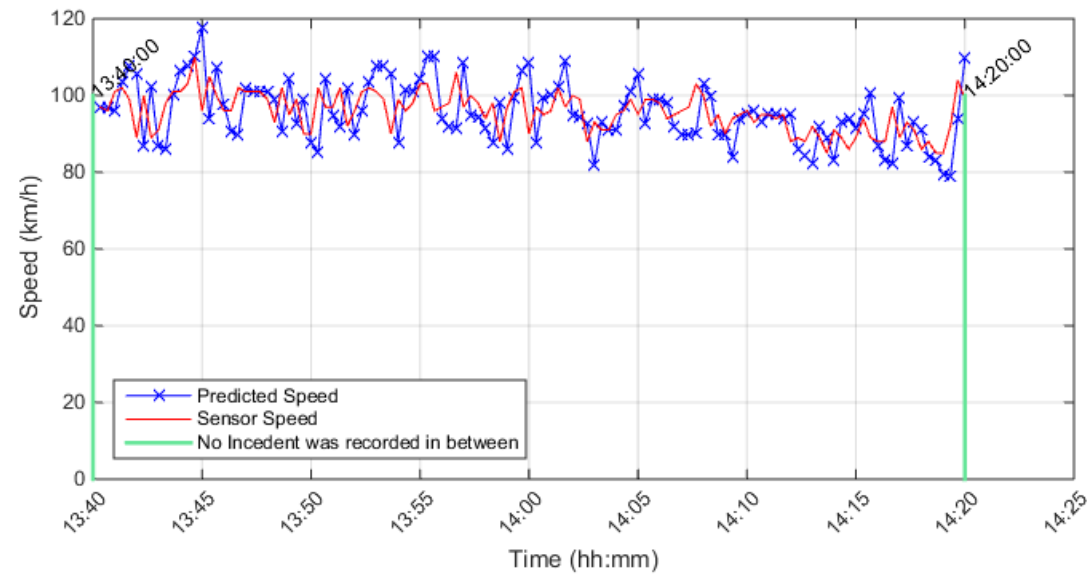

Upstream Detector \# is 401DE0050DWC, Detector Rank = 1, Distance from incident free location in $(\mathrm{km})=0.45946$ Incident free Season $=$ Winter, Stream $=$ C, Direction $=$ W, Date \& Time $=2011-12-26$ 22:38:09

Selected incident free Date/Time $=2011-12-26$ 14:00:00

Conditional Probability level $=0.6$, Cond. Prob. $r 3 s 2=0.2$, Cond. Prob. $r 3 s 1=0.1$, Percent drop in speed $\%=-56$

Max. Posterior Probability level $=0.97$, Percent range between speed states $=7 \%$

(No Incident was Detected) 


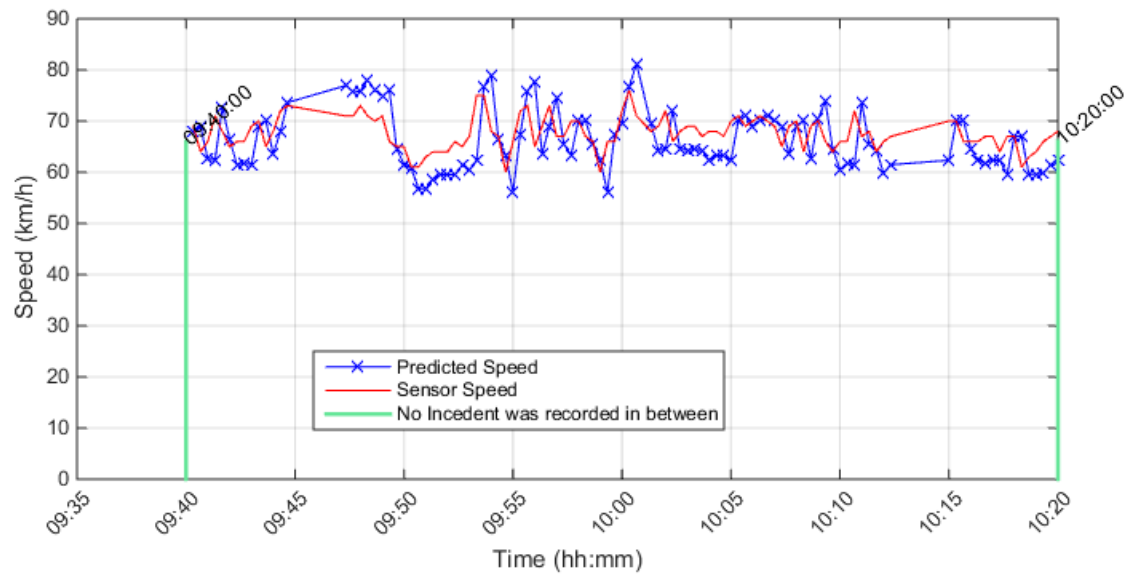

Upstream Detector \# is 401DE0060DWE, Detector Rank $=1$, Distance from incident free location in $(\mathrm{km})=0.17254$ Incident free Season $=$ Winter, Stream $=E$, Direction $=W$, Date \& Time $=$ 2011-01-12 06:05:49

Selected incident free Date/Time $=2011-01-12$ 10:00:00

Conditional Probability level $=0.6$, Cond.Prob. $r 3 \mathrm{~s} 2=0.2$, Cond. Prob. $r 3 \mathrm{~s} 1=0.1$, Percent drop in speed $\%=-56$

Max. Posterior Probability level $=0.97$, Percent range between speed states $=7 \%$

(No Incident was Detected)

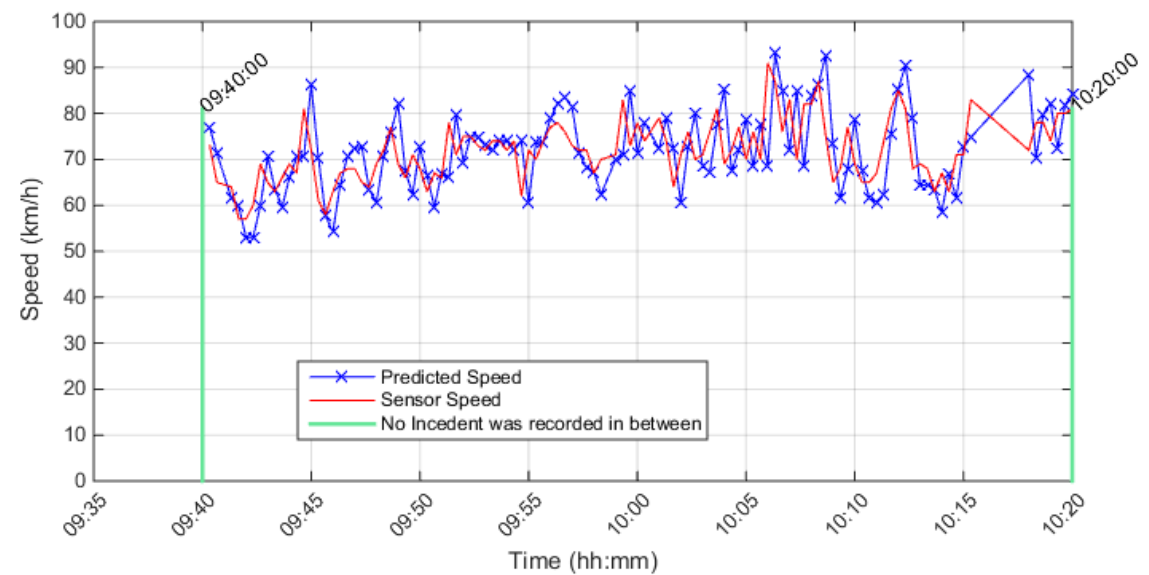

Upstream Detector \# is 401DE0290DWE, Detector Rank = 1, Distance from incident free location in $(\mathrm{km})=0.27431$ Incident free Season $=$ Winter, Stream $=E$, Direction $=W$, Date \& Time $=$ 2011-02-02 16:57:43

Selected incident free Date/Time $=$ 2011-02-02 10:00:00

Conditional Probability level $=0.6$, Cond.Prob. $r 3 \mathrm{~s} 2=0.2$, Cond.Prob. $r 3 \mathrm{~s} 1=0.1$, Percent drop in speed $\%=-56$

Max. Posterior Probability level $=0.97$, Percent range between speed states $=7 \%$

(No Incident was Detected) 


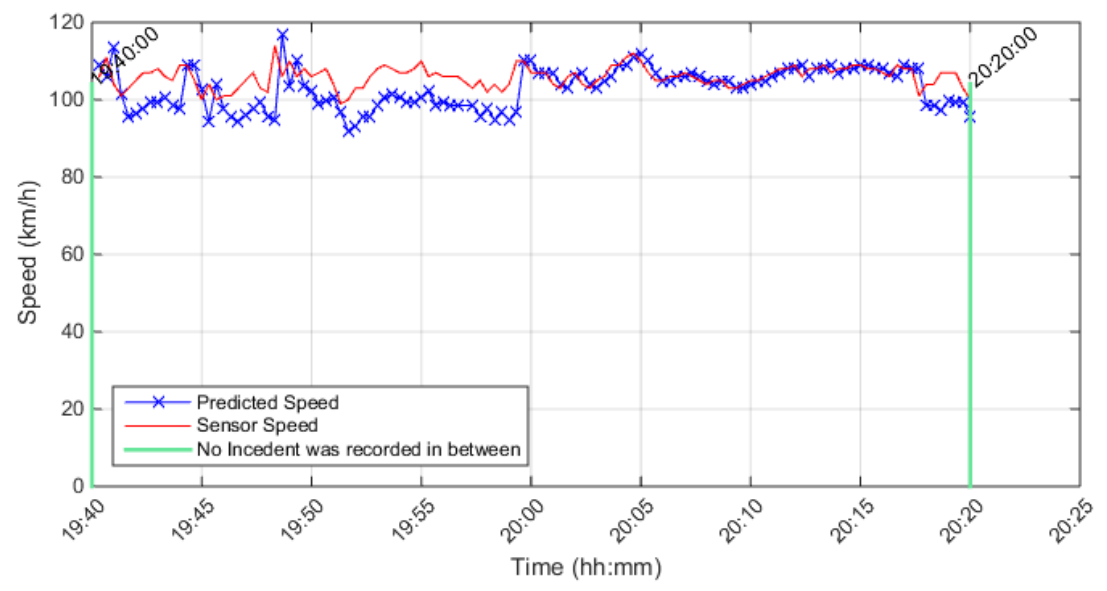

Upstream Detector \# is 401DE0020DWE, Detector Rank = 1, Distance from incident free location in $(\mathrm{km})=0.39496$ Incident free Season $=$ Spring, Stream $=E$, Direction $=W$, Date \& Time $=$ 2011-03-06 08:29:36 Selected incident free Date/Time $=$ 2011-03-06 20:00:00

Conditional Probability level $=0.6$, Cond.Prob. $r 3 \mathrm{~s} 2=0.2$, Cond.Prob. $r 3 \mathrm{~s} 1=0.1$, Percent drop in speed $\%=-56$

Max. Posterior Probability level $=0.97$, Percent range between speed states $=7 \%$

(No Incident was Detected)

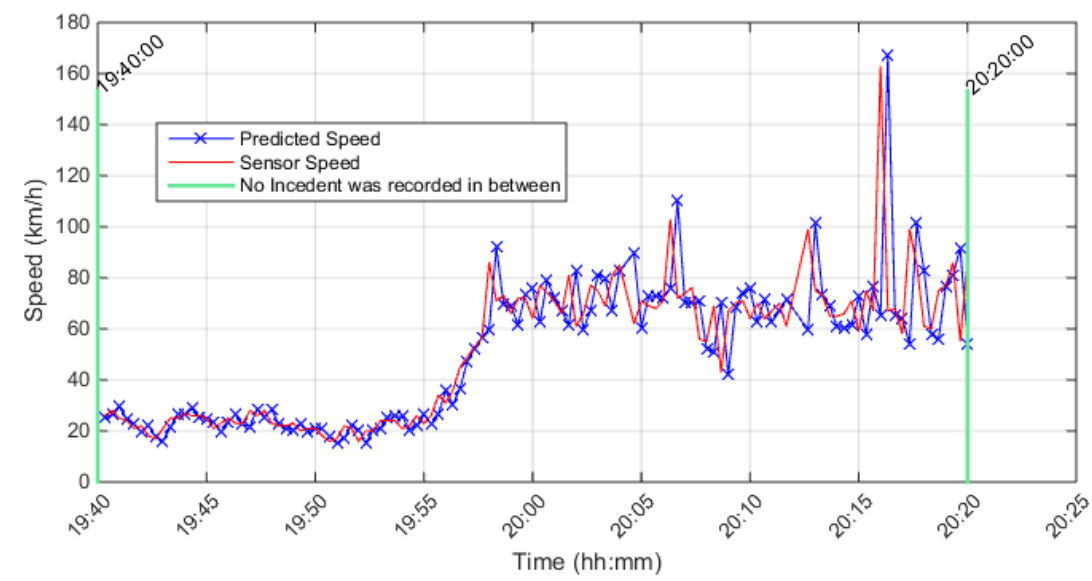

Upstream Detector \# is 401DW0260DWE, Detector Rank = 1, Distance from incident free location in $(\mathrm{km})=0.17943$ Incident free Season $=$ Spring, Stream $=E$, Direction $=W$, Date \& Time $=2011-03-23$ 10:46:20 Selected incident free Date/Time $=2011-03-23$ 20:00:00

Conditional Probability level $=0.6$, Cond. Prob. $r 3 s 2=0.2$, Cond. Prob. $r 3 s 1=0.1$, Percent drop in speed $\%=-56$

Max. Posterior Probability level $=0.97$, Percent range between speed states $=7 \%$

(No Incident was Detected) 


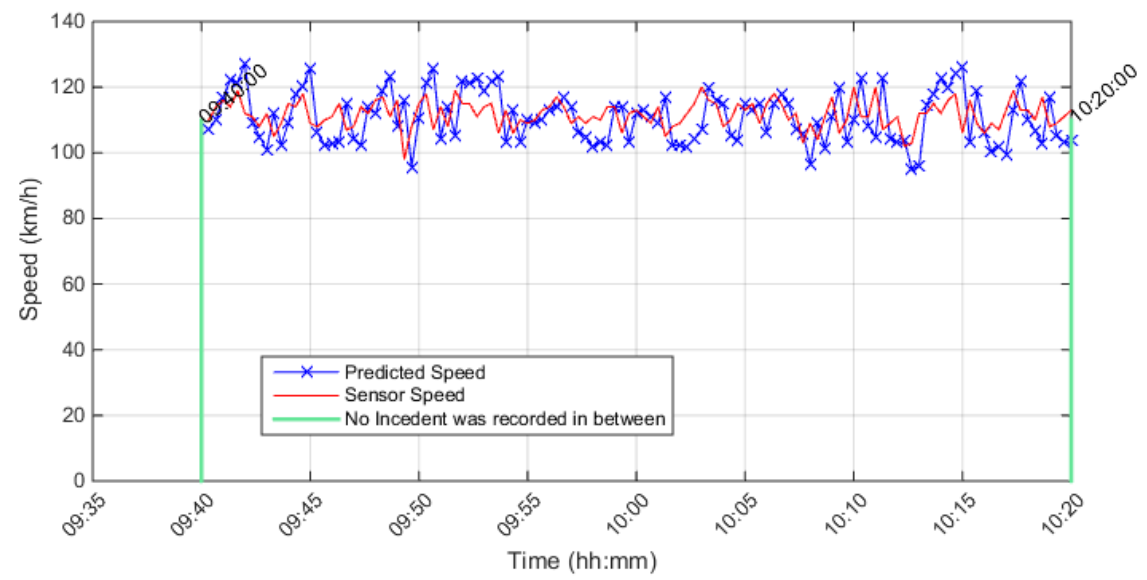

Upstream Detector \# is 401DW0020DWE, Detector Rank = 1, Distance from incident free location in $(\mathrm{km})=0.13067$ Incident free Season $=$ Spring, Stream $=E$, Direction $=W$, Date \& Time $=$ 2011-04-09 06:53:14

Selected incident free Date/Time $=2011-04-09$ 10:00:00

Conditional Probability level $=0.6$, Cond. Prob. $r 3 s 2=0.2$, Cond. Prob. $r 3 s 1=0.1$, Percent drop in speed $\%=-56$ Max. Posterior Probability level $=0.97$, Percent range between speed states $=7 \%$

(No Incident was Detected)

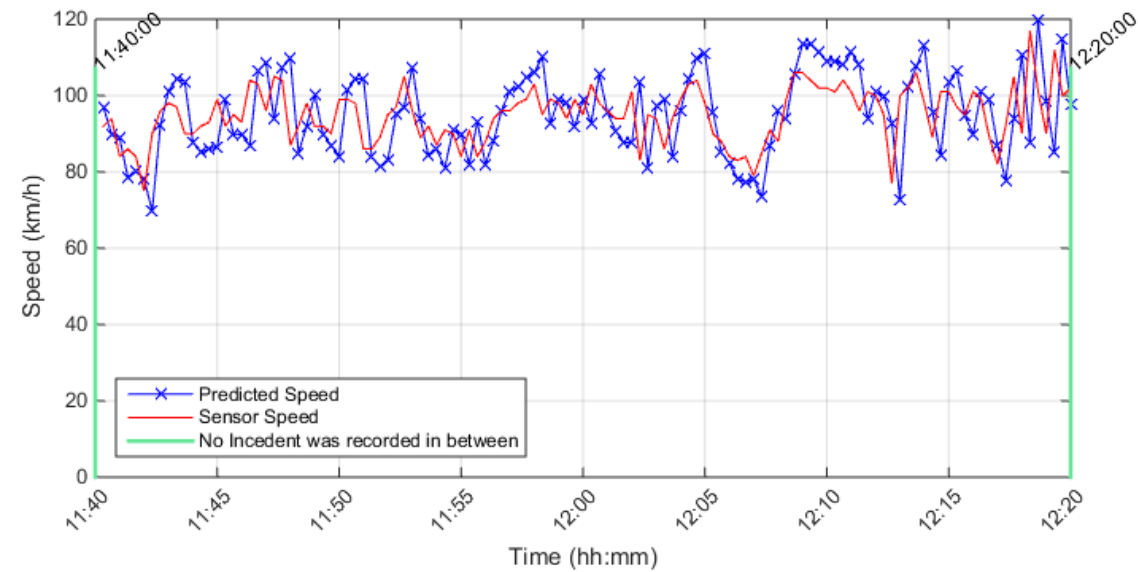

Upstream Detector \# is 401DW0090DWE, Detector Rank = 1, Distance from incident free location in $(\mathrm{km})=5.4576$ Incident free Season $=$ Spring, Stream $=E$, Direction $=$ W, Date \& Time $=2011-04-20$ 07:34:51 Selected incident free Date/Time $=2011-04-2012: 00: 00$

Conditional Probability level $=0.6$, Cond.Prob. $r 3 \mathrm{~s} 2=0.2$, Cond.Prob. $r 3 \mathrm{~s} 1=0.1$, Percent drop in speed $\%=-56$ Max. Posterior Probability level $=0.97$, Percent range between speed states $=7 \%$ (No Incident was Detected) 


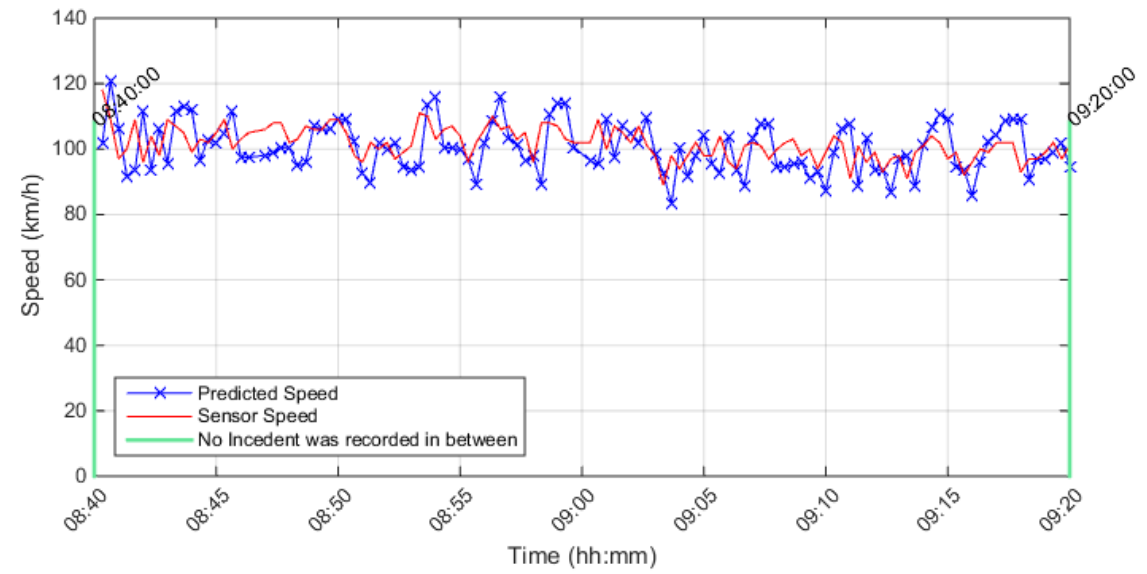

Upstream Detector \# is 401DE0160DWE, Detector Rank = 1, Distance from incident free location in $(\mathrm{km})=0.12782$ Incident free Season $=$ Spring. Stream $=E$, Direction $=$ W, Date \& Time $=2011-05-21$ 16:00:37 Selected incident free Date/Time $=2011-05-2109: 00: 00$

Conditional Probability level $=0.6$, Cond.Prob. $r 3 \mathrm{~s} 2=0.2$, Cond.Prob. $r 3 \mathrm{~s} 1=0.1$, Percent drop in speed $\%=-56$ Max. Posterior Probability level $=0.97$, Percent range between speed states $=7 \%$ (No Incident was Detected)

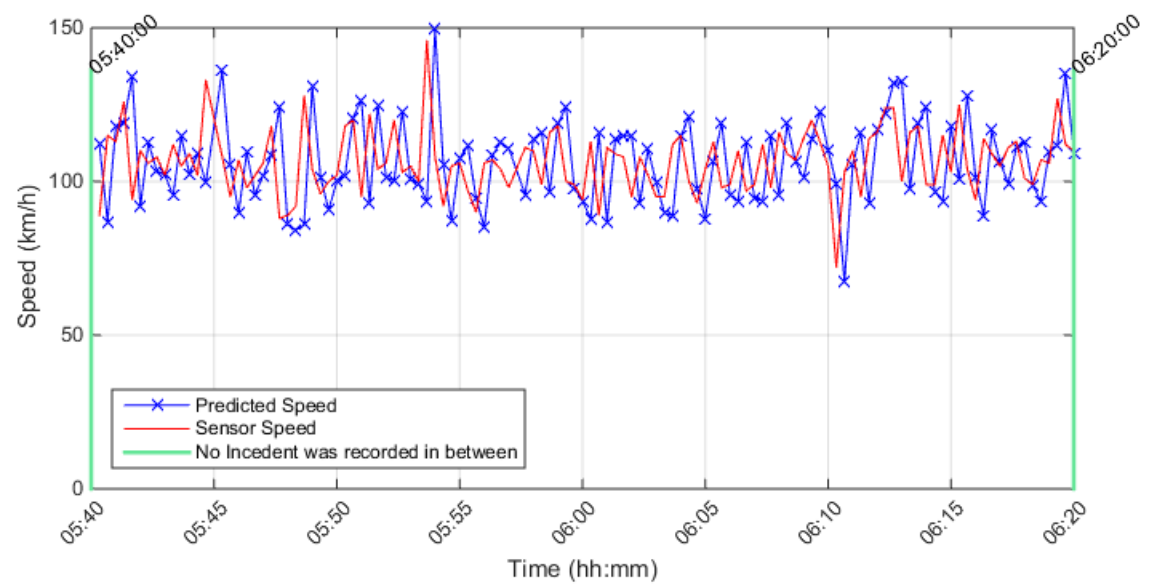

Upstream Detector \# is 401DW0270DWE, Detector Rank = 3, Distance from incident free location in $(\mathrm{km})=1.7666$ Incident free Season $=$ Spring, Stream $=E$, Direction $=W$, Date \& Time $=2011-05-28$ 01:46:20 Selected incident free Date/Time $=2011-05-28$ 06:00:00

Conditional Probability level $=0.6$, Cond.Prob. $r 3 \mathrm{~s} 2=0.2$, Cond. Prob. $r 3 \mathrm{~s} 1=0.1$, Percent drop in speed $\%=-56$ Max. Posterior Probability level $=0.97$, Percent range between speed states $=7 \%$ (No Incident was Detected) 


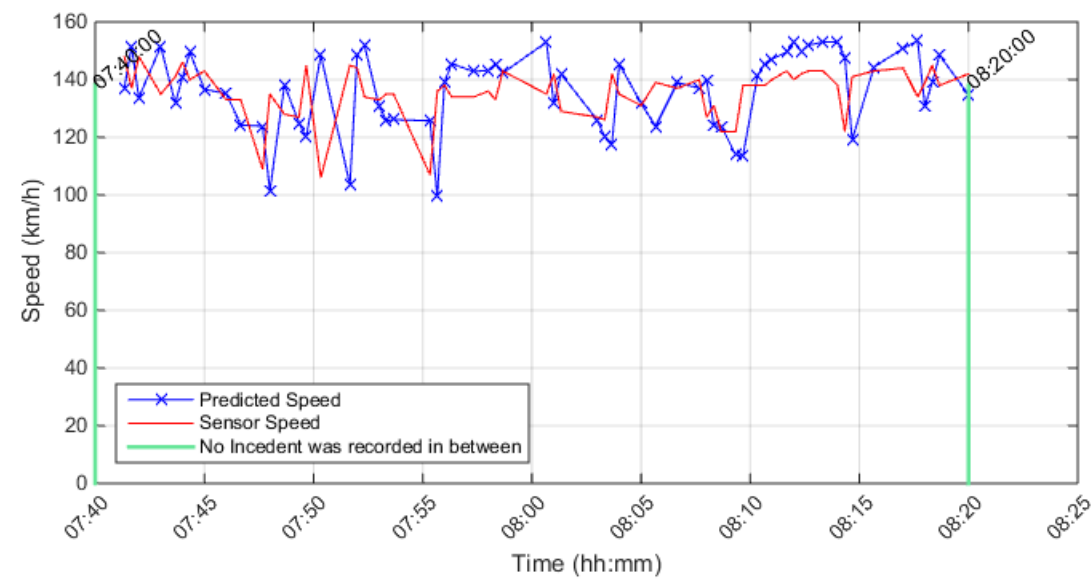

Upstream Detector \# is 401DE0020DWE, Detector Rank = 1, Distance from incident free location in $(\mathrm{km})=0.39496$ Incident free Season $=$ Summer, Stream $=E$, Direction $=W$, Date \& Time $=$ 2011-06-05 17:33:31

Selected incident free Date/Time $=2011-06-05$ 8:00:00

Conditional Probability level $=0.6$, Cond.Prob. $r 3 \mathrm{~s} 2=0.2$, Cond.Prob. $r 3 \mathrm{~s} 1=0.1$, Percent drop in speed $\%=-56$

Max. Posterior Probability level $=0.97$, Percent range between speed states $=7 \%$

(No Incident was Detected)

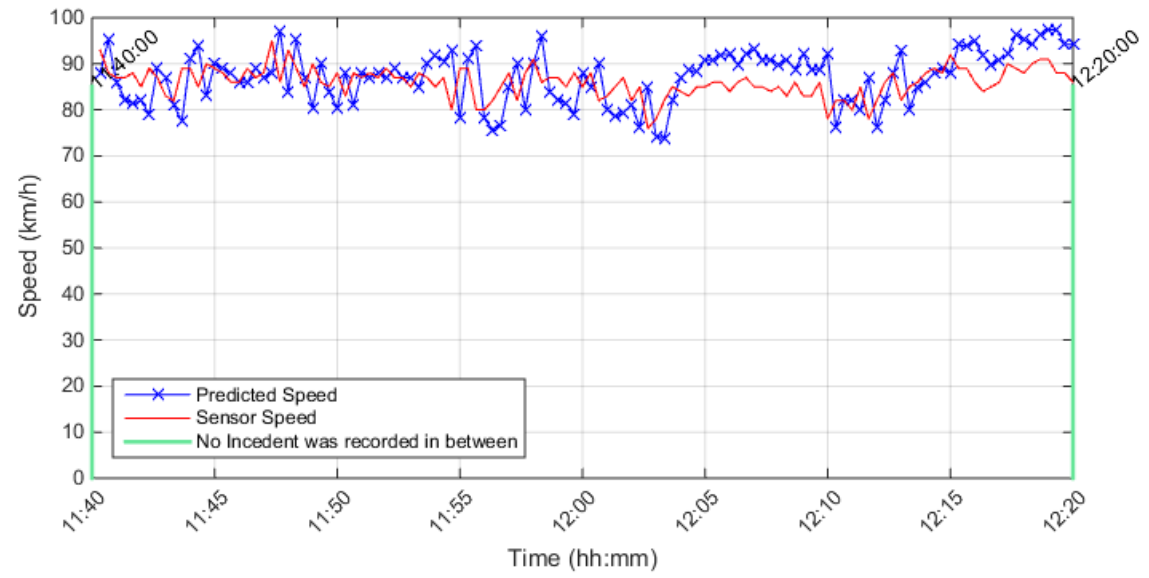

Upstream Detector \# is 401DW0040DWE, Detector Rank = 1, Distance from incident free location in $(\mathrm{km})=0.22683$ Incident free Season $=$ Summer, Stream $=\mathrm{E}$, Direction $=\mathrm{W}$, Date \& Time $=$ 2011-06-17 23:56:57 Selected incident free Date/Time $=2011-06-17$ 12:00:00

Conditional Probability level $=0.6$, Cond.Prob. $r 3 \mathrm{~s} 2=0.2$, Cond.Prob. $r 3 \mathrm{~s} 1=0.1$, Percent drop in speed $\%=-56$ Max. Posterior Probability level $=0.97$, Percent range between speed states $=7 \%$ (No Incident was Detected) 


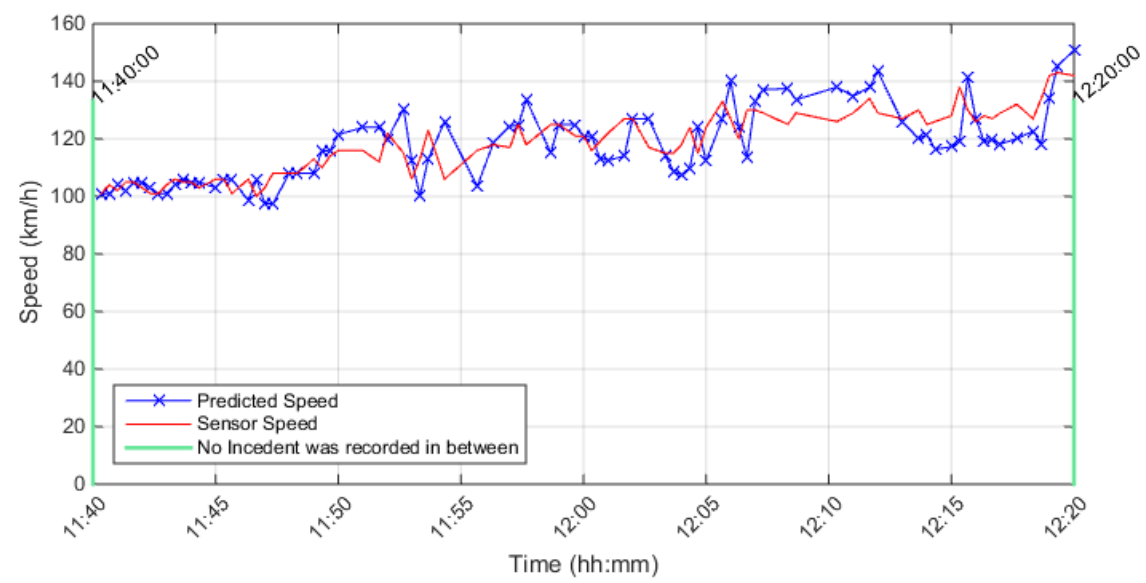

Upstream Detector \# is 401DE0020DWE, Detector Rank = 1, Distance from incident free location in $(\mathrm{km})=0.39496$ Incident free Season $=$ Summer, Stream $=E$, Direction $=W$, Date \& Time $=2011-06-2015: 46: 45$

Selected incident free Date/Time $=2011-06-2012: 00: 00$

Conditional Probability level $=0.6$, Cond.Prob. $r 3 \mathrm{~s} 2=0.2$, Cond.Prob. $r 3 \mathrm{~s} 1=0.1$, Percent drop in speed $\%=-56$ Max. Posterior Probability level $=0.97$, Percent range between speed states $=7 \%$

(No Incident was Detected)

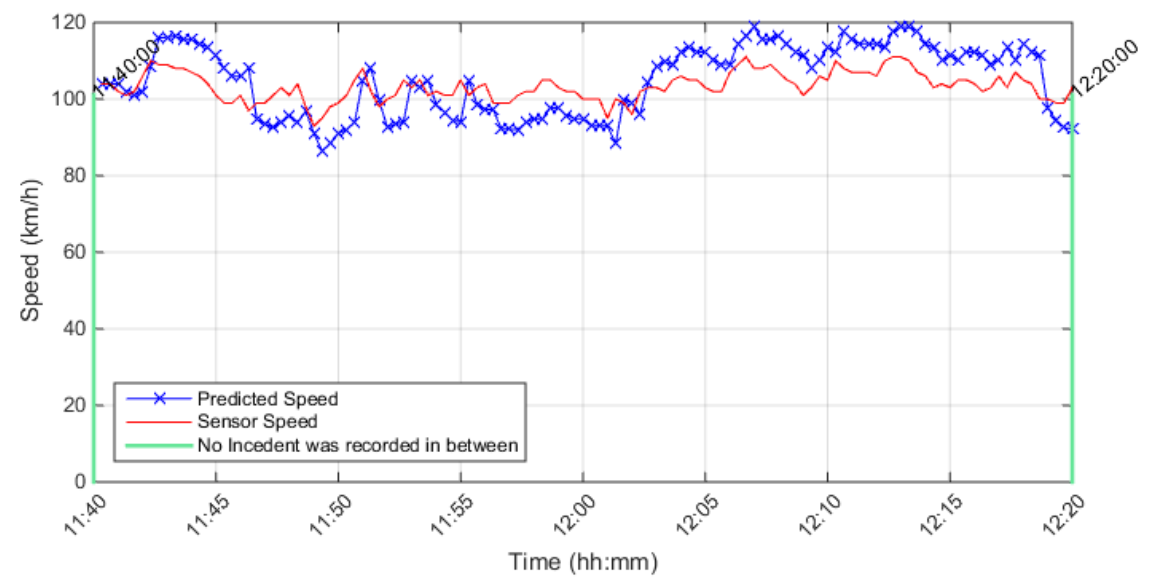

Upstream Detector \# is 401DE0130DWE, Detector Rank = 1, Distance from incident free location in $(\mathrm{km})=0.14797$ Incident free Season $=$ Summer, Stream $=E$, Direction $=W$, Date \& Time $=$ 2011-07-05 00:48:29 Selected incident free Date/Time $=2011-07-05$ 12:00:00

Conditional Probability level $=0.6$, Cond.Prob. $r 3 s 2=0.2$, Cond.Prob. $r 3 s 1=0.1$, Percent drop in speed $\%=-56$ Max. Posterior Probability level $=0.97$, Percent range between speed states $=7 \%$ (No Incident was Detected) 


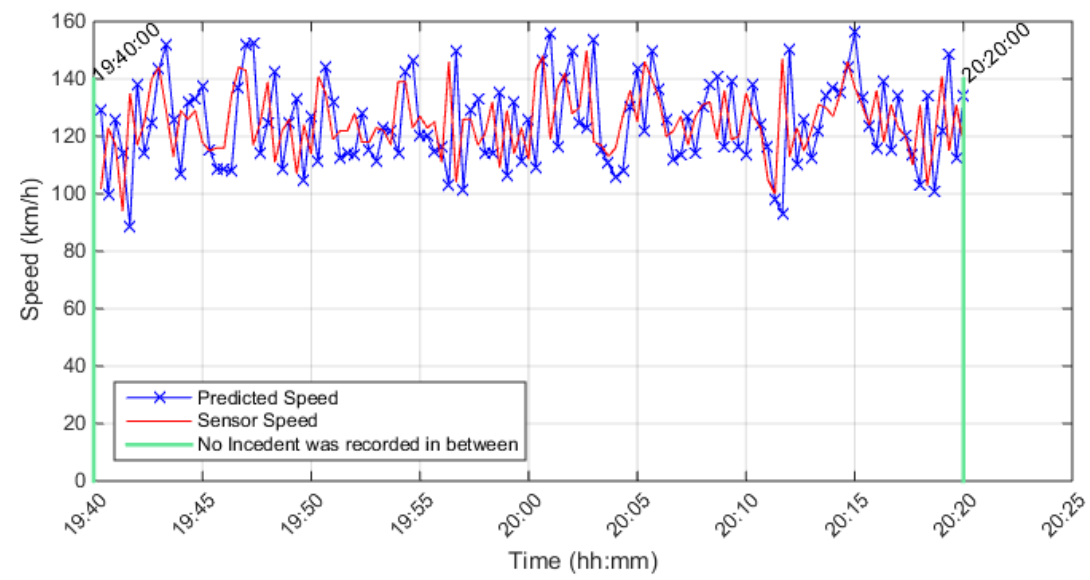

Upstream Detector \# is 401DW0280DWE, Detector Rank $=2$, Distance from incident free location in $(\mathrm{km})=1.0705$ Incident free Season $=$ Summer, Stream $=E$, Direction $=W$, Date \& Time $=$ 2011-07-26 11:41:15

Selected incident free Date/Time $=$ 2011-07-26 20:00:00

Conditional Probability level $=0.6$, Cond. Prob. $r 3 \mathrm{~s} 2=0.2$, Cond.Prob. $r 3 \mathrm{~s} 1=0.1$, Percent drop in speed $\%=-56$ Max. Posterior Probability level $=0.97$, Percent range between speed states $=7 \%$

(No Incident was Detected)

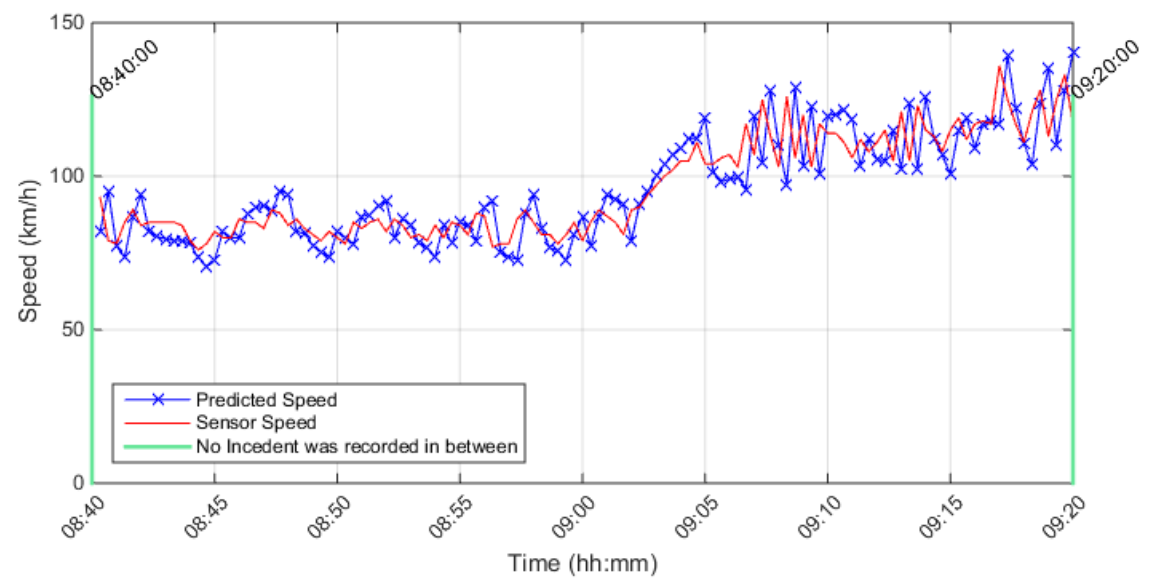

Upstream Detector \# is 401DE0020DWE, Detector Rank = 1, Distance from incident free location in $(\mathrm{km})=0.39496$ Incident free Season $=$ Summer, Stream $=E$, Direction $=W$, Date \& Time $=$ 2011-07-29 17:20:12

Selected incident free Date/Time $=2011-07-29$ 09:00:00

Conditional Probability level $=0.6$, Cond.Prob. $r 3 s 2=0.2$, Cond. Prob. $r 3 s 1=0.1$, Percent drop in speed $\%=-56$

Max. Posterior Probability level $=0.97$, Percent range between speed states $=7 \%$

(No Incident was Detected) 


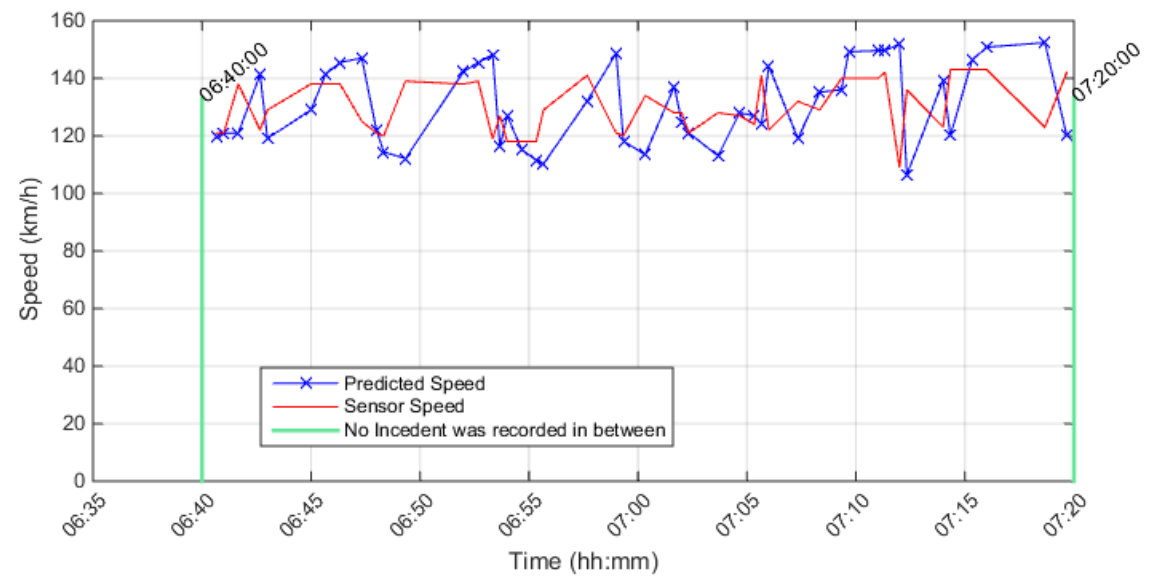

Upstream Detector \# is 401DE0020DWE, Detector Rank = 1, Distance from incident free location in $(\mathrm{km})=0.39496$ Incident free Season $=$ Summer, Stream $=\mathrm{E}$, Direction $=\mathrm{W}$, Date $\&$ Time $=2011-08-2811: 58: 55$

Selected incident free Date/Time $=2011-08-28$ 07:00:00

Conditional Probability level $=0.6$, Cond.Prob. $r 3 \mathrm{~s} 2=0.2$, Cond.Prob. $r 3 \mathrm{~s} 1=0.1$, Percent drop in speed $\%=-56$ Max. Posterior Probability level $=0.97$, Percent range between speed states $=7 \%$

(No Incident was Detected)

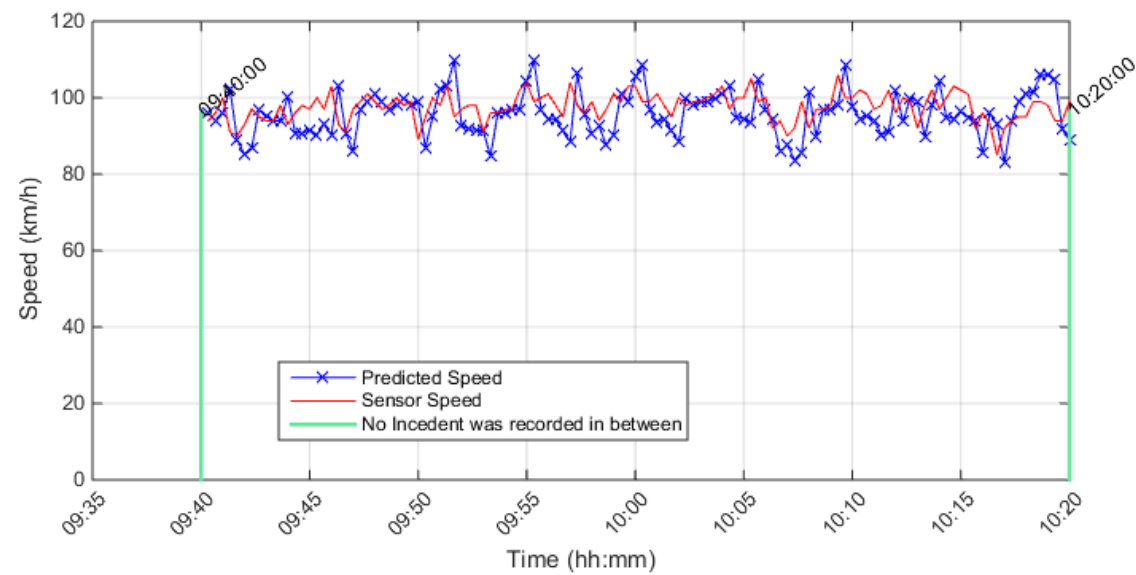

Upstream Detector \# is 401DE0160DWE, Detector Rank = 1, Distance from incident free location in $(\mathrm{km})=0.12782$ Incident free Season $=$ Summer, Stream $=E$, Direction $=$ W, Date $\&$ Time $=$ 2011-09-17 17:38:12

Selected incident free Date/Time $=2011-09-17$ 10:00:00

Conditional Probability level $=0.6$, Cond. Prob. $r 3 \mathrm{~s} 2=0.2$, Cond. Prob. $r 3 \mathrm{~s} 1=0.1$, Percent drop in speed $\%=-56$

Max. Posterior Probability level $=0.97$, Percent range between speed states $=7 \%$

(No Incident was Detected) 


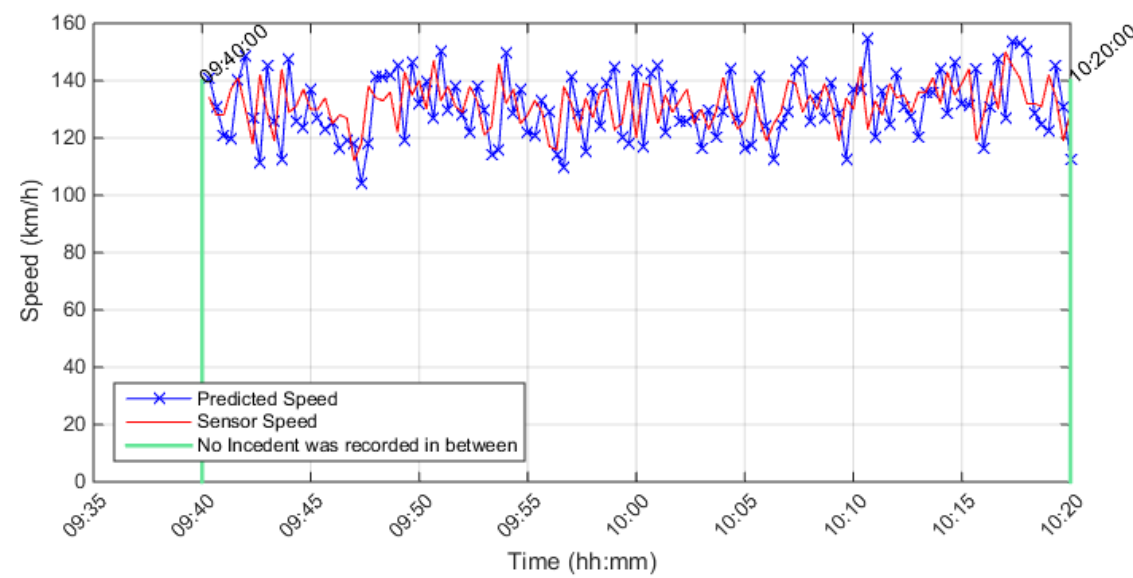

Upstream Detector \# is 401DE0050DWE, Detector Rank = 1, Distance from incident free location in $(\mathrm{km})=0.36941$ Incident free Season $=$ Summer, Stream $=E$, Direction $=W$, Date $\&$ Time $=2011-09-1719: 15: 50$ Selected incident free Date/Time $=2011-09-17$ 10:00:00

Conditional Probability level $=0.6$, Cond.Prob. $r 3 s 2=0.2$, Cond.Prob. $r 3 s 1=0.1$, Percent drop in speed $\%=-56$ Max. Posterior Probability level $=0.97$, Percent range between speed states $=7 \%$ (No Incident was Detected)

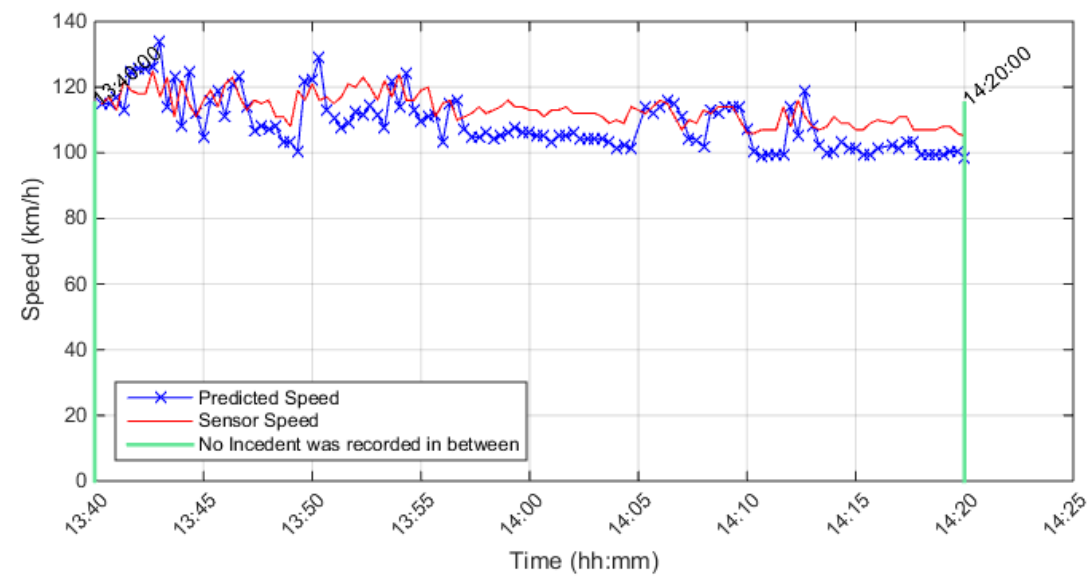

Upstream Detector \# is 401DE0020DWE, Detector Rank = 1, Distance from incident free location in $(\mathrm{km})=0.28759$ Incident free Season $=$ Summer, Stream $=E$, Direction $=W$, Date $\&$ Time $=2011-09-2007: 37: 47$ Selected incident free Date/Time $=2011-09-2014: 00: 00$

Conditional Probability level $=0.6$, Cond. Prob. $r 3 s 2=0.2$, Cond. Prob. $r 3 s 1=0.1$, Percent drop in speed $\%=-56$ Max. Posterior Probability level $=0.97$, Percent range between speed states $=7 \%$ (No Incident was Detected) 


\section{Appendix D Vissim Simulation}

\section{Appendix D.1 No Lane Blockage Scenario}

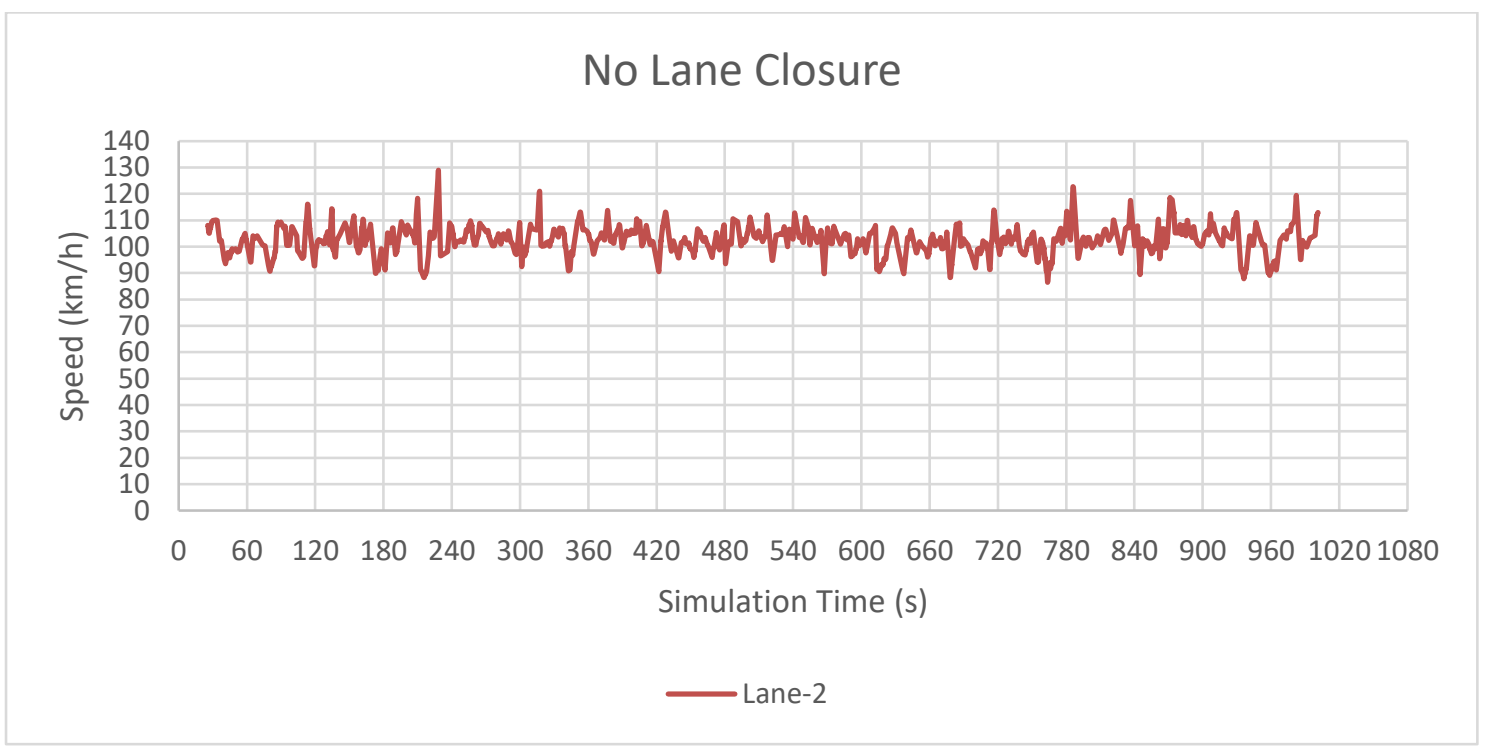

Speed Data Collected from Lane-2 on the Right

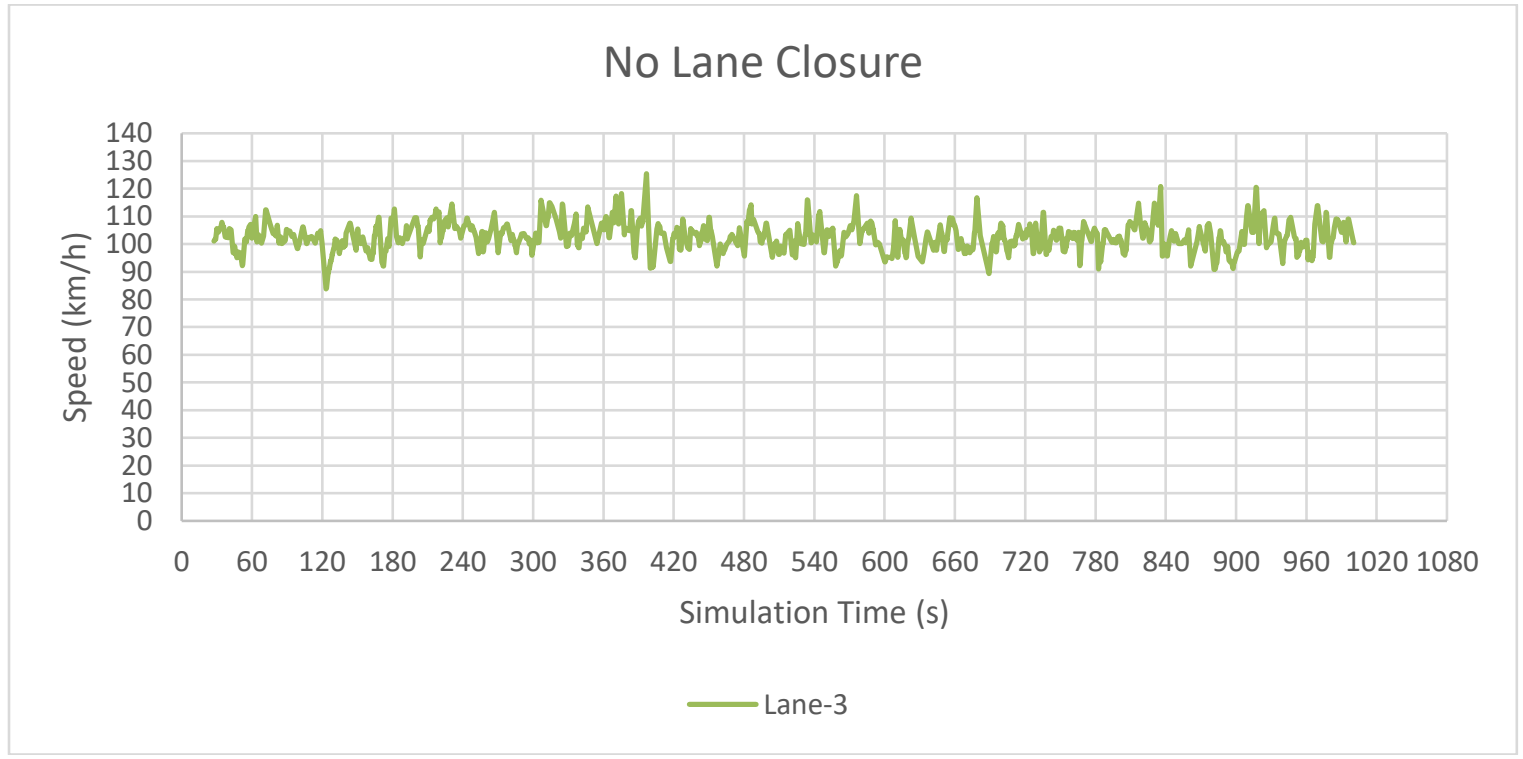

Speed Data Collected from Lane-3 on the Right 


\section{No Lane Closure}

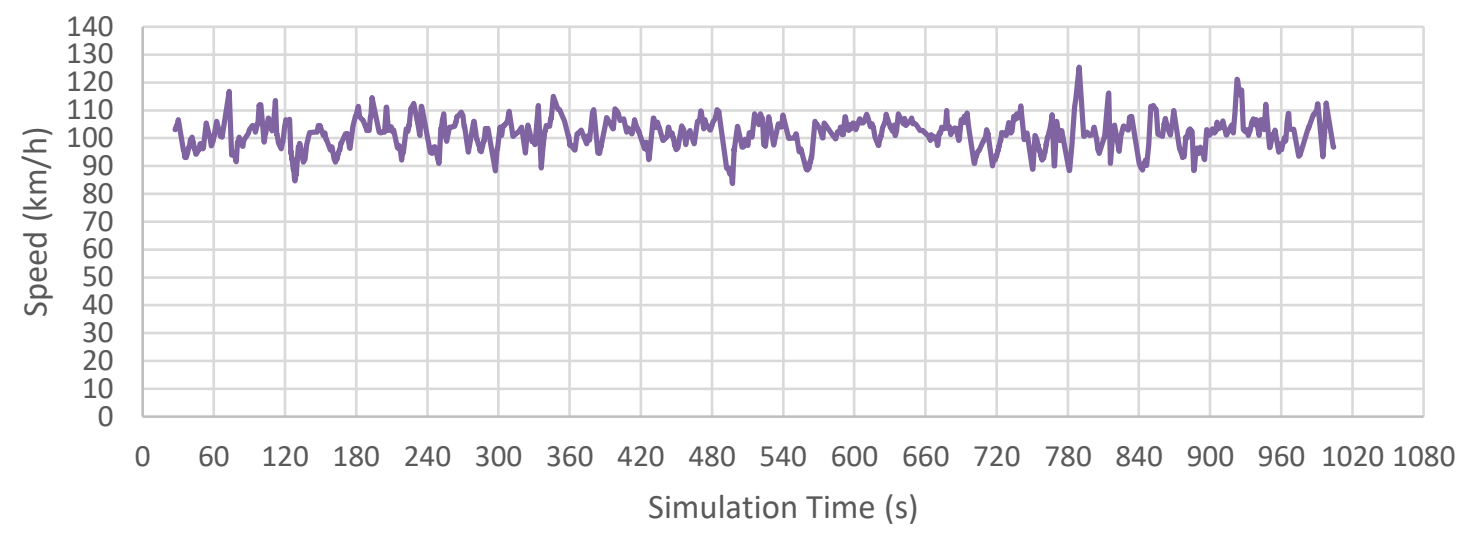

Lane-4

\section{Speed Data Collected from Lane-4 on the Right}

Incident detection application, no lane blockage, $v=2000 \mathrm{veh} / \mathrm{l} / \mathrm{h}$

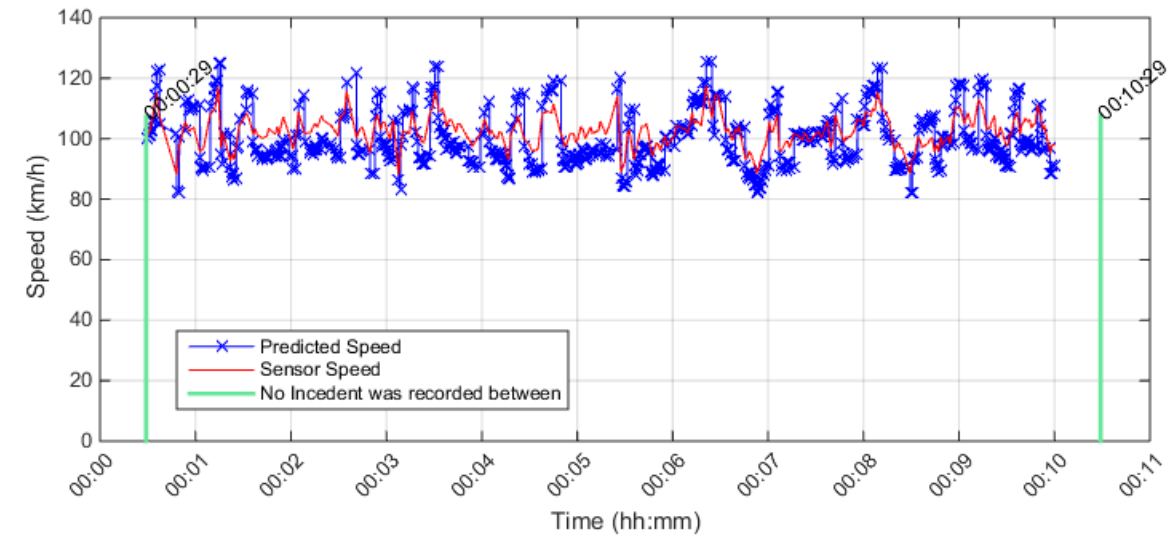

Upstream Detector \# is 401DE0010DEE, Detector Rank $=1$, Distance from incident free location in $(\mathrm{km})=0.35605$ Incident free Season $=$ Winter, Stream $=E$, Direction $=E$, Date \& Time $=$ 2011-02-01 14:30:30 Selected incident free Date/Time $=2011-02-1$ 00:00:29:00

Conditional Probability level $=0.6$, Cond.Prob. $r 3 \mathrm{~s} 2=0.2$, Cond.Prob. $r 3 \mathrm{~s} 1=0.1$, Percent drop in speed $\%=-56$

Max. Posterior Probability level $=0.97$, Percent range between speed states $=7 \%$

(No Incident was Detected)

\section{Incident Detection from Lane-1 on the Right-Run-2}




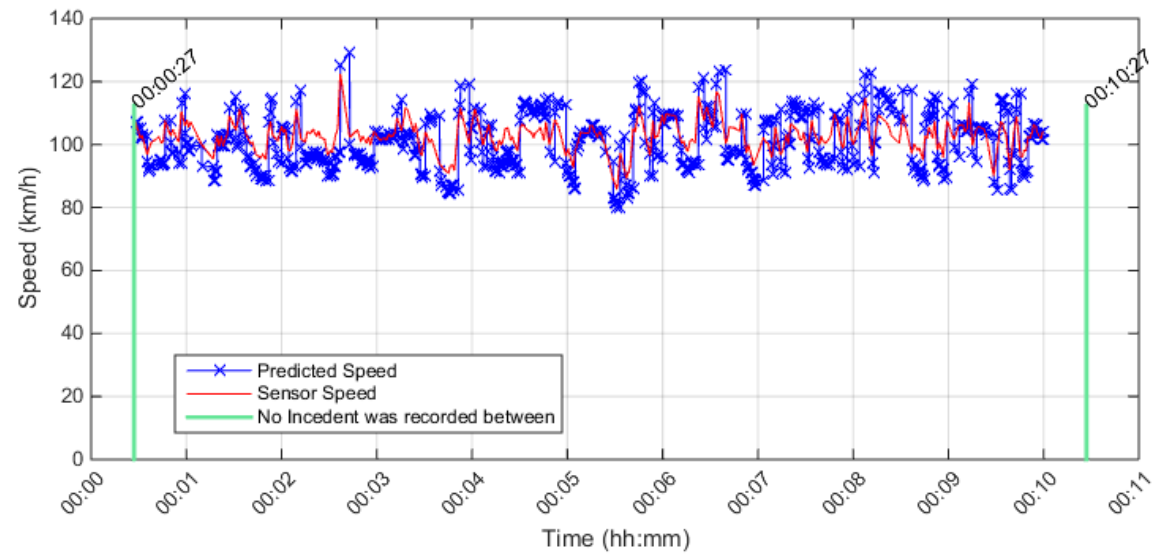

Upstream Detector \# is 401DE0010DEE, Detector Rank = 1, Distance from incident free location in $(\mathrm{km})=0.35605$ Incident free Season $=$ Winter, Stream $=E$, Direction $=E$, Date $\&$ Time $=2011-02-01$ 14:30:30 Selected incident free Date/Time $=2011-02-1$ 00:00:27:00

Conditional Probability level $=0.6$, Cond.Prob. $r 3 s 2=0.2$, Cond. Prob. $r 3 s 1=0.1$, Percent drop in speed $\%=-56$

Max. Posterior Probability level $=0.97$, Percent range between speed states $=7 \%$

(No Incident was Detected)

\section{Incident Detection from Lane-1 on the Right-Run-3}

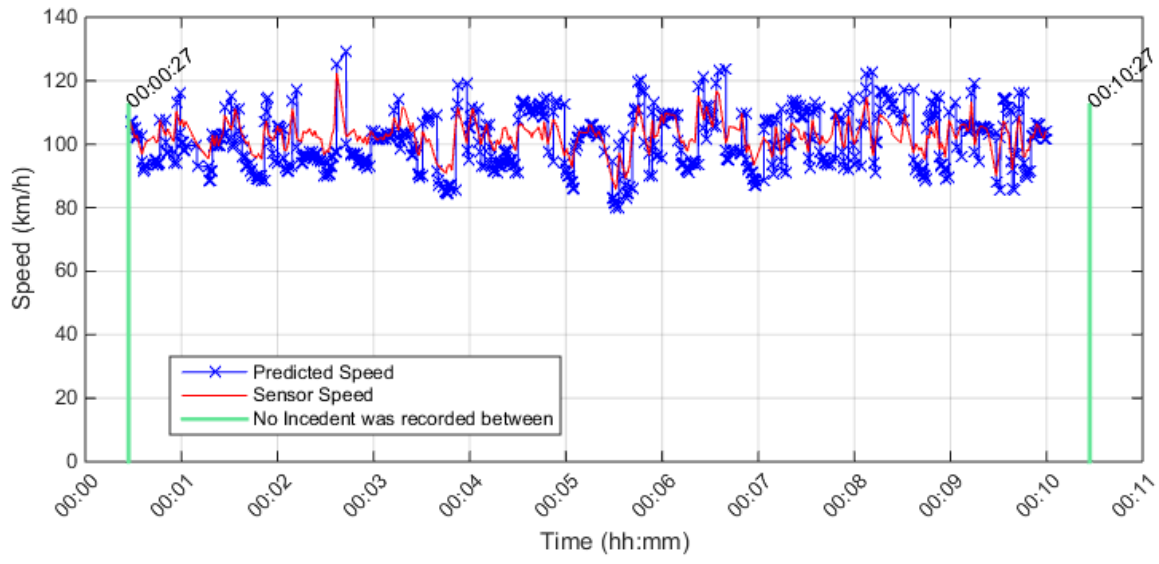

Upstream Detector \# is 401DE0010DEE, Detector Rank $=1$, Distance from incident free location in $(\mathrm{km})=0.35605$

Incident free Season $=$ Winter, Stream $=E$, Direction $=E$, Date \& Time $=$ 2011-02-01 14:30:30

Selected incident free Date/Time $=2011-02-1$ 00:00:27:00

Conditional Probability level $=0.6$, Cond.Prob. $r 3 s 2=0.2$, Cond.Prob. $r 3 s 1=0.1$, Percent drop in speed $\%=-56$

Max. Posterior Probability level $=0.97$, Percent range between speed states $=7 \%$

(No Incident was Detected)

\section{Incident Detection from Lane-1 on the Right-Run-4}




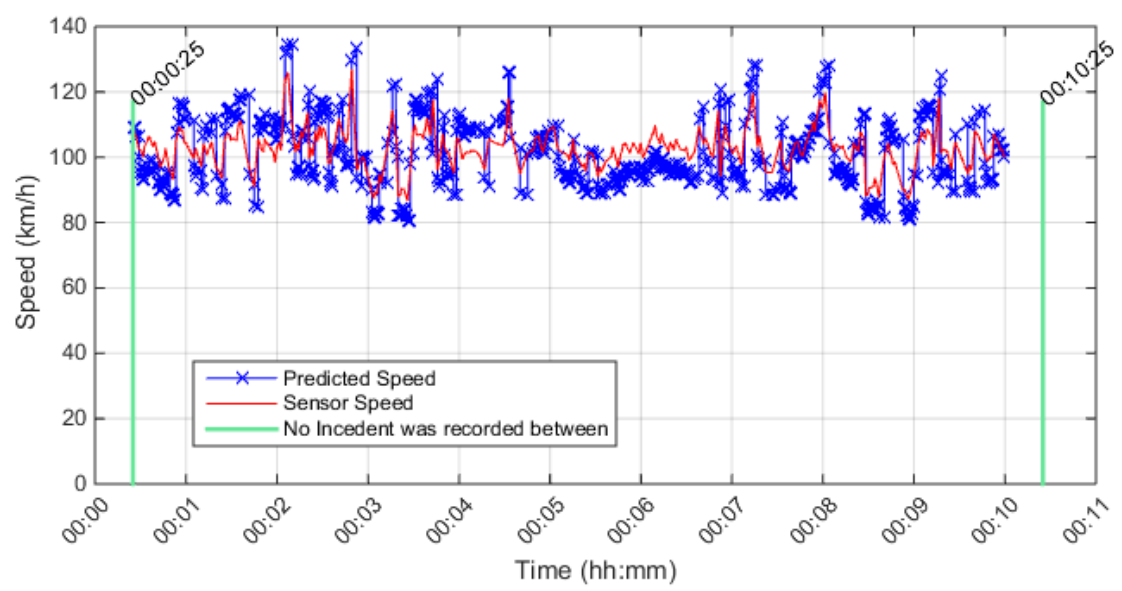

Upstream Detector \# is 401DE0010DEE, Detector Rank $=1$, Distance from incident free location in $(\mathrm{km})=0.35605$ Incident free Season $=$ Winter, Stream $=E$, Direction $=E$, Date $\&$ Time $=2011-02-0114: 30: 30$ Selected incident free Date/Time $=2011-02-100: 00: 25: 00$

Conditional Probability level $=0.6$, Cond.Prob. $r 3 s 2=0.2$, Cond. Prob. $r 3 s 1=0.1$, Percent drop in speed $\%=-56$

Max. Posterior Probability level $=0.97$, Percent range between speed states $=7 \%$

(No Incident was Detected)

\section{Incident Detection from Lane-1 on the Right-Run-5}

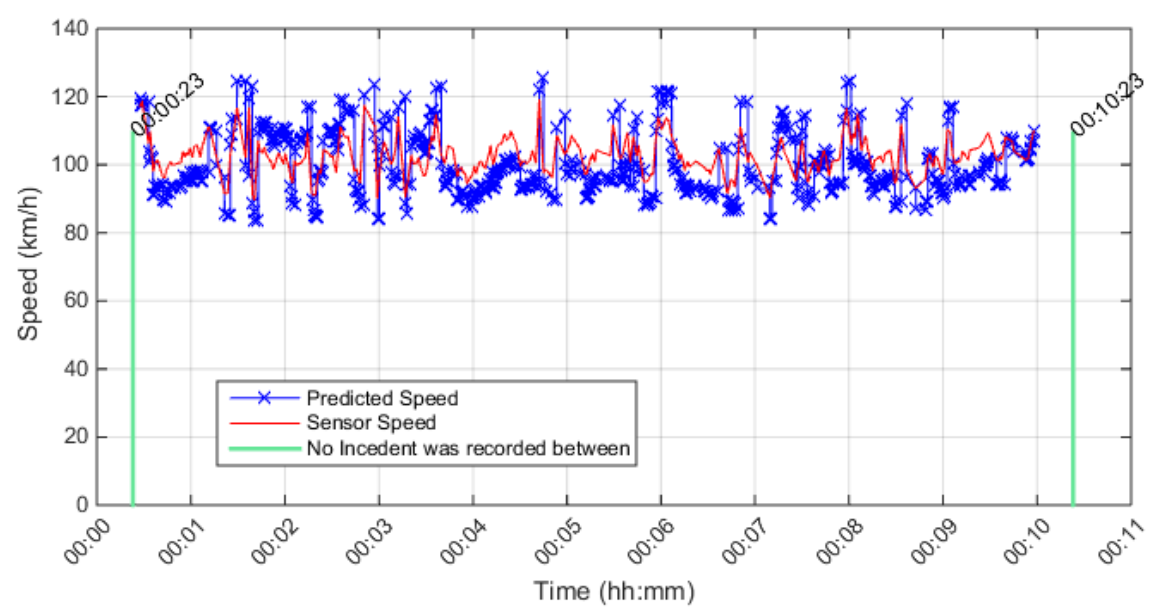

Upstream Detector \# is 401DE0010DEE, Detector Rank = 1, Distance from incident free location in $(\mathrm{km})=0.35605$ Incident free Season $=$ Winter, Stream $=E$, Direction $=E$, Date \& Time $=2011-02-0114: 30: 30$

Selected incident free Date/Time $=2011-02-1$ 00:00:23:00

Conditional Probability level $=0.6$, Cond.Prob. $r 3 s 2=0.2$, Cond.Prob. $r 3 s 1=0.1$, Percent drop in speed $\%=-56$

Max. Posterior Probability level $=0.97$, Percent range between speed states $=7 \%$

(No Incident was Detected)

\section{Incident Detection from Lane-1 on the Right-Run-6}




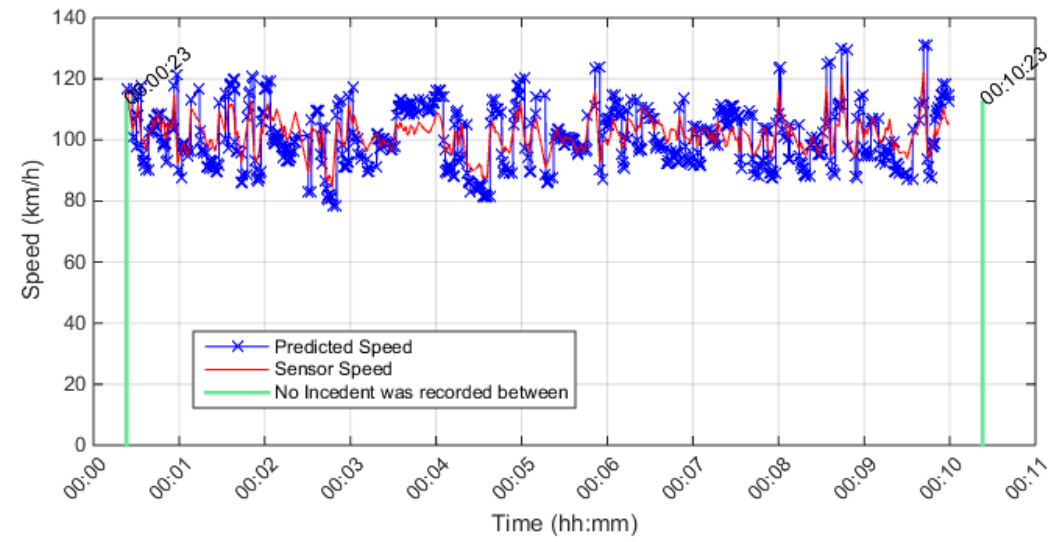

Upstream Detector \# is 401DE0010DEE, Detector Rank = 1, Distance from incident free location in $(\mathrm{km})=0.35605$ Incident free Season $=$ Winter, Stream $=E$, Direction $=E$, Date \& Time $=$ 2011-02-01 14:30:30 Selected incident free Date/Time $=$ 2011-02-1 00:00:23:00

Conditional Probability level $=0.6$, Cond. Prob. $r 3 s 2=0.2$, Cond. Prob. $r 3 s 1=0.1$, Percent drop in speed $\%=-56$ Max. Posterior Probability level $=0.97$, Percent range between speed states $=7 \%$ (No Incident was Detected)

\section{Incident Detection from Lane-1 on the Right-Run-7}

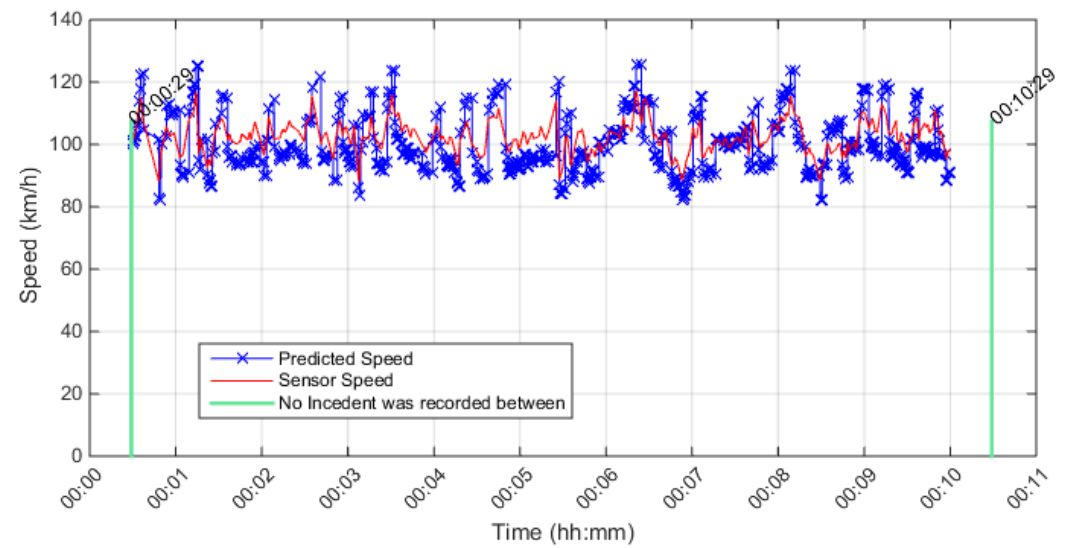

Upstream Detector \# is 401DE0010DEE, Detector Rank $=1$, Distance from incident free location in $(\mathrm{km})=0.35605$ Incident free Season $=$ Winter, Stream $=E$, Direction $=E$, Date \& Time $=$ 2011-02-01 14:30:30

Selected incident free Date/Time $=2011-02-1$ 00:00:29:00

Conditional Probability level $=0.6$, Cond. Prob. $r 3 \mathrm{~s} 2=0.2$, Cond. Prob. $r 3 \mathrm{~s} 1=0.1$, Percent drop in speed $\%=-56$

Max. Posterior Probability level $=0.97$, Percent range between speed states $=7 \%$

(No Incident was Detected)

\section{Incident Detection from Lane-1 on the Right-Run-8}




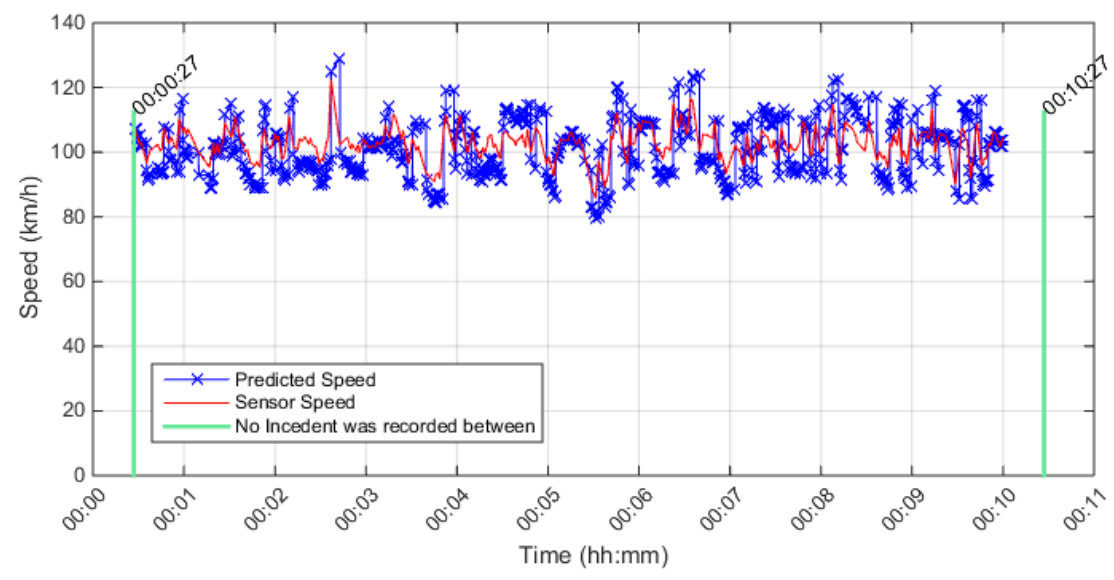

Upstream Detector \# is 401DE0010DEE, Detector Rank = 1, Distance from incident free location in $(\mathrm{km})=0.35605$ Incident free Season $=$ Winter, Stream $=E$, Direction $=E$, Date \& Time $=$ 2011-02-01 14:30:30

Selected incident free Date/Time $=$ 2011-02-1 00:00:27:00

Conditional Probability level $=0.6$, Cond.Prob. $r 3 \mathrm{~s} 2=0.2$, Cond. Prob. $\mathrm{r} 3 \mathrm{~s} 1=0.1$, Percent drop in speed $\%=-56$

Max. Posterior Probability level $=0.97$, Percent range between speed states $=7 \%$

(No Incident was Detected)

\section{Incident Detection from Lane-1 on the Right-Run-9}

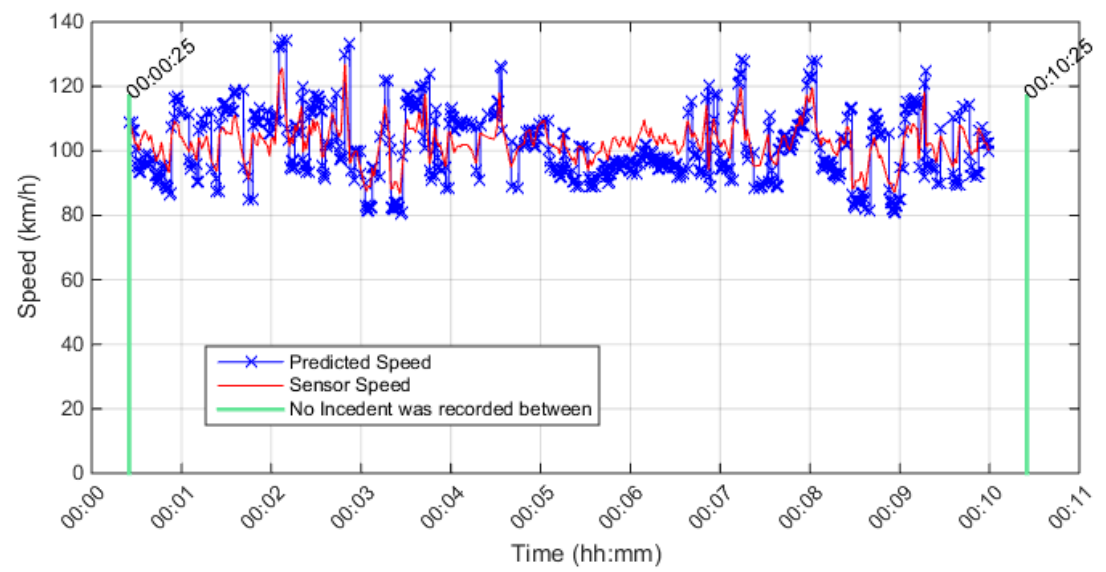

Upstream Detector \# is 401DE0010DEE, Detector Rank $=1$, Distance from incident free location in $(\mathrm{km})=0.35605$ Incident free Season $=$ Winter, Stream $=E$, Direction $=E$, Date \& Time $=2011-02-0114: 30: 30$

Selected incident free Date/Time $=2011-02-1$ 00:00:25:00

Conditional Probability level $=0.6$, Cond.Prob. $r 3 s 2=0.2$, Cond.Prob. $r 3 s 1=0.1$, Percent drop in speed $\%=-56$

Max. Posterior Probability level $=0.97$, Percent range between speed states $=7 \%$

(No Incident was Detected)

\section{Incident Detection from Lane-1 on the Right-Run-10}




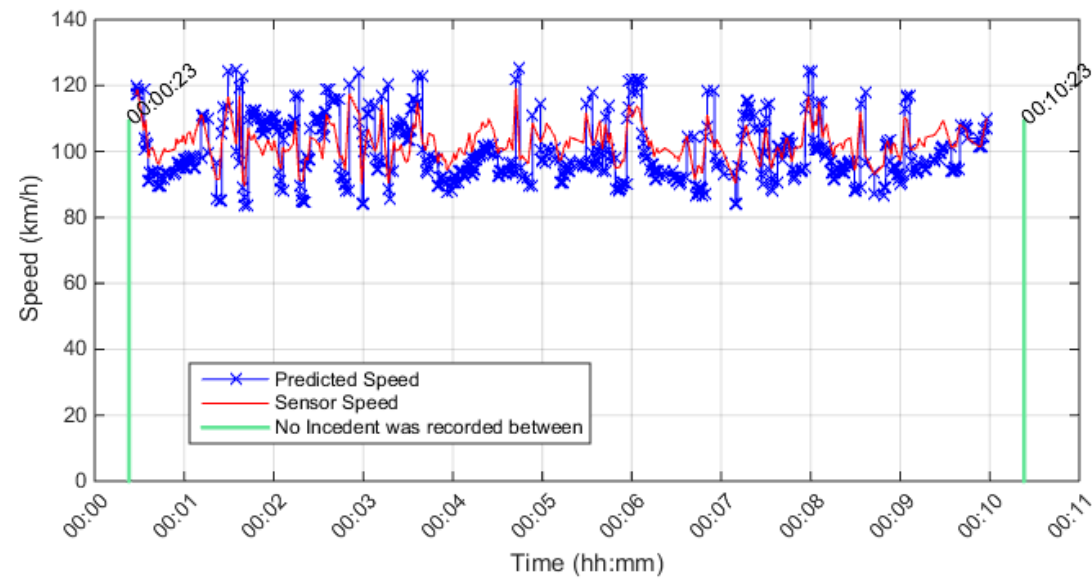

Upstream Detector \# is 401DE0010DEE, Detector Rank = 1, Distance from incident free location in $(\mathrm{km})=0.35605$ Incident free Season $=$ Winter, Stream $=E$, Direction $=E$, Date \& Time $=$ 2011-02-01 14:30:30 Selected incident free Date/Time $=2011-02-1$ 00:00:23:00

Conditional Probability level $=0.6$, Cond.Prob. $r 3 \mathrm{~s} 2=0.2$, Cond. Prob. $r 3 \mathrm{~s} 1=0.1$, Percent drop in speed $\%=-56$

Max. Posterior Probability level $=0.97$, Percent range between speed states $=7 \%$

(No Incident was Detected)

\section{Incident Detection from Lane-1 on the Right-Run-11}

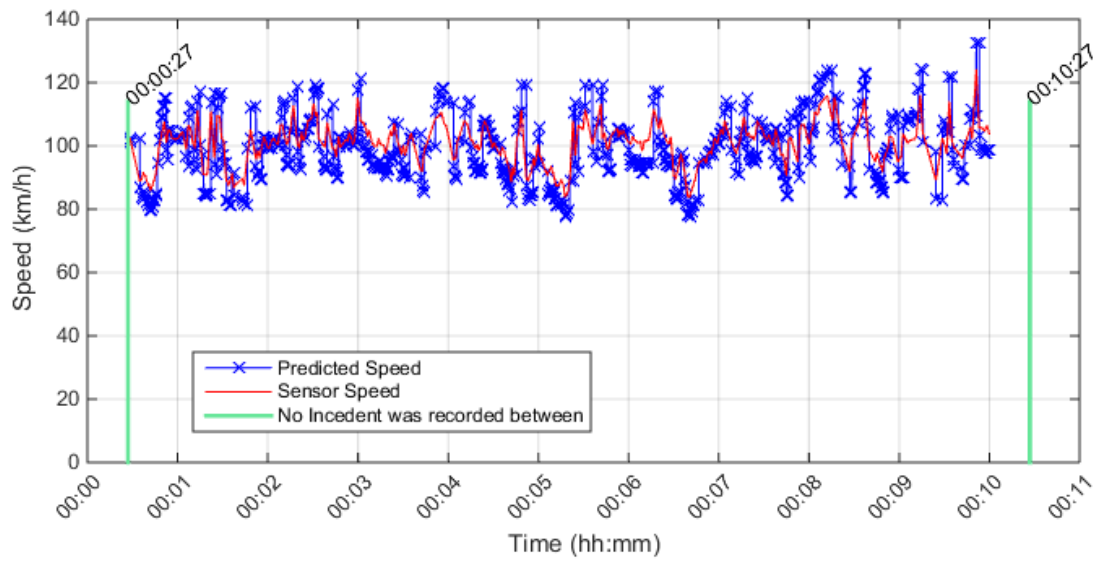

Upstream Detector \# is 401DE0010DEE, Detector Rank $=1$, Distance from incident free location in $(\mathrm{km})=0.35605$ Incident free Season $=$ Winter, Stream $=E$, Direction $=E$, Date $\&$ Time $=2011-02-0114: 30: 30$ Selected incident free Date/Time $=2011-02-1$ 00:00:27:00

Conditional Probability level $=0.6$, Cond.Prob. $r 3 \mathrm{~s} 2=0.2$, Cond. Prob. $r 3 \mathrm{~s} 1=0.1$, Percent drop in speed $\%=-56$

Max. Posterior Probability level $=0.97$, Percent range between speed states $=7 \%$

(No Incident was Detected)

\section{Incident Detection from Lane-1 on the Right-Run-12}




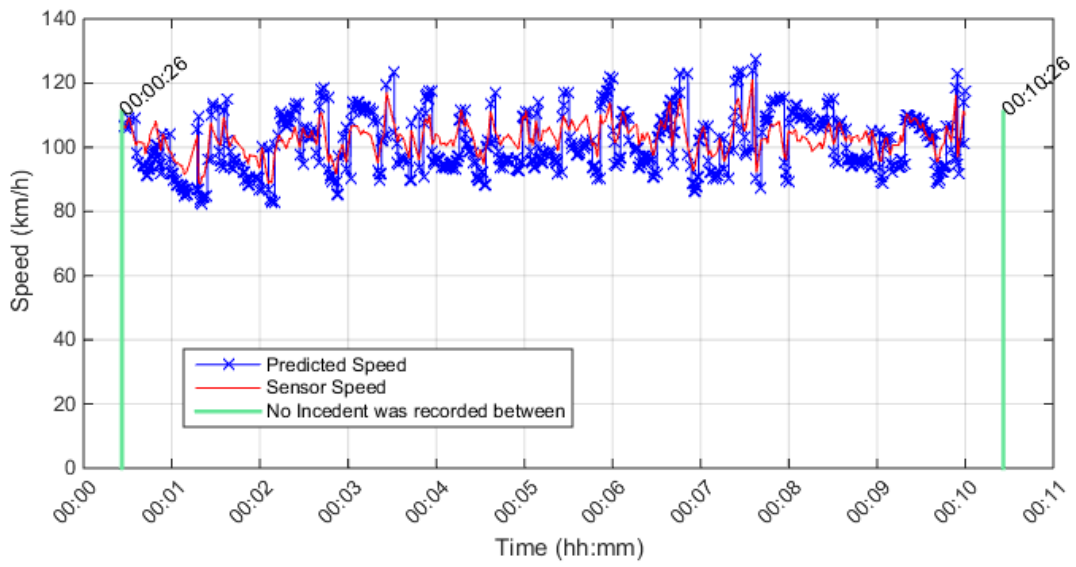

Upstream Detector \# is 401DE0010DEE, Detector Rank $=1$, Distance from incident free location in $(\mathrm{km})=0.35605$ Incident free Season $=$ Winter, Stream $=E$, Direction $=E$, Date \& Time $=$ 2011-02-01 14:30:30

Selected incident free Date/Time $=2011-02-1$ 00:00:26:00

Conditional Probability level $=0.6$, Cond.Prob. $r 3 s 2=0.2$, Cond.Prob. $r 3 s 1=0.1$, Percent drop in speed $\%=-56$ Max. Posterior Probability level $=0.97$, Percent range between speed states $=7 \%$ (No Incident was Detected)

\section{Incident Detection from Lane-1 on the Right-Run-13}

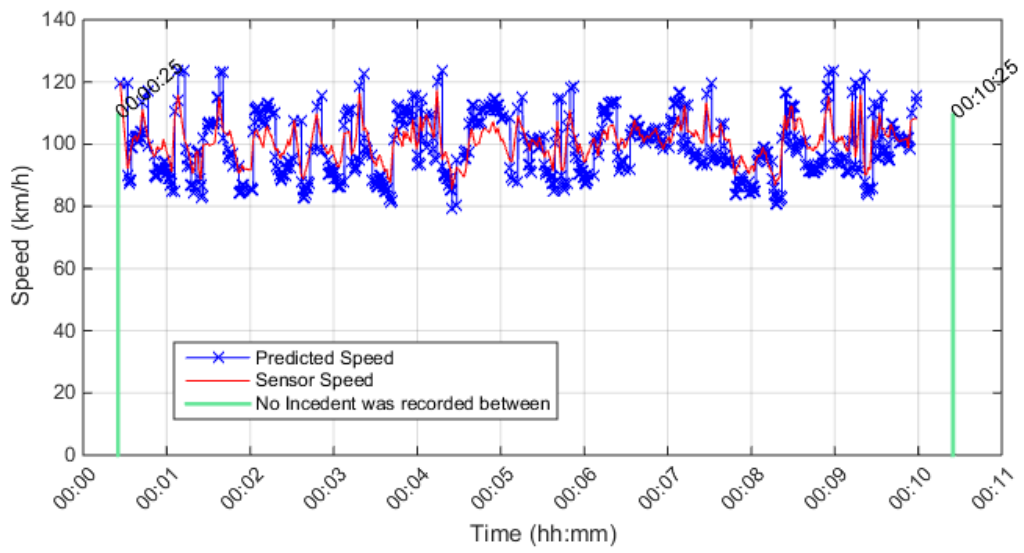

Upstream Detector \# is 401DE0010DEE, Detector Rank = 1, Distance from incident free location in $(\mathrm{km})=0.35605$ Incident free Season $=$ Winter, Stream $=E$, Direction $=E$, Date \& Time $=$ 2011-02-01 14:30:30 Selected incident free Date/Time $=2011-02-1$ 00:00:25:00

Conditional Probability level $=0.6$, Cond.Prob. $\mathrm{r} 3 \mathrm{~s} 2=0.2$, Cond. Prob. $\mathrm{r} 3 \mathrm{~s} 1=0.1$, Percent drop in speed $\%=-56$ Max. Posterior Probability level $=0.97$, Percent range between speed states $=7 \%$ (No Incident was Detected)

\section{Incident Detection from Lane-1 on the Right-Run-14}




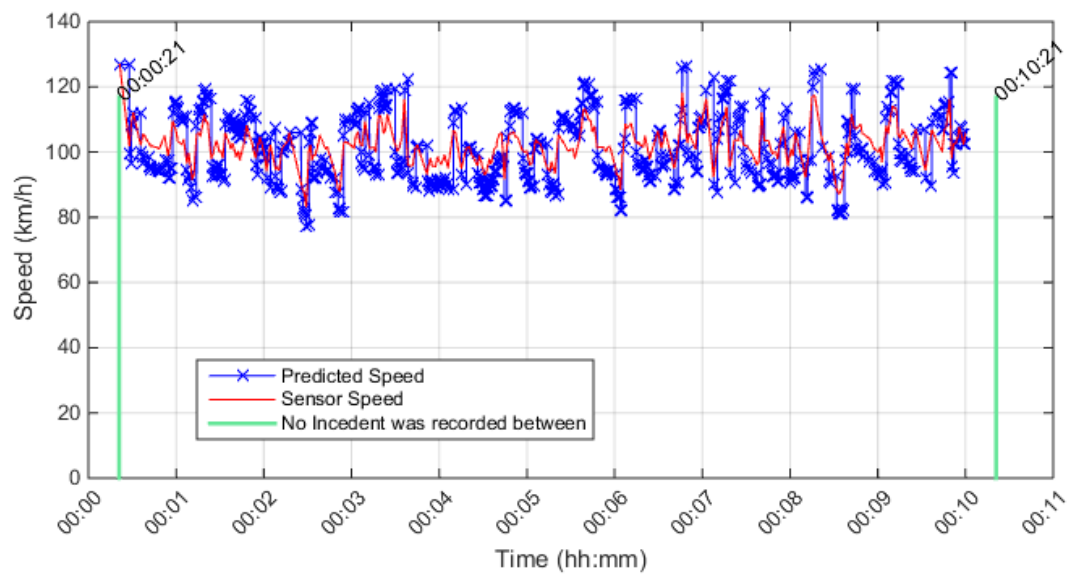

Upstream Detector \# is 401DE0010DEE, Detector Rank = 1, Distance from incident free location in $(\mathrm{km})=0.35605$ Incident free Season $=$ Winter, Stream $=E$, Direction $=E$, Date \& Time $=2011-02-0114: 30: 30$

Selected incident free Date/Time $=2011-02-1$ 00:00:21:00

Conditional Probability level $=0.6$, Cond. Prob. $r 3 s 2=0.2$, Cond.Prob. $r 3 s 1=0.1$, Percent drop in speed $\%=-56$

Max. Posterior Probability level $=0.97$, Percent range between speed states $=7 \%$

(No Incident was Detected)

\section{Incident Detection from Lane-1 on the Right-Run-15}

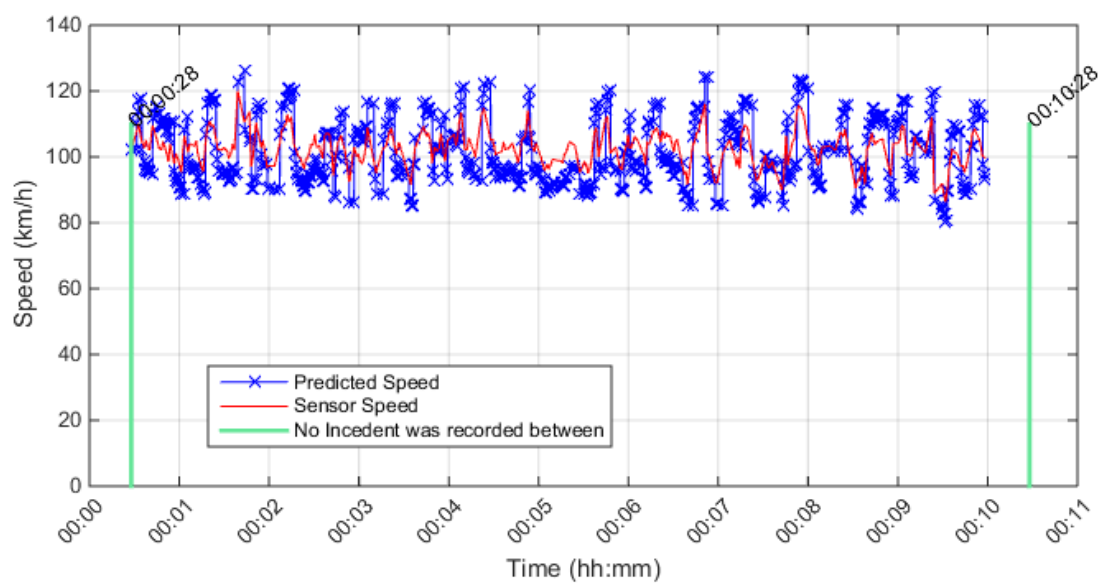

Upstream Detector \# is 401DE0010DEE, Detector Rank = 1, Distance from incident free location in $(\mathrm{km})=0.35605$ Incident free Season $=$ Winter, Stream $=E$, Direction $=E$, Date $\&$ Time $=2011-02-01$ 14:30:30 Selected incident free Date/Time $=$ 2011-02-1 00:00:28:00

Conditional Probability level $=0.6$, Cond. Prob. $r 3 s 2=0.2$, Cond. Prob. $r 3 s 1=0.1$, Percent drop in speed $\%=-56$

Max. Posterior Probability level $=0.97$, Percent range between speed states $=7 \%$ (No Incident was Detected)

Incident Detection from Lane-1 on the Right-Run-16 


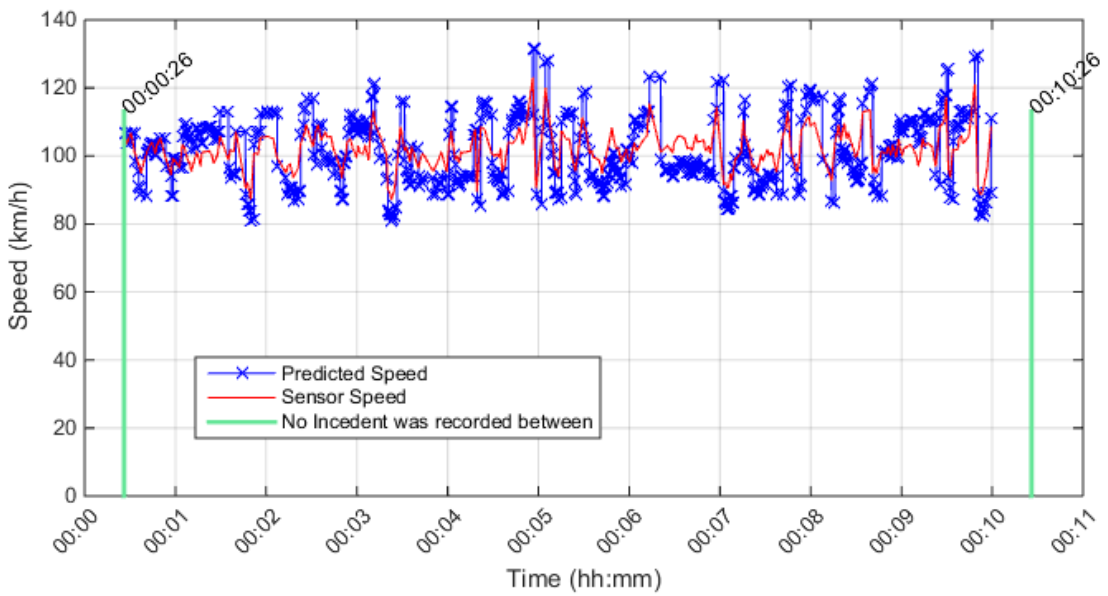

Upstream Detector \# is 401DE0010DEE, Detector Rank = 1, Distance from incident free location in $(\mathrm{km})=0.35605$ Incident free Season $=$ Winter, Stream $=E$, Direction $=E$, Date $\&$ Time $=2011-02-01$ 14:30:30 Selected incident free Date/Time $=$ 2011-02-1 00:00:26:00

Conditional Probability level $=0.6$, Cond.Prob. $r 3 s 2=0.2$, Cond.Prob. $r 3 s 1=0.1$, Percent drop in speed $\%=-56$

Max. Posterior Probability level $=0.97$, Percent range between speed states $=7 \%$ (No Incident was Detected)

\section{Incident Detection from Lane-1 on the Right-Run-17}

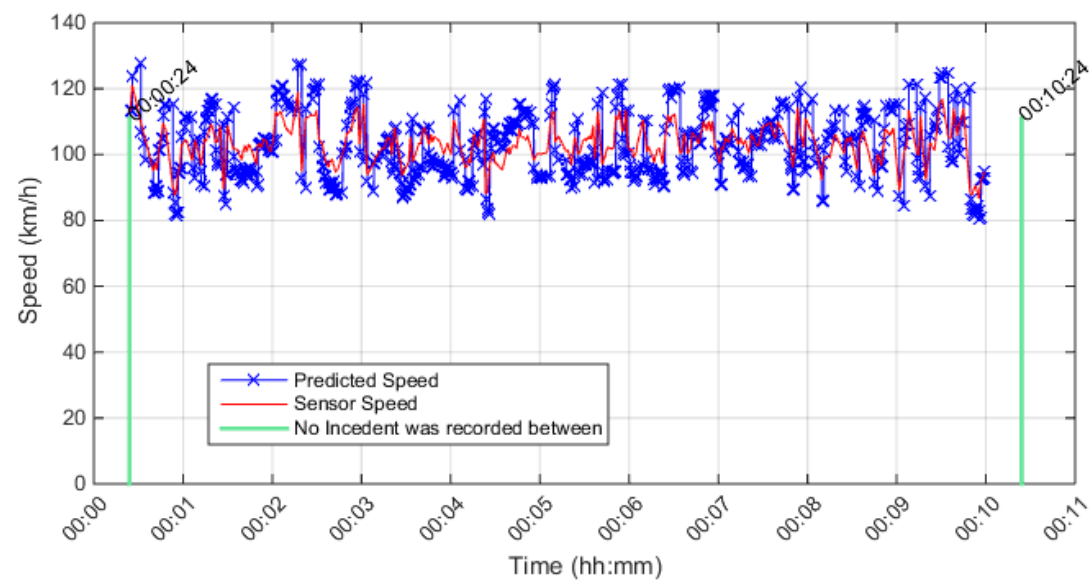

Upstream Detector \# is 401DE0010DEE, Detector Rank $=1$, Distance from incident free location in $(\mathrm{km})=0.35605$ Incident free Season $=$ Winter, Stream $=E$, Direction $=E$, Date $\&$ Time $=2011-02-0114: 30: 30$ Selected incident free Date/Time $=2011-02-1$ 00:00:24:00

Conditional Probability level $=0.6$, Cond. Prob. $r 3 s 2=0.2$, Cond. Prob. $r 3 s 1=0.1$, Percent drop in speed $\%=-56$ Max. Posterior Probability level $=0.97$, Percent range between speed states $=7 \%$ (No Incident was Detected)

\section{Incident Detection from Lane-1 on the Right-Run-18}




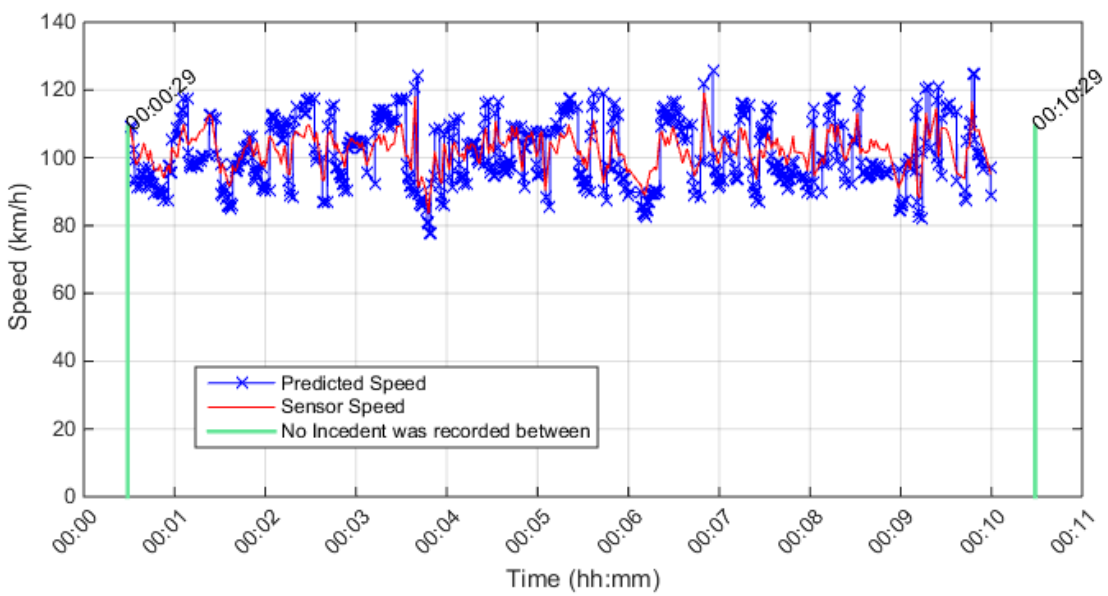

Upstream Detector \# is 401DE0010DEE, Detector Rank = 1, Distance from incident free location in $(\mathrm{km})=0.35605$ Incident free Season $=$ Winter, Stream $=E$, Direction $=E$, Date $\&$ Time $=$ 2011-02-01 14:30:30 Selected incident free Date/Time $=$ 2011-02-1 00:00:29:00

Conditional Probability level $=0.6$, Cond.Prob. $r 3 \mathrm{~s} 2=0.2$, Cond. Prob. $r 3 \mathrm{~s} 1=0.1$, Percent drop in speed $\%=-56$

Max. Posterior Probability level $=0.97$, Percent range between speed states $=7 \%$

(No Incident was Detected)

\section{Incident Detection from Lane-1 on the Right-Run-19}

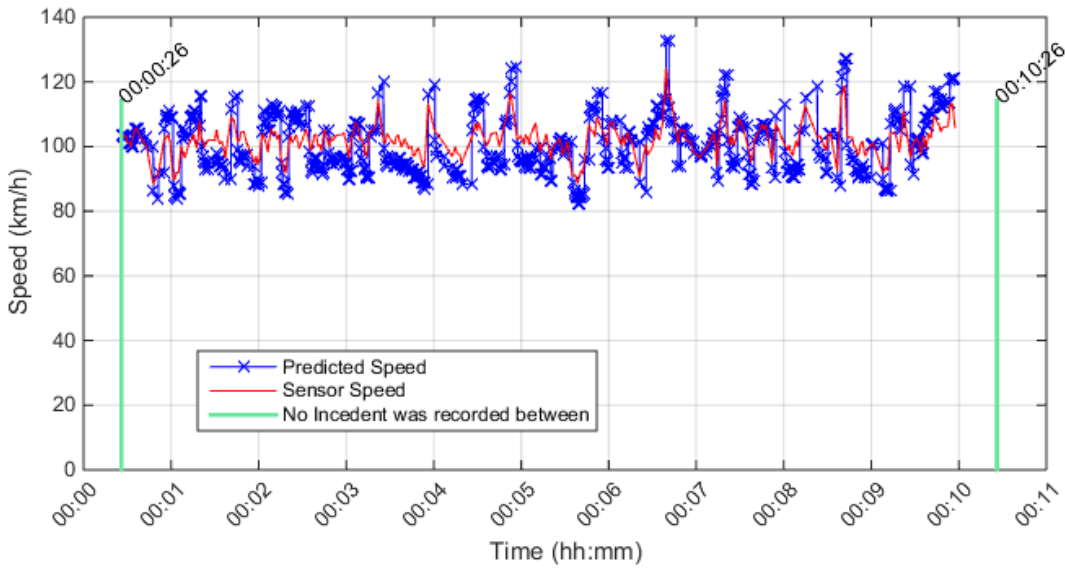

Upstream Detector \# is 401DE0010DEE, Detector Rank = 1, Distance from incident free location in $(\mathrm{km})=0.35605$ Incident free Season $=$ Winter, Stream $=E$, Direction $=E$, Date \& Time $=2011-02-0114: 30: 30$ Selected incident free Date/Time $=2011-02-1$ 00:00:26:00

Conditional Probability level $=0.6$, Cond.Prob. $\mathrm{r} 3 \mathrm{~s} 2=0.2$, Cond. Prob. $\mathrm{r} 3 \mathrm{~s} 1=0.1$, Percent drop in speed $\%=-56$

Max. Posterior Probability level $=0.97$, Percent range between speed states $=7 \%$

(No Incident was Detected)

\section{Incident Detection from Lane-1 on the Right-Run-20}




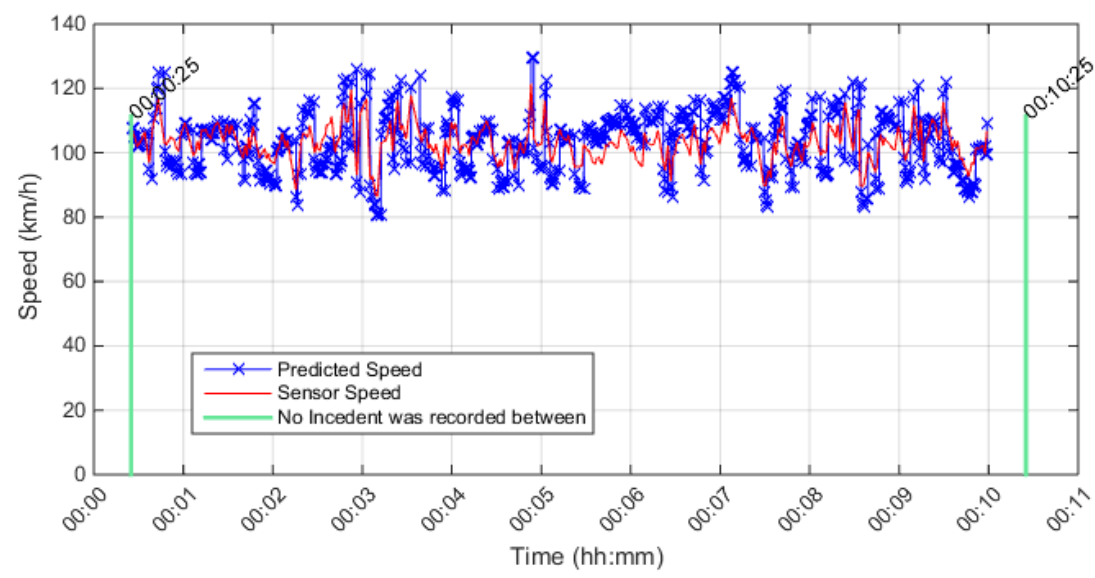

Upstream Detector \# is 401DE0010DEE, Detector Rank = 1, Distance from incident free location in $(\mathrm{km})=0.35605$ Incident free Season $=$ Winter, Stream $=E$, Direction $=E$, Date $\&$ Time $=2011-02-01$ 14:30:30 Selected incident free Date/Time $=2011-02-1$ 00:00:25:00

Conditional Probability level $=0.6$, Cond. Prob. $r 3 s 2=0.2$, Cond. Prob. $r 3 s 1=0.1$, Percent drop in speed $\%=-56$ Max. Posterior Probability level $=0.97$, Percent range between speed states $=7 \%$ (No Incident was Detected)

\section{Incident Detection from Lane-1 on the Right-Run-21}

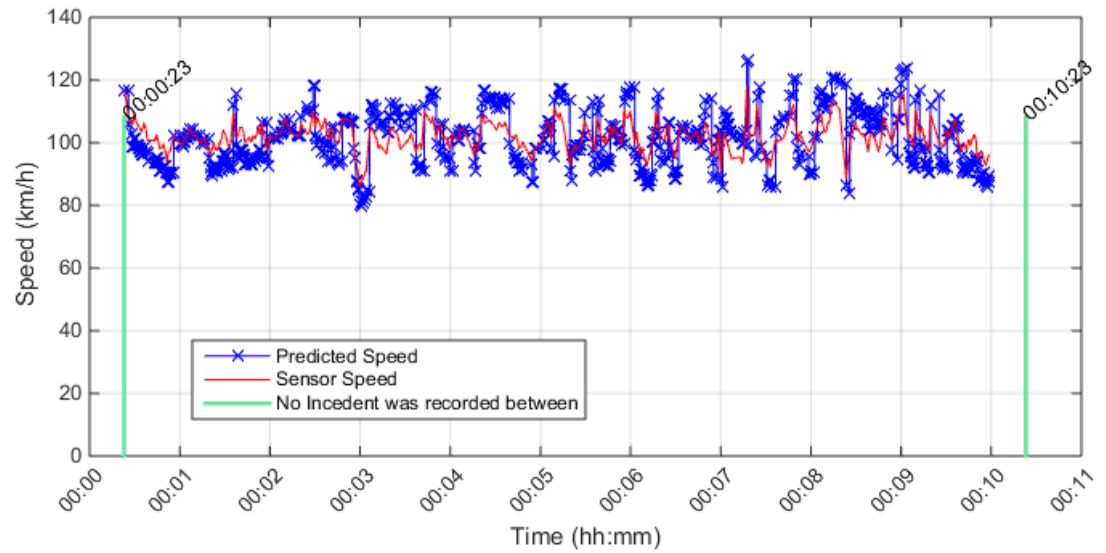

Upstream Detector \# is 401DE0010DEE, Detector Rank = 1, Distance from incident free location in $(\mathrm{km})=0.35605$ Incident free Season $=$ Winter, Stream $=$ E, Direction $=E$, Date \& Time $=2011-02-0114: 30: 30$ Selected incident free Date/Time $=$ 2011-02-1 00:00:23:00

Conditional Probability level $=0.6$, Cond.Prob. $r 3 s 2=0.2$, Cond. Prob. $r 3 s 1=0.1$, Percent drop in speed $\%=-56$

Max. Posterior Probability level $=0.97$, Percent range between speed states $=7 \%$

(No Incident was Detected)

\section{Incident Detection from Lane-1 on the Right-Run-22}




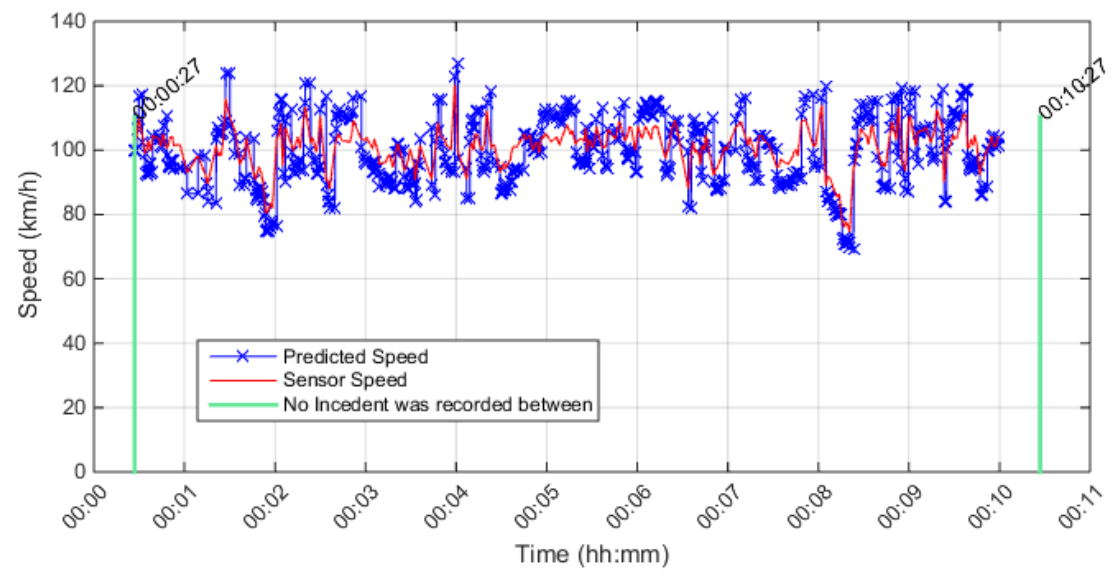

Upstream Detector \# is 401DE0010DEE, Detector Rank = 1, Distance from incident free location in $(\mathrm{km})=0.35605$ Incident free Season $=$ Winter, Stream $=E$, Direction $=E$, Date \& Time $=$ 2011-02-01 14:30:30

Selected incident free Date/Time $=2011-02-1$ 00:00:27:00

Conditional Probability level $=0.6$, Cond.Prob. $r 3 s 2=0.2$, Cond.Prob. $r 3 s 1=0.1$, Percent drop in speed $\%=-56$

Max. Posterior Probability level $=0.97$, Percent range between speed states $=7 \%$

(No Incident was Detected)

Incident Detection from Lane-1 on the Right-Run-23

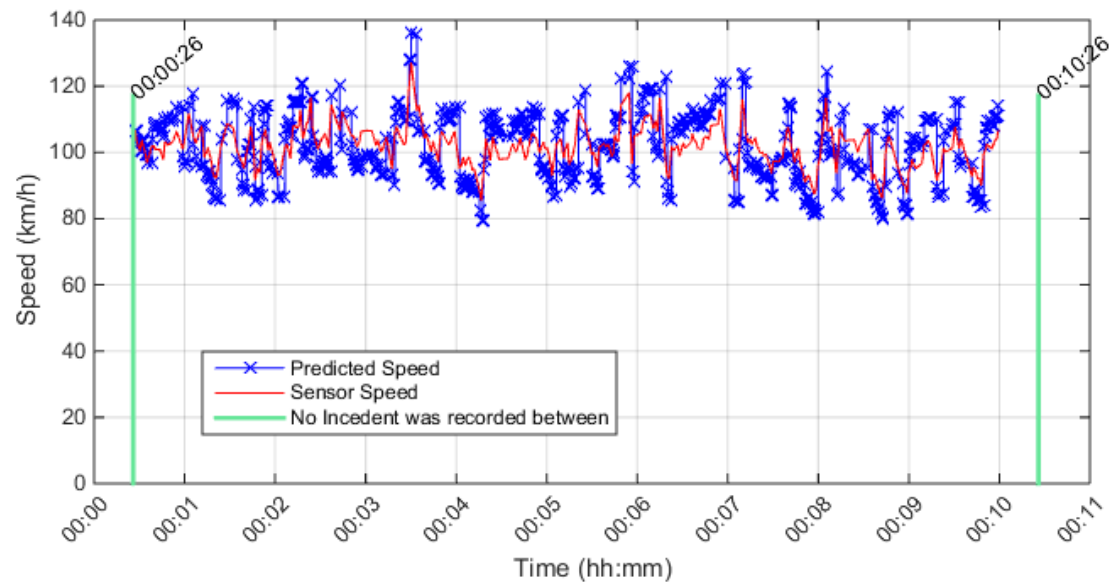

Upstream Detector \# is 401DE0010DEE, Detector Rank $=1$, Distance from incident free location in $(\mathrm{km})=0.35605$ Incident free Season $=$ Winter, Stream $=E$, Direction $=E$, Date $\&$ Time $=2011-02-0114: 30: 30$ Selected incident free Date/Time $=2011-02-1$ 00:00:26:00

Conditional Probability level $=0.6$, Cond.Prob. $r 3 s 2=0.2$, Cond. Prob. $r 3 s 1=0.1$, Percent drop in speed $\%=-56$

Max. Posterior Probability level $=0.97$, Percent range between speed states $=7 \%$

(No Incident was Detected)

\section{Incident Detection from Lane-1 on the Right-Run-24}




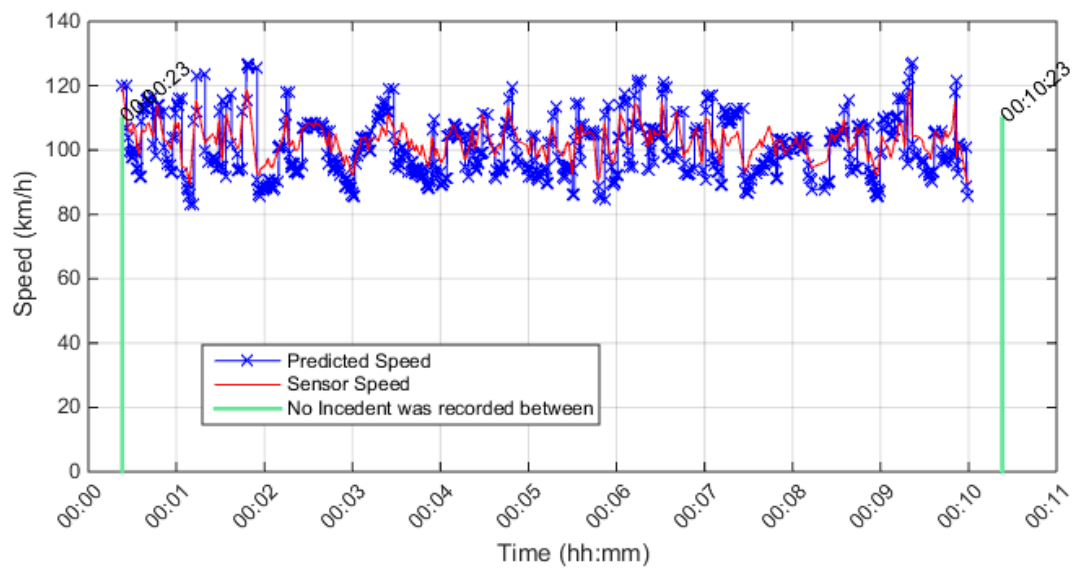

Upstream Detector \# is 401DE0010DEE, Detector Rank $=1$, Distance from incident free location in $(\mathrm{km})=0.35605$ Incident free Season $=$ Winter, Stream $=E$, Direction $=E$, Date $\&$ Time $=2011-02-0114: 30: 30$ Selected incident free Date/Time $=2011-02-1$ 00:00:23:00

Conditional Probability level $=0.6$, Cond.Prob. $r 3 s 2=0.2$, Cond. Prob. $r 3 s 1=0.1$, Percent drop in speed $\%=-56$ Max. Posterior Probability level $=0.97$, Percent range between speed states $=7 \%$ (No Incident was Detected)

Incident Detection from Lane-1 on the Right-Run-25

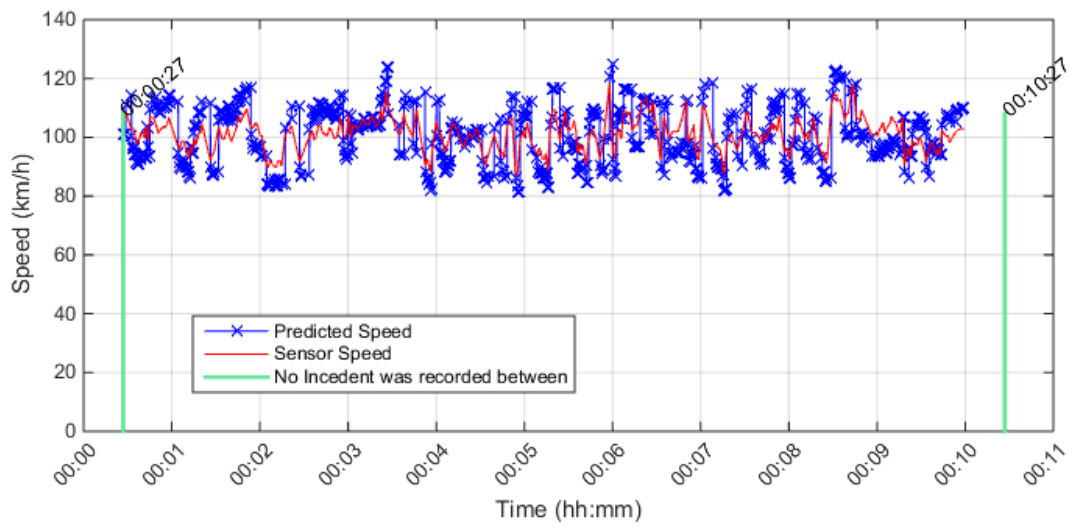

Upstream Detector \# is 401DE0010DEE, Detector Rank = 1, Distance from incident free location in $(\mathrm{km})=0.35605$ Incident free Season $=$ Winter, Stream $=E$, Direction $=E$, Date \& Time $=$ 2011-02-01 14:30:30 Selected incident free Date/Time $=2011-02-1$ 00:00:27:00

Conditional Probability level $=0.6$, Cond.Prob. $r 3 \mathrm{~s} 2=0.2$, Cond. Prob. $r 3 \mathrm{~s} 1=0.1$, Percent drop in speed $\%=-56$

Max. Posterior Probability level $=0.97$, Percent range between speed states $=7 \%$

(No Incident was Detected)

Incident Detection from Lane-1 on the Right-Run-26 


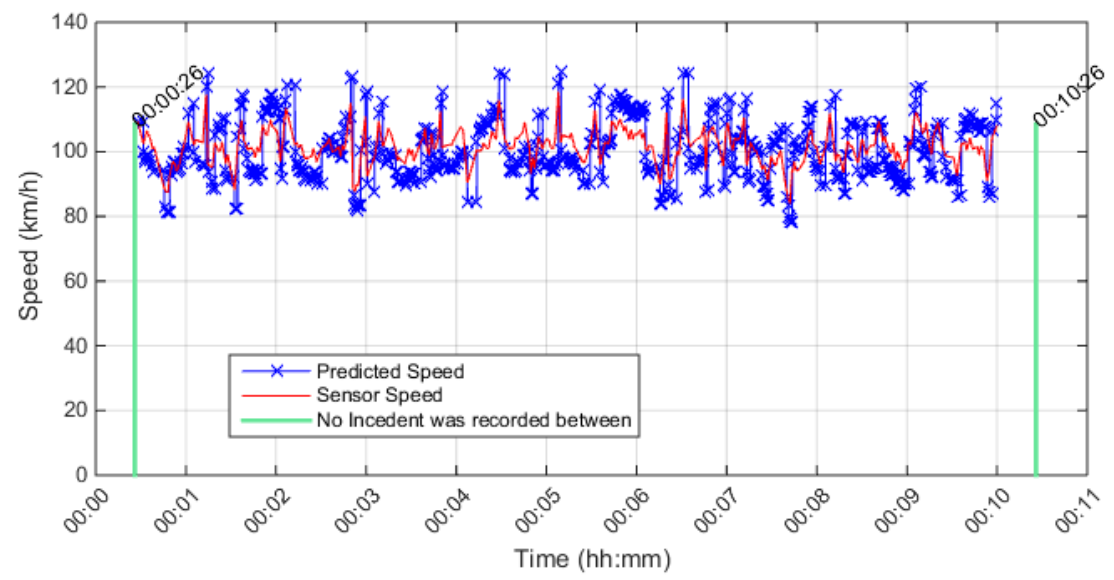

Upstream Detector \# is 401DE0010DEE, Detector Rank = 1, Distance from incident free location in $(\mathrm{km})=0.35605$ Incident free Season $=$ Winter, Stream $=E$, Direction $=E$, Date $\&$ Time $=2011-02-01$ 14:30:30

Selected incident free Date/Time $=$ 2011-02-1 00:00:26:00

Conditional Probability level $=0.6$, Cond.Prob. $r 3 s 2=0.2$, Cond.Prob. $r 3 s 1=0.1$, Percent drop in speed $\%=-56$

Max. Posterior Probability level $=0.97$, Percent range between speed states $=7 \%$

(No Incident was Detected)

\section{Incident Detection from Lane-1 on the Right-Run-27}

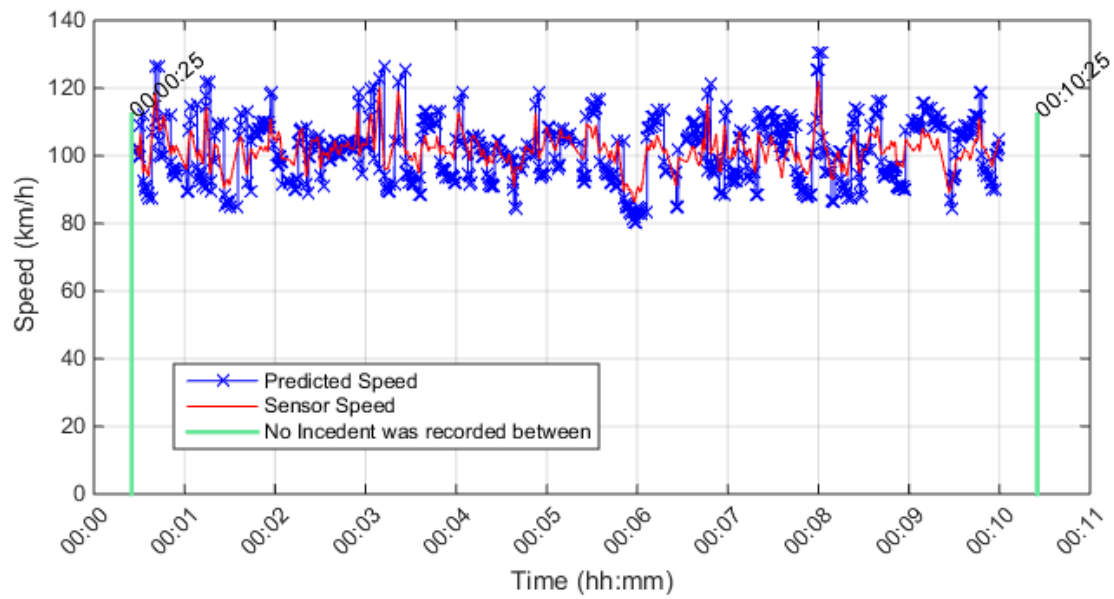

Upstream Detector \# is 401DE0010DEE, Detector Rank $=1$, Distance from incident free location in $(\mathrm{km})=0.35605$ Incident free Season $=$ Winter, Stream $=E$, Direction $=E$, Date \& Time $=2011-02-0114: 30: 30$ Selected incident free Date/Time $=$ 2011-02-1 00:00:25:00

Conditional Probability level $=0.6$, Cond.Prob. $r 3 s 2=0.2$, Cond. Prob. $r 3 s 1=0.1$, Percent drop in speed $\%=-56$ Max. Posterior Probability level $=0.97$, Percent range between speed states $=7 \%$ (No Incident was Detected)

\section{Incident Detection from Lane-1 on the Right-Run-28}




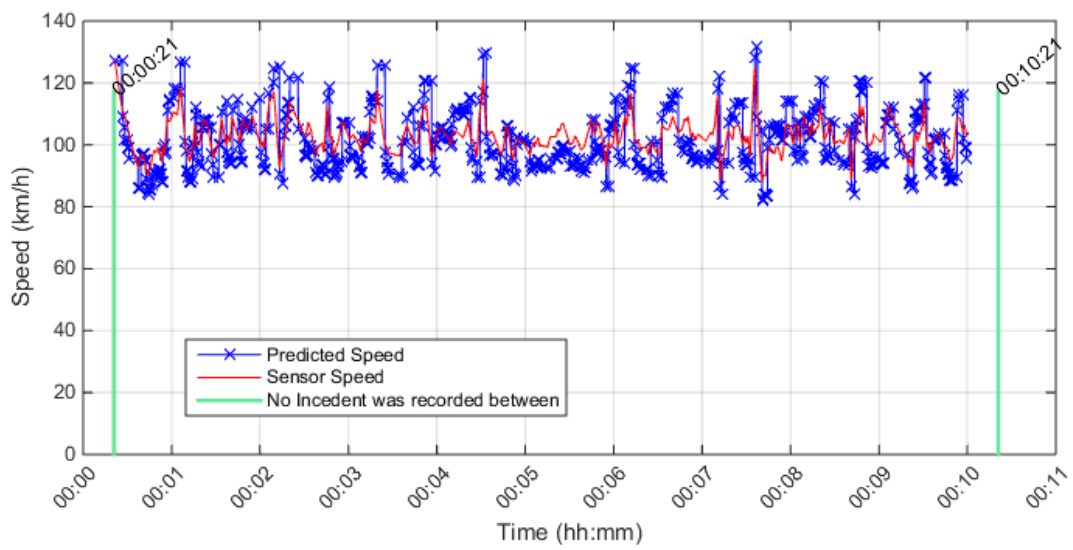

Upstream Detector \# is 401DE0010DEE, Detector Rank = 1, Distance from incident free location in $(\mathrm{km})=0.35605$ Incident free Season $=$ Winter, Stream $=E$, Direction $=E$, Date \& Time $=2011-02-0114: 30: 30$ Selected incident free Date/Time $=2011-02-1$ 00:00:21:00

Conditional Probability level $=0.6$, Cond. Prob. $r 3 \mathrm{~s} 2=0.2$, Cond.Prob. $r 3 \mathrm{~s} 1=0.1$, Percent drop in speed $\%=-56$

Max. Posterior Probability level $=0.97$, Percent range between speed states $=7 \%$

(No Incident was Detected)

\section{Incident Detection from Lane-1 on the Right-Run-29}

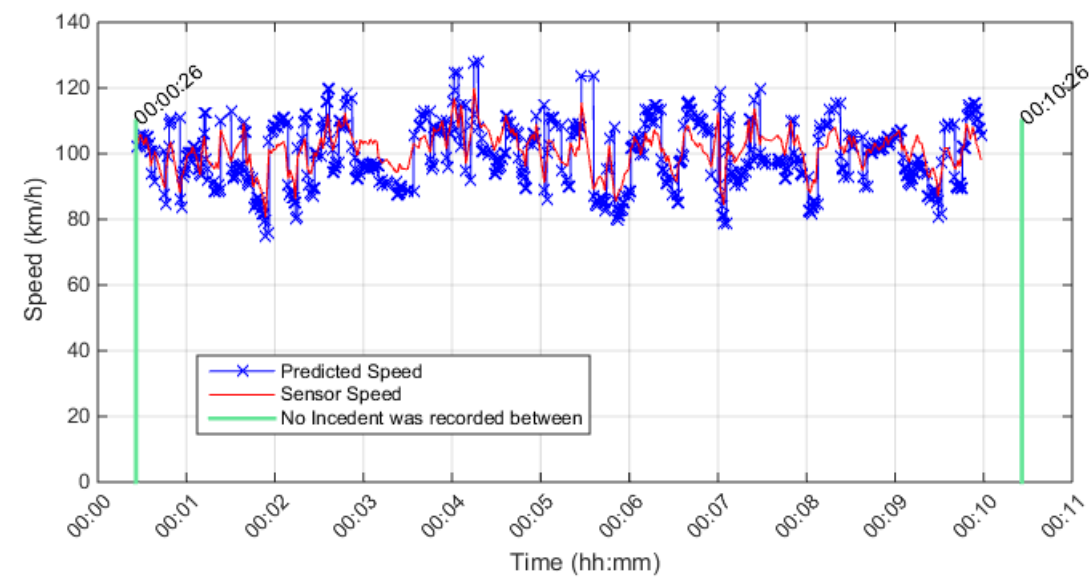

Upstream Detector \# is 401DE0010DEE, Detector Rank = 1, Distance from incident free location in $(\mathrm{km})=0.35605$ Incident free Season $=$ Winter, Stream $=E$, Direction $=E$, Date \& Time $=$ 2011-02-01 14:30:30 Selected incident free Date/Time $=$ 2011-02-1 00:00:26:00

Conditional Probability level $=0.6$, Cond. Prob. $r 3 \mathrm{~s} 2=0.2$, Cond. Prob. $r 3 \mathrm{~s} 1=0.1$, Percent drop in speed $\%=-56$

Max. Posterior Probability level $=0.97$, Percent range between speed states $=7 \%$

(No Incident was Detected)

\section{Incident Detection from Lane-1 on the Right-Run-30}


Incident detection application, no lane blockage, $v=1000 \mathrm{veh} / \mathrm{l} / \mathrm{h}$

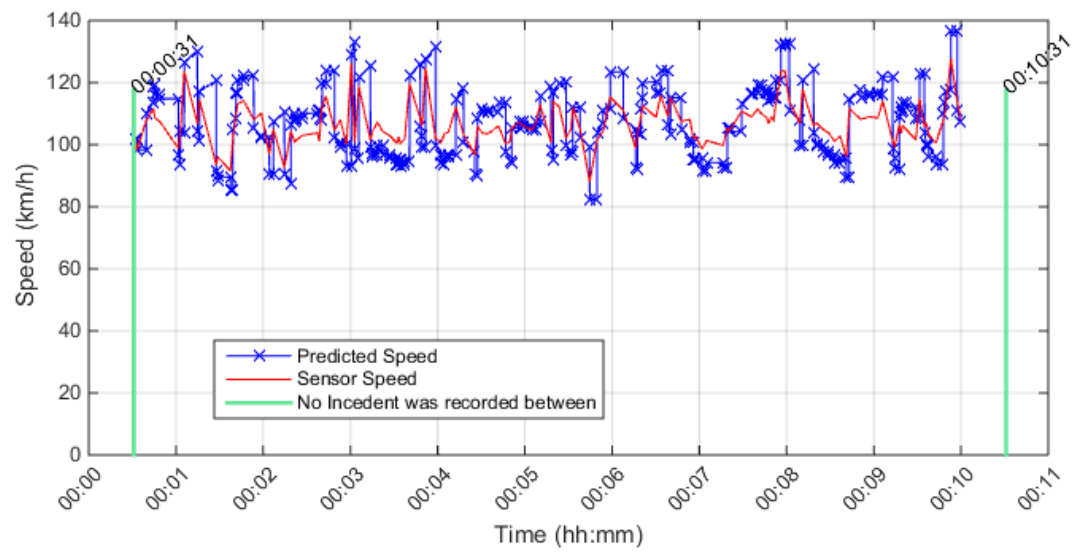

Upstream Detector \# is 401DE0010DEE, Detector Rank = 1, Distance from incident free location in $(\mathrm{km})=0.35605$ Incident free Season $=$ Winter, Stream $=E$, Direction $=E$, Date \& Time $=$ 2011-02-01 00:00:29

Selected incident free Date/Time $=2011-02-0100: 00: 31: 00$

Conditional Probability level $=0.6$, Cond.Prob. $r 3 s 2=0.2$, Cond.Prob. $r 3 s 1=0.1$, Percent drop in speed $\%=-56$

Max. Posterior Probability level $=0.97$, Percent range between speed states $=7 \%$

(No Incident was Detected)

Incident Detection from Lane-1 on the Right, Run-2, v = $1000 \mathrm{veh} / \mathrm{l} / \mathrm{h}$

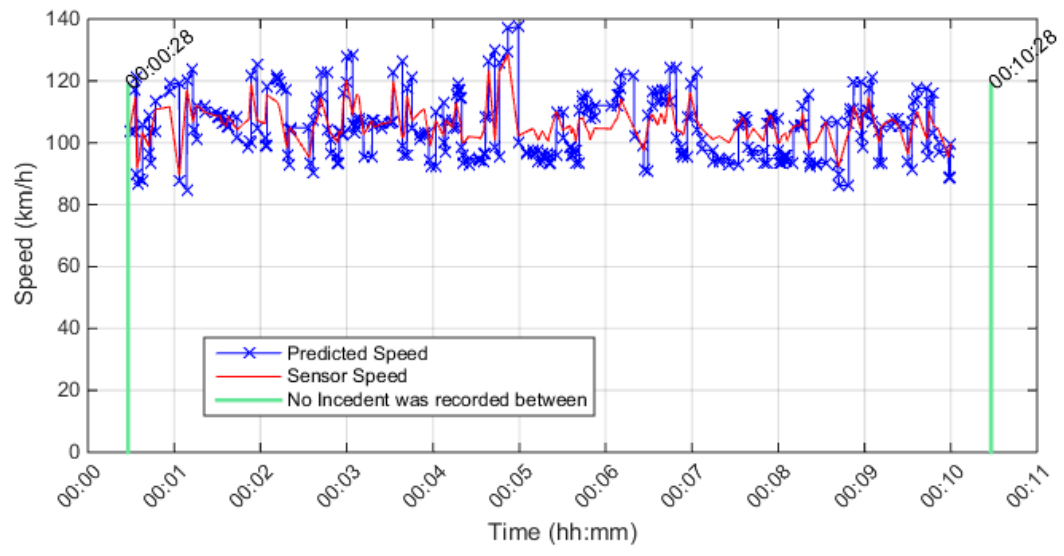

Upstream Detector \# is 401DE0010DEE, Detector Rank = 1, Distance from incident free location in $(\mathrm{km})=0.35605$ Incident free Season $=$ Winter, Stream $=E$, Direction $=E$, Date \& Time $=2011-02-0100: 00: 29$ Selected incident free Date/Time $=2011-02-0100: 00: 28: 00$

Conditional Probability level $=0.6$, Cond.Prob. $r 3 \mathrm{~s} 2=0.2$, Cond.Prob. $r 3 \mathrm{~s} 1=0.1$, Percent drop in speed $\%=-56$

Max. Posterior Probability level $=0.97$, Percent range between speed states $=7 \%$

(No Incident was Detected)

Incident Detection from Lane-1 on the Right, Run-3, v= $1000 \mathrm{veh} / \mathrm{l} / \mathrm{h}$ 


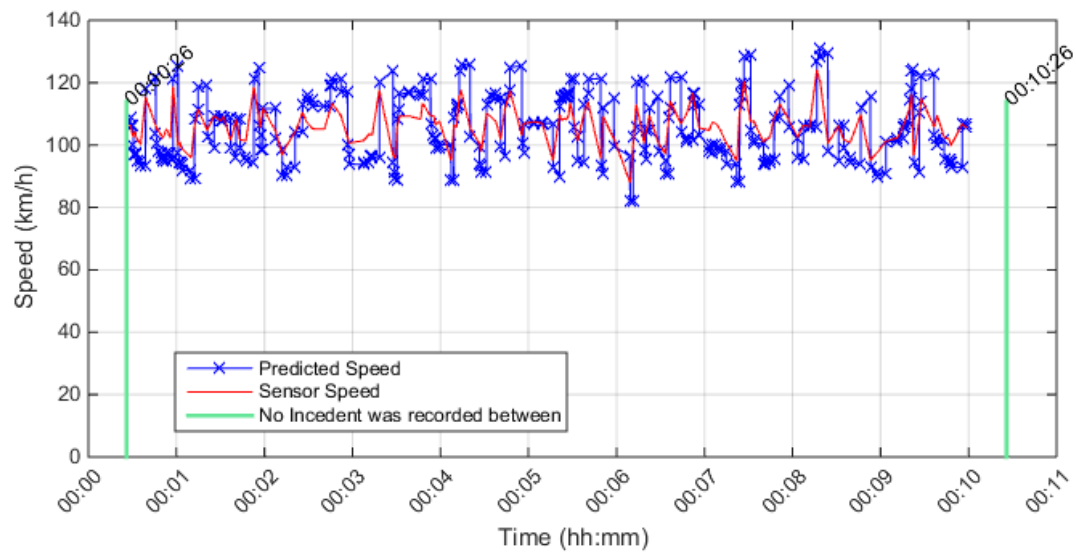

Upstream Detector \# is 401DE0010DEE, Detector Rank = 1, Distance from incident free location in $(\mathrm{km})=0.35605$ Incident free Season $=$ Winter, Stream $=E$, Direction $=E$, Date $\&$ Time $=$ 2011-02-01 00:00:29

Selected incident free Date/Time $=2011-02-0100: 00: 26: 00$

Conditional Probability level $=0.6$, Cond. Prob. $r 3 s 2=0.2$, Cond. Prob. $r 3 s 1=0.1$, Percent drop in speed $\%=-56$

Max. Posterior Probability level $=0.97$, Percent range between speed states $=7 \%$

(No Incident was Detected)

Incident Detection from Lane-1 on the Right, Run-4, v = $1000 \mathrm{veh} / \mathrm{l} / \mathrm{h}$

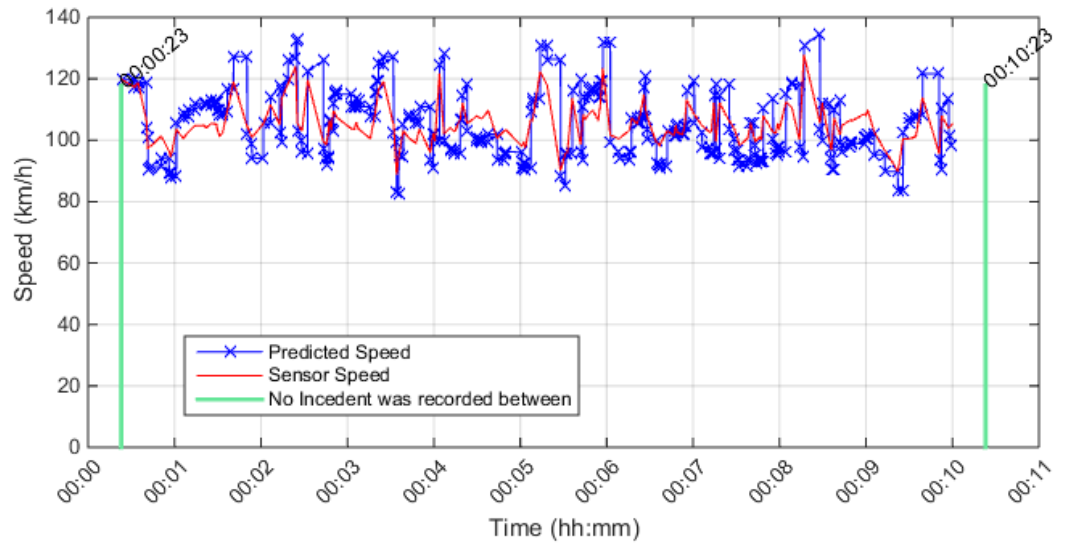

Upstream Detector \# is 401DE0010DEE, Detector Rank = 1, Distance from incident free location in $(\mathrm{km})=0.35605$ Incident free Season $=$ Winter, Stream $=E$, Direction $=E$, Date \& Time $=2011-02-01$ 00:00:29 Selected incident free Date/Time $=2011-02-0100: 00: 23: 00$

Conditional Probability level $=0.6$, Cond.Prob. $r 3 \mathrm{~s} 2=0.2$, Cond.Prob. $r 3 \mathrm{~s} 1=0.1$, Percent drop in speed $\%=-56$ Max. Posterior Probability level $=0.97$, Percent range between speed states $=7 \%$

(No Incident was Detected)

Incident Detection from Lane-1 on the Right, Run-5, v= $1000 \mathrm{veh} / \mathrm{l} / \mathrm{h}$ 


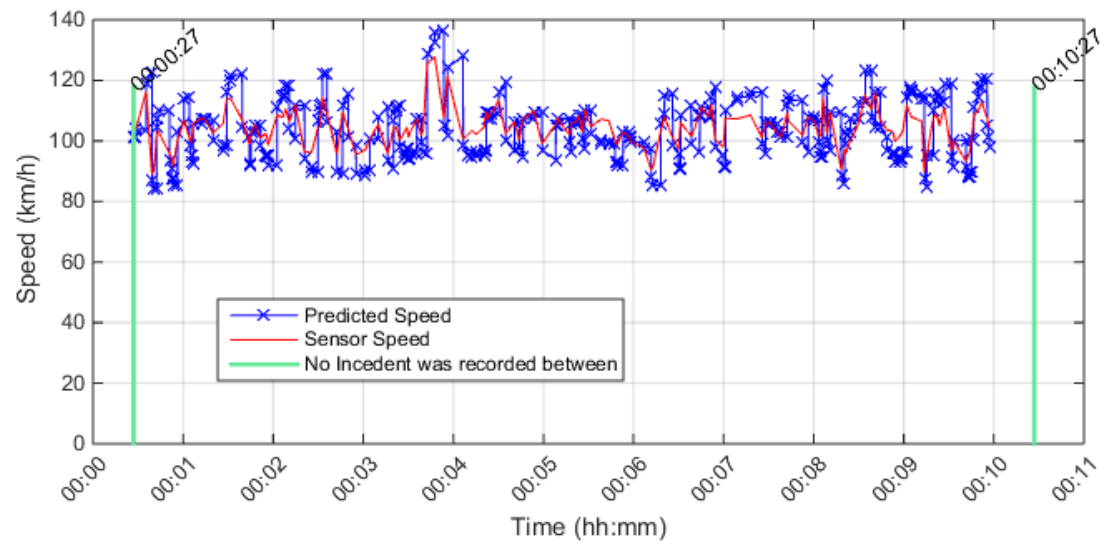

Upstream Detector \# is 401DE0010DEE, Detector Rank = 1, Distance from incident free location in $(\mathrm{km})=0.35605$ Incident free Season $=$ Winter, Stream $=E$, Direction $=E$, Date \& Time $=$ 2011-02-01 00:00:29 Selected incident free Date/Time $=2011-02-01$ 00:00:27:00

Conditional Probability level $=0.6$, Cond. Prob. $r 3 s 2=0.2$, Cond. Prob. $r 3 s 1=0.1$, Percent drop in speed $\%=-56$ Max. Posterior Probability level $=0.97$, Percent range between speed states $=7 \%$ (No Incident was Detected)

Incident Detection from Lane-1 on the Right, Run-6, v = $1000 \mathrm{veh} / \mathrm{l} / \mathrm{h}$

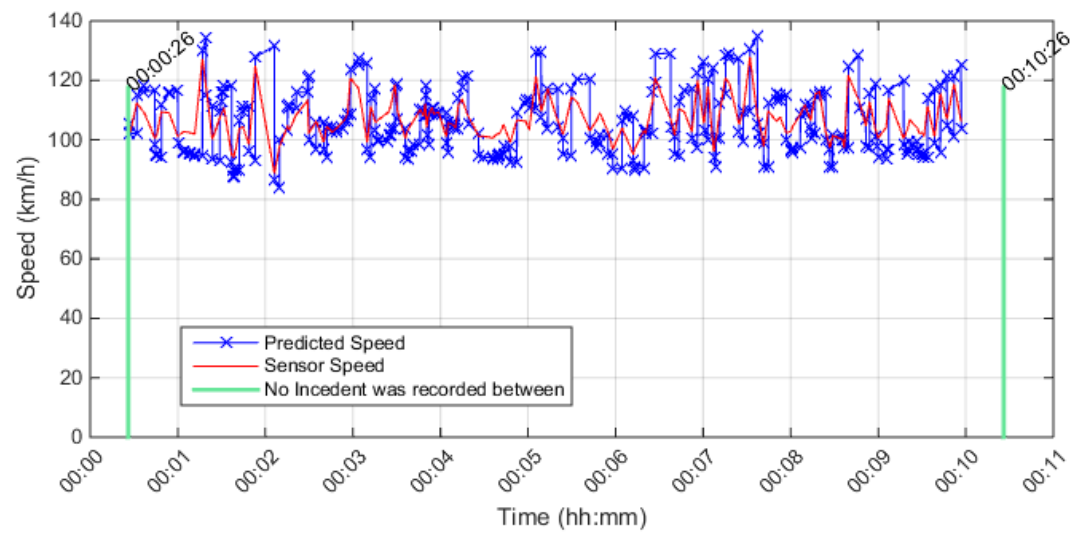

Upstream Detector \# is 401DE0010DEE, Detector Rank = 1, Distance from incident free location in $(\mathrm{km})=0.35605$ Incident free Season $=$ Winter, Stream $=E$, Direction $=E$, Date \& Time $=$ 2011-02-01 00:00:29

Selected incident free Date/Time $=2011-02-0100: 00: 26: 00$

Conditional Probability level $=0.6$, Cond.Prob. $\mathrm{r} 3 \mathrm{~s} 2=0.2$, Cond.Prob. $\mathrm{r} 3 \mathrm{~s} 1=0.1$, Percent drop in speed $\%=-56$

Max. Posterior Probability level $=0.97$, Percent range between speed states $=7 \%$

(No Incident was Detected)

Incident Detection from Lane-1 on the Right, Run-7, v= $1000 \mathrm{veh} / \mathrm{l} / \mathrm{h}$ 


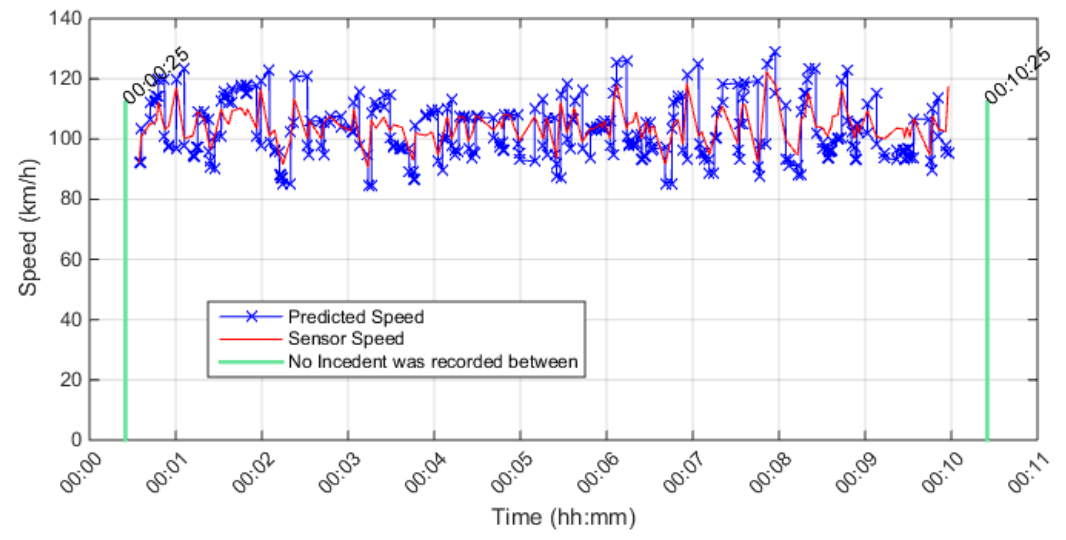

Upstream Detector \# is 401DE0010DEE, Detector Rank = 1, Distance from incident free location in $(\mathrm{km})=0.35605$ Incident free Season $=$ Winter, Stream $=E$, Direction $=E$, Date \& Time $=2011-02-0100: 00: 29$ Selected incident free Date/Time $=2011-02-0100: 00: 25: 00$

Conditional Probability level $=0.6$, Cond.Prob. $r 3 \mathrm{~s} 2=0.2$, Cond. Prob. $r 3 \mathrm{~s} 1=0.1$, Percent drop in speed $\%=-56$

Max. Posterior Probability level $=0.97$, Percent range between speed states $=7 \%$

(No Incident was Detected)

Incident Detection from Lane-1 on the Right, Run-8, v $=1000 \mathrm{veh} / \mathrm{l} / \mathrm{h}$

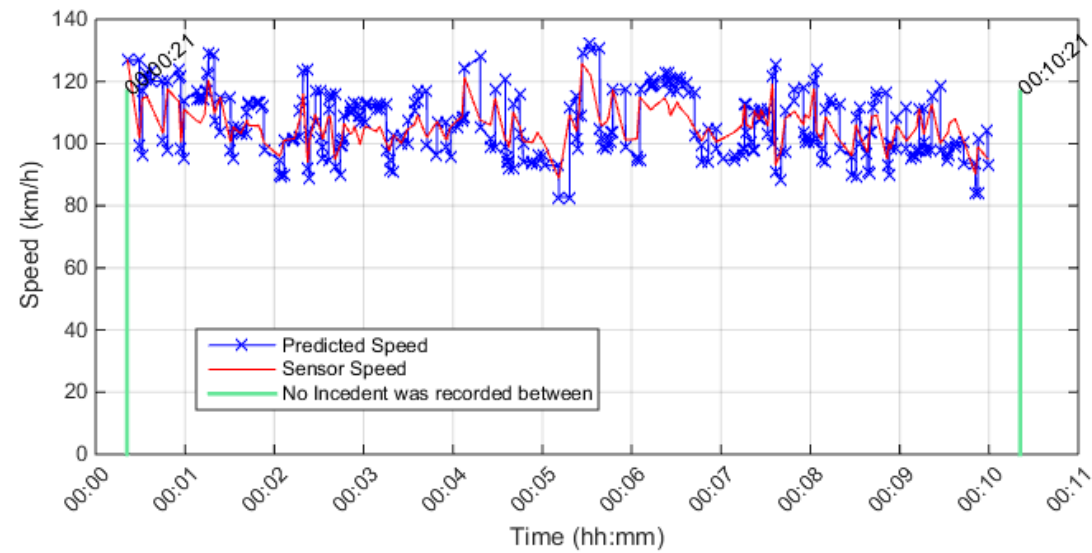

Upstream Detector \# is 401DE0010DEE, Detector Rank $=1$, Distance from incident free location in $(\mathrm{km})=0.35605$ Incident free Season $=$ Winter, Stream $=E$, Direction $=E$, Date $\&$ Time $=$ 2011-02-01 00:00:29 Selected incident free Date/Time $=2011-02-0100: 00: 21: 00$

Conditional Probability level $=0.6$, Cond.Prob. $\mathrm{r} 3 \mathrm{~s} 2=0.2$, Cond. Prob. $\mathrm{r} 3 \mathrm{~s} 1=0.1$, Percent drop in speed $\%=-56$ Max. Posterior Probability level $=0.97$, Percent range between speed states $=7 \%$

(No Incident was Detected)

Incident Detection from Lane-1 on the Right, Run-9, v = $1000 \mathrm{veh} / \mathrm{l} / \mathrm{h}$ 


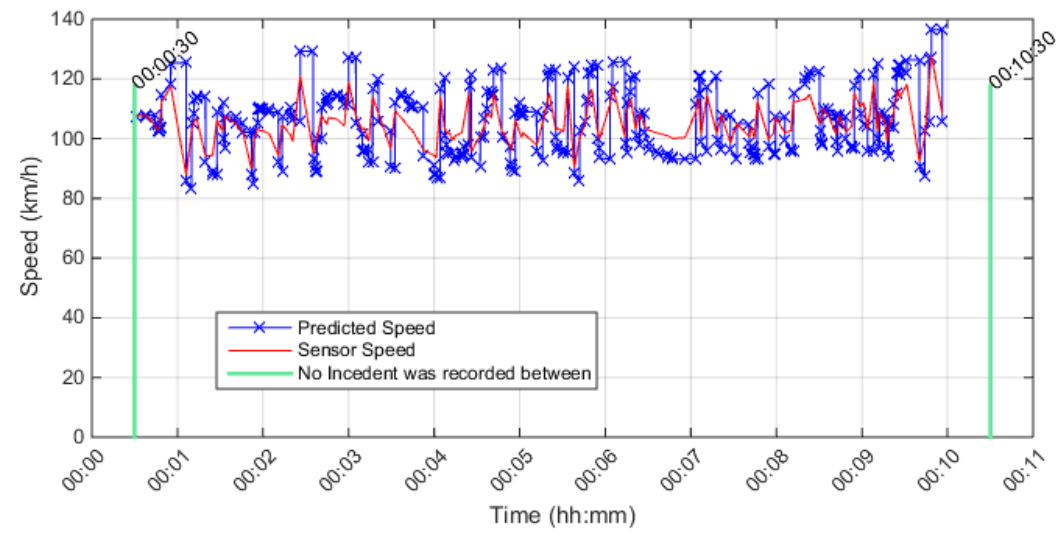

Upstream Detector \# is 401DE0010DEE, Detector Rank = 1, Distance from incident free location in $(\mathrm{km})=0.35605$ Incident free Season $=$ Winter, Stream $=E$, Direction $=E$, Date \& Time $=2011-02-0100: 00: 29$ Selected incident free Date/Time $=2011-02-0100: 00: 30: 00$

Conditional Probability level $=0.6$, Cond.Prob. $r 3 \mathrm{~s} 2=0.2$, Cond. Prob. $\mathrm{r} 3 \mathrm{~s} 1=0.1$, Percent drop in speed $\%=-56$ Max. Posterior Probability level $=0.97$, Percent range between speed states $=7 \%$

(No Incident was Detected)

Incident Detection from Lane-1 on the Right, Run-10, v = $1000 \mathrm{veh} / \mathrm{l} / \mathrm{h}$

\section{Appendix D.2 Two Lane Blockage Scenario}

Incident detection application, two-lane blockage, $v=2000 \mathrm{veh} / \mathrm{l} / \mathrm{h}$, data obtained from the first lane.

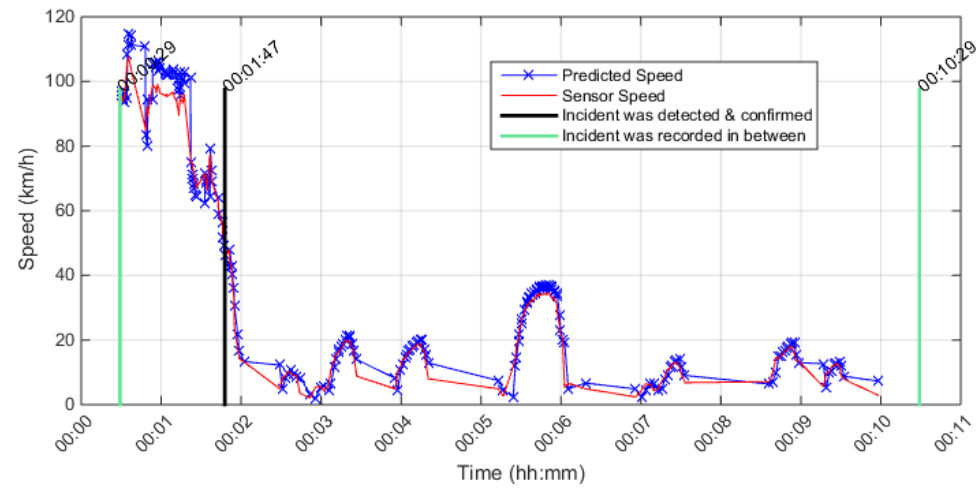

Upstream Detector \# is 401DE0010DEE, Detector Rank $=1$, Distance from incident location in $(\mathrm{km})=0.35605$ Season $=$ Winter, Stream $=E$, Direction $=E$, Date \& Time $=2011-02-0100: 00: 29$

Incident Reason $=$ Collisio

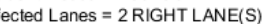

Conditional Probability level $=0.6$, Cond. Prob. $r 3 \mathrm{~s} 2=0.2$, Cond. Prob. $r 3 \mathrm{~s} 1=0.1$, Percent drop in speed $\%=-56$

Max. Posterior Probability level $=0.97$, Percent range between speed states $=7 \%$

Incident was detected at 00:10 minutes from drop in speed point. Incident was confirmed after 5 minutes from the detection time

Incident Detection Utilizing the Algorithm, Data obtained from Right Lane \#1, Run- 


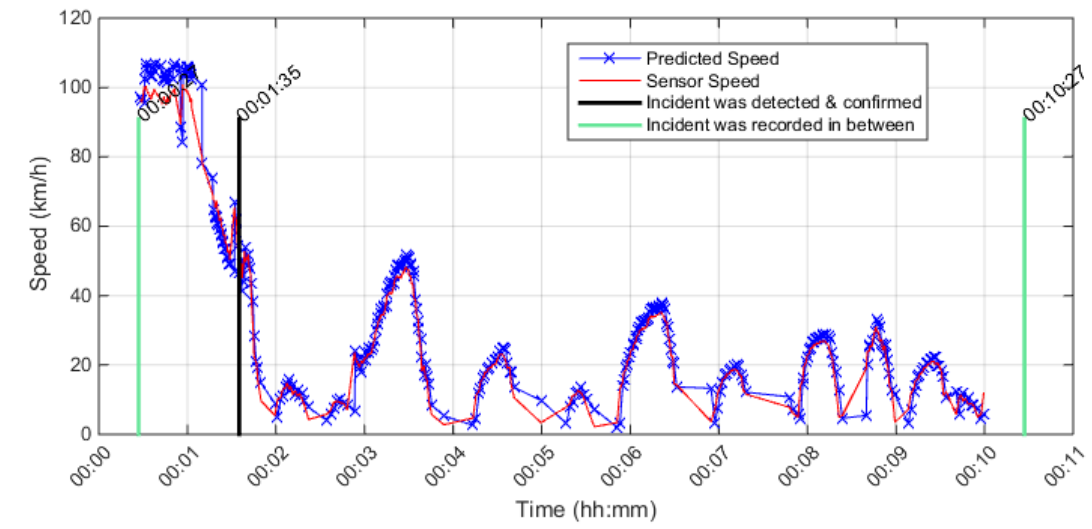

Upstream Detector \# is 401DE0010DEE, Detector Rank $=1$, Distance from incident location in $(\mathrm{km})=0.35605$ Season $=$ Winter, Stream $=E$, Direction $=E$, Date \& Time $=2011-02-0100: 00: 27$ Incident Reason $=$ Collision

Affected Lanes $=2$ RIGHT LANE(S)

Conditional Probability level $=0.6$, Cond. Prob. $r 3 s 2=0.2$, Cond. Prob. $r 3 s 1=0.1$, Percent drop in speed $\%=-56$

Max. Posterior Probability level $=0.97$, Percent range between speed states $=7 \%$

Incident was detected at 00:25 minutes from drop in speed point. Incident was confirmed after 5 minutes from the detection time

Incident Detection Utilizing the Algorithm, Data obtained from Right Lane \#1, Run3

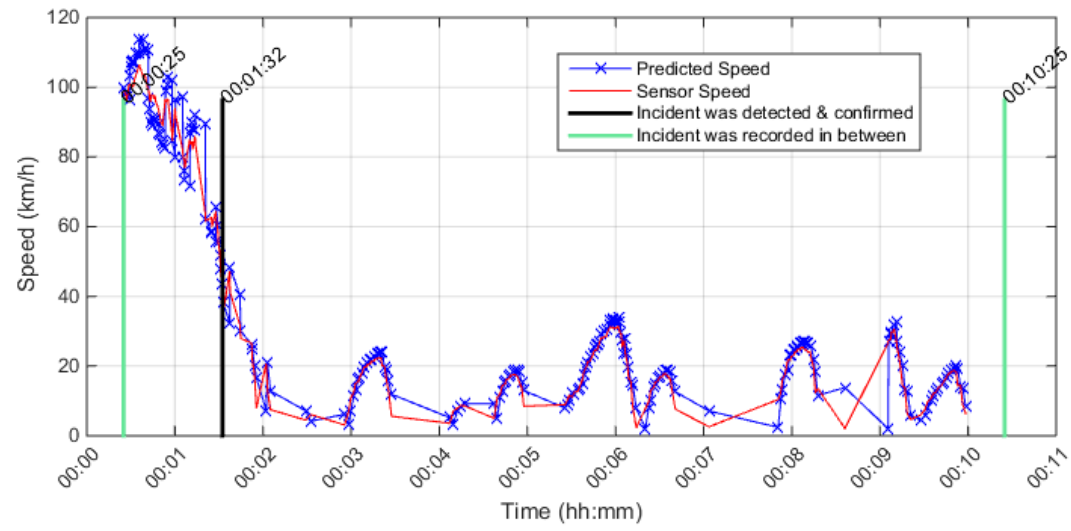

Upstream Detector \# is 401DE0010DEE, Detector Rank $=1$, Distance from incident location in $(\mathrm{km})=0.35605$ Season $=$ Winter, Stream $=E$, Direction $=E$, Date $\&$ Time $=2011-02-0100: 00: 25$ Incident Reason $=$ Collision

Affected Lanes = 2 RIGHT LANE(S)

Conditional Probability level $=0.6$, Cond.Prob. $r 3 s 2=0.2$, Cond.Prob. $r 3 s 1=0.1$, Percent drop in speed $\%=-56$

Max. Posterior Probability level $=0.97$, Percent range between speed states $=7 \%$

Incident was detected at 00:11 minutes from drop in speed point. Incident was confirmed after 5 minutes from the detection time

Incident Detection Utilizing the Algorithm, Data obtained from Right Lane \#1, Run4 


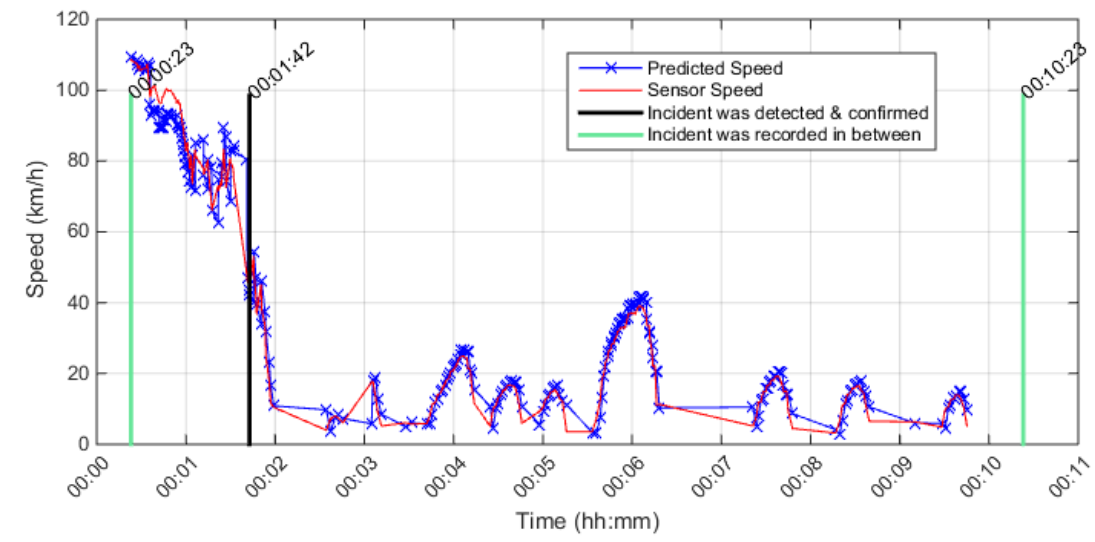

Upstream Detector \# is 401DE0010DEE, Detector Rank $=1$, Distance from incident location in $(\mathrm{km})=0.35605$ Season $=$ Winter, Stream $=E$, Direction $=E$, Date \& Time $=2011-02-0100: 00: 23$ Incident Reason $=$ Collision

Affected Lanes = 2 RIGHT LANE(S)

Conditional Probability level $=0.6$, Cond. Prob. $r 3 s 2=0.2$, Cond. Prob. $r 3 s 1=0.1$, Percent drop in speed $\%=-56$

Max. Posterior Probability level $=0.97$, Percent range between speed states $=7 \%$

Incident was detected at 00:01 minutes from drop in speed point. Incident was confirmed after 5 minutes from the detection time

Incident Detection Utilizing the Algorithm, Data obtained from Right Lane \#1, Run5

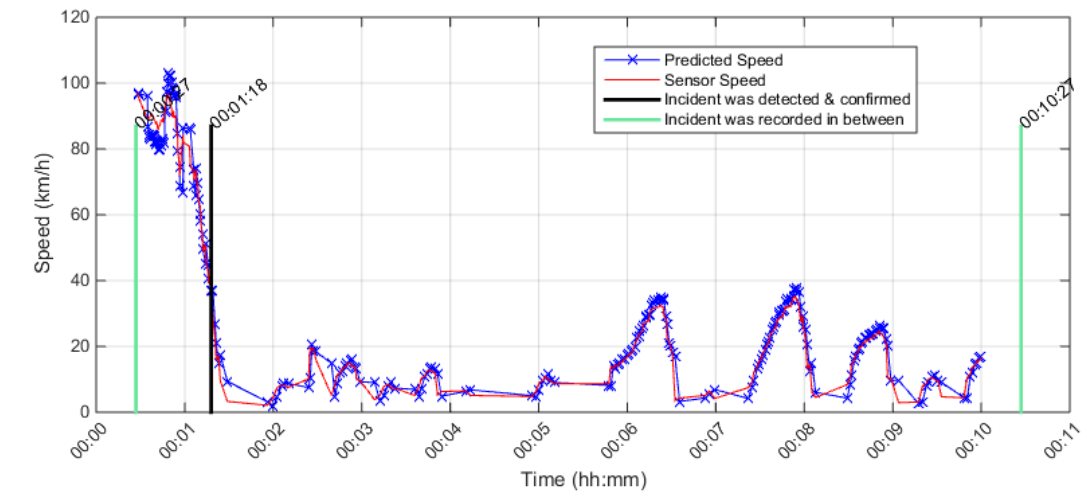

Upstream Detector \# is 401DE0010DEE, Detector Rank $=1$, Distance from incident location in $(\mathrm{km})=0.35605$ Season $=$ Winter, Stream $=E$, Direction $=E$, Date \& Time $=2011-02-0100: 00: 27$ ncident Reason $=$ Collisio

Conditional Probability level $=0.6$, Cond. Prob. $r 3 \mathrm{~s} 2=0.2$, Cond. Prob. $\mathrm{r} 3 \mathrm{~s} 1=0.1$, Percent drop in speed $\%=-56$

Conditional Probability level $=0.6$, Cond. Prob. $r 3 \mathrm{~s} 2=0.2$, Cond.Prob. $r 3 \mathrm{~s} 1=0.1$, Pe
Max. Posterior Probability level $=0.97$, Percent range between speed states $=7 \%$

Max. Posterior Probability level $=0.97$, Percent range between speed states $=7 \%$

Incident Detection Utilizing the Algorithm, Data obtained from Right Lane \#1, Run6 


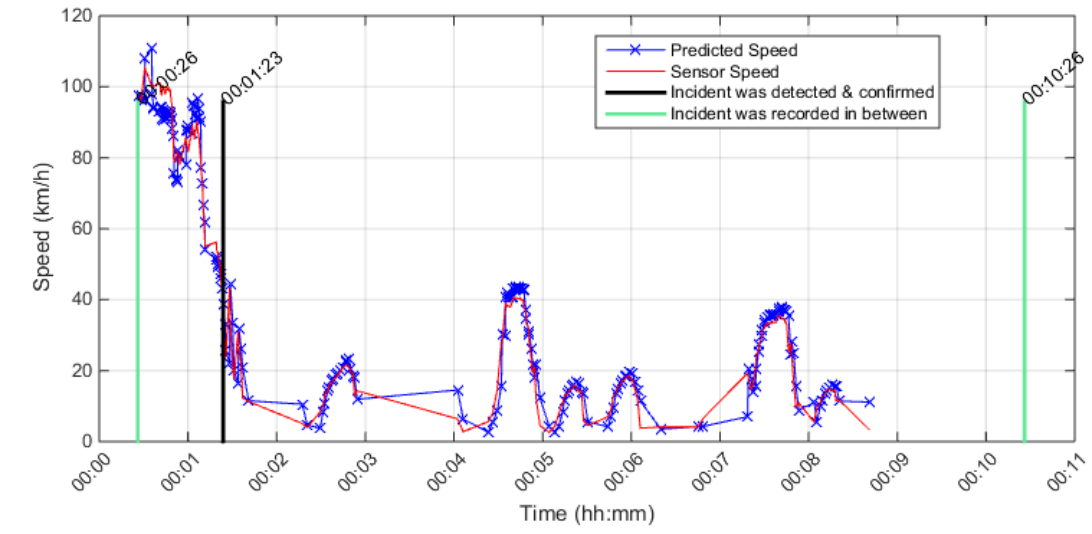

Upstream Detector \# is 401DE0010DEE, Detector Rank $=1$, Distance from incident location in $(\mathrm{km})=0.35605$ Season $=$ Winter, Stream $=E$, Direction $=E$, Date \& Time $=2011-02-0100: 00: 26$ Incident Reason $=$ Collision

Affected Lanes $=2$ RIGHT LANE(S)

Conditional Probability level $=0.6$, Cond. Prob. $r 3 \mathrm{~s} 2=0.2$, Cond.Prob. $r 3 \mathrm{~s} 1=0.1$, Percent drop in speed $\%=-56$ Max. Posterior Probability level $=0.97$, Percent range between speed states $=7 \%$

Incident was detected at 00:15 minutes from drop in speed point. Incident was confirmed after 5 minutes from the detection time

Incident Detection Utilizing the Algorithm, Data obtained from Right Lane \#1, Run7

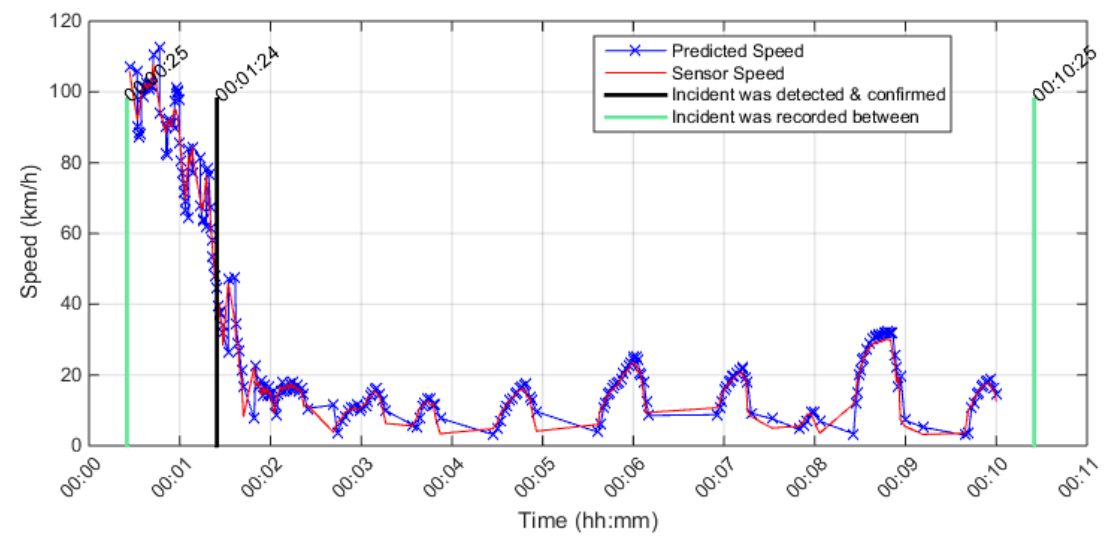

Upstream Detector \# is 401DE0010DEE, Detector Rank $=1$, Distance from incident location in $(\mathrm{km})=0.35605$ Season $=$ Winter, Stream $=E$, Direction $=E$, Date \& Time $=2011-02-0100: 00: 25$ Incident Reason $=$ Collision

Affected Lanes = 2RIGHT LANE(S)

Conditional Probability level $=0.6$, Cond. Prob. $r 3 \mathrm{~s} 2=0.2$, Cond. Prob. $r 3 \mathrm{~s} 1=0.1$, Percent drop in $\mathrm{speed} \%=-56$

Max. Posterior Probability level $=0.97$, Percent range between speed states $=7 \%$

Incident was detected at 00:05 minutes from drop in speed point. Incident was confirmed after 5 minutes from the detection time

Incident Detection Utilizing the Algorithm, Data obtained from Right Lane \#1, Run- 


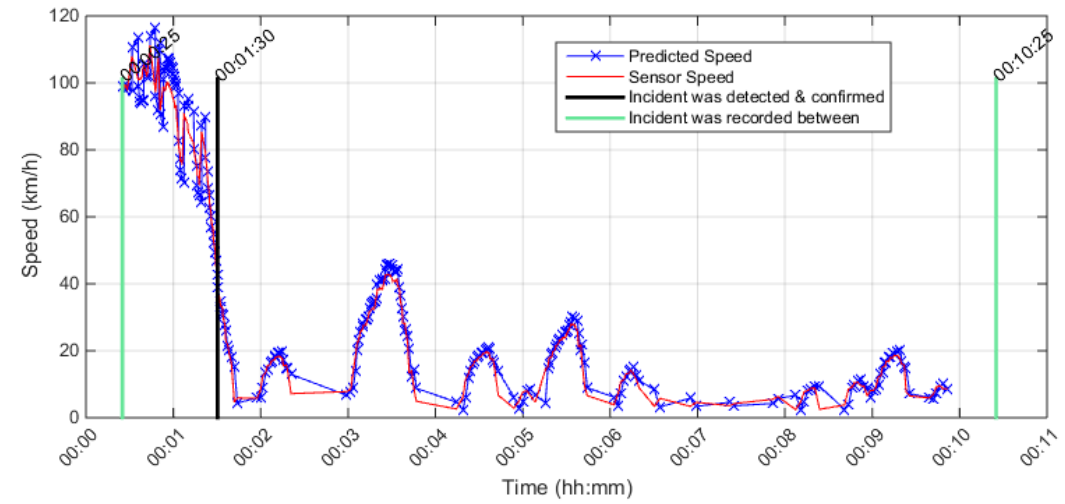

Upstream Detector \# is 401DE0010DEE, Detector Rank $=1$, Distance from incident location in $(\mathrm{km})=0.35605$ Season $=$ Winter, Stream $=E$, Direction $=E$, Date \& Time $=2011-02-0100: 00: 25$ Incident Reason $=$ Collision

Affected Lanes $=2$ RIGHT LANE(S)

Conditional Probability level $=0.6$, Cond.Prob. $r 3 \mathrm{~s} 2=0.2$, Cond. Prob. $r 3 \mathrm{~s} 1=0.1$, Percent drop in speed $\%=-56$

Max. Posterior Probability level $=0.97$, Percent range between speed states $=7 \%$

Incident was detected at 00:08 minutes from drop in speed point. Incident was confirmed after 5 minutes from the detection time

\section{Incident Detection Utilizing the Algorithm, Data obtained from Right Lane \#1, Run-} 9

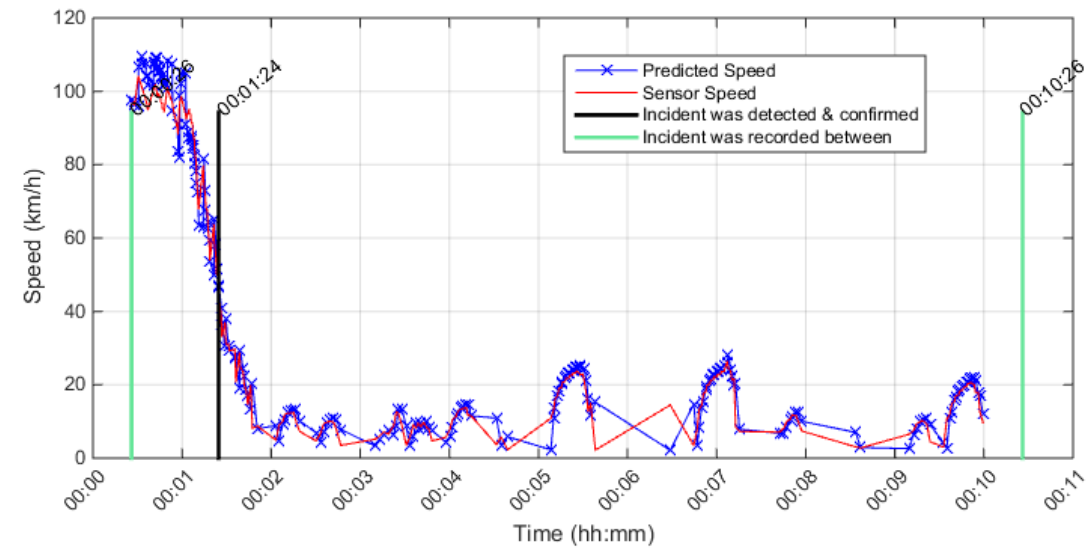

Upstream Detector \# is 401DE0010DEE, Detector Rank $=1$, Distance from incident location in $(\mathrm{km})=0.35605$ Season $=$ Winter, Stream $=E$, Direction $=E$, Date \& Time $=2011-02-0100: 00: 26$ Incident Reason $=$ Collision

Affected Lanes $=2$ RIGHT LANE(S)

Conditional Probability level $=0.6$, Cond.Prob. $r 3 \mathrm{~s} 2=0.2$, Cond.Prob. $r 3 \mathrm{~s} 1=0.1$, Percent drop in speed $\%=-56$

Max. Posterior Probability level $=0.97$, Percent range between speed states $=7 \%$

Incident was detected at 00:09 minutes from drop in speed point. Incident was confirmed after 5 minutes from the detection time

Incident Detection Utilizing the Algorithm, Data obtained from Right Lane \#1, Run10 


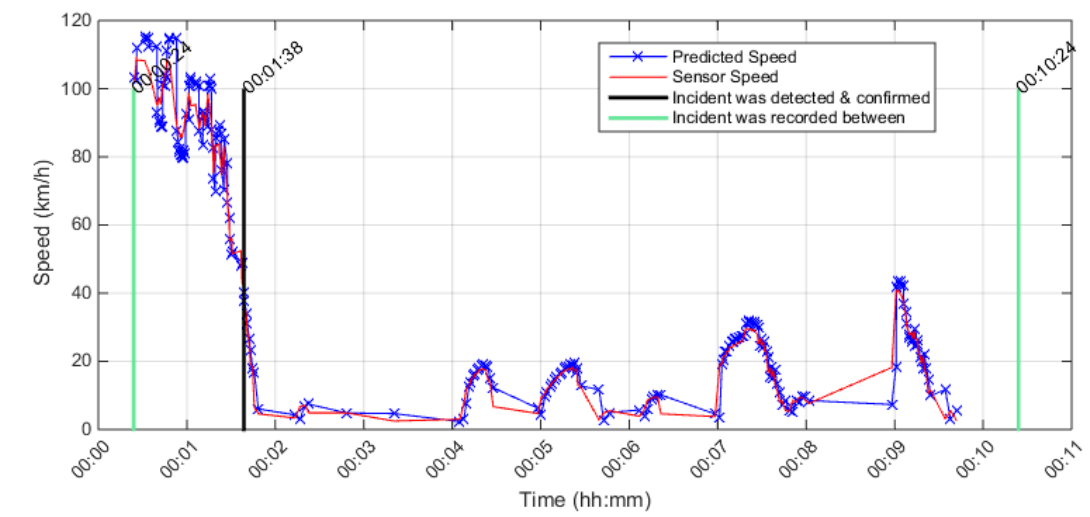

Upstream Detector \# is 401DE0010DEE, Detector Rank $=1$, Distance from incident location in $(\mathrm{km})=0.35605$ Season $=$ Winter, Stream $=E$, Direction $=E$, Date \& Time $=2011-02-0100: 00: 24$ Incident Reason $=$ Collision

Affected Lanes = 2RIGHT LANE(S)

Conditional Probability level $=0.6$, Cond.Prob. $r 3 \mathrm{~s} 2=0.2$, Cond. Prob. $r 3 \mathrm{~s} 1=0.1$, Percent drop in speed $\%=-56$

Max. Posterior Probability level $=0.97$, Percent range between speed states $=7 \%$

Incident was detected at 00:13 minutes from drop in speed point. Incident was confirmed after 5 minutes from the detection time

\section{Incident Detection Utilizing the Algorithm, Data obtained from Right Lane \#1, Run-} 11

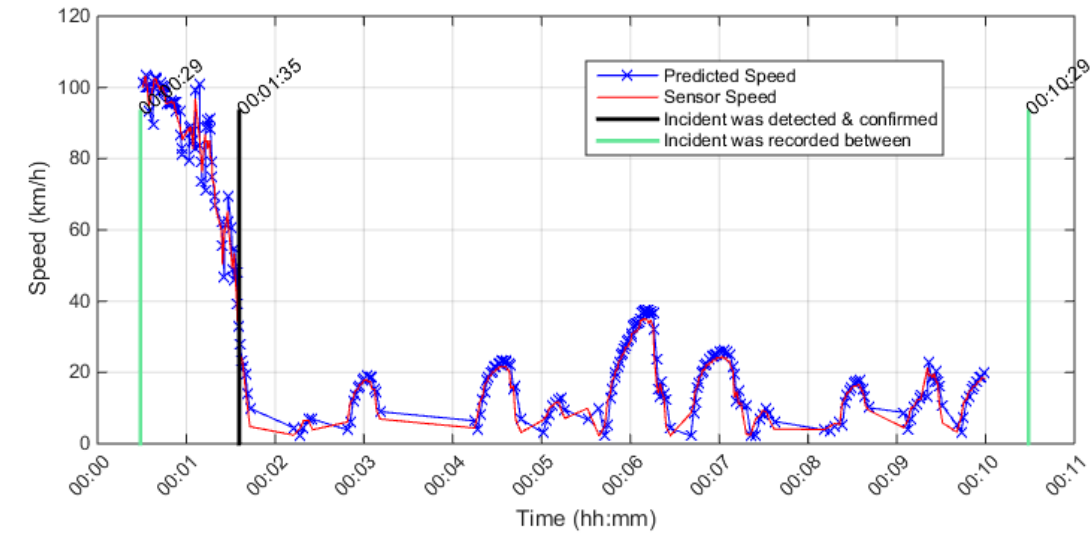

Upstream Detector \# is 401DE0010DEE, Detector Rank $=1$, Distance from incident location in $(\mathrm{km})=0.35605$ Season $=$ Winter, Stream $=E$, Direction $=E$, Date \& Time $=2011-02-0100: 00: 29$ Incident Reason $=$ Collision

Affected Lanes = 2 RIGHT LANE(S)

Conditional Probability level $=0.6$, Cond.Prob. $r 3 s 2=0.2$, Cond. Prob. $r 3 s 1=0.1$, Percent drop in speed $\%=-56$ Max. Posterior Probability level $=0.97$, Percent range between speed states $=7 \%$

Incident was detected at 00:19 minutes from drop in speed point. Incident was confirmed after 5 minutes from the detection time

Incident Detection Utilizing the Algorithm, Data obtained from Right Lane \#1, Run12 


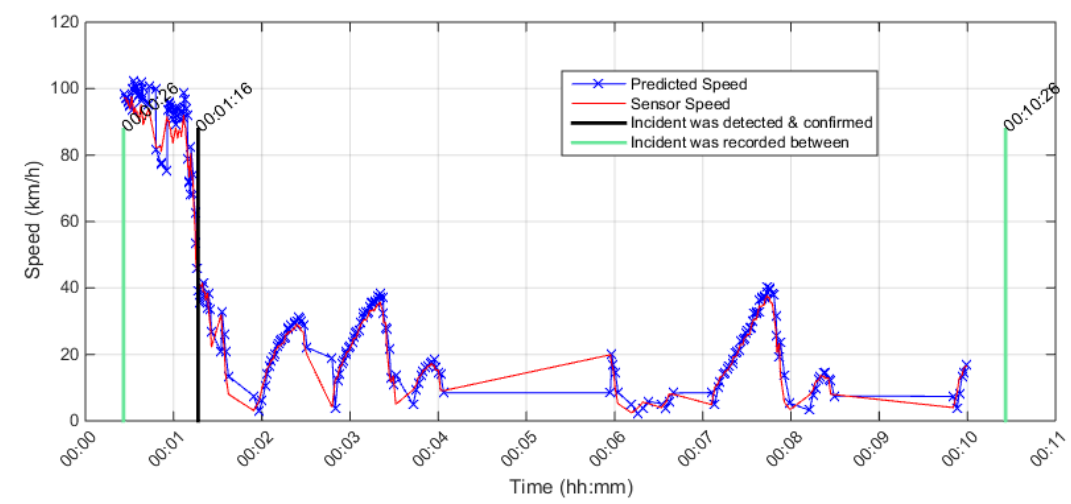

Upstream Detector \# is 401DE0010DEE, Detector Rank $=1$, Distance from incident location in $(\mathrm{km})=0.35605$ Season $=$ Winter, Stream $=E$, Direction $=E$, Date \& Time $=2011-02-0100: 00: 26$ Incident Reason = Collision

Affected Lanes $=2$ RIGHT LANE(S)

Conditional Probability level $=0.6$, Cond. Prob. $r 3 \mathrm{~s} 2=0.2$, Cond. Prob. $r 3 \mathrm{~s} 1=0.1$, Percent drop in speed $\%=-56$

Max. Posterior Probability level $=0.97$, Percent range between speed states $=7 \%$

Incident was detected at 00:04 minutes from drop in speed point. Incident was confirmed after 5 minutes from the detection time

\section{Incident Detection Utilizing the Algorithm, Data obtained from Right Lane \#1, Run-} 13

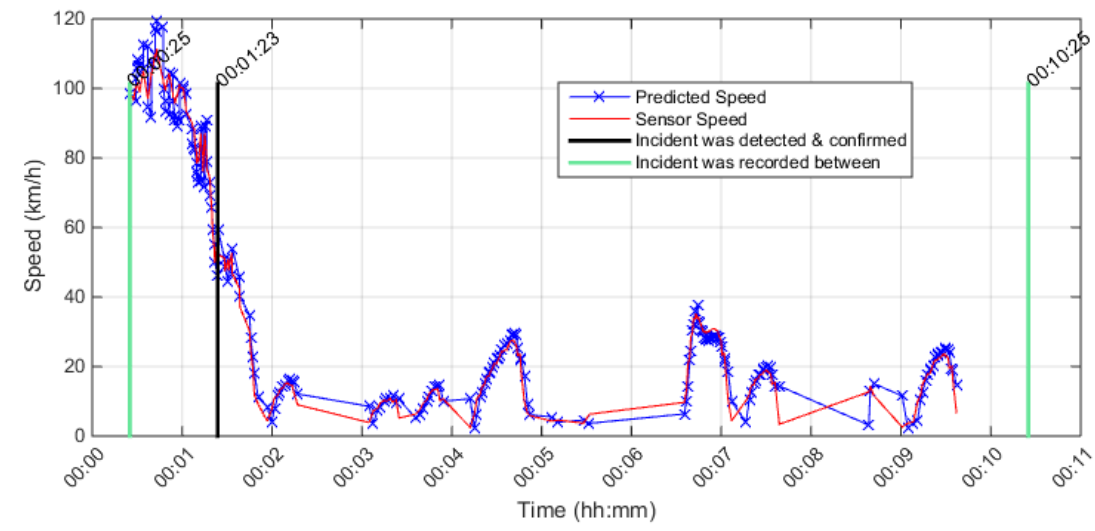

Upstream Detector \# is 401DE0010DEE, Detector Rank $=1$, Distance from incident location in $(\mathrm{km})=0.35605$ Season $=$ Winter, Stream $=E$, Direction $=E$, Date \& Time $=2011-02-0100: 00: 25$ Incident Reason = Collision

Affected Lanes = 2 RIGHT LANE(S)

Conditional Probability level $=0.6$, Cond.Prob. $r 3 s 2=0.2$, Cond.Prob. $r 3 s 1=0.1$, Percent drop in speed $\%=-56$ Max. Posterior Probability level $=0.97$, Percent range between speed states $=7 \%$

Incident was detected at 00:07 minutes from drop in speed point. Incident was confirmed after 5 minutes from the detection time

Incident Detection Utilizing the Algorithm, Data obtained from Right Lane \#1, Run14 


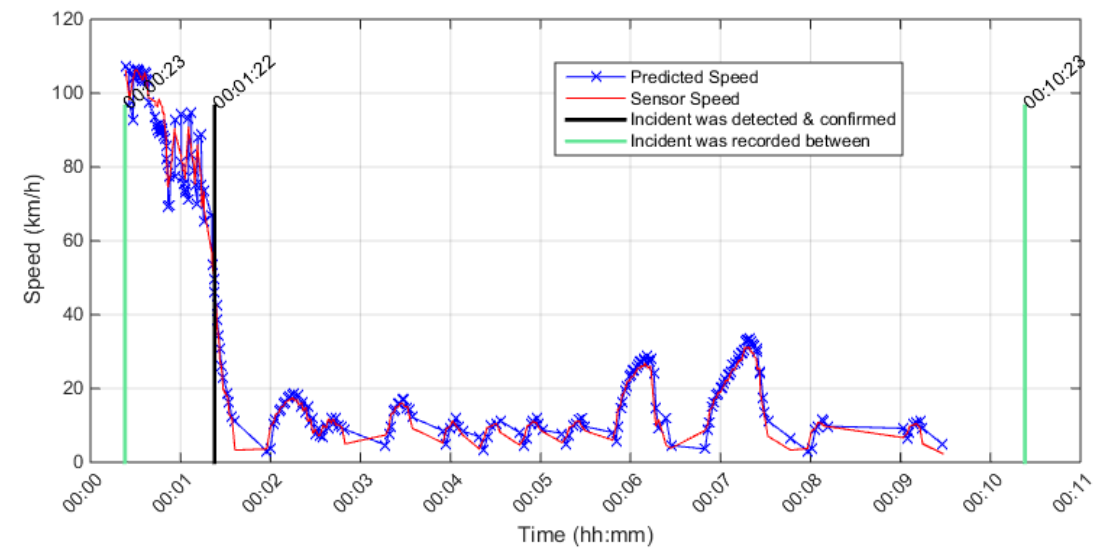

Upstream Detector \# is 401DE0010DEE, Detector Rank $=1$, Distance from incident location in $(\mathrm{km})=0.35605$ Season $=$ Winter, Stream $=E$, Direction $=E$, Date \& Time $=2011-02-0100: 00: 23$

Incident Reason $=$ Collision

Affected Lanes $=2$ RIGHT LANE(S)

Conditional Probability level $=0.6$, Cond.Prob. $r 3 \mathrm{~s} 2=0.2$, Cond. Prob. $r 3 \mathrm{~s} 1=0.1$, Percent drop in speed $\%=-56$

Max. Posterior Probability level $=0.97$, Percent range between speed states $=7 \%$

Incident was detected at 00:08 minutes from drop in speed point. Incident was confirmed after 5 minutes from the detection time

\section{Incident Detection Utilizing the Algorithm, Data obtained from Right Lane \#1, Run-} 15

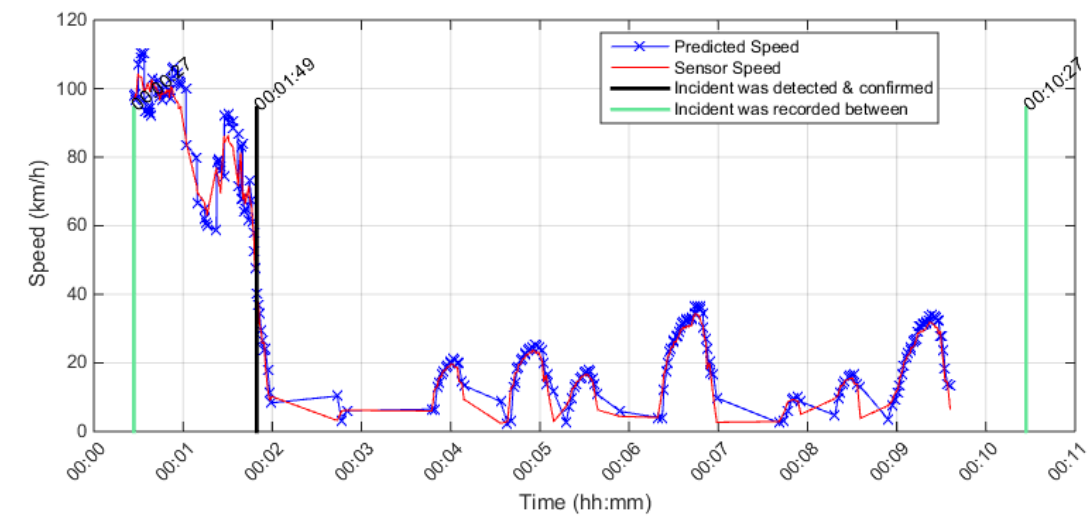

Upstream Detector \# is 401DE0010DEE, Detector Rank $=1$, Distance from incident location in $(\mathrm{km})=0.35605$ Season $=$ Winter, Stream $=E$, Direction $=E$, Date \& Time $=2011-02-0100: 00: 27$ Incident Reason $=$ Collision

Affected Lanes = 2RIGHT LANE(S)

Conditional Probability level $=0.6$, Cond.Prob. $r 3 s 2=0.2$, Cond. Prob. $r 3 s 1=0.1$, Percent drop in speed $\%=-56$

Max. Posterior Probability level $=0.97$, Percent range between speed states $=7 \%$

Incident was detected at 00:09 minutes from drop in speed point. Incident was confirmed after 5 minutes from the detection time

Incident Detection Utilizing the Algorithm, Data obtained from Right Lane \#1, Run16 


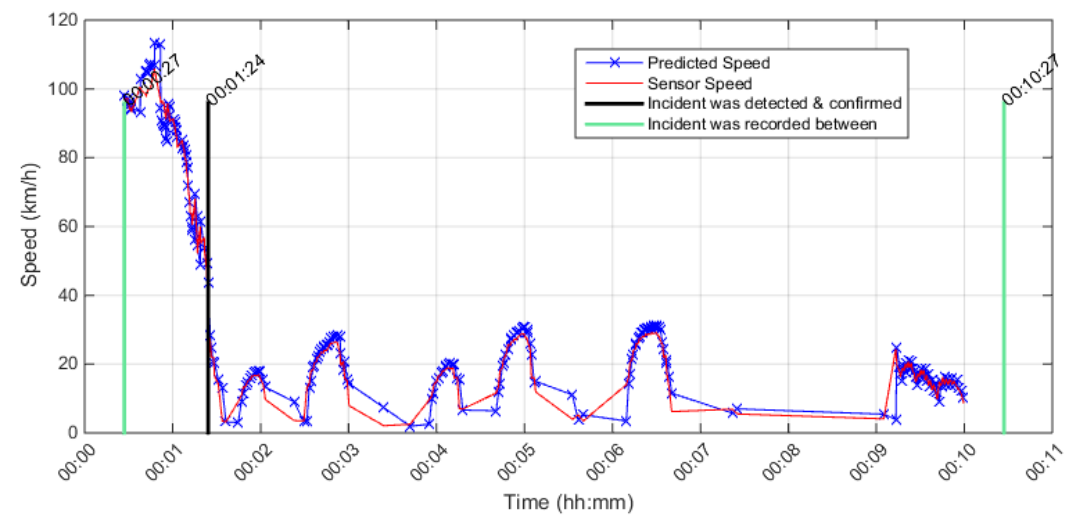

Upstream Detector \# is 401DE0010DEE, Detector Rank $=1$, Distance from incident location in $(\mathrm{km})=0.35605$

Season $=$ Winter, Stream $=E$, Direction $=E$, Date \& Time $=2011-02-0100: 00: 27$

Incident Reason $=$ Collision

Affected Lanes $=2$ RIGHT LANE(S)

Conditional Probability level $=0.6$, Cond.Prob. $r 3 \mathrm{~s} 2=0.2$, Cond.Prob. $r 3 \mathrm{~s} 1=0.1$, Percent drop in speed $\%=-56$

Max. Posterior Probability level $=0.97$, Percent range between speed states $=7 \%$

Incident was detected at 00:14 minutes from drop in speed point. Incident was confirmed after 5 minutes from the detection time

Incident Detection Utilizing the Algorithm, Data obtained from Right Lane \#1, Run17

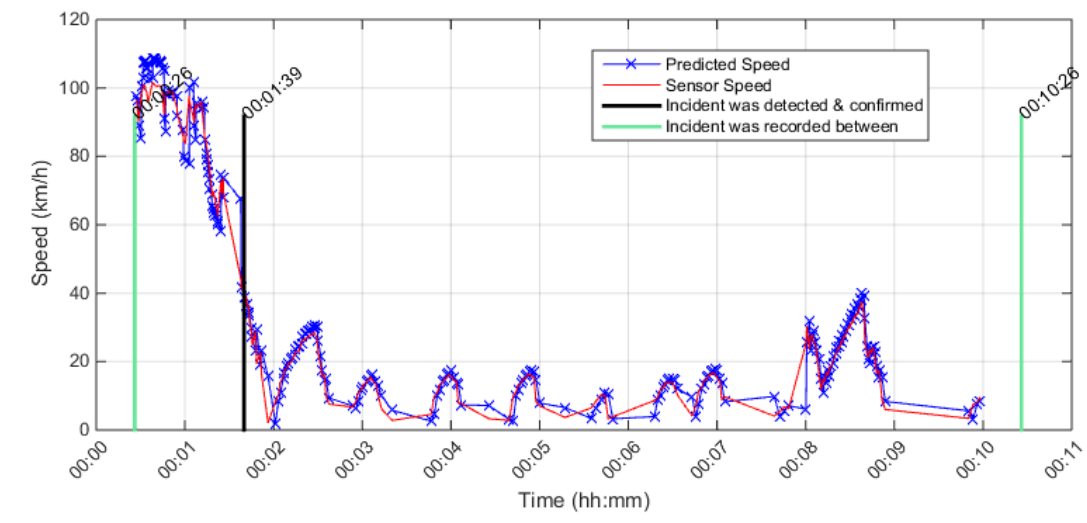

Upstream Detector \# is 401DE0010DEE, Detector Rank $=1$, Distance from incident location in $(\mathrm{km})=0.35605$ Season $=$ Winter, Stream $=E$, Direction $=E$, Date \& Time $=2011-02-0100: 00: 26$ Incident Reason = Collision

Affected Lanes $=2$ RIGHT LANE(S)

Conditional Probability level $=0.6$, Cond. Prob. $r 3 \mathrm{~s} 2=0.2$, Cond.Prob. $r 3 \mathrm{~s} 1=0.1$, Percent drop in speed $\%=-56$

Max. Posterior Probability level $=0.97$, Percent range between speed states $=7 \%$

Incident was detected at 00:26 minutes from drop in speed point. Incident was confirmed after 5 minutes from the detection time

Incident Detection Utilizing the Algorithm, Data obtained from Right Lane \#1, Run18 


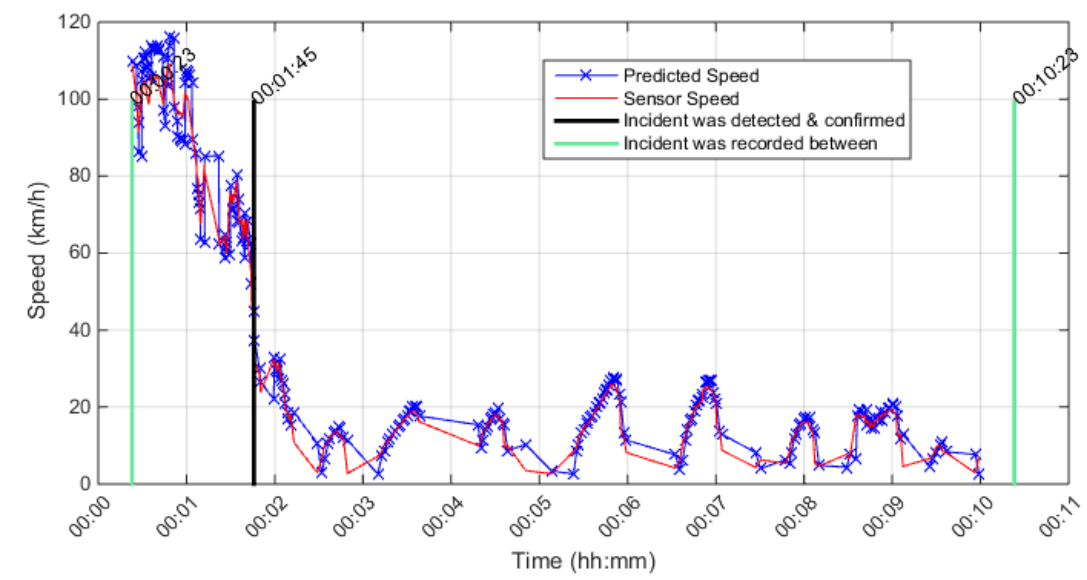

Upstream Detector \# is 401DE0010DEE, Detector Rank $=1$, Distance from incident location in $(\mathrm{km})=0.35605$ Season $=$ Winter, Stream $=E$, Direction $=E$, Date \& Time $=2011-02-0100: 00: 23$ Incident Reason $=$ Collision

Affected Lanes = 2 RIGHT LANE(S)

Conditional Probability level $=0.6$, Cond.Prob. $r 3 s 2=0.2$, Cond. Prob. $r 3 s 1=0.1$, Percent drop in speed $\%=-56$

Max. Posterior Probability level $=0.97$, Percent range between speed states $=7 \%$

Incident was detected at 00:23 minutes from drop in speed point. Incident was confirmed after 5 minutes from the detection time

Incident Detection Utilizing the Algorithm, Data obtained from Right Lane \#1, Run19

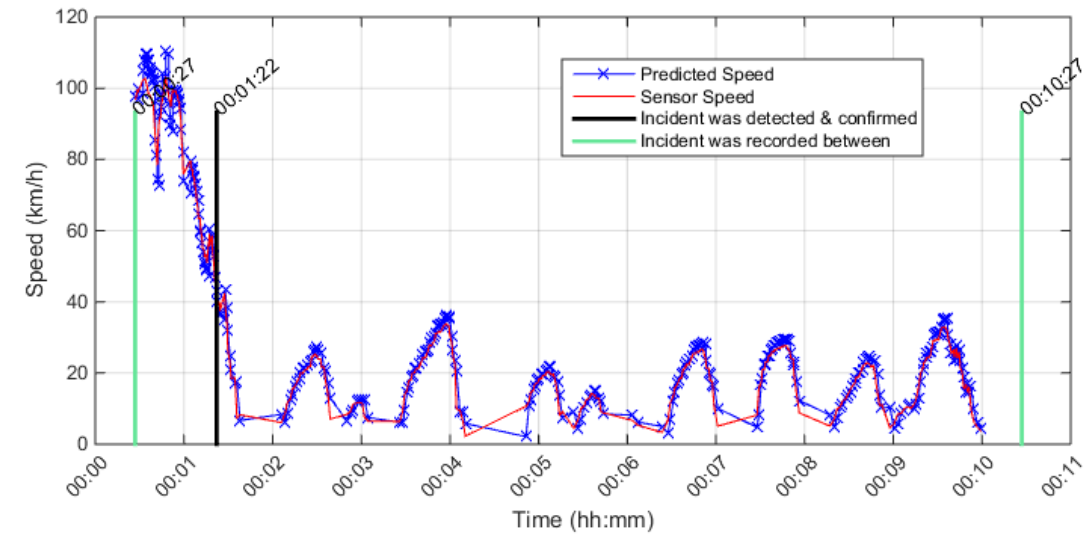

Upstream Detector \# is 401DE0010DEE, Detector Rank $=1$, Distance from incident location in $(\mathrm{km})=0.35605$ Season $=$ Winter, Stream $=E$, Direction $=E$, Date \& Time $=$ 2011-02-01 00:00:27 Incident Reason $=$ Collision

Affected Lanes = 2 RIGHT LANE(S)

Conditional Probability level $=0.6$, Cond.Prob. $r 3 s 2=0.2$, Cond. Prob. $r 3 s 1=0.1$, Percent drop in speed $\%=-56$

Max. Posterior Probability level $=0.97$, Percent range between speed states $=7 \%$

Incident was detected at 00:17 minutes from drop in speed point. Incident was confirmed after 5 minutes from the detection time

Incident Detection Utilizing the Algorithm, Data obtained from Right Lane \#1, Run20 


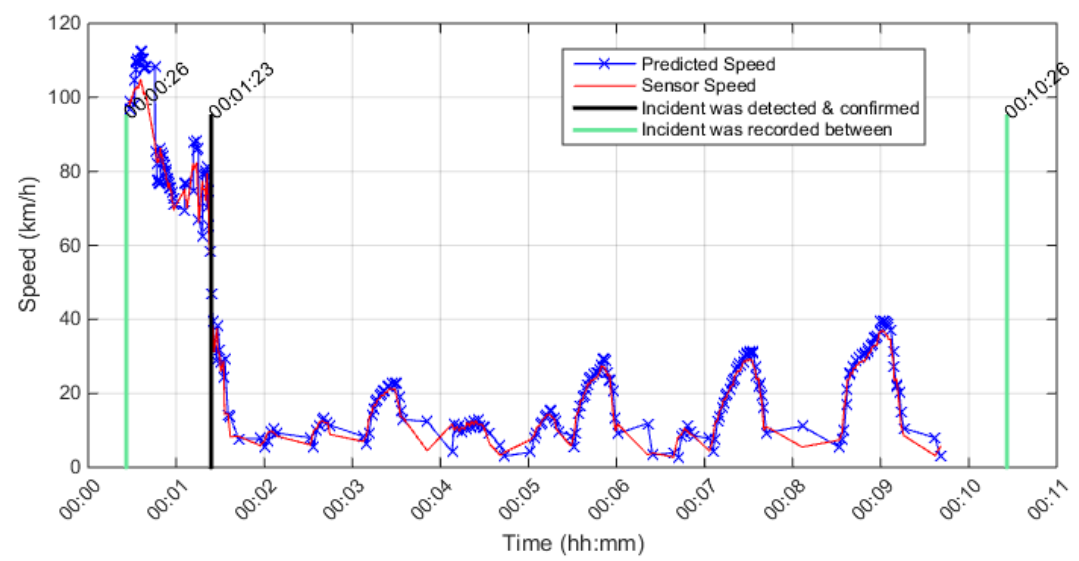

Upstream Detector \# is 401DE0010DEE, Detector Rank = 1, Distance from incident location in $(\mathrm{km})=0.35605$ Season $=$ Winter, Stream $=E$, Direction $=E$, Date \& Time $=2011-02-0100: 00: 26$ Incident Reason $=$ Collision

Affected Lanes $=2$ RIGHT LANE(S)

Conditional Probability level $=0.6$, Cond.Prob. $r 3 s 2=0.2$, Cond. Prob. $r 3 s 1=0.1$, Percent drop in speed $\%=-56$

Max. Posterior Probability level $=0.97$, Percent range between speed states $=7 \%$

Incident was detected at 00:02 minutes from drop in speed point. Incident was confirmed after 5 minutes from the detection time

\section{Incident Detection Utilizing the Algorithm, Data obtained from Right Lane \#1, Run-} 21

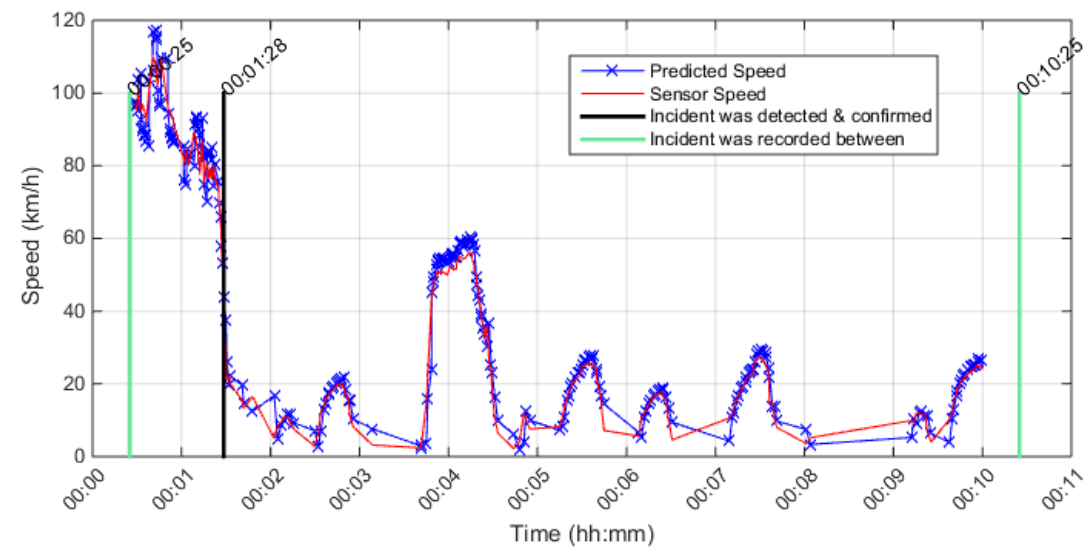

Upstream Detector \# is 401DE0010DEE, Detector Rank = 1, Distance from incident location in $(\mathrm{km})=0.35605$ Season $=$ Winter, Stream $=$ E, Direction $=E$, Date \& Time $=2011-02-0100: 00: 25$ Incident Reason $=$ Collision

Affected Lanes = 2 RIGHT LANE(S)

Conditional Probability level $=0.6$, Cond. Prob. $r 3 s 2=0.2$, Cond. Prob. $r 3 s 1=0.1$, Percent drop in speed $\%=-56$

Max. Posterior Probability level $=0.97$, Percent range between speed states $=7 \%$

Incident was detected at 00:06 minutes from drop in speed point. Incident was confirmed after 5 minutes from the detection time

Incident Detection Utilizing the Algorithm, Data obtained from Right Lane \#1, Run22 


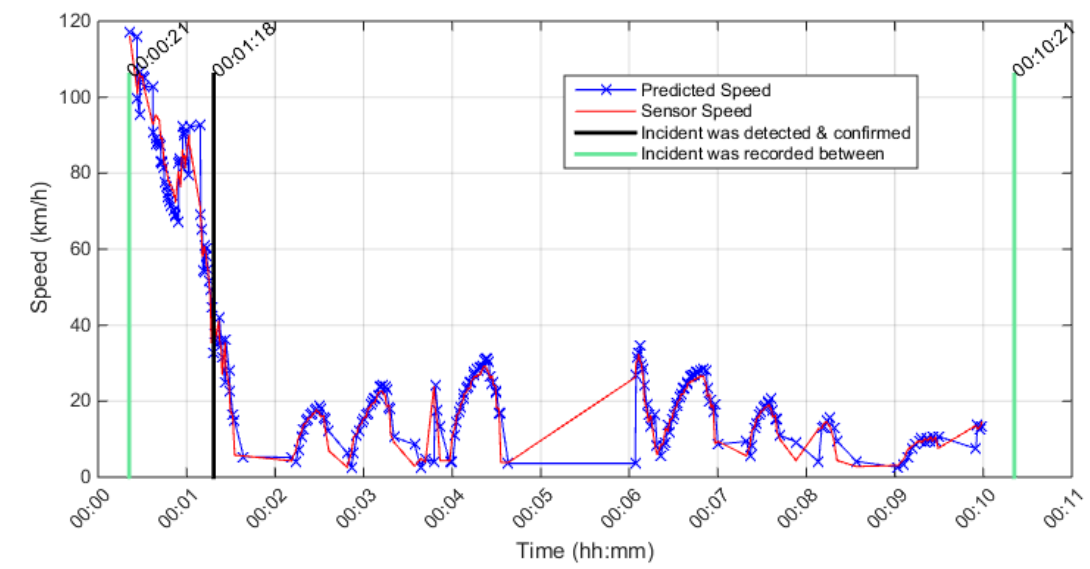

Upstream Detector \# is 401DE0010DEE, Detector Rank $=1$, Distance from incident location in $(\mathrm{km})=0.35605$ Season $=$ Winter, Stream $=E$, Direction $=E$, Date $\&$ Time $=2011-02-0100: 00: 21$

Incident Reason $=$ Collision

Affected Lanes $=2$ RIGHT LANE(S)

Conditional Probability level $=0.6$, Cond. Prob. $r 3 s 2=0.2$, Cond. Prob. $r 3 s 1=0.1$, Percent drop in speed $\%=-56$

Max. Posterior Probability level $=0.97$, Percent range between speed states $=7 \%$

Incident was detected at 00:09 minutes from drop in speed point. Incident was confirmed after 5 minutes from the detection time

\section{Incident Detection Utilizing the Algorithm, Data obtained from Right Lane \#1, Run-} 23

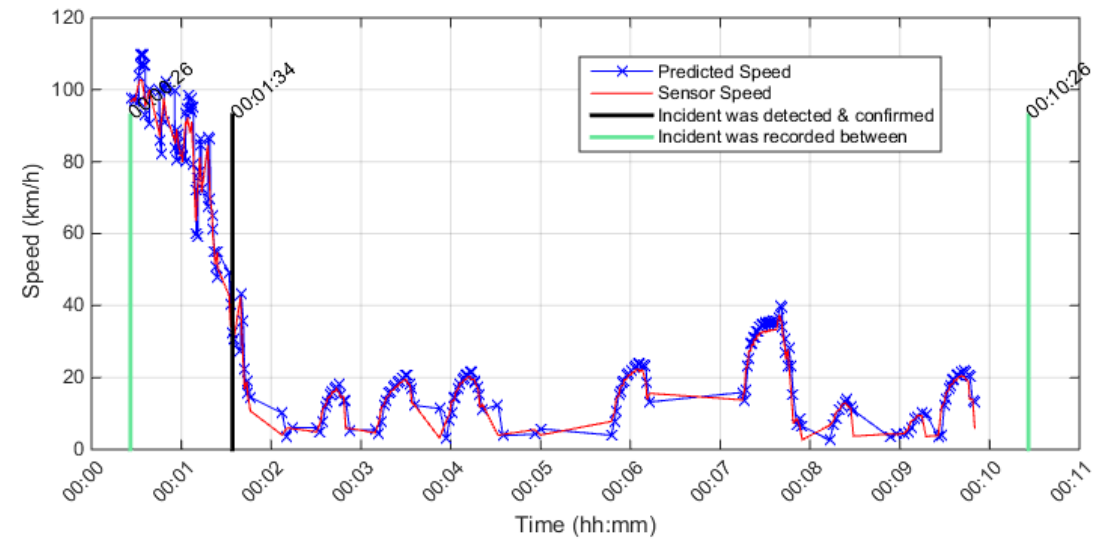

Upstream Detector \# is 401DE0010DEE, Detector Rank $=1$, Distance from incident location in $(\mathrm{km})=0.35605$ Season $=$ Winter, Stream $=E$, Direction $=E$, Date \& Time $=2011-02-0100: 00: 26$ Incident Reason $=$ Collision

Affected Lanes $=2$ RIGHT LANE(S)

Conditional Probability level $=0.6$, Cond. Prob. $r 3 s 2=0.2$, Cond. Prob. $r 3 s 1=0.1$, Percent drop in speed $\%=-56$

Max. Posterior Probability level $=0.97$, Percent range between speed states $=7 \%$

Incident was detected at 00:14 minutes from drop in speed point. Incident was confirmed after 5 minutes from the detection time

\section{Incident Detection Utilizing the Algorithm, Data obtained from Right Lane \#1, Run-}




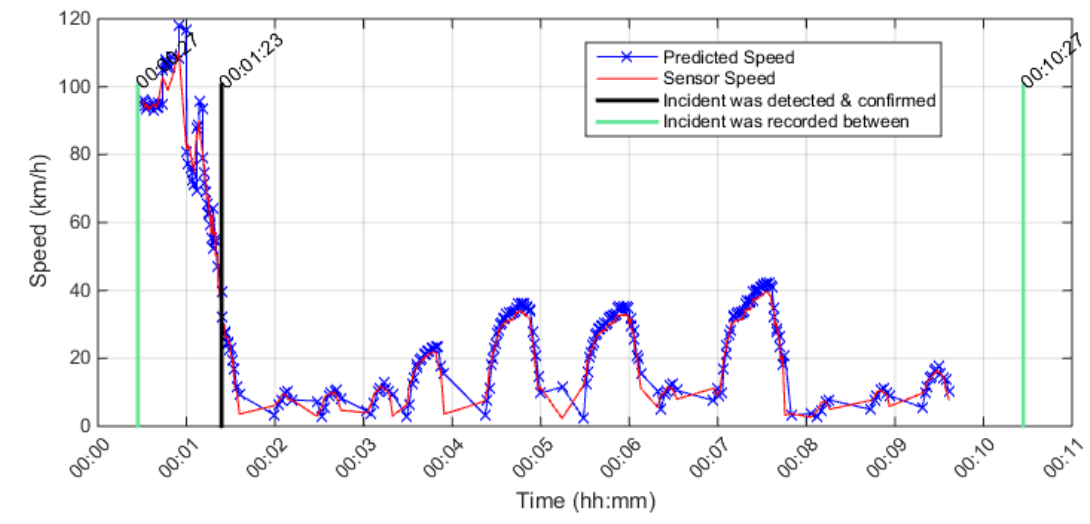

Upstream Detector \# is 401DE0010DEE, Detector Rank = 1, Distance from incident location in $(\mathrm{km})=0.35605$ Season $=$ Winter, Stream $=E$, Direction $=E$, Date $\&$ Time $=2011-02-0100: 00: 27$ Incident Reason $=$ Collision
Affected Lanes $=2$ RIGHT LANE(S)

Conditional Probability level $=0.6$, Cond.Prob. $r 3 s 2=0.2$, Cond. Prob. $r 3 s 1=0.1$, Percent drop in speed $\%=-56$ Max. Posterior Probability level $=0.97$, Percent range between speed states $=7 \%$

Incident was detected at 00:12 minutes from drop in speed point. Incident was confirmed after 5 minutes from the detection time

\section{Incident Detection Utilizing the Algorithm, Data obtained from Right Lane \#1, Run-} 25

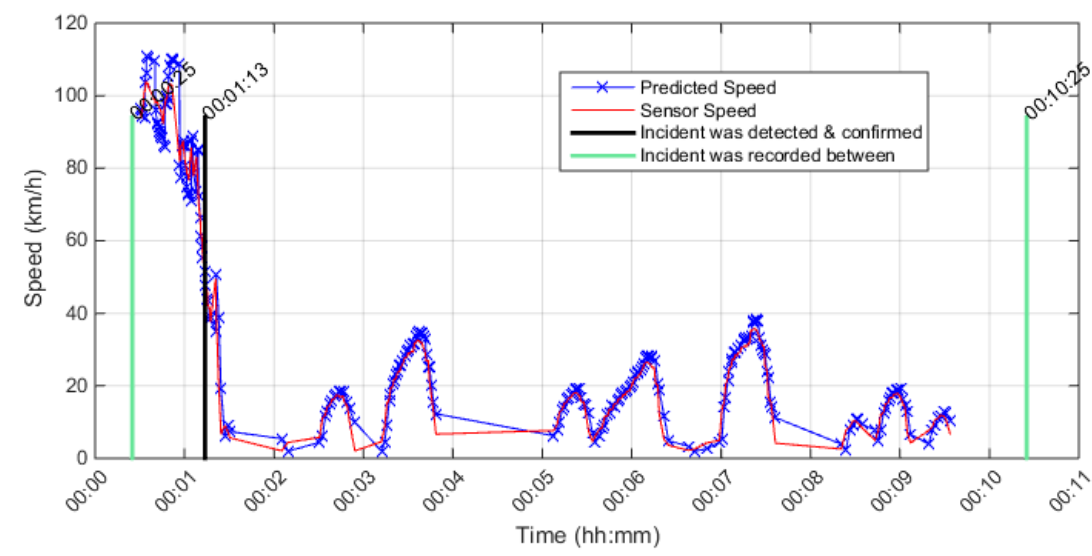

Upstream Detector \# is 401DE0010DEE, Detector Rank $=1$, Distance from incident location in $(\mathrm{km})=0.35605$ Season $=$ Winter, Stream $=E$, Direction $=E$, Date $\&$ Time $=2011-02-0100: 00: 25$ Incident Reason $=$ Collision

Affected Lanes = 2 RIGHT LANE(S)

Conditional Probability level $=0.6$, Cond.Prob. $r 3 s 2=0.2$, Cond. Prob. $r 3 s 1=0.1$, Percent drop in speed $\%=-56$

Max. Posterior Probability level $=0.97$, Percent range between speed states $=7 \%$

Incident was detected at 00:04 minutes from drop in speed point. Incident was confirmed after 5 minutes from the detection time

Incident Detection Utilizing the Algorithm, Data obtained from Right Lane \#1, Run26 


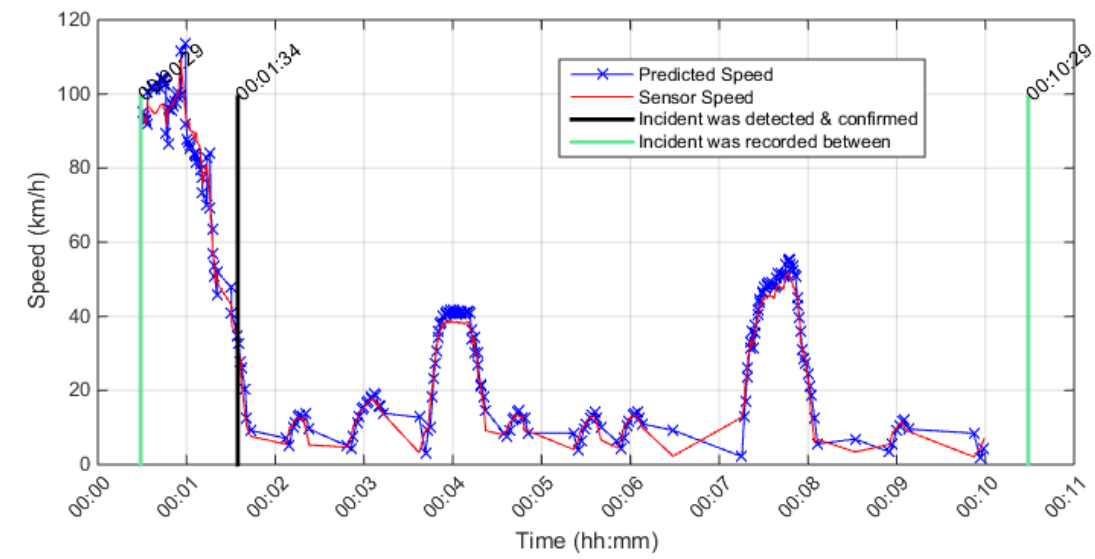

Upstream Detector \# is 401DE0010DEE, Detector Rank = 1, Distance from incident location in $(\mathrm{km})=0.35605$ Season $=$ Winter, Stream $=E$, Direction $=E$, Date \& Time $=2011-02-0100: 00: 29$ Incident Reason $=$ Collision

Affected Lanes = 2RIGHT LANE(S)

Conditional Probability level $=0.6$, Cond.Prob. $\mathrm{r} 3 \mathrm{~s} 2=0.2$, Cond.Prob. $\mathrm{r} 3 \mathrm{~s} 1=0.1$, Percent drop in speed $\%=-56$

Max. Posterior Probability level $=0.97$, Percent range between speed states $=7 \%$

Incident was detected at 00:18 minutes from drop in speed point. Incident was confirmed after 5 minutes from the detection time

\section{Incident Detection Utilizing the Algorithm, Data obtained from Right Lane \#1, Run-} 27

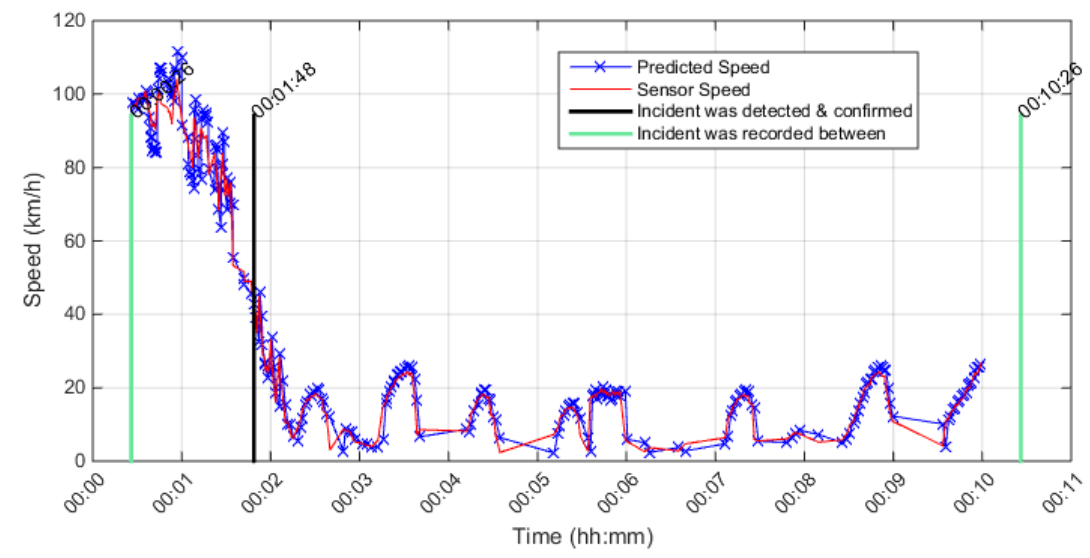

Upstream Detector \# is 401DE0010DEE, Detector Rank $=1$, Distance from incident location in $(\mathrm{km})=0.35605$ Season $=$ Winter, Stream $=E$, Direction $=E$, Date \& Time $=2011-02-0100: 00: 26$ Incident Reason $=$ Collision

Affected Lanes $=2$ RIGHT LANE(S)

Conditional Probability level $=0.6$, Cond.Prob. $r 3 \mathrm{~s} 2=0.2$, Cond.Prob. $r 3 \mathrm{~s} 1=0.1$, Percent drop in speed $\%=-56$

Max. Posterior Probability level $=0.97$, Percent range between speed states $=7 \%$

Incident was detected at 00:17 minutes from drop in speed point. Incident was confirmed after 5 minutes from the detection time

Incident Detection Utilizing the Algorithm, Data obtained from Right Lane \#1, Run28 


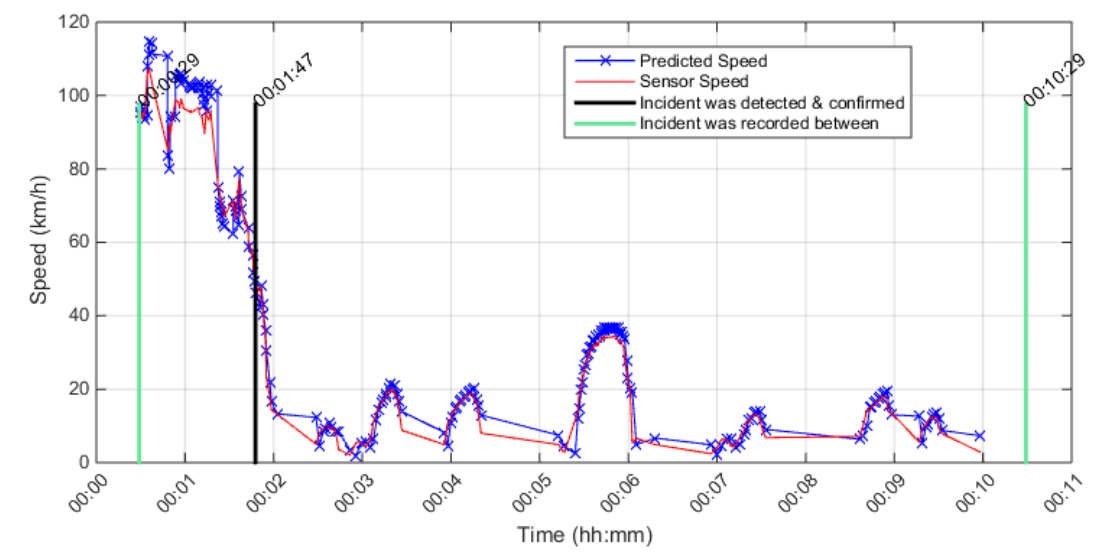

Upstream Detector \# is 401DE0010DEE, Detector Rank = 1, Distance from incident location in $(\mathrm{km})=0.35605$ Season $=$ Winter, Stream $=E$, Direction $=E$, Date $\&$ Time $=2011-02-0100: 00: 29$ Incident Reason $=$ Collision

Affected Lanes $=2$ RIGHT LANE(S)

Conditional Probability level $=0.6$, Cond. Prob. $r 3 \mathrm{~s} 2=0.2$, Cond. Prob. $r 3 \mathrm{~s} 1=0.1$, Percent drop in speed $\%=-56$

Max. Posterior Probability level $=0.97$, Percent range between speed states $=7 \%$

Incident was detected at 00:10 minutes from drop in speed point. Incident was confirmed after 5 minutes from the detection time

Incident Detection Utilizing the Algorithm, Data obtained from Right Lane \#1, Run29

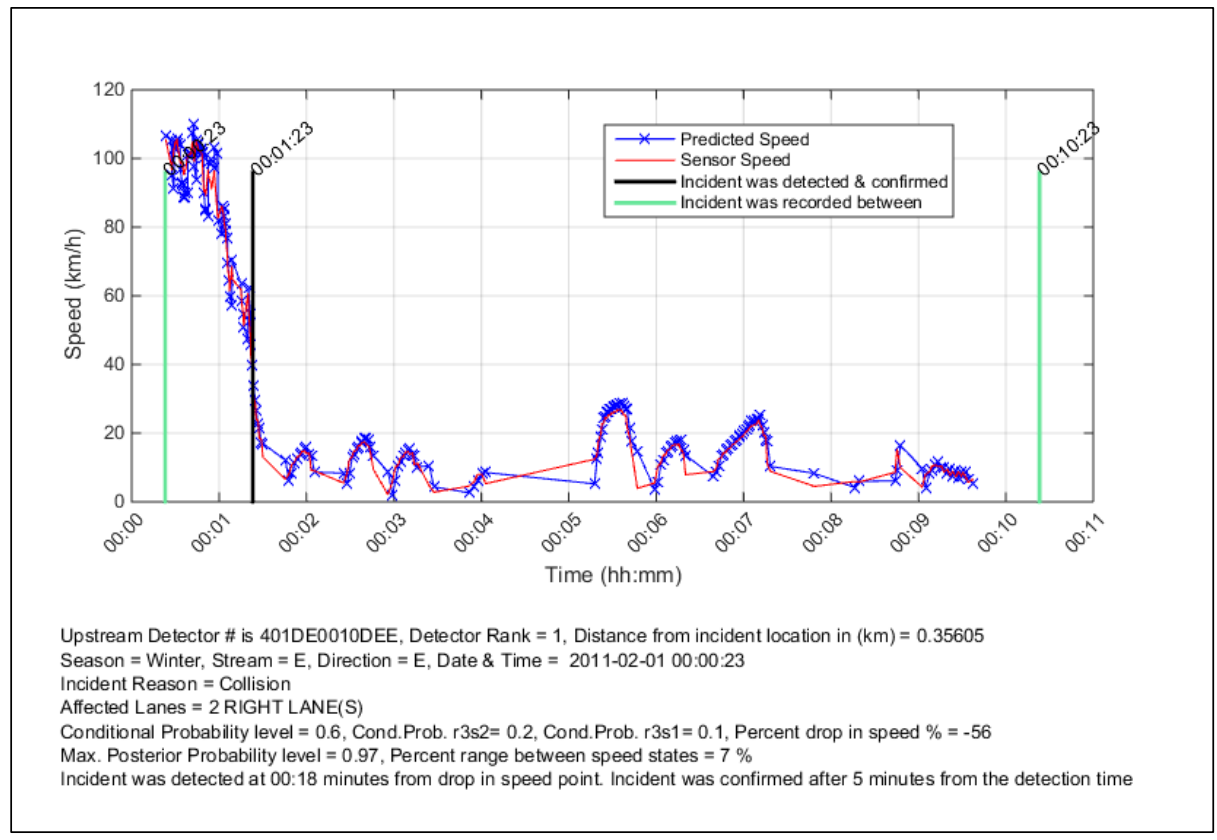

Incident Detection Utilizing the Algorithm, Data obtained from Right Lane \#1, Run30 


\section{Incident detection application, two lane blockage, $v=2000 \mathrm{veh} / \mathrm{l} / \mathrm{h}$, data obtained from the second lane.}

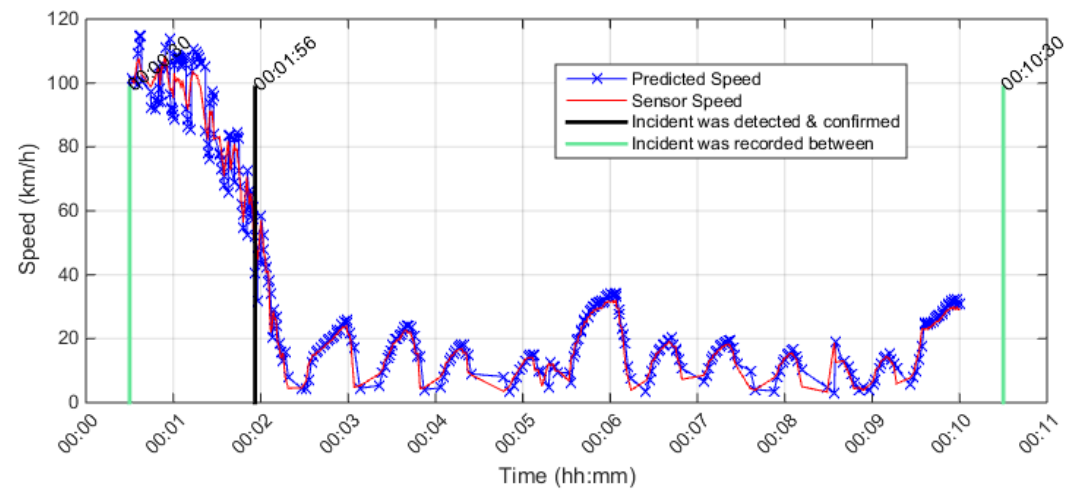

Upstream Detector \# is 401DE0010DEE, Detector Rank $=1$, Distance from incident location in $(\mathrm{km})=0.35605$

Season $=$ Winter, Stream $=E$, Direction $=E$, Date $\&$ Time $=2011-02-0100: 00: 30$

Incident Reason $=$ Collision

Affected Lanes $=2$ RIGHT LANE $($ S)

Conditional Probability level $=0.6$, Cond.Prob. $r 3 s 2=0.2$, Cond. Prob. $r 3 s 1=0.1$, Percent drop in speed $\%=-56$

Max. Posterior Probability level $=0.97$, Percent range between speed states $=7 \%$

Incident was detected at 00:11 minutes from drop in speed point. Incident was confirmed after 5 minutes from the detection time

Incident Detection Utilizing the Algorithm, Data obtained from Right Lane \#2, Run2

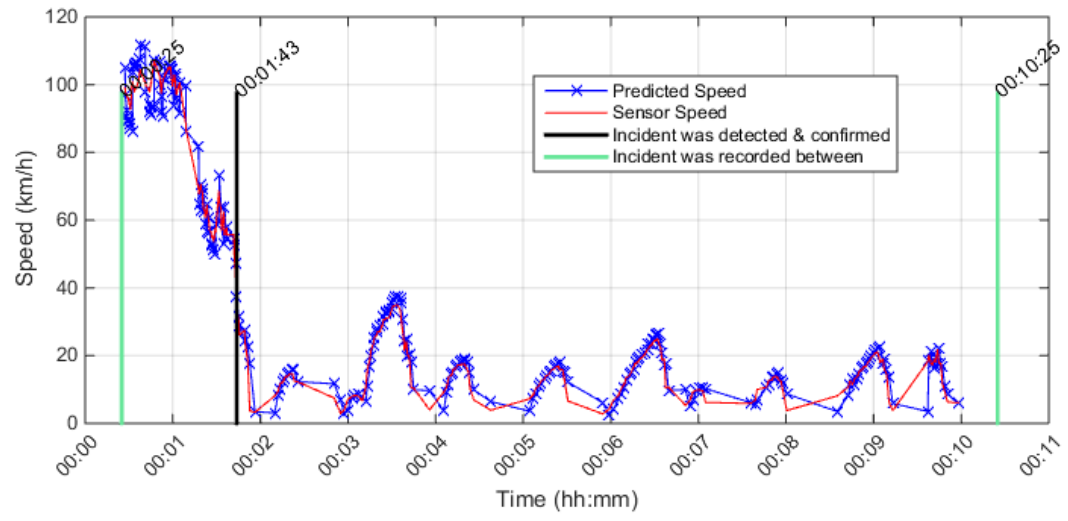

Upstream Detector \# is 401DE0010DEE, Detector Rank $=1$, Distance from incident location in $(\mathrm{km})=0.35605$

Season $=$ Winter, Stream $=E$, Direction $=E$, Date \& Time $=2011-02-0100: 00: 25$

Incident Reason $=$ Collision

Incident Reason $=$ Collision
Affected Lanes $=2$ RIGHT LANE(S)

Conditional Probability level $=0.6$, Cond.Prob. $r 3 s 2=0.2$, Cond.Prob. $r 3 s 1=0.1$, Percent drop in speed $\%=-56$

Max. Posterior Probability level $=0.97$, Percent range between speed states $=7 \%$

Incident was detected at 00:34 minutes from drop in speed point. Incident was confirmed after 5 minutes from the detection time

Incident Detection Utilizing the Algorithm, Data obtained from Right Lane \#2, Run- 


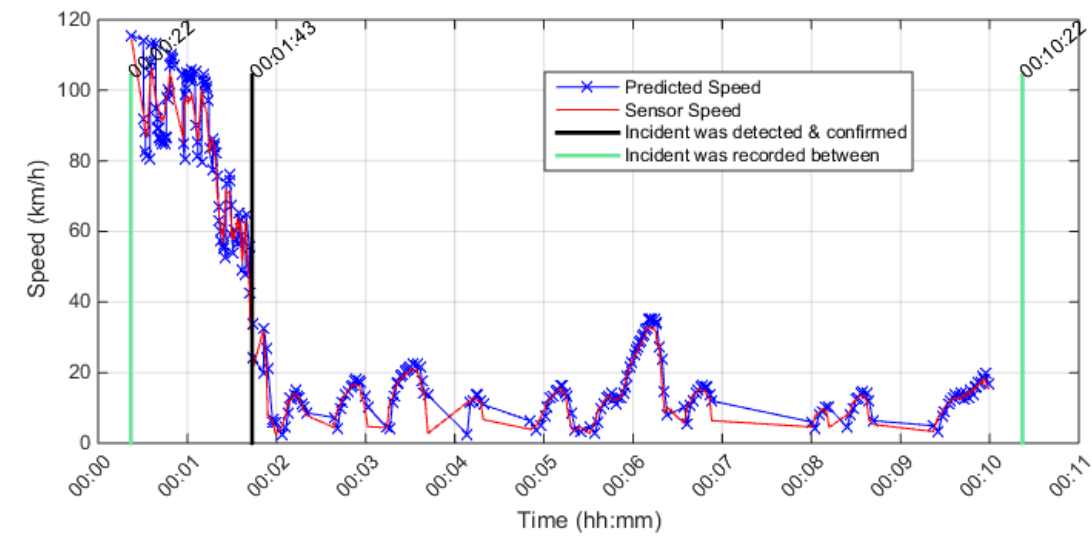

Upstream Detector \# is 401DE0010DEE, Detector Rank $=1$, Distance from incident location in $(\mathrm{km})=0.35605$ Season $=$ Winter, Stream $=E$, Direction $=E$, Date $\&$ Time $=2011-02-0100: 00: 22$ Incident Reason $=$ Collision

Affected Lanes = 2RIGHT LANE(S)

Conditional Probability level $=0.6$, Cond.Prob. r $3 \mathrm{~s} 2=0.2$, Cond.Prob. $\mathrm{r} 3 \mathrm{~s} 1=0.1$, Percent drop in speed $\%=-56$ Max. Posterior Probability level $=0.97$, Percent range between speed states $=7 \%$

Incident was detected at 00:24 minutes from drop in speed point. Incident was confirmed after 5 minutes from the detection time

\section{Incident Detection Utilizing the Algorithm, Data obtained from Right Lane \#2, Run-} 4

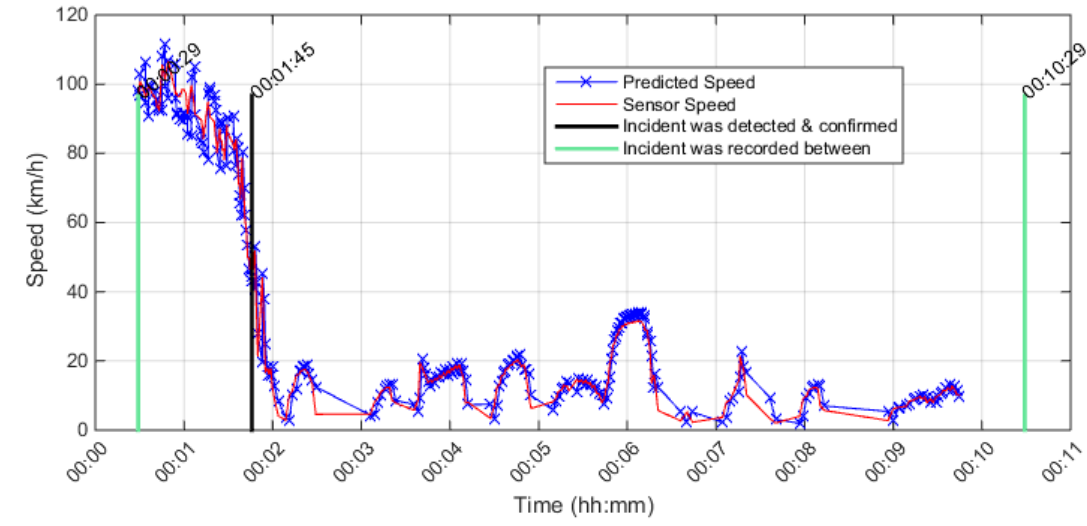

Upstream Detector \# is 401DE0010DEE, Detector Rank $=1$, Distance from incident location in $(\mathrm{km})=0.35605$ Season $=$ Winter, Stream $=E$, Direction $=E$, Date $\&$ Time $=2011-02-0100: 00: 29$ Incident Reason $=$ Collision

Affected Lanes $=2$ RIGHT LANE(S)

Conditional Probability level $=0.6$, Cond.Prob. $r 3 \mathrm{~s} 2=0.2$, Cond.Prob. $r 3 \mathrm{~s} 1=0.1$, Percent drop in speed $\%=-56$

Max. Posterior Probability level $=0.97$, Percent range between speed states $=7 \%$

Incident was detected at 00:06 minutes from drop in speed point. Incident was confirmed after 5 minutes from the detection time

Incident Detection Utilizing the Algorithm, Data obtained from Right Lane \#2, Run5 


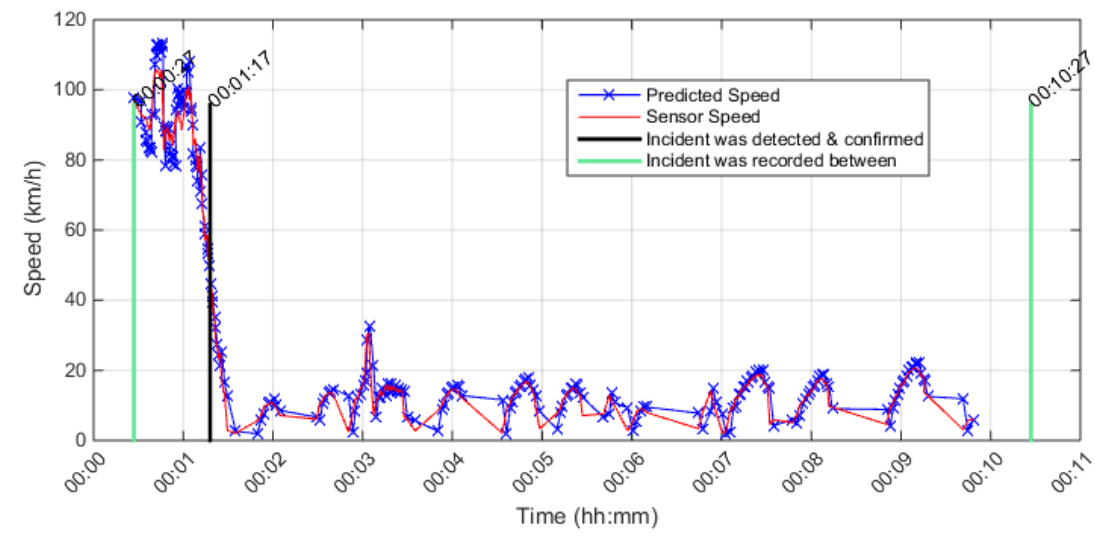

Upstream Detector \# is 401DE0010DEE, Detector Rank $=1$, Distance from incident location in $(\mathrm{km})=0.35605$ Season $=$ Winter, Stream $=E$, Direction $=E$, Date $\&$ Time $=2011-02-0100: 00: 27$ Incident Reason $=$ Collision

Affected Lanes $=2$ RIGHT LANE(S)

Conditional Probability level $=0.6$, Cond. Prob. $r 3 \mathrm{~s} 2=0.2$, Cond. Prob. $r 3 \mathrm{~s} 1=0.1$, Percent drop in speed $\%=-56$

Max. Posterior Probability level $=0.97$, Percent range between speed states $=7 \%$

Incident was detected at 00:06 minutes from drop in speed point. Incident was confirmed after 5 minutes from the detection time

\section{Incident Detection Utilizing the Algorithm, Data obtained from Right Lane \#2, Run-} 6

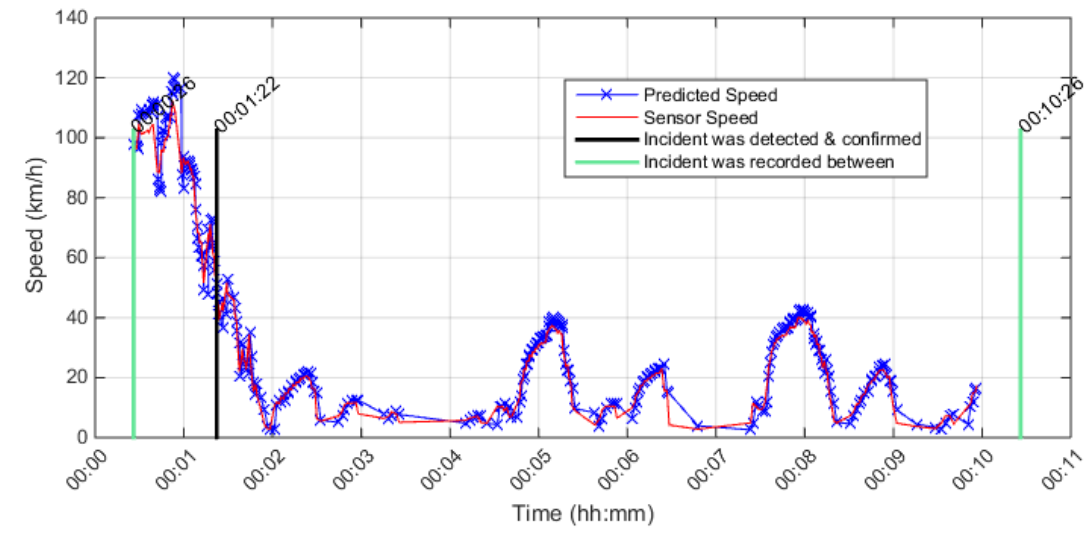

Upstream Detector \# is 401DE0010DEE, Detector Rank = 1, Distance from incident location in $(\mathrm{km})=0.35605$ Season $=$ Winter, Stream $=E$, Direction $=E$, Date $\&$ Time $=2011-02-0100: 00: 26$ Incident Reason $=$ Collision

Affected Lanes $=2$ RIGHT LANE(S)

Conditional Probability level $=0.6$, Cond.Prob. $r 3 s 2=0.2$, Cond.Prob. $r 3 s 1=0.1$, Percent drop in speed $\%=-56$

Max. Posterior Probability level $=0.97$, Percent range between speed states $=7 \%$

Incident was detected at 00:14 minutes from drop in speed point. Incident was confirmed after 5 minutes from the detection time

Incident Detection Utilizing the Algorithm, Data obtained from Right Lane \#2, Run- 


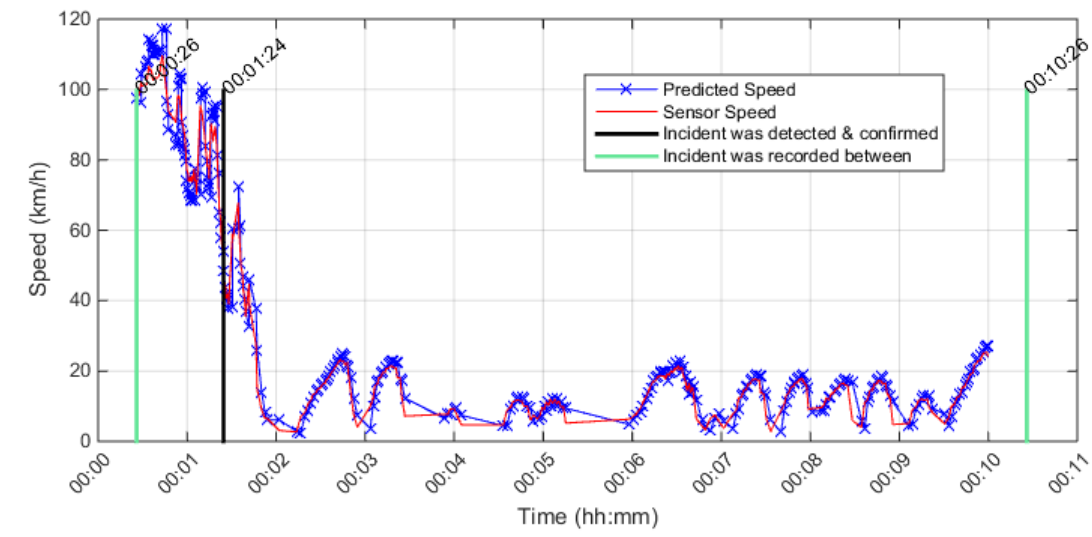

Upstream Detector \# is 401DE0010DEE, Detector Rank $=1$, Distance from incident location in $(\mathrm{km})=0.35605$ Season $=$ Winter, Stream $=E$, Direction $=E$, Date $\&$ Time $=2011-02-0100: 00: 26$ Incident Reason $=$ Collision

Affected Lanes $=2$ RIGHT LANE(S)

Conditional Probability level $=0.6$, Cond.Prob. $r 3 s 2=0.2$, Cond.Prob. $r 3 s 1=0.1$, Percent drop in speed $\%=-56$

Max. Posterior Probability level $=0.97$, Percent range between speed states $=7 \%$

Incident was detected at 00:04 minutes from drop in speed point. Incident was confirmed after 5 minutes from the detection time

Incident Detection Utilizing the Algorithm, Data obtained from Right Lane \#2, Run8

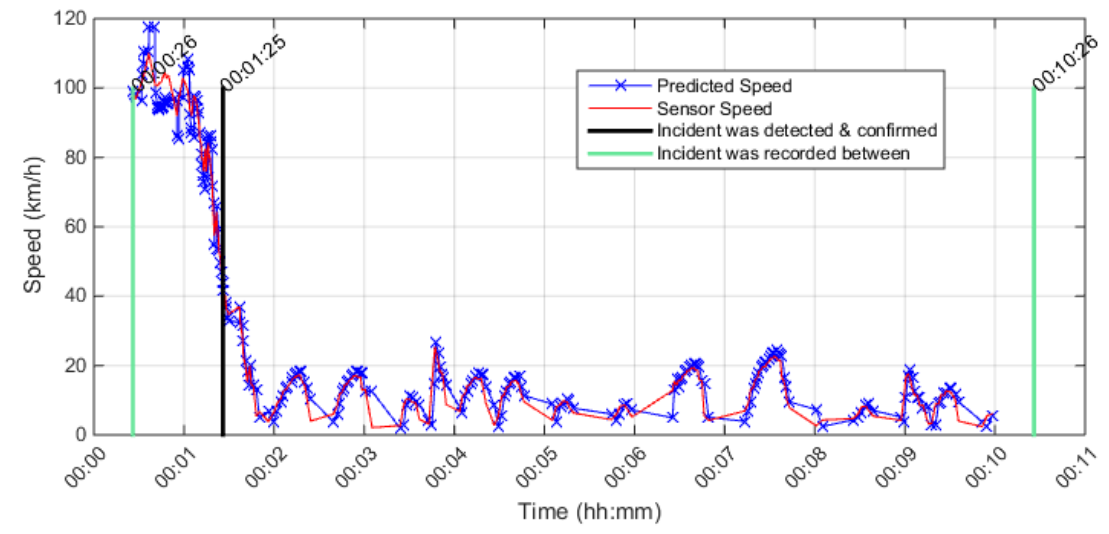

Upstream Detector \# is 401DE0010DEE, Detector Rank $=1$, Distance from incident location in $(\mathrm{km})=0.35605$ Season $=$ Winter, Stream $=E$, Direction $=E$, Date \& Time $=2011-02-0100: 00: 26$

Incident Reason $=$ Collision

Affected Lanes = 2 RIGHT LANE(S)

Conditional Probability level $=0.6$, Cond.Prob. $r 3 s 2=0.2$, Cond.Prob. $r 3 s 1=0.1$, Percent drop in speed $\%=-56$

Max. Posterior Probability level $=0.97$, Percent range between speed states $=7 \%$

Incident was detected at 00:07 minutes from drop in speed point. Incident was confirmed after 5 minutes from the detection time

Incident Detection Utilizing the Algorithm, Data obtained from Right Lane \#2, Run- 


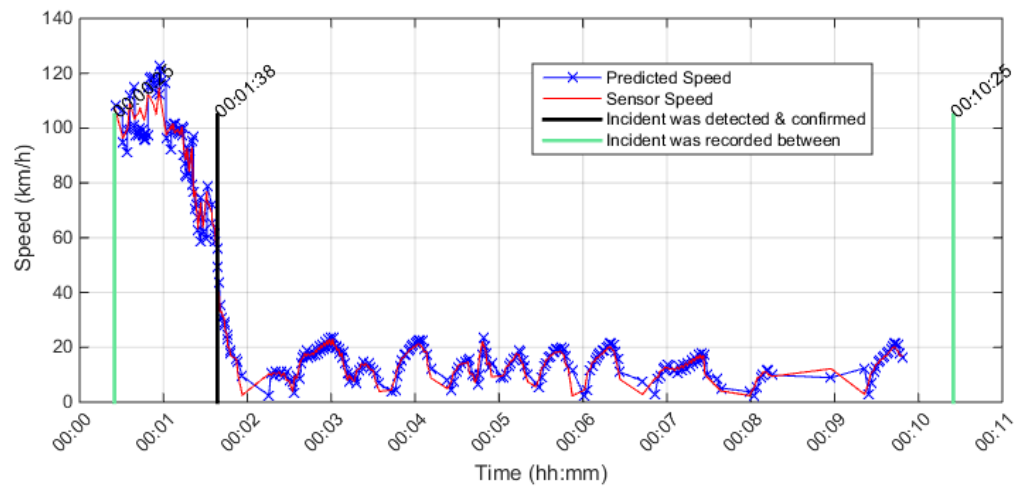

Upstream Detector \# is 401DE0010DEE, Detector Rank $=1$, Distance from incident location in $(\mathrm{km})=0.35605$ Season $=$ Winter, Stream $=E$, Direction $=E$, Date \& Time $=2011-02-0100: 00: 25$

Incident Reason = Collision

Incident Reason = Collision
Affected Lanes = 2RIGHT LANE(S)

Conditional Probability level $=0.6$, Cond.Prob. $r 3 \mathrm{~s} 2=0.2$, Cond. Prob. $r 3 \mathrm{~s} 1=0.1$, Percent drop in speed $\%=-56$

Conditional Probability level $=0.6$, Cond. Prob. r3s $2=0.2$, Cond. Prob. r $3 \mathrm{~s} 1=0.1$, Percen
Max. Posterior Probability level $=0.97$, Percent range between speed states $=7 \%$

Incident was detected at 00:17 minutes from drop in speed point. Incident was confirmed after 5 minutes from the detection time

Incident Detection Utilizing the Algorithm, Data obtained from Right Lane \#2, Run10

\section{Incident Detection from Lane \#1, two-lane blockage and data was collected from the first lane, traffic volume level of $1000 \mathrm{veh} / \mathrm{l} / \mathrm{h}$}

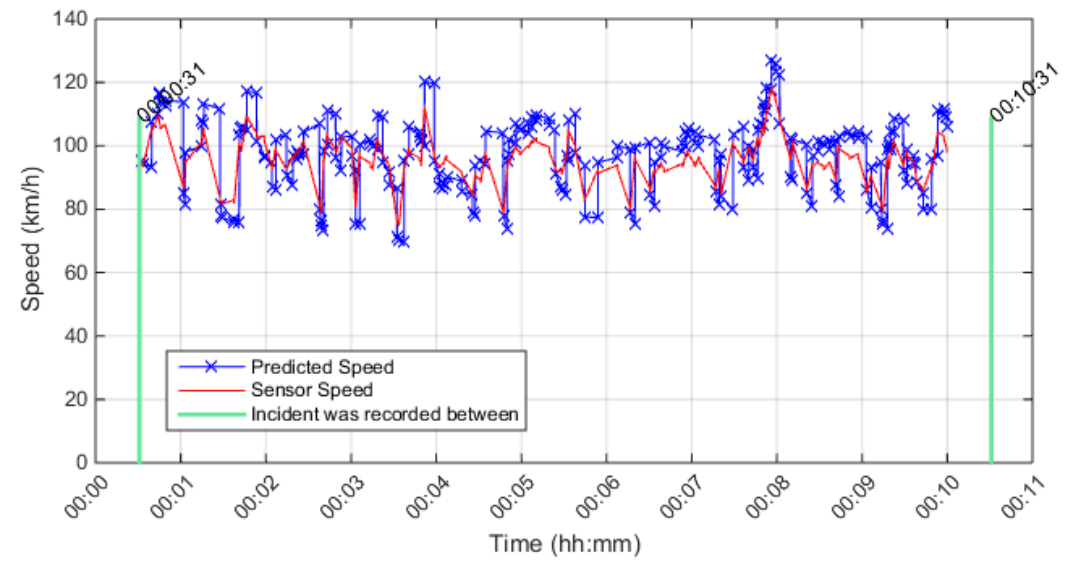

Upstream Detector \# is 401DE0010DEE, Detector Rank = 1, Distance from incident location in $(\mathrm{km})=0.35605$ Season $=$ Winter, Stream $=E$, Direction $=E$, Date \& Time $=$ 2011-02-01 00:00:31 Incident Reason $=$ Collision

Affected Lanes = 2 RIGHT LANE(S)

Conditional Probability level $=0.6$, Cond.Prob. $r 3 s 2=0.2$, Cond. Prob. $r 3 s 1=0.1$, Percent drop in speed $\%=-56$ Max. Posterior Probability level $=0.97$, Percent range between speed states $=7 \%$

(No Incident was Detected)

Incident Detection Utilizing the Algorithm, Data obtained from Right Lane \#1, Run2 


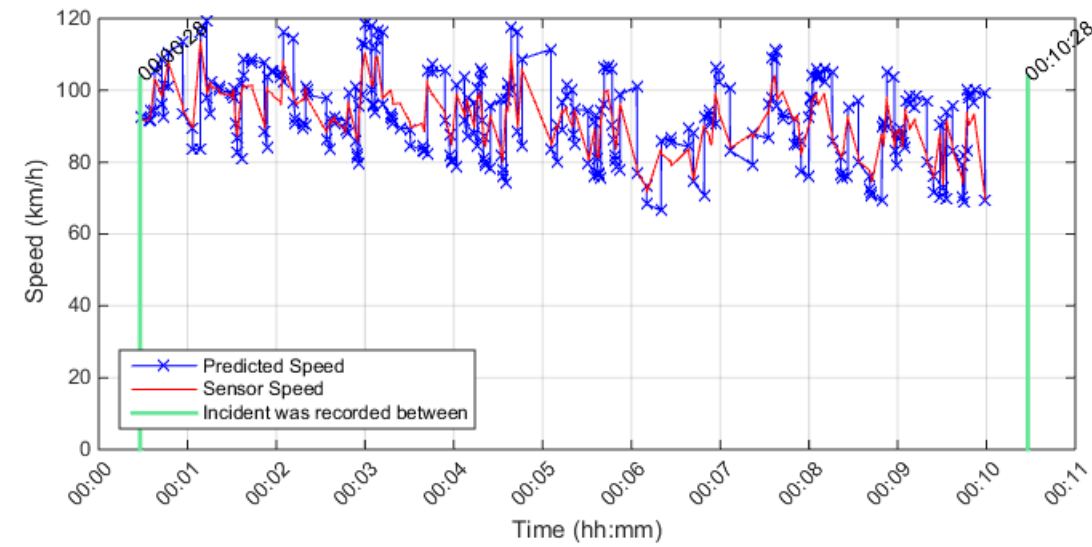

Upstream Detector \# is 401DE0010DEE, Detector Rank = 1, Distance from incident location in $(\mathrm{km})=0.35605$ Season $=$ Winter, Stream $=E$, Direction $=E$, Date $\&$ Time $=2011-02-0100: 00: 28$ Incident Reason $=$ Collision

Affected Lanes $=2$ RIGHT LANE(S)

Conditional Probability level $=0.6$, Cond.Prob. $r 3 \mathrm{~s} 2=0.2$, Cond. Prob. $r 3 \mathrm{~s} 1=0.1$, Percent drop in speed $\%=-56$ Max. Posterior Probability level $=0.97$, Percent range between speed states $=7 \%$ (No Incident was Detected)

\section{Incident Detection Utilizing the Algorithm, Data obtained from Right Lane \#1, Run-}

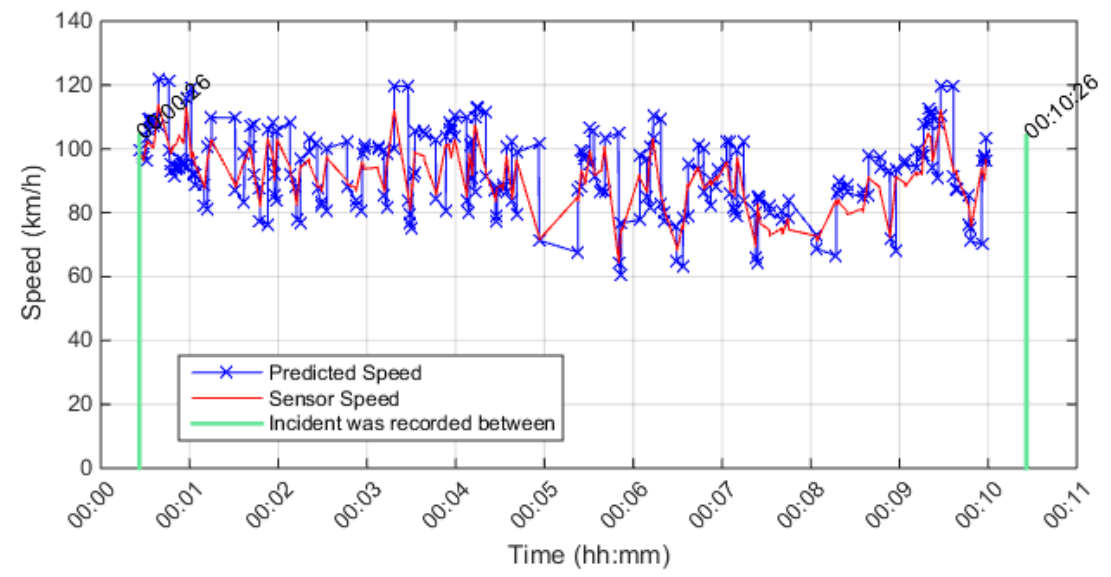

Upstream Detector \# is 401DE0010DEE, Detector Rank = 1, Distance from incident location in $(\mathrm{km})=0.35605$ Season $=$ Winter, Stream $=E$, Direction $=E$, Date \& Time $=2011-02-01$ 00:00:26 Incident Reason $=$ Collision

Affected Lanes $=2$ RIGHT LANE(S)

Conditional Probability level $=0.6$, Cond.Prob. $r 3 s 2=0.2$, Cond. Prob. $r 3 s 1=0.1$, Percent drop in $s p e e d \%=-56$ Max. Posterior Probability level $=0.97$, Percent range between speed states $=7 \%$ (No Incident was Detected)

\section{Incident Detection Utilizing the Algorithm, Data obtained from Right Lane \#1, Run-}




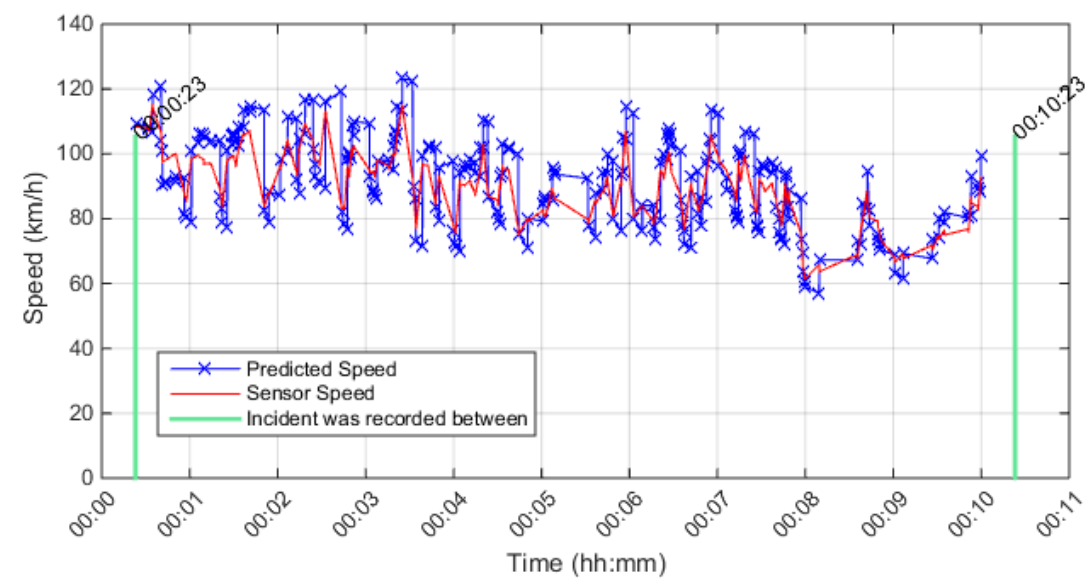

Upstream Detector \# is 401DE0010DEE, Detector Rank = 1, Distance from incident location in $(\mathrm{km})=0.35605$ Season $=$ Winter, Stream $=E$, Direction $=E$, Date \& Time $=2011-02-0100: 00: 23$ Incident Reason $=$ Collision

Affected Lanes $=2$ RIGHT LANE(S)

Conditional Probability level $=0.6$, Cond.Prob. $r 3 s 2=0.2$, Cond.Prob. $r 3 s 1=0.1$, Percent drop in $s p e e d \%=-56$ Max. Posterior Probability level $=0.97$, Percent range between speed states $=7 \%$ (No Incident was Detected)

\section{Incident Detection Utilizing the Algorithm, Data obtained from Right Lane \#1, Run-} 5

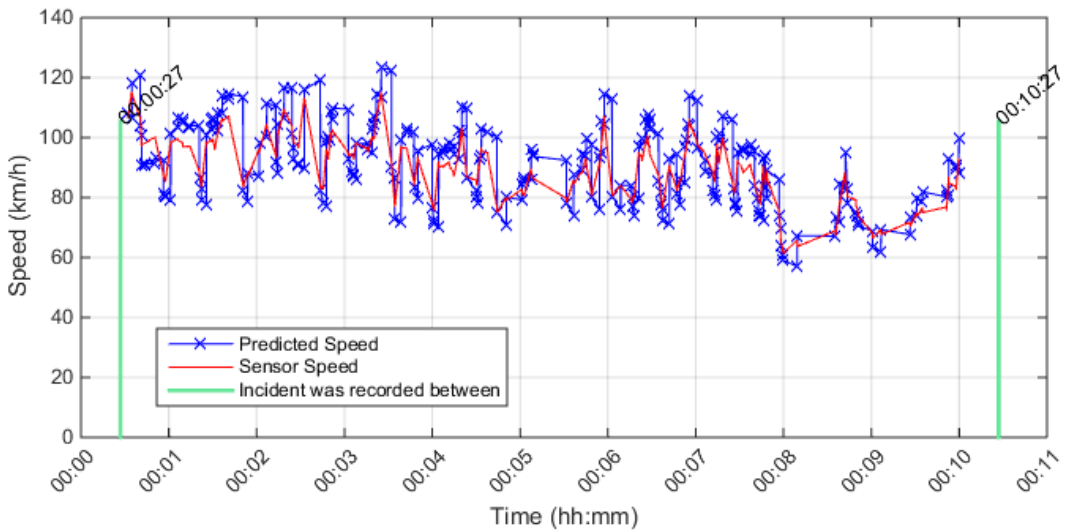

Upstream Detector \# is 401DE0010DEE, Detector Rank $=1$, Distance from incident location in $(\mathrm{km})=0.35605$ Season $=$ Winter, Stream $=E$, Direction $=E$, Date $\&$ Time $=2011-02-0100: 00: 27$ Incident Reason $=$ Collision

Affected Lanes $=2$ RIGHT LANE(S)

Conditional Probability level $=0.6$, Cond.Prob. $r 3 s 2=0.2$, Cond.Prob. $r 3 s 1=0.1$, Percent drop in speed $\%=-56$

Max. Posterior Probability level $=0.97$, Percent range between speed states $=7 \%$

(No Incident was Detected)

Incident Detection Utilizing the Algorithm, Data obtained from Right Lane \#1, Run- 


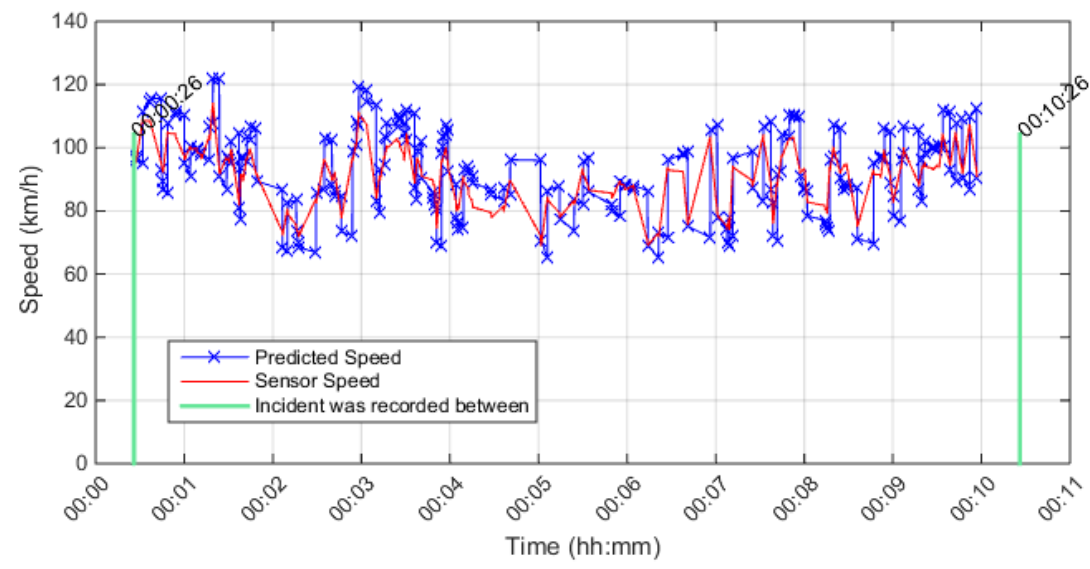

Upstream Detector \# is 401DE0010DEE, Detector Rank $=1$, Distance from incident location in $(\mathrm{km})=0.35605$ Season $=$ Winter, Stream $=E$, Direction $=E$, Date \& Time $=2011-02-0100: 00: 26$ Incident Reason $=$ Collision

Affected Lanes $=2$ RIGHT LANE(S)

Conditional Probability level $=0.6$, Cond.Prob. $r 3 s 2=0.2$, Cond. Prob. $r 3 s 1=0.1$, Percent drop in speed $\%=-56$ Max. Posterior Probability level $=0.97$, Percent range between speed states $=7 \%$ (No Incident was Detected)

\section{Incident Detection Utilizing the Algorithm, Data obtained from Right Lane \#1, Run-}

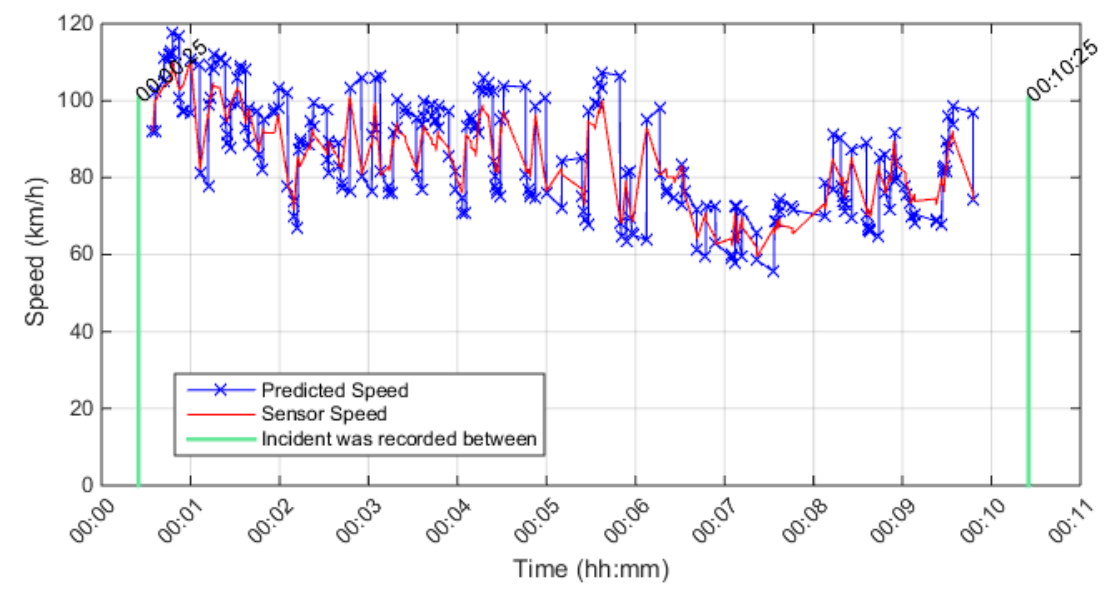

Upstream Detector \# is 401DE0010DEE, Detector Rank = 1, Distance from incident location in $(\mathrm{km})=0.35605$ Season $=$ Winter, Stream $=E$, Direction $=E$, Date \& Time $=$ 2011-02-01 00:00:25 Incident Reason $=$ Collision

Affected Lanes $=2$ RIGHT LANE(S)

Conditional Probability level $=0.6$, Cond.Prob. $r 3 s 2=0.2$, Cond.Prob. $r 3 s 1=0.1$, Percent drop in speed $\%=-56$

Max. Posterior Probability level $=0.97$, Percent range between speed states $=7 \%$

(No Incident was Detected)

Incident Detection Utilizing the Algorithm, Data obtained from Right Lane \#1, Run- 


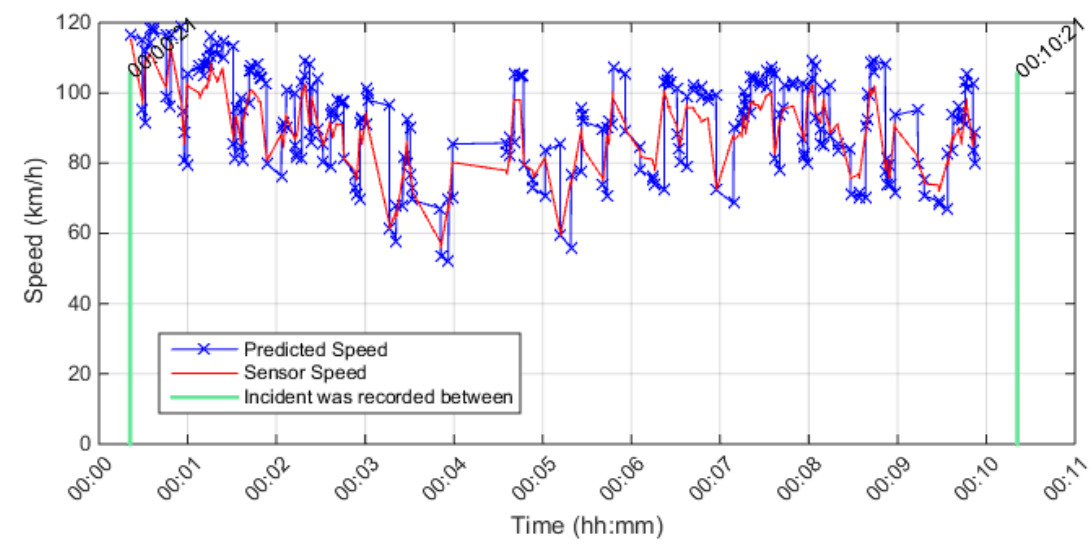

Upstream Detector \# is 401DE0010DEE, Detector Rank = 1, Distance from incident location in $(\mathrm{km})=0.35605$ Season $=$ Winter, Stream $=E$, Direction $=E$, Date \& Time $=2011-02-01$ 00:00:21 Incident Reason $=$ Collision

Affected Lanes = 2RIGHT LANE(S)

Conditional Probability level $=0.6$, Cond.Prob. $r 3 s 2=0.2$, Cond.Prob. $r 3 s 1=0.1$, Percent drop in speed $\%=-56$ Max. Posterior Probability level $=0.97$, Percent range between speed states $=7 \%$

(No Incident was Detected)

\section{Incident Detection Utilizing the Algorithm, Data obtained from Right Lane \#1, Run-} 9

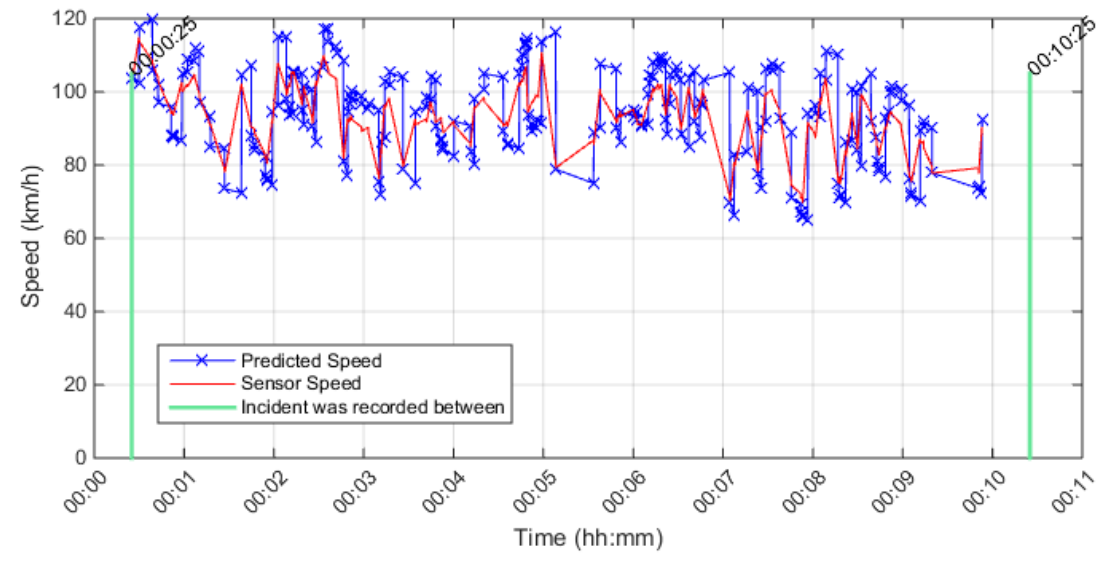

Upstream Detector \# is 401DE0010DEE, Detector Rank $=1$, Distance from incident location in $(\mathrm{km})=0.35605$

Season $=$ Winter, Stream $=E$, Direction $=E$, Date $\&$ Time $=2011-02-0100: 00: 25$

Incident Reason $=$ Collision

Affected Lanes $=2$ RIGHT LANE(S)

Conditional Probability level $=0.6$, Cond.Prob. $r 3 s 2=0.2$, Cond.Prob. $r 3 s 1=0.1$, Percent drop in speed $\%=-56$

Max. Posterior Probability level $=0.97$, Percent range between speed states $=7 \%$

(No Incident was Detected)

Incident Detection Utilizing the Algorithm, Data obtained from Right Lane \#1, Run- 


\section{Incident Detection from Lane \#2, two-lane blockage, and data was collected from the first lane, traffic volume level of $1000 \mathrm{veh} / \mathrm{l} / \mathrm{h}$}

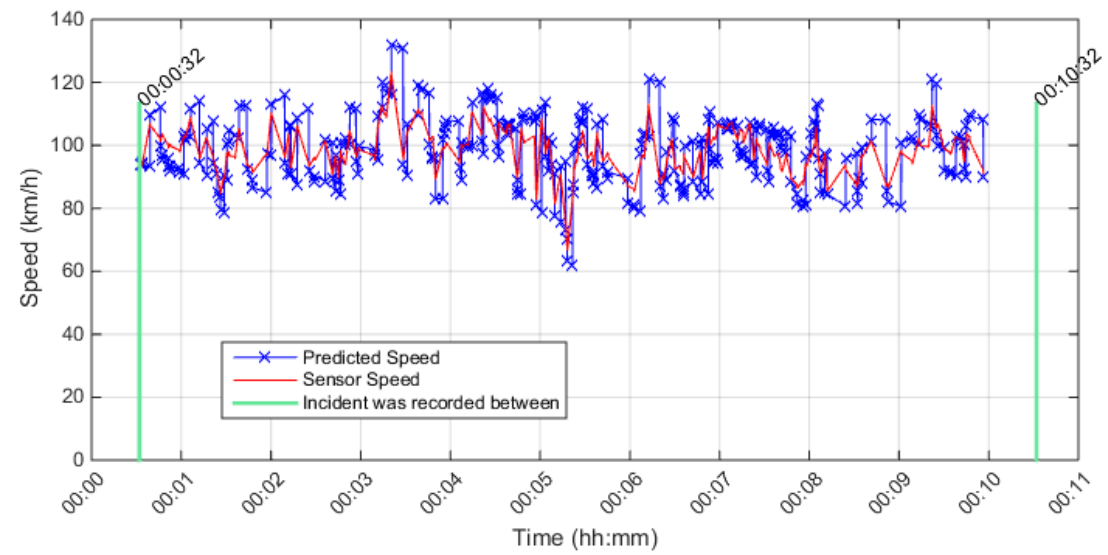

Upstream Detector \# is 401DE0010DEE, Detector Rank $=1$, Distance from incident location in $(\mathrm{km})=0.35605$ Season $=$ Winter, Stream $=E$, Direction $=E$, Date $\&$ Time $=2011-02-0100: 00: 32$ Incident Reason $=$ Collision

Affected Lanes $=2$ RIGHT LANE(S)

Conditional Probability level $=0.6$, Cond.Prob. $r 3 s 2=0.2$, Cond.Prob. $r 3 s 1=0.1$, Percent drop in speed $\%=-56$ Max. Posterior Probability level $=0.97$, Percent range between speed states $=7 \%$

(No Incident was Detected)

Incident Detection Utilizing the Algorithm, Data obtained from Right Lane \#2, Run2

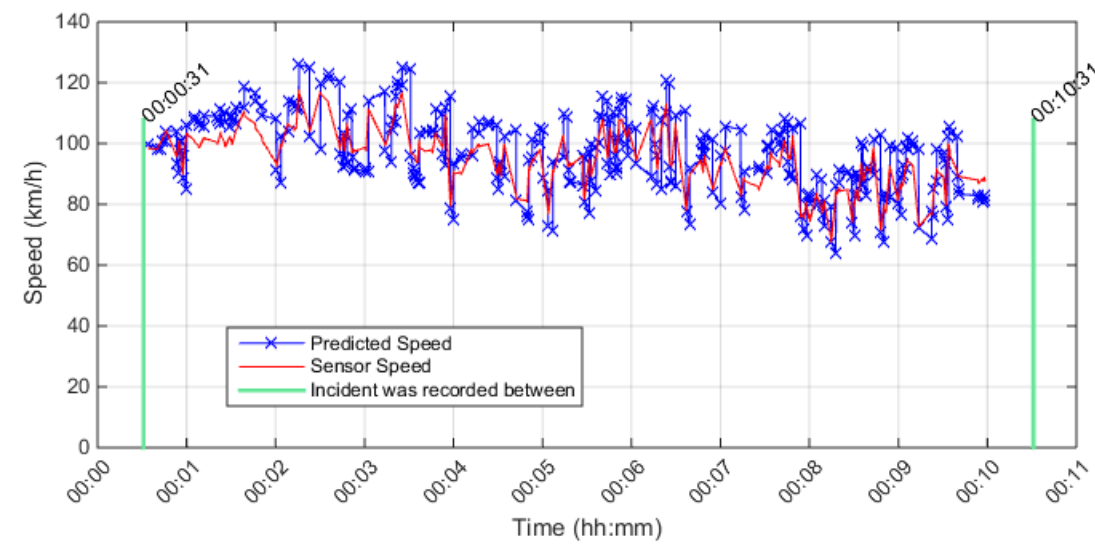

Upstream Detector \# is 401DE0010DEE, Detector Rank $=1$, Distance from incident location in $(\mathrm{km})=0.35605$ Season $=$ Winter, Stream $=E$, Direction $=E$, Date $\&$ Time $=$ 2011-02-01 00:00:31 Incident Reason $=$ Collision Affected Lanes $=2$ RIGHT LANE(S)

Conditional Probability level $=0.6$, Cond.Prob. $r 3 \mathrm{~s} 2=0.2$, Cond.Prob. $r 3 \mathrm{~s} 1=0.1$, Percent drop in speed $\%=-56$ Max. Posterior Probability level $=0.97$, Percent range between speed states $=7 \%$ (No Incident was Detected)

Incident Detection Utilizing the Algorithm, Data obtained from Right Lane \#2, Run3 


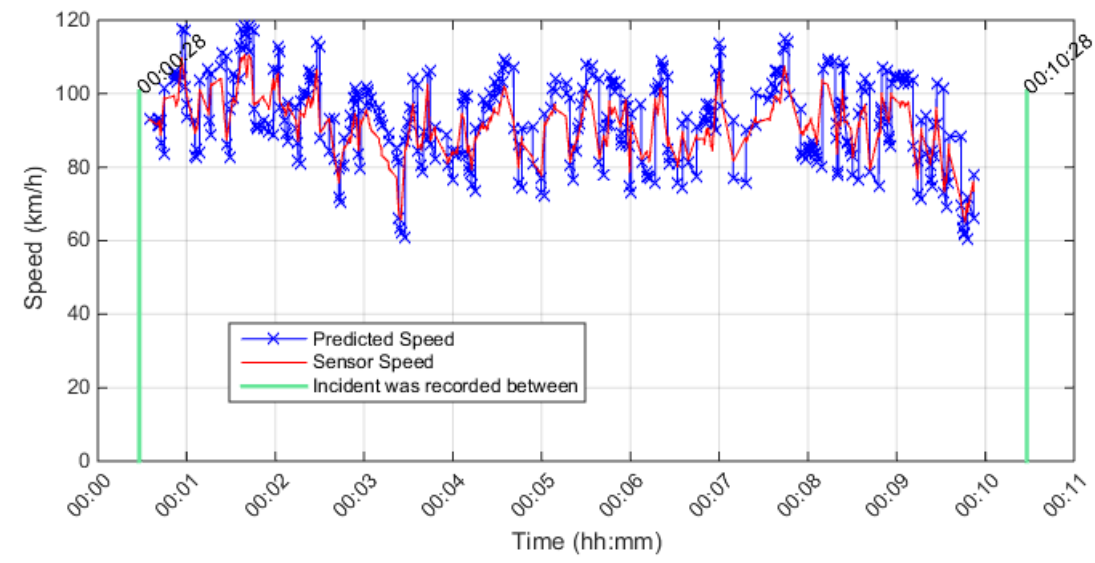

Upstream Detector \# is 401DE0010DEE, Detector Rank = 1, Distance from incident location in $(\mathrm{km})=0.35605$ Season $=$ Winter, Stream $=E$, Direction $=E$, Date $\&$ Time $=2011-02-0100: 00: 28$ Incident Reason $=$ Collision

Affected Lanes $=2$ RIGHT LANE(S)

Conditional Probability level $=0.6$, Cond.Prob. $r 3 \mathrm{~s} 2=0.2$, Cond. Prob. $r 3 \mathrm{~s} 1=0.1$, Percent drop in speed $\%=-56$

Max. Posterior Probability level $=0.97$, Percent range between speed states $=7 \%$

(No Incident was Detected)

Incident Detection Utilizing the Algorithm, Data obtained from Right Lane \#2, Run4

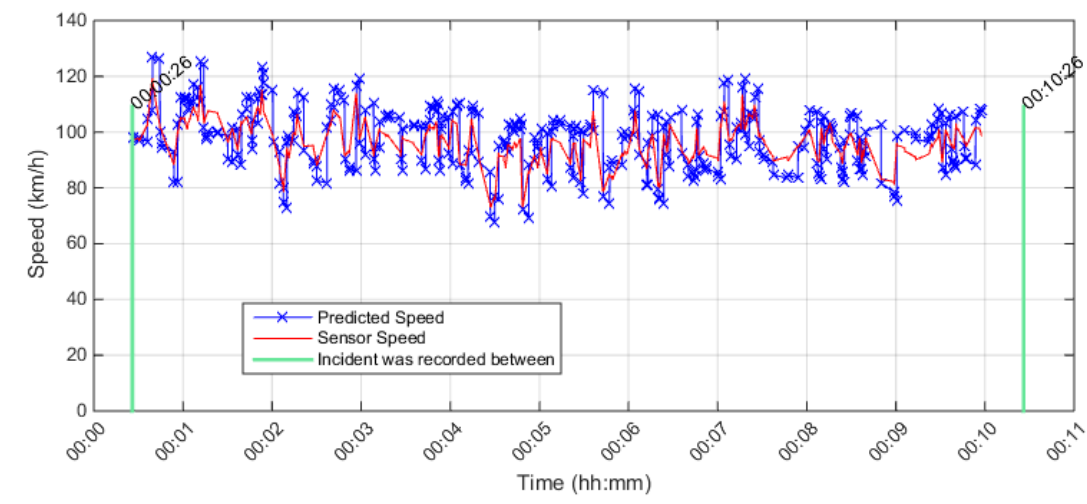

Upstream Detector \# is 401DE0010DEE, Detector Rank $=1$, Distance from incident location in $(\mathrm{km})=0.35605$ Season $=$ Winter, Stream $=E$, Direction $=E$, Date \& Time $=2011-02-0100: 00: 26$ Incident Reason $=$ Collision

Affected Lanes $=2$ RIGHT LANE(S)

Conditional Probability level $=0.6$, Cond.Prob. $r 3 s 2=0.2$, Cond.Prob. $r 3 s 1=0.1$, Percent drop in speed $\%=-56$

Max. Posterior Probability level $=0.97$, Percent range between speed states $=7 \%$

(No Incident was Detected)

Incident Detection Utilizing the Algorithm, Data obtained from Right Lane \#2, Run- 


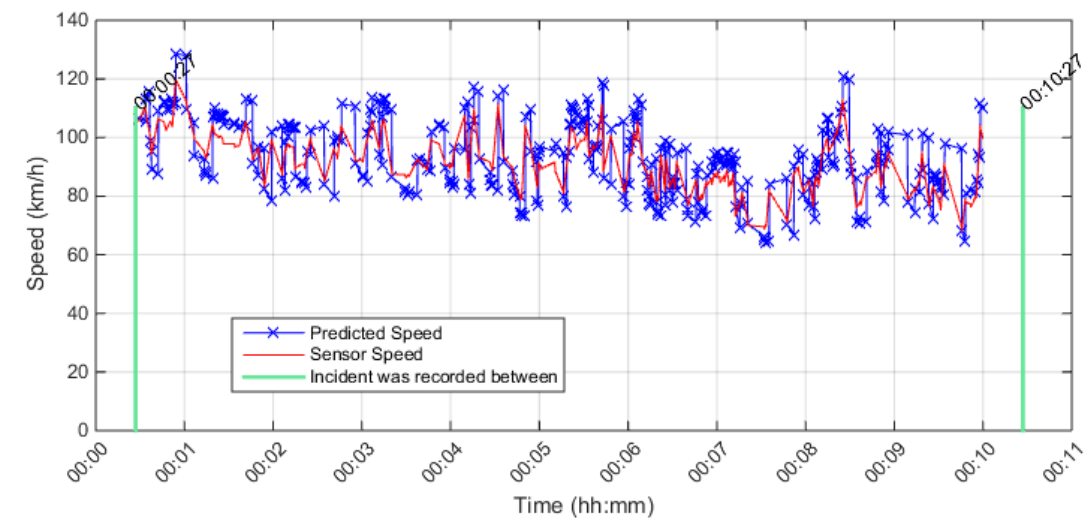

Upstream Detector \# is 401DE0010DEE, Detector Rank $=1$, Distance from incident location in $(\mathrm{km})=0.35605$ Season $=$ Winter, Stream $=E$, Direction $=E$, Date \& Time $=2011-02-0100: 00: 27$ Incident Reason $=$ Collision

Affected Lanes = 2 RIGHT LANE(S)

Conditional Probability level $=0.6$, Cond.Prob. $r 3 s 2=0.2$, Cond.Prob. $r 3 s 1=0.1$, Percent drop in speed $\%=-56$ Max. Posterior Probability level $=0.97$, Percent range between speed states $=7 \%$ (No Incident was Detected)

\section{Incident Detection Utilizing the Algorithm, Data obtained from Right Lane \#2, Run-} 6

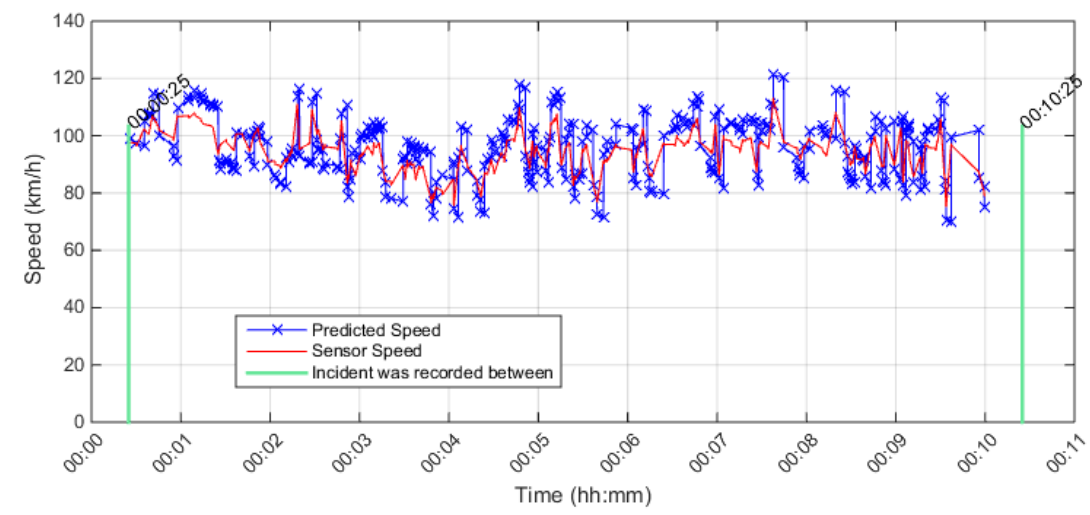

Upstream Detector \# is 401DE0010DEE, Detector Rank $=1$, Distance from incident location in $(\mathrm{km})=0.35605$ Season $=$ Winter, Stream $=E$, Direction $=E$, Date $\&$ Time $=2011-02-0100: 00: 25$ Incident Reason $=$ Collision Affected Lanes $=2$ RIGHT LANE(S)

Conditional Probability level $=0.6$, Cond. Prob. $r 3 \mathrm{~s} 2=0.2$, Cond. Prob. $r 3 s 1=0.1$, Percent drop in speed $\%=-56$ Max. Posterior Probability level $=0.97$, Percent range between speed states $=7 \%$ (No Incident was Detected)

Incident Detection Utilizing the Algorithm, Data obtained from Right Lane \#2, Run- 


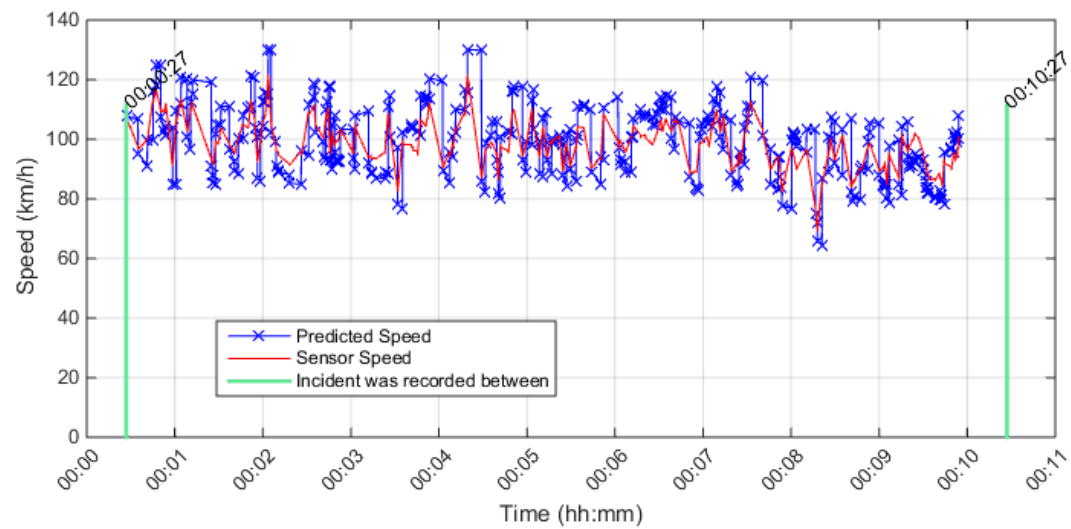

Upstream Detector \# is 401DE0010DEE, Detector Rank $=1$, Distance from incident location in $(\mathrm{km})=0.35605$ Season $=$ Winter, Stream $=E$, Direction $=E$, Date \& Time $=2011-02-0100: 00: 27$ Incident Reason $=$ Collision

Affected Lanes = 2 RIGHT LANE(S)

Conditional Probability level $=0.6$, Cond.Prob. $r 3 s 2=0.2$, Cond.Prob. $r 3 s 1=0.1$, Percent drop in speed $\%=-56$

Max. Posterior Probability level $=0.97$, Percent range between speed states $=7 \%$

(No Incident was Detected)

\section{Incident Detection Utilizing the Algorithm, Data obtained from Right Lane \#2, Run-} 8

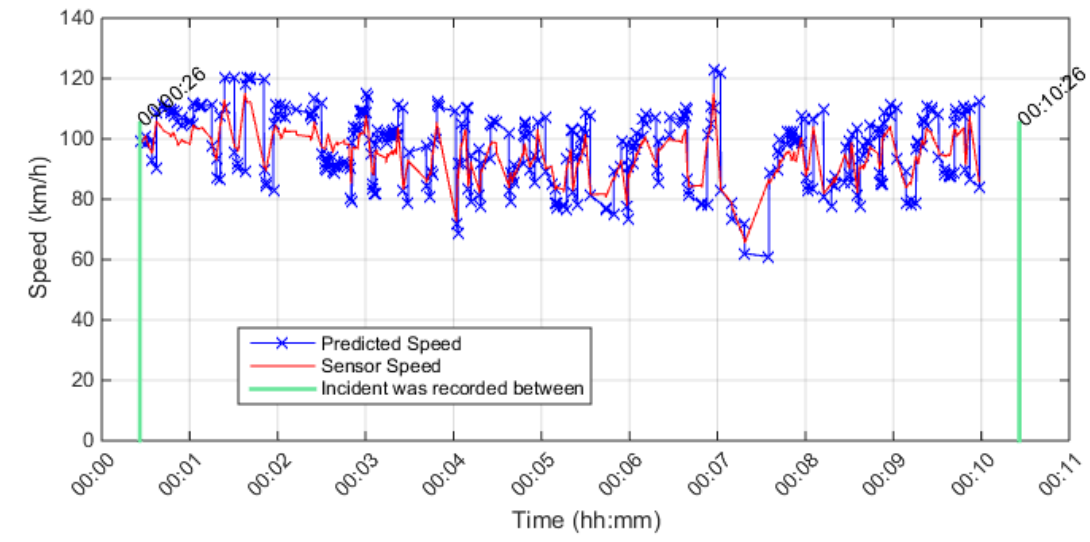

Upstream Detector \# is 401DE0010DEE, Detector Rank = 1, Distance from incident location in $(\mathrm{km})=0.35605$ Season $=$ Winter, Stream $=E$, Direction $=E$, Date \& Time $=2011-02-0100: 00: 26$ Incident Reason $=$ Collision

Affected Lanes = 2RIGHT LANE(S)

Conditional Probability level $=0.6$, Cond.Prob. $\mathrm{r} 3 \mathrm{~s} 2=0.2$, Cond. Prob. $\mathrm{r} 3 \mathrm{~s} 1=0.1$, Percent drop in speed $\%=-56$ Max. Posterior Probability level $=0.97$, Percent range between speed states $=7 \%$ (No Incident was Detected)

\section{Incident Detection Utilizing the Algorithm, Data obtained from Right Lane \#2, Run-}




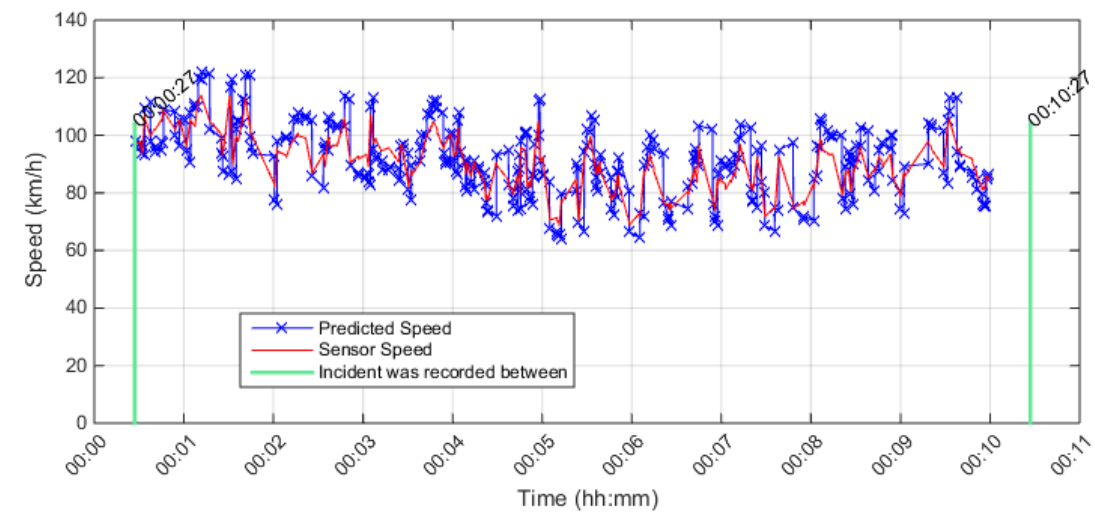

Upstream Detector \# is 401DE0010DEE, Detector Rank $=1$, Distance from incident location in $(\mathrm{km})=0.35605$ Season $=$ Winter, Stream $=E$, Direction $=E$, Date $\&$ Time $=$ 2011-02-01 00:00:27 Incident Reason $=$ Collision

Affected Lanes $=2$ RIGHT LANE(S)

Conditional Probability level $=0.6$, Cond. Prob. $r 3 s 2=0.2$, Cond. Prob. $r 3 s 1=0.1$, Percent drop in speed $\%=-56$

Max. Posterior Probability level $=0.97$, Percent range between speed states $=7 \%$

(No Incident was Detected)

Incident Detection Utilizing the Algorithm, Data obtained from Right Lane \#2, Run10

\section{Incident Detection from Lane \#1, two-lane blockage and data was collected from the first lane, traffic volume level of $1500 \mathrm{veh} / \mathrm{l} / \mathrm{h}$}

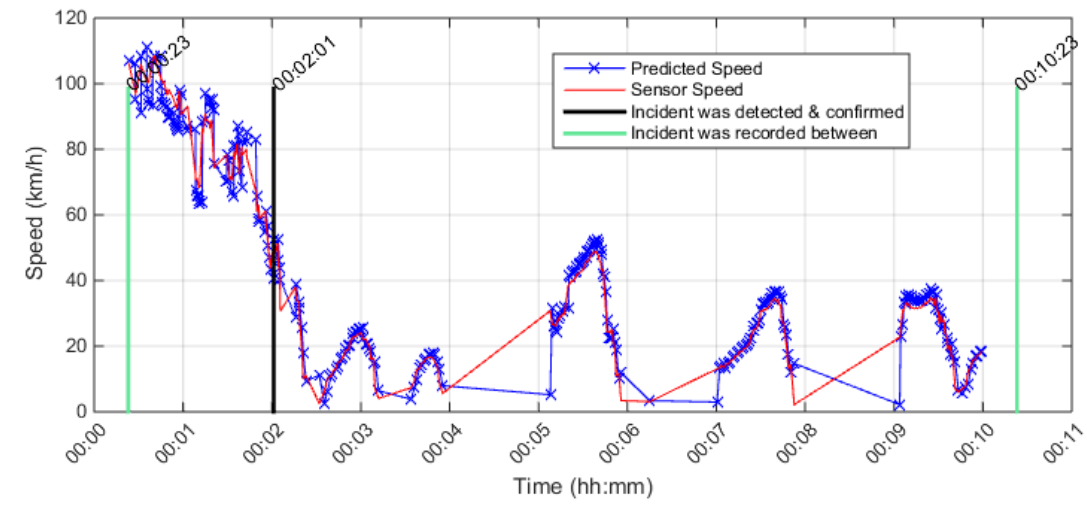

Upstream Detector \# is 401DE0010DEE, Detector Rank $=1$, Distance from incident location in $(\mathrm{km})=0.35605$ Season $=$ Winter, Stream $=E$, Direction $=E$, Date $\&$ Time $=2011-02-0100: 00: 23$ Incident Reason $=$ Collision Affected Lanes $=2$ RIGHT LANE(S)

Conditional Probability level $=0.6$, Cond. Prob. $r 3 s 2=0.2$, Cond. Prob. $r 3 s 1=0.1$, Percent drop in speed $\%=-56$ Max. Posterior Probability level $=0.97$, Percent range between speed states $=7 \%$

Incident was detected at 00:12 minutes from drop in speed point. Incident was confirmed after 5 minutes from the detection time 
Incident Detection Utilizing the Algorithm, Data obtained from Right Lane \#1, Run2

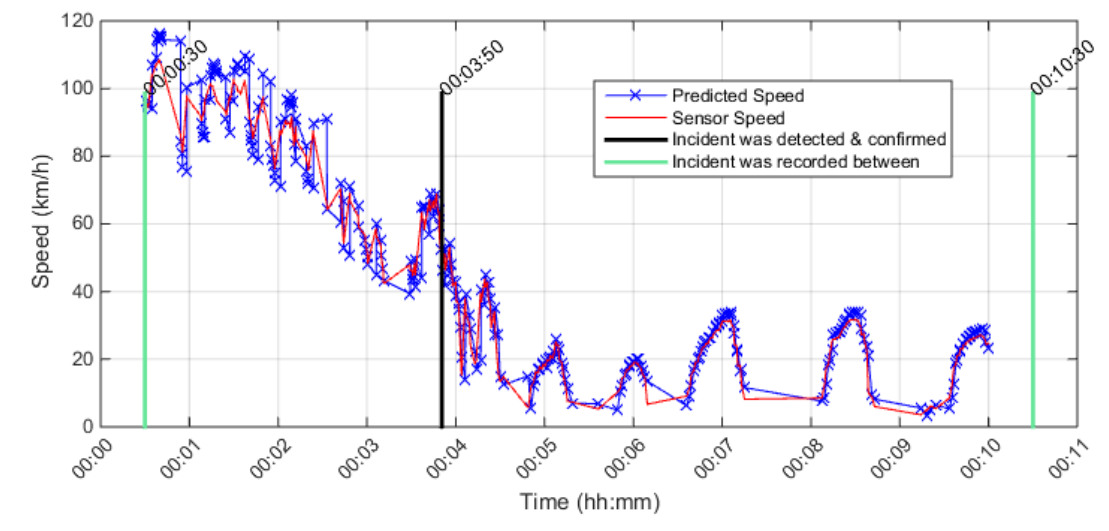

Upstream Detector \# is 401DE0010DEE, Detector Rank = 1, Distance from incident location in $(\mathrm{km})=0.35605$ Season $=$ Winter, Stream $=E$, Direction $=E$, Date \& Time $=2011-02-0100: 00: 30$ Incident Reason $=$ Collision

Affected Lanes $=2$ RIGHT LANE(S)

Conditional Probability level $=0.6$, Cond. Prob. $r 3 \mathrm{~s} 2=0.2$, Cond. Prob. $r 3 \mathrm{~s} 1=0.1$, Percent drop in speed $\%=-56$

Max. Posterior Probability level $=0.97$, Percent range between speed states $=7 \%$

Incident was detected at 01:17 minutes from drop in speed point. Incident was confirmed after 5 minutes from the detection time

Incident Detection Utilizing the Algorithm, Data obtained from Right Lane \#1, Run3

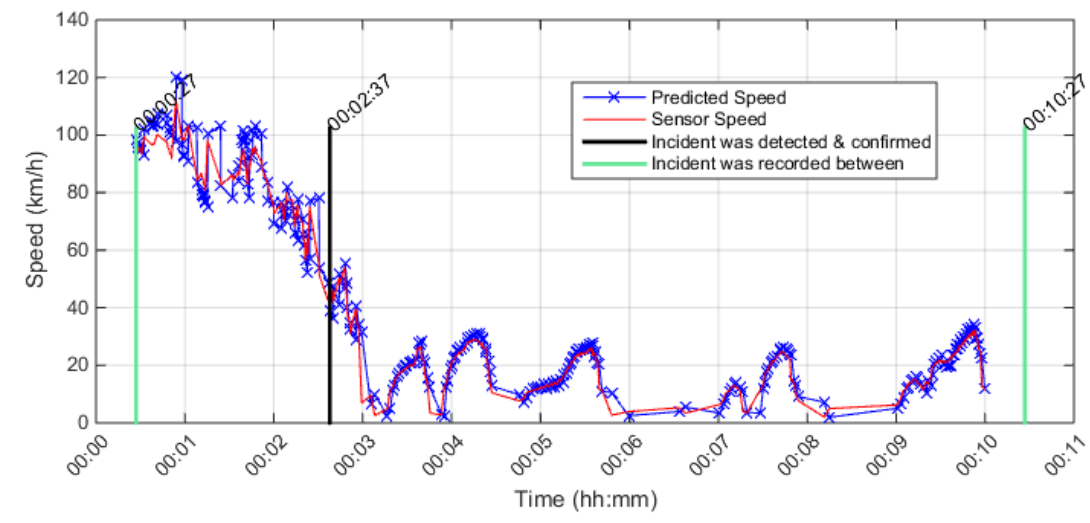

Upstream Detector \# is 401DE0010DEE, Detector Rank = 1, Distance from incident location in $(\mathrm{km})=0.35605$ Season $=$ Winter, Stream $=E$, Direction $=E$, Date \& Time $=$ 2011-02-01 00:00:27

Incident Reason = Collision
Affected Lanes $=2$ RIGHT LANE(S)

Conditional Probability level $=0.6$, Cond.Prob. $r 3 s 2=0.2$, Cond. Prob. $r 3 s 1=0.1$, Percent drop in speed $\%=-56$

Max. Posterior Probability level $=0.97$, Percent range between speed states $=7 \%$

Incident was detected at 00:07 minutes from drop in speed point. Incident was confirmed after 5 minutes from the detection time

Incident Detection Utilizing the Algorithm, Data obtained from Right Lane \#1, Run- 


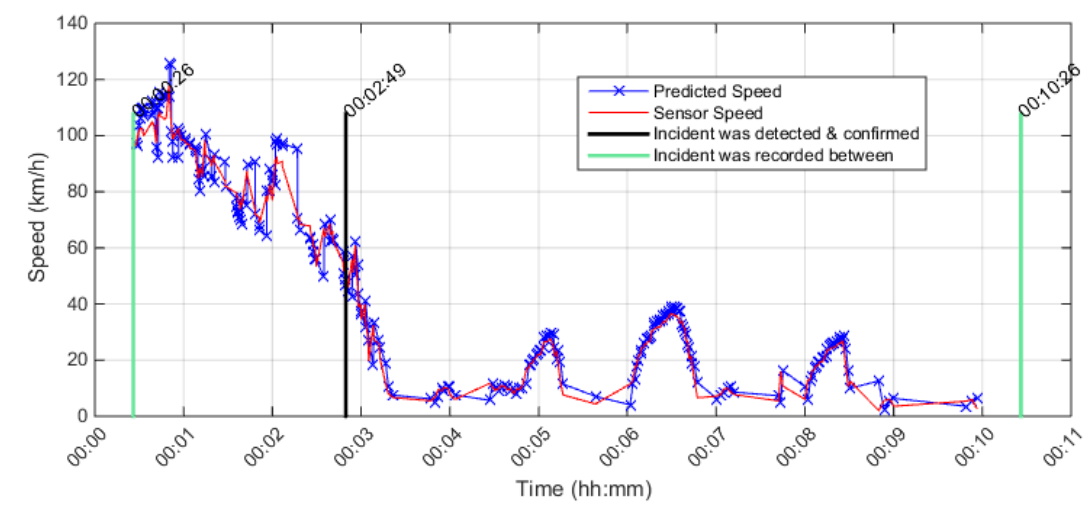

Upstream Detector \# is 401DE0010DEE, Detector Rank $=1$, Distance from incident location in $(\mathrm{km})=0.35605$ Season $=$ Winter, Stream $=E$, Direction $=E$, Date \& Time $=2011-02-0100: 00: 26$

Incident Reason $=$ Collision

Affected $\operatorname{Lanes}=2$ RIGHT LANE(S)

Conditional Probability level $=0.6$, Cond. Prob. $r 3 s 2=0.2$, Cond. Prob. $r 3 s 1=0.1$, Percent drop in speed $\%=-56$

Max. Posterior Probability level $=0.97$. Percent range between speed states $=7 \%$

Incident was detected at 00:32 minutes from drop in speed point. Incident was confirmed after 5 minutes from the detection time

\section{Incident Detection Utilizing the Algorithm, Data obtained from Right Lane \#1, Run-} 5

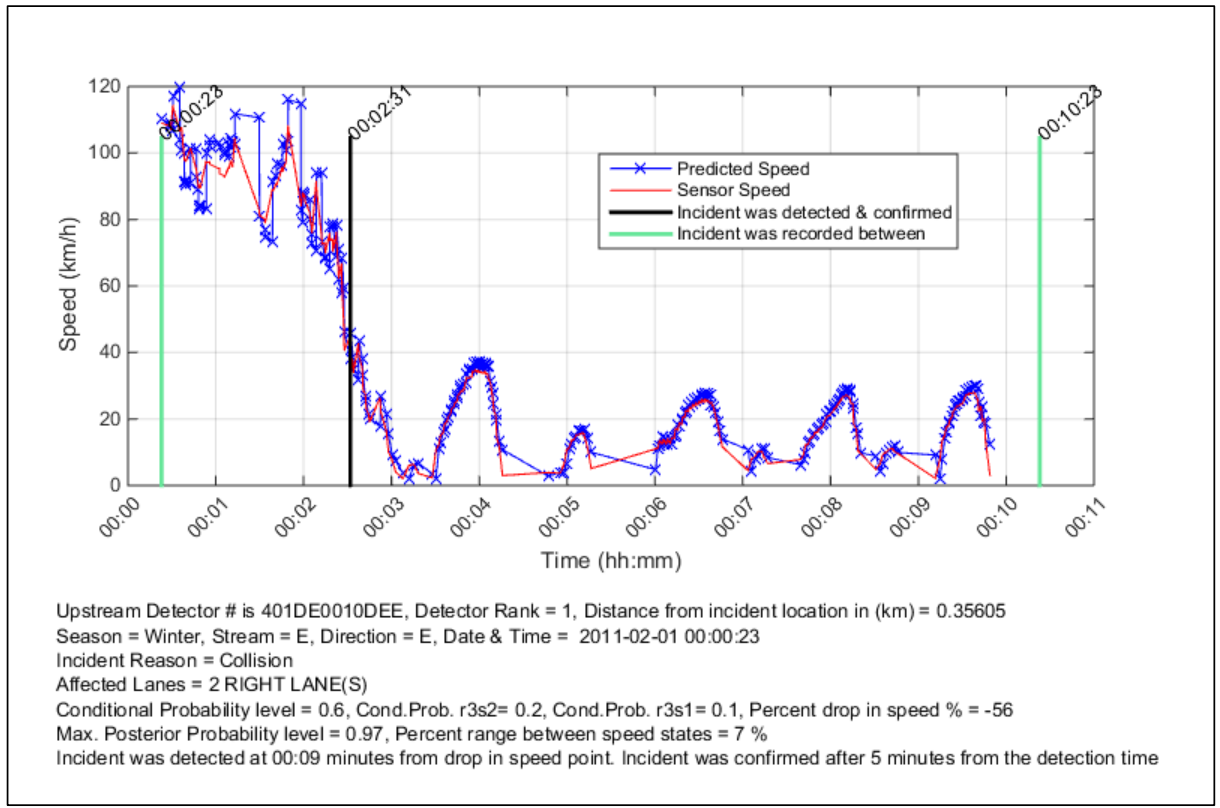

Incident Detection Utilizing the Algorithm, Data obtained from Right Lane \#1, Run6 


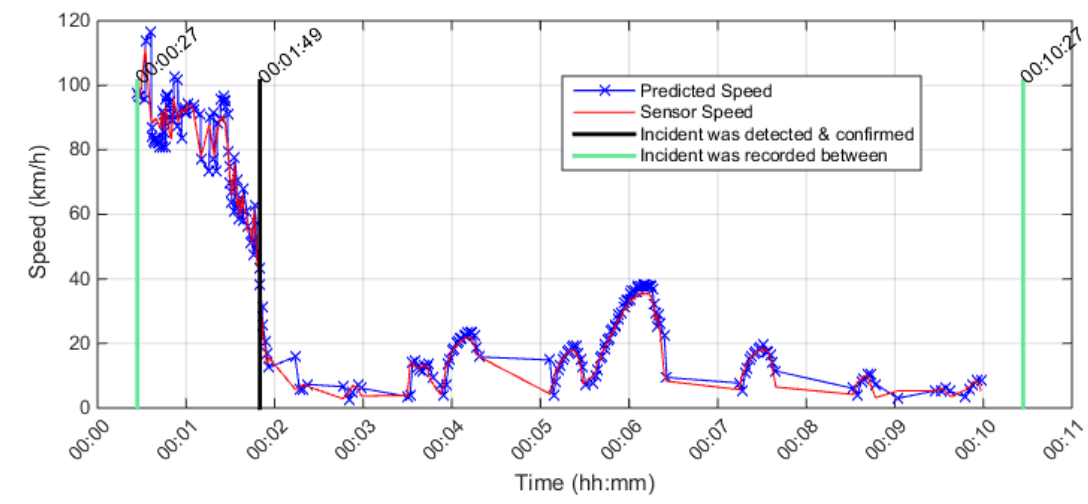

Upstream Detector \# is 401DE0010DEE, Detector Rank = 1, Distance from incident location in $(\mathrm{km})=0.35605$ Season $=$ Winter, Stream $=E$, Direction $=E$, Date \& Time $=2011-02-0100: 00: 27$ Incident Reason $=$ Collision

Affected Lanes = 2 RIGHT LANE(S)

Conditional Probability level $=0.6$, Cond.Prob. $r 3 \mathrm{~s} 2=0.2$, Cond. Prob. $r 3 \mathrm{~s} 1=0.1$, Percent drop in speed $\%=-56$

Max. Posterior Probability level $=0.97$, Percent range between speed states $=7 \%$

Incident was detected at 00:16 minutes from drop in speed point. Incident was confirmed after 5 minutes from the detection time

\section{Incident Detection Utilizing the Algorithm, Data obtained from Right Lane \#1, Run-}

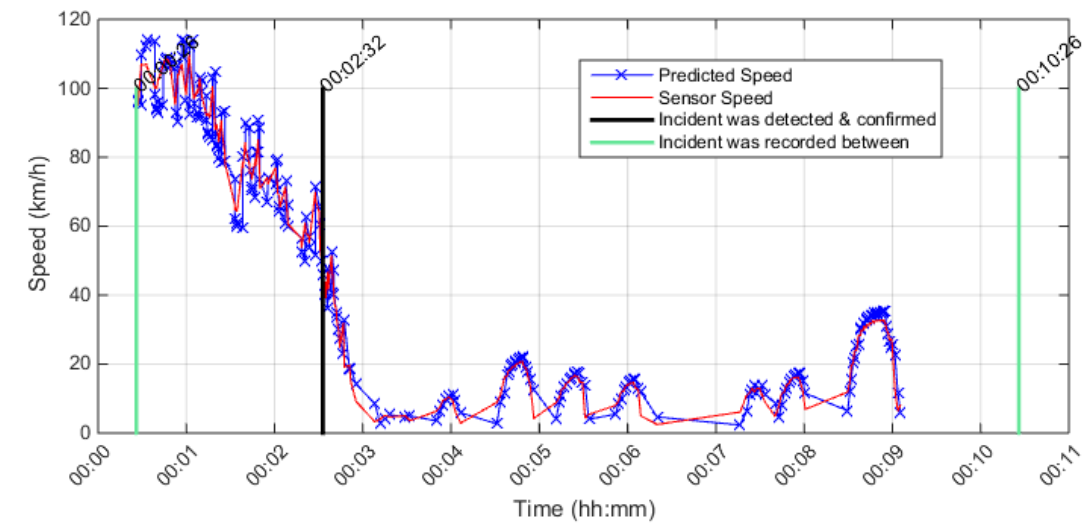

Upstream Detector \# is 401DE0010DEE, Detector Rank $=1$, Distance from incident location in $(\mathrm{km})=0.35605$ Season $=$ Winter, Stream $=E$, Direction $=E$, Date $\&$ Time $=2011-02-0100: 00: 26$ Incident Reason $=$ Collision

Affected Lanes = 2RIGHT LANE(S)

Conditional Probability level $=0.6$, Cond. Prob. $r 3 \mathrm{~s} 2=0.2$, Cond.Prob. $r 3 s 1=0.1$, Percent drop in speed $\%=-56$ Max. Posterior Probability level $=0.97$, Percent range between speed states $=7 \%$

Incident was detected at 00:42 minutes from drop in speed point. Incident was confirmed after 5 minutes from the detection time

\section{Incident Detection Utilizing the Algorithm, Data obtained from Right Lane \#1, Run-} 8 


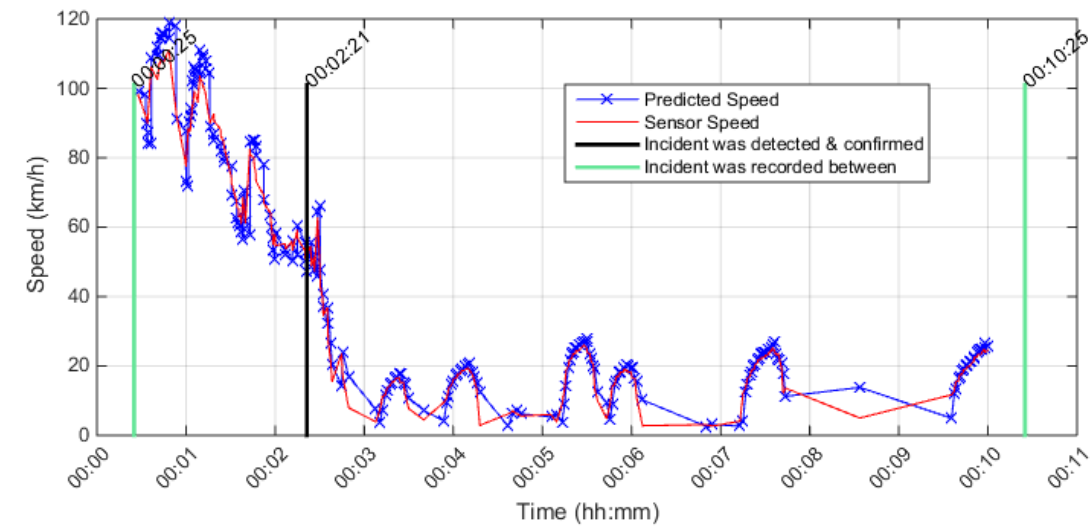

Upstream Detector \# is 401DE0010DEE, Detector Rank = 1, Distance from incident location in $(\mathrm{km})=0.35605$ Season $=$ Winter, Stream $=E$, Direction $=E$, Date \& Time $=2011-02-0100: 00: 25$ Incident Reason $=$ Collision

Affected Lanes $=2$ RIGHT LANE(S)

Conditional Probability level $=0.6$, Cond. Prob. $r 3 s 2=0.2$, Cond. Prob. $r 3 s 1=0.1$, Percent drop in speed $\%=-56$

Max. Posterior Probability level $=0.97$, Percent range between speed states $=7 \%$

Incident was detected at 00:34 minutes from drop in speed point. Incident was confirmed after 5 minutes from the detection time

Incident Detection Utilizing the Algorithm, Data obtained from Right Lane \#1, Run9

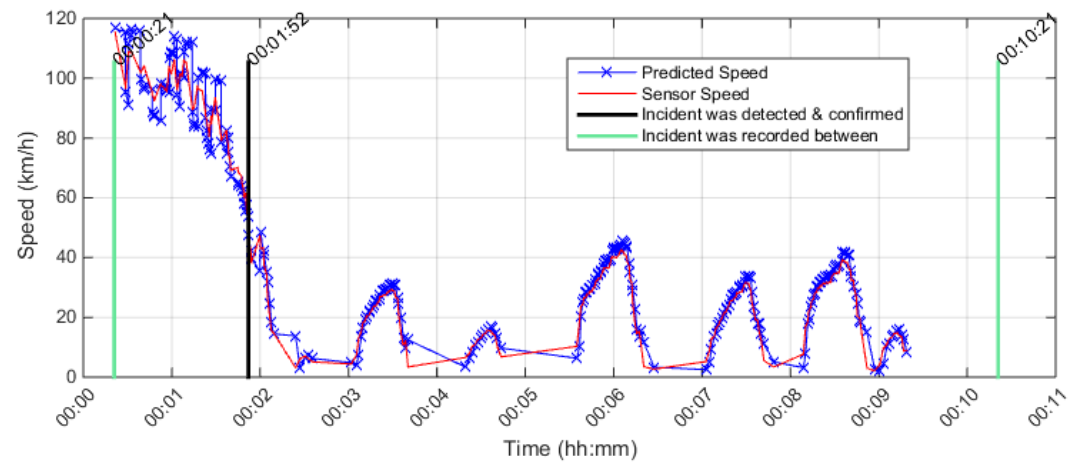

Upstream Detector \# is 401DE0010DEE, Detector Rank $=1$, Distance from incident location in $(\mathrm{km})=0.35605$

Season $=$ Winter, Stream $=E$, Direction $=E$, Date \& Time $=2011-02-0100: 00: 21$

Incident Reason $=$ Collision

Affected Lanes $=2$ RIGHT LANE(S)

Conditional Probability level $=0.6$, Cond.Prob. $r 3 \mathrm{~s} 2=0.2$, Cond.Prob. $r 3 s 1=0.1$, Percent drop in speed $\%=-56$

Max. Posterior Probability level $=0.97$, Percent range between speed states $=7 \%$

Incident was detected at 00:18 minutes from drop in speed point. Incident was confirmed after 5 minutes from the detection time

Incident Detection Utilizing the Algorithm, Data obtained from Right Lane \#1, Run10

Incident Detection from Lane \#2, two-lane blockage, and data was collected from the first lane, traffic volume level of $1500 \mathrm{veh} / \mathrm{l} / \mathrm{h}$ 


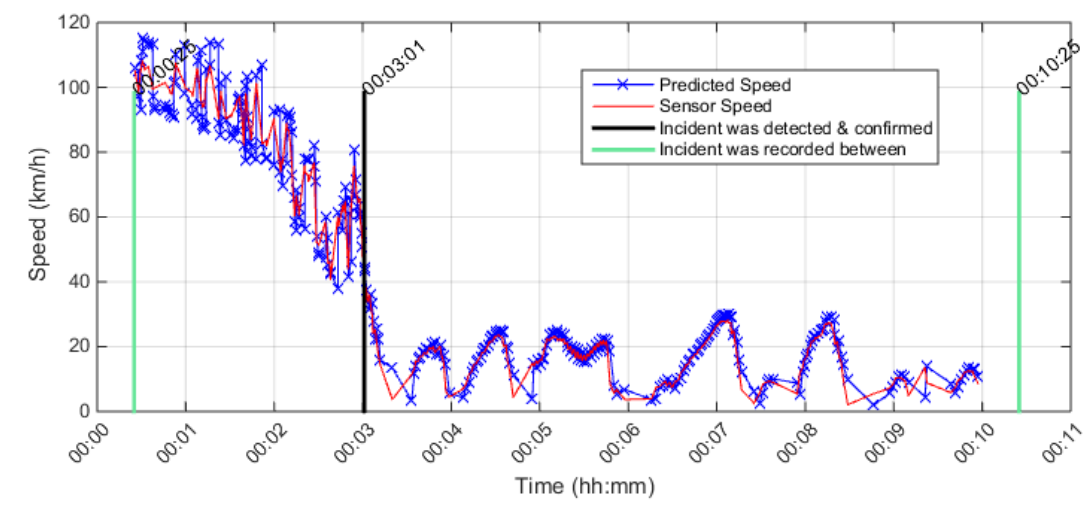

Upstream Detector \# is 401DE0010DEE, Detector Rank $=1$, Distance from incident location in $(\mathrm{km})=0.35605$ Season $=$ Winter, Stream $=E$, Direction $=E$, Date $\&$ Time $=2011-02-0100: 00: 25$ Incident Reason $=$ Collision

Affected Lanes = 2RIGHT LANE(S)

Conditional Probability level $=0.6$, Cond.Prob. $r 3 s 2=0.2$, Cond.Prob. $r 3 s 1=0.1$, Percent drop in speed $\%=-56$

Max. Posterior Probability level $=0.97$, Percent range between speed states $=7 \%$

Incident was detected at 00:06 minutes from drop in speed point. Incident was confirmed after 5 minutes from the detection time

Incident Detection Utilizing the Algorithm, Data obtained from Right Lane \#2, Run2

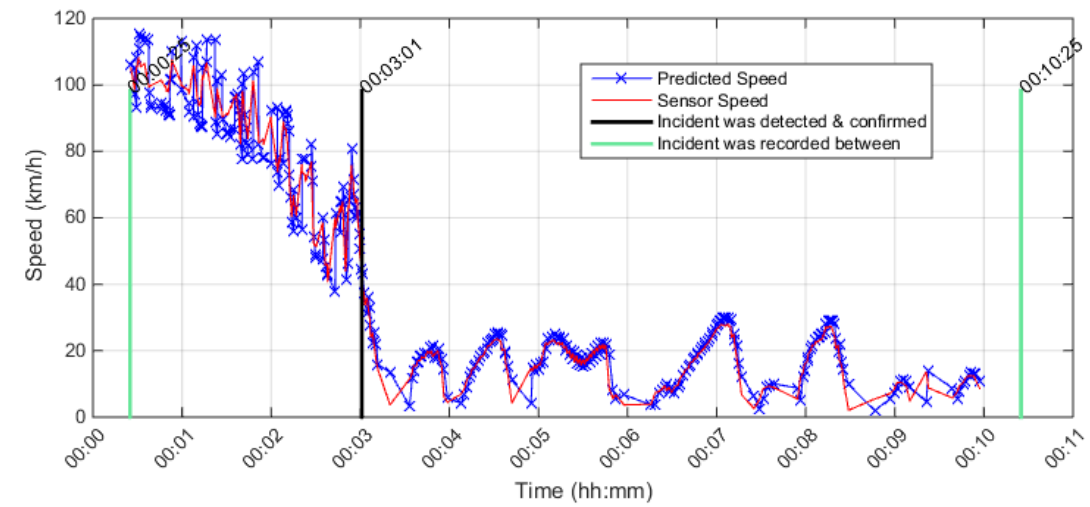

Upstream Detector \# is 401DE0010DEE, Detector Rank $=1$, Distance from incident location in $(\mathrm{km})=0.35605$ Season $=$ Winter, Stream $=E$, Direction $=E$, Date \& Time $=2011-02-0100: 00: 25$ Incident Reason $=$ Collision

Affected Lanes $=2$ RIGHT LANE(S)

Conditional Probability level $=0.6$, Cond.Prob. r3s2 $=0.2$, Cond.Prob. r3s1 $=0.1$, Percent drop in speed $\%=-56$

Max. Posterior Probability level $=0.97$, Percent range between speed states $=7 \%$

Incident was detected at 00:06 minutes from drop in speed point. Incident was confirmed after 5 minutes from the detection time

Incident Detection Utilizing the Algorithm, Data obtained from Right Lane \#2, Run3 


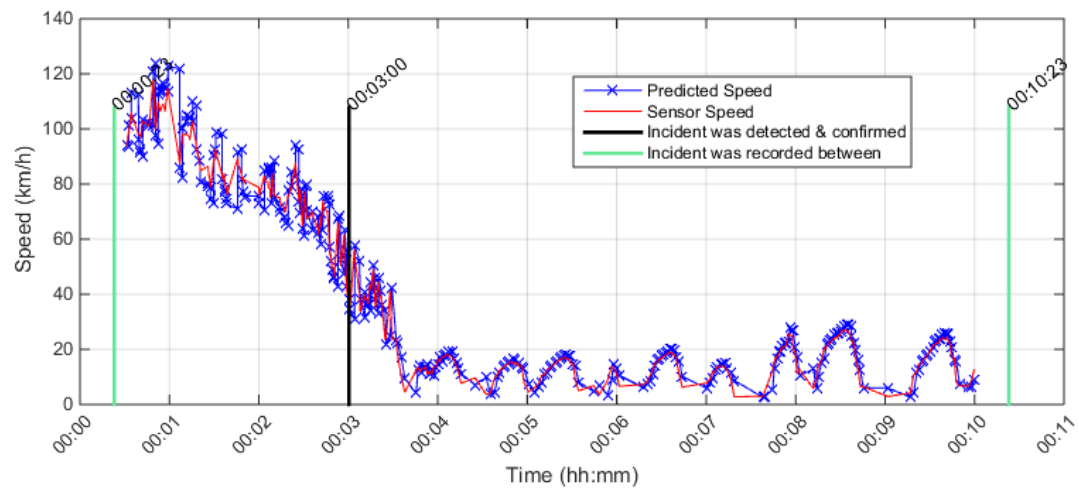

Upstream Detector \# is 401DE0010DEE, Detector Rank $=1$, Distance from incident location in $(\mathrm{km})=0.35605$ Season $=$ Winter, Stream $=E$, Direction $=E$, Date \& Time $=2011-02-0100: 00: 23$ Incident Reason $=$ Collision

Affected Lanes $=2$ RIGHT LANE(S)

Conditional Probability level $=0.6$, Cond. Prob. $r 3 \mathrm{~s} 2=0.2$, Cond. Prob. $r 3 \mathrm{~s} 1=0.1$, Percent drop in speed $\%=-56$

Max. Posterior Probability level $=0.97$, Percent range between speed states $=7 \%$

Incident was detected at 00:28 minutes from drop in speed point. Incident was confirmed after 5 minutes from the detection time

Incident Detection Utilizing the Algorithm, Data obtained from Right Lane \#2, Run4

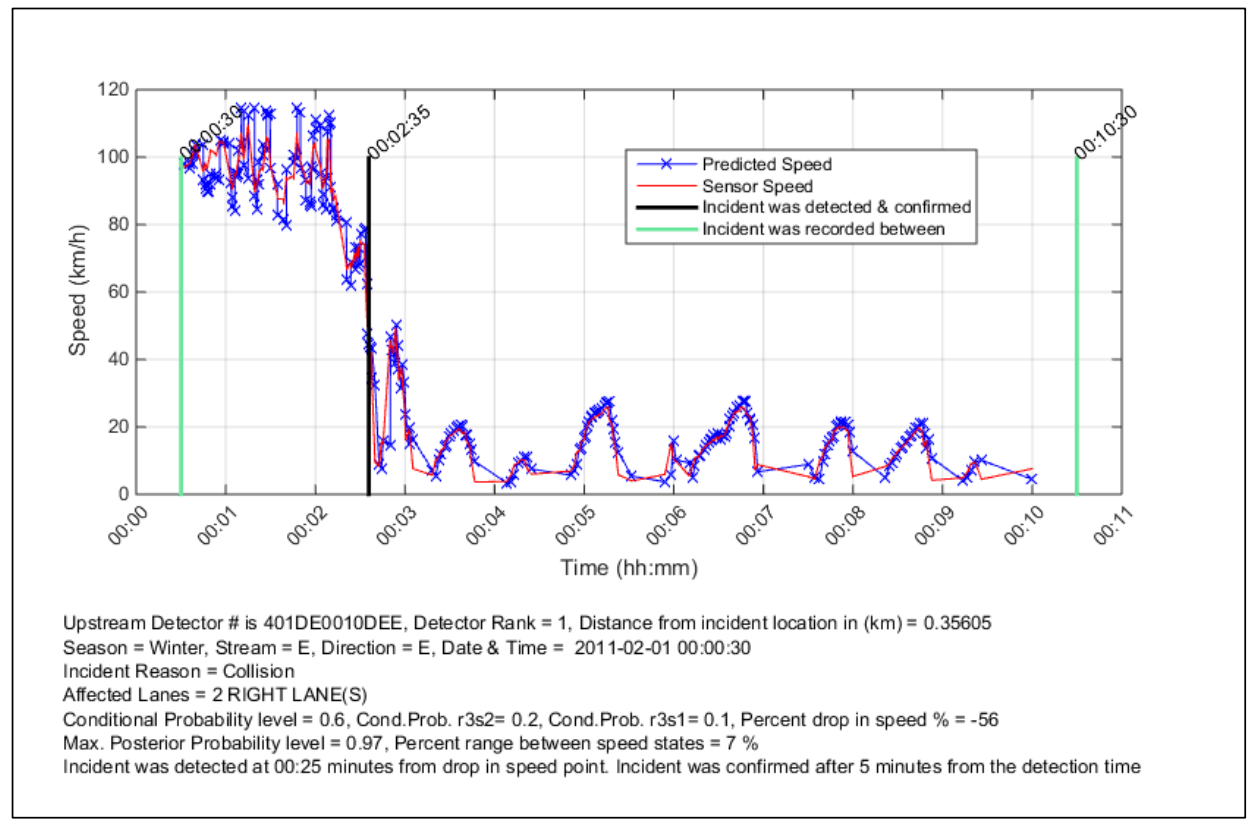

Incident Detection Utilizing the Algorithm, Data obtained from Right Lane \#2, Run5 


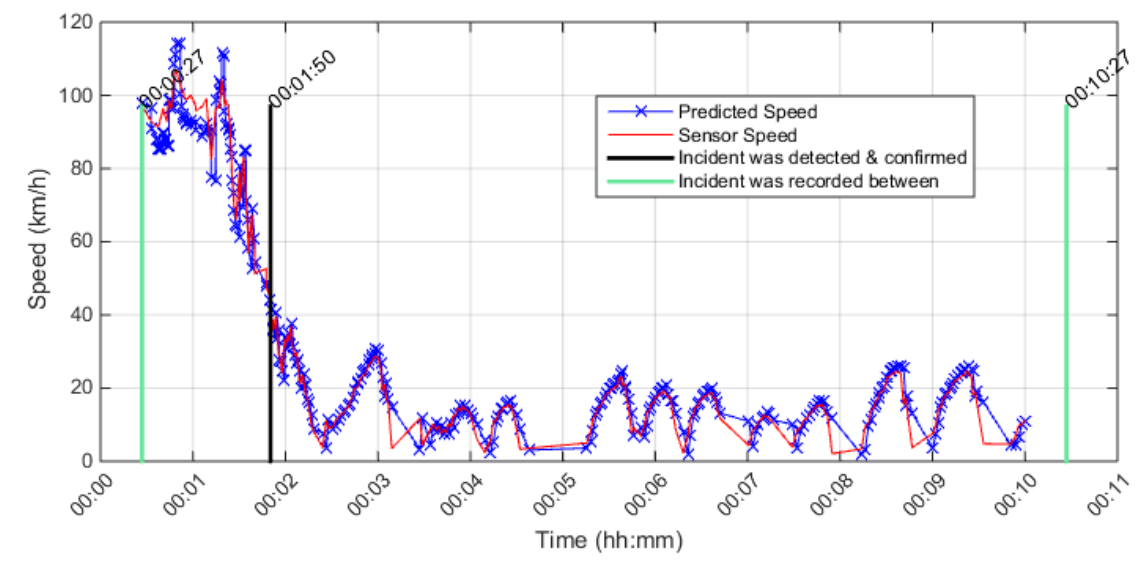

Upstream Detector \# is 401DE0010DEE, Detector Rank = 1, Distance from incident location in $(\mathrm{km})=0.35605$ Season $=$ Winter, Stream $=E$, Direction $=E$, Date $\&$ Time $=2011-02-0100: 00: 27$ Incident Reason $=$ Collision

Affected Lanes = 2 RIGHT LANE(S)

Conditional Probability level $=0.6$, Cond. Prob. $r 3 \mathrm{~s} 2=0.2$, Cond.Prob. $r 3 \mathrm{~s} 1=0.1$, Percent drop in speed $\%=-56$

Max. Posterior Probability level $=0.97$, Percent range between speed states $=7 \%$

Incident was detected at 00:16 minutes from drop in speed point. Incident was confirmed after 5 minutes from the detection time

\section{Incident Detection Utilizing the Algorithm, Data obtained from Right Lane \#2, Run-}

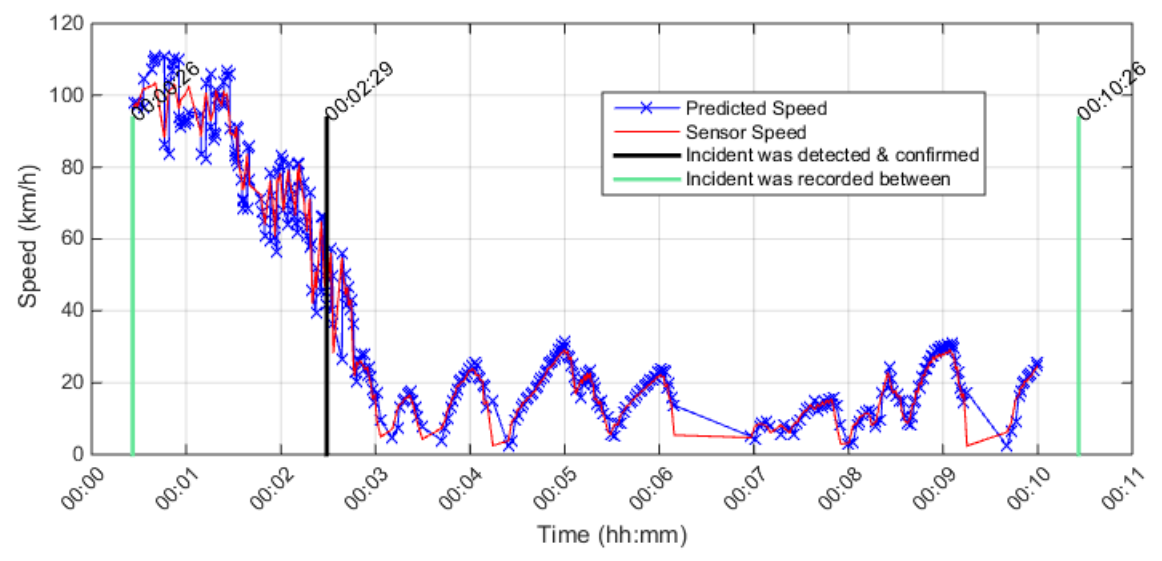

Upstream Detector \# is 401DE0010DEE, Detector Rank = 1, Distance from incident location in $(\mathrm{km})=0.35605$ Season $=$ Winter, Stream $=E$, Direction $=E$, Date \& Time $=2011-02-0100: 00: 26$

Incident Reason $=$ Collision

Affected Lanes = 2 RIGHT LANE(S)

Conditional Probability level $=0.6$, Cond.Prob. $r 3 \mathrm{~s} 2=0.2$, Cond.Prob. $\mathrm{r} 3 \mathrm{~s} 1=0.1$, Percent drop in $\mathrm{speed} \%=-56$

Max. Posterior Probability level $=0.97$, Percent range between speed states $=7 \%$

Incident was detected at 00:17 minutes from drop in speed point. Incident was confirmed after 5 minutes from the detection time

Incident Detection Utilizing the Algorithm, Data obtained from Right Lane \#2, Run- 


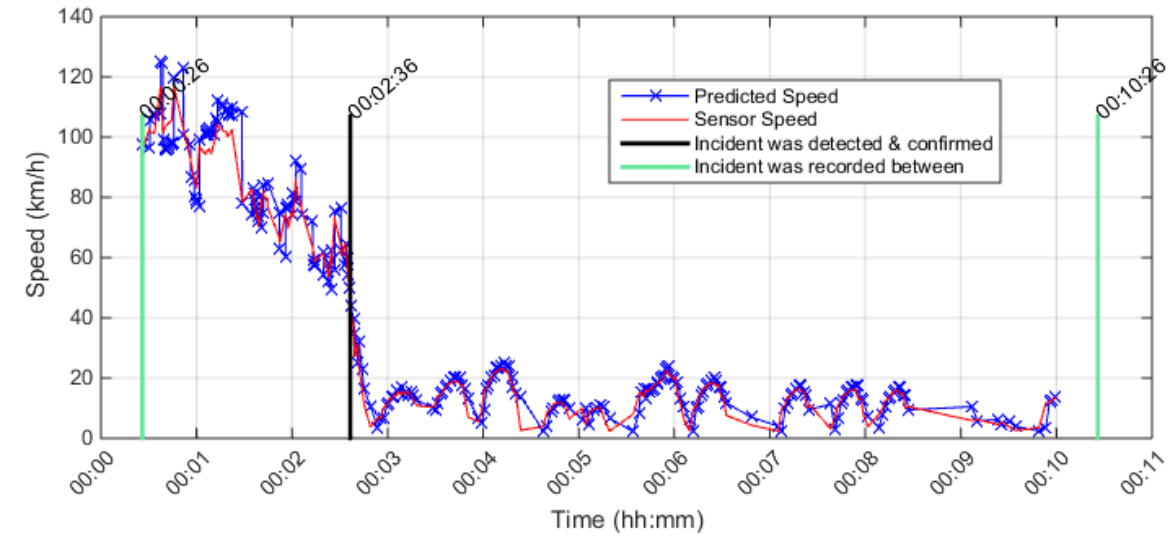

Upstream Detector \# is 401DE0010DEE, Detector Rank = 1, Distance from incident location in $(\mathrm{km})=0.35605$ Season $=$ Winter, Stream $=$ E, Direction $=E$, Date \& Time $=$ 2011-02-01 00:00:26 Incident Reason $=$ Collision

Affected Lanes = 2RIGHT LANE(S)

Conditional Probability level $=0.6$, Cond.Prob. $r 3 s 2=0.2$, Cond.Prob. $r 3 s 1=0.1$, Percent drop in speed $\%=-56$

Max. Posterior Probability level $=0.97$, Percent range between speed states $=7 \%$

Incident was detected at 00:30 minutes from drop in speed point. Incident was confirmed after 5 minutes from the detection time

\section{Incident Detection Utilizing the Algorithm, Data obtained from Right Lane \#2, Run-} 8

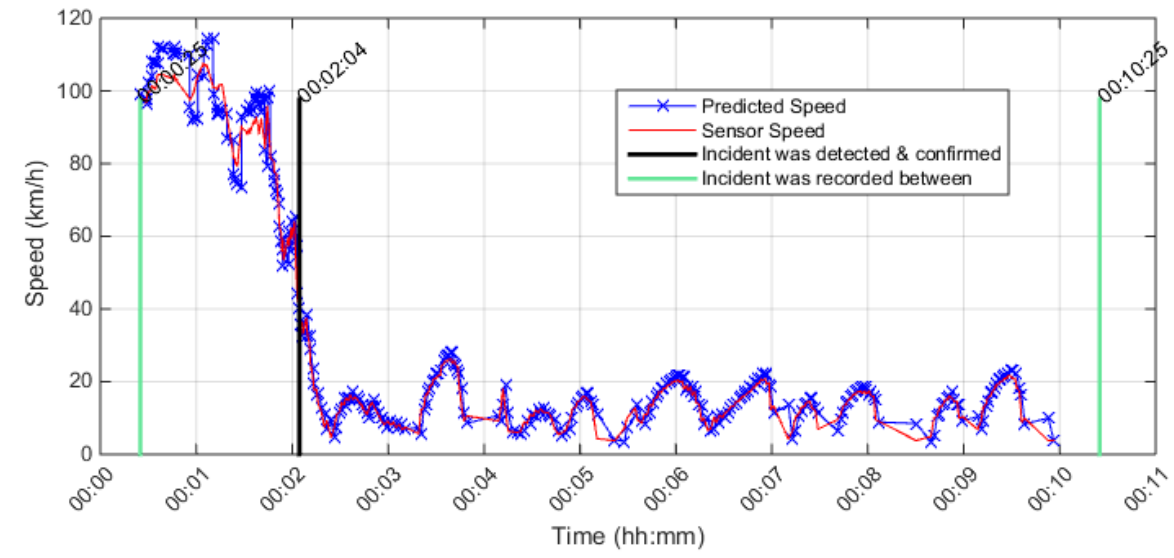

Upstream Detector \# is 401DE0010DEE, Detector Rank = 1, Distance from incident location in $(\mathrm{km})=0.35605$ Season $=$ Winter, Stream $=E$, Direction $=E$, Date \& Time $=2011-02-0100: 00: 25$ Incident Reason $=$ Collision

Affected Lanes = 2RIGHT LANE(S)

Conditional Probability level $=0.6$, Cond.Prob. $r 3 s 2=0.2$, Cond.Prob. $r 3 s 1=0.1$, Percent drop in speed $\%=-56$ Max. Posterior Probability level $=0.97$, Percent range between speed states $=7 \%$

Incident was detected at 00:18 minutes from drop in speed point. Incident was confirmed after 5 minutes from the detection time

\section{Incident Detection Utilizing the Algorithm, Data obtained from Right Lane \#2, Run-}




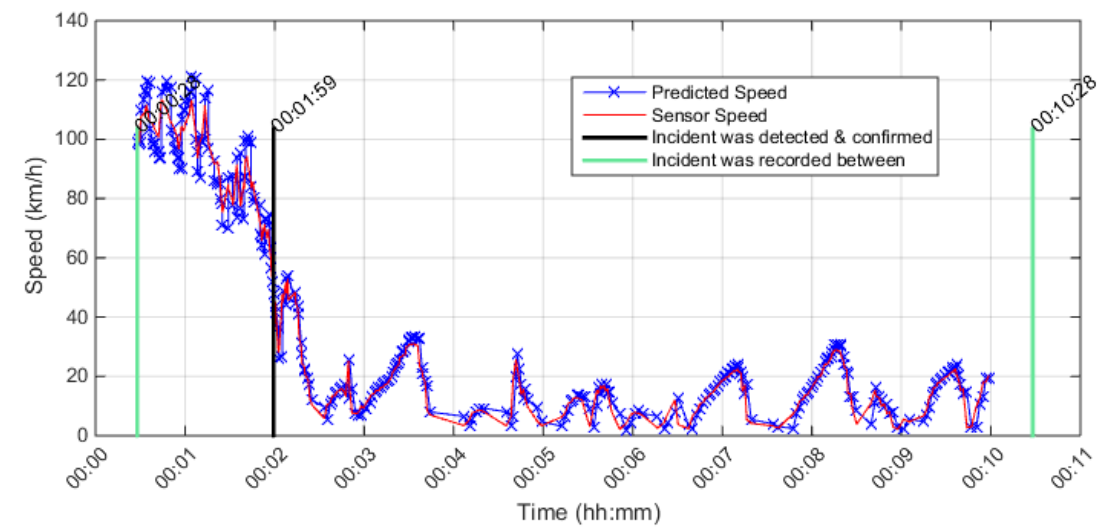

Upstream Detector \# is 401DE0010DEE, Detector Rank $=1$, Distance from incident location in $(\mathrm{km})=0.35605$

Season $=$ Winter, Stream $=E$, Direction $=E$, Date $\&$ Time $=2011-02-0100: 00: 28$

Incident Reason $=$ Collision

Affected Lanes $=2$ RIGHT LANE(S)

Conditional Probability level $=0.6$, Cond.Prob. $r 3 s 2=0.2$, Cond.Prob. $r 3 s 1=0.1$, Percent drop in speed $\%=-56$

Max. Posterior Probability level $=0.97$, Percent range between speed states $=7 \%$

Incident was detected at 00:15 minutes from drop in speed point. Incident was confirmed after 5 minutes from the detection time

Incident Detection Utilizing the Algorithm, Data obtained from Right Lane \#2, Run10

\section{Incident Detection from Lane \#1, two-lane blockage and data was collected from the first lane, traffic volume level of $1250 \mathrm{veh} / \mathrm{l} / \mathrm{h}$}

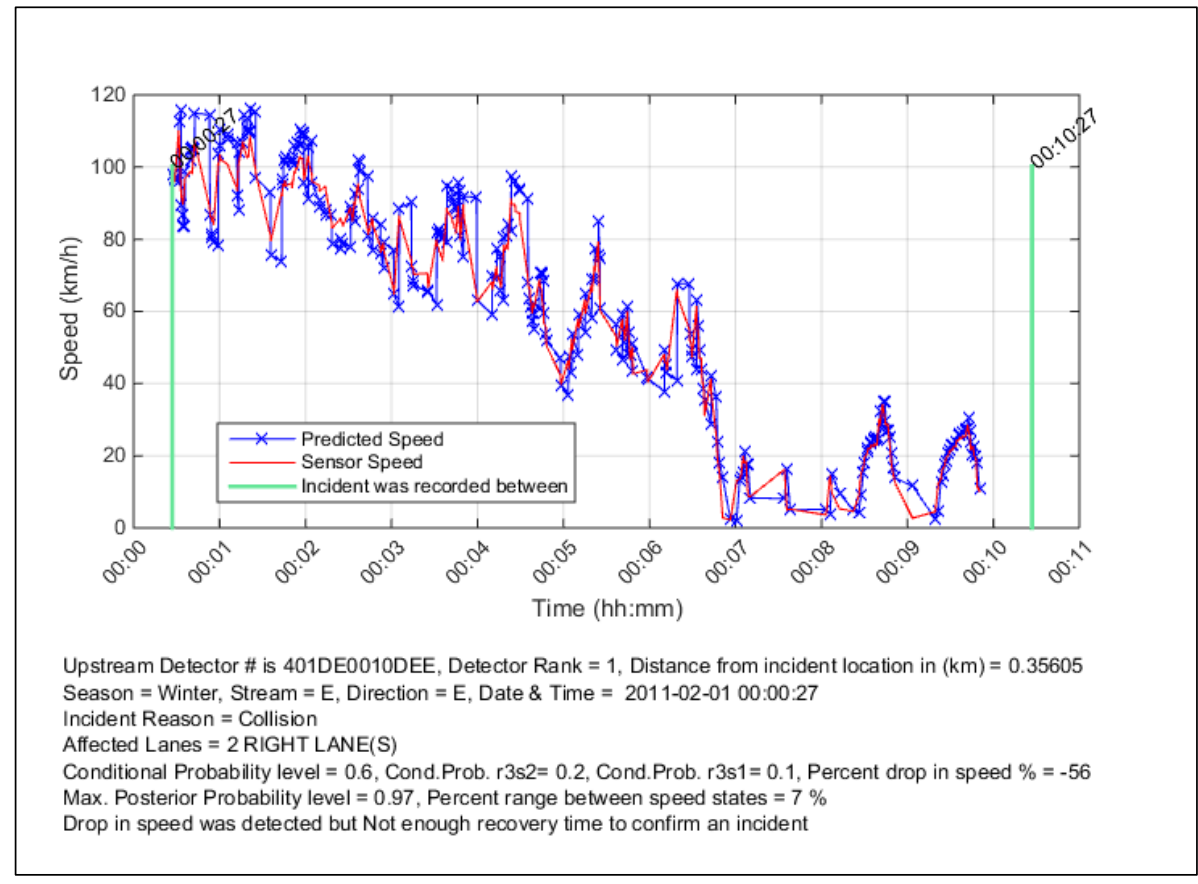

Incident Detection Utilizing the Algorithm, Data obtained from Right Lane \#1, Run3 


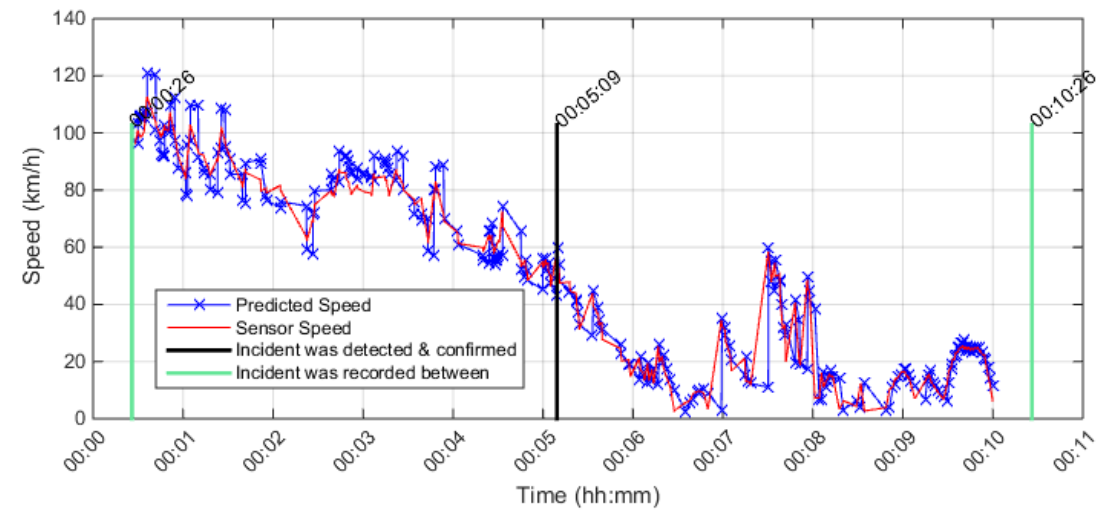

Upstream Detector \# is 401DE0010DEE, Detector Rank $=1$, Distance from incident location in $(\mathrm{km})=0.35605$ Season $=$ Winter, Stream $=E$, Direction $=E$, Date $\&$ Time $=2011-02-0100: 00: 26$ Incident Reason $=$ Collision

Affected Lanes = 2 RIGHT LANE(S)

Conditional Probability level $=0.6$, Cond.Prob. $r 3 s 2=0.2$, Cond.Prob. $r 3 s 1=0.1$, Percent drop in speed $\%=-56$

Max. Posterior Probability level $=0.97$, Percent range between speed states $=7 \%$

Incident was detected at 01:15 minutes from drop in speed point. Incident was confirmed after 5 minutes from the detection time

\section{Incident Detection Utilizing the Algorithm, Data obtained from Right Lane \#1, Run-} 4

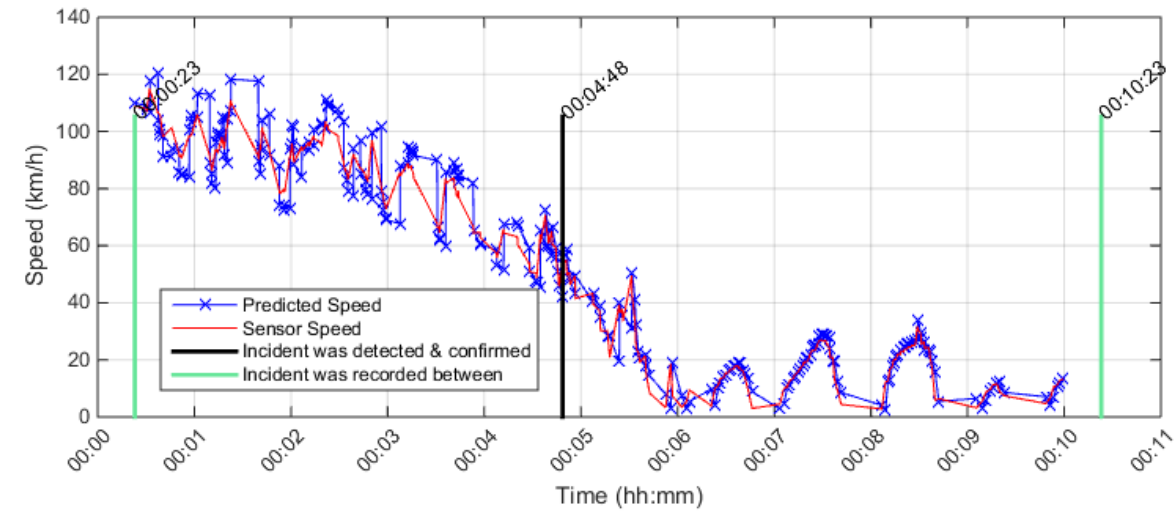

Upstream Detector \# is 401DE0010DEE, Detector Rank = 1, Distance from incident location in $(\mathrm{km})=0.35605$ Season $=$ Winter, Stream $=E$, Direction $=E$, Date $\&$ Time $=2011-02-0100: 00: 23$ Incident Reason $=$ Collision

Affected Lanes $=2$ RIGHT LANE(S)

Conditional Probability level $=0.6$, Cond.Prob. $r 3 \mathrm{~s} 2=0.2$, Cond.Prob. $r 3 \mathrm{~s} 1=0.1$, Percent drop in speed $\%=-56$

Max. Posterior Probability level $=0.97$, Percent range between speed states $=7 \%$

Incident was detected at 00:55 minutes from drop in speed point. Incident was confirmed after 5 minutes from the detection time

\section{Incident Detection Utilizing the Algorithm, Data obtained from Right Lane \#1, Run-}




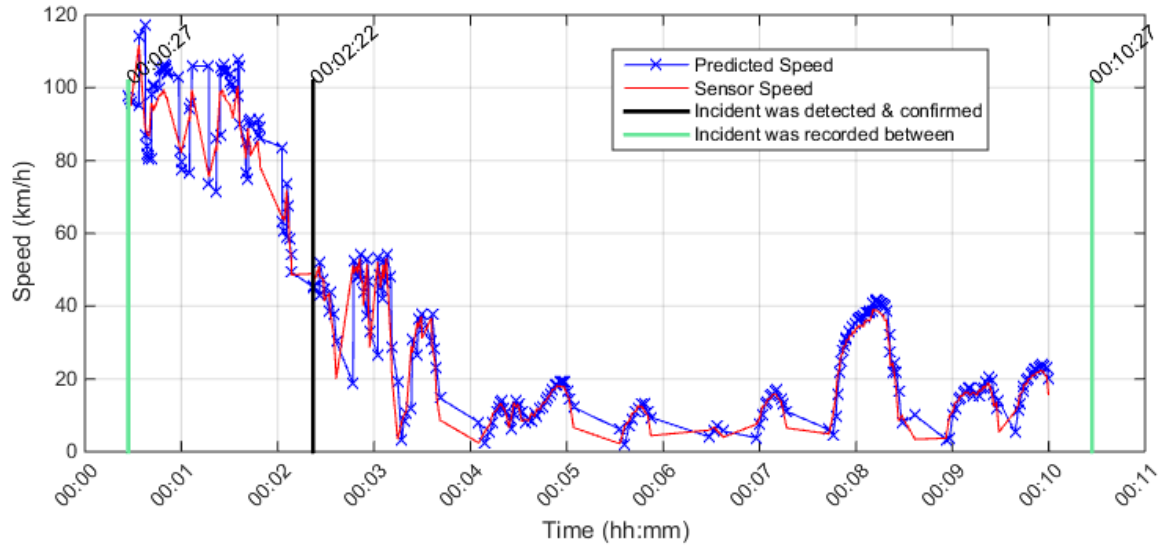

Upstream Detector \# is 401DE0010DEE, Detector Rank = 1, Distance from incident location in $(\mathrm{km})=0.35605$

Season $=$ Winter, Stream $=E$, Direction $=E$, Date $\&$ Time $=2011-02-0100: 00: 27$

Incident Reason $=$ Collision

Affected Lanes = 2 RIGHT LANE (S)

Conditional Probability level $=0.6$, Cond.Prob. $r 3 s 2=0.2$, Cond.Prob. $r 3 s 1=0.1$, Percent drop in speed $\%=-56$

Max. Posterior Probability level $=0.97$, Percent range between speed states $=7 \%$

Incident was detected at 00:19 minutes from drop in speed point. Incident was confirmed after 5 minutes from the detection time

Incident Detection Utilizing the Algorithm, Data obtained from Right Lane \#1, Run6

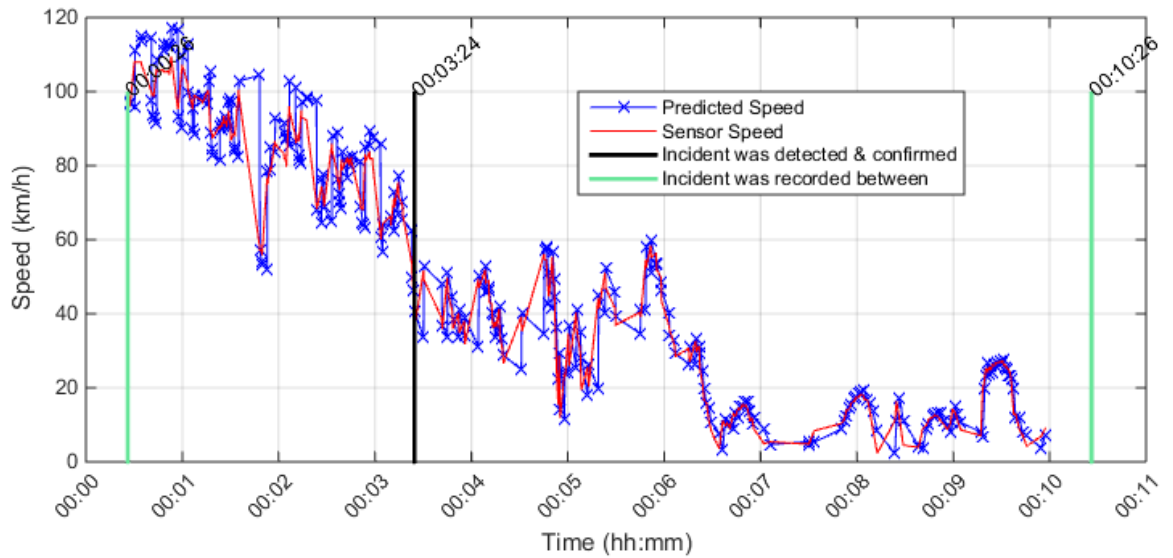

Upstream Detector \# is 401DE0010DEE, Detector Rank = 1, Distance from incident location in $(\mathrm{km})=0.35605$

Season $=$ Winter, Stream $=E$, Direction $=E$, Date $\&$ Time $=2011-02-0100: 00: 26$

Incident Reason $=$ Collision

Affected Lanes $=2$ RIGHT LANE (S)

Conditional Probability level $=0.6$, Cons. Prob. $r 3 s 2=0.2$, Conc. Prob. $r 3 s 1=0.1$, Percent drop in speed $\%=-56$

Max. Posterior Probability level $=0.97$, Percent range between speed states $=7 \%$

Incident was detected at 00:10 minutes from drop in speed point. Incident was confirmed after 5 minutes from the detection time

Incident Detection Utilizing the Algorithm, Data obtained from Right Lane \#1, Run7

593 


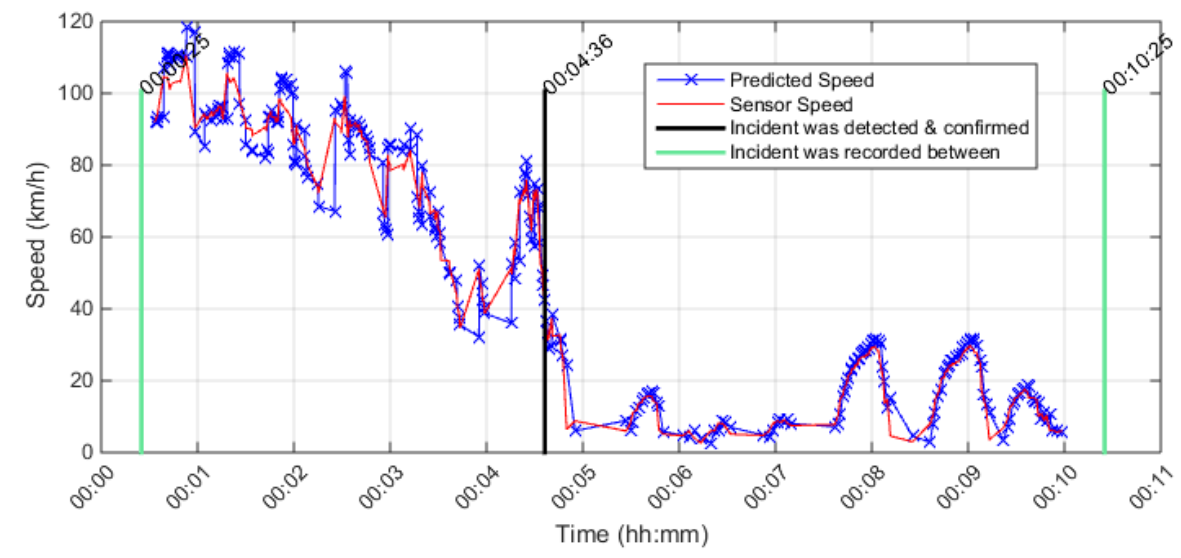

Upstream Detector \# is 401DE0010DEE, Detector Rank $=1$, Distance from incident location in $(\mathrm{km})=0.35605$ Season $=$ Winter, Stream $=E$, Direction $=E$, Date \& Time $=$ 2011-02-01 00:00:25 Incident Reason $=$ Collision

Affected Lanes $=2$ RIGHT LANE(S)

Conditional Probability level $=0.6$, Cond. Prob. $r 3 s 2=0.2$, Cond.Prob. $r 3 s 1=0.1$, Percent drop in speed $\%=-56$

Max. Posterior Probability level $=0.97$, Percent range between speed states $=7 \%$

Incident was detected at 00:11 minutes from drop in speed point. Incident was confirmed after 5 minutes from the detection time

\section{Incident Detection Utilizing the Algorithm, Data obtained from Right Lane \#1, Run-} 8

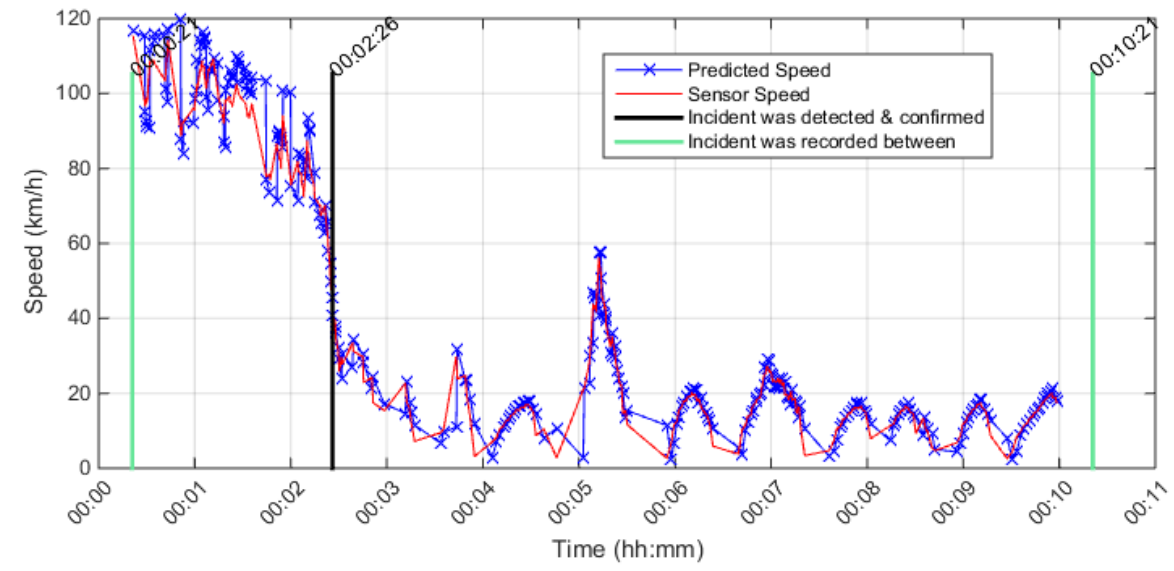

Upstream Detector \# is 401DE0010DEE, Detector Rank $=1$, Distance from incident location in $(\mathrm{km})=0.35605$ Season $=$ Winter, Stream $=E$, Direction $=E$, Date \& Time $=2011-02-0100: 00: 21$ Incident Reason $=$ Collision Affected Lanes $=2$ RIGHT LANE(S)

Conditional Probability level $=0.6$, Cond.Prob. $r 3 s 2=0.2$, Cond.Prob. $r 3 s 1=0.1$, Percent drop in speed $\%=-56$ Max. Posterior Probability level $=0.97$, Percent range between speed states $=7 \%$

Incident was detected at 00:14 minutes from drop in speed point. Incident was confirmed after 5 minutes from the detection time

\section{Incident Detection Utilizing the Algorithm, Data obtained from Right Lane \#1, Run-} 9 


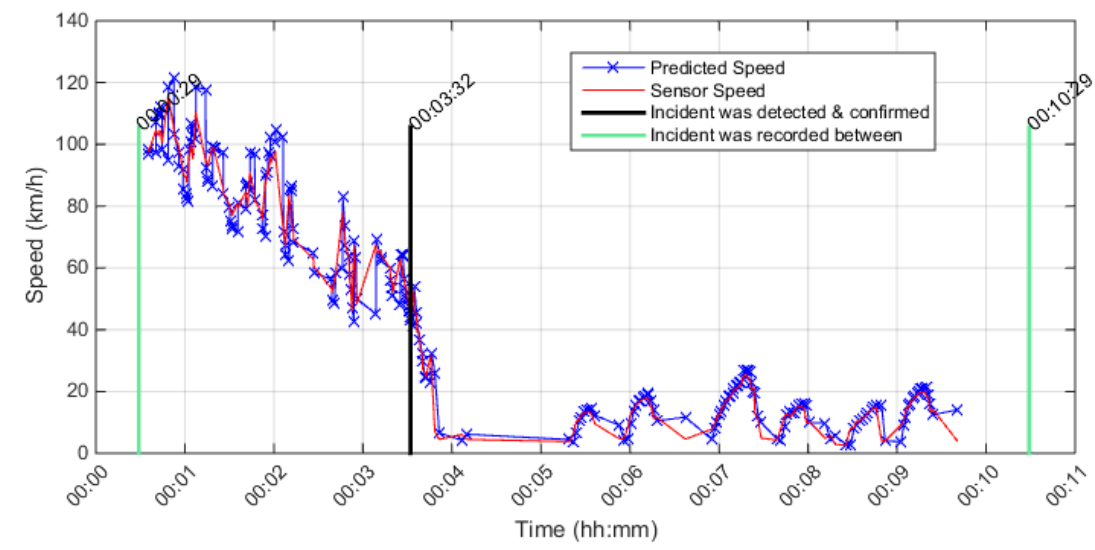

Upstream Detector \# is 401DE0010DEE, Detector Rank $=1$, Distance from incident location in $(\mathrm{km})=0.35605$ Season $=$ Winter, Stream $=E$, Direction $=E$, Date \& Time $=$ 2011-02-01 00:00:29 Incident Reason $=$ Collision

Affected Lanes = 2RIGHT LANE(S)

Conditional Probability level $=0.6$, Cond.Prob. $r 3 s 2=0.2$, Cond. Prob. $r 3 s 1=0.1$, Percent drop in speed $\%=-56$

Max. Posterior Probability level $=0.97$, Percent range between speed states $=7 \%$

Incident was detected at 00:45 minutes from drop in speed point. Incident was confirmed after 5 minutes from the detection time

Incident Detection Utilizing the Algorithm, Data obtained from Right Lane \#1, Run10

\section{Incident Detection from Lane \#2, two-lane blockage, and data was collected from the first lane, traffic volume level of $1250 \mathrm{veh} / \mathrm{l} / \mathrm{h}$}

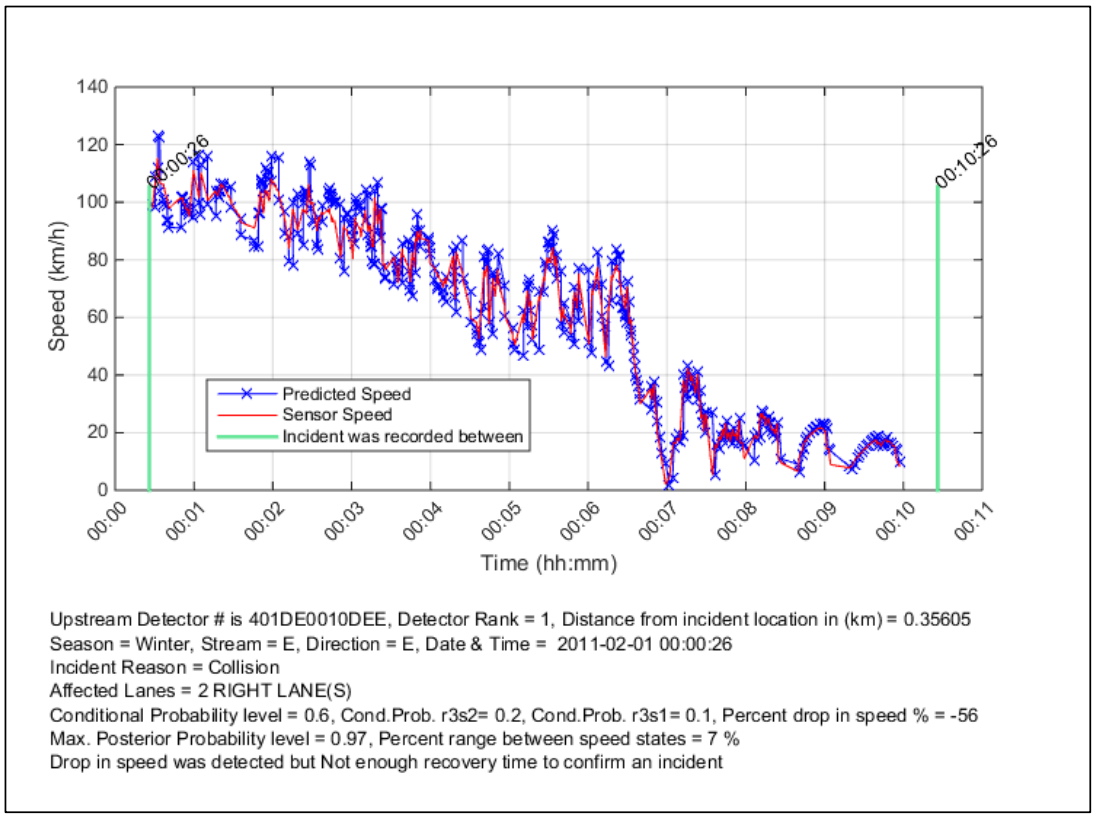

Incident Detection Utilizing the Algorithm, Data obtained from Right Lane \#2, Run- 


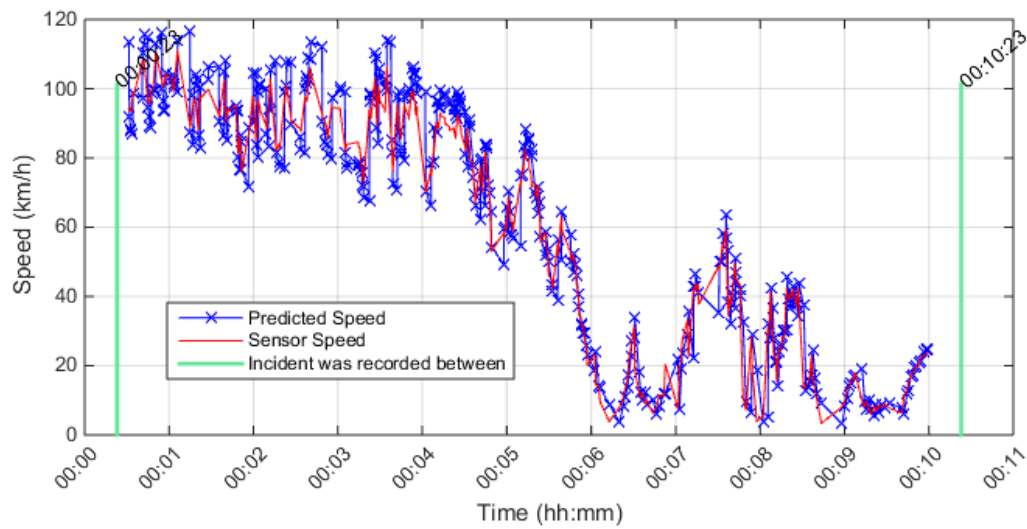

Upstream Detector \# is 401DE0010DEE, Detector Rank = 1, Distance from incident location in $(\mathrm{km})=0.35605$ Season $=$ Winter, Stream $=E$, Direction $=E$, Date \& Time $=2011-02-0100: 00: 23$ Incident Reason $=$ Collision

Affected Lanes = 2 RIGHT LANE(S)

Conditional Probability level $=0.6$, Cond. Prob. $r 3 s 2=0.2$, Cond. Prob. $r 3 s 1=0.1$, Percent drop in speed $\%=-56$

Max. Posterior Probability level $=0.97$, Percent range between speed states $=7 \%$

Drop in speed was detected but Not enough recovery time to confirm an incident

\section{Incident Detection Utilizing the Algorithm, Data obtained from Right Lane \#2, Run-}

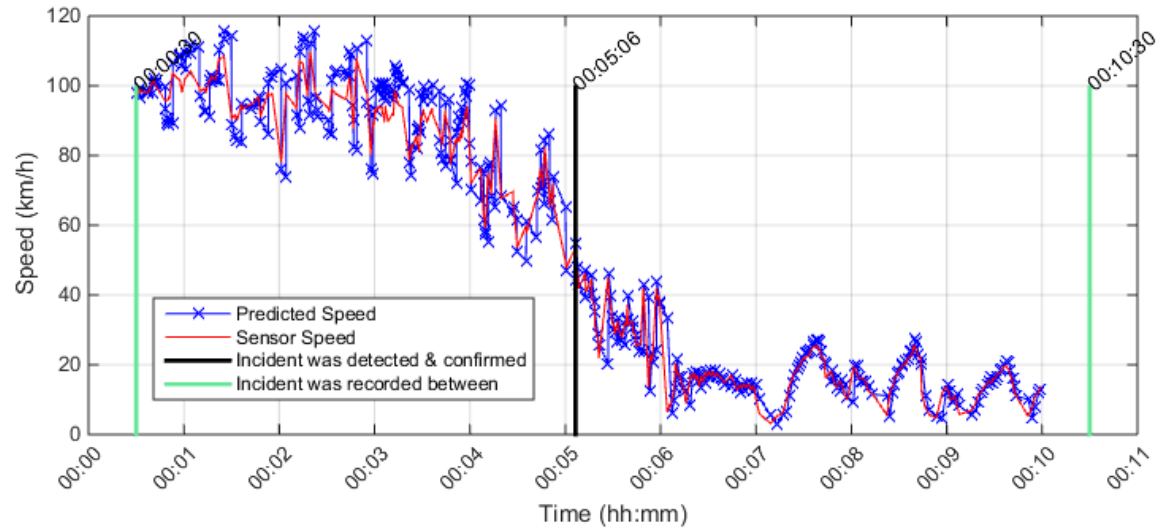

Upstream Detector \# is 401DE0010DEE, Detector Rank = 1, Distance from incident location in $(\mathrm{km})=0.35605$ Season $=$ Winter, Stream $=E$, Direction $=E$, Date $\&$ Time $=2011-02-0100: 00: 30$ Incident Reason $=$ Collision

Affected Lanes $=2$ RIGHT LANE(S)

Conditional Probability level $=0.6$, Cond.Prob. $r 3 \mathrm{~s} 2=0.2$, Cond.Prob. $r 3 \mathrm{~s} 1=0.1$, Percent drop in speed $\%=-56$

Max. Posterior Probability level $=0.97$, Percent range between speed states $=7 \%$

Incident was detected at 00:16 minutes from drop in speed point. Incident was confirmed after 5 minutes from the detection time

Incident Detection Utilizing the Algorithm, Data obtained from Right Lane \#2, Run5 


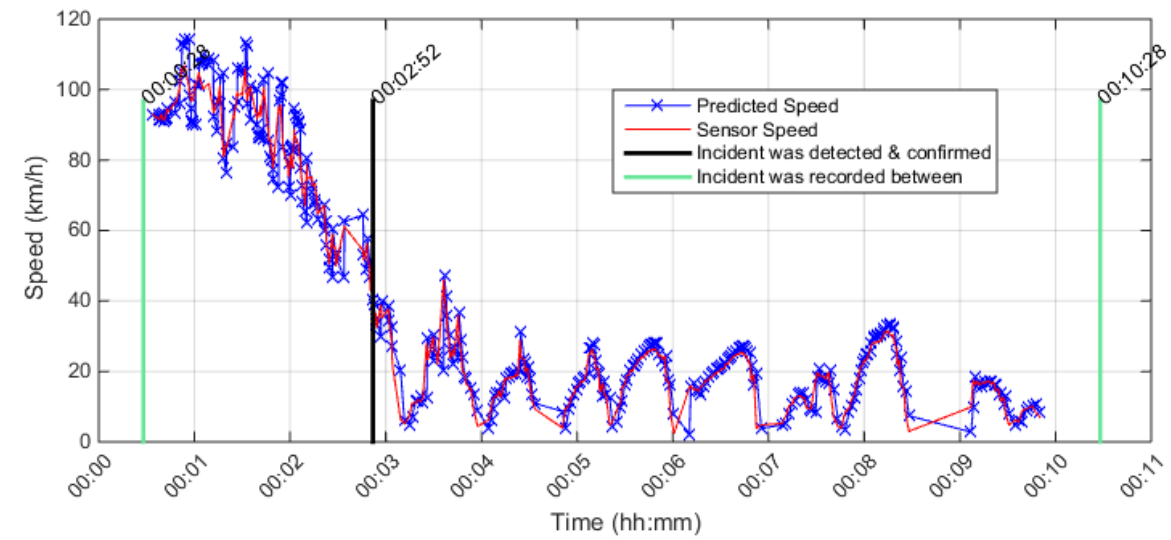

Upstream Detector \# is 401DE0010DEE, Detector Rank = 1, Distance from incident location in $(\mathrm{km})=0.35605$ Season $=$ Winter, Stream $=E$, Direction $=E$, Date \& Time $=2011-02-0100: 00: 28$ Incident Reason $=$ Collision

Affected Lanes $=2$ RIGHT LANE(S)

Conditional Probability level $=0.6$, Cond.Prob. $r 3 s 2=0.2$, Cond. Prob. $r 3 s 1=0.1$, Percent drop in speed $\%=-56$

Max. Posterior Probability level $=0.97$. Percent range between speed states $=7 \%$

Incident was detected at 00:41 minutes from drop in speed point. Incident was confirmed after 5 minutes from the detection time

\section{Incident Detection Utilizing the Algorithm, Data obtained from Right Lane \#2, Run-}

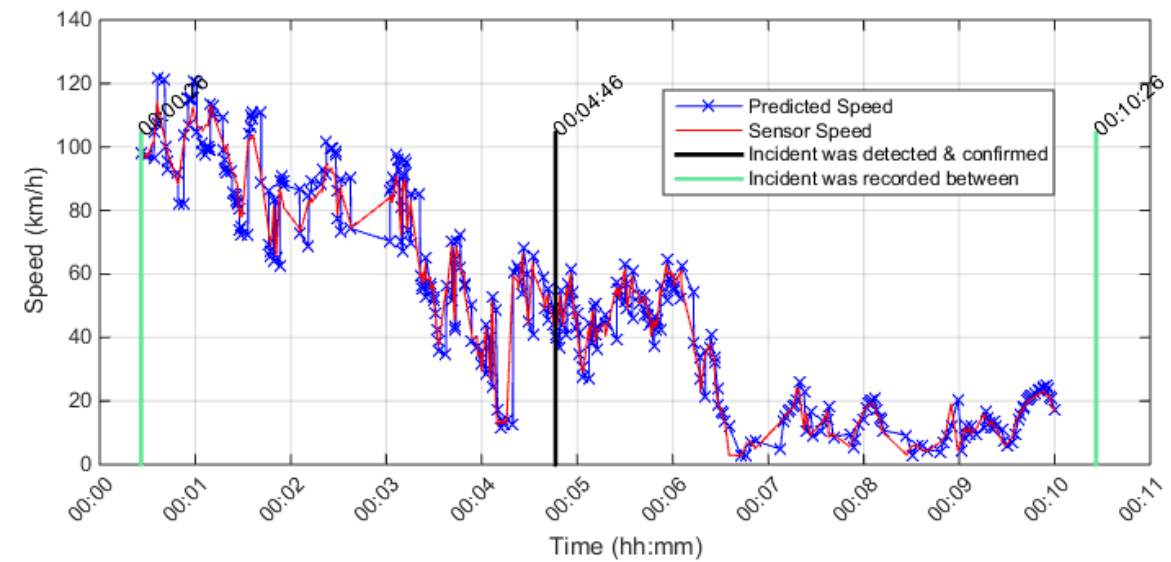

Upstream Detector \# is 401DE0010DEE, Detector Rank = 1, Distance from incident location in $(\mathrm{km})=0.35605$ Season $=$ Winter, Stream $=E$, Direction $=E$, Date $\&$ Time $=2011-02-0100: 00: 26$ Incident Reason $=$ Collision

Affected Lanes = 2RIGHT LANE(S)

Conditional Probability level $=0.6$, Cond.Prob. $r 3 s 2=0.2$, Cond.Prob. $r 3 s 1=0.1$, Percent drop in speed $\%=-56$ Max. Posterior Probability level $=0.97$, Percent range between speed states $=7 \%$

Incident was detected at 01:25 minutes from drop in speed point. Incident was confirmed after 5 minutes from the detection time

Incident Detection Utilizing the Algorithm, Data obtained from Right Lane \#2, Run7 


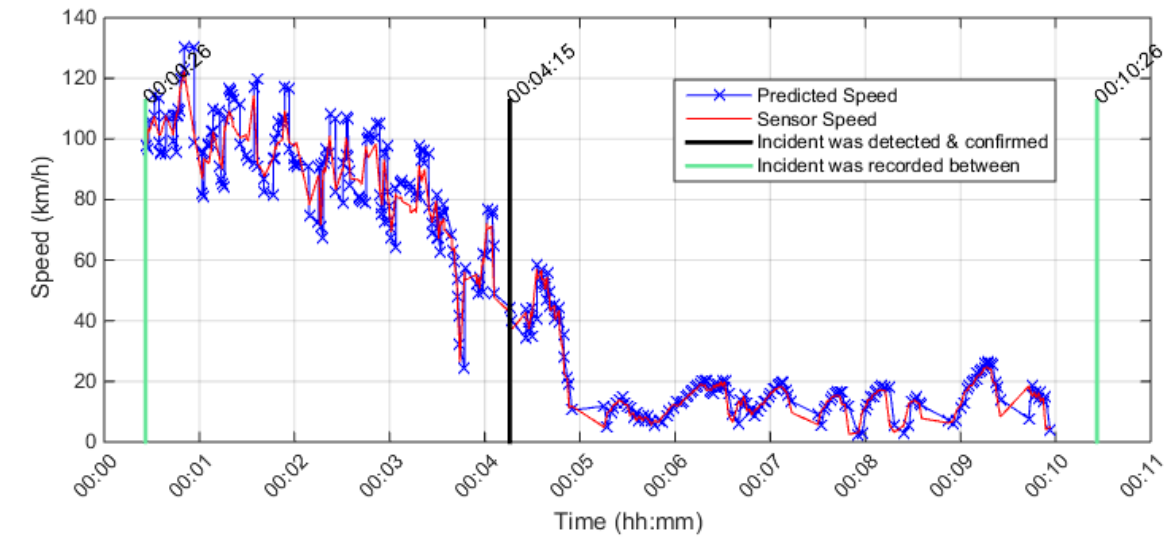

Upstream Detector \# is 401DE0010DEE, Detector Rank $=1$, Distance from incident location in $(\mathrm{km})=0.35605$ Season $=$ Winter, Stream $=E$, Direction $=E$, Date $\&$ Time $=2011-02-0100: 00: 26$

Incident Reason $=$ Collision

Affected Lanes = 2 RIGHT LANE(S)

Conditional Probability level $=0.6$, Cond.Prob. $r 3 \mathrm{~s} 2=0.2$, Cond.Prob. $r 3 \mathrm{~s} 1=0.1$, Percent drop in speed $\%=-56$

Max. Posterior Probability level $=0.97$, Percent range between speed states $=7 \%$

Incident was detected at 00:46 minutes from drop in speed point. Incident was confirmed after 5 minutes from the detection time

\section{Incident Detection Utilizing the Algorithm, Data obtained from Right Lane \#2, Run-}

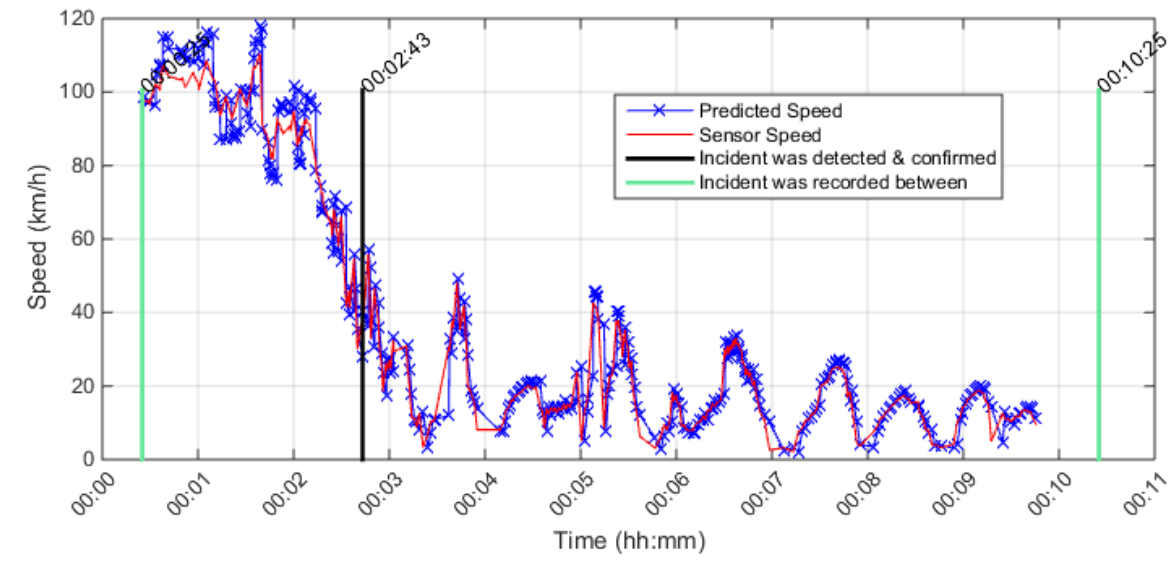

Upstream Detector \# is 401DE0010DEE, Detector Rank = 1, Distance from incident location in $(\mathrm{km})=0.35605$ Season $=$ Winter, Stream $=E$, Direction $=E$, Date \& Time $=$ 2011-02-01 00:00:25 Incident Reason $=$ Collision

Affected Lanes = 2 RIGHT LANE(S)

Conditional Probability level $=0.6$, Cond.Prob. $r 3 s 2=0.2$, Cond. Prob. $r 3 s 1=0.1$, Percent drop in speed $\%=-56$

Max. Posterior Probability level $=0.97$, Percent range between speed states $=7 \%$

Incident was detected at 00:29 minutes from drop in speed point. Incident was confirmed after 5 minutes from the detection time

Incident Detection Utilizing the Algorithm, Data obtained from Right Lane \#2, Run- 


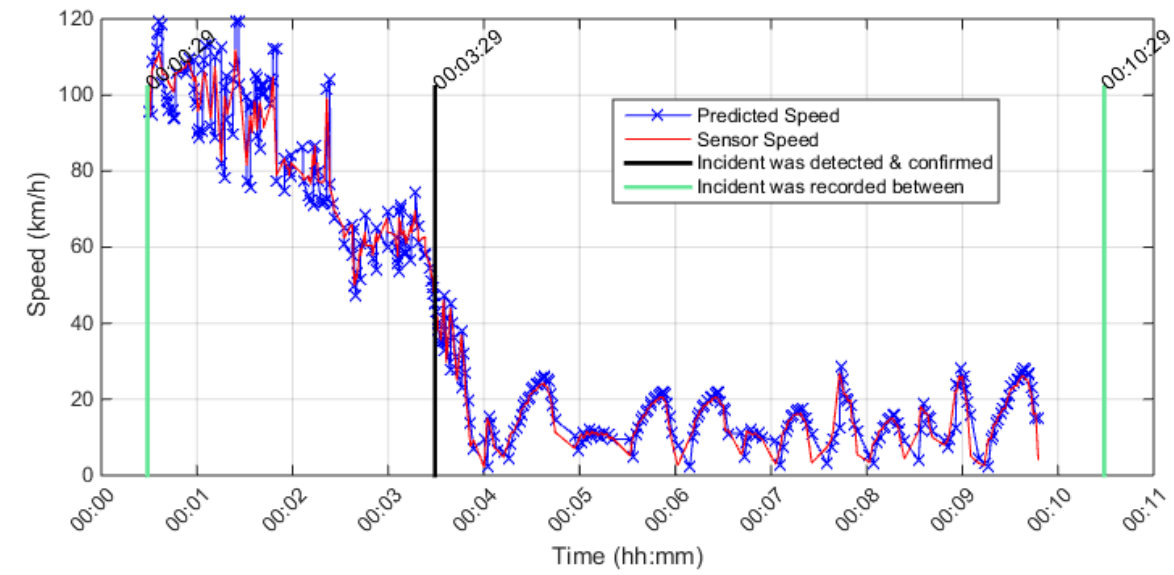

Upstream Detector \# is 401DE0010DEE, Detector Rank = 1, Distance from incident location in $(\mathrm{km})=0.35605$ Season $=$ Winter, Stream $=E$, Direction $=E$, Date $\&$ Time $=2011-02-0100: 00: 29$ Incident Reason $=$ Collision

Affected Lanes $=2$ RIGHT LANE(S)

Conditional Probability level $=0.6$, Cond.Prob. $r 3 \mathrm{~s} 2=0.2$, Cond.Prob. $r 3 \mathrm{~s} 1=0.1$, Percent drop in speed $\%=-56$

Max. Posterior Probability level $=0.97$, Percent range between speed states $=7 \%$

Incident was detected at 01:05 minutes from drop in speed point. Incident was confirmed after 5 minutes from the detection time

\section{Incident Detection Utilizing the Algorithm, Data obtained from Right Lane \#2, Run-} 10 